\title{
Complementarities in the Implementation of Advanced Manufacturing Technologies
}

\author{
by \\ Jennifer Percival
}

\begin{abstract}
A thesis
presented to the University of Waterloo

in fulfillment of the

thesis requirement for the degree of

Doctor of Philosophy

in

Management Sciences
\end{abstract}

Waterloo, Ontario, Canada, 2004

(C) Jennifer Percival 2004 
I hereby declare that I am the sole author of this thesis. This is a true copy of the thesis, including any required final revisions, as accepted by my examiners.

I understand that my thesis may be made electronically available to the public. 


\begin{abstract}
Within the last decade, the importance of flexibility and efficiency has increased in the manufacturing sector. The rising level of uncertainty in consumer preferences has caused many organizations to aggressively search for cost reductions and other sources of competitive advantage. This fact has resulted in an increased implementation of advanced manufacturing technologies (AMT). A number of studies propose that the implementation of AMT must be accompanied by a shift in supporting organizational practices to realize the greatest performance enhancement. As yet, the complementarities between organizational policies and AMT have not been determined.
\end{abstract}

Using assumptions about complementarities in manufacturing made by Milgrom and Roberts (1995) in conjunction with a comprehensive AMT survey (Survey of Advanced Technology in Canadian Manufacturing-1998) a model of manufacturing plant productivity was developed. Constrained regression analysis reveals that the use of AMT, as well as various organizational policies, depends both on the size of the plant as well as the industry in which it operates.

Factor analysis of the over 70 variables found that regardless of the nature of the variable (business strategy, source of implementation support, AMT, etc.), all design elements factored together. The factor analysis also shows that large firms who use AMT also have many design technologies. This result differs for smaller firms where the use of AMT is highly correlated with perceived benefits of the technology and a large number of sources of implementation support. The analysis also supports the distinction of high technology (highly innovative) industries and low technology (low levels of innovation) industries since electronics, chemicals and automotive 
have a large percentage of plants with all of the model factors whereas the textile, non-metal and lumber industries have very few plants with all of the model factors. The results show that there are important differences that should be considered when creating policies to encourage innovation and the use of AMT for the various manufacturing industries and plant sizes. 


\section{Acknowledgements}

I would like to extend my deepest appreciation to Professor B.P. Cozzarin, the supervisor, for his insightful guidance in developing this thesis, and his encouragement throughout my studies. I would also like to thank Professors David Fuller, Raymond Vickson, Rod McNaughton, Greg Bennett, and Pierre Mohnen for their thought provoking comments, challenging questions and valuable suggestions.

I would also like to thank the members of the Science, Innovation, and Electronic Information Division of Statistics Canada for their help in obtaining the data required to apply the complementarity methodology to Canadian manufacturing plants and for the Statistics Canada Research Fellowship. Further, acknowledgement is due to the Natural Sciences and Engineering Research Council of Canada for their PGS B scholarship support and the Social

Sciences and Humanities Research Council of Canada, Standard Research Grant "Advanced Manufacturing Technologies and Organizational Complementarity.”

Finally, my deepest love and gratitude go to my husband, Nathan, for his constant support, understanding, and patience. Without his encouragement and love, I could never have completed my research. 


\section{Table of Contents}

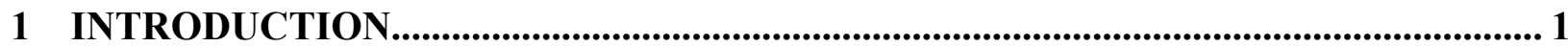

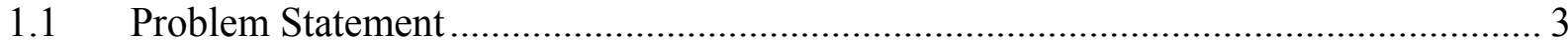

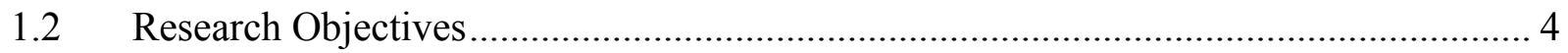

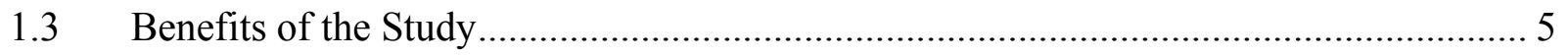

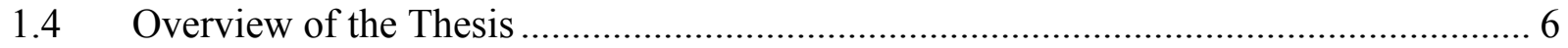

2 LITERATURE REVIEW ON ADVANCED MANUFACTURING TECHNOLOGIES 8

2.1 Organizational Factors Critical for Success................................................................ 9

2.2 Market Structure and Competitive Advantage ………………………………............ 11

3 LITERATURE REVIEW ON COMPLEMENTARITY .............................................. 13

3.1 Applications of Complementarity Analysis............................................................ 16

3.1.1 Implication for Competitive Advantage Strategies................................................ 17

3.1.2 Complementarities in the Implementation of Information Technology ................... 19

3.1.3 Interaction Between Human Resource Policies and Productivity ............................ 20

3.1.4 Co-ordination of Innovation Policies.................................................................... 22

3.1.5 Complementarity Between Advanced Manufacturing Technologies ....................... 24

3.2 An Overview of Basic Lattice Theory ..................................................................... 26

3.2.1 The Basic Concepts of a Lattice …………………................................................ 27

3.2.2 The Basic Solution Strategies for Optimization on a Lattice ……........................ 30

3.2.3 The Basic Comparative Statics Results ................................................................ 31

3.3 Techniques to Determine Complementarity ………….............................................. 32

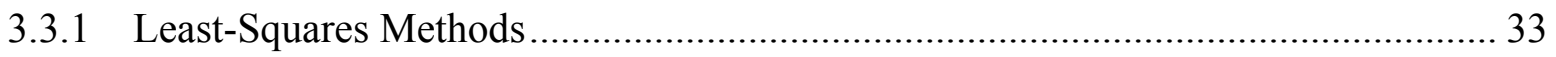

3.3.2 Parametric Method............................................................................................. 35

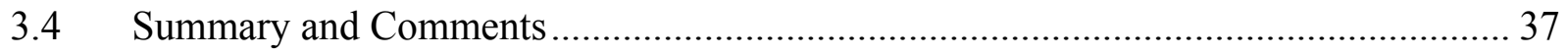

4 RESEARCH DESIGN ................................................................................................................. 39

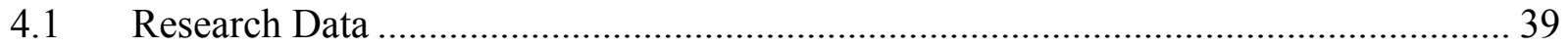

4.1.1 Survey of Advanced Technology in Canadian Manufacturing.................................. 39

4.1.2 Annual Survey of Advanced Manufactures............................................................. 40 


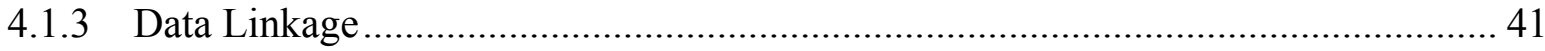

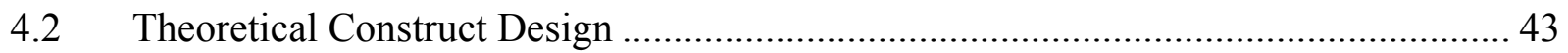

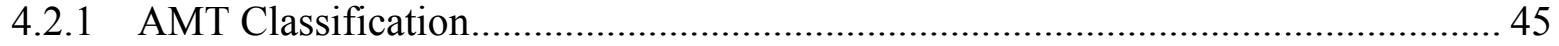

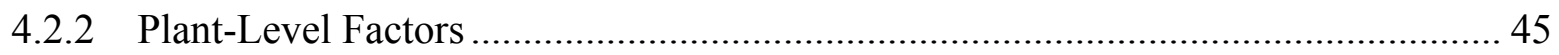

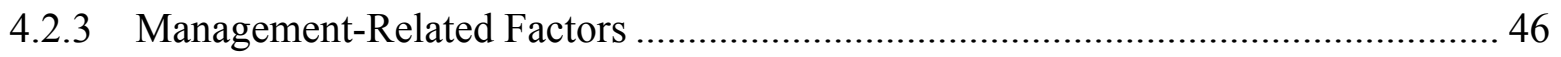

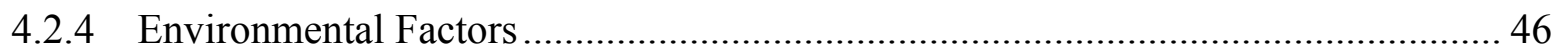

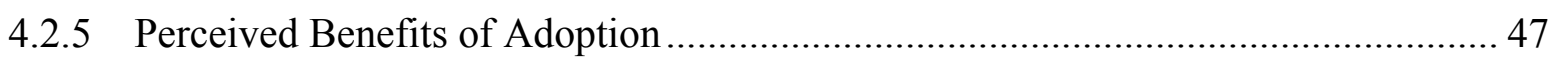

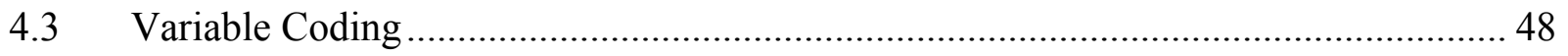

4.4 Factor Analysis Results for Variable Reduction.......................................................... 49

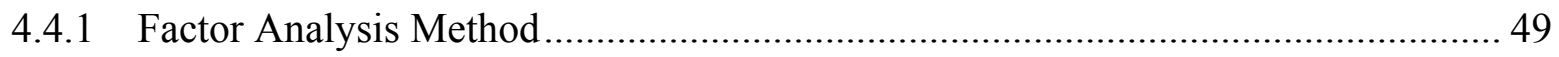

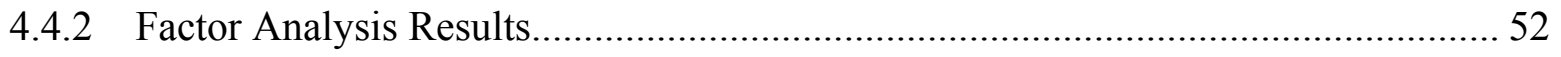

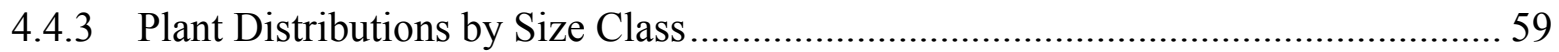

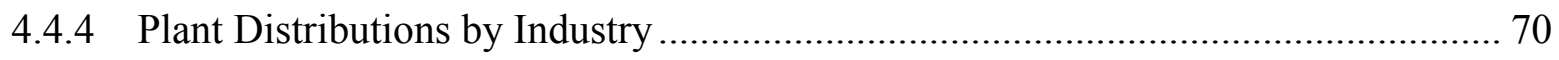

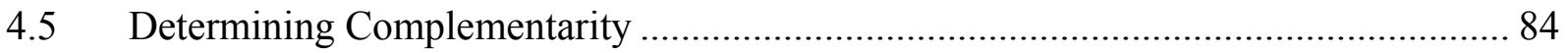

4.6 Finding the Optimal Set of Organizational Policies ..................................................... 88

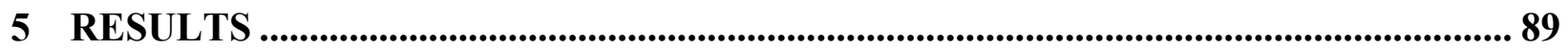

5.1 Constrained Regression Model ............................................................................. 90

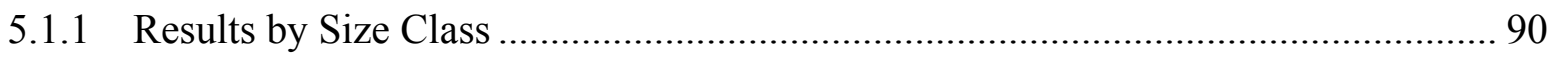

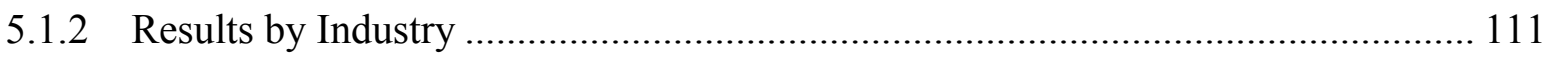

$5.2 \quad$ Ordinary Least Squares Model ................................................................................. 147

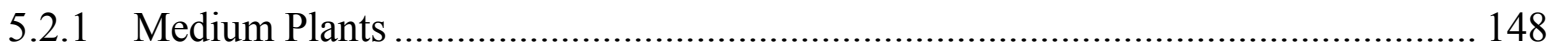

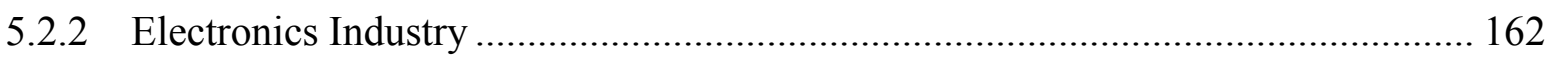

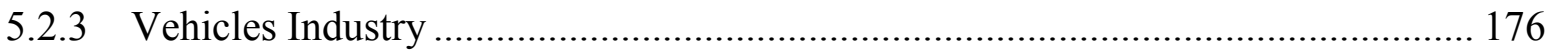

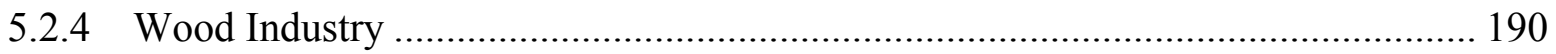

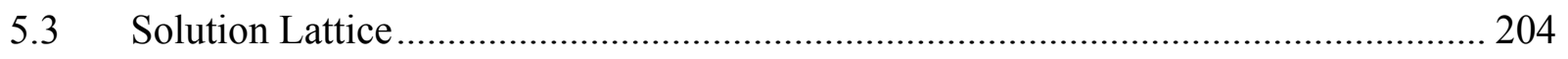

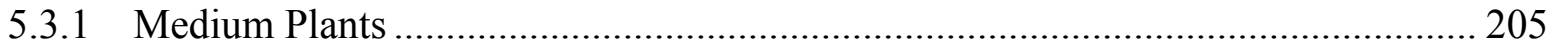

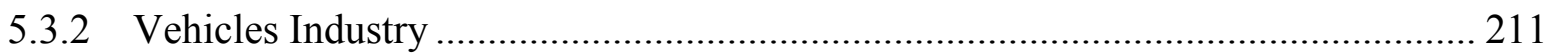

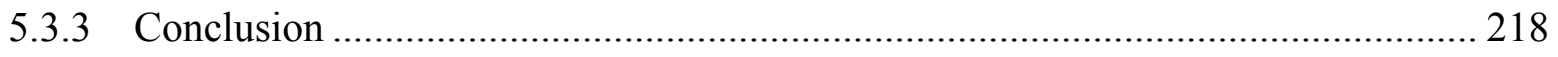


6 CONCLUSION AND FUTURE RESEARCH ................................................................ 219

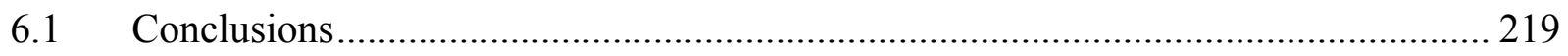

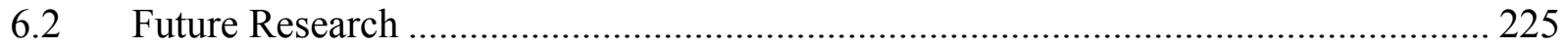

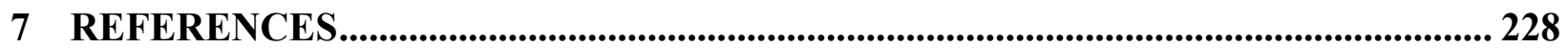

APPENDIX A - DEFINITION OF ADVANCED TECHNOLOGIES................................... 234

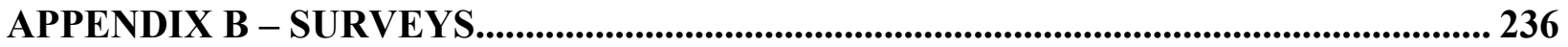

B1 Survey of Advanced Technology in Canadian Manufacturing - 1998 ............................. 236

B2 Annual Survey of Manufactures ........................................................................... 248

APPENDIX C - INDUSTRY STRATIFICATION ..................................................................... 256

APPENDIX D - ADVANCED TECHNOLOGY USE ........................................................... 257

APPENDIX E - SIGNIFICANCE VALUES ............................................................................ 259

APPENDIX F - EXAMPLE SAS CODE ........................................................................... 262

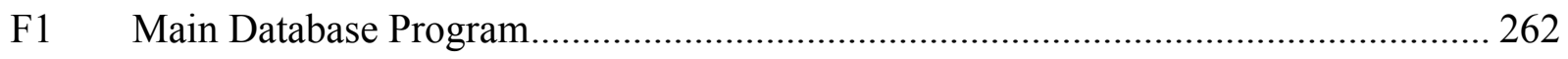

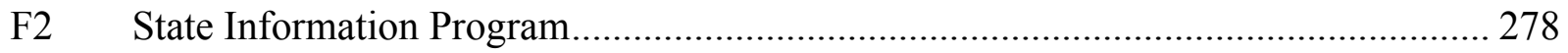

F3 Business Practices Constrained Regression - Supermodularity Constraints.............. 280

F4 AMT Constrained Regression - Submodularity Constraints...................................... 284

F5 Sources of Implementation Support OLS Regression - Medium Plants ..................... 302

F6 Perceived Benefits of AMT OLS Regression - Wood Industry ................................... 305 


\section{List of Tables}

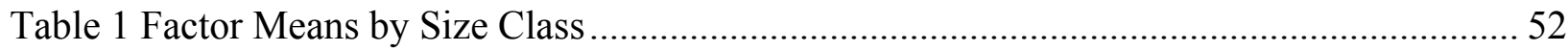

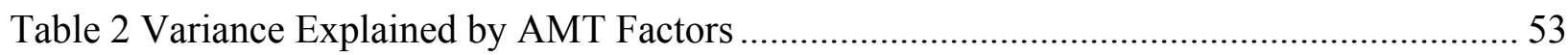

Table 3 Variance Explained by the Business Practices Factors ……………………………...... 54

Table 4 Variance Explained by the Sources of Implementation Support Factors ......................... 55

Table 5 Variance Explained by the Perceived Benefits Factors ..................................................... 57

Table 6 Variance Explained by the High-Level Factors............................................................ 58

Table 7 Plant Distributions for Advanced Manufacturing Technologies by Size Class................ 60

Table 8 Plant Distributions for Business Practices by Size Class ................................................ 61

Table 9 Plant Distributions for Human Resources Practices by Size Class ................................... 62

Table 10 Plant Distributions for Research and Development Practices by Size Class.................. 63

Table 11 Plant Distributions for Sources of Implementation Support by Size Class .................... 65

Table 12 Plant Distributions for Perceived Benefits of AMT by Size Class................................ 66

Table 13 Plant Distributions for High-Level Factors by Size Class ............................................... 68

Table 14 Plant Distributions for Advanced Manufacturing Technologies by Industry................. 71

Table 15 Plant Distributions for Business Practices by Industry.............................................. 74

Table 16 Plant Distributions for Human Resources Practices by Industry .................................... 75

Table 17 Plant Distributions for Research and Development Practices by Industry ..................... 76

Table 18 Plant Distributions for Sources of Implementation Support by Industry ...................... 78

Table 19 Plant Distributions for Perceived Benefits of AMT by Industry .................................... 80

Table 20 Plant Distributions for High-Level Factors by Industry .............................................. 83

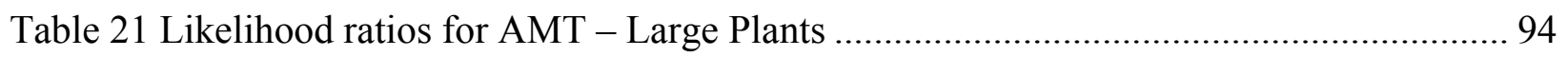

Table 22 Likelihood ratios for AMT - Medium Plants .................................................................... 94

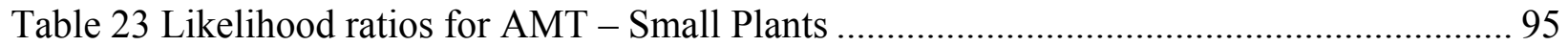

Table 24 Likelihood ratios for Business Practices - Large Plants.................................................. 97

Table 25 Likelihood ratios for Business Practices - Medium Plants ............................................. 97

Table 26 Likelihood ratios for Business Practices - Small Plants.................................................. 97

Table 27 Likelihood ratios for Implementation Support - Large Plants ..................................... 100

Table 28 Likelihood ratios for Implementation Support - Medium Plants ................................ 100

Table 29 Likelihood ratios for Implementation Support - Small Plants ...................................... 100

Table 30 Likelihood ratios for Perceived Benefits of AMT - Large Plants ................................ 103

Table 31 Likelihood ratios for Perceived Benefits of AMT - Medium Plants............................ 103 
Table 32 Likelihood ratios for Perceived Benefits of AMT - Small Plants 104

Table 33 Likelihood ratios for High-Level Factors - Large Plants ....................................... 106

Table 34 Likelihood ratios for High-Level Factors - Medium Plants.................................... 107

Table 35 Likelihood ratios for High-Level Factors - Small Plants ...................................... 107

Table 36 Likelihood ratios for AMT - Chemical Industry ................................................ 116

Table 37 Likelihood ratios for AMT - Electronics Industry ............................................ 116

Table 38 Likelihood ratios for AMT - Furniture and Other Industries ................................ 116

Table 39 Likelihood ratios for AMT - Machinery Industry ................................................. 116

Table 40 Likelihood ratios for AMT - Metals Industry .................................................... 117

Table 41 Likelihood ratios for AMT - Non-Metals Industry ............................................ 117

Table 42 Likelihood ratios for AMT - Plastics Industry ................................................... 117

Table 43 Likelihood ratios for AMT - Textiles Industry ................................................. 117

Table 44 Likelihood ratios for AMT - Vehicles Industry .................................................. 118

Table 45 Likelihood ratios for AMT - Wood Industry .................................................. 118

Table 46 Likelihood ratios for Business Practices - Chemical Industry ................................. 121

Table 47 Likelihood ratios for Business Practices - Electronics Industry ............................. 121

Table 48 Likelihood ratios for Business Practices - Furniture and Other Industries ............... 121

Table 49 Likelihood ratios for Business Practices - Machinery Industry ................................ 122

Table 50 Likelihood ratios for Business Practices - Metals Industry ................................... 122

Table 51 Likelihood ratios for Business Practices - Non-Metals Industry ............................ 122

Table 52 Likelihood ratios for Business Practices - Plastics Industry .................................. 122

Table 53 Likelihood ratios for Business Practices - Textiles Industry ................................ 123

Table 54 Likelihood ratios for Business Practices - Vehicles Industry ................................ 123

Table 55 Likelihood ratios for Business Practices - Wood Industry..................................... 123

Table 56 Likelihood ratios for Implementation Support - Chemical Industry........................ 127

Table 57 Likelihood ratios for Implementation Support - Electronics Industry ..................... 127

Table 58 Likelihood ratios for Implementation Support - Furniture and Other Industries....... 127

Table 59 Likelihood ratios for Implementation Support - Machinery Industry...................... 127

Table 60 Likelihood ratios for Implementation Support - Metals Industry ........................... 128

Table 61 Likelihood ratios for Implementation Support - Non-Metals Industry.................... 128

Table 62 Likelihood ratios for Implementation Support - Plastics Industry.......................... 128

Table 63 Likelihood ratios for Implementation Support - Textiles Industry ......................... 128

Table 64 Likelihood ratios for Implementation Support - Vehicles Industry ......................... 129 
Table 65 Likelihood ratios for Implementation Support - Wood Industry 129

Table 66 Likelihood ratios for Perceived Benefits of AMT - Chemicals Industry ..................... 133

Table 67 Likelihood ratios for Perceived Benefits of AMT - Electronics Industry.................... 133

Table 68 Likelihood ratios for Perceived Benefits of AMT - Furniture and Other Industries .. 133

Table 69 Likelihood ratios for Perceived Benefits of AMT - Machinery Industry .................... 133

Table 70 Likelihood ratios for Perceived Benefits of AMT - Metals Industry ............................ 134

Table 71 Likelihood ratios for Perceived Benefits of AMT - Non-Metals Industry................... 134

Table 72 Likelihood ratios for Perceived Benefits of AMT - Plastics Industry ......................... 134

Table 73 Likelihood ratios for Perceived Benefits of AMT - Textiles Industry ......................... 134

Table 74 Likelihood ratios for Perceived Benefits of AMT - Vehicles Industry......................... 135

Table 75 Likelihood ratios for Perceived Benefits of AMT - Wood Industry ............................ 135

Table 76 Likelihood ratios for High-Level Factors - Chemicals Industry ................................... 141

Table 77 Likelihood ratios for High-Level Factors - Electronics Industry ................................. 141

Table 78 Likelihood ratios for High-Level Factors - Furniture and Other Industries................. 142

Table 79 Likelihood ratios for High-Level Factors - Machinery Industry ................................ 142

Table 80 Likelihood ratios for High-Level Factors - Metals Industry ........................................ 142

Table 81 Likelihood ratios for High-Level Factors - Non-Metals Industry ............................... 143

Table 82 Likelihood ratios for High-Level Factors - Plastics Industry...................................... 143

Table 83 Likelihood ratios for High-Level Factors - Textiles Industry ..................................... 143

Table 84 Likelihood ratios for High-Level Factors - Vehicles Industry ..................................... 144

Table 85 Likelihood ratios for High-Level Factors - Wood Industry ....................................... 144

Table 86 Coefficients with individual variables for AMT - Medium Plants .............................. 152

Table 87 Coefficients with individual variables for business practices - Medium Plants ......... 153

Table 88 Coefficients with individual variables for source of implementation support -

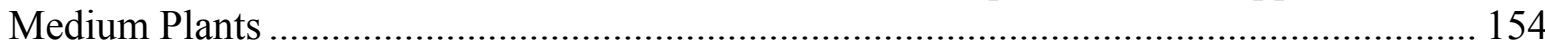

Table 89 Coefficients with individual variables for perceived benefits of AMT Medium Plants . 155

Table 90 Coefficients with individual variables for high-level factors - Medium Plants .......... 156

Table 91 Coefficients with individual variables for AMT - Electronics Industry ....................... 166

Table 92 Coefficients with individual variables for business practices - Electronics Industry . 167

Table 93 Coefficients with individual variables for source of implementation support -

Electronics Industry 168

Table 94 Coefficients with individual variables for perceived benefits of AMT -

Electronics Industry 
Table 95 Coefficients with individual variables for high-level factors - Electronics Industry .. 170

Table 96 Coefficients with individual variables for AMT - Vehicles Industry ....................... 180

Table 97 Coefficients with individual variables for business practices - Vehicles Industry ..... 181

Table 98 Coefficients with individual variables for source of implementation support Vehicles Industry

Table 99 Coefficients with individual variables for perceived benefits of AMT -

Vehicles Industry ..... 183

Table 100 Coefficients with individual variables for high-level factors - Vehicles Industry .... 184

Table 101 Coefficients with individual variables for AMT - Wood Industry ........................ 194

Table 102 Coefficients with individual variables for business practices - Wood Industry

Table 103 Coefficients with individual variables for source of implementation support Wood Industry 196

Table 104 Coefficients with individual variables for perceived benefits of AMT Wood Industry

Table 105 Coefficients with individual variables for high-level factors - Wood Industry ........ 198

Table 106 Hypotheses Tested from the Literature Review ................................................ 221

Table 107 Significance values for likelihood ratios of AMT ............................................. 259

Table 108 Significance values for likelihood ratios of business practices ............................. 259

Table 109 Significance values for likelihood ratios of sources of implementation support....... 260

Table 110 Significance values for likelihood ratios of perceived benefits .............................. 260

Table 111 Significance values for likelihood ratios of high-level factors ............................. 261 


\section{Table of Figures}

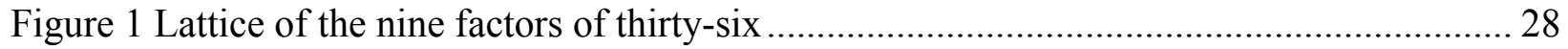

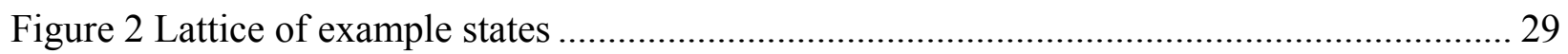

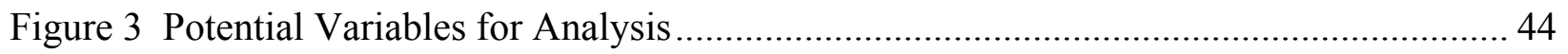

Figure 4 Plot of lattice for AMT - Medium Plants.................................................................. 206

Figure 5 Plot of lattice of business practices - Medium Plants .............................................. 207

Figure 6 Plot of lattice of source of implementation support - Medium Plants ......................... 208

Figure 7 Plot of lattice of perceived benefits of AMT - Medium Plants ................................... 210

Figure 8 Plot of lattice of high-level factors - Medium Plants.................................................... 211

Figure 9 Plot of lattice of AMT - Vehicles Industry .............................................................. 212

Figure 10 Plot of lattice of business practices - Vehicles Industry ......................................... 214

Figure 11 Plot of lattice of sources of implementation support - Vehicles Industry .................. 215

Figure 12 Plot of lattice of perceived benefits of AMT - Vehicles Industry .............................. 216

Figure 13 Plot of lattice of high-level factors - Vehicles Industry ............................................. 217 


\section{INTRODUCTION}

With the emphasis on reducing costs and increasing manufacturing efficiency, a record number of companies are embarking on different forms of advanced manufacturing technologies (AMT) (Udo and Ehie, 1997). AMT are broadly defined by many authors (Small and Chen (1995), Sheppard, McDermott, and Stock (2000), and Alcorta (1994)) as "an automated production system of people, machines, and tools for the planning and control of the production process, including the procurement of raw materials, parts, and components, and the shipment and service of finished products". In particular, AMT are a group of computer-based technologies including: computer-aided design, robotics, group technology, flexible manufacturing systems, automated materials handling systems, storage and retrieval systems, computer numerically controlled machine tools, and bar-coding or other automated identification techniques (refer to Appendix A for more examples).

In 1998, Frohlich found that sales of AMT in the United States were typically between $\$ 600$ and $\$ 850$ million (U.S.) per month and were growing at over $10 \%$ per year. Due to the economic climate today, these numbers are probably smaller but the importance of AMT to manufacturing has not changed. The uncertainty in today's environment, the increasing demands by consumers, and the rapid change in consumer preferences, increases the value of the implementation of AMT because of the increasing need for flexibility, greater variety, and increased quality and efficiency. Unfortunately, Chung (1996) found in his review of over 15 studies that the balance of implementations of AMT result in failures (no substantial increase in process flexibility, responsiveness, reliability, or quality) $50 \%$ to $75 \%$ of the time. This represents a large number of firms that invested millions of dollars only to obtain minimal returns; in some cases, organizations had a negative return on their investment in AMT. 
Many organizational and managerial procedures and policies have been developed to decrease uncertainty in traditional manufacturing systems (e.g., standardizing product designs, lengthening the product life cycle, using statistical control methods, and the trading off of flexibility and costs) (Stevenson and Hojati, 2001). These constraints are reduced or possibly eliminated with the use of advanced technology. The use of computer networks and automated machines provides efficient information feedback mechanisms for even the physically longest manufacturing system. Automated processing times are extremely reliable and data reports from the machines are very accurate, unbiased, and timely. This allows management to make better decisions in a much faster timeframe. "Thus most of our traditional manufacturing management, industrial engineering, and operations research techniques for increasing the efficiency and effectiveness of manufacturing systems are irrelevant in the factory-of-the-future."(Goldhar and Jelinek, 1985)

The implementation of AMT affects not only the manufacturing division of a plant, but also the marketing, human resource, research and development, and engineering design divisions. This technology alters the design of a plant as well as the relationship between the various units. It also affects the relationship between the plant and its suppliers, as reliability and flexibility are currently more important. The relationship between the firm and its customers also changes: for example, firms can adjust to changes in demand more quickly and are able to offer better quality, shorter lead times, and improved reliability.

For organizations that have successfully implemented AMT, the benefits have been astounding. General Electric modernized its locomotive plant with a flexible manufacturing system and reduced machining time for multi-ton engine frame parts from 16 days to 16 hours (Goldhar and Jelinek, 1985). The Ingersoll Milling Machine Company can now produce custom 
parts in single batch sizes for the same production cost as for long-run standard parts (Goldhar and Jelinek, 1985). AMT have given some adopters a strong competitive advantage in their industry. The above success stories demonstrate the potential of AMT under the right circumstances.

\subsection{Problem Statement}

The purpose of this study is to identify complementary characteristics of organization structures to aid in the explanation of the success and failure of the implementation of AMT. The characteristics that are found to be complementary will be used to assist organizations in maximizing the benefits from the use of new technology. The results will help companies plan future implementations as well as assist them in obtaining greater benefits from existing implementations. The conclusions of this study could in turn lead organizations to create a sustainable competitive advantage by having a complex set of integrated strategies. An AMTbased competitive advantage could block imitation because of the complexity of the operational strategy (Rivkin, 2000).

Recently, due to the poor economic outlook of international markets, the importance of flexibility and efficiency has increased in the manufacturing sector. It is widely recognized that AMT assist firms in decreasing materials costs, increasing flexibility, and improving productivity. The improvement of efficiency and productivity, as well as the decrease in cost, has resulted in an increased number of implementations of AMT (Frolich, 1998).

Some organizations are obtaining great benefits from AMT while others do not obtain the productivity gains that were expected. The "management problem" is becoming a focal point in order to extract value from AMT. Milgrom and Roberts (1995) proposed that the implementation 
of AMT must be accompanied by a shift in the supporting aspects of the organization including marketing, human resource, engineering design, and production policies. The amount of cooperation and integration required to obtain a successful implementation is extremely high compared with more traditional forms of production machinery.

\subsection{Research Objectives}

There is a voluminous and diverse literature dealing with the adoption of advanced manufacturing technologies, much of it theoretical (Milgrom and Roberts, 1990, 1995; Meredith, 1987; Chang, 1993; Eaton and Schmitt, 1994). To date the bulk of empirical studies have relied on limited data that concentrate on one industry, or that have small sample size, or both. The average sample size from the literature (Gupta et al., 1997; McDermott and Stock, 1999; Chung, 1996; Shepherd et al., 1997; Frolich, 1998; Udo and Ehie, 1996, Small and Yasin, 1997), as calculated by the author, is 106 firms; representing an average survey response rate of only $21.3 \%$. The majority of these studies have focused on U.S. firms. Few studies have been done on European firms and fewer on Canadian firms.

This study uses a unique and comprehensive data set (The 1998 Survey of Advanced Technology in Canadian Manufacturing) to do ex post tests of hypotheses pertaining to the use of AMT (such as computer-aided design/computer-aided manufacturing, computer integrated manufacturing, automated storage and retrieval system, materials resource planning II/enterprise resource planning systems, flexible manufacturing systems, rapid prototyping systems) across all 3-digit standard industrial classification (SIC) codes for manufacturing industries in Canada. The 1998 Survey of Advanced Technology in Canadian Manufacturing was linked to the Annual Survey of Manufactures so that plant level productivity and profitability information would be 
available to test the benefits of AMT. A total of 3,702 out of 3,757 in scope plants (plants that were still manufacturing goods at the time of the study) responded to the 1998 Survey of Advanced Technology in Canadian Manufacturing - a response rate of $98.5 \%$ of in scope plants.

There were three overall objectives of the research. The first was to determine which characteristics of the organization are complementary with AMT. The complementarities were determined by industry in order to determine if there were global or local complementarities. Comparisons were also done by plant size to determine if there were some characteristics that were more important for smaller firms given their tighter resource constraints. This aided in the second goal, which was to determine the optimal set of strategic policies for the organization to maximize profit. This set of strategies forms the basis of sustainable competitive advantage due to the complex nature of the variable interactions. It may not be feasible for plants to move directly to the optimal solution. For that reason, the final goal, the lattice ${ }^{1}$ (refer to Chapter 2) was determined (for an industry and medium sized plants) so that managers will be able to decide what set of policies offers the optimal benefit given their constraints. The creation of the lattice involved consideration of both the complementarities determined between the various factors, as well as the profitability data of the firms using the different strategy configurations.

\subsection{Benefits of the Study}

This study extends the research of Milgrom and Roberts $(1990,1995)$ by testing the complementarity assumptions made in their previous work. The assumptions on complementarity are important as they allow the authors (Milgrom and Roberts $(1990,1995)$ ), as

\footnotetext{
${ }^{1}$ A Lattice is a set $\mathrm{X}$ partially ordered by a binary relation $\mathrm{R}$ with a meet and a join.
} 
well as the author, to apply results from lattice theory ${ }^{2}$ (refer to Chapter 2) to a profit function. Milgrom and Roberts (1995) then made conclusions about the behaviour of the optimal solution under exogenous changes. This thesis also complements the work on innovation by Mohnen and Roller (2003) by determining the industry-specific complementarities for the implementation of AMT (a form of innovation) at the plant level. Using the profitability values from the various plants, this study was able to measure the increased benefit derived from the various complementarities and determine an optimal set of strategic policies for the organization. This is the first analysis (known to the author) to go past simply checking for complementarity of AMT and the organizational factors.

With the results from this study, managers will have a better idea of how to plan a successful implementation of AMT in their plant or of how to gain efficiencies from their installed base of AMT. This study provides empirical insight into why implementations of AMT have failed in the past and might provide insight into overcoming obstacles to the implementation of other process innovations. Finally, the results from this study provide insight into the different factors acting at the industry level and how these affect the implementation of new manufacturing techniques.

\subsection{Overview of the Thesis}

The following chapter provides a literature review on advanced manufacturing technologies research. This includes research on the factors critical for successful implementations and the use

\footnotetext{
${ }^{2}$ Lattice theory is the branch of mathematics that deals with partially ordered sets. It is the theoretical basis for the methodology used in this study.
} 
of AMT for gaining competitive advantage. The specific characteristics of AMT are also described in detail.

Chapter 3 provides a literature review on complementarities, which includes both traditional and discrete definitions of complementarity. This section also contains a review of current complementarities research in the areas of human resources management, information technology, innovation, and AMT. The chapter ends with a review of lattice theory and techniques for determining complementarities.

Chapter 4 describes the research design. The chapter begins by introducing the secondary data and the theoretical constructs that were used in the survey. The results of factor analysis on the variables are then analysed and distributions of the plants by size class and industry are then discussed. The section concludes with the description of the constrained regression method used to determine complementarities.

Chapter 5 shows the results from the constrained regression analysis of both the complementarity and substitutability hypotheses. Each size class and industry exhibits its own unique set of complementary factors. The chapter then proceeds with ordinary least squares analysis of models including both factor state variables and individual variables for a representative sample of size classes and industries. This analysis demonstrates that there are some limitations to the use of factors for variable reduction in this form of analysis. The chapter concludes with the construction of sample lattices for an industry and plant size class.

Finally, the author gives conclusions and identifies directions for future research. 


\section{LITERATURE REVIEW ON ADVANCED MANUFACTURING TECHNOLOGIES}

AMT represent a wide variety of modern manufacturing systems, mainly computer based that are devoted to the improvement of manufacturing operations. There is a continuum of possible AMT that can be implemented by a firm. This ranges from stand-alone units such as a robot, to more integrated systems such as flexible manufacturing systems and ultimately to fully integrated systems called Computer Integrated Manufacturing (CIM). The amount of integration of AMT is one of the key determinants for organizational changes that are complementary to the implementation process. The implementation of AMT changes the characteristics of any manufacturing plant. The degree to which a plant will change is dependent on the number of AMT implemented as well as the level of integration. Goldhar and Jelinek (1985) summarize the operating capabilities of the advanced factory as follows:

1. The economic order quantity approaches one.

2. Variety has no-cost penalty on the factory floor.

3. Revenue per unit is highly sensitive to total production volume (not volume for this design only) because fixed conversion costs approach $100 \%$ of all conversion costs.

4. Joint-cost economics obtain: the value of the system (its potential return on investment) is a function of the bundle of products it produces, the range of processes it can perform.

5. Rapid response to changes in product design, market demand, and production mix are possible.

6. Unmanned and continuous operation is standard.

7. Closely-coupled and highly integrated production systems and close supplier-user links will result in minimal inventory levels - and greater vulnerability to error:

8. Consistently high levels of quality, accuracy, and repeatability will be demanded, as well as permitted by the technology. 
It has been shown that flexibility often reduces the engineering cost of design changes and the attendant systems modifications. However, these savings are normally offset by increased costs for programming the equipment. The labour changes are also normally offset, as there will be a decrease in unskilled labour requirements but an increase in those for skilled labour (Sanchez, 1996). An implementation of AMT normally results in a decrease in total human resource costs, because AMT reduce turnover rates by increasing employee satisfaction (thus decreasing recruiting and training costs). AMT have also been shown to reduce the amount of rework and scrap, which translates into improved quality and reliability for the customer. This decrease has been attributed to the elimination of the operator (the process is automated with AMT), as well as the elimination of operator fatigue and boredom (Troxler, 1990).

In order to demonstrate the importance of AMT to firms, Chapter 2 will review the current literature focussing on the organizational factors critical for the successful implementation of AMT as well as their impact on the market structure and competitive advantage of the firms. This section will set the foundation for the complementarity analysis to be performed by introducing some of the components already known to aid in the successful implementation of AMT. It will also demonstrate the impact that AMT can have in a plant, firm, and even industry in order to aid in the justification of the importance of the application of the results from this study.

\subsection{Organizational Factors Critical for Success}

Certain organizational factors are necessary conditions for the implementation of AMT. The first of these factors is a team-based project management approach (Small and Yasin, 1997). This approach is necessary because of the complementary nature of AMT with so many different 
departments in the organization. The more integrated the system, the more critical the need for input from multiple departments during the planning stages.

A second factor for determining the successful of an implementation of AMT is commitment from both a project champion and from the organization as a whole. It has been found that without solid commitment from management and workers the full potential of AMT cannot be achieved. The faith of the organization in AMT affects many types of benefits derived from the implementation such as return on equity, level of enhanced competitiveness, amount of cost reductions, work conditions, and improvement in control (Udo and Ehie, 1996).

The employees are the third focus of organizational factors. Employee empowerment, defined as giving workers more responsibility and control over the manufacturing process, was shown to be positively correlated to increased AMT performance (Boyer et al., 1997). Worker empowerment also requires training so that workers are able to take on added responsibility and become comfortable with the new technology. Furthermore, it is important that training occur before, during, and after the implementation of AMT (Frohlich, 1998). The increase in employee empowerment requires a complementary change in hiring and training policies, as well as a change in the incentive structure of the firm. With increased responsibility, workers will expect, and demand, increased salaries and other benefits. All of the human resource policies must be set to agree with the changes to the new expectations placed on the workers (Chung, 1996; Sun and Gertsen, 1995).

Frohlich (1998) also found that increased coordination of worker's efforts through nontechnical means (e.g., facilitating communication) was positively correlated to increased performance and growth with AMT. This increase in communication facilitates the required 
interaction between the various groups of the organization to help solve problems more efficiently. It is also critical to the integration of the systems so that the entire organization is able to achieve the maximum benefit from AMT.

\subsection{Market Structure and Competitive Advantage}

The impact of advanced manufacturing systems on the size of markets has been examined in different forms over the past ten years. Roller and Tombak (1993) showed that there is an inverse relationship between the number of firms in the market and the proportion using flexible manufacturing systems. U.S. and Japanese data from the metalworking industries support this finding, where a larger fraction of flexible manufacturing systems was correlated with a higher concentration of firms in the industry. Doms et al. (1995) also supported this finding, as they discovered that AMT increased firm growth rates and decreased the likelihood of a firm exiting the market.

AMT are not just for larger firms, Meredith (1986) studied the use of AMT by small firms and demonstrates that small firms can gain a significant competitive advantage from their implementation. Large firms tend to have rigid structures, and consequently may have difficulty adapting to the organizational requirements necessary for achieving optimal benefits from AMT. Small firms are typically much more flexible than large firms and can adapt to harnessing the increased flexibility offered by AMT. Smaller firms also tend to involve most departments in decision-making since cost is more of a binding constraint. For example, departments in small firms cooperate, determine which components are critical, and implement them first. Hence, they are more likely to overcome the high cost of implementing AMT and thus may gain a competitive advantage over larger firms in their industry. 
Because AMT increase the flexibility of the firm they can act as a barrier to entry. A flexible organization can more easily adapt to customer demand, and shift production to emerging markets. The potential for this behaviour increases business and financial risk for new firms wishing to challenge the incumbent. It may even be optimal for a firm to hold excess flexibility purely for the sake of deterring entry into an emerging market (Chang, 1993). Moorman and Slotegraaf (1999) also found that high levels of technological and marketing capabilities are complementary to an increased amount of quality improvements. Helfat (1997) also found that technological and marketing capabilities are complementary to an increased amount of R\&D in related fields. Complementarities further aid organizations in maintaining a competitive advantage once it is achieved. 


\section{LITERATURE REVIEW ON COMPLEMENTARITY}

The first definition of complementarity was by Fisher in 1893. This is a polar definition because only two goods are involved. If you selected only one of the goods then they were substitutes (like coffee and tea) or you selected both and they were complements (like bread and butter). The second definition is the Edgeworth-Pareto complementarity based on introspective utility's cross derivative. This method presupposes that the consumer has in mind one cardinal indicator of utility and that it is known. The third definition is to define complementarity using the sign of the cross-elasticities, $\partial q_{i} / \partial p_{j}{ }^{3}$ or by $\partial q_{j} / \partial p_{i}$. This method is intuitive but suffers from the problem that the cross-elasticities may not be the same, so for example lemon might be complementary to salt and salt a substitute for lemon (Samuelson, 1974).

Hicks and Allen (1934) established a framework for complementary goods, which revolutionized demand theory. This definition, based on the work of Slutsky, Hicks, Allen, and Schultz in the 1930s, was created to deal with the inconsistency of previous definitions. Instead of using the sign of the cross-elasticity, $\partial q_{i} / \partial p_{j}$ a compensated price change was used. In effect, increasing $p_{j}$, there is a simultaneous increase in money income by an amount just sufficient to keep the consumer on the same indifference contour and then determine the sign of the change in $q_{i}$, namely of $\left(\partial q_{i} / \partial p_{j}\right)_{\bar{u}}=\left(\partial q_{i} / \partial p_{j}\right)+q_{j} \partial q_{i} / \partial$ income $=s_{i j}=s_{j i}$. If $s_{i j}>0$ this implies $i$ and $j$ are substitutes, $s_{i j}<0$ this implies $i$ and $j$ are complements, and $s_{i j}=0$ then $i$ and $j$ are independent (Samuelson, 1974). Since then, the notions of complements and substitutes have become widely used in demand theory.

${ }^{3} p j$ is the price for item $j, q_{i}$ is the quantity of iterm $i$. The elasticity of substitution is a unitless measure 
The elasticity of substitution is often used instead of the cross-elasticity of demand. This is primarily because the most common measure is symmetric, thereby allowing the use of theorems and operations relating to symmetric matrices (Cahill, 1999). There are three principal definitions for the elasticity of substitution $(\sigma)$. The first (1) is for two inputs $x_{1}$ and $x_{2}$, where $f$ is the production function and $\sigma$ is the elasticity of the input ratio with respect to the marginal rate of technical substitution (Hicks, 1970).

$$
\sigma \equiv \frac{\partial\left(x_{2} / x_{1}\right)}{\partial\left(f_{1} / f_{2}\right)} \cdot \frac{f_{1} / f_{2}}{x_{2} / x_{1}}(1)
$$

The second definition is the Allen partial elasticity of substitution $\left(\sigma_{i j}{ }^{A}\right)$ and is defined by (2), where $F$ is the bordered Hessian ${ }^{4}$ determinant, and $F_{i j}$ is the cofactor associated with $f_{i j}$ (the $i j^{\text {th }}$ element of the Hessian matrix). Goods are complements if the elasticity of substitution is negative and substitutes if it is positive (Chambers, 1988).

$$
\sigma_{i j}^{A}=\frac{\sum_{i} x_{i} f_{i}}{x_{i} x_{j}} \cdot \frac{F_{i j}}{F}
$$

Uzawa (1962) applied Allen's elasticity of substitution measure to a cost function dual to an ninput technology. This created the Allen-Uzawa form of $\sigma_{i j}{ }^{A V}(3), i \neq j ; i, j \in N$, where $C$ is the total minimum cost and is evaluated at some vector of prices $w$ and output level $y$; $C_{k}=\frac{\partial C(w, y)}{\partial w_{k}}, k=i, j ;$ and $C_{i j}=\frac{\partial^{2} C(w, y)}{\partial w_{i} \partial w_{j}}$ (Cahill, 1999)

\footnotetext{
${ }^{4}$ A bordered Hessian is the $(n+1) *(n+1)$ matrix defined as having the upper left cell as 0 , the remainder of the first row and column containing the first derivatives and the lower quadrant containing the usual Hessian matrix.
} 


$$
\sigma_{i j}^{A V}(w, y)=\frac{C \cdot C_{i j}}{C_{i} C_{j}}
$$

The Allen and Allen-Uzawa elasticities of substitution measure only the change in one input due to a change in price in one other input, this means that one should not use it to measure changes in input ratios (Cahill, 1999). It does show that a factor of production cannot be a complement for all other factors of production in terms of the Allen measure (Chambers, 1988). This coincides with the results for the two-good case by Hicks (1963).

The final measure is the Morishima elasticity of substitution $\left(\sigma_{i j}^{M}\right)$. This measure was derived in 1967 in Japanese and has not been translated into English (Cahill, 1999). This is a two factor, one price measure and is defined as (4). Using this definition, one can relate $\sigma_{i j}^{M}$ directly to the corresponding Allen measure $\sigma_{i j}{ }^{A}\left(\sigma_{i j}{ }^{M}=\frac{f_{j} x_{j}}{f_{i} x_{i}}\left(\sigma_{i j}{ }^{A}-\sigma_{j j}{ }^{A}\right)\right)$ (Chambers, 1988).

$$
\sigma_{i j}^{M}=\frac{f_{j}}{x_{i}} \cdot \frac{F_{i j}}{F}-\frac{f_{j}}{x_{j}} \cdot \frac{F_{i j}}{F}
$$

This formulation demonstrates two facts, $\sigma_{i j}^{M}$ is not symmetric, and a pair of goods that are complements in terms of the Allen measure could be substitutes using the Morishima measure. According to Chambers (1988), this highlights the somewhat arbitrary nature of any elasticity of substitution in the many-input case.

The remainder of this chapter reviews the existing methods to determine and analyze complementarity. The traditional continuous variable case is not considered, as it cannot be used to measure the complementarity between organizational activities. Not only is the use of various strategies not a continuous measure, there are no observed input prices for these types of 
activities (e.g., total quality management, concurrent engineering, team work, etc.). Without input prices, it is impossible to use a cost or production function elasticity to determine complementarity. Emphasis is given to the current applications of complementarity in the discrete variable case; the various measurement techniques; and the underlying theory allowing for the use of discrete measures.

Chapter 3 is divided into three main sections. The first section identifies complementarities from current literature. This includes complementarities used to gain a competitive advantage, those found in human resources management literature, those found in the implementation of information technology, innovations, and AMT. The second section introduces the basic concepts from lattice theory that are the foundation for the methodology used in this study. This section includes many of the theorems that allow for the optimization of discrete measures that is not possible with traditional economic methods. The final section presents various techniques used to determine complementarity in order to provide a balanced evaluation of the techniques currently used as well as some of the problems associated with such methods. The end of this section presents all of the underlying theory presented in order for the reader to proceed to the research design with an understanding of the foundational elements of the methodology.

\subsection{Applications of Complementarity Analysis}

Fundamentally, the impact of complementarity means that there is a marked benefit for making changes in groups. This implies that implementing a new "cost saving" measure may result in the opposite effect, as the fit between the new method and existing practice does not match. In particular, it has been shown that managers working independently, and whose decisions are not 
co-ordinated, will systematically under respond to external changes and may never find the global optimum set of policies (Milgrom and Roberts, 1995).

\subsubsection{Implication for Competitive Advantage Strategies}

\subsubsection{Competitive Advantage Strategy Literature}

Complementarity has seen the greatest application in the domain of dynamic capabilities (Teece et al., 1997). Dynamic capabilities explain how to achieve and allow the firm to maintain a competitive advantage. Teece et al. (1997) argued that a new entrant into the market cannot imitate a complex strategy overnight but will take time to piece together the policies required to match the incumbent. The authors also stress the path dependencies involved in attaining a complex strategy where many of the policies are intertwined and complementary to one another. Tyler (2001) stated three reasons for complexities related to imitation: the historical path taken to achieve the current state, the causal linkages between resources and a firm's competitive advantage is ambiguous, and the resource generating the advantage is socially complex (e.g., there are many complementary policies).

Rivkin (2000) also argued that complexity due to a high level of complementarity between strategies and internal policies creates a competitive advantage. He argues that due to the complexity of the problem, there is no polynomial-time algorithm that a competitor can use to imitate the incumbent's success. The problem under consideration by the imitator is NPComplete, and thus all the competitor can do is to iteratively alter his strategy to be more like the incumbent. Using simulation, Rivkin (2000) showed that the majority of firms will become trapped at a local maximum but not replicate the strategy profile of the incumbent. Even the incumbent may not reach a global maximum due to the complexity of the problem (if $\mathrm{N}$ is the 
number of variables or policies, there are $2^{\mathrm{N}}$ possible solutions to check in order to find the optimum policy set).

Argyres (1995) examined the success of new technology strategy implementation with two different case studies, IBM and General Motors (GM). He determined that the firm's governance structure had to be complementary to the incentive structure in order for the strategy to be successful. The GM case demonstrates that when incentives and governance are not aligned then the strategy will be a failure. Conversely, IBM implemented a cooperative structure with team bonus incentives, and the technology strategies were a success. The knowledge of the type of governance structure that complements a given type of incentive policy gave IBM a great advantage over many of its competitors. The lack of this knowledge was a major reason for GM's problems. Massive losses occurred from 1980 to 1987 until this issue was recognized and resolved (Argyres, 1995).

\subsubsection{Hypotheses Derived from the Competitive Advantage Literature}

There are a number of hypotheses regarding complementarities from the literature on competitive advantage strategies that will be tested in this research. These relate primarily to the benefits that the firms gain from implementing AMT. These include the hypothesis that increased efficiency, increased productivity, and product improvements are complementary (Goldhar and Jelinek, 1985), as well as the hypothesis that cross-functional teams are complementary with product improvements (Teece, 1988). Much of the current research is not specific about the organizational variables that are complementary stating only that complementarities or complex interactions between variables are a source of competitive advantage (Amit and Zott, 2001, Teece, 1986, and Rivkin, 2000). 


\subsubsection{Complementarities in the Implementation of Information Technology}

The implementation of IT has been investigated in many studies. In particular, researchers have strived to determine why large re-engineering initiatives have resulted in less than satisfactory results. Barua et al. (1996) determined that radical change is supported by the theory on complementarity, as many complementary elements must be changed in order to maintain the optimal set of strategies. The authors also found that these complementarities play an important role in determining the success of the re-engineering venture. This conclusion was also supported in a European study on innovation by Whittington et al. (1999) where they conclude that radical change results in greater benefits than small, uncoordinated incremental changes.

Barua and Lee (1997) and Brynjolsson and Hitt (1996) examine the "productivity paradox" of information technology (IT). Brynjolsson and Hitt (1996) determine that the implementation of IT improves productivity but also that technology strategy is an important determinant of the size of the returns. They determine that it is not the amount of money spent on the technology that is implemented that determines success but that it is the fit with the rest of the strategic policies that matters. Barua and Lee (1997) showed that any of the deflators traditionally used in economic evaluations of IT are too high and that these are the main source of bias when considering the profitability effect of IT implementations. Both studies explained that the difficulty in determining the true effect of IT on productivity is that IT is complementary to so many other functions that these indirect effects are often not included, thus understating the benefits. This conclusion is supported by Lee (2001), who looked at the implementation of IT within U.S. mortgage firms. She concluded that unless the proper complementarities were identified and managed, then the IT implementation would not attain all of its potential benefits. 
Davern and Kauffman (2000) went further in their study to explain (using locus of value) that business process redesign and human capital are two key variables that must be complementary to the IT policies for them to be effective. They also determined that the complementary assets of appropriate incentive structures and knowledge-sharing culture were critical factors in determining the success of the IT implementation. Since IT is similar in technological competency requirements to Advanced Manufacturing Technologies, it is expected that similar policies would be complementary for a successful implementation of AMT.

\subsubsection{Interaction Between Human Resource Policies and Productivity}

\subsubsection{Human Resource Policies and Productivity Literature}

Drake et al. (1999) examined one of the contributing factors to the success of implementing activity-based costing - having complementary human resource incentive policies. It is believed that this new costing policy will provide more information that can lead to an increase in process improvements. The authors showed that there is complementarity between activity-based costing and the incentive structure of the firm. If an incentive structure based on cooperation is not in place, then the effect of implementing activity-based costing will be negative. Moreover, it has been shown that performance-based incentives are also complementary to information asymmetry (Seidmann and Sundararajan, 1997). The recognition of this complementarity is important for the redesign of human resource policies when new technologies are implemented.

Negotiated transfer prices are an important aspect of many corporate policies, especially for large corporations. The negotiation process is often slow and takes a significant portion of managers' time. Once again, the knowledge of complementary factors has been shown to create significant advantages for aiding in this process. Ghosh (2000) showed that internal sourcing is complementary with a division manager having his/her compensation based on the performance 
of the entire organization. However, external sourcing is complementary with divisional performance compensation (the division is given a bonus based on their performance and not based on individual or company wide performance). He also showed that complementary arrangements between organizational factors (e.g., human resource policies, organizational design, and communication procedures) increased the perception that the transfer pricing was fair, reduced conflict between the trading partners, and significantly reduced the time taken to negotiate the contract. All of these benefits equate to an increase in productivity and a reduction in costs for all divisions involved in the price transfer negotiations.

Chenhall and Langsfield-Smith (1998) examined the fit between strategic priority (differentiation or low price), and human resource and management policies (e.g., quality systems, integration, team-based structures) using cluster analysis methods. The study found that different human resource and management policies cluster with the different strategic priorities. The results showed that there were some management policies that were required for either differentiation or low price strategies to be successful, and others only related to the success of one of the strategic types.

\subsubsection{Hypotheses Derived from the Human Resources Literature}

There are a wide variety of organizational variables that have been studied in human resource literature. The hypotheses derived from the literature for this research included: cross functional teams and human resource incentives are complementary (Drake, Haka and Ravenscroft, 1999), that cross functional teams, benchmarking, and training are complementary (Chenhall and Langfield-Smith, 1998), and that human resource incentives and training are complementary (Ichniowski, Shaw, and Prennushi, 1997). These hypotheses use variables from different parts of 
the organization and together link other variables as complementary (e.g., incentives and benchmarking).

\subsubsection{Co-ordination of Innovation Policies}

\subsubsection{Innovation Policy Literature}

When studying the relationship between various forms of integrating mechanisms required for a successful innovation environment, Ettlie and Reza (1992) found weak evidence that some integrating mechanisms counter the effect of others. They found that there was strong correlation between supplier integration and low scrap value, low rework rates, and high target-cycle-time percentages when using new flexible automation, but it was not significant in reducing the change over time measures. More importantly, the study determined that implementing innovations such as AMT can be managed and that different integrating mechanisms are complementary to unique environments. In particular, Miller and O'Leary (1997) found that capital budgeting practices had to be altered at Caterpillar when the company implemented flexible manufacturing systems. The study found that complementary capital budgeting procedures were necessary to encourage departments to purchase diverse but complementary assets instead of the previous policy of incremental uncoordinated purchases.

A decentralized corporate environment is typically viewed as beneficial for introducing innovation. The premise is that if people make independent decisions, then if one person makes a poor choice then it will be offset by another's insightful decision (Dietl, 1999). This is not the case when complementarities are involved. Dietl (1999) showed that when there are numerous complementarities, the systematic error of a centralized, coordinated system would be less costly than the random errors of a decentralized organization. The error is less costly since all of the 
complementary elements are still coordinated and the supermodularity of the function is being exploited even with a sub-optimal solution (Milgrom and Roberts, 1995).

Athey and Schmutzler (1995) examined the relationship between product flexibility (a long run variable representing the ability to adapt a product to make it lighter, sturdier, improve its quality, and alter its design) and process flexibility (a long run variable representing the ability to adapt to different environments, and alter manufacturing organization based on human resource policies, managements styles, and communication channels) in an innovative environment. Using a two-stage game theoretic model, they found that product and process flexibility were complementary. In particular, they found that implementing an innovation was complementary to increasing a firm's research capabilities which in turn increases the return to both process and product flexibility. This is an important study as it is one of the few that considers a multi-period time horizon.

More recently, Mohnen and Roller (2003) studied complementarities in innovation policy. They looked at the obstacles (lack of appropriate sources of finance, lack of skilled personnel, lack of opportunities for cooperation with other firms and technological institutions, legislation, norms, regulations, standards, taxation) that could be reduced by government policies and determined that the complementarity between innovation policies was significant. Mohnen and Roller (2003) split the data into two groups, probability to innovate (e.g., the probability the organization creates any innovations) and intensity of innovation (the amount of innovation activity done by an organization given that it does do some amount of innovation activity), to avoid censoring bias. They also showed that access to qualified labour was the primary obstacle that had to be overcome in order for an innovative environment to exist; it displayed macro- 
complementarity. There were other factors, such as access to financial capital, which only exhibited complementarity in a few industries (micro-complementarity).

\subsubsection{Hypotheses Derived from the Innovation Literature}

The innovation literature discusses various forms of innovation including both process and product innovations. This area of research has many hypotheses that will be tested including: the hypothesis that just-in-time, certifications, AMT, and cross functional teams are complementary (Ettlie and Reza, 1992), that internal research and development and AMT are complementary (Chenhall and Langfield-Smith, 1998), and that diverse forms of research and development are complementary (Helfat, 1997). These hypotheses represent complementarities between a number of business practices and environmental factors that occur at a plant.

\subsubsection{Complementarity Between Advanced Manufacturing Technologies}

\subsubsection{Advanced Manufacturing Technologies Literature}

The complementary nature of AMT increases the benefit of implementing the technologies in groups e.g., the introduction of computer-aided design (CAD)/computer-aided manufacturing (CAM) technology makes it cheaper to improve products and design new ones. For this to be effective, however, it requires a flexible manufacturing system that can efficiently handle the frequent design changes (Milgrom and Roberts, 1990). Schlie and Goldhar (1995) showed that implementation of computer-integrated manufacturing (a form of AMT) must be accompanied by a complementary change of competitive strategy in order for the organization to derive the maximum benefit. It has also been found that the adoption of AMT may create pressure to scaleup facilities as many of the personnel and equipment resources can be used for a greater variety of products and thus increase the efficiency of the plant (Alcorta, 1994). 
Lei, Hitt and Goldhar (1996) proposed and tested nine hypotheses that relate to AMT and what they call "strategic flexibility" (the ability to rapidly exploit new product markets using economies of scope inherent in AMT). Three of them relate to complementarity with AMT and were accepted during the study. The first is their hypothesis that investment in AMT is an option that increases strategic flexibility and that a greater product range increases the value of the option. The second hypothesis asserts that a more competitive environment will increase the value of the firm's AMT investment. The third hypothesis says that CAD / CAM systems require an integrated network of employees in the firm (design, manufacturing, and marketing) in connection with outside stakeholders (customers and suppliers). All of these are complementarities between various strategic forces within the organization and can be exploited to gain significant productivity improvement when using AMT.

Few studies have examined the complementarities that exist between different forms of AMT themselves. The studies that investigate complementarities between forms of AMT are theoretical using deductive logic to pose hypotheses. Most studies that have empirical data have concentrated on a single type of advanced manufacturing technology such as flexible manufacturing systems. This study attempts to provide more insight into multiple forms of AMT and to test some of the hypotheses from previous studies such as Milgrom and Roberts (1990, 1995) and Lei, Hitt and Goldhar (1996).

\subsubsection{Hypotheses Derived from the Advanced Manufacturing Technology Literature}

Due to the wide variety of topics in this literature, there are a number of different types of hypotheses that will be tested. These include hypotheses based on the size of the firm such as Meredith's (1987) claim that small organizations can used AMT more effectively as well as Small and Yasin's (1997) claim that large organizations obtain a greater benefit from AMT. 
These claims would suggest the hypothesis that large plants will have more AMT but that small plants will get a large percentage increase in productivity than larger plants, as they are more efficient.

Other forms of hypotheses involve the different types of AMT as well as other organizational variables. These include: the hypothesis that all forms of AMT are complementary (Alcorta, 1994), that AMT and cross functional teams are complementary (Sun and Gertsen, 1995), that design technologies, productivity improvements, information exchange (including all sources both internal and external of information), and product improvements are complementary (Schlie and Goldhar 1995), that AMT and training are complementary (Alcorta, 1994), and that design technologies, external linkages, and plant organizational changes are complementary (Lei, Hitt, and Goldhar, 1996). These hypotheses include variables from different forms of literature that will allow for a large web of inter-connected hypotheses to be formed.

\subsection{An Overview of Basic Lattice Theory}

In order to consider the complementarity of various policies, discrete variables must be used. Traditional economic techniques cannot be used for discrete measures and so lattice theory must be used in order to be able to determine the complementarity of the variables. Lattice theory enables the optimization of functions over a partially ordered set so that complementarities can be determined.

Over the past 70 years a considerable amount of research has been done about partially ordered sets and in particular lattices. This has been for the most part done in the theoretical realm of pure mathematics and combinatorics. Lattice theory is the branch of mathematics that 
studies the choices from sets of objects that are partially ordered. More recently, lattice theory has been applied by Topkis (1978) and Milgrom and Roberts $(1990,1995)$ to economic problems in the realm of profit optimization. The structure imposed by lattice theory allows for the use of discrete measures and variables in the optimization process, something that is not possible using conventional economic theory. It is important as it permits researchers to obtain clear comparative statics results that enable them to interpret observed changes in the strategies and structures of firms as optimizing responses to environmental changes (Milgrom and Roberts, 1995). This is the underlying theory for the methods used in this analysis.

\subsubsection{The Basic Concepts of a Lattice}

The initial starting point of lattice theory is the notion of a partially ordered set. Donnellan (1968) defined a partial ordering as a binary relation $R$ where the elements of a set $S$ are reflexive ( $\forall a \in S, a \leq a)$, antisymmetric ( $\forall a, b \in S$, if $a \leq b$ and $b \leq a$, then $a=b$ ), and transitive ( $\forall a, b \in S$, if $a \leq b$ and $b \leq c$, then $a \leq c$ ). Then the set $S$ is said to be a partially ordered set on $R$. These assumptions are consistent with the assumptions in standard economic theory for consumer utility functions. Any set of variables that is a partially ordered set has the potential to be a lattice. There are no restrictions on the partial derivatives or on the continuity of the variables.

The general theory of lattices is based on two additional relations or operations, a meet and a join. Lattice theory has been studied from the point of view of algebra and in terms of set theory. The first definition is to look at a lattice as an algebra with two binary operations that are idempotent ( $a \cap a=a$ and $a \cup a=a$ ), commutative, associative, and have the property of "absorption" ( $a \cap(a \cup b)=a$ and $a \cup(a \cap b)=a)$ (Birkoff, 1948). Another is to consider it in 
terms of a meet being the greatest lower bound between two elements (represented as $\cap$ ) and a join as the least upper bound between the variables (represented as $\cup$ ). A complete lattice is a partially ordered set where any two variables from the set have both of their meet and their join also contained in the set (Birkoff, 1948). If some of the meets and/or joins are not in the set, then the partially ordered set is still a lattice but it is not complete. Any variables that can form a partially ordered set can also form a lattice (e.g., real, natural, or integers may for a lattice).

An example of a lattice is the set $\mathrm{L}$ containing all nine factors of the natural number 36, namely $1,2,3,4,6,9,12,18,36$. This set is also a sub-lattice of the set of factors for 216 .

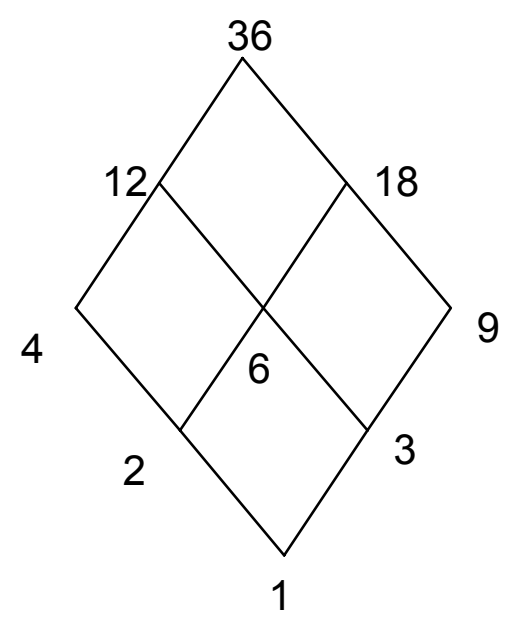

\section{Figure 1 Lattice of the nine factors of thirty-six ${ }^{5}$}

For the purposes of this research, the nodes of the lattice will represent different sets of policies or AMT devices. Let us consider a small example of firms with respect to two possible policies: they perform research and development, and they believe that AMT increase

\footnotetext{
${ }^{5}$ In this case the binary relation $R$ is $\geq$, the meet is the greatest common factor, and the join is the least common multiple.
} 
productivity. A firm can have none, one, or both of these attributes resulting in four possible states. If the two attributes are complementary then doing both at the same time is better than either one individually, and definitely better than doing neither. The lattice for this would look like figure 1. From this we will be able to tell the optimal path for a firm to follow in order to increase the benefits from implementing AMT. In this example, the optimal path would be to use communications technologies and then both if only one of the forms of technology could be implemented initially as the average price-cost margin in state 1 is larger than that in state 2 .

Both (11) - State 3

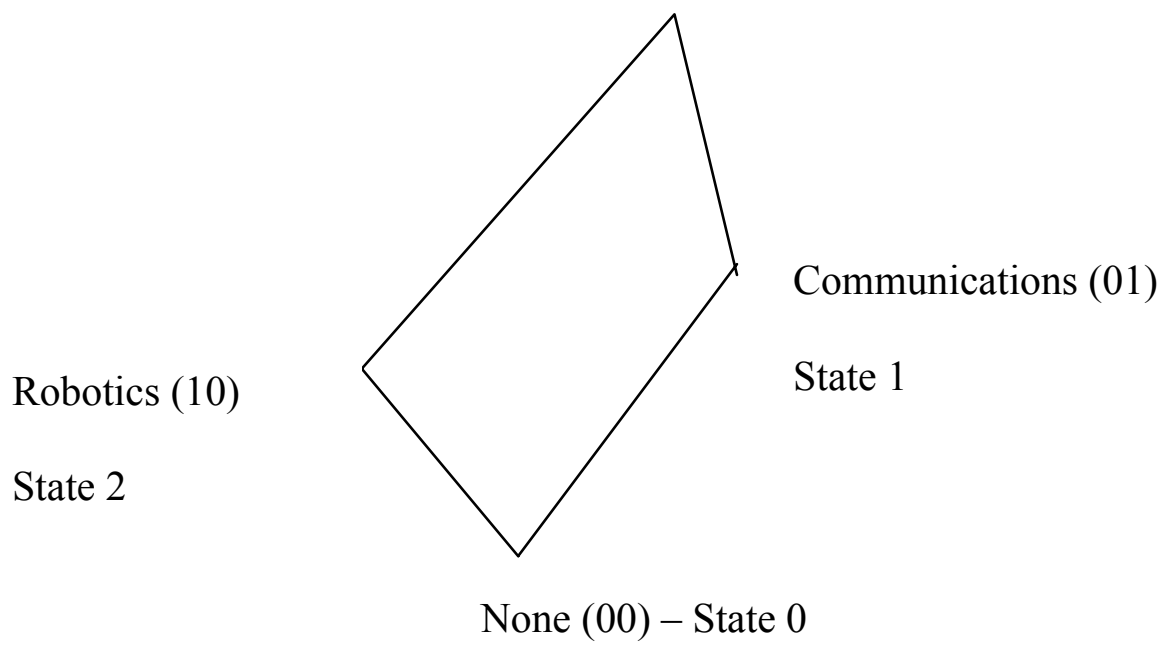

Figure 2 Lattice of example states ${ }^{6}$

The component of the theoretical framework that is most often used in the economic literature is the sub-lattice. A sub-lattice is a subset of the original lattice that satisfies the properties of a complete lattice. Using this structure, it is possible to consider solutions on a

\footnotetext{
${ }^{6}$ In this example, $a \geq b$ implies that the profit margin of $a$ is at least that of $b$. The meet is the component wise minimum and the join is the component wise maximum.
} 
reduced problem and still have application of the underlying lattice theory remain valid. This is particularly useful when looking at multivariate problems where the size of the lattice grows exponentially as it does with the complementarity of strategic policies in a firm.

\subsubsection{The Basic Solution Strategies for Optimization on a Lattice}

The method used to determine optimal solutions on a lattice is supermodularity. According to Milgrom and Roberts (1990), a function is supermodular when "the sum of the changes in the function when several arguments are increased separately is less than the change resulting from increasing all the arguments together." In essence, it is a function that exhibits the property of complementarity as increasing one or more inputs raises the return to increasing additional variables. The supermodularity of profit functions is one of the reasons supporting radical change because a large transformation is required to move all of the complementary strategies simultaneously. When innovations are implemented, there is normally a mismatch between the existing procedural and structural requirements and those of the new innovation (Teece, 1997). The construction of the lattice supports this result as the effects from initiating change in multiple areas at once rises the firm further up the lattice than simply changing a single aspect.

The primary results in the existing research used to show supermodularity of a function are centred on the decomposition of the problem. A function is supermodular if for every pair of inputs the function is supermodular in those inputs. The sum of two or more supermodular functions is supermodular but the product is not necessarily supermodular. These theorems are important for the decomposition of complex functions such as the profit function where there are numerous relationships between subsets of the variables relating to productivity, internal costs, marketing, and labour. They are used in order to create supermodular functions to demonstrate 
the effect of complementarity on the organizations output (Topkis, 1978; Milgrom and Roberts, 1990, 1995; and Mohnen and Roller, 2003).

Milgrom and Roberts (1995) also show that the search for the optimal solution can be restricted to recursively searching those solutions that are strictly above or below the current best solution. By restricting the search in this way, it still assures at least $50 \%$ of the gains realized by an unrestricted search. The time savings involved using this technique is quite significant and is due to the nature of the supermodular function. If the function is not supermodular then these significant gains are not likely to occur when simply searching the neighbouring solutions.

\subsubsection{The Basic Comparative Statics Results}

Incorporating lattice theory into economics has created new techniques called monotone comparative statics. These techniques assume that the problem is formulated such that the set being used as inputs is a sub-lattice and that the function being considered is supermodular. Again, there are no requirements for the variables to be continuous or to form a convex set as is required in neoclassical production theory (Chambers, 1988); they must simply form a lattice. This is a change from the most common comparative static methods that are based on applying the explicit function theorem, which requires a convex set of preferences (Milgrom and Shannon, 1994).

With monotone comparative statics, a given supermodular function, and a sub-lattice, the set of maximizers of the function over the sub-lattice is also a sub-lattice itself. This implies that the set of optimizers rises as the parameter values increase. This is particularly useful for determining the effect of exogenous variables on the optimal solution as in Milgrom and Roberts $(1990,1995)$. To ensure such a result, the function requires an additional assumption that it has 
the single crossing point property as defined by Milgrom and Shannon (1994) e.g., the function defined as the difference between the function at any two points only crosses zero once. This ensures the monotonicity of the functional differences (the differences are non-decreasing) as well as of the optimizers. The proof that the function will exhibit monotone increasing optimizers has been used in various models to show how the optimal results will vary when exposed to external change (Topkis, 1995, and Milgrom and Roberts, 1995).

More recently, the stochastic case of monotone comparative statics has been explored. This area requires a slight adjustment to the assumptions made about the function. Here, logsupermodularity is the requirement and not supermodularity (Athey, 2000). This is because under uncertainty there are more instances of multiplicative functions (due to the nature of expected values), and supermodularity is not normally retained under multiplication. By taking the $\log$ of the profit function, this transformation causes the multiplicative functions to become additive, which preserves the supermodularity characteristic. The log-supermodularity property is much more flexible in this regard and is used in risk aversion and investment and portfolio theory (Athey, 2000).

\subsection{Techniques to Determine Complementarity}

Two or more variables are called (Edgeworth) complementary if a higher value in any variable increases the marginal returns to higher values in the remaining variables. In more mathematical terms, two elements are complementary in the objective function if they satisfy the supermodularity restrictions (e.g., increasing both values at once has a greater return than increasing the variables one at a time). Given a real-valued function $f$ on a lattice $\mathrm{X}, f$ is supermodular and its arguments are (Edgeworth) complements if and only if for any $x, y$ in $\mathrm{X}$, $f(x)-f(x \cap y) \leq f(x \cup y)-f(y) \quad(x \cap y$ is the greatest lower bound between $x$ and $y, x \cup y$ 
is the least upper bound between $x$ and $y$ ). If $f$ is twice continuously differentiable, the defining condition is equivalent to nonnegative mixed-partial derivatives (Milgrom and Roberts, 1995). These two perspectives are important, as each has led to different techniques to determine complementarities.

All use of complementarity in this study refers to the Edgeworth definition of complementarity. Specifically, it does not refer to the idea of complementarity expressed by Bohr where he calls 'complementarity' the logical relation between two descriptions or sets of concepts which, though mutually exclusive, are nevertheless both necessary for an exhaustive description of the situation (Hitchcock, 2002). It also does not refer to the notion of complementarity in sociology concerning interpersonal theory of personality (Acton, 2002).

There are two levels of complementarity when considering complementarities within firms. One is that the complementarity is industry specific; in this case, it is called microcomplementarity. At the other extreme are complementarities that exist across all industries, or macro-complementarity (Mohnen and Roller, 2003). Macro-complementarity is quite rare, as very few studies have considered multiple industries and those that have, found major difference between various manufacturing environments.

\subsubsection{Least-Squares Methods}

Regression methods are the most commonly used technique for determining a complementary relationship similar to those in the proposed study. These models are used to determine which variables are positively or negatively correlated and therefore which elements are complementary to one another. The primary methods employed are ordinary least squares (Shephard et al., 2000) and step-wise regression models, as most studies have multiple variables 
to consider, e.g., Chung (1996), Udo and Ehie (1996), Boyer et al. (1997). Kotha and Swamidass (2000), and Gupta et al. (1997) use moderated and moderated subgroup regression analysis in their study. A moderated or moderated subgroup regression includes variables representing the joint effect between pairs of elements. This method provides a straightforward test of contingency hypotheses in which interactions are implied. It is also an efficient method for testing interaction effects since the interaction terms will not be tested for significance until the main effect independent variables have entered into the regression equation (Gupta et al., 1997). This allows Gupta et al. (1997) to determine some of the important groupings of variables found in their research.

Sheppard et al. (2000), Chung (1996), and Kotha and Swamidass (2000), mention that they check for collinearity between variables. If any independent variables are strongly correlated, then it will be impossible to separate the effects of each variable (Chung, 1996). In each study, it was found to be insignificant. Thus, the coefficients in the multiple regression models will be able to estimate the predictive effect of changing one independent variable while holding the others constant. Collinearity of the variables was therefore disregarded as a limitation to the study results. None of the remaining studies that were reviewed mentioned collinearity and there is a concern that these results could be biased.

The problem with linear regression techniques according to Athey and Stern (1998) is that a positive correlation in the unobservables results in a positive bias in the estimate of the interaction effects. As well, if the practices are complementary in the design phases, then the interaction effect will be understated. The meaning is that regression approaches may find complementarity when none exists or not find it when it is present. 
Reduced-form regression models have also been proposed for determining complementarity. Reduced-form regression models are non-structural models where the objective is to determine the direction of the effect a number of independent variables have on a particular dependent variable. The reduced-form model is derived by expressing the endogenous variables as a function of the exogenous variables and the error term (Verbeek, et al., 2000). These models are easier to construct and estimate than structural models. The method also avoids some of the problem related to missing data or data that cannot easily be quantified such as the price of imported technology or information transfer (Arora, 1996). The problem encountered by this technique is that reduced-form tests cannot disentangle the nature of interaction between pairs of variables if there are more than two endogenous variables (Arora, 1996). In particular, it does not provide a general solution for testing complementarity when there are more than two choices, which is the case for most studies (Athey and Stern, 1998). Arora (1996) suggests that the reduced-form regression tests need more restrictions so that they can be used. In particular, it was suggested that a one-sided test for complementarity based on the variance-covariance matrix of the residuals would be required.

\subsubsection{Parametric Method}

This is a relatively new method to determine complementarity. This method involves proving that the function under consideration is supermodular in each pair of elements. If true, then the pair of elements is complementary. The method is very different from regression models and as such does not suffer from the same limitations and possible biases.

The parametric method relies on the random practice model, a derivative of the random systems model, which is a specific application of "switching regression." The switching regression is a model of an agent choosing between several discrete choices. In this type of 
model, only the distribution of interactions among practices is identified; practices may be complements for some plants and substitutes for others (Athey and Stern, 1998). Switching regression models are either Bernoulli (where the current state is independent of all others and the probability of being in a state remains constant), or Markov (where the current state depends only on the previous state) (Knuping, 1997). There are also models that combine Markov and Bernoulli states of nature. The method of switching regression used by Athey and Stern (1998) places a restriction on the variation of the interactions between variables by assuming a constant elasticity of substitution between inputs; they call this model the random practice model. When the restrictions of the random practice model are satisfied, it allows the researcher to draw unambiguous policy conclusions about the interaction effects between variables (Athey and Stern, 1998).

This method requires pair-wise tests of the variables for computational ease so that only a single linear inequality constraint needs to be considered. The test for complementarity is performed by proving that the data satisfies the set of parametric equations required for supermodularity. Normally, an assumption about the distribution of the covariance will need to be made (such as normally distributed). The complementarity hypothesis is then tested using a one-tailed t-test of the inequality constraint. However, two null hypotheses should be tested, one with supermodularity of the function as the null hypothesis, to test for complementarity, and one with submodularity as the null hypothesis to test if the elements are substitutes. Complementarity between variables (or if the variables are substitutes) was tested by industry to determine the local industry-specific complementarities (substitutes) that exist and those that are exhibited on a global level. 
This method was used by Mohnen and Roller (2003) to determine complementarities between problems related to innovation and the probability of having an innovation. To compute the test statistics Mohnen and Roller (2003) computed a Wald Statistic. Upper and lower bounds calculated by Kodde and Palm (1986) were used to determine significance. The results are heavily dependent on accurate estimations of the coefficients of the objective function (Mohnen and Roller, 2003). A descriptive clustering test was also conducted to confirm the results of the ttests.

\subsection{Summary and Comments}

The theory of complementarity and supermodular functions is well documented. Many comparative statics techniques have been developed to take advantage of the supermodularity condition. In particular, the theory focuses on the behaviour of the optimal solution under exogenous changes in parameters. This is only useful if an optimal solution can indeed be found. It has been shown that in general the problem is NP-complete as the size of the solution set increases exponentially in the number of variables (Rivkin, 2000). This means that there is no polynomial time algorithm to find the optimal solution and as such, it is rather irrelevant what occurs after that point. The supermodularity property reduces the solution set for improvements to those directly above or below on the lattice. This is a much smaller number of possible solutions to consider and there exists a polynomial time algorithm to solve the problem. Without a polynomial time algorithm to find the optimal solution, there is no way to apply any of the comparative statics results, as they require an optimal solution.

The previous survey of complementarity research is a representative sample of the current literature on the subject. Complementarity research is gaining popularity and is being applied to an increasing number of areas. The majority of the research is centred on human 
resource policies and information technology implementations. Most studies are theoretical in nature and very few conduct empirical tests. The primary reason for this is the lack of data. Human resource policy interactions and their benefits are very hard to measure and normally rely on managers' perceptions. Thus, the data are costly to obtain. IT has been a focus because of its high profile and the large number of firms that have implemented the technology and not obtained the desired benefits. Many companies have been searching for explanations and ideas on how to improve their performance. The measures used for this type of analysis are for the most part easier to obtain than the human resource benefits but are also considered to be a source of competitive advantage making them slightly harder for the researcher to obtain.

Recent studies in AMT have focused on two main areas, the first are methods to evaluate implementations of AMT, and the second is identifying critical organizational factors necessary for AMT implementation. The evaluation techniques stress the importance of the strategic nature of AMT and the impact they have on an organization's competitive situation. The studies related to organizational factors have focused on human resource policies as well as competitive advantages. Both branches of research have for the most part relied on small sample sizes (between 40 and 200) and have not considered any industry specific effects.

This study extends the work of Milgrom and Roberts (1995) using data from the 1998 Survey of Advanced Technology in Canadian Manufacturing to test which characteristics of the firm are complementary in the context of implementing AMT. This large data set will allow the results to be generalized. It will also allow industry specific complementarities to be determined as well as those that occur at the macro-level. This study also brings together the two streams of research by empirically testing assumptions made in various theoretical studies. 


\section{RESEARCH DESIGN}

This study is one of the first to use unique and comprehensive data to conduct ex post tests of hypotheses pertaining to the use of AMT across all 3-digit SIC codes for Canadian manufacturing industries (refer to Appendix C for SIC codes and definitions). The study determined pair-wise complementarity between a number of organizational factors in order to derive an optimal set of firm-level policies to enable organizations to gain a competitive advantage from the implementation of AMT.

\subsection{Research Data}

This study uses secondary data sources from Statistics Canada. No primary data sources were created, as two different surveys existed with a large number of variables pertaining to the use of AMT, management practices, and plant economic performance. A much larger proportion of the population was sampled by Statistics Canada than would have otherwise have been feasible privately.

\subsubsection{Survey of Advanced Technology in Canadian Manufacturing}

The research used Statistics Canada's 1998 Survey of Advanced Technology in Canadian Manufacturing. The survey consists of nine main sections with information collected on over 175 variables - general firm and establishment characteristics; adoption of advanced technologies; use of business practices; the development and implementation of advanced technologies; skill requirements; results of adoption; obstacles to adoption; research and development; and electronic communication (refer to Appendix B for a copy of the survey). Plant managers were asked to report the adoption or whether they planned to adopt 26 specific advanced technologies (refer to Appendix A for definitions) grouped into six functional categories 
(Design and engineering; processing, fabrication and assembly; automated material handling; inspection; network communications; integration and control).

The survey design was a stratified random sample drawn from 43 Standard Industrial Classification (SIC) industry categories as well as by plant size. The total sample size was 3,757 with an overall response rate of $98.5 \%$. Response rates were similar across the three plant size categories - for small plants (with between 10 to 49 employees) the response rate was $98.8 \%$ out of a sample of 1,503 , for medium plants (50-249 employees) the response rate was $98.3 \%$ out of a sample of 1,431 , and for large plants the response rate was $98.6 \%$ out of a sample size of 823 (Sabourin and Beckstead 1999). For the purpose of the study, there are a total of 1,455 small plants, 1,376 medium plants, and 798 large plants. These response rates are much higher then the average of $20 \%-25 \%$ found in most literature. The sample size is also much larger than the average size of 106 respondents found during the literature review (Gupta et al., 1997; McDermott and Stock, 1999; Chung, 1996; Shepherd et al., 1997; Frolich, 1998; Udo and Ehie, 1996, Small and Yasin, 1997).

The sample was taken disproportionately to ensure that each stratum would be adequately represented. For this reason all analysis in this research was done using population weights. The population weights for the survey are equal to the inverse of the sampling rate. Results for the occurrence of states in this report are population estimates, these represent the percentage of establishments in the population that exhibit a particular set of characteristics.

\subsubsection{Annual Survey of Advanced Manufactures}

The data from the technology survey was linked to the plant-level Annual Survey of Manufactures. Approximately 35,000 plants are surveyed each year. Plant-level variables from 
the Survey of Manufactures include: industry code for each firm, value of shipments or sales, value-added, output price deflator, total number of workers and production workers, skilled workers, total hours worked by production workers, total wages and salaries, total energy cost, total material cost, and value of exports. This allowed the study to test theoretical increases in productivity and profitability of the plant by industry SIC code.

\subsubsection{Data Linkage}

The two Statistics Canada surveys were linked using a unique plant code identifier found in both datasets. Theoretically, all of the plants surveyed in the 1998 Survey of Advanced Technology in Canadian Manufacturing were also surveyed in the 1998 Annual Survey of Manufactures. There were a number of difficulties (described below) encountered during this process and thus 2,498 of the 3,702 plants were able to be linked (representing a loss of $32.5 \%$ of the sample).

The first problem was that the 1998 Survey of Advanced Technology in Canadian Manufacturing used SIC codes whereas the 1998 Annual Survey of Manufactures used the newer North American Industry Classification System (NAICS) codes. Under NAICS the printing, publishing and allied industries are no longer in manufacturing but are classified as part of the information and cultural industries. For this reason, these plants were not included in the 1998 Annual Survey of Manufactures, and hence could not be linked.

The second problem was that some of the plants received a short form version of the 1998 Survey of Manufactures and so much of the economic information was omitted. The economic information for these plants is only available using tax information, which is not feasible to obtain from Canada Customs and Revenue Agency. Thus, the linkage between the datasets for these plants was infeasible. 
The final problem was the requirement to also link these two datasets with a third file, the 1995 Annual Survey of Manufactures. This linkage is required for a second study being done under the SSHRC funding. Both the 1998 Survey of Advanced Technology in Canadian Manufacturing and the 1998 Annual Survey of Manufactures datasets were linked together before the linkage to the 1995 Annual Survey of Manufactures was attempted. This resulted in a reduction in the linked dataset size to 2,273 plants.

The only new problem encountered with the 1995 Annual Survey of Manufactures survey was that some of the firms were not in existence in 1995 or were not part of the 1995 Annual Survey of Manufactures. There was no information in either of the surveys that would identify the date the plants were first opened so there was no way of ascertaining either explanation as to why the plant was not in the 1995 Annual Survey of Manufactures. As a result, these plants were not included in the linkage (reducing the sample a total of $38.6 \%$ from the initial 3,702 to 2,273 ).

After numerous consultations with a Statistics Canada methodologist, it was decided that plants that were not linked to both annual surveys of manufactures would be treated as nonresponse to the combined survey process. For this reason the weights for the plants had to be readjusted and new weights for the data were calculated. These weights were constructed so that the reduced dataset, when weighted, would properly represent the 1998 population from which it was taken.

The sample was reduced further when the food industry. The survey was did not include a representative sample of the food industry as there was a separate survey conducted for food manufacturers. As well, any plants with fewer than $\$ 50,000$ in shipments in 1998 or fewer than ten employees were removed, as these were not in the scope of the survey design and would 
represent outlier data points. This resulted in a total sample size of 2,191 plants. The distribution of the sample data by size class is 686 large plants, 1,006 medium plants, and 499 small plants. The distribution of the sample by industry is 258 plants in the chemical industry, 218 plants in the electronics industry, 187 plants in the furniture and other industry, 95 plants in the machinery industry, 219 plants in the metal industry, 117 plants in the non-metals industry, 133 plants in the plastics industry, 296 plants in the textiles industry, 239 plants in the vehicles industry, and 429 plants in the wood industry.

\subsection{Theoretical Construct Design}

The survey contains a large number of variables. To show complementarity, pair-wise comparisons were performed. This causes the number of comparisons to become a constraint to the research rather quickly as there are $\left(\begin{array}{l}N \\ 2\end{array}\right)$ complementarity tests to perform (where $\mathrm{N}$ is the number of variables). For this reason, as well as the similarity between some of the measures taken in the survey, groupings of measures had to be created. Many of the survey questions about specific obstacles to implementation were omitted, as these were not the focus of the study. The following is an outline of the variables that were used (refer to Figure 3) along with reasoning for their inclusion in the study. The grouping of the measures presented in this section was derived from the survey design; the actual grouping was determined using factor analysis and is presented in section 4.3 . 


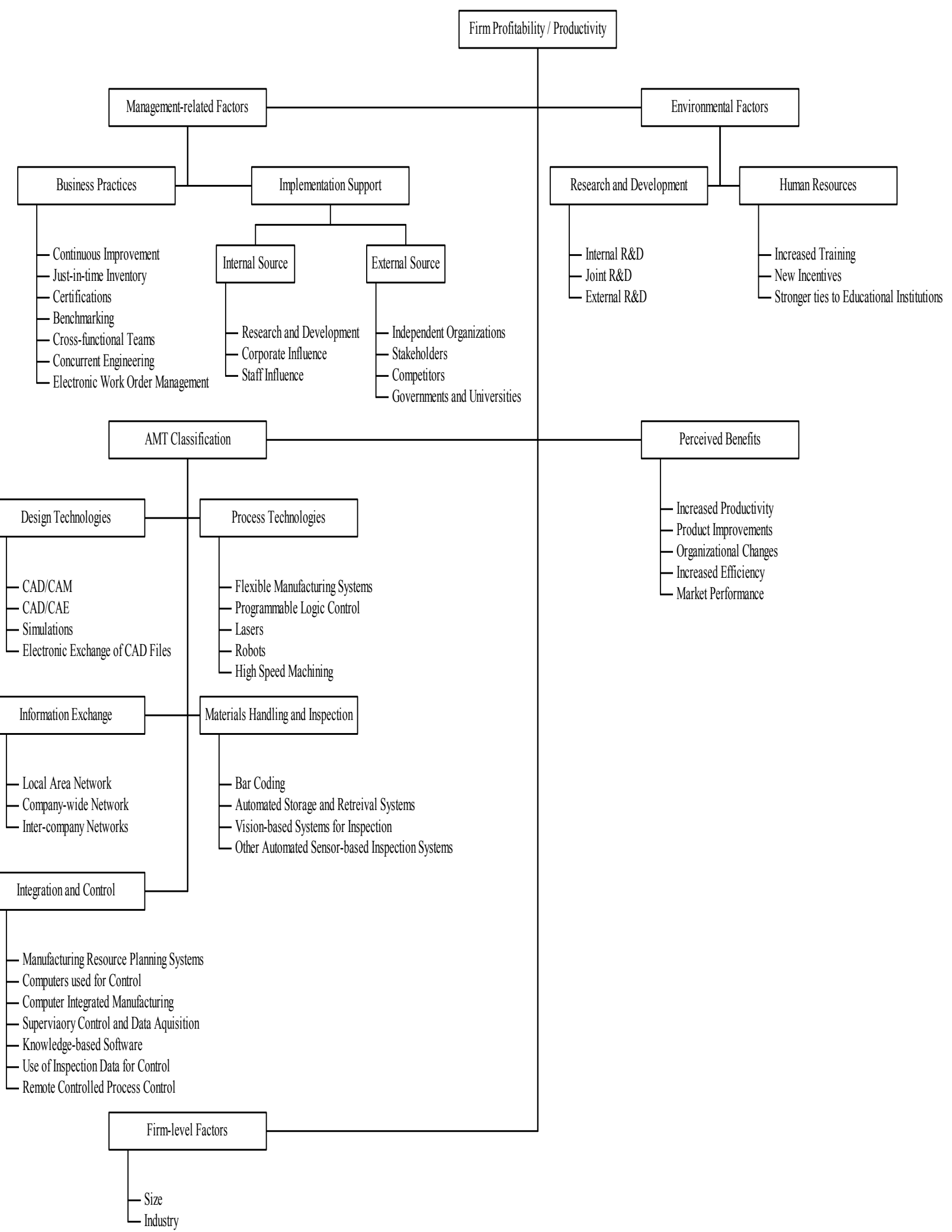

Figure 3 Potential Variables for Analysis 


\subsubsection{AMT Classification}

Kotha and Swamidass (2000) classify AMT in four categories: product design technologies, process technologies, logistics/planning technologies, and information exchange technologies. Product design technologies consist primarily of computer-aided design (CAD), computer-aided engineering (CAE) and automated drafting technologies. Process technologies include flexible manufacturing systems, numerically controlled machines (NC), and programmable controllers. Logistics and planning technologies entail production scheduling systems, shop floor control systems, retrieval systems, and materials requirements planning (MRP) systems. Finally, information exchange technologies are any computer technology that facilitates the storage and exchange of information such as a database, computer networks, or a personal computer. In this study, logistics and planning technologies are separated into two groups - materials handling and inspection, and integration and control. Therefore, there were five AMT groupings because of the increased number of logistics and planning systems over the study considered by Kotha and Swamidass (2000).

\subsubsection{Plant-Level Factors}

The factors in this category were derived from both the Annual Survey of Manufactures and the Advanced Technology Survey. The three primary factors were the size of the plant, the SIC code for the plant's industry, and profitability (measured as the price-cost margin or profit margin). Although the plant's industry was determined by SIC, some SIC codes were combined to form an industry that was sufficiently large to analyze. The grouping followed that of Mohnen and Roller (2003), and resulted in eleven industries. 


\subsubsection{Management-Related Factors}

According to Udo and Ehie (1996), business strategies are extremely important to the successful implementation of AMT. Seven practices were studied in the Statistics Canada survey: continuous improvement, just-in-time inventory, certifications (both certification of suppliers and of the plant), benchmarking, electronic work order management, cross-functional teams, and concurrent engineering. These were the most cited practices from the AMT survey, while others had less than $25 \%$ of respondents using them (Sabourin and Beckstead, 1999).

Research and development is a source of innovation for the firm. Theoretically, a firm that is more innovative will be willing to perform more research and development and will be more interested in using the latest technologies. Innovators are also associated with higher levels of risk taking due to their early adoption of new methods and techniques. Since the implementation of AMT involves a high degree of risk, the level of research and development is an important indicator to AMT implementation. Three variables expressing the source of research and development were used in the study: internal (in-house), joint (with an external company), and external (contract research and development out to an external company).

\subsubsection{Environmental Factors}

The sources for ideas in terms of implementing AMT can be influential. It is widely recognized that the support of a project champion is crucial to the success of any change (Teece, 1988, Chenhall and Langsfield-Smith, 1998). The external support for new technology is also important as it helps to reduce the risk of implementation. Both internal and external sources for the support of AMT implementation were surveyed by Statistics Canada. The internal variable was comprised of three components: research and development (research, experimentation, production engineering), corporation characteristics (corporate head office, other plants, 
technology watch program), and staff characteristics (production staff, design staff, and sales and

marketing). The external variable was comprised of four components: independent organizations (trade fairs and conferences, patents, and consultants), stakeholders (customers and suppliers), competitors (related firms, other producers), and government and universities. These sources of support can be critical for the successful implementation of AMT.

A number of human resource issues were also studied in the Statistics Canada survey. The primary variable was increased training. This included training for computer literacy, technical skills, quality control skills, and safety. An improved incentive package and stronger links to educational institutions were surveyed as well. These aspects are important determining factors for the skill level of the work force implementing and using AMT in the plant.

\subsubsection{Perceived Benefits of Adoption}

The perceived benefits of adoption are extremely important, as these will play an important role for future adoptions and for the use of the current set of AMT. If managers do not perceive a benefit for the technology then they will not champion its implementation or approve the budgetary requests for necessary capital expenditures. Five main categories were studied: improved productivity, product improvements, organizational changes, plant efficiencies, and market performance. Improved productivity included improvements due to reduced labour, reduced material consumption, reduced capital requirements, reduced set-up time, and reduced scrap. Product improvements resulted from new product features, reduced time to market, and improved quality. Organization changes were measured by increased production flexibility and increased skills requirements. Plant efficiency was determined by an increased machine utilization rate. Finally, market performance was calculated by an increase in market share and increase in profitability. 


\subsection{Variable Coding}

The first step was to code the individual variables. The states of the plants were then represented as binary strings. If a plant demonstrated a particular element then this was represented by a 1 in the position of the variable in the string, otherwise it was 0 . Factor analysis was performed in order to reduce the number of variables being considered and thus the total number of states that a plant can exhibit.

For those variables that were measured on a five point Likert scale, three possible techniques may be used to convert them to binary values. The first is to use extreme scores (4 or 5) and convert those to 1 and all others to 0 . The second method is to use the most important scores. In this case, only half the values for each category may be 1 and the rest 0 . Those variables with the highest importance are given the value of 1 until a tie cannot be broken in determining the next value of 1 cut-off and still obey the $50 \%$ constraint. The third technique is to determine the mean value for the variable, plants that have a greater score are coded as 1 , and the remainder are 0 . Each of these methods has benefits and problems.

According to Arundel and Sonntag (1999), the smaller firms tend to have lower average scores. If the averages were determined based on the control variables, then using the most important or difference compared to the average would alleviate this bias. The second issue for bias results when a plant responds the same for all questions; it is then impossible to determine the most important issues to that plant. In this case, the extreme values and the difference from the average will be less biased. This study used the same method as Mohnen and Roller (2003) and Arundel and Sonntag (1999) to convert the Likert scale values to binary using the comparison to the group average. For any variable that was a combination of more than a single measure in the survey, the average for the total in the variable construct was calculated and any 
plant that had a value greater than the average was coded as 1 and all others were coded as 0 . For these variables, the actual values in the Likert scale were used and the initial coding step was eliminated. This reduced the amount of approximations occurring during the coding phase.

There are a total of $2^{\mathrm{n}}$ ( $\mathrm{n}$ is the number of variables) possible states for a plant, some of these states did not occur, in that case, they were removed from the analysis. The occurrences of each state were counted by firm size and by industry. The analysis of the distribution of plants demonstrated the clustering of variables that occurred in the manufacturing sector. It also demonstrated if further variable reduction was necessary due to high levels of plant cohesiveness. If there was a high level of cohesiveness of plants then the distribution of plants in the factor states has a large number of empty states. In this case, further variable reduction was necessary.

\subsection{Factor Analysis Results for Variable Reduction}

The variables were considered for factors based on the questions designed in the Statistics Canada 1998 Survey of Advanced Technology in Canadian Manufacturing. Each question was considered for variable reduction on its own and then all the variables were considered together in order to create what is referred to as high-level factors. All of the factor analysis was completed using survey weights as required by Statistics Canada.

\subsubsection{Factor Analysis Method}

In order to determine the factors for this study PROC FACTOR from the SAS programming language version 8.2 was used. This procedure was used in order to conduct both Principle Component factor analysis as well as Maximum Likelihood factor analysis. After multiple variations were tested, an oblique rotation using the promax parameter was selected in the factor 
procedure. This form of rotation does not restrict the factors to be independent from one another, allowing complementarities to exist. The prior communality estimates were done using the squared multiple correlation between the variable and all other variables. The number of factors retained in the procedure was first determined by the number of eigenvalues greater than one or set at a maximum of seven if there were more than seven eigenvalues greater than one. Variables that had a weight of .3 or higher on a factor in the standardized regression coefficients were determined to be part of that factor. If a variable did not have a score of at least .3 then it was placed as part of the factor in which it had its maximum absolute score.

An iterative process was used to create the factors for this study. After the initial factor analysis was complete, it was apparent that the binary variables would have to be created using means for each size class of plant and not an overall mean (refer to Table 1). The initial results had few of the factors in small plants and most of the factors used in larger plants. Since the remainder of the analysis was going to be done using plant size as a control variable, creating the binary variables by size was considered an acceptable method.

The next stage of the factor analysis focused on the large number of occurrences of states with no plants in them. Two different solutions were used to deal with this issue. The first was to look at the occurrences that happened only when looking at the plants by industry classification. Using two-digit SIC codes provided a large number of occurrences of empty states (there were twenty-one industries, including food related industries). For this reason, the two-digit SIC codes were aggregated to form a total of ten industries (Chemical, Electronic, Machinery, Metals, NonMetals, Plastics, Textiles, Vehicles, Wood, and Furniture and Other). This reduced the number of very small or empty states. 
A second method was used when there were a large number of empty states in both the analysis by plant size and by industry. In these cases, the number of factors determined in the factor analysis procedure had to be forced to be a smaller value using the max factor criteria. This occurred primarily in the factors that had a large number of variables including sources of implementation support, advanced manufacturing technologies used, and the high-level factors. In all cases, the proportion of variance explained by the factors remained greater than $75 \%$.

The factor analysis was completed using the reduced dataset as well as the original Statistics Canada 1998 Survey of Advanced Technology in Canadian Manufacturing. This was done to verify that the reduction of the dataset had been unbiased, as well as a verification of the factor analysis itself. Both sets of analysis produced very similar results. In cases where the results differed it was with variables that had been moderately weighted on a couple of factors or were not weighted heavily on any one factor in either case.

Binary variables were then created for each plant to represent the factor. If a plant had a total score from the responses to the survey questions that loaded heavily on the factor greater than the mean for its respective size class then it was coded as 1 , otherwise it was coded as 0 . This process was completed for each of the factors in the study. The following is a table with all of the size class means for each of the factors described above. The means reported in table 1 were the cut-off values for determining if a plant exhibited the factor. 


\begin{tabular}{|c|c|c|c|}
\hline Factor & Large Plant & Medium Plant & Small Plant \\
\hline \multicolumn{4}{|l|}{ Implemented AMT } \\
\hline Production Design & 5.2334 & 2.8589 & 1.9870 \\
\hline Communication & 5.1268 & 2.7426 & 1.7138 \\
\hline Inspection and Storage & 1.6739 & 0.5422 & 0.3370 \\
\hline Robotics & 1.0240 & 0.3372 & 0.2844 \\
\hline \multicolumn{4}{|l|}{ Business Practices } \\
\hline Quality Control & 3.9510 & 2.3569 & 1.5087 \\
\hline Planning & 2.2126 & 1.2990 & 0.9244 \\
\hline Design & 2.2003 & 1.2632 & 0.8692 \\
\hline \multicolumn{4}{|l|}{ Sources of Implementation Support } \\
\hline Primary Stakeholders & 5.9096 & 4.2986 & 2.8610 \\
\hline Research and Development & 3.1293 & 2.0230 & 1.0992 \\
\hline External Information Sources & 2.9022 & 2.2305 & 1.5373 \\
\hline Corporate Organizations & 1.9847 & 1.2442 & 0.7220 \\
\hline \multicolumn{4}{|l|}{ Human Resources Practices } \\
\hline Human Resources & 1.7874 & 1.0948 & 0.9075 \\
\hline \multicolumn{4}{|l|}{ Research and Development Practices } \\
\hline Research and Development & 1.4705 & 1.0555 & 0.6848 \\
\hline \multicolumn{4}{|l|}{ Perceived Benefits of AMT } \\
\hline Improved Time Usage & 21.6682 & 17.8889 & 13.9126 \\
\hline Improved Productivity & 10.8436 & 8.7161 & 6.1236 \\
\hline Improved Flexibility & 11.6430 & 9.4633 & 6.9600 \\
\hline Improved Profitability & 11.7809 & 9.7180 & 7.1841 \\
\hline \multicolumn{4}{|l|}{ High-Level Factors } \\
\hline Perceived Benefits & 55.9358 & 45.7863 & 34.1803 \\
\hline AMT \& Business Practices & 13.4766 & 7.0622 & 4.5541 \\
\hline Sources of Implementation Support and R\&D & 14.3291 & 10.1386 & 6.2588 \\
\hline Design & 5.7934 & 2.9262 & 2.3291 \\
\hline Human Resources Practices & 1.7874 & 1.0948 & 0.9075 \\
\hline
\end{tabular}

Table 1 Factor Means by Size Class

\subsubsection{Factor Analysis Results}

The results of the factor analysis for each of the higher-level factor types described in the theoretical constructs are examined in this section. For each factor type, the variables loading heavily on each factor are identified and the proportion of variance, as well as the variance 
explained, by each factor are presented. Finally, the high-level factors themselves are determined and their statistical output analysed.

\subsubsection{AMT Classification}

There are four factors used to describe the AMT classifications used by the plants. These were obtained using Maximum Likelihood factor analysis but similar results were also obtained using the Principle Components method. All factors with an eigenvalue greater than one were retained. The four factors explain $99.93 \%$ of the variance (refer to Table 2). The hypothesis that these four factors adequately explain the variance between plants could not be rejected at a $95 \%$ confidence level.

\begin{tabular}{|l|c|c|}
\hline \multicolumn{1}{|c|}{ Factor } & $\begin{array}{c}\text { Variance Explained } \\
\text { (Weighted) }\end{array}$ & $\begin{array}{c}\text { Variance Explained } \\
\text { (Unweighted) }\end{array}$ \\
\hline 1 - Production Design & $40.13 \%$ & $36.63 \%$ \\
\hline 2 - Communications & $16.92 \%$ & $24.54 \%$ \\
\hline 3 - Inspection and Storage & $16.32 \%$ & $18.95 \%$ \\
\hline 4 - Robotics & $26.56 \%$ & $19.78 \%$ \\
\hline
\end{tabular}

Table 2 Variance Explained by AMT Factors

The first factor represents the production design technologies. These include the use of local area networks (LANs), $\mathrm{CAD} / \mathrm{CAE}, \mathrm{CAD} / \mathrm{CAM}$, computer modeling, electronic exchange of CAD files, programmable logic controllers (PLC), lasers, rapid prototyping, and high speed machining. The second factor represents the communication technologies. These include the use of wide area networks (WANs), electronic data interchange (EDI), electronic part identification, computer integrated manufacturing (CIM), supervisory control and data acquisition (SCADA), computers on the factory floor, the use of inspection data, remote controlled processes, knowledge-based software, and MRPII. The third factor represents the inspection and automated storage technologies. These include the use of automated storage and retrieval systems, as well 
as vision based and sensor based inspection systems. The final factor represents robotic technologies. These include flexible manufacturing systems and flexible manufacturing cells (FMS/FMC), as well as robots with and without sensing capabilities.

\subsubsection{Management Related Factors}

There are two different types of management related factors. The first relates to the business practices used by the plants and the second relates to the plants' sources of implementation support. These two factors were treated separately for the variable reduction during the factor analysis as they measure different aspects of the management techniques used within the plant.

\subsection{Business Practices}

There are three factors to describe the business practices used by the plants. These were obtained using the Principle Components factor analysis. Three factors were retained to provide sufficient meaning to the results, therefore factors with eigenvalue greater than 0.3 were retained. Since eigenvalues less than one were used, the set of factors over identifies the model. The three factors explain $115.95 \%$ of the variance (refer to Table 3). The hypothesis that these three factors adequately explain the variance between plants could not be rejected at a $95 \%$ confidence level.

\begin{tabular}{|l|c|}
\hline \multicolumn{1}{|c|}{ Factor } & $\begin{array}{c}\text { Variance Explained } \\
\text { (Unweighted) }\end{array}$ \\
\hline 1 - Quality Control & $49.10 \%$ \\
\hline 2 - Planning & $35.80 \%$ \\
\hline 3 - Design & $31.05 \%$ \\
\hline
\end{tabular}

Table 3 Variance Explained by the Business Practices Factors

The first factor represents the quality control practices. These include the use of continuous improvement, benchmarking, certifications of suppliers and plant, and statistical control. The second factor represents the planning practices used at the plant. These include the 
use of just-in-time inventory planning (JIT), electronic work order, process simulation, distribution resource planning, and quality function deployment. The third factor represents the design practices used at the plant. These include the use of cross-functional teams, concurrent engineering, and continuous product improvement.

\subsection{Sources of Implementation Support}

There are four factors used to describe the sources of implementation support used by the plants. These were obtained using the Maximum Likelihood factor analysis but similar results were also obtained using the Principle Components analysis method. Four factors were retained in order to provide a better description of the results, therefore factors with an eigenvalue greater than 0.6 were retained. The four factors explain $110.05 \%$ of the variance, as factors with eigenvalues less than one were retained (refer to Table 4). The hypothesis that these four factors adequately explain the variance between plants could not be rejected at a $95 \%$ confidence level.

\begin{tabular}{|l|c|c|}
\hline \multicolumn{1}{|c|}{ Factor } & $\begin{array}{c}\text { Variance Explained } \\
\text { (Weighted) }\end{array}$ & $\begin{array}{c}\text { Variance Explained } \\
\text { (Unweighted) }\end{array}$ \\
\hline 1 - Primary Stakeholders & $7.75 \%$ & $37.85 \%$ \\
\hline 2 - Research and Development & $7.76 \%$ & $26.99 \%$ \\
\hline 3 - External Information Sources & $5.16 \%$ & $24.01 \%$ \\
\hline 4 - Corporate Organizations & $89.38 \%$ & $21.20 \%$ \\
\hline
\end{tabular}

Table 4 Variance Explained by the Sources of Implementation Support Factors

The first factor represents the primary stakeholders. This includes support for implementation of advanced technologies from the production engineering staff, the production staff, the design teams, sales and marketing staff, direct suppliers, customers, and patents. The second factor represents support from research and development. This includes support for AMT from internal research, experimental development, production engineering, trade fairs, governments, and other producers. The third factor represents support from external information 
sources. These include sales and marketing, customers, as well as related firms. The final factor represents sources of implementation support from corporate organizations. These include the head office of the plant, related plants within the larger organization, and universities.

\subsubsection{Environmental Factors}

There are two different types of environmental factors. The first relates to the human resources practices used by the plants and the second relates to the conduct of the research and development. These two types of factors were treated separately for the variable reduction during the factor analysis as they measure different aspects of the management techniques used at the plant.

\subsection{Human Resources Practices}

There is only one factor used to describe the human resources practices used within a plant. This was obtained using the Maximum Likelihood method but similar results were also obtained using Principle Components. The factor with an eigenvalue greater than one was retained. The factor explains $109.25 \%$ of the variance as all of the variables weight very heavily on the factor. The hypothesis that this factor adequately explains the variance between plants could not be rejected at a $95 \%$ confidence level. The factor includes such human resources practices as increased training, improved wages, and benefits, established stronger links with educational institutions, and searched for new skilled personnel.

\subsection{Research and Development Practices}

There is only one factor to describe the research and development practices within plants. The factor represents research and development practices, which include in-house, joint, and contracted out research and development. This factor was obtained using Maximum Likelihood (but similar results were also obtained using Principle Components). The factor with an 
eigenvalue greater than one was retained. The factor explains $100.00 \%$ of the variance. The hypothesis that this factor adequately explains the variance between plants could not be rejected at a $95 \%$ confidence level.

\subsubsection{Perceived Benefits}

There are four factors used to describe the perceived benefits of implementing AMT. These were obtained using the Maximum Likelihood method. Four factors were retained for the analysis, therefore factors with an eigenvalue greater than 0.4 were retained. The four factors explain $107.67 \%$ of the variance (refer to Table 5). The hypothesis that these four factors adequately explain the variance between plants could not be rejected at a 95\% confidence level.

\begin{tabular}{|l|c|c|}
\hline \multicolumn{1}{|c|}{ Factor } & $\begin{array}{c}\text { Variance Explained } \\
\text { (Weighted) }\end{array}$ & $\begin{array}{c}\text { Variance Explained } \\
\text { (Unweighted) }\end{array}$ \\
\hline 1 - Improved Time Usage & $30.54 \%$ & $32.41 \%$ \\
\hline 2- Improved Productivity & $27.22 \%$ & $28.13 \%$ \\
\hline 3 - Improved Flexibility & $23.19 \%$ & $24.36 \%$ \\
\hline $4-$ Improved Profitability & $26.72 \%$ & $22.77 \%$ \\
\hline
\end{tabular}

Table 5 Variance Explained by the Perceived Benefits Factors

The first factor represents perceived time and money savings. These include reduced capital, reduced rejection time, reduced set-up time, increased quality, new product features, and reduced time to market. The second factor represents perceived productivity improvements. These include reduced labour, reduced material, reduced capital, increased utilization rate, and increased profitability. The third factor represents perceived flexibility improvements. These include increased flexibility, increased skill requirements, and increased quality. The final factor represents perceived increase in profitability. These include increased equipment utilization rate, increased market share, and increased profitability. 


\subsubsection{High-Level Factors}

There are five factors used to describe the high-level components used by the plants. The highlevel components are analysed in order to incorporate all of the various business practices, sources of implementation support, perceived benefits, and AMT into one model. Over 70 variables are used to create the five factors. These factors were obtained using the Maximum Likelihood factor analysis but similar results were also obtained using the Principle Components analysis method. Five of the sixteen factors with an eigenvalue greater than one were retained; all factors with an eigenvalue greater than three were retained. The five factors explain $78.37 \%$ of the variance (refer to Table 6). The hypothesis that these four factors adequately explain the variance between plants could not be rejected at a $95 \%$ confidence level.

\begin{tabular}{|l|c|c|}
\hline \multicolumn{1}{|c|}{ Factor } & $\begin{array}{c}\text { Variance } \\
\text { Explained } \\
\text { (Weighted) }\end{array}$ & $\begin{array}{c}\text { Variance Explained } \\
\text { (Unweighted) }\end{array}$ \\
\hline 1 - Perceived Benefits & $26.51 \%$ & $24.34 \%$ \\
\hline 2 - AMT \& Business Practices & $14.69 \%$ & $20.02 \%$ \\
\hline 3 - Sources of Implementation Support and R\&D & $12.05 \%$ & $14.82 \%$ \\
\hline 4 - Design & $10.32 \%$ & $11.06 \%$ \\
\hline 5 - Human Resources Practices & $14.81 \%$ & $8.14 \%$ \\
\hline
\end{tabular}

Table 6 Variance Explained by the High-Level Factors

The first factor represents the perceived benefits of implementing AMT. The second factor represents the advanced manufacturing technologies except for those related to production design and the business practices used by the plants. The third factor represents the source of implementation support used by the plants, cross functional teams, and the research and development practices of the plants. The fourth factor represents the design elements of the plants. These include the production design technologies from the AMT classification factor as 
well as concurrent engineering. The final factor represents the human resources practices of the plants.

\subsubsection{Plant Distributions by Size Class $^{7}$}

The following is an analysis of the distribution of plants by size class in the various factor states. Any state that did not contain any percentage of the population for a size class was treated as a special case in the regression analysis, as there was no way to approximate the coefficient for that state. Each of the factor types previously described is presented with some of the similarities and differences highlighted between the plant distributions.

\subsubsection{Advanced Manufacturing Technologies}

The distribution of the plants throughout the various states of AMT is spread over all states (refer to Table 7). Each of the size classes has a large percentage of plants in the initial state but only around $10 \%$ in the state containing all implemented AMT factors. There were no states that needed to be treated as a special case; although there were a few states that had a rather small percentage of plants.

The distribution of the medium and small plants was once again very similar. Close to $30 \%$ of medium plants and $40 \%$ of small plants did not exhibit any of the AMT factors (they are in state zero). There were some states, however, that did contain a significant proportion of medium and small plants. The states that included only the communication factor (state four) or only the production design factor (state eight) each represent around 10\% of the respective plants. The majority of the other plants that exhibited at least one of the AMT factors were found

\footnotetext{
${ }^{7}$ All the results presented in this section were calculated using weighted variables. The variable weights were created based on the population in a random sample stratified by both size class and industry.
} 
in states that included both of the communication and the production design factors (states twelve to fifteen). These results are not surprising as the types of AMT found in the inspection and storage and the robotics factors are used when there is a large manufacturing volume and are more costly to purchase and implement.

\begin{tabular}{|c|c|c|c|}
\hline State & 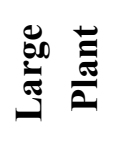 & $\underset{\sum}{\stackrel{\Xi}{\Xi}} \stackrel{\Xi}{\Xi}$ & 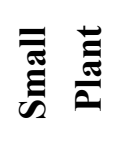 \\
\hline 0 - 0000 (no factors) & $18.39 \%$ & $27.76 \%$ & $39.22 \%$ \\
\hline 1 - 0001 (factor 4) & $2.68 \%$ & $1.27 \%$ & $1.54 \%$ \\
\hline 2 - 0010 (factor 3) & $10.03 \%$ & $2.77 \%$ & $3.27 \%$ \\
\hline $3-0011$ (factor $3 \& 4)$ & $1.26 \%$ & $0.30 \%$ & $0.03 \%$ \\
\hline $4-0100$ (factor 2) & $8.38 \%$ & $6.29 \%$ & $10.99 \%$ \\
\hline 5 - 0101 (factor $2 \& 4)$ & $1.14 \%$ & $0.43 \%$ & $1.92 \%$ \\
\hline $6-0110$ (factor $2 \& 3$ ) & $9.54 \%$ & $3.48 \%$ & $1.48 \%$ \\
\hline 7 - 0111 (factor $2,3 \& 4$ ) & $2.52 \%$ & $4.37 \%$ & $0.16 \%$ \\
\hline $8-1000$ (factor 1 ) & $5.63 \%$ & $10.44 \%$ & $10.08 \%$ \\
\hline $9-1001$ (factor $1 \& 4)$ & $3.10 \%$ & $4.15 \%$ & $1.64 \%$ \\
\hline $10-1010$ (factor $1 \& 3)$ & $10.62 \%$ & $3.14 \%$ & $1.57 \%$ \\
\hline 11 - 1011 (factor $1,3 \& 4$ ) & $3.02 \%$ & $0.48 \%$ & $0.71 \%$ \\
\hline $12-1100$ (factor $1 \& 2$ ) & $3.81 \%$ & $13.28 \%$ & $8.68 \%$ \\
\hline $13-1101$ (factor $1,2 \& 4)$ & $2.52 \%$ & $3.24 \%$ & $6.26 \%$ \\
\hline $14-1110$ (factor $1,2 \& 3$ ) & $6.18 \%$ & $8.68 \%$ & $5.24 \%$ \\
\hline 15 - 1111 (all factors) & $11.18 \%$ & $9.93 \%$ & $7.20 \%$ \\
\hline
\end{tabular}

Factor 1= Production Design (LAN, CAD/CAE, CAD/CAM, Modeling, Electronic exchange of CAD files, PLC, Lasers, Rapid prototyping, High speed machining)

Factor 2 = Communications (WAN, EDI, Electronic part identification, CIM, SCADA, Computer on factory floor, Use of inspection data, Remote controlled processes, Knowledge-based software, MRPII)

Factor 3 = Inspection, and Storage (Automated storage and retrieval, Vision based, Sensor based)

Factor 4 = Robotics (FMS/FMC, Robots with and without sensing)

Table 7 Plant Distributions for Advanced Manufacturing Technologies by Size Class

The distribution of the large plants was heavily linked to the use of inspection and storage

AMT. The states that included the inspection and storage factor on its own (state two), or with 
the communication or production design factors (states six and ten), contained approximately $10 \%$ of the large plants. The strong use of this form of AMT by large plants was consistent with their reliance on quality control business practices. Once again, the robotics factor was not exhibited by most large plants.

\subsubsection{Business Practices}

The distribution of the plants throughout the various states of business practices resulted in at least $1.30 \%$ of plants in any state (refer to Table 8 ). Each of the size classes had a large percentage of plants in states at both ends of the spectrum. There were no states that needed to be treated as a special case, but there were some interesting features.

\begin{tabular}{|c|c|c|c|}
\hline State & 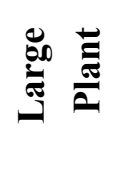 & 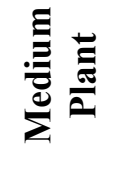 & పే \\
\hline 0 - 000 (no factors) & $18.12 \%$ & $32.51 \%$ & $37.95 \%$ \\
\hline $1-001$ (factor 3) & $3.27 \%$ & $7.11 \%$ & $8.21 \%$ \\
\hline $2-010$ (factor 2) & $4.39 \%$ & $8.95 \%$ & $5.67 \%$ \\
\hline $3-011($ factor $2 \& 3)$ & $1.30 \%$ & $4.20 \%$ & $7.78 \%$ \\
\hline $4-100($ factor 1$)$ & $18.26 \%$ & $12.85 \%$ & $1.47 \%$ \\
\hline 5 - 101 (factor $1 \& 3)$ & $21.94 \%$ & $10.81 \%$ & $4.60 \%$ \\
\hline $6-110$ (factor $1 \& 2)$ & $9.83 \%$ & $5.87 \%$ & $4.48 \%$ \\
\hline 7 - 111 (all factors) & $22.89 \%$ & $17.70 \%$ & $29.84 \%$ \\
\hline
\end{tabular}

Factor 1 = Quality Control (Continuous improvement, benchmarking, Certifications of suppliers and plant, and statistical control)

Factor 2 = Planning (JIT, Electronic work order, process simulation, distribution resource planning, quality function deployment)

Factor 3 = Design (Cross functional teams, concurrent engineering, continuous improvement)

Table 8 Plant Distributions for Business Practices by Size Class

The first state examined was the one that contains both the planning and design factors (state three). This state contained a relatively small percentage of large and medium plants but 
has almost $8 \%$ of the small plants. This was the fourth most common state for small plants. The reason such a large percentage of small plants were found in this state was the apparent lack of use of the quality control factor without the plant also using all of the other business practices factors. Almost $30 \%$ of the small plants used all of the factors but very few with only the quality control factor on its own or with one other factor. The large and medium plants exhibited a much more even distribution between the plants that have the quality control factor. The majority of the larger plants, $72.92 \%$, had the quality control factor as part of their business practice state. This demonstrates the importance that larger plants place on this aspect of their business.

\subsubsection{Human Resources}

\begin{tabular}{|c|c|c|c|}
\hline State & 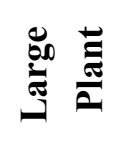 & $\underset{\sum}{\stackrel{\Xi}{\Xi}} \stackrel{\Xi}{\Xi}$ & 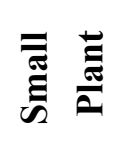 \\
\hline $0-0$ (no factor) & $46.39 \%$ & $64.91 \%$ & $68.23 \%$ \\
\hline $1-1$ (factor 1$)$ & $53.61 \%$ & $35.09 \%$ & $31.77 \%$ \\
\hline
\end{tabular}

Factor 1 = Human Resource Policies (Increased training, Improved benefits, Increased education links, Hired more skilled personnel)

Table 9 Plant Distributions for Human Resources Practices by Size Class

The distribution of the sole human resources factor was consistent over the different plant sizes (refer to Table 9). It did not appear that the proportionate use of human resource initiatives differed dramatically by plant size. It must be stressed again however, that the variables used to determine these values were based on comparisons to individual means for each size class. 


\subsubsection{Research and Development}

\begin{tabular}{|c|c|c|c|}
\hline State & 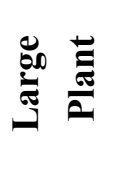 & 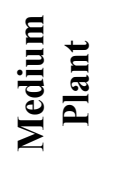 & 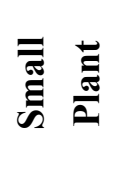 \\
\hline $0-0$ (no factor) & $53.28 \%$ & $74.39 \%$ & $53.49 \%$ \\
\hline $1-1$ (factor 1$)$ & $46.72 \%$ & $25.61 \%$ & $46.51 \%$ \\
\hline
\end{tabular}

Factor $1=$ Research \& Development (Conduct internal R\&D, Conduct Joint R\&D, Hire consultants for $\mathrm{R} \& \mathrm{D})$

Table 10 Plant Distributions for Research and Development Practices by Size Class

The distribution of the research and development factor was not consistent over the different plant sizes (refer to Table 10). The distribution for the large and small plants was very similar but the distribution of medium plants was dramatically different. As with the all the factors, the variables used to determine these values were based on comparisons to individual means for each size class. This therefore implies that the distribution for the plants should have been close to a 50-50 split between the two states. The distribution found in the medium plants would imply that there was a small number of medium plants that had a high amount of R\&D occurring and a much larger percentage that were only pursuing one or no R\&D avenues.

\subsubsection{Implementation Support}

The distribution of the plants throughout the various states of sources of implementation support was uneven (refer to Table 11). Each of the size classes had a large percentage of plants in states at both ends of the spectrum, but due to the large number of states, there were many states containing small percentages of plants. In particular, the state containing only the research and development group and external information as sources for support (state six) needed to be 
treated as a special case as there were very few, if any, plants in this state. Since there were such small percentages of plants in this state, it was removed entirely for the regression of analysis done by size class.

Looking at the distribution of large plants, there were a much larger percentage of plants exhibiting all of the factors of sources of implementation support (primary stakeholders, research and development, external information sources, and corporate organizations) than those of medium and small plants. The large and medium plants seemed to rely more heavily on external sources and were not as dependent on having primary stakeholders involved in the implementation. This was exhibited by the fact that almost $10 \%$ of the large and medium plants were found in the state that contains only external information sources and corporate organizations as factors for implementation support (state three).

The distribution of smaller plants also supported their need for primary stakeholder involvement as approximately $85 \%$ of small plants that exhibited one of the implementation support factors exhibited at least the primary stakeholder factor (states eight to fifteen). Both large and medium plants also had over $70 \%$ of plants (that exhibited at least one source of implementation support factor) exhibiting the primary stakeholder factor as part of their implementation support state. This distribution supports the belief that an internal project champion and commitment from the entire organization is important for the implementation of AMT. 


\begin{tabular}{|c|c|c|c|}
\hline State & 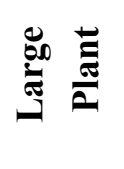 & 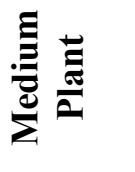 & 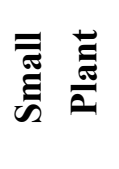 \\
\hline $0-0000$ (no factors) & $11.16 \%$ & $25.71 \%$ & $37.71 \%$ \\
\hline $1-0001$ (factor 4) & $7.54 \%$ & $4.56 \%$ & $4.71 \%$ \\
\hline $2-0010$ (factor 3$)$ & $1.62 \%$ & $1.67 \%$ & $1.76 \%$ \\
\hline $3-0011$ (factor $3 \& 4)$ & $10.29 \%$ & $9.45 \%$ & $0.83 \%$ \\
\hline $4-0100$ (factor 2$)$ & $1.09 \%$ & $2.52 \%$ & $1.22 \%$ \\
\hline 5 - 0101 (factor $2 \& 4)$ & $1.91 \%$ & $2.03 \%$ & $0.37 \%$ \\
\hline $6-0110$ (factor $2 \& 3)$ & $0.34 \%$ & $0.17 \%$ & $0.00 \%$ \\
\hline 7 - 0111 (factor $2,3 \& 4)$ & $1.13 \%$ & $1.63 \%$ & $0.43 \%$ \\
\hline $8-1000($ factor 1$)$ & $4.47 \%$ & $5.16 \%$ & $2.97 \%$ \\
\hline 9 - 1001 (factor 1\& 4) & $4.03 \%$ & $1.54 \%$ & $1.74 \%$ \\
\hline $10-1010($ factor $1 \& 3)$ & $4.60 \%$ & $8.49 \%$ & $7.83 \%$ \\
\hline $11-1011$ (factor $1,3 \& 4)$ & $14.27 \%$ & $6.14 \%$ & $12.72 \%$ \\
\hline $12-1100$ (factor $1 \& 2$ ) & $1.04 \%$ & $4.81 \%$ & $0.91 \%$ \\
\hline $13-1101$ (factor $1,2 \& 4$ ) & $1.55 \%$ & $2.22 \%$ & $4.63 \%$ \\
\hline $14-1110$ (factor $1,2 \& 3$ ) & $5.02 \%$ & $10.75 \%$ & $4.34 \%$ \\
\hline $15-1111$ (all factors) & $29.94 \%$ & $13.14 \%$ & $17.85 \%$ \\
\hline
\end{tabular}

Factor 1 = Primary Stakeholders (Production Engineering, Production, Design, Sales and Marketing staff, Suppliers, Customers, Patents)

Factor 2 = Research \& Development (Research, Experimental development, Production engineering, Trade Fairs, Governments, Other producers)

Factor 3 = External Information Sources (Sales and Marketing, Customers, Related Firms)

Factor 4 = Corporate Organizations (Head Office, Related plants, Universities)

Table 11 Plant Distributions for Sources of Implementation Support by Size Class

\subsubsection{Perceived Benefits}

The distribution of the plants throughout the various states of perceived benefits included plants in every state (refer to Table 12). However, each of the size classes had a large percentage of plants in states at both ends of the spectrum. Due to the large number of states there were many states containing small percentages of plants. In particular, the state containing only the improved time and productivity factors as perceived benefits (state twelve) needed to be treated 
as a special case as there were very few plants in this state. Since there were no occurrences of small plants in this state, it was removed from the regression analysis pertaining to small plants.

\begin{tabular}{|c|c|c|c|}
\hline State & 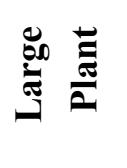 & 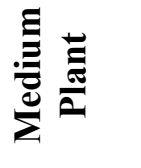 & 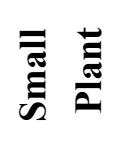 \\
\hline 0 - 0000 (no factors) & $15.86 \%$ & $19.18 \%$ & $35.57 \%$ \\
\hline $1-0001$ (factor 4) & $4.98 \%$ & $2.76 \%$ & $0.92 \%$ \\
\hline $2-0010$ (factor 3) & $2.52 \%$ & $2.08 \%$ & $2.87 \%$ \\
\hline 3 - 0011 (factor $3 \&$ 4) & $9.98 \%$ & $2.05 \%$ & $0.77 \%$ \\
\hline 4 - 0100 (factor 2) & $2.81 \%$ & $4.25 \%$ & $0.56 \%$ \\
\hline 5 - 0101 (factor $2 \& 4$ ) & $1.96 \%$ & $1.11 \%$ & $0.74 \%$ \\
\hline $6-0110$ (factor $2 \& 3$ ) & $2.73 \%$ & $0.36 \%$ & $0.61 \%$ \\
\hline 7 - 0111 (factor 2, $3 \& 4$ ) & $2.65 \%$ & $1.84 \%$ & $0.88 \%$ \\
\hline $8-1000$ (factor 1$)$ & $1.58 \%$ & $0.40 \%$ & $0.02 \%$ \\
\hline 9 - 1001 (factor 1\& 4) & $1.22 \%$ & $0.62 \%$ & $0.10 \%$ \\
\hline $10-1010$ (factor $1 \& 3$ ) & $3.71 \%$ & $1.86 \%$ & $1.23 \%$ \\
\hline $11-1011$ (factor $1,3 \& 4$ ) & $6.60 \%$ & $5.78 \%$ & $8.33 \%$ \\
\hline $12-1100$ (factor $1 \& 2$ ) & $1.76 \%$ & $1.64 \%$ & $0.00 \%$ \\
\hline $13-1101$ (factor $1,2 \& 4)$ & $10.65 \%$ & $7.68 \%$ & $3.02 \%$ \\
\hline $14-1110$ (factor $1,2 \& 3)$ & $4.20 \%$ & $3.56 \%$ & $1.92 \%$ \\
\hline 15 - 1111 (all factors) & $26.77 \%$ & $44.80 \%$ & $42.47 \%$ \\
\hline
\end{tabular}

Factor 1 = Time (Reduced Capital, Reduced rejection time, Reduced set-up time, Increased quality, New product features, Reduced time to market)

Factor 2 = Productivity (Reduced labour, Reduced material, Reduced capital) Increased utilization rate, Increased Profitability)

Factor 3 = Flexibility (Increased flexibility, Increased skill requirements, Increased quality)

Factor 4 = Profitability (Increased equipment utilization rate, Increased market share, Increased Profitability)

Table 12 Plant Distributions for Perceived Benefits of AMT by Size Class

The distribution of the medium and small plants was fairly similar. There were a large number of plants that exhibited none of the perceived benefit factors and an even larger percentage that exhibit all of the factors. The only other states that contained a significant number of medium and small plants are those that included the perceived benefit factor of time 
improvements as well as two of the remaining factors (states thirteen to fifteen). For medium sized plants, the state that consisted of the productivity factor on its own (state four) was also significant. The occurrences of the large percentage of plants in the extreme ends of the spectrum indicate that the medium and small plants perceived that AMT assisted them in either none or all of their competitive manufacturing goals. The fact that such a large percentage believed AMT will benefit so many aspects of their manufacturing process could be one of the reasons so many perceive the implementation of AMT as a failure as it would be virtually impossible for an implementation of AMT to achieve such a large impact on its own.

The distribution of the large plants was quite different. It was much more evenly distributed among the states but still retained the emphasis on the states including the perceived benefit factor of time improvements as well as two of the remaining factors (states thirteen to fifteen). The state including the perceived benefits of flexibility and profitability (state three) was also an important one for larger plants as almost $10 \%$ of the large plants occupied this state. These results demonstrate the diversity of goals that large plants have for AMT.

\subsubsection{High-Level Factors}

The distribution of the plants throughout the various states of high-level factors was relatively concentrated on those states that included at least the perceived benefit factor or the AMT and business practices factor (refer to Table13). Thirty percent of the small plants exhibited none of the high-level factors, decreasing by a factor of two as the plant size increases. Only around 10\% of the plants, irrespective of size class, were in the state containing all high-level factors. There were no states that needed to be treated as a special case; although there were a few states that had a rather small percentage of plants due the large number of possible states. 


\begin{tabular}{|c|c|c|c|c|c|c|c|}
\hline State & 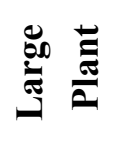 & 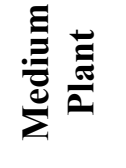 & 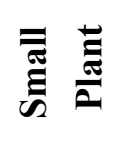 & State & 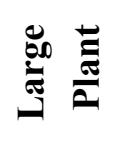 & $\stackrel{\Xi}{\sum^{e}}$ & 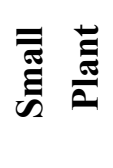 \\
\hline $0-00000$ (no factors) & $8.52 \%$ & $16.79 \%$ & $30.69 \%$ & $16-10000$ (factor 1$)$ & $5.08 \%$ & $4.01 \%$ & $3.19 \%$ \\
\hline $1-00001$ (factor 5) & $4.85 \%$ & $0.49 \%$ & $0.54 \%$ & $17-10001$ (factor 1\& 5) & $7.72 \%$ & $2.42 \%$ & $1.02 \%$ \\
\hline $2-00010$ (factor 4) & $0.77 \%$ & $2.57 \%$ & $3.52 \%$ & 18 - 10010 (factor 1\& 4) & $0.58 \%$ & $2.07 \%$ & $0.72 \%$ \\
\hline $3-00011$ (factor $4 \& 5)$ & $1.08 \%$ & $0.18 \%$ & $0.04 \%$ & $19-10011$ (factor1,4\&5) & $4.00 \%$ & $2.63 \%$ & $0.57 \%$ \\
\hline $4-00100$ (factor 3) & $0.50 \%$ & $1.68 \%$ & $0.81 \%$ & $20-10100$ (factor $1 \& 3$ ) & $0.84 \%$ & $3.66 \%$ & $3.57 \%$ \\
\hline $5-00101$ (factor $3 \& 5)$ & $1.63 \%$ & $1.25 \%$ & $0.06 \%$ & $21-10101($ factor $1,3 \& 5)$ & $1.62 \%$ & $6.03 \%$ & $4.79 \%$ \\
\hline $6-00110($ factor $3 \& 4)$ & $0.31 \%$ & $1.32 \%$ & $0.41 \%$ & $22-10110$ (factor1,3\&4) & $0.52 \%$ & $9.01 \%$ & $4.20 \%$ \\
\hline $7-00111$ (factor3,4\&5) & $0.67 \%$ & $0.78 \%$ & $0.29 \%$ & 23-10111(factor1,3,4\&5) & $3.95 \%$ & $3.11 \%$ & $3.97 \%$ \\
\hline $8-01000$ (factor 2) & $1.79 \%$ & $2.12 \%$ & $3.67 \%$ & $24-11000$ (factor $1 \& 2$ ) & $2.07 \%$ & $2.33 \%$ & $3.16 \%$ \\
\hline $9-01001$ (factor $2 \& 5)$ & $1.48 \%$ & $0.02 \%$ & $0.02 \%$ & 25-11001 (factor $1,2 \& 5)$ & $2.27 \%$ & $1.00 \%$ & $5.19 \%$ \\
\hline $10-01010$ (factor $2 \& 4)$ & $2.24 \%$ & $1.54 \%$ & $0.32 \%$ & $26-11010$ (factor $1,2 \& 4)$ & $1.53 \%$ & $2.71 \%$ & $0.65 \%$ \\
\hline $11-01011$ (factor2,4\&5) & $3.28 \%$ & $0.86 \%$ & $0.16 \%$ & 27-11011(factor1,2,4\&5) & $3.50 \%$ & $2.65 \%$ & $0.05 \%$ \\
\hline $12-01100$ (factor $2 \& 3)$ & $2.35 \%$ & $0.60 \%$ & $0.55 \%$ & $28-11100$ (factor $1,2 \& 3)$ & $4.97 \%$ & $2.55 \%$ & $3.96 \%$ \\
\hline 13-01101(factor2,3\&5) & $1.53 \%$ & $0.12 \%$ & $0.07 \%$ & 29-11101(factor $1,2,3 \& 5)$ & $2.85 \%$ & $2.78 \%$ & $2.98 \%$ \\
\hline 14-01110(factor2,3\&4) & $11.42 \%$ & $1.66 \%$ & $0.27 \%$ & $3-11110$ (factor1,2,3\&4) & $3.12 \%$ & $10.30 \%$ & $8.54 \%$ \\
\hline 15-01111(factor2,3,4\&5) & $2.14 \%$ & $1.38 \%$ & $0.05 \%$ & $31-11111$ (all factors) & $10.84 \%$ & $9.38 \%$ & $11.96 \%$ \\
\hline
\end{tabular}

Factor $1=$ Perceived Benefits

Factor 2 = Advanced Manufacturing Technologies (Except Design) \& Business Practices

Factor 3 = Implementation Support, Cross Functional Teams \& Research and Development

Factor $4=$ Design (Production Design Technologies, Concurrent Engineering)

Factor $5=$ Human Resources

Table 13 Plant Distributions for High-Level Factors by Size Class

The distribution of the large plants was marked by a relatively regular distribution of plants once the factors of AMT and business practices or perceived benefits were included in the state (states eight to thirty-one). In particular the state that exhibited the AMT and business practices, sources of implementation support and the design factor (state fourteen) contained over $11 \%$ of the large plants. There were also a large number of plants found in the states that included both the AMT and business practices factor as well as the perceived benefits factor (states twenty-three to thirty-one). Another state in which the distribution of large plants differed dramatically from that of the medium and small plants was state one. This state consists of only 
the human resources practices factor and contained ten times more large plants than either medium or small plants at almost $5 \%$ of the population of large plants. The higher percentage of large plants in this state could be due to the fact that larger plants normally have a department that is solely dedicated to human resources, which may not exist in the smaller plants.

The distribution of medium plants once again sees the large proportion of plants exhibiting at least the perceived benefits factor. The highest concentrations of plants, other than at the states with all or all but the human resources factor, were found in the states that included the perceived benefits and the sources of implementation support factors as well as one of the design or human resources factors (states twenty-one and twenty-two). There were not as many medium plants as large plants in the states containing the AMT factor without the perceived benefits factor. The inclusion of the perceived benefits factor appears to be important for medium plants that exhibit any of the remaining high-level factors.

The importance of the perceived benefits factor was highlighted once again in the distribution of the small plants. The inclusion of this factor, along with the sources of implementation support factor, was critical for the small plants that exhibit at least one of the high-level factors. There were few small plants the exhibit the human resources factor or the design factor unless the implementation support factor was present. This distribution demonstrates the additional monetary constraint that small plants have for AMT implementations as they put a high emphasis on perceiving a large number of benefits and had a significant level of support for the implementation before it was conducted. 


\subsubsection{Plant Distributions by Industry ${ }^{8}$}

Whether a particular plant had a factor was calculated using means based on the size class of the plant. Thus, the size class of the plant still had an impact on this portion of the analysis. Any state that did not contain any percentage of the sample for a size class was treated as a special case in the regression analysis, as there was no way to approximate the coefficient for that state. Each of the factor types previously described is presented with some of the similarities and differences highlighted between the plant distributions.

\subsubsection{Advanced Manufacturing Technologies}

The distribution of the plants throughout the various states of AMT was very sporadic (refer to Table 14). Each of the industries, except the metals and textile industries had a large percentage of plants in states at both ends of the spectrum but there remain states that contained very few plants. In particular, state nine was empty in the chemical industry, states three, five, and seven were empty in the machinery industry, state five was empty in the plastics industry, and state eleven was empty in the textiles industry. All of these states were dropped in the regression analysis for the respective industries.

There were a number of industries that had an extremely high proportion of plants without any of the AMT factors. These were in the traditional "low-technology" industries including furniture and other, non-metals, textiles. These industries had at least $39 \%$ of their population reside in the state without any AMT factors. In particular, the textile industry had over $57 \%$ of its plant population exhibiting none of the AMT factors. On the other extreme, the traditional high-technology or medium-technology industries including electronics, machinery,

\footnotetext{
${ }^{8}$ All the results presented in this section were once again calculated using weighted variables.
} 
plastics, and vehicles, had a high proportion of plants with all of the AMT factors (state fifteen).

These industries had at least $13.8 \%$ of their population found in this state.

\begin{tabular}{|c|c|c|c|c|c|c|c|c|c|c|}
\hline State & 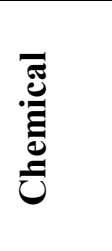 & 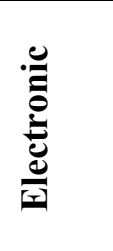 & 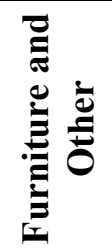 & 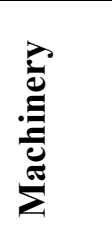 & $\sum_{\sum}^{\frac{n}{\pi}}$ & 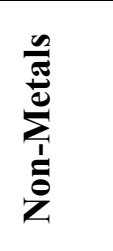 & 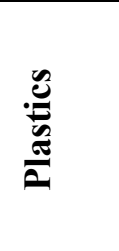 & 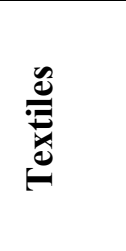 & $\frac{\tilde{e}}{\stackrel{0}{e}}$ & $\begin{array}{l}\overrightarrow{8} \\
\dot{8} \\
\dot{3}\end{array}$ \\
\hline 0-0000 (no factors) & $33.97 \%$ & $22.53 \%$ & $41.63 \%$ & $21.66 \%$ & $29.86 \%$ & $39.31 \%$ & $27.48 \%$ & $57.46 \%$ & $22.13 \%$ & $33.65 \%$ \\
\hline 1-0001 (factor 4) & $0.69 \%$ & $0.32 \%$ & $1.49 \%$ & $1.18 \%$ & $0.02 \%$ & $2.88 \%$ & $1.08 \%$ & $2.62 \%$ & $1.01 \%$ & $3.03 \%$ \\
\hline 2-0010 (factor 3) & $12.34 \%$ & $0.97 \%$ & $1.19 \%$ & $0.20 \%$ & $0.29 \%$ & $0.32 \%$ & $1.79 \%$ & $5.00 \%$ & $4.52 \%$ & $7.79 \%$ \\
\hline 3-0011 (factor 3 \& 4) & $0.14 \%$ & $0.10 \%$ & $0.27 \%$ & $0.00 \%$ & $0.07 \%$ & $0.61 \%$ & $0.51 \%$ & $0.17 \%$ & $0.40 \%$ & $0.20 \%$ \\
\hline 4-0100 (factor 2) & $9.12 \%$ & $4.09 \%$ & $5.08 \%$ & $6.87 \%$ & $13.22 \%$ & $9.77 \%$ & $15.39 \%$ & $7.54 \%$ & $3.93 \%$ & $9.97 \%$ \\
\hline 5-0101 (factor $2 \& 4)$ & $0.68 \%$ & $0.19 \%$ & $0.17 \%$ & $0.00 \%$ & $2.57 \%$ & $2.48 \%$ & $0.00 \%$ & $3.02 \%$ & $0.10 \%$ & $1.42 \%$ \\
\hline 6-0110 (factor $2 \& 3)$ & $7.58 \%$ & $2.12 \%$ & $1.66 \%$ & $0.08 \%$ & $0.48 \%$ & $5.02 \%$ & $5.59 \%$ & $3.98 \%$ & $0.07 \%$ & $3.94 \%$ \\
\hline 7-0111 (factor 2,3\&4) & $0.62 \%$ & $1.63 \%$ & $0.10 \%$ & $0.00 \%$ & $0.05 \%$ & $2.72 \%$ & $1.96 \%$ & $1.31 \%$ & $9.08 \%$ & $3.10 \%$ \\
\hline $8-1000$ (factor 1) & $3.66 \%$ & $16.38 \%$ & $11.63 \%$ & $16.54 \%$ & $15.07 \%$ & $4.60 \%$ & $7.74 \%$ & $1.07 \%$ & $11.07 \%$ & $7.11 \%$ \\
\hline 9-1001 (factor $1 \&$ 4) & $0.00 \%$ & $4.69 \%$ & $5.40 \%$ & $8.03 \%$ & $1.98 \%$ & $0.60 \%$ & $0.95 \%$ & $0.05 \%$ & $1.11 \%$ & $2.37 \%$ \\
\hline 10-1010 (factor $1 \& 3$ ) & $1.15 \%$ & $0.88 \%$ & $1.69 \%$ & $6.94 \%$ & $0.74 \%$ & $1.31 \%$ & $5.79 \%$ & $2.34 \%$ & $9.48 \%$ & $2.45 \%$ \\
\hline 11-1011(factor1,3\&4) & $0.39 \%$ & $4.51 \%$ & $1.59 \%$ & $0.08 \%$ & $0.17 \%$ & $2.24 \%$ & $0.45 \%$ & $0.00 \%$ & $2.06 \%$ & $0.18 \%$ \\
\hline $12-1100$ (factor $1 \& 2)$ & $13.27 \%$ & $9.76 \%$ & $11.59 \%$ & $14.15 \%$ & $14.68 \%$ & $14.13 \%$ & $5.39 \%$ & $2.74 \%$ & $6.50 \%$ & $7.07 \%$ \\
\hline 13-1101(factor1,2\&4) & $1.35 \%$ & $9.26 \%$ & $4.92 \%$ & $3.61 \%$ & $9.12 \%$ & $3.34 \%$ & $6.28 \%$ & $1.38 \%$ & $3.13 \%$ & $3.35 \%$ \\
\hline 14-1110(factor1,2\&3) & $8.07 \%$ & $7.29 \%$ & $5.47 \%$ & $1.08 \%$ & $8.33 \%$ & $2.59 \%$ & $3.12 \%$ & $6.58 \%$ & $11.53 \%$ & $6.60 \%$ \\
\hline 15-1111 (all factors) & $6.98 \%$ & $15.27 \%$ & $6.13 \%$ & $19.58 \%$ & $3.34 \%$ & $8.09 \%$ & $16.48 \%$ & $4.70 \%$ & $13.89 \%$ & $7.77 \%$ \\
\hline
\end{tabular}

Factor $1=$ Production Design (LAN, CAD/CAE, CAD/CAM, Modeling, Electronic exchange of CAD files, PLC, Lasers, Rapid prototyping, High speed machining)

Factor 2 = Communications (WAN, EDI, Electronic part identification, CIM, SCADA, Computer on factory floor, Use of inspection data, Remote controlled processes, Knowledge-based software, MRPII)

Factor 3 = Inspection, and Storage (Automated storage and retrieval, Vision based, Sensor based)

Factor 4 = Robotics (FMS/FMC, Robots with and without sensing)

Table 14 Plant Distributions for Advanced Manufacturing Technologies by Industry

The chemical industry was unique in the high percentage of plants it had in the state consisting of only the inspection and storage AMT factor (state two). This state contained over $12 \%$ of the plants in the chemical industry. The chemical industry does have a lot of government regulated standards that it must conform to thus the inspection of the manufactured product is 
very important. The other interesting feature of the chemical industry was that the proportion of plants in the various states that included at least one AMT factor were relatively evenly distributed when compared to any of the other industries. There did not appear to be a dominant factor that is included in all plants.

This was not the case for the electronics industry though. The majority of the plants that exhibited at least one AMT factor were found in the states that included at least the production design factor (states eight to fifteen). Almost every plant that had implemented an AMT factor had implemented the production design factor. This result exhibits the need for production design technologies in the highly competitive and rapidly changing electronics market.

The machinery industry was unique in the high percentage of plants found in the state consisting of the production design and the robotics factors (state nine). There was eight percent of the machinery plants were resident in this state but only a small percentage of plants found in the majority of the other states.

Another state where there were a large proportion of the plants from the machinery industry was the state 10 . This state includes the production design and the inspection and storage factors. There were at least $6 \%$ of the machinery, plastics, and vehicle industries' plants found in this state. These industries produce larger manufactured pieces than many of the other industries and would therefore be able to efficiently use automated retrieval systems. The surprising fact was that when the robotics factor was added to the makeup of this state there were very few plants from any of these industries resident there (state eleven).

As in the analysis performed by size class, the production design and communication factors on their own contained a significant number of plants. The state consisting of the 
communication factor (state four) contained close to $10 \%$ of the chemical, metals, plastics, and wood industries. The state exhibiting only the production design factor (state eight) contained over $11 \%$ of the electronics, furniture and other, machinery, metals, and vehicles industries. These two factors were more widely used by the plants regardless of industry. In fact, the majority of the plants that did have at least one AMT factor were found in the states that included both the production design and communication factors.

\subsubsection{Business Practices}

The distribution of plants throughout the various states of business practices included representation of every state by the plants in each industry (refer to Table 15). Each of the industries had a large percentage of plants in states at both ends of the spectrum. There were no states that needed to be treated as a special case but there were some interesting features.

The first feature to note is that there were three distinct groupings of industries if one examined only the extreme ends of the state spectrum. The furniture, non-metals, wood, and textile industries had a large proportion of plants that exhibited none of the business factors. The proportion of plants in this state from these industries ranged from $38 \%$ in the non-metals industry, all the way to $53 \%$ of the textile plants. These are industries that are typically described as low-technology and as a result it is not surprising that they were not incorporating some of the more innovative business practices into there manufacturing processes. At the other extreme were the chemical, electronic, plastic and vehicle industries. These industries had a large proportion of plants exhibiting all the business factors. The proportion of plants in this state ranged from $32 \%$ in the plastics industry all the way to $48 \%$ in the chemical industry. This grouping coincides with typical "high-technology" industries. The remaining industries consisting of machinery and metals had a more balanced distribution between the two extremes. 


\begin{tabular}{|c|c|c|c|c|c|c|c|c|c|c|}
\hline State & & 异 & 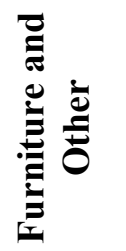 & 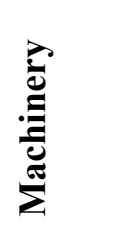 & $\frac{\frac{n}{\pi}}{\sum}$ & 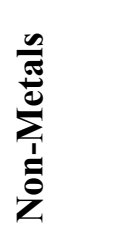 & $\begin{array}{l}\cdot \stackrel{0}{0} \\
\frac{\tilde{\sigma}}{a} \\
\underline{a}\end{array}$ & 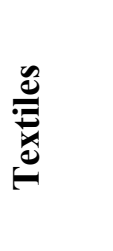 & $\frac{\frac{e}{e}}{\stackrel{e}{e}}$ & $\begin{array}{l}\dot{8} \\
\dot{0}\end{array}$ \\
\hline $0-000$ (no factors) & $15.34 \%$ & $19.25 \%$ & $42.84 \%$ & $21.05 \%$ & $34.87 \%$ & $38.24 \%$ & $20.26 \%$ & $53.08 \%$ & $22.87 \%$ & $39.46 \%$ \\
\hline 1-001 (factor 3) & $2.32 \%$ & $7.27 \%$ & $10.71 \%$ & $20.87 \%$ & $7.99 \%$ & $3.96 \%$ & $6.40 \%$ & $9.12 \%$ & $3.81 \%$ & $3.84 \%$ \\
\hline 2-010 (factor 2) & $7.10 \%$ & $4.40 \%$ & $7.77 \%$ & $1.29 \%$ & $7.73 \%$ & $4.72 \%$ & $1.99 \%$ & $7.42 \%$ & $5.69 \%$ & $8.93 \%$ \\
\hline 3-011 (factor $2 \& 3$ ) & $3.35 \%$ & $4.12 \%$ & $6.60 \%$ & $15.43 \%$ & $0.19 \%$ & $4.38 \%$ & $13.28 \%$ & $12.28 \%$ & $4.21 \%$ & $6.17 \%$ \\
\hline $4-100$ (factor 1$)$ & $5.30 \%$ & $7.40 \%$ & $1.72 \%$ & $5.84 \%$ & $9.11 \%$ & $8.54 \%$ & $12.85 \%$ & $2.42 \%$ & $5.29 \%$ & $6.40 \%$ \\
\hline 5-101 (factor $1 \& 3$ ) & $11.41 \%$ & $8.22 \%$ & $7.04 \%$ & $12.35 \%$ & $6.43 \%$ & $11.33 \%$ & $11.14 \%$ & $1.71 \%$ & $16.42 \%$ & $6.50 \%$ \\
\hline 6-110 (factor $1 \& 2$ ) & $7.25 \%$ & $6.26 \%$ & $3.12 \%$ & $0.30 \%$ & $8.36 \%$ & $6.78 \%$ & $1.90 \%$ & $4.91 \%$ & $5.06 \%$ & $5.33 \%$ \\
\hline 7-111 (all factors) & $47.93 \%$ & $43.09 \%$ & $20.20 \%$ & $22.86 \%$ & $25.32 \%$ & $22.04 \%$ & $32.18 \%$ & $9.07 \%$ & $36.65 \%$ & $23.39 \%$ \\
\hline
\end{tabular}

Factor 1 = Quality Control (Continuous improvement, benchmarking, Certifications of suppliers and plant, and statistical control)

Factor 2 = Planning (JIT, Electronic work order, process simulation, distribution resource planning, quality function deployment)

Factor 3 = Design (Cross functional teams, concurrent engineering, continuous improvement)

Table 15 Plant Distributions for Business Practices by Industry

Another grouping that occurred was in the state consisting of the planning and design business factors (state three). This state contained at least $12 \%$ of the machinery, plastics, and textile industries but not even $0.5 \%$ of the metals industry. These industries are not commonly grouped together as similar manufacturing processes.

A similar type of grouping also exists in the state consisting of the quality control and design business practices (state five). This state contained over $11 \%$ of plants in the chemical, machinery, non-metals, plastics, and vehicle industries. In these industries, the manufacturing process creates very specific components within tight tolerances in order to meet contractual standards and government regulations. 


\subsubsection{Human Resources}

The distribution of the sole human resources factor was inconsistent over the different industries (refer to Table 16). It appears that there were two different types of industries that occur with respect to the use of human resources policies. There are those that, for the most part, do not exhibit the human resources factor such as the chemical, furniture, machinery, non-metals, textiles, vehicles, and wood industries and those where it was more uniformly distributed such as the electronics, metals, and plastics industries. The reason why some of the more traditional industries may not have a high level of the human resources factor is that they do not find new personnel from educational institutions and do not increase training (as the techniques are taught primarily through apprenticeship programs).

\begin{tabular}{|c|c|c|c|c|c|c|c|c|c|c|}
\hline State & 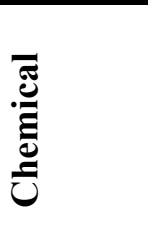 & 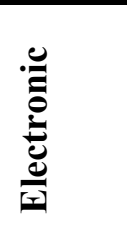 & 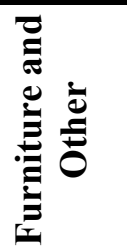 & 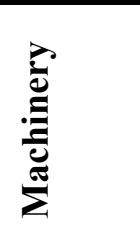 & 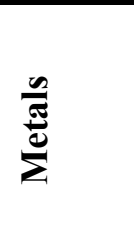 & 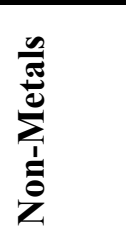 & 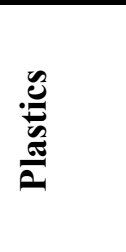 & 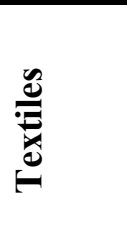 & 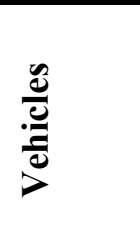 & $\begin{array}{l}\tilde{E} \\
\dot{8}\end{array}$ \\
\hline 0 (no factor) & $72.39 \%$ & $56.04 \%$ & $72.79 \%$ & $64.54 \%$ & $51.65 \%$ & $77.07 \%$ & $58.83 \%$ & $81.49 \%$ & $64.72 \%$ & $69.47 \%$ \\
\hline 1 (factor 1$)$ & $27.61 \%$ & $43.96 \%$ & $27.21 \%$ & $35.46 \%$ & $48.35 \%$ & $22.93 \%$ & $41.17 \%$ & $18.51 \%$ & $35.28 \%$ & $30.53 \%$ \\
\hline
\end{tabular}

Factor 1 = Human Resource Policies (Increased training, Improved benefits, Increased education links, Hired more skilled personnel)

Table 16 Plant Distributions for Human Resources Practices by Industry

\subsubsection{Research and Development}

The distribution of the research and development states can once again be broken down into three similar groups (refer to Table 17). The first grouping was that of the traditional lowtechnology industries including metals, textiles, and wood. These industries had at least $69 \%$ of their plants in the zero state (did not exhibit the R\&D factor). The second grouping was the moderate one where there was a balance between the proportion of plants with and without the 
R\&D factor. These included the furniture and other industry, the non-metals industry, and the vehicles industry. Finally, there were the traditional high-technology industries such as chemicals, electronics, and plastics, where at least $60 \%$ of the plants exhibited the R\&D factor.

\begin{tabular}{|c|c|c|c|c|c|c|c|c|c|c|}
\hline State & 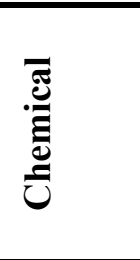 & 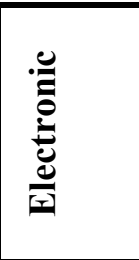 & 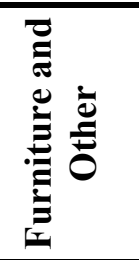 & 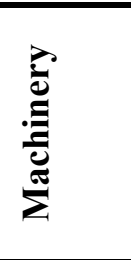 & $\frac{\frac{n}{\pi}}{\sum^{\frac{\pi}{0}}}$ & 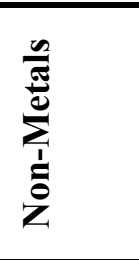 & 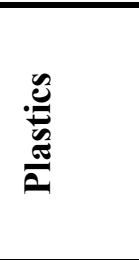 & 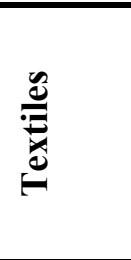 & 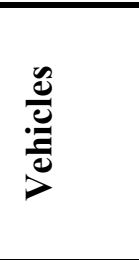 & $\begin{array}{l}\square \\
\dot{0}\end{array}$ \\
\hline 0 (no factor) & $28.60 \%$ & $35.16 \%$ & $59.10 \%$ & $44.15 \%$ & $69.03 \%$ & $57.83 \%$ & $39.86 \%$ & $73.03 \%$ & $50.49 \%$ & $74.01 \%$ \\
\hline 1 (factor 1 ) & $71.40 \%$ & $64.84 \%$ & $40.90 \%$ & $55.85 \%$ & $30.97 \%$ & $42.17 \%$ & $60.14 \%$ & $26.97 \%$ & $49.51 \%$ & $25.99 \%$ \\
\hline
\end{tabular}

Factor $1=$ Research \& Development (Conduct internal R\&D, Conduct Joint R\&D, Hire consultants for $R \& D)$

Table 17 Plant Distributions for Research and Development Practices by Industry

\subsubsection{Implementation Support}

The distribution of plants throughout the various states of sources of implementation support was uneven (refer to Table 18). Each industry, with the exception of the textiles industry, had a large percentage of plants in states at both ends of the spectrum but due to the large number of states, there were many cells containing small percentages of plants. In particular, the state containing only the research and development employees and external information as sources of support (state six), needed to be treated as a special case as there were very few plants in this state. Due to the small sample of plants in this state, it was removed entirely for the regression analysis done by industry. State seven was also empty in the machinery and textile industries and was removed from the regression analysis for those industries.

The majority of plants in all industries that had at least one factor were in states that contained the primary stakeholder factor. The importance of this factor was highlighted even more in the distribution of the plants by industry than by size class. The only industries that have 
any significant proportion of plants outside this definition were the metals and non-metals industries. These industries both had over $8 \%$ of their total population in the state consisting of only the corporate organizations factor (state one). Based on the analysis from the distribution by size class this would support the fact that most of the metals and non-metals plants are part of larger organizations and are not small manufacturing plants. In particular, the metals industry also had $12 \%$ of its population in the state consisting of only the external information and corporate organizations (state three), a state with a large proportion of large plants but very few small plants. 


\begin{tabular}{|c|c|c|c|c|c|c|c|c|c|c|}
\hline State & 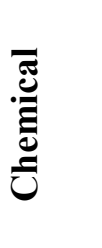 & 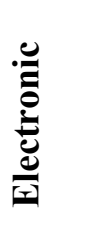 & ס气 & 总 & 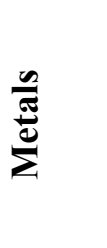 & 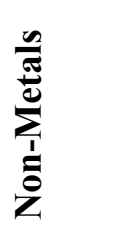 & 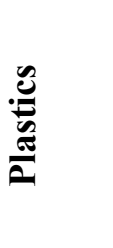 & 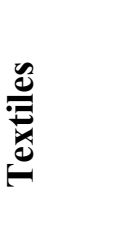 & $\frac{\frac{o}{e}}{\stackrel{e}{e}}$ & $\frac{7}{8}$ \\
\hline 0-0000 (no factors) & $38.00 \%$ & $23.55 \%$ & $38.70 \%$ & $29.30 \%$ & $23.80 \%$ & $38.40 \%$ & $26.31 \%$ & $58.50 \%$ & $23.00 \%$ & $28.26 \%$ \\
\hline 1-0001 (factor 4) & $2.71 \%$ & $1.53 \%$ & $1.86 \%$ & $3.63 \%$ & $8.43 \%$ & $8.50 \%$ & $4.59 \%$ & $4.69 \%$ & $2.79 \%$ & $4.58 \%$ \\
\hline 2-0010 (factor 3) & $0.74 \%$ & $1.74 \%$ & $2.66 \%$ & $2.67 \%$ & $0.34 \%$ & $0.24 \%$ & $0.08 \%$ & $0.13 \%$ & $1.84 \%$ & $3.86 \%$ \\
\hline 3-0011 (factor 3\&4) & $0.67 \%$ & $3.74 \%$ & $1.59 \%$ & $0.17 \%$ & $12.05 \%$ & $0.15 \%$ & $1.87 \%$ & $1.05 \%$ & $2.77 \%$ & $3.99 \%$ \\
\hline 4-0100 (factor 2) & $2.64 \%$ & $2.55 \%$ & $2.71 \%$ & $1.19 \%$ & $0.64 \%$ & $0.61 \%$ & $0.45 \%$ & $3.69 \%$ & $3.78 \%$ & $0.82 \%$ \\
\hline 5-0101 (factor 2\&4) & $0.74 \%$ & $2.05 \%$ & $0.39 \%$ & $0.17 \%$ & $1.07 \%$ & $0.70 \%$ & $1.29 \%$ & $1.61 \%$ & $3.69 \%$ & $0.46 \%$ \\
\hline $6-0110$ (factor $2 \& 3)$ & $0.13 \%$ & $0.23 \%$ & $0.00 \%$ & $0.07 \%$ & $0.00 \%$ & $0.00 \%$ & $0.00 \%$ & $0.36 \%$ & $0.00 \%$ & $0.07 \%$ \\
\hline 7-0111 (factor2,3\&4) & $0.87 \%$ & $0.95 \%$ & $1.56 \%$ & $0.00 \%$ & $0.41 \%$ & $2.85 \%$ & $0.45 \%$ & $0.00 \%$ & $1.68 \%$ & $1.18 \%$ \\
\hline 8-1000 (factor 1) & $2.38 \%$ & $1.46 \%$ & $6.41 \%$ & $7.38 \%$ & $1.32 \%$ & $0.55 \%$ & $5.39 \%$ & $2.63 \%$ & $3.74 \%$ & $5.33 \%$ \\
\hline 9-1001 (factor $1 \& 4)$ & $3.53 \%$ & $1.81 \%$ & $0.53 \%$ & $0.63 \%$ & $1.23 \%$ & $3.37 \%$ & $1.05 \%$ & $3.33 \%$ & $0.27 \%$ & $2.69 \%$ \\
\hline $10-1010$ (factor $1 \& 3$ ) & $1.68 \%$ & $3.90 \%$ & $4.48 \%$ & $8.94 \%$ & $7.61 \%$ & $3.46 \%$ & $6.53 \%$ & $7.48 \%$ & $1.50 \%$ & $14.67 \%$ \\
\hline 11-1011 (factor1,3\&4) & $3.92 \%$ & $11.01 \%$ & $8.73 \%$ & $5.06 \%$ & $14.30 \%$ & $13.08 \%$ & $6.99 \%$ & $7.89 \%$ & $12.78 \%$ & $12.23 \%$ \\
\hline $12-1100$ (factor $1 \& 2$ ) & $4.46 \%$ & $5.74 \%$ & $4.13 \%$ & $4.85 \%$ & $1.00 \%$ & $2.89 \%$ & $3.65 \%$ & $0.90 \%$ & $1.69 \%$ & $0.55 \%$ \\
\hline 13-1101 (factor $1,2 \& 4$ ) & $4.06 \%$ & $1.96 \%$ & $3.72 \%$ & $7.06 \%$ & $0.63 \%$ & $3.19 \%$ & $5.46 \%$ & $1.42 \%$ & $1.99 \%$ & $6.63 \%$ \\
\hline $14-1110$ (factor $1,2 \& 3$ ) & $5.78 \%$ & $9.24 \%$ & $5.95 \%$ & $4.20 \%$ & $14.71 \%$ & $1.47 \%$ & $7.10 \%$ & $1.81 \%$ & $5.70 \%$ & $2.48 \%$ \\
\hline 15-1111 (all factors) & $27.71 \%$ & $28.54 \%$ & $16.60 \%$ & $24.67 \%$ & $12.48 \%$ & $20.52 \%$ & $28.77 \%$ & $4.52 \%$ & $32.77 \%$ & $12.20 \%$ \\
\hline
\end{tabular}

Factor 1 = Primary Stakeholders (Production Engineering, Production, Design, Sales and Marketing staff, Suppliers, Customers, Patents)

Factor 2 = Research \& Development (Research, Experimental development, Production engineering, Trade Fairs, Governments, Other producers)

Factor 3 = External Information Sources (Sales and Marketing, Customers, Related Firms)

Factor 4 = Corporate Organizations (Head Office, Related plants, Universities)

Table 18 Plant Distributions for Sources of Implementation Support by Industry

\subsubsection{Perceived Benefits}

The distribution of the plants throughout the various states of perceived benefits was very sporadic except for the extreme points of the spectrum (refer to Table 19). Each of the industries had a large percentage of plants in states at both ends of the spectrum but due to the large number of states, there were many states containing small percentages of plants. In particular, 
state eight was empty in the chemical industry, state four was empty in the electronics industry, state nine was empty in the furniture and other industry, states four, six, and nine were empty in the machinery industry, states six and eight were empty in the non-metals industry, and states two, five, and six were empty in the plastics industry. All of these states were dropped in the regression analysis for the respective industries. Some of these states had very small proportions of plants in all industries but it was decided that since special cases were made due to some of the state being both empty in some industries and significant in others that all non-empty states remained in the analysis.

The state consisting of only the perceived benefit of improved flexibility (state two) contained very few plants from the chemical, metals, plastics, and textiles industries but it did contain at least $7 \%$ of the electronics, machinery, and vehicles industries. This result is supported by the fact that many of the electronics, machinery, and vehicle plants create multiple products on a single line and the improvement in flexibility was one of their major objectives when implementing AMT in order to gain a competitive advantage when keeping up with changing consumer demands. In particular, the vehicles industry was where AMT had reduced the change over time for new car models from weeks to days.

The remaining states where there was a significant proportion of the plant population in any industry were states that contain at least the perceived benefit of time savings along with two of the remaining factors (states thirteen to fifteen). As with the analysis of the perceived benefits by size class most plants perceived a large number of benefits to implementing AMT or none of the factors, very few plants fall outside these extremes. 


\begin{tabular}{|c|c|c|c|c|c|c|c|c|c|c|}
\hline State & $\frac{\bar{\Xi}}{\stackrel{\Xi}{\Xi}}$ & 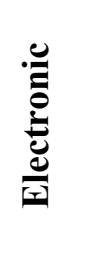 & 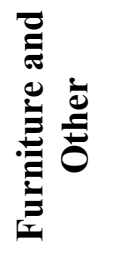 & 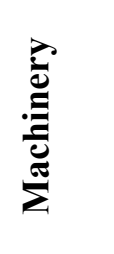 & $\frac{n}{\sum}$ & 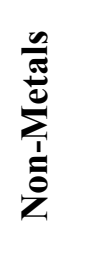 & 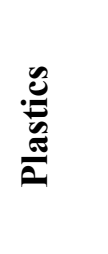 & $\underset{f}{\stackrel{e}{e}}$ & $\frac{\frac{\pi}{e}}{\frac{e}{e}}$ & $\begin{array}{l}\overline{8} \\
8 \\
8\end{array}$ \\
\hline $0-0000$ (no factors) & $36.29 \%$ & $22.08 \%$ & $34.76 \%$ & $20.13 \%$ & $24.16 \%$ & $33.31 \%$ & $25.72 \%$ & $44.88 \%$ & $18.18 \%$ & $27.55 \%$ \\
\hline 1-0001 (factor 4) & $4.85 \%$ & $1.42 \%$ & $0.40 \%$ & $0.20 \%$ & $0.88 \%$ & $2.15 \%$ & $2.53 \%$ & $4.31 \%$ & $1.58 \%$ & $3.21 \%$ \\
\hline 2-0010 (factor 3) & $0.65 \%$ & $8.10 \%$ & $2.64 \%$ & $6.87 \%$ & $0.17 \%$ & $4.79 \%$ & $0.00 \%$ & $0.34 \%$ & $8.21 \%$ & $2.44 \%$ \\
\hline 3-0011 (factor 3 \& 4) & $2.31 \%$ & $1.00 \%$ & $0.64 \%$ & $0.64 \%$ & $0.30 \%$ & $0.61 \%$ & $0.57 \%$ & $2.67 \%$ & $9.81 \%$ & $2.42 \%$ \\
\hline 4-0100 (factor 2) & $2.40 \%$ & $0.44 \%$ & $1.49 \%$ & $0.00 \%$ & $5.89 \%$ & $0.40 \%$ & $0.89 \%$ & $1.09 \%$ & $0.76 \%$ & $0.56 \%$ \\
\hline 5-0101 (factor $2 \& 4)$ & $2.00 \%$ & $0.00 \%$ & $0.81 \%$ & $0.07 \%$ & $1.21 \%$ & $0.00 \%$ & $0.00 \%$ & $0.67 \%$ & $0.30 \%$ & $1.75 \%$ \\
\hline $6-0110$ (factor $2 \& 3)$ & $1.16 \%$ & $0.17 \%$ & $0.05 \%$ & $0.00 \%$ & $0.05 \%$ & $0.24 \%$ & $0.00 \%$ & $3.84 \%$ & $0.39 \%$ & $0.66 \%$ \\
\hline 7-0111 (factor 2,3\&4) & $2.71 \%$ & $0.62 \%$ & $0.56 \%$ & $0.55 \%$ & $1.23 \%$ & $3.64 \%$ & $1.08 \%$ & $0.60 \%$ & $1.27 \%$ & $1.93 \%$ \\
\hline $8-1000$ (factor 1$)$ & $0.00 \%$ & $0.67 \%$ & $0.34 \%$ & $0.17 \%$ & $0.26 \%$ & $0.00 \%$ & $0.17 \%$ & $0.23 \%$ & $0.38 \%$ & $0.21 \%$ \\
\hline 9-1001 (factor 1\& 4) & $1.69 \%$ & $0.13 \%$ & $0.00 \%$ & $0.00 \%$ & $0.10 \%$ & $0.40 \%$ & $0.26 \%$ & $1.10 \%$ & $0.20 \%$ & $0.35 \%$ \\
\hline 10-1010 (factor $1 \& 3$ ) & $0.46 \%$ & $2.40 \%$ & $4.19 \%$ & $3.96 \%$ & $0.35 \%$ & $3.43 \%$ & $0.54 \%$ & $1.58 \%$ & $1.02 \%$ & $0.86 \%$ \\
\hline 11-1011 (factor $1,3 \& 4$ ) & $1.51 \%$ & $6.23 \%$ & $8.18 \%$ & $9.40 \%$ & $2.71 \%$ & $2.93 \%$ & $14.03 \%$ & $7.20 \%$ & $4.23 \%$ & $12.03 \%$ \\
\hline $12-1100$ (factor $1 \& 2$ ) & $0.52 \%$ & $1.40 \%$ & $1.10 \%$ & $0.64 \%$ & $0.46 \%$ & $0.21 \%$ & $0.50 \%$ & $0.72 \%$ & $1.39 \%$ & $0.44 \%$ \\
\hline 13-1101 (factor $1,2 \& 4)$ & $2.21 \%$ & $2.43 \%$ & $0.84 \%$ & $8.39 \%$ & $13.05 \%$ & $2.24 \%$ & $6.76 \%$ & $1.01 \%$ & $3.62 \%$ & $2.24 \%$ \\
\hline $14-1110$ (factor $1,2 \& 3$ ) & $1.22 \%$ & $5.08 \%$ & $5.19 \%$ & $0.17 \%$ & $1.18 \%$ & $7.99 \%$ & $3.69 \%$ & $2.53 \%$ & $2.91 \%$ & $1.76 \%$ \\
\hline 15-1111 (all factors) & $40.00 \%$ & $47.82 \%$ & $38.82 \%$ & $48.81 \%$ & $48.02 \%$ & $37.67 \%$ & $43.27 \%$ & $27.23 \%$ & $45.75 \%$ & $41.60 \%$ \\
\hline
\end{tabular}

Factor 1 = Time (Reduced Capital, Reduced rejection time, Reduced set-up time, Increased quality, New product features, Reduced time to market)

Factor 2 = Productivity (Reduced labour, Reduced material, Reduced capital) Increased utilization rate, Increased Profitability)

Factor 3 = Flexibility (Increased flexibility, Increased skill requirements, Increased quality)

Factor 4 = Profitability (Increased equipment utilization rate, Increased market share, Increased Profitability)

Table 19 Plant Distributions for Perceived Benefits of AMT by Industry

\subsubsection{High-Level Factors}

The distribution of the plants throughout the various states of high-level factors was very irregular (refer to Table 20). Each of the industries had a large percentage of plants in states exhibiting none of the high-level factors but there were many of the states that contain very few plants. In particular, states six, seven, eighteen, and nineteen were empty in the chemical industry, states nine, twelve, thirteen, and twenty were empty in the electronics industry, states 
thirteen and fourteen were empty in the furniture and other industry, states four six nine twelve thirteen, twenty-three, and twenty-nine were empty in the machinery industry, states one, four, seven, and nine were empty in the non-metals industry, the fourth, ninth, tenth, and eleventh states were empty in the plastics industry, and state five was empty in the wood industry. All of these states were dropped in the regression analysis for the respective industries.

The textile industry had the largest percentage of plants exhibiting none of the high-level factors at $49 \%$. This was greater than the next closest industry by over $15 \%$ demonstrating the traditional nature of this industry. The only other state that exhibited a significant proportion of textile plants was the state consisting of only the perceived benefits factor (state sixteen).

The machinery and metals industries both had a significant representation in state two. This state consisted only of the design high-level factor. The machinery and metals industries had over $6 \%$ of their population in this state, over twice as much as the next nearest industry. This was consistent with the high proportion of plants having the production design factor in the implemented AMT analysis.

The chemical and non-metals industries had a significant proportion of plants in state eight. Over $8.5 \%$ of the plants in these industries exhibited the AMT and business practices factor on its own. These industries were relatively unique as the majority of the remaining industries had less that $3 \%$ of their plants in this state.

The vehicles industry also had a strongly idiosyncratic state. Over $11 \%$ of its plants were found in state fourteen. This state consisted of the AMT and business practices, the sources of implementation support, and the design factor. This highly complex state demonstrates the innovative nature of the vehicles industry. 
Finally, there was a grouping of industries including the chemical, electronics, machinery, plastics and vehicles industries. These industries had over $30 \%$ of their populations in the states containing at least the perceived benefits, AMT and business practices and sources of implementation support factors (states twenty-eight to thirty-one). These industries are considered as the more traditional high-technology industries and did seem to perform the more innovative practices.

The factor that appeared to be included the least in these industries was the human resources factor. It appeared to be the least added factor to any other grouping of all the factors. States that did include this factor, with the exception of the final state including all factors, exhibited very small proportions of the plant population. The results could be due to the fact that the person responding to the survey was the plant manager and may have had less experience with human resources issues if they were done out of an offsite head office. 


\begin{tabular}{|c|c|c|c|c|c|c|c|c|c|c|}
\hline & 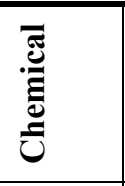 & 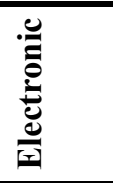 & 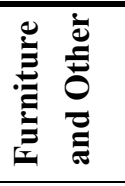 & 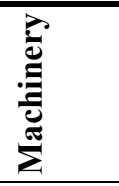 & $\frac{n}{\sum}$ & $\sum_{\substack{0 \\
0}}^{\frac{0}{\pi}}$ & $\begin{array}{l}\stackrel{\tilde{U}}{0} \\
\frac{\tilde{\sigma}}{2}\end{array}$ & 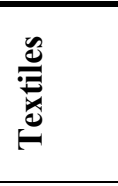 & $\frac{\frac{\pi}{e}}{\stackrel{0}{e}}$ & $\begin{array}{l}\overrightarrow{0} \\
\stackrel{0}{0}\end{array}$ \\
\hline 0-00000 (no factors) & $18.96 \%$ & $18.55 \%$ & $32.30 \%$ & $18.56 \%$ & $21.06 \%$ & $26.26 \%$ & $21.76 \%$ & $49.09 \%$ & $17.83 \%$ & $19.39 \%$ \\
\hline $1-00001$ (factor 5) & $1.87 \%$ & $1.30 \%$ & $0.20 \%$ & 0.007 & $0.07 \%$ & $0.00 \%$ & $0.28 \%$ & $1.43 \%$ & $0.46 \%$ & $1.79 \%$ \\
\hline 2-00010 (factor 4) & $0.21 \%$ & $0.59 \%$ & $1.75 \%$ & $6.95 \%$ & $6.01 \%$ & $0.40 \%$ & $0.57 \%$ & $1.25 \%$ & $0.20 \%$ & $3.48 \%$ \\
\hline 3-00011 (factor 4 \& 5) & $0.10 \%$ & $0.10 \%$ & $0.15 \%$ & $0.13 \%$ & $0.12 \%$ & $0.54 \%$ & $0.00 \%$ & $0.05 \%$ & $0.49 \%$ & $0.14 \%$ \\
\hline 4-00100 (factor 3) & $2.15 \%$ & $4.38 \%$ & $0.59 \%$ & $0.00 \%$ & $0.22 \%$ & $0.00 \%$ & $0.57 \%$ & $1.76 \%$ & $4.17 \%$ & $0.73 \%$ \\
\hline $5-00101$ (factor $3 \&$ 5) & $0.39 \%$ & $0.57 \%$ & $0.17 \%$ & $0.55 \%$ & $0.05 \%$ & $0.54 \%$ & $0.36 \%$ & $0.10 \%$ & $1.04 \%$ & $1.46 \%$ \\
\hline $6-00110$ (factor $3 \& 4)$ & $0.00 \%$ & $1.18 \%$ & $0.17 \%$ & $0.00 \%$ & $0.90 \%$ & $0.61 \%$ & $4.04 \%$ & $0.05 \%$ & $0.13 \%$ & $0.63 \%$ \\
\hline $7-00111$ (factor $3,4 \& 5$ ) & $0.00 \%$ & $3.08 \%$ & $0.56 \%$ & $0.72 \%$ & $0.61 \%$ & $0.00 \%$ & $0.45 \%$ & $0.13 \%$ & $0.29 \%$ & $0.00 \%$ \\
\hline 8-01000 (factor 2) & $14.48 \%$ & $2.31 \%$ & $3.15 \%$ & $0.08 \%$ & $1.01 \%$ & $8.51 \%$ & $0.00 \%$ & $0.34 \%$ & $0.42 \%$ & $5.16 \%$ \\
\hline $9-01001$ (factor $2 \& 5)$ & $0.29 \%$ & $0.00 \%$ & $0.05 \%$ & $0.00 \%$ & $0.07 \%$ & $0.00 \%$ & $0.00 \%$ & $0.18 \%$ & $0.20 \%$ & $0.23 \%$ \\
\hline $10-01010$ (factor $2 \& 4)$ & $2.69 \%$ & $1.27 \%$ & $1.49 \%$ & $0.55 \%$ & $0.41 \%$ & $0.45 \%$ & $0.00 \%$ & $0.13 \%$ & $1.27 \%$ & $1.07 \%$ \\
\hline $11-01011$ (factor $2,4 \& 5)$ & $0.27 \%$ & $0.63 \%$ & $0.05 \%$ & $0.80 \%$ & $0.32 \%$ & $2.24 \%$ & $0.74 \%$ & $0.09 \%$ & $2.05 \%$ & $0.69 \%$ \\
\hline $12-01100($ factor $2 \& 3)$ & $3.98 \%$ & $0.00 \%$ & $0.17 \%$ & $0.00 \%$ & $0.05 \%$ & $4.48 \%$ & $0.38 \%$ & $0.77 \%$ & $1.49 \%$ & $0.38 \%$ \\
\hline $13-0$ & 1 . & 0 & 0 & 0 & 6 & 02 & 0 & $\%$ & $\%$ & $0.27 \%$ \\
\hline $14-01110$ (factor $2,3 \& 4$ ) & $1.69 \%$ & $0.72 \%$ & $0.00 \%$ & $0.72 \%$ & $0.71 \%$ & $0.24 \%$ & $0.86 \%$ & $2.17 \%$ & $11.23 \%$ & $0.95 \%$ \\
\hline $15-01111$ (factor $2,3,4 \& 5)$ & $0.90 \%$ & $1.83 \%$ & $1.08 \%$ & $0.41 \%$ & $0.43 \%$ & $0.25 \%$ & $0.82 \%$ & $0.13 \%$ & $1.26 \%$ & $0.43 \%$ \\
\hline $16-10000($ factor 1$)$ & $1.98 \%$ & $0.88 \%$ & $4.47 \%$ & $0.28 \%$ & $0.84 \%$ & $7.01 \%$ & $3.29 \%$ & $10.11 \%$ & $3.00 \%$ & $4.40 \%$ \\
\hline $17-10001$ (factor $1 \& 5)$ & $0.35 \%$ & $0.73 \%$ & $1.13 \%$ & $2.67 \%$ & $0.77 \%$ & $2.85 \%$ & $0.64 \%$ & $4.02 \%$ & $0.99 \%$ & $3.30 \%$ \\
\hline $18-10010$ (factor $1 \& 4)$ & $0.00 \%$ & $1.37 \%$ & $1.61 \%$ & $2.94 \%$ & $0.34 \%$ & $0.48 \%$ & $0.45 \%$ & $0.74 \%$ & $0.94 \%$ & $1.96 \%$ \\
\hline 19-10011 (factor $1,4 \& 5)$ & $0.00 \%$ & $1.77 \%$ & $4.11 \%$ & $2.92 \%$ & $1.22 \%$ & $0.15 \%$ & $0.57 \%$ & $0.34 \%$ & $0 \%$ & $0.38 \%$ \\
\hline 20-10100 (factor $1 \&$ 3) & $2.04 \%$ & $0.00 \%$ & $2.02 \%$ & $0.08 \%$ & $1.24 \%$ & $9.95 \%$ & $3.85 \%$ & $4.95 \%$ & $1.78 \%$ & $6.77 \%$ \\
\hline 21-10101 (factor $1,3 \& 5$ ) & $3.48 \%$ & $0.65 \%$ & $5.85 \%$ & $4.28 \%$ & $11.92 \%$ & $2.27 \%$ & $1.42 \%$ & $2.16 \%$ & $2.62 \%$ & $2.94 \%$ \\
\hline 22-10110 (factor $1,3 \& 4$ ) & $1.55 \%$ & $4.50 \%$ & $5.92 \%$ & $14.59 \%$ & $5.79 \%$ & $0.86 \%$ & $5.92 \%$ & $2.67 \%$ & $1.82 \%$ & $6.66 \%$ \\
\hline $23-10111$ (factor $1,3,4 \& 5)$ & $1.08 \%$ & $0.96 \%$ & $2.92 \%$ & $4.19 \%$ & $8.66 \%$ & $3.39 \%$ & $0.08 \%$ & $0.72 \%$ & $3.75 \%$ & $2.70 \%$ \\
\hline $24-1$ & $3.47 \%$ & $\%$ & $\%$ & $\%$ & $\%$ & $1.14 \%$ & $\%$ & $\%$ & $\%$ & $6.24 \%$ \\
\hline $25-11001$ (factor $1,2 \& 5)$ & $1.21 \%$ & $0.13 \%$ & $3.14 \%$ & $6.87 \%$ & $6.29 \%$ & $0.24 \%$ & $5.08 \%$ & $1.81 \%$ & $0.87 \%$ & $3.30 \%$ \\
\hline 26-11010 (factor $1,2 \& 4)$ & $0.79 \%$ & $3.00 \%$ & $0.17 \%$ & $0.24 \%$ & $0.62 \%$ & $3.26 \%$ & $0.73 \%$ & $0.30 \%$ & $0.71 \%$ & $3.40 \%$ \\
\hline $27-11011$ (factor $1,2,4 \& 5)$ & $0.40 \%$ & $1.59 \%$ & $0.15 \%$ & $2.01 \%$ & $0.32 \%$ & $0.24 \%$ & $0.80 \%$ & $0.91 \%$ & $3.16 \%$ & $2.14 \%$ \\
\hline $28-11100$ (factor $1,2 \& 3$ ) & $10.51 \%$ & $2.25 \%$ & $6.05 \%$ & $0.08 \%$ & $1.96 \%$ & $8.04 \%$ & $5.62 \%$ & $1.58 \%$ & $0.68 \%$ & $3.86 \%$ \\
\hline $29-11101$ (factor $1,2,3 \& 5)$ & $7.56 \%$ & $1.43 \%$ & $1.02 \%$ & $0.00 \%$ & $0.73 \%$ & $3.33 \%$ & $13.86 \%$ & $3.72 \%$ & $0.81 \%$ & $3.39 \%$ \\
\hline $30-11110$ (factor $1,2,3 \& 4)$ & $7.88 \%$ & $13.33 \%$ & $12.72 \%$ & $19.46 \%$ & $9.05 \%$ & $5.36 \%$ & $5.52 \%$ & $2.37 \%$ & $18.13 \%$ & $4.40 \%$ \\
\hline 31-11111 (all factors) & $8.05 \%$ & $29.19 \%$ & $6.63 \%$ & $9.82 \%$ & $16.72 \%$ & $6.69 \%$ & $15.97 \%$ & $2.62 \%$ & $12.41 \%$ & $7.37 \%$ \\
\hline
\end{tabular}

Factor $1=$ Perceived Benefits

Factor 2 = Advanced Manufacturing Technologies (Except Design) \& Business Practices

Factor 3 = Implementation Support, Cross Functional Teams \& Research and Development

Factor 4 = Design (Production Design Technologies, Concurrent Engineering)

Factor $5=$ Human Resources

\section{Table 20 Plant Distributions for High-Level Factors by Industry}




\subsection{Determining Complementarity}

Two different control variables, plant size, and industry were used. The study controlled for industry by estimating a separate model for each industry sector. This is important since the type of AMT implemented by each industry is very different (refer to Appendix D). For example, integration and control systems are used by over $60 \%$ of plants in the beverage, primary textile, paper and allied products, primary metal, and electronic products plants, but less than $40 \%$ of plants in the furniture and fixture, and clothing industries (Sabourin and Beckstead, 1999). By controlling for industry, potential bias due to the amount of variation in the use of AMT and the types of AMT used is avoided.

Plant size was also used as a control. As discussed in Meredith (1987), smaller firms can benefit from different forms of AMT than those used by larger firms. In the 1998 Survey of Advanced Technology in Canadian Manufacturing, Sabourin and Beckstead (1999) found that there is a wide range of implementation levels when the different plant sizes are compared. For example, CAD software is used by $81 \%$ of large plants but only $37 \%$ of small plants (refer to Appendix D). A similar discrepancy was found when automated materials handling systems, network communication, and processing systems are considered.

It is hypothesised that all of the variables described in the previous section are pair-wise complementary with the exception of the firm-level factors that will be used as control variables. The method for determining complementarity is the framework set forth in Athey and Stern (1998). This is a form of constrained regression and involves pair-wise tests for all variables. This method was used by Mohnen and Roller (2003) to show complementarity between government innovation policies in the European Union. 
Using the Athey and Stern method, profit and productivity functions were specified using the variables defined in the previous section. The profit (productivity) function for a given industry $j$ is $P_{j}=\sum_{i=0}^{2^{n}-1} \gamma_{i j} s_{i j}+\varepsilon_{j}$, where $n$ is the number of endogenous variables. The variables $s_{i j}$ define a set of state dummy variables representing state $i$ in industry $j$. The dummy variables are defined using binary algebra convention (e.g., state three of a four variate problem 0011 , would be represented by $s_{3 j}$ ). Using this function, the supermodularity constraints were then used as a set of restrictions on the coefficients of the variables. Consider a smaller example with only four variables. There are then 16 states ranging from 0000 (where none of the variables are implemented), to 1111 (where all elements are implemented). The complementarity conditions for the first two elements to be complementary are written as: $\gamma_{8+s}+\gamma_{4+s} \leq \gamma_{0+s}+\gamma_{12+s}$, where $s$ $=0,1,2,3$. Similarly, the remaining 20 restrictions for the other five pairs of variables can be expressed. For the entire set to be complementary, all 24 restrictions must be satisfied. Since pair-wise complementarity between any subset of variables implies supermodularity over the subset, this implies the joint testing of four inequality constraints (Mohnen and Roller, 2003). The profit function could be submodular, in which case the elements are substitutes. This property was tested by changing the sign of the inequalities.

Two types of hypothesis testing were conducted. The first tested for strict complementarity by testing for supermodularity of the function as the null hypothesis. The second tested for strict substitutes by testing for submodularity of the function as the null hypothesis. These two tests determined which set of elements should be adopted simultaneously in order for a plant to obtain the optimal benefits and which set of elements should never be adopted simultaneously. 
The first hypothesis has as the null hypothesis strict equality and for the alternative that the inequality is negative, that is for elements 1 and 2 in the example,

$$
\begin{aligned}
& \mathrm{H}_{0}:-\gamma_{0+s}+\gamma_{4+s}+\gamma_{8+s}-\gamma_{12+s}=0, \text { for all } s=0,1,2,3 \\
& \mathrm{H}_{1}:-\gamma_{0+s}+\gamma_{4+s}+\gamma_{8+s}-\gamma_{12+s}<0, \text { for all } s=0,1,2,3
\end{aligned}
$$

Note that this test is a joint one-sided test whether two obstacles are strict complements. Under the hypothesis $S \gamma=0$, where $S$ is a $4 x 8$ matrix in the example partitioned as $\left[S^{0}\left|S^{1}\right| S^{2} \mid S^{3}\right]$, the joint restrictions are distributed as $v=S \hat{\gamma} \sim N\left(0, S \operatorname{cov}(\hat{\gamma}) S^{\prime}\right)$, where $\operatorname{cov}(\hat{\gamma})$ is the variancecovariance matrix of the estimated $\gamma$ coefficients. The base model was created without any of the complementarity restrictions. A constrained regression model, with the complementarity restrictions as constraints was then calculated for each pair-wise test. To obtain the significance using this test a likelihood ratio (LR) was calculated ${ }^{9}$. Gourieroux, Holly, and Monfort (1982) show that the LR is similar to the Wald statistic method used by Mohnen and Roller (2003).

Similarly, the second hypothesis had as the null hypothesis strict equality and as the alternative that the inequality is positive, that is for elements 1 and 2 in the example,

$$
\begin{aligned}
& \mathrm{H}_{0}:-\gamma_{0+s}+\gamma_{4+s}+\gamma_{8+s}-\gamma_{12+s}=0, \text { for all } s=0,1,2,3 \\
& \mathrm{H}_{1}:-\gamma_{0+s}+\gamma_{4+s}+\gamma_{8+s}-\gamma_{12+s}>0, \text { for all } s=0,1,2,3
\end{aligned}
$$

\footnotetext{
${ }^{9}$ The likelihood ratio test statistic is of the form $\operatorname{LR}=2\left[\mathrm{~L}\left(\theta_{\mathrm{U}}\right)-\mathrm{L}\left(\theta_{\mathrm{R}}\right)\right]$, where $\theta_{\mathrm{U}}$ is the unrestricted Maximum Likelihood estimate of $\theta$, and $\theta_{\mathrm{R}}$ is the restricted Maximum Likelihood estimate of $\theta$. To implement the test we use the following: $L R=n \log \left(\mathrm{SSR}_{U}\right) / \log \left(\mathrm{SSR}_{\mathrm{R}}\right)$, where $\mathrm{SSR}_{\mathrm{U}}$ is the unrestricted sum of squared residuals and $\mathrm{SSR}_{\mathrm{R}}$ is the restricted sum of squared residuals.
} 
This test accepts $\mathrm{H}_{1}$ when the constraints are jointly positive, and the elements are therefore strict substitutes. To obtain the significance using this test, a likelihood ratio was once again calculated.

There are therefore three alternatives for the relationship between a pair of elements: (i) the elements are strict complements, (ii) the elements are strict substitutes, (iii) the elements have intermediate p-values for both tests and are neither strict complements, nor strict substitutes. These tests were performed for each industry and each size class of plants.

Athey and Stern (1998) show that this method of determining complementarity does not suffer from the potential bias that an ordinary least squares type of analysis does. The parametric approach is beneficial, as many of the industries had relatively small sample sizes. In addition, allowing for a distribution of unobservables with a variance-covariance matrix that is unrestricted provides an efficient specification of the complementarities (Athey and Stern, 1998). This method involves performing many hypothesis tests, as all comparisons must be done in pairs. Each pair must be tested for complementarity in each scenario.

Due to the computational complexity of these calculations, the number of variables in the regression equation had to be limited. The regression must be calculated with $2^{(\mathrm{n}-2)}$ constraints, which is theoretically but not computationally possible for mid to large values of $\mathrm{n}$. For this reason, the problem was broken down into sub-problems. A regression equation, as described above, was created for each of the factors previously identified. Only variables of these factors were in the regression equation. Tests of pair-wise complementarity were then conducted as described above. 
Once the complementary variables for each factor type were determined, the factors for the high-level elements themselves were calculated using factor analysis. Using these variables representing the high-level factors, a regression equation was created that includes only a few comprehensive variables representing each of the high-level factor types. Analysis was then conducted to determine the pair-wise complementarity between these factor variables in order to complete the study. The high-level analysis incorporates all of the elements of factor types including AMT, business practices, sources of implementation support, human resources practices, R\&D methods, and perceived benefits of AMT. The same control variables (size and industry type) were used for all regression equations throughout.

\subsection{Finding the Optimal Set of Organizational Policies}

Once the complementarities between the variables were known, the optimal set of technologies and business practices was determined by considering all complementary variables as the basis for the optimal state. A choice had to be made between the substitute variables as to which is more beneficial. This decision was determined from plots of the lattice of state variables. The optimal state was confirmed by looking at the actual profit values from the 1998 Annual Survey of Manufacturers to determine if the companies within this state performed the best.

Due to the number of dimensions in the analysis, the lattices were plotted on a two dimensional graph in order to determine the relative rankings of the states. The average marginal profit for each state was used as the value of the lattice point on the vertical axis. The profit values enable the ranking of the various other states so that managers will be able to see which changes to make if financial or other factors constrain the amount they can implement. This will enable managers to efficiently move their plants to higher states in the lattice. 


\section{RESULTS}

This section begins by analyzing the results from the constrained regression tests for supermodularity (complements) and submodularity (substitutes). The results are once again presented by the variable factor types concluding with the analysis of the high-level factors. Following this is a second form of complementarity analysis that looks at the individual variables themselves. This method is conducted for a few representative industries in order to determine which of the individual variables within the factors have the largest impact on the complementarities found. The final analysis in this section is the creation of a lattice for medium plants and plants in the vehicles industry. These lattices demonstrate how the results can be used to produce recommendations to plant managers by plant size or industry.

Throughout this section, the following terminology will be used. Any reference to two complementary variables means that the supermodularity hypothesis for these two variables cannot be rejected but the submodularity hypothesis is rejected. Similarly, two variables will be said to be substitutes if the submodularity hypothesis for the two variables cannot be rejected but the supermodularity hypothesis is rejected. If both the supermodularity and the submodularity hypotheses are rejected, then the variables will be said to be independent.

The dependent variables for the analysis are either plant profit or plant labour productivity. The profit measure used for this analysis is the plant manufacturing value added divided by the number of shipments in order to normalize the measure. The labour productivity measure is the manufacturing value added divided by the number of employees. For the purposes of the analysis, the natural $\log$ of labour productivity was used as the dependent variable. 


\subsection{Constrained Regression Model}

The constrained regression models were altered to coincide with the variable states that were removed from the analysis. For this reason, the values used to determine the significance of the likelihood ratio tests are different for some of the industries and plant size classes. Only the machinery, non-metal, and plastics industries have different values for significance due to their small sample size. All significance cut-off values can be found in Appendix E.

\subsubsection{Results by Size Class}

Very few exceptions had to be made when undertaking the analysis by size class. The sample size for each size class is large enough that the same cut-off values for significance can be used. The analysis below will focus on the supermodularity and submodularity tests that were determined to be significant by factor type. Overall conclusions about the constrained regression analysis by size class will be made at the end of this section.

\subsubsection{Advanced Manufacturing Technologies}

First, consider the constrained regression results with labour productivity as the dependent variable. There are a number of significant results found in the analysis, although not all AMT are found to be complementary. This conclusion goes against the results found by Alcorta (1994) and the hypotheses posed by Milgrom and Roberts (1995). The reasons for the discrepancy may be due the larger number of technologies surveyed.

For large plants, production design technologies and inspection and storage technologies were found to be substitutes (refer to Table 21). This result is not too surprising as the design process does not normally take advantage or utilize inspection and storage technologies. The use of the automated inspection and retrieval systems would only be added to the plan after the 
design phase of the manufacturing process is complete. Communications technologies and the robotics technologies were also found to be substitutes for one another. This result demonstrates that when the complex technology of robotics is used, along with remote controlled processes through the communications network, labour productivity is decreased due perhaps to the increase in skills and time required to monitor and control the robotics.

There are also a large number of complementarities between AMT factors in large plants. The production design technologies and robotics technologies are complementary. This would coincide with the added abilities that the robotics impart on the design process, as well as the increase in labour productivity resulting from designs that maximize the use of the functionalities included in the robotics at the plant. The communication technologies and the inspection and storage technologies were also found to be complements. This result is very intuitive as without a strong communication system within the plant there would be no way to take advantage of automated systems as they need to have network communication with the manufacturing machinery. Inspection technologies must also have communication with managers to alert them to any quality problems. The final complementarity involves the inspection and storage technologies and robotics. The complementarity between these two technologies can be explained since robotic systems are the primary users of automated storage and retrieval systems.

Medium plants have fairly different results with respect to labour productivity (refer to Table 22). The production design technologies and the inspection and storage technologies remain substitutes. For medium plants, the production design and robotics technologies are also substitutes. Here the reason may be the added complexity required to design products using robotics. Since there are a smaller number of employees within medium plants compared to large plants, robotics experts are less likely to participate in the design process, particularly with 
respect to taking advantage to all of the features robotics can provide. The results might also be due to the economies of scale that the large plants can take advantage of making it more cost effect to utilize the robotics technologies.

Complementarities are also found between the factors for the medium plants. Once again, the inspection and storage technologies and the robotics technologies are found to be complementary. The communications technologies and the robotics technologies are also found to be complements. These two forms of technology are complementary in medium plants due to the labour-saving nature of the technology. The decrease in the number of employees makes the importance of a good communication infrastructure extremely important for the monitoring and use of robotics within medium plants.

There are very few conclusions that can be made about complementarity between factors with respect to labour productivity in small plants (refer to Table 23). Some of this may be due to the distribution of small plants in the various AMT states (only seven of the states are highly inhabited). Another reason may be that due to the small number of employees, technology is used as a base requirement for competitiveness and not to replace human labour. For these plants, the production design technologies and the communications technologies are found to be substitutes for each other. This may be because of the size of the plant; since the majority of small plants have limited floor space, the need for a large number of computers is reduced. In some small plants, the design technologies would overlap in applications with other computers or remote processes on the plant floor. Small plants are also less likely to use wide area networks to communicate with other corporate facilities, as many of these plants are not associated with other facilities. 
With respect to labour productivity in small plants, there is one pair of complementary technologies. Communications technologies and the robotics technologies are once again complementary. As in medium plants, the labour-saving nature of the technologies is important to the competitive advantage of small plants.

With respect to profit, some different results are obtained. For large plants, only one conclusion can be made: the inspection and storage technologies and the robotics technologies are substitutes (refer to Table 21). This result implies that although these two technologies may be complementary with respect to labour productivity, they are expensive and result in lower profit per shipment. This result could be because the cost of technology per shipment is quite large if the economies of size are not leveraged to there fullest, or from having insufficient time for full implementation based on when the technology was implemented with respect to the time of the study. If the technology was newly purchased and has not been fully integrated into the manufacturing process then the capital cost will have a large effect as the benefits of the technology will not be incorporated into profits. There is a greater time lag between the laboursaving benefits and the capital cost reductions.

The results for medium plants with respect to profit are similar to those found relating to labour productivity (refer to Table 22). The production design and robotics technologies are found to be substitutes and the communication technologies and robotics are found to be complements. The remainder of the results are inconclusive as they have moderate values for every test.

There are a much larger number of significant test results for small plants with respect to profit (refer to Table 23). In this case, the communications technologies and the inspection and 
storage technologies are substitutes. An explanation is that small plants can obtain as much useful information and ability from having a good communication network as they can by using an automated storage system to keep track of inventory. Inspection and storage technologies and robotics technologies are found to be independent from one another with respect to profit for small plants. An explanation is that few small plants use these technologies so they are neither complementary or substitutes for one another.

Small plants also exhibit a couple complementary pairings. Production design and communications technologies are complementary. This is a much more intuitive result than that found with respect to labour productivity. Here, the use of communications technologies and production design technologies combine to give the plant more efficient use of resources and a competitive advantage by being able to better manage the processes occurring in the plant. Communications technologies are also complementary to robotics technologies, just as in the analysis with respect to labour productivity.

\begin{tabular}{|c|c|c|c|c|c|c|c|c|c|c|c|c|}
\hline & \multicolumn{6}{|c|}{ Labour Productivity } & \multicolumn{6}{|c|}{ Profit } \\
\hline \multirow[t]{5}{*}{$\begin{array}{c}\text { Obstacle } \\
\text { Pairs }\end{array}$} & $1-2$ & $1-3$ & $1-4$ & $2-3$ & $2-4$ & $3-4$ & $1-2$ & $1-3$ & $1-4$ & $2-3$ & $2-4$ & $3-4$ \\
\hline & \multicolumn{12}{|c|}{ Supermodularity Test } \\
\hline & 0.849 & $8.618^{*}$ & 0.000 & 0.472 & $3.617^{*}$ & 0.000 & 1.921 & 0.480 & 0.000 & 0.000 & 0.000 & $143.08^{*}$ \\
\hline & \multicolumn{12}{|c|}{ Submodularity Test } \\
\hline & 0.377 & 0.063 & $4.623 *$ & $2.013 \$$ & 0.000 & $5.315^{*}$ & 0.000 & 0.000 & 1.280 & 1.440 & 0.640 & 0.000 \\
\hline
\end{tabular}

$*$ Significant at the 0.01 level $†$ Significant at the 0.05 level $\$$ Significant at the 0.10 level

Table 21 Likelihood ratios for AMT - Large Plants

\begin{tabular}{|c|c|c|c|c|c|c|c|c|c|c|c|c|}
\hline & \multicolumn{6}{|c|}{ Labour Productivity } & \multicolumn{6}{|c|}{ Profit } \\
\hline \multirow[t]{5}{*}{$\begin{array}{c}\text { Obstacle } \\
\text { Pairs }\end{array}$} & $1-2$ & $1-3$ & $1-4$ & $2-3$ & $2-4$ & $3-4$ & $1-2$ & $1-3$ & $1-4$ & $2-3$ & $2-4$ & $3-4$ \\
\hline & \multicolumn{12}{|c|}{ Supermodularity Test } \\
\hline & 0.018 & $2.384^{\dagger}$ & $4.260^{*}$ & 0.254 & 0.105 & 0.044 & 0.11 & 1.025 & $2.278^{\ddagger}$ & 0.342 & 0.000 & 0.114 \\
\hline & \multicolumn{12}{|c|}{ Submodularity Test } \\
\hline & 0.780 & 0.272 & 0.035 & 0.657 & $44.509^{*}$ & $77.018^{*}$ & 0.911 & 0.342 & 0.114 & 0.683 & $4.442^{*}$ & 1.139 \\
\hline
\end{tabular}

$*$ Significant at the 0.01 level $†$ Significant at the 0.05 level $\$$ Significant at the 0.10 level

Table 22 Likelihood ratios for AMT - Medium Plants 


\begin{tabular}{|c|c|c|c|c|c|c|c|c|c|c|c|c|}
\hline & \multicolumn{6}{|c|}{ Labour Productivity } & \multicolumn{6}{|c|}{ Profit } \\
\hline \multirow[t]{5}{*}{$\begin{array}{c}\text { Obstacle } \\
\text { Pairs }\end{array}$} & $1-2$ & $1-3$ & $1-4$ & $2-3$ & $2-4$ & $3-4$ & $1-2$ & $1-3$ & $1-4$ & $2-3$ & $2-4$ & $3-4$ \\
\hline & \multicolumn{12}{|c|}{ Supermodularity Test } \\
\hline & $2.320^{*}$ & 1.036 & 0.814 & 1.165 & 0.002 & 1.215 & 1.301 & 0.021 & 1.652 & $5.618^{*}$ & 0.248 & $3.140^{\dagger}$ \\
\hline & \multicolumn{12}{|c|}{ Submodularity Test } \\
\hline & 0.681 & 0.000 & 1.234 & 0.057 & $3.944^{*}$ & 0.372 & $2.169^{*}$ & 1.074 & 1.694 & 0.454 & $8.840^{*}$ & $2.189^{*}$ \\
\hline
\end{tabular}

* Significant at the 0.01 level $\dagger$ Significant at the 0.05 level $\$$ Significant at the 0.10 level

Table 23 Likelihood ratios for AMT - Small Plants

Factor 1= Production Design (LAN, CAD/CAE, CAD/CAM, Modeling, Electronic exchange of CAD files, PLC, Lasers, Rapid prototyping, High speed machining)

Factor 2 = Communications (WAN, EDI, Electronic part identification, CIM, SCADA, Computer on factory floor, Use of inspection data, Remote controlled processes, Knowledge-based software, MRPII)

Factor 3 = Inspection, and Storage (Automated storage and retrieval, Vision based, Sensor based)

Factor 4 = Robotics (FMS/FMC, Robots with and without sensing)

\subsubsection{Business Practices}

The results from the business practices analysis demonstrates rather mixed results. Many business practices are neither complements nor substitutes for one another for the small plants. There are also a large number of substitutes found in the results. This could be because many of the business practices do not directly relate to one another.

With respect to labour productivity, large plants have quality control practices and design practices as substitutes (refer to Table 24). The two types of practices may be substitutes in terms of labour productivity, as it would take more time in order to design new products and maintain high levels of quality control. This combination of business practices would reduce the productivity of those in the design processes. The planning and design practices were found to be complementary with respect to labour productivity in large plants. Many of the planning practices are related to the design phase. 
Medium plants exhibit different characteristics than large plants (refer to Table 25). In this case, planning business practices were found to be substitutes with both the quality control practices as well as the design practice. In contrast with large plants, the quality control practices and the design practices are neither strict complements nor strict substitutes. In medium plants, using the various planning practices such as JIT and electronic work orders, in conjunction with the quality control or the design practices, results in more time being required and thus lower labour productivity. This result is due to the increased complexity that the planning business practices require including simulating any design changes and having to alter plans to maintain high quality standards.

The results for small plants with respect to labour productivity are also unique (refer to Table 26). The design practices are substitutes with both the quality control practices and the planning practices. While the quality control, practices and planning practices are complements. Here, the design practices, when paired with the quality control or design practices, results in lower labour productivity due to the added processes included in the design process. As with large plants, the quality control practices add a significant amount of effort due to tighter control standards to the design process. Similarly, the simulations included as part of the planning practices will increase the time required during the design phase of production thus reducing labour productivity.

Fewer conclusions can be drawn with respect to profit. Large plants have no conclusive results and medium plants demonstrate only that quality control and design practices are substitutes (refer to Table 24 and table 25). Once again, this relates back to the additional effort and time required to create new designs while maintaining high levels of quality control checks and balances. 


\begin{tabular}{|c|c|c|c|c|c|c|}
\hline & \multicolumn{3}{|c|}{$\begin{array}{c}\text { Labour } \\
\text { Productivity }\end{array}$} & \multicolumn{3}{c|}{ Profit } \\
\cline { 2 - 7 } $\begin{array}{c}\text { Obstacle } \\
\text { Pairs }\end{array}$ & $1-2$ & $1-3$ & $2-3$ & $1-2$ & $1-3$ & $2-3$ \\
\hline & \multicolumn{6}{|c|}{ Supermodularity Test } \\
\cline { 2 - 7 } & 1.673 & $3.231^{\dagger}$ & 0.289 & 0.317 & 0.000 & 1.585 \\
\cline { 2 - 7 } & \multicolumn{6}{|c|}{ Submodularity Test } \\
\cline { 2 - 7 } & 1.154 & 0.577 & $8.021^{*}$ & 0.000 & 0.000 & 0.000 \\
\hline
\end{tabular}

Table 24 Likelihood ratios for Business Practices - Large Plants

\begin{tabular}{|c|c|c|c|c|c|c|}
\hline & \multicolumn{3}{|c|}{$\begin{array}{c}\text { Labour } \\
\text { Productivity }\end{array}$} & \multicolumn{3}{c|}{ Profit } \\
\hline $\begin{array}{c}\text { Obstacle } \\
\text { Pairs }\end{array}$ & $1-2$ & $1-3$ & $2-3$ & $1-2$ & $1-3$ & $2-3$ \\
\hline \multirow{6}{*}{ Supermodularity Test } \\
\cline { 2 - 7 } & $8.453^{*}$ & $4.399^{\dagger}$ & $7.980^{*}$ & 0.000 & $2.712^{\ddagger}$ & 0.000 \\
\cline { 2 - 7 } & \multicolumn{6}{|c|}{ Submodularity Test } \\
\cline { 2 - 7 } & 1.800 & $4.236^{\dagger}$ & 1.418 & 0.000 & 0.000 & 0.000 \\
\hline
\end{tabular}

* Significant at the 0.01 level $\dagger$ Significant at the 0.05 level $\$$ Significant at the 0.10 level

Table 25 Likelihood ratios for Business Practices - Medium Plants

\begin{tabular}{|c|c|c|c|c|c|c|}
\hline & \multicolumn{3}{|c|}{$\begin{array}{c}\text { Labour } \\
\text { Productivity }\end{array}$} & \multicolumn{3}{c|}{ Profit } \\
\hline $\begin{array}{c}\text { Obstacle } \\
\text { Pairs }\end{array}$ & $1-2$ & $1-3$ & $2-3$ & $1-2$ & $1-3$ & $2-3$ \\
\hline \multirow{5}{*}{} & \multicolumn{6}{|c|}{ Supermodularity Test } \\
\cline { 2 - 7 } & 0.000 & $3.451^{\dagger}$ & $5.010^{*}$ & 1.322 & $3.524^{\dagger}$ & $3.164^{\dagger}$ \\
\cline { 2 - 7 } & \multicolumn{6}{|c|}{ Submodularity Test } \\
\cline { 2 - 7 } & $4.593^{\dagger}$ & 0.739 & 0.000 & $7.009^{*}$ & $4.566^{\dagger}$ & $6.288^{*}$ \\
\hline
\end{tabular}

* Significant at the 0.01 level $†$ Significant at the 0.05 level $\$$ Significant at the 0.10 level

Table 26 Likelihood ratios for Business Practices - Small Plants

Factor 1 = Quality Control (Continuous improvement, benchmarking, Certifications of suppliers and plant, and statistical control)

Factor 2 = Planning (JIT, Electronic work order, process simulation, distribution resource planning, quality function deployment)

Factor 3 = Design (Cross functional teams, concurrent engineering, continuous improvement)

Small plants have a much more significant set of results with respect to profit than either large or medium plants (refer to Table 26). The small plant results are similar to those for small 
plants with respect to labour productivity. The only difference is that for the pair-wise comparisons involving planning practices, both pairs are independent. This could be because the planning practices have an indirect effect on profit as most of the practices involve how information flows throughout the manufacturing process.

\subsubsection{Sources of Implementation Support}

The analysis of sources of information support does not have many significant results with respect to labour productivity (refer to Table 27, table 28 and table 29). There are a much greater number of significant results for sources of implementation support with respect to profit. A strong set of sources of implementation support may allow plant managers to make better choices in terms of the types of technology to implement and decrease the amount of conflict during the implementation process. This would obviously result in a larger effect on profit than on labour productivity. Since many of the sources of implementation support are found outside the plant, the participation and support of these individuals during the implementation process would have little or no effect on the labour productivity of the plant.

With respect to labour productivity in large plants, it was found that support from those involved with research and development and corporate organizations are substitutes (refer to Table 27). This result demonstrates the innovative qualities of the research and development employees and their drive to support the use of new technologies. The support that most plants would obtain from outside organizations would be R\&D related thus not noticeably laboursaving to the plant.

Medium plants exhibit two pairs of substitute sources of implementation support (refer to Table 28). Primary stakeholders and the corporate organizations are substitutes. This 
demonstrates that either medium plants have implementations of technologies championed from within the plant or they are forced upon them from head office. There is no added productivity benefit from having support from both groups. Support from those involved with research and development and support from external information sources are substitutes. Since the research and development group includes trade shows and government, there is overlap in the information being transferred to employees at the plant. Therefore, using two of these sources of support at once would not significantly increase the knowledge or ability of the workers.

Medium plants also exhibit a complementary pair of sources of implementation support. External information sources and corporate organizations are complementary. This result stems from the fact that all of these sources of support do not use internal resources and thus will increase the labour productivity of the plant since with the large amount of external support the implementation will require less internal resources for success.

The results for small plants with respect to labour productivity have some similarities to those for the medium plants (refer to Table 29). The primary stakeholders and the corporate organizations remain substitutes. Small plants also have a couple of complementary pairs of sources of implementation support. The first is the primary stakeholders and the research and development group. These two groups represent everyone within the facility so it is intuitive that if everyone within the plant supports the implementation the process of changing to the new technology will flow more smoothly. The second is that the primary stakeholders and external information sources are complementary. These are complementary since if there is support from the stakeholders and the market then the sales of the company should increase with the same number of employees thus increasing the labour productivity of the plant. 


\begin{tabular}{|c|c|c|c|c|c|c|c|c|c|c|c|c|}
\hline & \multicolumn{8}{|c|}{ Labour Productivity } & \multicolumn{1}{c|}{ Profit } \\
\hline $\begin{array}{c}\text { Obstacle } \\
\text { Pairs }\end{array}$ & $1-2$ & $1-3$ & $1-4$ & $2-3$ & $2-4$ & $3-4$ & $1-2$ & $1-3$ & $1-4$ & $2-3$ & $2-4$ & $3-4$ \\
\hline & \multicolumn{10}{|c|}{ Supermodularity Test } \\
\cline { 2 - 11 } & 1.169 & 0.868 & 0.367 & 0.234 & $2.304^{\ddagger}$ & 2.037 & $10.529^{*}$ & 0.448 & 0.506 & 0.172 & 0.403 & $10.987^{*}$ \\
\cline { 2 - 3 } & \multicolumn{10}{|c|}{ Submodularity Test } \\
\cline { 2 - 11 } & 0.000 & 0.000 & 0.100 & 0.668 & 0.067 & 0.000 & 0.125 & 0.053 & $7.097^{*}$ & $10.071^{*}$ & $9.385^{*}$ & 0.002 \\
\hline
\end{tabular}

$*$ Significant at the 0.01 level $†$ Significant at the 0.05 level $\$$ Significant at the 0.10 level

Table 27 Likelihood ratios for Implementation Support - Large Plants

\begin{tabular}{|c|c|c|c|c|c|c|c|c|c|c|c|c|}
\hline & \multicolumn{6}{|c|}{ Labour Productivity } & \multicolumn{6}{|c|}{ Profit } \\
\hline \multirow[t]{5}{*}{$\begin{array}{c}\text { Obstacle } \\
\text { Pairs } \\
\end{array}$} & $1-2$ & $1-3$ & $1-4$ & $2-3$ & $2-4$ & $3-4$ & $1-2$ & $1-3$ & $1-4$ & $2-3$ & $2-4$ & $3-4$ \\
\hline & \multicolumn{12}{|c|}{ Supermodularity Test } \\
\hline & 0.130 & 0.511 & $72.158^{*}$ & $2.467^{\ddagger}$ & 0.000 & 0.000 & 1.195 & 0.000 & 1.493 & $7.765^{*}$ & 0.000 & 0.896 \\
\hline & \multicolumn{12}{|c|}{ Submodularity Test } \\
\hline & 1.532 & 1.532 & 0.000 & 0.000 & 1.119 & $2.728^{4}$ & $2.688^{\dagger}$ & $3.434^{4}$ & $130.51^{*}$ & 0.747 & $4.480^{*}$ & $2.389^{\ddagger}$ \\
\hline
\end{tabular}

* Significant at the 0.01 level $†$ Significant at the 0.05 level $\$$ Significant at the 0.10 level

Table 28 Likelihood ratios for Implementation Support - Medium Plants

\begin{tabular}{|c|c|c|c|c|c|c|c|c|c|c|c|c|}
\hline & \multicolumn{6}{|c|}{ Labour Productivity } & \multicolumn{6}{|c|}{ Profit } \\
\hline \multirow[t]{5}{*}{$\begin{array}{c}\text { Obstacle } \\
\text { Pairs } \\
\end{array}$} & $1-2$ & $1-3$ & $1-4$ & $2-3$ & $2-4$ & $3-4$ & $1-2$ & $1-3$ & $1-4$ & $2-3$ & $2-4$ & $3-4$ \\
\hline & \multicolumn{12}{|c|}{ Supermodularity Test } \\
\hline & 0.013 & 0.003 & $71.358^{*}$ & 1.083 & 1.178 & 1.005 & 0.000 & 0.000 & 0.000 & 1.535 & 1.249 & 1.665 \\
\hline & \multicolumn{12}{|c|}{ Submodularity Test } \\
\hline & 2.304 & $46.500^{*}$ & 0.000 & 0.786 & 0.036 & 0.480 & 10.563 & 1.821 & 0.989 & 0.052 & 0.416 & 0.572 \\
\hline
\end{tabular}

$*$ Significant at the 0.01 level $\dagger$ Significant at the 0.05 level $\$$ Significant at the 0.10 level

Table 29 Likelihood ratios for Implementation Support - Small Plants

Factor 1 = Primary Stakeholders (Production Engineering, Production, Design, Sales and Marketing staff, Suppliers, Customers, Patents)

Factor 2 = Research \& Development (Research, Experimental development, Production engineering, Trade Fairs, Governments, Other producers)

Factor 3 = External Information Sources (Sales and Marketing, Customers, Related Firms)

Factor 4 = Corporate Organizations (Head Office, Related plants, Universities)

With respect to profit, large plants exhibit two sets of substitute sources of implementation support (refer to Table 27). The primary stakeholders and the research and development group are substitutes. This result may be because in large plants there are research 
and development employees that determine what new technology is required in order to make new products and remain competitive so having more people involved will only increase expenses. Once again, the external information sources and the corporate organizations are substitutes.

Large plants also exhibit a number of complementary pairs related to sources of implementation support. The first is that primary stakeholders and corporate organizations are complementary. This result is due to the fact that if all the corporate organizations support the technologies being used, then they will be able to align the products and marketing campaign for the entire company to take advantage of the features inherent in the chosen technology. As well, corporate organizations are complementary with the research and development group. The complementarity between these two groups derives from eliminating the duplication of research into the technologies between company plants and increasing the sharing of information between the company plants in order to troubleshoot any problems during the implementations. Finally, the research and development group and external information sources are also complementary. Once again, by using outside sources, the members of the research and development group will be able to gain greater knowledge about the technologies, thus allowing them to find the proper technology for the plant's needs and troubleshoot implementation problems more easily.

Medium plants exhibit a number of significant results with respect to the profit function (refer to Table 28). As in the analysis related to labour productivity, the research and development group and the external information sources are substitutes. As in large plants, primary stakeholders and the research and development group are both complementary with the corporate organizations. There are a number of other complementary pairs found in the medium plant results. First, the primary stakeholders are complementary with both the research and 
development group and external information sources. This is a very intuitive result since when there is support from primary stakeholders any additional support is beneficial to the plant's profit margin. The other pair of complementary sources of implementation support is the external information sources and corporate organizations. This support does not affect the costs of the plant, thus these two combined sources of support will offer a better set of information to the plant for very little cost.

The results for small plants with respect to profit are mostly inconclusive (refer to Table 29). There is one significant result. Primary stakeholders and the research and development group are complementary. This is similar to the results found with respect to labour productivity, and those of the medium plants with respect to profit.

\subsubsection{Perceived Benefits of AMT}

Very few perceived benefit pairings exhibited significant test results. This is due primarily to the fact that the majority of plants in any of the size classes fall within three to five states (refer to Table 12). Since there are few plants in most of the states, the results for the regression analysis are largely inconclusive.

There is one significant result for large plants with respect to labour productivity (refer to Table 30). The perceived benefit of flexibility improvements and profitability improvements are substitutes. This could be because the elements of the flexibility component include increased quality and flexibility that would lead to creating more high quality, custom product, which in turn would increase productivity.

There is also a significant pairing for small plants with respect to labour productivity (refer to Table 32). In this case, a perceived reduction of time usage and increases in productivity 
are complementary. This result is intuitive; as if a plant is able to reduce the amount of time it takes to set-up and deal with quality issues and at the same time increase the utilization rate of equipment then there will be an overall increase in labour productivity.

With respect to profit, large and small plants both exhibit the same significant pairing (refer to Table 30 and table 32). The perceived benefit of productivity improvements and the perceived benefit of profitability increases are substitutes. Medium plants also exhibit a significant pairing of perceived benefits (refer to Table 31). The perceived benefit of productivity increases and the perceived benefit of flexibility improvements are substitutes. These may be substitutes as increased flexibility involves increased skill requirements that will increase labour costs and may cause the labour requirements and capital costs included in the productivity measure to rise.

\begin{tabular}{|c|c|c|c|c|c|c|c|c|c|c|c|c|}
\hline & \multicolumn{6}{|c|}{ Labour Productivity } & \multicolumn{6}{|c|}{ Profit } \\
\hline \multirow[t]{5}{*}{$\begin{array}{c}\text { Obstacle } \\
\text { Pairs }\end{array}$} & $1-2$ & $1-3$ & $1-4$ & $2-3$ & $2-4$ & $3-4$ & $1-2$ & $1-3$ & $1-4$ & $2-3$ & $2-4$ & $3-4$ \\
\hline & \multicolumn{12}{|c|}{ Supermodularity Test } \\
\hline & 0.541 & 0.000 & 0.180 & 0.861 & 0.621 & $2.664^{\dagger}$ & 0.000 & 0.000 & 0.000 & 0.616 & $174.68^{*}$ & 0.097 \\
\hline & \multicolumn{12}{|c|}{ Submodularity Test } \\
\hline & 0.000 & 1.342 & 0.100 & 0.300 & 0.581 & 0.020 & 0.205 & 0.411 & 0.000 & 0.000 & 0.000 & 0.411 \\
\hline
\end{tabular}

Table 30 Likelihood ratios for Perceived Benefits of AMT - Large Plants

\begin{tabular}{|c|c|c|c|c|c|c|c|c|c|c|c|c|}
\hline & \multicolumn{6}{|c|}{ Labour Productivity } & \multicolumn{6}{|c|}{ Profit } \\
\hline \multirow[t]{5}{*}{$\begin{array}{c}\text { Obstacle } \\
\text { Pairs } \\
\end{array}$} & $1-2$ & $1-3$ & $1-4$ & $2-3$ & $2-4$ & $3-4$ & $1-2$ & $1-3$ & $1-4$ & $2-3$ & $2-4$ & $3-4$ \\
\hline & \multicolumn{12}{|c|}{ Supermodularity Test } \\
\hline & 1.500 & 0.474 & 0.32 & 0.688 & 0.013 & 0.286 & 1.771 & 0.000 & 0.443 & 2.214 & 0.295 & 0.148 \\
\hline & \multicolumn{12}{|c|}{ Submodularity Test } \\
\hline & 0.013 & 0.006 & 0.870 & 0.000 & 0.565 & 0.039 & 0.148 & 0.886 & 0.590 & 0.000 & 0.886 & 0.738 \\
\hline
\end{tabular}

Table 31 Likelihood ratios for Perceived Benefits of AMT - Medium Plants 


\begin{tabular}{|c|c|c|c|c|c|c|c|c|c|c|c|c|}
\hline & \multicolumn{6}{|c|}{ Labour Productivity } & \multicolumn{6}{|c|}{ Profit } \\
\hline \multirow[t]{5}{*}{$\begin{array}{c}\text { Obstacle } \\
\text { Pairs }\end{array}$} & $1-2$ & $1-3$ & $1-4$ & $2-3$ & $2-4$ & $3-4$ & $1-2$ & $1-3$ & $1-4$ & $2-3$ & $2-4$ & $3-4$ \\
\hline & \multicolumn{12}{|c|}{ Supermodularity Test } \\
\hline & 0.155 & 0.062 & 0.000 & 0.258 & 0.538 & 0.115 & 0.053 & 0.319 & 0.000 & 0.000 & $3.220^{\dagger}$ & 0.053 \\
\hline & \multicolumn{12}{|c|}{ Submodularity Test } \\
\hline & $28.675^{*}$ & 0.118 & 0.211 & 0.224 & 0.000 & 0.068 & 0.346 & 0.000 & 0.958 & 0.452 & 0.000 & 0.293 \\
\hline
\end{tabular}

$*$ Significant at the 0.01 level $†$ Significant at the 0.05 level $\$$ Significant at the 0.10 level

Table 32 Likelihood ratios for Perceived Benefits of AMT - Small Plants

Factor 1 = Time (Reduced Capital, Reduced rejection time, Reduced set-up time, Increased quality, New product features, Reduced time to market)

Factor 2 = Productivity (Reduced labour, Reduced material, Reduced capital) Increased utilization rate, Increased Profitability)

Factor 3 = Flexibility (Increased flexibility, Increased skill requirements, Increased quality)

Factor 4 = Profitability (Increased equipment utilization rate, Increased market share, Increased Profitability)

\subsubsection{High-Level Factors}

There are a number of significant results for all plant sizes for the analysis of the high-level factors (refer to Table 33, table 34, and table 35). Each plant size exhibits its own unique set of complementary and substitute pairs, although there are a couple of similarities as well. For example, all plant sizes have the AMT and business practices factor and the implementation support and R\&D factor complementary with respect to both labour productivity and profit.

With respect to labour productivity, large plants exhibit a number of substitute pairings (refer to Table 33). The perceived benefits factor is a substitute for both the design factor and the human resources factor. These results show that the perception of AMT by the plant manager does not appear to increase the effect of the measurable elements of design technology usage or increased human resources initiatives. The AMT and business practices factor and the human resources factor are also substitutes demonstrating that the use of technology is a substitute for additional training that might be offered by human resources in order to improve labour 
productivity. Finally, the implementation support and R\&D factor and the design factor are substitutes with respect to labour productivity. This result may be due to duplication of effort between the research and development group and the design group.

Large plants also exhibit another complementary pair with respect to labour productivity, the design factor and human resources factor. The majority of the human resources initiatives surveyed were geared towards the design group. By hiring workers with greater skill sets that have closer ties to universities and colleges, the efficiency of the people in the design group will increase.

The results for medium plants with respect to labour productivity include more complementary pairs than those of large plants (refer to Table 34). There are only two substitute pairs for medium plants. The first is the perceived benefits factor and the implementation support and R\&D factor. The second is the human resources and the design factor. This result could be because not many of the medium plants used the survey's human resource policies (only $35 \%$ of medium plants exhibit the human resources factor (refer to Table 9).

The perceived benefits factor is complementary with both the design factor and the human resources factor with respect to labour productivity. In medium plants, having the perception of benefits of AMT appears to increase the acceptance of the technology and make it easier for the human resource policies to be effectively implemented and the design team to better utilize its resources. The other complementary pairing is between the implementation support and R\&D factor and human resources factor. Once again, the ability to obtain highly skilled personnel through improved human resource initiatives increases the research and development capabilities of the plant. 
The results for small plants with respect to labour productivity exhibit similarities with both the large plants and the medium plants (refer to Table 35). As in the large plant analysis, the perceived benefits and human resources factors are substitutes. As well, the design factor and human resources factor are complements. Small plants also have the perceived benefits and implementation support and R\&D factors as substitutes similar to the results for medium plants.

Unique to the small plants though, are a few other significant results. First, the AMT and business practices and the design factor are substitutes. It could be that the more technology that is included in the manufacturing process, the more complex the design phase becomes, thus decreasing labour productivity. Small plants also have the pair-wise comparison of implementation support and R\&D factor with the human resources factor rejecting both strict complementarity and substitutability. The significance level for supermodularity is low relative to that of submodularity, which implies that there is probably some level of weak complementarity similar to the results for medium plants.

\begin{tabular}{|c|c|c|c|c|c|c|c|c|c|c|}
\hline & \multicolumn{10}{|c|}{ Labour Productivity } \\
\hline \multirow{6}{*}{$\begin{array}{c}\text { Obstacle } \\
\text { Pairs }\end{array}$} & $1-2$ & $1-3$ & $1-4$ & $1-5$ & $2-3$ & $2-4$ & $2-5$ & $3-4$ & $3-5$ & $4-5$ \\
\hline & \multicolumn{10}{|c|}{ Supermodularity Test } \\
\hline & 0.016 & 0.574 & $4348.1^{*}$ & $4348.1^{*}$ & 0.031 & 0.931 & $1.801^{*}$ & $1.847^{\ddagger}$ & 0.186 & 0.031 \\
\hline & \multicolumn{10}{|c|}{ Submodularity Test } \\
\hline & 0.310 & 0.388 & 1.273 & 0.202 & $4348.1^{*}$ & 0.248 & 0.000 & 0.062 & 0.792 & $2.546^{*}$ \\
\hline & \multicolumn{10}{|c|}{ Profit } \\
\hline \multirow{5}{*}{$\begin{array}{c}\text { Obstacle } \\
\text { Pairs }\end{array}$} & $1-2$ & $1-3$ & $1-4$ & $1-5$ & $2-3$ & $2-4$ & $2-5$ & $3-4$ & $3-5$ & $4-5$ \\
\hline & \multicolumn{10}{|c|}{ Supermodularity Test } \\
\hline & $80.860^{*}$ & 0.535 & 1.447 & $80.860^{*}$ & 0.021 & $80.860^{*}$ & 1.438 & $80.860^{*}$ & $3.766^{*}$ & 0.113 \\
\hline & \multicolumn{10}{|c|}{ Submodularity Test } \\
\hline & 0.005 & $2.064^{\dagger}$ & $80.860^{*}$ & 1.445 & $80.860^{*}$ & 0.247 & 1.049 & $2.068^{\dagger}$ & 0.879 & $80.860^{*}$ \\
\hline
\end{tabular}

$*$ Significant at the 0.01 level $\dagger$ Significant at the 0.05 level $\$$ Significant at the 0.10 level

Table 33 Likelihood ratios for High-Level Factors - Large Plants 


\begin{tabular}{|c|c|c|c|c|c|c|c|c|c|c|}
\hline & \multicolumn{10}{|c|}{ Labour Productivity } \\
\hline \multirow{6}{*}{$\begin{array}{c}\text { Obstacle } \\
\text { Pairs }\end{array}$} & $1-2$ & $1-3$ & $1-4$ & $1-5$ & $2-3$ & $2-4$ & $2-5$ & $3-4$ & $3-5$ & $4-5$ \\
\hline & \multicolumn{10}{|c|}{ Supermodularity Test } \\
\hline & 1.010 & $51.013^{*}$ & 0.022 & 0.140 & 0.599 & 1.260 & 0.315 & 0.599 & 0.905 & $49.539^{*}$ \\
\hline & \multicolumn{10}{|c|}{ Submodularity Test } \\
\hline & 0.114 & 0.035 & $2.108^{\dagger}$ & $49.329^{*}$ & $5705.1^{*}$ & 0.118 & 0.595 & 0.630 & $43.472^{*}$ & 0.328 \\
\hline & \multicolumn{10}{|c|}{ Profit } \\
\hline \multirow{5}{*}{$\begin{array}{l}\text { Obstacle } \\
\text { Pairs }\end{array}$} & $1-2$ & $1-3$ & $1-4$ & $1-5$ & $2-3$ & $2-4$ & $2-5$ & $3-4$ & $3-5$ & $4-5$ \\
\hline & \multicolumn{10}{|c|}{ Supermodularity Test } \\
\hline & 0.417 & 1.488 & 0.417 & 0.417 & 0.774 & 0.357 & 0.952 & $297.70^{*}$ & 0.179 & 1.488 \\
\hline & \multicolumn{10}{|c|}{ Submodularity Test } \\
\hline & 0.595 & 0.179 & $1.964^{\dagger}$ & 0.774 & $297.70^{*}$ & $3.749^{*}$ & 0.655 & 0.298 & $4.106^{*}$ & 0.476 \\
\hline
\end{tabular}

$*$ Significant at the 0.01 level $†$ Significant at the 0.05 level $\$$ Significant at the 0.10 level

Table 34 Likelihood ratios for High-Level Factors - Medium Plants

\begin{tabular}{|c|c|c|c|c|c|c|c|c|c|c|}
\hline & \multicolumn{10}{|c|}{ Labour Productivity } \\
\hline \multirow{6}{*}{$\begin{array}{c}\text { Obstacle } \\
\text { Pairs }\end{array}$} & $1-2$ & $1-3$ & $1-4$ & $1-5$ & $2-3$ & $2-4$ & $2-5$ & $3-4$ & $3-5$ & $4-5$ \\
\hline & \multicolumn{10}{|c|}{ Supermodularity Test } \\
\hline & 0.858 & $49.083^{*}$ & 0.499 & $17.716^{*}$ & 0.728 & $1.961^{\dagger}$ & 0.278 & 1.325 & $2.131^{\dagger}$ & 0.724 \\
\hline & \multicolumn{10}{|c|}{ Submodularity Test } \\
\hline & 0.209 & 0.607 & 0.650 & 0.176 & $2525.3^{*}$ & 0.265 & 0.781 & 0.730 & $46.231^{*}$ & $1.783^{*}$ \\
\hline & \multicolumn{10}{|c|}{ Profit } \\
\hline \multirow{5}{*}{$\begin{array}{c}\text { Obstacle } \\
\text { Pairs }\end{array}$} & $1-2$ & $1-3$ & $1-4$ & $1-5$ & $2-3$ & $2-4$ & $2-5$ & $3-4$ & $3-5$ & $4-5$ \\
\hline & \multicolumn{10}{|c|}{ Supermodularity Test } \\
\hline & 1.337 & 0.316 & $11.424^{*}$ & 0.704 & 0.949 & $11.056^{*}$ & 0.316 & 0.745 & 1.256 & 1.317 \\
\hline & \multicolumn{10}{|c|}{ Submodularity Test } \\
\hline & 0.368 & $4.094^{*}$ & 0.235 & 0.184 & $75.087^{*}$ & 0.858 & $11.271^{*}$ & 1.613 & 0.439 & 0.061 \\
\hline
\end{tabular}

* Significant at the 0.01 level $†$ Significant at the 0.05 level $\$$ Significant at the 0.10 level

Table 35 Likelihood ratios for High-Level Factors - Small Plants

Factor $1=$ Perceived Benefits

Factor 2 = Advanced Manufacturing Technologies (Except Design) \& Business Practices

Factor 3 = Implementation Support, Cross Functional Teams \& Research and Development

Factor 4 = Design (Production Design Technologies, Concurrent Engineering)

Factor $5=$ Human Resources

With respect to profit, the results for large plants differ slightly from those for labour productivity and include many additional significant results (refer to Table 33). The design factor 
and human resources factor remain complements, furthermore, the perceived benefits and the human resources factors remain substitutes. Similar to the medium plant analysis, the perceived benefits and the design factor are complements. As well, the perceived benefits factor and the AMT and business practices factor are substitutes. This result demonstrates that a large perception in the benefits of AMT and their use does not necessarily lead to increased profits. This result supports the findings of Barua and Lee (1997). The technology must be utilized properly for it to have a positive effect on profit and the reputation of the technology within the plant does not guarantee success. The AMT and business practices factor and the design factor are also substitutes with respect to profit. Once again, the additional complexities and costs associated with the use of AMT seem to have negatively affected profit. This could be due to many companies not taking advantage of AMT features that coincide with their market position. The higher cost of designing a standard product using the more sophisticated technology and the capital cost of the technology itself decrease profit for plants that manufacture large standard batch sizes (Troxler, 1990).

The results for medium plants with respect to profit are very similar to those with respect to labour productivity (refer to Table 34). The perceived benefits factor and the design factor remain complementary, as do the implementation support and R\&D factor and the human resources factor. In this case, the AMT and business practices and the design factor are complementary. Medium plants seem to be able to better utilize the technology to increase their profits when they have integrated AMT and design. This could be because the labour requirements for the technology is lower, allowing the medium firms to have higher profit margins than large plants due to more efficient use of resources. There is also a substitute pairing found in the medium plant analysis, the implementation support and R\&D factor and the design 
factor are substitutes. Once again, this could be because there are very few medium plants that conduct a large amount of $R \& D$. That $R \& D$ which is done may be a duplication of effort between the research and development group and the design group within the plant.

Finally, small plants exhibit a few substitute and complementary pairings (refer to Table 35). The AMT and business practices and the design factors remain substitutes. The perceived benefits factor and the design factor are also substitutes with respect to profit. This result could stem from the fact that the perception of the technology does not aid the designer in using it effectively and may cause conflict between management and the designers when the technology does not work as effectively as predicted. Similar to large plants, the perceived benefits and implementation support and R\&D factors are complementary for small plants. The AMT and business practices and human resources factors are also complementary. This result supports the intuitive idea that higher skilled workers are required to effectively operate more complex forms of technology. Hiring more skilled workers and providing better incentives and training will bring (and help retain) the most qualified personnel, who in turn are better able to utilize technology to its optimum capacity.

\subsubsection{Conclusions}

The analysis of complementarities by plant size demonstrates that size matters with respect to optimal states for plants to inhabit. The difference in complementary and substitute pairs of factors supports the results found by Meredith (1996) that small plants will operate differently from large plants and they will use different technology than large plants. The fact that different complementarities are found with respect to labour productivity and profit demonstrate that there are other important factors, such as the amount of excess plant capacity, not included in the analysis that helps explain the transformation of labour productivity into profit. The 
complementarities found with respect to labour productivity relate to inconclusive pairings in the profit analysis or often exhibit identical results. The number of significant results is not consistently greater using labour productivity rather than profit. It appears that some of the factors, such as sources of implementation support have more significant results for profit, while the use of AMT and business practices has a greater impact on labour productivity.

The hypothesis from Alcorta (1994) that all forms of AMT are complementary was rejected in this analysis. Presumably this is because of the large selection of AMT analyzed and many of these technologies were not prevalent during Alcorta's 1994 study (only seven of the twenty-six technologies were included in the study) or because Alcorta (1994) only studied one form of industry. As there are many industries in each size class, if industry is the stronger effect many of the results by size class will be confounded due to the mixing of industries. I believe this may explain the large number of inconclusive results found in the AMT analysis, as well as the large number of independent pairings found in the business practices analysis.

The perceived benefits analysis has a large number of inconclusive results. In this case, I believe the results are due to the subjective nature of the questions and the high level of similarity between the questions themselves. Managers must have a large number of perceived benefits of the technology or they would not have paid to implement it. The benefits they perceive may be due to actual observations of the technology at work in their plant or from sales representatives' comments. These two sources of perception of benefits can lead to opposite conclusions and thus those who answered based on sales hype and not the results of actual implementations may confound the results. 
Large firms must be careful when deciding to incorporate many of the quality control business practices into their plants. These practices are substitutes with both of the other forms of business practices (planning and design) as they increase the time and effort required to accomplish all other tasks. It may be that the quality control business practices are substitutes for other business practices in large plants simply due to the amount of interactions between different groups that must occur for the business practice to be successful. The efficient interaction of groups is something that is much easier to achieve in smaller plants where there is less hierarchy and fewer people involved in the process.

The sources of implementation support analysis demonstrates how beneficial it is to have a large number of primary stakeholders involved with the AMT implementation for medium and small plants. The support of primary stakeholders increases the benefits of all other forms of support and is thus critical for small and medium plants to have successful implementations of AMT. The results from the high-level analysis support the results from Udo and Ehie (1996) that the successful implementation of AMT requires strong support from within the organization and a strong champion to lead the implementation within any size plant.

\subsubsection{Results by Industry}

Many exceptions had to be made when considering the analysis by industry. Many of these exceptions occurred in the machinery industry, which experienced a concentration of plants in only a few states, and the remaining states where very sparsely populated. The sample sizes for the various industries are large enough that the same cut-off values for significance level can be used for most industries (except in the non-metals, plastics, and machinery industries that have smaller sample sizes). The analysis below will focus on the supermodularity and submodularity tests that were determined to be significant by factor type. Unlike the analysis presented for the 
size classes, both the analysis with respect to labour productivity and profit will be done at once for each industry. The results will be presented in this manner since there are a large number of similarities between both models. Overall conclusions about the constrained regression analysis by industry will be made at the end of the section.

\subsubsection{Advanced Manufacturing Technologies}

The results of AMT implementations by industry demonstrate that there are strong industry effects that must be considered. The usage of some forms of AMT in low-tech industries results in substitute pairings, whereas these same pairings are complementary in medium and high-tech plants. Due to the smaller sample sizes for each industry, there are also a greater number of inconclusive results that are very close to the significance level cut-off value.

The chemical industry is an example of an industry with a large number of inconclusive results (refer to Table 36). This industry has only one significant pairing with respect to profit. Production design and inspection and storage technologies are complementary. This result is intuitive based on the types of products produced and the high level of quality control that must be maintained in order to meet government and safety regulations.

The electronics industry exhibits significantly different results from the chemical industry (refer to Table 37). In this case, the production design and inspection and storage technologies are substitutes with respect to labour productivity. This result may be due to the fact that the integration of the various forms of technology is complex. It may also be that there are a large number of inspection processes that are built-in during the design phased and included within the design technologies so that additional inspection equipment may be a duplication of effort. The inspection and storage and robotics technologies were found to be complementary though, with 
respect to profit. This result, as found in the size class analysis, is because robotics in manufacturing relies heavily on the automated storage and retrieval systems in order to obtain raw material and shelve finished goods.

The furniture and other industries have a large number of significant results (refer to Table 38). These results are consistent from the analysis with respect to both labour productivity and profit. The communications and the inspection and storage technologies are found to be substitutes in both models. As in the analysis by size class, this may be because many furniture plants would be smaller operations that cannot effectively use inspection and storage technologies due to the artistic nature of the product. In this case, the information obtained from a good communications network and inventory tracking may be sufficient for the plant's success. Production design technologies and robotics are also found to be substitutes. Once again, the nature of the manufactured product results in low robotics use by plants in this industry. The communications and robotics technologies are found to be complementary with respect to both models. These results stem primarily from the larger plants in this industry which produce more standard product lines and rely on good network technologies to communicate the subtle line changes they need in their automated manufacturing process. The furniture and other industries also exhibit a final complementary but only with respect to profit. As with the electronics industry, the inspection and storage and robotics technologies are complementary.

The machinery industry has only two significant pairings but these results are significant in the analysis of both models (refer to Table 39). As in the furniture industry, the production design technologies and the robotics technologies are substitutes and the communication and robotics technologies are complements. These results demonstrate that as in the furniture 
industry, the use of technology to create very similar products has a strong effect on the complementarities exhibited in the industry.

The metals industry also has similar results to those in the furniture and other industries (refer to Table 40). The analysis with respect to labour productivity finds that both the design technologies and robotics technologies and the communication and inspection and storage technologies are substitutes. This industry has the communication and inspection and storage technologies as substitutes and the communication and robotics technologies as complements with respect to profit as in the furniture analysis. The metals industry also exhibits an additional significant pair with respect to profit; the production design technologies and the communications technologies are substitutes.

The non-metals industry has completely different results from every other industry (refer to Table 41). Here, the production design technologies and the inspection and storage technologies are complementary and the inspection and storage and robotics technologies are substitutes with respect to labour productivity. These results demonstrate that the implementation of AMT must be considered separately for each industry and that by doing analysis with a mixture of industries may result in inconclusive tests.

The plastics industry is somewhat similar to the electronics industry (refer to Table 42). Production design technologies and inspection and storage technologies are substitutes with respect to profit. Another significant result can be found in the analysis with respect to profit. The production design technologies and the robotics technologies are complementary. This finding is somewhat intuitive, since if the majority of the design process is done electronically 
then it will be very simple to transfer the design to robots and optimally use their features in production.

There is only one significant pairing in the textiles industry, although it is significant for both models (refer to Table 43). As in the plastics industry, the production design technologies and the inspection and storage technologies are substitutes. This may once again be a result of the nature of the manufactured product, as many of the design technologies are not appropriate for textiles.

The vehicles industry has a large number of significant findings (refer to Table 44). Once again, the design technologies and communication technologies are found to be substitutes with respect to both models. Communications and inspection and storage technologies reject both the complementarity hypothesis as well as the substitutability hypothesis with respect to labour productivity. Inspection and storage technologies and robotics are also independent with respect to profit. These results may be due the fact that there are plants that specialize in different stages of the vehicle manufacturing process and their needs and ability to use storage and retrieval technologies varies greatly. The communications and robotics technologies are found to be substitutes with respect to labour productivity. This result is primarily due to the fact that the increased complexity of the operations when all of these technologies are used requires higher skilled workers or more time for the average worker to perform changes or problem solving tasks. As in the analysis by size class, the inspection and storage and the robotics technologies are complementary with respect to labour productivity. This complementarity stems in part from the fact that both of these forms of technology have a similar level of technical complexity that results in knowledge transfer in the learning process. There is also a complementarity pairing of production design and inspection and storage technologies unique to the analysis with respect to 
profit. This result is similar to that found with respect to labour productivity in the non-metals industry.

\begin{tabular}{|c|c|c|c|c|c|c|c|c|c|c|c|c|}
\hline & \multicolumn{6}{|c|}{ Labour Productivity } & \multicolumn{6}{|c|}{ Profit } \\
\hline $\begin{array}{c}\text { Obstacle } \\
\text { Pairs }\end{array}$ & $1-2$ & $1-3$ & $1-4$ & $2-3$ & $2-4$ & $3-4$ & $1-2$ & $1-3$ & $1-4$ & $2-3$ & $2-4$ & $3-4$ \\
\hline & \multicolumn{12}{|c|}{ Supermodularity Test } \\
\hline & 0.571 & 0.051 & 0.286 & 0.796 & 1.602 & 0.418 & 0.061 & 0.000 & 0.033 & 0.126 & 2.066 & 0.403 \\
\hline & \multicolumn{12}{|c|}{ Submodularity Test } \\
\hline & 0.000 & 0.214 & 0.031 & 0.275 & 0.010 & 0.082 & 0.028 & $115.01^{*}$ & 0.032 & 0.230 & 0.000 & 0.000 \\
\hline
\end{tabular}

* Significant at the 0.01 level $\dagger$ Significant at the 0.05 level $\$$ Significant at the 0.10 level

Table 36 Likelihood ratios for AMT - Chemical Industry

\begin{tabular}{|c|c|c|c|c|c|c|c|c|c|c|c|c|}
\hline & \multicolumn{6}{|c|}{ Labour Productivity } & \multicolumn{6}{|c|}{ Profit } \\
\hline $\begin{array}{l}\text { Obstacle } \\
\text { Pairs }\end{array}$ & $1-2$ & $1-3$ & $1-4$ & $2-3$ & $2-4$ & $3-4$ & $1-2$ & $1-3$ & $1-4$ & $2-3$ & $2-4$ & $3-4$ \\
\hline & \multicolumn{12}{|c|}{ Supermodularity Test } \\
\hline & 0.543 & $2.209^{\ddagger}$ & 0.153 & 0.653 & 0.722 & 0.861 & 0.154 & 0.061 & 0.092 & 0.277 & 0.000 & 0.061 \\
\hline & \multicolumn{12}{|c|}{ Submodularity Test } \\
\hline & 0.431 & 0.028 & 1.056 & 0.597 & 0.917 & 0.236 & 0.031 & 0.092 & 0.061 & 0.000 & 0.184 & $21.761^{*}$ \\
\hline
\end{tabular}

* Significant at the 0.01 level $\dagger$ Significant at the 0.05 level $\quad$ Significant at the 0.10 level

Table 37 Likelihood ratios for AMT - Electronics Industry

\begin{tabular}{|c|c|c|c|c|c|c|c|c|c|c|c|c|}
\hline & \multicolumn{6}{|c|}{ Labour Productivity } & \multicolumn{6}{|c|}{ Profit } \\
\hline $\begin{array}{c}\text { Obstacle } \\
\text { Pairs }\end{array}$ & $1-2$ & $1-3$ & $1-4$ & $2-3$ & $2-4$ & $3-4$ & $1-2$ & $1-3$ & $1-4$ & $2-3$ & $2-4$ & $3-4$ \\
\hline & \multicolumn{12}{|c|}{ Supermodularity Test } \\
\hline & 1.267 & 0.538 & $1.961^{\ddagger}$ & $33.928^{*}$ & 0.087 & 0.772 & 1.463 & 1.705 & $32.182^{*}$ & $3.240^{\circ}$ & 1.182 & 0.667 \\
\hline & \multicolumn{12}{|c|}{ Submodularity Test } \\
\hline & 0.017 & 0.165 & 0.035 & 0.416 & $3.019^{4}$ & 0.668 & 0.213 & 1.267 & 1.232 & 1.514 & $5.762^{*}$ & $5.089^{*}$ \\
\hline
\end{tabular}

* Significant at the 0.01 level $†$ Significant at the 0.05 level $\$$ Significant at the 0.10 level

Table 38 Likelihood ratios for AMT - Furniture and Other Industries

\begin{tabular}{|c|c|c|c|c|c|c|c|c|c|c|c|c|}
\hline & \multicolumn{6}{|c|}{ Labour Productivity } & \multicolumn{6}{|c|}{ Profit } \\
\hline \multirow[t]{5}{*}{$\begin{array}{c}\text { Obstacle } \\
\text { Pairs } \\
\end{array}$} & $1-2$ & $1-3$ & $1-4$ & $2-3$ & $2-4$ & $3-4$ & $1-2$ & $1-3$ & $1-4$ & $2-3$ & $2-4$ & $3-4$ \\
\hline & \multicolumn{12}{|c|}{ Supermodularity Test } \\
\hline & 1.315 & 1.185 & $8.473^{*}$ & 0.433 & 0.097 & 0.016 & 0.000 & 0.185 & $8.292^{*}$ & 0.243 & 0.000 & 0.206 \\
\hline & \multicolumn{12}{|c|}{ Submodularity Test } \\
\hline & 0.081 & 0.000 & 0.000 & 0.308 & $8.279^{\prime}$ & 0.779 & 0.231 & 0.000 & 0.000 & 0.041 & $10.072^{*}$ & 0.248 \\
\hline
\end{tabular}

* Significant at the 0.01 level $\dagger$ Significant at the 0.05 level $\$$ Significant at the 0.10 level

Table 39 Likelihood ratios for AMT - Machinery Industry 


\begin{tabular}{|c|c|c|c|c|c|c|c|c|c|c|c|c|}
\hline & \multicolumn{6}{|c|}{ Labour Productivity } & \multicolumn{6}{|c|}{ Profit } \\
\hline \multirow[t]{5}{*}{$\begin{array}{c}\text { Obstacle } \\
\text { Pairs }\end{array}$} & $1-2$ & $1-3$ & $1-4$ & $2-3$ & $2-4$ & $3-4$ & $1-2$ & $1-3$ & $1-4$ & $2-3$ & $2-4$ & $3-4$ \\
\hline & \multicolumn{12}{|c|}{ Supermodularity Test } \\
\hline & 1.516 & 0.572 & $17.881^{*}$ & $10.300^{*}$ & 0.012 & 0.042 & $2.857^{\dagger}$ & 0.348 & 0.056 & $2.700^{\dagger}$ & 0.093 & $2.020^{\ddagger}$ \\
\hline & \multicolumn{12}{|c|}{ Submodularity Test } \\
\hline & 0.115 & 0.012 & 0.039 & 0.000 & 0.835 & 0.148 & 0.738 & 0.906 & 4.635 & 0.036 & $2.229^{\ddagger}$ & 0.000 \\
\hline
\end{tabular}

* Significant at the 0.01 level $†$ Significant at the 0.05 level $\$$ Significant at the 0.10 level

Table 40 Likelihood ratios for AMT - Metals Industry

\begin{tabular}{|c|c|c|c|c|c|c|c|c|c|c|c|c|}
\hline & \multicolumn{6}{|c|}{ Labour Productivity } & \multicolumn{6}{|c|}{ Profit } \\
\hline \multirow[t]{5}{*}{$\begin{array}{c}\text { Obstacle } \\
\text { Pairs }\end{array}$} & $1-2$ & $1-3$ & $1-4$ & $2-3$ & $2-4$ & $3-4$ & $1-2$ & $1-3$ & $1-4$ & $2-3$ & $2-4$ & $3-4$ \\
\hline & \multicolumn{12}{|c|}{ Supermodularity Test } \\
\hline & 0.879 & 0.117 & 0.059 & 0.142 & 0.519 & $22.162^{*}$ & 0.410 & 0.000 & 0.046 & 0.200 & 0.047 & 0.153 \\
\hline & \multicolumn{12}{|c|}{ Submodularity Test } \\
\hline & 0.318 & $2.202^{\$}$ & 0.343 & 0.603 & 0.167 & 0.000 & 0.124 & 1.509 & 0.085 & 0.017 & 0.013 & 0.001 \\
\hline
\end{tabular}

$*$ Significant at the 0.01 level $†$ Significant at the 0.05 level $\$$ Significant at the 0.10 level

Table 41 Likelihood ratios for AMT - Non-Metals Industry

\begin{tabular}{|c|c|c|c|c|c|c|c|c|c|c|c|c|}
\hline & \multicolumn{6}{|c|}{ Labour Productivity } & \multicolumn{6}{|c|}{ Profit } \\
\hline \multirow[t]{5}{*}{$\begin{array}{c}\text { Obstacle } \\
\text { Pairs } \\
\end{array}$} & $1-2$ & $1-3$ & $1-4$ & $2-3$ & $2-4$ & $3-4$ & $1-2$ & $1-3$ & $1-4$ & $2-3$ & $2-4$ & $3-4$ \\
\hline & \multicolumn{12}{|c|}{ Supermodularity Test } \\
\hline & 0.140 & 1.270 & 0.676 & 0.396 & 0.851 & 0.023 & 2.129 & $2.443^{\ddagger}$ & 0.330 & 0.581 & 1.494 & 1.451 \\
\hline & \multicolumn{12}{|c|}{ Submodularity Test } \\
\hline & 0.070 & 0.152 & 0.117 & 0.210 & 0.000 & 0.758 & 0.330 & 1.643 & $2.655^{\ddagger}$ & 1.292 & 1.019 & 1.347 \\
\hline
\end{tabular}

$*$ Significant at the 0.01 level $\dagger$ Significant at the 0.05 level $\$$ Significant at the 0.10 level

Table 42 Likelihood ratios for AMT - Plastics Industry

\begin{tabular}{|c|c|c|c|c|c|c|c|c|c|c|c|c|}
\hline & \multicolumn{6}{|c|}{ Labour Productivity } & \multicolumn{6}{|c|}{ Profit } \\
\hline \multirow[t]{5}{*}{$\begin{array}{c}\text { Obstacle } \\
\text { Pairs }\end{array}$} & $1-2$ & $1-3$ & $1-4$ & $2-3$ & $2-4$ & $3-4$ & $1-2$ & $1-3$ & $1-4$ & $2-3$ & $2-4$ & $3-4$ \\
\hline & \multicolumn{12}{|c|}{ Supermodularity Test } \\
\hline & 0.000 & $2.604^{\sharp}$ & 0.999 & 0.077 & 1.192 & 0.010 & 0.423 & $2.680^{*}$ & 0.494 & 0.635 & 0.000 & 0.846 \\
\hline & \multicolumn{12}{|c|}{ Submodularity Test } \\
\hline & 1.153 & 0.000 & 0.000 & 0.202 & 0.019 & 0.971 & 1.411 & 0.423 & 0.000 & 1.834 & 1.199 & 0.000 \\
\hline
\end{tabular}

* Significant at the 0.01 level $†$ Significant at the 0.05 level $\$$ Significant at the 0.10 level

Table 43 Likelihood ratios for AMT - Textiles Industry 


\begin{tabular}{|c|c|c|c|c|c|c|c|c|c|c|c|c|}
\hline & \multicolumn{6}{|c|}{ Labour Productivity } & \multicolumn{6}{|c|}{ Profit } \\
\hline \multirow[t]{5}{*}{$\begin{array}{c}\text { Obstacle } \\
\text { Pairs }\end{array}$} & $1-2$ & $1-3$ & $1-4$ & $2-3$ & $2-4$ & $3-4$ & $1-2$ & $1-3$ & $1-4$ & $2-3$ & $2-4$ & $3-4$ \\
\hline & \multicolumn{12}{|c|}{ Supermodularity Test } \\
\hline & $2.918^{\dagger}$ & 0.016 & 0.032 & $60.957^{*}$ & $2.805^{\dagger}$ & 0.048 & $3.401^{*}$ & 0.215 & 1.072 & 0.082 & 1.071 & $35.862^{*}$ \\
\hline & \multicolumn{12}{|c|}{ Submodularity Test } \\
\hline & 0.000 & 0.742 & 0.355 & $1.999^{*}$ & 0.032 & 2.144 & 0.036 & $8.761^{*}$ & 0.785 & 0.962 & 0.051 & $2.283^{*}$ \\
\hline
\end{tabular}

* Significant at the 0.01 level $\dagger$ Significant at the 0.05 level $\$$ Significant at the 0.10 level

Table 44 Likelihood ratios for AMT - Vehicles Industry

\begin{tabular}{|c|c|c|c|c|c|c|c|c|c|c|c|c|}
\hline & \multicolumn{6}{|c|}{ Labour Productivity } & \multicolumn{6}{|c|}{ Profit } \\
\hline \multirow[t]{5}{*}{$\begin{array}{c}\text { Obstacle } \\
\text { Pairs }\end{array}$} & $1-2$ & $1-3$ & $1-4$ & $2-3$ & $2-4$ & $3-4$ & $1-2$ & $1-3$ & $1-4$ & $2-3$ & $2-4$ & $3-4$ \\
\hline & \multicolumn{12}{|c|}{ Supermodularity Test } \\
\hline & 0.000 & $3.932^{*}$ & 0.030 & 0.142 & 0.022 & 1.641 & 0.399 & $4.947^{*}$ & 0.638 & 0.160 & 0.000 & 1.037 \\
\hline & \multicolumn{12}{|c|}{ Submodularity Test } \\
\hline & 1.776 & 0.000 & $89.724^{*}$ & 1.947 & $3.670^{*}$ & 0.179 & 0.957 & 0.000 & 0.160 & 3.112 & $9.414^{*}$ & 0.000 \\
\hline
\end{tabular}

* Significant at the 0.01 level $\dagger$ Significant at the 0.05 level $\$$ Significant at the 0.10 level

\section{Table 45 Likelihood ratios for AMT - Wood Industry}

Factor 1= Production Design (LAN, CAD/CAE, CAD/CAM, Modeling, Electronic exchange of CAD files, PLC, Lasers, Rapid prototyping, High speed machining)

Factor 2 = Communications (WAN, EDI, Electronic part identification, CIM, SCADA, Computer on factory floor, Use of inspection data, Remote controlled processes, Knowledge-based software, MRPII)

Factor 3 = Inspection, and Storage (Automated storage and retrieval, Vision based, Sensor based)

Factor $4=$ Robotics (FMS/FMC, Robots with and without sensing)

Finally, the wood industry has some findings similar to those in the furniture and other industry (refer to Table 45). Communications and robotics technologies are once again complementary for both models. The production design and inspection and storage technologies are substitutes in both models. This result may be because very few wood plants use both forms of technology. The last significant pairing is found in the analysis with respect to labour productivity. Unlike the furniture and other industries, the production design and robotics technologies are complementary. This result demonstrates that when wood plants use robotics they rely on having an integrated design process in order to maximize the benefits of the robotics technology. 


\subsubsection{Business Practices}

Once again, the results of this analysis support the conclusion that there is a strong industry effect with respect to determining complementary business practices or technology use. The results from the two models remain consistent for each industry, but between industries, there is no real consensus on the complementarity of factor pairs. These results demonstrate that the market conditions of a particular industry have a large impact on the business practices that should be followed.

The chemical and metals industry have the quality control and planning business practices as substitutes with respect to labour productivity (refer to Table 46 and table 50). Similar results with respect to labour productivity were found in the machinery industry (refer to Table 49) and with respect to both models in the vehicles industry (refer to Table 54). These industries all have a number of tight contractual specifications on their raw materials and finished goods so that many of the quality control elements are featured in the agreements and are thus included in the planning process (Stevenson and Hojati, 2001). Also, in order to maintain a high level of planning checks and balances, along with a number of quality control practices, it takes longer to design and plan a new product or product changes resulting in lower labour productivity during the design phase of a better quality product. (Stevenson and Hojati, 2001)

On the other hand, the electronics industry (with respect to profit) (refer to Table 47), and the non-metals and wood industry (results for both models) (refer to Table 51 and table 55), have the quality control and planning business practices as complementary. This result may be due to the more flexible constraints that are placed on the finished goods in these industries and, due to high levels of competition, there is less product differentiation other than price and quality 
available. For example, computers are extremely similar to one another in design and functionality. The reasons that consumers choose particular brands are due to the quality reputation of the manufacture and the price of the configured item. There are also less certifications and government restrictions placed on the products in these industries making the quality control practices important features for a plant's competitive advantage.

The quality control and design business practices are found to be substitutes in the majority of the industries. The furniture and other industries and the metals industry (refer to Table 48 and table 50) find substitutes with respect to labour productivity, the plastics industry (refer to Table 52) find substitutes with respect to profit, and the non-metals, vehicles, and wood industries find substitutes with respect to both models (refer to Table 51, table 54, and table 55). This result reflects the additional amount of effort and time that is required to incorporate a large number of continuous improvement elements of design and maintain the level of quality needed to ensure compliance with the quality control practices.

The textiles industry (refer to Table 53) with respect to labour productivity, the electronics industry (refer to Table 47) with respect to profit, and the machinery industry (refer to Table 49) with respect to both models, find the quality control practices and design business practices to be complementary. In these industries, there are continual new designs being produced in order to remain competitive. For this reason, and the fact that quality is one of the consumers' primary decision variables for these products, the combined use of quality control and design practices is required for a plant to succeed.

The electronics industry with respect to profit (refer to Table 47) and the wood industry with respect to both models (refer to Table 55) find that the planning and design business 
practices are substitutes. The electronics industry is constantly in the process of creating new designs and many of the planning practices are not applicable to the wood industry. These results demonstrate that complementarities in industries are not bound to the traditional low-tech and high-tech groupings.

\begin{tabular}{|c|c|c|c|c|c|c|}
\hline & \multicolumn{3}{|c|}{$\begin{array}{c}\text { Labour } \\
\text { Productivity }\end{array}$} & \multicolumn{4}{c|}{ Profit } \\
\hline $\begin{array}{c}\text { Obstacle } \\
\text { Pairs }\end{array}$ & $1-2$ & $1-3$ & $2-3$ & $1-2$ & $1-3$ & $2-3$ \\
\hline \multirow{6}{*}{} & \multicolumn{6}{|c|}{ Supermodularity Test } \\
\cline { 2 - 7 } & $2.974^{\ddagger}$ & 0.026 & 0.209 & 1.011 & 0.328 & 0.002 \\
\cline { 2 - 7 } & \multicolumn{6}{|c|}{ Submodularity Test } \\
\cline { 2 - 7 } & 0.000 & 1.591 & 0.939 & 0.616 & 1.416 & 2.284 \\
\hline
\end{tabular}

* Significant at the 0.01 level $†$ Significant at the 0.05 level $\$$ Significant at the 0.10 level

Table 46 Likelihood ratios for Business Practices - Chemical Industry

\begin{tabular}{|c|c|c|c|c|c|c|}
\hline & \multicolumn{3}{|c|}{$\begin{array}{c}\text { Labour } \\
\text { Productivity }\end{array}$} & \multicolumn{3}{c|}{ Profit } \\
\hline $\begin{array}{c}\text { Obstacle } \\
\text { Pairs }\end{array}$ & $1-2$ & $1-3$ & $2-3$ & $1-2$ & $1-3$ & $2-3$ \\
\hline \multirow{6}{*}{} & \multicolumn{6}{|c|}{ Supermodularity Test } \\
\cline { 2 - 7 } & 0.917 & 0.940 & 1.947 & 0.586 & 0.846 & $3.776^{\dagger}$ \\
\cline { 2 - 7 } & \multicolumn{6}{|c|}{ Submodularity Test } \\
\cline { 2 - 7 } & 0.604 & 0.559 & 0.201 & $3.580^{\dagger}$ & $3.385^{\dagger}$ & 0.716 \\
\hline
\end{tabular}

* Significant at the 0.01 level $\dagger$ Significant at the 0.05 level $\$$ Significant at the 0.10 level

Table 47 Likelihood ratios for Business Practices - Electronics Industry

\begin{tabular}{|c|c|c|c|c|c|c|}
\hline & \multicolumn{3}{|c|}{$\begin{array}{c}\text { Labour } \\
\text { Productivity }\end{array}$} & \multicolumn{3}{|c|}{ Profit } \\
\hline $\begin{array}{c}\text { Obstacle } \\
\text { Pairs }\end{array}$ & $1-2$ & $1-3$ & $2-3$ & $1-2$ & $1-3$ & $2-3$ \\
\hline & \multicolumn{6}{|c|}{ Supermodularity Test } \\
\hline & 1.589 & $3.064^{\dagger}$ & 0.000 & 0.634 & 0.536 & 0.000 \\
\hline & \multicolumn{6}{|c|}{ Submodularity Test } \\
\hline & 0.000 & 0.000 & $4.997^{*}$ & 0.000 & 0.000 & $3.371^{\dagger}$ \\
\hline
\end{tabular}

Table 48 Likelihood ratios for Business Practices - Furniture and Other Industries 


\begin{tabular}{|c|c|c|c|c|c|c|}
\hline & \multicolumn{3}{|c|}{$\begin{array}{c}\text { Labour } \\
\text { Productivity }\end{array}$} & \multicolumn{4}{|c|}{ Profit } \\
\hline \multirow{3}{*}{$\begin{array}{c}\text { Obstacle } \\
\text { Pairs }\end{array}$} & $1-2$ & $1-3$ & $2-3$ & $1-2$ & $1-3$ & $2-3$ \\
\hline & \multicolumn{5}{|c|}{ Supermodularity Test } \\
\cline { 2 - 7 } & 0.553 & 0.000 & 0.889 & $4.244^{\dagger}$ & 0.089 & 0.080 \\
\cline { 2 - 7 } & \multicolumn{6}{|c|}{ Submodularity Test } \\
\cline { 2 - 7 } & 0.000 & $4.444^{\dagger}$ & 0.000 & 0.386 & $8.159^{*}$ & $3.291^{\dagger}$ \\
\hline
\end{tabular}

Table 49 Likelihood ratios for Business Practices - Machinery Industry

\begin{tabular}{|c|c|c|c|c|c|c|}
\hline & \multicolumn{3}{|c|}{$\begin{array}{c}\text { Labour } \\
\text { Productivity }\end{array}$} & \multicolumn{3}{c|}{ Profit } \\
\hline $\begin{array}{c}\text { Obstacle } \\
\text { Pairs }\end{array}$ & $1-2$ & $1-3$ & $2-3$ & $1-2$ & $1-3$ & $2-3$ \\
\hline \multirow{6}{*}{ Supermodularity Test } \\
\cline { 2 - 7 } & $6.673^{*}$ & $8.756^{*}$ & $2.329^{*}$ & 0.592 & 1.382 & 0.099 \\
\cline { 2 - 7 } & \multicolumn{6}{|c|}{ Submodularity Test } \\
\cline { 2 - 7 } & 0.000 & 0.000 & 0.000 & 0.000 & 0.000 & 0.197 \\
\hline
\end{tabular}

* Significant at the 0.01 level $\quad \dagger$ Significant at the 0.05 level $\$$ Significant at the 0.10 level

Table 50 Likelihood ratios for Business Practices - Metals Industry

\begin{tabular}{|c|c|c|c|c|c|c|}
\hline & \multicolumn{3}{|c|}{$\begin{array}{c}\text { Labour } \\
\text { Productivity }\end{array}$} & \multicolumn{4}{c|}{ Profit } \\
\cline { 2 - 7 } $\begin{array}{c}\text { Obstacle } \\
\text { Pairs }\end{array}$ & $1-2$ & $1-3$ & $2-3$ & $1-2$ & $1-3$ & $2-3$ \\
\hline \multirow{5}{*}{} & \multicolumn{6}{|c|}{ Supermodularity Test } \\
\cline { 2 - 7 } & 0.000 & $3.341^{\dagger}$ & 0.371 & 0.000 & $3.064^{\ddagger}$ & 0.000 \\
\cline { 2 - 7 } & \multicolumn{6}{|c|}{ Submodularity Test } \\
\cline { 2 - 8 } & $5.754^{*}$ & 0.000 & 0.101 & $5.845^{*}$ & 0.000 & 0.226 \\
\hline
\end{tabular}

* Significant at the 0.01 level $\dagger$ Significant at the 0.05 level $\$$ Significant at the 0.10 level

Table 51 Likelihood ratios for Business Practices - Non-Metals Industry

\begin{tabular}{|c|c|c|c|c|c|c|}
\hline & \multicolumn{3}{|c|}{$\begin{array}{c}\text { Labour } \\
\text { Productivity }\end{array}$} & \multicolumn{3}{c|}{ Profit } \\
\cline { 2 - 7 } $\begin{array}{c}\text { Obstacle } \\
\text { Pairs }\end{array}$ & $1-2$ & $1-3$ & $2-3$ & $1-2$ & $1-3$ & $2-3$ \\
\hline & \multicolumn{5}{|c|}{ Supermodularity Test } \\
\cline { 2 - 7 } & 0.000 & 1.465 & 1.017 & 0.002 & $5.277^{*}$ & 0.059 \\
\cline { 2 - 7 } & \multicolumn{6}{|c|}{ Submodularity Test } \\
\cline { 2 - 7 } & 0.671 & 0.000 & 0.000 & 0.765 & 0.000 & 0.175 \\
\hline
\end{tabular}

Table 52 Likelihood ratios for Business Practices - Plastics Industry 


\begin{tabular}{|c|c|c|c|c|c|c|}
\hline \multirow{4}{*}{} & \multicolumn{3}{|c|}{$\begin{array}{c}\text { Labour } \\
\text { Productivity }\end{array}$} & \multicolumn{3}{c|}{ Profit } \\
\cline { 2 - 7 } $\begin{array}{c}\text { Obstacle } \\
\text { Pairs }\end{array}$ & $1-2$ & $1-3$ & $2-3$ & $1-2$ & $1-3$ & $2-3$ \\
\hline & \multicolumn{5}{|c|}{ Supermodularity Test } \\
\cline { 2 - 7 } & 0.456 & 0.000 & 0.699 & 0.105 & 0.316 & 0.210 \\
\cline { 2 - 7 } & \multicolumn{6}{|c|}{ Submodularity Test } \\
\cline { 2 - 7 } & 0.927 & $5.256^{*}$ & 0.760 & 1.578 & 0.841 & $2.419^{\ddagger}$ \\
\hline
\end{tabular}

Table 53 Likelihood ratios for Business Practices - Textiles Industry

\begin{tabular}{|c|c|c|c|c|c|c|}
\hline & \multicolumn{2}{|c|}{ Labour Productivity } & \multicolumn{3}{|c|}{ Profit } \\
\hline $\begin{array}{c}\text { Obstacle } \\
\text { Pairs }\end{array}$ & $1-2$ & $1-3$ & $2-3$ & $1-2$ & $1-3$ & $2-3$ \\
\hline \multirow{6}{*}{ Supermodularity Test } \\
\cline { 2 - 7 } & $71.502^{*}$ & $11.216^{*}$ & 0.000 & $7.273^{*}$ & $9.154^{*}$ & 0.000 \\
\cline { 2 - 7 } & \multicolumn{6}{|c|}{ Submodularity Test } \\
\cline { 2 - 7 } & 0.000 & 0.000 & $2.337^{\ddagger}$ & 0.000 & 0.000 & $3.614^{\dagger}$ \\
\hline
\end{tabular}

* Significant at the 0.01 level $†$ Significant at the 0.05 level $\$$ Significant at the 0.10 level

Table 54 Likelihood ratios for Business Practices - Vehicles Industry

\begin{tabular}{|c|c|c|c|c|c|c|}
\hline \multirow[b]{2}{*}{$\begin{array}{c}\text { Obstacle } \\
\text { Pairs }\end{array}$} & \multicolumn{3}{|c|}{$\begin{array}{c}\text { Labour } \\
\text { Productivity }\end{array}$} & \multicolumn{3}{|c|}{ Profit } \\
\hline & $1-2$ & $1-3$ & $2-3$ & $1-2$ & $1-3$ & $2-3$ \\
\hline & \multicolumn{6}{|c|}{ Supermodularity Test } \\
\hline & 0.000 & $6.619^{*}$ & $3.925^{\dagger}$ & 1.560 & $5.105^{*}$ & $10.493^{*}$ \\
\hline & \multicolumn{6}{|c|}{ Submodularity Test } \\
\hline & $7.215^{*}$ & 0.000 & 0.272 & $11.485^{*}$ & $4.821^{*}$ & 1.560 \\
\hline
\end{tabular}

* Significant at the 0.01 level $†$ Significant at the 0.05 level $\quad \$$ Significant at the 0.10 level

Table 55 Likelihood ratios for Business Practices - Wood Industry

Factor 1 = Quality Control (Continuous improvement, benchmarking, Certifications of suppliers and plant, and statistical control)

Factor 2 = Planning (JIT, Electronic work order, process simulation, distribution resource planning, quality function deployment)

Factor 3 = Design (Cross functional teams, concurrent engineering, continuous improvement)

The planning and design business practices are found to be complementary with respect to profit in the machinery and textiles industries (refer to Table 49 and table 53), and with respect to both models in the furniture and other industries and the vehicles industry (refer to Table 48 
and table 54). These industries demonstrate that the benefits of increased planning and improved planning management facilitate the design process. These industries are typically creating updates to existing models and not entirely new or innovative products.

\subsubsection{Sources of Implementation Support}

The results of the analysis by industry for the sources of implementation support exhibit very different findings. The majority of industries have very few significant results although there are a couple such as the vehicles and wood industries that have many. In this set of analysis in particular, the sample size seems to have had a major impact on the number of significant results. Only the larger industries have more than a single significant pairing.

The electronics industry has only one significant result (refer to Table 57). The external information sources and the corporate organizations are substitutes with respect to labour productivity. This result may be due to the number of interactions that occur and the differing opinions that may result in this competitive market between the head office of a company and outside market pressures.

The furniture and other industries exhibit two significant results that are consistent for both models (refer to Table 58). Primary stakeholders and external information sources are complementary. This result stems from the custom nature of the furniture industry where the needs and style choices of customers are the driving forces of the market. Primary stakeholders are also found to be substitutes for support from corporate organizations. This is an occurrence where the technology is either pushed down into the plant from head office or the desire from change is championed from within the plant. In this industry there does not appear to be any added benefit to having both sources of implementation support. 
The machinery industry has the research and development group and the corporate organizations as substitutes for one another with respect to both models (refer to Table 59). An explanation is that head office may be performing much of the research and development effort so the two measures have significant overlaps. Similarly, the research and development group and the external information sources are also found to be substitutes with respect to profit. Finally, the primary stakeholders are complementary with all other sources of implementation support. As with the small and medium plants, having primary stakeholders involved with the implementation is a critical component for success.

The research and development employees and the corporate organizations are found to be complements for the metals industry with respect to both models (refer to Table 60). The research and development group is also a complement for the external information sources with respect to profit. These results demonstrate that in the metals industry, the research and development group appears to be the internal champion for the use of AMT and that any support from outside the plant that they can obtain increases the effectiveness of the implementation. The external information sources and the corporate organizations are substitutes though with respect to profit. This result implies that a plant should only use one source of external support, as using both simultaneously may lead to conflict and confusion.

The plastics industry exhibits only one significant result (refer to Table 62). As in the furniture industry, primary stakeholders and corporate organizations, as sources of implementation support, are substitutes. Once again, technology should either be chosen from within the plant or pushed via head office. There is no additional benefit to having head office support an internally championed implementation. 
The textiles industry has the opposite result to that of the plastics industry (refer to Table 63 ), in that the primary stakeholders are complementary to the external information sources with respect to labour productivity, and complementary to corporate organizations with respect to both models. As in the machinery industry, the primary stakeholders in the textiles industry are better able to champion change when they have support from outside the plant. This result demonstrates the importance of primary stakeholders in the textiles industry.

There are only substitute pairings found in the vehicles industry (refer to Table 64). The research and development group is a substitute for all other sources of implementation support. These results imply that if the research and development group decides it requires new technology, then the effort required to gain support from any other source results in increased costs and a slower implementation process. The vehicles industry also exhibits a significant substitute pairing with respect to profit. As in the furniture and plastics industries, the primary stakeholders and corporate organizations are substitutes.

Finally, the wood industry reveals a large number of significant results (refer to Table 65). The primary stakeholders are found to reject both complementarity and substitutability when paired with either the research and development group or the external information sources with respect to labour productivity. In the analysis using profit as the dependent variable, these two pairings are found to be complementary. As with many of the other industries, the primary stakeholders and the corporate organizations are found to be substitutes with respect to labour productivity. Both models have two other results pertaining to external information sources. As with the vehicles industry, the external information sources are substitutes for the research and development employees. The pairing where the wood industry is unique however is that it has the external information sources and the corporate organizations as a complementary pairing. 
This result demonstrates that support for implementation from head office must be accompanied with support from the market for the optimal benefit to be obtained.

\begin{tabular}{|c|c|c|c|c|c|c|c|c|c|c|c|c|}
\hline & \multicolumn{6}{|c|}{ Labour Productivity } & \multicolumn{6}{|c|}{ Profit } \\
\hline \multirow[t]{5}{*}{$\begin{array}{c}\text { Obstacle } \\
\text { Pairs } \\
\end{array}$} & $1-2$ & $1-3$ & $1-4$ & $2-3$ & $2-4$ & $3-4$ & $1-2$ & $1-3$ & $1-4$ & $2-3$ & $2-4$ & $3-4$ \\
\hline & \multicolumn{12}{|c|}{ Supermodularity Test } \\
\hline & 0.064 & 0.319 & 1.687 & 0.000 & 0.009 & 0.137 & 0.150 & 0.028 & 1.438 & 0.003 & 0.000 & 0.003 \\
\hline & \multicolumn{12}{|c|}{ Submodularity Test } \\
\hline & 0.119 & 0.146 & 0.046 & 0.182 & 0.046 & 0.966 & 0.054 & 0.156 & 0.000 & 0.539 & 0.506 & 0.055 \\
\hline
\end{tabular}

$*$ Significant at the 0.01 level $†$ Significant at the 0.05 level $\$$ Significant at the 0.10 level

Table 56 Likelihood ratios for Implementation Support - Chemical Industry

\begin{tabular}{|c|c|c|c|c|c|c|c|c|c|c|c|c|}
\hline & \multicolumn{6}{|c|}{ Labour Productivity } & \multicolumn{6}{|c|}{ Profit } \\
\hline \multirow[t]{5}{*}{$\begin{array}{c}\text { Obstacle } \\
\text { Pairs } \\
\end{array}$} & $1-2$ & $1-3$ & $1-4$ & $2-3$ & $2-4$ & $3-4$ & $1-2$ & $1-3$ & $1-4$ & $2-3$ & $2-4$ & $3-4$ \\
\hline & \multicolumn{12}{|c|}{ Supermodularity Test } \\
\hline & 0.618 & 0.633 & 0.783 & 0.226 & 1.416 & $3.269^{\dagger}$ & 1.234 & 0.132 & 0.000 & 0.044 & 0.000 & 0.793 \\
\hline & \multicolumn{12}{|c|}{ Submodularity Test } \\
\hline & 1.009 & 1.567 & 0.949 & 1.988 & 0.331 & 0.075 & 0.132 & 1.411 & 0.793 & 1.058 & 0.264 & 0.000 \\
\hline
\end{tabular}

* Significant at the 0.01 level $\dagger$ Significant at the 0.05 level \$Significant at the 0.10 level

Table 57 Likelihood ratios for Implementation Support - Electronics Industry

\begin{tabular}{|c|c|c|c|c|c|c|c|c|c|c|c|c|}
\hline & \multicolumn{6}{|c|}{ Labour Productivity } & \multicolumn{6}{|c|}{ Profit } \\
\hline \multirow[t]{5}{*}{$\begin{array}{c}\text { Obstacle } \\
\text { Pairs } \\
\end{array}$} & $1-2$ & $1-3$ & $1-4$ & $2-3$ & $2-4$ & $3-4$ & $1-2$ & $1-3$ & $1-4$ & $2-3$ & $2-4$ & $3-4$ \\
\hline & \multicolumn{12}{|c|}{ Supermodularity Test } \\
\hline & 2.049 & 0.363 & $4.109^{*}$ & 0.773 & 0.878 & 0.609 & 0.969 & 0.060 & $2.258^{*}$ & 0.934 & 0.616 & 0.604 \\
\hline & \multicolumn{12}{|c|}{ Submodularity Test } \\
\hline & 0.433 & $2.939^{\dagger}$ & 0.000 & 0.293 & 0.621 & 1.990 & 0.171 & $2.302^{\ddagger}$ & 0.001 & 1.113 & 1.100 & 1.291 \\
\hline
\end{tabular}

* Significant at the 0.01 level $†$ Significant at the 0.05 level $\$$ Significant at the 0.10 level

Table 58 Likelihood ratios for Implementation Support - Furniture and Other Industries

\begin{tabular}{|c|c|c|c|c|c|c|c|c|c|c|c|c|}
\hline & \multicolumn{1}{c|}{ Labour Productivity } & \multicolumn{1}{c|}{ Profit } \\
\hline $\begin{array}{c}\text { Obstacle } \\
\text { Pairs }\end{array}$ & $1-2$ & $1-3$ & $1-4$ & $2-3$ & $2-4$ & $3-4$ & $1-2$ & $1-3$ & $1-4$ & $2-3$ & $2-4$ & $3-4$ \\
\hline & \multicolumn{10}{|c|}{ Supermodularity Test } \\
\cline { 2 - 11 } & 0.000 & 0.000 & 0.000 & 1.461 & $3.742^{\dagger}$ & 0.356 & 0.000 & 0.000 & 0.000 & $6.891^{*}$ & $92.189^{*}$ & 1.119 \\
\cline { 2 - 3 } & \multicolumn{10}{|c|}{ Submodularity Test } \\
\cline { 2 - 11 } & 0.837 & 0.606 & 0.760 & 0.000 & 0.000 & 0.000 & $3.503^{\dagger}$ & $5.643^{*}$ & $2.993^{\dagger}$ & 0.000 & 0.000 & 0.102 \\
\hline
\end{tabular}

* Significant at the 0.01 level $\dagger$ Significant at the 0.05 level $\$$ Significant at the 0.10 level

Table 59 Likelihood ratios for Implementation Support - Machinery Industry 


\begin{tabular}{|c|c|c|c|c|c|c|c|c|c|c|c|c|}
\hline & \multicolumn{6}{|c|}{ Labour Productivity } & \multicolumn{6}{|c|}{ Profit } \\
\hline \multirow[t]{5}{*}{$\begin{array}{c}\text { Obstacle } \\
\text { Pairs }\end{array}$} & $1-2$ & $1-3$ & $1-4$ & $2-3$ & $2-4$ & $3-4$ & $1-2$ & $1-3$ & $1-4$ & $2-3$ & $2-4$ & $3-4$ \\
\hline & \multicolumn{12}{|c|}{ Supermodularity Test } \\
\hline & 0.817 & 1.225 & 1.836 & 0.058 & 0.380 & 0.817 & 0.695 & 1.258 & 0.744 & 0.000 & 1.022 & $2.574^{\ddagger}$ \\
\hline & \multicolumn{12}{|c|}{ Submodularity Test } \\
\hline & 1.456 & 0.005 & 0.173 & 0.264 & $5.579^{*}$ & 0.000 & 1.482 & 0.076 & 0.351 & $65.333^{*}$ & $7.904^{*}$ & 0.000 \\
\hline
\end{tabular}

$*$ Significant at the 0.01 level $\dagger$ Significant at the 0.05 level $\$$ Significant at the 0.10 level

Table 60 Likelihood ratios for Implementation Support - Metals Industry

\begin{tabular}{|c|c|c|c|c|c|c|c|c|c|c|c|c|}
\hline & \multicolumn{6}{|c|}{ Labour Productivity } & \multicolumn{6}{|c|}{ Profit } \\
\hline \multirow[t]{5}{*}{$\begin{array}{c}\text { Obstacle } \\
\text { Pairs } \\
\end{array}$} & $1-2$ & $1-3$ & $1-4$ & $2-3$ & $2-4$ & $3-4$ & $1-2$ & $1-3$ & $1-4$ & $2-3$ & $2-4$ & $3-4$ \\
\hline & \multicolumn{12}{|c|}{ Supermodularity Test } \\
\hline & 0.155 & 0.099 & 0.718 & 0.851 & 0.210 & 0.144 & 0.029 & 0.069 & 0.060 & 0.237 & 0.043 & 0.002 \\
\hline & \multicolumn{12}{|c|}{ Submodularity Test } \\
\hline & 0.585 & 1.491 & 0.000 & 0.420 & 0.652 & 0.740 & 0.210 & 0.242 & 0.000 & 0.000 & 0.000 & 0.002 \\
\hline
\end{tabular}

* Significant at the 0.01 level † Significant at the 0.05 level \$Significant at the 0.10 level

Table 61 Likelihood ratios for Implementation Support - Non-Metals Industry

\begin{tabular}{|c|c|c|c|c|c|c|c|c|c|c|c|c|}
\hline \multirow{2}{*}{\begin{tabular}{|c|} 
Obstacle \\
Pairs
\end{tabular}} & \multicolumn{6}{|c|}{ Labour Productivity } & \multicolumn{6}{|c|}{ Profit } \\
\hline & $1-2$ & $1-3$ & $1-4$ & $2-3$ & $2-4$ & $3-4$ & $1-2$ & $1-3$ & $1-4$ & $2-3$ & $2-4$ & $3-4$ \\
\hline & \multicolumn{12}{|c|}{ Supermodularity Test } \\
\hline & 0.323 & 0.515 & 0.299 & 0.335 & 1.090 & 0.671 & 0.310 & 0.586 & $11.087^{*}$ & 0.262 & 0.147 & $12.986^{*}$ \\
\hline & \multicolumn{12}{|c|}{ Submodularity Test } \\
\hline & 0.587 & 0.419 & 0.000 & 1.665 & 0.719 & 1.377 & 0.800 & 0.333 & 0.019 & 1.714 & 0.000 & 0.000 \\
\hline
\end{tabular}

* Significant at the 0.01 level $†$ Significant at the 0.05 level $\$$ Significant at the 0.10 level

Table 62 Likelihood ratios for Implementation Support - Plastics Industry

\begin{tabular}{|c|c|c|c|c|c|c|c|c|c|c|c|c|}
\hline & \multicolumn{6}{|c|}{ Labour Productivity } & \multicolumn{6}{|c|}{ Profit } \\
\hline \multirow[t]{5}{*}{$\begin{array}{c}\text { Obstacle } \\
\text { Pairs }\end{array}$} & $1-2$ & $1-3$ & $1-4$ & $2-3$ & $2-4$ & $3-4$ & $1-2$ & $1-3$ & $1-4$ & $2-3$ & $2-4$ & $3-4$ \\
\hline & \multicolumn{12}{|c|}{ Supermodularity Test } \\
\hline & 2.098 & 0.000 & 0.385 & 0.648 & 1.070 & 1.098 & 0.246 & 0.614 & 0.000 & 0.614 & 0.000 & 0.573 \\
\hline & \multicolumn{12}{|c|}{ Submodularity Test } \\
\hline & 0.859 & $200.06^{*}$ & 3.004 & 0.690 & 0.873 & 0.225 & 0.368 & 0.246 & $4.503^{*}$ & 0.000 & 0.901 & 0.246 \\
\hline
\end{tabular}

* Significant at the 0.01 level $\dagger$ Significant at the 0.05 level $\$$ Significant at the 0.10 level

Table 63 Likelihood ratios for Implementation Support - Textiles Industry 


\begin{tabular}{|c|c|c|c|c|c|c|c|c|c|c|c|c|}
\hline & \multicolumn{6}{|c|}{ Labour Productivity } & \multicolumn{6}{|c|}{ Profit } \\
\hline \multirow[t]{5}{*}{$\begin{array}{c}\text { Obstacle } \\
\text { Pairs }\end{array}$} & $1-2$ & $1-3$ & $1-4$ & $2-3$ & $2-4$ & $3-4$ & $1-2$ & $1-3$ & $1-4$ & $2-3$ & $2-4$ & $3-4$ \\
\hline & \multicolumn{12}{|c|}{ Supermodularity Test } \\
\hline & $2.208^{\ddagger}$ & 0.567 & 0.528 & $41.718^{*}$ & $2.814^{\dagger}$ & 0.684 & 1.530 & 0.041 & $18.929^{*}$ & 0.672 & 0.864 & 0.120 \\
\hline & \multicolumn{12}{|c|}{ Submodularity Test } \\
\hline & 0.000 & 0.410 & 0.020 & 0.020 & 0.000 & 0.195 & 0.000 & 0.346 & 0.061 & 0.000 & 0.017 & 0.278 \\
\hline
\end{tabular}

* Significant at the 0.01 level $†$ Significant at the 0.05 level $\$$ Significant at the 0.10 level

Table 64 Likelihood ratios for Implementation Support - Vehicles Industry

\begin{tabular}{|c|c|c|c|c|c|c|c|c|c|c|c|c|}
\hline & \multicolumn{6}{|c|}{ Labour Productivity } & \multicolumn{6}{|c|}{ Profit } \\
\hline \multirow[t]{5}{*}{$\begin{array}{c}\text { Obstacle } \\
\text { Pairs }\end{array}$} & $1-2$ & $1-3$ & $1-4$ & $2-3$ & $2-4$ & $3-4$ & $1-2$ & $1-3$ & $1-4$ & $2-3$ & $2-4$ & $3-4$ \\
\hline & \multicolumn{12}{|c|}{ Supermodularity Test } \\
\hline & $2.107^{\ddagger}$ & 2.985 & $3.531^{\dagger}$ & $28.652^{*}$ & 0.401 & 0.000 & 0.097 & 0.581 & 0.484 & $4.550^{*}$ & 0.000 & 0.000 \\
\hline & \multicolumn{12}{|c|}{ Submodularity Test } \\
\hline & $4.572^{*}$ & 3.113 & 0.060 & 0.486 & 1.970 & $4.333^{*}$ & $5.906^{*}$ & $2.227^{\ddagger}$ & 0.387 & 0.000 & 1.355 & $3.485^{\dagger}$ \\
\hline
\end{tabular}

* Significant at the 0.01 level $\dagger$ Significant at the 0.05 level $\$$ Significant at the 0.10 level

Table 65 Likelihood ratios for Implementation Support - Wood Industry

Factor 1 = Primary Stakeholders (Production Engineering, Production, Design, Sales and Marketing staff, Suppliers, Customers, Patents)

Factor 2 = Research \& Development (Research, Experimental development, Production engineering, Trade Fairs, Governments, Other producers)

Factor 3 = External information (Sales and Marketing, Customers, Related Firms)

Factor 4 = Corporate Organizations (Head Office, Related plants, Universities)

\subsubsection{Perceived Benefits of AMT}

The analysis of the perceived benefits of AMT exhibits a number of inconclusive results (refer to Table 66 to table 75). This problem is due to the limited sample size of the smaller industries and the distribution of the plants within the various states. A number of industries have a distribution where there is a large percentage of plants in only a couple of states and then very few in each of the remaining states. The plastics, non-metals, and machinery industries all have a number of states with no plants and thus a number of constraints had to be removed from the analysis making it almost impossible to reject any of the hypotheses. 
The electronics industry has one of the highest number of significant test results relative to the remaining industries (refer to Table 67). The perception of decreased time usage and increased flexibility were found to be substitutes with respect to both labour productivity and profit. This result may be because if there is a significant amount of time savings in the set-up and rejection times then this implicitly increases the flexibility of plant so the additional perception of increased flexibility offers no additional savings. The perception of productivity improvements and increased profitability are also found to be substitutes with respect to both models. In this case, the productivity improvements are such that they will lead to the profitability increases, therefore these two measures are substitutes. This is supported by the fact that there are no plants in the electronics industry that exhibit only these two perceived benefits factors. The perception of improved productivity is found to be complementary with increased flexibility. The increases in flexibility allow for greater use of the productivity improvements such as increased utilization rates and reduced materials usage. Assuming the perceived benefits are those actually obtained in the plants then the various improvements would support one another and an overall increase in both profit and productivity. As in large plants, the perception of improved flexibility and profitability are substitutes with respect to labour productivity. With respect to profit, the perception of increased profitability is complementary with the perception of improved time usage. This result is intuitive since if the amount of time used to produce a high quality product decreases this will lead to a greater increase in profitability.

The furniture and other industries have only two significant pair-wise results (refer to Table 68). The first is that the perception of improved productivity and improved profitability are complementary with respect to both models. In this case, the increase in market share is amplifying the effect of the productivity improvements. The perceived benefit of productivity 
increases is also found to be complementary with improved flexibility with respect to profit. The increases in flexibility, including higher skilled labour, results in products that are more artistically complex and trendy. If at the same time there are productivity increases then the plant will be able to create a more marketable product for lower marginal cost, thus gaining them a significant competitive advantage.

The metals industry exhibits only one significant result found in the model with profit as the dependent variable (refer to Table 70). The perceived benefit of better time usage and the perception of increased profitability are substitutes. Since the improved usage of time would implicitly yield increases in profits as costs are being decreased, the time factor has a significant overlap with the profitability measure resulting in the two factors being substitutes.

The machinery industry also has only a single significant result, although this time with respect to labour productivity (refer to Table 69). As in the metals industry, the perceived benefit of better time usage and the perception of increased profitability are substitutes. In this case, the finding may be due in part to the fact that there are no plants in the machinery industry that exhibit only these two perceived benefit factors.

The textiles industry, like the electronics industry, has a large number of significant results (refer to Table 73). As in the electronics industry, the perceived benefit of improved productivity and increased profitability are substitutes and the improved time usage and increased profitability are found to be complements with respect to both models. As well, the pairing of increased productivity and improved flexibility is both found to be complementary and the pairing of improved time usage and increased flexibility are found to be a substitute with respect to profit. The result that is unique to the textiles industry is that the perception of time 
usage improvements and flexibility increases are found to be substitutes with respect to labour productivity. These results demonstrate that the flexibility improvements are implicitly incorporated in the set-up, reject time, and capital reductions. The final significant result in the textiles industry is that the perception of increased flexibility and increased profitability are complementary.

The vehicles industry exhibits three significant results (refer to Table 74). As in the textiles and electronics industries, the perceived benefit of productivity improvements and increases in profitability are substitutes with respect to labour productivity. The second result is that the perception of increased profitability and increased flexibility are also complementary with respect to labour productivity. This result is similar to that found in the electronics industry, and demonstrates that there is a strong link between flexibility and profitability causing an overlap of the measures. The final significant result is found in the profit model where the perceived benefit of productivity improvements is complementary with the perception of improved flexibility. This result mirrors that of the textiles industry.

Finally, the wood industry exhibits one significant result (refer to Table 75). With respect to profit, the perceived benefit of increased productivity and flexibility improvements are substitutes. This opposes the results found in the vehicles and textiles industries. The reason for the difference may be due to the fact that there are only a small number of plants in any of the states containing these two perceived benefits factors or maybe due to the fact that most wood products are relatively standard and so flexibility improvements do not offer relevant benefits to wood manufacturers. 


\begin{tabular}{|c|c|c|c|c|c|c|c|c|c|c|c|c|}
\hline & \multicolumn{6}{|c|}{ Labour Productivity } & \multicolumn{6}{|c|}{ Profit } \\
\hline \multirow[t]{5}{*}{$\begin{array}{c}\text { Obstacle } \\
\text { Pairs }\end{array}$} & $1-2$ & $1-3$ & $1-4$ & $2-3$ & $2-4$ & $3-4$ & $1-2$ & $1-3$ & $1-4$ & $2-3$ & $2-4$ & $3-4$ \\
\hline & \multicolumn{12}{|c|}{ Supermodularity Test } \\
\hline & 0.305 & 0.000 & 0.000 & 0.553 & 1.077 & 0.095 & 0.017 & 0.000 & 0.000 & 0.323 & 0.688 & 0.650 \\
\hline & \multicolumn{12}{|c|}{ Submodularity Test } \\
\hline & 0.000 & 0.638 & 0.343 & 0.000 & 0.000 & 0.362 & 0.502 & 0.875 & 1.037 & 0.007 & 0.052 & 0.000 \\
\hline
\end{tabular}

* Significant at the 0.01 level $†$ Significant at the 0.05 level $\$$ Significant at the 0.10 level

Table 66 Likelihood ratios for Perceived Benefits of AMT - Chemicals Industry

\begin{tabular}{|c|c|c|c|c|c|c|c|c|c|c|c|c|}
\hline & \multicolumn{6}{|c|}{ Labour Productivity } & \multicolumn{6}{|c|}{ Profit } \\
\hline \multirow[t]{5}{*}{\begin{tabular}{|c|} 
Obstacle \\
Pairs \\
\end{tabular}} & $1-2$ & $1-3$ & $1-4$ & $2-3$ & $2-4$ & $3-4$ & $1-2$ & $1-3$ & $1-4$ & $2-3$ & $2-4$ & $3-4$ \\
\hline & \multicolumn{12}{|c|}{ Supermodularity Test } \\
\hline & 0.890 & $5.573^{*}$ & 0.094 & 0.000 & $3.138^{\dagger}$ & $3321.6^{*}$ & 0.000 & $3.270^{\dagger}$ & 0.000 & 0.000 & $7.983^{*}$ & 0.433 \\
\hline & \multicolumn{12}{|c|}{ Submodularity Test } \\
\hline & 0.250 & 0.000 & 1.140 & $3.434^{\dagger}$ & 0.000 & 0.156 & 0.000 & 0.000 & $4.857^{*}$ & $2.405^{\ddagger}$ & 0.00 & 0.481 \\
\hline
\end{tabular}

* Significant at the 0.01 level $\dagger$ Significant at the 0.05 level $\$$ Significant at the 0.10 level

Table 67 Likelihood ratios for Perceived Benefits of AMT - Electronics Industry

\begin{tabular}{|c|c|c|c|c|c|c|c|c|c|c|c|c|}
\hline & \multicolumn{6}{|c|}{ Labour Productivity } & \multicolumn{6}{|c|}{ Profit } \\
\hline \multirow[t]{5}{*}{$\begin{array}{c}\text { Obstacle } \\
\text { Pairs } \\
\end{array}$} & $1-2$ & $1-3$ & $1-4$ & $2-3$ & $2-4$ & $3-4$ & $1-2$ & $1-3$ & $1-4$ & $2-3$ & $2-4$ & $3-4$ \\
\hline & \multicolumn{12}{|c|}{ Supermodularity Test } \\
\hline & 0.346 & 0.346 & 0.779 & 1.187 & 0.297 & 0.557 & 0.069 & 0.053 & 0.651 & 0.569 & 0.000 & 0.192 \\
\hline & \multicolumn{12}{|c|}{ Submodularity Test } \\
\hline & 0.161 & 0.260 & 0.161 & 0.210 & $3.130^{\dagger}$ & 0.990 & 0.124 & 0.569 & 0.029 & $110.65^{*}$ & $2.878^{\dagger}-10$ & 0.741 \\
\hline
\end{tabular}

$*$ Significant at the 0.01 level $†$ Significant at the 0.05 level $\$$ Significant at the 0.10 level

Table 68 Likelihood ratios for Perceived Benefits of AMT - Furniture and Other Industries

\begin{tabular}{|c|c|c|c|c|c|c|c|c|c|c|c|c|}
\hline & \multicolumn{6}{|c|}{ Labour Productivity } & \multicolumn{6}{|c|}{ Profit } \\
\hline \multirow[t]{5}{*}{$\begin{array}{c}\text { Obstacle } \\
\text { Pairs }\end{array}$} & $1-2$ & $1-3$ & $1-4$ & $2-3$ & $2-4$ & $3-4$ & $1-2$ & $1-3$ & $1-4$ & $2-3$ & $2-4$ & $3-4$ \\
\hline & \multicolumn{12}{|c|}{ Supermodularity Test } \\
\hline & 0.000 & 0.083 & $4.973^{\dagger}$ & 0.050 & 0.000 & 0.315 & 0.000 & 0.000 & 2.027 & 0.005 & 0.000 & 0.410 \\
\hline & \multicolumn{12}{|c|}{ Submodularity Test } \\
\hline & 0.928 & 0.050 & 0.000 & 0.746 & 0.564 & 0.149 & 1.219 & 0.028 & 0.000 & 0.145 & 0.828 & 0.013 \\
\hline
\end{tabular}

* Significant at the 0.01 level $†$ Significant at the 0.05 level $\$$ Significant at the 0.10 level

Table 69 Likelihood ratios for Perceived Benefits of AMT - Machinery Industry 


\begin{tabular}{|c|c|c|c|c|c|c|c|c|c|c|c|c|}
\hline \multirow[b]{2}{*}{$\begin{array}{c}\text { Obstacle } \\
\text { Pairs }\end{array}$} & \multicolumn{6}{|c|}{ Labour Productivity } & \multicolumn{6}{|c|}{ Profit } \\
\hline & $1-2$ & $1-3$ & $1-4$ & $2-3$ & $2-4$ & $3-4$ & $1-2$ & $1-3$ & $1-4$ & $2-3$ & $2-4$ & $3-4$ \\
\hline & \multicolumn{12}{|c|}{ Supermodularity Test } \\
\hline & 0.252 & 0.295 & 0.076 & 0.308 & 0.028 & 0.008 & 0.611 & 0.047 & $144.31^{*}$ & 0.141 & 0.094 & 0.000 \\
\hline & \multicolumn{12}{|c|}{ Submodularity Test } \\
\hline & 0.000 & 0.000 & 0.003 & 0.020 & 0.187 & 0.068 & 0.000 & 0.094 & 0.047 & 0.000 & 0.000 & 0.188 \\
\hline
\end{tabular}

* Significant at the 0.01 level $\dagger$ Significant at the 0.05 level $\$$ Significant at the 0.10 level

Table 70 Likelihood ratios for Perceived Benefits of AMT - Metals Industry

\begin{tabular}{|c|c|c|c|c|c|c|c|c|c|c|c|c|}
\hline & \multicolumn{6}{|c|}{ Labour Productivity } & \multicolumn{6}{|c|}{ Profit } \\
\hline \multirow[t]{5}{*}{$\begin{array}{c}\text { Obstacle } \\
\text { Pairs } \\
\end{array}$} & $1-2$ & $1-3$ & $1-4$ & $2-3$ & $2-4$ & $3-4$ & $1-2$ & $1-3$ & $1-4$ & $2-3$ & $2-4$ & $3-4$ \\
\hline & \multicolumn{12}{|c|}{ Supermodularity Test } \\
\hline & 0.000 & 0.028 & 0.213 & 0.128 & 0.000 & 0.000 & 0.000 & 0.000 & 0.164 & 0.024 & 0.000 & 0.000 \\
\hline & \multicolumn{12}{|c|}{ Submodularity Test } \\
\hline & 0.156 & 0.000 & 0.000 & 0.242 & 0.171 & 0.398 & 0.111 & 0.000 & 0.000 & 0.258 & 0.008 & 0.268 \\
\hline
\end{tabular}

$*$ Significant at the 0.01 level $†$ Significant at the 0.05 level $\$$ Significant at the 0.10 level

Table 71 Likelihood ratios for Perceived Benefits of AMT - Non-Metals Industry

\begin{tabular}{|c|c|c|c|c|c|c|c|c|c|c|c|c|}
\hline & \multicolumn{6}{|c|}{ Labour Productivity } & \multicolumn{6}{|c|}{ Profit } \\
\hline $\begin{array}{c}\text { Obstacle } \\
\text { Pairs }\end{array}$ & $1-2$ & $1-3$ & $1-4$ & $2-3$ & $2-4$ & $3-4$ & $1-2$ & $1-3$ & $1-4$ & $2-3$ & $2-4$ & $3-4$ \\
\hline & \multicolumn{12}{|c|}{ Supermodularity Test } \\
\hline & 0.038 & 0.958 & 0.077 & 0.288 & 0.000 & 0.345 & 0.000 & 0.719 & 0.000 & 0.360 & 0.198 & 0.561 \\
\hline & \multicolumn{12}{|c|}{ Submodularity Test } \\
\hline & 0.000 & 0.000 & 0.000 & 0.063 & 1.389 & 0.025 & 0.562 & 0.000 & 0.739 & 0.098 & 0.375 & 0.000 \\
\hline
\end{tabular}

$*$ Significant at the 0.01 level $†$ Significant at the 0.05 level $\$$ Significant at the 0.10 level

Table 72 Likelihood ratios for Perceived Benefits of AMT - Plastics Industry

\begin{tabular}{|c|c|c|c|c|c|c|c|c|c|c|c|c|}
\hline & \multicolumn{6}{|c|}{ Labour Productivity } & \multicolumn{6}{|c|}{ Profit } \\
\hline \multirow[t]{5}{*}{$\begin{array}{c}\text { Obstacle } \\
\text { Pairs } \\
\end{array}$} & $1-2$ & $1-3$ & $1-4$ & $2-3$ & $2-4$ & $3-4$ & $1-2$ & $1-3$ & $1-4$ & $2-3$ & $2-4$ & $3-4$ \\
\hline & \multicolumn{12}{|c|}{ Supermodularity Test } \\
\hline & $2.633^{\circ}$ & 1.134 & 0.022 & 0.073 & $2.318^{\ddagger}$ & 0.410 & 1.689 & $5.766^{*}$ & 0.016 & 0.000 & $27.813^{*}$ & 0.712 \\
\hline & \multicolumn{12}{|c|}{ Submodularity Test } \\
\hline & 0.205 & 0.219 & $2.531^{\dagger}$ & 1.368 & 0.534 & 0.483 & $6.559^{*}$ & 0.298 & $11.995^{*}$ & $7.685^{*}$ & 0.000 & $5.094^{*}$ \\
\hline
\end{tabular}

* Significant at the 0.01 level $\dagger$ Significant at the 0.05 level $†$ Significant at the 0.10 level

Table 73 Likelihood ratios for Perceived Benefits of AMT - Textiles Industry 


\begin{tabular}{|c|c|c|c|c|c|c|c|c|c|c|c|c|}
\hline & \multicolumn{6}{|c|}{ Labour Productivity } & \multicolumn{6}{|c|}{ Profit } \\
\hline $\begin{array}{c}\text { Obstacle } \\
\text { Pairs }\end{array}$ & $1-2$ & $1-3$ & $1-4$ & $2-3$ & $2-4$ & $3-4$ & $1-2$ & $1-3$ & $1-4$ & $2-3$ & $2-4$ & $3-4$ \\
\hline & \multicolumn{12}{|c|}{ Supermodularity Test } \\
\hline & 0.343 & 0.000 & 0.102 & 0.064 & $45.869^{*}$ & $70.874^{4}$ & 0.247 & 0.035 & 1.109 & 0.033 & 0.326 & 0.003 \\
\hline & \multicolumn{12}{|c|}{ Submodularity Test } \\
\hline & 0.000 & 0.572 & 0.051 & 0.038 & 0.013 & 0.000 & 0.259 & 0.227 & 0.012 & $40.183^{*}$ & 0.254 & 1.413 \\
\hline
\end{tabular}

$*$ Significant at the 0.01 level $†$ Significant at the 0.05 level $\$$ Significant at the 0.10 level

Table 74 Likelihood ratios for Perceived Benefits of AMT - Vehicles Industry

\begin{tabular}{|c|c|c|c|c|c|c|c|c|c|c|c|c|}
\hline & \multicolumn{6}{|c|}{ Labour Productivity } & \multicolumn{6}{|c|}{ Profit } \\
\hline \multirow[t]{5}{*}{\begin{tabular}{|c|} 
Obstacle \\
Pairs \\
\end{tabular}} & $1-2$ & $1-3$ & $1-4$ & $2-3$ & $2-4$ & $3-4$ & $1-2$ & $1-3$ & $1-4$ & $2-3$ & $2-4$ & $3-4$ \\
\hline & \multicolumn{12}{|c|}{ Supermodularity Test } \\
\hline & 0.348 & 0.272 & 0.000 & 0.449 & 1.405 & 0.089 & 0.000 & 1.881 & 0.075 & $2.107^{\ddagger}$ & 0.451 & 0.677 \\
\hline & \multicolumn{12}{|c|}{ Submodularity Test } \\
\hline & 0.000 & 0.101 & 0.279 & 0.057 & 0.000 & 0.146 & 0.828 & 0.075 & 0.677 & 0.000 & 0.828 & 0.752 \\
\hline
\end{tabular}

Table 75 Likelihood ratios for Perceived Benefits of AMT - Wood Industry

Factor 1 = Time (Reduced Capital, Reduced rejection time, Reduced set-up time, Increased quality, New product features, Reduced time to market)

Factor 2 = Productivity (Reduced labour, Reduced material, Reduced capital) Increased utilization rate, Increased Profitability)

Factor 3 = Flexibility (Increased flexibility, Increased skill requirements, Increased quality)

Factor 4 = Profitability (Increased equipment utilization rate, Increased market share, Increased Profitability)

\subsubsection{High-Level Factors}

Once again, the sample size and the distribution of plants within the various states is a more limiting factor to the results in this study. Only three industries have plants existing in all possible states. There are a number of exceptions that had to be dealt with resulting in many of the constraints for the analysis being removed due to lack of data. For this reason, each of the industries has different cut-off values for significance, and within many of the industries, the various pairings have different cut-off values as well (refer to Appendix E for values). 
The chemicals industry exhibits a number of significant results (refer to Table 76). The perceived benefits factor and the human resources factor were found to be substitutes with respect to both models. This result indicates that there is no added benefit to hiring skilled personnel if there is only the perception that AMT are useful, the technologies must be implemented before benefits from the human resources practices can be obtained. The AMT and business practices factor and the implementation support and $R \& D$ factor were found to be complementary in both models. This result supports those of Udo and Ehie (1996) who state that having support from many sources within the plant will aid in the implementation of AMT. There were also a number of results that are significant only with respect to profit. These include the substitute pairings of the AMT and business practices factor and the human resource factor, and the implementation support and R\&D factor and the design factor. The R\&D portion of the implementation support and $R \& D$ factor may be a substitute for the design factor due to duplicate effort by the groups resulting in less profitability for the plant. The chemical industry does a significant amount of R\&D that is a sunk cost. For example, the pharmaceutical industry has low success rates on drug research; even when a viable drug is discovered it takes many years to recover costs due to the drug approval process. The design factor was found to be complementary with the human resources factor. This result demonstrates the need to attract and retain highly qualified personnel in the chemical industry.

The electronics industry exhibits substitutability (with respect to labour productivity) between the perceived benefits factor and the implementation support and R\&D factor (refer to Table 77). These two factors measure very similar features, since if there is a lot of support for the AMT then there should also be a large number of perceived benefits. When both of these factors are exhibited simultaneously, the information from the sources of support and the 
perceptual biases may cause unwanted conflict during the implementation process reducing the productivity of the workers. On the other hand, the implementation support and R\&D factor was found to be complementary with the human resources factor with respect to labour productivity. This result demonstrates that if the majority of the support is going to be obtained from within the company then it is increasingly beneficial to have a highly skilled workforce providing that support.

With respect to profit, there are also a number of significant results in the electronics industry. The perceived benefits and the AMT that were implemented are found to be complements. This demonstrates that if perceived benefits for AMT were high then the plant would have already implemented the technology. If not, then the high level of perceived benefits would have caused conflict within the plant since the technology was not being implemented. Therefore, a high level of both AMT and perceived benefits results in higher profitability as the plant management championed the implementation of the technology. Finally, the AMT and business practices factor is a substitute for the implementation support and R\&D factor. This result may be because there are not many plants in the electronics industry that exhibit the implementation support and R\&D factor, and in particular, none that exhibit only these two factors simultaneously.

The furniture and others industries exhibit a number of significant results with respect to profit but only one with respect to labour productivity (refer to Table 78). With respect to labour productivity, the implementation support and R\&D factor and the human resources factor were found to be substitutes. This could be because of the artistic nature of the research and development that takes place in the furniture industry resulting in long apprenticeship training programs that reduce the labour productivity of new employees, especially when there are a 
number of technological changes being supported at the time. With respect to profit, the perceived benefits factor and the design factor were found to be substitutes. This model also demonstrates complementarities between the AMT and business practices factor and either the implementation support and R\&D factor, the design factor, or the human resources factor. The large number of complementarities with the AMT and business practices supports the many statements by Milgrom and Roberts $(1990,1995)$ and Ettlie and Reza (1992) that AMT are complementary with design, innovative human resources approaches, and many sources of implementation support. The AMT and business practices factor is complementary with other factors in six of the ten industries.

The machinery industry exhibits only two significant results and those are found in the model with labour productivity as the dependent variable (refer to Table 79). As in the chemical industry, the AMT and business practices factor and the human resources factor are substitutes as are the implementation support and $R \& D$ factor and the design factor. This industry has seven states with no data and so a large number of constraints had to be removed from the models (which is a large limiting factor for the results presented in this section).

The metals industry has a number of significant results (refer to Table 80). As in the furniture industry, the AMT and business practices factor and the human resources factor are complementary. In this case, the human resources industry is found to be complementary with both the design factor and the implementation support and R\&D factor with respect to profit. This demonstrates the strong need to hire highly skilled workers in the metals industry and the competitive advantage that such a workforce brings to all aspects of the implementation of AMT. There was also a couple of substitute pairing identified in the analysis. With respect to labour productivity, the AMT and business practices are substitutes for the design factor. This result 
could be due to the increased complexity to designing new products to work with high levels of AMT. It is also supported by the results found in the AMT analysis (presented at the beginning of the constrained regression analysis by industry) where the design technologies were substitutes for two of the three other forms of technologies. With respect to profit, the perceived benefits factor and the AMT and business practices factor are also found to be substitutes. This result may be due to the recent purchase of a large amount of AMT as the perception of its value are high and the initial capital costs of AMT are normally quite large.

The non-metals industry exhibits only two substitute pairings with respect to profit (refer to Table 81). The implementation support and R\&D factor is a substitute for both the design factor and the human resources factor. This result implies that the time it takes the various groups to support the implementations is reducing their capacity for training and design work thus reducing the profit of the plant. Once again, there are a large number of empty states that are limiting factors for the scope of the results of this analysis.

The textiles industry also has very few significant results (refer to Table 83). As in the furniture industry, the AMT and business practices factor and the human resources factor are complements and the implementation support and R\&D factor and human resources factor are substitutes. The textiles industry also exhibits one other complementary pair in the analysis with respect to profit, the perceived benefits factor and the human resources factor. This result demonstrates that if there is a strong belief in the benefits of AMT and highly skilled personnel are hired or trained, then there will be increased benefits to the plant.

The analysis for the vehicles industry demonstrates an extremely large number of significant results (refer to Table 84). A number of the significance tests result in both the 
complementarity hypothesis and the substitutability hypothesis being rejected (variables are independent). This occurs with respect to labour productivity with the AMT and business practices factor and both the perceived benefits factor and the implementation support and R\&D factor, and with respect to profit with the perceived benefits factor and both the design factor and the human resources factor. With respect to labour productivity, the vehicles industry exhibits a number of complementarities. The perceived benefits factor and the design factor are complementary as well as the human resources factor and either the design factor or the implementation support and research and development factor. These results demonstrate that having highly skilled personnel increases the ability of the workers to utilize the new design technologies and support change within the plant. With respect to profit, the AMT and business practices are complementary with both the implementation support and R\&D factor and the human resources practices but a substitute for the design factor. These results support the findings that a large base of support and highly skilled workers are required for successful implementations of AMT but reject the Alcorta (1994) hypothesis that all forms of AMT are complementary.

Finally, the wood industry had a number of significant results, although the majority of them are with respect to profit (refer to Table 85). As in the furniture industry, the AMT and business practices factor is complementary with both the design factor and the human resources factor for both models. The AMT and business practices factor is also found to be a substitute for the implementation support and R\&D factor in both models. This result may stem from the fact that if there is a large amount of AMT already implemented in the plant then having support for changes only serves to cause conflict over upgrading existing technologies. 
There are a number of results involving the perceived benefits factor with respect to profit in the wood industry. The perceived benefits factor is complementary with the AMT and business practices factor, a substitute for the implementation support and R\&D factor, and independent from the human resources factor. The mixed conclusions found with the perceived benefits factor may be due to the subjective nature of the responses. The human resources factor is also found to be a substitute for the design factor. This result demonstrates that there is a large amount of learning-by-doing that occurs while workers design the product making increased training less cost effective.

\begin{tabular}{|c|c|c|c|c|c|c|c|c|c|c|}
\hline & \multicolumn{10}{|c|}{ Labour Productivity } \\
\hline \multirow{5}{*}{$\begin{array}{c}\text { Obstacle } \\
\text { Pairs }\end{array}$} & $1-2$ & $1-3$ & $1-4$ & $1-5$ & $2-3$ & $2-4$ & $2-5$ & $3-4$ & $3-5$ & $4-5$ \\
\hline & \multicolumn{10}{|c|}{ Supermodularity Test } \\
\hline & 1.644 & 1.150 & 0.127 & $865.47^{*}$ & 0.168 & 0.359 & 0.610 & 1.748 & 0.482 & 0.050 \\
\hline & \multicolumn{10}{|c|}{ Submodularity Test } \\
\hline & 0.000 & 0.069 & 0.538 & 0.216 & $3.438^{*}$ & 0.087 & 0.413 & 0.000 & 0.548 & 0.756 \\
\hline & \multicolumn{10}{|c|}{ Profit } \\
\hline \multirow{5}{*}{$\begin{array}{c}\text { Obstacle } \\
\text { Pairs }\end{array}$} & $1-2$ & $1-3$ & $1-4$ & $1-5$ & $2-3$ & $2-4$ & $2-5$ & $3-4$ & $3-5$ & $4-5$ \\
\hline & \multicolumn{10}{|c|}{ Supermodularity Test } \\
\hline & 0.731 & 0.381 & 0.179 & $59.823^{*}$ & 0.359 & 0.558 & $59.823^{*}$ & $4.322^{*}$ & 0.490 & 0.227 \\
\hline & \multicolumn{10}{|c|}{ Submodularity Test } \\
\hline & 0.124 & 0.849 & 0.881 & 0.291 & $2.449^{\dagger}$ & 0.254 & 0.423 & 0.037 & 0.900 & $59.823^{*}$ \\
\hline
\end{tabular}

* Significant at the 0.01 level $†$ Significant at the 0.05 level $\$$ Significant at the 0.10 level

Table 76 Likelihood ratios for High-Level Factors - Chemicals Industry

\begin{tabular}{|c|c|c|c|c|c|c|c|c|c|c|}
\hline & \multicolumn{10}{|c|}{ Labour Productivity } \\
\hline \multirow{5}{*}{$\begin{array}{c}\text { Obstacle } \\
\text { Pairs }\end{array}$} & $1-2$ & $1-3$ & $1-4$ & $1-5$ & $2-3$ & $2-4$ & $2-5$ & $3-4$ & $3-5$ & $4-5$ \\
\hline & \multicolumn{10}{|c|}{ Supermodularity Test } \\
\hline & 0.081 & $1067.8^{*}$ & 0.127 & 0.020 & 0.005 & 0.293 & 0.204 & 0.143 & 0.136 & 0.097 \\
\hline & \multicolumn{10}{|c|}{ Submodularity Test } \\
\hline & 0.143 & 0.158 & 0.032 & 0.188 & 0.188 & 0.000 & 0.020 & 0.020 & $889.80^{*}$ & 0.025 \\
\hline & \multicolumn{10}{|c|}{ Profit } \\
\hline \multirow{5}{*}{$\begin{array}{c}\text { Obstacle } \\
\text { Pairs }\end{array}$} & $1-2$ & $1-3$ & $1-4$ & $1-5$ & $2-3$ & $2-4$ & $2-5$ & $3-4$ & $3-5$ & $4-5$ \\
\hline & \multicolumn{10}{|c|}{ Supermodularity Test } \\
\hline & 0.224 & 0.025 & 0.062 & 0.124 & $20.391^{*}$ & 0.031 & 0.099 & 0.124 & 0.124 & 0.099 \\
\hline & \multicolumn{10}{|c|}{ Submodularity Test } \\
\hline & $20.391^{*}$ & 0.298 & 0.031 & 0.174 & 0.497 & 0.062 & 0.025 & 0.199 & 0.041 & 0.050 \\
\hline
\end{tabular}

Table 77 Likelihood ratios for High-Level Factors - Electronics Industry 


\begin{tabular}{|c|c|c|c|c|c|c|c|c|c|c|}
\hline & \multicolumn{10}{|c|}{ Labour Productivity } \\
\hline \multirow{5}{*}{$\begin{array}{c}\text { Obstacle } \\
\text { Pairs }\end{array}$} & $1-2$ & $1-3$ & $1-4$ & $1-5$ & $2-3$ & $2-4$ & $2-5$ & $3-4$ & $3-5$ & $4-5$ \\
\hline & \multicolumn{10}{|c|}{ Supermodularity Test } \\
\hline & 0.041 & 0.269 & 0.344 & 0.103 & 0.131 & 0.021 & 0.069 & 0.158 & $2735.9^{*}$ & 0.779 \\
\hline & \multicolumn{10}{|c|}{ Submodularity Test } \\
\hline & 0.186 & 0.028 & 0.007 & 0.000 & 0.358 & 0.551 & 1.157 & 0.434 & 0.124 & 0.012 \\
\hline & \multicolumn{10}{|c|}{ Profit1.157 } \\
\hline \multirow{5}{*}{$\begin{array}{c}\text { Obstacle } \\
\text { Pairs }\end{array}$} & $1-2$ & $1-3$ & $1-4$ & $1-5$ & $2-3$ & $2-4$ & $2-5$ & $3-4$ & $3-5$ & $4-5$ \\
\hline & \multicolumn{10}{|c|}{ Supermodularity Test } \\
\hline & 0.016 & 0.868 & $73.464^{*}$ & 0.007 & 0.788 & 0.064 & 0.095 & 0.490 & 0.171 & 0.363 \\
\hline & \multicolumn{10}{|c|}{ Submodularity Test } \\
\hline & 0.978 & 0.043 & 0.089 & 0.066 & $73.464^{*}$ & $1.968^{\ddagger}$ & $1.839^{\ddagger}$ & 0.197 & 0.492 & 0.085 \\
\hline
\end{tabular}

* Significant at the 0.01 level $\quad \dagger$ Significant at the 0.05 level $\quad$ Significant at the 0.10 level

Table 78 Likelihood ratios for High-Level Factors - Furniture and Other Industries

\begin{tabular}{|c|c|c|c|c|c|c|c|c|c|c|}
\hline & \multicolumn{10}{|c|}{ Labour Productivity } \\
\hline \multirow{5}{*}{$\begin{array}{l}\text { Obstacle } \\
\text { Pairs }\end{array}$} & $1-2$ & $1-3$ & $1-4$ & $1-5$ & $2-3$ & $2-4$ & $2-5$ & $3-4$ & $3-5$ & $4-5$ \\
\hline & \multicolumn{10}{|c|}{ Supermodularity Test } \\
\hline & 1.489 & 0.000 & 0.000 & 0.000 & 0.000 & 0.000 & $3.073^{\dagger}$ & $2.911^{\dagger}$ & 0.051 & 0.000 \\
\hline & \multicolumn{10}{|c|}{ Submodularity Test } \\
\hline & 0.000 & 0.183 & 0.271 & 0.142 & 1.992 & 0.000 & 0.000 & 0.000 & 0.315 & 0.000 \\
\hline & \multicolumn{10}{|c|}{ Profit } \\
\hline \multirow{5}{*}{$\begin{array}{c}\text { Obstacle } \\
\text { Pairs }\end{array}$} & $1-2$ & $1-3$ & $1-4$ & $1-5$ & $2-3$ & $2-4$ & $2-5$ & $3-4$ & $3-5$ & $4-5$ \\
\hline & \multicolumn{10}{|c|}{ Supermodularity Test } \\
\hline & 0.368 & 0.003 & 0.003 & 0.034 & 0.071 & 0.026 & 1.037 & 0.071 & 0.003 & 0.087 \\
\hline & \multicolumn{10}{|c|}{ Submodularity Test } \\
\hline & 0.004 & 0.302 & 0.064 & 0.024 & 0.039 & 0.000 & 0.075 & 0.000 & 1.807 & 0.195 \\
\hline
\end{tabular}

$*$ Significant at the 0.01 level $\dagger$ Significant at the 0.05 level $\$$ Significant at the 0.10 level

Table 79 Likelihood ratios for High-Level Factors - Machinery Industry

\begin{tabular}{|c|c|c|c|c|c|c|c|c|c|c|}
\hline & \multicolumn{10}{|c|}{ Labour Productivity } \\
\hline \multirow{6}{*}{$\begin{array}{c}\text { Obstacle } \\
\text { Pairs }\end{array}$} & $1-2$ & $1-3$ & $1-4$ & $1-5$ & $2-3$ & $2-4$ & $2-5$ & $3-4$ & $3-5$ & $4-5$ \\
\hline & \multicolumn{10}{|c|}{ Supermodularity Test } \\
\hline & 0.351 & 0.208 & 0.006 & 0.465 & 0.951 & $3.493^{*}$ & 0.164 & 1.355 & 0.629 & 0.286 \\
\hline & \multicolumn{10}{|c|}{ Submodularity Test } \\
\hline & 0.407 & 0.316 & 0.282 & 0.089 & 0.560 & 0.017 & $1.908^{\ddagger}$ & 0.548 & 0.365 & 0.087 \\
\hline & \multicolumn{10}{|c|}{ Profit } \\
\hline \multirow{5}{*}{$\begin{array}{c}\text { Obstacle } \\
\text { Pairs }\end{array}$} & $1-2$ & $1-3$ & $1-4$ & $1-5$ & $2-3$ & $2-4$ & $2-5$ & $3-4$ & $3-5$ & $4-5$ \\
\hline & \multicolumn{10}{|c|}{ Supermodularity Test } \\
\hline & $78.476^{*}$ & 0.103 & 0.072 & 0.170 & 0.220 & 0.128 & 0.078 & 0.641 & 0.228 & 0.175 \\
\hline & \multicolumn{10}{|c|}{ Submodularity Test } \\
\hline & 0.966 & 0.195 & 0.083 & 0.095 & 0.047 & 0.009 & $78.476^{*}$ & 0.001 & $78.476^{*}$ & $78.476^{*}$ \\
\hline
\end{tabular}

* Significant at the 0.01 level $†$ Significant at the 0.05 level $\$$ Significant at the 0.10 level

Table 80 Likelihood ratios for High-Level Factors - Metals Industry 


\begin{tabular}{|c|c|c|c|c|c|c|c|c|c|c|}
\hline & \multicolumn{10}{|c|}{ Labour Productivity } \\
\hline \multirow{5}{*}{$\begin{array}{l}\text { Obstacle } \\
\text { Pairs }\end{array}$} & $1-2$ & $1-3$ & $1-4$ & $1-5$ & $2-3$ & $2-4$ & $2-5$ & $3-4$ & $3-5$ & $4-5$ \\
\hline & \multicolumn{10}{|c|}{ Supermodularity Test } \\
\hline & 0.155 & 0.468 & 0.792 & 0.288 & 0.466 & 0.380 & 0.426 & 0.184 & 0.155 & 0.967 \\
\hline & \multicolumn{10}{|c|}{ Submodularity Test } \\
\hline & 0.132 & 0.022 & 0.000 & 0.295 & 0.006 & 1.739 & 1.347 & 0.484 & 0.046 & 0.806 \\
\hline & \multicolumn{10}{|c|}{ Profit } \\
\hline \multirow{5}{*}{$\begin{array}{c}\text { Obstacle } \\
\text { Pairs }\end{array}$} & $1-2$ & $1-3$ & $1-4$ & $1-5$ & $2-3$ & $2-4$ & $2-5$ & $3-4$ & $3-5$ & $4-5$ \\
\hline & \multicolumn{10}{|c|}{ Supermodularity Test } \\
\hline & 0.105 & 0.228 & 0.264 & 0.091 & 0.112 & 0.173 & 0.143 & $28.945^{*}$ & $28.945^{*}$ & 0.078 \\
\hline & \multicolumn{10}{|c|}{ Submodularity Test } \\
\hline & 0.161 & 0.068 & 0.061 & 0.078 & 0.002 & 0.814 & 0.465 & 0.207 & 0.017 & 0.887 \\
\hline
\end{tabular}

* Significant at the 0.01 level $†$ Significant at the 0.05 level $\$$ Significant at the 0.10 level

Table 81 Likelihood ratios for High-Level Factors - Non-Metals Industry

\begin{tabular}{|c|c|c|c|c|c|c|c|c|c|c|}
\hline & \multicolumn{10}{|c|}{ Labour Productivity } \\
\hline \multirow{5}{*}{$\begin{array}{c}\text { Obstacle } \\
\text { Pairs }\end{array}$} & $1-2$ & $1-3$ & $1-4$ & $1-5$ & $2-3$ & $2-4$ & $2-5$ & $3-4$ & $3-5$ & $4-5$ \\
\hline & \multicolumn{10}{|c|}{ Supermodularity Test } \\
\hline & 0.015 & 0.739 & 0.024 & 0.072 & 0.030 & 0.060 & 0.448 & 0.681 & 0.000 & 0.341 \\
\hline & \multicolumn{10}{|c|}{ Submodularity Test } \\
\hline & 0.015 & 0.000 & 0.066 & 0.000 & 0.463 & 0.284 & 0.085 & 0.006 & 0.352 & 0.000 \\
\hline & \multicolumn{10}{|c|}{ Profit } \\
\hline \multirow{5}{*}{$\begin{array}{c}\text { Obstacle } \\
\text { Pairs }\end{array}$} & $1-2$ & $1-3$ & $1-4$ & $1-5$ & $2-3$ & $2-4$ & $2-5$ & $3-4$ & $3-5$ & $4-5$ \\
\hline & \multicolumn{10}{|c|}{ Supermodularity Test } \\
\hline & 0.032 & 1.892 & 0.805 & 0.332 & 0.759 & 0.540 & 0.360 & 0.714 & 0.342 & 1.073 \\
\hline & \multicolumn{10}{|c|}{ Submodularity Test } \\
\hline & 0.164 & 0.033 & 0.201 & 0.039 & 0.819 & 0.240 & 1.160 & 0.924 & 1.605 & 0.022 \\
\hline
\end{tabular}

* Significant at the 0.01 level $\dagger$ Significant at the 0.05 level $\$$ Significant at the 0.10 level

Table 82 Likelihood ratios for High-Level Factors - Plastics Industry

\begin{tabular}{|c|c|c|c|c|c|c|c|c|c|c|}
\hline & \multicolumn{10}{|c|}{ Labour Productivity } \\
\hline \multirow{6}{*}{$\begin{array}{c}\text { Obstacle } \\
\text { Pairs }\end{array}$} & $1-2$ & $1-3$ & $1-4$ & $1-5$ & $2-3$ & $2-4$ & $2-5$ & $3-4$ & $3-5$ & $4-5$ \\
\hline & \multicolumn{10}{|c|}{ Supermodularity Test } \\
\hline & 0.546 & 0.236 & 0.090 & 0.138 & 0.908 & 0.251 & 1.654 & 0.892 & $1.977^{\dagger}$ & 0.699 \\
\hline & \multicolumn{10}{|c|}{ Submodularity Test } \\
\hline & 0.307 & 0.189 & 0.558 & 1.399 & 0.621 & 0.063 & $19.259^{*}$ & 0.291 & 0.432 & 0.377 \\
\hline & \multicolumn{10}{|c|}{ Profit } \\
\hline \multirow{5}{*}{$\begin{array}{c}\text { Obstacle } \\
\text { Pairs }\end{array}$} & $1-2$ & $1-3$ & $1-4$ & $1-5$ & $2-3$ & $2-4$ & $2-5$ & $3-4$ & $3-5$ & $4-5$ \\
\hline & \multicolumn{10}{|c|}{ Supermodularity Test } \\
\hline & 0.124 & 0.155 & 0.620 & 0.031 & 0.310 & 0.124 & 0.310 & 0.930 & $2.109^{\dagger}$ & 0.465 \\
\hline & \multicolumn{10}{|c|}{ Submodularity Test } \\
\hline & 0.062 & 0.248 & 0.248 & $2.109^{\dagger}$ & 0.558 & 0.031 & 0.279 & 0.062 & 0.124 & 0.062 \\
\hline
\end{tabular}

* Significant at the 0.01 level $\dagger$ Significant at the 0.05 level $†$ Significant at the 0.10 level

Table 83 Likelihood ratios for High-Level Factors - Textiles Industry 


\begin{tabular}{|c|c|c|c|c|c|c|c|c|c|c|}
\hline & \multicolumn{10}{|c|}{ Labour Productivity } \\
\hline \multirow{5}{*}{$\begin{array}{c}\text { Obstacle } \\
\text { Pairs }\end{array}$} & $1-2$ & $1-3$ & $1-4$ & $1-5$ & $2-3$ & $2-4$ & $2-5$ & $3-4$ & $3-5$ & $4-5$ \\
\hline & \multicolumn{10}{|c|}{ Supermodularity Test } \\
\hline & $41.734^{*}$ & 0.582 & 0.279 & 0.886 & $97.985^{*}$ & 0.394 & 0.993 & 1.017 & 0.033 & 0.993 \\
\hline & \multicolumn{10}{|c|}{ Submodularity Test } \\
\hline & $14.521^{*}$ & 0.090 & $40.913^{*}$ & 0.279 & $3.413^{*}$ & 0.451 & 0.435 & 0.468 & $40.729^{*}$ & $40.733^{*}$ \\
\hline & \multicolumn{10}{|c|}{ Profit } \\
\hline \multirow{5}{*}{$\begin{array}{c}\text { Obstacle } \\
\text { Pairs }\end{array}$} & $1-2$ & $1-3$ & $1-4$ & $1-5$ & $2-3$ & $2-4$ & $2-5$ & $3-4$ & $3-5$ & $4-5$ \\
\hline & \multicolumn{10}{|c|}{ Supermodularity Test } \\
\hline & 1.097 & 0.091 & $26.178^{*}$ & $29.301^{*}$ & 0.006 & $9.182^{*}$ & 0.281 & 0.024 & $28.885^{*}$ & 0.100 \\
\hline & \multicolumn{10}{|c|}{ Submodularity Test } \\
\hline & 0.002 & 0.100 & $30.176^{*}$ & $28.593^{*}$ & $30.134^{*}$ & 0.354 & $28.552^{*}$ & 0.578 & 1.261 & 0.352 \\
\hline
\end{tabular}

* Significant at the 0.01 level $\dagger$ Significant at the 0.05 level $\$$ Significant at the 0.10 level

Table 84 Likelihood ratios for High-Level Factors - Vehicles Industry

\begin{tabular}{|c|c|c|c|c|c|c|c|c|c|c|}
\hline & \multicolumn{10}{|c|}{ Labour Productivity } \\
\hline \multirow{6}{*}{$\begin{array}{c}\text { Obstacle } \\
\text { Pairs }\end{array}$} & $1-2$ & $1-3$ & $1-4$ & $1-5$ & $2-3$ & $2-4$ & $2-5$ & $3-4$ & $3-5$ & $4-5$ \\
\hline & \multicolumn{10}{|c|}{ Supermodularity Test } \\
\hline & 0.959 & 1.308 & 0.123 & 0.491 & $3069.8^{*}$ & 0.740 & 0.219 & 0.728 & 0.647 & 1.126 \\
\hline & \multicolumn{10}{|c|}{ Submodularity Test } \\
\hline & 0.327 & 0.082 & 0.684 & 0.260 & 0.740 & $1.761^{\ddagger}$ & $2.557^{\dagger}$ & 0.472 & 0.758 & 0.855 \\
\hline & \multicolumn{10}{|c|}{ Profit } \\
\hline \multirow{5}{*}{$\begin{array}{c}\text { Obstacle } \\
\text { Pairs }\end{array}$} & $1-2$ & $1-3$ & $1-4$ & $1-5$ & $2-3$ & $2-4$ & $2-5$ & $3-4$ & $3-5$ & $4-5$ \\
\hline & \multicolumn{10}{|c|}{ Supermodularity Test } \\
\hline & 0.810 & $152.15^{*}$ & 0.315 & $152.15^{*}$ & $3.058^{*}$ & 0.315 & 0.450 & 0.495 & 0.495 & $3.238^{*}$ \\
\hline & \multicolumn{10}{|c|}{ Submodularity Test } \\
\hline & $152.15^{*}$ & 0.810 & 1.529 & $152.15^{*}$ & 0.450 & $3.598^{*}$ & $152.15^{*}$ & 0.450 & 0.270 & 0.135 \\
\hline
\end{tabular}

* Significant at the 0.01 level $\dagger$ Significant at the 0.05 level $\$$ Significant at the 0.10 level

Table 85 Likelihood ratios for High-Level Factors - Wood Industry

Factor $1=$ Perceived Benefits

Factor 2 = Advanced Manufacturing Technologies (Except Design) \& Business Practices

Factor 3 = Implementation Support, Cross Functional Teams \& Research and Development

Factor 4 = Design (Production Design Technologies, Concurrent Engineering)

Factor 5 = Human Resources

\subsubsection{Conclusions}

The analysis of the constrained regression models by industry demonstrates the importance of the industry effect. Each industry has its own set of complementarities, especially when considering the high-level factors. These results demonstrate that analysis done with a high level 
of industry aggregation may be misleading and may not offer any useable results for plant managers. The industry effect appears to be stronger than that of plant size, indicating the market in which a plant operates has a strong effect on the types of AMT and business practices it should adopt in order to obtain a competitive advantage.

A few limitations appear to have had a significant impact on the findings. The primary limitation is that the sample size by industry is primarily between 100 and 250 plants. This small sample sizes leads to empty states or states with very few observations. This limitation forces the removal of constraints as there is no way to estimate their value. The reduction in constraints not only decreases the degrees of freedom of the significance test; it also makes it hard to reject any hypothesis, as there are fewer differences from the unconstrained model itself.

One industry in which there were few significant results combined with adequate sample size was the chemical industry. This industry is the most diverse, as it contains all types of chemical producers from pharmaceuticals to petroleum products, from fertilizers to cleaning products. The wide range of products and their associated differences in government regulations, patent protection, and research and development needs may have caused some of the inconclusive results. This industry should be broken down into smaller segments when a larger sample size is available.

Some common elements emerged throughout the various sections of the analysis. For example, many of the industries had very similar results in terms of business practices. The exception to this was the electronics industry, which was diametrically opposite to the general trend. The electronics industry, perhaps due to the intense competition and short product life cycle, requires its own set of optimal business practices in order to remain competitive. 
There was also a common complementary pair found in the AMT analysis. In these models, the industries such as machinery and vehicles that made similar products with only small variations year after year (or had many standard parts), the communications and robotics technologies were complementary. The communications infrastructure allowed small changes to be made to how the robotics operated with little cost thus improving the labour productivity and profitability of the plant.

Similarities in the perceived benefits factors were primarily confined to perceived productivity improvements and perceived profit increases being substitutes. This was the case in all significant results excluding the furniture industry. This result seems to indicate that some of the perceived benefits were not translating to actual profit. An explanation is the benefits perceived by the managers were a mixture of those they observed at their plant from the technologies and those they had been told would happen from conferences and technology sales representatives. These two different sources of perceived benefits caused confounding of the results.

Finally, there was a common pairing in the sources of implementation support analysis. The results of this analysis demonstrated that in many industries there is either a push of the technology down to the plants from head office or there is a pull for the technology to be implemented from within the plant. These two types of implementations do not rely on one another and are substitutes in many cases. This result indicates that if the plants are forced to include the technology then they will find a way to implement it without additional championing from primary stakeholders. Otherwise, if left to their own devices, primary stakeholders will need to be the driving force behind the implementation. 


\subsection{Ordinary Least Squares Model}

In order to support the constrained regression analysis presented above an ordinary least squares model was developed that included the individual variables. This method is similar to the analysis performed by Ichniowski, Shaw, and Prennushi (1997) for human resources policies. Each individual variable was added one at a time to the unrestricted model previously presented in section 4.5. The formulation of the profit function will now include an individual variable $\left(Z_{j}\right)$

as well as all of the state variables: $P_{j}=\sum_{i=0}^{2^{n}-1} \gamma_{i j} s_{i j}+\alpha_{j} Z_{j}+\varepsilon_{j}$. By considering the individual effects of the variables to the unrestricted model with the factor states, the significance of each variable will be determined. When there is strong support for complementarity the interaction effects will be larger than that of the individual variable, and in most cases, the individual variables will be insignificant (Ichniowski, Shaw, and Prennushi, 1997). This model will support the existence of complementarities and demonstrate if any individual variable deserves special attention due to its potential impact on the profit function.

This method involves a large number of regressions in order to complete the analysis as there are over 75 variables used in this study. A representative sample was chosen for this method in order to retain the focus on the constrained regression analysis. This section will analyse the medium plant size class, the electronics industry representing high-technology industries, the vehicles industry representing medium-technology industries, and the wood industry representing low-technology industries with profit as the dependent variable. These industries were chosen as they have relatively large sample sizes for the technology type and have low occurrences of states with insufficient data. 


\subsubsection{Medium Plants}

In general, when the individual variables are added to the regression equation including the various factor states, the individual variables have no significant impact on the profit of the plant. Most of the coefficients for individual variables are insignificant (refer to Table 86 to table 90). For those variables that do have a significant coefficient, the coefficient is relatively small compared to the size of the coefficients for the state variables. There are also a few instances where the coefficients for the state variables are also insignificant such as in the high-level analysis.

In the analysis of AMT that were implemented by the plants, all the states were found to have coefficients that were significant. There were a number of individual variables with positive and significant coefficients (refer to Table 86). The use of FMC/FMS, PLC machines, lasers, and high speed machining technologies all have significant coefficients that are smaller than $20 \%$ of magnitude of coefficients for the state variables. A couple of the communications technologies such as wide area networks and electronic data interchange also have small significant and positive coefficients. These forms of AMT demonstrate that the benefits to being able to receive and process information from external sources are greater than the amount captured by the interaction effects in the state variables. There are also a few small negative coefficients that were significant including the automated material handling and inspection technologies, as well as $\mathrm{CAD} / \mathrm{CAE}$ and $\mathrm{CIM}$ systems. These small negative coefficients demonstrate that the interaction effect may be overstated if there is a large installation of the material handling and inspection technologies or a large installed base of AMT as would be found in a plant using computer integrated manufacturing. 
The analysis of the business practices has only three variables with significant coefficients (refer to Table 87). The use of process simulations has a significant positive coefficient while the use of statistical process control and just-in-time inventory control has small negative coefficients. None of these variables will have a large effect on effects of the state variables on profit (even though the coefficients are statistically significant they are not very large compared to the coefficients for the state variables).

In the analysis of the sources of implementation support, there were three clusters of significant variables (refer to Table 88). The first cluster is support from the experimental design group and corporate head office. These variables have small negative coefficients that are approximately $20 \%$ the size of the coefficients for the state variables. These two results appear to support the idea that the amount of benefit obtained from groups of people that are not primary stakeholders is overstated in the interaction effects contained within the factor states. The second grouping involves sources of support from within the market. These include consultants, customers, and the government. These variables have small positive coefficients that are approximately $30 \%$ the size as those for the state variable coefficients. The support obtained from outside the plant is greater than what is contained in the analysis using only the state variables. One of the reasons that these would be important sources of support is that the government and customers can dictate many of the technologies that must be implemented in order to comply with contracts and government regulations. Finally, support from trade fairs has a significant positive coefficient that is approximately $50 \%$ of the coefficient for a state variable. This result may be due to the fact that the trade fair variable does not weight heavily on any of the factors and that the support obtained from trade fairs would aid primarily in the selection of the technologies to implement and not significantly in the implementation phase itself. 
The analysis of the perceived benefits model has the state consisting of the second and third factors (increased productivity and flexibility) exhibiting an insignificant coefficient (refer to Table 89 ). This result is not too surprising, as only $0.36 \%$ of medium plants are found in this state. Four individual variables have small coefficients. The perceived benefits of reduced set-up time, new product features, and increased skill requirement all have small positive coefficients. The perceived benefit of increased profitability is overstated in the state variables as it has a small negative coefficient. None of these variables has a coefficient that is greater than $20 \%$ of the coefficient for a state variable.

The analysis of the high-level factor model has two states that have insignificant coefficients. In particular, the state including only the human resources factor and the state consisting of the human resources factor and the AMT and business practices factor are insignificant (refer to Table 90). These states have only $0.49 \%$ and $0.02 \%$ of medium plants respectively.

The individual variables that have significant coefficients in the high-level factor model are very similar to those that were significant in the analysis of the factor types. The coefficients are all very small for the individual variable and usually are no more than $10 \%$ of the coefficients for the state variables. A few individual variables have significant coefficients in the high-level analysis that are not significant in the previous analysis. In terms of business practices, these include cross-functional design, concurrent engineering, continuous improvement, benchmarking, plant certifications, and supplier certifications. These variables primarily represent the design business practices and the certification processes that a plant would use. The reason that these variables would have a greater impact on profit than what is represented in the factor states is that many of these variables have intangible benefits such as certifications 
increasing the public image of the products being manufactured, and the design practices increasing the speed to market or creating high quality or more innovative products.

The ability to create new innovative products not being adequately represented in the factor state model is also supported by the fact that both the in-house $R \& D$ and contracted $R \& D$ variables have small significant coefficients. The remaining individual variables that are significant are very similar to those discussed in the previous analysis except that a couple of the support variables have changed slightly: support from experimental design no longer has a significant coefficient but now production staff does. Once again, support from trade fairs has the largest significant coefficient, although this time it is only approximately $30 \%$ of the coefficient for a state variable.

Overall, the regression analysis using the state and individual variables supports the concept of many of the individual variables being complementary. When the individual variables are added to the model containing the state variables, the individual variables have very little impact. There are a number of variables that have negative coefficients implying that the state variables may be overstating the effect of the individual variables within the factor. Another reason that the coefficient may be negative is that the variable may not be complementary with the rest of the variables included in the factor. The complementarity analysis from section 5.1 was not performed for the individual variables within a factor and some of the variables included in a factor may not be complementary with one another. 


\begin{tabular}{|c|c|c|c|c|c|c|c|c|c|c|c|c|c|c|c|c|c|c|c|c|c|c|c|c|c|}
\hline $\begin{array}{l}\text { Individu } \\
\text { included i } \\
\text { in the }\end{array}$ & 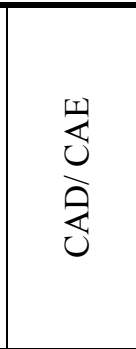 & 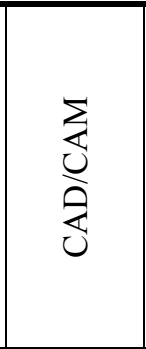 & 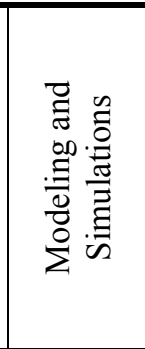 & 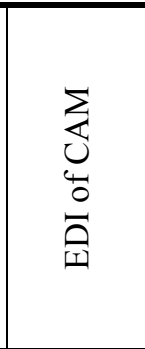 & $\sum_{i=1}^{\sum_{i=1}^{n}}$ & 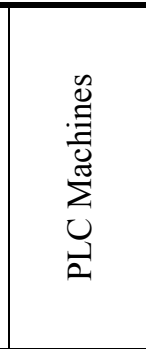 & 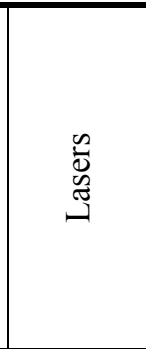 & 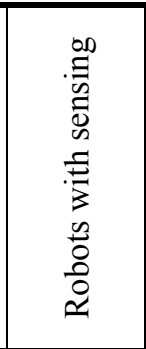 & 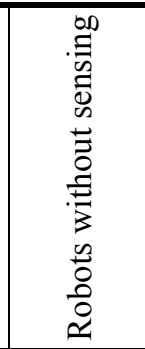 & 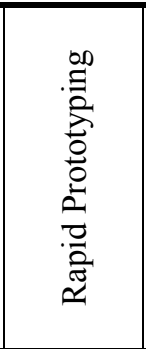 & 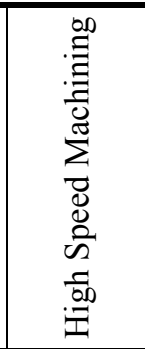 & 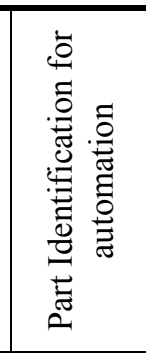 & 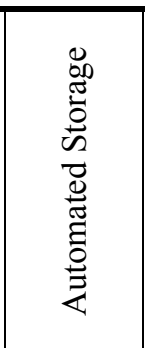 & 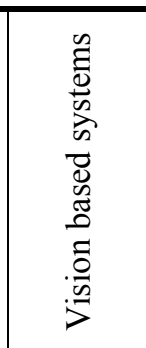 & 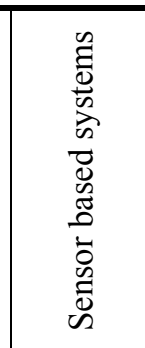 & 杂 & $\frac{z}{3}$ & 贫 & $\begin{array}{l}= \\
\tilde{\Xi}\end{array}$ & 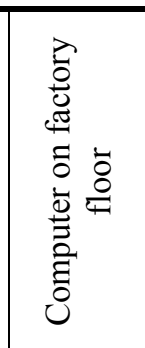 & $\sum$ & 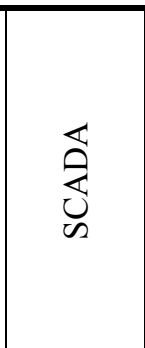 & 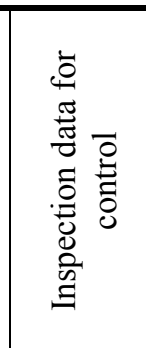 & 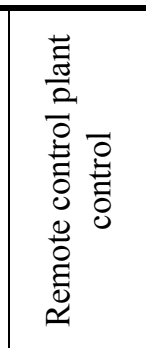 & 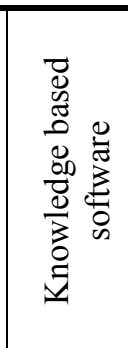 \\
\hline No factors & $\begin{array}{l}609^{\circ} \\
01061) \\
\end{array}$ & 1047) & \begin{tabular}{|l|l|}
$0.25619^{*}$ \\
$(0.01044)$ \\
\end{tabular} & \begin{tabular}{|l}
$0.25601^{*}$ \\
$(0.01045)$ \\
\end{tabular} & 1042) & 1043) & \begin{tabular}{|l|l|l}
$0.25594^{*}$ \\
$(0.01042)$ \\
\end{tabular} & \begin{tabular}{|l|l|}
$0.25625^{*}$ \\
$(0.01045)$ \\
\end{tabular} & $\begin{array}{l}0.25625^{*} \\
(0.01045) \\
\end{array}$ & \begin{tabular}{|l|}
$0.25625^{*}$ \\
$(0.01045)$ \\
\end{tabular} & \begin{tabular}{|l|l}
$0.25594^{*}$ \\
$(0.01043)$ \\
\end{tabular} & \begin{tabular}{|l|l|}
$0.25625^{*}$ \\
$(0.01044)$
\end{tabular} & \begin{tabular}{|l|}
$0.25625^{*}$ \\
$(0.01042)$ \\
\end{tabular} & \begin{tabular}{|l|l}
$0.25625^{*}$ \\
$(0.01042)$ \\
\end{tabular} & $\begin{array}{l}0.25625^{*} \\
(0.01043) \\
\end{array}$ & \begin{tabular}{|l|l|}
$0.26401^{*}$ \\
$(0.01085)$ \\
\end{tabular} & \begin{tabular}{|l|l}
$0.2533^{*}$ \\
$(0.01052)$ \\
\end{tabular} & $\begin{array}{l}24996^{*} \\
.01082) \\
\end{array}$ & \begin{tabular}{|l|l|}
$0.25588^{*}$ \\
$(0.01047)$ \\
\end{tabular} & \begin{tabular}{|l|l|}
$0.25529^{*}$ \\
$(0.01049)$ \\
\end{tabular} & \begin{tabular}{|l|l|}
$25637^{\circ}$ \\
$.01042)$ \\
\end{tabular} & \begin{tabular}{|l|l|}
$0.25634^{*}$ \\
$(0.01044)$ \\
\end{tabular} & \begin{tabular}{|l|l|}
$0.25624^{*}$ \\
$(0.0047)$
\end{tabular} & $\begin{array}{l}25625^{\circ} \\
.001045) \\
\end{array}$ & \begin{tabular}{|l|l|}
$\begin{array}{l}0.2563^{*} \\
(0.01045)\end{array}$ \\
\end{tabular} \\
\hline Factor 4 & & & \begin{tabular}{|l|l|}
$.28039^{*}$ \\
$(0.04872)$
\end{tabular} & $\begin{array}{l}0.28072^{*} \\
(0.04875) \\
\end{array}$ & & $\begin{array}{l}6259^{*} \\
64977)\end{array}$ & \begin{tabular}{|l|l|}
$.27819^{*}$ \\
$(0.04864)$
\end{tabular} & \begin{tabular}{|l|l|}
$0.27951^{*}$ \\
$(0.04881)$
\end{tabular} & $\begin{array}{l}27685^{*} \\
.04943)\end{array}$ & \begin{tabular}{|l|}
$0.28129^{*}$ \\
$(0.04873)$ \\
\end{tabular} & \begin{tabular}{|l|l|}
$0.27876^{*}$ \\
$(0.04866)$
\end{tabular} & \begin{tabular}{|l}
$0.28153^{*}$ \\
$(0.04873)$
\end{tabular} & \begin{tabular}{|l|}
$0.28153^{*}$ \\
$(0.04859)$ \\
\end{tabular} & \begin{tabular}{|l|}
$0.28153^{*}$ \\
$(0.0486)$
\end{tabular} & $\begin{array}{l}0.28153^{*} \\
(0.04865)\end{array}$ & \begin{tabular}{|l|}
$0.2862^{*}$ \\
$(0.04862)$ \\
\end{tabular} & & $\begin{array}{ll}0.27759^{*} \\
(0.04866)\end{array}$ & & \begin{tabular}{|l|l|}
$0.28012^{*}$ \\
$(0.04874)$
\end{tabular} & & & & \begin{tabular}{|l|l|}
$0.28153^{*}$ \\
$(0.04874)$
\end{tabular} & \begin{tabular}{|l|l}
$0.28186^{*}$ \\
$(0.04877)$
\end{tabular} \\
\hline Factor 3 & $\begin{array}{l}111{ }^{*} \\
3319)\end{array}$ & $1309^{\circ}$ & \begin{tabular}{|l|l|}
$0.31256^{*}$ \\
$(0.03305)$ \\
\end{tabular} & \begin{tabular}{|l|l|}
$0.31233^{*}$ \\
$(0.03307)$
\end{tabular} & \begin{tabular}{|l|l}
$0.31256^{*}$ \\
$(0.03298)$ \\
\end{tabular} & $\begin{array}{l}0.29925^{*} \\
(0.03324) \\
\end{array}$ & \begin{tabular}{|l}
$0.31256^{*}$ \\
$(0.03298)$ \\
\end{tabular} & \begin{tabular}{|l|l|}
$0.31256^{*}$ \\
$(0.03306)$ \\
\end{tabular} & $\begin{array}{l}0.31256^{*} \\
(0.03307) \\
\end{array}$ & \begin{tabular}{|l|}
$0.31256^{*}$ \\
$(0.03306)$ \\
\end{tabular} & \begin{tabular}{|l|l}
$\begin{array}{l}0.31144^{*} \\
(0.033)\end{array}$ \\
\end{tabular} & $\begin{array}{l}3.32602^{*} \\
0.03742) \\
\end{array}$ & \begin{tabular}{|l|}
$0.31775^{*}$ \\
$(0.03303)$ \\
\end{tabular} & \begin{tabular}{|l|l|}
$0.29978^{*}$ \\
$(0.0333)$ \\
\end{tabular} & $\begin{array}{l}0.31909^{*} \\
(0.03316) \\
\end{array}$ & \begin{tabular}{|l|}
$0.32387^{*}$ \\
$(0.03326)$ \\
\end{tabular} & \begin{tabular}{|l|l|}
$0.30385^{*}$ \\
$(0.0333)$ \\
\end{tabular} & & \begin{tabular}{|l|}
$0.31234^{*}$ \\
$(0.03307)$ \\
\end{tabular} & \begin{tabular}{|l}
$0.30678^{*}$ \\
$(0.03357)$ \\
\end{tabular} & & \begin{tabular}{|l|l}
$0.31256^{*}$ \\
$(0.03306)$ \\
\end{tabular} & $\begin{array}{l}0.31251^{*} \\
(0.03324) \\
\end{array}$ & $\begin{array}{l}0.31256^{*} \\
(0.03307) \\
\end{array}$ & \begin{tabular}{|l|l}
$0.31266^{*}$ \\
$(0.03307)$ \\
\end{tabular} \\
\hline & & $\begin{array}{l}5394^{4} \\
9992) \\
\end{array}$ & $\begin{array}{l}25394^{\dagger} \\
.09986) \\
\end{array}$ & \begin{tabular}{|l|l}
$0.25394^{\dagger}$ \\
$(0.09991)$
\end{tabular} & \begin{tabular}{|l|l}
$0.24517^{\dagger}$ \\
$(0.09973)$
\end{tabular} & $\begin{array}{l}2298^{4} \\
10006)\end{array}$ & \begin{tabular}{|l|}
$0.25394^{\dagger}$ \\
$(0.09966)$
\end{tabular} & \begin{tabular}{|l|l}
$0.24395^{\dagger}$ \\
$(0.10087)$
\end{tabular} & $\begin{array}{l}4705^{\dagger} \\
10065)\end{array}$ & \begin{tabular}{|l|l}
0.25394 \\
$(0.09991)$
\end{tabular} & \begin{tabular}{|l|l}
$0.25394^{\dagger}$ \\
$(0.09972)$
\end{tabular} & \begin{tabular}{l|l}
$0.25824^{\dagger}$ \\
$(0.10006)$
\end{tabular} & \begin{tabular}{|l|l|}
$0.28689^{\circ}$ \\
$(0.10049)$
\end{tabular} & \begin{tabular}{|l}
$0.24364^{\dagger}$ \\
$(0.09973)$
\end{tabular} & \begin{tabular}{|l|l}
$0.26875^{*}$ \\
$(0.1)$
\end{tabular} & \begin{tabular}{|l|}
$0.26697^{*}$ \\
$(0.09974)$
\end{tabular} & \begin{tabular}{|l}
$0.24761^{\dagger}$ \\
$(0.09984)$
\end{tabular} & & \begin{tabular}{|l|l}
$0.25394^{\dagger}$ \\
$(0.09992)$
\end{tabular} & $\begin{array}{l}0.25207^{\dagger} \\
(0.0999)\end{array}$ & & \begin{tabular}{|l|l}
$0.25677^{\dagger}$ \\
$(0.09994)$
\end{tabular} & & \begin{tabular}{|l|l|}
$0.25394^{\dagger}$ \\
$(0.09993)$
\end{tabular} & $\begin{array}{l}0.25394^{4} \\
(0.09993)\end{array}$ \\
\hline Factor 2 & & & $\begin{array}{l}2318^{*} \\
.02193) \\
\end{array}$ & & & & $\begin{array}{l}0.23121^{*} \\
(0.02188) \\
\end{array}$ & & & \begin{tabular}{|l|l|}
$0.2318^{*}$ \\
$(0.02194)$ \\
\end{tabular} & & & \begin{tabular}{|l|l|l|l|l|} 
\\
$(0.02187)$ \\
\end{tabular} & & & & & & & $\begin{array}{l}0.2226^{*} \\
(0.02383)\end{array}$ & & \begin{tabular}{|l|l|}
$0.23903^{*}$ \\
$(0.02331)$ \\
\end{tabular} & & $\begin{array}{l}0.23191^{*} \\
(0.02195) \\
\end{array}$ & \begin{tabular}{|l}
$\begin{array}{l}0.23239^{*} \\
(0.02212)\end{array}$ \\
\end{tabular} \\
\hline & 8379) & $\begin{array}{l}1032^{\prime} \\
8401)\end{array}$ & \begin{tabular}{|l|l}
$0.31032^{*}$ \\
$(0.08396)$
\end{tabular} & \begin{tabular}{|l|l}
$0.31032^{*}$ \\
$(0.084)$
\end{tabular} & $\begin{array}{l}0.28792^{*} \\
(0.08433)\end{array}$ & $88866^{\circ}$ & $\begin{array}{l}0.31032^{*} \\
(0.08379)\end{array}$ & $\begin{array}{l}0.30098^{*} \\
(0.08499)\end{array}$ & $\begin{array}{l}0882^{*} \\
08408)\end{array}$ & \begin{tabular}{|l|l|}
$0.30895^{*}$ \\
$(0.08402)$
\end{tabular} & $\begin{array}{l}0.31032^{*} \\
(0.08384)\end{array}$ & $\begin{array}{l}0.31032^{*} \\
(0.08399)\end{array}$ & $\begin{array}{l}0.31032^{*} \\
(0.08375) \\
\end{array}$ & $\begin{array}{l}0.31032^{*} \\
(0.08377)\end{array}$ & $\begin{array}{l}0.31032^{*} \\
(0.08384)\end{array}$ & \begin{tabular}{l|l}
$0.32564^{*}$ \\
$(0.08396)$
\end{tabular} & $\begin{array}{l}0.29979^{*} \\
(0.0841)\end{array}$ & & $\begin{array}{l}0.30652^{*} \\
(0.08426)\end{array}$ & $\begin{array}{l}0.29625^{*} \\
(0.08518)\end{array}$ & & $\begin{array}{l}0.31805^{*} \\
(0.0844)\end{array}$ & & $\begin{array}{l}0.31178^{*} \\
(0.08422)\end{array}$ & $\begin{array}{l}0.31332^{2} \\
(0.08519)\end{array}$ \\
\hline & & 2952) & \begin{tabular}{|l|l}
$0.25736^{*}$ \\
$(0.02949)$ \\
\end{tabular} & \begin{tabular}{|l|l|}
$0.25727^{*}$ \\
$(0.02951)$ \\
\end{tabular} & \begin{tabular}{|l|l|}
$0.25736^{*}$ \\
$(0.02943)$ \\
\end{tabular} & & \begin{tabular}{|l|l}
$0.2475^{*}$ \\
$(0.02974)$ \\
\end{tabular} & \begin{tabular}{|l}
$0.25736^{\circ}$ \\
$(0.0295)$
\end{tabular} & 25736" 02951$)$ & \begin{tabular}{|l}
$0.25723^{*}$ \\
$(0.0295)$ \\
\end{tabular} & 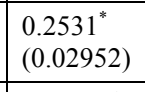 & \begin{tabular}{|l|l}
$0.27133^{*}$ \\
$(0.03466)$ \\
\end{tabular} & \begin{tabular}{|l|l|}
$0.27896^{*}$ \\
$(0.03067)$ \\
\end{tabular} & \begin{tabular}{|l|l}
$0.24221^{*}$ \\
$(0.03008)$ \\
\end{tabular} & \begin{tabular}{|l|l}
$0.27323^{*}$ \\
$(0.03048)$ \\
\end{tabular} & \begin{tabular}{|l|}
$0.27383^{*}$ \\
$(0.03012)$ \\
\end{tabular} & \begin{tabular}{|l|l|}
$0.23817^{*}$ \\
$(0.03141)$ \\
\end{tabular} & & \begin{tabular}{|l|l|}
$0.25263^{*}$ \\
$(0.03062)$ \\
\end{tabular} & $\begin{array}{l}0.24315^{*} \\
(0.03283) \\
\end{array}$ & & \begin{tabular}{|l|l}
$0.26068^{*}$ \\
$(0.02972)$ \\
\end{tabular} & \begin{tabular}{|l|l}
$0.25723^{*}$ \\
$(0.03066)$ \\
\end{tabular} & \begin{tabular}{|l|l|}
$0.25782^{*}$ \\
$(0.02957)$ \\
\end{tabular} & \begin{tabular}{|l}
$0.25836^{*}$ \\
$(0.02989)$
\end{tabular} \\
\hline Fact & $\begin{array}{l}199^{*} \\
2626) \\
\end{array}$ & $\begin{array}{ll}15321^{\circ} \\
2632)\end{array}$ & $\begin{array}{l}4048^{*} \\
2632)\end{array}$ & \begin{tabular}{|l|l}
$0.34142^{*}$ \\
$(0.02632)$
\end{tabular} & \begin{tabular}{|l|l}
$0.28896^{*}$ \\
$(0.03458)$
\end{tabular} & $\begin{array}{l}04811^{*} \\
2906)\end{array}$ & $\begin{array}{l}0.34151^{*} \\
(0.02626) \\
\end{array}$ & $\begin{array}{l}0.33612^{*} \\
(0.02736)\end{array}$ & $\begin{array}{l}4017^{*} \\
22643)\end{array}$ & \begin{tabular}{|l|}
$0.33827^{*}$ \\
$(0.02669)$ \\
\end{tabular} & \begin{tabular}{|l}
$0.32791^{*}$ \\
$(0.02708)$
\end{tabular} & $\begin{array}{l}0.34798^{*} \\
(0.02763)\end{array}$ & $\begin{array}{l}0.34247^{*} \\
(0.02625) \\
\end{array}$ & \begin{tabular}{|l|l}
$0.31778^{*}$ \\
$(0.02802)$ \\
\end{tabular} & $\begin{array}{l}0.36811^{*} \\
(0.02938)\end{array}$ & \begin{tabular}{|l|}
$0.35831^{*}$ \\
$(0.02706)$ \\
\end{tabular} & \begin{tabular}{|l|l}
$0.31983^{*}$ \\
$(0.02902)$ \\
\end{tabular} & \begin{tabular}{|l}
$0.32365^{*}$ \\
$(0.02751)$
\end{tabular} & \begin{tabular}{|l|l}
$0.3376^{*}$ \\
$(0.02718)$
\end{tabular} & \begin{tabular}{|l|l|l}
$0.32744^{*}$ \\
$(0.02993)$
\end{tabular} & \begin{tabular}{|l|l|}
$0.35009^{*}$ \\
$(0.02649)$ \\
\end{tabular} & \begin{tabular}{|l|}
$0.34969^{*}$ \\
$(0.0278)$
\end{tabular} & $\begin{array}{l}0.34129^{*} \\
(0.02942) \\
\end{array}$ & \begin{tabular}{|l|l}
$0.3433^{*}$ \\
$(0.02729)$ \\
\end{tabular} & $\begin{array}{l}0.34356^{\circ} \\
(0.02805)\end{array}$ \\
\hline & & & $\begin{array}{l}6218^{*} \\
11739) \\
\end{array}$ & \begin{tabular}{|l}
$0.26063^{*}$ \\
$(0.01912)$
\end{tabular} & \begin{tabular}{|l|l}
$0.26639^{*}$ \\
$(0.01698)$
\end{tabular} & 4987" & $\begin{array}{l}0.25641^{*} \\
(0.01752) \\
\end{array}$ & \begin{tabular}{|l|l}
$0.26639^{*}$ \\
$(0.01703)$
\end{tabular} & $\begin{array}{l}6069^{*} \\
1703) \\
\end{array}$ & \begin{tabular}{|l|}
$0.26628^{*}$ \\
$(0.01703)$ \\
\end{tabular} & \begin{tabular}{|l|l}
$0.26233^{*}$ \\
$(0.01711)$
\end{tabular} & & \begin{tabular}{|l|l}
$0.26639^{*}$ \\
$(0.01698)$
\end{tabular} & \begin{tabular}{|l}
$0.26639^{*}$ \\
$(0.01698)$ \\
\end{tabular} & & \begin{tabular}{|l|l}
$0.29072^{*}$ \\
$(0.01949)$ \\
\end{tabular} & \begin{tabular}{|l|l}
$0.25585^{*}$ \\
$(0.01802)$
\end{tabular} & & \begin{tabular}{|l|l}
$0.2649^{*}$ \\
$(0.01722)$
\end{tabular} & \begin{tabular}{|l|l}
$0.26473^{*}$ \\
$(0.01711)$
\end{tabular} & & \begin{tabular}{|l}
$0.26659^{*}$ \\
$(0.01703)$
\end{tabular} & & $\begin{array}{l}0.26642^{*} \\
(0.01703) \\
\end{array}$ & $\begin{array}{l}0.26672^{*} \\
(0.0171)\end{array}$ \\
\hline & $\begin{array}{l}5223^{*} \\
3065) \\
\end{array}$ & \begin{tabular}{|l|}
$0.12295^{*}$ \\
$(0.02892)$ \\
\end{tabular} & $\begin{array}{l}1038^{*} \\
02784) \\
\end{array}$ & \begin{tabular}{|l|l}
$0.111133^{*}$ \\
$(0.02902)$
\end{tabular} & \begin{tabular}{|l|l|}
$0.08549^{*}$ \\
$(0.0304)$
\end{tabular} & $\begin{array}{l}0.08948^{*} \\
(0.02866) \\
\end{array}$ & \begin{tabular}{|l|l}
$0.11062^{*}$ \\
$(0.02716)$
\end{tabular} & 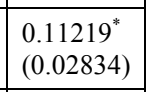 & $\begin{array}{l}0.11485^{*} \\
(0.02771)\end{array}$ & \begin{tabular}{|l|}
$0.11835^{*}$ \\
$(0.02702)$ \\
\end{tabular} & \begin{tabular}{|l|l}
$0.10541^{*}$ \\
$(0.02769)$
\end{tabular} & $\begin{array}{l}0.11835^{*} \\
(0.02702) \\
\end{array}$ & \begin{tabular}{|l|}
$0.11835^{*}$ \\
$(0.02694)$ \\
\end{tabular} & \begin{tabular}{|l|l}
$0.11835^{*}$ \\
$(0.02695)$
\end{tabular} & $\begin{array}{l}0.11833^{*} \\
(0.02697)\end{array}$ & \begin{tabular}{|l|l|}
$0.13599^{*}$ \\
$(0.02782)$ \\
\end{tabular} & \begin{tabular}{|l|l}
$0.11239^{*}$ \\
$(0.02719)$
\end{tabular} & \begin{tabular}{|l|l}
$0.11657^{*}$ \\
$(0.02697)$
\end{tabular} & \begin{tabular}{|l|l}
$0.11684^{*}$ \\
$(0.02715)$
\end{tabular} & 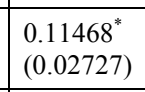 & & \begin{tabular}{|l|l|}
$0.11887^{*}$ \\
$(0.02702)$
\end{tabular} & $\begin{array}{l}0.11833^{*} \\
(0.02705) \\
\end{array}$ & $\begin{array}{l}0.11883^{*} \\
(0.02709) \\
\end{array}$ & \begin{tabular}{|l|l}
$0.1184^{*}$ \\
$(0.02703)$
\end{tabular} \\
\hline & & & & \begin{tabular}{|l|l|l}
$0.21675^{*}$ \\
$(0.03197)$
\end{tabular} & \begin{tabular}{|l|l}
$0.22181^{*}$ \\
$(0.03096)$
\end{tabular} & \begin{tabular}{|l|l|}
$0.204^{*}$ \\
$(0.03151)$
\end{tabular} & \begin{tabular}{|l|l|}
$0.22008^{*}$ \\
$(0.03097)$
\end{tabular} & \begin{tabular}{|l|l|}
$0.22181^{*}$ \\
$(0.03104)$
\end{tabular} & $\begin{array}{l}0.22181^{*} \\
(0.03104)\end{array}$ & \begin{tabular}{|l|l|}
$0.22181^{*}$ \\
$(0.03104)$
\end{tabular} & \begin{tabular}{|l|l|l}
$0.2132^{*}$ \\
$(0.03126)$
\end{tabular} & & $\begin{array}{l}0.22433^{*} \\
(0.03097) \\
\end{array}$ & \begin{tabular}{|l|l}
$0.21425^{*}$ \\
$(0.03111)$
\end{tabular} & & & \begin{tabular}{|l|l}
$0.20943^{*}$ \\
$(0.03178)$
\end{tabular} & & & \begin{tabular}{|l|l}
$0.2167^{*}$ \\
$(0.03146)$
\end{tabular} & & & & & \\
\hline & \begin{tabular}{|l}
$0.29434^{*}$ \\
$(0.08091)$ \\
\end{tabular} & \begin{tabular}{|l|}
$0.25991^{*}$ \\
$(0.07987)$ \\
\end{tabular} & \begin{tabular}{|l}
$0.24726^{*}$ \\
$(0.07975)$ \\
\end{tabular} & \begin{tabular}{|l|l|}
$.24892^{*}$ \\
$(0.08019)$ \\
\end{tabular} & \begin{tabular}{|l}
$0.20911^{\dagger}$ \\
$(0.08176)$ \\
\end{tabular} & $\begin{array}{l}0.21544^{*} \\
(0.08032) \\
\end{array}$ & \begin{tabular}{|l}
$0.24334^{*}$ \\
$(0.07943)$ \\
\end{tabular} & \begin{tabular}{|l}
$0.25349^{*}$ \\
$(0.07952)$ \\
\end{tabular} & $\begin{array}{l}0.25317^{*} \\
(0.07962) \\
\end{array}$ & \begin{tabular}{|l|}
$0.25623^{*}$ \\
$(0.07942)$ \\
\end{tabular} & \begin{tabular}{|l}
$0.25473^{*}$ \\
$(0.07928)$ \\
\end{tabular} & $\begin{array}{l}0.26714^{*} \\
(0.08068)\end{array}$ & \begin{tabular}{|l|}
$0.28239^{*}$ \\
$(0.07989)$ \\
\end{tabular} & \begin{tabular}{|l}
$0.24903^{*}$ \\
$(0.07927)$ \\
\end{tabular} & $\begin{array}{l}0.26635^{*} \\
(0.07944) \\
\end{array}$ & \begin{tabular}{|l|}
$0.27607^{*}$ \\
$(0.07957)$ \\
\end{tabular} & \begin{tabular}{|l|l}
$0.242 *^{*}$ \\
$(0.07972)$ \\
\end{tabular} & \begin{tabular}{|l|l}
$0.25048^{*}$ \\
$(0.0793)$ \\
\end{tabular} & \begin{tabular}{|l|}
$0.25558^{*}$ \\
$(0.07944)$ \\
\end{tabular} & \begin{tabular}{|l|l|}
$.24989^{*}$ \\
$(0.07967)$
\end{tabular} & \begin{tabular}{|l|}
$0.26228^{*}$ \\
$(0.07925)$ \\
\end{tabular} & \begin{tabular}{|l|l}
$0.25623^{*}$ \\
$(0.07941)$
\end{tabular} & $\begin{array}{l}0.25622^{*} \\
(0.07945) \\
\end{array}$ & $\begin{array}{l}0.25649^{*} \\
(0.07945) \\
\end{array}$ & \begin{tabular}{|l}
$0.25622^{*}$ \\
$(0.07944)$
\end{tabular} \\
\hline Factor $1 \& 2$ & $\begin{array}{l}6212^{*} \\
02079) \\
\end{array}$ & \begin{tabular}{|l|}
$0.23145^{*}$ \\
$(0.01711)$ \\
\end{tabular} & \begin{tabular}{|l|l}
$0.22501^{*}$ \\
$(0.01529)$
\end{tabular} & \begin{tabular}{|l|l|}
$.22025^{*}$ \\
$(0.01897)$
\end{tabular} & \begin{tabular}{|l|l|}
$0.22787^{*}$ \\
$(0.01506)$
\end{tabular} & $\begin{array}{l}57^{\circ} \\
1862) \\
\end{array}$ & \begin{tabular}{|l|l|}
$0.22149^{*}$ \\
$(0.01531)$
\end{tabular} & \begin{tabular}{|l}
$0.22787^{\circ}$ \\
$(0.0151)$
\end{tabular} & $\begin{array}{l}0.22787^{\circ} \\
(0.0151)\end{array}$ & \begin{tabular}{|l|}
$0.22598^{*}$ \\
$(0.01532)$ \\
\end{tabular} & \begin{tabular}{|l|l}
$0.21946^{*}$ \\
$(0.01561)$
\end{tabular} & \begin{tabular}{|l|}
$0.22787^{\circ}$ \\
$(0.0151)$
\end{tabular} & \begin{tabular}{|l|}
$0.22787^{*}$ \\
$(0.01506)$
\end{tabular} & \begin{tabular}{|l|l|}
$0.22787^{*}$ \\
$(0.01506)$
\end{tabular} & & \begin{tabular}{|l|l|}
$0.25701^{*}$ \\
$(0.01893)$
\end{tabular} & & & & \begin{tabular}{|l|l|}
$0.21571^{*}$ \\
$(0.01948)$
\end{tabular} & & & & & \\
\hline & \begin{tabular}{|l}
$0.29997^{*}$ \\
$(0.03398)$ \\
\end{tabular} & \begin{tabular}{|l|l}
$0.26802^{*}$ \\
$(0.03124)$ \\
\end{tabular} & \begin{tabular}{|l|l}
$0.25983^{*}$ \\
$(0.03087)$ \\
\end{tabular} & \begin{tabular}{|l}
$0.2566^{*}$ \\
$(0.03303)$ \\
\end{tabular} & \begin{tabular}{|l|l|}
$0.21219^{*}$ \\
$(0.03798)$ \\
\end{tabular} & \begin{tabular}{|l|l|}
$0.22872^{*}$ \\
$(0.03287)$ \\
\end{tabular} & \begin{tabular}{|l|l}
$0.25218^{*}$ \\
$(0.0309)$
\end{tabular} & \begin{tabular}{|l|l}
$\begin{array}{l}0.2623^{\circ} \\
(0.0308)\end{array}$ \\
\end{tabular} & $\begin{array}{l}0.26191^{*} \\
(0.03107)\end{array}$ & \begin{tabular}{|l}
$\begin{array}{l}0.25600^{*} \\
(0.033)\end{array}$ \\
\end{tabular} & \begin{tabular}{|l|l|}
$0.25109^{*}$ \\
$(0.03124)$ \\
\end{tabular} & $\begin{array}{l}0.26512^{*} \\
(0.03055) \\
\end{array}$ & \begin{tabular}{|l|}
$0.26512^{*}$ \\
$(0.03046)$ \\
\end{tabular} & \begin{tabular}{|l|l|}
$0.26512^{*}$ \\
$(0.03047)$ \\
\end{tabular} & \begin{tabular}{|l|l}
$0.26511^{*}$ \\
$(0.0305)$ \\
\end{tabular} & \begin{tabular}{|l|l|}
$0.29477^{*}$ \\
$(0.03262)$ \\
\end{tabular} & \begin{tabular}{|l|l|}
$.24689^{*}$ \\
$(0.03221)$ \\
\end{tabular} & \begin{tabular}{|l|l}
$0.24112^{*}$ \\
$(0.03242)$ \\
\end{tabular} & \begin{tabular}{|l|l}
$0.25896^{*}$ \\
$(0.03236)$ \\
\end{tabular} & $\begin{array}{l}0.25395^{*} \\
(0.03258) \\
\end{array}$ & \begin{tabular}{|l|}
$0.28574^{*}$ \\
$(0.03164)$ \\
\end{tabular} & \begin{tabular}{|l|l|}
$.27299^{*}$ \\
$(0.03174)$ \\
\end{tabular} & \begin{tabular}{|l|l}
$0.265^{*}$ \\
$(0.03134)$ \\
\end{tabular} & \begin{tabular}{|l}
$0.26661^{*}$ \\
$(0.03114)$ \\
\end{tabular} & \begin{tabular}{|l|l}
$0.26644^{*}$ \\
$(0.0312)$ \\
\end{tabular} \\
\hline Factor $1,2 \& 3$ & & \begin{tabular}{|l|}
$0.25306^{*}$ \\
$(0.02109)$ \\
\end{tabular} & \begin{tabular}{|l}
$0.24063^{*}$ \\
$(0.01988)$
\end{tabular} & \begin{tabular}{|l|l|}
$0.24193^{*}$ \\
$(0.02127)$
\end{tabular} & \begin{tabular}{|l|l|}
$0.24869^{*}$ \\
$(0.01862)$
\end{tabular} & $\begin{array}{l}0.21853^{*} \\
(0.02126)\end{array}$ & \begin{tabular}{|l|l}
$0.24069^{*}$ \\
$(0.01894)$
\end{tabular} & \begin{tabular}{|l|l|}
$0.24869^{*}$ \\
$(0.01867)$
\end{tabular} & $\begin{array}{l}0.24869^{*} \\
(0.01867)\end{array}$ & \begin{tabular}{|l|}
$0.24767^{*}$ \\
$(0.01872)$ \\
\end{tabular} & \begin{tabular}{|l|l|}
$0.23982^{*}$ \\
$(0.01912)$
\end{tabular} & & \begin{tabular}{|l|l|}
$0.26429^{*}$ \\
$(0.01964)$ \\
\end{tabular} & \begin{tabular}{|l|l|}
$.22427^{*}$ \\
$(0.02117)$
\end{tabular} & & \begin{tabular}{|l|}
$0.27824^{*}$ \\
$(0.02195)$ \\
\end{tabular} & & & & $\begin{array}{l}0.23736^{*} \\
(0.02191)\end{array}$ & & & & & \\
\hline & \begin{tabular}{|l}
$0.34167^{*}$ \\
$(0.02217)$ \\
\end{tabular} & \begin{tabular}{|l|}
$0.31443^{*}$ \\
$(0.02028)$ \\
\end{tabular} & \begin{tabular}{|l}
$0.29936^{*}$ \\
$(0.01959)$ \\
\end{tabular} & \begin{tabular}{|l|l}
$0.30201^{*}$ \\
$(0.02108)$
\end{tabular} & \begin{tabular}{|l|l}
$0.27219^{*}$ \\
$(0.02373)$ \\
\end{tabular} & $\begin{array}{l}0.27027^{*} \\
(0.02202) \\
\end{array}$ & $\begin{array}{l}0.29955^{*} \\
(0.01798) \\
\end{array}$ & \begin{tabular}{|l|l}
$0.30067^{*}$ \\
$(0.02161)$
\end{tabular} & $\begin{array}{l}0.30257^{*} \\
(0.02163) \\
\end{array}$ & \begin{tabular}{|l|}
$0.30795^{*}$ \\
$(0.01765)$ \\
\end{tabular} & \begin{tabular}{|l|l}
$0.29694^{*}$ \\
$(0.01851)$
\end{tabular} & $\begin{array}{l}0.32112^{*} \\
(0.02282) \\
\end{array}$ & \begin{tabular}{|l|}
$0.31608^{*}$ \\
$(0.01759)$ \\
\end{tabular} & 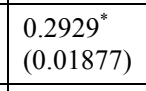 & $\begin{array}{l}0.33568^{*} \\
(0.02162) \\
\end{array}$ & \begin{tabular}{|l}
$\begin{array}{l}0.33822^{*} \\
(0.0207)\end{array}$ \\
\end{tabular} & \begin{tabular}{|l|l}
$0.28988^{*}$ \\
$(0.02078)$ \\
\end{tabular} & \begin{tabular}{|l|l|}
$0.28854^{*}$ \\
$(0.01998)$ \\
\end{tabular} & \begin{tabular}{|l|}
$0.30391^{*}$ \\
$(0.02025)$ \\
\end{tabular} & $\begin{array}{l}0.29767^{*} \\
(0.02137) \\
\end{array}$ & \begin{tabular}{|l|}
$0.33286^{*}$ \\
$(0.01984)$ \\
\end{tabular} & \begin{tabular}{|l|l|l}
$0.31759^{*}$ \\
$(0.01941)$ \\
\end{tabular} & $\begin{array}{l}0.30965^{*} \\
(0.02082) \\
\end{array}$ & $\begin{array}{l}0.31065^{*} \\
(0.01777) \\
\end{array}$ & \begin{tabular}{|l}
$0.31134^{*}$ \\
$(0.01886)$ \\
\end{tabular} \\
\hline Individual Variable & $\begin{array}{l}-0.0381^{\dagger} \\
(0.01642) \\
\end{array}$ & \begin{tabular}{|l|}
-0.00647 \\
$(0.01453)$ \\
\end{tabular} & \begin{tabular}{|l|l|}
0.01963 \\
$(0.01666)$ \\
\end{tabular} & \begin{tabular}{|l|l}
0.0104 \\
$(0.01568)$ \\
\end{tabular} & \begin{tabular}{|l|l|}
$0.05968^{\dagger}$ \\
$(0.02555)$ \\
\end{tabular} & \begin{tabular}{|l|l}
$0.042 *^{*}$ \\
$(0.01438)$
\end{tabular} & \begin{tabular}{|l|l}
$0.041466^{\dagger}$ \\
$(0.01789)$
\end{tabular} & \begin{tabular}{|l|}
0.01743 \\
$(0.0242)$ \\
\end{tabular} & \begin{tabular}{|l|l}
0.01452 \\
$(0.02552)$ \\
\end{tabular} & \begin{tabular}{|l|}
0.022044 \\
$(0.028)$
\end{tabular} & $\begin{array}{l}03211^{\dagger} \\
0.01558) \\
\end{array}$ & \begin{tabular}{|l}
-0.01632 \\
$(0.02125)$
\end{tabular} & \begin{tabular}{|c}
$-0.0733^{\dagger}$ \\
$(0.0294)$ \\
\end{tabular} & \begin{tabular}{|l|l|}
$0.04918^{\dagger}$ \\
$(0.02031)$
\end{tabular} & \begin{tabular}{|l}
$-0.0415^{\dagger}$ \\
$(0.02053)$
\end{tabular} & \begin{tabular}{|c|c}
$-0.0335^{\dagger}$ \\
$(0.01321)$ \\
\end{tabular} & \begin{tabular}{|l|l}
$0.02402^{\ddagger}$ \\
$(0.01361)$
\end{tabular} & \begin{tabular}{|l}
$0.0287^{\dagger}$ \\
$(0.01318)$
\end{tabular} & & \begin{tabular}{|l}
$\begin{array}{l}0.01509 \\
(0.01529)\end{array}$ \\
\end{tabular} & & \begin{tabular}{|l|l}
-0.01453 \\
$(0.01589)$
\end{tabular} & & & \\
\hline
\end{tabular}

Table 86 Coefficients with individual variables for AMT - Medium Plants 


\begin{tabular}{|c|c|c|c|c|c|c|c|c|c|c|c|c|}
\hline $\begin{array}{l}\text { Individual variable } \\
\text { included in the } \\
\text { model in the column }\end{array}$ & 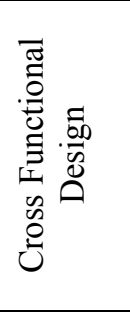 & 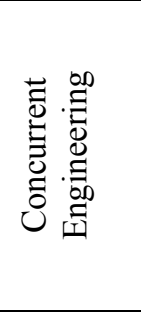 & 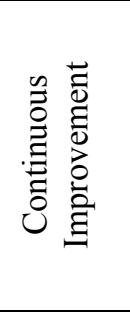 & 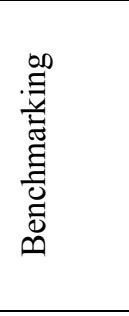 & 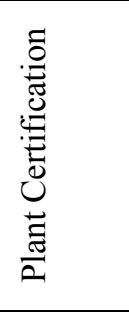 & 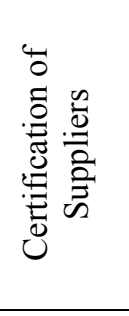 & 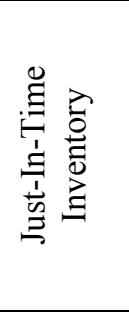 & 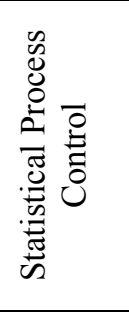 & 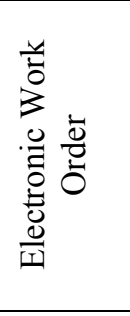 & 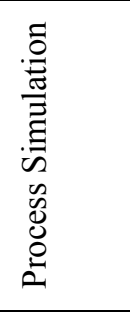 & 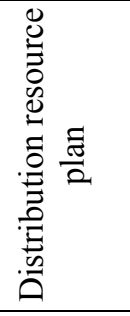 & 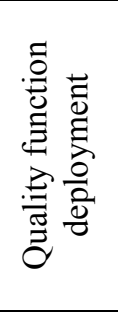 \\
\hline No factors & $\begin{array}{l}0.22287^{*} \\
(0.00972) \\
\end{array}$ & $\begin{array}{l}0.22373^{*} \\
(0.00972)\end{array}$ & $\begin{array}{l}0.22194^{*} \\
(0.01006)\end{array}$ & $\begin{array}{l}0.22446^{*} \\
(0.00975)\end{array}$ & $\begin{array}{l}0.22609^{*} \\
(0.00992)\end{array}$ & $\begin{array}{l}0.22033^{*} \\
(0.01037)\end{array}$ & $\begin{array}{l}0.23137^{*} \\
(0.01061)\end{array}$ & $\begin{array}{l}0.22484^{*} \\
(0.00965)\end{array}$ & $\begin{array}{l}0.22404^{*} \\
(0.00972)\end{array}$ & $\begin{array}{l}0.22321^{*} \\
(0.00967)\end{array}$ & $\begin{array}{l}0.22375^{*} \\
(0.00969)\end{array}$ & $\begin{array}{l}0.22417^{*} \\
(0.00972)\end{array}$ \\
\hline Factor 3 & $\begin{array}{l}0.24655^{*} \\
(0.02493)\end{array}$ & $\begin{array}{l}0.26185^{*} \\
(0.02386)\end{array}$ & $\begin{array}{l}0.25269^{*} \\
(0.02509)\end{array}$ & $\begin{array}{l}0.26467^{*} \\
(0.02107)\end{array}$ & $\begin{array}{l}0.26571^{*} \\
(0.02097)\end{array}$ & $\begin{array}{l}0.26053^{*} \\
(0.02078)\end{array}$ & $\begin{array}{l}0.26477^{*} \\
(0.02075)\end{array}$ & $\begin{array}{l}0.26343^{*} \\
(0.02063)\end{array}$ & $\begin{array}{l}0.26396^{*} \\
(0.02118)\end{array}$ & $\begin{array}{l}0.26114^{*} \\
(0.02067)\end{array}$ & $\begin{array}{l}0.26209^{*} \\
(0.02072)\end{array}$ & $\begin{array}{l}0.2628^{*} \\
(0.02076)\end{array}$ \\
\hline Factor 2 & $\begin{array}{l}0.19053^{*} \\
(0.01994)\end{array}$ & $\begin{array}{l}0.19898^{*} \\
(0.0185)\end{array}$ & $\begin{array}{l}0.19699^{*} \\
(0.01871)\end{array}$ & $\begin{array}{l}0.20402^{*} \\
(0.01991)\end{array}$ & $\begin{array}{l}0.20238^{*} \\
(0.01871)\end{array}$ & $\begin{array}{l}0.19766^{*} \\
(0.01851)\end{array}$ & $\begin{array}{l}0.21803^{*} \\
(0.02139)\end{array}$ & $\begin{array}{l}0.20373^{*} \\
(0.01844)\end{array}$ & $\begin{array}{l}0.2038^{*} \\
(0.02165)\end{array}$ & $\begin{array}{l}0.19255^{*} \\
(0.01864)\end{array}$ & $\begin{array}{l}0.1993^{*} \\
(0.01865)\end{array}$ & $\begin{array}{l}0.20236^{*} \\
(0.01937)\end{array}$ \\
\hline Factor $2 \& 3$ & $\begin{array}{l}0.23095^{*} \\
(0.03107)\end{array}$ & $\begin{array}{l}0.24805^{*} \\
(0.02879)\end{array}$ & $\begin{array}{l}0.23776^{*} \\
(0.03126)\end{array}$ & $\begin{array}{l}0.2518^{*} \\
(0.02747)\end{array}$ & $\begin{array}{l}0.25131^{*} \\
(0.0271)\end{array}$ & $\begin{array}{l}0.24645^{*} \\
(0.02703)\end{array}$ & $\begin{array}{l}0.26605^{*} \\
(0.02877)\end{array}$ & $\begin{array}{l}0.24991^{*} \\
(0.02685)\end{array}$ & $\begin{array}{l}0.2516^{*} \\
(0.02809)\end{array}$ & $\begin{array}{l}0.242^{*} \\
(0.02705)\end{array}$ & $\begin{array}{l}0.249^{*} \\
(0.02772)\end{array}$ & $\begin{array}{l}0.25555^{*} \\
(0.02981)\end{array}$ \\
\hline Factor 1 & $\begin{array}{l}0.30377^{*} \\
(0.0154)\end{array}$ & $\begin{array}{l}0.30383^{*} \\
(0.01541)\end{array}$ & $\begin{array}{l}0.29393^{*} \\
(0.02145)\end{array}$ & $\begin{array}{l}0.3112^{*} \\
(0.01889)\end{array}$ & $\begin{array}{l}0.31955^{*} \\
(0.02108)\end{array}$ & $\begin{array}{l}0.29084^{*} \\
(0.02089)\end{array}$ & $\begin{array}{l}0.30811^{*} \\
(0.01558)\end{array}$ & $\begin{array}{l}0.32159^{*} \\
(0.01642)\end{array}$ & $\begin{array}{l}0.30504^{*} \\
(0.01567)\end{array}$ & $\begin{array}{l}0.30362^{*} \\
(0.01537)\end{array}$ & $\begin{array}{l}0.30383^{*} \\
(0.01541)\end{array}$ & $\begin{array}{l}0.30427^{*} \\
(0.01543)\end{array}$ \\
\hline Factor $1 \& 2$ & $\begin{array}{l}0.29896^{*} \\
(0.02278) \\
\end{array}$ & $\begin{array}{l}0.29904^{*} \\
(0.02279) \\
\end{array}$ & $\begin{array}{l}0.28873^{*} \\
(0.02758) \\
\end{array}$ & $\begin{array}{l}0.30637^{*} \\
(0.02525) \\
\end{array}$ & $\begin{array}{l}0.31418^{*} \\
(0.02666) \\
\end{array}$ & $\begin{array}{l}0.28664^{*} \\
(0.02647)\end{array}$ & $\begin{array}{l}0.31773^{*} \\
(0.02512) \\
\end{array}$ & $\begin{array}{l}0.32295^{*} \\
(0.02402) \\
\end{array}$ & $\begin{array}{l}0.30281^{*} \\
(0.02445) \\
\end{array}$ & $\begin{array}{l}0.28996^{*} \\
(0.02309) \\
\end{array}$ & $\begin{array}{l}0.29939^{*} \\
(0.02298) \\
\end{array}$ & $\begin{array}{l}0.30587^{*} \\
(0.0257) \\
\end{array}$ \\
\hline All Factors & $\begin{array}{l}0.25561^{*} \\
(0.01989) \\
\end{array}$ & $\begin{array}{l}0.2721^{*} \\
(0.01793) \\
\end{array}$ & $\begin{array}{l}0.26065^{*} \\
(0.02198) \\
\end{array}$ & $\begin{array}{l}0.2806^{*} \\
(0.01796) \\
\end{array}$ & $\begin{array}{l}0.2875^{*} \\
(0.0191) \\
\end{array}$ & $\begin{array}{l}0.25962^{*} \\
(0.01906)\end{array}$ & $\begin{array}{l}0.29202^{*} \\
(0.01725) \\
\end{array}$ & $\begin{array}{l}0.30492^{*} \\
(0.01693) \\
\end{array}$ & $\begin{array}{l}0.2774^{*} \\
(0.01772) \\
\end{array}$ & $\begin{array}{l}0.25176^{*} \\
(0.01602)\end{array}$ & $\begin{array}{l}0.27341^{*} \\
(0.01601) \\
\end{array}$ & $\begin{array}{l}0.2789^{*} \\
(0.0174) \\
\end{array}$ \\
\hline Individual Variable & $\begin{array}{l}0.01961 \\
(0.01752)\end{array}$ & $\begin{array}{l}0.0003437 \\
(0.01706)\end{array}$ & $\begin{array}{l}0.01179 \\
(0.01777)\end{array}$ & $\begin{array}{l}-0.00977 \\
(0.01451)\end{array}$ & $\begin{array}{l}-0.01714 \\
(0.0157)\end{array}$ & $\begin{array}{l}0.01422 \\
(0.01546)\end{array}$ & $\begin{array}{l}-0.02339^{*} \\
(0.01333)\end{array}$ & $\begin{array}{l}-0.0466^{*} \\
(0.01539)\end{array}$ & $\begin{array}{l}-0.00634 \\
(0.01493)\end{array}$ & $\begin{array}{l}0.04413^{\dagger} \\
(0.01976)\end{array}$ & $\begin{array}{l}-0.00242 \\
(0.02076)\end{array}$ & $\begin{array}{c}-0.00968 \\
(0.01688)\end{array}$ \\
\hline
\end{tabular}

* Significant at the 0.01 level $†$ Significant at the 0.05 level $\$$ Significant at the 0.10 level

Table 87 Coefficients with individual variables for business practices - Medium Plants 


\begin{tabular}{|c|c|c|c|c|c|c|c|c|c|c|c|c|c|c|c|c|c|c|}
\hline $\begin{array}{l}\text { Individual variable } \\
\text { included in the model } \\
\text { in the column }\end{array}$ & 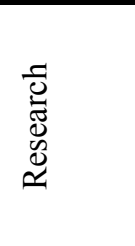 & 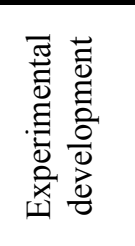 & 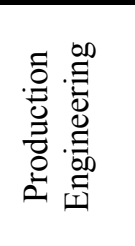 & 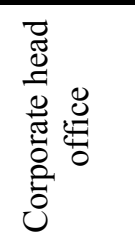 & $\begin{array}{l}\frac{n}{0} \\
\frac{\pi}{2} \\
0 \\
0 \\
\frac{0}{0} \\
\frac{\pi}{0} \\
\simeq\end{array}$ & 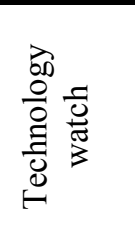 & 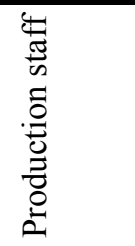 & 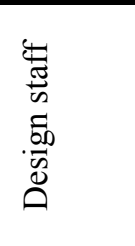 & 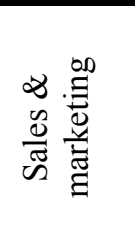 & 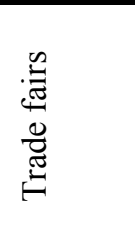 & 莺 & 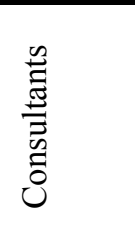 & 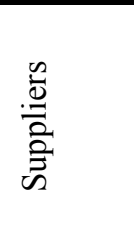 & 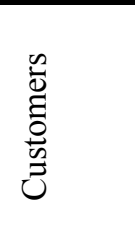 & 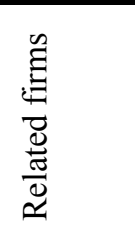 & 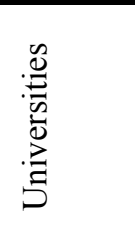 & 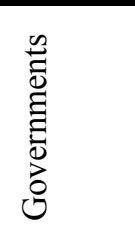 & 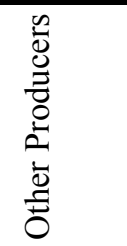 \\
\hline No factors & $\begin{array}{l}0.23887^{*} \\
(0.01124)\end{array}$ & $\begin{array}{l}0.24428^{\prime \prime} \\
(0.01097)\end{array}$ & $\begin{array}{l}0.23922^{*} \\
(0.01117)\end{array}$ & $\begin{array}{l}0.24478^{*} \\
(0.01108)\end{array}$ & $\begin{array}{l}0.24015^{*} \\
(0.01098)\end{array}$ & $\begin{array}{l}0.24067^{*} \\
(0.01095)\end{array}$ & $\begin{array}{l}0.23408^{*} \\
(0.012)\end{array}$ & $\begin{array}{l}0.24501^{*} \\
(0.0113)\end{array}$ & $\begin{array}{l}0.23905^{*} \\
(0.0111)\end{array}$ & $\begin{array}{l}0.23868^{*} \\
(0.01093)\end{array}$ & $\begin{array}{l}0.23898^{*} \\
(0.01203)\end{array}$ & $\begin{array}{l}0.24037^{*} \\
(0.01088)\end{array}$ & $\begin{array}{l}0.24122^{*} \\
(0.01108)\end{array}$ & $\begin{array}{l}0.22792^{*} \\
(0.01139)\end{array}$ & $\begin{array}{l}0.24372^{*} \\
(0.01132)\end{array}$ & $\begin{array}{l}0.24003^{*} \\
(0.01094)\end{array}$ & $\begin{array}{l}0.23965^{*} \\
(0.01084)\end{array}$ & $\begin{array}{l}0.24057^{*} \\
(0.01095)\end{array}$ \\
\hline Factor 4 & $\begin{array}{l}0.22993^{*} \\
(0.02664)\end{array}$ & $\begin{array}{l}0.24948^{*} \\
(0.02642)\end{array}$ & $\begin{array}{l}0.23162^{\prime \prime} \\
(0.02623)\end{array}$ & $\begin{array}{l}0.26044^{\prime} \\
(0.02857)\end{array}$ & $\begin{array}{l}0.22547^{7} \\
(0.03119)\end{array}$ & $\begin{array}{l}0.23419^{*} \\
(0.02603)\end{array}$ & $\begin{array}{l}0.22177^{*} \\
(0.02753)\end{array}$ & $\begin{array}{l}0.24312^{*} \\
(0.02663)\end{array}$ & $\begin{array}{l}0.23244^{*} \\
(0.02603)\end{array}$ & $\begin{array}{l}0.23061^{*} \\
(0.02592)\end{array}$ & $\begin{array}{l}0.23122^{*} \\
(0.02723)\end{array}$ & $\begin{array}{l}0.23385^{\circ} \\
(0.02584)\end{array}$ & $\begin{array}{l}0.23546^{7} \\
(0.02637)\end{array}$ & $\begin{array}{l}0.20672^{2} \\
(0.02682)\end{array}$ & $\begin{array}{l}0.24066^{*} \\
(0.02674)\end{array}$ & $\begin{array}{l}0.22295^{\circ} \\
(0.02743)\end{array}$ & $\begin{array}{l}0.23295^{\circ} \\
(0.02572)\end{array}$ & $\begin{array}{l}0.23384^{*} \\
(0.02598)\end{array}$ \\
\hline Fact & $\begin{array}{l}0.18266^{*} \\
(0.0432)\end{array}$ & $\begin{array}{l}0.19093^{*} \\
(0.04283)\end{array}$ & $\begin{array}{l}0.18437^{*} \\
(0.04302)\end{array}$ & $\begin{array}{l}0.19536^{*} \\
(0.0431)\end{array}$ & $\begin{array}{l}0.18508^{*} \\
(0.04299)\end{array}$ & $\begin{array}{l}0.18563^{*} \\
(0.04298)\end{array}$ & $\begin{array}{l}0.18041^{*} \\
(0.04312)\end{array}$ & $\begin{array}{l}0.18796^{*} \\
(0.04295)\end{array}$ & $\begin{array}{l}0.17774^{*} \\
(0.04403)\end{array}$ & $\begin{array}{l}0.18563^{*} \\
(0.04282)\end{array}$ & $\begin{array}{l}0.1825^{*} \\
(0.04405)\end{array}$ & $\begin{array}{l}0.18563^{*} \\
(0.04274)\end{array}$ & $\begin{array}{l}0.18734^{*} \\
(0.04324)\end{array}$ & $\begin{array}{l}0.12159^{*} \\
(0.04604)\end{array}$ & $\begin{array}{l}0.20484^{*} \\
(0.04656)\end{array}$ & $\begin{array}{l}0.1752^{*} \\
(0.04377)\end{array}$ & $\begin{array}{l}0.18563^{*} \\
(0.04255)\end{array}$ & $\begin{array}{l}0.1856^{*} \\
(0.04298) \\
\end{array}$ \\
\hline Fact & $\begin{array}{l}0.28385^{*} \\
(0.01922)\end{array}$ & $\begin{array}{l}0.30648^{*} \\
(0.01903)\end{array}$ & $\begin{array}{l}0.2874^{*} \\
(0.0181)\end{array}$ & $\begin{array}{l}0.31817^{*} \\
(0.02251)\end{array}$ & $\begin{array}{l}0.28355^{*} \\
(0.02048)\end{array}$ & $\begin{array}{l}0.28829^{*} \\
(0.01805)\end{array}$ & $\begin{array}{l}0.27827^{*} \\
(0.01955)\end{array}$ & $\begin{array}{l}0.28867^{*} \\
(0.01803)\end{array}$ & $\begin{array}{l}0.28261^{*} \\
(0.01931)\end{array}$ & $\begin{array}{l}0.28826^{*} \\
(0.01798)\end{array}$ & $\begin{array}{l}0.28533^{*} \\
(0.02018)\end{array}$ & $\begin{array}{l}0.28805^{*} \\
(0.01795)\end{array}$ & $\begin{array}{l}0.28902^{*} \\
(0.01817)\end{array}$ & $\begin{array}{l}0.22469^{*} \\
(0.02479)\end{array}$ & $\begin{array}{l}0.30746^{*} \\
(0.02546)\end{array}$ & $\begin{array}{l}0.26711^{*} \\
(0.02489)\end{array}$ & $\begin{array}{l}0.28826^{*} \\
(0.01787)\end{array}$ & $\begin{array}{l}0.28824^{*} \\
(0.01805)\end{array}$ \\
\hline Factor 2 & $\begin{array}{l}0.25328^{*} \\
(0.03727)\end{array}$ & $\begin{array}{l}0.3058^{*} \\
(0.03795)\end{array}$ & $\begin{array}{l}0.25257^{*} \\
(0.03813)\end{array}$ & $\begin{array}{l}0.26905^{*} \\
(0.03504)\end{array}$ & $\begin{array}{l}0.25959^{*} \\
(0.03529)\end{array}$ & $\begin{array}{l}0.26192^{*} \\
(0.03498)\end{array}$ & $\begin{array}{l}0.25012^{*} \\
(0.03606)\end{array}$ & $\begin{array}{l}0.27607^{*} \\
(0.03612)\end{array}$ & $\begin{array}{l}0.259^{\circ} \\
(0.03514)\end{array}$ & $\begin{array}{l}0.2603^{*} \\
(0.03485)\end{array}$ & $\begin{array}{l}0.25798^{*} \\
(0.03698)\end{array}$ & $\begin{array}{l}0.23932^{*} \\
(0.03545)\end{array}$ & $\begin{array}{l}0.26256^{*} \\
(0.03503)\end{array}$ & $\begin{array}{l}0.24262^{*} \\
(0.03512)\end{array}$ & $\begin{array}{l}0.27105^{*} \\
(0.03599)\end{array}$ & $\begin{array}{l}0.25799^{*} \\
(0.03509)\end{array}$ & $\begin{array}{l}0.24339^{*} \\
(0.03487)\end{array}$ & $\begin{array}{l}0.26163^{*} \\
(0.03509)\end{array}$ \\
\hline Factor $2 \& 4$ & $\begin{array}{l}0.36901^{*} \\
(0.0411)\end{array}$ & $\begin{array}{l}0.4157^{*} \\
(0.0409)\end{array}$ & $\begin{array}{l}0.36834^{*} \\
(0.04188)\end{array}$ & $\begin{array}{l}0.39887^{\prime \prime} \\
(0.03999)\end{array}$ & $\begin{array}{l}0.36994^{*} \\
(0.04216)\end{array}$ & $\begin{array}{l}0.3785^{*} \\
(0.03908)\end{array}$ & $\begin{array}{l}0.36513^{*} \\
(0.04007)\end{array}$ & $\begin{array}{l}0.38818^{*} \\
(0.03946)\end{array}$ & $\begin{array}{l}0.37579^{*} \\
(0.039)\end{array}$ & $\begin{array}{l}0.37416^{*} \\
(0.03882)\end{array}$ & & $\begin{array}{l}0.35822^{*} \\
(0.03917)\end{array}$ & $\begin{array}{l}0.37901^{*} \\
(0.03908) \\
\end{array}$ & $\begin{array}{l}0.35645^{*} \\
(0.03909)\end{array}$ & $\begin{array}{l}0.38811^{*} \\
(0.04009)\end{array}$ & $\begin{array}{l}0.36585^{*} \\
(0.04009)\end{array}$ & $\begin{array}{l}0.36393^{*} \\
(0.03867)\end{array}$ & $\begin{array}{l}0.37754^{4} \\
(0.03905)\end{array}$ \\
\hline Factor $2 \& 3$ & & & & & & & & & Insuffic & ent Data & & & & & & & & \\
\hline Factor $2,3 \& 4$ & $\begin{array}{l}0.12942^{*} \\
(0.0457) \\
\end{array}$ & $\begin{array}{l}0.18168^{*} \\
(0.04565) \\
\end{array}$ & $\begin{array}{l}0.12991^{*} \\
(0.04584) \\
\end{array}$ & $\begin{array}{l}0.16872^{*} \\
(0.04535) \\
\end{array}$ & $\begin{array}{l}0.13083^{*} \\
(0.04652) \\
\end{array}$ & $\begin{array}{l}0.1391^{*} \\
(0.04342)\end{array}$ & $\begin{array}{l}0.1347^{*} \\
(0.04349)\end{array}$ & $\begin{array}{l}0.1443^{*} \\
(0.0435) \\
\end{array}$ & $\begin{array}{l}0.13005^{*} \\
(0.04474) \\
\end{array}$ & $\begin{array}{l}0.13895^{*} \\
(0.04325) \\
\end{array}$ & $\begin{array}{l}0.13788^{*} \\
(0.04353) \\
\end{array}$ & $\begin{array}{l}0.11635^{*} \\
(0.04371) \\
\end{array}$ & $\begin{array}{l}0.13895^{*} \\
(0.04341) \\
\end{array}$ & $\begin{array}{l}0.1084^{\dagger} \\
(0.04389)\end{array}$ & $\begin{array}{l}0.15815^{*} \\
(0.04696)\end{array}$ & & & $\begin{array}{l}0.13885^{*} \\
(0.04342) \\
\end{array}$ \\
\hline $\mathrm{F}$ & $\begin{array}{l}0.26109^{*} \\
(0.02588)\end{array}$ & $\begin{array}{l}0.27707^{*} \\
(0.02459)\end{array}$ & $\begin{array}{l}0.26067^{*} \\
(0.02638)\end{array}$ & $\begin{array}{l}0.28692^{*} \\
(0.02602)\end{array}$ & $\begin{array}{l}0.2663^{*} \\
(0.02445)\end{array}$ & $\begin{array}{l}0.2676^{\circ} \\
(0.02477)\end{array}$ & $\begin{array}{l}0.24861^{*} \\
(0.02802)\end{array}$ & $\begin{array}{l}0.28758^{*} \\
(0.02787)\end{array}$ & $\begin{array}{l}0.26204^{*} \\
(0.0251)\end{array}$ & $\begin{array}{l}0.26677^{*} \\
(0.02435)\end{array}$ & $\begin{array}{l}0.26183^{*} \\
(0.0288)\end{array}$ & $\begin{array}{l}0.26677^{*} \\
(0.0243)\end{array}$ & $\begin{array}{l}0.26922^{*} \\
(0.02539)\end{array}$ & $\begin{array}{l}0.21048^{*} \\
(0.02862)\end{array}$ & $\begin{array}{l}0.27049^{*} \\
(0.02467)\end{array}$ & $\begin{array}{l}0.26495^{*} \\
(0.02446)\end{array}$ & $\begin{array}{l}0.26622^{*} \\
(0.02419)\end{array}$ & $\begin{array}{l}0.26663^{*} \\
(0.02449)\end{array}$ \\
\hline Factor $1 \& 4$ & $\begin{array}{l}0.18065^{\circ} \\
(0.04467)\end{array}$ & $\begin{array}{l}0.20691^{*} \\
(0.0453)\end{array}$ & $\begin{array}{l}0.17318^{\prime \prime} \\
(0.04673)\end{array}$ & $\begin{array}{l}0.20189^{\prime \prime} \\
(0.04547)\end{array}$ & $\begin{array}{l}0.17407^{\prime \prime} \\
(0.04729)\end{array}$ & $\begin{array}{l}0.18212^{*} \\
(0.04471)\end{array}$ & $\begin{array}{l}0.16149^{*} \\
(0.04715)\end{array}$ & $\begin{array}{l}0.2005^{\circ} \\
(0.04623)\end{array}$ & $\begin{array}{l}\left(0.0217735^{*}\right. \\
(0.04494)\end{array}$ & $\begin{array}{l}0.17904^{*} \\
(0.0445)\end{array}$ & $\begin{array}{l}(0.0200) \\
0.17677^{*} \\
(0.04711)\end{array}$ & $\begin{array}{l}\left(0.1787^{*}\right. \\
(0.04442) \\
\end{array}$ & $\begin{array}{l}0.18585^{*} \\
(0.04619)\end{array}$ & $\begin{array}{l}0.023736^{*} \\
(0.04592)\end{array}$ & $\begin{array}{l}0.18206 \\
(0.04463) \\
\end{array}$ & $\begin{array}{l}0.16763^{*} \\
(0.04604)\end{array}$ & $\begin{array}{l}\left(0.17402^{*}\right. \\
(0.04424)\end{array}$ & $\begin{array}{l}0.18163^{*} \\
(0.04465)\end{array}$ \\
\hline Facto & $\begin{array}{l}0.26076^{\prime \prime} \\
(0.01936)\end{array}$ & $\begin{array}{l}0.27658^{*} \\
(0.01952)\end{array}$ & $\begin{array}{l}0.25897^{\prime \prime} \\
(0.02019)\end{array}$ & $\begin{array}{l}0.27501^{*} \\
(0.01975)\end{array}$ & $\begin{array}{l}0.26283^{\circ} \\
(0.01905)\end{array}$ & $\begin{array}{l}0.26397^{*} \\
(0.01953)\end{array}$ & $\begin{array}{l}0.24306^{\prime \prime} \\
(0.02433)\end{array}$ & $\begin{array}{l}0.2797^{*} \\
(0.02184)\end{array}$ & $\begin{array}{l}0.25229^{\prime \prime} \\
(0.02316)\end{array}$ & $\begin{array}{l}0.26309^{*} \\
(0.01897)\end{array}$ & $\begin{array}{l}0.25821^{*} \\
(0.02429)\end{array}$ & $\begin{array}{l}0.25652^{*} \\
(0.01904)\end{array}$ & $\begin{array}{l}0.26581^{*} \\
(0.02052)\end{array}$ & $\begin{array}{l}0.19793^{*} \\
(0.02581)\end{array}$ & $\begin{array}{l}0.28152^{*} \\
(0.02568)\end{array}$ & $\begin{array}{l}0.25684^{\prime \prime} \\
(0.01969)\end{array}$ & $\begin{array}{l}0.25129^{\prime \prime} \\
(0.01904)\end{array}$ & $\begin{array}{l}0.26267^{*} \\
(0.01965)\end{array}$ \\
\hline Factor $1,3 \& 4$ & $\begin{array}{l}0.27574^{*} \\
(0.02251)\end{array}$ & $\begin{array}{l}0.28311^{*} \\
(0.0239)\end{array}$ & $\begin{array}{l}0.27024^{*} \\
(0.02517)\end{array}$ & $\begin{array}{l}0.2978^{*} \\
(0.02419)\end{array}$ & $\begin{array}{l}0.26983^{*} \\
(0.02716)\end{array}$ & $\begin{array}{l}0.27801^{*} \\
(0.02267)\end{array}$ & $\begin{array}{l}0.25722^{*} \\
(0.02705)\end{array}$ & $\begin{array}{l}0.29203^{*} \\
(0.02431)\end{array}$ & $\begin{array}{l}0.26667^{*} \\
(0.02587)\end{array}$ & $\begin{array}{l}0.27643^{*} \\
(0.02231)\end{array}$ & $\begin{array}{l}0.27243^{*} \\
(0.02696)\end{array}$ & $\begin{array}{l}0.27405^{\circ} \\
(0.02229)\end{array}$ & $\begin{array}{l}0.27981^{*} \\
(0.02348)\end{array}$ & $\begin{array}{l}0.21383^{*} \\
(0.02805)\end{array}$ & $\begin{array}{l}0.29627^{7} \\
(0.02857)\end{array}$ & $\begin{array}{l}0.25688^{*} \\
(0.02784)\end{array}$ & $\begin{array}{l}0.26961^{*} \\
(0.02244)\end{array}$ & $\begin{array}{l}0.27705^{*} \\
(0.02257) \\
\end{array}$ \\
\hline Factor $1 \& 2$ & $\begin{array}{l}0.18455^{*} \\
(0.0281)\end{array}$ & $\begin{array}{l}0.23505^{*} \\
(0.02908)\end{array}$ & $\begin{array}{l}0.18266^{*} \\
(0.03015)\end{array}$ & $\begin{array}{l}0.20138^{*} \\
(0.02553)\end{array}$ & $\begin{array}{l}0.19195^{*} \\
(0.02534)\end{array}$ & $\begin{array}{l}0.19335^{*} \\
(0.02548)\end{array}$ & $\begin{array}{l}0.1739^{*} \\
(0.02901)\end{array}$ & $\begin{array}{l}0.21438^{*} \\
(0.02888)\end{array}$ & $\begin{array}{l}0.18806^{*} \\
(0.02592)\end{array}$ & $\begin{array}{l}0.18655^{*} \\
(0.02531)\end{array}$ & $\begin{array}{l}0.18798^{*} \\
(0.02922)\end{array}$ & $\begin{array}{l}0.17651^{*} \\
(0.02563)\end{array}$ & $\begin{array}{l}0.1956^{*} \\
(0.02656)\end{array}$ & $\begin{array}{l}0.14519^{*} \\
(0.0282) \\
\end{array}$ & $\begin{array}{l}0.20317^{*} \\
(0.02711)\end{array}$ & $\begin{array}{l}0.19236^{*} \\
(0.02528)\end{array}$ & $\begin{array}{l}0.16437^{*} \\
(0.02583)\end{array}$ & $\begin{array}{l}0.19231^{*} \\
(0.02573)\end{array}$ \\
\hline Factor $1,2 \& 4$ & $\begin{array}{l}0.30044^{*} \\
(0.03945)\end{array}$ & $\begin{array}{l}0.34418^{*} \\
(0.039)\end{array}$ & $\begin{array}{l}0.29893^{*} \\
(0.04079)\end{array}$ & $\begin{array}{l}0.33983^{*} \\
(0.03967)\end{array}$ & $\begin{array}{l}0.30124^{*} \\
(0.04062)\end{array}$ & $\begin{array}{l}0.3099^{\circ} \\
(0.03745)\end{array}$ & $\begin{array}{l}0.28905^{*} \\
(0.0402)\end{array}$ & $\begin{array}{l}0.33106^{\prime \prime} \\
(0.03982)\end{array}$ & $\begin{array}{l}0.30417^{\circ} \\
(0.03772)\end{array}$ & $\begin{array}{l}0.30828^{*} \\
(0.03711)\end{array}$ & $\begin{array}{l}0.30441^{*} \\
(0.03998)\end{array}$ & $\begin{array}{l}0.28063^{\prime \prime} \\
(0.03804)\end{array}$ & $\begin{array}{l}0.31165^{*} \\
(0.03792)\end{array}$ & $\begin{array}{l}0.26927^{*} \\
(0.03852)\end{array}$ & $\begin{array}{l}0.31824^{*} \\
(0.03819)\end{array}$ & $\begin{array}{l}0.30142^{*} \\
(0.03774)\end{array}$ & $\begin{array}{l}0.28353^{*} \\
(0.03732)\end{array}$ & $\begin{array}{l}0.30859^{*} \\
(0.03774)\end{array}$ \\
\hline $1,2 \& 3$ & $\begin{array}{l}0.24164^{*} \\
(0.02151)\end{array}$ & $\begin{array}{l}0.29223^{*} \\
(0.02212)\end{array}$ & $\begin{array}{l}\left(0.24057^{*}\right. \\
(0.02341)\end{array}$ & $\begin{array}{l}\left(0.25521^{*}\right. \\
(0.01701)\end{array}$ & $\begin{array}{l}0.2494^{*} \\
(0.01707)\end{array}$ & $\begin{array}{l}\left(0.25217^{*}\right. \\
(0.01884)\end{array}$ & $\begin{array}{l}0.23146^{*} \\
(0.02221)\end{array}$ & & $\begin{array}{l}0.23853^{*} \\
(0.02234)\end{array}$ & & & & & & $\begin{array}{l}0.26912^{*} \\
(0.02428)\end{array}$ & $\begin{array}{l}0.24662^{*} \\
(0.0172)\end{array}$ & $\begin{array}{l}0.225^{\circ} \\
(0.01769)\end{array}$ & $\begin{array}{l}0.24959^{*} \\
(0.01984)\end{array}$ \\
\hline All Factors & $\begin{array}{l}0.27497^{\prime \prime} \\
(0.01957)\end{array}$ & $\begin{array}{l}0.32486^{\prime \prime} \\
(0.02094)\end{array}$ & $\begin{array}{l}0.27348^{\prime \prime} \\
(0.02192)\end{array}$ & $\begin{array}{l}0.31274^{\prime \prime} \\
(0.02033)\end{array}$ & $\begin{array}{l}0.27615^{\circ} \\
(0.02093)\end{array}$ & $\begin{array}{l}0.28468^{\prime \prime} \\
(0.01722)\end{array}$ & $\begin{array}{l}0.26304^{*} \\
(0.02155)\end{array}$ & $\begin{array}{l}0.30286^{\prime \prime} \\
(0.01993)\end{array}$ & $\begin{array}{l}0.27267^{\prime \prime} \\
(0.0199)\end{array}$ & $\begin{array}{l}0.27633^{*} \\
(0.01546)\end{array}$ & $\begin{array}{l}0.27819^{\prime \prime} \\
(0.0215)\end{array}$ & $\begin{array}{l}0.25909^{\prime \prime} \\
(0.01687)\end{array}$ & $\begin{array}{l}0.28678^{*} \\
(0.01851)\end{array}$ & $\begin{array}{l}0.21897^{\prime \prime} \\
(0.02302)\end{array}$ & $\begin{array}{l}0.30171^{\prime \prime} \\
(0.02319)\end{array}$ & $\begin{array}{l}0.26404^{*} \\
(0.02175)\end{array}$ & $\begin{array}{l}0.25471^{\prime \prime} \\
(0.01643)\end{array}$ & $\begin{array}{l}0.28239^{*} \\
(0.01727)\end{array}$ \\
\hline $\mathrm{Ir}$ & $\begin{array}{l}0.00953 \\
(0.0143)\end{array}$ & $\begin{array}{c}-0.04575^{*} \\
(0.01572)\end{array}$ & $\begin{array}{l}0.01019 \\
(0.01662)\end{array}$ & $\begin{array}{c}-0.03261^{\dagger} \\
(0.01474)\end{array}$ & $\begin{array}{l}0.00861 \\
(0.01773)\end{array}$ & $\begin{array}{c}-0.00317 \\
(0.01562)\end{array}$ & $\begin{array}{l}0.0204 \\
(0.01544)\end{array}$ & $\begin{array}{c}-0.02323 \\
(0.01502)\end{array}$ & $\begin{array}{l}0.01221 \\
(0.0149)\end{array}$ & $\begin{array}{l}0.11085^{*} \\
(0.04102)\end{array}$ & $\begin{array}{l}0.00501 \\
(0.01546)\end{array}$ & $\begin{array}{l}0.06052^{*} \\
(0.01831)\end{array}$ & $\begin{array}{c}-0.00475 \\
(0.01336)\end{array}$ & $\begin{array}{l}0.06517^{*} \\
(0.01756)\end{array}$ & $\begin{array}{c}-0.01921 \\
(0.01797)\end{array}$ & $\begin{array}{l}0.02176 \\
(0.01765)\end{array}$ & $\begin{array}{l}0.07671^{*} \\
(0.01712)\end{array}$ & $\begin{array}{l}0.00139 \\
(0.01602)\end{array}$ \\
\hline
\end{tabular}

Significant at the 0.01 level $†$ Significant at the 0.05 level $\$$ Significant at the 0.10 level
Table 88 Coefficients with individual variables for source of implementation support - Medium Plants 


\begin{tabular}{|c|c|c|c|c|c|c|c|c|c|c|c|c|c|}
\hline $\begin{array}{l}\text { Individual variable } \\
\text { included in the } \\
\text { model in the } \\
\text { column }\end{array}$ & 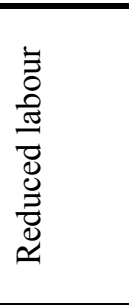 & 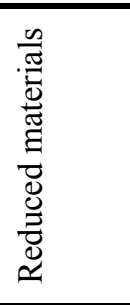 & 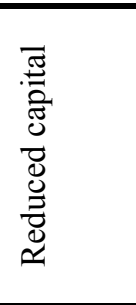 & 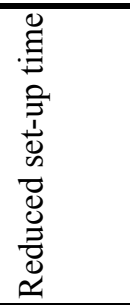 & 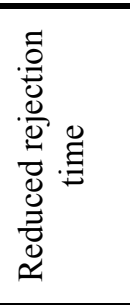 & 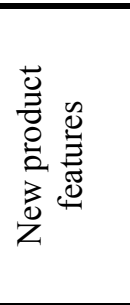 & 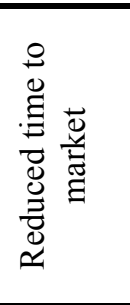 & 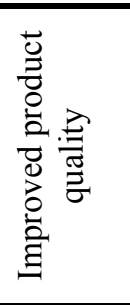 & 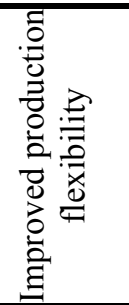 & 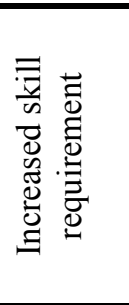 & 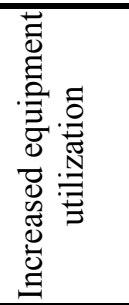 & 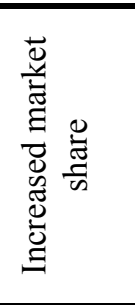 & 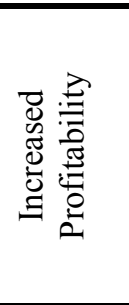 \\
\hline No factors & $\begin{array}{l}0.20813^{*} \\
(0.01273)\end{array}$ & $\begin{array}{l}0.20773^{*} \\
(0.01275)\end{array}$ & $\begin{array}{l}0.20767^{*} \\
(0.01275)\end{array}$ & $\begin{array}{l}0.20711^{*} \\
(0.01271)\end{array}$ & $\begin{array}{l}0.2073^{*} \\
(0.01275)\end{array}$ & $\begin{array}{l}0.20538^{*} \\
(0.01274)\end{array}$ & $\begin{array}{l}0.20812^{*} \\
(0.01275)\end{array}$ & $\begin{array}{l}0.20952^{*} \\
(0.01278)\end{array}$ & $\begin{array}{l}0.20766^{*} \\
(0.01275)\end{array}$ & $\begin{array}{l}0.20092^{*} \\
(0.01281)\end{array}$ & $\begin{array}{l}0.20751^{*} \\
(0.01274)\end{array}$ & $\begin{array}{l}0.20751^{*} \\
(0.01274)\end{array}$ & $\begin{array}{l}0.20879^{*} \\
(0.0127)\end{array}$ \\
\hline Factor 4 & $\begin{array}{l}0.20169^{*} \\
(0.03367)\end{array}$ & $\begin{array}{l}0.19797^{*} \\
(0.03381)\end{array}$ & $\begin{array}{l}0.1978^{*} \\
(0.0337)\end{array}$ & $\begin{array}{l}0.18708^{*} \\
(0.03383)\end{array}$ & $\begin{array}{l}0.1925^{*} \\
(0.03437)\end{array}$ & $\begin{array}{l}0.18643^{*} \\
(0.03381)\end{array}$ & $\begin{array}{l}0.20483^{*} \\
(0.03448)\end{array}$ & $\begin{array}{l}0.20924^{*} \\
(0.03427)\end{array}$ & $\begin{array}{l}0.19676^{*} \\
(0.03397)\end{array}$ & $\begin{array}{l}0.18241^{*} \\
(0.03369)\end{array}$ & $\begin{array}{l}0.19081^{*} \\
(0.03461)\end{array}$ & $\begin{array}{l}0.19081^{*} \\
(0.03461)\end{array}$ & $\begin{array}{l}0.23871^{*} \\
(0.03675)\end{array}$ \\
\hline $\mathrm{Fac}$ & $\begin{array}{l}0.23983^{*} \\
(0.03995)\end{array}$ & $\begin{array}{l}0.2265^{*} \\
(0.0387) \\
\end{array}$ & $\begin{array}{l}0.22651^{*} \\
(0.0387)\end{array}$ & $\begin{array}{l}0.22036^{*} \\
(0.03868)\end{array}$ & $\begin{array}{l}0.21761^{*} \\
(0.0403)\end{array}$ & $\begin{array}{l}0.22029^{*} \\
(0.03865)\end{array}$ & $\begin{array}{l}0.22841^{*} \\
(0.03875)\end{array}$ & $\begin{array}{l}0.24995^{*} \\
(0.04142)\end{array}$ & $\begin{array}{l}0.22287^{*} \\
(0.04051)\end{array}$ & $\begin{array}{l}0.19529^{*} \\
(0.03947)\end{array}$ & $\begin{array}{l}0.22454^{*} \\
(0.03875)\end{array}$ & $\begin{array}{l}0.22454^{*} \\
(0.03875)\end{array}$ & $\begin{array}{l}0.23718^{*} \\
(0.03877)\end{array}$ \\
\hline Factor $3 \& 4$ & $\begin{array}{l}0.17064^{*} \\
(0.03898)\end{array}$ & $\begin{array}{l}0.1676^{*} \\
(0.03899)\end{array}$ & $\begin{array}{l}0.1674^{*} \\
(0.03899)\end{array}$ & $\begin{array}{l}0.16335^{*} \\
(0.03888)\end{array}$ & $\begin{array}{l}0.1624^{*} \\
(0.03952)\end{array}$ & $\begin{array}{l}0.15005^{*} \\
(0.03944)\end{array}$ & $\begin{array}{l}0.17453^{*} \\
(0.03977)\end{array}$ & $\begin{array}{l}0.18484^{*} \\
(0.04039)\end{array}$ & $\begin{array}{l}0.16295^{*} \\
(0.04199)\end{array}$ & $\begin{array}{l}0.1149^{*} \\
(0.04151)\end{array}$ & $\begin{array}{l}0.16032^{*} \\
(0.03981)\end{array}$ & $\begin{array}{l}0.16032^{*} \\
(0.03981)\end{array}$ & $\begin{array}{l}0.21802^{*} \\
(0.04315)\end{array}$ \\
\hline Factor 2 & $\begin{array}{l}0.36846^{*} \\
(0.03129)\end{array}$ & $\begin{array}{l}0.34641^{*} \\
(0.03077)\end{array}$ & $\begin{array}{l}0.34729^{*} \\
(0.02712)\end{array}$ & $\begin{array}{l}0.34739^{*} \\
(0.02698)\end{array}$ & $\begin{array}{l}0.34 \\
(0.0\end{array}$ & $\begin{array}{l}0.34763^{*} \\
(0.02697)\end{array}$ & $\begin{array}{l}0.34 \\
(0.0\end{array}$ & $\begin{array}{l}0.34943^{*} \\
(0.02705)\end{array}$ & $\begin{array}{l}0.34732^{*} \\
(0.02708)\end{array}$ & $\begin{array}{l}0.29665^{*} \\
(0.03053)\end{array}$ & $\begin{array}{l}0.34725^{*} \\
(0.02705)\end{array}$ & $\begin{array}{l}0.3 \\
0.0\end{array}$ & $\begin{array}{l}0.35399^{*} \\
(0.02707)\end{array}$ \\
\hline $\mathrm{Fa}$ & $\begin{array}{l}0.30904^{*} \\
(0.0543) \\
\end{array}$ & $\begin{array}{l}0.29137^{*} \\
(0.05463)\end{array}$ & $\begin{array}{l}0.29105^{*} \\
(0.05345)\end{array}$ & $\begin{array}{l}0.29201^{*} \\
(0.05274)\end{array}$ & $\begin{array}{l}0.28893^{*} \\
(0.05307)\end{array}$ & $\begin{array}{l}0.28419^{*} \\
(0.05282)\end{array}$ & $\begin{array}{l}0.30105^{*} \\
(0.05385)\end{array}$ & $\begin{array}{l}0.30779^{*} \\
(0.05371)\end{array}$ & $\begin{array}{l}0.29053^{*} \\
(0.05328)\end{array}$ & $\begin{array}{l}0.28208^{*} \\
(0.05264)\end{array}$ & $\begin{array}{l}0.2813^{*} \\
(0.05434)\end{array}$ & $\begin{array}{l}0.2813^{*} \\
(0.05434)\end{array}$ & $\begin{array}{l}0.34166^{*} \\
(0.05583)\end{array}$ \\
\hline Factor $2 \& 3$ & $\begin{array}{l}0.16369^{\ddagger} \\
(0.09402)\end{array}$ & & $\begin{array}{l}0.14004 \\
(0.09323)\end{array}$ & $\begin{array}{l}0.13997 \\
(0.09239)\end{array}$ & $\begin{array}{l}0.13406 \\
(0.09317)\end{array}$ & $\begin{array}{l}0.12439 \\
(0.09261)\end{array}$ & $\begin{array}{l}0.14314 \\
(0.09263)\end{array}$ & $\begin{array}{l}0.16802^{\ddagger} \\
(0.09401)\end{array}$ & $\begin{array}{l}0.13761 \\
(0.09376)\end{array}$ & $\begin{array}{l}58 \\
356) \\
\end{array}$ & $\begin{array}{l}0.1348 \\
(0.09297)\end{array}$ & $\begin{array}{l}0.1348 \\
(0.09297)\end{array}$ & $\begin{array}{l}0.18325^{\ddagger} \\
(0.09361)\end{array}$ \\
\hline & $\begin{array}{l}0.288 \\
(0.04 \\
\end{array}$ & $\begin{array}{l}0.26 \\
(0.0 \\
\end{array}$ & $\begin{array}{l}0.26 \\
0.04 \\
\end{array}$ & $\begin{array}{l}0.256 \\
(0.04 \\
\end{array}$ & $\begin{array}{l}0.26 \\
(0.0 \\
\end{array}$ & $\begin{array}{l}0.25796^{*} \\
(0.04121)\end{array}$ & $\begin{array}{l}0.27 \\
(0.04 \\
\end{array}$ & $\begin{array}{l}0.28821^{*} \\
(0.0428)\end{array}$ & $\begin{array}{l}0.26473^{*} \\
(0.04362)\end{array}$ & $\begin{array}{l}0.21206^{*} \\
(0.04395)\end{array}$ & $\begin{array}{l}0.26023^{*} \\
(0.0423)\end{array}$ & $\begin{array}{l}0.2 \\
(0.0\end{array}$ & $\begin{array}{l}0.32285^{*} \\
(0.04563)\end{array}$ \\
\hline Fac & $\begin{array}{l}0.17282^{\ddagger} \\
(0.09072)\end{array}$ & $\begin{array}{l}0.16319^{\ddagger} \\
(0.09051)\end{array}$ & $\begin{array}{l}0.16263^{\ddagger} \\
(0.09056)\end{array}$ & $\begin{array}{l}0.15076^{\ddagger} \\
(0.0904)\end{array}$ & $\begin{array}{l}0.14992 \\
(0.09202)\end{array}$ & $\begin{array}{l}0.13525 \\
(0.09088)\end{array}$ & $\begin{array}{l}0.17475^{\ddagger} \\
(0.09152)\end{array}$ & $\begin{array}{l}0.17515^{\ddagger} \\
(0.09071)\end{array}$ & $\begin{array}{l}0.16216^{\ddagger} \\
(0.09057)\end{array}$ & $\begin{array}{l}0.16319^{\ddagger} \\
(0.08995)\end{array}$ & $\begin{array}{l}0.16319^{\sharp} \\
(0.09047)\end{array}$ & $\begin{array}{l}0.16319^{\ddagger} \\
(0.09047)\end{array}$ & $\begin{array}{l}0.16319^{\ddagger} \\
(0.09019)\end{array}$ \\
\hline Factor & $\begin{array}{l}0.21437^{*} \\
(0.07078)\end{array}$ & $\begin{array}{l}0.20841^{*} \\
(0.07089)\end{array}$ & $\begin{array}{l}0.20767^{*} \\
(0.07098)\end{array}$ & $\begin{array}{l}0.19224^{*} \\
(0.07086)\end{array}$ & $\begin{array}{l}0.20315^{*} \\
(0.07106)\end{array}$ & $\begin{array}{l}0.17444^{\dagger} \\
(0.07177)\end{array}$ & $\begin{array}{l}0.22053^{*} \\
(0.07207)\end{array}$ & $\begin{array}{l}0.22489^{*} \\
(0.07137)\end{array}$ & $\begin{array}{l}0.20842^{*} \\
(0.07073)\end{array}$ & $\begin{array}{l}0.19681^{*} \\
(0.07036)\end{array}$ & $\begin{array}{l}0.20182^{*} \\
(0.07113)\end{array}$ & $\begin{array}{l}0.20182^{*} \\
(0.07113)\end{array}$ & $\begin{array}{l}0.26846^{*} \\
(0.07393)\end{array}$ \\
\hline Fact & $\begin{array}{l}0.28077^{*} \\
(0.04096)\end{array}$ & $\begin{array}{l}0.27699^{*} \\
(0.0409) \\
\end{array}$ & $\begin{array}{l}0.27528^{*} \\
(0.04192)\end{array}$ & $\begin{array}{l}0.25151^{*} \\
(0.04215)\end{array}$ & $\begin{array}{l}0.26907^{*} \\
(0.0421)\end{array}$ & $\begin{array}{l}0.24474^{*} \\
(0.04269)\end{array}$ & $\begin{array}{l}0.28934^{*} \\
(0.04345)\end{array}$ & $\begin{array}{l}0.30424^{*} \\
(0.04436)\end{array}$ & $\begin{array}{l}0.27288^{*} \\
(0.04308)\end{array}$ & $\begin{array}{l}0.23074^{*} \\
(0.04271)\end{array}$ & $\begin{array}{l}0.27589^{*} \\
(0.0409)\end{array}$ & $\begin{array}{l}0.27589^{*} \\
(0.0409) \\
\end{array}$ & $\begin{array}{l}0.28063^{*} \\
(0.04078)\end{array}$ \\
\hline Factor $1,3 \& 4$ & $\begin{array}{l}0.31038^{*} \\
(0.02337)\end{array}$ & $\begin{array}{l}0.30636^{*} \\
(0.02324)\end{array}$ & $\begin{array}{l}0.30508^{*} \\
(0.02434)\end{array}$ & $\begin{array}{l}0.28601^{*} \\
(0.02467)\end{array}$ & $\begin{array}{l}0.29439^{*} \\
(0.02776)\end{array}$ & $\begin{array}{l}0.26913^{*} \\
(0.02738)\end{array}$ & $\begin{array}{l}0.31828^{*} \\
(0.02716)\end{array}$ & $\begin{array}{l}0.32895^{*} \\
(0.02723)\end{array}$ & $\begin{array}{l}0.30317^{*} \\
(0.02558)\end{array}$ & $\begin{array}{l}0.25316^{*} \\
(0.02757)\end{array}$ & $\begin{array}{l}0.29657^{*} \\
(0.0257) \\
\end{array}$ & $\begin{array}{l}0.29657^{*} \\
(0.0257)\end{array}$ & $\begin{array}{l}0.36168^{*} \\
(0.03103)\end{array}$ \\
\hline Factor $1 \& 2$ & \multicolumn{13}{|c|}{ Insufficient Data } \\
\hline Factor $1,2 \& 4$ & $\begin{array}{l}0.29853^{*} \\
(0.02305) \\
\end{array}$ & $\begin{array}{l}0.2833^{*} \\
(0.02061)\end{array}$ & $\begin{array}{l}0.28089^{*} \\
(0.02496)\end{array}$ & $\begin{array}{l}0.25472^{*} \\
(0.02344)\end{array}$ & $\begin{array}{l}0.2723^{*} \\
(0.02473)\end{array}$ & $\begin{array}{l}0.25131^{*} \\
(0.02376)\end{array}$ & $\begin{array}{l}0.29439^{*} \\
(0.02386)\end{array}$ & $\begin{array}{l}0.28976^{*} \\
(0.02049)\end{array}$ & $\begin{array}{l}0.28315^{*} \\
(0.02021)\end{array}$ & $\begin{array}{l}0.26579^{*} \\
(0.02065)\end{array}$ & $\begin{array}{l}0.27321^{*} \\
(0.02329)\end{array}$ & $\begin{array}{l}0.27321^{*} \\
(0.02329)\end{array}$ & $\begin{array}{l}0.33585^{*} \\
(0.02802)\end{array}$ \\
\hline Factor $1,2 \& 3$ & $\begin{array}{l}0.28869^{*} \\
(0.03145)\end{array}$ & $\begin{array}{l}0.27346^{*} \\
(0.03171)\end{array}$ & $\begin{array}{l}0.27206^{*} \\
(0.03211)\end{array}$ & $\begin{array}{l}0.25458^{*} \\
(0.03063)\end{array}$ & $\begin{array}{l}0.26428^{*} \\
(0.03221)\end{array}$ & $\begin{array}{l}0.24604^{*} \\
(0.03151)\end{array}$ & $\begin{array}{l}0.28692^{*} \\
(0.03312)\end{array}$ & $\begin{array}{l}0.29608^{*} \\
(0.03258)\end{array}$ & $\begin{array}{l}0.27077^{*} \\
(0.03189)\end{array}$ & $\begin{array}{l}0.21475^{*} \\
(0.03391)\end{array}$ & $\begin{array}{l}0.27156^{*} \\
(0.02973)\end{array}$ & $\begin{array}{l}0.27156^{*} \\
(0.02973)\end{array}$ & $\begin{array}{l}0.28491^{*} \\
(0.02972)\end{array}$ \\
\hline 1 acto & $\begin{array}{l}0.28512^{*} \\
(0.01657)\end{array}$ & $\begin{array}{l}0.26512^{*} \\
(0.01513)\end{array}$ & $\begin{array}{l}0.2639^{*} \\
(0.01466)\end{array}$ & $\begin{array}{l}0.24005^{*} \\
(0.01372)\end{array}$ & $\begin{array}{l}0.2544^{*} \\
(0.01704)\end{array}$ & $\begin{array}{l}0.23375^{*} \\
(0.0152)\end{array}$ & $\begin{array}{l}0.27827^{*} \\
(0.01668)\end{array}$ & $\begin{array}{l}0.2913^{*} \\
(0.01801)\end{array}$ & $\begin{array}{l}0.26164^{*} \\
(0.01702)\end{array}$ & $\begin{array}{l}0.20913^{*} \\
(0.01817)\end{array}$ & $\begin{array}{l}0.25612^{*} \\
(0.01401)\end{array}$ & $\begin{array}{l}0.25612^{*} \\
(0.01401)\end{array}$ & $\begin{array}{l}0.32339^{*} \\
(0.023)\end{array}$ \\
\hline al Va & $\begin{array}{l}-0.02169 \\
(0.0164) \\
\end{array}$ & $\begin{array}{l}0.00131 \\
(0.01574) \\
\end{array}$ & $\begin{array}{l}0.00285 \\
(0.01515) \\
\end{array}$ & $\begin{array}{l}0.03473^{\dagger} \\
(0.01451) \\
\end{array}$ & $\begin{array}{l}0.01327 \\
(0.01675) \\
\end{array}$ & $\begin{array}{l}0.03902^{\dagger} \\
(0.01532) \\
\end{array}$ & $\begin{array}{l}-0.01376 \\
(0.01643) \\
\end{array}$ & $\begin{array}{l}-0.02723 \\
(0.0173) \\
\end{array}$ & $\begin{array}{l}0.00522 \\
(0.01709)\end{array}$ & $\begin{array}{l}0.06122^{*} \\
(0.01736) \\
\end{array}$ & $\begin{array}{l}0.01327 \\
(0.01486) \\
\end{array}$ & $\begin{array}{l}0.01327 \\
(0.01486) \\
\end{array}$ & $\begin{array}{l}-0.05964^{*} \\
(0.02235) \\
\end{array}$ \\
\hline
\end{tabular}

* Significant at the 0.01 level $†$ Significant at the 0.05 level $\$$ Significant at the 0.10 level

Table 89 Coefficients with individual variables for perceived benefits of AMT - Medium Plants 


\begin{tabular}{|c|c|c|c|c|c|c|c|c|c|c|c|c|c|}
\hline $\begin{array}{l}\text { Individual variable } \\
\text { included in the } \\
\text { model in the } \\
\text { column }\end{array}$ & 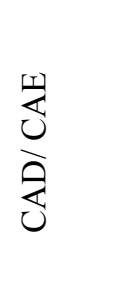 & 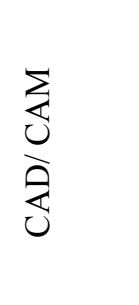 & 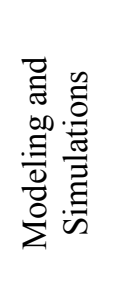 & 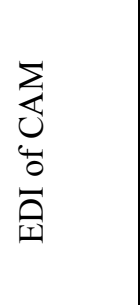 & 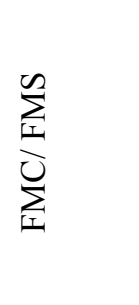 & 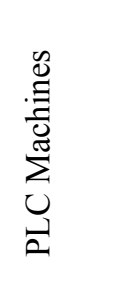 & 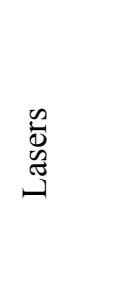 & 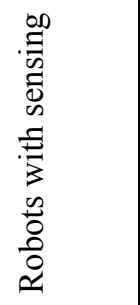 & 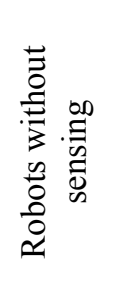 & 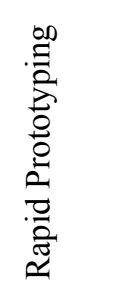 & 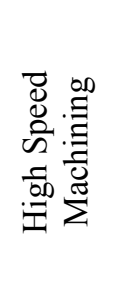 & 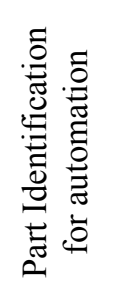 & 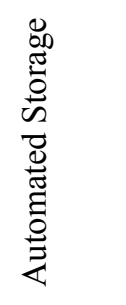 \\
\hline No factors & $\begin{array}{l}0.27248^{*} \\
(0.01309)\end{array}$ & $\begin{array}{l}0.2704^{*} \\
(0.01303)\end{array}$ & $\begin{array}{l}0.26967^{*} \\
(0.01301)\end{array}$ & $\begin{array}{l}0.26959^{*} \\
(0.01303)\end{array}$ & $\begin{array}{l}0.26967^{*} \\
(0.013)\end{array}$ & $\begin{array}{l}0.2687^{*} \\
(0.01298)\end{array}$ & $\begin{array}{l}0.26964^{*} \\
(0.01301)\end{array}$ & $\begin{array}{l}0.26966^{*} \\
(0.01303)\end{array}$ & $\begin{array}{l}0.26954^{*} \\
(0.01302)\end{array}$ & $\begin{array}{l}0.26967^{*} \\
(0.01303)\end{array}$ & $\begin{array}{l}0.26927^{*} \\
(0.01302)\end{array}$ & $\begin{array}{l}0.26927^{*} \\
(0.01304)\end{array}$ & $\begin{array}{l}0.26967^{*} \\
(0.01301)\end{array}$ \\
\hline Factor 5 & $\begin{array}{l}0.12664^{\ddagger} \\
(0.06968)\end{array}$ & $\begin{array}{l}0.11427^{\ddagger} \\
(0.06937)\end{array}$ & $\begin{array}{l}0.11011 \\
(0.06934)\end{array}$ & $\begin{array}{l}0.11216 \\
(0.06942) \\
\end{array}$ & $\begin{array}{l}0.11275 \\
(0.06924)\end{array}$ & $\begin{array}{l}0.10546 \\
(0.0692) \\
\end{array}$ & $\begin{array}{l}0.11275 \\
(0.06932)\end{array}$ & $\begin{array}{l}0.11275 \\
(0.06942) \\
\end{array}$ & $\begin{array}{l}0.10745 \\
(0.06948)\end{array}$ & $\begin{array}{l}0.11275 \\
(0.0694) \\
\end{array}$ & $\begin{array}{l}0.11275 \\
(0.06934)\end{array}$ & $\begin{array}{l}0.11204 \\
(0.06942) \\
\end{array}$ & $\begin{array}{l}0.11275 \\
(0.06932) \\
\end{array}$ \\
\hline Factor 4 & $\begin{array}{l}0.17764^{*} \\
(0.03576)\end{array}$ & $\begin{array}{l}0.16671^{*} \\
(0.03508)\end{array}$ & $\begin{array}{l}0.14654^{*} \\
(0.03308)\end{array}$ & $\begin{array}{l}0.14824^{*} \\
(0.03356)\end{array}$ & $\begin{array}{l}0.13487^{*} \\
(0.03369)\end{array}$ & $\begin{array}{l}0.12329^{*} \\
(0.03438)\end{array}$ & $\begin{array}{l}0.14802^{*} \\
(0.033)\end{array}$ & $\begin{array}{l}0.15114^{*} \\
(0.033)\end{array}$ & $\begin{array}{l}0.15129^{*} \\
(0.03297)\end{array}$ & $\begin{array}{l}0.15129^{*} \\
(0.03298)\end{array}$ & $\begin{array}{l}0.13953^{*} \\
(0.03389)\end{array}$ & $\begin{array}{l}0.15122^{*} \\
(0.03299)\end{array}$ & $\begin{array}{l}0.15267^{*} \\
(0.03296)\end{array}$ \\
\hline Factor $4 \& 5$ & $\begin{array}{l}0.26587^{\dagger} \\
(0.12869)\end{array}$ & $\begin{array}{l}0.25346^{\dagger} \\
(0.12857)\end{array}$ & $\begin{array}{l}0.22942^{\ddagger} \\
(0.12779)\end{array}$ & $\begin{array}{l}0.22897^{\ddagger} \\
(0.12855)\end{array}$ & $\begin{array}{l}0.2284^{\ddagger} \\
(0.12761)\end{array}$ & $\begin{array}{l}0.22574^{\ddagger} \\
(0.12746)\end{array}$ & $\begin{array}{l}0.23267^{\ddagger} \\
(0.12773)\end{array}$ & $\begin{array}{l}0.23543^{\ddagger} \\
(0.12791)\end{array}$ & $\begin{array}{l}0.23543^{\ddagger} \\
(0.1278)\end{array}$ & $\begin{array}{l}0.23543^{\ddagger} \\
(0.12787)\end{array}$ & $\begin{array}{l}0.2205^{\ddagger} \\
(0.12816)\end{array}$ & $\begin{array}{l}0.23429^{\ddagger} \\
(0.1279) \\
\end{array}$ & $\begin{array}{l}0.23543^{\ddagger} \\
(0.12773)\end{array}$ \\
\hline Factor 3 & $\begin{array}{l}0.25201^{*} \\
(0.04486)\end{array}$ & $\begin{array}{l}0.23402^{*} \\
(0.04384)\end{array}$ & $\begin{array}{l}0.23367^{*} \\
(0.04382)\end{array}$ & $\begin{array}{l}0.2299^{*} \\
(0.04452)\end{array}$ & $\begin{array}{l}0.23179^{*} \\
(0.04377)\end{array}$ & $\begin{array}{l}0.22443^{*} \\
(0.04383)\end{array}$ & $\begin{array}{l}0.23183^{*} \\
(0.04383)\end{array}$ & $\begin{array}{l}0.23367^{*} \\
(0.04388)\end{array}$ & $\begin{array}{l}0.23367^{*} \\
(0.04384)\end{array}$ & $\begin{array}{l}0.23367^{*} \\
(0.04386)\end{array}$ & $\begin{array}{l}0.23367^{*} \\
(0.04383)\end{array}$ & $\begin{array}{l}0.23278^{*} \\
(0.04389)\end{array}$ & $\begin{array}{l}0.23367^{*} \\
(0.04382)\end{array}$ \\
\hline Factor $3 \& 5$ & $\begin{array}{l}0.15893^{*} \\
(0.05072)\end{array}$ & $\begin{array}{l}0.15879^{*} \\
(0.05077)\end{array}$ & $\begin{array}{l}0.15852^{*} \\
(0.05075)\end{array}$ & $\begin{array}{l}0.15852^{*} \\
(0.05081)\end{array}$ & $\begin{array}{l}0.15852^{*} \\
(0.05068)\end{array}$ & $\begin{array}{l}0.14661^{*} \\
(0.0508)\end{array}$ & $\begin{array}{l}0.15852^{*} \\
(0.05074)\end{array}$ & $\begin{array}{l}0.15852^{*} \\
(0.05082)\end{array}$ & $\begin{array}{l}0.15852^{*} \\
(0.05077)\end{array}$ & $\begin{array}{l}0.15852^{*} \\
(0.0508)\end{array}$ & $\begin{array}{l}0.1529^{*} \\
(0.0509) \\
\end{array}$ & $\begin{array}{l}0.15852^{*} \\
(0.05081)\end{array}$ & $\begin{array}{l}0.15852^{*} \\
(0.05074)\end{array}$ \\
\hline Facto & $\begin{array}{l}0.25555^{*} \\
(0.05089)\end{array}$ & $\begin{array}{l}0.24065^{*} \\
(0.04982)\end{array}$ & $\begin{array}{l}0.221^{*} \\
(0.04837)\end{array}$ & $\begin{array}{l}0.22141^{*} \\
(0.04894)\end{array}$ & $\begin{array}{l}0.21026^{*} \\
(0.04868)\end{array}$ & $\begin{array}{l}0.20278^{*} \\
(0.04885)\end{array}$ & $\begin{array}{l}0.22203^{*} \\
(0.04833)\end{array}$ & $\begin{array}{l}0.22511^{*} \\
(0.04837)\end{array}$ & $\begin{array}{l}0.2246^{*} \\
(0.04833)\end{array}$ & $\begin{array}{l}0.22121^{*} \\
(0.04857)\end{array}$ & $\begin{array}{l}0.21795^{*} \\
(0.04855)\end{array}$ & $\begin{array}{l}0.225^{*} \\
(0.04836)\end{array}$ & $\begin{array}{l}0.22572^{*} \\
(0.0483)\end{array}$ \\
\hline Factor & $\begin{array}{l}0.20033^{*} \\
(0.06192)\end{array}$ & $\begin{array}{l}0.17829^{*} \\
(0.06021)\end{array}$ & $\begin{array}{l}0.14762^{\dagger} \\
(0.06131)\end{array}$ & $\begin{array}{l}0.16246^{*} \\
(0.06173)\end{array}$ & $\begin{array}{l}0.16428^{*} \\
(0.0598)\end{array}$ & $\begin{array}{l}0.13959^{\dagger} \\
(0.06066)\end{array}$ & $\begin{array}{l}0.15439^{\dagger} \\
(0.0605)\end{array}$ & $\begin{array}{l}0.16989^{*} \\
(0.0599)\end{array}$ & $\begin{array}{l}0.1578^{*} \\
(0.06056)\end{array}$ & $\begin{array}{l}0.16989^{*} \\
(0.05988)\end{array}$ & $\begin{array}{l}0.16989^{*} \\
(0.05984)\end{array}$ & $\begin{array}{l}0.16831^{*} \\
(0.05994)\end{array}$ & $\begin{array}{l}0.17337^{*} \\
(0.05985)\end{array}$ \\
\hline Factor 2 & $\begin{array}{l}0.18071^{*} \\
(0.03795)\end{array}$ & $\begin{array}{l}0.18076^{*} \\
(0.03799)\end{array}$ & $\begin{array}{l}0.18017^{*} \\
(0.03797)\end{array}$ & $\begin{array}{l}0.18048^{*} \\
(0.03802)\end{array}$ & $\begin{array}{l}0.18048^{*} \\
(0.03792)\end{array}$ & $\begin{array}{l}0.17094^{*} \\
(0.03803)\end{array}$ & $\begin{array}{l}0.18048^{*} \\
(0.03796)\end{array}$ & $\begin{array}{l}0.18048^{*} \\
(0.03802)\end{array}$ & $\begin{array}{l}0.18023^{*} \\
(0.03799)\end{array}$ & $\begin{array}{l}0.18048^{*} \\
(0.03801)\end{array}$ & $\begin{array}{l}0.18029^{*} \\
(0.03798)\end{array}$ & $\begin{array}{l}0.1804^{*} \\
(0.03801)\end{array}$ & $\begin{array}{l}0.18122^{*} \\
(0.03797)\end{array}$ \\
\hline Factor $2 \& 5$ & $\begin{array}{l}0.37022 \\
(0.22928) \\
\end{array}$ & $\begin{array}{l}0.36771 \\
(0.22955) \\
\end{array}$ & $\begin{array}{l}0.35732 \\
(0.22928) \\
\end{array}$ & $\begin{array}{l}0.35291 \\
(0.22975) \\
\end{array}$ & $\begin{array}{l}0.35732 \\
(0.229) \\
\end{array}$ & $\begin{array}{l}0.34092 \\
(0.22877) \\
\end{array}$ & $\begin{array}{l}0.35732 \\
(0.22926) \\
\end{array}$ & $\begin{array}{l}0.35732 \\
(0.2296) \\
\end{array}$ & $\begin{array}{l}0.35732 \\
(0.2294) \\
\end{array}$ & $\begin{array}{l}0.35732 \\
(0.22952) \\
\end{array}$ & $\begin{array}{l}0.35732 \\
(0.22934) \\
\end{array}$ & $\begin{array}{l}0.35206 \\
(0.22971) \\
\end{array}$ & $\begin{array}{l}0.35732 \\
(0.22927) \\
\end{array}$ \\
\hline Factor $2 \& 4$ & $\begin{array}{l}0.26361^{*} \\
(0.04315)\end{array}$ & $\begin{array}{l}0.25799^{*} \\
(0.04323)\end{array}$ & $\begin{array}{l}0.23446^{*} \\
(0.04262)\end{array}$ & $\begin{array}{l}0.23884^{*} \\
(0.04421)\end{array}$ & $\begin{array}{l}0.23106^{*} \\
(0.04252)\end{array}$ & $\begin{array}{l}0.216^{*} \\
(0.04331)\end{array}$ & $\begin{array}{l}0.24193^{*} \\
(0.04214)\end{array}$ & $\begin{array}{l}0.24426^{*} \\
(0.0424)\end{array}$ & $\begin{array}{l}0.23693^{*} \\
(0.04262)\end{array}$ & $\begin{array}{l}0.24549^{*} \\
(0.04214)\end{array}$ & $\begin{array}{l}0.2419^{*} \\
(0.04218)\end{array}$ & $\begin{array}{l}0.24057^{*} \\
(0.04285)\end{array}$ & $\begin{array}{l}0.24952^{*} \\
(0.04216)\end{array}$ \\
\hline Factor $2,4 \& 5$ & $\begin{array}{l}0.14606^{\dagger} \\
(0.05917)\end{array}$ & $\begin{array}{l}0.13448^{\dagger} \\
(0.05864)\end{array}$ & $\begin{array}{l}0.11849^{\dagger} \\
(0.05798)\end{array}$ & $\begin{array}{l}0.12007^{\dagger} \\
(0.05827)\end{array}$ & $\begin{array}{l}0.12293^{\dagger} \\
(0.05784)\end{array}$ & $\begin{array}{l}0.0962 \\
(0.05856)\end{array}$ & $\begin{array}{l}0.12136^{\dagger} \\
(0.05792)\end{array}$ & $\begin{array}{l}0.1222^{\dagger} \\
(0.05806)\end{array}$ & $\begin{array}{l}0.12236^{\dagger} \\
(0.05795)\end{array}$ & $\begin{array}{l}0.12293^{\dagger} \\
(0.05797)\end{array}$ & $\begin{array}{l}0.1053^{\ddagger} \\
(0.05912)\end{array}$ & $\begin{array}{l}0.11773^{\dagger} \\
(0.05856)\end{array}$ & $\begin{array}{l}0.13763^{\dagger} \\
(0.05857)\end{array}$ \\
\hline Facto & $\begin{array}{l}0.28348^{*} \\
(0.0694)\end{array}$ & $\begin{array}{l}0.28437^{*} \\
(0.06947)\end{array}$ & $\begin{array}{l}0.28252^{*} \\
(0.06943)\end{array}$ & $\begin{array}{l}0.28321^{*} \\
(0.06952)\end{array}$ & $\begin{array}{l}0.26837^{*} \\
(0.06966)\end{array}$ & $\begin{array}{l}0.26095^{*} \\
(0.06972)\end{array}$ & $\begin{array}{l}0.28348^{*} \\
(0.06942)\end{array}$ & $\begin{array}{l}0.28348^{*} \\
(0.06952)\end{array}$ & $\begin{array}{l}0.28348^{*} \\
(0.06946)\end{array}$ & $\begin{array}{l}0.28348^{*} \\
(0.0695)\end{array}$ & $\begin{array}{l}0.28348^{*} \\
(0.06945)\end{array}$ & $\begin{array}{l}0.28181^{*} \\
(0.06956)\end{array}$ & $\begin{array}{l}0.28579^{*} \\
(0.06944)\end{array}$ \\
\hline Facto & $\begin{array}{l}0.39724^{\dagger} \\
(0.17999)\end{array}$ & $\begin{array}{l}0.37281^{\dagger} \\
(0.1797)\end{array}$ & $\begin{array}{l}0.37281^{\dagger} \\
(0.1796)\end{array}$ & $\begin{array}{l}0.37281^{\dagger} \\
(0.17983)\end{array}$ & $\begin{array}{l}0.37281^{\dagger} \\
(0.17938)\end{array}$ & $\begin{array}{l}0.34174^{\ddagger} \\
(0.17949)\end{array}$ & $\begin{array}{l}0.37281^{\dagger} \\
(0.17959)\end{array}$ & $\begin{array}{l}0.37281^{\dagger} \\
(0.17985)\end{array}$ & $\begin{array}{l}0.37281^{\dagger} \\
(0.1797)\end{array}$ & $\begin{array}{l}0.37281^{\dagger} \\
(0.17979)\end{array}$ & $\begin{array}{l}0.37281^{\dagger} \\
(0.17965)\end{array}$ & $\begin{array}{l}0.36816^{\dagger} \\
(0.17997)\end{array}$ & $\begin{array}{l}0.37281^{\dagger} \\
(0.17959)\end{array}$ \\
\hline Factor & $\begin{array}{l}0.24256^{*} \\
(0.04537)\end{array}$ & $\begin{array}{l}0.23109^{*} \\
(0.04482)\end{array}$ & $\begin{array}{l}0.20014^{*} \\
(0.04376)\end{array}$ & $\begin{array}{l}0.20908^{*} \\
(0.0443)\end{array}$ & $\begin{array}{l}0.19441^{*} \\
(0.04374)\end{array}$ & $\begin{array}{l}0.1863^{*} \\
(0.04397)\end{array}$ & $\begin{array}{l}0.21335^{*} \\
(0.04289)\end{array}$ & $\begin{array}{l}0.21322^{*} \\
(0.04321)\end{array}$ & $\begin{array}{l}0.20964^{*} \\
(0.04307)\end{array}$ & $\begin{array}{l}0.21448^{*} \\
(0.04293)\end{array}$ & $\begin{array}{l}0.20672^{*} \\
(0.04322)\end{array}$ & $\begin{array}{l}0.20957^{*} \\
(0.04363)\end{array}$ & $\begin{array}{l}0.23253^{*} \\
(0.04421)\end{array}$ \\
\hline Factor $2,3,4 \& 5$ & $\begin{array}{l}0.24994^{*} \\
(0.04828)\end{array}$ & $\begin{array}{l}0.23495^{*} \\
(0.04711)\end{array}$ & $\begin{array}{l}0.21638^{*} \\
(0.04641)\end{array}$ & $\begin{array}{l}0.21708^{*} \\
(0.048)\end{array}$ & $\begin{array}{l}0.21685^{*} \\
(0.04625)\end{array}$ & $\begin{array}{l}0.19316^{*} \\
(0.04737)\end{array}$ & $\begin{array}{l}0.22106^{*} \\
(0.04624)\end{array}$ & $\begin{array}{l}0.22319^{*} \\
(0.04629)\end{array}$ & $\begin{array}{l}0.21899^{*} \\
(0.04637)\end{array}$ & $\begin{array}{l}0.22341^{*} \\
(0.04627)\end{array}$ & $\begin{array}{l}0.214^{*} \\
(0.04666)\end{array}$ & $\begin{array}{l}0.22165^{*} \\
(0.04636) \\
\end{array}$ & $\begin{array}{l}0.23195^{*} \\
(0.0465)\end{array}$ \\
\hline Factor 1 & $\begin{array}{l}0.27111^{*} \\
(0.02691)\end{array}$ & $\begin{array}{l}0.26992^{*} \\
(0.02695)\end{array}$ & $\begin{array}{l}0.26673^{*} \\
(0.02682)\end{array}$ & $\begin{array}{l}0.2665^{*} \\
(0.02686)\end{array}$ & $\begin{array}{l}0.26216^{*} \\
(0.02687)\end{array}$ & $\begin{array}{l}0.25884^{*} \\
(0.02691)\end{array}$ & $\begin{array}{l}0.26535^{*} \\
(0.02683)\end{array}$ & $\begin{array}{l}0.26649^{*} \\
(0.02688)\end{array}$ & $\begin{array}{l}0.26616^{*} \\
(0.02684)\end{array}$ & $\begin{array}{l}0.26663^{*} \\
(0.02685)\end{array}$ & $\begin{array}{l}0.26589^{*} \\
(0.02684)\end{array}$ & $\begin{array}{l}0.26557^{*} \\
(0.02692)\end{array}$ & $\begin{array}{l}0.2676^{*} \\
(0.02683)\end{array}$ \\
\hline Factor $1 \& 5$ & $\begin{array}{l}0.28259^{*} \\
(0.03197) \\
\end{array}$ & $\begin{array}{l}0.28044^{*} \\
(0.03202)\end{array}$ & $\begin{array}{l}0.27515^{*} \\
(0.03174) \\
\end{array}$ & $\begin{array}{l}0.27515^{*} \\
(0.03178) \\
\end{array}$ & $\begin{array}{l}0.27515^{*} \\
(0.0317) \\
\end{array}$ & $\begin{array}{l}0.27206^{*} \\
(0.03168) \\
\end{array}$ & $\begin{array}{l}0.27515^{*} \\
(0.03174) \\
\end{array}$ & $\begin{array}{l}0.27515^{*} \\
(0.03178) \\
\end{array}$ & $\begin{array}{l}0.27515^{*} \\
(0.03175) \\
\end{array}$ & $\begin{array}{l}0.27515^{*} \\
(0.03177) \\
\end{array}$ & $\begin{array}{l}0.27121^{*} \\
(0.03186) \\
\end{array}$ & $\begin{array}{l}0.27373^{*} \\
(0.03185) \\
\end{array}$ & $\begin{array}{l}0.27811^{*} \\
(0.03179) \\
\end{array}$ \\
\hline Factor 1 & $\begin{array}{l}0.304^{*} \\
(0.03708)\end{array}$ & $\begin{array}{l}0.2898^{*} \\
(0.03567)\end{array}$ & $\begin{array}{l}0.26708^{*} \\
(0.03526)\end{array}$ & $\begin{array}{l}0.27458^{*} \\
(0.03553)\end{array}$ & $\begin{array}{l}0.27182^{*} \\
(0.03465)\end{array}$ & $\begin{array}{l}0.24913^{*} \\
(0.03607)\end{array}$ & $\begin{array}{l}0.26722^{*} \\
(0.0352)\end{array}$ & $\begin{array}{l}0.27857^{*} \\
(0.03462)\end{array}$ & $\begin{array}{l}0.27857^{*} \\
(0.03459)\end{array}$ & $\begin{array}{l}0.2777^{*} \\
(0.03462)\end{array}$ & $\begin{array}{l}0.27162^{*} \\
(0.03489)\end{array}$ & $\begin{array}{l}0.2783^{*} \\
(0.03461)\end{array}$ & $\begin{array}{l}0.2805^{*} \\
(0.03459)\end{array}$ \\
\hline Fac & $\begin{array}{l}0.24137^{*} \\
(0.03559)\end{array}$ & $\begin{array}{l}0.2251^{*} \\
(0.03363)\end{array}$ & $\begin{array}{l}0.20573^{*} \\
(0.03207)\end{array}$ & $\begin{array}{l}0.20943^{*} \\
(0.03217)\end{array}$ & $\begin{array}{l}0.2112^{*} \\
(0.03184)\end{array}$ & $\begin{array}{l}0.19496^{*} \\
(0.03235)\end{array}$ & $\begin{array}{l}0.20048^{*} \\
(0.03252)\end{array}$ & $\begin{array}{l}0.21091^{*} \\
(0.03198)\end{array}$ & $\begin{array}{l}0.20911^{*} \\
(0.03195)\end{array}$ & $\begin{array}{l}0.21141^{*} \\
(0.03192)\end{array}$ & $\begin{array}{l}0.21^{*} \\
(0.03191)\end{array}$ & $\begin{array}{l}0.21112^{*} \\
(0.03193)\end{array}$ & $\begin{array}{l}0.21141^{*} \\
(0.03188)\end{array}$ \\
\hline Fact & $\begin{array}{l}0.29365^{*} \\
(0.02815)\end{array}$ & $\begin{array}{l}0.28507^{*} \\
(0.02771)\end{array}$ & $\begin{array}{l}0.28381^{*} \\
(0.02768)\end{array}$ & $\begin{array}{l}0.28295^{*} \\
(0.02777)\end{array}$ & $\begin{array}{l}0.2836^{*} \\
(0.02765)\end{array}$ & $\begin{array}{l}0.27677^{*} \\
(0.02773)\end{array}$ & $\begin{array}{l}0.28357^{*} \\
(0.02768)\end{array}$ & $\begin{array}{l}0.2833^{*} \\
(0.02778)\end{array}$ & $\begin{array}{l}0.28304^{*} \\
(0.0277)\end{array}$ & $\begin{array}{l}0.28381^{*} \\
(0.02771)\end{array}$ & $\begin{array}{l}0.27794^{*} \\
(0.02797)\end{array}$ & $\begin{array}{l}0.28135^{*} \\
(0.02798)\end{array}$ & $\begin{array}{l}0.28535^{*} \\
(0.0277)\end{array}$ \\
\hline Factor & $\begin{array}{l}0.27149^{*} \\
(0.02278)\end{array}$ & $\begin{array}{l}0.27145^{*} \\
(0.02286)\end{array}$ & $\begin{array}{l}0.26852^{*} \\
(0.02275) \\
\end{array}$ & $\begin{array}{l}0.26858^{*} \\
(0.02278) \\
\end{array}$ & $\begin{array}{l}0.26859^{*} \\
(0.02272)\end{array}$ & $\begin{array}{l}0.26034^{*} \\
(0.02289) \\
\end{array}$ & $\begin{array}{l}0.2687^{*} \\
(0.02275)\end{array}$ & $\begin{array}{l}0.26867^{*} \\
(0.02278)\end{array}$ & $\begin{array}{l}0.2687^{*} \\
(0.02276)\end{array}$ & $\begin{array}{l}0.2687^{*} \\
(0.02277)\end{array}$ & $\begin{array}{l}0.26838^{*} \\
(0.02275)\end{array}$ & $\begin{array}{l}0.26847^{*} \\
(0.02278)\end{array}$ & $\begin{array}{l}0.2687^{*} \\
(0.02275)\end{array}$ \\
\hline Factor $1,3 \& 4$ & $\begin{array}{l}0.21944^{*} \\
(0.02348)\end{array}$ & $\begin{array}{l}0.19674^{*} \\
(0.01856)\end{array}$ & $\begin{array}{l}0.18227^{*} \\
(0.01869)\end{array}$ & $\begin{array}{l}0.18435^{*} \\
(0.0222) \\
\end{array}$ & $\begin{array}{l}0.18856^{*} \\
(0.01797)\end{array}$ & $\begin{array}{l}0.16806^{*} \\
(0.01971)\end{array}$ & $\begin{array}{l}0.18732^{*} \\
(0.01808)\end{array}$ & $\begin{array}{l}0.19042^{*} \\
(0.01805)\end{array}$ & $\begin{array}{l}0.18939^{*} \\
(0.01801)\end{array}$ & $\begin{array}{l}0.19055^{*} \\
(0.01799)\end{array}$ & $\begin{array}{l}0.18962^{*} \\
(0.01799)\end{array}$ & $\begin{array}{l}0.18935^{*} \\
(0.01814) \\
\end{array}$ & $\begin{array}{l}0.19083^{*} \\
(0.01796)\end{array}$ \\
\hline Factor $1,3,4 \& 5$ & $\begin{array}{l}0.25378^{*} \\
(0.03345)\end{array}$ & $\begin{array}{l}0.23629^{*} \\
(0.03103)\end{array}$ & $\begin{array}{l}0.21918^{*} \\
(0.02949)\end{array}$ & $\begin{array}{l}0.21816^{*} \\
(0.03131)\end{array}$ & $\begin{array}{l}0.22207^{*} \\
(0.02935)\end{array}$ & $\begin{array}{l}0.20163^{*} \\
(0.03034)\end{array}$ & $\begin{array}{l}0.21705^{*} \\
(0.02962)\end{array}$ & $\begin{array}{l}0.22323^{*} \\
(0.02943)\end{array}$ & $\begin{array}{l}0.22349^{*} \\
(0.02939)\end{array}$ & $\begin{array}{l}0.22349^{*} \\
(0.02941)\end{array}$ & $\begin{array}{l}0.21498^{*} \\
(0.02993)\end{array}$ & $\begin{array}{l}0.22127^{*} \\
(0.02962)\end{array}$ & $\begin{array}{l}0.22349^{*} \\
(0.02937)\end{array}$ \\
\hline Factor $1 \& 2$ & $\begin{array}{l}0.19941^{*} \\
(0.0354)\end{array}$ & $\begin{array}{l}0.20027^{*} \\
(0.03545)\end{array}$ & $\begin{array}{l}0.19885^{*} \\
(0.03541)\end{array}$ & $\begin{array}{l}0.19876^{*} \\
(0.03546)\end{array}$ & $\begin{array}{l}0.19623^{*} \\
(0.03539)\end{array}$ & $\begin{array}{l}0.17283^{*} \\
(0.03654)\end{array}$ & $\begin{array}{l}0.19885^{*} \\
(0.03541)\end{array}$ & $\begin{array}{l}0.19872^{*} \\
(0.03547)\end{array}$ & $\begin{array}{l}0.19751^{*} \\
(0.03545)\end{array}$ & $\begin{array}{l}0.19885^{*} \\
(0.03545)\end{array}$ & $\begin{array}{l}0.19885^{*} \\
(0.03542)\end{array}$ & $\begin{array}{l}0.19582^{*} \\
(0.03577)\end{array}$ & $\begin{array}{l}0.20528^{*} \\
(0.03562)\end{array}$ \\
\hline Factor $1,2 \& 5$ & $\begin{array}{l}0.20385^{*} \\
(0.05385)\end{array}$ & $\begin{array}{l}0.20344^{*} \\
(0.0539)\end{array}$ & $\begin{array}{l}0.20208^{*} \\
(0.05386)\end{array}$ & $\begin{array}{l}0.20177^{*} \\
(0.05393)\end{array}$ & $\begin{array}{l}0.19464^{*} \\
(0.05389)\end{array}$ & $\begin{array}{l}0.18192^{*} \\
(0.05421)\end{array}$ & $\begin{array}{l}0.20208^{*} \\
(0.05386)\end{array}$ & $\begin{array}{l}0.20106^{*} \\
(0.05407)\end{array}$ & $\begin{array}{l}0.19895^{*} \\
(0.05394)\end{array}$ & $\begin{array}{l}0.20136^{*} \\
(0.05392)\end{array}$ & $\begin{array}{l}0.19782^{*} \\
(0.05395)\end{array}$ & $\begin{array}{l}0.19875^{*} \\
(0.05418)\end{array}$ & $\begin{array}{l}0.20289^{*} \\
(0.05386)\end{array}$ \\
\hline Factor & $\begin{array}{l}0.47435^{*} \\
(0.02969)\end{array}$ & $\begin{array}{l}0.47001^{*} \\
(0.02963)\end{array}$ & $\begin{array}{l}0.46176^{*} \\
(0.02904)\end{array}$ & $\begin{array}{l}0.45994^{*} \\
(0.02953)\end{array}$ & $\begin{array}{l}0.44719^{*} \\
(0.02978)\end{array}$ & $\begin{array}{l}0.42664^{*} \\
(0.03171)\end{array}$ & $\begin{array}{l}0.45443^{*} \\
(0.02942)\end{array}$ & $\begin{array}{l}0.46077^{*} \\
(0.0298)\end{array}$ & $\begin{array}{l}0.46087^{*} \\
(0.02908)\end{array}$ & $\begin{array}{l}0.4622^{*} \\
(0.02907)\end{array}$ & $\begin{array}{l}0.45262^{*} \\
(0.02979)\end{array}$ & $\begin{array}{l}0.45957^{*} \\
(0.02944)\end{array}$ & $\begin{array}{l}0.4632^{*} \\
(0.02904)\end{array}$ \\
\hline Factor $1,2,4 \& 5$ & $\begin{array}{l}0.26502^{*} \\
(0.03479)\end{array}$ & $\begin{array}{l}0.25084^{*} \\
(0.03348)\end{array}$ & $\begin{array}{l}0.24028^{*} \\
(0.03326)\end{array}$ & $\begin{array}{l}0.24032^{*} \\
(0.03446)\end{array}$ & $\begin{array}{l}0.2206^{*} \\
(0.03479)\end{array}$ & $\begin{array}{l}0.21072^{*} \\
(0.03528)\end{array}$ & $\begin{array}{l}0.23393^{*} \\
(0.03376)\end{array}$ & $\begin{array}{l}0.24438^{*} \\
(0.03325)\end{array}$ & $\begin{array}{l}0.24262^{*} \\
(0.03321)\end{array}$ & $\begin{array}{l}0.23035^{*} \\
(0.0373)\end{array}$ & $\begin{array}{l}0.22871^{*} \\
(0.03489)\end{array}$ & $\begin{array}{l}0.24105^{*} \\
(0.03374)\end{array}$ & $\begin{array}{l}0.24625^{*} \\
(0.03315)\end{array}$ \\
\hline Factor & $\begin{array}{l}0.24525^{*} \\
(0.04378) \\
\end{array}$ & $\begin{array}{l}0.24685^{*} \\
(0.04394)\end{array}$ & $\begin{array}{l}0.24091^{*} \\
(0.04377)\end{array}$ & $\begin{array}{l}0.24169^{*} \\
(0.04384) \\
\end{array}$ & $\begin{array}{l}0.2415^{*} \\
(0.04371) \\
\end{array}$ & $\begin{array}{l}0.21864^{*} \\
(0.04448)\end{array}$ & $\begin{array}{l}0.24106^{*} \\
(0.04377) \\
\end{array}$ & $\begin{array}{l}0.24198^{*} \\
(0.04384) \\
\end{array}$ & $\begin{array}{l}0.24006^{*} \\
(0.04382)\end{array}$ & $\begin{array}{l}0.24226^{*} \\
(0.04381) \\
\end{array}$ & $\begin{array}{l}0.24017^{*} \\
(0.0438) \\
\end{array}$ & $\begin{array}{l}0.23702^{*} \\
(0.04459) \\
\end{array}$ & $\begin{array}{l}0.2609^{*} \\
(0.04515) \\
\end{array}$ \\
\hline Factor $1,2,3 \& 5$ & $\begin{array}{l}0.32607^{*} \\
(0.03362)\end{array}$ & $\begin{array}{l}0.3199^{*} \\
(0.03345)\end{array}$ & $\begin{array}{l}0.31885^{*} \\
(0.03342)\end{array}$ & $\begin{array}{l}0.31727^{*} \\
(0.03365)\end{array}$ & $\begin{array}{l}0.31327^{*} \\
(0.03348)\end{array}$ & $\begin{array}{l}0.30324^{*} \\
(0.03382)\end{array}$ & $\begin{array}{l}0.31901^{*} \\
(0.03342)\end{array}$ & $\begin{array}{l}0.31857^{*} \\
(0.03351)\end{array}$ & $\begin{array}{l}0.31629^{*} \\
(0.03351)\end{array}$ & $\begin{array}{l}0.31878^{*} \\
(0.03346)\end{array}$ & $\begin{array}{l}0.31886^{*} \\
(0.03343)\end{array}$ & $\begin{array}{l}0.31509^{*} \\
(0.03403)\end{array}$ & $\begin{array}{l}0.32168^{*} \\
(0.03346)\end{array}$ \\
\hline Factor $1,2,3 \& 4$ & $\begin{array}{l}0.29213^{*} \\
(0.02109)\end{array}$ & $\begin{array}{l}0.27788^{*} \\
(0.01849)\end{array}$ & $\begin{array}{l}0.2571^{*} \\
(0.01805)\end{array}$ & $\begin{array}{l}0.26322^{*} \\
(0.01938)\end{array}$ & $\begin{array}{l}0.25364^{*} \\
(0.01794)\end{array}$ & $\begin{array}{l}0.23551^{*} \\
(0.02044)\end{array}$ & $\begin{array}{l}0.25827^{*} \\
(0.01775)\end{array}$ & $\begin{array}{l}0.26666^{*} \\
(0.01756)\end{array}$ & $\begin{array}{l}0.26222^{*} \\
(0.01738)\end{array}$ & $\begin{array}{l}0.26513^{*} \\
(0.01716)\end{array}$ & $\begin{array}{l}0.26209^{*} \\
(0.01727)\end{array}$ & $\begin{array}{l}0.26429^{*} \\
(0.01781)\end{array}$ & $\begin{array}{l}0.27038^{*} \\
(0.01687)\end{array}$ \\
\hline All Factors & $\begin{array}{l}0.33504^{*} \\
(0.02261)\end{array}$ & $\begin{array}{l}0.32197^{*} \\
(0.02061)\end{array}$ & $\begin{array}{l}0.29538^{*} \\
(0.01949)\end{array}$ & $\begin{array}{l}0.30283^{*} \\
(0.02124)\end{array}$ & $\begin{array}{l}0.29391^{*} \\
(0.0189)\end{array}$ & $\begin{array}{l}0.27522^{*} \\
(0.02142)\end{array}$ & $\begin{array}{l}0.30288^{*} \\
(0.01809)\end{array}$ & $\begin{array}{l}0.30748^{*} \\
(0.01828)\end{array}$ & $\begin{array}{l}0.30171^{*} \\
(0.01855)\end{array}$ & $\begin{array}{l}0.30426^{*} \\
(0.01851)\end{array}$ & $\begin{array}{l}0.29944^{*} \\
(0.01881)\end{array}$ & $\begin{array}{l}0.30424^{*} \\
(0.01908)\end{array}$ & $\begin{array}{l}0.31253^{*} \\
(0.01793)\end{array}$ \\
\hline $\begin{array}{l}\text { Individual } \\
\text { Variable }\end{array}$ & $\begin{array}{l}-0.03044^{\ddagger} \\
(0.01609)\end{array}$ & $\begin{array}{l}-0.01803 \\
(0.01402)\end{array}$ & $\begin{array}{l}0.0272^{\ddagger} \\
(0.01642)\end{array}$ & $\begin{array}{l}0.00765 \\
(0.01537)\end{array}$ & $\begin{array}{l}0.03781^{\dagger} \\
(0.01665)\end{array}$ & $\begin{array}{l}0.03871^{*} \\
(0.01394)\end{array}$ & $\begin{array}{l}0.03079^{\ddagger} \\
(0.01809)\end{array}$ & $\begin{array}{l}0.0053 \\
(0.01985)\end{array}$ & $\begin{array}{l}0.02812 \\
(0.02145)\end{array}$ & $\begin{array}{l}0.02474 \\
(0.02891)\end{array}$ & $\begin{array}{l}0.02369 \\
(0.01588)\end{array}$ & $\begin{array}{l}0.00913 \\
(0.01439)\end{array}$ & $\begin{array}{c}-0.04751^{\ddagger} \\
(0.02825)\end{array}$ \\
\hline
\end{tabular}

Table 90 Coefficients with individual variables for high-level factors - Medium Plants 


\begin{tabular}{|c|c|c|c|c|c|c|c|c|c|c|c|c|c|}
\hline $\begin{array}{l}\text { Individual variable } \\
\text { included in the } \\
\text { model in the } \\
\text { column }\end{array}$ & 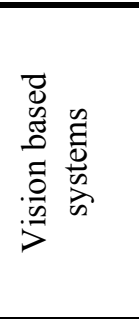 & 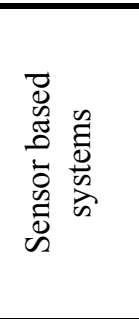 & 㤂 & $\underset{3}{z}$ & 馬 & $\begin{array}{l}= \\
\vec{a} \\
\tilde{\Sigma}\end{array}$ & 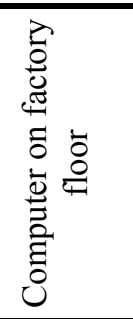 & $\sum_{U}$ & 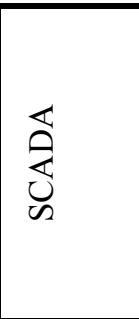 & 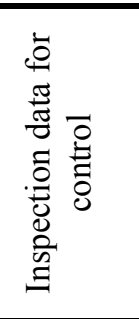 & 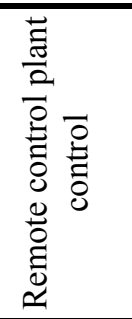 & 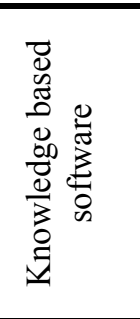 & 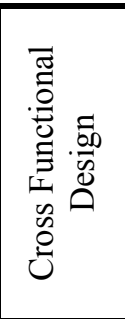 \\
\hline No factors & $\begin{array}{l}0.26859^{*} \\
(0.01301)\end{array}$ & $\begin{array}{l}0.26925^{*} \\
(0.01303)\end{array}$ & $\begin{array}{l}0.27131^{*} \\
(0.01303)\end{array}$ & $\begin{array}{l}0.26917^{*} \\
(0.01309)\end{array}$ & $\begin{array}{l}0.26058^{*} \\
(0.01342)\end{array}$ & $\begin{array}{l}0.26951^{*} \\
(0.01303)\end{array}$ & $\begin{array}{l}0.27031^{*} \\
(0.01311)\end{array}$ & $\begin{array}{l}0.27138^{*} \\
(0.01304)\end{array}$ & $\begin{array}{l}0.26994^{*} \\
(0.01304)\end{array}$ & $\begin{array}{l}0.26968^{*} \\
(0.01304)\end{array}$ & $\begin{array}{l}0.26967^{*} \\
(0.01303)\end{array}$ & $\begin{array}{l}26958^{*} \\
.01302)\end{array}$ & $\begin{array}{l}0.26713^{*} \\
(0.01299)\end{array}$ \\
\hline Factor 5 & $\begin{array}{l}0.11275 \\
(0.06925)\end{array}$ & $\begin{array}{l}0.11275 \\
(0.06939)\end{array}$ & $\begin{array}{l}0.12385^{\ddagger} \\
(0.06949)\end{array}$ & $\begin{array}{l}0.10985 \\
(0.06976)\end{array}$ & $\begin{array}{l}0.10044 \\
(0.06932)\end{array}$ & $\begin{array}{l}0.11138 \\
(0.0694)\end{array}$ & $\begin{array}{l}0.1132 \\
(0.06942)\end{array}$ & $\begin{array}{l}0.11275 \\
(0.0693)\end{array}$ & \begin{tabular}{|l|}
0.11275 \\
$(0.06941)$
\end{tabular} & $\begin{array}{l}0.1128 \\
(0.06955)\end{array}$ & $\begin{array}{l}0.11275 \\
(0.06942)\end{array}$ & $\begin{array}{l}0.11275 \\
(0.06938)\end{array}$ & \begin{tabular}{|l|}
0.09843 \\
$(0.06922)$
\end{tabular} \\
\hline Factor 4 & $\begin{array}{l}0.15018^{*} \\
(0.03292)\end{array}$ & $\begin{array}{l}0.1512^{*} \\
(0.03298)\end{array}$ & $\begin{array}{l}0.16331^{*} \\
(0.03346)\end{array}$ & $\begin{array}{l}0.14943^{*} \\
(0.0333)\end{array}$ & $\begin{array}{l}0.14366^{*} \\
(0.033)\end{array}$ & $\begin{array}{l}0.15105^{*} \\
(0.03298)\end{array}$ & $\begin{array}{l}0.15166^{*} \\
(0.03301)\end{array}$ & $\begin{array}{l}0.15605^{*} \\
(0.03303)\end{array}$ & \begin{tabular}{|l|}
$0.15203^{*}$ \\
$(0.03302)$
\end{tabular} & $\begin{array}{l}0.1513^{*} \\
(0.033)\end{array}$ & $\begin{array}{l}0.15129^{*} \\
(0.033)\end{array}$ & $\begin{array}{l}0.15119^{*} \\
(0.03298)\end{array}$ & \begin{tabular}{|l}
$0.14723^{*}$ \\
$(0.03286)$
\end{tabular} \\
\hline Factor $4 \& 5$ & $\begin{array}{l}0.22054^{\ddagger} \\
(0.12779)\end{array}$ & $\begin{array}{l}0.23543^{\ddagger} \\
(0.12786)\end{array}$ & $\begin{array}{l}0.2569^{\dagger} \\
(0.12808)\end{array}$ & $\begin{array}{l}0.23404^{\ddagger} \\
(0.12795)\end{array}$ & $\begin{array}{l}0.22413^{\ddagger} \\
(0.12752) \\
\end{array}$ & $\begin{array}{l}0.23543^{\ddagger} \\
(0.12785)\end{array}$ & $\begin{array}{l}0.23543^{\ddagger} \\
(0.1279) \\
\end{array}$ & $\begin{array}{l}0.23543^{\ddagger} \\
(0.12768)\end{array}$ & $\begin{array}{l}0.23543^{\ddagger} \\
(0.12789)\end{array}$ & $\begin{array}{l}0.23554^{\ddagger} \\
(0.12816)\end{array}$ & $\begin{array}{l}0.23543^{\ddagger} \\
(0.12791)\end{array}$ & $\begin{array}{l}0.23543^{\ddagger} \\
(0.12784)\end{array}$ & $\begin{array}{l}0.22484^{*} \\
(0.12732)\end{array}$ \\
\hline Factor 3 & $\begin{array}{l}0.22982^{*} \\
(0.04381)\end{array}$ & $\begin{array}{l}0.23367^{*} \\
(0.04386)\end{array}$ & $\begin{array}{l}0.25245^{*} \\
(0.04475)\end{array}$ & $\begin{array}{l}0.23249^{*} \\
(0.04397)\end{array}$ & $\begin{array}{l}0.23116^{*} \\
(0.04373)\end{array}$ & $\begin{array}{l}0.22619^{*} \\
(0.04456)\end{array}$ & $\begin{array}{l}0.23504^{*} \\
(0.04399)\end{array}$ & $\begin{array}{l}0.23367^{*} \\
(0.0438)\end{array}$ & $\begin{array}{l}0.23421^{*} \\
(0.04388)\end{array}$ & $\begin{array}{l}0.2337^{*} \\
(0.04392)\end{array}$ & $\begin{array}{l}0.23367^{*} \\
(0.04388)\end{array}$ & $\begin{array}{l}0.23178^{*} \\
(0.04389)\end{array}$ & \begin{tabular}{|l}
$0.23287^{*}$ \\
$(0.04366)$
\end{tabular} \\
\hline Facto & $\begin{array}{l}0.15852^{*} \\
(0.05069)\end{array}$ & $\begin{array}{l}0.15852^{*} \\
(0.0508)\end{array}$ & $\begin{array}{l}0.18451^{*} \\
(0.0523)\end{array}$ & $\begin{array}{l}0.15667^{*} \\
(0.05101)\end{array}$ & $\begin{array}{l}0.14814^{*} \\
(0.05078)\end{array}$ & $\begin{array}{l}0.15852^{*} \\
(0.05079)\end{array}$ & $\begin{array}{l}0.15896^{*} \\
(0.05082)\end{array}$ & $\begin{array}{l}0.15852^{*} \\
(0.05073)\end{array}$ & $\begin{array}{l}0.15903^{*} \\
(0.05082)\end{array}$ & $\begin{array}{l}0.15854^{*} \\
(0.05083)\end{array}$ & $\begin{array}{l}0.15852^{*} \\
(0.05082)\end{array}$ & & $\begin{array}{l}0.12998^{\dagger} \\
(0.05137)\end{array}$ \\
\hline Facto & $\begin{array}{l}0.22511^{*} \\
(0.04825)\end{array}$ & $\begin{array}{l}0.22238^{*} \\
(0.04845)\end{array}$ & $\begin{array}{l}0.24941^{*} \\
(0.04973)\end{array}$ & $\begin{array}{l}0.22115^{*} \\
(0.0493)\end{array}$ & $\begin{array}{l}0.21588^{*} \\
(0.04832)\end{array}$ & $\begin{array}{l}0.22191^{*} \\
(0.04847)\end{array}$ & $\begin{array}{l}0.22741^{*} \\
(0.04866)\end{array}$ & $\begin{array}{l}0.22759^{*} \\
(0.0483)\end{array}$ & $\begin{array}{l}0.22511^{*} \\
(0.04836)\end{array}$ & $\begin{array}{l}0.22514^{*} \\
(0.04841)\end{array}$ & $\begin{array}{l}0.22511^{*} \\
(0.04837)\end{array}$ & $\begin{array}{l}0.22511^{*} \\
(0.04834)\end{array}$ & $\begin{array}{l}0.21647^{*} \\
(0.04821)\end{array}$ \\
\hline Factor $3,4 \& 5$ & $\begin{array}{l}0.16989^{*} \\
(0.05976)\end{array}$ & $\begin{array}{l}0.16989^{*} \\
(0.05988)\end{array}$ & $\begin{array}{l}0.18358^{*} \\
(0.06016)\end{array}$ & $\begin{array}{l}0.16823^{*} \\
(0.06003)\end{array}$ & $\begin{array}{l}0.16056^{*} \\
(0.05979)\end{array}$ & $\begin{array}{l}0.16804^{*} \\
(0.05991)\end{array}$ & $\begin{array}{l}0.17132^{*} \\
(0.05999)\end{array}$ & $\begin{array}{l}0.17204^{*} \\
(0.05981)\end{array}$ & $\begin{array}{l}0.16989^{*} \\
(0.05989)\end{array}$ & $\begin{array}{l}0.16991^{*} \\
(0.05992)\end{array}$ & $\begin{array}{l}0.16762^{*} \\
(0.06115)\end{array}$ & $\begin{array}{l}0.1673^{*} \\
(0.05992)\end{array}$ & \begin{tabular}{|l}
$0.15481^{*}$ \\
$(0.0598)$
\end{tabular} \\
\hline Factor 2 & $\begin{array}{l}0.18003^{*} \\
(0.03793)\end{array}$ & $\begin{array}{l}0.17999^{*} \\
(0.03801)\end{array}$ & $\begin{array}{l}0.19087^{*} \\
(0.03828)\end{array}$ & $\begin{array}{l}0.18028^{*} \\
(0.03802)\end{array}$ & $\begin{array}{l}0.17891^{*} \\
(0.03789)\end{array}$ & $\begin{array}{l}0.17754^{*} \\
(0.03813)\end{array}$ & $\begin{array}{l}0.18378^{*} \\
(0.03877)\end{array}$ & $\begin{array}{l}0.19076^{*} \\
(0.03834)\end{array}$ & $\begin{array}{l}0.18372^{*} \\
(0.03844)\end{array}$ & $\begin{array}{l}0.18056^{*} \\
(0.03841)\end{array}$ & $\begin{array}{l}0.18038^{*} \\
(0.03802)\end{array}$ & $\begin{array}{l}0.18019^{*} \\
(0.038)\end{array}$ & \begin{tabular}{|l}
$0.1632^{*}$ \\
$(0.03823)$
\end{tabular} \\
\hline Facto & $\begin{array}{l}0.34071 \\
(0.22917) \\
\end{array}$ & $\begin{array}{l}0.35732 \\
(0.22951) \\
\end{array}$ & $\begin{array}{l}0.36899 \\
(0.22919) \\
\end{array}$ & $\begin{array}{l}0.35161 \\
(0.22999) \\
\end{array}$ & $\begin{array}{l}0.32408 \\
(0.2291) \\
\end{array}$ & $\begin{array}{l}0.35732 \\
(0.2295) \\
\end{array}$ & $\begin{array}{l}0.36069 \\
(0.22971) \\
\end{array}$ & $\begin{array}{l}0.37372 \\
(0.22935) \\
\end{array}$ & \begin{tabular}{|l|}
0.36107 \\
$(0.22966)$ \\
\end{tabular} & $\begin{array}{l}0.35752 \\
(0.23008) \\
\end{array}$ & $\begin{array}{l}0.35551 \\
(0.22981) \\
\end{array}$ & $\begin{array}{l}0.3401 \\
(0.23002) \\
\end{array}$ & \begin{tabular}{|l|}
0.33939 \\
$(0.22852)$ \\
\end{tabular} \\
\hline Factor $2 \& 4$ & $\begin{array}{l}0.23398^{*} \\
(0.04238)\end{array}$ & $\begin{array}{l}0.24054^{*} \\
(0.04249)\end{array}$ & $\begin{array}{l}0.26243^{*} \\
(0.04289)\end{array}$ & $\begin{array}{l}0.24115^{*} \\
(0.04342)\end{array}$ & $\begin{array}{l}0.21861^{*} \\
(0.04318)\end{array}$ & $\begin{array}{l}0.24072^{*} \\
(0.04243)\end{array}$ & $\begin{array}{l}0.25015^{*} \\
(0.04351)\end{array}$ & $\begin{array}{l}0.25351^{*} \\
(0.0423)\end{array}$ & $\begin{array}{l}0.25003^{*} \\
(0.04291)\end{array}$ & $\begin{array}{l}0.24557^{*} \\
(0.04255)\end{array}$ & $\begin{array}{l}0.24521^{*} \\
(0.04218)\end{array}$ & & \begin{tabular}{|l|}
$0.2319^{*}$ \\
$(0.04217)$
\end{tabular} \\
\hline Factor $2,4 \& 5$ & $\begin{array}{l}0.11^{\ddagger} \\
(0.05816)\end{array}$ & $\begin{array}{l}0.10876^{\ddagger} \\
(0.06005)\end{array}$ & $\begin{array}{l}0.13872^{\dagger} \\
(0.05839) \\
\end{array}$ & $\begin{array}{l}0.11737^{\dagger} \\
(0.05951)\end{array}$ & $\begin{array}{l}0.10004^{\ddagger} \\
(0.05841)\end{array}$ & $\begin{array}{l}0.11791^{\dagger} \\
(0.05821)\end{array}$ & $\begin{array}{l}0.12696^{\dagger} \\
(0.05873) \\
\end{array}$ & $\begin{array}{l}0.14984^{\dagger} \\
(0.05964) \\
\end{array}$ & $\begin{array}{l}0.12603^{\dagger} \\
(0.05824) \\
\end{array}$ & $\begin{array}{l}0.12303^{\dagger} \\
(0.05839)\end{array}$ & $\begin{array}{l}0.12169^{\dagger} \\
(0.05838)\end{array}$ & & \begin{tabular}{|l}
$0.11977^{\dagger}$ \\
$(0.05771)$
\end{tabular} \\
\hline Facto & $\begin{array}{l}0.28158^{*} \\
(0.06936)\end{array}$ & $\begin{array}{l}0.27721^{*} \\
(0.06984)\end{array}$ & $\begin{array}{l}0.30666^{*} \\
(0.07031)\end{array}$ & $\begin{array}{l}0.27856^{*} \\
(0.07052)\end{array}$ & $\begin{array}{l}0.25317^{*} \\
(0.07019)\end{array}$ & $\begin{array}{l}0.28255^{*} \\
(0.0695)\end{array}$ & $\begin{array}{l}0.28811^{*} \\
(0.07034)\end{array}$ & $\begin{array}{l}0.29335^{*} \\
(0.0696)\end{array}$ & \begin{tabular}{|l|}
$0.2873^{*}$ \\
$(0.06984)$
\end{tabular} & $\begin{array}{l}0.28366^{*} \\
(0.07077)\end{array}$ & $\begin{array}{l}0.28312^{*} \\
(0.06955)\end{array}$ & & \begin{tabular}{|l|}
$0.27411^{*}$ \\
$(0.06924)$
\end{tabular} \\
\hline $\mathrm{F}$ & $\begin{array}{l}0.37281^{\dagger} \\
(0.17942)\end{array}$ & $\begin{array}{l}0.37281^{\dagger} \\
(0.17978)\end{array}$ & $\begin{array}{l}0.39228^{\dagger} \\
(0.17973)\end{array}$ & $\begin{array}{l}0.3671^{\dagger} \\
(0.18036)\end{array}$ & $\begin{array}{l}0.33957^{\ddagger} \\
(0.17963)\end{array}$ & $\begin{array}{l}0.35963^{\dagger} \\
(0.1803)\end{array}$ & $\begin{array}{l}0.37452^{\dagger} \\
(0.17988)\end{array}$ & $\begin{array}{l}0.38731^{\dagger} \\
(0.1797)\end{array}$ & $\begin{array}{l}0.38166^{\dagger} \\
(0.18051)\end{array}$ & $\begin{array}{l}0.37295^{\dagger} \\
(0.18016)\end{array}$ & $\begin{array}{l}0.37281^{\dagger} \\
(0.17985)\end{array}$ & $\begin{array}{l}0.36403^{\dagger} \\
(0.17993)\end{array}$ & $\begin{array}{l}0.35124 \\
(0.17908)\end{array}$ \\
\hline Factor $2,3 \& 4$ & $\begin{array}{l}0.20818^{*} \\
(0.04294)\end{array}$ & $\begin{array}{l}0.21077^{*} \\
(0.04313)\end{array}$ & $\begin{array}{l}0.23736^{*} \\
(0.04432)\end{array}$ & $\begin{array}{l}0.20984^{*} \\
(0.04437)\end{array}$ & $\begin{array}{l}0.19474^{*} \\
(0.04342)\end{array}$ & $\begin{array}{l}0.21068^{*} \\
(0.04312)\end{array}$ & $\begin{array}{l}0.21802^{*} \\
(0.04372)\end{array}$ & $\begin{array}{l}0.2253^{*} \\
(0.04326)\end{array}$ & $\begin{array}{l}0.21945^{*} \\
(0.04384)\end{array}$ & $\begin{array}{l}0.21464^{*} \\
(0.04451)\end{array}$ & $\begin{array}{l}0.21397^{*} \\
(0.04304)\end{array}$ & $\begin{array}{l}0.20763^{*} \\
(0.04339)\end{array}$ & \begin{tabular}{|l}
$0.18541^{*}$ \\
$(0.04373)$
\end{tabular} \\
\hline Factor $2,3,4 \& 5$ & $\begin{array}{l}0.21619^{*} \\
(0.04629)\end{array}$ & $\begin{array}{l}0.21595^{*} \\
(0.047)\end{array}$ & $\begin{array}{l}0.2506^{*} \\
(0.04809)\end{array}$ & $\begin{array}{l}0.21822^{*} \\
(0.04793)\end{array}$ & $\begin{array}{l}0.19721^{*} \\
(0.04714)\end{array}$ & $\begin{array}{l}0.21614^{*} \\
(0.04689)\end{array}$ & $\begin{array}{l}0.22815^{*} \\
(0.04756)\end{array}$ & $\begin{array}{l}0.23273^{*} \\
(0.04647)\end{array}$ & $\begin{array}{l}0.22618^{*} \\
(0.04654)\end{array}$ & $\begin{array}{l}0.22352^{*} \\
(0.04698)\end{array}$ & $\begin{array}{l}0.22281^{*} \\
(0.0464)\end{array}$ & $\begin{array}{l}0.21452^{*} \\
(0.04699)\end{array}$ & \begin{tabular}{|l}
$0.19783^{*}$ \\
$(0.04677)$
\end{tabular} \\
\hline Factor 1 & $\begin{array}{l}0.266^{*} \\
(0.0268) \\
\end{array}$ & $\begin{array}{l}0.26658^{*} \\
(0.02685)\end{array}$ & $\begin{array}{l}0.27271^{*} \\
(0.02697)\end{array}$ & $\begin{array}{l}0.26491^{*} \\
(0.02721)\end{array}$ & $\begin{array}{l}0.25566^{*} \\
(0.02708)\end{array}$ & $\begin{array}{l}0.26606^{*} \\
(0.02686)\end{array}$ & $\begin{array}{l}0.26791^{*} \\
(0.027)\end{array}$ & $\begin{array}{l}0.2677^{*} \\
(0.02682)\end{array}$ & $\begin{array}{l}0.26736^{*} \\
(0.02688)\end{array}$ & $\begin{array}{l}0.26677^{*} \\
(0.02703)\end{array}$ & $\begin{array}{l}0.26673^{*} \\
(0.02686)\end{array}$ & $\begin{array}{l}0.26517^{*} \\
(0.02689)\end{array}$ & $\begin{array}{l}0.26432^{*} \\
(0.02674)\end{array}$ \\
\hline Factor $1 \& 5$ & $\begin{array}{l}0.27515^{*} \\
(0.03171)\end{array}$ & $\begin{array}{l}0.27497^{*} \\
(0.03177)\end{array}$ & $\begin{array}{l}0.28638^{*} \\
(0.03219)\end{array}$ & $\begin{array}{l}0.27279^{*} \\
(0.03228)\end{array}$ & $\begin{array}{l}0.26125^{*} \\
(0.03209)\end{array}$ & $\begin{array}{l}0.27288^{*} \\
(0.03186)\end{array}$ & $\begin{array}{l}0.27703^{*} \\
(0.03208)\end{array}$ & $\begin{array}{l}0.27779^{*} \\
(0.03176)\end{array}$ & $\begin{array}{l}0.27737^{*} \\
(0.03202)\end{array}$ & $\begin{array}{l}0.27518^{*} \\
(0.03189)\end{array}$ & $\begin{array}{l}0.27515^{*} \\
(0.03178)\end{array}$ & $\begin{array}{l}0.27264^{*} \\
(0.03185)\end{array}$ & \begin{tabular}{|l}
$0.27347^{*}$ \\
$(0.03163)$
\end{tabular} \\
\hline Factor & $\begin{array}{l}0.27789^{*} \\
(0.03454)\end{array}$ & $\begin{array}{l}0.27808^{*} \\
(0.03461)\end{array}$ & $\begin{array}{l}0.29202^{*} \\
(0.03517)\end{array}$ & $\begin{array}{l}0.2766^{*} \\
(0.03494)\end{array}$ & $\begin{array}{l}0.27366^{*} \\
(0.03454)\end{array}$ & $\begin{array}{l}0.2761^{*} \\
(0.0347)\end{array}$ & $\begin{array}{l}0.28022^{*} \\
(0.03482)\end{array}$ & $\begin{array}{l}0.28135^{*} \\
(0.03459)\end{array}$ & $\begin{array}{l}0.27868^{*} \\
(0.03461)\end{array}$ & $\begin{array}{l}0.2786^{*} \\
(0.03467)\end{array}$ & $\begin{array}{l}0.27854^{*} \\
(0.03462)\end{array}$ & $\begin{array}{l}0.27366^{*} \\
(0.0349)\end{array}$ & \begin{tabular}{|l}
$0.26607^{*}$ \\
$(0.03467)$
\end{tabular} \\
\hline $\mathrm{Fs}$ & $\begin{array}{l}0.20926^{*} \\
(0.03187)\end{array}$ & $\begin{array}{l}0.21074^{*} \\
(0.03192)\end{array}$ & $\begin{array}{l}0.23422^{*} \\
(0.03379) \\
\end{array}$ & $\begin{array}{l}0.20775^{*} \\
(0.03311) \\
\end{array}$ & $\begin{array}{l}0.20263^{*} \\
(0.03198) \\
\end{array}$ & $\begin{array}{l}0.20982^{*} \\
(0.03196) \\
\end{array}$ & $\begin{array}{l}0.21192^{*} \\
(0.03195) \\
\end{array}$ & $\begin{array}{l}0.21627^{*} \\
(0.03198) \\
\end{array}$ & $\begin{array}{l}0.21148^{*} \\
(0.03192) \\
\end{array}$ & $\begin{array}{l}0.21143^{*} \\
(0.03196)\end{array}$ & $\begin{array}{l}0.21135^{*} \\
(0.03193) \\
\end{array}$ & $\begin{array}{l}0.21044^{*} \\
(0.03192)\end{array}$ & \begin{tabular}{|l}
$0.19598^{*}$ \\
$(0.03215)$ \\
\end{tabular} \\
\hline $\mathrm{F}$ & $\begin{array}{l}0.28359^{*} \\
(0.02765)\end{array}$ & $\begin{array}{l}0.28364^{*} \\
(0.02771)\end{array}$ & $\begin{array}{l}0.30462^{*} \\
(0.0295)\end{array}$ & $\begin{array}{l}0.27972^{*} \\
(0.02941)\end{array}$ & $\begin{array}{l}0.27131^{*} \\
(0.02801)\end{array}$ & $\begin{array}{l}0.28182^{*} \\
(0.02779)\end{array}$ & $\begin{array}{l}0.28663^{*} \\
(0.02847)\end{array}$ & $\begin{array}{l}0.28644^{*} \\
(0.02771)\end{array}$ & \begin{tabular}{|l|}
$0.28564^{*}$ \\
$(0.0279)$
\end{tabular} & $\begin{array}{l}0.28385^{*} \\
(0.02789)\end{array}$ & $\begin{array}{l}0.28367^{*} \\
(0.02773)\end{array}$ & $\begin{array}{l}0.28232^{*} \\
(0.02774)\end{array}$ & $\begin{array}{l}0.26686^{*} \\
(0.02811)\end{array}$ \\
\hline Factor & $\begin{array}{l}0.2687^{*} \\
(0.02272)\end{array}$ & $\begin{array}{l}0.2687^{*} \\
(0.02277)\end{array}$ & $\begin{array}{l}0.29337^{*} \\
(0.02577)\end{array}$ & $\begin{array}{l}0.26732^{*} \\
(0.02302)\end{array}$ & $\begin{array}{l}0.26596^{*} \\
(0.02272)\end{array}$ & $\begin{array}{l}0.26845^{*} \\
(0.02277)\end{array}$ & $\begin{array}{l}0.26962^{*} \\
(0.02288)\end{array}$ & $\begin{array}{l}0.2687^{*} \\
(0.02274)\end{array}$ & \begin{tabular}{|l|}
$0.2688^{*}$ \\
$(0.02278)$
\end{tabular} & $\begin{array}{l}0.26872^{*} \\
(0.0228)\end{array}$ & $\begin{array}{l}0.2687^{*} \\
(0.02278)\end{array}$ & $\begin{array}{l}0.2687^{*} \\
(0.02277)\end{array}$ & $\begin{array}{l}0.26272^{*} \\
(0.02274)\end{array}$ \\
\hline Factor $1,3 \& 4$ & $\begin{array}{l}0.1905^{*} \\
(0.01795)\end{array}$ & $\begin{array}{l}0.19056^{*} \\
(0.01799)\end{array}$ & $\begin{array}{l}0.21134^{*} \\
(0.0206) \\
\end{array}$ & $\begin{array}{l}0.18979^{*} \\
(0.01816)\end{array}$ & $\begin{array}{l}0.17511^{*} \\
(0.01886)\end{array}$ & $\begin{array}{l}0.18363^{*} \\
(0.01951)\end{array}$ & $\begin{array}{l}0.19361^{*} \\
(0.01911)\end{array}$ & $\begin{array}{l}0.19299^{*} \\
(0.018)\end{array}$ & $\begin{array}{l}0.19092^{*} \\
(0.01799) \\
\end{array}$ & $\begin{array}{l}0.19084^{*} \\
(0.01801)\end{array}$ & $\begin{array}{l}0.1908^{*} \\
(0.01799) \\
\end{array}$ & $\begin{array}{l}0.19072^{*} \\
(0.01798)\end{array}$ & $\begin{array}{l}0.16223^{*} \\
(0.02009) \\
\end{array}$ \\
\hline Factor $1,3,4 \& 5$ & $\begin{array}{l}0.22241^{*} \\
(0.02935)\end{array}$ & $\begin{array}{l}0.22306^{*} \\
(0.02941)\end{array}$ & $\begin{array}{l}0.24344^{*} \\
(0.03096)\end{array}$ & $\begin{array}{l}0.221^{*} \\
(0.03002)\end{array}$ & $\begin{array}{l}0.21971^{*} \\
(0.02934)\end{array}$ & $\begin{array}{l}0.21932^{*} \\
(0.02973)\end{array}$ & $\begin{array}{l}0.22539^{*} \\
(0.02974)\end{array}$ & $\begin{array}{l}0.22468^{*} \\
(0.02937)\end{array}$ & \begin{tabular}{|l|}
$0.22463^{*}$ \\
$(0.02948)$
\end{tabular} & $\begin{array}{l}0.22355^{*} \\
(0.0297)\end{array}$ & $\begin{array}{l}0.22347^{*} \\
(0.02942)\end{array}$ & $\begin{array}{l}0.22123^{*} \\
(0.02948)\end{array}$ & \begin{tabular}{|l}
$0.19979^{*}$ \\
$(0.03023)$
\end{tabular} \\
\hline Factor $1 \& 2$ & $\begin{array}{l}0.19115^{*} \\
(0.03555)\end{array}$ & $\begin{array}{l}0.19337^{*} \\
(0.03596)\end{array}$ & $\begin{array}{l}0.21068^{*} \\
(0.03586)\end{array}$ & $\begin{array}{l}0.19571^{*} \\
(0.03625)\end{array}$ & $\begin{array}{l}0.17005^{*} \\
(0.03694)\end{array}$ & $\begin{array}{l}0.19121^{*} \\
(0.03634)\end{array}$ & $\begin{array}{l}0.20213^{*} \\
(0.03626)\end{array}$ & $\begin{array}{l}0.20364^{*} \\
(0.03549)\end{array}$ & \begin{tabular}{|l|}
$0.20098^{*}$ \\
$(0.03566)$
\end{tabular} & $\begin{array}{l}0.19902^{*} \\
(0.0374)\end{array}$ & $\begin{array}{l}0.19844^{*} \\
(0.03553)\end{array}$ & $\begin{array}{l}0.19377^{*} \\
(0.03575)\end{array}$ & $\begin{array}{l}0.17453^{*} \\
(0.03613)\end{array}$ \\
\hline Factor $1,2 \& 5$ & $\begin{array}{l}0.19108^{*} \\
(0.05404)\end{array}$ & $\begin{array}{l}0.19492^{*} \\
(0.05449)\end{array}$ & $\begin{array}{l}0.22652^{*} \\
(0.05515)\end{array}$ & $\begin{array}{l}0.19833^{*} \\
(0.05468)\end{array}$ & $\begin{array}{l}0.19606^{*} \\
(0.05379)\end{array}$ & $\begin{array}{l}0.19558^{*} \\
(0.05434)\end{array}$ & $\begin{array}{l}0.20528^{*} \\
(0.05444)\end{array}$ & $\begin{array}{l}0.21172^{*} \\
(0.05408)\end{array}$ & \begin{tabular}{|l|}
$0.20561^{*}$ \\
$(0.05429)$
\end{tabular} & $\begin{array}{l}0.2022^{*} \\
(0.05465)\end{array}$ & $\begin{array}{l}0.20077^{*} \\
(0.0544)\end{array}$ & $\begin{array}{l}0.19192^{*} \\
(0.05472)\end{array}$ & \begin{tabular}{|l}
$0.19914^{*}$ \\
$(0.05367)$
\end{tabular} \\
\hline Fact & $\begin{array}{l}0.44658^{*} \\
(0.02992)\end{array}$ & $\begin{array}{l}0.46055^{*} \\
(0.02915)\end{array}$ & $\begin{array}{l}0.47573^{*} \\
(0.02974)\end{array}$ & $\begin{array}{l}0.45707^{*} \\
(0.0319)\end{array}$ & $\begin{array}{l}0.44905^{*} \\
(0.02941)\end{array}$ & $\begin{array}{l}0.45614^{*} \\
(0.02983)\end{array}$ & $\begin{array}{l}0.4678^{*} \\
(0.03153)\end{array}$ & $\begin{array}{l}0.46723^{*} \\
(0.02913)\end{array}$ & \begin{tabular}{|l|}
$0.46408^{*}$ \\
$(0.0292)$
\end{tabular} & $\begin{array}{l}0.46263^{*} \\
(0.03008)\end{array}$ & $\begin{array}{l}0.46199^{*} \\
(0.02922)\end{array}$ & $\begin{array}{l}0.46071^{*} \\
(0.02911)\end{array}$ & \begin{tabular}{|l}
$0.45754^{*}$ \\
$(0.02898)$
\end{tabular} \\
\hline Factor & $\begin{array}{l}0.23202^{*} \\
(0.03363)\end{array}$ & $\begin{array}{l}0.23847^{*} \\
(0.03394)\end{array}$ & $\begin{array}{l}0.26218^{*} \\
(0.03419) \\
\end{array}$ & $\begin{array}{l}0.2401^{*} \\
(0.03517)\end{array}$ & $\begin{array}{l}0.22138^{*} \\
(0.03422)\end{array}$ & $\begin{array}{l}0.23746^{*} \\
(0.03409)\end{array}$ & $\begin{array}{l}0.25049^{*} \\
(0.03558)\end{array}$ & $\begin{array}{l}0.26057^{*} \\
(0.03416)\end{array}$ & $\begin{array}{l}0.25229^{*} \\
(0.03564)\end{array}$ & $\begin{array}{l}0.24502^{*} \\
(0.03369)\end{array}$ & $\begin{array}{l}0.24371^{*} \\
(0.03384)\end{array}$ & $\begin{array}{l}0.23353^{*} \\
(0.03482)\end{array}$ & $\begin{array}{l}0.23781^{*} \\
(0.0331)\end{array}$ \\
\hline $\mathrm{F}$ & $\begin{array}{l}0.23313^{*} \\
(0.04392) \\
\end{array}$ & $\begin{array}{l}0.23805^{*} \\
(0.04406)\end{array}$ & $\begin{array}{l}0.26664^{*} \\
(0.04535) \\
\end{array}$ & $\begin{array}{l}0.23779^{*} \\
(0.04513) \\
\end{array}$ & $\begin{array}{l}0.21515^{*} \\
(0.04483) \\
\end{array}$ & $\begin{array}{l}0.23401^{*} \\
(0.04466) \\
\end{array}$ & $\begin{array}{l}0.2473^{*} \\
(0.04534) \\
\end{array}$ & $\begin{array}{l}0.24906^{*} \\
(0.0439) \\
\end{array}$ & \begin{tabular}{|l|l|}
$0.24705^{*}$ \\
$(0.04463)$ \\
\end{tabular} & $\begin{array}{l}0.24242^{*} \\
(0.04521)\end{array}$ & $\begin{array}{l}0.24218^{*} \\
(0.04383) \\
\end{array}$ & $\begin{array}{l}0.23725^{*} \\
(0.04405)\end{array}$ & \begin{tabular}{|l}
$0.21527^{*}$ \\
$(0.04445)$ \\
\end{tabular} \\
\hline Factor $1,2,3 \& 5$ & $\begin{array}{l}0.30561^{*} \\
(0.03395)\end{array}$ & $\begin{array}{l}0.31498^{*} \\
(0.03375)\end{array}$ & $\begin{array}{l}0.3416^{*} \\
(0.03521)\end{array}$ & $\begin{array}{l}0.31453^{*} \\
(0.03516)\end{array}$ & $\begin{array}{l}0.30454^{*} \\
(0.03378)\end{array}$ & $\begin{array}{l}0.31418^{*} \\
(0.03384)\end{array}$ & $\begin{array}{l}0.32251^{*} \\
(0.03443)\end{array}$ & $\begin{array}{l}0.32852^{*} \\
(0.03379)\end{array}$ & $\begin{array}{l}0.32028^{*} \\
(0.03354)\end{array}$ & $\begin{array}{l}0.3191^{*} \\
(0.03416)\end{array}$ & $\begin{array}{l}0.31858^{*} \\
(0.03355)\end{array}$ & $\begin{array}{l}0.313^{*} \\
(0.03392)\end{array}$ & \begin{tabular}{|l}
$0.30358^{*}$ \\
$(0.03366)$
\end{tabular} \\
\hline Factor $1,2,3 \& 4$ & $\begin{array}{l}0.25593^{*} \\
(0.01768)\end{array}$ & $\begin{array}{l}0.26129^{*} \\
(0.01838)\end{array}$ & $\begin{array}{l}0.29145^{*} \\
(0.02038)\end{array}$ & $\begin{array}{l}0.26309^{*} \\
(0.02055)\end{array}$ & $\begin{array}{l}0.24302^{*} \\
(0.01919)\end{array}$ & $\begin{array}{l}0.25893^{*} \\
(0.01933)\end{array}$ & $\begin{array}{l}0.27283^{*} \\
(0.0202)\end{array}$ & $\begin{array}{l}0.28131^{*} \\
(0.01823)\end{array}$ & $\begin{array}{l}0.27294^{*} \\
(0.01897)\end{array}$ & $\begin{array}{l}0.26814^{*} \\
(0.01994)\end{array}$ & $\begin{array}{l}0.26707^{*} \\
(0.01756)\end{array}$ & $\begin{array}{l}0.26039^{*} \\
(0.01825)\end{array}$ & $\begin{array}{l}0.23783^{*} \\
(0.01931)\end{array}$ \\
\hline All Factors & $\begin{array}{l}0.29869^{*} \\
(0.01833)\end{array}$ & $\begin{array}{l}0.30291^{*} \\
(0.01887)\end{array}$ & $\begin{array}{l}0.3287^{*} \\
(0.02034)\end{array}$ & $\begin{array}{l}0.30418^{*} \\
(0.02073)\end{array}$ & $\begin{array}{l}0.28423^{*} \\
(0.01994)\end{array}$ & $\begin{array}{l}0.3008^{*} \\
(0.01959)\end{array}$ & $\begin{array}{l}0.31328^{*} \\
(0.02084)\end{array}$ & $\begin{array}{l}0.3219^{*} \\
(0.01914)\end{array}$ & $\begin{array}{l}0.31299^{*} \\
(0.01943)\end{array}$ & $\begin{array}{l}0.30875^{*} \\
(0.02122)\end{array}$ & $\begin{array}{l}0.30809^{*} \\
(0.01801)\end{array}$ & $\begin{array}{l}0.30233^{*} \\
(0.01872)\end{array}$ & $\begin{array}{l}0.27758^{*} \\
(0.02029)\end{array}$ \\
\hline $\begin{array}{l}\text { Individual } \\
\text { Variable }\end{array}$ & $\begin{array}{l}0.03921^{\dagger} \\
(0.01801)\end{array}$ & $\begin{array}{l}0.01569 \\
(0.01735)\end{array}$ & $\begin{array}{l}-0.02755^{\dagger} \\
(0.01357)\end{array}$ & $\begin{array}{l}0.00571 \\
(0.01372)\end{array}$ & $\begin{array}{l}0.03324^{*} \\
(0.01242)\end{array}$ & $\begin{array}{l}0.01318 \\
(0.01384)\end{array}$ & $\begin{array}{l}-0.00584 \\
(0.0135)\end{array}$ & $\begin{array}{c}-0.02845^{\ddagger} \\
(0.01515)\end{array}$ & \begin{tabular}{|c|}
-0.00886 \\
$(0.01568)$
\end{tabular} & $\begin{array}{l}-0.00020 \\
(0.01479)\end{array}$ & $\begin{array}{l}0.00429 \\
(0.02315)\end{array}$ & $\begin{array}{l}0.01722 \\
(0.01601)\end{array}$ & \begin{tabular}{|l}
$0.04233^{*}$ \\
$(0.01349)$
\end{tabular} \\
\hline
\end{tabular}

* Significant at the 0.01 level † Significant at the 0.05 level $\$$ Significant at the 0.10 level

Table 90 Coefficients with individual variables for high-level factors - Medium Plants 


\begin{tabular}{|c|c|c|c|c|c|c|c|c|c|c|c|c|c|}
\hline $\begin{array}{l}\text { Individual variable } \\
\text { included in the } \\
\text { model in the } \\
\text { column }\end{array}$ & 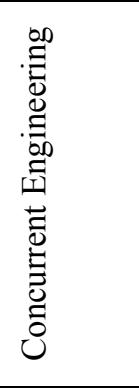 & 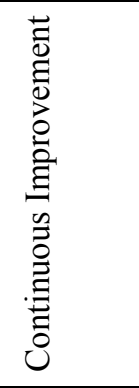 & 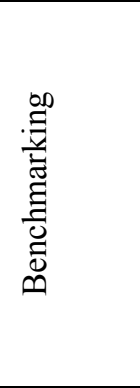 & 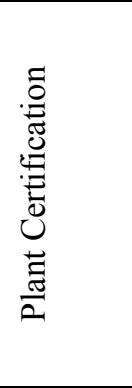 & 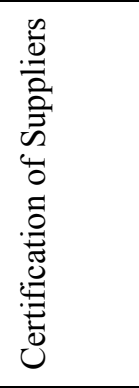 & 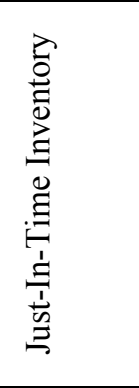 & 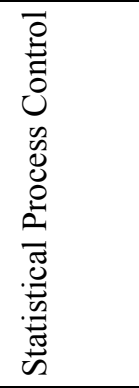 & 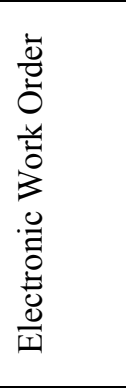 & 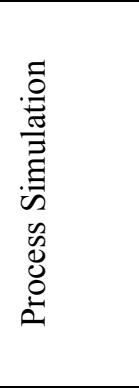 & 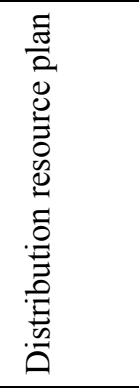 & 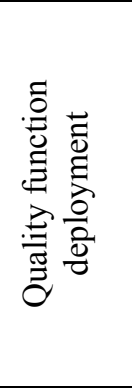 & 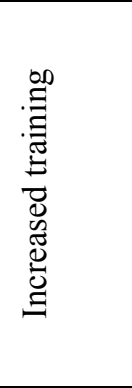 & 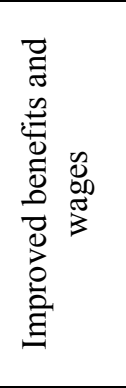 \\
\hline No factors & $\begin{array}{l}0.26868^{*} \\
(0.01302)\end{array}$ & $\begin{array}{l}0.25146^{*} \\
(0.01396)\end{array}$ & $\begin{array}{l}0.25925^{*} \\
(0.01354)\end{array}$ & $\begin{array}{l}0.25854^{*} \\
(0.01388)\end{array}$ & $\begin{array}{l}0.25613^{*} \\
(0.0137)\end{array}$ & $\begin{array}{l}0.27565^{*} \\
(0.0133)\end{array}$ & $\begin{array}{l}0.27065^{*} \\
(0.01307)\end{array}$ & $\begin{array}{l}0.26935^{*} \\
(0.01304)\end{array}$ & $\begin{array}{l}0.26889^{*} \\
(0.013)\end{array}$ & $\begin{array}{l}0.26968^{*} \\
(0.01303)\end{array}$ & $\begin{array}{l}0.27058^{*} \\
(0.01313)\end{array}$ & $\begin{array}{l}0.26835^{*} \\
(0.01304)\end{array}$ & $\begin{array}{l}0.26967^{*} \\
(0.013)\end{array}$ \\
\hline Factor 5 & $\begin{array}{l}0.10633 \\
(0.06941) \\
\end{array}$ & $\begin{array}{l}0.0857 \\
(0.06942) \\
\end{array}$ & $\begin{array}{l}0.11167 \\
(0.06916)\end{array}$ & $\begin{array}{l}0.09248 \\
(0.06981)\end{array}$ & $\begin{array}{l}0.09128 \\
(0.06944) \\
\end{array}$ & $\begin{array}{l}0.11341 \\
(0.06926)\end{array}$ & $\begin{array}{l}0.12119^{\ddagger} \\
(0.06996) \\
\end{array}$ & $\begin{array}{l}0.11275 \\
(0.0694) \\
\end{array}$ & $\begin{array}{l}0.1084 \\
(0.06924) \\
\end{array}$ & $\begin{array}{l}0.11275 \\
(0.06942) \\
\end{array}$ & $\begin{array}{l}0.11502^{\ddagger} \\
(0.06954)\end{array}$ & $\begin{array}{l}0.06928 \\
(0.07441) \\
\end{array}$ & $\begin{array}{l}0.09235 \\
(0.06993) \\
\end{array}$ \\
\hline Factor 4 & $\begin{array}{l}0.14529^{*} \\
(0.03313)\end{array}$ & $\begin{array}{l}0.14127^{*} \\
(0.03292)\end{array}$ & $\begin{array}{l}0.14508^{*} \\
(0.03295)\end{array}$ & $\begin{array}{l}0.14864^{*} \\
(0.03293)\end{array}$ & $\begin{array}{l}0.14821^{*} \\
(0.03285)\end{array}$ & $\begin{array}{l}0.16567^{*} \\
(0.03361)\end{array}$ & $\begin{array}{l}0.15188^{*} \\
(0.03299)\end{array}$ & $\begin{array}{l}0.14993^{*} \\
(0.03305)\end{array}$ & $\begin{array}{l}0.14896^{*} \\
(0.03292)\end{array}$ & $\begin{array}{l}0.15129^{*} \\
(0.033)\end{array}$ & $\begin{array}{l}0.15135^{*} \\
(0.03299) \\
\end{array}$ & $\begin{array}{l}0.14876^{*} \\
(0.03299) \\
\end{array}$ & $\begin{array}{l}0.15129^{*} \\
(0.03292)\end{array}$ \\
\hline Factor $4 \& 5$ & $\begin{array}{l}0.22741^{\ddagger} \\
(0.1278)\end{array}$ & $\begin{array}{l}0.21249^{\ddagger} \\
(0.12729)\end{array}$ & $\begin{array}{l}0.23211^{\ddagger} \\
(0.12744) \\
\end{array}$ & $\begin{array}{l}0.23289^{\ddagger} \\
(0.12758)\end{array}$ & $\begin{array}{l}0.23543^{\ddagger} \\
(0.1273)\end{array}$ & $\begin{array}{l}0.23932^{\ddagger} \\
(0.12763)\end{array}$ & $\begin{array}{l}0.23543^{\ddagger} \\
(0.12786) \\
\end{array}$ & $\begin{array}{l}0.23543^{\ddagger} \\
(0.12788)\end{array}$ & $\begin{array}{l}0.2311^{\ddagger} \\
(0.12755)\end{array}$ & $\begin{array}{l}0.23576^{\ddagger} \\
(0.12793)\end{array}$ & $\begin{array}{l}0.23616^{\ddagger} \\
(0.1279) \\
\end{array}$ & $\begin{array}{l}0.20717 \\
(0.12894) \\
\end{array}$ & $\begin{array}{l}0.2198^{\ddagger} \\
(0.12783) \\
\end{array}$ \\
\hline Factor 3 & $\begin{array}{l}0.2325^{*} \\
(0.04382) \\
\end{array}$ & $\begin{array}{l}0.22945^{*} \\
(0.04362) \\
\end{array}$ & $\begin{array}{l}0.22921^{*} \\
(0.04375) \\
\end{array}$ & $\begin{array}{l}0.2197^{*} \\
(0.04419) \\
\end{array}$ & $\begin{array}{l}0.21206^{*} \\
(0.04424) \\
\end{array}$ & $\begin{array}{l}0.23664^{*} \\
(0.0438) \\
\end{array}$ & $\begin{array}{l}0.23537^{*} \\
(0.0439) \\
\end{array}$ & $\begin{array}{l}0.23355^{*} \\
(0.04387) \\
\end{array}$ & $\begin{array}{l}0.23367^{*} \\
(0.04375) \\
\end{array}$ & $\begin{array}{l}0.23367^{*} \\
(0.04388)\end{array}$ & $\begin{array}{l}0.23405^{*} \\
(0.04388) \\
\end{array}$ & $\begin{array}{l}0.23367^{*} \\
(0.04382) \\
\end{array}$ & $\begin{array}{l}0.23367^{*} \\
(0.04378) \\
\end{array}$ \\
\hline Factor $3 \& 5$ & $\begin{array}{l}0.15852^{*} \\
(0.05074)\end{array}$ & $\begin{array}{l}0.12662^{\dagger} \\
(0.05132)\end{array}$ & $\begin{array}{l}0.14631^{*} \\
(0.05082)\end{array}$ & $\begin{array}{l}0.14767^{*} \\
(0.05091)\end{array}$ & $\begin{array}{l}0.15347^{*} \\
(0.0506) \\
\end{array}$ & $\begin{array}{l}0.18014^{*} \\
(0.05172)\end{array}$ & $\begin{array}{l}0.15935^{*} \\
(0.0508) \\
\end{array}$ & $\begin{array}{l}0.15838^{*} \\
(0.05081)\end{array}$ & $\begin{array}{l}0.15852^{*} \\
(0.05067)\end{array}$ & $\begin{array}{l}0.15852^{*} \\
(0.05082)\end{array}$ & $\begin{array}{l}0.16322^{*} \\
(0.05154)\end{array}$ & $\begin{array}{l}0.11296^{\ddagger} \\
(0.05811)\end{array}$ & $\begin{array}{l}0.14725^{*} \\
(0.05098) \\
\end{array}$ \\
\hline Factor $3 \& 4$ & $\begin{array}{l}0.20957^{*} \\
(0.04911) \\
\end{array}$ & $\begin{array}{l}0.19737^{*} \\
(0.04872) \\
\end{array}$ & $\begin{array}{l}0.22211^{*} \\
(0.0482) \\
\end{array}$ & $\begin{array}{l}0.21757^{*} \\
(0.04836) \\
\end{array}$ & $\begin{array}{l}0.22247^{*} \\
(0.04815) \\
\end{array}$ & $\begin{array}{l}0.22914^{*} \\
(0.0483) \\
\end{array}$ & $\begin{array}{l}0.22948^{*} \\
(0.04857) \\
\end{array}$ & $\begin{array}{l}0.22361^{*} \\
(0.04841) \\
\end{array}$ & $\begin{array}{l}0.21544^{*} \\
(0.0484) \\
\end{array}$ & $\begin{array}{l}0.22584^{*} \\
(0.04855) \\
\end{array}$ & $\begin{array}{l}0.22675^{*} \\
(0.04846) \\
\end{array}$ & $\begin{array}{l}0.22511^{*} \\
(0.04831) \\
\end{array}$ & $\begin{array}{l}0.22511^{*} \\
(0.04826) \\
\end{array}$ \\
\hline Factor $3,4 \& 5$ & $\begin{array}{l}0.16266^{*} \\
(0.05995)\end{array}$ & $\begin{array}{l}0.1583^{*} \\
(0.05962) \\
\end{array}$ & $\begin{array}{l}0.1477^{\dagger} \\
(0.06023)\end{array}$ & $\begin{array}{l}0.15267^{\dagger} \\
(0.06022)\end{array}$ & $\begin{array}{l}0.14903^{\dagger} \\
(0.06001)\end{array}$ & $\begin{array}{l}0.18455^{*} \\
(0.06017)\end{array}$ & $\begin{array}{l}0.16989^{*} \\
(0.05988)\end{array}$ & $\begin{array}{l}0.16847^{*} \\
(0.05992)\end{array}$ & $\begin{array}{l}0.16989^{*} \\
(0.05973)\end{array}$ & $\begin{array}{l}0.16989^{*} \\
(0.0599) \\
\end{array}$ & $\begin{array}{l}0.17373^{*} \\
(0.06031)\end{array}$ & $\begin{array}{l}0.12565^{\ddagger} \\
(0.06584)\end{array}$ & $\begin{array}{l}0.13986^{\dagger} \\
(0.06142)\end{array}$ \\
\hline Factor 2 & $\begin{array}{l}0.17086^{*} \\
(0.03836) \\
\end{array}$ & $\begin{array}{l}0.134566^{*} \\
(0.04001)\end{array}$ & $\begin{array}{l}0.14629^{*} \\
(0.03991)\end{array}$ & $\begin{array}{l}0.15261^{*} \\
(0.03984) \\
\end{array}$ & $\begin{array}{l}0.14272^{*} \\
(0.0398) \\
\end{array}$ & $\begin{array}{l}0.2007^{*} \\
(0.03911) \\
\end{array}$ & $\begin{array}{l}0.18971^{*} \\
(0.03923)\end{array}$ & $\begin{array}{l}0.17434^{*} \\
(0.03901)\end{array}$ & $\begin{array}{l}0.16375^{*} \\
(0.03855) \\
\end{array}$ & $\begin{array}{l}0.18186^{*} \\
(0.03882) \\
\end{array}$ & $\begin{array}{l}0.18366^{*} \\
(0.03846) \\
\end{array}$ & $\begin{array}{l}0.18048^{*} \\
(0.03797) \\
\end{array}$ & $\begin{array}{l}0.18048^{*} \\
(0.03793) \\
\end{array}$ \\
\hline Factor & $\begin{array}{l}0.35732 \\
(0.22925) \\
\end{array}$ & $\begin{array}{l}0.33771 \\
(0.22825) \\
\end{array}$ & $\begin{array}{l}0.34273 \\
(0.2288)\end{array}$ & $\begin{array}{l}0.34531 \\
(0.22905) \\
\end{array}$ & $\begin{array}{l}0.31897 \\
(0.22885) \\
\end{array}$ & $\begin{array}{l}0.36803 \\
(0.22913) \\
\end{array}$ & $\begin{array}{l}0.35732 \\
(0.2295)\end{array}$ & $\begin{array}{l}0.35311 \\
(0.22962)\end{array}$ & $\begin{array}{l}0.35732 \\
(0.22893) \\
\end{array}$ & $\begin{array}{l}0.35875 \\
(0.22974) \\
\end{array}$ & $\begin{array}{l}0.3617 \\
(0.22971) \\
\end{array}$ & $\begin{array}{l}0.31176 \\
(0.23104) \\
\end{array}$ & $\begin{array}{l}0.35732 \\
(0.22908) \\
\end{array}$ \\
\hline Fact & $\begin{array}{l}0.23437^{*} \\
(0.04257) \\
\end{array}$ & $\begin{array}{l}0.20231^{*} \\
(0.04368) \\
\end{array}$ & $\begin{array}{l}0.2192^{*} \\
(0.0431) \\
\end{array}$ & $\begin{array}{l}0.22303^{*} \\
(0.04318) \\
\end{array}$ & $\begin{array}{l}0.21187^{*} \\
(0.04337) \\
\end{array}$ & $\begin{array}{l}0.26183^{*} \\
(0.04276) \\
\end{array}$ & $\begin{array}{l}0.25736^{*} \\
(0.04396) \\
\end{array}$ & $\begin{array}{l}0.23785^{*} \\
(0.04353) \\
\end{array}$ & $\begin{array}{l}0.23292^{*} \\
(0.04236) \\
\end{array}$ & $\begin{array}{l}0.246^{*} \\
(0.04225) \\
\end{array}$ & $\begin{array}{l}0.24885^{*} \\
(0.0426) \\
\end{array}$ & $\begin{array}{l}0.24549^{*} \\
(0.0421) \\
\end{array}$ & $\begin{array}{l}0.24549^{*} \\
(0.04206) \\
\end{array}$ \\
\hline Facto & $\begin{array}{l}0.11509^{\dagger} \\
(0.05808)\end{array}$ & $\begin{array}{l}0.09579^{\ddagger} \\
(0.05816)\end{array}$ & $\begin{array}{l}0.10245^{\ddagger} \\
(0.05827)\end{array}$ & $\begin{array}{l}0.09861^{\ddagger} \\
(0.05882)\end{array}$ & $\begin{array}{l}0.09479 \\
(0.05845) \\
\end{array}$ & $\begin{array}{l}0.14032^{\dagger} \\
(0.05844)\end{array}$ & $\begin{array}{l}0.13153^{\dagger} \\
(0.05868) \\
\end{array}$ & $\begin{array}{l}0.11509^{\ddagger} \\
(0.05905)\end{array}$ & $\begin{array}{l}0.11237^{\ddagger} \\
(0.05799) \\
\end{array}$ & $\begin{array}{l}0.12496^{\dagger} \\
(0.05912)\end{array}$ & $\begin{array}{l}0.13015^{\dagger} \\
(0.05948) \\
\end{array}$ & $\begin{array}{l}0.07835 \\
(0.0642) \\
\end{array}$ & $\begin{array}{l}0.09759^{\ddagger} \\
(0.05908) \\
\end{array}$ \\
\hline Factor & $\begin{array}{l}0.28003^{*} \\
(0.06945) \\
\end{array}$ & $\begin{array}{l}0.23883^{*} \\
(0.07027) \\
\end{array}$ & $\begin{array}{l}0.26343^{*} \\
(0.06965) \\
\end{array}$ & $\begin{array}{l}0.26131^{*} \\
(0.07002) \\
\end{array}$ & $\begin{array}{l}0.25161^{*} \\
(0.06997) \\
\end{array}$ & $\begin{array}{l}0.3054^{*} \\
(0.07013) \\
\end{array}$ & $\begin{array}{l}0.29139^{*} \\
(0.06999) \\
\end{array}$ & $\begin{array}{l}0.27837^{*} \\
(0.06989) \\
\end{array}$ & $\begin{array}{l}0.2819^{*} \\
(0.06932) \\
\end{array}$ & $\begin{array}{l}0.28372^{*} \\
(0.06954) \\
\end{array}$ & $\begin{array}{l}0.28734^{*} \\
(0.06988) \\
\end{array}$ & $\begin{array}{l}0.28348^{*} \\
(0.06943) \\
\end{array}$ & $\begin{array}{l}0.28348^{*} \\
(0.06937) \\
\end{array}$ \\
\hline Factor $2,3 \& 5$ & $\begin{array}{l}0.37281^{\dagger} \\
(0.17958)\end{array}$ & $\begin{array}{l}0.34009^{\ddagger} \\
(0.17898)\end{array}$ & $\begin{array}{l}0.33836^{\ddagger} \\
(0.17962)\end{array}$ & $\begin{array}{l}0.34446^{\ddagger} \\
(0.17981) \\
\end{array}$ & $\begin{array}{l}0.3457^{\ddagger} \\
(0.17921)\end{array}$ & $\begin{array}{l}0.39068^{\dagger} \\
(0.17964)\end{array}$ & $\begin{array}{l}0.38441^{\dagger} \\
(0.18019)\end{array}$ & $\begin{array}{l}0.3699^{\dagger} \\
(0.17986)\end{array}$ & $\begin{array}{l}0.37281^{\dagger} \\
(0.17933)\end{array}$ & $\begin{array}{l}0.3752^{\dagger} \\
(0.18036)\end{array}$ & $\begin{array}{l}0.37818^{\dagger} \\
(0.1801) \\
\end{array}$ & $\begin{array}{l}0.32725^{\ddagger} \\
(0.18183) \\
\end{array}$ & $\begin{array}{l}0.34281^{\ddagger} \\
(0.18)\end{array}$ \\
\hline Factor $2,3 \& 4$ & $\begin{array}{l}0.19617^{*} \\
(0.04415)\end{array}$ & $\begin{array}{l}0.16888^{*} \\
(0.04464) \\
\end{array}$ & $\begin{array}{l}0.18815^{*} \\
(0.04387) \\
\end{array}$ & $\begin{array}{l}0.18838^{*} \\
(0.04434)\end{array}$ & $\begin{array}{l}0.17927^{*} \\
(0.04427) \\
\end{array}$ & $\begin{array}{l}0.22924^{*} \\
(0.04341)\end{array}$ & $\begin{array}{l}0.22104^{*} \\
(0.04349) \\
\end{array}$ & $\begin{array}{l}0.20934^{*} \\
(0.04356)\end{array}$ & $\begin{array}{l}0.19336^{*} \\
(0.04373)\end{array}$ & $\begin{array}{l}0.21525^{*} \\
(0.04317) \\
\end{array}$ & $\begin{array}{l}0.21937^{*} \\
(0.04387)\end{array}$ & $\begin{array}{l}0.21448^{*} \\
(0.04289)\end{array}$ & $\begin{array}{l}0.21448^{*} \\
(0.04285)\end{array}$ \\
\hline Factor $2,3,4 \& 5$ & $\begin{array}{l}0.21099^{*} \\
(0.04676) \\
\end{array}$ & $\begin{array}{l}0.18238^{*} \\
(0.04748) \\
\end{array}$ & $\begin{array}{l}0.20058^{*} \\
(0.04687) \\
\end{array}$ & $\begin{array}{l}0.20277^{*} \\
(0.04704) \\
\end{array}$ & $\begin{array}{l}0.19429^{*} \\
(0.04704) \\
\end{array}$ & $\begin{array}{l}0.23647^{*} \\
(0.04659) \\
\end{array}$ & $\begin{array}{l}0.23022^{*} \\
(0.04682) \\
\end{array}$ & $\begin{array}{l}0.21768^{*} \\
(0.04699) \\
\end{array}$ & $\begin{array}{l}0.21298^{*} \\
(0.04636) \\
\end{array}$ & $\begin{array}{l}0.2236^{*} \\
(0.0463) \\
\end{array}$ & $\begin{array}{l}0.22744^{*} \\
(0.04687) \\
\end{array}$ & $\begin{array}{l}0.18727^{*} \\
(0.05139) \\
\end{array}$ & $\begin{array}{l}0.19202^{*} \\
(0.0485) \\
\end{array}$ \\
\hline Factor 1 & $\begin{array}{l}0.26557^{*} \\
(0.02683)\end{array}$ & $\begin{array}{l}0.25292^{*} \\
(0.02699)\end{array}$ & $\begin{array}{l}0.26449^{*} \\
(0.02677)\end{array}$ & $\begin{array}{l}0.26081^{*} \\
(0.02692)\end{array}$ & $\begin{array}{l}0.2545^{*} \\
(0.02703)\end{array}$ & $\begin{array}{l}0.27369^{*} \\
(0.027)\end{array}$ & $\begin{array}{l}0.26733^{*} \\
(0.02686)\end{array}$ & $\begin{array}{l}0.26532^{*} \\
(0.02693)\end{array}$ & $\begin{array}{l}0.26673^{*} \\
(0.02678)\end{array}$ & $\begin{array}{l}0.26674^{*} \\
(0.02686)\end{array}$ & $\begin{array}{l}0.2671^{*} \\
(0.02687)\end{array}$ & $\begin{array}{l}0.26588^{*} \\
(0.02683)\end{array}$ & $\begin{array}{l}0.26673^{*} \\
(0.0268) \\
\end{array}$ \\
\hline Fac & $\begin{array}{l}0.27231^{*} \\
(0.03178) \\
\end{array}$ & $\begin{array}{l}0.26188^{*} \\
(0.03181) \\
\end{array}$ & $\begin{array}{l}0.26802^{*} \\
(0.03177) \\
\end{array}$ & $\begin{array}{l}0.26305^{*} \\
(0.03214) \\
\end{array}$ & $\begin{array}{l}0.263^{*} \\
(0.03188) \\
\end{array}$ & $\begin{array}{l}0.28283^{*} \\
(0.03192) \\
\end{array}$ & $\begin{array}{l}0.27515^{*} \\
(0.03177) \\
\end{array}$ & $\begin{array}{l}0.27485^{*} \\
(0.03178) \\
\end{array}$ & $\begin{array}{l}0.27211^{*} \\
(0.03172) \\
\end{array}$ & $\begin{array}{l}0.27515^{*} \\
(0.03178)\end{array}$ & $\begin{array}{l}0.27537^{*} \\
(0.03178) \\
\end{array}$ & $\begin{array}{l}0.22959^{*} \\
(0.04253) \\
\end{array}$ & $\begin{array}{l}0.25062^{*} \\
(0.03376) \\
\end{array}$ \\
\hline Facto & $\begin{array}{l}0.27232^{*} \\
(0.03475)\end{array}$ & $\begin{array}{l}0.26945^{*} \\
(0.0345)\end{array}$ & $\begin{array}{l}0.26861^{*} \\
(0.03468)\end{array}$ & $\begin{array}{l}0.2706^{*} \\
(0.0347) \\
\end{array}$ & $\begin{array}{l}0.26768^{*} \\
(0.03464)\end{array}$ & $\begin{array}{l}0.28138^{*} \\
(0.03456)\end{array}$ & $\begin{array}{l}0.28051^{*} \\
(0.03466)\end{array}$ & $\begin{array}{l}0.27579^{*} \\
(0.03484)\end{array}$ & $\begin{array}{l}0.27717^{*} \\
(0.03452)\end{array}$ & $\begin{array}{l}0.27857^{*} \\
(0.03462)\end{array}$ & $\begin{array}{l}0.27914^{*} \\
(0.03463)\end{array}$ & $\begin{array}{l}0.27717^{*} \\
(0.03458)\end{array}$ & $\begin{array}{l}0.27857^{*} \\
(0.03454)\end{array}$ \\
\hline Factor $1,4 \& 5$ & $\begin{array}{l}0.19918^{*} \\
(0.03264) \\
\end{array}$ & $\begin{array}{l}0.19014^{*} \\
(0.03231)\end{array}$ & $\begin{array}{l}0.19658^{*} \\
(0.03227) \\
\end{array}$ & $\begin{array}{l}0.19838^{*} \\
(0.03235) \\
\end{array}$ & $\begin{array}{l}0.19765^{*} \\
(0.03209) \\
\end{array}$ & $\begin{array}{l}0.21949^{*} \\
(0.03208)\end{array}$ & $\begin{array}{l}0.21165^{*} \\
(0.03192) \\
\end{array}$ & $\begin{array}{l}0.20994^{*} \\
(0.03199)\end{array}$ & $\begin{array}{l}0.21141^{*} \\
(0.03184)\end{array}$ & $\begin{array}{l}0.21146^{*} \\
(0.03193) \\
\end{array}$ & $\begin{array}{l}0.21216^{*} \\
(0.03195) \\
\end{array}$ & $\begin{array}{l}0.169^{*} \\
(0.04137) \\
\end{array}$ & $\begin{array}{l}0.18813^{*} \\
(0.0337) \\
\end{array}$ \\
\hline Factor $1 \& 3$ & $\begin{array}{l}0.28255^{*} \\
(0.02769) \\
\end{array}$ & $\begin{array}{l}0.25491^{*} \\
(0.02877) \\
\end{array}$ & $\begin{array}{l}0.27929^{*} \\
(0.02767) \\
\end{array}$ & $\begin{array}{l}0.27585^{*} \\
(0.02787) \\
\end{array}$ & $\begin{array}{l}0.2694^{*} \\
(0.02799) \\
\end{array}$ & $\begin{array}{l}0.29079^{*} \\
(0.02785) \\
\end{array}$ & $\begin{array}{l}0.28445^{*} \\
(0.02772) \\
\end{array}$ & $\begin{array}{l}0.28226^{*} \\
(0.0278) \\
\end{array}$ & $\begin{array}{l}0.28347^{*} \\
(0.02764) \\
\end{array}$ & $\begin{array}{l}0.28381^{*} \\
(0.02772) \\
\end{array}$ & $\begin{array}{l}0.28433^{*} \\
(0.02773) \\
\end{array}$ & $\begin{array}{l}0.28381^{*} \\
(0.02768) \\
\end{array}$ & $\begin{array}{l}0.28381^{*} \\
(0.02766) \\
\end{array}$ \\
\hline Factor $1,3 \& 5$ & $\begin{array}{l}0.26696^{*} \\
(0.02277) \\
\end{array}$ & $\begin{array}{l}0.25624^{*} \\
(0.02292) \\
\end{array}$ & $\begin{array}{l}0.26471^{*} \\
(0.02274)\end{array}$ & $\begin{array}{l}0.26328^{*} \\
(0.02284) \\
\end{array}$ & $\begin{array}{l}0.23702^{*} \\
(0.02492) \\
\end{array}$ & $\begin{array}{l}0.28723^{*} \\
(0.02435) \\
\end{array}$ & $\begin{array}{l}0.27035^{*} \\
(0.02284) \\
\end{array}$ & $\begin{array}{l}0.26757^{*} \\
(0.02283) \\
\end{array}$ & $\begin{array}{l}0.2687^{*} \\
(0.02271) \\
\end{array}$ & $\begin{array}{l}0.2687^{*} \\
(0.02278) \\
\end{array}$ & $\begin{array}{l}0.26931^{*} \\
(0.0228) \\
\end{array}$ & $\begin{array}{l}0.25229^{*} \\
(0.02493) \\
\end{array}$ & $\begin{array}{l}0.26237^{*} \\
(0.02292) \\
\end{array}$ \\
\hline Factor $1,3 \& 4$ & $\begin{array}{l}0.18383^{*} \\
(0.01841) \\
\end{array}$ & $\begin{array}{l}0.17107^{*} \\
(0.01875) \\
\end{array}$ & $\begin{array}{l}0.16932^{*} \\
(0.01959)\end{array}$ & $\begin{array}{l}0.1852^{*} \\
(0.01811) \\
\end{array}$ & $\begin{array}{l}0.18424^{*} \\
(0.01803)\end{array}$ & $\begin{array}{l}0.20316^{*} \\
(0.01887)\end{array}$ & $\begin{array}{l}0.19124^{*} \\
(0.01799) \\
\end{array}$ & $\begin{array}{l}0.18491^{*} \\
(0.01987)\end{array}$ & $\begin{array}{l}0.19083^{*} \\
(0.01794) \\
\end{array}$ & $\begin{array}{l}0.19091^{*} \\
(0.018)\end{array}$ & $\begin{array}{l}0.19198^{*} \\
(0.01811) \\
\end{array}$ & $\begin{array}{l}0.19034^{*} \\
(0.01797) \\
\end{array}$ & $\begin{array}{l}0.19083^{*} \\
(0.01795) \\
\end{array}$ \\
\hline Factor $1,3,4 \& 5$ & $\begin{array}{l}0.21249^{*} \\
(0.03004) \\
\end{array}$ & $\begin{array}{l}0.19609^{*} \\
(0.03027) \\
\end{array}$ & $\begin{array}{l}0.21217^{*} \\
(0.0296) \\
\end{array}$ & $\begin{array}{l}0.20957^{*} \\
(0.02997) \\
\end{array}$ & $\begin{array}{l}0.21328^{*} \\
(0.02947) \\
\end{array}$ & $\begin{array}{l}0.22861^{*} \\
(0.02945) \\
\end{array}$ & $\begin{array}{l}0.2247^{*} \\
(0.02943) \\
\end{array}$ & $\begin{array}{l}0.22081^{*} \\
(0.02966) \\
\end{array}$ & $\begin{array}{l}0.22034^{*} \\
(0.02936) \\
\end{array}$ & $\begin{array}{l}0.22366^{*} \\
(0.02943) \\
\end{array}$ & $\begin{array}{l}0.22374^{*} \\
(0.02942) \\
\end{array}$ & $\begin{array}{l}0.18224^{*} \\
(0.03899) \\
\end{array}$ & $\begin{array}{l}0.19103^{*} \\
(0.03311) \\
\end{array}$ \\
\hline Facto & $\begin{array}{l}0.19035^{*} \\
(0.03574) \\
\end{array}$ & $\begin{array}{l}0.16675^{*} \\
(0.03642) \\
\end{array}$ & $\begin{array}{l}0.17043^{*} \\
(0.03684) \\
\end{array}$ & $\begin{array}{l}0.1862^{*} \\
(0.0358) \\
\end{array}$ & $\begin{array}{l}0.17169^{*} \\
(0.03639) \\
\end{array}$ & $\begin{array}{l}0.21531^{*} \\
(0.03622) \\
\end{array}$ & $\begin{array}{l}0.20853^{*} \\
(0.03689) \\
\end{array}$ & $\begin{array}{l}0.19675^{*} \\
(0.03558) \\
\end{array}$ & $\begin{array}{l}0.19885^{*} \\
(0.03536) \\
\end{array}$ & $\begin{array}{l}0.19905^{*} \\
(0.03548) \\
\end{array}$ & $\begin{array}{l}0.20049^{*} \\
(0.03558) \\
\end{array}$ & $\begin{array}{l}0.19828^{*} \\
(0.03542) \\
\end{array}$ & $\begin{array}{l}0.19885^{*} \\
(0.03538) \\
\end{array}$ \\
\hline Factor $1,2 \& 5$ & $\begin{array}{l}0.19953^{*} \\
(0.05387)\end{array}$ & $\begin{array}{l}0.1587^{*} \\
(0.05502)\end{array}$ & $\begin{array}{l}0.17942^{*} \\
(0.05438)\end{array}$ & $\begin{array}{l}0.18012^{*} \\
(0.05465)\end{array}$ & $\begin{array}{l}0.18264^{*} \\
(0.05405)\end{array}$ & $\begin{array}{l}0.22036^{*} \\
(0.0545) \\
\end{array}$ & $\begin{array}{l}0.20939^{*} \\
(0.05446)\end{array}$ & $\begin{array}{l}0.19789^{*} \\
(0.05425)\end{array}$ & $\begin{array}{l}0.19162^{*} \\
(0.05396)\end{array}$ & $\begin{array}{l}0.20265^{*} \\
(0.05403)\end{array}$ & $\begin{array}{l}0.20568^{*} \\
(0.05433)\end{array}$ & $\begin{array}{l}0.15845^{*} \\
(0.0603)\end{array}$ & $\begin{array}{l}0.17426^{*} \\
(0.05539)\end{array}$ \\
\hline Factor & $\begin{array}{l}0.45339^{*} \\
(0.0295) \\
\end{array}$ & $\begin{array}{l}0.41782^{*} \\
(0.03161) \\
\end{array}$ & $\begin{array}{l}0.44023^{*} \\
(0.03011) \\
\end{array}$ & $\begin{array}{l}0.44462^{*} \\
(0.03005) \\
\end{array}$ & $\begin{array}{l}0.43492^{*} \\
(0.03031) \\
\end{array}$ & $\begin{array}{l}0.47504^{*} \\
(0.02961) \\
\end{array}$ & $\begin{array}{l}0.46565^{*} \\
(0.02925) \\
\end{array}$ & $\begin{array}{l}0.46112^{*} \\
(0.02914) \\
\end{array}$ & $\begin{array}{l}0.46047^{*} \\
(0.02901) \\
\end{array}$ & $\begin{array}{l}0.4629^{*} \\
(0.02916) \\
\end{array}$ & $\begin{array}{l}0.46572^{*} \\
(0.02966) \\
\end{array}$ & $\begin{array}{l}0.46252^{*} \\
(0.02904) \\
\end{array}$ & $\begin{array}{l}0.46252^{*} \\
(0.02901) \\
\end{array}$ \\
\hline Factor $1,2,4 \& 5$ & $\begin{array}{l}0.24088^{*} \\
(0.03322) \\
\end{array}$ & $\begin{array}{l}0.21023^{*} \\
(0.03445) \\
\end{array}$ & $\begin{array}{l}0.22911^{*} \\
(0.03357) \\
\end{array}$ & $\begin{array}{l}0.23586^{*} \\
(0.03334)\end{array}$ & $\begin{array}{l}0.22304^{*} \\
(0.03379) \\
\end{array}$ & $\begin{array}{l}0.25233^{*} \\
(0.03329) \\
\end{array}$ & $\begin{array}{l}0.24703^{*} \\
(0.03325)\end{array}$ & $\begin{array}{l}0.24133^{*} \\
(0.03358)\end{array}$ & $\begin{array}{l}0.24323^{*} \\
(0.0331)\end{array}$ & $\begin{array}{l}0.24535^{*} \\
(0.03327)\end{array}$ & $\begin{array}{l}0.24648^{*} \\
(0.0333)\end{array}$ & $\begin{array}{l}0.20003^{*} \\
(0.04333)\end{array}$ & $\begin{array}{l}0.2101^{*} \\
(0.03697)\end{array}$ \\
\hline Factor $1,2 \& 3$ & $\begin{array}{l}0.23357^{*} \\
(0.04404) \\
\end{array}$ & $\begin{array}{l}0.20099^{*} \\
(0.04513)\end{array}$ & $\begin{array}{l}0.21266^{*} \\
(0.045) \\
\end{array}$ & $\begin{array}{l}0.22786^{*} \\
(0.04417) \\
\end{array}$ & $\begin{array}{l}0.22469^{*} \\
(0.04399) \\
\end{array}$ & $\begin{array}{l}0.25971^{*} \\
(0.0445) \\
\end{array}$ & $\begin{array}{l}0.24837^{*} \\
(0.04428) \\
\end{array}$ & $\begin{array}{l}0.23461^{*} \\
(0.04516) \\
\end{array}$ & $\begin{array}{l}0.23226^{*} \\
(0.0439) \\
\end{array}$ & $\begin{array}{l}0.24363^{*} \\
(0.0445) \\
\end{array}$ & $\begin{array}{l}0.24575^{*} \\
(0.04429) \\
\end{array}$ & $\begin{array}{l}0.24226^{*} \\
(0.04377) \\
\end{array}$ & $\begin{array}{l}0.24226^{*} \\
(0.04373) \\
\end{array}$ \\
\hline Factor $1,2,3 \& 5$ & $\begin{array}{l}0.31367^{*} \\
(0.03356)\end{array}$ & $\begin{array}{l}0.27447^{*} \\
(0.03563)\end{array}$ & $\begin{array}{l}0.29167^{*} \\
(0.03483)\end{array}$ & $\begin{array}{l}0.30028^{*} \\
(0.03438)\end{array}$ & $\begin{array}{l}0.28832^{*} \\
(0.03479)\end{array}$ & $\begin{array}{l}0.33096^{*} \\
(0.03387)\end{array}$ & $\begin{array}{l}0.32768^{*} \\
(0.03469)\end{array}$ & $\begin{array}{l}0.31062^{*} \\
(0.03555)\end{array}$ & $\begin{array}{l}0.31684^{*} \\
(0.03339)\end{array}$ & $\begin{array}{l}0.31985^{*} \\
(0.03381)\end{array}$ & $\begin{array}{l}0.32293^{*} \\
(0.03423)\end{array}$ & $\begin{array}{l}0.27477^{*} \\
(0.04328)\end{array}$ & $\begin{array}{l}0.28838^{*} \\
(0.03639) \\
\end{array}$ \\
\hline Factor $1,2,3 \& 4$ & $\begin{array}{l}0.25488^{*} \\
(0.01841)\end{array}$ & $\begin{array}{l}0.22671^{*} \\
(0.02049)\end{array}$ & $\begin{array}{l}0.24187^{*} \\
(0.01933)\end{array}$ & $\begin{array}{l}0.24431^{*} \\
(0.01974) \\
\end{array}$ & $\begin{array}{l}0.23708^{*} \\
(0.01956)\end{array}$ & $\begin{array}{l}0.28365^{*} \\
(0.01834)\end{array}$ & $\begin{array}{l}0.2769^{*} \\
(0.01928)\end{array}$ & $\begin{array}{l}0.26014^{*} \\
(0.02023)\end{array}$ & $\begin{array}{l}0.24929^{*} \\
(0.01851)\end{array}$ & $\begin{array}{l}0.26914^{*} \\
(0.01804)\end{array}$ & $\begin{array}{l}0.27156^{*} \\
(0.01806) \\
\end{array}$ & $\begin{array}{l}0.26749^{*} \\
(0.01681) \\
\end{array}$ & $\begin{array}{l}0.268^{*} \\
(0.01679) \\
\end{array}$ \\
\hline All Factors & $\begin{array}{l}0.29409^{*} \\
(0.01963)\end{array}$ & $\begin{array}{l}0.26401^{*} \\
(0.02182)\end{array}$ & $\begin{array}{l}0.28245^{*} \\
(0.02018)\end{array}$ & $\begin{array}{l}0.28504^{*} \\
(0.02054)\end{array}$ & $\begin{array}{l}0.27986^{*} \\
(0.02005)\end{array}$ & $\begin{array}{l}0.32731^{*} \\
(0.01984)\end{array}$ & $\begin{array}{l}0.31838^{*} \\
(0.02059) \\
\end{array}$ & $\begin{array}{l}0.30257^{*} \\
(0.01977)\end{array}$ & $\begin{array}{l}0.29303^{*} \\
(0.01891)\end{array}$ & $\begin{array}{l}0.30954^{*} \\
(0.0186)\end{array}$ & $\begin{array}{l}0.3123^{*} \\
(0.01906)\end{array}$ & $\begin{array}{l}0.26495^{*} \\
(0.03243) \\
\end{array}$ & $\begin{array}{l}0.28662^{*} \\
(0.02057)\end{array}$ \\
\hline $\begin{array}{l}\text { Individual } \\
\text { Variable }\end{array}$ & $\begin{array}{l}0.02348^{\ddagger} \\
(0.01348)\end{array}$ & $\begin{array}{l}0.04629^{*} \\
(0.01326)\end{array}$ & $\begin{array}{l}0.03445^{*} \\
(0.01268)\end{array}$ & $\begin{array}{l}0.02835^{\dagger} \\
(0.01244)\end{array}$ & $\begin{array}{l}0.03836^{*} \\
(0.01253)\end{array}$ & $\begin{array}{c}-0.02528^{\dagger} \\
(0.01193)\end{array}$ & $\begin{array}{l}-0.01446 \\
(0.01529)\end{array}$ & $\begin{array}{l}0.00993 \\
(0.01419)\end{array}$ & $\begin{array}{l}0.04493^{\dagger} \\
(0.01878)\end{array}$ & $\begin{array}{l}-0.00338 \\
(0.01917)\end{array}$ & $\begin{array}{l}-0.0076 \\
(0.01396)\end{array}$ & $\begin{array}{l}0.04556 \\
(0.02831)\end{array}$ & $\begin{array}{l}0.04244^{\dagger} \\
(0.02003)\end{array}$ \\
\hline
\end{tabular}

* Significant at the 0.01 level $\quad$ Significant at the 0.05 level $\quad$ Significant at the 0.10 level

Table 90 Coefficients with individual variables for high-level factors - Medium Plants 


\begin{tabular}{|c|c|c|c|c|c|c|c|c|c|c|c|c|c|}
\hline & 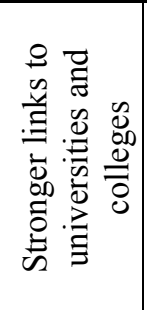 & 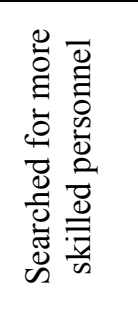 & 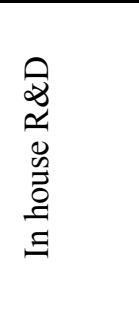 & 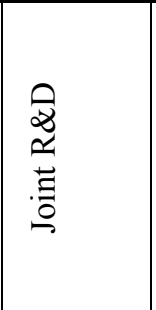 & 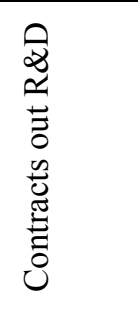 & 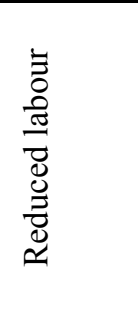 & 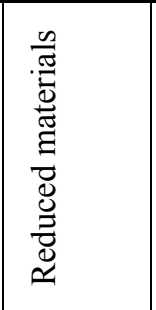 & 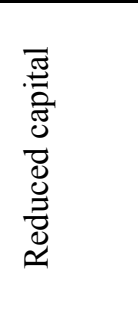 & 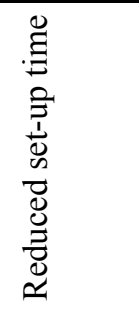 & 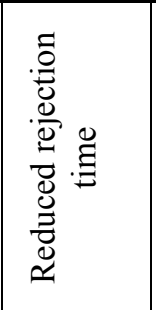 & 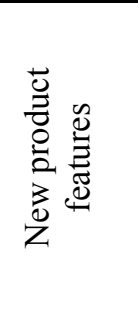 & 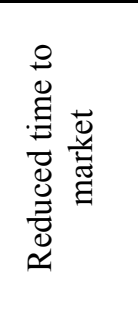 & 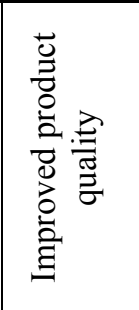 \\
\hline No factors & $\begin{array}{l}.26967^{*} \\
(0.01303)\end{array}$ & $\begin{array}{l}0.26979^{*} \\
(0.01303)\end{array}$ & $\begin{array}{l}0.2562^{*} \\
(0.01413)\end{array}$ & \begin{tabular}{|l|}
$0.26898^{*}$ \\
$(0.01308)$ \\
\end{tabular} & $\begin{array}{l}0.27076^{*} \\
(0.01303)\end{array}$ & $\begin{array}{l}0.26566^{*} \\
(0.0134)\end{array}$ & \begin{tabular}{|l|}
$0.26738^{*}$ \\
$(0.01335)$ \\
\end{tabular} & $\begin{array}{l}0.26988^{*} \\
(0.01308)\end{array}$ & $\begin{array}{l}0.26801^{*} \\
(0.01299)\end{array}$ & \begin{tabular}{|l|}
$0.26894^{*}$ \\
$(0.01307)$ \\
\end{tabular} & $\begin{array}{l}0.26766^{*} \\
(0.01304)\end{array}$ & $\begin{array}{l}0.27013^{*} \\
(0.01308)\end{array}$ & \begin{tabular}{|l|l}
$0.27172^{*}$ \\
$(0.01302)$
\end{tabular} \\
\hline Factor 5 & $\begin{array}{l}0.11089 \\
(0.07008) \\
\end{array}$ & $\begin{array}{l}0.12832^{\ddagger} \\
(0.07276) \\
\end{array}$ & $\begin{array}{l}0.09431 \\
(0.06963) \\
\end{array}$ & \begin{tabular}{|l|}
0.11117 \\
$(0.06945)$ \\
\end{tabular} & $\begin{array}{l}0.12481^{\ddagger} \\
(0.06966) \\
\end{array}$ & $\begin{array}{l}0.10292 \\
(0.0698) \\
\end{array}$ & \begin{tabular}{|l|}
0.10815 \\
$(0.06965)$ \\
\end{tabular} & $\begin{array}{l}0.11395 \\
(0.06972) \\
\end{array}$ & $\begin{array}{l}0.10841 \\
(0.06916) \\
\end{array}$ & \begin{tabular}{|l|}
0.10786 \\
$(0.06973)$ \\
\end{tabular} & $\begin{array}{l}0.10815 \\
(0.0693) \\
\end{array}$ & \begin{tabular}{|l}
0.11348 \\
$(0.06944)$ \\
\end{tabular} & 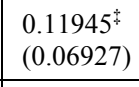 \\
\hline Factor 4 & $\begin{array}{l}\begin{array}{l}0.15129^{*} \\
(0.033)\end{array} \\
\end{array}$ & $\begin{array}{l}0.15173^{*} \\
(0.03299) \\
\end{array}$ & $\begin{array}{l}0.12714^{*} \\
(0.03438)\end{array}$ & \begin{tabular}{|l|}
$0.15086^{*}$ \\
$(0.033)$
\end{tabular} & $\begin{array}{l}0.15129^{*} \\
(0.03295)\end{array}$ & $\begin{array}{l}0.14732^{*} \\
(0.03312)\end{array}$ & \begin{tabular}{|l|}
$0.15001^{*}$ \\
$(0.03303)$
\end{tabular} & $\begin{array}{l}0.15144^{*} \\
(0.03301)\end{array}$ & $\begin{array}{l}0.1481^{*} \\
(0.03288)\end{array}$ & \begin{tabular}{|l|}
$0.15006^{*}$ \\
$(0.03303)$
\end{tabular} & $\begin{array}{l}0.14913^{*} \\
(0.03294)\end{array}$ & $\begin{array}{l}0.1524^{*} \\
(0.03311)\end{array}$ & $\begin{array}{l}0.15821^{*} \\
(0.03302)\end{array}$ \\
\hline Factor $4 \& 5$ & $\begin{array}{l}0.23401^{\ddagger} \\
(0.12812)\end{array}$ & $\begin{array}{l}0.25461^{\ddagger} \\
(0.13068) \\
\end{array}$ & $\begin{array}{l}0.22146^{\ddagger} \\
(0.12766) \\
\end{array}$ & \begin{tabular}{|l|}
$0.23543^{\ddagger}$ \\
$(0.12789)$ \\
\end{tabular} & $\begin{array}{l}0.2386^{\ddagger} \\
(0.12773) \\
\end{array}$ & $\begin{array}{l}0.22743^{\ddagger} \\
(0.12797) \\
\end{array}$ & \begin{tabular}{|l|}
$0.23447^{\ddagger}$ \\
$(0.12788)$
\end{tabular} & $\begin{array}{l}0.23589^{\ddagger} \\
(0.12794)\end{array}$ & $\begin{array}{l}0.22942^{\ddagger} \\
(0.12742)\end{array}$ & \begin{tabular}{|l|}
$0.23365^{\ddagger}$ \\
$(0.1279)$
\end{tabular} & $\begin{array}{l}0.23247^{\ddagger} \\
(0.12763)\end{array}$ & $\begin{array}{l}0.2378^{\ddagger} \\
(0.12804)\end{array}$ & \begin{tabular}{|l|l}
$0.26329^{\dagger}$ \\
$(0.12806)$
\end{tabular} \\
\hline Factor 3 & $\begin{array}{l}0.23331^{*} \\
(0.04392)\end{array}$ & $\begin{array}{l}0.23367^{*} \\
(0.04387) \\
\end{array}$ & $\begin{array}{l}0_{0.20999^{*}} \\
(0.04483)\end{array}$ & \begin{tabular}{|l|}
$0.23261^{*}$ \\
$(0.04391)$ \\
\end{tabular} & $\begin{array}{l}0.25021^{*} \\
(0.04483) \\
\end{array}$ & $\begin{array}{l}0.22175^{*} \\
(0.04485) \\
\end{array}$ & \begin{tabular}{|l|}
$0.23072^{*}$ \\
$(0.04403)$ \\
\end{tabular} & $\begin{array}{l}0.23421^{*} \\
(0.04397) \\
\end{array}$ & $\begin{array}{l}0.23307^{*} \\
(0.0437) \\
\end{array}$ & \begin{tabular}{|l|l}
$0.22676^{*}$ \\
$(0.0449)$
\end{tabular} & $\begin{array}{l}0.22273^{*} \\
(0.04409) \\
\end{array}$ & $\begin{array}{l}0.23509^{*} \\
(0.04402) \\
\end{array}$ & \begin{tabular}{|l}
$0.26122^{*}$ \\
$(0.04522)$ \\
\end{tabular} \\
\hline Factor $3 \& 5$ & $\begin{array}{l}0.15532^{*} \\
(0.05343)\end{array}$ & $\begin{array}{l}0.1777^{*} \\
(0.05748)\end{array}$ & $\begin{array}{l}0.14988^{*} \\
(0.05079)\end{array}$ & \begin{tabular}{|l|}
$0.15852^{*}$ \\
$(0.05081)$ \\
\end{tabular} & $\begin{array}{l}0.15852^{*} \\
(0.05074)\end{array}$ & $\begin{array}{l}0.15752^{*} \\
(0.05078)\end{array}$ & \begin{tabular}{|l|l|l}
$0.15852^{*}$ \\
$(0.0508)$ \\
\end{tabular} & $\begin{array}{l}0.15869^{*} \\
(0.05082)\end{array}$ & $\begin{array}{l}0.14633^{*} \\
(0.0508)\end{array}$ & \begin{tabular}{|l|}
$0.15491^{*}$ \\
$(0.05105)$ \\
\end{tabular} & $\begin{array}{l}0.14939^{*} \\
(0.05089)\end{array}$ & $\begin{array}{l}0.1588^{*} \\
(0.05082)\end{array}$ & \begin{tabular}{|l}
$0.17006^{*}$ \\
$(0.05089)$
\end{tabular} \\
\hline Fact & $\begin{array}{l}0.22511^{*} \\
(0.04837)\end{array}$ & $\begin{array}{l}0.22511^{*} \\
(0.04836)\end{array}$ & $\begin{array}{l}0.20465^{*} \\
(0.04896)\end{array}$ & \begin{tabular}{|l|}
$0.22431^{*}$ \\
$(0.04838)$
\end{tabular} & $\begin{array}{l}0.23054^{*} \\
(0.0484)\end{array}$ & $\begin{array}{l}0.22378^{*} \\
(0.04834)\end{array}$ & \begin{tabular}{|l|}
$0.22493^{*}$ \\
$(0.04836)$
\end{tabular} & $\begin{array}{l}0.22571^{*} \\
(0.04848)\end{array}$ & $\begin{array}{l}0.21979^{*} \\
(0.04821)\end{array}$ & \begin{tabular}{|l|}
$0.22283^{*}$ \\
$(0.04846)$
\end{tabular} & $\begin{array}{l}0.21114^{*} \\
(0.04871)\end{array}$ & $\begin{array}{l}0.22747^{*} \\
(0.04872)\end{array}$ & \begin{tabular}{|l|l}
$0.23707^{*}$ \\
$(0.04848)$
\end{tabular} \\
\hline Factor 3 & $\begin{array}{l}0.16709^{*} \\
(0.06161)\end{array}$ & $\begin{array}{l}0.18907^{*} \\
(0.06565) \\
\end{array}$ & $\begin{array}{l}0.14389^{\dagger} \\
(0.06068) \\
\end{array}$ & \begin{tabular}{|l|}
$0.16711^{*}$ \\
$(0.06006)$ \\
\end{tabular} & $\begin{array}{l}0.18158^{*} \\
(0.06019) \\
\end{array}$ & $\begin{array}{l}0.16514^{*} \\
(0.05997) \\
\end{array}$ & \begin{tabular}{|l|}
$0.16914^{*}$ \\
$(0.05989)$ \\
\end{tabular} & $\begin{array}{l}0.17072^{*} \\
(0.06007) \\
\end{array}$ & $\begin{array}{l}0.15965^{*} \\
(0.05978) \\
\end{array}$ & \begin{tabular}{|l|}
$0.16773^{*}$ \\
$(0.05996)$ \\
\end{tabular} & $\begin{array}{l}0.15693^{*} \\
(0.06008) \\
\end{array}$ & $\begin{array}{l}0.17173^{*} \\
(0.06007) \\
\end{array}$ & \begin{tabular}{|l}
$0.17543^{*}$ \\
$(0.05977)$ \\
\end{tabular} \\
\hline Factor 2 & $\begin{array}{l}0.18048^{*} \\
(0.03802) \\
\end{array}$ & $\begin{array}{l}0.18048^{*} \\
(0.03801) \\
\end{array}$ & $\begin{array}{l}0.1525^{*} \\
(0.03963) \\
\end{array}$ & \begin{tabular}{|l|}
$0.1769^{*}$ \\
$(0.03845)$ \\
\end{tabular} & $\begin{array}{l}0.18139^{*} \\
(0.03796) \\
\end{array}$ & $\begin{array}{l}0.18029^{*} \\
(0.03799) \\
\end{array}$ & \begin{tabular}{|l|}
$0.18029^{*}$ \\
$(0.03801)$ \\
\end{tabular} & $\begin{array}{l}0.18051^{*} \\
(0.03802) \\
\end{array}$ & $\begin{array}{l}0.18017^{*} \\
(0.03787) \\
\end{array}$ & \begin{tabular}{|l|}
$0.18036^{*}$ \\
$(0.03801)$ \\
\end{tabular} & $\begin{array}{l}0.17987^{*} \\
(0.03793) \\
\end{array}$ & $\begin{array}{l}0.18059^{*} \\
(0.03802) \\
\end{array}$ & \begin{tabular}{|l}
$0.19406^{*}$ \\
$(0.03832)$ \\
\end{tabular} \\
\hline Factor $2 \& 5$ & $\begin{array}{ll}.35573 \\
(0.22974) \\
\end{array}$ & $\begin{array}{ll}0.36837 \\
(0.23007)\end{array}$ & $\begin{array}{l}0.34041 \\
(0.22902)\end{array}$ & \begin{tabular}{|l|l|}
0.3523 \\
$(0.2297)$ \\
\end{tabular} & $\begin{array}{l}0.35732 \\
(0.22925)\end{array}$ & $\begin{array}{ll}0.3502 \\
(0.22948)\end{array}$ & \begin{tabular}{|l|}
0.35732 \\
$(0.22953)$ \\
\end{tabular} & $\begin{array}{ll}0.35904 \\
(0.22979)\end{array}$ & $\begin{array}{l}0.35732 \\
(0.22868)\end{array}$ & \begin{tabular}{|l|}
0.35066 \\
$(0.22973)$ \\
\end{tabular} & $\begin{array}{l}0.35732 \\
(0.22908)\end{array}$ & $\begin{array}{l}0.35732 \\
(0.22958)\end{array}$ & \begin{tabular}{|l|l}
$0.3786^{\ddagger}$ \\
$(0.22909)$
\end{tabular} \\
\hline Factor $2 \& 4$ & $\begin{array}{l}0.24549^{*} \\
(0.04215) \\
\end{array}$ & $\begin{array}{ll}0.2458^{*} \\
(0.04215) \\
\end{array}$ & $\begin{array}{l}0.23371^{*} \\
(0.04231) \\
\end{array}$ & \begin{tabular}{|l|l|}
$0.24169^{*}$ \\
$(0.04259)$ \\
\end{tabular} & $\begin{array}{l}0.2466^{*} \\
(0.04209) \\
\end{array}$ & $\begin{array}{l}0.24403^{*} \\
(0.04214) \\
\end{array}$ & \begin{tabular}{|l|}
$0.24462^{*}$ \\
$(0.04216)$ \\
\end{tabular} & $\begin{array}{l}0.24549^{*} \\
(0.04215) \\
\end{array}$ & $\begin{array}{l}0.24107^{*} \\
(0.04202) \\
\end{array}$ & \begin{tabular}{|l|}
$0.2438^{*}$ \\
$(0.04221)$ \\
\end{tabular} & $\begin{array}{l}0.23444^{*} \\
(0.04238) \\
\end{array}$ & $\begin{array}{l}0.24695^{*} \\
(0.04231) \\
\end{array}$ & \begin{tabular}{|l|}
$0.26068^{*}$ \\
$(0.0425)$ \\
\end{tabular} \\
\hline Factor $2,4 \& 5$ & $\begin{array}{l}0.12124^{\dagger} \\
(0.05865) \\
\end{array}$ & $\begin{array}{l}0.14211^{\dagger} \\
(0.06391) \\
\end{array}$ & $\begin{array}{l}0.10487^{\ddagger} \\
(0.0583) \\
\end{array}$ & \begin{tabular}{|l|}
$0.11796^{\dagger}$ \\
$(0.05854)$ \\
\end{tabular} & $\begin{array}{l}0.13158^{\dagger} \\
(0.05812) \\
\end{array}$ & $\begin{array}{l}0.11905^{\dagger} \\
(0.05803) \\
\end{array}$ & \begin{tabular}{|l|}
$0.12248^{\dagger}$ \\
$(0.05798)$ \\
\end{tabular} & $\begin{array}{ll}0.12301^{\dagger} \\
(0.058) \\
\end{array}$ & $\begin{array}{ll}0.11472^{\dagger} \\
(0.05784) \\
\end{array}$ & \begin{tabular}{|l|}
$0.11962^{\dagger}$ \\
$(0.05816)$ \\
\end{tabular} & $\begin{array}{l}0.10982^{\ddagger} \\
(0.0582) \\
\end{array}$ & $\begin{array}{l}0.12514^{\dagger} \\
(0.05825) \\
\end{array}$ & \begin{tabular}{|l}
$0.14495^{\dagger}$ \\
$(0.05854)$ \\
\end{tabular} \\
\hline Fact & $\begin{array}{l}0.28348^{*} \\
(0.06952)\end{array}$ & $\begin{array}{l}0.28348^{*} \\
(0.06951)\end{array}$ & $\begin{array}{l}0.27605^{*} \\
(0.06938)\end{array}$ & \begin{tabular}{|l|}
$0.28287^{*}$ \\
$(0.06952)$
\end{tabular} & $\begin{array}{l}0.28689^{*} \\
(0.06944)\end{array}$ & $\begin{array}{l}0.27676^{*} \\
(0.06967)\end{array}$ & \begin{tabular}{|l|}
$0.28239^{*}$ \\
$(0.06952)$
\end{tabular} & $\begin{array}{l}0.28499^{*} \\
(0.07)\end{array}$ & $\begin{array}{l}0.28196^{*} \\
(0.06925)\end{array}$ & \begin{tabular}{|l|}
$0.27886^{*}$ \\
$(0.0698)$
\end{tabular} & $\begin{array}{l}0.26175^{*} \\
(0.07013)\end{array}$ & $\begin{array}{l}0.28615^{*} \\
(0.06983)\end{array}$ & \begin{tabular}{|l|l}
$0.30265^{*}$ \\
$(0.06977)$
\end{tabular} \\
\hline Factor $2,3 \& 5$ & $\begin{array}{l}0.37016^{\dagger} \\
(0.18037)\end{array}$ & $\begin{array}{l}0.39199^{\dagger} \\
(0.18181)\end{array}$ & $\begin{array}{l}0.34347^{\ddagger} \\
(0.17972)\end{array}$ & \begin{tabular}{|l|}
$0.37025^{\dagger}$ \\
$(0.17987)$ \\
\end{tabular} & $\begin{array}{l}0.385511^{\dagger} \\
(0.17972)\end{array}$ & $\begin{array}{l}0.36424^{\dagger} \\
(0.17983)\end{array}$ & \begin{tabular}{|l|}
$0.36773^{\dagger}$ \\
$(0.17991)$ \\
\end{tabular} & $\begin{array}{l}0.37521^{\dagger} \\
(0.18031)\end{array}$ & $\begin{array}{l}0.36511^{\dagger} \\
(0.17915)\end{array}$ & \begin{tabular}{|l|}
$0.36464^{\dagger}$ \\
$(0.18016)$ \\
\end{tabular} & $\begin{array}{l}0.34209^{\ddagger} \\
(0.18004)\end{array}$ & $\begin{array}{l}0.37591^{\dagger} \\
(0.18)\end{array}$ & \begin{tabular}{|l|l}
$0.3989^{\dagger}$ \\
$(0.17965)$
\end{tabular} \\
\hline Factor 2,3\& 4 & & $\begin{array}{l}0.21506^{*} \\
(0.04295) \\
\end{array}$ & $\begin{array}{l}0.18827^{*} \\
(0.04417) \\
\end{array}$ & \begin{tabular}{|l|}
$0.2075^{*}$ \\
$(0.04441)$ \\
\end{tabular} & $\begin{array}{l}0.22444^{*} \\
(0.04326) \\
\end{array}$ & $\begin{array}{l}0.21347^{*} \\
(0.04292) \\
\end{array}$ & \begin{tabular}{|l|}
$0.21092^{*}$ \\
$(0.04317)$ \\
\end{tabular} & $\begin{array}{l}0.21489^{*} \\
(0.04301) \\
\end{array}$ & $\begin{array}{l}0.20428^{*} \\
(0.04293) \\
\end{array}$ & \begin{tabular}{|l|}
$0.20939^{*}$ \\
$(0.04351)$ \\
\end{tabular} & $\begin{array}{l}0.20618^{*} \\
(0.04303) \\
\end{array}$ & $\begin{array}{l}0.21733^{*} \\
(0.04352) \\
\end{array}$ & \begin{tabular}{|l}
$0.22782^{*}$ \\
$(0.04318)$ \\
\end{tabular} \\
\hline Factor 2,3,4\&5 & $\begin{array}{l}0.22151^{*} \\
(0.04731)\end{array}$ & $\begin{array}{l}0.24169^{*} \\
(0.0529)\end{array}$ & $\begin{array}{l}0.20118^{*} \\
(0.04705)\end{array}$ & \begin{tabular}{|l|}
$0.21981^{*}$ \\
$(0.04664)$
\end{tabular} & $\begin{array}{l}0.23381^{*} \\
(0.0466)\end{array}$ & $\begin{array}{l}0.21655^{*} \\
(0.04656)\end{array}$ & \begin{tabular}{|l|}
$0.22177^{*}$ \\
$(0.04632)$
\end{tabular} & $\begin{array}{l}0.22481^{*} \\
(0.04689)\end{array}$ & $\begin{array}{l}0.21468^{*} \\
(0.04621)\end{array}$ & \begin{tabular}{|l|}
$0.21936^{*}$ \\
$(0.04661)$
\end{tabular} & $\begin{array}{l}0.21312^{*} \\
(0.04644)\end{array}$ & $\begin{array}{l}0.22642^{*} \\
(0.04688)\end{array}$ & \begin{tabular}{|l|l}
$0.23914^{*}$ \\
$(0.04661)$
\end{tabular} \\
\hline Factor 1 & $\begin{array}{l}0.26673^{*} \\
(0.02686)\end{array}$ & $\begin{array}{l}0.26708^{*} \\
(0.02686)\end{array}$ & $\begin{array}{l}0.25425^{*} \\
(0.02727)\end{array}$ & \begin{tabular}{|l|l|}
$0.265^{*}$ \\
$(0.027)$
\end{tabular} & $\begin{array}{l}0.27088^{*} \\
(0.02693)\end{array}$ & $\begin{array}{l}0.25369^{*} \\
(0.02875)\end{array}$ & \begin{tabular}{|l|}
$0.2602^{*}$ \\
$(0.02811)$ \\
\end{tabular} & $\begin{array}{l}0.26925^{*} \\
(0.0301)\end{array}$ & $\begin{array}{l}0.24309^{*} \\
(0.02805)\end{array}$ & \begin{tabular}{|l|}
$0.25763^{*}$ \\
$(0.02966)$ \\
\end{tabular} & $\begin{array}{l}0.24387^{*} \\
(0.02891)\end{array}$ & $\begin{array}{l}0.27176^{*} \\
(0.0296)\end{array}$ & \begin{tabular}{|l|l}
$0.30063^{*}$ \\
$(0.03025)$
\end{tabular} \\
\hline Factor $1 \& 5$ & $\begin{array}{l}0.27342^{*} \\
(0.03301)\end{array}$ & $\begin{array}{l}0.29417^{*} \\
(0.04149) \\
\end{array}$ & $\begin{array}{l}\begin{array}{l}0.265^{*} \\
(0.03196)\end{array} \\
\end{array}$ & \begin{tabular}{|l|}
$0.27223^{*}$ \\
$0.03212)$ \\
\end{tabular} & $\begin{array}{l}.27583^{*} \\
(0.03174) \\
\end{array}$ & $\begin{array}{l}0.26404^{*} \\
(0.03295) \\
\end{array}$ & \begin{tabular}{|l|}
$0.26826^{*}$ \\
$(0.03296)$
\end{tabular} & $\begin{array}{l}.278^{*} \\
(0.03529) \\
\end{array}$ & $\begin{array}{l}0.2461^{*} \\
(0.03331) \\
\end{array}$ & \begin{tabular}{|l|}
$0.26609^{*}$ \\
$(0.03416)$ \\
\end{tabular} & $\begin{array}{l}0.25018^{*} \\
(0.03385) \\
\end{array}$ & $\begin{array}{l}0.28088^{*} \\
(0.03479)\end{array}$ & \begin{tabular}{|l}
$0.31186^{*}$ \\
$(0.03516)$
\end{tabular} \\
\hline Facto & $\begin{array}{l}0.27857^{*} \\
(0.03462) \\
\end{array}$ & $\begin{array}{l}0.27991^{*} \\
(0.03466) \\
\end{array}$ & $\begin{array}{l}0.2589^{*} \\
(0.03546)\end{array}$ & \begin{tabular}{|l|}
$0.27625^{*}$ \\
$(0.03481)$ \\
\end{tabular} & $\begin{array}{l}0.28416^{*} \\
(0.03471) \\
\end{array}$ & $\begin{array}{l}0.26937^{*} \\
(0.03535) \\
\end{array}$ & \begin{tabular}{|l|}
$0.27273^{*}$ \\
$(0.03539)$ \\
\end{tabular} & $\begin{array}{l}0.28128^{*} \\
(0.03756) \\
\end{array}$ & $\begin{array}{l}0.25414^{*} \\
(0.03556)\end{array}$ & \begin{tabular}{|l|}
$0.26867^{*}$ \\
$(0.03722)$ \\
\end{tabular} & $\begin{array}{l}0.25117^{*} \\
(0.0369) \\
\end{array}$ & $\begin{array}{l}0.28399^{*} \\
(0.03711)\end{array}$ & \begin{tabular}{|l}
$0.31232^{*}$ \\
$(0.03725)$ \\
\end{tabular} \\
\hline Factor $1,4 \& 5$ & $\begin{array}{l}.20947^{*} \\
(0.03345)\end{array}$ & $\begin{array}{l}0.23058^{*} \\
(0.04174) \\
\end{array}$ & $\begin{array}{l}0.18924^{*} \\
(0.03312) \\
\end{array}$ & \begin{tabular}{|l|l|}
$0.20935^{*}$ \\
$(0.0321)$ \\
\end{tabular} & $\begin{array}{l}0.21711^{*} \\
(0.03205) \\
\end{array}$ & $\begin{array}{l}0.19918^{*} \\
(0.03333) \\
\end{array}$ & \begin{tabular}{|l|}
$0.20522^{*}$ \\
$(0.03288)$
\end{tabular} & $\begin{array}{l}0.21395^{*} \\
(0.03474)\end{array}$ & $\begin{array}{l}0.18656^{*} \\
(0.03301)\end{array}$ & \begin{tabular}{|l|}
$0.20089^{*}$ \\
$(0.03508)$
\end{tabular} & $\begin{array}{l}0.18636^{*} \\
(0.034)\end{array}$ & $\begin{array}{l}0.21689^{*} \\
(0.03468)\end{array}$ & \begin{tabular}{|l|l}
$0.24259^{*}$ \\
$(0.03436)$
\end{tabular} \\
\hline Fact & $\begin{array}{l}0.28373^{*} \\
(0.02772) \\
\end{array}$ & $\begin{array}{l}0.28521^{*} \\
(0.02778) \\
\end{array}$ & $\begin{array}{ll}0.2696^{*} \\
(0.02825) \\
\end{array}$ & \begin{tabular}{|l|l|}
$0.28243^{*}$ \\
$(0.02781)$ \\
\end{tabular} & $\begin{array}{l}0.28934^{*} \\
(0.02786) \\
\end{array}$ & $\begin{array}{l}0.26956^{*} \\
(0.0299) \\
\end{array}$ & \begin{tabular}{|l|}
$0.27458^{*}$ \\
$(0.0301)$ \\
\end{tabular} & $\begin{array}{l}0.28642^{*} \\
(0.03108) \\
\end{array}$ & $\begin{array}{l}0.25441^{*} \\
(0.02954) \\
\end{array}$ & \begin{tabular}{|l|}
$0.27296^{*}$ \\
$(0.03152)$ \\
\end{tabular} & $\begin{array}{l}0.25709^{*} \\
(0.03042) \\
\end{array}$ & \begin{tabular}{|l|l|l}
$.2893^{*}$ \\
$(0.03085)$ \\
\end{tabular} & \begin{tabular}{|l}
$0.31949^{*}$ \\
$(0.03135)$ \\
\end{tabular} \\
\hline Fa & $\begin{array}{l}0.26551^{*} \\
(0.0281)^{*}\end{array}$ & $\begin{array}{l}0.28767^{*} \\
(0.03502)\end{array}$ & $\begin{array}{l}0.26293^{*} \\
(0.02284)\end{array}$ & \begin{tabular}{|l|}
$0.26805^{*}$ \\
$(0.0228)$
\end{tabular} & $\begin{array}{l}0.28643^{*} \\
(0.02491)\end{array}$ & $\begin{array}{l}0.25282^{*} \\
(0.02599)\end{array}$ & \begin{tabular}{|l|}
$0.25971^{*}$ \\
$(0.02548)$ \\
\end{tabular} & $\begin{array}{l}0.26913^{*} \\
(0.0229)\end{array}$ & $\begin{array}{l}0.23554^{*} \\
(0.02559)\end{array}$ & \begin{tabular}{|l|}
$0.25771^{*}$ \\
$(0.02738)$ \\
\end{tabular} & $\begin{array}{l}0.24335^{*} \\
(0.02571)\end{array}$ & $\begin{array}{l}0.27434^{*} \\
(0.0267)\end{array}$ & \begin{tabular}{|l}
$0.30513^{*}$ \\
$(0.02728)$
\end{tabular} \\
\hline Factor & $\begin{array}{l}0.19083^{*} \\
(0.01799)\end{array}$ & $\begin{array}{l}0.19322^{*} \\
(0.0183)\end{array}$ & $\begin{array}{l}0.16763^{*} \\
(0.02034)\end{array}$ & \begin{tabular}{|l|}
$0.18987^{*}$ \\
$(0.01805)$
\end{tabular} & $\begin{array}{l}0.19384^{*} \\
(0.01805)\end{array}$ & $\begin{array}{l}0.17793^{*} \\
(0.02067)\end{array}$ & \begin{tabular}{|l|}
$0.18689^{*}$ \\
$(0.01867)$
\end{tabular} & $\begin{array}{l}0.19342^{*} \\
(0.02276)\end{array}$ & $\begin{array}{l}0.15707^{*} \\
(0.02159)\end{array}$ & \begin{tabular}{|l|l}
$0.18035^{*}$ \\
$(0.0231)$
\end{tabular} & $\begin{array}{l}0.16411^{*} \\
(0.02197)\end{array}$ & $\begin{array}{l}0.1967^{*} \\
(0.02311)\end{array}$ & \begin{tabular}{|l|l}
$0.20868^{*}$ \\
$(0.01941)$
\end{tabular} \\
\hline Factor 1 & $\begin{array}{l}0.22078^{*} \\
(0.03255) \\
\end{array}$ & $\begin{array}{l}0.24223^{*} \\
(0.03944) \\
\end{array}$ & $\begin{array}{l}0.19679^{*} \\
(0.03133) \\
\end{array}$ & \begin{tabular}{|l|l}
$0.22212^{*}$ \\
$(0.0295)$ \\
\end{tabular} & $\begin{array}{l}0.22768^{*} \\
(0.02947) \\
\end{array}$ & $\begin{array}{l}0.21033^{*} \\
(0.03118) \\
\end{array}$ & \begin{tabular}{|l|}
$0.2167^{*}$ \\
$(0.03065)$ \\
\end{tabular} & $\begin{array}{l}0.22591^{*} \\
(0.03216) \\
\end{array}$ & $\begin{array}{l}.20031^{*} \\
(0.03045) \\
\end{array}$ & \begin{tabular}{|l|}
$0.21417^{*}$ \\
$(0.03211)$ \\
\end{tabular} & $\begin{array}{l}0.19887^{*} \\
(0.03159) \\
\end{array}$ & $\begin{array}{l}0.22907^{*} \\
(0.03248) \\
\end{array}$ & \begin{tabular}{|l|l|}
$0.2555^{*}$ \\
$(0.03219)$ \\
\end{tabular} \\
\hline Fact & $\begin{array}{l}0.19885^{*} \\
(0.03546)\end{array}$ & $\begin{array}{l}0.20092^{*} \\
(0.03557) \\
\end{array}$ & $\begin{array}{l}0.1819^{*} \\
(0.03604)\end{array}$ & \begin{tabular}{|l|}
$0.19867^{*}$ \\
$(0.03546)$ \\
\end{tabular} & $\begin{array}{l}0.20775^{*} \\
(0.03577) \\
\end{array}$ & $\begin{array}{ll}0.1845^{*} \\
(0.03721) \\
\end{array}$ & \begin{tabular}{|l|}
$0.19215^{*}$ \\
$(0.03646)$ \\
\end{tabular} & $\begin{array}{l}0.20148^{*} \\
(0.03817) \\
\end{array}$ & $\begin{array}{l}0.18461^{*} \\
(0.03568)\end{array}$ & \begin{tabular}{|l|}
$0.19214^{*}$ \\
$(0.03665)$ \\
\end{tabular} & $\begin{array}{l}0.17272^{*} \\
(0.03749) \\
\end{array}$ & $\begin{array}{l}0.20436^{*} \\
(0.03798) \\
\end{array}$ & \begin{tabular}{|l}
$0.23472^{*}$ \\
$(0.03836)$ \\
\end{tabular} \\
\hline$F_{i}$ & $\begin{array}{l}0.20049^{*} \\
(0.05455)\end{array}$ & $\begin{array}{l}0.2158^{*} \\
(0.05725)\end{array}$ & $\begin{array}{l}0.19053^{*} \\
(0.05398)\end{array}$ & \begin{tabular}{|l|}
$0.20095^{*}$ \\
$(0.05396)$ \\
\end{tabular} & $\begin{array}{l}0.20349^{*} \\
(0.05386)\end{array}$ & $\begin{array}{l}0.18575^{*} \\
(0.05542)\end{array}$ & \begin{tabular}{|l|}
$0.19559^{*}$ \\
$(0.05455)$ \\
\end{tabular} & $\begin{array}{l}0.2048^{*} \\
(0.0559)\end{array}$ & $\begin{array}{l}0.17603^{*} \\
(0.05452)\end{array}$ & \begin{tabular}{|l|}
$0.1912^{*}$ \\
$(0.05598)$ \\
\end{tabular} & $\begin{array}{l}0.17804^{*} \\
(0.05501)\end{array}$ & $\begin{array}{l}0.20744^{*} \\
(0.05554)\end{array}$ & \begin{tabular}{|l}
$0.23834^{*}$ \\
$(0.05584)$
\end{tabular} \\
\hline Factor & $\begin{array}{l}0.46252^{*} \\
(0.02908)\end{array}$ & $\begin{array}{l}0.46269^{*} \\
(0.02907)\end{array}$ & $\begin{array}{l}0.45026^{*} \\
(0.02943)\end{array}$ & \begin{tabular}{|l|}
$0.46108^{*}$ \\
$(0.02917)$ \\
\end{tabular} & $\begin{array}{l}0.46296^{*} \\
(0.02903)\end{array}$ & $\begin{array}{l}0.45607^{*} \\
(0.0295)\end{array}$ & \begin{tabular}{|l|}
$0.45864^{*}$ \\
$(0.02949)$
\end{tabular} & $\begin{array}{l}0.46514^{*} \\
(0.03231)\end{array}$ & $\begin{array}{l}0.43281^{*} \\
(0.03084)\end{array}$ & \begin{tabular}{|l|}
$0.45168^{*}$ \\
$(0.03272)$ \\
\end{tabular} & $\begin{array}{l}0.43719^{*} \\
(0.0314)\end{array}$ & $\begin{array}{l}0.46634^{*} \\
(0.03056)\end{array}$ & \begin{tabular}{|l|l}
$0.48788^{*}$ \\
$(0.03084)$
\end{tabular} \\
\hline Factor $1,2,4 \&$ & $\begin{array}{l}0.24255^{*} \\
(0.03539) \\
\end{array}$ & $\begin{array}{l}0.26384^{*} \\
(0.04246) \\
\end{array}$ & $\begin{array}{l}0.22587^{*} \\
(0.03401)\end{array}$ & \begin{tabular}{|l|l}
$0.24257^{*}$ \\
$(0.0334)$ \\
\end{tabular} & $\begin{array}{l}0.25061^{*} \\
(0.0333) \\
\end{array}$ & $\begin{array}{l}0.23578^{*} \\
(0.03394) \\
\end{array}$ & \begin{tabular}{|l|}
$0.23982^{*}$ \\
$0.03381)$ \\
\end{tabular} & $\begin{array}{l}0.24695^{*} \\
(0.03491) \\
\end{array}$ & $\begin{array}{l}0.23413^{*} \\
(0.03328)\end{array}$ & \begin{tabular}{|l|}
$0.23441^{*}$ \\
$(0.03623)$ \\
\end{tabular} & $\begin{array}{l}0.21954^{*} \\
(0.03523) \\
\end{array}$ & $\begin{array}{l}0.24997^{*} \\
(0.03543) \\
\end{array}$ & \begin{tabular}{|l}
$0.26563^{*}$ \\
$(0.03418)$ \\
\end{tabular} \\
\hline Factor $1,2 \& 3$ & $\begin{array}{l}0.24226^{*} \\
(0.04383)\end{array}$ & $\begin{array}{l}0.24226^{*} \\
(0.04382)\end{array}$ & $\begin{array}{l}0.22007^{*} \\
(0.04465)\end{array}$ & \begin{tabular}{|l|}
$0.24118^{*}$ \\
$(0.04386)$
\end{tabular} & $\begin{array}{l}0.24514^{*} \\
(0.04379)\end{array}$ & $\begin{array}{l}0.22776^{*} \\
(0.04527)\end{array}$ & \begin{tabular}{|l|}
$0.23632^{*}$ \\
$(0.04446)$
\end{tabular} & $\begin{array}{l}0.24433^{*} \\
(0.04522)\end{array}$ & $\begin{array}{l}0.21721^{*} \\
(0.04456)\end{array}$ & \begin{tabular}{|l|}
$0.23253^{*}$ \\
$(0.04584)$
\end{tabular} & $\begin{array}{l}0.21572^{*} \\
(0.0455)\end{array}$ & $\begin{array}{l}0.24761^{*} \\
(0.04577)\end{array}$ & \begin{tabular}{|l|l|}
$0.27046^{*}$ \\
$(0.04524)$
\end{tabular} \\
\hline Factor $1,2,3 \& 5$ & $\begin{array}{l}0.31661^{*} \\
(0.03567)\end{array}$ & $\begin{array}{l}0.33819^{*} \\
(0.04293)\end{array}$ & $\begin{array}{l}0.29547^{*} \\
(0.03476)\end{array}$ & \begin{tabular}{|l|}
$0.31618^{*}$ \\
$(0.03378)$ \\
\end{tabular} & $\begin{array}{l}0.32448^{*} \\
(0.03357)\end{array}$ & $\begin{array}{l}0.30633^{*} \\
(0.03491)\end{array}$ & \begin{tabular}{|l|}
$0.31443^{*}$ \\
$(0.03396)$ \\
\end{tabular} & $\begin{array}{l}0.32162^{*} \\
(0.03629)\end{array}$ & $\begin{array}{l}0.29142^{*} \\
(0.03476)\end{array}$ & \begin{tabular}{|l|}
$0.3094^{*}$ \\
$(0.03601)$ \\
\end{tabular} & $\begin{array}{l}0.2955^{*} \\
(0.03521)\end{array}$ & $\begin{array}{l}0.32332^{*} \\
(0.03512)\end{array}$ & \begin{tabular}{|l|l}
$0.3502^{*}$ \\
$(0.03579)$
\end{tabular} \\
\hline Factor 1,2 & \begin{tabular}{l|l}
$0.268^{*}$ \\
$(0.01683)$
\end{tabular} & $\begin{array}{l}0.27036^{*} \\
(0.01715)\end{array}$ & $\begin{array}{l}0.24548^{*} \\
(0.01918)\end{array}$ & \begin{tabular}{|l|}
$0.2651^{*}$ \\
$(0.01747)$ \\
\end{tabular} & $\begin{array}{l}0.27734^{*} \\
(0.01764)\end{array}$ & $\begin{array}{l}0.25503^{*} \\
(0.0197)\end{array}$ & \begin{tabular}{|l|}
$0.26044^{*}$ \\
$(0.01938)$
\end{tabular} & $\begin{array}{l}0.2707^{*} \\
(0.02225)\end{array}$ & $\begin{array}{l}0.23826^{*} \\
(0.01984)\end{array}$ & \begin{tabular}{|l|}
$0.25935^{*}$ \\
$(0.02064)$
\end{tabular} & $\begin{array}{l}0.24763^{*} \\
(0.01937)\end{array}$ & $\begin{array}{l}0.27385^{*} \\
(0.02218)\end{array}$ & \begin{tabular}{|l|l}
$0.29774^{*}$ \\
$(0.02083)$
\end{tabular} \\
\hline All Factor & $\begin{array}{l}0.30649^{*} \\
(0.02082) \\
\end{array}$ & $\begin{array}{l}0.32692^{*} \\
(0.03126) \\
\end{array}$ & $\begin{array}{l}0.28704^{*} \\
(0.01986)\end{array}$ & \begin{tabular}{|l|}
$0.30391^{*}$ \\
$(0.01935)$ \\
\end{tabular} & $\begin{array}{l}0.31667^{*} \\
(0.01837) \\
\end{array}$ & $\begin{array}{l}0.29491^{*} \\
(0.02082) \\
\end{array}$ & \begin{tabular}{|l|}
$0.30138^{*}$ \\
$(0.02022)$ \\
\end{tabular} & $\begin{array}{l}0.31076^{*} \\
(0.0213)\end{array}$ & $\begin{array}{l}0.27947^{*} \\
(0.02055)\end{array}$ & \begin{tabular}{|l|l|}
$0.29799^{*}$ \\
$(0.02306)$ \\
\end{tabular} & $\begin{array}{l}0.28143^{*} \\
(0.02194) \\
\end{array}$ & $\begin{array}{l}0.31369^{*} \\
(0.02181) \\
\end{array}$ & \begin{tabular}{|l|l}
$0.34271^{*}$ \\
$(0.0227)$ \\
\end{tabular} \\
\hline $\begin{array}{l}\text { Individual } \\
\text { Variable }\end{array}$ & .0.0193) & $\begin{array}{l}-0.01918 \\
(0.02689)\end{array}$ & $\begin{array}{l}0.02934^{\dagger} \\
(0.01211)\end{array}$ & \begin{tabular}{|l|}
0.00872 \\
$(0.01411)$
\end{tabular} & $\begin{array}{l}-0.02548^{\ddagger} \\
(0.01462)\end{array}$ & $(0.01329)$ & $(0.01268)$ & $(0.01608)$ & $\begin{array}{l}0.03903^{*} \\
(0.01393)\end{array}$ & $(0.016)$ & $\begin{array}{l}0.03072^{\dagger} \\
(0.01457)\end{array}$ & $\begin{array}{l}-0.00633 \\
(0.01564)\end{array}$ & \begin{tabular}{|l}
$-0.03692^{\dagger}$ \\
$(0.01531)$
\end{tabular} \\
\hline
\end{tabular}

* Significant at the 0.01 level $†$ Significant at the 0.05 level $\quad$ Significant at the 0.10 level

Table 90 Coefficients with individual variables for high-level factors - Medium Plants 


\begin{tabular}{|c|c|c|c|c|c|c|c|c|c|c|c|c|c|}
\hline $\begin{array}{l}\text { Individual variable } \\
\text { included in the } \\
\text { model in the } \\
\text { column }\end{array}$ & 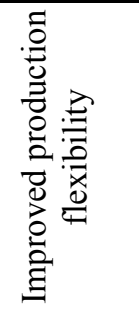 & 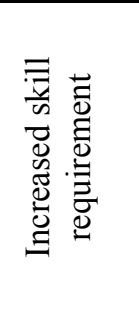 & 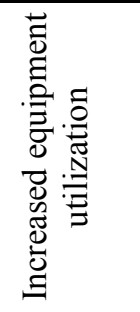 & 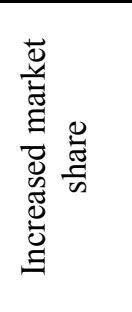 & 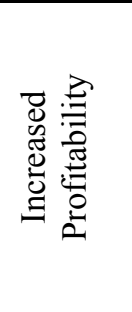 & 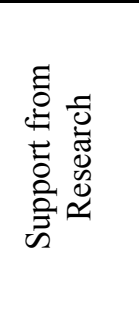 & 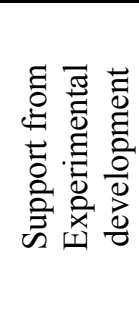 & 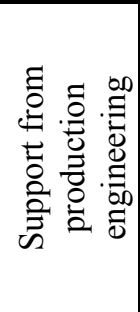 & 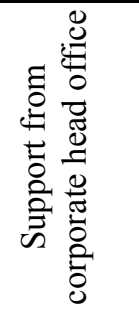 & 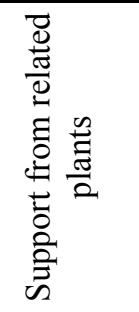 & 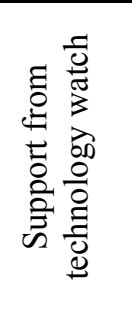 & 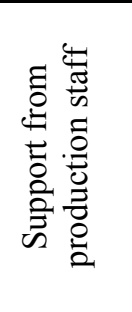 & 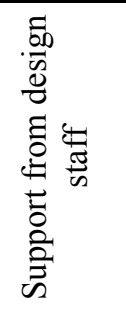 \\
\hline No factors & $\begin{array}{l}0.26962^{*} \\
(0.01305)\end{array}$ & $\begin{array}{l}0.25722^{*} \\
(0.01346)\end{array}$ & $\begin{array}{l}0.26734^{*} \\
(0.01305)\end{array}$ & $\begin{array}{l}0.27043^{*} \\
(0.01304)\end{array}$ & $\begin{array}{l}0.26992^{*} \\
(0.01307)\end{array}$ & $\begin{array}{l}0.26747^{*} \\
(0.01332)\end{array}$ & $\begin{array}{l}0.26997^{*} \\
(0.01302)\end{array}$ & $\begin{array}{l}0.26985^{*} \\
(0.01304)\end{array}$ & $\begin{array}{l}0.26535^{*} \\
(0.01339)\end{array}$ & $\begin{array}{l}0.26929^{*} \\
(0.013)\end{array}$ & $\begin{array}{l}0.26977^{*} \\
(0.01303)\end{array}$ & $\begin{array}{l}0.26049^{*} \\
(0.01367)\end{array}$ & $\begin{array}{l}0.2691^{*} \\
(0.01302)\end{array}$ \\
\hline Factor 5 & $\begin{array}{l}0.11218 \\
(0.0698)\end{array}$ & $\begin{array}{l}0.10212 \\
(0.06908)\end{array}$ & $\begin{array}{l}0.0995 \\
(0.06957)\end{array}$ & $\begin{array}{l}0.1132 \\
(0.06938)\end{array}$ & $\begin{array}{l}0.11493 \\
(0.06998)\end{array}$ & $\begin{array}{l}0.10819 \\
(0.06964)\end{array}$ & $\begin{array}{l}0.1174^{\ddagger} \\
(0.06941)\end{array}$ & $\begin{array}{l}0.11545^{\ddagger} \\
(0.06998)\end{array}$ & $\begin{array}{l}0.10994 \\
(0.06938)\end{array}$ & $\begin{array}{l}0.10018 \\
(0.0695)\end{array}$ & $\begin{array}{l}0.11275 \\
(0.06941)\end{array}$ & $\begin{array}{l}0.08694 \\
(0.07027)\end{array}$ & $\begin{array}{l}0.09622 \\
(0.07021)\end{array}$ \\
\hline Factor 4 & $\begin{array}{l}0.15106^{*} \\
(0.03312)\end{array}$ & $\begin{array}{l}0.14003^{*} \\
(0.03297)\end{array}$ & $\begin{array}{l}0.14551^{*} \\
(0.03305)\end{array}$ & $\begin{array}{l}0.15341^{*} \\
(0.03304)\end{array}$ & $\begin{array}{l}0.15218^{*} \\
(0.03319)\end{array}$ & $\begin{array}{l}0.14711^{*} \\
(0.03341)\end{array}$ & $\begin{array}{l}0.16187^{*} \\
(0.03369)\end{array}$ & $\begin{array}{l}0.15217^{*} \\
(0.03312)\end{array}$ & $\begin{array}{l}0.14708^{*} \\
(0.03311)\end{array}$ & $\begin{array}{l}0.14805^{*} \\
(0.03295)\end{array}$ & $\begin{array}{l}0.15159^{*} \\
(0.033)\end{array}$ & $\begin{array}{l}0.13158^{*} \\
(0.03415)\end{array}$ & $\begin{array}{l}0.13372^{*} \\
(0.03498)\end{array}$ \\
\hline Facto & $\begin{array}{l}0.2347^{\ddagger} \\
(0.12825)\end{array}$ & $\begin{array}{l}0.21721^{\ddagger} \\
(0.12727)\end{array}$ & $\begin{array}{l}0.23112^{\ddagger} \\
(0.12765)\end{array}$ & $\begin{array}{l}0.23543^{\ddagger} \\
(0.12784)\end{array}$ & $\begin{array}{l}0.23734^{\ddagger} \\
(0.12814)\end{array}$ & $\begin{array}{l}0.22747^{\ddagger} \\
(0.12827)\end{array}$ & $\begin{array}{l}0.23543^{\ddagger} \\
(0.12776)\end{array}$ & $\begin{array}{l}0.23819^{\ddagger} \\
(0.12823)\end{array}$ & $\begin{array}{l}0.22478^{\ddagger} \\
(0.12802)\end{array}$ & $\begin{array}{l}0.23543^{\ddagger} \\
(0.12761)\end{array}$ & $\begin{array}{l}0.23663^{\ddagger} \\
(0.12792)\end{array}$ & $\begin{array}{l}0.22505^{\ddagger} \\
(0.12769)\end{array}$ & $\begin{array}{l}0.22792^{\ddagger} \\
(0.12786)\end{array}$ \\
\hline Factor 3 & $\begin{array}{l}0.23293^{*} \\
(0.0449)\end{array}$ & $\begin{array}{l}0.2228^{*} \\
(0.04374)\end{array}$ & $\begin{array}{l}0.22718^{*} \\
(0.0439)\end{array}$ & $\begin{array}{l}0.24243^{*} \\
(0.04466)\end{array}$ & $\begin{array}{l}0.23487^{*} \\
(0.04414)\end{array}$ & $\begin{array}{l}0.23114^{*} \\
(0.04398)\end{array}$ & $\begin{array}{l}0.23898^{*} \\
(0.04397)\end{array}$ & $\begin{array}{l}0.23712^{*} \\
(0.04529)\end{array}$ & $\begin{array}{l}0.22797^{*} \\
(0.04403)\end{array}$ & $\begin{array}{l}0.21525^{*} \\
(0.0446)\end{array}$ & $\begin{array}{l}0.23414^{*} \\
(0.04388)\end{array}$ & $\begin{array}{l}0.2042^{*} \\
(0.04583)\end{array}$ & $\begin{array}{l}0.22574^{*} \\
(0.04415)\end{array}$ \\
\hline Fact & $\begin{array}{l}0.15818^{*} \\
(0.05101)\end{array}$ & $\begin{array}{l}0.11185^{\dagger} \\
(0.05235)\end{array}$ & $\begin{array}{l}0.15685^{*} \\
(0.05071)\end{array}$ & $\begin{array}{l}0.16222^{*} \\
(0.05091)\end{array}$ & $\begin{array}{l}0.15955^{*} \\
(0.05099)\end{array}$ & $\begin{array}{l}0.15175^{*} \\
(0.05151)\end{array}$ & $\begin{array}{l}0.16356^{*} \\
(0.05087)\end{array}$ & $\begin{array}{l}0.16022^{*} \\
(0.05112)\end{array}$ & $\begin{array}{l}0.14719^{*} \\
(0.05143)\end{array}$ & $\begin{array}{l}0.14845^{*} \\
(0.05091)\end{array}$ & $\begin{array}{l}0.15852^{*} \\
(0.05081)\end{array}$ & $\begin{array}{l}0.12974^{\dagger} \\
(0.0524)\end{array}$ & $\begin{array}{l}0.138^{*} \\
(0.05257)\end{array}$ \\
\hline Fact & $\begin{array}{l}0.22478^{*} \\
(0.04856)\end{array}$ & $\begin{array}{l}0.20867^{*} \\
(0.04833)\end{array}$ & $\begin{array}{l}0.22268^{*} \\
(0.04828)\end{array}$ & $\begin{array}{l}0.2307^{*} \\
(0.04864)\end{array}$ & $\begin{array}{l}0.22669^{*} \\
(0.04879)\end{array}$ & $\begin{array}{l}0.22318^{*} \\
(0.04842)\end{array}$ & $\begin{array}{l}0.24173^{*} \\
(0.04954)\end{array}$ & $\begin{array}{l}0.2279^{*} \\
(0.04922)\end{array}$ & $\begin{array}{l}0.21812^{*} \\
(0.04859)\end{array}$ & $\begin{array}{l}0.21204^{*} \\
(0.04864)\end{array}$ & $\begin{array}{l}0.22894^{*} \\
(0.04892)\end{array}$ & $\begin{array}{l}0.19885^{*} \\
(0.04975)\end{array}$ & $\begin{array}{l}0.20412^{*} \\
(0.0503)\end{array}$ \\
\hline Factor & $\begin{array}{l}0.16969^{*} \\
(0.05996)\end{array}$ & $\begin{array}{l}0.16246^{*} \\
(0.05959)\end{array}$ & $\begin{array}{l}0.168^{*} \\
(0.05978)\end{array}$ & $\begin{array}{l}0.17206^{*} \\
(0.05991)\end{array}$ & $\begin{array}{l}0.17077^{*} \\
(0.06001)\end{array}$ & $\begin{array}{l}0.1622^{*} \\
(0.06066)\end{array}$ & $\begin{array}{l}0.18755^{*} \\
(0.06096)\end{array}$ & $\begin{array}{l}0.17388^{*} \\
(0.0613)\end{array}$ & $\begin{array}{l}0.16178^{*} \\
(0.06014)\end{array}$ & $\begin{array}{l}0.16206^{*} \\
(0.05987)\end{array}$ & $\begin{array}{l}0.17101^{*} \\
(0.05993)\end{array}$ & $\begin{array}{l}0.15907^{*} \\
(0.05997)\end{array}$ & $\begin{array}{l}0.14781^{\dagger} \\
(0.06162)\end{array}$ \\
\hline Factor 2 & $\begin{array}{l}0.18046^{*} \\
(0.03802)\end{array}$ & $\begin{array}{l}0.17862^{*} \\
(0.0378)\end{array}$ & $\begin{array}{l}0.17986^{*} \\
(0.03794)\end{array}$ & $\begin{array}{l}0.18048^{*} \\
(0.038)\end{array}$ & $\begin{array}{l}0.18053^{*} \\
(0.03802)\end{array}$ & $\begin{array}{l}0.17511^{*} \\
(0.03861)\end{array}$ & $\begin{array}{l}0.18863^{*} \\
(0.03835)\end{array}$ & $\begin{array}{l}0.18221^{*} \\
(0.03844)\end{array}$ & $\begin{array}{l}0.17435^{*} \\
(0.03824)\end{array}$ & $\begin{array}{l}0.17033^{*} \\
(0.03822)\end{array}$ & $\begin{array}{l}0.18048^{*} \\
(0.03802)\end{array}$ & $\begin{array}{l}0.17892^{*} \\
(0.03794)\end{array}$ & $\begin{array}{l}0.17605^{*} \\
(0.03809)\end{array}$ \\
\hline Facto & $\begin{array}{l}0.35732 \\
(0.2296)\end{array}$ & $\begin{array}{l}0.32879 \\
(0.22841)\end{array}$ & $\begin{array}{l}0.34121 \\
(0.22924)\end{array}$ & $\begin{array}{l}0.35732 \\
(0.22948)\end{array}$ & $\begin{array}{l}0.35966 \\
(0.22979)\end{array}$ & $\begin{array}{l}0.35168 \\
(0.22964)\end{array}$ & $\begin{array}{l}0.35732 \\
(0.22933)\end{array}$ & $\begin{array}{l}0.36175 \\
(0.23005)\end{array}$ & $\begin{array}{l}0.35732 \\
(0.22938)\end{array}$ & $\begin{array}{l}0.34577 \\
(0.22912)\end{array}$ & $\begin{array}{l}0.35732 \\
(0.22957)\end{array}$ & $\begin{array}{l}0.32679 \\
(0.22948)\end{array}$ & $\begin{array}{l}0.34797 \\
(0.22942)\end{array}$ \\
\hline Facto & $\begin{array}{l}0.24512^{*} \\
(0.04241)\end{array}$ & $\begin{array}{l}0.23767^{*} \\
(0.04197)\end{array}$ & $\begin{array}{l}0.23977^{*} \\
(0.04216)\end{array}$ & $\begin{array}{l}0.24949^{*} \\
(0.04231) \\
\end{array}$ & $\begin{array}{l}0.24737^{*} \\
(0.04283) \\
\end{array}$ & $\begin{array}{l}0.24419^{*} \\
(0.04217)\end{array}$ & $\begin{array}{l}0.24719^{*} \\
(0.04212)\end{array}$ & $\begin{array}{l}0.24752^{*} \\
(0.04267)\end{array}$ & $\begin{array}{l}0.23481^{*} \\
(0.04282)\end{array}$ & $\begin{array}{l}0.23569^{*} \\
(0.0423)\end{array}$ & $\begin{array}{l}0.24586^{*} \\
(0.04216) \\
\end{array}$ & $\begin{array}{l}0.23108^{*} \\
(0.04257) \\
\end{array}$ & $\begin{array}{l}0.2401^{*} \\
(0.04226) \\
\end{array}$ \\
\hline $\mathrm{F}$ & $\begin{array}{l}0.1227^{\dagger} \\
(0.05807)\end{array}$ & $\begin{array}{l}0.0925 \\
(0.05835)\end{array}$ & $\begin{array}{l}0.12225^{\dagger} \\
(0.05787)\end{array}$ & $\begin{array}{l}0.12293^{\dagger} \\
(0.05796)\end{array}$ & $\begin{array}{l}0.12312^{\dagger} \\
(0.058)\end{array}$ & $\begin{array}{l}0.12272^{\dagger} \\
(0.05798)\end{array}$ & $\begin{array}{l}0.12382^{\dagger} \\
(0.05793)\end{array}$ & $\begin{array}{l}0.12525^{\dagger} \\
(0.05848)\end{array}$ & $\begin{array}{l}0.11556^{\dagger} \\
(0.05819)\end{array}$ & $\begin{array}{l}0.11251^{\ddagger} \\
(0.05806)\end{array}$ & $\begin{array}{l}0.12293^{\dagger} \\
(0.05799)\end{array}$ & $\begin{array}{l}0.09974^{\ddagger} \\
(0.05883)\end{array}$ & $\begin{array}{l}0.11682^{\dagger} \\
(0.05807)\end{array}$ \\
\hline $\mathrm{F}$ & $\begin{array}{l}0.28344^{*} \\
(0.06953)\end{array}$ & $\begin{array}{l}0.24672^{*} \\
(0.06996)\end{array}$ & $\begin{array}{l}0.27104^{*} \\
(0.06964)\end{array}$ & $\begin{array}{l}0.284^{*} \\
(0.06949)\end{array}$ & $\begin{array}{l}0.28509^{*} \\
(0.06983)\end{array}$ & $\begin{array}{l}0.28149^{*} \\
(0.06955)\end{array}$ & $\begin{array}{l}0.29421^{*} \\
(0.0698)\end{array}$ & $\begin{array}{l}0.2862^{*} \\
(0.07009)\end{array}$ & $\begin{array}{l}0.27035^{*} \\
(0.07011)\end{array}$ & $\begin{array}{l}0.26359^{*} \\
(0.06997)\end{array}$ & $\begin{array}{l}0.28379^{*} \\
(0.06952)\end{array}$ & $\begin{array}{l}0.26206^{*} \\
(0.07006)\end{array}$ & $\begin{array}{l}0.27782^{*} \\
(0.06955)\end{array}$ \\
\hline Facto & $\begin{array}{l}0.37225^{\dagger} \\
(0.18)\end{array}$ & $\begin{array}{l}0.32332^{\ddagger} \\
(0.17939)\end{array}$ & $\begin{array}{l}0.35305^{\dagger} \\
(0.17972)\end{array}$ & $\begin{array}{l}0.37281^{\dagger} \\
(0.17976)\end{array}$ & $\begin{array}{l}0.37281^{\dagger} \\
(0.17985)\end{array}$ & $\begin{array}{l}0.36303^{\dagger} \\
(0.18022)\end{array}$ & $\begin{array}{l}0.39403^{\dagger} \\
(0.18019)\end{array}$ & $\begin{array}{l}0.37411^{\dagger} \\
(0.1799)\end{array}$ & $\begin{array}{l}0.35667^{\dagger} \\
(0.18006)\end{array}$ & $\begin{array}{l}0.35092^{\ddagger} \\
(0.17972)\end{array}$ & $\begin{array}{l}0.37281^{\dagger} \\
(0.17983)\end{array}$ & $\begin{array}{l}0.34228^{\ddagger} \\
(0.17997)\end{array}$ & $\begin{array}{l}0.36198^{\dagger} \\
(0.17979)\end{array}$ \\
\hline Factor & $\begin{array}{l}0.21414^{*} \\
(0.04318)\end{array}$ & $\begin{array}{l}0.16998^{*} \\
(0.04466)\end{array}$ & $\begin{array}{l}0.21006^{*} \\
(0.04291)\end{array}$ & $\begin{array}{l}0.21999^{*} \\
(0.04326)\end{array}$ & $\begin{array}{l}0.2168^{*} \\
(0.04397)\end{array}$ & $\begin{array}{l}0.20708^{*} \\
(0.04394)\end{array}$ & $\begin{array}{l}0.22879^{*} \\
(0.04392)\end{array}$ & $\begin{array}{l}0.21883^{*} \\
(0.04523)\end{array}$ & $\begin{array}{l}0.20251^{*} \\
(0.04378)\end{array}$ & $\begin{array}{l}0.19993^{*} \\
(0.04338)\end{array}$ & $\begin{array}{l}0.21766^{*} \\
(0.04338)\end{array}$ & $\begin{array}{l}0.18395^{*} \\
(0.04509)\end{array}$ & $\begin{array}{l}0.19308^{*} \\
(0.04521)\end{array}$ \\
\hline Factor2, & $\begin{array}{l}0.22303^{*} \\
(0.04654)\end{array}$ & $\begin{array}{l}0.20736^{*} \\
(0.04626)\end{array}$ & $\begin{array}{l}0.21702^{*} \\
(0.04629)\end{array}$ & $\begin{array}{l}0.22573^{*} \\
(0.04631)\end{array}$ & $\begin{array}{l}0.22508^{*} \\
(0.04678)\end{array}$ & $\begin{array}{l}0.21491^{*} \\
(0.0475)\end{array}$ & $\begin{array}{l}0.24332^{*} \\
(0.04806)\end{array}$ & $\begin{array}{l}0.22693^{*} \\
(0.04769)\end{array}$ & $\begin{array}{l}0.21386^{*} \\
(0.04676)\end{array}$ & $\begin{array}{l}0.2093^{*} \\
(0.04664)\end{array}$ & $\begin{array}{l}0.2277^{*} \\
(0.04701)\end{array}$ & $\begin{array}{l}0.19783^{*} \\
(0.04765)\end{array}$ & $\begin{array}{l}0.20133^{*} \\
(0.04852)\end{array}$ \\
\hline Factor 1 & $\begin{array}{l}0.26595^{*} \\
(0.02866)\end{array}$ & $\begin{array}{l}0.22777^{*} \\
(0.02906)\end{array}$ & $\begin{array}{l}0.24542^{*} \\
(0.02874)\end{array}$ & $\begin{array}{l}0.27813^{*} \\
(0.02902)\end{array}$ & $\begin{array}{l}0.27014^{*} \\
(0.03022)\end{array}$ & $\begin{array}{l}0.26309^{*} \\
(0.02724)\end{array}$ & $\begin{array}{l}0.27252^{*} \\
(0.0271) \\
\end{array}$ & $\begin{array}{l}0.26803^{*} \\
(0.02719)\end{array}$ & $\begin{array}{l}0.26231^{*} \\
(0.02703)\end{array}$ & $\begin{array}{l}0.26122^{*} \\
(0.02692)\end{array}$ & $\begin{array}{l}0.26707^{*} \\
(0.02687)\end{array}$ & $\begin{array}{l}0.24817^{*} \\
(0.02813)\end{array}$ & $\begin{array}{l}0.26037^{*} \\
(0.02716) \\
\end{array}$ \\
\hline Factc & $\begin{array}{l}0.27417^{*} \\
(0.03416)\end{array}$ & $\begin{array}{l}0.23306^{*} \\
(0.03394)\end{array}$ & $\begin{array}{l}0.25854^{*} \\
(0.03273)\end{array}$ & $\begin{array}{l}0.28607^{*} \\
(0.03347)\end{array}$ & $\begin{array}{l}0.27821^{*} \\
(0.03411)\end{array}$ & $\begin{array}{l}0.27093^{*} \\
(0.03222)\end{array}$ & $\begin{array}{l}0.28415^{*} \\
(0.0323)\end{array}$ & $\begin{array}{l}0.27694^{*} \\
(0.03231)\end{array}$ & $\begin{array}{l}0.27034^{*} \\
(0.03194)\end{array}$ & $\begin{array}{l}0.26786^{*} \\
(0.03189)\end{array}$ & $\begin{array}{l}0.27703^{*} \\
(0.03198)\end{array}$ & $\begin{array}{l}0.25053^{*} \\
(0.03367)\end{array}$ & $\begin{array}{l}0.27129^{*} \\
(0.03185)\end{array}$ \\
\hline $\mathrm{F}$ & $\begin{array}{l}0.27775^{*} \\
(0.03619)\end{array}$ & $\begin{array}{l}0.24191^{*} \\
(0.03607)\end{array}$ & $\begin{array}{l}0.26173^{*} \\
(0.0355)\end{array}$ & $\begin{array}{l}0.28853^{*} \\
(0.03591)\end{array}$ & $\begin{array}{l}0.28171^{*} \\
(0.03687)\end{array}$ & $\begin{array}{l}0.27706^{*} \\
(0.03466)\end{array}$ & $\begin{array}{l}0.28341^{*} \\
(0.03472)\end{array}$ & $\begin{array}{l}0.28124^{*} \\
(0.03569)\end{array}$ & $\begin{array}{l}0.27263^{*} \\
(0.03485)\end{array}$ & $\begin{array}{l}0.27283^{*} \\
(0.03464)\end{array}$ & $\begin{array}{l}0.27887^{*} \\
(0.03462)\end{array}$ & $\begin{array}{l}0.26004^{*} \\
(0.03557)\end{array}$ & $\begin{array}{l}0.27166^{*} \\
(0.03488)\end{array}$ \\
\hline $\mathrm{F}$ & $\begin{array}{l}0.21047^{*} \\
(0.03413)\end{array}$ & $\begin{array}{l}0.16598^{*} \\
(0.03444)\end{array}$ & $\begin{array}{l}0.19161^{*} \\
(0.03329)\end{array}$ & $\begin{array}{l}0.21812^{*} \\
(0.03256)\end{array}$ & $\begin{array}{l}0.21497^{*} \\
(0.03504)\end{array}$ & $\begin{array}{l}0.20516^{*} \\
(0.03288)\end{array}$ & $\begin{array}{l}0.21413^{*} \\
(0.03194)\end{array}$ & $\begin{array}{l}0.21279^{*} \\
(0.03224)\end{array}$ & $\begin{array}{l}0.20147^{*} \\
(0.0327)\end{array}$ & $\begin{array}{l}0.20298^{*} \\
(0.03209)\end{array}$ & $\begin{array}{l}0.21155^{*} \\
(0.03193)\end{array}$ & $\begin{array}{l}0.19254^{*} \\
(0.03302)\end{array}$ & $\begin{array}{l}0.19066^{*} \\
(0.03476)\end{array}$ \\
\hline Fact & $\begin{array}{l}0.28302^{*} \\
(0.02953)\end{array}$ & $\begin{array}{l}0.25176^{*} \\
(0.02913)\end{array}$ & $\begin{array}{l}0.26123^{*} \\
(0.02977)\end{array}$ & $\begin{array}{l}0.2946^{*} \\
(0.0296)\end{array}$ & $\begin{array}{l}0.2878^{*} \\
(0.03208)\end{array}$ & $\begin{array}{l}0.27762^{*} \\
(0.02879)\end{array}$ & $\begin{array}{l}0.29966^{*} \\
(0.02959)\end{array}$ & $\begin{array}{l}0.28722^{*} \\
(0.02986)\end{array}$ & $\begin{array}{l}0.27246^{*} \\
(0.0289)\end{array}$ & $\begin{array}{l}0.26829^{*} \\
(0.02858)\end{array}$ & $\begin{array}{l}0.28636^{*} \\
(0.02815)\end{array}$ & $\begin{array}{l}0.2561^{*} \\
(0.03046)\end{array}$ & $\begin{array}{l}0.27011^{*} \\
(0.02915)\end{array}$ \\
\hline Factor 1 & $\begin{array}{l}0.26776^{*} \\
(0.02579)\end{array}$ & $\begin{array}{l}0.22175^{*} \\
(0.02653)\end{array}$ & $\begin{array}{l}0.26041^{*} \\
(0.02309)\end{array}$ & $\begin{array}{l}0.28077^{*} \\
(0.02557) \\
\end{array}$ & $\begin{array}{l}0.27253^{*} \\
(0.02755) \\
\end{array}$ & $\begin{array}{l}0.26047^{*} \\
(0.02503)\end{array}$ & $\begin{array}{l}0.28539^{*} \\
(0.02527)\end{array}$ & $\begin{array}{l}0.27308^{*} \\
(0.02688)\end{array}$ & $\begin{array}{l}0.26502^{*} \\
(0.02291)\end{array}$ & $\begin{array}{l}0.26461^{*} \\
(0.0228)\end{array}$ & $\begin{array}{l}0.2742^{*} \\
(0.02511) \\
\end{array}$ & $\begin{array}{l}0.23898^{*} \\
(0.02653) \\
\end{array}$ & $\begin{array}{l}0.24788^{*} \\
(0.02665) \\
\end{array}$ \\
\hline Factor & $\begin{array}{l}.19032^{*} \\
0.01915)\end{array}$ & $\begin{array}{l}0.16231^{*} \\
(0.01976)\end{array}$ & $\begin{array}{l}0.1675^{*} \\
(0.02124)\end{array}$ & $\begin{array}{l}0.20441^{*} \\
(0.02225)\end{array}$ & $\begin{array}{l}0.19435^{*} \\
(0.02297)\end{array}$ & $\begin{array}{l}0.18751^{*} \\
(0.01846)\end{array}$ & $\begin{array}{l}0.20638^{*} \\
(0.02069)\end{array}$ & $\begin{array}{l}0.19252^{*} \\
(0.01882)\end{array}$ & $\begin{array}{l}0.17913^{*} \\
(0.01988)\end{array}$ & $\begin{array}{l}0.17496^{*} \\
(0.0194)\end{array}$ & $\begin{array}{l}0.19205^{*} \\
(0.01814)\end{array}$ & $\begin{array}{l}0.17371^{*} \\
(0.0196)\end{array}$ & $\begin{array}{l}0.17799^{*} \\
(0.0199)\end{array}$ \\
\hline Factor $1,3,4 \& 5$ & $\begin{array}{l}.22245^{*} \\
0.03229)\end{array}$ & $\begin{array}{l}0.17993^{*} \\
(0.03193)\end{array}$ & $\begin{array}{l}0.20305^{*} \\
(0.03099)\end{array}$ & $\begin{array}{l}0.23605^{*} \\
(0.0318)\end{array}$ & $\begin{array}{l}0.22728^{*} \\
(0.03318)\end{array}$ & $\begin{array}{l}0.21731^{*} \\
(0.03043)\end{array}$ & $\begin{array}{l}0.2393^{*} \\
(0.03118)\end{array}$ & $\begin{array}{l}0.22683^{*} \\
(0.03137)\end{array}$ & $\begin{array}{l}0.21562^{*} \\
(0.02994)\end{array}$ & $\begin{array}{l}0.21055^{*} \\
(0.02996)\end{array}$ & $\begin{array}{l}0.22588^{*} \\
(0.02977)\end{array}$ & $\begin{array}{l}0.19435^{*} \\
(0.03227)\end{array}$ & $\begin{array}{l}0.20385^{*} \\
(0.03216)\end{array}$ \\
\hline Factor & $\begin{array}{l}.19794^{*} \\
0.03732)\end{array}$ & $\begin{array}{l}0.15352^{*} \\
(0.0377)\end{array}$ & $\begin{array}{l}0.17511^{*} \\
(0.03723)\end{array}$ & $\begin{array}{l}0.21245^{*} \\
(0.0378)\end{array}$ & $\begin{array}{l}0.20281^{*} \\
(0.03891)\end{array}$ & $\begin{array}{l}0.19257^{*} \\
(0.03633)\end{array}$ & $\begin{array}{l}0.20093^{*} \\
(0.03545)\end{array}$ & $\begin{array}{l}0.19946^{*} \\
(0.03552)\end{array}$ & $\begin{array}{l}0.19219^{*} \\
(0.03576)\end{array}$ & $\begin{array}{l}0.1935^{*} \\
(0.03547)\end{array}$ & $\begin{array}{l}0.19932^{*} \\
(0.03547)\end{array}$ & $\begin{array}{l}0.17965^{*} \\
(0.03647)\end{array}$ & $\begin{array}{l}0.18922^{*} \\
(0.036)\end{array}$ \\
\hline $\mathrm{F}$ & $\begin{array}{l}0.20171^{*} \\
(0.05414)\end{array}$ & $\begin{array}{l}0.17463^{*} \\
(0.05422)\end{array}$ & $\begin{array}{l}0.18449^{*} \\
(0.0545)\end{array}$ & $\begin{array}{l}0.21039^{*} \\
(0.0545)\end{array}$ & $\begin{array}{l}0.20505^{*} \\
(0.05526)\end{array}$ & $\begin{array}{l}0.20074^{*} \\
(0.05394)\end{array}$ & $\begin{array}{l}0.21063^{*} \\
(0.05417)\end{array}$ & $\begin{array}{l}0.20462^{*} \\
(0.05457)\end{array}$ & $\begin{array}{l}0.19225^{*} \\
(0.05435)\end{array}$ & $\begin{array}{l}0.19691^{*} \\
(0.05386)\end{array}$ & $\begin{array}{l}0.20226^{*} \\
(0.05393)\end{array}$ & $\begin{array}{l}0.17526^{*} \\
(0.0552) \\
\end{array}$ & $\begin{array}{l}0.19475^{*} \\
(0.05409)\end{array}$ \\
\hline $\mathrm{Fr}$ & $\begin{array}{l}0.46191^{*} \\
(0.03013)\end{array}$ & $\begin{array}{l}0.41412^{*} \\
(0.03223)\end{array}$ & $\begin{array}{l}0.44464^{*} \\
(0.03029)\end{array}$ & $\begin{array}{l}0.47585^{*} \\
(0.03178)\end{array}$ & $\begin{array}{l}0.46523^{*} \\
(0.03108)\end{array}$ & $\begin{array}{l}0.45949^{*} \\
(0.02932)\end{array}$ & $\begin{array}{l}0.46579^{*} \\
(0.02912)\end{array}$ & $\begin{array}{l}0.46431^{*} \\
(0.02966)\end{array}$ & $\begin{array}{l}0.46041^{*} \\
(0.02909)\end{array}$ & $\begin{array}{l}0.45153^{*} \\
(0.02945)\end{array}$ & $\begin{array}{l}0.46409^{*} \\
(0.02923)\end{array}$ & $\begin{array}{l}0.44683^{*} \\
(0.02989)\end{array}$ & $\begin{array}{l}0.46085^{*} \\
(0.02907)\end{array}$ \\
\hline$\& 5$ & $\begin{array}{l}0.24459^{*} \\
(0.03348)\end{array}$ & $\begin{array}{l}0.20417^{*} \\
(0.03511)\end{array}$ & $\begin{array}{l}0.22907^{*} \\
(0.03401)\end{array}$ & $\begin{array}{l}0.25475^{*} \\
(0.0345)\end{array}$ & $\begin{array}{l}0.2488^{*} \\
(0.03669)\end{array}$ & $\begin{array}{l}0.23831^{*} \\
(0.03422)\end{array}$ & $\begin{array}{l}0.25019^{*} \\
(0.03333)\end{array}$ & $\begin{array}{l}0.24639^{*} \\
(0.03352)\end{array}$ & $\begin{array}{l}0.23747^{*} \\
(0.0336) \\
\end{array}$ & $\begin{array}{l}0.234566^{*} \\
(0.03346)\end{array}$ & $\begin{array}{l}0.247^{*} \\
(0.03342) \\
\end{array}$ & $\begin{array}{l}0.21936^{*} \\
(0.03514)\end{array}$ & $\begin{array}{l}0.23399^{*} \\
(0.03394) \\
\end{array}$ \\
\hline Factor & $\begin{array}{l}0.24135^{*} \\
(0.04536)\end{array}$ & $\begin{array}{l}0.19642^{*} \\
(0.04561)\end{array}$ & $\begin{array}{l}0.22012^{*} \\
(0.04504)\end{array}$ & $\begin{array}{l}0.25424^{*} \\
(0.0453)\end{array}$ & $\begin{array}{l}0.24617^{*} \\
(0.04659)\end{array}$ & $\begin{array}{l}0.23554^{*} \\
(0.04463)\end{array}$ & $\begin{array}{l}0.25513^{*} \\
(0.04459)\end{array}$ & $\begin{array}{l}0.24561^{*} \\
(0.04516)\end{array}$ & $\begin{array}{l}0.23023^{*} \\
(0.04465)\end{array}$ & $\begin{array}{l}0.22021^{*} \\
(0.04491)\end{array}$ & $\begin{array}{l}0.24495^{*} \\
(0.04413)\end{array}$ & $\begin{array}{l}0.21511^{*} \\
(0.04548)\end{array}$ & $\begin{array}{l}0.22723^{*} \\
(0.04491)\end{array}$ \\
\hline Factor $1,2,3 \& 5$ & $\begin{array}{l}0.318^{*} \\
(0.03589)\end{array}$ & $\begin{array}{l}0.27397^{*} \\
(0.03582)\end{array}$ & $\begin{array}{l}0.29306^{*} \\
(0.03571)\end{array}$ & $\begin{array}{l}0.32857^{*} \\
(0.0347) \\
\end{array}$ & $\begin{array}{l}0.32278^{*} \\
(0.03679) \\
\end{array}$ & $\begin{array}{l}0.31023^{*} \\
(0.03525)\end{array}$ & $\begin{array}{l}0.33713^{*} \\
(0.0355)\end{array}$ & $\begin{array}{l}0.32243^{*} \\
(0.03528)\end{array}$ & $\begin{array}{l}0.30678^{*} \\
(0.0346)\end{array}$ & $\begin{array}{l}0.30083^{*} \\
(0.03444)\end{array}$ & $\begin{array}{l}0.32089^{*} \\
(0.03366)\end{array}$ & $\begin{array}{l}0.29508^{*} \\
(0.03516)\end{array}$ & $\begin{array}{l}0.30715^{*} \\
(0.03435)\end{array}$ \\
\hline Factor 1, & $\begin{array}{l}0.26712^{*} \\
(0.02027)\end{array}$ & $\begin{array}{l}0.22323^{*} \\
(0.0213)\end{array}$ & $\begin{array}{l}0.24598^{*} \\
(0.01992)\end{array}$ & $\begin{array}{l}0.2779^{*} \\
(0.01935)\end{array}$ & $\begin{array}{l}0.27176^{*} \\
(0.0227)\end{array}$ & $\begin{array}{l}0.26075^{*} \\
(0.01915)\end{array}$ & $\begin{array}{l}0.28601^{*} \\
(0.02058)\end{array}$ & $\begin{array}{l}0.27211^{*} \\
(0.02152)\end{array}$ & $\begin{array}{l}0.25771^{*} \\
(0.0184)\end{array}$ & $\begin{array}{l}0.25317^{*} \\
(0.01815)\end{array}$ & $\begin{array}{l}0.27089^{*} \\
(0.01773)\end{array}$ & $\begin{array}{l}0.23934^{*} \\
(0.02136)\end{array}$ & $\begin{array}{l}0.24949^{*} \\
(0.02085)\end{array}$ \\
\hline All Factors & $\begin{array}{l}0.30765^{*} \\
(0.02151)\end{array}$ & $\begin{array}{l}0.26161^{*} \\
(0.02246)\end{array}$ & $\begin{array}{l}0.28875^{*} \\
(0.02022)\end{array}$ & $\begin{array}{l}0.31789^{*} \\
(0.01993)\end{array}$ & $\begin{array}{l}0.31192^{*} \\
(0.02233)\end{array}$ & $\begin{array}{l}0.30104^{*} \\
(0.02019)\end{array}$ & $\begin{array}{l}0.32215^{*} \\
(0.0199)\end{array}$ & $\begin{array}{l}0.3127^{*} \\
(0.02228)\end{array}$ & $\begin{array}{l}0.29884^{*} \\
(0.01915)\end{array}$ & $\begin{array}{l}0.29178^{*} \\
(0.0194)\end{array}$ & $\begin{array}{l}0.31123^{*} \\
(0.01851)\end{array}$ & $\begin{array}{l}0.27931^{*} \\
(0.0223)\end{array}$ & $\begin{array}{l}0.29002^{*} \\
(0.02166)\end{array}$ \\
\hline $\begin{array}{l}\text { Individual } \\
\text { Variable }\end{array}$ & $\begin{array}{l}0.0011 \\
(0.01412)\end{array}$ & $\begin{array}{l}0.04949^{*} \\
(0.01457)\end{array}$ & $\begin{array}{l}0.02796^{\dagger} \\
(0.01361)\end{array}$ & $(0.01414)$ & $\begin{array}{l}-0.004000 \\
(0.01644)\end{array}$ & $\begin{array}{l}0.00978 \\
(0.01234)\end{array}$ & $\begin{array}{l}-0.02122 \\
(0.01399)\end{array}$ & $\begin{array}{l}-0.00443 \\
(0.01447)\end{array}$ & $\begin{array}{l}0.01614 \\
(0.01173)\end{array}$ & $\begin{array}{l}0.02727^{\dagger} \\
(0.01266)\end{array}$ & $\begin{array}{l}-0.00781 \\
(0.01503)\end{array}$ & $\begin{array}{l}0.03053^{\dagger} \\
(0.01406)\end{array}$ & $\begin{array}{l}0.02208 \\
(0.01471)\end{array}$ \\
\hline
\end{tabular}

* Significant at the 0.01 level $†$ Significant at the 0.05 level $\$$ Significant at the 0.10 level

Table 90 Coefficients with individual variables for high-level factors - Medium Plants 


\begin{tabular}{|c|c|c|c|c|c|c|c|c|c|c|}
\hline $\begin{array}{l}\text { Individual variable } \\
\text { included in the model } \\
\text { in the column }\end{array}$ & 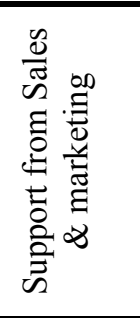 & 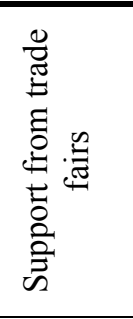 & 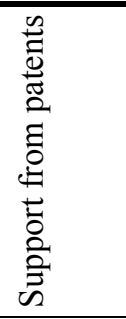 & 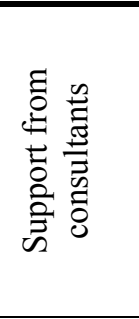 & 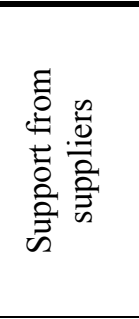 & 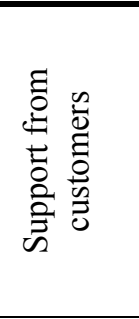 & 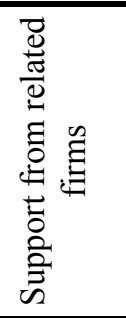 & 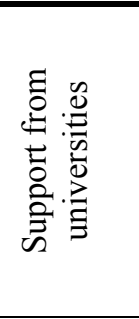 & 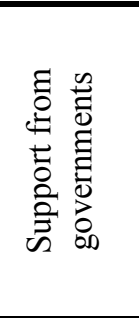 & 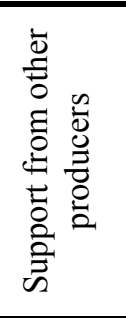 \\
\hline No factors & $\begin{array}{l}0.26494^{*} \\
(0.01342)\end{array}$ & $\begin{array}{l}0.26959^{*} \\
(0.01299)\end{array}$ & $\begin{array}{l}0.26685^{*} \\
(0.01323)\end{array}$ & $\begin{array}{l}0.26967^{*} \\
(0.01297)\end{array}$ & $\begin{array}{l}0.27126^{*} \\
(0.01305)\end{array}$ & $\begin{array}{l}0.25308^{*} \\
(0.01346)\end{array}$ & $\begin{array}{l}0.26594^{*} \\
(0.01356)\end{array}$ & $\begin{array}{l}0.26124^{*} \\
(0.0133)\end{array}$ & $\begin{array}{l}0.26967^{*} \\
(0.01297)\end{array}$ & $\begin{array}{l}0.26969^{*} \\
(0.01303)\end{array}$ \\
\hline Factor 5 & $\begin{array}{l}0.11011 \\
(0.06937)\end{array}$ & $\begin{array}{l}0.11275 \\
(0.06922)\end{array}$ & $\begin{array}{l}0.1004 \\
(0.07012)\end{array}$ & $\begin{array}{l}0.11275 \\
(0.06907)\end{array}$ & $\begin{array}{l}0.11706^{\ddagger} \\
(0.06938)\end{array}$ & $\begin{array}{l}0.07116 \\
(0.06941)\end{array}$ & $\begin{array}{l}0.10777 \\
(0.06957)\end{array}$ & $\begin{array}{l}0.10865 \\
(0.06914)\end{array}$ & $\begin{array}{l}0.11118 \\
(0.06912)\end{array}$ & $\begin{array}{l}0.11421 \\
(0.06956)\end{array}$ \\
\hline Factor 4 & $\begin{array}{l}0.14195^{*} \\
(0.03359) \\
\end{array}$ & $\begin{array}{l}0.15057^{*} \\
(0.0329) \\
\end{array}$ & $\begin{array}{l}0.14172^{*} \\
(0.03391)\end{array}$ & $\begin{array}{l}0.15129^{*} \\
(0.03283)\end{array}$ & $\begin{array}{l}0.15565^{*} \\
(0.03306) \\
\end{array}$ & $\begin{array}{l}0.12776^{*} \\
(0.03312) \\
\end{array}$ & $\begin{array}{l}0.14325^{*} \\
(0.03397) \\
\end{array}$ & $\begin{array}{l}0.1413^{*} \\
(0.03304) \\
\end{array}$ & $\begin{array}{l}0.1493^{*} \\
(0.03286) \\
\end{array}$ & $\begin{array}{l}0.15129^{*} \\
(0.033) \\
\end{array}$ \\
\hline Factor $4 \& 5$ & $\begin{array}{l}0.23273^{\ddagger} \\
(0.12779)\end{array}$ & $\begin{array}{l}0.23543^{\ddagger} \\
(0.12754)\end{array}$ & $\begin{array}{l}0.23221^{\ddagger} \\
(0.12785)\end{array}$ & $\begin{array}{l}0.23543^{\ddagger} \\
(0.12727) \\
\end{array}$ & $\begin{array}{l}0.23543^{\ddagger} \\
(0.12774)\end{array}$ & $\begin{array}{l}0.21638^{\ddagger} \\
(0.12676)\end{array}$ & $\begin{array}{l}0.23543^{\ddagger} \\
(0.12785) \\
\end{array}$ & $\begin{array}{l}0.23543^{\ddagger} \\
(0.12737) \\
\end{array}$ & $\begin{array}{l}0.23543^{\ddagger} \\
(0.12735)\end{array}$ & $\begin{array}{l}0.23543^{\ddagger} \\
(0.12791) \\
\end{array}$ \\
\hline Factor 3 & $\begin{array}{l}0.21729^{*} \\
(0.04529)\end{array}$ & $\begin{array}{l}0.23367^{*} \\
(0.04375)\end{array}$ & $\begin{array}{l}0.21503^{*} \\
(0.04649)\end{array}$ & $\begin{array}{l}0.21993^{*} \\
(0.04388)\end{array}$ & $\begin{array}{l}0.24158^{*} \\
(0.04409)\end{array}$ & $\begin{array}{l}0.17504^{*} \\
(0.0455)\end{array}$ & $\begin{array}{l}0.22228^{*} \\
(0.04535)\end{array}$ & $\begin{array}{l}0.20378^{*} \\
(0.04491)\end{array}$ & $\begin{array}{l}0.23289^{*} \\
(0.04369)\end{array}$ & $\begin{array}{l}0.23715^{*} \\
(0.04516)\end{array}$ \\
\hline Factor & $\begin{array}{l}0.14304^{*} \\
(0.05189) \\
\end{array}$ & $\begin{array}{l}0.15852^{*} \\
(0.05067)\end{array}$ & $\begin{array}{l}0.13815^{\dagger} \\
(0.05351)\end{array}$ & $\begin{array}{l}0.1578^{*} \\
(0.05056)\end{array}$ & $\begin{array}{l}0.1746^{*} \\
(0.05171) \\
\end{array}$ & $\begin{array}{l}0.09701^{\ddagger} \\
(0.05227)\end{array}$ & $\begin{array}{l}0.14532^{*} \\
(0.05253)\end{array}$ & $\begin{array}{l}0.15339^{*} \\
(0.05063)\end{array}$ & $\begin{array}{l}0.15483^{*} \\
(0.05061) \\
\end{array}$ & $\begin{array}{l}0.16032^{*} \\
(0.05111) \\
\end{array}$ \\
\hline Facto & $\begin{array}{l}0.21023^{*} \\
(0.04941)\end{array}$ & $\begin{array}{l}0.22511^{*} \\
(0.04823) \\
\end{array}$ & $\begin{array}{l}0.21085^{*} \\
(0.04976) \\
\end{array}$ & $\begin{array}{l}0.22174^{*} \\
(0.04814) \\
\end{array}$ & $\begin{array}{l}0.23842^{*} \\
(0.049) \\
\end{array}$ & $\begin{array}{l}0.17382^{*} \\
(0.04933) \\
\end{array}$ & $\begin{array}{l}0.21271^{*} \\
(0.04996) \\
\end{array}$ & $\begin{array}{l}0.21634^{*} \\
(0.04826) \\
\end{array}$ & $\begin{array}{l}0.20337^{*} \\
(0.04872) \\
\end{array}$ & $\begin{array}{l}0.22619^{*} \\
(0.04848) \\
\end{array}$ \\
\hline Facto & $\begin{array}{l}0.15503^{\dagger} \\
(0.06072)\end{array}$ & $\begin{array}{l}0.16989^{*} \\
(0.05973)\end{array}$ & $\begin{array}{l}0.14921^{\dagger} \\
(0.06226)\end{array}$ & $\begin{array}{l}0.1424^{\dagger} \\
(0.06024)\end{array}$ & $\begin{array}{l}0.17917^{*} \\
(0.0601)\end{array}$ & $\begin{array}{l}0.12626^{\dagger} \\
(0.06017)\end{array}$ & $\begin{array}{l}0.15669^{\dagger} \\
(0.06135)\end{array}$ & $\begin{array}{l}0.16089^{*} \\
(0.05973)\end{array}$ & $\begin{array}{l}0.13923^{\dagger} \\
(0.06054)\end{array}$ & $\begin{array}{l}0.17286^{*} \\
(0.06059)\end{array}$ \\
\hline Factor 2 & $\begin{array}{l}0.17388^{*} \\
(0.03826) \\
\end{array}$ & $\begin{array}{l}0.18048^{*} \\
(0.03791) \\
\end{array}$ & $\begin{array}{l}0.1799^{*} \\
(0.03799) \\
\end{array}$ & $\begin{array}{l}0.18048^{*} \\
(0.03783) \\
\end{array}$ & $\begin{array}{l}0.18048^{*} \\
(0.03797) \\
\end{array}$ & $\begin{array}{l}0.16822^{*} \\
(0.03776) \\
\end{array}$ & $\begin{array}{l}0.17555^{*} \\
(0.03833) \\
\end{array}$ & $\begin{array}{l}0.16799^{*} \\
(0.03811) \\
\end{array}$ & $\begin{array}{l}0.18048^{*} \\
(0.03785) \\
\end{array}$ & $\begin{array}{l}0.18061^{*} \\
(0.03802) \\
\end{array}$ \\
\hline Factor & $\begin{array}{l}0.34989 \\
(0.22942) \\
\end{array}$ & $\begin{array}{l}0.35732 \\
(0.22893) \\
\end{array}$ & $\begin{array}{l}0.3454 \\
(0.22964) \\
\end{array}$ & $\begin{array}{l}0.30293 \\
(0.2291) \\
\end{array}$ & $\begin{array}{l}0.35732 \\
(0.22929) \\
\end{array}$ & $\begin{array}{l}0.33126 \\
(0.22747) \\
\end{array}$ & $\begin{array}{l}0.34971 \\
(0.22962) \\
\end{array}$ & $\begin{array}{l}0.33693 \\
(0.22874) \\
\end{array}$ & $\begin{array}{l}0.33598 \\
(0.2287) \\
\end{array}$ & $\begin{array}{l}0.36013 \\
(0.22975) \\
\end{array}$ \\
\hline Factor $2 \& 4$ & $\begin{array}{l}0.24166^{*} \\
(0.04219)\end{array}$ & $\begin{array}{l}0.24549^{*} \\
(0.04203) \\
\end{array}$ & $\begin{array}{l}0.24169^{*} \\
(0.04224)\end{array}$ & $\begin{array}{l}0.24549^{*} \\
(0.04194)\end{array}$ & $\begin{array}{l}0.24672^{*} \\
(0.04211)\end{array}$ & $\begin{array}{l}0.2106^{*} \\
(0.04251)\end{array}$ & $\begin{array}{l}0.24087^{*} \\
(0.04239)\end{array}$ & $\begin{array}{l}0.24293^{*} \\
(0.04199)\end{array}$ & $\begin{array}{l}0.24411^{*} \\
(0.04197) \\
\end{array}$ & $\begin{array}{l}0.24566^{*} \\
(0.04216) \\
\end{array}$ \\
\hline Factor $2,4 \& 5$ & $\begin{array}{l}0.12087^{\dagger} \\
(0.05795) \\
\end{array}$ & $\begin{array}{l}0.12293^{\dagger} \\
(0.05782) \\
\end{array}$ & $\begin{array}{l}0.10927^{\ddagger} \\
(0.05905)\end{array}$ & $\begin{array}{l}0.12293^{\dagger} \\
(0.0577) \\
\end{array}$ & $\begin{array}{l}0.13456^{\dagger} \\
(0.05836) \\
\end{array}$ & $\begin{array}{l}0.07963 \\
(0.05829) \\
\end{array}$ & $\begin{array}{l}0.11701^{\dagger} \\
(0.05828)\end{array}$ & $\begin{array}{l}0.11112^{\ddagger} \\
(0.05789) \\
\end{array}$ & $\begin{array}{l}0.12293^{\dagger} \\
(0.05774) \\
\end{array}$ & $\begin{array}{l}0.12396^{\dagger} \\
(0.05808) \\
\end{array}$ \\
\hline Fac & $\begin{array}{l}0.27436^{*} \\
(0.06974)\end{array}$ & $\begin{array}{l}0.28348^{*} \\
(0.06932)\end{array}$ & $\begin{array}{l}0.2628^{*} \\
(0.07155)\end{array}$ & $\begin{array}{l}0.27944^{*} \\
(0.06919)\end{array}$ & $\begin{array}{l}0.30423^{*} \\
(0.0706)\end{array}$ & $\begin{array}{l}0.22413^{*} \\
(0.07019)\end{array}$ & $\begin{array}{l}0.27126^{*} \\
(0.07059)\end{array}$ & $\begin{array}{l}0.25878^{*} \\
(0.06976)\end{array}$ & $\begin{array}{l}0.28101^{*} \\
(0.06922)\end{array}$ & $\begin{array}{l}0.28437^{*} \\
(0.06958)\end{array}$ \\
\hline Factc & $\begin{array}{l}0.36387^{\dagger} \\
(0.17977)\end{array}$ & $\begin{array}{l}0.37281^{\dagger} \\
(0.17933) \\
\end{array}$ & $\begin{array}{l}0.35819^{\dagger} \\
(0.18013)\end{array}$ & $\begin{array}{l}0.3451^{\ddagger} \\
(0.17917)\end{array}$ & $\begin{array}{l}0.38336^{\dagger} \\
(0.17973)\end{array}$ & $\begin{array}{l}0.36067^{\dagger} \\
(0.17815)\end{array}$ & $\begin{array}{l}0.35961^{\dagger} \\
(0.18026)\end{array}$ & $\begin{array}{l}0.37281^{\dagger} \\
(0.17909)\end{array}$ & $\begin{array}{l}0.37281^{\dagger} \\
(0.17906)\end{array}$ & $\begin{array}{l}0.37281^{\dagger} \\
(0.17985)\end{array}$ \\
\hline Factor & $\begin{array}{l}0.20038^{*} \\
(0.04401)\end{array}$ & $\begin{array}{l}0.21348^{*} \\
(0.04283) \\
\end{array}$ & $\begin{array}{l}0.19657^{*} \\
(0.04541)\end{array}$ & $\begin{array}{l}0.19348^{*} \\
(0.04325) \\
\end{array}$ & $\begin{array}{l}0.23369^{*} \\
(0.0445) \\
\end{array}$ & $\begin{array}{l}0.16752^{*} \\
(0.04388) \\
\end{array}$ & $\begin{array}{l}0.2044^{*} \\
(0.04413)\end{array}$ & $\begin{array}{l}0.20382^{*} \\
(0.04293)\end{array}$ & $\begin{array}{l}0.19444^{*} \\
(0.0433) \\
\end{array}$ & $\begin{array}{l}0.21629^{*} \\
(0.04331)\end{array}$ \\
\hline Factor $2,3,4 \& 5$ & $\begin{array}{l}0.21051^{*} \\
(0.0471) \\
\end{array}$ & $\begin{array}{l}0.2222^{*} \\
(0.04615)\end{array}$ & $\begin{array}{l}0.20472^{*} \\
(0.04877)\end{array}$ & $\begin{array}{l}0.20626^{*} \\
(0.04638)\end{array}$ & $\begin{array}{l}0.23713^{*} \\
(0.04699)\end{array}$ & $\begin{array}{l}0.17489^{*} \\
(0.04717)\end{array}$ & $\begin{array}{l}0.21526^{*} \\
(0.047)\end{array}$ & $\begin{array}{l}0.20375^{*} \\
(0.04659)\end{array}$ & $\begin{array}{l}0.20749^{*} \\
(0.0464)\end{array}$ & $\begin{array}{l}0.22573^{*} \\
(0.04683)\end{array}$ \\
\hline Factor 1 & $\begin{array}{l}0.26354^{*} \\
(0.02692)\end{array}$ & $\begin{array}{l}0.2661^{*} \\
(0.02678)\end{array}$ & $\begin{array}{l}0.25083^{*} \\
(0.02989)\end{array}$ & $\begin{array}{l}0.26673^{*} \\
(0.02673)\end{array}$ & $\begin{array}{l}0.26966^{*} \\
(0.02689) \\
\end{array}$ & $\begin{array}{l}0.242^{*} \\
(0.0272) \\
\end{array}$ & $\begin{array}{l}0.26136^{*} \\
(0.02739) \\
\end{array}$ & $\begin{array}{l}0.26351^{*} \\
(0.02677)\end{array}$ & $\begin{array}{l}0.26673^{*} \\
(0.02674) \\
\end{array}$ & $\begin{array}{l}0.26687^{*} \\
(0.02686) \\
\end{array}$ \\
\hline Factor $1 \& 5$ & $\begin{array}{l}0.26683^{*} \\
(0.03227) \\
\end{array}$ & $\begin{array}{l}0.27515^{*} \\
(0.03169) \\
\end{array}$ & $\begin{array}{l}0.2619^{*} \\
(0.0336) \\
\end{array}$ & $\begin{array}{l}0.25808^{*} \\
(0.03209) \\
\end{array}$ & $\begin{array}{l}0.28112^{*} \\
(0.03195) \\
\end{array}$ & $\begin{array}{l}0.2371^{*} \\
(0.03267) \\
\end{array}$ & $\begin{array}{l}0.26771^{*} \\
(0.03265) \\
\end{array}$ & $\begin{array}{l}0.265^{*} \\
(0.03184) \\
\end{array}$ & $\begin{array}{l}0.27515^{*} \\
(0.03164) \\
\end{array}$ & $\begin{array}{l}0.27545^{*} \\
(0.03179) \\
\end{array}$ \\
\hline Factor $1 \& 4$ & $\begin{array}{l}0.27546^{*} \\
(0.03465)\end{array}$ & $\begin{array}{l}0.25786^{*} \\
(0.03558)\end{array}$ & $\begin{array}{l}0.2632^{*} \\
(0.03686)\end{array}$ & $\begin{array}{l}0.27114^{*} \\
(0.03452)\end{array}$ & $\begin{array}{l}0.28863^{*} \\
(0.03512)\end{array}$ & $\begin{array}{l}0.24874^{*} \\
(0.03496)\end{array}$ & $\begin{array}{l}0.27473^{*} \\
(0.03482)\end{array}$ & $\begin{array}{l}0.27456^{*} \\
(0.0345)\end{array}$ & $\begin{array}{l}0.27765^{*} \\
(0.03447)\end{array}$ & $\begin{array}{l}0.27872^{*} \\
(0.03462)\end{array}$ \\
\hline $\mathrm{Fac}$ & $\begin{array}{l}0.20437^{*} \\
(0.03227) \\
\end{array}$ & $\begin{array}{l}0.21141^{*} \\
(0.03184) \\
\end{array}$ & $\begin{array}{l}0.19968^{*} \\
(0.03335) \\
\end{array}$ & $\begin{array}{l}0.2107^{*} \\
(0.03177) \\
\end{array}$ & $\begin{array}{l}0.21489^{*} \\
(0.03196) \\
\end{array}$ & $\begin{array}{l}0.17597^{*} \\
(0.03265) \\
\end{array}$ & $\begin{array}{l}0.20838^{*} \\
(0.03206) \\
\end{array}$ & $\begin{array}{l}0.20758^{*} \\
(0.03182) \\
\end{array}$ & $\begin{array}{l}0.21141^{*} \\
(0.03179) \\
\end{array}$ & $\begin{array}{l}0.21191^{*} \\
(0.03196) \\
\end{array}$ \\
\hline Fac & $\begin{array}{l}0.27349^{*} \\
(0.0286) \\
\end{array}$ & $\begin{array}{l}0.28381^{*} \\
(0.02764)\end{array}$ & $\begin{array}{l}0.26363^{*} \\
(0.03235)\end{array}$ & $\begin{array}{l}0.27135^{*} \\
(0.02787)\end{array}$ & $\begin{array}{l}0.29833^{*} \\
(0.02909)\end{array}$ & $\begin{array}{l}0.23375^{*} \\
(0.02976)\end{array}$ & $\begin{array}{l}0.27371^{*} \\
(0.02954)\end{array}$ & $\begin{array}{l}0.25941^{*} \\
(0.02887)\end{array}$ & $\begin{array}{l}0.27127^{*} \\
(0.02793)\end{array}$ & $\begin{array}{l}0.28526^{*} \\
(0.02807) \\
\end{array}$ \\
\hline Factor & $\begin{array}{l}0.25269^{*} \\
(0.02532) \\
\end{array}$ & $\begin{array}{l}0.26424^{*} \\
(0.02279) \\
\end{array}$ & $\begin{array}{l}0.24802^{*} \\
(0.02848) \\
\end{array}$ & $\begin{array}{l}0.26441^{*} \\
(0.02271) \\
\end{array}$ & $\begin{array}{l}0.28807^{*} \\
(0.02569) \\
\end{array}$ & $\begin{array}{l}0.20876^{*} \\
(0.02643)\end{array}$ & $\begin{array}{l}0.2561^{*} \\
(0.02611)\end{array}$ & $\begin{array}{l}0.2601^{*} \\
(0.02288)\end{array}$ & $\begin{array}{l}0.26676^{*} \\
(0.02269) \\
\end{array}$ & $\begin{array}{l}0.27201^{*} \\
(0.02495)\end{array}$ \\
\hline Factor $1,3 \& 4$ & $\begin{array}{l}0.18287^{*} \\
(0.0188) \\
\end{array}$ & $\begin{array}{l}0.19083^{*} \\
(0.01794) \\
\end{array}$ & $\begin{array}{l}0.1702^{*} \\
(0.0248) \\
\end{array}$ & $\begin{array}{l}0.18482^{*} \\
(0.018) \\
\end{array}$ & $\begin{array}{l}0.19615^{*} \\
(0.01826) \\
\end{array}$ & $\begin{array}{l}0.13243^{*} \\
(0.0223) \\
\end{array}$ & $\begin{array}{l}0.17908^{*} \\
(0.02157)\end{array}$ & $\begin{array}{l}0.16343^{*} \\
(0.02028) \\
\end{array}$ & $\begin{array}{l}0.18786^{*} \\
(0.01794) \\
\end{array}$ & $\begin{array}{l}0.19156^{*} \\
(0.01813) \\
\end{array}$ \\
\hline Factor $1,3,4 \& 5$ & $\begin{array}{l}0.21215^{*} \\
(0.03042) \\
\end{array}$ & $\begin{array}{l}0.22349^{*} \\
(0.02933) \\
\end{array}$ & $\begin{array}{l}0.20509^{*} \\
(0.03311) \\
\end{array}$ & $\begin{array}{l}0.21617^{*} \\
(0.02936) \\
\end{array}$ & $\begin{array}{l}0.2398^{*} \\
(0.03105) \\
\end{array}$ & $\begin{array}{l}0.16483^{*} \\
(0.0321) \\
\end{array}$ & $\begin{array}{l}0.2118^{*} \\
(0.0317) \\
\end{array}$ & $\begin{array}{l}0.20781^{*} \\
(0.02979) \\
\end{array}$ & $\begin{array}{l}0.20288^{*} \\
(0.03011) \\
\end{array}$ & $\begin{array}{l}0.22461^{*} \\
(0.02962) \\
\end{array}$ \\
\hline Factor $1 \& 2$ & $\begin{array}{l}0.19377^{*} \\
(0.0356)\end{array}$ & $\begin{array}{l}0.19427^{*} \\
(0.03541)\end{array}$ & $\begin{array}{l}0.18451^{*} \\
(0.03737)\end{array}$ & $\begin{array}{l}0.19885^{*} \\
(0.03528)\end{array}$ & $\begin{array}{l}0.21094^{*} \\
(0.03619) \\
\end{array}$ & $\begin{array}{l}0.18145^{*} \\
(0.03535)\end{array}$ & $\begin{array}{l}0.19187^{*} \\
(0.03614)\end{array}$ & $\begin{array}{l}0.19073^{*} \\
(0.03542)\end{array}$ & $\begin{array}{l}0.19803^{*} \\
(0.03531)\end{array}$ & $\begin{array}{l}0.19912^{*} \\
(0.03547)\end{array}$ \\
\hline Factor $1,2 \& 5$ & $\begin{array}{l}0.19901^{*} \\
(0.05392)\end{array}$ & $\begin{array}{l}0.20208^{*} \\
(0.05378)\end{array}$ & $\begin{array}{l}0.18691^{*} \\
(0.05534)\end{array}$ & $\begin{array}{l}0.20208^{*} \\
(0.05366) \\
\end{array}$ & $\begin{array}{l}0.21371^{*} \\
(0.05434) \\
\end{array}$ & $\begin{array}{l}0.15228^{*} \\
(0.05463)\end{array}$ & $\begin{array}{l}0.19259^{*} \\
(0.05476)\end{array}$ & $\begin{array}{l}0.18465^{*} \\
(0.05405) \\
\end{array}$ & $\begin{array}{l}0.20208^{*} \\
(0.0537) \\
\end{array}$ & $\begin{array}{l}0.20234^{*} \\
(0.05394)\end{array}$ \\
\hline Factor & $\begin{array}{l}0.45587^{*} \\
(0.02941)\end{array}$ & $\begin{array}{l}0.46205^{*} \\
(0.02899) \\
\end{array}$ & $\begin{array}{l}0.44573^{*} \\
(0.03221) \\
\end{array}$ & $\begin{array}{l}0.46252^{*} \\
(0.02893) \\
\end{array}$ & $\begin{array}{l}0.47599^{*} \\
(0.0302) \\
\end{array}$ & $\begin{array}{l}0.41432^{*} \\
(0.03085) \\
\end{array}$ & $\begin{array}{l}0.45484^{*} \\
(0.03009)\end{array}$ & $\begin{array}{l}0.44882^{*} \\
(0.02934) \\
\end{array}$ & $\begin{array}{l}0.44375^{*} \\
(0.02964) \\
\end{array}$ & $\begin{array}{l}0.46393^{*} \\
(0.0294) \\
\end{array}$ \\
\hline Factor 1, & $\begin{array}{l}0.23751^{*} \\
(0.03355)\end{array}$ & $\begin{array}{l}0.24411^{*} \\
(0.03309)\end{array}$ & $\begin{array}{l}0.2296^{*} \\
(0.03551) \\
\end{array}$ & $\begin{array}{l}0.24417^{*} \\
(0.03302)\end{array}$ & $\begin{array}{l}0.24942^{*} \\
(0.03326)\end{array}$ & $\begin{array}{l}0.19689^{*} \\
(0.03467)\end{array}$ & $\begin{array}{l}0.24265^{*} \\
(0.03325)\end{array}$ & $\begin{array}{l}0.23966^{*} \\
(0.0331) \\
\end{array}$ & $\begin{array}{l}0.24368^{*} \\
(0.03304)\end{array}$ & $\begin{array}{l}0.24494^{*} \\
(0.03319) \\
\end{array}$ \\
\hline Factor & $\begin{array}{l}0.23137^{*} \\
(0.04443)\end{array}$ & $\begin{array}{l}0.24226^{*} \\
(0.0437) \\
\end{array}$ & $\begin{array}{l}0.22397^{*} \\
(0.04634) \\
\end{array}$ & $\begin{array}{l}0.23083^{*} \\
(0.04376) \\
\end{array}$ & $\begin{array}{l}0.25287^{*} \\
(0.04426) \\
\end{array}$ & $\begin{array}{l}0.18075^{*} \\
(0.04565) \\
\end{array}$ & $\begin{array}{l}0.23563^{*} \\
(0.04432) \\
\end{array}$ & $\begin{array}{l}0.22774^{*} \\
(0.04393) \\
\end{array}$ & \begin{tabular}{|l|l}
$0.2348^{*}$ \\
$(0.04371)$ \\
\end{tabular} & $\begin{array}{l}0.24334^{*} \\
(0.04395) \\
\end{array}$ \\
\hline Factor $1,2,3 \& 5$ & $\begin{array}{l}0.30631^{*} \\
(0.03458) \\
\end{array}$ & $\begin{array}{l}0.31901^{*} \\
(0.03337)\end{array}$ & $\begin{array}{l}0.29859^{*} \\
(0.03748) \\
\end{array}$ & $\begin{array}{l}0.30564^{*} \\
(0.03357) \\
\end{array}$ & $\begin{array}{l}0.33611^{*} \\
(0.03505)\end{array}$ & $\begin{array}{l}0.26433^{*} \\
(0.03545)\end{array}$ & $\begin{array}{l}0.30754^{*} \\
(0.03542)\end{array}$ & $\begin{array}{l}0.29938^{*} \\
(0.03402)\end{array}$ & $\begin{array}{l}0.30353^{*} \\
(0.03374)\end{array}$ & $\begin{array}{l}0.32085^{*} \\
(0.03395)\end{array}$ \\
\hline Factor $1,2,3 \& 4$ & $\begin{array}{l}0.25389^{*} \\
(0.01946)\end{array}$ & $\begin{array}{l}0.26173^{*} \\
(0.01698) \\
\end{array}$ & $\begin{array}{l}0.24829^{*} \\
(0.02343) \\
\end{array}$ & $\begin{array}{l}0.25068^{*} \\
(0.01763)\end{array}$ & $\begin{array}{l}0.28334^{*} \\
(0.01929) \\
\end{array}$ & $\begin{array}{l}0.21263^{*} \\
(0.02096) \\
\end{array}$ & $\begin{array}{l}0.25689^{*} \\
(0.02025) \\
\end{array}$ & $\begin{array}{l}0.24879^{*} \\
(0.01804)\end{array}$ & $\begin{array}{l}0.24938^{*} \\
(0.01791) \\
\end{array}$ & $\begin{array}{l}0.26995^{*} \\
(0.01787) \\
\end{array}$ \\
\hline All Factors & $\begin{array}{l}0.29762^{*} \\
(0.01935)\end{array}$ & $\begin{array}{l}0.30606^{*} \\
(0.01778)\end{array}$ & $\begin{array}{l}0.28903^{*} \\
(0.02406)\end{array}$ & $\begin{array}{l}0.29114^{*} \\
(0.01857)\end{array}$ & $\begin{array}{l}0.31976^{*} \\
(0.01907) \\
\end{array}$ & $\begin{array}{l}0.25634^{*} \\
(0.02133)\end{array}$ & $\begin{array}{l}0.29727^{*} \\
(0.02118)\end{array}$ & $\begin{array}{l}0.28536^{*} \\
(0.01948)\end{array}$ & $\begin{array}{l}0.29172^{*} \\
(0.01863)\end{array}$ & $\begin{array}{l}0.31034^{*} \\
(0.0186) \\
\end{array}$ \\
\hline $\begin{array}{l}\text { Individual } \\
\text { Variable }\end{array}$ & $\begin{array}{l}0.01754 \\
(0.01218)\end{array}$ & $\begin{array}{l}0.09793^{\dagger} \\
(0.0409)\end{array}$ & $\begin{array}{l}0.02068 \\
(0.01712)\end{array}$ & $\begin{array}{l}0.0544^{*} \\
(0.01732)\end{array}$ & $\begin{array}{l}-0.02151 \\
(0.01327)\end{array}$ & $\begin{array}{l}0.06152^{*} \\
(0.01412)\end{array}$ & $\begin{array}{l}0.0132 \\
(0.01339)\end{array}$ & $\begin{array}{l}0.03538^{*} \\
(0.01228)\end{array}$ & $\begin{array}{l}0.05038^{*} \\
(0.01713) \\
\end{array}$ & $\begin{array}{l}-0.00488 \\
(0.015)\end{array}$ \\
\hline
\end{tabular}

* Significant at the 0.01 level $†$ Significant at the 0.05 level $\$$ Significant at the 0.10 level

Table 90 Coefficients with individual variables for high-level factors - Medium Plants 


\subsubsection{Electronics Industry}

The analysis of the electronics industry has many states that have insignificant results and a few individual variables that have coefficients with large significant results. The results from the electronics industry are quite different from those of medium plants. For example, there are fewer individual variables that have significant coefficients but those that do have much larger coefficients than those in the medium plant analysis. This may be because of the much smaller sample size or the idiosyncrasies of the electronics industry itself.

The analysis of AMT implemented by the plants has a number of states that are insignificant (refer to Table 91). All the states that do not include the production design technologies, except the state that contains none of the technologies or only the communication technologies, have insignificant coefficients. This demonstrates the importance of the production design technologies in the electronics industry.

The AMT analysis exhibited four significant and negative coefficients. The use of high speed machining, part identification for automation, local area networks, and computers on the factor floor are all approximately $70 \%-80 \%$ of the state coefficients. These variables have a significant impact on the outcome of the profit function and are largely overstated in the interaction effects included in the state variables. This could be because they are substitutes for some of the other variables included in the factor or that the use of these technologies in the plants has not improved the profitability of the plants but has incurred large implementation costs.

The analysis of the business practices including the individual variables has two states with insignificant coefficients: the state consisting of planning practices, and the state consisting 
of quality control practices (refer to Table 92). Within these states, business practices are primarily implemented as a larger group of changes and not individually. This is supported by the fact that none of the individual variables had a significant coefficient.

The analysis of the model for the sources of implementation support has a number of states that have insignificant coefficients: the state consisting of only corporate organizations, only external information sources, only primary stakeholders, research and development group and corporate organizations, research and development group and primary stakeholders, and the state consisting of primary stakeholders and corporate organizations (refer to Table 93). Once again, the hypothesis that multiple sources of implementation support are required for change is support by the analysis.

In the sources of implementation support model, there are only three variables that have significant coefficients. The use of patents and customers as sources of implementation support both have rather large positive coefficients that are approximately $50-70 \%$ of the magnitude of the state coefficients. The results demonstrate once again that the effect of external sources of support, particularly from those that can specify requirements, are understated in the interaction effects included in the state variables. The remaining variable that has a significant coefficient is the use of trade fairs as a source of implementation support. In this case, the coefficient is negative and is larger than those for the various factor states. This coefficient is probably a large negative quantity because very few plants used it as a source of implementation support and those that did had poor results. It may also be because the support obtained at a trade fair may be very misleading since trade fairs consist of sales representatives trying to sell their products, and do not impart information that can be readily applied to the implementation of the technology in the individual plants. 
The analysis of the perceived benefits model also has a number of states with insignificant coefficients. The states consisting of only improved time usage, only increased productivity, increased flexibility and profitability, increased productivity and flexibility, increased productivity, flexibility, and profitability, improved time usage and increased profitability, and improved time usage and increased productivity all have insignificant coefficients (refer to Table 94). The results are not surprising as most of these states contain less than $1 \%$ of electronics plants. The only individual variable that has a significant coefficient is the perceived benefit of new product features. This benefit has a coefficient that is approximately $50 \%$ of those for state variables. The ability to produce new product features is an important aspect in the electronics industry, due to the high turnover rate of technology and short production life-cycles.

In the analysis of the high-level factor model, very few states have significant coefficients in the electronics industry. The state containing none of the factors, the state consisting of only the AMT factor, the state consisting of all the factors except the human resources factor and the state consisting of all the factors are the only four states with significant coefficients (refer to Table 95). None of the individual AMT, business practices, or human resources variables had significant coefficients. Once again, the effect of research and development has been understated in the high-level factor states. The use of contract R\&D, support from production engineers and from other producers all have large significant coefficients.

There are also a number of large negative coefficients in the high-level factor model analysis. As in the sources of implementation support, the use of trade fairs has an extremely large negative coefficient. The perceived benefit of the reduction in set-up time, increased skill requirements, and increased equipment utilization rates all have significant coefficients 
demonstrated that the increased labour costs are not accounted for in the factor model and greater manufacturing capacity is not translating into increased profits.

The analysis of the electronics industry using the individual variables and the state variables indicates that there are some individual variables that must be considered along with the state variables in order to obtain a more accurate profit function. These results also point to a limitation due to the small sample size of the electronics industry as many of the states have insignificant coefficients. Since two plants may have the same factor but due to a different composition of variables, this will lead to some of the individual variables having significant coefficients. It is the size of some of these coefficients in the electronics industry that makes the individual variables such as support from trade fairs so important. 


\begin{tabular}{|c|c|c|c|c|c|c|c|c|c|c|c|c|c|c|c|c|c|c|c|c|c|c|c|c|c|}
\hline $\begin{array}{l}\text { Individual } \\
\text { variable } \\
\text { included in } \\
\text { the model in } \\
\text { the column }\end{array}$ & 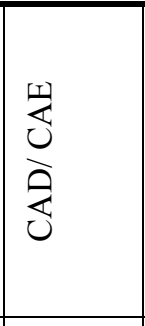 & 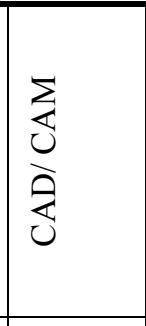 & 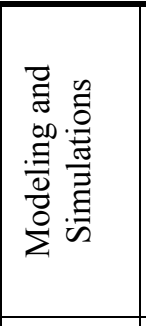 & 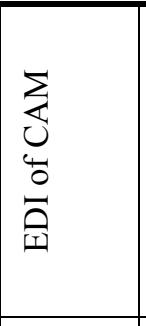 & $\begin{array}{l}\sum_{I}^{\infty} \\
\sum_{\Sigma}^{\infty}\end{array}$ & 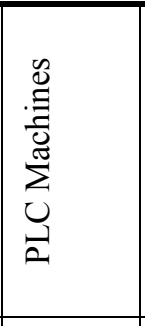 & 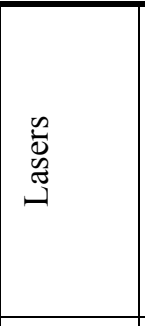 & 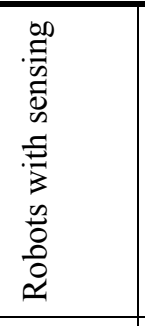 & 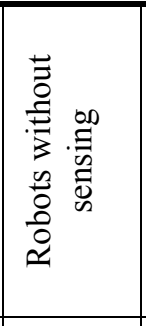 & 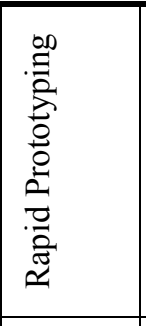 & 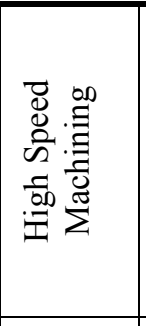 & 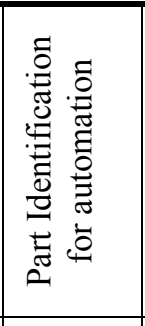 & 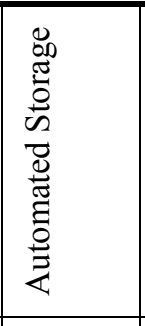 & 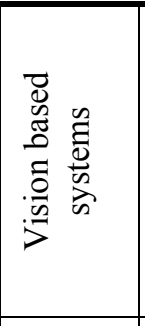 & 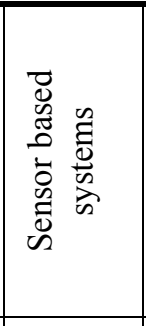 & 杂 & 玄 & 公 & $\begin{array}{l}= \\
\hat{\tilde{z}} \\
\text { 芝 }\end{array}$ & 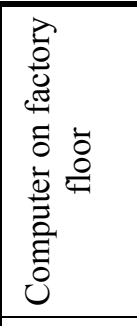 & $\sum_{J}$ & 离 & 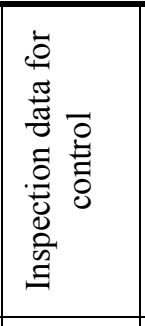 & 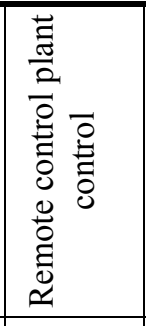 & 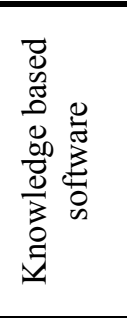 \\
\hline No factors & $\begin{array}{l}026^{\ddagger} \\
8222) \\
\end{array}$ & \begin{tabular}{|l|}
$0.09336^{\ddagger}$ \\
$(0.05525)$
\end{tabular} & \begin{tabular}{|l|}
$0.09292^{\ddagger}$ \\
$(0.05499)$
\end{tabular} & \begin{tabular}{|l|}
$0.09400^{\ddagger}$ \\
$(0.05498)$
\end{tabular} & \begin{tabular}{|l|}
$0.09315^{\ddagger}$ \\
$(0.05498)$
\end{tabular} & $\begin{array}{l}0.09608^{\ddagger} \\
(0.05518) \\
\end{array}$ & \begin{tabular}{|l|}
$0.09296^{\ddagger}$ \\
$(0.05499)$
\end{tabular} & \begin{tabular}{|l|}
$0.09296^{\ddagger}$ \\
$(0.05496)$
\end{tabular} & \begin{tabular}{|l}
$0.09297^{\ddagger}$ \\
$(0.05499)$
\end{tabular} & \begin{tabular}{|l|}
$0.09296^{\ddagger}$ \\
$(0.05449)$
\end{tabular} & \begin{tabular}{|l|}
$0.09296^{\ddagger}$ \\
$(0.05496)$ \\
\end{tabular} & \begin{tabular}{|l|l}
$0.09376^{\ddagger}$ \\
$(0.05449)$ \\
\end{tabular} & \begin{tabular}{|l|}
$0.09296^{\ddagger}$ \\
$(0.05490)$
\end{tabular} & \begin{tabular}{|l|}
$\begin{array}{l}0.09296^{\ddagger} \\
(0.05494)\end{array}$ \\
\end{tabular} & \begin{tabular}{|c|c}
$0.09296^{\ddagger}$ \\
$(0.05498)$
\end{tabular} & & & & & \begin{tabular}{|l|l}
$0.12217^{\dagger}$ \\
$(0.05457)$
\end{tabular} & & & \begin{tabular}{|l|l}
$0.09332^{\ddagger}$ \\
$(0.05501)$ \\
\end{tabular} & $\begin{array}{l}0.09296^{\ddagger} \\
(0.05494) \\
\end{array}$ & $\begin{array}{l}.09324^{\ddagger} \\
.05494) \\
\end{array}$ \\
\hline Factor 4 & $\begin{array}{l}0995 \\
5844) \\
\end{array}$ & \begin{tabular}{|l|l}
-0.02897 \\
$(0.45660)$
\end{tabular} & \begin{tabular}{|l|}
-0.03188 \\
$(0.45496)$
\end{tabular} & \begin{tabular}{|l|}
-0.00448 \\
$(0.45726)$
\end{tabular} & $\begin{array}{l}-0.00037 \\
(0.46735)\end{array}$ & $\begin{array}{l}0.00930 \\
(0.45973) \\
\end{array}$ & \begin{tabular}{|l|}
-0.03188 \\
$(0.45496)$ \\
\end{tabular} & $\begin{array}{l}0.01116 \\
(0.46499)\end{array}$ & \begin{tabular}{|c|}
-0.03056 \\
$(0.46698)$
\end{tabular} & \begin{tabular}{|l|}
-0.03188 \\
$(0.45081)$
\end{tabular} & $\begin{array}{l}-0.03188 \\
(0.45470)\end{array}$ & \begin{tabular}{|l|}
-0.03188 \\
$(0.45087)$
\end{tabular} & \begin{tabular}{|l|}
-0.03188 \\
$(0.45426)$
\end{tabular} & \begin{tabular}{|l|}
-0.03188 \\
$(0.45455)$
\end{tabular} & \begin{tabular}{|l|}
-0.03188 \\
$(0.45493)$
\end{tabular} & \begin{tabular}{|l|l|}
0.11391 \\
$(0.44632)$ \\
\end{tabular} & $\begin{array}{l}-0.03396 \\
(0.45706)\end{array}$ & \begin{tabular}{|c|}
-0.05057 \\
$(0.45618)$
\end{tabular} & \begin{tabular}{|l|}
-0.02170 \\
$(0.46077)$
\end{tabular} & $\begin{array}{l}0.17743 \\
(0.44978) \\
\end{array}$ & \begin{tabular}{|l|}
-0.03188 \\
$(0.45445)$
\end{tabular} & \begin{tabular}{|l|}
-0.03188 \\
$(0.45282)$
\end{tabular} & \begin{tabular}{|l|}
-0.01762 \\
$(0.46051)$
\end{tabular} & \begin{tabular}{|l|}
-0.03188 \\
$(0.45460)$ \\
\end{tabular} & $\begin{array}{l}-0.03188 \\
(0.45458) \\
\end{array}$ \\
\hline Factor 3 & $\begin{array}{ll}2217 \\
6931) \\
\end{array}$ & \begin{tabular}{|l|l}
0.25344 \\
$(0.26468)$
\end{tabular} & \begin{tabular}{|l|}
0.25236 \\
$(0.26471)$ \\
\end{tabular} & \begin{tabular}{|l|}
0.26405 \\
$(0.26509)$ \\
\end{tabular} & \begin{tabular}{|l|l}
0.25714 \\
$(0.26489)$
\end{tabular} & \begin{tabular}{|l|}
0.29393 \\
$(0.27310)$
\end{tabular} & \begin{tabular}{|l|}
0.25293 \\
$(0.26478)$ \\
\end{tabular} & \begin{tabular}{|l|l}
0.25276 \\
$(0.26439)$
\end{tabular} & \begin{tabular}{|l|l}
0.25294 \\
$(0.26493)$ \\
\end{tabular} & \begin{tabular}{|l|}
0.27857 \\
$(0.26245)$ \\
\end{tabular} & \begin{tabular}{|l|}
0.25787 \\
$0.26459)$ \\
\end{tabular} & \begin{tabular}{|l|l}
0.34655 \\
$(0.26669)$ \\
\end{tabular} & \begin{tabular}{|l|}
0.25276 \\
$(0.26411)$ \\
\end{tabular} & \begin{tabular}{|l|}
0.24292 \\
$(0.26478)$ \\
\end{tabular} & \begin{tabular}{|l|}
0.23994 \\
$(0.27686)$ \\
\end{tabular} & \begin{tabular}{|l|}
0.38182 \\
$(0.26130)$ \\
\end{tabular} & \begin{tabular}{|l|l}
0.24922 \\
$(0.27478)$
\end{tabular} & \begin{tabular}{|l|l}
0.23622 \\
$(0.26638)$ \\
\end{tabular} & \begin{tabular}{|l|l|}
0.25559 \\
$(0.26529)$ \\
\end{tabular} & \begin{tabular}{|l|l}
0.30351 \\
$(0.25903)$ \\
\end{tabular} & \begin{tabular}{|l|l}
0.25276 \\
$(0.26423)$ \\
\end{tabular} & \begin{tabular}{|l|l}
0.25276 \\
$(0.26328)$ \\
\end{tabular} & \begin{tabular}{|l|}
0.25871 \\
$(0.26618)$
\end{tabular} & \begin{tabular}{|l|}
0.25276 \\
$(0.26431)$ \\
\end{tabular} & $\begin{array}{l}.25276 \\
(0.26430) \\
\end{array}$ \\
\hline tor & 2589 & $\begin{array}{l}05898 \\
82305)\end{array}$ & \begin{tabular}{|c|}
-0.05898 \\
$(0.82306)$
\end{tabular} & \begin{tabular}{|c|}
-0.01241 \\
$(0.82667)$
\end{tabular} & $\begin{array}{l}-0.02747 \\
(0.82986)\end{array}$ & \begin{tabular}{|l|}
-0.01780 \\
$(0.82521)$ \\
\end{tabular} & \begin{tabular}{|l|}
-0.05771 \\
$(0.82741)$
\end{tabular} & $\begin{array}{l}-0.01594 \\
(0.82838)\end{array}$ & \begin{tabular}{|c|}
-0.05765 \\
$(0.82977)$ \\
\end{tabular} & \begin{tabular}{|c|}
-0.05898 \\
$(0.81557)$
\end{tabular} & \begin{tabular}{|l|}
-0.05898 \\
$(0.82261)$
\end{tabular} & \begin{tabular}{|l|l}
0.12110 \\
$(0.82108)$ \\
\end{tabular} & \begin{tabular}{|l|l}
-0.05898 \\
$(0.82180)$
\end{tabular} & $\begin{array}{l}-0.05898 \\
(0.82232)\end{array}$ & \begin{tabular}{|c|}
-0.07386 \\
$(0.82848)$
\end{tabular} & \begin{tabular}{|c|}
-0.05898 \\
$(0.80316)$
\end{tabular} & \begin{tabular}{|c|}
-0.05898 \\
$(0.82306)$ \\
\end{tabular} & \begin{tabular}{|c|}
-0.09074 \\
$(0.82495)$ \\
\end{tabular} & \begin{tabular}{|c|}
-0.04879 \\
$(0.82626)$
\end{tabular} & \begin{tabular}{|l|l}
0.15033 \\
$(0.80721)$
\end{tabular} & \begin{tabular}{|c|}
-0.05898 \\
$(0.82215)$ \\
\end{tabular} & & \begin{tabular}{|c|}
-0.05898 \\
$(0.82298)$ \\
\end{tabular} & \begin{tabular}{|l|}
-0.005898 \\
$(0.82241)$ \\
\end{tabular} & $\begin{array}{l}-0.05898 \\
(0.82238) \\
(\end{array}$ \\
\hline Factor 2 & $\begin{array}{l}9933^{*} \\
2914) \\
\end{array}$ & $\begin{array}{l}0.23683^{\ddagger} \\
(0.12902) \\
\end{array}$ & \begin{tabular}{|l|}
$0.23645^{\ddagger}$ \\
$(0.12903)$
\end{tabular} & \begin{tabular}{|l|}
$0.23887^{\ddagger}$ \\
$(0.12895)$ \\
\end{tabular} & \begin{tabular}{|l|}
$0.23813^{\ddagger}$ \\
$(0.12906)$ \\
\end{tabular} & \begin{tabular}{|l|}
$0.25043^{\ddagger}$ \\
$(0.13092)$ \\
\end{tabular} & \begin{tabular}{|l|}
$0.23659^{\ddagger}$ \\
$(0.12898)$
\end{tabular} & \begin{tabular}{|l|l}
$0.23659^{\ddagger}$ \\
$(0.12892)$
\end{tabular} & \begin{tabular}{|l}
$0.23659^{\ddagger}$ \\
$(0.12898)$ \\
\end{tabular} & \begin{tabular}{|l|}
$0.24536^{\ddagger}$ \\
$(0.12789)$
\end{tabular} & \begin{tabular}{|l|}
$0.23659^{\ddagger}$ \\
$(0.12891)$ \\
\end{tabular} & \begin{tabular}{|l|l}
$0.23659^{\ddagger}$ \\
$(0.12782)$ \\
\end{tabular} & \begin{tabular}{|l}
$\begin{array}{l}0.23659^{\ddagger} \\
(0.12878)\end{array}$ \\
\end{tabular} & \begin{tabular}{|l|}
$0.23659^{\ddagger}$ \\
$(0.12886)$
\end{tabular} & \begin{tabular}{|l|l}
$0.23659^{\ddagger}$ \\
$(0.12897)$ \\
\end{tabular} & \begin{tabular}{|l|}
$0.28296^{\dagger}$ \\
$(0.12670)$ \\
\end{tabular} & & \begin{tabular}{|l|l}
$0.22914^{\ddagger}$ \\
$(0.12974)$ \\
\end{tabular} & & 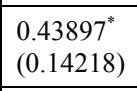 & \begin{tabular}{|l|l}
$0.26659^{\ddagger}$ \\
$(0.13641)$ \\
\end{tabular} & & \begin{tabular}{|l|}
$0.24628^{\ddagger}$ \\
$(0.13785)$ \\
\end{tabular} & $\begin{array}{l}0.23659^{\ddagger} \\
(0.12888) \\
\end{array}$ & $\begin{array}{l}25245^{\ddagger} \\
.13176) \\
\end{array}$ \\
\hline Factor $2 \& 4$ & 9295) & \begin{tabular}{|l}
0.60642 \\
$(0.59315)$ \\
\end{tabular} & \begin{tabular}{|l|}
0.60642 \\
$(0.59316)$
\end{tabular} & \begin{tabular}{|l|}
0.60642 \\
$(0.59270)$
\end{tabular} & \begin{tabular}{|l|}
7793 \\
$0267)$ \\
\end{tabular} & \begin{tabular}{|l|l}
0.60642 \\
$(0.59263)$
\end{tabular} & \begin{tabular}{|l|l|}
0.60642 \\
$(0.59316)$
\end{tabular} & \begin{tabular}{|l|l}
0.60642 \\
$(0.59287)$
\end{tabular} & \begin{tabular}{|l|}
0.60642 \\
$(0.59316)$ \\
\end{tabular} & \begin{tabular}{|l|}
0.60642 \\
$(0.58776)$
\end{tabular} & \begin{tabular}{|l|}
0.60642 \\
$(0.59283)$
\end{tabular} & \begin{tabular}{|l|}
0.60642 \\
$(0.58783)$ \\
\end{tabular} & \begin{tabular}{l|l|}
0642 \\
$69225)$ \\
\end{tabular} & \begin{tabular}{|l|}
0.60642 \\
$(0.59262)$
\end{tabular} & \begin{tabular}{|l|}
0.60642 \\
$(0.59313)$ \\
\end{tabular} & \begin{tabular}{|l|}
0.60642 \\
$(0.57882)$ \\
\end{tabular} & \begin{tabular}{|l|l|}
$\begin{array}{l}0.60642 \\
(0.59316)\end{array}$ \\
\end{tabular} & \begin{tabular}{|l|l}
0.60642 \\
$(0.59279)$
\end{tabular} & \begin{tabular}{|l|}
0.61661 \\
$(0.59762)$ \\
\end{tabular} & \begin{tabular}{|l}
0.81573 \\
$(0.58365)$ \\
\end{tabular} & \begin{tabular}{|l|}
0.60642 \\
$(0.59250)$
\end{tabular} & & \begin{tabular}{|l|l}
0.60642 \\
$(0.59310)$
\end{tabular} & \begin{tabular}{|l|}
0.60642 \\
$(0.59269)$ \\
\end{tabular} & $\begin{array}{l}0.60642 \\
(0.59267) \\
\end{array}$ \\
\hline & 7978) & 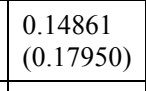 & \begin{tabular}{|l|}
0.14829 \\
$(0.17969)$ \\
\end{tabular} & \begin{tabular}{|l|}
0.15381 \\
$(0.17961)$ \\
\end{tabular} & 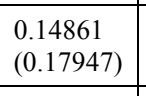 & \begin{tabular}{|l|}
0.18405 \\
$(0.18888)$ \\
\end{tabular} & \begin{tabular}{|l|l|}
0.14861 \\
$(0.17951)$ \\
\end{tabular} & \begin{tabular}{|l|l}
0.14861 \\
$(0.17942)$ \\
\end{tabular} & 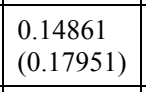 & \begin{tabular}{|l|l|}
0.14861 \\
$(0.17787)$
\end{tabular} & \begin{tabular}{|l|l|}
0.14861 \\
$(0.17941)$ \\
\end{tabular} & 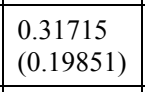 & \begin{tabular}{|l|}
0.24323 \\
$(0.21580)$ \\
\end{tabular} & \begin{tabular}{|l|l|}
0.14525 \\
$(0.17943)$ \\
\end{tabular} & \begin{tabular}{|l|}
0.13604 \\
$(0.19661)$ \\
\end{tabular} & \begin{tabular}{|l|}
$0.37373^{\dagger}$ \\
$(0.18896)$ \\
\end{tabular} & & & \begin{tabular}{|l|l|}
0.15040 \\
$(0.17996)$ \\
\end{tabular} & \begin{tabular}{|l|l}
$0.35792^{\ddagger}$ \\
$(0.18815)$
\end{tabular} & \begin{tabular}{|l|}
0.15131 \\
$(0.17935)$ \\
\end{tabular} & & & \begin{tabular}{|l|}
0.14861 \\
$(0.17936)$ \\
\end{tabular} & $\begin{array}{l}.18750 \\
(0.19154)\end{array}$ \\
\hline Factor $2,3 \& 4$ & $\begin{array}{l}5406 \\
5406) \\
\end{array}$ & & \begin{tabular}{|l|l|}
0.22084 \\
$(0.21212)$ \\
\end{tabular} & \begin{tabular}{|l|}
0.22598 \\
$(0.20995)$ \\
\end{tabular} & & \begin{tabular}{|l|}
0.22905 \\
$(0.20410)$ \\
\end{tabular} & \begin{tabular}{|l|}
0.22311 \\
$(0.20404)$ \\
\end{tabular} & & & \begin{tabular}{|l|l|}
0.22311 \\
$(0.20219)$ \\
\end{tabular} & \begin{tabular}{|l|}
0.22311 \\
$(0.20393)$ \\
\end{tabular} & & \begin{tabular}{|l|l|}
0.22311 \\
$0.20373)$ \\
\end{tabular} & \begin{tabular}{|l|}
0.21292 \\
$(0.20456)$ \\
\end{tabular} & \begin{tabular}{|l|}
0.22188 \\
$(0.20418)$ \\
\end{tabular} & & & & & & & & & & $\begin{array}{l}0.22992 \\
(0.20421) \\
\end{array}$ \\
\hline Factor 1 & 1531 & $\begin{array}{l}1055^{*} \\
77275) \\
\end{array}$ & \begin{tabular}{|l|}
$0.20790^{*}$ \\
$(0.06458)$
\end{tabular} & $\begin{array}{l}1300^{\circ} \\
9006) \\
\end{array}$ & $8622^{\circ}$ & \begin{tabular}{|l|l|}
$0.22405^{*}$ \\
$(0.06978)$ \\
\end{tabular} & \begin{tabular}{|l|}
$0.20805^{*}$ \\
$(0.06451)$
\end{tabular} & 68447) & 680499) & \begin{tabular}{|l|}
$0.21144^{*}$ \\
$(0.06393)$
\end{tabular} & \begin{tabular}{|l|}
$0.21454^{*}$ \\
$(0.06591)$ \\
\end{tabular} & \begin{tabular}{|l|}
$0.20803^{*}$ \\
$(0.06391)$ \\
\end{tabular} & \begin{tabular}{|l|}
$0.20803^{*}$ \\
$(0.06439)$
\end{tabular} & \begin{tabular}{|l|}
$0.20803^{*}$ \\
$(0.06444)$
\end{tabular} & \begin{tabular}{|l|}
$0.20784^{*}$ \\
$(0.06450)$ \\
\end{tabular} & 227) & \begin{tabular}{|l|}
$0.20773^{*}$ \\
$(0.06480)$
\end{tabular} & \begin{tabular}{|l|}
$0.20166^{*}$ \\
$(0.0658)$ \\
\end{tabular} & \begin{tabular}{|l|}
$0.21259^{*}$ \\
$(0.07232)$ \\
\end{tabular} & \begin{tabular}{|l|l|}
$.22735^{*}$ \\
$(0.06334)$ \\
\end{tabular} & \begin{tabular}{|l|}
$0.20803^{*}$ \\
$(0.06442)$ \\
\end{tabular} & \begin{tabular}{|l|}
$0.20803^{*}$ \\
$(0.06419)$ \\
\end{tabular} & \begin{tabular}{|l|}
$0.20951^{*}$ \\
$(0.06492)$ \\
\end{tabular} & \begin{tabular}{|l|l|}
$0.20954^{*}$ \\
$(0.06450)$ \\
\end{tabular} & $\begin{array}{l}0.21170^{\circ} \\
(0.06475) \\
\end{array}$ \\
\hline $\mathrm{Fa}$ & \begin{tabular}{ll|}
610 \\
$6310)$ \\
3
\end{tabular} & & \begin{tabular}{|l|}
0.18937 \\
$(0.12220)$ \\
\end{tabular} & \begin{tabular}{|l|}
$0.22284^{\ddagger}$ \\
$(0.13396)$
\end{tabular} & \begin{tabular}{|l|l}
0.21732 \\
$(0.15190)$
\end{tabular} & \begin{tabular}{|l|l}
$0.21070^{\ddagger}$ \\
$(0.12520)$
\end{tabular} & \begin{tabular}{|l|}
0.19038 \\
$(0.12135)$ \\
\end{tabular} & \begin{tabular}{|l}
0.19972 \\
$(0.12236)$
\end{tabular} & \begin{tabular}{|l|l|}
0.19040 \\
$(0.12201)$ \\
\end{tabular} & \begin{tabular}{|l|}
0.19016 \\
$(0.11941)$
\end{tabular} & \begin{tabular}{|l|}
0.19938 \\
$(0.12200)$ \\
\end{tabular} & \begin{tabular}{|l|l}
$0.20262^{\ddagger}$ \\
$(0.11960)$ \\
\end{tabular} & \begin{tabular}{|l|}
0.19016 \\
$(0.12032)$ \\
\end{tabular} & \begin{tabular}{|l|}
0.19016 \\
$(0.12040)$ \\
\end{tabular} & \begin{tabular}{|l|l|}
0.19016 \\
$(0.12050)$ \\
\end{tabular} & & \begin{tabular}{|l|}
0.18900 \\
$(0.12296)$
\end{tabular} & & \begin{tabular}{|l|l|}
0.19245 \\
$(0.12162)$ \\
\end{tabular} & \begin{tabular}{|l|l|l}
$0.24874^{\dagger}$ \\
$(0.11929)$ \\
\end{tabular} & \begin{tabular}{|l|l|}
0.19288 \\
$(0.12044)$ \\
\end{tabular} & \begin{tabular}{|l|}
0.19622 \\
$(0.12002)$ \\
\end{tabular} & \begin{tabular}{|l|}
0.19207 \\
$(0.12088)$ \\
\end{tabular} & \begin{tabular}{|l|l|l}
0.19016 \\
$(0.12041)$ \\
\end{tabular} & $\begin{array}{l}.19289 \\
(0.12050) \\
\end{array}$ \\
\hline Factor $1 \& 3$ & \begin{tabular}{ll|}
$4452)$ \\
4
\end{tabular} & $\begin{array}{l}2082 \\
8255) \\
2\end{array}$ & \begin{tabular}{|l|l|}
0.21568 \\
$(0.27974)$ \\
\end{tabular} & \begin{tabular}{|l|}
0.25331 \\
$(0.28519)$ \\
\end{tabular} & $\begin{array}{l}8007) \\
876\end{array}$ & $\begin{array}{l}0.22344 \\
(0.28420) \\
\end{array}$ & \begin{tabular}{|l|l|}
0.216966 \\
$(0.27783)$ \\
\end{tabular} & \begin{tabular}{l|l|}
1696 \\
$27770)$ \\
\end{tabular} & \begin{tabular}{|l|}
0.21696 \\
$(0.27783)$ \\
\end{tabular} & 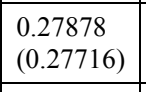 & \begin{tabular}{|l|}
0.21696 \\
$(0.27768)$ \\
\end{tabular} & \begin{tabular}{|l|}
0.39704 \\
$(0.29097)$ \\
\end{tabular} & \begin{tabular}{|l|}
0.25247 \\
$(0.28105)$ \\
\end{tabular} & \begin{tabular}{|l|}
0.21696 \\
$(0.27758)$ \\
\end{tabular} & \begin{tabular}{|l|l|}
0.21200 \\
$(0.27961)$ \\
\end{tabular} & & \begin{tabular}{|l|l|}
0.21538 \\
$(0.27981)$ \\
\end{tabular} & & \begin{tabular}{|l|}
0.21812 \\
$(0.27794)$ \\
\end{tabular} & \begin{tabular}{|l|l|}
0.31058 \\
$(0.27321)$ \\
\end{tabular} & \begin{tabular}{|l|l|}
0.22944 \\
$(0.27815)$ \\
\end{tabular} & & \begin{tabular}{|l|}
0.22009 \\
$(0.27825)$ \\
\end{tabular} & \begin{tabular}{|l|}
0.21696 \\
$(0.27761)$ \\
\end{tabular} & $\begin{array}{l}0.21696 \\
(0.27760) \\
\end{array}$ \\
\hline Factor $1,3 \& 4$ & $\begin{array}{l}122^{\dagger} \\
12671) \\
\end{array}$ & $\begin{array}{l}93677^{\dagger} \\
3531) \\
\end{array}$ & \begin{tabular}{|l|l|}
$0.28901^{\dagger}$ \\
$(0.12335)$
\end{tabular} & \begin{tabular}{|l|l}
$0.295288^{\dagger}$ \\
$(0.12325)$ \\
\end{tabular} & \begin{tabular}{|l|}
$0.29783^{\dagger}$ \\
$(0.12616)$ \\
\end{tabular} & $\begin{array}{l}0.33060^{\dagger} \\
(0.14075)\end{array}$ & \begin{tabular}{|l|}
$0.29030^{\dagger}$ \\
$(0.13587)$
\end{tabular} & \begin{tabular}{|l|}
$0.32228^{\dagger}$ \\
$(0.14348)$
\end{tabular} & \begin{tabular}{|c|}
$0.28960^{\dagger}$ \\
$(0.12361)$
\end{tabular} & \begin{tabular}{|c|}
$0.28943^{\dagger}$ \\
$(0.12178)$
\end{tabular} & \begin{tabular}{|l|}
$0.31914^{\dagger}$ \\
$(0.13792)$ \\
\end{tabular} & \begin{tabular}{|l|l}
$0.34639^{*}$ \\
$(0.12538)$
\end{tabular} & \begin{tabular}{|l|}
$0.30490^{\dagger}$ \\
$(0.12427)$
\end{tabular} & \begin{tabular}{|l|}
0.23901 \\
$(0.14851)$ \\
\end{tabular} & \begin{tabular}{|l|l}
$0.27925^{\dagger}$ \\
$(0.13901)$ \\
\end{tabular} & \begin{tabular}{|l|l}
$0.35552^{*}$ \\
$(0.12172)$ \\
\end{tabular} & \begin{tabular}{|l|}
$0.28842^{\dagger}$ \\
$(0.12473)$
\end{tabular} & \begin{tabular}{|l|l}
$0.28482^{\dagger}$ \\
$(0.12316)$ \\
\end{tabular} & \begin{tabular}{|l|}
$0.29071^{\dagger}$ \\
$(0.12323)$
\end{tabular} & $\begin{array}{l}0.33896^{*} \\
(0.12118)\end{array}$ & \begin{tabular}{|l|}
$0.28943^{\dagger}$ \\
$(0.12276)$
\end{tabular} & \begin{tabular}{|l|}
$0.28943^{\dagger}$ \\
$(0.12232)$
\end{tabular} & \begin{tabular}{|l|}
$0.30054^{\dagger}$ \\
$(0.13496)$
\end{tabular} & $\begin{array}{l}0.28943^{\dagger} \\
(0.12280)\end{array}$ & $\begin{array}{l}0.28943^{\dagger} \\
(0.12280)\end{array}$ \\
\hline Factor $1 \& 2$ & 2292" & 19991" & \begin{tabular}{|l|}
$0.31509^{*}$ \\
$(0.08984)$
\end{tabular} & \begin{tabular}{|l|}
$0.34737^{*}$ \\
$(0.10035)$ \\
\end{tabular} & 1779" & \begin{tabular}{|l|}
$0.34850^{*}$ \\
$(0.09925)$
\end{tabular} & \begin{tabular}{|l|}
$0.31668^{*}$ \\
$(0.08585)$ \\
\end{tabular} & \begin{tabular}{|l|}
$0.31638^{*}$ \\
$(0.08352)$ \\
\end{tabular} & \begin{tabular}{|l|}
$0.31638^{*}$ \\
$(0.08356)$
\end{tabular} & \begin{tabular}{|l|}
$0.34183^{*}$ \\
$(0.08385)$
\end{tabular} & \begin{tabular}{|l|}
$0.32118^{*}$ \\
$(0.08413)$
\end{tabular} & \begin{tabular}{|l|}
$0.32445^{*}$ \\
$(0.08292)$ \\
\end{tabular} & \begin{tabular}{|l|}
$0.31638^{*}$ \\
$(0.08343)$
\end{tabular} & \begin{tabular}{|l|}
$0.31638^{*}$ \\
$(0.08349)$ \\
\end{tabular} & \begin{tabular}{|l|}
$0.31638^{*}$ \\
$(0.08356)$
\end{tabular} & \begin{tabular}{|l|}
$0.54804^{*}$ \\
$(0.10940)$
\end{tabular} & \begin{tabular}{|l|}
$.31307^{*}$ \\
$(0.10872)$
\end{tabular} & \begin{tabular}{|l|}
$0.28983^{*}$ \\
$(0.09873)$ \\
\end{tabular} & \begin{tabular}{|l|}
$0.32017^{*}$ \\
$(0.08787)$
\end{tabular} & \begin{tabular}{|l|l|}
$0.47756^{\circ}$ \\
$(0.09702)$
\end{tabular} & \begin{tabular}{|l|}
$0.32411^{*}$ \\
$(0.08426)$
\end{tabular} & \begin{tabular}{|l|l|}
$0.34368^{*}$ \\
$(0.08549)$
\end{tabular} & \begin{tabular}{|l|}
$0.32479^{*}$ \\
$(0.09361)$
\end{tabular} & \begin{tabular}{|l|l|}
$0.322299^{*}$ \\
$(0.08431)$
\end{tabular} & $\begin{array}{l}0.32423^{*} \\
(0.08459)\end{array}$ \\
\hline Factor $1,2 \& 4$ & \begin{tabular}{|l|l|}
$6959^{*}$ \\
$12917)$ \\
\end{tabular} & $\begin{array}{l}0.33541^{*} \\
(0.09234)\end{array}$ & \begin{tabular}{|l|}
$0.33075^{*}$ \\
$(0.10115)$
\end{tabular} & \begin{tabular}{|l|}
$0.37491^{*}$ \\
$(0.11426)$
\end{tabular} & $\begin{array}{l}0.35875^{*} \\
(0.12304)\end{array}$ & \begin{tabular}{|l|}
$0.35219^{*}$ \\
$(0.09161)$ \\
\end{tabular} & \begin{tabular}{|l|}
$0.33317^{*}$ \\
$(0.08842)$
\end{tabular} & \begin{tabular}{|l}
$0.34145^{*}$ \\
$(0.08792)$
\end{tabular} & \begin{tabular}{|l}
$0.33298^{*}$ \\
$(0.08644)$
\end{tabular} & \begin{tabular}{|l|}
$\begin{array}{l}0.38539^{*} \\
(0.08928)\end{array}$ \\
\end{tabular} & \begin{tabular}{|l|}
$0.33925^{*}$ \\
$(0.08680)$
\end{tabular} & \begin{tabular}{|l|l}
$0.33676^{*}$ \\
$(0.08505)$ \\
\end{tabular} & \begin{tabular}{|l|}
$0.33285^{*}$ \\
$(0.08566)$
\end{tabular} & \begin{tabular}{|l|}
$0.33285^{*}$ \\
$(0.08571)$
\end{tabular} & \begin{tabular}{|l}
$0.33253^{*}$ \\
$(0.08581)$
\end{tabular} & \begin{tabular}{|l|}
$0.58067^{*}$ \\
$(0.11444)$
\end{tabular} & \begin{tabular}{|l|}
$0.32966^{*}$ \\
$(0.10889)$
\end{tabular} & \begin{tabular}{|l|}
$0.31481^{*}$ \\
$(0.09291)$
\end{tabular} & \begin{tabular}{|l|l}
$0.34125^{*}$ \\
$(0.10486)$
\end{tabular} & $\begin{array}{l}0.42426^{*} \\
(0.08895)\end{array}$ & \begin{tabular}{|l|l}
$0.35089^{*}$ \\
$(0.08983)$
\end{tabular} & \begin{tabular}{|l|}
$0.36509^{*}$ \\
$(0.08853)$ \\
\end{tabular} & \begin{tabular}{|l|l}
$0.33591^{*}$ \\
$(0.08715)$ \\
\end{tabular} & \begin{tabular}{|l|}
$0.33567^{*}$ \\
$(0.08587)$
\end{tabular} & $\begin{array}{l}\begin{array}{l}0.34339^{*} \\
(0.08763)\end{array} \\
\end{array}$ \\
\hline Factor $1,2 \& 3$ & \begin{tabular}{|l|l}
$0.25077^{\ddagger}$ \\
$(0.13763)$
\end{tabular} & \begin{tabular}{|l|l|}
$0.21710^{\dagger}$ \\
$(0.10800)$
\end{tabular} & \begin{tabular}{|l|}
$0.21281^{\dagger}$ \\
$(0.09815)$
\end{tabular} & \begin{tabular}{|l|}
$0.25601^{\dagger}$ \\
$(0.12312)$
\end{tabular} & \begin{tabular}{|l|}
$0.21479^{\dagger}$ \\
$(0.09672)$
\end{tabular} & \begin{tabular}{|l|l}
$0.25237^{\dagger}$ \\
$(0.11638)$
\end{tabular} & \begin{tabular}{|l|}
$0.21370^{\dagger}$ \\
$(0.09763)$
\end{tabular} & $\begin{array}{l}0.21408^{\dagger} \\
(0.09660)\end{array}$ & \begin{tabular}{|l|l}
$0.21349^{\dagger}$ \\
$(0.09664)$
\end{tabular} & $\left|\begin{array}{l}0.21604^{\dagger} \\
(0.09577)\end{array}\right|$ & \begin{tabular}{|l|l}
$0.23103^{\dagger}$ \\
$(0.10344)$
\end{tabular} & \begin{tabular}{|l|l|}
$0.27897^{*}$ \\
$(0.10170)$
\end{tabular} & $\left|\begin{array}{l}0.22960^{\dagger} \\
(0.09863)\end{array}\right|$ & \begin{tabular}{|l|}
$0.20542^{\dagger}$ \\
$(0.09747)$
\end{tabular} & \begin{tabular}{|l|l}
$0.20400^{\ddagger}$ \\
$(0.11404)$
\end{tabular} & \begin{tabular}{|l|l|}
$0.45789^{*}$ \\
$(0.12171)$
\end{tabular} & & & & \begin{tabular}{|l|l|l}
$0.29734^{*}$ \\
$(0.09829)$
\end{tabular} & \begin{tabular}{|l|l}
$0.22104^{7}$ \\
$(0.09719)$
\end{tabular} & \begin{tabular}{|l|l}
$0.23284^{\dagger}$ \\
$(0.09720)$
\end{tabular} & \begin{tabular}{|l|l|}
$0.22509^{\dagger}$ \\
$(0.11283)$
\end{tabular} & \begin{tabular}{|l|}
$0.21501^{\dagger}$ \\
$(0.09660)$
\end{tabular} & $\begin{array}{l}0.23822^{\dagger} \\
(0.10564)\end{array}$ \\
\hline & \begin{tabular}{|l|l}
$0.35613^{*}$ \\
$(0.11193)$ \\
\end{tabular} & 2621" & \begin{tabular}{|l|}
$0.32035^{*}$ \\
$(0.07845)$
\end{tabular} & \begin{tabular}{|l|l|}
$0.36782^{*}$ \\
$(0.10601)$
\end{tabular} & \begin{tabular}{|l}
$0.34953^{*}$ \\
$(0.11522)$
\end{tabular} & \begin{tabular}{|l|}
$0.35716^{*}$ \\
$(0.08896)$
\end{tabular} & \begin{tabular}{|l|}
$0.32230^{*}$ \\
$(0.07044)$
\end{tabular} & \begin{tabular}{|l}
$0.34406^{*}$ \\
$(0.08333)$
\end{tabular} & \begin{tabular}{|l|l}
$0.32259^{*}$ \\
$(0.08326)$ \\
\end{tabular} & \begin{tabular}{|l|}
$0.38040^{*}$ \\
$(0.07281)$
\end{tabular} & \begin{tabular}{|l|}
$0.33174^{*}$ \\
$(0.06988)$
\end{tabular} & \begin{tabular}{|l|}
$0.44245^{*}$ \\
$(0.09137)$ \\
\end{tabular} & \begin{tabular}{|l|}
$0.33660^{*}$ \\
$(0.06924)$
\end{tabular} & \begin{tabular}{|l|l}
$0.30406^{*}$ \\
$(0.07305)$
\end{tabular} & \begin{tabular}{|l|}
$0.31533^{*}$ \\
$(0.07911)$ \\
\end{tabular} & \begin{tabular}{|l|}
$0.54907^{*}$ \\
$(0.09676)$ \\
\end{tabular} & \begin{tabular}{|l|l|}
$0.31898^{*}$ \\
$(0.09170)$
\end{tabular} & \begin{tabular}{|l|}
$0.30089^{*}$ \\
$(0.07878)$ \\
\end{tabular} & \begin{tabular}{|l|}
$0.33151^{*}$ \\
$(0.09567)$ \\
\end{tabular} & 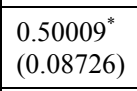 & \begin{tabular}{|l|}
$0.35417^{*}$ \\
$(0.08227)$ \\
\end{tabular} & \begin{tabular}{|l|}
$0.37488^{*}$ \\
$(0.07677)$ \\
\end{tabular} & \begin{tabular}{|l}
$0.33170^{*}$ \\
$(0.08277)$
\end{tabular} & $\begin{array}{l}0.32718^{*} \\
(0.06739) \\
\end{array}$ & $\begin{array}{l}0.33151^{*} \\
(0.06876)\end{array}$ \\
\hline & 19803) & .00494 & . & 08365) & \begin{tabular}{|l|}
-0.03151 \\
$(0.10732)$
\end{tabular} & 06881) & 08473) & \begin{tabular}{|c|}
-0.04304 \\
$(0.09712)$
\end{tabular} & \begin{tabular}{|l}
-0.00133 \\
$(0.10530)$
\end{tabular} & $\left.\mid \begin{array}{c}-0.18546^{\ddagger} \\
(0.09625)\end{array}\right)$ & $\left(\begin{array}{c}-0.03672 \\
(0.07751)\end{array}\right)$ & 99411) & $\mid$ & \begin{tabular}{|l|}
0.07064 \\
$(0.11706)$
\end{tabular} & .0950 & .07803) & & & \begin{tabular}{l|}
0.01019 \\
$0.07309)$
\end{tabular} & & 0.08 & & $\begin{array}{l}-0.01426 \\
(0.07160)\end{array}$ & $(0.19528)$ & \\
\hline
\end{tabular}

Table 91 Coefficients with individual variables for AMT - Electronics Industry 


\begin{tabular}{|c|c|c|c|c|c|c|c|c|c|c|c|c|}
\hline $\begin{array}{l}\text { Individual variable } \\
\text { included in the } \\
\text { model in the } \\
\text { column }\end{array}$ & 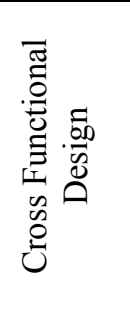 & 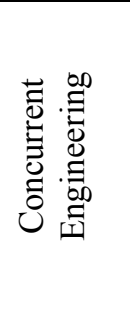 & 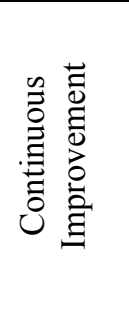 & 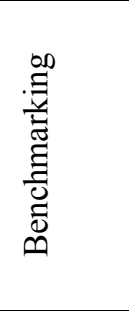 & 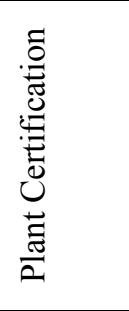 & 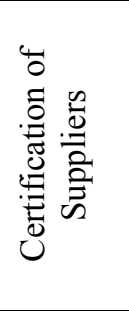 & 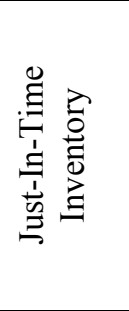 & 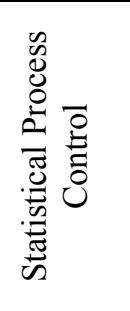 & 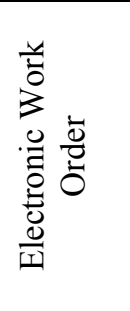 & 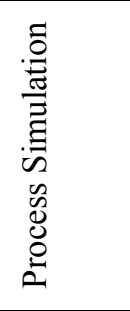 & 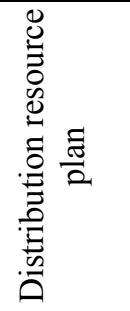 & 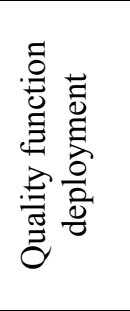 \\
\hline No factors & $\begin{array}{l}0.22587^{*} \\
(0.05798)\end{array}$ & $\begin{array}{l}0.22305^{*} \\
(0.05788)\end{array}$ & $\begin{array}{l}0.22211^{*} \\
(0.05838)\end{array}$ & $\begin{array}{l}0.22019^{*} \\
(0.05701)\end{array}$ & $\begin{array}{l}0.25725^{*} \\
(0.06382)\end{array}$ & $\begin{array}{l}0.19104^{*} \\
(0.06258)\end{array}$ & $\begin{array}{l}0.21983^{*} \\
(0.05767)\end{array}$ & $\begin{array}{l}0.22115^{*} \\
(0.05776)\end{array}$ & $\begin{array}{l}0.22044^{*} \\
(0.05786)\end{array}$ & $\begin{array}{l}0.22172^{*} \\
(0.0578)\end{array}$ & $\begin{array}{l}0.22172^{*} \\
(0.0578)\end{array}$ & $\begin{array}{l}0.22222^{*} \\
(0.05778)\end{array}$ \\
\hline Factor 3 & $\begin{array}{l}0.21947^{\dagger} \\
(0.10312)\end{array}$ & $\begin{array}{l}0.20988^{\ddagger} \\
(0.11008) \\
\end{array}$ & $\begin{array}{l}0.18891^{\ddagger} \\
(0.10099)\end{array}$ & $\begin{array}{l}0.11364 \\
(0.09757) \\
\end{array}$ & $\begin{array}{l}0.19525^{\dagger} \\
(0.0939) \\
\end{array}$ & $\begin{array}{l}0.18551^{\dagger} \\
(0.09372)\end{array}$ & $\begin{array}{l}0.18376^{\ddagger} \\
(0.09385) \\
\end{array}$ & $\begin{array}{l}0.1866^{\dagger} \\
(0.09398)\end{array}$ & $\begin{array}{l}0.18633^{\dagger} \\
(0.09404)\end{array}$ & $\begin{array}{l}0.18716^{\dagger} \\
(0.09406)\end{array}$ & $\begin{array}{l}0.18716^{\dagger} \\
(0.09406)\end{array}$ & $\begin{array}{l}0.19108^{\dagger} \\
(0.09436)\end{array}$ \\
\hline Factor 2 & $\begin{array}{l}0.13845 \\
(0.12074) \\
\end{array}$ & $\begin{array}{l}0.15093 \\
(0.12586) \\
\end{array}$ & $\begin{array}{l}0.1371 \\
(0.12092) \\
\end{array}$ & $\begin{array}{l}0.13696 \\
(0.11921) \\
\end{array}$ & $\begin{array}{l}0.13994 \\
(0.12043) \\
\end{array}$ & $\begin{array}{l}0.13423 \\
(0.12045)\end{array}$ & $\begin{array}{l}0.08529 \\
(0.12969)\end{array}$ & $\begin{array}{l}0.13696 \\
(0.12077)\end{array}$ & $\begin{array}{l}0.12271 \\
(0.12536)\end{array}$ & $\begin{array}{l}0.13696 \\
(0.12087)\end{array}$ & $\begin{array}{l}0.13685 \\
(0.12091)\end{array}$ & $\begin{array}{l}0.15626 \\
(0.12723)\end{array}$ \\
\hline Factor $2 \& 3$ & $\begin{array}{l}0.3857^{*} \\
(0.1359)\end{array}$ & $\begin{array}{l}0.36043^{*} \\
(0.13099)\end{array}$ & $\begin{array}{l}0.34691^{\dagger} \\
(0.13383)\end{array}$ & $\begin{array}{l}0.32435^{*} \\
(0.12339)\end{array}$ & $\begin{array}{l}0.38361^{*} \\
(0.12796)\end{array}$ & $\begin{array}{l}0.31311^{\dagger} \\
(0.12689)\end{array}$ & $\begin{array}{l}0.30939^{\dagger} \\
(0.12869)\end{array}$ & $\begin{array}{l}0.34461^{*} \\
(0.12472)\end{array}$ & $\begin{array}{l}0.33307^{*} \\
(0.12768)\end{array}$ & $\begin{array}{l}0.34617^{*} \\
(0.12507)\end{array}$ & $\begin{array}{l}0.34461^{*} \\
(0.12483)\end{array}$ & $\begin{array}{l}0.36607^{*} \\
(0.13241)\end{array}$ \\
\hline Factor 1 & $\begin{array}{l}-0.10727 \\
(0.0931)\end{array}$ & $\begin{array}{l}-0.10759 \\
(0.09319)\end{array}$ & $\begin{array}{l}-0.10664 \\
(0.09851)\end{array}$ & $\begin{array}{l}-0.2224^{\dagger} \\
(0.10326)\end{array}$ & $\begin{array}{l}-0.02671 \\
(0.11227)\end{array}$ & $\begin{array}{l}-0.19432^{\ddagger} \\
(0.11558)\end{array}$ & $\begin{array}{l}-0.11501 \\
(0.09317)\end{array}$ & $\begin{array}{l}-0.1266 \\
(0.09761)\end{array}$ & $\begin{array}{l}-0.11147 \\
(0.0935)\end{array}$ & $\begin{array}{l}-0.10816 \\
(0.09321)\end{array}$ & $\begin{array}{l}-0.10816 \\
(0.09322)\end{array}$ & $\begin{array}{l}-0.10816 \\
(0.09317)\end{array}$ \\
\hline Factor $1 \& 3$ & $\begin{array}{l}0.32592^{*} \\
(0.09748)\end{array}$ & $\begin{array}{l}0.31582^{*} \\
(0.10326)\end{array}$ & $\begin{array}{l}0.29801^{*} \\
(0.11272)\end{array}$ & $\begin{array}{l}0.20264^{\dagger} \\
(0.09514)\end{array}$ & $\begin{array}{l}0.39151^{*} \\
(0.11576)\end{array}$ & $\begin{array}{l}0.21078^{\ddagger} \\
(0.11073)\end{array}$ & $\begin{array}{l}0.27541^{*} \\
(0.09003)\end{array}$ & $\begin{array}{l}0.26846^{*} \\
(0.09769)\end{array}$ & $\begin{array}{l}0.27754^{*} \\
(0.09714)\end{array}$ & $\begin{array}{l}0.29486^{*} \\
(0.08849)\end{array}$ & $\begin{array}{l}0.29468^{*} \\
(0.08849)\end{array}$ & $\begin{array}{l}0.29508^{*} \\
(0.08845)\end{array}$ \\
\hline Factor $1 \& 2$ & $\begin{array}{l}0.3236^{*} \\
(0.10146)\end{array}$ & $\begin{array}{l}0.32088^{*} \\
(0.10151) \\
\end{array}$ & $\begin{array}{l}0.31969^{*} \\
(0.1035) \\
\end{array}$ & $\begin{array}{l}0.22382^{\dagger} \\
(0.10735)\end{array}$ & $\begin{array}{l}0.41553^{*} \\
(0.12582) \\
\end{array}$ & $\begin{array}{l}0.23023^{\ddagger} \\
(0.12327)\end{array}$ & $\begin{array}{l}0.2827^{*} \\
(0.10646) \\
\end{array}$ & $\begin{array}{l}0.3101^{*} \\
(0.10221)\end{array}$ & $\begin{array}{l}0.30069^{*} \\
(0.10979) \\
\end{array}$ & $\begin{array}{l}0.32091^{*} \\
(0.102)\end{array}$ & $\begin{array}{l}0.31835^{*} \\
(0.1017) \\
\end{array}$ & $\begin{array}{l}0.32494^{*} \\
(0.10216)\end{array}$ \\
\hline All Factors & $\begin{array}{l}0.29427^{*} \\
(0.0581)\end{array}$ & $\begin{array}{l}0.28935^{*} \\
(0.08058)\end{array}$ & $\begin{array}{l}0.26414^{*} \\
(0.07144)\end{array}$ & $\begin{array}{l}0.16955^{*} \\
(0.05364)\end{array}$ & $\begin{array}{l}0.34753^{*} \\
(0.07713)\end{array}$ & $\begin{array}{l}0.1896^{*} \\
(0.06898)\end{array}$ & $\begin{array}{l}0.20399^{*} \\
(0.06554)\end{array}$ & $\begin{array}{l}0.24035^{*} \\
(0.05089)\end{array}$ & $\begin{array}{l}0.2466^{*} \\
(0.05171)\end{array}$ & $\begin{array}{l}0.26687^{*} \\
(0.04797)\end{array}$ & $\begin{array}{l}0.25992^{*} \\
(0.04883)\end{array}$ & $\begin{array}{l}0.28206^{*} \\
(0.05775)\end{array}$ \\
\hline $\begin{array}{l}\text { Individual } \\
\text { Variable }\end{array}$ & $\begin{array}{l}-0.04848 \\
(0.06384)\end{array}$ & $\begin{array}{l}-0.03111 \\
(0.07837)\end{array}$ & $\begin{array}{l}-0.00333 \\
(0.06982)\end{array}$ & $\begin{array}{l}0.14647^{\dagger} \\
(0.06029)\end{array}$ & $\begin{array}{l}-0.09683 \\
(0.07504)\end{array}$ & $\begin{array}{l}0.08848 \\
(0.07065)\end{array}$ & $\begin{array}{l}0.07332 \\
(0.06785)\end{array}$ & $\begin{array}{l}0.04014 \\
(0.0636)\end{array}$ & $\begin{array}{l}0.02568 \\
(0.06015)\end{array}$ & $\begin{array}{l}-0.01475 \\
(0.07503)\end{array}$ & $\begin{array}{l}0.00348 \\
(0.07664)\end{array}$ & $\begin{array}{l}-0.03268 \\
(0.06752)\end{array}$ \\
\hline
\end{tabular}

* Significant at the 0.01 level $†$ Significant at the 0.05 level $\$$ Significant at the 0.10 level

Table 92 Coefficients with individual variables for business practices - Electronics Industry 


\begin{tabular}{|c|c|c|c|c|c|c|c|c|c|c|c|c|c|c|c|c|c|c|}
\hline $\begin{array}{c}\text { Individual } \\
\text { variable } \\
\text { included in the } \\
\text { model in the } \\
\text { column }\end{array}$ & 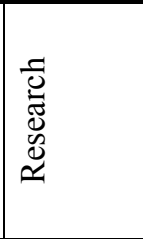 & 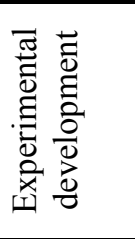 & 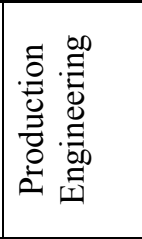 & 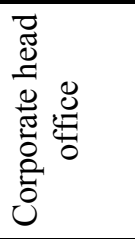 & 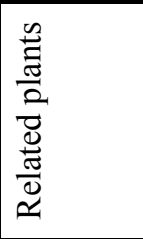 & 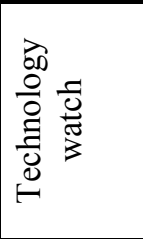 & 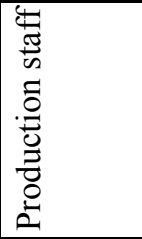 & 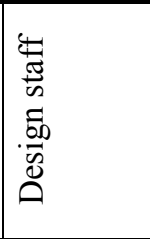 & 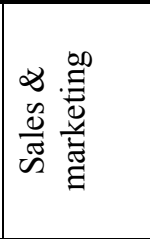 & 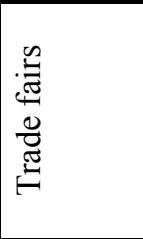 & 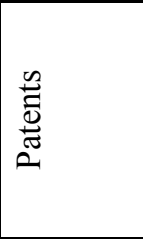 & 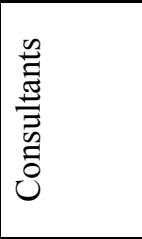 & 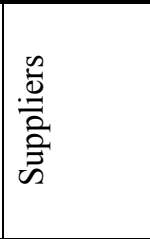 & 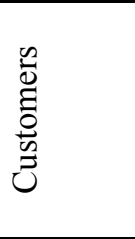 & 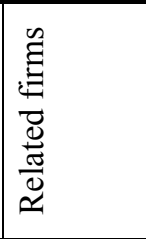 & 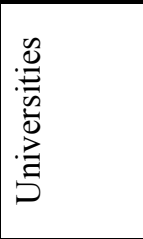 & 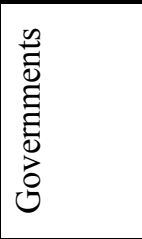 & 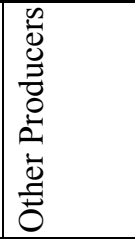 \\
\hline No factc & $\begin{array}{l}0.20807^{*} \\
(0.05212) \\
\end{array}$ & $\begin{array}{l}0.20582^{*} \\
(0.05193)\end{array}$ & $\begin{array}{l}0.19557^{*} \\
(0.05308) \\
\end{array}$ & $\begin{array}{l}0.21283^{*} \\
(0.05233)\end{array}$ & $\begin{array}{l}0.20466^{*} \\
(0.05186) \\
\end{array}$ & \begin{tabular}{|l}
$0.20457^{*}$ \\
$(0.05183)$ \\
\end{tabular} & $\begin{array}{l}0.20418^{*} \\
(0.05230) \\
\end{array}$ & \begin{tabular}{|l|}
$0.20512^{*}$ \\
$(0.05249)$ \\
\end{tabular} & \begin{tabular}{|l|}
$0.20831^{*}$ \\
$(0.05214)$ \\
\end{tabular} & \begin{tabular}{|l}
$0.20443^{*}$ \\
$(0.04950)$ \\
\end{tabular} & \begin{tabular}{|l|}
$0.17414^{*}$ \\
$(0.05316)$ \\
\end{tabular} & $\begin{array}{l}0.20443^{*} \\
(0.05185) \\
\end{array}$ & \begin{tabular}{|l|}
$0.20351^{*}$ \\
$(0.05198)$ \\
\end{tabular} & $\begin{array}{l}0.19478^{*} \\
(0.05168)\end{array}$ & \begin{tabular}{|l|l|}
$0.20124^{*}$ \\
$(0.05212)$ \\
\end{tabular} & $\begin{array}{l}.20443^{*} \\
0.05184)\end{array}$ & $\begin{array}{l}0.20443^{*} \\
(0.05185) \\
\end{array}$ & $\begin{array}{l}0.20415^{*} \\
(0.05175) \\
\end{array}$ \\
\hline Factor 4 & \begin{tabular}{|l|l|l}
0.23409 \\
$(0.21040)$ \\
\end{tabular} & $\begin{array}{l}0.21016 \\
(0.20482)\end{array}$ & \begin{tabular}{|l}
0.15132 \\
$(0.21281)$ \\
\end{tabular} & $\begin{array}{l}0.26532 \\
(0.21240)\end{array}$ & \begin{tabular}{|l}
0.21547 \\
$(0.21595)$ \\
\end{tabular} & \begin{tabular}{|l|}
0.19940 \\
$(0.20335)$ \\
\end{tabular} & \begin{tabular}{|l|}
0.19799 \\
$(0.20709)$ \\
\end{tabular} & & & & & & & & & & & \begin{tabular}{|l}
0.19940 \\
$(0.20302)$ \\
\end{tabular} \\
\hline $\mathrm{F}$ & & $\begin{array}{l}0.09131 \\
(0.19053) \\
\end{array}$ & \begin{tabular}{|l|l|}
0.09131 \\
$(0.19035)$ \\
\end{tabular} & $\begin{array}{l}0.16187 \\
(0.20165) \\
\end{array}$ & \begin{tabular}{|l|}
0.09131 \\
$(0.19060)$ \\
\end{tabular} & \begin{tabular}{|l|}
0.09131 \\
$(0.19053)$ \\
\end{tabular} & \begin{tabular}{|l|}
0.09098 \\
$(0.19084)$ \\
\end{tabular} & \begin{tabular}{|l|}
0.09131 \\
$(0.19062)$ \\
\end{tabular} & \begin{tabular}{|l|}
0.14370 \\
$(0.20672)$ \\
\end{tabular} & \begin{tabular}{|l|}
0.09131 \\
$(0.18196)$ \\
\end{tabular} & & & \begin{tabular}{|l|}
0.09131 \\
$(0.19060)$ \\
\end{tabular} & & & & & \begin{tabular}{|l|}
0.09131 \\
$(0.19022)$ \\
\end{tabular} \\
\hline Factor $3 \& 4$ & $\begin{array}{l}-0.31221^{\dagger} \\
(0.13005)\end{array}$ & $\begin{array}{l}-0.31109^{\dagger} \\
(0.13040)\end{array}$ & $\begin{array}{l}-0.32442^{\dagger} \\
(0.13043)\end{array}$ & $\begin{array}{l}-0.25062^{*} \\
(0.14370)\end{array}$ & $\begin{array}{l}-0.29942^{\dagger} \\
(0.14890)\end{array}$ & $\begin{array}{l}-0.31461^{\dagger} \\
(0.13003)\end{array}$ & \begin{tabular}{|l|}
$-0.31744^{\dagger}$ \\
$(0.14069)$
\end{tabular} & $\begin{array}{c}-0.31487^{\dagger} \\
(0.13028) \\
\end{array}$ & \begin{tabular}{|c|}
$-0.26641^{*}$ \\
$(0.15022)$
\end{tabular} & $\begin{array}{l}-0.13209 \\
(0.13087) \\
\end{array}$ & $\begin{array}{l}-0.42970^{*} \\
(0.13910)\end{array}$ & $\begin{array}{l}-0.31642^{\dagger} \\
(0.13020)\end{array}$ & $\begin{array}{c}-0.31549^{\dagger} \\
(0.13006)\end{array}$ & $\begin{array}{l}-0.35787^{*} \\
(0.13099)\end{array}$ & $\begin{array}{l}-0.33254^{\prime} \\
(0.13344)\end{array}$ & $\begin{array}{l}-0.28646^{\ddagger} \\
(0.15204)\end{array}$ & $\begin{array}{l}-0.31549^{\dagger} \\
(0.13007)\end{array}$ & $\begin{array}{l}-0.31549^{\dagger} \\
(0.12980) \\
\end{array}$ \\
\hline Factor 2 & $\begin{array}{l}0.32984^{\ddagger} \\
(0.1802)\end{array}$ & $\begin{array}{l}0.30417^{\ddagger} \\
(0.17164)\end{array}$ & $\begin{array}{l}0.20442 \\
(0.18225)\end{array}$ & $\begin{array}{l}0.27696^{*} \\
(0.15718)\end{array}$ & $\begin{array}{l}0.275 \\
(0.15 \\
\end{array}$ & $\begin{array}{l}0.27704^{7} \\
(0.15764)\end{array}$ & $\begin{array}{l}0.27258^{\ddagger} \\
(0.16353)\end{array}$ & 0.27 & \begin{tabular}{|c|}
$0.28276^{\ddagger}$ \\
$(0.15797)$
\end{tabular} & & & & & & & & & $\begin{array}{l}0.25791 \\
(0.15823)\end{array}$ \\
\hline Factor $2 \& 4$ & \begin{tabular}{|l|}
0.29588 \\
$(0.19707)$ \\
\end{tabular} & $\begin{array}{l}0.26796 \\
(0.18774) \\
\end{array}$ & \begin{tabular}{|l|l|}
0.16242 \\
$(0.20243)$ \\
\end{tabular} & $\begin{array}{l}0.26429 \\
(0.17699) \\
\end{array}$ & \begin{tabular}{|l|}
0.24102 \\
$(0.17605)$ \\
\end{tabular} & \begin{tabular}{|l|}
0.23889 \\
$(0.17572)$ \\
\end{tabular} & \begin{tabular}{|l|}
0.23850 \\
$(0.17614)$ \\
\end{tabular} & $\begin{array}{l}0.24216 \\
(0.18001) \\
\end{array}$ & \begin{tabular}{|l|}
0.24236 \\
$(0.17570)$ \\
\end{tabular} & \begin{tabular}{|l|}
0.27382 \\
$(0.16800)$ \\
\end{tabular} & \begin{tabular}{|l|}
0.07344 \\
$(0.19002)$ \\
\end{tabular} & \begin{tabular}{|l|}
0.23889 \\
$(0.17580)$ \\
\end{tabular} & \begin{tabular}{|l|}
0.23788 \\
$(0.17583)$ \\
\end{tabular} & & \begin{tabular}{|l|}
0.23889 \\
$(0.17567)$ \\
\end{tabular} & \begin{tabular}{|l|}
0.27156 \\
$(0.19684)$ \\
\end{tabular} & \begin{tabular}{|l|}
0.24151 \\
$(0.17664)$ \\
\end{tabular} & \begin{tabular}{|l|}
0.23455 \\
$(0.17550)$ \\
\end{tabular} \\
\hline $\mathrm{F}$ & & & & & & & & & 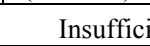 & 2 & & & & & & & & \\
\hline & & & & & & & & & & & & & & & & & & \begin{tabular}{|l|}
0.41172 \\
$(0.25838)$ \\
\end{tabular} \\
\hline $\mathrm{F}$ & $\begin{array}{l}0.11126 \\
(0.20851)\end{array}$ & $\begin{array}{l}0.12523 \\
(0.21316)\end{array}$ & $\begin{array}{l}0.03623 \\
(0.22741) \\
\end{array}$ & $\begin{array}{l}0.11702 \\
(0.20824)\end{array}$ & & & \begin{tabular}{|l|l|}
0.102 \\
$(0.22$
\end{tabular} & & & & & & & & & & & $\begin{array}{l}0.10559 \\
(0.20809)\end{array}$ \\
\hline Factc & \begin{tabular}{|l|}
0.09919 \\
$(0.18745)$
\end{tabular} & $\begin{array}{l}0.09807 \\
(0.18778)\end{array}$ & \begin{tabular}{|l|}
0.01900 \\
$(0.20979)$
\end{tabular} & $\begin{array}{l}0.15784 \\
(0.19722)\end{array}$ & & \begin{tabular}{|l|l|}
0.09547 \\
$(0.18738)$
\end{tabular} & \begin{tabular}{|l|l|}
0.08825 \\
$(0.20414)$
\end{tabular} & & \begin{tabular}{|l|}
0.10098 \\
$(0.18763)$
\end{tabular} & & & & & & & \begin{tabular}{|l|l|}
0.09691 \\
$(0.18780)$
\end{tabular} & \begin{tabular}{|l|}
0.09186 \\
$(0.18726)$
\end{tabular} & \begin{tabular}{|l|l|}
0.08419 \\
$(0.18698)$
\end{tabular} \\
\hline 3 & $\begin{array}{l}0.25733^{\ddagger} \\
(0.13608) \\
\end{array}$ & $\begin{array}{l}0.23357^{\ddagger} \\
(0.12830) \\
\end{array}$ & $\begin{array}{l}0.16419 \\
(0.15145) \\
\end{array}$ & $\begin{array}{l}0.24922^{\ddagger} \\
(0.12885) \\
\end{array}$ & $\begin{array}{l}0.22658^{\ddagger} \\
(0.12736) \\
\end{array}$ & $\begin{array}{l}0.23228^{\ddagger} \\
(0.12795) \\
\end{array}$ & \begin{tabular}{|l|}
0.22369 \\
$(0.15002)$ \\
\end{tabular} & \begin{tabular}{|l|}
0.23246 \\
$(0.14513)$ \\
\end{tabular} & \begin{tabular}{|c|}
$0.27579^{\ddagger}$ \\
$(0.14799)$ \\
\end{tabular} & $\begin{array}{l}0.22658^{\ddagger} \\
(0.12158) \\
\end{array}$ & \begin{tabular}{|l|}
0.04081 \\
$(0.15260)$ \\
\end{tabular} & $\begin{array}{l}0.22658^{\ddagger} \\
(0.12736) \\
\end{array}$ & \begin{tabular}{|l|}
$0.21934^{\ddagger}$ \\
$(0.13069)$ \\
\end{tabular} & & & $\begin{array}{l}0.22869^{\ddagger} \\
(0.12746) \\
\end{array}$ & $\begin{array}{l}0.22658^{\ddagger} \\
(0.12736) \\
\end{array}$ & $\begin{array}{l}0.22658^{\ddagger} \\
(0.12710) \\
\end{array}$ \\
\hline $3 \& 4$ & $\begin{array}{l}0.18011^{\dagger} \\
(0.07668) \\
\end{array}$ & $\begin{array}{l}0.17500^{\dagger} \\
(0.07599)\end{array}$ & \begin{tabular}{|l|l|}
0.10238 \\
$(0.11961)$ \\
\end{tabular} & $\begin{array}{l}0.20884^{\dagger} \\
(0.08313)\end{array}$ & $\begin{array}{l}0.18517^{\ddagger} \\
(0.09462) \\
\end{array}$ & $\begin{array}{l}0.17508^{\dagger} \\
(0.07600) \\
\end{array}$ & \begin{tabular}{|l|}
0.16972 \\
$(0.10995)$ \\
\end{tabular} & & \begin{tabular}{|c|}
$0.22185^{\dagger}$ \\
$(0.10702)$ \\
\end{tabular} & & & $\begin{array}{l}0.17206^{\dagger} \\
(0.07591) \\
\end{array}$ & \begin{tabular}{|l|l|}
$0.16823^{\dagger}$ \\
$(0.07789)$ \\
\end{tabular} & & & $\begin{array}{l}0.20517^{\ddagger} \\
(0.11640) \\
\end{array}$ & $\begin{array}{l}73^{\dagger} \\
584) \\
\end{array}$ & $\begin{array}{l}0.14463^{\ddagger} \\
(0.08151) \\
\end{array}$ \\
\hline Factor $1 \& 2$ & \begin{tabular}{|l|l|}
0.18632 \\
$(0.13466)$ \\
\end{tabular} & $\begin{array}{l}0.17013 \\
(0.13543)\end{array}$ & $\begin{array}{l}0.05784 \\
(0.14381) \\
\end{array}$ & $\begin{array}{l}0.13726 \\
(0.10483) \\
\end{array}$ & \begin{tabular}{|l|}
0.13371 \\
$(0.10514)$ \\
\end{tabular} & \begin{tabular}{|l|}
0.13571 \\
$(0.10521)$ \\
\end{tabular} & \begin{tabular}{|l|}
0.12 \\
$(0.12$ \\
\end{tabular} & $\begin{array}{l}0.1 \\
(0.1 \\
\end{array}$ & \begin{tabular}{|l|}
0.15 \\
$(0.1$ \\
\end{tabular} & \begin{tabular}{|l|}
0.13250 \\
$(0.10024)$ \\
\end{tabular} & $\begin{array}{l}-0.0 \\
(0.1 \\
\end{array}$ & \begin{tabular}{|l|}
0.12 \\
$(0.10$ \\
\end{tabular} & \begin{tabular}{|l|}
0.12310 \\
$(0.11171)$ \\
\end{tabular} & $\begin{array}{l}2 \\
89)\end{array}$ & $53)$ & \begin{tabular}{|l|}
0.13250 \\
$(0.10498)$ \\
\end{tabular} & \begin{tabular}{|l|}
0.13538 \\
$(0.10670)$ \\
\end{tabular} & $\begin{array}{l}0.11607 \\
(0.10629) \\
\end{array}$ \\
\hline 4 & $\begin{array}{l}0.42228^{7} \\
(0.20348)\end{array}$ & & $\begin{array}{l}0.28479 \\
(0.20585)\end{array}$ & & & & & & & & & & & & & & & $\begin{array}{l}0.36126^{\dagger} \\
(0.17936)\end{array}$ \\
\hline Factor 1 & $\begin{array}{l}0.35827^{\circ} \\
(0.12571)\end{array}$ & $\begin{array}{l}0.33555^{*} \\
(0.11903)\end{array}$ & $\begin{array}{l}0.22531^{7} \\
(0.12645)\end{array}$ & $\begin{array}{l}0.29895^{\prime \prime} \\
(0.08256)\end{array}$ & $\begin{array}{l}0.30013^{*} \\
(0.08337)\end{array}$ & \begin{tabular}{|l}
$0.30571^{*}$ \\
$(0.08458)$
\end{tabular} & $\begin{array}{l}0.29513^{*} \\
(0.11260)\end{array}$ & \begin{tabular}{|c|}
$0.30559^{\dagger}$ \\
$(0.12285)$
\end{tabular} & \begin{tabular}{|l|}
$0.33014^{*}$ \\
$(0.09637)$
\end{tabular} & $\begin{array}{l}0.30566^{*} \\
(0.07904)\end{array}$ & \begin{tabular}{|l|l|}
0.11610 \\
$(0.11754)$
\end{tabular} & $\begin{array}{l}0.29601^{*} \\
(0.08361)\end{array}$ & \begin{tabular}{|l|}
$0.29461^{*}$ \\
$(0.08384)$
\end{tabular} & $\begin{array}{l}0.16355 \\
(0.10946)\end{array}$ & \begin{tabular}{|l|}
$0.24887^{\dagger}$ \\
$(0.11992)$ \\
\end{tabular} & $\begin{array}{l}0.30100^{*} \\
(0.08317)\end{array}$ & $\begin{array}{l}0.30182^{2} \\
(0.08668)\end{array}$ & $\begin{array}{l}0.28814^{\prime \prime} \\
(0.08328)\end{array}$ \\
\hline All Factors & $\begin{array}{l}0.39063^{*} \\
(0.10273) \\
\end{array}$ & & $\begin{array}{l}0.25710^{\dagger} \\
(0.10984) \\
\end{array}$ & $\begin{array}{l}0.38240^{*} \\
(0.06691)\end{array}$ & & $\begin{array}{l}0.34600^{*} \\
(0.05613) \\
\end{array}$ & $\begin{array}{l}0.32993^{*} \\
(0.08264)\end{array}$ & & \begin{tabular}{|l|}
$0.38148^{*}$ \\
$(0.08884)$
\end{tabular} & & & & & & & & & \\
\hline \begin{tabular}{|l} 
Individual \\
Variable
\end{tabular} & \begin{tabular}{|l|l}
-0.06102 \\
$(0.09572)$
\end{tabular} & $\begin{array}{l}.03763 \\
.08558)\end{array}$ & \begin{tabular}{|l}
0.07647 \\
$(0.10078)$
\end{tabular} & $\begin{array}{l}07055 \\
06724)\end{array}$ & \begin{tabular}{|l}
-0.01607 \\
$(0.07249)$
\end{tabular} & \begin{tabular}{|l}
-0.03253 \\
$(0.07321)$
\end{tabular} & $\begin{array}{l}0.00297 \\
(0.08136)\end{array}$ & \begin{tabular}{|l|l}
-0.00767 \\
$(0.09078)$
\end{tabular} & \begin{tabular}{|l|}
-0.05238 \\
$(0.08045)$
\end{tabular} & \begin{tabular}{|l|}
$-0.52810^{*}$ \\
$(0.11902)$
\end{tabular} & $\begin{array}{l}0.19068^{\dagger} \\
(0.08847)\end{array}$ & \begin{tabular}{|l|}
0.01189 \\
$(0.07391)$
\end{tabular} & \begin{tabular}{|l}
0.01538 \\
$(0.06237)$
\end{tabular} & $\begin{array}{l}0.14880^{\ddagger} \\
(0.08019)\end{array}$ & $\begin{array}{l}0.05036 \\
(0.08916)\end{array}$ & \begin{tabular}{|l|}
-0.03498 \\
$(0.09493)$
\end{tabular} & $\begin{array}{l}-0.01151 \\
(0.07572)\end{array}$ & \begin{tabular}{|l|}
0.06560 \\
$(0.07094)$
\end{tabular} \\
\hline
\end{tabular}

* Significant at the 0.01 level $†$ Significant at the 0.05 level $\$$ Significant at the 0.10 level

Table 93 Coefficients with individual variables for source of implementation support - Electronics Industry 


\begin{tabular}{|c|c|c|c|c|c|c|c|c|c|c|c|c|c|}
\hline $\begin{array}{l}\text { Individual variable } \\
\text { included in the model in } \\
\text { the column }\end{array}$ & 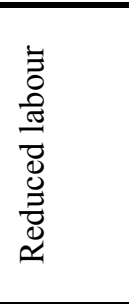 & 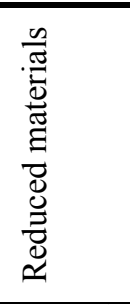 & 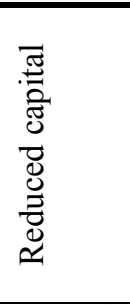 & 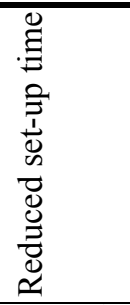 & 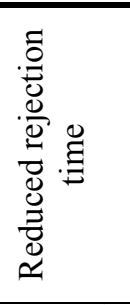 & 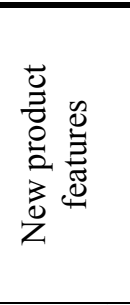 & 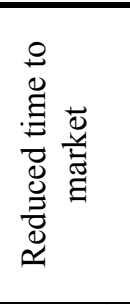 & 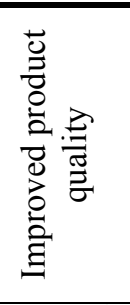 & 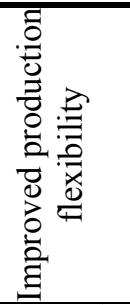 & 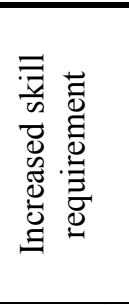 & 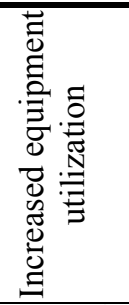 & 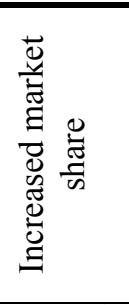 & 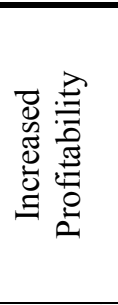 \\
\hline No factors & $\begin{array}{l}0.20411^{*} \\
(0.05126)\end{array}$ & $\begin{array}{l}0.20375^{*} \\
(0.05124)\end{array}$ & $\begin{array}{l}0.20398^{*} \\
(0.05126)\end{array}$ & $\begin{array}{l}0.20496 \\
(0.05096)\end{array}$ & $\begin{array}{l}0.20362^{*} \\
(0.0515)\end{array}$ & $\begin{array}{l}0.20016^{*} \\
(0.05087)\end{array}$ & $\begin{array}{l}0.20176^{*} \\
(0.05103)\end{array}$ & $\begin{array}{l}0.19861^{*} \\
(0.05167)\end{array}$ & $\begin{array}{l}0.20363^{*} \\
(0.05126)\end{array}$ & $\begin{array}{l}0.20251^{*} \\
(0.05163)\end{array}$ & $\begin{array}{l}0.20846^{*} \\
(0.05126)\end{array}$ & $\begin{array}{l}0.20730^{*} \\
(0.05148)\end{array}$ & $\begin{array}{l}0.20342^{*} \\
(0.05119)\end{array}$ \\
\hline Factor 4 & $\begin{array}{l}0.33568^{\ddagger} \\
(0.19270)\end{array}$ & $\begin{array}{l}0.31086 \\
(0.19335)\end{array}$ & $\begin{array}{l}0.32618^{\ddagger} \\
(0.19114)\end{array}$ & $\begin{array}{l}0.33932^{\ddagger} \\
(0.19017)\end{array}$ & $\begin{array}{l}0.32104 \\
(0.20783)\end{array}$ & $\begin{array}{l}0.24632 \\
(0.19419)\end{array}$ & $\begin{array}{l}0.32618^{7} \\
(0.19020)\end{array}$ & $\begin{array}{l}0.27064 \\
(0.20413)\end{array}$ & $\begin{array}{l}0.31310 \\
(0.19420)\end{array}$ & $\begin{array}{l}0.32185^{\ddagger} \\
(0.19198)\end{array}$ & $\begin{array}{l}0.38608^{7} \\
(0.19761)\end{array}$ & $\begin{array}{l}0.37936^{*} \\
(0.20816)\end{array}$ & $\begin{array}{l}0.23600 \\
(0.21823)\end{array}$ \\
\hline Factor 3 & $\begin{array}{l}0.23459^{\dagger} \\
(0.09395)\end{array}$ & $\begin{array}{l}0.21839^{\dagger} \\
(0.08458)\end{array}$ & $\begin{array}{l}0.21886^{\dagger} \\
(0.08461)\end{array}$ & $\begin{array}{l}0.22459^{*} \\
(0.08418) \\
\end{array}$ & $\begin{array}{l}0.21351^{\ddagger} \\
(0.12032)\end{array}$ & $\begin{array}{l}0.16699^{\ddagger} \\
(0.08828)\end{array}$ & $\begin{array}{l}0.21705^{\dagger} \\
(0.08420)\end{array}$ & $\begin{array}{l}0.13842 \\
(0.13454) \\
\end{array}$ & $\begin{array}{l}0.18801 \\
(0.11736)\end{array}$ & $\begin{array}{l}0.21029^{\dagger} \\
(0.09173)\end{array}$ & $\begin{array}{l}0.21980^{*} \\
(0.08436) \\
\end{array}$ & $\begin{array}{l}0.24828^{\dagger} \\
(0.09615)\end{array}$ & $\begin{array}{l}0.21886^{\dagger} \\
(0.08448)\end{array}$ \\
\hline Factor $3 \& 4$ & $\begin{array}{l}0.03996 \\
(0.24304)\end{array}$ & $\begin{array}{l}0.02739 \\
(0.24077)\end{array}$ & $\begin{array}{l}0.02739 \\
(0.24088)\end{array}$ & $\begin{array}{l}0.07386 \\
(0.24121)\end{array}$ & $\begin{array}{l}0.02739 \\
(0.2405)\end{array}$ & $\begin{array}{l}-0.03420 \\
(0.24106)\end{array}$ & $\begin{array}{l}-0.10353 \\
(0.25624)\end{array}$ & $\begin{array}{l}-0.01116 \\
(0.24576)\end{array}$ & $\begin{array}{l}-0.00519 \\
(0.25571)\end{array}$ & $\begin{array}{l}0.00627 \\
(0.25621)\end{array}$ & $\begin{array}{l}0.10271 \\
(0.24900)\end{array}$ & $\begin{array}{l}0.08058 \\
(0.25453)\end{array}$ & $\begin{array}{l}-0.02982 \\
(0.24970)\end{array}$ \\
\hline Factor 2 & $\begin{array}{l}0.14497 \\
(0.36616)\end{array}$ & $\begin{array}{l}0.09298 \\
(0.36587)\end{array}$ & $\begin{array}{l}0.12786 \\
(0.36197)\end{array}$ & $\begin{array}{l}0.14690 \\
(0.35969)\end{array}$ & $\begin{array}{l}0.12248 \\
(0.36096)\end{array}$ & $\begin{array}{l}0.12248 \\
(0.35846)\end{array}$ & $\begin{array}{l}0.12248 \\
(0.35975)\end{array}$ & $\begin{array}{l}0.05871 \\
(0.37049)\end{array}$ & $\begin{array}{l}0.11509 \\
(0.36200)\end{array}$ & $\begin{array}{l}0.12248 \\
(0.36155)\end{array}$ & $\begin{array}{l}0.13957 \\
(0.36074)\end{array}$ & $\begin{array}{l}0.12248 \\
(0.36123)\end{array}$ & $\begin{array}{l}0.09470 \\
(0.36243)\end{array}$ \\
\hline Factor $2 \& 4$ & \multicolumn{13}{|c|}{ Insufficient Data } \\
\hline Factor $2 \& 3$ & $\begin{array}{l}0.28668 \\
(0.29449)\end{array}$ & $\begin{array}{l}0.28668 \\
(0.29441)\end{array}$ & $\begin{array}{l}0.28668 \\
(0.29453)\end{array}$ & $\begin{array}{l}0.31910 \\
(0.29348)\end{array}$ & $\begin{array}{l}0.04881 \\
(0.30684)\end{array}$ & $\begin{array}{l}0.17474 \\
(0.29801)\end{array}$ & $\begin{array}{l}0.14110 \\
(0.30991)\end{array}$ & $\begin{array}{l}0.28668 \\
(0.29417)\end{array}$ & $\begin{array}{l}0.27687 \\
(0.29563)\end{array}$ & $\begin{array}{l}0.28668 \\
(0.29456)\end{array}$ & $\begin{array}{l}0.28668 \\
(0.29365)\end{array}$ & $\begin{array}{l}0.28668 \\
(0.29430)\end{array}$ & $\begin{array}{l}0.28668 \\
(0.29407)\end{array}$ \\
\hline Factor $2,3 \& 4$ & $\begin{array}{l}0.34098 \\
(0.58955) \\
\end{array}$ & $\begin{array}{l}0.31190 \\
(0.58452)\end{array}$ & $\begin{array}{l}0.31190 \\
(0.58477) \\
\end{array}$ & $\begin{array}{l}0.31190 \\
(0.58127) \\
\end{array}$ & $\begin{array}{l}0.15261 \\
(0.09155) \\
\end{array}$ & $\begin{array}{l}0.18010 \\
(0.58401)\end{array}$ & $\begin{array}{l}0.31190 \\
(0.58190)\end{array}$ & $\begin{array}{l}0.22940 \\
(0.59384)\end{array}$ & $\begin{array}{l}0.31190 \\
(0.58469) \\
\end{array}$ & $\begin{array}{l}0.29078 \\
(0.59129) \\
\end{array}$ & $\begin{array}{l}0.31190 \\
(0.58301)\end{array}$ & $\begin{array}{l}0.31190 \\
(0.58431)\end{array}$ & $\begin{array}{l}0.31190 \\
(0.58385)\end{array}$ \\
\hline Factor 1 & $\begin{array}{l}0.07968 \\
(0.31514)\end{array}$ & $\begin{array}{l}0.01243 \\
(0.31469)\end{array}$ & $\begin{array}{l}0.05801 \\
(0.30698)\end{array}$ & $\begin{array}{l}0.06808 \\
(0.30435)\end{array}$ & $\begin{array}{l}0.28202 \\
(0.3034) \\
\end{array}$ & $\begin{array}{l}0.00775 \\
(0.30424)\end{array}$ & $\begin{array}{l}0.02693 \\
(0.30492)\end{array}$ & $\begin{array}{l}0.00733 \\
(0.31075)\end{array}$ & $\begin{array}{l}0.01801 \\
(0.31778)\end{array}$ & $\begin{array}{l}0.02947 \\
(0.31820)\end{array}$ & $\begin{array}{l}0.11367 \\
(0.31000)\end{array}$ & $\begin{array}{l}0.08453 \\
(0.31027)\end{array}$ & $\begin{array}{l}-0.07182 \\
(0.33761) \\
\end{array}$ \\
\hline Factor $1 \& 4$ & $\begin{array}{l}0.40003 \\
(0.65370)\end{array}$ & $\begin{array}{l}0.40003 \\
(0.65351)\end{array}$ & $\begin{array}{l}0.42373 \\
(0.65855)\end{array}$ & $\begin{array}{l}0.50762 \\
(0.65340)\end{array}$ & $\begin{array}{l}0.39454 \\
(0.65867)\end{array}$ & $\begin{array}{l}0.26824 \\
(0.65201)\end{array}$ & $\begin{array}{l}0.25444 \\
(0.65834)\end{array}$ & $\begin{array}{l}0.31754 \\
(0.66175)\end{array}$ & $\begin{array}{l}0.36745 \\
(0.65933)\end{array}$ & $\begin{array}{l}0.40003 \\
(0.65385)\end{array}$ & $\begin{array}{l}0.40003 \\
(0.65183)\end{array}$ & $\begin{array}{l}0.45322 \\
(0.65850)\end{array}$ & $\begin{array}{l}0.27762 \\
(0.66840)\end{array}$ \\
\hline Factor $1 \& 3$ & $\begin{array}{c}-0.78903^{*} \\
(0.15546) \\
\end{array}$ & $\begin{array}{l}-0.79185^{*} \\
(0.15541)\end{array}$ & $\begin{array}{l}-0.78792^{*} \\
(0.15564) \\
\end{array}$ & $\begin{array}{l}-0.72132^{*} \\
(0.16048) \\
\end{array}$ & $\begin{array}{l}-0.79483^{*} \\
(0.17168) \\
\end{array}$ & $\begin{array}{l}-0.81940^{*} \\
(0.15490)\end{array}$ & $\begin{array}{l}-0.85079^{*} \\
(0.16025)\end{array}$ & $\begin{array}{l}-0.86928^{*} \\
(0.18624)\end{array}$ & $\begin{array}{l}-0.82283^{*} \\
(0.17759) \\
\end{array}$ & $\begin{array}{l}-0.81137^{*} \\
(0.17827) \\
\end{array}$ & $\begin{array}{c}-0.78709^{*} \\
(0.15500)\end{array}$ & $\begin{array}{l}-0.78802^{*} \\
(0.15536) \\
\end{array}$ & $\begin{array}{l}-0.80014^{*} \\
(0.15564)\end{array}$ \\
\hline Factor $1,3 \& 4$ & $\begin{array}{l}0.22300^{\dagger} \\
(0.09710)\end{array}$ & $\begin{array}{l}0.19557^{\ddagger} \\
(0.10630)\end{array}$ & $\begin{array}{l}0.22143^{\dagger} \\
(0.09690)\end{array}$ & $\begin{array}{l}0.25346^{\dagger} \\
(0.09835)\end{array}$ & $\begin{array}{l}0.21445^{\ddagger} \\
(0.11733)\end{array}$ & $\begin{array}{l}0.11599 \\
(0.11003)\end{array}$ & $\begin{array}{l}0.14291 \\
(0.10935)\end{array}$ & $\begin{array}{l}0.14147 \\
(0.13916)\end{array}$ & $\begin{array}{l}0.18938 \\
(0.12349)\end{array}$ & $\begin{array}{l}0.19786 \\
(0.12912)\end{array}$ & $\begin{array}{l}0.27137^{\dagger} \\
(0.10662)\end{array}$ & $\begin{array}{l}0.26727^{\dagger} \\
(0.12256)\end{array}$ & $\begin{array}{l}0.11919 \\
(0.15134)\end{array}$ \\
\hline Factor $1 \& 2$ & $\begin{array}{l}0.30357 \\
(0.20390) \\
\end{array}$ & $\begin{array}{l}0.28247 \\
(0.20618)\end{array}$ & $\begin{array}{l}0.31927 \\
(0.21417) \\
\end{array}$ & $\begin{array}{l}0.33986^{\ddagger} \\
(0.20402)\end{array}$ & $\begin{array}{l}0.29695 \\
(0.20701) \\
\end{array}$ & $\begin{array}{l}0.23151 \\
(0.20509)\end{array}$ & $\begin{array}{l}0.18446 \\
(0.21768)\end{array}$ & $\begin{array}{l}0.23470 \\
(0.22012)\end{array}$ & $\begin{array}{l}0.29469 \\
(0.20399) \\
\end{array}$ & $\begin{array}{l}0.29786 \\
(0.20375) \\
\end{array}$ & $\begin{array}{l}0.31145 \\
(0.20330) \\
\end{array}$ & $\begin{array}{l}0.31910 \\
(0.20578)\end{array}$ & $\begin{array}{l}0.29938 \\
(0.20332)\end{array}$ \\
\hline Factor $1,2 \& 4$ & $\begin{array}{l}0.45635^{*} \\
(0.15869)\end{array}$ & $\begin{array}{l}0.42722^{*} \\
(0.15742)\end{array}$ & $\begin{array}{l}0.46496^{*} \\
(0.17168)\end{array}$ & $\begin{array}{l}0.52696^{*} \\
(0.16261)\end{array}$ & $\begin{array}{l}0.43872^{*} \\
(0.16633)\end{array}$ & $\begin{array}{l}0.31627^{\ddagger} \\
(0.16731)\end{array}$ & $\begin{array}{l}0.30305^{\ddagger} \\
(0.18166)\end{array}$ & $\begin{array}{l}0.38132^{\dagger} \\
(0.17381)\end{array}$ & $\begin{array}{l}0.44124^{*} \\
(0.15465)\end{array}$ & $\begin{array}{l}0.42859^{\dagger} \\
(0.16510)\end{array}$ & $\begin{array}{l}0.44884^{*} \\
(0.15426)\end{array}$ & $\begin{array}{l}0.49059^{*} \\
(0.17163)\end{array}$ & $\begin{array}{l}0.32017 \\
(0.21092)\end{array}$ \\
\hline Factor $1,2 \& 3$ & $\begin{array}{l}0.32711^{*} \\
(0.11588)\end{array}$ & $\begin{array}{l}0.28296^{\dagger} \\
(0.11881)\end{array}$ & $\begin{array}{l}0.32553^{*} \\
(0.11899)\end{array}$ & $\begin{array}{l}0.36605^{*} \\
(0.11191)\end{array}$ & $\begin{array}{l}0.30468^{\dagger} \\
(0.13455)\end{array}$ & $\begin{array}{l}0.20000^{\prime} \\
(0.12085)\end{array}$ & $\begin{array}{l}0.16422 \\
(0.14644)\end{array}$ & $\begin{array}{l}0.23046 \\
(0.14848)\end{array}$ & $\begin{array}{l}0.29137^{\dagger} \\
(0.11734)\end{array}$ & $\begin{array}{l}0.28910^{\dagger} \\
(0.13684)\end{array}$ & $\begin{array}{l}0.33024^{*} \\
(0.10798)\end{array}$ & $\begin{array}{l}0.31086^{*} \\
(0.10675)\end{array}$ & $\begin{array}{l}0.30496^{*} \\
(0.10680)\end{array}$ \\
\hline All Factors & $\begin{array}{l}0.29417^{*} \\
(0.07611)\end{array}$ & $\begin{array}{l}0.23592^{*} \\
(0.07153)\end{array}$ & $\begin{array}{l}0.28777^{*} \\
(0.07419) \\
\end{array}$ & $\begin{array}{l}0.35559^{*} \\
(0.06499)\end{array}$ & $\begin{array}{l}0.26301^{*} \\
(0.089)\end{array}$ & $\begin{array}{l}0.15434^{\dagger} \\
(0.06950)\end{array}$ & $\begin{array}{l}0.13089 \\
(0.10108)\end{array}$ & $\begin{array}{l}0.18792^{\ddagger} \\
(0.11003)\end{array}$ & $\begin{array}{l}0.23899^{*} \\
(0.08435)\end{array}$ & $\begin{array}{l}0.24850^{*} \\
(0.08824)\end{array}$ & $\begin{array}{l}0.32517^{*} \\
(0.06073)\end{array}$ & $\begin{array}{l}0.31670^{*} \\
(0.08324)\end{array}$ & $\begin{array}{l}0.14764 \\
(0.14564) \\
\end{array}$ \\
\hline Individual Variable & $\begin{array}{l}-0.02909 \\
(0.07557)\end{array}$ & $\begin{array}{l}0.03816 \\
(0.07405)\end{array}$ & $\begin{array}{l}-0.02369 \\
(0.07899)\end{array}$ & $\begin{array}{l}-0.10759 \\
(0.06765)\end{array}$ & $\begin{array}{l}0.00549 \\
(0.08786)\end{array}$ & $\begin{array}{l}0.13179^{\ddagger} \\
(0.06986)\end{array}$ & $\begin{array}{l}0.14559 \\
(0.10073)\end{array}$ & $\begin{array}{l}0.08249 \\
(0.10736)\end{array}$ & $\begin{array}{l}0.03258 \\
(0.08591)\end{array}$ & $\begin{array}{l}0.02112 \\
(0.08725)\end{array}$ & $\begin{array}{l}-0.07532 \\
(0.06578)\end{array}$ & $\begin{array}{l}-0.05319 \\
(0.08280)\end{array}$ & $\begin{array}{l}0.12242 \\
(0.14370)\end{array}$ \\
\hline
\end{tabular}

* Significant at the 0.01 level

$\uparrow$ Significant at the 0.05 level

t Significant at the 0.10 level

Table 94 Coefficients with individual variables for perceived benefits of AMT - Electronics Industry 


\begin{tabular}{|c|c|c|c|c|c|c|c|c|c|c|c|c|c|}
\hline $\begin{array}{l}\text { Individual variable } \\
\text { included in the } \\
\text { model in the } \\
\text { column }\end{array}$ & 忢 & 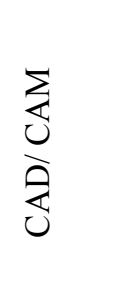 & 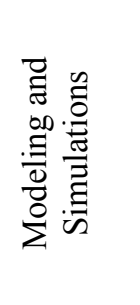 & 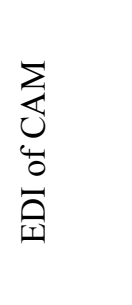 & 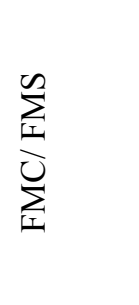 & 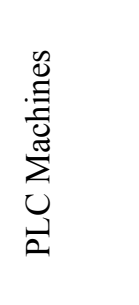 & $\begin{array}{l}\tilde{\omega} \\
\tilde{\Xi} \\
\tilde{\Xi}\end{array}$ & 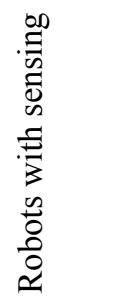 & 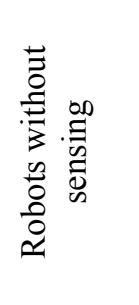 & 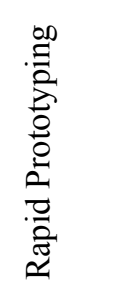 & 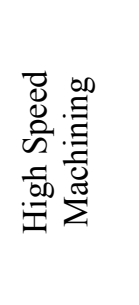 & 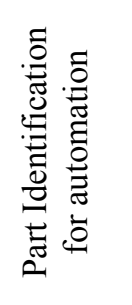 & 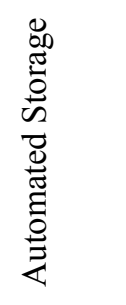 \\
\hline No factors & $\begin{array}{l}0.16490^{*} \\
(0.06249)\end{array}$ & $\begin{array}{l}0.18373^{*} \\
(0.06178)\end{array}$ & $\begin{array}{l}0.18353^{*} \\
(0.06154)\end{array}$ & $\begin{array}{l}0.18306^{*} \\
(0.06135)\end{array}$ & $\begin{array}{l}0.18347^{*} \\
(0.06155)\end{array}$ & $\begin{array}{l}0.18919^{*} \\
(0.06178)\end{array}$ & $\begin{array}{l}0.18353^{*} \\
(0.06155)\end{array}$ & $\begin{array}{l}0.18353^{*} \\
(0.06150)\end{array}$ & $\begin{array}{l}0.18353^{*} \\
(0.06155)\end{array}$ & $\begin{array}{l}0.18353^{*} \\
(0.06120)\end{array}$ & $\begin{array}{l}0.18353^{*} \\
(0.06144)\end{array}$ & $\begin{array}{l}0.18353^{*} \\
(0.06129)\end{array}$ & $\begin{array}{l}0.18353^{*} \\
(0.06152)\end{array}$ \\
\hline Factor 5 & $\begin{array}{l}0.34886 \\
(0.22594)\end{array}$ & $\begin{array}{l}0.34886 \\
(0.22723)\end{array}$ & $\begin{array}{l}0.34886 \\
(0.22720)\end{array}$ & $\begin{array}{l}0.34886 \\
(0.22648)\end{array}$ & $\begin{array}{l}0.34886 \\
(0.22722)\end{array}$ & $\begin{array}{l}0.34886 \\
(0.22678)\end{array}$ & $\begin{array}{l}0.34886 \\
(0.22722)\end{array}$ & $\begin{array}{l}0.34886 \\
(0.22706) \\
\end{array}$ & $\begin{array}{l}0.34886 \\
(0.22722)\end{array}$ & $\begin{array}{l}0.34886 \\
(0.22595)\end{array}$ & $\begin{array}{l}0.34886 \\
(0.22683)\end{array}$ & $\begin{array}{l}0.34886 \\
(0.22627) \\
\end{array}$ & $\begin{array}{l}0.34886 \\
(0.22711) \\
\end{array}$ \\
\hline Factor 4 & $\begin{array}{l}0.01107 \\
(0.34580)\end{array}$ & $\begin{array}{l}0.13456 \\
(0.34111)\end{array}$ & $\begin{array}{l}0.12302 \\
(0.34088)\end{array}$ & $\begin{array}{l}0.04910 \\
(0.34481)\end{array}$ & $\begin{array}{l}0.13136 \\
(0.33791)\end{array}$ & $\begin{array}{l}0.17370 \\
(0.34039)\end{array}$ & $\begin{array}{l}0.13458 \\
(0.33798)\end{array}$ & $\begin{array}{l}0.13279 \\
(0.33746)\end{array}$ & $\begin{array}{l}0.13279 \\
(0.33771)\end{array}$ & $\begin{array}{l}0.13279 \\
(0.33581)\end{array}$ & $\begin{array}{l}0.14348 \\
(0.33738)\end{array}$ & $\begin{array}{l}0.13279 \\
(0.33629)\end{array}$ & $\begin{array}{l}0.14319 \\
(0.33837)\end{array}$ \\
\hline Factor $4 \& 5$ & $\begin{array}{l}0.30501 \\
(0.58025)\end{array}$ & $\begin{array}{l}0.42937 \\
(0.58203) \\
\end{array}$ & $\begin{array}{l}0.41216 \\
(0.58172) \\
\end{array}$ & $\begin{array}{l}0.34304 \\
(0.58056)\end{array}$ & $\begin{array}{l}0.41837 \\
(0.58174) \\
\end{array}$ & $\begin{array}{l}0.45721 \\
(0.57758) \\
\end{array}$ & $\begin{array}{l}0.42672 \\
(0.57759) \\
\end{array}$ & $\begin{array}{l}0.42672 \\
(0.57718)\end{array}$ & $\begin{array}{l}0.42672 \\
(0.57761)\end{array}$ & $\begin{array}{l}0.42672 \\
(0.57437)\end{array}$ & $\begin{array}{l}0.42672 \\
(0.57661)\end{array}$ & $\begin{array}{l}0.47404 \\
(0.57639)\end{array}$ & $\begin{array}{l}0.42672 \\
(0.57732) \\
\end{array}$ \\
\hline Factor 3 & $\begin{array}{l}-0.26670^{\dagger} \\
(0.12549)\end{array}$ & $\begin{array}{l}-0.17286 \\
(0.10875)\end{array}$ & $\begin{array}{l}-0.17286 \\
(0.10873)\end{array}$ & $\begin{array}{l}-0.23738^{\ddagger} \\
(0.12277)\end{array}$ & $\begin{array}{l}-0.17286 \\
(0.10874)\end{array}$ & $\begin{array}{l}-0.17286 \\
(0.10854)\end{array}$ & $\begin{array}{l}-0.17286 \\
(0.10874)\end{array}$ & $\begin{array}{l}-0.17286 \\
(0.10867)\end{array}$ & $\begin{array}{l}-0.17286 \\
(0.10875)\end{array}$ & $\begin{array}{l}-0.17286 \\
(0.10814)\end{array}$ & $\begin{array}{l}-0.17286 \\
(0.10856)\end{array}$ & $\begin{array}{l}-0.17286 \\
(0.10829)\end{array}$ & $\begin{array}{l}-0.17286 \\
(0.10869)\end{array}$ \\
\hline Factor $3 \& 5$ & $\begin{array}{l}-0.00931 \\
(0.35187)\end{array}$ & $\begin{array}{l}0.11288 \\
(0.34422)\end{array}$ & $\begin{array}{l}0.10982 \\
(0.34417)\end{array}$ & $\begin{array}{l}0.09757 \\
(0.34311)\end{array}$ & $\begin{array}{l}0.11241 \\
(0.34397)\end{array}$ & $\begin{array}{l}0.11241 \\
(0.34332)\end{array}$ & $\begin{array}{l}0.11241 \\
(0.34397)\end{array}$ & $\begin{array}{l}0.11241 \\
(0.34373)\end{array}$ & $\begin{array}{l}0.11241 \\
(0.34398)\end{array}$ & $\begin{array}{l}0.11241 \\
(0.34205)\end{array}$ & $\begin{array}{l}0.11241 \\
(0.34339)\end{array}$ & $\begin{array}{l}0.11241 \\
(0.34254)\end{array}$ & $\begin{array}{l}0.11241 \\
(0.34381)\end{array}$ \\
\hline Factor $3 \& 4$ & $\begin{array}{l}0.09021 \\
(0.25182) \\
\end{array}$ & $\begin{array}{l}0.21223 \\
(0.23938) \\
\end{array}$ & $\begin{array}{l}0.19905 \\
(0.24699) \\
\end{array}$ & $\begin{array}{l}0.14422 \\
(0.24601) \\
\end{array}$ & $\begin{array}{l}0.20710 \\
(0.24256) \\
\end{array}$ & $\begin{array}{l}0.27290 \\
(0.24908) \\
\end{array}$ & $\begin{array}{l}0.21192 \\
(0.23923) \\
\end{array}$ & $\begin{array}{l}0.21687 \\
(0.23924) \\
\end{array}$ & $\begin{array}{l}0.21043 \\
(0.24017) \\
\end{array}$ & $\begin{array}{l}0.30722 \\
(0.24660) \\
\end{array}$ & $\begin{array}{l}0.21915 \\
(0.23899) \\
\end{array}$ & $\begin{array}{l}0.21192 \\
(0.23823) \\
\end{array}$ & $\begin{array}{l}0.21192 \\
(0.23912) \\
\end{array}$ \\
\hline Factor $3,4 \& 5$ & $\begin{array}{l}0.07725 \\
(0.16833)\end{array}$ & $\begin{array}{l}0.20161 \\
(0.16396)\end{array}$ & $\begin{array}{l}0.19897 \\
(0.14750)\end{array}$ & $\begin{array}{l}0.11528 \\
(0.16496)\end{array}$ & $\begin{array}{l}0.19897 \\
(0.14751)\end{array}$ & $\begin{array}{l}0.19897 \\
(0.14723)\end{array}$ & $\begin{array}{l}0.19897 \\
(0.14751)\end{array}$ & $\begin{array}{l}0.19897 \\
(0.14740)\end{array}$ & $\begin{array}{l}0.19897 \\
(0.14751)\end{array}$ & $\begin{array}{l}0.19897 \\
(0.14668)\end{array}$ & $\begin{array}{l}0.19897 \\
(0.14726)\end{array}$ & $\begin{array}{l}0.19897 \\
(0.14689)\end{array}$ & $\begin{array}{l}0.19897 \\
(0.14744)\end{array}$ \\
\hline Factor 2 & $\begin{array}{l}0.34304^{\ddagger} \\
(0.19514)\end{array}$ & $\begin{array}{l}0.34304^{\ddagger} \\
(0.19625)\end{array}$ & $\begin{array}{l}0.34304^{\ddagger} \\
(0.19623)\end{array}$ & $\begin{array}{l}0.34304^{\ddagger} \\
(0.19560) \\
\end{array}$ & $\begin{array}{l}0.34304^{\ddagger} \\
(0.19624)\end{array}$ & $\begin{array}{l}0.38893^{\ddagger} \\
(0.20300)\end{array}$ & $\begin{array}{l}0.34304^{\ddagger} \\
(0.19624)\end{array}$ & $\begin{array}{l}0.34304^{\ddagger} \\
(0.19610)\end{array}$ & $\begin{array}{l}0.34304^{\ddagger} \\
(0.19625)\end{array}$ & $\begin{array}{l}0.34304^{\ddagger} \\
(0.19515)\end{array}$ & $\begin{array}{l}0.34304^{\ddagger} \\
(0.19591)\end{array}$ & $\begin{array}{l}0.34304^{\ddagger} \\
(0.19542) \\
\end{array}$ & $\begin{array}{l}0.34304^{\ddagger} \\
(0.19615) \\
\end{array}$ \\
\hline Factor $2 \& 5$ & \multicolumn{13}{|c|}{ Insufficient Data } \\
\hline Factor $2 \& 4$ & $\begin{array}{l}0.08813 \\
(0.22448) \\
\end{array}$ & $\begin{array}{l}0.20411 \\
(0.22231) \\
\end{array}$ & $\begin{array}{l}0.19526 \\
(0.21421) \\
\end{array}$ & $\begin{array}{l}0.16619 \\
(0.21372) \\
\end{array}$ & $\begin{array}{l}0.19811 \\
(0.21407) \\
\end{array}$ & $\begin{array}{l}0.26262 \\
(0.22320) \\
\end{array}$ & $\begin{array}{l}0.20538 \\
(0.21399) \\
\end{array}$ & $\begin{array}{l}0.21095 \\
(0.21261) \\
\end{array}$ & $\begin{array}{l}0.20078 \\
(0.21240) \\
\end{array}$ & $\begin{array}{l}0.20165 \\
(0.21086) \\
\end{array}$ & $\begin{array}{l}0.20165 \\
(0.21168) \\
\end{array}$ & $\begin{array}{l}0.22860 \\
(0.21222) \\
\end{array}$ & $\begin{array}{l}0.21078 \\
(0.21296) \\
\end{array}$ \\
\hline Factor $2,4 \& 5$ & $\begin{array}{l}-0.12870 \\
(0.36277)\end{array}$ & $\begin{array}{l}-0.00435 \\
(0.36239)\end{array}$ & $\begin{array}{l}-0.02155 \\
(0.36195)\end{array}$ & $\begin{array}{l}-0.09067 \\
(0.36190)\end{array}$ & $\begin{array}{l}-0.00857 \\
(0.35548)\end{array}$ & $\begin{array}{l}0.05399 \\
(0.36158)\end{array}$ & $\begin{array}{l}-0.00699 \\
(0.35524)\end{array}$ & $\begin{array}{l}-0.00699 \\
(0.35498)\end{array}$ & $\begin{array}{l}-0.00699 \\
(0.35525)\end{array}$ & $\begin{array}{l}0.01900 \\
(0.35370)\end{array}$ & $\begin{array}{l}-0.00699 \\
(0.35464)\end{array}$ & $\begin{array}{l}0.08765 \\
(0.36154)\end{array}$ & $\begin{array}{l}0.04236 \\
(0.37239)\end{array}$ \\
\hline Factor $2 \& 3$ & \multicolumn{13}{|c|}{ Insufficient Data } \\
\hline Factor $2,3 \& 5$ & \multicolumn{13}{|c|}{ Insufficient Data } \\
\hline Factor $2,3 \& 4$ & $\begin{array}{l}0.11631 \\
(0.34375) \\
\end{array}$ & $\begin{array}{l}0.23977 \\
(0.33891) \\
\end{array}$ & $\begin{array}{l}0.23065 \\
(0.33738) \\
\end{array}$ & $\begin{array}{l}0.16846 \\
(0.34020) \\
\end{array}$ & $\begin{array}{l}0.23249 \\
(0.33869) \\
\end{array}$ & $\begin{array}{l}0.28870 \\
(0.34007) \\
\end{array}$ & $\begin{array}{l}0.23979 \\
(0.33584) \\
\end{array}$ & $\begin{array}{l}0.24525 \\
(0.33560) \\
\end{array}$ & $\begin{array}{l}0.23693 \\
(0.33593) \\
\end{array}$ & $\begin{array}{l}0.30587 \\
(0.33688) \\
\end{array}$ & $\begin{array}{l}0.23802 \\
(0.33500) \\
\end{array}$ & $\begin{array}{l}0.30072 \\
(0.33780) \\
\end{array}$ & $\begin{array}{l}0.25780 \\
(0.33841) \\
\end{array}$ \\
\hline Factor $2,3,4 \& 5$ & $\begin{array}{l}0.12377 \\
(0.20764)\end{array}$ & $\begin{array}{l}0.24773 \\
(0.20107)\end{array}$ & $\begin{array}{l}0.23772 \\
(0.19513)\end{array}$ & $\begin{array}{l}0.16640 \\
(0.20362)\end{array}$ & $\begin{array}{l}0.24456 \\
(0.19174)\end{array}$ & $\begin{array}{l}0.29745 \\
(0.20054)\end{array}$ & $\begin{array}{l}0.24703 \\
(0.19196)\end{array}$ & $\begin{array}{l}0.25351 \\
(0.19204)\end{array}$ & $\begin{array}{l}0.24240 \\
(0.19652)\end{array}$ & $\begin{array}{l}0.25304 \\
(0.19059)\end{array}$ & $\begin{array}{l}0.26065 \\
(0.19217)\end{array}$ & $\begin{array}{l}0.26466 \\
(0.19139)\end{array}$ & $\begin{array}{l}0.25447 \\
(0.19259)\end{array}$ \\
\hline Factor 1 & $\begin{array}{l}0.12640 \\
(0.28448)\end{array}$ & $\begin{array}{l}0.22921 \\
(0.27736)\end{array}$ & $\begin{array}{l}0.22753 \\
(0.27745)\end{array}$ & $\begin{array}{l}0.21956 \\
(0.27659)\end{array}$ & $\begin{array}{l}0.22921 \\
(0.27735)\end{array}$ & $\begin{array}{l}0.23623 \\
(0.27695)\end{array}$ & $\begin{array}{l}0.22921 \\
(0.27735)\end{array}$ & $\begin{array}{l}0.22921 \\
(0.27715)\end{array}$ & $\begin{array}{l}0.22921 \\
(0.27736)\end{array}$ & $\begin{array}{l}0.22921 \\
(0.27580)\end{array}$ & $\begin{array}{l}0.22921 \\
(0.27688)\end{array}$ & $\begin{array}{l}0.24012 \\
(0.27633)\end{array}$ & $\begin{array}{l}0.22921 \\
(0.27722)\end{array}$ \\
\hline Facto & $\begin{array}{l}-0.00969 \\
(0.28201) \\
\end{array}$ & $\begin{array}{l}0.06648 \\
(0.28125)\end{array}$ & $\begin{array}{l}0.06346 \\
(0.27907)\end{array}$ & $\begin{array}{l}0.02685 \\
(0.28016)\end{array}$ & $\begin{array}{l}0.06329 \\
(0.27940)\end{array}$ & $\begin{array}{l}0.11902 \\
(0.28539)\end{array}$ & $\begin{array}{l}0.06681 \\
(0.27926)\end{array}$ & $\begin{array}{l}0.07478 \\
(0.27935)\end{array}$ & $\begin{array}{l}0.06270 \\
(0.28115)\end{array}$ & $\begin{array}{l}0.06516 \\
(0.27741)\end{array}$ & $\begin{array}{l}0.06516 \\
(0.27850)\end{array}$ & $\begin{array}{l}0.09107 \\
(0.27856)\end{array}$ & $\begin{array}{l}0.06516 \\
(0.27884)\end{array}$ \\
\hline Facto & $\begin{array}{l}0.12704 \\
(0.23510)\end{array}$ & $\begin{array}{l}0.24875 \\
(0.22137)\end{array}$ & $\begin{array}{l}0.24875 \\
(0.22135)\end{array}$ & $\begin{array}{l}0.23692 \\
(0.22090)\end{array}$ & $\begin{array}{l}0.24875 \\
(0.22136)\end{array}$ & $\begin{array}{l}0.30111 \\
(0.22917)\end{array}$ & $\begin{array}{l}0.25023 \\
(0.22166)\end{array}$ & $\begin{array}{l}0.24875 \\
(0.22120)\end{array}$ & $\begin{array}{l}0.24875 \\
(0.22137)\end{array}$ & $\begin{array}{l}0.24875 \\
(0.22013)\end{array}$ & $\begin{array}{l}0.24875 \\
(0.22099)\end{array}$ & $\begin{array}{l}0.24875 \\
(0.22044)\end{array}$ & $\begin{array}{l}0.24875 \\
(0.22126)\end{array}$ \\
\hline Factor 1 & $\begin{array}{l}0.18293 \\
(0.34386) \\
\end{array}$ & $\begin{array}{l}0.30729 \\
(0.34324) \\
\end{array}$ & $\begin{array}{l}0.29556 \\
(0.33845) \\
\end{array}$ & $\begin{array}{l}0.25242 \\
(0.33784) \\
\end{array}$ & $\begin{array}{l}0.30275 \\
(0.33606) \\
\end{array}$ & $\begin{array}{l}0.36562 \\
(0.34247) \\
\end{array}$ & $\begin{array}{l}0.30641 \\
(0.33596) \\
\end{array}$ & $\begin{array}{l}0.32162 \\
(0.33695) \\
\end{array}$ & $\begin{array}{l}0.30318 \\
(0.33634) \\
\end{array}$ & $\begin{array}{l}0.32786 \\
(0.33419) \\
\end{array}$ & $\begin{array}{l}0.31521 \\
(0.33537) \\
\end{array}$ & $\begin{array}{l}0.30465 \\
(0.33429) \\
\end{array}$ & $\begin{array}{l}0.30465 \\
(0.33553) \\
\end{array}$ \\
\hline Factor $1 \& 3$ & \multicolumn{13}{|c|}{ Insufficient Data } \\
\hline Factor $1,3 \& 5$ & $\begin{array}{l}0.35016 \\
(0.32077) \\
\end{array}$ & $\begin{array}{l}0.40626 \\
(0.32239) \\
\end{array}$ & $\begin{array}{l}0.40495 \\
(0.32039) \\
\end{array}$ & $\begin{array}{l}0.39207 \\
(0.31958) \\
\end{array}$ & $\begin{array}{l}0.40321 \\
(0.32074) \\
\end{array}$ & $\begin{array}{l}0.42697 \\
(0.32083) \\
\end{array}$ & $\begin{array}{l}0.40495 \\
(0.32041) \\
\end{array}$ & $\begin{array}{l}0.41382 \\
(0.32062) \\
\end{array}$ & $\begin{array}{l}0.40262 \\
(0.32212) \\
\end{array}$ & $\begin{array}{l}0.40495 \\
(0.31862) \\
\end{array}$ & $\begin{array}{l}0.40495 \\
(0.31987) \\
\end{array}$ & $\begin{array}{l}0.40495 \\
(0.31908) \\
\end{array}$ & $\begin{array}{l}0.40495 \\
(0.32026) \\
\end{array}$ \\
\hline Factor $1,3 \& 4$ & $\begin{array}{l}0.06311 \\
(0.14488)\end{array}$ & $\begin{array}{l}0.18110 \\
(0.12878)\end{array}$ & $\begin{array}{l}0.17808 \\
(0.12231)\end{array}$ & $\begin{array}{l}0.13533 \\
(0.12797)\end{array}$ & $\begin{array}{l}0.17815 \\
(0.12268)\end{array}$ & $\begin{array}{l}0.23162^{\ddagger} \\
(0.13606)\end{array}$ & $\begin{array}{l}0.18112 \\
(0.12268)\end{array}$ & $\begin{array}{l}0.17959 \\
(0.12202)\end{array}$ & $\begin{array}{l}0.17959 \\
(0.12211)\end{array}$ & $\begin{array}{l}0.19383 \\
(0.12181)\end{array}$ & $\begin{array}{l}0.21387^{\ddagger} \\
(0.12899)\end{array}$ & $\begin{array}{l}0.19296 \\
(0.12205)\end{array}$ & $\begin{array}{l}0.17959 \\
(0.12205)\end{array}$ \\
\hline Factor $1,3,4 \& 5$ & $\begin{array}{l}0.10592 \\
(0.17716)\end{array}$ & $\begin{array}{l}0.22870 \\
(0.16027)\end{array}$ & $\begin{array}{l}0.22350 \\
(0.15884)\end{array}$ & $\begin{array}{l}0.15906 \\
(0.16863)\end{array}$ & $\begin{array}{l}0.22212 \\
(0.16413)\end{array}$ & $\begin{array}{l}0.26206 \\
(0.16233)\end{array}$ & $\begin{array}{l}0.22838 \\
(0.15772)\end{array}$ & $\begin{array}{l}0.23082 \\
(0.15762)\end{array}$ & $\begin{array}{l}0.22763 \\
(0.15762)\end{array}$ & $\begin{array}{l}0.24476 \\
(0.15717)\end{array}$ & $\begin{array}{l}0.23938 \\
(0.15801)\end{array}$ & $\begin{array}{l}0.24947 \\
(0.15790)\end{array}$ & $\begin{array}{l}0.22763 \\
(0.15754)\end{array}$ \\
\hline Factor $1 \& 2$ & $\begin{array}{l}0.09131 \\
(0.19512)\end{array}$ & $\begin{array}{l}0.09131 \\
(0.19623)\end{array}$ & $\begin{array}{l}0.09131 \\
(0.19621)\end{array}$ & $\begin{array}{l}0.09131 \\
(0.19559)\end{array}$ & $\begin{array}{l}0.09131 \\
(0.19622)\end{array}$ & $\begin{array}{l}0.14551 \\
(0.20574)\end{array}$ & $\begin{array}{l}0.09131 \\
(0.19622)\end{array}$ & $\begin{array}{l}0.09131 \\
(0.19608)\end{array}$ & $\begin{array}{l}0.09131 \\
(0.19623)\end{array}$ & $\begin{array}{l}0.09131 \\
(0.19513)\end{array}$ & $\begin{array}{l}0.09131 \\
(0.19589)\end{array}$ & $\begin{array}{l}0.18595 \\
(0.20916)\end{array}$ & $\begin{array}{l}0.15217 \\
(0.24006)\end{array}$ \\
\hline Factor $1,2 \& 5$ & \multicolumn{13}{|c|}{ Insufficient Data } \\
\hline Factor $1,2 \& 4$ & $\begin{array}{l}-0.13643 \\
(0.16615)\end{array}$ & $\begin{array}{l}-0.02487 \\
(0.16101)\end{array}$ & $\begin{array}{l}-0.02888 \\
(0.14974)\end{array}$ & $\begin{array}{l}-0.06042 \\
(0.15197)\end{array}$ & $\begin{array}{l}-0.03131 \\
(0.15358)\end{array}$ & $\begin{array}{l}0.00727 \\
(0.15447)\end{array}$ & $\begin{array}{l}-0.02625 \\
(0.14964)\end{array}$ & $\begin{array}{l}-0.02273 \\
(0.14962)\end{array}$ & $\begin{array}{l}-0.02785 \\
(0.14991)\end{array}$ & $\begin{array}{l}-0.02087 \\
(0.14873)\end{array}$ & $\begin{array}{l}-0.01174 \\
(0.15044)\end{array}$ & $\begin{array}{l}-0.00177 \\
(0.15021)\end{array}$ & $\begin{array}{l}-0.02708 \\
(0.14943)\end{array}$ \\
\hline Factor $1,2,4 \& 5$ & $\begin{array}{l}0.06850 \\
(0.22048)\end{array}$ & $\begin{array}{l}0.19248 \\
(0.21459)\end{array}$ & $\begin{array}{l}0.18177 \\
(0.20949)\end{array}$ & $\begin{array}{l}0.11183 \\
(0.21656) \\
\end{array}$ & $\begin{array}{l}0.18583 \\
(0.20878)\end{array}$ & $\begin{array}{l}0.24375 \\
(0.21443)\end{array}$ & $\begin{array}{l}0.19154 \\
(0.20584)\end{array}$ & $\begin{array}{l}0.20997 \\
(0.20875) \\
\end{array}$ & $\begin{array}{l}0.18764 \\
(0.20879)\end{array}$ & $\begin{array}{l}0.25365 \\
(0.20896)\end{array}$ & $\begin{array}{l}0.21116 \\
(0.20685)\end{array}$ & $\begin{array}{l}0.23989 \\
(0.20844) \\
\end{array}$ & $\begin{array}{l}0.20563 \\
(0.20846) \\
\end{array}$ \\
\hline Factor $1,2 \& 3$ & $\begin{array}{l}0.32521 \\
(0.47573) \\
\end{array}$ & $\begin{array}{l}0.36681 \\
(0.47759) \\
\end{array}$ & $\begin{array}{l}0.36184 \\
(0.47813) \\
\end{array}$ & $\begin{array}{l}0.36681 \\
(0.47603) \\
\end{array}$ & $\begin{array}{l}0.36681 \\
(0.47758) \\
\end{array}$ & $\begin{array}{l}0.38766 \\
(0.47728) \\
\end{array}$ & $\begin{array}{l}0.36681 \\
(0.47757) \\
\end{array}$ & $\begin{array}{l}0.36681 \\
(0.47724)\end{array}$ & $\begin{array}{l}0.36681 \\
(0.47759) \\
\end{array}$ & $\begin{array}{l}0.36681 \\
(0.47491) \\
\end{array}$ & $\begin{array}{l}0.36681 \\
(0.47677) \\
\end{array}$ & $\begin{array}{l}0.36681 \\
(0.47558) \\
\end{array}$ & $\begin{array}{l}0.36681 \\
(0.47735) \\
\end{array}$ \\
\hline Factor $1,2,3 \& 5$ & $\begin{array}{l}0.24308 \\
(0.22056)\end{array}$ & $\begin{array}{l}0.31165 \\
(0.21682)\end{array}$ & $\begin{array}{l}0.30924 \\
(0.21710)\end{array}$ & $\begin{array}{l}0.29191 \\
(0.21683)\end{array}$ & $\begin{array}{l}0.30992 \\
(0.21728)\end{array}$ & $\begin{array}{l}0.33740 \\
(0.21846)\end{array}$ & $\begin{array}{l}0.31165 \\
(0.21681)\end{array}$ & $\begin{array}{l}0.31165 \\
(0.21666)\end{array}$ & $\begin{array}{l}0.31165 \\
(0.21682)\end{array}$ & $\begin{array}{l}0.33029 \\
(0.21598)\end{array}$ & $\begin{array}{l}0.31758 \\
(0.21657)\end{array}$ & $\begin{array}{l}0.36496^{\ddagger} \\
(0.21996)\end{array}$ & $\begin{array}{l}0.31742 \\
(0.21711)\end{array}$ \\
\hline Factor $1,2,3 \& 4$ & $\begin{array}{l}0.20294^{\dagger} \\
(0.10203)\end{array}$ & $\begin{array}{l}0.31347^{*} \\
(0.08796)\end{array}$ & $\begin{array}{l}0.30594^{*} \\
(0.07584)\end{array}$ & $\begin{array}{l}0.24991^{*} \\
(0.08964)\end{array}$ & $\begin{array}{l}0.30554^{*} \\
(0.08678)\end{array}$ & $\begin{array}{l}0.34389^{*} \\
(0.08018)\end{array}$ & $\begin{array}{l}0.31303^{*} \\
(0.07186)\end{array}$ & $\begin{array}{l}0.31950^{*} \\
(0.07244) \\
\end{array}$ & $\begin{array}{l}0.31052^{*} \\
(0.07244)\end{array}$ & $\begin{array}{l}0.33398^{*} \\
(0.07219) \\
\end{array}$ & $\begin{array}{l}0.31754^{*} \\
(0.07121)\end{array}$ & $\begin{array}{l}0.35222^{*} \\
(0.07758)\end{array}$ & $\begin{array}{l}0.31692^{*} \\
(0.07196)\end{array}$ \\
\hline All Factors & $\begin{array}{l}0.22876^{*} \\
(0.08497)\end{array}$ & $\begin{array}{l}0.33385^{*} \\
(0.07095)\end{array}$ & $\begin{array}{l}0.32480^{*} \\
(0.05919)\end{array}$ & $\begin{array}{l}0.26293^{*} \\
(0.07831)\end{array}$ & $\begin{array}{l}0.32833^{*} \\
(0.05692)\end{array}$ & $\begin{array}{l}0.38116^{*} \\
(0.07491)\end{array}$ & $\begin{array}{l}0.33545^{*} \\
(0.05555)\end{array}$ & $\begin{array}{l}0.34647^{*} \\
(0.05552)\end{array}$ & $\begin{array}{l}0.33079^{*} \\
(0.05103)\end{array}$ & $\begin{array}{l}0.35280^{*} \\
(0.05020)\end{array}$ & $\begin{array}{l}0.35788^{*} \\
(0.05793)\end{array}$ & $\begin{array}{l}0.35506^{*} \\
(0.05155)\end{array}$ & $\begin{array}{l}0.33480^{*} \\
(0.04884)\end{array}$ \\
\hline $\begin{array}{l}\text { Individual } \\
\text { Variable }\end{array}$ & $\begin{array}{l}0.12171 \\
(0.08259)\end{array}$ & $\begin{array}{l}-0.00264 \\
(0.07157)\end{array}$ & $\begin{array}{l}0.01456 \\
(0.06954)\end{array}$ & $\begin{array}{l}0.08368 \\
(0.07478)\end{array}$ & $\begin{array}{l}0.00835 \\
(0.06934)\end{array}$ & $\begin{array}{l}-0.06097 \\
(0.07090)\end{array}$ & $\begin{array}{l}-0.01047 \\
(0.08094)\end{array}$ & $\begin{array}{l}-0.04281 \\
(0.08017)\end{array}$ & $\begin{array}{l}0.00645 \\
(0.09144)\end{array}$ & $\begin{array}{l}-0.13743 \\
(0.09363)\end{array}$ & $\begin{array}{l}-0.06256 \\
(0.07699)\end{array}$ & $\begin{array}{l}-0.09464 \\
(0.07460)\end{array}$ & $\begin{array}{l}-0.06086 \\
(0.13842)\end{array}$ \\
\hline
\end{tabular}

* Significant at the 0.01 level $†$ Significant at the 0.05 level $\ddagger$ Significant at the 0.10 level

Table 95 Coefficients with individual variables for high-level factors - Electronics Industry 


\begin{tabular}{|c|c|c|c|c|c|c|c|c|c|c|c|c|c|}
\hline & 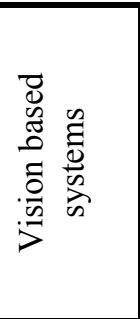 & 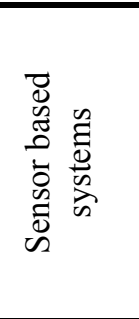 & 㤂 & $\underset{3}{Z}$ & $\overrightarrow{\widehat{\mid}}$ & $\begin{array}{l}\exists \\
\vec{z} \\
\Sigma\end{array}$ & 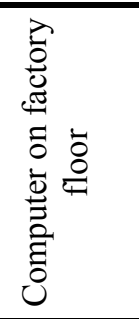 & $\sum_{U}$ & 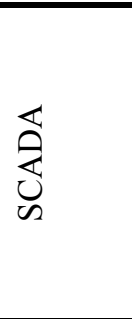 & 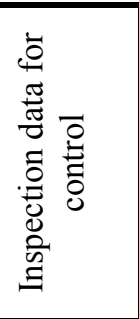 & 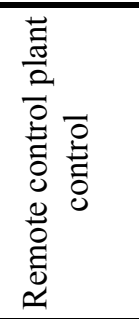 & 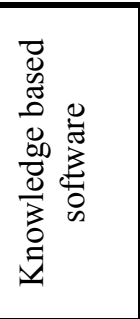 & 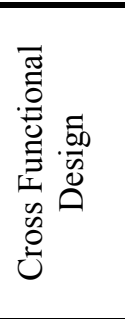 \\
\hline No factors & $\begin{array}{l}0.18353^{*} \\
(0.06152) \\
\end{array}$ & $\begin{array}{l}0.18394^{*} \\
(0.06158) \\
\end{array}$ & $\begin{array}{l}0.18973^{*} \\
(0.06163) \\
\end{array}$ & $\begin{array}{l}0.18270^{*} \\
(0.06189) \\
\end{array}$ & $\begin{array}{l}0.17370^{*} \\
(0.06303)\end{array}$ & $\begin{array}{l}0.18286^{*} \\
(0.06126) \\
\end{array}$ & $\begin{array}{l}0.19049^{*} \\
(0.06129) \\
\end{array}$ & $\begin{array}{l}0.18353^{*} \\
(0.06154) \\
\end{array}$ & $\begin{array}{l}0.18353^{*} \\
(0.06142) \\
\end{array}$ & $\begin{array}{l}0.18323^{*} \\
(0.06154) \\
\end{array}$ & $\begin{array}{l}0.18353^{*} \\
(0.06150) \\
\end{array}$ & $\begin{array}{l}0.18410^{*} \\
(0.06142) \\
\end{array}$ & $\begin{array}{l}0.19800^{*} \\
(0.06267) \\
\end{array}$ \\
\hline Factor 5 & $\begin{array}{l}0.34886 \\
(0.22711) \\
\end{array}$ & $\begin{array}{l}0.34886 \\
(0.22720) \\
\end{array}$ & $\begin{array}{l}0.34886 \\
(0.22654) \\
\end{array}$ & $\begin{array}{l}0.34041 \\
(0.23668) \\
\end{array}$ & $\begin{array}{l}0.34886 \\
(0.22693) \\
\end{array}$ & $\begin{array}{l}0.34886 \\
(0.22617) \\
\end{array}$ & $\begin{array}{l}0.34886 \\
(0.22572) \\
\end{array}$ & $\begin{array}{l}0.34886 \\
(0.22718) \\
\end{array}$ & $\begin{array}{l}0.34886 \\
(0.22676) \\
\end{array}$ & $\begin{array}{l}0.34886 \\
(0.22715) \\
\end{array}$ & $\begin{array}{l}0.34886 \\
(0.22703) \\
\end{array}$ & $\begin{array}{l}0.34886 \\
(0.22673) \\
\end{array}$ & $\begin{array}{l}0.34886 \\
(0.22647) \\
\end{array}$ \\
\hline Factor 4 & $\begin{array}{l}0.13279 \\
(0.33753)\end{array}$ & $\begin{array}{l}0.13810 \\
(0.33872)\end{array}$ & $\begin{array}{l}0.20408 \\
(0.34314)\end{array}$ & $\begin{array}{l}0.12434 \\
(0.34414)\end{array}$ & $\begin{array}{l}0.10264 \\
(0.33996)\end{array}$ & $\begin{array}{l}0.08863 \\
(0.33776)\end{array}$ & $\begin{array}{l}0.17978 \\
(0.33675)\end{array}$ & $\begin{array}{l}0.12945 \\
(0.33787)\end{array}$ & $\begin{array}{l}0.14504 \\
(0.33729)\end{array}$ & $\begin{array}{l}0.12131 \\
(0.33919)\end{array}$ & $\begin{array}{l}0.13279 \\
(0.33742)\end{array}$ & $\begin{array}{l}0.14553 \\
(0.33727)\end{array}$ & $\begin{array}{l}0.15762 \\
(0.33730)\end{array}$ \\
\hline Factor $4 \& 5$ & $\begin{array}{l}0.42672 \\
(0.57731) \\
\end{array}$ & $\begin{array}{l}0.43449 \\
(0.57887) \\
\end{array}$ & $\begin{array}{l}0.51272 \\
(0.58138) \\
\end{array}$ & $\begin{array}{l}0.41828 \\
(0.58138) \\
\end{array}$ & $\begin{array}{l}0.38179 \\
(0.58036) \\
\end{array}$ & $\begin{array}{l}0.38257 \\
(0.57587) \\
\end{array}$ & $\begin{array}{l}0.52071 \\
(0.57678) \\
\end{array}$ & $\begin{array}{l}0.41697 \\
(0.57863) \\
\end{array}$ & $\begin{array}{l}0.42672 \\
(0.57642)\end{array}$ & $\begin{array}{l}0.41524 \\
(0.57835) \\
\end{array}$ & $\begin{array}{l}0.42672 \\
(0.57712) \\
\end{array}$ & $\begin{array}{l}0.42672 \\
(0.57636) \\
\end{array}$ & $\begin{array}{l}0.49937 \\
(0.57927) \\
\end{array}$ \\
\hline Factor 3 & $\begin{array}{l}-0.17286 \\
(0.10869)\end{array}$ & $\begin{array}{l}-0.17286 \\
(0.10874)\end{array}$ & $\begin{array}{l}-0.08687 \\
(0.13469)\end{array}$ & $\begin{array}{l}-0.17286 \\
(0.10874)\end{array}$ & $\begin{array}{l}-0.17286 \\
(0.10861)\end{array}$ & $\begin{array}{l}-0.24095^{\dagger} \\
(0.11965)\end{array}$ & $\begin{array}{l}-0.15134 \\
(0.10886)\end{array}$ & $\begin{array}{l}-0.17286 \\
(0.10873)\end{array}$ & $\begin{array}{l}-0.17286 \\
(0.10852)\end{array}$ & $\begin{array}{l}-0.17286 \\
(0.10871)\end{array}$ & $\begin{array}{l}-0.17286 \\
(0.10865)\end{array}$ & $\begin{array}{l}-0.17286 \\
(0.10851)\end{array}$ & $\begin{array}{l}-0.17286 \\
(0.10838)\end{array}$ \\
\hline Factor $3 \& 5$ & $\begin{array}{l}0.11241 \\
(0.34380)\end{array}$ & $\begin{array}{l}0.11241 \\
(0.34395)\end{array}$ & $\begin{array}{l}0.12766 \\
(0.34323)\end{array}$ & $\begin{array}{l}0.11091 \\
(0.34417)\end{array}$ & $\begin{array}{l}0.10444 \\
(0.34372)\end{array}$ & $\begin{array}{l}0.09675 \\
(0.34258)\end{array}$ & $\begin{array}{l}0.12907 \\
(0.34186)\end{array}$ & $\begin{array}{l}0.11241 \\
(0.34392)\end{array}$ & $\begin{array}{l}0.11241 \\
(0.34327)\end{array}$ & $\begin{array}{l}0.11241 \\
(0.34387)\end{array}$ & $\begin{array}{l}0.11241 \\
(0.34369)\end{array}$ & $\begin{array}{l}0.11241 \\
(0.34324)\end{array}$ & $\begin{array}{l}0.12529 \\
(0.34303)\end{array}$ \\
\hline Factor $3 \& 4$ & $\begin{array}{l}0.21192 \\
(0.23911) \\
\end{array}$ & $\begin{array}{l}0.21192 \\
(0.23922) \\
\end{array}$ & $\begin{array}{l}0.29792 \\
(0.25155) \\
\end{array}$ & $\begin{array}{l}0.20836 \\
(0.24086) \\
\end{array}$ & $\begin{array}{l}0.19296 \\
(0.24044) \\
\end{array}$ & $\begin{array}{l}0.13382 \\
(0.24521) \\
\end{array}$ & $\begin{array}{l}0.27710 \\
(0.24113) \\
\end{array}$ & $\begin{array}{l}0.21192 \\
(0.23920) \\
\end{array}$ & $\begin{array}{l}0.21192 \\
(0.23875) \\
\end{array}$ & $\begin{array}{l}0.21192 \\
(0.23916) \\
\end{array}$ & $\begin{array}{l}0.21192 \\
(0.23904) \\
\end{array}$ & $\begin{array}{l}0.21192 \\
(0.23872) \\
\end{array}$ & $\begin{array}{l}0.22580 \\
(0.23876)\end{array}$ \\
\hline Factor $3,4 \& 5$ & $\begin{array}{l}0.19897 \\
(0.14744)\end{array}$ & $\begin{array}{l}0.19897 \\
(0.14750)\end{array}$ & $\begin{array}{l}0.28496^{\ddagger} \\
(0.16738)\end{array}$ & $\begin{array}{l}0.19897 \\
(0.14751)\end{array}$ & $\begin{array}{l}0.15404 \\
(0.16050)\end{array}$ & $\begin{array}{l}0.19897 \\
(0.14683)\end{array}$ & $\begin{array}{l}0.19897 \\
(0.14653)\end{array}$ & $\begin{array}{l}0.19897 \\
(0.14749)\end{array}$ & $\begin{array}{l}0.19897 \\
(0.14721)\end{array}$ & $\begin{array}{l}0.19897 \\
(0.14747)\end{array}$ & $\begin{array}{l}0.19897 \\
(0.14739)\end{array}$ & $\begin{array}{l}0.19897 \\
(0.14719)\end{array}$ & $\begin{array}{l}0.27161^{\ddagger} \\
(0.16048)\end{array}$ \\
\hline Factor 2 & $\begin{array}{l}0.34304^{\ddagger} \\
(0.19614)\end{array}$ & $\begin{array}{l}0.34425^{\ddagger} \\
(0.19632)\end{array}$ & $\begin{array}{l}0.34973^{\ddagger} \\
(0.19575)\end{array}$ & $\begin{array}{l}0.34238^{\ddagger} \\
(0.19631)\end{array}$ & $\begin{array}{l}0.33955^{\ddagger} \\
(0.19605)\end{array}$ & $\begin{array}{l}0.33617^{\ddagger} \\
(0.19540)\end{array}$ & $\begin{array}{l}0.41377^{\dagger} \\
(0.19991)\end{array}$ & $\begin{array}{l}0.34304^{\ddagger} \\
(0.19621)\end{array}$ & $\begin{array}{l}0.34861^{\ddagger} \\
(0.19594)\end{array}$ & $\begin{array}{l}0.34304^{\ddagger} \\
(0.19618)\end{array}$ & $\begin{array}{l}0.34304^{\ddagger} \\
(0.19608)\end{array}$ & $\begin{array}{l}0.39333^{\ddagger} \\
(0.20346)\end{array}$ & $\begin{array}{l}0.36102^{\ddagger} \\
(0.19624)\end{array}$ \\
\hline Factor $2 \& 5$ & \multicolumn{13}{|c|}{ Insufficient Data } \\
\hline Factor $2 \& 4$ & $\begin{array}{l}0.19856 \\
(0.21205) \\
\end{array}$ & $\begin{array}{l}0.20269 \\
(0.21209) \\
\end{array}$ & $\begin{array}{l}0.23229 \\
(0.21332) \\
\end{array}$ & $\begin{array}{l}0.19737 \\
(0.21468) \\
\end{array}$ & $\begin{array}{l}0.15672 \\
(0.22114) \\
\end{array}$ & $\begin{array}{l}0.16423 \\
(0.21291) \\
\end{array}$ & $\begin{array}{l}0.28153 \\
(0.21649) \\
\end{array}$ & $\begin{array}{l}0.19470 \\
(0.21357) \\
\end{array}$ & $\begin{array}{l}0.21131 \\
(0.21189) \\
\end{array}$ & $\begin{array}{l}0.18367 \\
(0.21809) \\
\end{array}$ & $\begin{array}{l}0.20165 \\
(0.21187) \\
\end{array}$ & $\begin{array}{l}0.20165 \\
(0.21159) \\
\end{array}$ & $\begin{array}{l}0.20654 \\
(0.21139) \\
\end{array}$ \\
\hline Factor $2,4 \& 5$ & $\begin{array}{l}-0.00699 \\
(0.35506)\end{array}$ & $\begin{array}{l}-0.00699 \\
(0.35521)\end{array}$ & $\begin{array}{l}0.07900 \\
(0.36308)\end{array}$ & $\begin{array}{l}-0.01544 \\
(0.36136)\end{array}$ & $\begin{array}{l}-0.05192 \\
(0.36046)\end{array}$ & $\begin{array}{l}-0.09530 \\
(0.35973)\end{array}$ & $\begin{array}{l}0.08699 \\
(0.35776)\end{array}$ & $\begin{array}{l}-0.02281 \\
(0.36000)\end{array}$ & $\begin{array}{l}0.06468 \\
(0.36359)\end{array}$ & $\begin{array}{l}-0.02996 \\
(0.36113)\end{array}$ & $\begin{array}{l}-0.00699 \\
(0.35495)\end{array}$ & $\begin{array}{l}0.00711 \\
(0.35482)\end{array}$ & $\begin{array}{l}0.06565 \\
(0.35986)\end{array}$ \\
\hline Factor $2 \& 3$ & \multicolumn{13}{|c|}{ Insufficient Data } \\
\hline Factor 2, $3 \& 5$ & \multicolumn{13}{|c|}{ Insufficient Data } \\
\hline Factor $2,3 \& 4$ & $\begin{array}{l}0.23029 \\
(0.33584)\end{array}$ & $\begin{array}{l}0.24569 \\
(0.33774)\end{array}$ & $\begin{array}{l}0.32401 \\
(0.34397)\end{array}$ & $\begin{array}{l}0.23232 \\
(0.33853)\end{array}$ & $\begin{array}{l}0.19309 \\
(0.34114)\end{array}$ & $\begin{array}{l}0.17840 \\
(0.33699)\end{array}$ & $\begin{array}{l}0.30028 \\
(0.33562)\end{array}$ & $\begin{array}{l}0.22509 \\
(0.33892)\end{array}$ & $\begin{array}{l}0.25012 \\
(0.33516)\end{array}$ & $\begin{array}{l}0.22639 \\
(0.33710)\end{array}$ & $\begin{array}{l}0.25807 \\
(0.33712)\end{array}$ & $\begin{array}{l}0.26318 \\
(0.33599)\end{array}$ & $\begin{array}{l}0.31066 \\
(0.34059)\end{array}$ \\
\hline Factor $2,3,4 \& 5$ & $\begin{array}{l}0.24296 \\
(0.19158)\end{array}$ & $\begin{array}{l}0.25085 \\
(0.19346)\end{array}$ & $\begin{array}{l}0.33148 \\
(0.20706)\end{array}$ & $\begin{array}{l}0.23903 \\
(0.19817)\end{array}$ & $\begin{array}{l}0.21710 \\
(0.19553)\end{array}$ & $\begin{array}{l}0.19704 \\
(0.19413)\end{array}$ & $\begin{array}{l}0.29914 \\
(0.19326)\end{array}$ & $\begin{array}{l}0.24441 \\
(0.19160)\end{array}$ & $\begin{array}{l}0.27297 \\
(0.19369)\end{array}$ & $\begin{array}{l}0.23415 \\
(0.19424)\end{array}$ & $\begin{array}{l}0.28339 \\
(0.20259)\end{array}$ & $\begin{array}{l}0.27336 \\
(0.19362)\end{array}$ & $\begin{array}{l}0.27461 \\
(0.19269)\end{array}$ \\
\hline Factor 1 & $\begin{array}{l}0.22921 \\
(0.27722)\end{array}$ & $\begin{array}{l}0.22921 \\
(0.27733)\end{array}$ & $\begin{array}{l}0.31520 \\
(0.28784)\end{array}$ & $\begin{array}{l}0.22692 \\
(0.27793)\end{array}$ & $\begin{array}{l}0.21705 \\
(0.27754)\end{array}$ & $\begin{array}{l}0.20530 \\
(0.27665)\end{array}$ & $\begin{array}{l}0.22921 \\
(0.27552)\end{array}$ & $\begin{array}{l}0.22696 \\
(0.27744)\end{array}$ & $\begin{array}{l}0.22921 \\
(0.27679)\end{array}$ & $\begin{array}{l}0.22921 \\
(0.27727)\end{array}$ & $\begin{array}{l}0.22921 \\
(0.27712)\end{array}$ & $\begin{array}{l}0.22921 \\
(0.27676)\end{array}$ & $\begin{array}{l}0.23758 \\
(0.27654)\end{array}$ \\
\hline Factor & $\begin{array}{l}0.06516 \\
(0.27883)\end{array}$ & $\begin{array}{l}0.06941 \\
(0.27977)\end{array}$ & $\begin{array}{l}0.11805 \\
(0.28244)\end{array}$ & $\begin{array}{l}0.05997 \\
(0.28193)\end{array}$ & $\begin{array}{l}0.03753 \\
(0.28135)\end{array}$ & $\begin{array}{l}0.02115 \\
(0.27963)\end{array}$ & $\begin{array}{l}0.14437 \\
(0.28153)\end{array}$ & $\begin{array}{l}0.06516 \\
(0.27893)\end{array}$ & $\begin{array}{l}0.06516 \\
(0.27840)\end{array}$ & $\begin{array}{l}0.05639 \\
(0.28001)\end{array}$ & $\begin{array}{l}0.06516 \\
(0.27874)\end{array}$ & $\begin{array}{l}0.06516 \\
(0.27837)\end{array}$ & $\begin{array}{l}0.09352 \\
(0.27918)\end{array}$ \\
\hline Factol & $\begin{array}{l}0.24875 \\
(0.22125)\end{array}$ & $\begin{array}{l}0.24875 \\
(0.22135)\end{array}$ & $\begin{array}{l}0.33475 \\
(0.23472)\end{array}$ & $\begin{array}{l}0.24756 \\
(0.22156)\end{array}$ & $\begin{array}{l}0.24240 \\
(0.22126)\end{array}$ & $\begin{array}{l}0.17293 \\
(0.22754)\end{array}$ & $\begin{array}{l}0.34274 \\
(0.22763)\end{array}$ & $\begin{array}{l}0.24875 \\
(0.22133)\end{array}$ & $\begin{array}{l}0.24875 \\
(0.22091)\end{array}$ & $\begin{array}{l}0.24875 \\
(0.22130)\end{array}$ & $\begin{array}{l}0.24875 \\
(0.22118)\end{array}$ & $\begin{array}{l}0.24875 \\
(0.22089)\end{array}$ & $\begin{array}{l}0.24875 \\
(0.22063)\end{array}$ \\
\hline Factor $1,4 \& 5$ & $\begin{array}{l}0.30465 \\
(0.33552) \\
\end{array}$ & $\begin{array}{l}0.30465 \\
(0.33567) \\
\end{array}$ & $\begin{array}{l}0.39064 \\
(0.34409) \\
\end{array}$ & $\begin{array}{l}0.29620 \\
(0.34216) \\
\end{array}$ & $\begin{array}{l}0.30465 \\
(0.33526) \\
\end{array}$ & $\begin{array}{l}0.26963 \\
(0.33516) \\
\end{array}$ & $\begin{array}{l}0.34742 \\
(0.33454) \\
\end{array}$ & $\begin{array}{l}0.30465 \\
(0.33564) \\
\end{array}$ & $\begin{array}{l}0.32096 \\
(0.33551) \\
\end{array}$ & $\begin{array}{l}0.29942 \\
(0.33592) \\
\end{array}$ & $\begin{array}{l}0.34932 \\
(0.34439) \\
\end{array}$ & $\begin{array}{l}0.33419 \\
(0.33654) \\
\end{array}$ & $\begin{array}{l}0.34998 \\
(0.33698) \\
\end{array}$ \\
\hline Factor $1 \& 3$ & \multicolumn{13}{|c|}{ Insufficient Data } \\
\hline Factor $1,3 \& 5$ & $\begin{array}{l}0.40495 \\
(0.32026)\end{array}$ & $\begin{array}{l}0.40495 \\
(0.32039)\end{array}$ & $\begin{array}{l}0.44763 \\
(0.32191)\end{array}$ & $\begin{array}{l}0.40365 \\
(0.32057)\end{array}$ & $\begin{array}{l}0.39803 \\
(0.32016)\end{array}$ & $\begin{array}{l}0.31663 \\
(0.32572)\end{array}$ & $\begin{array}{l}0.42443 \\
(0.31853)\end{array}$ & $\begin{array}{l}0.40495 \\
(0.32036)\end{array}$ & $\begin{array}{l}0.40495 \\
(0.31976)\end{array}$ & $\begin{array}{l}0.38878 \\
(0.32362)\end{array}$ & $\begin{array}{l}0.40495 \\
(0.32015)\end{array}$ & $\begin{array}{l}0.40495 \\
(0.31973)\end{array}$ & $\begin{array}{l}0.47759 \\
(0.32577)\end{array}$ \\
\hline Factor $1,3 \& 4$ & $\begin{array}{l}0.17959 \\
(0.12204)\end{array}$ & $\begin{array}{l}0.17959 \\
(0.12210)\end{array}$ & $\begin{array}{l}0.23420^{\ddagger} \\
(0.13189)\end{array}$ & $\begin{array}{l}0.17602 \\
(0.12526)\end{array}$ & $\begin{array}{l}0.17959 \\
(0.12195)\end{array}$ & $\begin{array}{l}0.15426 \\
(0.12301)\end{array}$ & $\begin{array}{l}0.20949^{*} \\
(0.12273)\end{array}$ & $\begin{array}{l}0.17875 \\
(0.12212) \\
\end{array}$ & $\begin{array}{l}0.18267 \\
(0.12191)\end{array}$ & $\begin{array}{l}0.17860 \\
(0.12210)\end{array}$ & $\begin{array}{l}0.17959 \\
(0.12200)\end{array}$ & $\begin{array}{l}0.19905 \\
(0.12371)\end{array}$ & $\begin{array}{l}0.23938^{\ddagger} \\
(0.13272)\end{array}$ \\
\hline Factor $1,3,4 \& 5$ & $\begin{array}{l}0.22763 \\
(0.15754) \\
\end{array}$ & $\begin{array}{l}0.22899 \\
(0.15776) \\
\end{array}$ & $\begin{array}{l}0.29883^{\ddagger} \\
(0.17051) \\
\end{array}$ & $\begin{array}{l}0.22505 \\
(0.15891) \\
\end{array}$ & $\begin{array}{l}0.21881 \\
(0.15791) \\
\end{array}$ & $\begin{array}{l}0.19929 \\
(0.15832) \\
\end{array}$ & $\begin{array}{l}0.24056 \\
(0.15679) \\
\end{array}$ & $\begin{array}{l}0.22763 \\
(0.15759) \\
\end{array}$ & $\begin{array}{l}0.22763 \\
(0.15730) \\
\end{array}$ & $\begin{array}{l}0.22197 \\
(0.15840) \\
\end{array}$ & $\begin{array}{l}0.22763 \\
(0.15749) \\
\end{array}$ & $\begin{array}{l}0.22763 \\
(0.15728) \\
\end{array}$ & $\begin{array}{l}0.25824 \\
(0.15942) \\
\end{array}$ \\
\hline Factor $1 \& 2$ & $\begin{array}{l}0.09131 \\
(0.19613)\end{array}$ & $\begin{array}{l}0.10512 \\
(0.20809)\end{array}$ & $\begin{array}{l}0.16775 \\
(0.20813)\end{array}$ & $\begin{array}{l}0.08287 \\
(0.20711)\end{array}$ & $\begin{array}{l}0.04638 \\
(0.20606)\end{array}$ & $\begin{array}{l}0.09131 \\
(0.19532)\end{array}$ & $\begin{array}{l}0.18530 \\
(0.20361)\end{array}$ & $\begin{array}{l}0.09131 \\
(0.19619)\end{array}$ & $\begin{array}{l}0.09131 \\
(0.19583)\end{array}$ & $\begin{array}{l}0.07090 \\
(0.20463)\end{array}$ & $\begin{array}{l}0.09131 \\
(0.19606)\end{array}$ & $\begin{array}{l}0.16584 \\
(0.21223)\end{array}$ & $\begin{array}{l}.09131 \\
(0.19558)\end{array}$ \\
\hline Factor $1,2 \&$ & \multicolumn{13}{|c|}{ Insufficient Data } \\
\hline Factor $1,2 \& 4$ & $\begin{array}{l}-0.03256 \\
(0.14993)\end{array}$ & $\begin{array}{l}-0.02522 \\
(0.14978)\end{array}$ & $\begin{array}{l}0.02136 \\
(0.15570)\end{array}$ & $\begin{array}{l}-0.03073 \\
(0.15222)\end{array}$ & $\begin{array}{l}-0.04005 \\
(0.15044)\end{array}$ & $\begin{array}{l}-0.06785 \\
(0.15191)\end{array}$ & $\begin{array}{l}0.02309 \\
(0.15180)\end{array}$ & $\begin{array}{l}-0.03229 \\
(0.15073)\end{array}$ & $\begin{array}{l}-0.00639 \\
(0.15101)\end{array}$ & $\begin{array}{l}-0.04797 \\
(0.16090)\end{array}$ & $\begin{array}{l}-0.02708 \\
(0.14938)\end{array}$ & $\begin{array}{l}-0.01785 \\
(0.14953)\end{array}$ & $\begin{array}{l}-0.00725 \\
(0.15004)\end{array}$ \\
\hline Factor $1,2,4 \& 5$ & $\begin{array}{l}0.16908 \\
(0.21080) \\
\end{array}$ & $\begin{array}{l}0.20055 \\
(0.21203) \\
\end{array}$ & $\begin{array}{l}0.27620 \\
(0.22000)\end{array}$ & $\begin{array}{l}0.18230 \\
(0.21475) \\
\end{array}$ & $\begin{array}{l}0.15162 \\
(0.21249) \\
\end{array}$ & $\begin{array}{l}0.10190 \\
(0.21506) \\
\end{array}$ & $\begin{array}{l}0.26499 \\
(0.20952) \\
\end{array}$ & $\begin{array}{l}0.17830 \\
(0.21025) \\
\end{array}$ & $\begin{array}{l}0.20541 \\
(0.20588)\end{array}$ & $\begin{array}{l}0.17474 \\
(0.21021) \\
\end{array}$ & $\begin{array}{l}0.19021 \\
(0.20542) \\
\end{array}$ & $\begin{array}{l}0.20544 \\
(0.20583) \\
\end{array}$ & $\begin{array}{l}0.25825 \\
(0.21358) \\
\end{array}$ \\
\hline Factor $1,2 \& 3$ & $\begin{array}{l}0.36681 \\
(0.47734)\end{array}$ & $\begin{array}{l}0.36681 \\
(0.47754)\end{array}$ & $\begin{array}{l}0.45281 \\
(0.48281)\end{array}$ & $\begin{array}{l}0.35837 \\
(0.48215)\end{array}$ & $\begin{array}{l}0.32188 \\
(0.48120)\end{array}$ & $\begin{array}{l}0.27850 \\
(0.47995)\end{array}$ & $\begin{array}{l}0.46080 \\
(0.47805)\end{array}$ & $\begin{array}{l}0.36681 \\
(0.47750)\end{array}$ & $\begin{array}{l}0.36681 \\
(0.47661)\end{array}$ & $\begin{array}{l}0.34385 \\
(0.48191)\end{array}$ & $\begin{array}{l}0.36681 \\
(0.47718)\end{array}$ & $\begin{array}{l}0.39229 \\
(0.47738)\end{array}$ & $\begin{array}{l}0.39164 \\
(0.47651)\end{array}$ \\
\hline Factor $1,2,3 \& 5$ & $\begin{array}{l}0.30407 \\
(0.21736) \\
\end{array}$ & $\begin{array}{l}0.31274 \\
(0.21687) \\
\end{array}$ & $\begin{array}{l}0.37431^{\ddagger} \\
(0.22387)\end{array}$ & $\begin{array}{l}0.30434 \\
(0.22425)\end{array}$ & $\begin{array}{l}0.27281 \\
(0.22343)\end{array}$ & $\begin{array}{l}0.24153 \\
(0.22211)\end{array}$ & $\begin{array}{l}0.37715^{\ddagger} \\
(0.21925)\end{array}$ & $\begin{array}{l}0.31027 \\
(0.21684)\end{array}$ & $\begin{array}{l}0.33322 \\
(0.21773)\end{array}$ & $\begin{array}{l}0.29875 \\
(0.21985)\end{array}$ & $\begin{array}{l}0.31165 \\
(0.21664)\end{array}$ & $\begin{array}{l}0.31690 \\
(0.21643)\end{array}$ & $\begin{array}{l}0.35434 \\
(0.21938)\end{array}$ \\
\hline Factor $1,2,3 \& 4$ & $\begin{array}{l}0.31074^{*} \\
(0.07094)\end{array}$ & $\begin{array}{l}0.31368^{*} \\
(0.07174)\end{array}$ & $\begin{array}{l}0.38916^{*} \\
(0.10102)\end{array}$ & $\begin{array}{l}0.30587^{*} \\
(0.08381)\end{array}$ & $\begin{array}{l}0.29280^{*} \\
(0.07568)\end{array}$ & $\begin{array}{l}0.25624^{*} \\
(0.08188)\end{array}$ & $\begin{array}{l}0.36433^{*} \\
(0.07784)\end{array}$ & $\begin{array}{l}0.30550^{*} \\
(0.07441)\end{array}$ & $\begin{array}{l}0.33951^{*} \\
(0.07749)\end{array}$ & $\begin{array}{l}0.30048^{*} \\
(0.07765)\end{array}$ & $\begin{array}{l}0.31276^{*} \\
(0.07092)\end{array}$ & $\begin{array}{l}0.32014^{*} \\
(0.07142)\end{array}$ & $\begin{array}{l}0.37022^{*} \\
(0.08775)\end{array}$ \\
\hline All Factors & $\begin{array}{l}0.32149^{*} \\
(0.05369)\end{array}$ & $\begin{array}{l}0.33779^{*} \\
(0.05666)\end{array}$ & $\begin{array}{l}0.40129^{*} \\
(0.08053)\end{array}$ & $\begin{array}{l}0.32613^{*} \\
(0.06646)\end{array}$ & $\begin{array}{l}0.30921^{*} \\
(0.05810)\end{array}$ & $\begin{array}{l}0.27231^{*} \\
(0.06571)\end{array}$ & $\begin{array}{l}0.38201^{*} \\
(0.05741)\end{array}$ & $\begin{array}{l}0.32630^{*} \\
(0.05273)\end{array}$ & $\begin{array}{l}0.34682^{*} \\
(0.05115)\end{array}$ & $\begin{array}{l}0.31789^{*} \\
(0.06281)\end{array}$ & $\begin{array}{l}0.33533^{*} \\
(0.04874)\end{array}$ & $\begin{array}{l}0.35189^{*} \\
(0.05306)\end{array}$ & $\begin{array}{l}0.37117^{*} \\
(0.05947)\end{array}$ \\
\hline $\begin{array}{l}\text { Individual } \\
\text { Variable }\end{array}$ & $\begin{array}{l}0.04578 \\
(0.10193)\end{array}$ & $\begin{array}{l}-0.01553 \\
(0.07796)\end{array}$ & $\begin{array}{l}-0.08599 \\
(0.07992)\end{array}$ & $\begin{array}{l}0.00845 \\
(0.06627)\end{array}$ & $\begin{array}{l}0.04493 \\
(0.06368)\end{array}$ & $\begin{array}{l}0.08831 \\
(0.06615)\end{array}$ & $\begin{array}{l}-0.09398 \\
(0.05883)\end{array}$ & $\begin{array}{l}0.01951 \\
(0.07236)\end{array}$ & $\begin{array}{l}-0.07167 \\
(0.08074)\end{array}$ & $\begin{array}{l}0.02297 \\
(0.06550)\end{array}$ & $\begin{array}{l}-0.11882 \\
(0.20783)\end{array}$ & $\begin{array}{l}-0.07452 \\
(0.08187)\end{array}$ & $\begin{array}{l}-0.07264 \\
(0.06433)\end{array}$ \\
\hline
\end{tabular}

* Significant at the 0.01 level $\dagger$ Significant at the 0.05 level $\$$ Significant at the 0.10 level

Table 95 Coefficients with individual variables for high-level factors - Electronics Industry 


\begin{tabular}{|c|c|c|c|c|c|c|c|c|c|c|c|c|c|}
\hline $\begin{array}{l}\text { Individual variable } \\
\text { included in the } \\
\text { model in the } \\
\text { column }\end{array}$ & 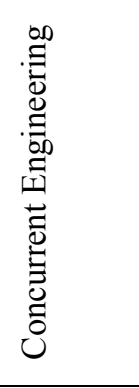 & 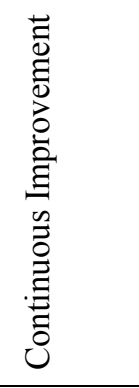 & 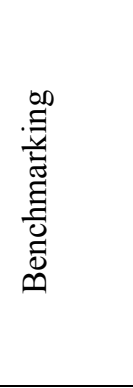 & 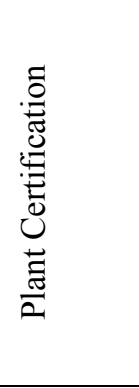 & 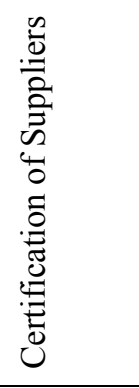 & 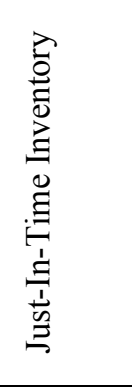 & 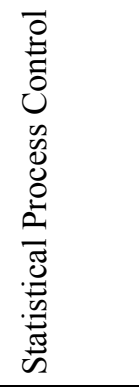 & 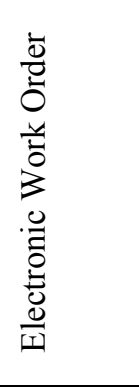 & 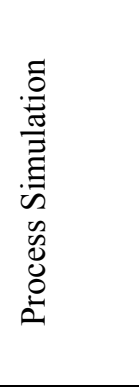 & 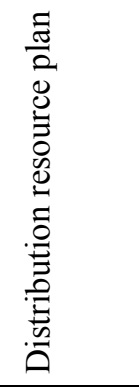 & 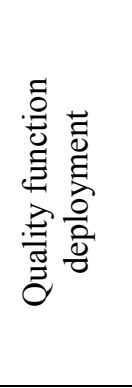 & 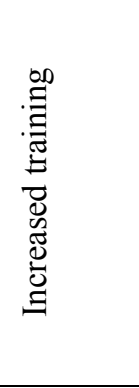 & 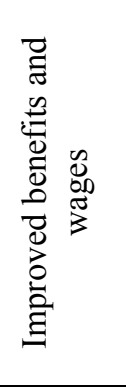 \\
\hline No factors & $\begin{array}{l}0.20087^{*} \\
(0.06303) \\
\end{array}$ & $\begin{array}{l}0.19678^{*} \\
(0.06316) \\
\end{array}$ & $\begin{array}{l}0.18381^{*} \\
(0.06175) \\
\end{array}$ & $\begin{array}{l}0.20412^{*} \\
(0.06671) \\
\end{array}$ & $\begin{array}{l}0.16126^{\dagger} \\
(0.06448)\end{array}$ & $\begin{array}{l}0.18016^{*} \\
(0.06391) \\
\end{array}$ & $\begin{array}{l}0.18685^{*} \\
(0.06186) \\
\end{array}$ & $\begin{array}{l}0.18377^{*} \\
(0.06155) \\
\end{array}$ & $\begin{array}{l}0.18353^{*} \\
(0.06137) \\
\end{array}$ & $\begin{array}{l}0.18353^{*} \\
(0.06139) \\
\end{array}$ & $\begin{array}{l}0.18991^{*} \\
(0.06152) \\
\end{array}$ & $\begin{array}{l}0.18353^{*} \\
(0.06147) \\
\end{array}$ & $\begin{array}{l}0.18353^{*} \\
(0.06153) \\
\end{array}$ \\
\hline Factor 5 & $\begin{array}{l}0.34886 \\
(0.22638)\end{array}$ & $\begin{array}{l}0.34886 \\
(0.22674)\end{array}$ & $\begin{array}{l}0.34886 \\
(0.22722)\end{array}$ & $\begin{array}{l}0.34886 \\
(0.22685)\end{array}$ & $\begin{array}{l}0.34886 \\
(0.22648)\end{array}$ & $\begin{array}{l}0.34886 \\
(0.22720)\end{array}$ & $\begin{array}{l}0.34886 \\
(0.22707)\end{array}$ & $\begin{array}{l}0.34886 \\
(0.22719)\end{array}$ & $\begin{array}{l}0.34886 \\
(0.22658)\end{array}$ & $\begin{array}{l}0.34886 \\
(0.22664)\end{array}$ & $\begin{array}{l}0.34886 \\
(0.22632)\end{array}$ & $\begin{array}{l}0.34886 \\
(0.22695)\end{array}$ & $\begin{array}{l}0.31274 \\
(0.24847)\end{array}$ \\
\hline Factor 4 & $\begin{array}{l}0.18755 \\
(0.33959)\end{array}$ & $\begin{array}{l}0.16306 \\
(0.33866)\end{array}$ & $\begin{array}{l}0.13345 \\
(0.33791) \\
\end{array}$ & $\begin{array}{l}0.19265 \\
(0.34550)\end{array}$ & $\begin{array}{l}0.11944 \\
(0.33681)\end{array}$ & $\begin{array}{l}0.13075 \\
(0.33784)\end{array}$ & $\begin{array}{l}0.13844 \\
(0.33767)\end{array}$ & $\begin{array}{l}0.14163 \\
(0.33943)\end{array}$ & $\begin{array}{l}0.13279 \\
(0.33675)\end{array}$ & $\begin{array}{l}0.13279 \\
(0.33684)\end{array}$ & $\begin{array}{l}0.14657 \\
(0.33655)\end{array}$ & $\begin{array}{l}0.13279 \\
(0.33730)\end{array}$ & $\begin{array}{l}0.13279 \\
(0.33760)\end{array}$ \\
\hline Factor $4 \& 5$ & $\begin{array}{l}0.50834 \\
(0.57954)\end{array}$ & $\begin{array}{l}0.48726 \\
(0.58029)\end{array}$ & $\begin{array}{l}0.43059 \\
(0.58171)\end{array}$ & $\begin{array}{l}0.48659 \\
(0.58158)\end{array}$ & $\begin{array}{l}0.34865 \\
(0.57991)\end{array}$ & $\begin{array}{l}0.41481 \\
(0.58075)\end{array}$ & $\begin{array}{l}0.44325 \\
(0.57816)\end{array}$ & $\begin{array}{l}0.44440 \\
(0.58167)\end{array}$ & $\begin{array}{l}0.42672 \\
(0.57597)\end{array}$ & $\begin{array}{l}0.42672 \\
(0.57612)\end{array}$ & $\begin{array}{l}0.50735 \\
(0.57900)\end{array}$ & $\begin{array}{l}0.46841 \\
(0.58018)\end{array}$ & $\begin{array}{l}0.39060 \\
(0.58613)\end{array}$ \\
\hline Factor 3 & $\begin{array}{l}-0.17286 \\
(0.10834)\end{array}$ & $\begin{array}{l}-0.17286 \\
(0.10852)\end{array}$ & $\begin{array}{l}-0.17286 \\
(0.10875)\end{array}$ & $\begin{array}{l}-0.11300 \\
(0.13222)\end{array}$ & $\begin{array}{l}-0.25094^{\ddagger} \\
(0.12883)\end{array}$ & $\begin{array}{l}-0.17286 \\
(0.10874)\end{array}$ & $\begin{array}{l}-0.17286 \\
(0.10868)\end{array}$ & $\begin{array}{l}-0.17286 \\
(0.10873)\end{array}$ & $\begin{array}{l}-0.17286 \\
(0.10844)\end{array}$ & $\begin{array}{l}-0.17286 \\
(0.10847)\end{array}$ & $\begin{array}{l}-0.17286 \\
(0.10831)\end{array}$ & $\begin{array}{l}-0.17286 \\
(0.10862)\end{array}$ & $\begin{array}{l}-0.17286 \\
(0.10871)\end{array}$ \\
\hline Factor $3 \& 5$ & $\begin{array}{l}0.12688 \\
(0.34292) \\
\end{array}$ & $\begin{array}{l}0.12314 \\
(0.34346) \\
\end{array}$ & $\begin{array}{l}0.11309 \\
(0.34420) \\
\end{array}$ & $\begin{array}{l}0.12302 \\
(0.34368) \\
\end{array}$ & $\begin{array}{l}0.09856 \\
(0.34308) \\
\end{array}$ & $\begin{array}{l}0.10261 \\
(0.34757) \\
\end{array}$ & $\begin{array}{l}0.11241 \\
(0.34376) \\
\end{array}$ & $\begin{array}{l}0.11554 \\
(0.34415) \\
\end{array}$ & $\begin{array}{l}0.12721 \\
(0.34330) \\
\end{array}$ & $\begin{array}{l}0.12685 \\
(0.34341) \\
\end{array}$ & $\begin{array}{l}0.11241 \\
(0.34261) \\
\end{array}$ & $\begin{array}{l}0.12719 \\
(0.34426) \\
\end{array}$ & $\begin{array}{l}0.10600 \\
(0.34433) \\
\end{array}$ \\
\hline Factor $3 \& 4$ & $\begin{array}{l}0.29354 \\
(0.24802)\end{array}$ & $\begin{array}{l}0.22592 \\
(0.23924)\end{array}$ & $\begin{array}{l}0.21237 \\
(0.23937)\end{array}$ & $\begin{array}{l}0.21884 \\
(0.23900)\end{array}$ & $\begin{array}{l}0.20290 \\
(0.23859)\end{array}$ & $\begin{array}{l}0.20504 \\
(0.24178)\end{array}$ & $\begin{array}{l}0.21575 \\
(0.23920)\end{array}$ & $\begin{array}{l}0.22214 \\
(0.24254)\end{array}$ & $\begin{array}{l}0.21192 \\
(0.23856)\end{array}$ & $\begin{array}{l}0.21192 \\
(0.23862)\end{array}$ & $\begin{array}{l}0.21192 \\
(0.23829)\end{array}$ & $\begin{array}{l}0.21192 \\
(0.23895)\end{array}$ & $\begin{array}{l}0.21192 \\
(0.23916)\end{array}$ \\
\hline Factor $3,4 \& 5$ & $\begin{array}{l}0.28058^{\ddagger} \\
(0.16217)\end{array}$ & $\begin{array}{l}0.19897 \\
(0.14720)\end{array}$ & $\begin{array}{l}0.20284 \\
(0.16284) \\
\end{array}$ & $\begin{array}{l}0.19897 \\
(0.14727) \\
\end{array}$ & $\begin{array}{l}0.19897 \\
(0.14703)\end{array}$ & $\begin{array}{l}0.19897 \\
(0.14750) \\
\end{array}$ & $\begin{array}{l}0.19897 \\
(0.14742)\end{array}$ & $\begin{array}{l}0.19897 \\
(0.14749) \\
\end{array}$ & $\begin{array}{l}0.19897 \\
(0.14709)\end{array}$ & $\begin{array}{l}0.19897 \\
(0.14713)\end{array}$ & $\begin{array}{l}0.19897 \\
(0.14693) \\
\end{array}$ & $\begin{array}{l}0.28234 \\
(0.19189) \\
\end{array}$ & $\begin{array}{l}0.19897 \\
(0.14746) \\
\end{array}$ \\
\hline Factor 2 & $\begin{array}{l}0.36324^{\ddagger} \\
(0.19626) \\
\end{array}$ & $\begin{array}{l}0.40358^{\ddagger} \\
(0.20704)\end{array}$ & $\begin{array}{l}0.34691^{\ddagger} \\
(0.20801)\end{array}$ & $\begin{array}{l}0.40291^{\ddagger} \\
(0.20996)\end{array}$ & $\begin{array}{l}0.26496 \\
(0.20763) \\
\end{array}$ & $\begin{array}{l}0.33113 \\
(0.20543) \\
\end{array}$ & $\begin{array}{l}0.35379^{\ddagger} \\
(0.19728) \\
\end{array}$ & $\begin{array}{l}0.34879^{\ddagger} \\
(0.19751) \\
\end{array}$ & $\begin{array}{l}0.34304^{\ddagger} \\
(0.19569) \\
\end{array}$ & $\begin{array}{l}0.36320^{\ddagger} \\
(0.19679) \\
\end{array}$ & $\begin{array}{l}0.34931^{\ddagger} \\
(0.19553) \\
\end{array}$ & $\begin{array}{l}0.34304 \\
(0.19601) \\
\end{array}$ & $\begin{array}{l}0.34304^{\ddagger} \\
(0.19618) \\
\end{array}$ \\
\hline Factor $2 \& 5$ & \multicolumn{13}{|c|}{ Insufficient Data } \\
\hline Factor $2 \& 4$ & $\begin{array}{l}0.23623 \\
(0.21325)\end{array}$ & $\begin{array}{l}0.25311 \\
(0.21918)\end{array}$ & $\begin{array}{l}0.20494 \\
(0.22000)\end{array}$ & $\begin{array}{l}0.26151 \\
(0.22475)\end{array}$ & $\begin{array}{l}0.14613 \\
(0.21708)\end{array}$ & $\begin{array}{l}0.19481 \\
(0.21488)\end{array}$ & $\begin{array}{l}0.22020 \\
(0.21509)\end{array}$ & $\begin{array}{l}0.20403 \\
(0.21222)\end{array}$ & $\begin{array}{l}0.20727 \\
(0.21152)\end{array}$ & $\begin{array}{l}0.20714 \\
(0.21157)\end{array}$ & $\begin{array}{l}0.23037 \\
(0.21248)\end{array}$ & $\begin{array}{l}0.20165 \\
(0.21179)\end{array}$ & $\begin{array}{l}0.20165 \\
(0.21198)\end{array}$ \\
\hline Factor $2,4 \& 5$ & $\begin{array}{l}0.07463 \\
(0.36051)\end{array}$ & $\begin{array}{l}0.05355 \\
(0.36081)\end{array}$ & $\begin{array}{l}-0.00312 \\
(0.36188)\end{array}$ & $\begin{array}{l}0.05288 \\
(0.36260)\end{array}$ & $\begin{array}{l}-0.08506 \\
(0.36086)\end{array}$ & $\begin{array}{l}-0.00924 \\
(0.35540)\end{array}$ & $\begin{array}{l}0.02607 \\
(0.36105)\end{array}$ & $\begin{array}{l}0.01069 \\
(0.36191)\end{array}$ & $\begin{array}{l}0.00880 \\
(0.35456)\end{array}$ & $\begin{array}{l}0.00842 \\
(0.35467)\end{array}$ & $\begin{array}{l}0.00826 \\
(0.35405)\end{array}$ & $\begin{array}{l}0.07639 \\
(0.37552)\end{array}$ & $\begin{array}{l}-0.01382 \\
(0.35564)\end{array}$ \\
\hline Factor $2 \& 3$ & \multicolumn{13}{|c|}{ Insufficient Data } \\
\hline Factor $2,3 \& 5$ & \multicolumn{13}{|c|}{ Insufficient Data } \\
\hline Fact & $\begin{array}{l}0.31963 \\
(0.34129)\end{array}$ & $\begin{array}{l}0.29856 \\
(0.34154)\end{array}$ & $\begin{array}{l}0.24124 \\
(0.34044)\end{array}$ & $\begin{array}{l}0.29789 \\
(0.34342)\end{array}$ & $\begin{array}{l}0.15995 \\
(0.34165)\end{array}$ & $\begin{array}{l}0.22812 \\
(0.33933)\end{array}$ & $\begin{array}{l}0.26550 \\
(0.33978)\end{array}$ & $\begin{array}{l}0.25272 \\
(0.34044)\end{array}$ & $\begin{array}{l}0.29438 \\
(0.33897)\end{array}$ & $\begin{array}{l}0.27824 \\
(0.33716)\end{array}$ & $\begin{array}{l}0.26524 \\
(0.33497)\end{array}$ & $\begin{array}{l}0.23802 \\
(0.33517)\end{array}$ & $\begin{array}{l}0.23802 \\
(0.33546)\end{array}$ \\
\hline Factor2 & $\begin{array}{l}0.29208 \\
(0.19486)\end{array}$ & $\begin{array}{l}0.30602 \\
(0.20266)\end{array}$ & $\begin{array}{l}0.24663 \\
(0.19269) \\
\end{array}$ & $\begin{array}{l}0.29413 \\
(0.20087)\end{array}$ & $\begin{array}{l}0.17775 \\
(0.20029) \\
\end{array}$ & $\begin{array}{l}0.23803 \\
(0.19532) \\
\end{array}$ & $\begin{array}{l}0.25998 \\
(0.19363) \\
\end{array}$ & $\begin{array}{l}0.25518 \\
(0.19531) \\
\end{array}$ & $\begin{array}{l}0.27031 \\
(0.19253)\end{array}$ & $\begin{array}{l}0.25445 \\
(0.19131)\end{array}$ & $\begin{array}{l}0.24992 \\
(0.19087) \\
\end{array}$ & $\begin{array}{l}0.30919 \\
(0.21318)\end{array}$ & $\begin{array}{l}0.23019 \\
(0.19622) \\
\end{array}$ \\
\hline Factor 1 & $\begin{array}{l}0.29814 \\
(0.28234)\end{array}$ & $\begin{array}{l}0.28034 \\
(0.28254)\end{array}$ & $\begin{array}{l}0.23247 \\
(0.28341)\end{array}$ & $\begin{array}{l}0.27977 \\
(0.28415)\end{array}$ & $\begin{array}{l}0.17226 \\
(0.28108)\end{array}$ & $\begin{array}{l}0.21729 \\
(0.28392)\end{array}$ & $\begin{array}{l}0.23302 \\
(0.27728)\end{array}$ & $\begin{array}{l}0.23399 \\
(0.27795)\end{array}$ & $\begin{array}{l}0.22921 \\
(0.27657)\end{array}$ & $\begin{array}{l}0.24186 \\
(0.27694)\end{array}$ & $\begin{array}{l}0.23850 \\
(0.27636)\end{array}$ & $\begin{array}{l}0.22921 \\
(0.27703)\end{array}$ & $\begin{array}{l}0.22921 \\
(0.27727)\end{array}$ \\
\hline Factor $1 \& 5$ & $\begin{array}{l}0.08420 \\
(0.27840)\end{array}$ & $\begin{array}{l}0.12570 \\
(0.28638)\end{array}$ & $\begin{array}{l}0.06754 \\
(0.28218) \\
\end{array}$ & $\begin{array}{l}0.12503 \\
(0.28856) \\
\end{array}$ & $\begin{array}{l}0.00462 \\
(0.28326)\end{array}$ & $\begin{array}{l}0.05464 \\
(0.28407) \\
\end{array}$ & $\begin{array}{l}0.07259 \\
(0.27918) \\
\end{array}$ & $\begin{array}{l}0.07397 \\
(0.28107) \\
\end{array}$ & $\begin{array}{l}0.06516 \\
(0.27819) \\
\end{array}$ & $\begin{array}{l}0.06516 \\
(0.27826)\end{array}$ & $\begin{array}{l}0.10561 \\
(0.27979) \\
\end{array}$ & $\begin{array}{l}0.14854 \\
(0.30456) \\
\end{array}$ & $\begin{array}{l}0.04862 \\
(0.28267)\end{array}$ \\
\hline Factor $1 \& 4$ & $\begin{array}{l}0.24875 \\
(0.22055)\end{array}$ & $\begin{array}{l}0.25731 \\
(0.22110)\end{array}$ & $\begin{array}{l}0.25208 \\
(0.22915)\end{array}$ & $\begin{array}{l}0.24875 \\
(0.22101)\end{array}$ & $\begin{array}{l}0.18172 \\
(0.22860)\end{array}$ & $\begin{array}{l}0.24875 \\
(0.22135)\end{array}$ & $\begin{array}{l}0.27714 \\
(0.22831)\end{array}$ & $\begin{array}{l}0.24875 \\
(0.22133)\end{array}$ & $\begin{array}{l}0.24875 \\
(0.22074)\end{array}$ & $\begin{array}{l}0.24875 \\
(0.22080)\end{array}$ & $\begin{array}{l}0.26016 \\
(0.22068)\end{array}$ & $\begin{array}{l}0.24875 \\
(0.22110)\end{array}$ & $\begin{array}{l}0.24875 \\
(0.22130)\end{array}$ \\
\hline Factor $1,4 \& 5$ & $\begin{array}{l}0.35557 \\
(0.33718)\end{array}$ & $\begin{array}{l}0.34118 \\
(0.33743)\end{array}$ & $\begin{array}{l}0.30618 \\
(0.33681)\end{array}$ & $\begin{array}{l}0.36451 \\
(0.34354)\end{array}$ & $\begin{array}{l}0.22657 \\
(0.34177)\end{array}$ & $\begin{array}{l}0.29746 \\
(0.33767)\end{array}$ & $\begin{array}{l}0.31217 \\
(0.33581)\end{array}$ & $\begin{array}{l}0.31568 \\
(0.33843)\end{array}$ & $\begin{array}{l}0.30465 \\
(0.33475)\end{array}$ & $\begin{array}{l}0.30465 \\
(0.33483)\end{array}$ & $\begin{array}{l}0.33496 \\
(0.33526)\end{array}$ & $\begin{array}{l}0.38802 \\
(0.35712)\end{array}$ & $\begin{array}{l}0.29643 \\
(0.33637)\end{array}$ \\
\hline $\mathrm{Fac}$ & \multicolumn{13}{|c|}{ Insufficient Data } \\
\hline Factor $1,3 \& 5$ & $\begin{array}{l}0.43442 \\
(0.32020)\end{array}$ & $\begin{array}{l}0.46549 \\
(0.32673)\end{array}$ & $\begin{array}{l}.40767 \\
(0.32408)\end{array}$ & $\begin{array}{l}0.44708 \\
(0.32428)\end{array}$ & $\begin{array}{l}0.36980 \\
(0.32091)\end{array}$ & $\begin{array}{l}0.39711 \\
(0.32288)\end{array}$ & $\begin{array}{l}0.41003 \\
(0.32037)\end{array}$ & $\begin{array}{l}0.40767 \\
(0.32055)\end{array}$ & $\begin{array}{l}0.40495 \\
(0.31951)\end{array}$ & $\begin{array}{l}0.40495 \\
(0.31960)\end{array}$ & $\begin{array}{l}0.42883 \\
(0.31973)\end{array}$ & $\begin{array}{l}0.46362 \\
(0.33153)\end{array}$ & $\begin{array}{l}0.36882 \\
(0.33578)\end{array}$ \\
\hline Factor $1,3 \& 4$ & $\begin{array}{l}0.22296^{\ddagger} \\
(0.12699)\end{array}$ & $\begin{array}{l}0.23752^{\ddagger} \\
(0.13778)\end{array}$ & $\begin{array}{l}0.17959 \\
(0.12211) \\
\end{array}$ & $\begin{array}{l}0.20476 \\
(0.12597)\end{array}$ & $\begin{array}{l}0.15583 \\
(0.12354) \\
\end{array}$ & $\begin{array}{l}0.17647 \\
(0.12312) \\
\end{array}$ & $\begin{array}{l}0.17959 \\
(0.12203) \\
\end{array}$ & $\begin{array}{l}0.18542 \\
(0.12422) \\
\end{array}$ & $\begin{array}{l}0.17959 \\
(0.12176)\end{array}$ & $\begin{array}{l}0.17959 \\
(0.12179) \\
\end{array}$ & $\begin{array}{l}0.22490^{\ddagger} \\
(0.12704)\end{array}$ & $\begin{array}{l}0.17959 \\
(0.12196) \\
\end{array}$ & $\begin{array}{l}0.17959 \\
(0.12207) \\
\end{array}$ \\
\hline Factor & $\begin{array}{l}0.25968 \\
(0.15933) \\
\end{array}$ & $\begin{array}{l}0.25574 \\
(0.16036) \\
\end{array}$ & $\begin{array}{l}0.22859 \\
(0.15854) \\
\end{array}$ & $\begin{array}{l}0.26845 \\
(0.16556) \\
\end{array}$ & $\begin{array}{l}0.16657 \\
(0.16628) \\
\end{array}$ & $\begin{array}{l}0.21961 \\
(0.16284) \\
\end{array}$ & $\begin{array}{l}0.23578 \\
(0.15835) \\
\end{array}$ & $\begin{array}{l}0.23087 \\
(0.15811) \\
\end{array}$ & $\begin{array}{l}0.23493 \\
(0.15733) \\
\end{array}$ & $\begin{array}{l}0.22763 \\
(0.15722) \\
\end{array}$ & $\begin{array}{l}0.23468 \\
(0.15710) \\
\end{array}$ & $\begin{array}{l}0.31101 \\
(0.19975) \\
\end{array}$ & $\begin{array}{l}0.19938 \\
(0.17616) \\
\end{array}$ \\
\hline Factor & $\begin{array}{l}0.09131 \\
(0.19550)\end{array}$ & $\begin{array}{l}0.09804 \\
(0.19596)\end{array}$ & $\begin{array}{l}0.09475 \\
(0.20558)\end{array}$ & $\begin{array}{l}0.14453 \\
(0.20707)\end{array}$ & $\begin{array}{l}0.02191 \\
(0.20515)\end{array}$ & $\begin{array}{l}0.08999 \\
(0.19633)\end{array}$ & $\begin{array}{l}0.09131 \\
(0.19610)\end{array}$ & $\begin{array}{l}0.09328 \\
(0.19635)\end{array}$ & $\begin{array}{l}0.09131 \\
(0.19567)\end{array}$ & $\begin{array}{l}0.09131 \\
(0.19573)\end{array}$ & $\begin{array}{l}0.09131 \\
(0.19545)\end{array}$ & $\begin{array}{l}0.09131 \\
(0.19599)\end{array}$ & $\begin{array}{l}0.09131 \\
(0.19617)\end{array}$ \\
\hline Factor $1,2 \& 5$ & \multicolumn{13}{|c|}{ Insufficient Data } \\
\hline Factor $1,2 \& 4$ & $\begin{array}{l}0.03453 \\
(0.15769)\end{array}$ & $\begin{array}{l}0.02800 \\
(0.16123)\end{array}$ & $\begin{array}{l}-0.02536 \\
(0.15260)\end{array}$ & $\begin{array}{l}0.03279 \\
(0.16725)\end{array}$ & $\begin{array}{l}-0.09810 \\
(0.16192)\end{array}$ & $\begin{array}{l}-0.03154 \\
(0.15122)\end{array}$ & $\begin{array}{l}0.00202 \\
(0.16022)\end{array}$ & $\begin{array}{l}-0.01251 \\
(0.16005)\end{array}$ & $\begin{array}{l}-0.01708 \\
(0.14939)\end{array}$ & $\begin{array}{l}-0.01365 \\
(0.14973)\end{array}$ & $\begin{array}{l}0.02413 \\
(0.15458)\end{array}$ & $\begin{array}{l}-0.02708 \\
(0.14933)\end{array}$ & $\begin{array}{l}-0.02708 \\
(0.14946)\end{array}$ \\
\hline Factor $1,2,4 \& 5$ & $\begin{array}{l}0.24966 \\
(0.21083)\end{array}$ & $\begin{array}{l}0.24337 \\
(0.21348)\end{array}$ & $\begin{array}{l}0.19312 \\
(0.21202)\end{array}$ & $\begin{array}{l}0.25008 \\
(0.21869)\end{array}$ & $\begin{array}{l}0.11214 \\
(0.21643)\end{array}$ & $\begin{array}{l}0.17905 \\
(0.21331)\end{array}$ & $\begin{array}{l}0.22045 \\
(0.21407)\end{array}$ & $\begin{array}{l}0.20789 \\
(0.21696)\end{array}$ & $\begin{array}{l}0.23523 \\
(0.20951)\end{array}$ & $\begin{array}{l}0.20865 \\
(0.20590)\end{array}$ & $\begin{array}{l}0.21357 \\
(0.20565)\end{array}$ & $\begin{array}{l}0.26647 \\
(0.23412)\end{array}$ & $\begin{array}{l}0.17045 \\
(0.21278)\end{array}$ \\
\hline Factor $1,2 \& 3$ & $\begin{array}{l}0.36681 \\
(0.47582)\end{array}$ & $\begin{array}{l}0.42735 \\
(0.48129)\end{array}$ & $\begin{array}{l}0.36936 \\
(0.47974)\end{array}$ & $\begin{array}{l}0.42668 \\
(0.48274)\end{array}$ & $\begin{array}{l}0.28874 \\
(0.48109)\end{array}$ & $\begin{array}{l}0.35490 \\
(0.48140)\end{array}$ & $\begin{array}{l}0.37811 \\
(0.47781)\end{array}$ & $\begin{array}{l}0.37286 \\
(0.47810)\end{array}$ & $\begin{array}{l}0.39535 \\
(0.47702)\end{array}$ & $\begin{array}{l}0.36681 \\
(0.47636)\end{array}$ & $\begin{array}{l}0.39437 \\
(0.47621)\end{array}$ & $\begin{array}{l}0.36681 \\
(0.47702)\end{array}$ & $\begin{array}{l}0.36681 \\
(0.47743)\end{array}$ \\
\hline Factor $1,2,3 \& 5$ & $\begin{array}{l}0.34971 \\
(0.21837)\end{array}$ & $\begin{array}{l}0.36644 \\
(0.22475)\end{array}$ & $\begin{array}{l}0.31435 \\
(0.22211)\end{array}$ & $\begin{array}{l}0.35105 \\
(0.22209)\end{array}$ & $\begin{array}{l}0.26216 \\
(0.22057)\end{array}$ & $\begin{array}{l}0.30296 \\
(0.22128)\end{array}$ & $\begin{array}{l}0.32713 \\
(0.21885)\end{array}$ & $\begin{array}{l}0.32319 \\
(0.22147)\end{array}$ & $\begin{array}{l}0.34826 \\
(0.21904)\end{array}$ & $\begin{array}{l}0.31165 \\
(0.21626)\end{array}$ & $\begin{array}{l}0.34883 \\
(0.21805)\end{array}$ & $\begin{array}{l}0.37784 \\
(0.23754)\end{array}$ & $\begin{array}{l}0.28297 \\
(0.23102)\end{array}$ \\
\hline Factor $1,2,3 \& 4$ & $\begin{array}{l}0.37909^{*} \\
(0.09064)\end{array}$ & $\begin{array}{l}0.36039^{*} \\
(0.08918)\end{array}$ & $\begin{array}{l}0.31455^{*} \\
(0.08880)\end{array}$ & $\begin{array}{l}0.35769^{*} \\
(0.09165)\end{array}$ & $\begin{array}{l}0.24850^{*} \\
(0.09035)\end{array}$ & $\begin{array}{l}0.30403^{*} \\
(0.08067)\end{array}$ & $\begin{array}{l}0.32577^{*} \\
(0.07633)\end{array}$ & $\begin{array}{l}0.32503^{*} \\
(0.08850)\end{array}$ & $\begin{array}{l}0.31973^{*} \\
(0.07118)\end{array}$ & $\begin{array}{l}0.31849^{*} \\
(0.07111)\end{array}$ & $\begin{array}{l}0.35517^{*} \\
(0.07901)\end{array}$ & $\begin{array}{l}0.31155^{*} \\
(0.07087)\end{array}$ & $\begin{array}{l}0.31155^{*} \\
(0.07093)\end{array}$ \\
\hline All Factors & $\begin{array}{l}0.39802^{*} \\
(0.07355)\end{array}$ & $\begin{array}{l}0.37841^{*} \\
(0.07069)\end{array}$ & $\begin{array}{l}0.33430^{*} \\
(0.06423)\end{array}$ & $\begin{array}{l}0.38605^{*} \\
(0.08362)\end{array}$ & $\begin{array}{l}0.26888^{*} \\
(0.07411)\end{array}$ & $\begin{array}{l}0.32497^{*} \\
(0.06005)\end{array}$ & $\begin{array}{l}0.34883^{*} \\
(0.05891)\end{array}$ & $\begin{array}{l}0.34310^{*} \\
(0.06532)\end{array}$ & $\begin{array}{l}0.37004^{*} \\
(0.06057)\end{array}$ & $\begin{array}{l}0.37236^{*} \\
(0.06318)\end{array}$ & $\begin{array}{l}0.38307^{*} \\
(0.06357)\end{array}$ & $\begin{array}{l}0.40352^{*} \\
(0.11612)\end{array}$ & $\begin{array}{l}0.30307^{*} \\
(0.09391)\end{array}$ \\
\hline $\begin{array}{l}\text { Individual } \\
\text { Variable }\end{array}$ & $\begin{array}{l}-0.08161 \\
(0.06856)\end{array}$ & $\begin{array}{l}-0.06054 \\
(0.06721)\end{array}$ & $\begin{array}{l}-0.00387 \\
(0.06897)\end{array}$ & $\begin{array}{l}-0.05987 \\
(0.07547)\end{array}$ & $\begin{array}{l}0.07808 \\
(0.06964)\end{array}$ & $\begin{array}{l}0.01191 \\
(0.06079)\end{array}$ & $\begin{array}{l}-0.03306 \\
(0.06572)\end{array}$ & $\begin{array}{l}-0.01768 \\
(0.06941)\end{array}$ & $\begin{array}{l}-0.08348 \\
(0.08012)\end{array}$ & $\begin{array}{l}-0.08147 \\
(0.08208)\end{array}$ & $\begin{array}{l}-0.08063 \\
(0.06530)\end{array}$ & $\begin{array}{l}-0.08337 \\
(0.12294)\end{array}$ & $\begin{array}{l}0.03612 \\
(0.10071)\end{array}$ \\
\hline
\end{tabular}

* Significant at the 0.01 level

$\dagger$ Significant at the 0.05 leve

$\$$ Significant at the 0.10 level

Table 95 Coefficients with individual variables for high-level factors - Electronics Industry 


\begin{tabular}{|c|c|c|c|c|c|c|c|c|c|c|c|c|c|}
\hline $\begin{array}{l}\text { Individual variable } \\
\text { included in the } \\
\text { model in the } \\
\text { column }\end{array}$ & 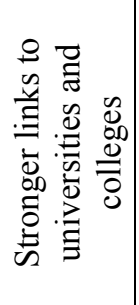 & 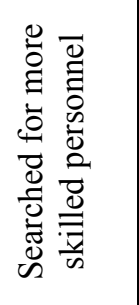 & 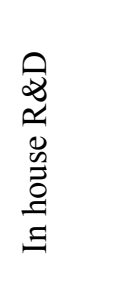 & 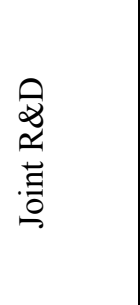 & 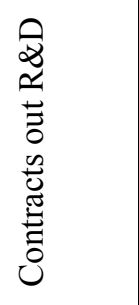 & 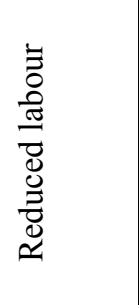 & 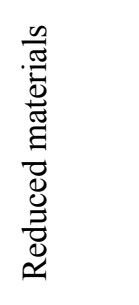 & 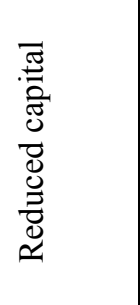 & 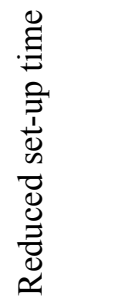 & 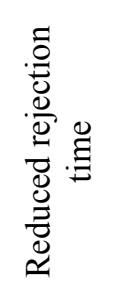 & 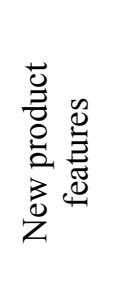 & 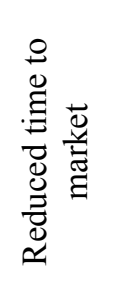 & 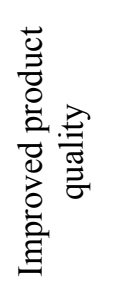 \\
\hline No factors & $\begin{array}{l}0.18353^{*} \\
(0.06155)\end{array}$ & $\begin{array}{l}0.18353^{*} \\
(0.06155)\end{array}$ & $\begin{array}{l}0.19139^{\dagger} \\
(0.07568)\end{array}$ & $\begin{array}{l}0.18596^{*} \\
(0.06271)\end{array}$ & $\begin{array}{l}0.17759^{*} \\
(0.06047)\end{array}$ & $\begin{array}{l}0.18289^{*} \\
(0.06113)\end{array}$ & $\begin{array}{l}0.18353^{*} \\
(0.06154)\end{array}$ & $\begin{array}{l}0.18353^{*} \\
(0.06154)\end{array}$ & $\begin{array}{l}0.18447^{*} \\
(0.06063)\end{array}$ & $\begin{array}{l}0.18254^{*} \\
(0.06157)\end{array}$ & $\begin{array}{l}0.18029^{*} \\
(0.06130)\end{array}$ & $\begin{array}{l}0.18202^{*} \\
(0.06153)\end{array}$ & $\begin{array}{l}0.18353^{*} \\
(0.06146)\end{array}$ \\
\hline Factor 5 & \begin{tabular}{l|}
0.34886 \\
$(0.22723)$ \\
\end{tabular} & $\begin{array}{l}0.34886 \\
(0.22723) \\
\end{array}$ & $\begin{array}{l}0.36162 \\
(0.23819) \\
\end{array}$ & $\begin{array}{l}0.36062 \\
(0.23458) \\
\end{array}$ & $\begin{array}{l}0.34886 \\
(0.22308) \\
\end{array}$ & $\begin{array}{l}0.34886 \\
(0.22569) \\
\end{array}$ & $\begin{array}{l}0.34886 \\
(0.22720) \\
\end{array}$ & \begin{tabular}{l|}
0.34886 \\
$(0.22721)$ \\
\end{tabular} & $\begin{array}{l}0.34886 \\
(0.22383) \\
\end{array}$ & $\begin{array}{l}0.31129 \\
(0.24515) \\
\end{array}$ & $\begin{array}{l}0.34886 \\
(0.22612) \\
\end{array}$ & $\begin{array}{l}0.34886 \\
(0.22698) \\
\end{array}$ & $\begin{array}{l}0.26518 \\
(0.25229) \\
\end{array}$ \\
\hline Factor 4 & $\begin{array}{l}0.13279 \\
(0.33771)\end{array}$ & $\begin{array}{l}0.13279 \\
(0.33771)\end{array}$ & $\begin{array}{l}0.14135 \\
(0.34107)\end{array}$ & $\begin{array}{l}0.13480 \\
(0.33782)\end{array}$ & $\begin{array}{l}0.13279 \\
(0.33154)\end{array}$ & $\begin{array}{l}0.13279 \\
(0.33543)\end{array}$ & $\begin{array}{l}0.13279 \\
(0.33766)\end{array}$ & $\begin{array}{l}0.13279 \\
(0.33769)\end{array}$ & $\begin{array}{l}0.16105 \\
(0.33286)\end{array}$ & $\begin{array}{l}0.13279 \\
(0.33756)\end{array}$ & $\begin{array}{l}0.05027 \\
(0.34146)\end{array}$ & $\begin{array}{l}0.10416 \\
(0.34033)\end{array}$ & $\begin{array}{l}0.10525 \\
(0.33915)\end{array}$ \\
\hline Factor & \begin{tabular}{l|}
0.42380 \\
$(0.58585)$ \\
\end{tabular} & $\begin{array}{l}0.42997 \\
(0.60980) \\
\end{array}$ & $\begin{array}{l}0.43948 \\
(0.58197) \\
\end{array}$ & $\begin{array}{l}0.43260 \\
(0.57829) \\
\end{array}$ & $\begin{array}{l}0.42672 \\
(0.56707) \\
\end{array}$ & $\begin{array}{l}0.42672 \\
(0.57371) \\
\end{array}$ & $\begin{array}{l}0.42672 \\
(0.57754) \\
\end{array}$ & $\begin{array}{l}0.42672 \\
(0.57757) \\
\end{array}$ & $\begin{array}{l}0.50940 \\
(0.57000)\end{array}$ & $\begin{array}{l}0.40794 \\
(0.57920) \\
\end{array}$ & $\begin{array}{l}0.42672 \\
(0.57480) \\
\end{array}$ & $\begin{array}{l}0.39809 \\
(0.57875) \\
\end{array}$ & $\begin{array}{l}0.42672 \\
(0.57674) \\
\end{array}$ \\
\hline Factor 3 & $\begin{array}{l}-0.17286 \\
(0.10875)\end{array}$ & $\begin{array}{l}-0.17286 \\
(0.10875)\end{array}$ & $\begin{array}{l}-0.16010 \\
(0.13013)\end{array}$ & $\begin{array}{l}-0.17017 \\
(0.10955)\end{array}$ & $\begin{array}{l}-0.30766^{*} \\
(0.11809)\end{array}$ & $\begin{array}{l}-0.25971^{\dagger} \\
(0.12071)\end{array}$ & $\begin{array}{l}-0.17286 \\
(0.10873)\end{array}$ & $\begin{array}{l}-0.17286 \\
(0.10874)\end{array}$ & $\begin{array}{l}-0.13499 \\
(0.10827)\end{array}$ & $\begin{array}{l}-0.21043 \\
(0.14258)\end{array}$ & $\begin{array}{l}-0.17286 \\
(0.10822)\end{array}$ & $\begin{array}{l}-0.17286 \\
(0.10863)\end{array}$ & $\begin{array}{l}-0.25654^{\ddagger} \\
(0.15480)\end{array}$ \\
\hline Factor $3 \& 5$ & $\begin{array}{l}0.11189 \\
(0.34442)\end{array}$ & $\begin{array}{l}0.11565 \\
(0.39566)\end{array}$ & $\begin{array}{l}0.12516 \\
(0.35131)\end{array}$ & $\begin{array}{l}0.11241 \\
(0.34395)\end{array}$ & $\begin{array}{l}0.08140 \\
(0.33790)\end{array}$ & $\begin{array}{l}0.11241 \\
(0.34166)\end{array}$ & $\begin{array}{l}0.11241 \\
(0.34394)\end{array}$ & $\begin{array}{l}0.11241 \\
(0.34396)\end{array}$ & $\begin{array}{l}0.11241 \\
(0.33884)\end{array}$ & $\begin{array}{l}0.11241 \\
(0.34384)\end{array}$ & $\begin{array}{l}0.01122 \\
(0.35025)\end{array}$ & $\begin{array}{l}0.06529 \\
(0.35149)\end{array}$ & $\begin{array}{l}0.04357 \\
(0.35526)\end{array}$ \\
\hline Factor $3 \& 4$ & $\begin{array}{l}0.21192 \\
(0.23924) \\
\end{array}$ & $\begin{array}{l}0.21192 \\
(0.23924) \\
\end{array}$ & $\begin{array}{l}0.22468 \\
(0.24967) \\
\end{array}$ & $\begin{array}{l}0.21328 \\
(0.23931) \\
\end{array}$ & $\begin{array}{l}0.11089 \\
(0.23790) \\
\end{array}$ & $\begin{array}{l}0.19041 \\
(0.23800) \\
\end{array}$ & $\begin{array}{l}0.20995 \\
(0.23937) \\
\end{array}$ & $\begin{array}{l}0.21877 \\
(0.24276) \\
\end{array}$ & $\begin{array}{l}0.21192 \\
(0.23566)\end{array}$ & $\begin{array}{l}0.20041 \\
(0.24080) \\
\end{array}$ & $\begin{array}{l}0.18844 \\
(0.23870) \\
\end{array}$ & $\begin{array}{l}0.17221 \\
(0.24698) \\
\end{array}$ & $\begin{array}{l}0.15390 \\
(0.25083)\end{array}$ \\
\hline Factor $3,4 \& 5$ & \begin{tabular}{l|}
0.19897 \\
$(0.14751)$
\end{tabular} & $\begin{array}{l}0.20221 \\
(0.24491)\end{array}$ & $\begin{array}{l}0.21173 \\
(0.16391)\end{array}$ & $\begin{array}{l}0.19897 \\
(0.14750)\end{array}$ & $\begin{array}{l}0.19897 \\
(0.14482)\end{array}$ & $\begin{array}{l}0.19897 \\
(0.14652)\end{array}$ & $\begin{array}{l}0.19897 \\
(0.14749)\end{array}$ & $\begin{array}{l}0.19897 \\
(0.14750)\end{array}$ & $\begin{array}{l}0.19897 \\
(0.14531)\end{array}$ & $\begin{array}{l}0.16140 \\
(0.17394)\end{array}$ & $\begin{array}{l}0.07598 \\
(0.17225)\end{array}$ & $\begin{array}{l}0.19897 \\
(0.14736)\end{array}$ & $\begin{array}{l}0.11529 \\
(0.18403)\end{array}$ \\
\hline Factor 2 & $\begin{array}{l}0.34304^{\ddagger} \\
(0.19625)\end{array}$ & $\begin{array}{l}0.34304^{\ddagger} \\
(0.19625)\end{array}$ & $\begin{array}{l}0.34620^{\ddagger} \\
(0.19703)\end{array}$ & $\begin{array}{l}0.35389^{\ddagger} \\
(0.20348)\end{array}$ & $\begin{array}{l}0.29978 \\
(0.19335)\end{array}$ & $\begin{array}{l}0.34304^{\ddagger} \\
(0.19492)\end{array}$ & $\begin{array}{l}0.34304^{\ddagger} \\
(0.19622)\end{array}$ & $\begin{array}{l}0.34304 \\
(0.19623)\end{array}$ & $\begin{array}{l}0.34304^{\ddagger} \\
(0.19331)\end{array}$ & $\begin{array}{l}0.34304^{\ddagger} \\
(0.19616)\end{array}$ & $\begin{array}{l}0.33348^{\ddagger} \\
(0.19542)\end{array}$ & $\begin{array}{l}0.34304 \\
(0.19604)\end{array}$ & $\begin{array}{l}0.34304^{\ddagger} \\
(0.19595)\end{array}$ \\
\hline Factor $2 \& 5$ & \multicolumn{13}{|c|}{ Insufficient Data } \\
\hline Factor $2 \& 4$ & \begin{tabular}{l|}
0.20165 \\
$(0.21205)$
\end{tabular} & $\begin{array}{l}0.20165 \\
(0.21205)\end{array}$ & $\begin{array}{l}0.20897 \\
(0.21596)\end{array}$ & $\begin{array}{l}0.21085 \\
(0.21689)\end{array}$ & $\begin{array}{l}0.13935 \\
(0.20948)\end{array}$ & $\begin{array}{l}0.17716 \\
(0.21116)\end{array}$ & $\begin{array}{l}0.19181 \\
(0.21644)\end{array}$ & $\begin{array}{l}0.20165 \\
(0.21203)\end{array}$ & $\begin{array}{l}0.26056 \\
(0.21030)\end{array}$ & $\begin{array}{l}0.17478 \\
(0.22199)\end{array}$ & $\begin{array}{l}0.14921 \\
(0.21449)\end{array}$ & $\begin{array}{l}0.20165 \\
(0.21182)\end{array}$ & $\begin{array}{l}0.12925 \\
(0.23225)\end{array}$ \\
\hline Factor $2,4 \& 5$ & $\begin{array}{l}-0.00754 \\
(0.35573)\end{array}$ & $\begin{array}{l}-0.00374 \\
(0.40549)\end{array}$ & $\begin{array}{l}0.00577 \\
(0.36234)\end{array}$ & $\begin{array}{l}0.00255 \\
(0.35835)\end{array}$ & $\begin{array}{l}-0.00699 \\
(0.34877)\end{array}$ & $\begin{array}{l}-0.11964 \\
(0.35971)\end{array}$ & $\begin{array}{l}-0.00699 \\
(0.35520)\end{array}$ & $\begin{array}{l}-0.00699 \\
(0.35523)\end{array}$ & $\begin{array}{l}0.12709 \\
(0.35433)\end{array}$ & $\begin{array}{l}-0.00699 \\
(0.35510)\end{array}$ & $\begin{array}{l}-0.03025 \\
(0.35393)\end{array}$ & $\begin{array}{l}-0.06426 \\
(0.36608)\end{array}$ & $\begin{array}{l}-0.00699 \\
(0.35471)\end{array}$ \\
\hline Factor $2 \& 3$ & \multicolumn{13}{|c|}{ Insufficient Data } \\
\hline Factor 2, $3 \& 5$ & \multicolumn{13}{|c|}{ Insufficient Data } \\
\hline Factor $2,3 \& 4$ & $\begin{array}{l}0.23802 \\
(0.33558)\end{array}$ & $\begin{array}{l}0.23908 \\
(0.34154)\end{array}$ & $\begin{array}{l}0.25078 \\
(0.34308)\end{array}$ & $\begin{array}{l}0.24383 \\
(0.33678)\end{array}$ & $\begin{array}{l}0.20851 \\
(0.32964)\end{array}$ & $\begin{array}{l}0.18240 \\
(0.33509)\end{array}$ & $\begin{array}{l}0.23514 \\
(0.33577)\end{array}$ & $\begin{array}{l}0.24002 \\
(0.33577)\end{array}$ & $\begin{array}{l}0.29175 \\
(0.33131)\end{array}$ & $\begin{array}{l}0.22581 \\
(0.33677)\end{array}$ & $\begin{array}{l}0.17575 \\
(0.33705)\end{array}$ & $\begin{array}{l}0.21869 \\
(0.33659)\end{array}$ & $\begin{array}{l}0.22390 \\
(0.33559)\end{array}$ \\
\hline Factor $2,3,4 \& 5$ & $\begin{array}{l}0.24327 \\
(0.20546)\end{array}$ & $\begin{array}{l}0.24830 \\
(0.25589)\end{array}$ & $\begin{array}{l}0.25417 \\
(0.19767)\end{array}$ & $\begin{array}{l}0.24613 \\
(0.19160)\end{array}$ & $\begin{array}{l}0.18501 \\
(0.18946)\end{array}$ & $\begin{array}{l}0.22013 \\
(0.19095)\end{array}$ & $\begin{array}{l}0.24228 \\
(0.19209)\end{array}$ & $\begin{array}{l}0.24548 \\
(0.19158)\end{array}$ & $\begin{array}{l}0.25458 \\
(0.18877)\end{array}$ & $\begin{array}{l}0.23662 \\
(0.19274)\end{array}$ & $\begin{array}{l}0.21379 \\
(0.19207)\end{array}$ & $\begin{array}{l}0.24233 \\
(0.19146)\end{array}$ & $\begin{array}{l}0.20691 \\
(0.19795)\end{array}$ \\
\hline Factor 1 & \begin{tabular}{l|}
0.22921 \\
$(0.27736)$ \\
\end{tabular} & $\begin{array}{l}0.22921 \\
(0.27736) \\
\end{array}$ & $\begin{array}{l}0.23998 \\
(0.28384) \\
\end{array}$ & $\begin{array}{l}0.23056 \\
(0.27741) \\
\end{array}$ & $\begin{array}{l}0.22921 \\
(0.27230) \\
\end{array}$ & $\begin{array}{l}0.19872 \\
(0.27614) \\
\end{array}$ & $\begin{array}{l}0.22655 \\
(0.27757) \\
\end{array}$ & $\begin{array}{l}0.23241 \\
(0.27802) \\
\end{array}$ & $\begin{array}{l}0.27395 \\
(0.27384) \\
\end{array}$ & $\begin{array}{l}0.19597 \\
(0.28901) \\
\end{array}$ & $\begin{array}{l}0.10621 \\
(0.29035) \\
\end{array}$ & $\begin{array}{l}0.21371 \\
(0.27813) \\
\end{array}$ & $\begin{array}{l}0.14553 \\
(0.29811) \\
\end{array}$ \\
\hline Fact & $\begin{array}{l}0.06382 \\
(0.28256)\end{array}$ & $\begin{array}{l}0.06841 \\
(0.34066)\end{array}$ & $\begin{array}{l}0.07792 \\
(0.28797)\end{array}$ & $\begin{array}{l}0.06838 \\
(0.27941)\end{array}$ & $\begin{array}{l}0.04477 \\
(0.27399)\end{array}$ & $\begin{array}{l}-0.00412 \\
(0.28041)\end{array}$ & $\begin{array}{l}0.04809 \\
(0.28898)\end{array}$ & $\begin{array}{l}0.07107 \\
(0.28122)\end{array}$ & $\begin{array}{l}0.19338 \\
(0.27990)\end{array}$ & $\begin{array}{l}0.04193 \\
(0.28463)\end{array}$ & $\begin{array}{l}-0.03021 \\
(0.28628)\end{array}$ & $\begin{array}{l}0.02976 \\
(0.28417)\end{array}$ & $\begin{array}{l}0.01980 \\
(0.28491)\end{array}$ \\
\hline Fact & $\begin{array}{l}0.24875 \\
(0.22137)\end{array}$ & $\begin{array}{l}0.24875 \\
(0.22137)\end{array}$ & $\begin{array}{l}0.25971 \\
(0.22971)\end{array}$ & $\begin{array}{l}0.24875 \\
(0.22135)\end{array}$ & $\begin{array}{l}0.24875 \\
(0.21733)\end{array}$ & $\begin{array}{l}0.23282 \\
(0.22010)\end{array}$ & $\begin{array}{l}0.23168 \\
(0.23386)\end{array}$ & $\begin{array}{l}0.26060 \\
(0.23261)\end{array}$ & $\begin{array}{l}0.24875 \\
(0.21806)\end{array}$ & $\begin{array}{l}0.21650 \\
(0.23503)\end{array}$ & $\begin{array}{l}0.12576 \\
(0.23801)\end{array}$ & $\begin{array}{l}0.19149 \\
(0.23871)\end{array}$ & $\begin{array}{l}0.16508 \\
(0.24704)\end{array}$ \\
\hline Factor 1 & $\begin{array}{l}0.30415 \\
(0.33611) \\
\end{array}$ & $\begin{array}{l}0.30734 \\
(0.37296) \\
\end{array}$ & $\begin{array}{l}0.31740 \\
(0.34320) \\
\end{array}$ & $\begin{array}{l}0.30663 \\
(0.33581) \\
\end{array}$ & $\begin{array}{l}0.30465 \\
(0.32957) \\
\end{array}$ & $\begin{array}{l}0.19199 \\
(0.34068) \\
\end{array}$ & $\begin{array}{l}0.29534 \\
(0.33817) \\
\end{array}$ & $\begin{array}{l}0.31380 \\
(0.34019) \\
\end{array}$ & $\begin{array}{l}0.43236 \\
(0.33489) \\
\end{array}$ & $\begin{array}{l}0.28197 \\
(0.34014) \\
\end{array}$ & $\begin{array}{l}0.18165 \\
(0.34601) \\
\end{array}$ & $\begin{array}{l}0.24738 \\
(0.34718) \\
\end{array}$ & $\begin{array}{l}0.25414 \\
(0.34174) \\
\end{array}$ \\
\hline Factor $1 \& 3$ & \multicolumn{13}{|c|}{ Insufficient Data } \\
\hline Factor $1,3 \& 5$ & $\begin{array}{l}0.40350 \\
(0.32409)\end{array}$ & $\begin{array}{l}0.40819 \\
(0.37536)\end{array}$ & $\begin{array}{l}0.41392 \\
(0.32432)\end{array}$ & $\begin{array}{l}0.41078 \\
(0.32170)\end{array}$ & $\begin{array}{l}0.28190 \\
(0.31793)\end{array}$ & $\begin{array}{l}0.29229 \\
(0.32585)\end{array}$ & $\begin{array}{l}0.39556 \\
(0.32306)\end{array}$ & $\begin{array}{l}0.41028 \\
(0.32201)\end{array}$ & $\begin{array}{l}0.57029^{\ddagger} \\
(0.32299)\end{array}$ & $\begin{array}{l}0.37316 \\
(0.32966)\end{array}$ & $\begin{array}{l}0.31839 \\
(0.32511)\end{array}$ & $\begin{array}{l}0.34768 \\
(0.33247)\end{array}$ & $\begin{array}{l}0.35149 \\
(0.32761)\end{array}$ \\
\hline Factor $1,3 \& 4$ & $\begin{array}{l}0.17959 \\
(0.12211) \\
\end{array}$ & $\begin{array}{l}0.17959 \\
(0.12211) \\
\end{array}$ & $\begin{array}{l}0.19196 \\
(0.14041)\end{array}$ & $\begin{array}{l}0.18080 \\
(0.12224)\end{array}$ & $\begin{array}{l}0.11579 \\
(0.12224)\end{array}$ & $\begin{array}{l}0.13691 \\
(0.12414)\end{array}$ & $\begin{array}{l}0.16376 \\
(0.14072)\end{array}$ & $\begin{array}{l}0.18711 \\
(0.13027)\end{array}$ & $\begin{array}{l}0.24720^{\dagger} \\
(0.12351)\end{array}$ & $\begin{array}{l}0.15989 \\
(0.13129)\end{array}$ & $\begin{array}{l}0.06934 \\
(0.14591)\end{array}$ & $\begin{array}{l}0.12232 \\
(0.15152)\end{array}$ & $\begin{array}{l}0.09591 \\
(0.16443)\end{array}$ \\
\hline Factor $1,3,4 \& 5$ & $\begin{array}{l}0.22659 \\
(0.16142)\end{array}$ & $\begin{array}{l}0.23088 \\
(0.25113)\end{array}$ & $\begin{array}{l}0.23356 \\
(0.16107)\end{array}$ & $\begin{array}{l}0.23053 \\
(0.15826)\end{array}$ & $\begin{array}{l}0.19104 \\
(0.15535)\end{array}$ & $\begin{array}{l}0.14001 \\
(0.16573)\end{array}$ & $\begin{array}{l}0.21205 \\
(0.17200)\end{array}$ & $\begin{array}{l}0.23715 \\
(0.16776)\end{array}$ & $\begin{array}{l}0.35624^{\dagger} \\
(0.16417)\end{array}$ & $\begin{array}{l}0.19572 \\
(0.17597)\end{array}$ & $\begin{array}{l}0.10464 \\
(0.18090)\end{array}$ & $\begin{array}{l}0.17250 \\
(0.17967)\end{array}$ & $\begin{array}{l}0.14395 \\
(0.19220)\end{array}$ \\
\hline Factor $1 \& 2$ & \begin{tabular}{l|}
0.09131 \\
$(0.19623)$
\end{tabular} & $\begin{array}{l}0.09131 \\
(0.19623)\end{array}$ & $\begin{array}{l}0.10265 \\
(0.20625)\end{array}$ & $\begin{array}{l}0.09131 \\
(0.19621)\end{array}$ & $\begin{array}{l}0.09131 \\
(0.19265)\end{array}$ & $\begin{array}{l}-0.02134 \\
(0.20706)\end{array}$ & $\begin{array}{l}0.08942 \\
(0.19638)\end{array}$ & $\begin{array}{l}0.10316 \\
(0.20883)\end{array}$ & $\begin{array}{l}0.10969 \\
(0.19345)\end{array}$ & $\begin{array}{l}0.05792 \\
(0.21260)\end{array}$ & $\begin{array}{l}-0.03168 \\
(0.21507)\end{array}$ & $\begin{array}{l}0.03405 \\
(0.21565)\end{array}$ & $\begin{array}{l}0.01693 \\
(0.21911)\end{array}$ \\
\hline Factor $1,2 \& 5$ & \multicolumn{13}{|c|}{ Insufficient Data } \\
\hline Factor $1,2 \& 4$ & \begin{tabular}{|l|}
-0.02708 \\
$(0.14951)$
\end{tabular} & $\begin{array}{l}-0.02708 \\
(0.14951)\end{array}$ & $\begin{array}{l}-0.02455 \\
(0.15017)\end{array}$ & $\begin{array}{l}-0.02509 \\
(0.14982)\end{array}$ & $\begin{array}{l}-0.04083 \\
(0.14687)\end{array}$ & $\begin{array}{l}-0.12625 \\
(0.16074)\end{array}$ & $\begin{array}{l}-0.03889 \\
(0.15834)\end{array}$ & $\begin{array}{l}-0.02299 \\
(0.15152)\end{array}$ & $\begin{array}{l}0.11452 \\
(0.15855)\end{array}$ & $\begin{array}{l}-0.06295 \\
(0.17348)\end{array}$ & $\begin{array}{l}-0.12429 \\
(0.16495)\end{array}$ & $\begin{array}{l}-0.08435 \\
(0.17432)\end{array}$ & $\begin{array}{l}-0.11076 \\
(0.18563)\end{array}$ \\
\hline Factor $1,2,4 \& 5$ & \begin{tabular}{l|}
0.18748 \\
$(0.22513)$ \\
\end{tabular} & $\begin{array}{l}0.19346 \\
(0.28371) \\
\end{array}$ & $\begin{array}{l}0.20297 \\
(0.21765) \\
\end{array}$ & $\begin{array}{l}0.19436 \\
(0.20660) \\
\end{array}$ & $\begin{array}{l}0.17914 \\
(0.20188) \\
\end{array}$ & $\begin{array}{l}0.10144 \\
(0.21150) \\
\end{array}$ & $\begin{array}{l}0.18087 \\
(0.20967) \\
\end{array}$ & $\begin{array}{l}0.19981 \\
(0.21358) \\
\end{array}$ & $\begin{array}{l}0.32128 \\
(0.20969) \\
\end{array}$ & $\begin{array}{l}0.15502 \\
(0.22293) \\
\end{array}$ & $\begin{array}{l}0.11620 \\
(0.21166) \\
\end{array}$ & $\begin{array}{l}0.15575 \\
(0.21238) \\
\end{array}$ & $\begin{array}{l}0.12204 \\
(0.22410) \\
\end{array}$ \\
\hline Factor $1,2 \& 3$ & $\begin{array}{l}0.36681 \\
(0.47759)\end{array}$ & $\begin{array}{l}0.36681 \\
(0.47759)\end{array}$ & $\begin{array}{l}0.37117 \\
(0.47818)\end{array}$ & $\begin{array}{l}0.37083 \\
(0.47796)\end{array}$ & $\begin{array}{l}0.30705 \\
(0.46941)\end{array}$ & $\begin{array}{l}0.29267 \\
(0.47659)\end{array}$ & $\begin{array}{l}0.34974 \\
(0.48346)\end{array}$ & $\begin{array}{l}0.37461 \\
(0.47987)\end{array}$ & $\begin{array}{l}0.53216 \\
(0.47542)\end{array}$ & $\begin{array}{l}0.34209 \\
(0.48123)\end{array}$ & $\begin{array}{l}0.24382 \\
(0.48374)\end{array}$ & $\begin{array}{l}0.30955 \\
(0.48548)\end{array}$ & $\begin{array}{l}0.31174 \\
(0.48237)\end{array}$ \\
\hline Factor $1,2,3 \& 5$ & $\begin{array}{l}0.31021 \\
(0.22213)\end{array}$ & $\begin{array}{l}0.31489 \\
(0.29195)\end{array}$ & $\begin{array}{l}0.32108 \\
(0.22316)\end{array}$ & $\begin{array}{l}0.31553 \\
(0.21766)\end{array}$ & $\begin{array}{l}0.28701 \\
(0.21306)\end{array}$ & $\begin{array}{l}0.24360 \\
(0.21946)\end{array}$ & $\begin{array}{l}0.30299 \\
(0.22015)\end{array}$ & $\begin{array}{l}0.31878 \\
(0.22104)\end{array}$ & $\begin{array}{l}0.41524^{\ddagger} \\
(0.21786)\end{array}$ & $\begin{array}{l}0.29056 \\
(0.22283)\end{array}$ & $\begin{array}{l}0.19732 \\
(0.23145)\end{array}$ & $\begin{array}{l}0.25438 \\
(0.23450)\end{array}$ & $\begin{array}{l}0.26467 \\
(0.22518)\end{array}$ \\
\hline Factor $1,2,3 \& 4$ & $\begin{array}{l}0.31155^{*} \\
(0.07095)\end{array}$ & $\begin{array}{l}0.31192^{*} \\
(0.07428)\end{array}$ & $\begin{array}{l}0.32252^{*} \\
(0.09386)\end{array}$ & $\begin{array}{l}0.31842^{*} \\
(0.07870)\end{array}$ & $\begin{array}{l}0.26560^{*} \\
(0.07175)\end{array}$ & $\begin{array}{l}0.21477^{\dagger} \\
(0.09259)\end{array}$ & $\begin{array}{l}0.29852^{*} \\
(0.09138)\end{array}$ & $\begin{array}{l}0.32173^{*} \\
(0.09383)\end{array}$ & $\begin{array}{l}0.43841^{*} \\
(0.08747)\end{array}$ & $\begin{array}{l}0.27503^{\dagger} \\
(0.11435)\end{array}$ & $\begin{array}{l}0.21487^{\dagger} \\
(0.10001)\end{array}$ & $\begin{array}{l}0.26020^{\dagger} \\
(0.10734)\end{array}$ & $\begin{array}{l}0.23121^{\ddagger} \\
(0.12743)\end{array}$ \\
\hline All Factors & $\begin{array}{l}0.32966^{*} \\
(0.09016)\end{array}$ & $\begin{array}{l}0.33518^{\ddagger} \\
(0.20141)\end{array}$ & $\begin{array}{l}0.34261^{*} \\
(0.07698)\end{array}$ & $\begin{array}{l}0.33705^{*} \\
(0.05469)\end{array}$ & $\begin{array}{l}0.25013^{*} \\
(0.05655)\end{array}$ & $\begin{array}{l}0.24404^{*} \\
(0.07272)\end{array}$ & $\begin{array}{l}0.31777^{*} \\
(0.07914)\end{array}$ & $\begin{array}{l}0.34050^{*} \\
(0.07081)\end{array}$ & $\begin{array}{l}0.46491^{*} \\
(0.07291)\end{array}$ & $\begin{array}{l}0.29583^{*} \\
(0.10102)\end{array}$ & $\begin{array}{l}0.22003^{\dagger} \\
(0.09510)\end{array}$ & $\begin{array}{l}0.28155^{*} \\
(0.09270)\end{array}$ & $\begin{array}{l}0.24979^{\dagger} \\
(0.11860)\end{array}$ \\
\hline $\begin{array}{l}\text { Individual } \\
\text { Variable }\end{array}$ & $\begin{array}{l}0.00292 \\
(0.09793)\end{array}$ & $\begin{array}{l}-0.00325 \\
(0.19551)\end{array}$ & $\begin{array}{l}-0.01276 \\
(0.07149)\end{array}$ & $\begin{array}{l}-0.01176 \\
(0.05837)\end{array}$ & $\begin{array}{l}0.17484^{*} \\
(0.06547)\end{array}$ & $\begin{array}{l}0.11266 \\
(0.06991)\end{array}$ & $\begin{array}{l}0.01708 \\
(0.07549)\end{array}$ & $\begin{array}{l}-0.01185 \\
(0.07148)\end{array}$ & $\begin{array}{l}-0.16535^{\dagger} \\
(0.06857)\end{array}$ & $\begin{array}{l}0.03757 \\
(0.09226)\end{array}$ & $\begin{array}{l}0.12299 \\
(0.09011)\end{array}$ & $\begin{array}{l}0.05727 \\
(0.08989)\end{array}$ & $\begin{array}{l}0.08368 \\
(0.11033)\end{array}$ \\
\hline
\end{tabular}

* Significant at the 0.01 level $†$ Significant at the 0.05 level $\$$ Significant at the 0.10 level

Table 95 Coefficients with individual variables for high-level factors - Electronics Industry 


\begin{tabular}{|c|c|c|c|c|c|c|c|c|c|c|c|c|c|}
\hline $\begin{array}{l}\text { Individual variable } \\
\text { included in the } \\
\text { model in the } \\
\text { column }\end{array}$ & 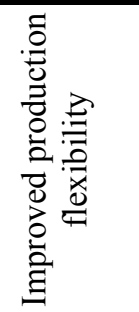 & 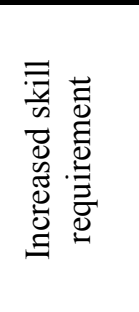 & 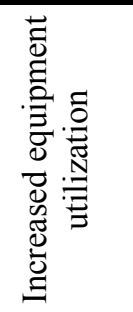 & 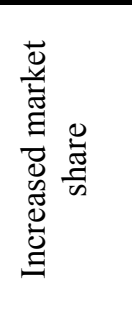 & 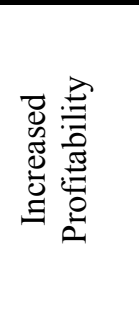 & 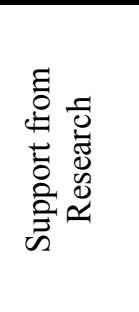 & 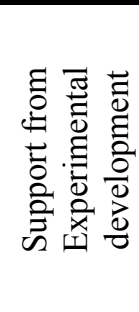 & 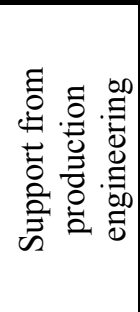 & 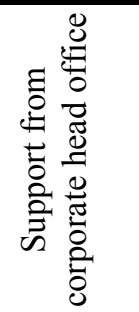 & 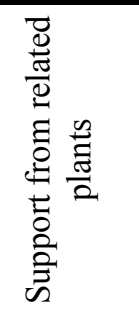 & 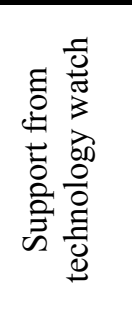 & 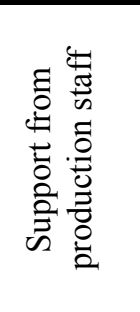 & 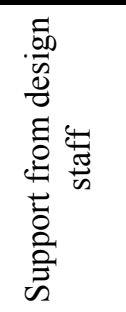 \\
\hline No factors & $\begin{array}{l}0.18431^{*} \\
(0.06139)\end{array}$ & $\begin{array}{l}0.18515^{*} \\
(0.06064)\end{array}$ & $\begin{array}{l}0.18353^{*} \\
(0.06102)\end{array}$ & $\begin{array}{l}0.17618^{*} \\
(0.06142)\end{array}$ & $\begin{array}{l}0.18347^{*} \\
(0.06155)\end{array}$ & $\begin{array}{l}0.18373^{*} \\
(0.06154)\end{array}$ & $\begin{array}{l}0.18353^{*} \\
(0.06150)\end{array}$ & $\begin{array}{l}0.17800^{*} \\
(0.06079)\end{array}$ & $\begin{array}{l}0.19408^{*} \\
(0.06131)\end{array}$ & $\begin{array}{l}0.18259^{*} \\
(0.06156)\end{array}$ & $\begin{array}{l}0.18353^{*} \\
(0.06145)\end{array}$ & $\begin{array}{l}0.18170^{*} \\
(0.06177)\end{array}$ & $\begin{array}{l}0.18419^{*} \\
(0.06152)\end{array}$ \\
\hline Factor 5 & $\begin{array}{l}0.34886 \\
(0.22661)\end{array}$ & $\begin{array}{l}0.56017^{\dagger} \\
(0.24054)\end{array}$ & $\begin{array}{l}0.34886 \\
(0.22526)\end{array}$ & $\begin{array}{l}0.34886 \\
(0.22598)\end{array}$ & $\begin{array}{l}0.34886 \\
(0.22722)\end{array}$ & $\begin{array}{l}0.34886 \\
(0.22717)\end{array}$ & $\begin{array}{l}0.34886 \\
(0.22706)\end{array}$ & $\begin{array}{l}0.34886 \\
(0.22423)\end{array}$ & $\begin{array}{l}0.34886 \\
(0.22531)\end{array}$ & $\begin{array}{l}0.34886 \\
(0.22711)\end{array}$ & $\begin{array}{l}0.34886 \\
(0.22686)\end{array}$ & $\begin{array}{l}0.34886 \\
(0.22716)\end{array}$ & $\begin{array}{l}0.34886 \\
(0.22707)\end{array}$ \\
\hline Factor 4 & $\begin{array}{l}0.18385 \\
(0.34052)\end{array}$ & $\begin{array}{l}0.20233 \\
(0.33396)\end{array}$ & $\begin{array}{l}0.13279 \\
(0.33479)\end{array}$ & $\begin{array}{l}0.11535 \\
(0.33608)\end{array}$ & $\begin{array}{l}0.12911 \\
(0.33882)\end{array}$ & $\begin{array}{l}0.14162 \\
(0.33895)\end{array}$ & $\begin{array}{l}0.13979 \\
(0.33772)\end{array}$ & $\begin{array}{l}-0.07739 \\
(0.34600)\end{array}$ & $\begin{array}{l}0.18638 \\
(0.33618)\end{array}$ & $\begin{array}{l}0.11891 \\
(0.33908)\end{array}$ & $\begin{array}{l}0.13279 \\
(0.33716)\end{array}$ & $\begin{array}{l}0.12465 \\
(0.33848)\end{array}$ & $\begin{array}{l}0.15731 \\
(0.34086)\end{array}$ \\
\hline Factor $4 \& 5$ & $\begin{array}{l}0.47779 \\
(0.57823)\end{array}$ & $\begin{array}{l}0.42672 \\
(0.56905)\end{array}$ & $\begin{array}{l}0.42672 \\
(0.57262)\end{array}$ & $\begin{array}{l}.42672 \\
(0.57446)\end{array}$ & $\begin{array}{l}0.42672 \\
(0.57759)\end{array}$ & $\begin{array}{l}0.42672 \\
(0.57748)\end{array}$ & $\begin{array}{l}0.42672 \\
(0.57719)\end{array}$ & $\begin{array}{l}0.21654 \\
(0.57754)\end{array}$ & $\begin{array}{l}0.42672 \\
(0.57274)\end{array}$ & $\begin{array}{l}0.42672 \\
(0.57733)\end{array}$ & $\begin{array}{l}.45791 \\
(0.57805)\end{array}$ & $\begin{array}{l}0.41435 \\
(0.57862)\end{array}$ & $\begin{array}{l}0.45125 \\
(0.57920)\end{array}$ \\
\hline Factor 3 & $\begin{array}{l}-0.07074 \\
(0.14782)\end{array}$ & $\begin{array}{l}-0.12446 \\
(0.10901) \\
\end{array}$ & $\begin{array}{l}-0.17286 \\
(0.10781)\end{array}$ & $\begin{array}{l}-0.25152^{\dagger} \\
(0.12105)\end{array}$ & $\begin{array}{l}-0.17286 \\
(0.10874)\end{array}$ & $\begin{array}{l}-0.17286 \\
(0.10872)\end{array}$ & $\begin{array}{l}-0.17286 \\
(0.10867)\end{array}$ & $\begin{array}{c}-0.33490^{\dagger} \\
(0.12906)\end{array}$ & $\begin{array}{l}-0.14831 \\
(0.10869)\end{array}$ & $\begin{array}{l}-0.20063 \\
(0.12632)\end{array}$ & $\begin{array}{l}-0.17286 \\
(0.10857)\end{array}$ & $\begin{array}{l}-0.19760 \\
(0.13138)\end{array}$ & $\begin{array}{l}-0.17286 \\
(0.10867)\end{array}$ \\
\hline Factor & $\begin{array}{l}0.19642 \\
(0.35286)\end{array}$ & $\begin{array}{l}0.28625 \\
(0.34653)\end{array}$ & $\begin{array}{l}0.22033 \\
(0.34611)\end{array}$ & $\begin{array}{l}0.02847 \\
(0.34699)\end{array}$ & $\begin{array}{l}0.10322 \\
(0.35084)\end{array}$ & $\begin{array}{l}0.13823 \\
(0.35484)\end{array}$ & $\begin{array}{l}0.11967 \\
(0.34401)\end{array}$ & $\begin{array}{l}0.07514 \\
(0.33985)\end{array}$ & $\begin{array}{l}0.11241 \\
(0.34108)\end{array}$ & $\begin{array}{l}0.10748 \\
(0.34401)\end{array}$ & $\begin{array}{l}0.12347 \\
(0.34372)\end{array}$ & $\begin{array}{l}0.08767 \\
(0.35171)\end{array}$ & $\begin{array}{l}0.12110 \\
(0.34417)\end{array}$ \\
\hline Factor 3 & $\begin{array}{l}0.21192 \\
(0.23859)\end{array}$ & $\begin{array}{l}0.25227 \\
(0.23629)\end{array}$ & $\begin{array}{l}0.22709 \\
(0.23732)\end{array}$ & $\begin{array}{l}0.19244 \\
(0.23831)\end{array}$ & $\begin{array}{l}0.21192 \\
(0.23923)\end{array}$ & $\begin{array}{l}0.22283 \\
(0.24202)\end{array}$ & $\begin{array}{l}0.25287 \\
(0.25137)\end{array}$ & $\begin{array}{l}0.00174 \\
(0.25375)\end{array}$ & $\begin{array}{l}0.22431 \\
(0.23732)\end{array}$ & $\begin{array}{l}0.20341 \\
(0.23994)\end{array}$ & $\begin{array}{l}0.21913 \\
(0.23903)\end{array}$ & $\begin{array}{l}0.19191 \\
(0.24651)\end{array}$ & $\begin{array}{l}0.26097 \\
(0.25753)\end{array}$ \\
\hline Factor $3,4 \& 5$ & $\begin{array}{l}0.30109^{\ddagger} \\
(0.17813)\end{array}$ & $\begin{array}{l}0.41028^{\dagger} \\
(0.16990)\end{array}$ & $\begin{array}{l}0.19897 \\
(0.14624)\end{array}$ & $\begin{array}{l}0.19897 \\
(0.14671)\end{array}$ & $\begin{array}{l}0.19897 \\
(0.14751)\end{array}$ & $\begin{array}{l}0.22480 \\
(0.17144)\end{array}$ & $\begin{array}{l}0.23992 \\
(0.16662)\end{array}$ & $\begin{array}{l}-0.01121 \\
(0.17274)\end{array}$ & $\begin{array}{l}0.19897 \\
(0.14627)\end{array}$ & $\begin{array}{l}0.19897 \\
(0.14744)\end{array}$ & $\begin{array}{l}0.19897 \\
(0.14728)\end{array}$ & $\begin{array}{l}0.17423 \\
(0.16490)\end{array}$ & $\begin{array}{l}0.24801 \\
(0.17578)\end{array}$ \\
\hline Factor 2 & $\begin{array}{l}0.34304^{\ddagger} \\
(0.19572)\end{array}$ & $\begin{array}{l}0.34304^{\ddagger} \\
(0.19334)\end{array}$ & $\begin{array}{l}0.43156^{\dagger} \\
(0.20051)\end{array}$ & $\begin{array}{l}0.34304^{\ddagger} \\
(0.19518)\end{array}$ & $\begin{array}{l}0.34304^{\ddagger} \\
(0.19624)\end{array}$ & $\begin{array}{l}0.34304^{\ddagger} \\
(0.19620)\end{array}$ & $\begin{array}{l}0.34623^{\ddagger} \\
(0.19620)\end{array}$ & $\begin{array}{l}0.32669^{\ddagger} \\
(0.19380)\end{array}$ & $\begin{array}{l}0.35138^{\ddagger} \\
(0.19465)\end{array}$ & $\begin{array}{l}0.34088^{\ddagger} \\
(0.19622)\end{array}$ & $\begin{array}{l}0.34304^{\ddagger} \\
(0.19593)\end{array}$ & $\begin{array}{l}0.34112^{\ddagger} \\
(0.19627)\end{array}$ & $\begin{array}{l}0.34685^{\ddagger} \\
(0.19625)\end{array}$ \\
\hline Factor $2 \& 5$ & \multicolumn{13}{|c|}{ Insufficient Data } \\
\hline Factor $2 \& 4$ & $\begin{array}{l}0.27427 \\
(0.22321)\end{array}$ & $\begin{array}{l}0.24759 \\
(0.20978)\end{array}$ & $\begin{array}{l}0.29493 \\
(0.21635)\end{array}$ & $\begin{array}{l}0.15815 \\
(0.21302)\end{array}$ & $\begin{array}{l}0.19521 \\
(0.21749)\end{array}$ & $\begin{array}{l}0.20552 \\
(0.21241)\end{array}$ & $\begin{array}{l}0.20779 \\
(0.21221)\end{array}$ & $\begin{array}{l}0.14179 \\
(0.21093)\end{array}$ & $\begin{array}{l}0.28553 \\
(0.21535)\end{array}$ & $\begin{array}{l}0.18794 \\
(0.21432)\end{array}$ & $\begin{array}{l}0.20165 \\
(0.21171)\end{array}$ & $\begin{array}{l}0.18062 \\
(0.22107)\end{array}$ & $\begin{array}{l}0.21231 \\
(0.21292)\end{array}$ \\
\hline Factor $2,4 \& 5$ & $\begin{array}{l}0.09514 \\
(0.36825)\end{array}$ & $\begin{array}{l}0.20432 \\
(0.36088)\end{array}$ & $\begin{array}{l}0.09939 \\
(0.35698)\end{array}$ & $\begin{array}{l}-0.08972 \\
(0.35791)\end{array}$ & $\begin{array}{l}-0.00699 \\
(0.35523)\end{array}$ & $\begin{array}{l}-0.00699 \\
(0.35517)\end{array}$ & $\begin{array}{l}0.03396 \\
(0.36339)\end{array}$ & $\begin{array}{l}-0.21717 \\
(0.36270)\end{array}$ & $\begin{array}{l}-0.00699 \\
(0.35225)\end{array}$ & $\begin{array}{l}-0.01224 \\
(0.35529)\end{array}$ & $\begin{array}{l}0.05539 \\
(0.36349)\end{array}$ & $\begin{array}{l}-0.03173 \\
(0.36273)\end{array}$ & $\begin{array}{l}0.04205 \\
(0.36769)\end{array}$ \\
\hline Factor $2 \& 3$ & \multicolumn{13}{|c|}{ Insufficient Data } \\
\hline Factor $2,3 \& 5$ & \multicolumn{13}{|c|}{ Insufficient Data } \\
\hline Factor $2,3 \& 4$ & $\begin{array}{l}0.23802 \\
(0.33467)\end{array}$ & $\begin{array}{l}0.27368 \\
(0.33093)\end{array}$ & $\begin{array}{l}0.28065 \\
(0.33350)\end{array}$ & $\begin{array}{l}0.18765 \\
(0.33556)\end{array}$ & $\begin{array}{l}0.23425 \\
(0.33676)\end{array}$ & $\begin{array}{l}0.25513 \\
(0.34046)\end{array}$ & $\begin{array}{l}0.25184 \\
(0.33636)\end{array}$ & $\begin{array}{l}0.02784 \\
(0.34397)\end{array}$ & $\begin{array}{l}0.29229 \\
(0.33410)\end{array}$ & $\begin{array}{l}0.21494 \\
(0.33965)\end{array}$ & $\begin{array}{l}0.24855 \\
(0.33530)\end{array}$ & $\begin{array}{l}0.22163 \\
(0.33902)\end{array}$ & $\begin{array}{l}0.28706 \\
(0.34875)\end{array}$ \\
\hline Factor $2,3,4 \& 5$ & $\begin{array}{l}0.26234 \\
(0.19179)\end{array}$ & $\begin{array}{l}0.29994 \\
(0.19011)\end{array}$ & $\begin{array}{l}0.28364 \\
(0.19109)\end{array}$ & $\begin{array}{l}0.21019 \\
(0.19210)\end{array}$ & $\begin{array}{l}0.24285 \\
(0.19261)\end{array}$ & $\begin{array}{l}0.25926 \\
(0.19714)\end{array}$ & $\begin{array}{l}0.26958 \\
(0.19683)\end{array}$ & $\begin{array}{l}0.10235 \\
(0.19940)\end{array}$ & $\begin{array}{l}0.27310 \\
(0.19059)\end{array}$ & $\begin{array}{l}0.23986 \\
(0.19195)\end{array}$ & $\begin{array}{l}0.27460 \\
(0.19486)\end{array}$ & $\begin{array}{l}0.22985 \\
(0.19713)\end{array}$ & $\begin{array}{l}0.27888 \\
(0.20226)\end{array}$ \\
\hline Factor 1 & $\begin{array}{l}0.25684 \\
(0.27794)\end{array}$ & $\begin{array}{l}0.44052 \\
(0.28707)\end{array}$ & $\begin{array}{l}0.36039 \\
(0.28422)\end{array}$ & $\begin{array}{l}0.12718 \\
(0.28472)\end{array}$ & $\begin{array}{l}0.22618 \\
(0.27828)\end{array}$ & $\begin{array}{l}0.25102 \\
(0.28696)\end{array}$ & $\begin{array}{l}0.22921 \\
(0.27716)\end{array}$ & $\begin{array}{l}0.07591 \\
(0.28199)\end{array}$ & $\begin{array}{l}0.24586 \\
(0.27518)\end{array}$ & $\begin{array}{l}0.22600 \\
(0.27733)\end{array}$ & $\begin{array}{l}0.22921 \\
(0.27691)\end{array}$ & $\begin{array}{l}0.22536 \\
(0.27752)\end{array}$ & $\begin{array}{l}0.26498 \\
(0.28583)\end{array}$ \\
\hline $\mathrm{Fa}$ & $\begin{array}{l}0.16729 \\
(0.29580)\end{array}$ & $\begin{array}{l}0.22718 \\
(0.28300)\end{array}$ & $\begin{array}{l}0.17573 \\
(0.28313)\end{array}$ & $\begin{array}{l}-0.03687 \\
(0.28628)\end{array}$ & $\begin{array}{l}0.05399 \\
(0.29133)\end{array}$ & $\begin{array}{l}0.08519 \\
(0.28703)\end{array}$ & $\begin{array}{l}0.08391 \\
(0.28103)\end{array}$ & $\begin{array}{l}-0.01507 \\
(0.27758)\end{array}$ & $\begin{array}{l}0.17234 \\
(0.28294)\end{array}$ & $\begin{array}{l}0.04064 \\
(0.28458)\end{array}$ & $\begin{array}{l}0.06516 \\
(0.27853)\end{array}$ & $\begin{array}{l}0.05008 \\
(0.28250)\end{array}$ & $\begin{array}{l}0.09532 \\
(0.28494)\end{array}$ \\
\hline Factor & $\begin{array}{l}0.24875 \\
(0.22077) \\
\end{array}$ & $\begin{array}{l}0.43018^{\ddagger} \\
(0.23081) \\
\end{array}$ & $\begin{array}{l}0.24875 \\
(0.21946) \\
\end{array}$ & $\begin{array}{l}0.14672 \\
(0.23118) \\
\end{array}$ & $\begin{array}{l}0.23758 \\
(0.23675) \\
\end{array}$ & $\begin{array}{l}0.27093 \\
(0.23370) \\
\end{array}$ & $\begin{array}{l}0.28970 \\
(0.23445) \\
\end{array}$ & $\begin{array}{l}0.03857 \\
(0.23743) \\
\end{array}$ & $\begin{array}{l}0.24875 \\
(0.21950) \\
\end{array}$ & $\begin{array}{l}0.24875 \\
(0.22126) \\
\end{array}$ & $\begin{array}{l}0.25757 \\
(0.22130) \\
\end{array}$ & $\begin{array}{l}0.22401 \\
(0.23328) \\
\end{array}$ & $\begin{array}{l}0.25569 \\
(0.22163) \\
\end{array}$ \\
\hline Factor 1 & $\begin{array}{l}0.36029 \\
(0.33923)\end{array}$ & $\begin{array}{l}0.43218 \\
(0.33496)\end{array}$ & $\begin{array}{l}0.35397 \\
(0.33390)\end{array}$ & $\begin{array}{l}0.22583 \\
(0.33828)\end{array}$ & $\begin{array}{l}0.29536 \\
(0.34286)\end{array}$ & $\begin{array}{l}0.31872 \\
(0.33899)\end{array}$ & $\begin{array}{l}0.32696 \\
(0.33811)\end{array}$ & $\begin{array}{l}0.17349 \\
(0.33632)\end{array}$ & $\begin{array}{l}0.30465 \\
(0.33287)\end{array}$ & $\begin{array}{l}0.28789 \\
(0.33778)\end{array}$ & $\begin{array}{l}0.32810 \\
(0.33649)\end{array}$ & $\begin{array}{l}0.29339 \\
(0.33728)\end{array}$ & $\begin{array}{l}0.35369 \\
(0.34886)\end{array}$ \\
\hline Factor $1 \& 3$ & \multicolumn{13}{|c|}{ Insufficient Data } \\
\hline Factor $1,3 \& 5$ & $\begin{array}{l}0.50707 \\
(0.33497)\end{array}$ & $\begin{array}{l}0.55365^{\ddagger} \\
(0.32169)\end{array}$ & $\begin{array}{l}0.53613 \\
(0.32570)\end{array}$ & $\begin{array}{l}0.30292 \\
(0.32638)\end{array}$ & $\begin{array}{l}0.39549 \\
(0.32820)\end{array}$ & $\begin{array}{l}0.42312 \\
(0.32621)\end{array}$ & $\begin{array}{l}0.44590 \\
(0.32948)\end{array}$ & $\begin{array}{l}0.19476 \\
(0.32960)\end{array}$ & $\begin{array}{l}0.51213 \\
(0.32323)\end{array}$ & $\begin{array}{l}0.38541 \\
(0.32346)\end{array}$ & $\begin{array}{l}0.43925 \\
(0.32288)\end{array}$ & $\begin{array}{l}0.38021 \\
(0.32872)\end{array}$ & $\begin{array}{l}0.43946 \\
(0.32722)\end{array}$ \\
\hline Factor $1,3 \& 4$ & $\begin{array}{l}0.26673^{\ddagger} \\
(0.14891)\end{array}$ & $\begin{array}{l}0.39090^{*} \\
(0.14906)\end{array}$ & $\begin{array}{l}0.22928^{\ddagger} \\
(0.12408)\end{array}$ & $\begin{array}{l}0.13182 \\
(0.12585)\end{array}$ & $\begin{array}{l}0.17210 \\
(0.13445)\end{array}$ & $\begin{array}{l}0.20098 \\
(0.14195)\end{array}$ & $\begin{array}{l}0.21506 \\
(0.13934)\end{array}$ & $\begin{array}{l}-0.00881 \\
(0.14652)\end{array}$ & $\begin{array}{l}0.21369^{\ddagger} \\
(0.12255)\end{array}$ & $\begin{array}{l}0.17959 \\
(0.12205)\end{array}$ & $\begin{array}{l}0.17959 \\
(0.12191)\end{array}$ & $\begin{array}{l}0.16091 \\
(0.13418)\end{array}$ & $\begin{array}{l}0.22863 \\
(0.15510)\end{array}$ \\
\hline Factor $1,3,4 \& 5$ & $\begin{array}{l}0.32244^{\ddagger} \\
(0.18277)\end{array}$ & $\begin{array}{l}0.42379^{\dagger} \\
(0.17547)\end{array}$ & $\begin{array}{l}0.32967^{\dagger} \\
(0.16597)\end{array}$ & $\begin{array}{l}0.12940 \\
(0.17083)\end{array}$ & $\begin{array}{l}0.21646 \\
(0.17859)\end{array}$ & $\begin{array}{l}0.23963 \\
(0.16273)\end{array}$ & $\begin{array}{l}0.24078 \\
(0.15947)\end{array}$ & $\begin{array}{l}0.03252 \\
(0.17790)\end{array}$ & $\begin{array}{l}0.29672^{\ddagger} \\
(0.16092)\end{array}$ & $\begin{array}{l}0.21836 \\
(0.15901)\end{array}$ & $\begin{array}{l}0.22763 \\
(0.15737)\end{array}$ & $\begin{array}{l}0.20473 \\
(0.17174)\end{array}$ & $\begin{array}{l}0.26824 \\
(0.17634)\end{array}$ \\
\hline Factor $1 \& 2$ & $\begin{array}{l}0.18209 \\
(0.21510)\end{array}$ & $\begin{array}{l}0.30262 \\
(0.21241)\end{array}$ & $\begin{array}{l}0.20792 \\
(0.20477)\end{array}$ & $\begin{array}{l}-0.01071 \\
(0.20751)\end{array}$ & $\begin{array}{l}0.08014 \\
(0.21344)\end{array}$ & $\begin{array}{l}0.11714 \\
(0.21478)\end{array}$ & $\begin{array}{l}0.09131 \\
(0.19609)\end{array}$ & $\begin{array}{l}0.09131 \\
(0.19365)\end{array}$ & $\begin{array}{l}0.19850 \\
(0.20346)\end{array}$ & $\begin{array}{l}0.09131 \\
(0.19614)\end{array}$ & $\begin{array}{l}0.09131 \\
(0.19592)\end{array}$ & $\begin{array}{l}0.08857 \\
(0.19635)\end{array}$ & $\begin{array}{l}0.09131 \\
(0.19610)\end{array}$ \\
\hline Factor $1,2 \& 5$ & \multicolumn{13}{|c|}{ Insufficient Data } \\
\hline Factor $1,2 \& 4$ & $\begin{array}{l}0.06167 \\
(0.17277)\end{array}$ & $\begin{array}{l}0.15894 \\
(0.16642)\end{array}$ & $\begin{array}{l}0.09227 \\
(0.16202)\end{array}$ & $\begin{array}{l}-0.11414 \\
(0.16041)\end{array}$ & $\begin{array}{l}-0.03468 \\
(0.16004)\end{array}$ & $\begin{array}{l}-0.01784 \\
(0.15271)\end{array}$ & $\begin{array}{l}-0.01831 \\
(0.15032)\end{array}$ & $\begin{array}{l}-0.13360 \\
(0.15488)\end{array}$ & $\begin{array}{l}0.03741 \\
(0.15250)\end{array}$ & $\begin{array}{l}-0.04013 \\
(0.15247)\end{array}$ & $\begin{array}{l}-0.02426 \\
(0.14931)\end{array}$ & $\begin{array}{l}-0.03116 \\
(0.14996)\end{array}$ & $\begin{array}{l}-0.00927 \\
(0.15340)\end{array}$ \\
\hline Factor 1 , & $\begin{array}{l}0.25029 \\
(0.21338) \\
\end{array}$ & $\begin{array}{l}0.40152^{\ddagger} \\
(0.22084)\end{array}$ & $\begin{array}{l}0.27758 \\
(0.20937) \\
\end{array}$ & $\begin{array}{l}0.11580 \\
(0.21084) \\
\end{array}$ & $\begin{array}{l}0.18206 \\
(0.21451) \\
\end{array}$ & $\begin{array}{l}0.21076 \\
(0.21700) \\
\end{array}$ & $\begin{array}{l}0.22020 \\
(0.21317) \\
\end{array}$ & $\begin{array}{l}-0.00666 \\
(0.22080) \\
\end{array}$ & $\begin{array}{l}0.20379 \\
(0.20400) \\
\end{array}$ & $\begin{array}{l}0.18608 \\
(0.20572) \\
\end{array}$ & $\begin{array}{l}0.20207 \\
(0.20582) \\
\end{array}$ & $\begin{array}{l}0.17421 \\
(0.21100) \\
\end{array}$ & $\begin{array}{l}0.23615 \\
(0.22417) \\
\end{array}$ \\
\hline Factor $1,2 \& 3$ & $\begin{array}{l}0.40172 \\
(0.47754)\end{array}$ & $\begin{array}{l}0.57812 \\
(0.47867)\end{array}$ & $\begin{array}{l}0.41166 \\
(0.47410)\end{array}$ & $\begin{array}{l}0.33194 \\
(0.47560)\end{array}$ & $\begin{array}{l}0.35564 \\
(0.48490)\end{array}$ & $\begin{array}{l}0.37564 \\
(0.47842)\end{array}$ & $\begin{array}{l}0.38081 \\
(0.47798)\end{array}$ & $\begin{array}{l}0.29497 \\
(0.47237)\end{array}$ & $\begin{array}{l}0.47400 \\
(0.47728)\end{array}$ & $\begin{array}{l}0.33905 \\
(0.48168)\end{array}$ & $\begin{array}{l}0.36681 \\
(0.47682)\end{array}$ & $\begin{array}{l}0.34207 \\
(0.48312)\end{array}$ & $\begin{array}{l}0.38358 \\
(0.47839)\end{array}$ \\
\hline Factor $1,2,3 \& 5$ & $\begin{array}{l}0.37617^{\ddagger} \\
(0.22535)\end{array}$ & $\begin{array}{l}0.47423^{\dagger} \\
(0.22408)\end{array}$ & $\begin{array}{l}0.40334^{\ddagger} \\
(0.22075)\end{array}$ & $\begin{array}{l}0.24053 \\
(0.22117) \\
\end{array}$ & $\begin{array}{l}0.30126 \\
(0.23044)\end{array}$ & $\begin{array}{l}0.33320 \\
(0.22872)\end{array}$ & $\begin{array}{l}0.34294 \\
(0.22465)\end{array}$ & $\begin{array}{l}0.17844 \\
(0.22194)\end{array}$ & $\begin{array}{l}0.39093^{\ddagger} \\
(0.21944)\end{array}$ & $\begin{array}{l}0.29668 \\
(0.21947)\end{array}$ & $\begin{array}{l}0.32349 \\
(0.21700)\end{array}$ & $\begin{array}{l}0.28925 \\
(0.22681)\end{array}$ & $\begin{array}{l}0.34712 \\
(0.22747)\end{array}$ \\
\hline Factor $1,2,3 \& 4$ & $\begin{array}{l}0.41110^{*} \\
(0.12080)\end{array}$ & $\begin{array}{l}0.48020^{*} \\
(0.09910)\end{array}$ & $\begin{array}{l}0.41854^{*} \\
(0.09158)\end{array}$ & $\begin{array}{l}0.23432^{*} \\
(0.08848)\end{array}$ & $\begin{array}{l}0.30143^{*} \\
(0.10404)\end{array}$ & $\begin{array}{l}0.33692^{*} \\
(0.11138)\end{array}$ & $\begin{array}{l}0.34393^{*} \\
(0.09379)\end{array}$ & $\begin{array}{l}0.10137 \\
(0.11641)\end{array}$ & $\begin{array}{l}0.37177^{*} \\
(0.07788)\end{array}$ & $\begin{array}{l}0.29624^{*} \\
(0.07931)\end{array}$ & $\begin{array}{l}0.32062^{*} \\
(0.07177)\end{array}$ & $\begin{array}{l}0.29168^{*} \\
(0.09243)\end{array}$ & $\begin{array}{l}0.35627^{*} \\
(0.11246)\end{array}$ \\
\hline All Factors & $\begin{array}{l}0.42212^{*} \\
(0.10099)\end{array}$ & $\begin{array}{l}0.53767^{*} \\
(0.09808)\end{array}$ & $\begin{array}{l}0.41960^{*} \\
(0.06793)\end{array}$ & $\begin{array}{l}0.24211^{*} \\
(0.07857)\end{array}$ & $\begin{array}{l}0.32203^{*} \\
(0.08879)\end{array}$ & $\begin{array}{l}0.35345^{*} \\
(0.08746)\end{array}$ & $\begin{array}{l}0.36303^{*} \\
(0.07629)\end{array}$ & $\begin{array}{l}0.12838 \\
(0.10196)\end{array}$ & $\begin{array}{l}0.39700^{*} \\
(0.06007)\end{array}$ & $\begin{array}{l}0.32211^{*} \\
(0.05349)\end{array}$ & $\begin{array}{l}0.35957^{*} \\
(0.05983)\end{array}$ & $\begin{array}{l}0.31042^{*} \\
(0.08036)\end{array}$ & $\begin{array}{l}0.37959^{*} \\
(0.10487)\end{array}$ \\
\hline $\begin{array}{l}\text { Individual } \\
\text { Variable }\end{array}$ & $\begin{array}{l}-0.10213 \\
(0.10044)\end{array}$ & $\begin{array}{c}-0.21131^{\dagger} \\
(0.08801)\end{array}$ & $\begin{array}{l}-0.13119^{\ddagger} \\
(0.07192)\end{array}$ & $\begin{array}{l}0.10203 \\
(0.07052)\end{array}$ & $\begin{array}{l}0.01117 \\
(0.08398)\end{array}$ & $\begin{array}{l}-0.02583 \\
(0.08742)\end{array}$ & $\begin{array}{l}-0.04095 \\
(0.07767)\end{array}$ & $\begin{array}{l}0.21018^{\dagger} \\
(0.09300)\end{array}$ & $\begin{array}{l}-0.10718^{\ddagger} \\
(0.05945)\end{array}$ & $\begin{array}{l}0.02776 \\
(0.06437)\end{array}$ & $\begin{array}{l}-0.06238 \\
(0.07954)\end{array}$ & $\begin{array}{l}0.02474 \\
(0.07378)\end{array}$ & $\begin{array}{l}-0.04904 \\
(0.09574)\end{array}$ \\
\hline
\end{tabular}

* Significant at the 0.01 level $\quad \dagger$ Significant at the 0.05 level $\$$ Significant at the 0.10 level

Table 95 Coefficients with individual variables for high-level factors - Electronics Industry 


\begin{tabular}{|c|c|c|c|c|c|c|c|c|c|c|}
\hline $\begin{array}{l}\text { Individual variable } \\
\text { included in the model } \\
\text { in the column }\end{array}$ & 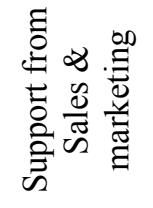 & 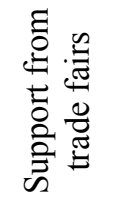 & 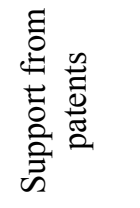 & 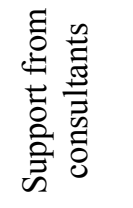 & 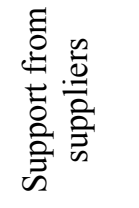 & 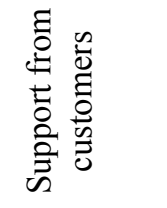 & 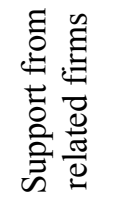 & 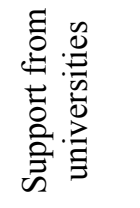 & 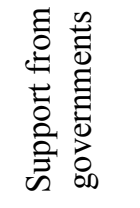 & 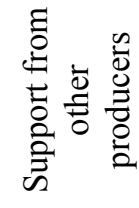 \\
\hline No factors & $\begin{array}{l}0.18466 * \\
(0.06180)\end{array}$ & $\begin{array}{l}0.18353^{*} \\
(0.05818)\end{array}$ & $\begin{array}{l}0.16819^{*} \\
(0.06110)\end{array}$ & $\begin{array}{l}0.18353^{*} \\
(0.06155) \\
\end{array}$ & $\begin{array}{l}0.18378^{*} \\
(0.06151)\end{array}$ & $\begin{array}{l}0.17778^{*} \\
(0.06060) \\
\end{array}$ & $\begin{array}{l}0.18353^{*} \\
(0.06115) \\
\end{array}$ & $\begin{array}{l}0.18353^{*} \\
(0.06148)\end{array}$ & $\begin{array}{l}0.18353^{*} \\
(0.06155) \\
\end{array}$ & $\begin{array}{l}0.18353^{*} \\
(0.06046)\end{array}$ \\
\hline Factor 5 & $\begin{array}{l}0.34886 \\
(0.22720) \\
\end{array}$ & $\begin{array}{l}0.34886 \\
(0.21479) \\
\end{array}$ & $\begin{array}{l}0.34886 \\
(0.22420) \\
\end{array}$ & $\begin{array}{l}0.34886 \\
(0.22722) \\
\end{array}$ & $\begin{array}{l}0.34886 \\
(0.22709) \\
\end{array}$ & \begin{tabular}{|l|l}
0.34886 \\
$(0.22358)$ \\
\end{tabular} & $\begin{array}{l}0.34886 \\
(0.22574) \\
\end{array}$ & $\begin{array}{l}0.34886 \\
(0.22696) \\
\end{array}$ & \begin{tabular}{|l|}
0.34886 \\
$(0.22722)$ \\
\end{tabular} & $\begin{array}{l}0.34886 \\
(0.22321) \\
\end{array}$ \\
\hline Factor 4 & $\begin{array}{l}0.13279 \\
(0.33767)\end{array}$ & $\begin{array}{l}0.13279 \\
(0.31922)\end{array}$ & $\begin{array}{l}0.06699 \\
(0.33447)\end{array}$ & $\begin{array}{l}0.13279 \\
(0.33770)\end{array}$ & $\begin{array}{l}0.13823 \\
(0.33771)\end{array}$ & $\begin{array}{l}0.10386 \\
(0.33249)\end{array}$ & $\begin{array}{l}0.13279 \\
(0.33550)\end{array}$ & $\begin{array}{l}0.12502 \\
(0.33751)\end{array}$ & $\begin{array}{l}0.13156 \\
(0.33796)\end{array}$ & $\begin{array}{l}0.09869 \\
(0.33200)\end{array}$ \\
\hline Factor $4 \& 5$ & $\begin{array}{l}0.42672 \\
(0.57755)\end{array}$ & $\begin{array}{l}0.42672 \\
(0.54599)\end{array}$ & $\begin{array}{l}0.23424 \\
(0.57618)\end{array}$ & $\begin{array}{l}0.42672 \\
(0.57760)\end{array}$ & $\begin{array}{l}0.44264 \\
(0.57826)\end{array}$ & $\begin{array}{l}0.34208 \\
(0.56935)\end{array}$ & $\begin{array}{l}0.42672 \\
(0.57384)\end{array}$ & $\begin{array}{l}0.42672 \\
(0.57693)\end{array}$ & $\begin{array}{l}0.42672 \\
(0.57760)\end{array}$ & $\begin{array}{l}0.42672 \\
(0.56741)\end{array}$ \\
\hline Factor 3 & $\begin{array}{l}-0.15759 \\
(0.13252)\end{array}$ & $\begin{array}{l}-0.04207 \\
(0.10640)\end{array}$ & $\begin{array}{l}-0.32126^{\dagger} \\
(0.12561)\end{array}$ & $\begin{array}{l}-0.17286 \\
(0.10874) \\
\end{array}$ & $\begin{array}{l}-0.17286 \\
(0.10868)\end{array}$ & $\begin{array}{l}-0.30337^{\dagger} \\
(0.11906)\end{array}$ & $\begin{array}{l}-0.26414^{\dagger} \\
(0.12245) \\
\end{array}$ & $\begin{array}{l}-0.21834 \\
(0.12793)\end{array}$ & $\begin{array}{l}-0.17286 \\
(0.10874) \\
\end{array}$ & $\begin{array}{l}-0.32669^{*} \\
(0.12183)\end{array}$ \\
\hline Factor $3 \& 5$ & $\begin{array}{l}0.12768 \\
(0.35219)\end{array}$ & $\begin{array}{l}0.11241 \\
(0.32515)\end{array}$ & $\begin{array}{l}-0.08008 \\
(0.34981)\end{array}$ & $\begin{array}{l}0.11241 \\
(0.34398)\end{array}$ & $\begin{array}{l}0.14424 \\
(0.35033)\end{array}$ & $\begin{array}{l}-0.05688 \\
(0.34517)\end{array}$ & $\begin{array}{l}-0.00599 \\
(0.34982)\end{array}$ & $\begin{array}{l}0.07500 \\
(0.34805)\end{array}$ & $\begin{array}{l}0.11113 \\
(0.34425)\end{array}$ & $\begin{array}{l}0.07702 \\
(0.33818)\end{array}$ \\
\hline Factor $3 \& 4$ & $\begin{array}{l}0.21837 \\
(0.24134)\end{array}$ & $\begin{array}{l}0.21192 \\
(0.22614)\end{array}$ & $\begin{array}{l}0.05620 \\
(0.24580)\end{array}$ & $\begin{array}{l}0.21192 \\
(0.23924)\end{array}$ & $\begin{array}{l}0.23768 \\
(0.24524)\end{array}$ & $\begin{array}{l}0.07496 \\
(0.24169)\end{array}$ & $\begin{array}{l}0.09352 \\
(0.24916)\end{array}$ & $\begin{array}{l}0.20324 \\
(0.23930)\end{array}$ & $\begin{array}{l}0.20776 \\
(0.24338)\end{array}$ & $\begin{array}{l}0.09662 \\
(0.23908)\end{array}$ \\
\hline Factor $3,4 \& 5$ & $\begin{array}{l}0.19897 \\
(0.14750) \\
\end{array}$ & $\begin{array}{l}0.19897 \\
(0.13944) \\
\end{array}$ & $\begin{array}{l}0.00648 \\
(0.16840) \\
\end{array}$ & $\begin{array}{l}0.19897 \\
(0.14751) \\
\end{array}$ & $\begin{array}{l}0.19897 \\
(0.14743) \\
\end{array}$ & \begin{tabular}{|l}
0.02968 \\
$(0.16016)$ \\
\end{tabular} & $\begin{array}{l}0.08057 \\
(0.16451) \\
\end{array}$ & $\begin{array}{l}0.19897 \\
(0.14734) \\
\end{array}$ & \begin{tabular}{|l}
0.19897 \\
$(0.14751)$ \\
\end{tabular} & $\begin{array}{l}0.19897 \\
(0.14491) \\
\end{array}$ \\
\hline Factor 2 & $\begin{array}{l}0.34304^{\ddagger} \\
(0.19623)\end{array}$ & $\begin{array}{l}0.34304 \\
(0.18550)\end{array}$ & $\begin{array}{l}0.32807^{\ddagger} \\
(0.19375)\end{array}$ & $\begin{array}{l}0.34304^{\ddagger} \\
(0.19624)\end{array}$ & $\begin{array}{l}0.34304^{\ddagger} \\
(0.19613)\end{array}$ & $\begin{array}{l}0.34304^{\ddagger} \\
(0.19310)\end{array}$ & $\begin{array}{l}0.34304^{\ddagger} \\
(0.19497)\end{array}$ & $\begin{array}{l}0.34304^{\ddagger} \\
(0.19601)\end{array}$ & $\begin{array}{l}0.34304^{\ddagger} \\
(0.19624)\end{array}$ & $\begin{array}{l}0.32752^{\ddagger} \\
(0.19287)\end{array}$ \\
\hline Factor $2 \& 5$ & \multicolumn{10}{|c|}{ Insufficient Data } \\
\hline Factor $2 \& 4$ & $\begin{array}{l}0.21589 \\
(0.22349)\end{array}$ & $\begin{array}{l}0.20165 \\
(0.20044)\end{array}$ & $\begin{array}{l}0.07774 \\
(0.21621)\end{array}$ & $\begin{array}{l}0.20165 \\
(0.21204)\end{array}$ & $\begin{array}{l}0.21071 \\
(0.21279)\end{array}$ & $\begin{array}{l}0.08127 \\
(0.21413)\end{array}$ & $\begin{array}{l}0.14319 \\
(0.21387)\end{array}$ & $\begin{array}{l}0.20165 \\
(0.21180)\end{array}$ & $\begin{array}{l}0.20165 \\
(0.21204)\end{array}$ & $\begin{array}{l}0.20165 \\
(0.20830)\end{array}$ \\
\hline Factor $2,4 \& 5$ & $\begin{array}{l}-0.00699 \\
(0.35521) \\
\end{array}$ & $\begin{array}{l}-0.00699 \\
(0.33580)\end{array}$ & $\begin{array}{l}-0.16307 \\
(0.35718) \\
\end{array}$ & $\begin{array}{l}-0.00568 \\
(0.35552) \\
\end{array}$ & $\begin{array}{l}-0.00699 \\
(0.35504) \\
\end{array}$ & $\begin{array}{l}-0.17627 \\
(0.35605) \\
\end{array}$ & $\begin{array}{l}-0.02938 \\
(0.35321) \\
\end{array}$ & $\begin{array}{l}-0.05246 \\
(0.36121)\end{array}$ & $\begin{array}{l}-0.00699 \\
(0.35524) \\
\end{array}$ & $\begin{array}{l}-0.00699 \\
(0.34897) \\
\end{array}$ \\
\hline Factor $2 \& 3$ & \multicolumn{10}{|c|}{ Insufficient Data } \\
\hline Factor $2,3 \& 5$ & \multicolumn{10}{|c|}{ Insufficient Data } \\
\hline Factor $2,3 \& 4$ & $\begin{array}{l}0.24575 \\
(0.33773) \\
\end{array}$ & $\begin{array}{l}0.23802 \\
(0.31721) \\
\end{array}$ & $\begin{array}{l}0.07802 \\
(0.33851) \\
\end{array}$ & $\begin{array}{l}0.24259 \\
(0.33910) \\
\end{array}$ & $\begin{array}{l}0.25911 \\
(0.33834) \\
\end{array}$ & \begin{tabular}{|l|l|}
0.12587 \\
$(0.33322)$ \\
\end{tabular} & $\begin{array}{l}0.13960 \\
(0.33913) \\
\end{array}$ & $\begin{array}{l}0.20790 \\
(0.33816) \\
\end{array}$ & \begin{tabular}{|l|}
0.23559 \\
$(0.33659)$ \\
\end{tabular} & $\begin{array}{l}0.17067 \\
(0.33064) \\
\end{array}$ \\
\hline Factor $2,3,4 \& 5$ & $\begin{array}{l}0.25490 \\
(0.19718) \\
\end{array}$ & $\begin{array}{l}0.24548 \\
(0.18111) \\
\end{array}$ & $\begin{array}{l}0.06359 \\
(0.20529) \\
\end{array}$ & $\begin{array}{l}0.24624 \\
(0.19176) \\
\end{array}$ & $\begin{array}{l}0.27310 \\
(0.20021) \\
\end{array}$ & $\begin{array}{l}0.12544 \\
(0.19454) \\
\end{array}$ & $\begin{array}{l}0.14927 \\
(0.19980) \\
\end{array}$ & $\begin{array}{l}0.21452 \\
(0.19682) \\
\end{array}$ & \begin{tabular}{|l}
0.24260 \\
$(0.19409)$ \\
\end{tabular} & $\begin{array}{l}0.17647 \\
(0.19004) \\
\end{array}$ \\
\hline Factor 1 & $\begin{array}{l}0.23158 \\
(0.27758)\end{array}$ & $\begin{array}{l}0.22921 \\
(0.26218)\end{array}$ & $\begin{array}{l}0.03672 \\
(0.28648)\end{array}$ & $\begin{array}{l}0.22921 \\
(0.27736) \\
\end{array}$ & $\begin{array}{l}0.23415 \\
(0.27740)\end{array}$ & $\begin{array}{l}0.07944 \\
(0.27941) \\
\end{array}$ & $\begin{array}{l}0.19716 \\
(0.27629) \\
\end{array}$ & $\begin{array}{l}0.22921 \\
(0.27703)\end{array}$ & $\begin{array}{l}0.22921 \\
(0.27736) \\
\end{array}$ & $\begin{array}{l}0.22921 \\
(0.27246)\end{array}$ \\
\hline Factor $1 \& 5$ & $\begin{array}{l}0.06756 \\
(0.27920)\end{array}$ & $\begin{array}{l}0.06516 \\
(0.26371)\end{array}$ & $\begin{array}{l}-0.05322 \\
(0.28015)\end{array}$ & $\begin{array}{l}0.06516 \\
(0.27897)\end{array}$ & $\begin{array}{l}0.08103 \\
(0.28083)\end{array}$ & $\begin{array}{l}0.00053935 \\
(0.27572)\end{array}$ & $\begin{array}{l}-0.01284 \\
(0.28150)\end{array}$ & $\begin{array}{l}0.03744 \\
(0.28168)\end{array}$ & $\begin{array}{l}0.06516 \\
(0.27897)\end{array}$ & $\begin{array}{l}-0.00292 \\
(0.27527)\end{array}$ \\
\hline Factor $1 \& 4$ & $\begin{array}{l}0.24875 \\
(0.22135)\end{array}$ & $\begin{array}{l}0.24875 \\
(0.20925)\end{array}$ & $\begin{array}{l}0.05627 \\
(0.23427)\end{array}$ & $\begin{array}{l}0.25467 \\
(0.23024)\end{array}$ & $\begin{array}{l}0.25326 \\
(0.22145)\end{array}$ & $\begin{array}{l}0.24875 \\
(0.21782)\end{array}$ & $\begin{array}{l}0.14710 \\
(0.22910)\end{array}$ & $\begin{array}{l}0.24875 \\
(0.22111)\end{array}$ & $\begin{array}{l}0.24875 \\
(0.22137)\end{array}$ & $\begin{array}{l}0.24875 \\
(0.21746)\end{array}$ \\
\hline Factor $1,4 \& 5$ & $\begin{array}{l}0.30465 \\
(0.33566)\end{array}$ & $\begin{array}{l}0.30465 \\
(0.31732)\end{array}$ & $\begin{array}{l}0.22833 \\
(0.33293)\end{array}$ & $\begin{array}{l}0.30840 \\
(0.33809)\end{array}$ & $\begin{array}{l}0.31189 \\
(0.33585)\end{array}$ & $\begin{array}{l}0.19901 \\
(0.33300)\end{array}$ & $\begin{array}{l}0.28465 \\
(0.33374)\end{array}$ & $\begin{array}{l}0.30465 \\
(0.33530)\end{array}$ & $\begin{array}{l}0.30343 \\
(0.33595)\end{array}$ & $\begin{array}{l}0.27095 \\
(0.33002)\end{array}$ \\
\hline Factor $1 \& 3$ & \multicolumn{10}{|c|}{ Insufficient Data } \\
\hline Factor $1,3 \& 5$ & $\begin{array}{l}0.42022 \\
(0.32922) \\
\end{array}$ & $\begin{array}{l}0.40495 \\
(0.30288) \\
\end{array}$ & $\begin{array}{l}0.21246 \\
(0.32731) \\
\end{array}$ & $\begin{array}{l}0.40731 \\
(0.32141) \\
\end{array}$ & $\begin{array}{l}0.42588 \\
(0.32329) \\
\end{array}$ & \begin{tabular}{|l|}
0.23566 \\
$(0.32247)$ \\
\end{tabular} & $\begin{array}{l}0.31109 \\
(0.32380) \\
\end{array}$ & $\begin{array}{l}0.38237 \\
(0.32180) \\
\end{array}$ & \begin{tabular}{|l|}
0.40384 \\
$(0.32064)$ \\
\end{tabular} & $\begin{array}{l}0.40495 \\
(0.31476) \\
\end{array}$ \\
\hline Factor $1,3 \& 4$ & $\begin{array}{l}0.18601 \\
(0.12618) \\
\end{array}$ & $\begin{array}{l}0.17959 \\
(0.11542) \\
\end{array}$ & $\begin{array}{l}-0.01290 \\
(0.14728) \\
\end{array}$ & $\begin{array}{l}0.18168 \\
(0.12415) \\
\end{array}$ & $\begin{array}{l}0.18641 \\
(0.12289) \\
\end{array}$ & $\begin{array}{l}0.10110 \\
(0.12418) \\
\end{array}$ & $\begin{array}{l}0.10796 \\
(0.12947) \\
\end{array}$ & $\begin{array}{l}0.17350 \\
(0.12230) \\
\end{array}$ & \begin{tabular}{|l}
0.17646 \\
$(0.12663)$ \\
\end{tabular} & $\begin{array}{l}0.17100 \\
(0.12000) \\
\end{array}$ \\
\hline Factor $1,3,4 \& 5$ & $\begin{array}{l}0.23492 \\
(0.16170)\end{array}$ & $\begin{array}{l}0.22763 \\
(0.14900) \\
\end{array}$ & $\begin{array}{l}0.05446 \\
(0.17319) \\
\end{array}$ & $\begin{array}{l}0.22899 \\
(0.15828) \\
\end{array}$ & $\begin{array}{l}0.23708 \\
(0.15880) \\
\end{array}$ & \begin{tabular}{|l}
0.16480 \\
$(0.15712)$ \\
\end{tabular} & $\begin{array}{l}0.16077 \\
(0.16218) \\
\end{array}$ & $\begin{array}{l}0.21173 \\
(0.15920) \\
\end{array}$ & $\begin{array}{l}0.22649 \\
(0.15810) \\
\end{array}$ & $\begin{array}{l}0.22020 \\
(0.15487) \\
\end{array}$ \\
\hline Factor $1 \& 2$ & $\begin{array}{l}0.10659 \\
(0.21032)\end{array}$ & $\begin{array}{l}0.09131 \\
(0.18549)\end{array}$ & $\begin{array}{l}-0.10117 \\
(0.21134)\end{array}$ & $\begin{array}{l}0.09131 \\
(0.19623)\end{array}$ & $\begin{array}{l}0.09131 \\
(0.19612)\end{array}$ & $\begin{array}{l}-0.07797 \\
(0.20461)\end{array}$ & $\begin{array}{l}-0.02708 \\
(0.20879)\end{array}$ & $\begin{array}{l}0.09131 \\
(0.19600)\end{array}$ & $\begin{array}{l}0.09131 \\
(0.19623)\end{array}$ & $\begin{array}{l}0.09131 \\
(0.19276)\end{array}$ \\
\hline Factor $1,2 \& 5$ & \multicolumn{10}{|c|}{ Insufficient Data } \\
\hline Factor $1,2 \& 4$ & $\begin{array}{l}-0.01669 \\
(0.15812)\end{array}$ & $\begin{array}{l}-0.02708 \\
(0.14132)\end{array}$ & $\begin{array}{l}-0.17619 \\
(0.16145)\end{array}$ & $\begin{array}{l}-0.02708 \\
(0.14950)\end{array}$ & $\begin{array}{l}-0.01726 \\
(0.15086)\end{array}$ & $\begin{array}{l}-0.06401 \\
(0.14785)\end{array}$ & $\begin{array}{l}-0.06222 \\
(0.15018)\end{array}$ & $\begin{array}{l}-0.04846 \\
(0.15268)\end{array}$ & $\begin{array}{l}-0.02794 \\
(0.14979)\end{array}$ & $\begin{array}{l}-0.02708 \\
(0.14687)\end{array}$ \\
\hline Factor $1,2,4 \& 5$ & $\begin{array}{l}0.20128 \\
(0.21277)\end{array}$ & $\begin{array}{l}0.23895 \\
(0.19461)\end{array}$ & $\begin{array}{l}0.06202 \\
(0.21055)\end{array}$ & $\begin{array}{l}0.19206 \\
(0.20653)\end{array}$ & $\begin{array}{l}0.19696 \\
(0.20597)\end{array}$ & $\begin{array}{l}0.08069 \\
(0.20698)\end{array}$ & $\begin{array}{l}0.12055 \\
(0.20893)\end{array}$ & $\begin{array}{l}0.17769 \\
(0.20619)\end{array}$ & $\begin{array}{l}0.18874 \\
(0.20620)\end{array}$ & $\begin{array}{l}0.19021 \\
(0.20196)\end{array}$ \\
\hline Factor $1,2 \& 3$ & $\begin{array}{l}0.38209 \\
(0.48351)\end{array}$ & $\begin{array}{l}0.36681 \\
(0.45145)\end{array}$ & $\begin{array}{l}0.17433 \\
(0.47878)\end{array}$ & $\begin{array}{l}0.37371 \\
(0.48324) \\
\end{array}$ & $\begin{array}{l}0.37770 \\
(0.47787)\end{array}$ & $\begin{array}{l}0.19753 \\
(0.47478)\end{array}$ & $\begin{array}{l}0.24841 \\
(0.48032)\end{array}$ & $\begin{array}{l}0.33688 \\
(0.47910)\end{array}$ & $\begin{array}{l}0.36681 \\
(0.47758)\end{array}$ & $\begin{array}{l}0.36681 \\
(0.46916)\end{array}$ \\
\hline Factor $1,2,3 \& 5$ & $\begin{array}{l}0.32192 \\
(0.22270) \\
\end{array}$ & $\begin{array}{l}0.31165 \\
(0.20495) \\
\end{array}$ & $\begin{array}{l}0.17138 \\
(0.22266) \\
\end{array}$ & $\begin{array}{l}0.31344 \\
(0.21766) \\
\end{array}$ & $\begin{array}{l}0.32509 \\
(0.21856) \\
\end{array}$ & $\begin{array}{l}0.19332 \\
(0.21853) \\
\end{array}$ & $\begin{array}{l}0.20449 \\
(0.22578) \\
\end{array}$ & $\begin{array}{l}0.27986 \\
(0.22166) \\
\end{array}$ & \begin{tabular}{|l}
0.30948 \\
$(0.21807)$ \\
\end{tabular} & $\begin{array}{l}0.29270 \\
(0.21311) \\
\end{array}$ \\
\hline Factor $1,2,3 \& 4$ & $\begin{array}{l}0.32383^{*} \\
(0.09347)\end{array}$ & $\begin{array}{l}0.31155^{*} \\
(0.06707)\end{array}$ & $\begin{array}{l}0.13750 \\
(0.10377)\end{array}$ & $\begin{array}{l}0.31391^{*} \\
(0.07530)\end{array}$ & $\begin{array}{l}0.32529^{*} \\
(0.07664)\end{array}$ & $\begin{array}{l}0.19137^{\dagger} \\
(0.08476)\end{array}$ & $\begin{array}{l}0.20449^{\dagger} \\
(0.09766)\end{array}$ & $\begin{array}{l}0.28582^{*} \\
(0.08053)\end{array}$ & $\begin{array}{l}0.31027^{*} \\
(0.07229)\end{array}$ & $\begin{array}{l}0.27091^{*} \\
(0.07139)\end{array}$ \\
\hline All Factors & $\begin{array}{l}0.34594^{*} \\
(0.08469) \\
\end{array}$ & $\begin{array}{l}0.40087^{*} \\
(0.04801) \\
\end{array}$ & $\begin{array}{l}0.14570 \\
(0.09486) \\
\end{array}$ & $\begin{array}{l}0.33436^{*} \\
(0.05493) \\
\end{array}$ & $\begin{array}{l}0.35017^{*} \\
(0.06191) \\
\end{array}$ & $\begin{array}{l}0.18590^{\dagger} \\
(0.07538) \\
\end{array}$ & $\begin{array}{l}0.22941^{*} \\
(0.08065)\end{array}$ & $\begin{array}{l}0.29862^{*} \\
(0.06921)\end{array}$ & $\begin{array}{l}0.32995^{*} \\
(0.05289)\end{array}$ & $\begin{array}{l}0.26772^{*} \\
(0.05348)\end{array}$ \\
\hline $\begin{array}{l}\text { Individual } \\
\text { Variable }\end{array}$ & $\begin{array}{l}-0.01527 \\
(0.07575)\end{array}$ & $\begin{array}{l}-0.57101^{*} \\
(0.11999)\end{array}$ & $\begin{array}{l}0.19249^{\dagger} \\
(0.08470)\end{array}$ & $\begin{array}{l}-0.00689 \\
(0.07372)\end{array}$ & $\begin{array}{l}-0.03184 \\
(0.06739)\end{array}$ & $\begin{array}{l}0.16929^{\dagger} \\
(0.06772)\end{array}$ & $\begin{array}{l}0.11840 \\
(0.07475)\end{array}$ & $\begin{array}{l}0.04547 \\
(0.06759)\end{array}$ & $\begin{array}{l}0.00720 \\
(0.07742)\end{array}$ & $\begin{array}{l}0.19953^{*} \\
(0.07598)\end{array}$ \\
\hline
\end{tabular}

$*$ Significant at the 0.01 level $†$ Significant at the 0.05 level $\$$ Significant at the 0.10 level

Table 95 Coefficients with individual variables for high-level factors - Electronics Industry 


\subsubsection{Vehicles Industry}

The regression analysis of the vehicles industry using both the individual variables and the factor state variables has similarities with both the medium plant analysis and the electronics industry analysis. As in the electronics industry, there are a number of states that have insignificant coefficients. The individual variable coefficients that are significant are not as large as those found in the electronics industry but are two to three times as large as those found in the medium plant analysis. This may be due to the sample size available for the vehicles industry, which is larger than that of the electronics industry but smaller than that of medium plants.

AMT implementations in the vehicles industry have a number of states with insignificant coefficients (refer to Table 96). In particular, any of the states containing only the robotics factor or the robotics factor and one other factor have insignificant coefficients. This result is due to the fact that most states that include in the robotics factor only contain $1 \%$ of the electronics industry. The state consisting of the communications and inspection and storage factors also has an insignificant coefficient. These results demonstrate that robotics is not normally the first form of technology implemented but that they rely heavily on the previous implementation of many other forms of technologies.

There are a number of individual variables that have significant coefficients. The use of modeling and simulations, FMC/FMS, sensor based systems, and inspection data for control all have positive coefficients that are approximately $50 \%$ of the state coefficients. The use of remote control processes for plant control has a negative and significant coefficient of similar size. These results demonstrate that for many of the planning and design technologies there is an additional benefit that is not included in the factor states. 
The analysis of business practices has a very interesting result. The state consisting of none of the factors is the only state that had an insignificant coefficient (refer to Table 97). This is unique to the vehicles industry and demonstrates that the business practices surveyed are in wide use in this industry. There are also a number of negative and significant coefficients for individual variables. These variables include continuous improvement, plant certifications, the use of just-in-time inventory control, and the use of electronic work orders. All of these variables have coefficients that are between $25 \%$ and $50 \%$ of the magnitude of state coefficients. As with medium plants, the JIT inventory method has a negative coefficient. This may be because of the longer distances that must be travelled in Canada between plants in order to move inventory making it much more difficult to implement JIT efficiently (Gonzalez-Benito, Suarez-Gonzalez, and Spring, 2000).

The model of the sources of implementation support has very few insignificant states or significant individual variables (refer to Table 98). The states consisting of only the primary stakeholders, only the external information sources, the primary stakeholders and the corporate organizations, and the state consisting of the primary stakeholders and the external information sources all have insignificant coefficients. These results demonstrate that the primary stakeholder cannot be the only source of support for implementations but that a large number of sources of support are required for successful AMT implementations. The only two variables that have significant coefficients are both positive and are approximately $35 \%-50 \%$ of the coefficients for the state variables. The variables representing consultants and other producers (two external sources of support) have understated benefits in the factor variables.

The model of perceived benefits has very few significant states (refer to Table 99). Only the state consisting of none of the factors, the state consisting of the perceived benefit of 
increased flexibility and profitability, and the state consisting of all the factors have significant coefficients. These three states contain approximately $74 \%$ of the plants in the vehicles industry so it is not too surprising that they are significant in the analysis. The perceived benefit of the reduction in labour costs, the reduction of rejection time, the increase in quality, and the increase in market share all have significant positive coefficients. Many of these variables are associated with bringing a new product to market in a timely fashion, supporting the need for innovation and high quality products. The reductions in material costs and the perceived benefit of reduced time to market both have negative coefficients that are significant. The effects of these two variables are overstated by the factor states, although the coefficients of the reduced time to market and increased market share do offset one another.

The analysis of the high-level factor model once again has a small number of significant states. The state consisting of none of the factors, of only the perceived benefits factor, only the implementation support and R\&D factor, the AMT and business practices and implementation support and R\&D factors, the perceived benefits, design, and human resources factor, all the factors except the AMT and business factor, and the state consisting of all the factors have significant coefficients. The majority of the significant states contain the design factor or the factor containing R\&D, supporting, once again, the need for innovation in the vehicles industry.

The significance of the individual variables also supports the importance of design innovation (refer to Table 100). All of the design AMT have positive significant coefficients that are approximately $30 \%-50 \%$ of the state coefficients. The certification of suppliers also has a large positive coefficient that is significant, demonstrating that quality throughout the supply chain is an important aspect of the vehicles industry. Performing in-house $R \& D$ has a positive coefficient, the opposite coefficient of contracting out $R \& D$. This demonstrates that the $R \& D$ 
aspect included in the state variables is more consistent with joint $R \& D$ and there are additional benefits to in-house $R \& D$ and fewer benefits from contracting out $R \& D$. The perceived benefits variables have coefficients similar to those in the factor type analysis and none of the human resource variables are significant. Similarly, variables for the sources of implementation support have significant coefficients except in this case the coefficients are negative. In particular, support from suppliers has replaced support from consultants demonstrating that suppliers do not have a strong ability to support the implementation of AMT in the supply chain but that support will come primarily through more of a pull technology effect.

Overall, the analysis supports complementarity, as the majority of the individual variables are insignificant when included in models containing the state variables. This demonstrates that the interaction effects are greater than the effect of the individual variables. Some of the individual variables do have significant coefficients that may be due to the use of the factor analysis and the different combination of variables that can be used to obtain the factor. The large number of insignificant states may be due to industry specific characteristics or simply to the sample size available for the analysis. In general, the variables representing the R\&D and design technologies in the vehicles industry must be included in the models as these have a significant impact on the profitability of the plant. 


\begin{tabular}{|c|c|c|c|c|c|c|c|c|c|c|c|c|c|c|c|c|c|c|c|c|c|c|c|c|c|}
\hline $\begin{array}{l}\text { Individual variable } \\
\text { included in the } \\
\text { model in the } \\
\text { column }\end{array}$ & 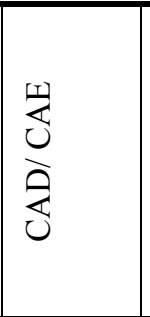 & 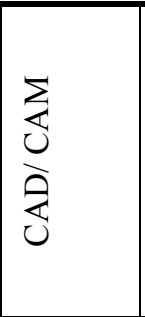 & 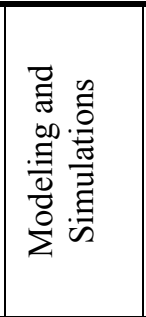 & 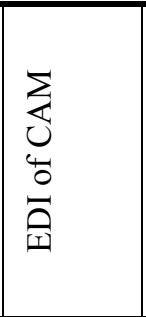 & $\sum_{\sum_{i}}^{\infty}$ & 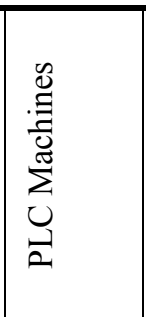 & 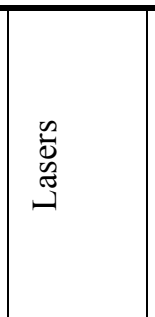 & 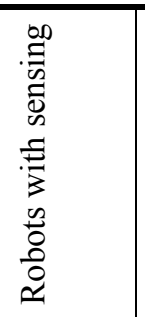 & 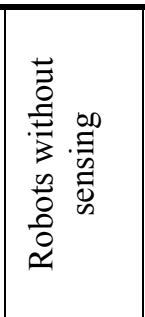 & 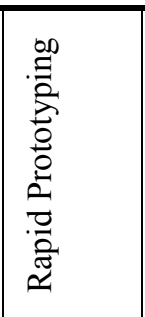 & 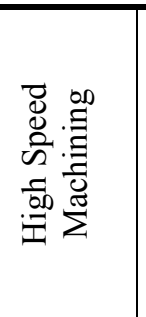 & 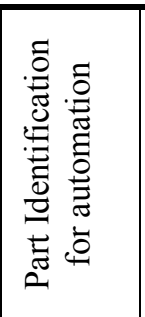 & 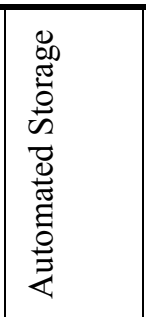 & 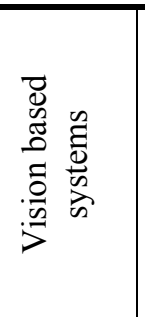 & 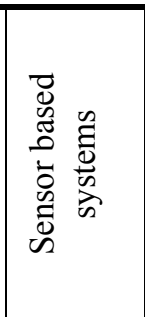 & 杂 & $\frac{z}{3}$ & 公 & $\begin{array}{l}= \\
\tilde{\tilde{\widetilde{\Sigma}}} \\
\Sigma\end{array}$ & 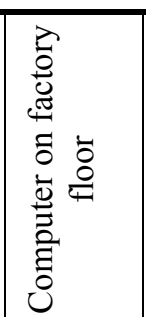 & $\sum$ & 念 & 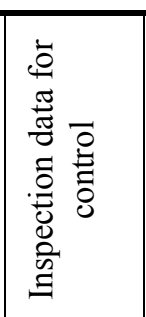 & 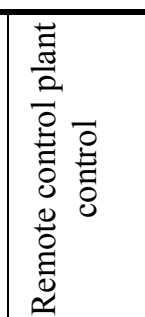 & 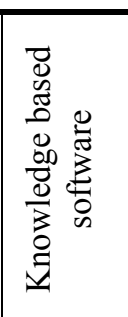 \\
\hline fac & $\begin{array}{l}528^{*} \\
3353) \\
\end{array}$ & \begin{tabular}{|l|}
$0.15927^{*}$ \\
$(0.03341)$ \\
\end{tabular} & \begin{tabular}{|l}
$0.15715^{*}$ \\
$(0.032)$
\end{tabular} & \begin{tabular}{|l|}
$0.15813^{*}$ \\
$(0.03302)$ \\
\end{tabular} & \begin{tabular}{|l|l}
$0.15701^{*}$ \\
$(0.03245)$ \\
\end{tabular} & \begin{tabular}{|l|l}
$0.15476^{*}$ \\
$(0.03257)$ \\
\end{tabular} & \begin{tabular}{|l|}
$0.1592^{*}$ \\
$(0.03261)$ \\
\end{tabular} & \begin{tabular}{l|l}
$0.1592^{*}$ \\
$(0.03252)$
\end{tabular} & \begin{tabular}{|l|l}
$0.15846^{*}$ \\
$(0.03263)$
\end{tabular} & \begin{tabular}{|l|l|}
$0.1592^{*}$ \\
$(0.03261)$ \\
\end{tabular} & \begin{tabular}{l|l|}
$0.1592^{*}$ \\
$(0.03262)$
\end{tabular} & \begin{tabular}{|l|}
$0.15944^{*}$ \\
$(0.03267)$ \\
\end{tabular} & \begin{tabular}{|l|l}
$0.1595^{*}$ \\
$(0.03266)$ \\
\end{tabular} & \begin{tabular}{|l|}
$0.1592^{*}$ \\
$(0.03265)$
\end{tabular} & $\begin{array}{l}0.15851^{*} \\
(0.03221)\end{array}$ & \begin{tabular}{|l|}
$0.14877^{*}$ \\
$(0.03339)$ \\
\end{tabular} & \begin{tabular}{|l|}
$0.16483^{*}$ \\
$(0.03298)$ \\
\end{tabular} & \begin{tabular}{|l|l}
$0.15417^{*}$ \\
$(0.03282)$
\end{tabular} & $\begin{array}{l}0.15308^{*} \\
(0.0329)\end{array}$ & \begin{tabular}{|l|l}
$0.15602^{*}$ \\
$(0.03277)$ \\
\end{tabular} & $\begin{array}{l}0.15887^{*} \\
(0.03252) \\
\end{array}$ & $\begin{array}{l}0.15787^{*} \\
(0.03253)\end{array}$ & \begin{tabular}{|l|l}
$0.15116^{*}$ \\
$(0.032)$
\end{tabular} & $\begin{array}{l}0.1592^{*} \\
(0.03227)\end{array}$ & $\begin{array}{l}0.15905^{*} \\
(0.03264)\end{array}$ \\
\hline Factor & $\begin{array}{l}1123 \\
563) \\
\end{array}$ & \begin{tabular}{|l|l}
-0.12849 \\
$(0.154)$
\end{tabular} & $\begin{array}{l}-0.18994 \\
(0.15127) \\
\end{array}$ & \begin{tabular}{|l|l}
-0.12996 \\
$(0.15312)$ \\
\end{tabular} & \begin{tabular}{|l|l}
-0.1901 \\
$(0.15587)$ \\
\end{tabular} & \begin{tabular}{|l|l}
-0.17994 \\
$(0.15528)$ \\
\end{tabular} & \begin{tabular}{|l|}
-0.12074 \\
$(0.15313)$ \\
\end{tabular} & \begin{tabular}{|l|}
-0.19172 \\
$(0.15961)$ \\
\end{tabular} & \begin{tabular}{|l|l}
-0.1741 \\
$(0.16439)$ \\
\end{tabular} & \begin{tabular}{|c|}
-0.12866 \\
$(0.1528)$ \\
\end{tabular} & \begin{tabular}{|l|}
-0.13739 \\
$(0.15345)$ \\
\end{tabular} & \begin{tabular}{|l|}
-0.12273 \\
$(0.15565)$ \\
\end{tabular} & \begin{tabular}{|l|l}
-0.12866 \\
$(0.15298)$ \\
\end{tabular} & \begin{tabular}{|c|}
-0.12866 \\
$(0.15301)$
\end{tabular} & \begin{tabular}{|l|l}
-0.12866 \\
$(0.15093)$ \\
\end{tabular} & \begin{tabular}{|l|l}
-0.1835 \\
$(0.15751)$
\end{tabular} & \begin{tabular}{|c|}
-0.09687 \\
$(0.15542)$ \\
\end{tabular} & \begin{tabular}{|l|l}
-0.16796 \\
$(0.15599)$
\end{tabular} & \begin{tabular}{|l|l}
-0.15412 \\
$(0.15379)$ \\
\end{tabular} & \begin{tabular}{|l|l}
-0.14688 \\
$(0.15397)$ \\
\end{tabular} & \begin{tabular}{|l|l}
-0.12866 \\
$(0.15239)$ \\
\end{tabular} & \begin{tabular}{|c}
-0.13732 \\
$(0.15251)$
\end{tabular} & \begin{tabular}{|l|l}
-0.19944 \\
$(0.15106)$
\end{tabular} & \begin{tabular}{|l|l}
-0.12866 \\
$(0.15121)$
\end{tabular} & \begin{tabular}{|l|l}
-0.12866 \\
$(0.15294)$
\end{tabular} \\
\hline Factor 3 & \begin{tabular}{|l|}
$6238^{\circ}$ \\
$7219)$
\end{tabular} & \begin{tabular}{|l|l}
$-0.3646^{*}$ \\
$(0.07221)$
\end{tabular} & \begin{tabular}{|c|}
$-0.36464^{*}$ \\
$(0.07066)$
\end{tabular} & \begin{tabular}{|c|}
$-0.36552^{*}$ \\
$(0.07222)$ \\
\end{tabular} & \begin{tabular}{|c|c}
$-0.37452^{*}$ \\
$(0.07183)$
\end{tabular} & $\begin{array}{c}-0.37288^{*} \\
(0.07186)\end{array}$ & \begin{tabular}{|c|}
$-0.36464^{*}$ \\
$(0.07202)$
\end{tabular} & \begin{tabular}{|c|}
$-0.364644^{*}$ \\
$(0.07183)$
\end{tabular} & \begin{tabular}{|l|l}
$-0.36464^{*}$ \\
$(0.07203)$
\end{tabular} & \begin{tabular}{|c|}
$-0.36464^{*}$ \\
$(0.07202)$ \\
\end{tabular} & \begin{tabular}{|c|c|}
$-0.364644^{*}$ \\
$(0.07205)$
\end{tabular} & \begin{tabular}{|l|l}
$-0.3561^{*}$ \\
$(0.08304)$
\end{tabular} & \begin{tabular}{|l|}
$-0.36464^{*}$ \\
$(0.0721)$
\end{tabular} & \begin{tabular}{|l|}
$-0.36685^{*}$ \\
$(0.0756)$
\end{tabular} & \begin{tabular}{|c|}
$-0.43166^{\circ}$ \\
$(0.07609)$
\end{tabular} & \begin{tabular}{|c|}
$-0.37158^{*}$ \\
$(0.07199)$
\end{tabular} & \begin{tabular}{|c|}
$-0.36061^{*}$ \\
$(0.07202)$
\end{tabular} & \begin{tabular}{|c|}
$-0.36837^{*}$ \\
$(0.07195)$
\end{tabular} & \begin{tabular}{|c|}
$-0.40254^{*}$ \\
$(0.07787)$
\end{tabular} & \begin{tabular}{|c|c|}
$-0.38192^{*}$ \\
$(0.07434)$
\end{tabular} & \begin{tabular}{|c|}
$-0.36464^{*}$ \\
$(0.07182)$
\end{tabular} & \begin{tabular}{|c}
$-0.36755^{*}$ \\
$(0.07185)$
\end{tabular} & \begin{tabular}{|c|}
$-0.37853^{*}$ \\
$(0.07058)$
\end{tabular} & \begin{tabular}{|c|}
$-0.36464^{*}$ \\
$(0.07126)$ \\
\end{tabular} & \begin{tabular}{|c|}
$-0.36464^{*}$ \\
$(0.07208)$
\end{tabular} \\
\hline Factor. & 6862) & \begin{tabular}{|l|}
0.2328 \\
$(0.24301)$ \\
\end{tabular} & \begin{tabular}{|l|l}
0.23267 \\
$(0.23772)$ \\
\end{tabular} & \begin{tabular}{|l|}
0.22274 \\
$(0.24688)$ \\
\end{tabular} & \begin{tabular}{|l|}
0.14881 \\
$(0.24555)$ \\
\end{tabular} & \begin{tabular}{|l|l|}
0.13935 \\
$(0.2478)$ \\
\end{tabular} & \begin{tabular}{|l|}
0.23267 \\
$(0.24229)$ \\
\end{tabular} & \begin{tabular}{|l|}
0.16702 \\
$(0.24665)$ \\
\end{tabular} & \begin{tabular}{|l|l|}
0.17785 \\
$(0.2531)$
\end{tabular} & \begin{tabular}{|l|}
0.23267 \\
$(0.24229)$ \\
\end{tabular} & \begin{tabular}{|l|}
0.22459 \\
$(0.24271)$ \\
\end{tabular} & \begin{tabular}{|l|l}
0.23925 \\
$(0.24468)$ \\
\end{tabular} & \begin{tabular}{|l|l}
0.23267 \\
$(0.24258)$ \\
\end{tabular} & \begin{tabular}{|l|}
0.22739 \\
$(0.2486)$
\end{tabular} & \begin{tabular}{|l|l}
0.15661 \\
$(0.24127)$ \\
\end{tabular} & \begin{tabular}{|l|l}
0.17369 \\
$(0.24538)$
\end{tabular} & \begin{tabular}{|l|}
0.26685 \\
$(0.24405)$ \\
\end{tabular} & \begin{tabular}{|l|l|l}
0.19041 \\
$(0.24439)$ \\
\end{tabular} & \begin{tabular}{|l|l|}
0.20438 \\
$(0.24279)$ \\
\end{tabular} & \begin{tabular}{|l|}
0.21087 \\
$(0.24329)$ \\
\end{tabular} & \begin{tabular}{|l|l}
0.19636 \\
$(0.24313)$ \\
\end{tabular} & \begin{tabular}{|l}
0.23267 \\
$(0.24161)$
\end{tabular} & \begin{tabular}{|l|l|}
0.15403 \\
$(0.23826)$
\end{tabular} & \begin{tabular}{|l|l}
0.23267 \\
$(0.23976)$ \\
\end{tabular} & \begin{tabular}{|l|l|}
.23267 \\
$(0.24251)$
\end{tabular} \\
\hline Factor 2 & 27794) & \begin{tabular}{|l|}
$0.47737^{*}$ \\
$(0.07731)$
\end{tabular} & \begin{tabular}{|l|}
$0.3853^{*}$ \\
$(0.08158)$ \\
\end{tabular} & \begin{tabular}{|l|}
$0.47702^{*}$ \\
$(0.0773)$ \\
\end{tabular} & \begin{tabular}{|l|}
$0.47452^{*}$ \\
$(0.07677)$ \\
\end{tabular} & \begin{tabular}{|l|l}
$0.47736^{*}$ \\
$(0.07683)$ \\
\end{tabular} & \begin{tabular}{|l|}
$0.47736^{*}$ \\
$(0.07719)$ \\
\end{tabular} & \begin{tabular}{|l|}
$0.47442^{*}$ \\
$(0.07702)$
\end{tabular} & \begin{tabular}{|l|}
$0.47736^{*}$ \\
$(0.0772)$ \\
\end{tabular} & \begin{tabular}{|l|}
$0.47937^{*}$ \\
$(0.07723)$ \\
\end{tabular} & \begin{tabular}{|l|}
$0.47736^{*}$ \\
$(0.07722)$ \\
\end{tabular} & \begin{tabular}{|l}
$0.47736^{*}$ \\
$(0.07729)$ \\
\end{tabular} & \begin{tabular}{|l|l}
$0.47736^{*}$ \\
$(0.07728)$ \\
\end{tabular} & \begin{tabular}{|l|l}
$0.47736^{*}$ \\
$(0.07729)$ \\
\end{tabular} & \begin{tabular}{|l|l}
$0.47736^{*}$ \\
$(0.07624)$ \\
\end{tabular} & \begin{tabular}{|l|}
$0.47272^{*}$ \\
$(0.07704)$
\end{tabular} & \begin{tabular}{|l|}
$0.48716^{*}$ \\
$(0.07762)$ \\
\end{tabular} & \begin{tabular}{|l|l}
$0.46459^{*}$ \\
$(0.07777)$ \\
\end{tabular} & \begin{tabular}{|l}
$0.42961^{*}$ \\
$(0.08579)$ \\
\end{tabular} & \begin{tabular}{|l}
$\begin{array}{l}0.46798^{*} \\
(0.0778)\end{array}$ \\
\end{tabular} & $\begin{array}{l}0.47736^{*} \\
(0.07698) \\
\end{array}$ & $\begin{array}{l}0.46799^{*} \\
(0.07728) \\
\end{array}$ & \begin{tabular}{|l|l}
$0.34466^{*}$ \\
$(0.08584)$ \\
\end{tabular} & $\begin{array}{l}0.47736^{*} \\
(0.07638) \\
\end{array}$ & \begin{tabular}{|l}
$.47309^{*}$ \\
$(0.07782)$ \\
\end{tabular} \\
\hline tor & 3833 & \begin{tabular}{|l|}
0.11973 \\
$(0.48831)$
\end{tabular} & \begin{tabular}{|l|}
0.1192 \\
$(0.47544)$ \\
\end{tabular} & \begin{tabular}{|l|l|}
0.1192 \\
$(0.4852)$
\end{tabular} & \begin{tabular}{|l|}
0.1192 \\
$(0.48186)$
\end{tabular} & \begin{tabular}{|l|}
0.02588 \\
$(0.48569)$ \\
\end{tabular} & \begin{tabular}{|l|}
0.1192 \\
$(0.48458)$
\end{tabular} & \begin{tabular}{|l|}
0.03167 \\
$(0.48778)$
\end{tabular} & \begin{tabular}{|l|l}
0.06438 \\
$(0.49012)$ \\
\end{tabular} & \begin{tabular}{|l|l|}
0.1192 \\
$(0.48457)$ \\
\end{tabular} & \begin{tabular}{|l|}
0.1192 \\
$(0.48479)$ \\
\end{tabular} & 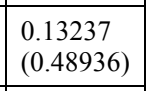 & 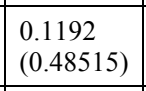 & \begin{tabular}{|l|}
0.1192 \\
$(0.48525)$ \\
\end{tabular} & \begin{tabular}{|l|l|l|l|l|}
$(0.47863)$ \\
\end{tabular} & \begin{tabular}{|l|}
0.04057 \\
$(0.48657)$
\end{tabular} & \begin{tabular}{|l|}
0.16477 \\
$(0.48581)$ \\
\end{tabular} & \begin{tabular}{|l}
0.06286 \\
$(0.48595)$ \\
\end{tabular} & \begin{tabular}{|l|l}
0.06262 \\
$(0.48558)$ \\
\end{tabular} & \begin{tabular}{|l|}
0.07561 \\
$(0.48657)$ \\
\end{tabular} & \begin{tabular}{|l|l|l}
0.04658 \\
$(0.48625)$
\end{tabular} & \begin{tabular}{|l|l}
0.053377 \\
$(0.48562)$ \\
\end{tabular} & \begin{tabular}{|c|}
-0.03807 \\
$(0.47652)$ \\
\end{tabular} & \begin{tabular}{|l|l|}
0.2724 \\
$(0.48409)$ \\
\end{tabular} & \begin{tabular}{|l|}
.099375 \\
$(0.48823)$ \\
\end{tabular} \\
\hline Factor $2 \& 3$ & $\begin{array}{l}3166 \\
5062) \\
\end{array}$ & \begin{tabular}{|l|}
.24806 \\
$(0.24868)$ \\
\end{tabular} & \begin{tabular}{|l|}
0.21349 \\
$(0.23799)$ \\
\end{tabular} & 24256 & \begin{tabular}{|l|l|}
0.1636767 \\
$(0.24555)$ \\
\end{tabular} & \begin{tabular}{|l|l|}
0.17754 \\
$(0.24492)$ \\
\end{tabular} & \begin{tabular}{|l|}
0.24753 \\
$(0.24229)$ \\
\end{tabular} & \begin{tabular}{|l|}
0.24753 \\
$(0.24166)$ \\
\end{tabular} & \begin{tabular}{|l|l}
0.24753 \\
$(0.24232)$ \\
\end{tabular} & \begin{tabular}{|l|}
0.24753 \\
$(0.24229)$ \\
\end{tabular} & \begin{tabular}{|l|}
0.23945 \\
$0.24271)$ \\
\end{tabular} & \begin{tabular}{|l|l|}
$\begin{array}{l}0.2607 \\
(0.2508)\end{array}$ \\
\end{tabular} & \begin{tabular}{|l|l|}
0.26497 \\
$(0.24915)$ \\
\end{tabular} & \begin{tabular}{|l|}
0.24489 \\
$(0.24413)$ \\
\end{tabular} & \begin{tabular}{|l}
0.13345 \\
$(0.24369)$ \\
\end{tabular} & \begin{tabular}{|l|}
0.18856 \\
$(0.24538)$ \\
\end{tabular} & \begin{tabular}{|l|}
0.2931 \\
$(0.24564)$ \\
\end{tabular} & \begin{tabular}{|l}
0.19118 \\
$(0.24635)$ \\
\end{tabular} & \begin{tabular}{|l|l|l}
0.19095 \\
$(0.24587)$ \\
\end{tabular} & \begin{tabular}{|l|}
0.20394 \\
$(0.24665)$ \\
\end{tabular} & \begin{tabular}{|l|l}
0.19306 \\
$(0.24498)$
\end{tabular} & \begin{tabular}{|l|l|}
0.19816 \\
$(0.2443)$
\end{tabular} & \begin{tabular}{|l|}
0.09026 \\
$(0.24192)$ \\
\end{tabular} & \begin{tabular}{|l|l}
0.28583 \\
$(0.24033)$
\end{tabular} & \begin{tabular}{|l|}
.23441 \\
$(0.24412)$ \\
\end{tabular} \\
\hline tor 2,3 & \begin{tabular}{ll|}
$386^{\circ}$ \\
$514)$
\end{tabular} & $\begin{array}{l}0.15978^{\circ} \\
(0.05108)\end{array}$ & \begin{tabular}{|l|}
$0.15824^{*}$ \\
$(0.04983)$
\end{tabular} & \begin{tabular}{|l|}
$0.15865^{*}$ \\
$(0.0511)$
\end{tabular} & \begin{tabular}{|l|}
0.05039 \\
$(0.07983)$ \\
\end{tabular} & \begin{tabular}{|l|}
$\begin{array}{l}0.06745 \\
(0.07569)\end{array}$ \\
\end{tabular} & $\begin{array}{l}0.15974^{*} \\
(0.05079)\end{array}$ & \begin{tabular}{|l|}
$\begin{array}{l}0.1482^{*} \\
(0.05139)\end{array}$ \\
\end{tabular} & \begin{tabular}{|l|l}
$0.15251^{*}$ \\
$(0.0517)$
\end{tabular} & $\begin{array}{l}16171^{\circ} \\
.05085)\end{array}$ & $\begin{array}{l}\begin{array}{l}0.15832^{\circ} \\
(0.05085)\end{array} \\
\end{array}$ & \begin{tabular}{|l|}
$\begin{array}{l}0.16163^{\circ} \\
(0.05143)\end{array}$ \\
\end{tabular} & \begin{tabular}{|l|l}
$0.16178^{*}$ \\
$(0.05128)$
\end{tabular} & $\begin{array}{l}0.15922^{\circ} \\
(0.05114)\end{array}$ & \begin{tabular}{|l|l}
$\begin{array}{l}0.01264 \\
(0.07765)\end{array}$ \\
\end{tabular} & \begin{tabular}{|l|}
0.08283 \\
$(0.07548)$ \\
\end{tabular} & $\begin{array}{l}0.20431^{\circ} \\
(0.06538)\end{array}$ & & \begin{tabular}{|l|}
$0.11308^{\ddagger}$ \\
$(0.06271)$
\end{tabular} & \begin{tabular}{|l|}
$0.11615^{\ddagger}$ \\
$(0.06908)$
\end{tabular} & & & \begin{tabular}{|l|l}
0.00247 \\
$(0.06935)$
\end{tabular} & $\begin{array}{l}0.27934^{\circ} \\
(0.07221)\end{array}$ & \begin{tabular}{|l|l}
$0.13653^{\ddagger}$ \\
$(0.07197)$
\end{tabular} \\
\hline Factor 1 & 7834) & \begin{tabular}{|l|}
$0.18851^{*}$ \\
$(0.06889)$
\end{tabular} & 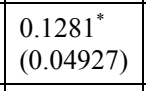 & \begin{tabular}{|l|}
$0.18068^{*}$ \\
$(0.05714)$ \\
\end{tabular} & \begin{tabular}{|l|}
$0.18499^{*}$ \\
$(0.04578)$ \\
\end{tabular} & \begin{tabular}{|l|l|}
$0.14223^{*}$ \\
$(0.05365)$ \\
\end{tabular} & \begin{tabular}{|l|}
$0.20159^{*}$ \\
$(0.04915)$ \\
\end{tabular} & \begin{tabular}{|l|}
$0.17699^{*}$ \\
$(0.04663)$
\end{tabular} & \begin{tabular}{|l|}
$0.18752^{*}$ \\
$(0.04602)$ \\
\end{tabular} & \begin{tabular}{|l|}
$0.18855^{*}$ \\
$(0.04601)$ \\
\end{tabular} & \begin{tabular}{|l}
$0.18543^{*}$ \\
$(0.0462)$ \\
\end{tabular} & \begin{tabular}{|l|}
$0.18837^{*}$ \\
$(0.0461)$ \\
\end{tabular} & \begin{tabular}{|l|l|}
$0.18801^{*}$ \\
$(0.04606)$ \\
\end{tabular} & \begin{tabular}{|l|l|}
$0.18801^{*}$ \\
$(0.04607)$ \\
\end{tabular} & \begin{tabular}{|l|l|}
$0.18801^{*}$ \\
$(0.04544)$ \\
\end{tabular} & \begin{tabular}{|l|}
$0.13106^{\dagger}$ \\
$(0.06183)$
\end{tabular} & \begin{tabular}{|l|l|}
$0.19929^{*}$ \\
$(0.04712)$ \\
\end{tabular} & \begin{tabular}{|l|l|}
$0.17259^{*}$ \\
$(0.04768)$
\end{tabular} & \begin{tabular}{|l|l|}
$0.18061^{*}$ \\
$(0.04628)$ \\
\end{tabular} & \begin{tabular}{|l|}
$0.18316^{*}$ \\
$(0.04628)$ \\
\end{tabular} & 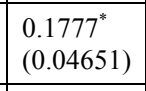 & $\begin{array}{l}0.18682^{*} \\
(0.04589)\end{array}$ & \begin{tabular}{|l|l|}
$0.17621^{*}$ \\
$(0.04515)$ \\
\end{tabular} & $\begin{array}{l}0.18801^{*} \\
(0.04553) \\
\end{array}$ & \begin{tabular}{|l} 
\\
$0.18691^{*}$ \\
$(0.04611)$ \\
\end{tabular} \\
\hline Factor $1 \& 4$ & 5855) & \begin{tabular}{|l|}
0.22563 \\
$(0.14748)$ \\
\end{tabular} & \begin{tabular}{|l|}
.164244 \\
$(0.1439)$ \\
\end{tabular} & \begin{tabular}{|l|}
0.21545 \\
$(0.15245)$ \\
\end{tabular} & \begin{tabular}{|l|}
0.13366 \\
$(0.15344)$ \\
\end{tabular} & \begin{tabular}{|l|l|}
0.13207 \\
$(0.15537)$ \\
\end{tabular} & \begin{tabular}{|l|l|}
0.23505 \\
$(0.14575)$ \\
\end{tabular} & \begin{tabular}{|l|}
0.18354 \\
$(0.14823)$ \\
\end{tabular} & \begin{tabular}{|l|l|}
0.18933 \\
$(0.15299)$ \\
\end{tabular} & \begin{tabular}{|l|}
0.23078 \\
$(0.14538)$ \\
\end{tabular} & \begin{tabular}{|l|}
0.21574 \\
$(0.14603)$ \\
\end{tabular} & \begin{tabular}{|l|l|}
0.23287 \\
$(0.14983)$ \\
\end{tabular} & \begin{tabular}{|l|}
0.22539 \\
$(0.1454)$ \\
\end{tabular} & \begin{tabular}{|l|l|}
.22539 \\
$(0.14542)$ \\
\end{tabular} & \begin{tabular}{|l|l|}
.22539 \\
$(0.14344)$ \\
\end{tabular} & \begin{tabular}{|l|}
0.14676 \\
$(0.15571)$ \\
\end{tabular} & \begin{tabular}{|l|}
$0.25868^{\ddagger}$ \\
$(0.14828)$ \\
\end{tabular} & \begin{tabular}{|l|l}
0.18996 \\
$(0.14793)$ \\
\end{tabular} & \begin{tabular}{|l|}
$\begin{array}{l}0.199988 \\
(0.1463)\end{array}$ \\
\end{tabular} & \begin{tabular}{|l|l|}
0.20846 \\
$(0.14628)$ \\
\end{tabular} & \begin{tabular}{|l|l|}
0.21886 \\
$(0.14491)$ \\
\end{tabular} & \begin{tabular}{|l|l}
0.21947 \\
$(0.14488)$
\end{tabular} & \begin{tabular}{|l|}
$\begin{array}{l}0.13608 \\
(0.1447)\end{array}$ \\
\end{tabular} & \begin{tabular}{|l|l|l}
$0.143791)$ \\
$(0.14371)$ \\
\end{tabular} & \begin{tabular}{|l|} 
\\
0.22539 \\
$(0.14536)$ \\
\end{tabular} \\
\hline Factor $1 \& 3$ & 8057) & \begin{tabular}{|l|}
$0.25353^{*}$ \\
$(0.07385)$ \\
\end{tabular} & \begin{tabular}{|l|}
$0.11684^{\ddagger}$ \\
$(0.06626)$ \\
\end{tabular} & \begin{tabular}{|l|}
$0.24306^{*}$ \\
$(0.06762)$ \\
\end{tabular} & \begin{tabular}{|l|}
$0.253^{*}$ \\
$(0.04943)$ \\
\end{tabular} & \begin{tabular}{|l|}
$0.16098^{\dagger}$ \\
$(0.07455)$ \\
\end{tabular} & \begin{tabular}{|l|l|}
$0.253^{*}$ \\
$(0.04971)$ \\
\end{tabular} & \begin{tabular}{|l|}
$0.253^{*}$ \\
$(0.04958)$ \\
\end{tabular} & \begin{tabular}{|l|l|}
$0.19894^{\dagger}$ \\
$(0.08755)$ \\
\end{tabular} & \begin{tabular}{|l|l|}
$0.253^{*}$ \\
$(0.04971)$ \\
\end{tabular} & \begin{tabular}{|l|l|}
$0.22114^{*}$ \\
$(0.06954)$ \\
\end{tabular} & \begin{tabular}{|l|l|}
$0.26617^{*}$ \\
$(0.08075)$ \\
\end{tabular} & \begin{tabular}{|l|}
$0.253^{*}$ \\
$(0.04977)$ \\
\end{tabular} & 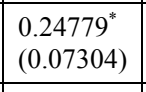 & \begin{tabular}{|l|l|l|l|l}
$(0.07787)$ \\
\end{tabular} & \begin{tabular}{|l|}
$0.17546^{\dagger}$ \\
$(0.07511)$
\end{tabular} & \begin{tabular}{|l|l|}
$.29793^{*}$ \\
$(0.06477)$ \\
\end{tabular} & \begin{tabular}{|l|l|}
$0.19744^{*}$ \\
$(0.06784)$ \\
\end{tabular} & \begin{tabular}{|l}
$\begin{array}{l}0.253^{*} \\
(0.0496)\end{array}$ \\
\end{tabular} & \begin{tabular}{|l|}
$0.21001^{*}$ \\
$(0.06785)$ \\
\end{tabular} & & \begin{tabular}{|l|l|l}
$0.253^{*}$ \\
$(0.04957)$ \\
\end{tabular} & \begin{tabular}{|l|l|}
0.09792 \\
$(0.06813)$ \\
\end{tabular} & \begin{tabular}{|l}
$0.253^{*}$ \\
$(0.04919)$ \\
\end{tabular} & \begin{tabular}{|l|l|} 
& $0.253^{*}$ \\
$(0.04975)$ & \\
\end{tabular} \\
\hline Factor $1,3 \& 4$ & 25911 & \begin{tabular}{|l|}
$0.27394^{\dagger}$ \\
$(0.11866)$ \\
\end{tabular} & \begin{tabular}{|l|l|}
0.14385 \\
$(0.11292)$ \\
\end{tabular} & \begin{tabular}{|l|l|}
$0.2635^{\dagger}$ \\
$(0.11609)$ \\
\end{tabular} & \begin{tabular}{|l|l|}
0.17243 \\
$(0.12036)$ \\
\end{tabular} & \begin{tabular}{|l|l|}
0.18012 \\
$(0.12038)$ \\
\end{tabular} & \begin{tabular}{|l|}
$0.31302^{*}$ \\
$(0.11788)$ \\
\end{tabular} & \begin{tabular}{|l|}
0.19013 \\
$(0.12335)$ \\
\end{tabular} & \begin{tabular}{|l|}
$0.25297^{\dagger}$ \\
$(0.11)$ \\
\end{tabular} & \begin{tabular}{|l|}
$0.27633^{\dagger}$ \\
$(0.10661)$ \\
\end{tabular} & \begin{tabular}{|l|}
$0.24425^{\dagger}$ \\
$(0.1152)$ \\
\end{tabular} & $\begin{array}{l}0.2866^{\dagger} \\
(0.1242) \\
\end{array}$ & \begin{tabular}{|l|l}
$0.288^{\circ}$ \\
$(0.11677)$ \\
\end{tabular} & \begin{tabular}{|l|}
$0.26892^{\dagger}$ \\
$(0.11633)$
\end{tabular} & \begin{tabular}{|l|l}
0.12132 \\
$(0.12179)$ \\
\end{tabular} & \begin{tabular}{|l|}
0.1948 \\
$(0.12068)$ \\
\end{tabular} & \begin{tabular}{|l|}
$0.3168^{*}$ \\
$(0.11374)$
\end{tabular} & \begin{tabular}{|l|l|}
$0.22254^{\ddagger}$ \\
$(0.11449)$
\end{tabular} & $\begin{array}{l}0.22702^{\dagger} \\
(0.11249)\end{array}$ & $\begin{array}{l}0.23406^{\dagger} \\
(0.1146)\end{array}$ & $\begin{array}{l}0.26388^{\dagger} \\
(0.1065)\end{array}$ & $\begin{array}{l}0.26706^{\dagger} \\
(0.10635)\end{array}$ & \begin{tabular}{|l|}
0.13897 \\
$(0.11215)$ \\
\end{tabular} & $\begin{array}{l}0.27343^{\dagger} \\
(0.10544)\end{array}$ & \begin{tabular}{|l|l|}
$0.2722^{+}$ \\
$(0.10668)$
\end{tabular} \\
\hline Factor $1 \& 2$ & $8306^{\dagger}$ & \begin{tabular}{|l|}
$0.15014^{\ddagger}$ \\
$(0.08001)$
\end{tabular} & \begin{tabular}{|l|}
$0.11864^{\dagger}$ \\
$(0.05982)$
\end{tabular} & \begin{tabular}{|l|}
$0.1443^{\dagger}$ \\
$(0.06495)$ \\
\end{tabular} & \begin{tabular}{|l|}
$0.14447^{\dagger}$ \\
$(0.05981)$
\end{tabular} & \begin{tabular}{|l|}
$0.12603^{\dagger}$ \\
$(0.0615)$ \\
\end{tabular} & \begin{tabular}{|l|}
0.15176 \\
$(0.06013)$
\end{tabular} & \begin{tabular}{|l|l|}
$0.14828^{\dagger}$ \\
$(0.05993)$
\end{tabular} & \begin{tabular}{|l|l|}
$0.14878^{\dagger}$ \\
$(0.06009)$
\end{tabular} & \begin{tabular}{|l|}
$0.15147^{\dagger}$ \\
$(0.06012)$
\end{tabular} & \begin{tabular}{|l|l|}
$0.144366^{\dagger}$ \\
$(0.06063)$
\end{tabular} & \begin{tabular}{|l}
$0.15023^{\dagger}$ \\
$(0.06022)$
\end{tabular} & \begin{tabular}{|l|}
$0.14962^{\dagger}$ \\
$(0.06014)$
\end{tabular} & $\begin{array}{l}0.14962^{\dagger} \\
(0.06016)\end{array}$ & \begin{tabular}{|l|l|}
$0.14728^{\dagger}$ \\
$(0.05934)$
\end{tabular} & \begin{tabular}{|l|}
0.07259 \\
$(0.08205)$
\end{tabular} & \begin{tabular}{|l|l|}
$0.18618^{\circ}$ \\
$(0.06888)$
\end{tabular} & $\begin{array}{l}0.11934^{\ddagger} \\
(0.06505)\end{array}$ & \begin{tabular}{|l|l|}
$0.13359^{\dagger}$ \\
$(0.06127)$
\end{tabular} & \begin{tabular}{|l|}
$0.12995^{\dagger}$ \\
$(0.06365)$
\end{tabular} & $\begin{array}{l}0.11684^{\ddagger} \\
(0.06464)\end{array}$ & \begin{tabular}{|l}
$0.12637^{\dagger}$ \\
$(0.06228)$
\end{tabular} & \begin{tabular}{|l|l|}
$0.10081^{\ddagger}$ \\
$(0.06066)$
\end{tabular} & \begin{tabular}{|l|l|}
$0.15509^{*}$ \\
$(0.05949)$
\end{tabular} & \begin{tabular}{|l}
$0.14666^{t}$ \\
$(0.06048)$
\end{tabular} \\
\hline Factor $1,2 \& 4$ & \begin{tabular}{l|l|}
$0439^{*}$ \\
$1073)$
\end{tabular} & \begin{tabular}{|l|}
$0.27051^{*}$ \\
$(0.09009)$
\end{tabular} & \begin{tabular}{|l|l}
$0.2407^{*}$ \\
$(0.08544)$
\end{tabular} & \begin{tabular}{|l|}
$0.26065^{*}$ \\
$(0.09731)$
\end{tabular} & \begin{tabular}{|l|l}
0.15846 \\
$(0.10676)$
\end{tabular} & $\begin{array}{l}0.18643^{\ddagger} \\
(0.10017)\end{array}$ & \begin{tabular}{|l|}
$0.27441^{*}$ \\
$(0.08688)$
\end{tabular} & \begin{tabular}{|l|l}
$0.20614^{\dagger}$ \\
$(0.0985)$
\end{tabular} & \begin{tabular}{|l|l}
$0.233966^{\dagger}$ \\
$(0.09914)$
\end{tabular} & $\begin{array}{l}0.27601^{*} \\
(0.08682) \\
\end{array}$ & $\begin{array}{l}0.26077^{*} \\
(0.08776) \\
\end{array}$ & $\begin{array}{l}0.27027^{*} \\
(0.08663)\end{array}$ & \begin{tabular}{|l|}
$0.27101^{*}$ \\
$(0.08665)$
\end{tabular} & $\begin{array}{l}0.2701^{*} \\
(0.08665)\end{array}$ & $\begin{array}{l}0.26542^{*} \\
(0.08548) \\
\end{array}$ & \begin{tabular}{|l|}
$0.19967^{\dagger}$ \\
$(0.10041)$
\end{tabular} & \begin{tabular}{|l|}
$0.2938^{*}$ \\
$(0.08911)$ \\
\end{tabular} & \begin{tabular}{|l|l}
$0.21572^{\dagger}$ \\
$(0.09758)$ \\
\end{tabular} & \begin{tabular}{|l|l}
$0.24285^{*}$ \\
$(0.08902)$
\end{tabular} & \begin{tabular}{|l|l}
$0.25053^{*}$ \\
$(0.08903)$
\end{tabular} & $\begin{array}{l}0.24939^{*} \\
(0.08766)\end{array}$ & \begin{tabular}{|l|}
$0.21464^{\dagger}$ \\
$(0.0954)$
\end{tabular} & \begin{tabular}{|l|}
0.13236 \\
$(0.09468)$ \\
\end{tabular} & $\begin{array}{l}0.27515^{*} \\
(0.08564) \\
\end{array}$ & \begin{tabular}{|l}
$.26455^{*}$ \\
$(0.08751)$ \\
\end{tabular} \\
\hline Factor & $\begin{array}{ll}1256^{\circ} \\
1778) \\
\end{array}$ & \begin{tabular}{|l|}
$0.37894^{*}$ \\
$(0.06913)$
\end{tabular} & \begin{tabular}{|l|}
$0.26907^{*}$ \\
$(0.05704)$
\end{tabular} & \begin{tabular}{|l|}
$0.36994^{*}$ \\
$0.05973)$
\end{tabular} & \begin{tabular}{|l|l}
$0.37328^{*}$ \\
$(0.04492)$
\end{tabular} & \begin{tabular}{|l|}
$0.29241^{*}$ \\
$(0.06906)$
\end{tabular} & \begin{tabular}{|l|}
$0.38122^{*}$ \\
$(0.04521)$
\end{tabular} & \begin{tabular}{|l|l|}
$0.37767^{*}$ \\
$(0.0449)$
\end{tabular} & \begin{tabular}{|l|}
$0.37843^{*}$ \\
$(0.04508)$
\end{tabular} & \begin{tabular}{|l|}
$0.37999^{\prime \prime}$ \\
$(0.04512)$
\end{tabular} & \begin{tabular}{|l}
$0.36768^{*}$ \\
$(0.04798)$
\end{tabular} & \begin{tabular}{|l|l}
$0.37997^{*}$ \\
$(0.04574)$
\end{tabular} & \begin{tabular}{|l|}
$0.37923^{*}$ \\
$(0.0452)$
\end{tabular} & \begin{tabular}{|l|l}
$0.37718^{*}$ \\
$(0.04692)$
\end{tabular} & \begin{tabular}{|l|l}
$0.25829^{*}$ \\
$(0.06577)$
\end{tabular} & \begin{tabular}{|l|}
$0.30165^{*}$ \\
$(0.07171)$
\end{tabular} & \begin{tabular}{|l|}
$0.39806^{\prime \prime}$ \\
$(0.04855)$
\end{tabular} & \begin{tabular}{|l}
$0.33041^{*}$ \\
$(0.06019)$
\end{tabular} & \begin{tabular}{|l|l}
$0.33263^{*}$ \\
$(0.05777)$
\end{tabular} & \begin{tabular}{|l|l}
$0.34217^{*}$ \\
$(0.05958)$
\end{tabular} & $\begin{array}{l}0.31237^{*} \\
(0.06645)\end{array}$ & \begin{tabular}{|l|l}
$0.32582^{*}$ \\
$(0.05919)$
\end{tabular} & \begin{tabular}{|l|l}
$0.25842^{*}$ \\
$(0.05751)$
\end{tabular} & \begin{tabular}{|l|l}
$0.40202^{*}$ \\
$(0.04576)$
\end{tabular} & \begin{tabular}{|l}
$.37600^{*}$ \\
$(0.04543)$
\end{tabular} \\
\hline All Factors & \begin{tabular}{|l|l|}
$30758^{*}$ \\
$07416)$ \\
\end{tabular} & \begin{tabular}{|l|l}
$0.30481^{*}$ \\
$(0.06274)$
\end{tabular} & \begin{tabular}{|l|l}
$0.2114^{*}$ \\
$(0.05061)$ \\
\end{tabular} & \begin{tabular}{|l|l|}
$0.2964^{*}$ \\
$(0.05507)$ \\
\end{tabular} & \begin{tabular}{|l|}
$0.22559^{*}$ \\
$(0.06043)$ \\
\end{tabular} & \begin{tabular}{|l|l}
$0.21192^{*}$ \\
$(0.06967)$ \\
\end{tabular} & \begin{tabular}{|l|}
$0.31634^{*}$ \\
$(0.04382)$
\end{tabular} & \begin{tabular}{|l|l}
$0.22792^{*}$ \\
$(0.07057)$ \\
\end{tabular} & \begin{tabular}{|l|}
$0.25453^{*}$ \\
$(0.0781)$ \\
\end{tabular} & \begin{tabular}{|l|}
$0.31923^{*}$ \\
$(0.0452)$ \\
\end{tabular} & \begin{tabular}{|l}
$0.28767^{*}$ \\
$(0.04833)$ \\
\end{tabular} & \begin{tabular}{|l}
$0.31646^{*}$ \\
$(0.07147)$
\end{tabular} & \begin{tabular}{|l|l}
$0.30852^{*}$ \\
$(0.04331)$
\end{tabular} & $\begin{array}{l}0.30139^{*} \\
(0.0511) \\
\end{array}$ & \begin{tabular}{|l|l}
$0.18168^{*}$ \\
$(0.06395)$
\end{tabular} & \begin{tabular}{|l|}
$0.22816^{*}$ \\
$(0.06894)$
\end{tabular} & \begin{tabular}{|l|l}
$0.34148^{*}$ \\
$(0.05352)$ \\
\end{tabular} & \begin{tabular}{|l}
$0.25507^{*}$ \\
$(0.05801)$ \\
\end{tabular} & \begin{tabular}{|l|l}
$0.25033^{*}$ \\
$(0.05923)$
\end{tabular} & \begin{tabular}{|l|l}
$0.26576^{*}$ \\
$(0.05837)$ \\
\end{tabular} & \begin{tabular}{|l}
$0.24589^{*}$ \\
$(0.05961)$ \\
\end{tabular} & \begin{tabular}{|l}
$0.2624^{*}$ \\
$(0.05119)$
\end{tabular} & \begin{tabular}{|l|l}
$0.17521^{*}$ \\
$(0.05651)$ \\
\end{tabular} & $\begin{array}{l}0.32699^{*} \\
(0.04181) \\
\end{array}$ & \begin{tabular}{|l}
$.28844^{*}$ \\
$(0.05396)$ \\
\end{tabular} \\
\hline Indivi & \begin{tabular}{|c|}
-0.03413 \\
$(0.06338)$ \\
\end{tabular} & \begin{tabular}{|l|l}
-0.00053 \\
$(0.05455)$ \\
\end{tabular} & \begin{tabular}{|l|l|l}
$0.13616^{*}$ \\
$(0.0445)$
\end{tabular} & \begin{tabular}{|l|}
0.00993 \\
$(0.04578)$ \\
\end{tabular} & \begin{tabular}{|l|}
$0.11181^{\ddagger}$ \\
$(0.06322)$ \\
\end{tabular} & \begin{tabular}{|l|l}
0.09331 \\
$(0.05696)$ \\
\end{tabular} & \begin{tabular}{|l|}
-0.0463 \\
$(0.05898)$ \\
\end{tabular} & \begin{tabular}{|l|l}
0.08753 \\
$(0.0651)$ \\
\end{tabular} & \begin{tabular}{|l|l}
0.05482 \\
$(0.07308)$ \\
\end{tabular} & \begin{tabular}{|c|}
-0.06003 \\
$(0.07609)$ \\
\end{tabular} & \begin{tabular}{|l|}
0.03231 \\
$(0.0493)$ \\
\end{tabular} & $\begin{array}{l}-0.01317 \\
(0.06358) \\
\end{array}$ & \begin{tabular}{|l|l}
-0.02325 \\
$(0.07579)$ \\
\end{tabular} & $\begin{array}{l}0.0052 \\
0.0542 \\
\end{array}$ & \begin{tabular}{|l|l|l}
$0.15211^{\dagger}$ \\
$(0.06129)$ \\
\end{tabular} & \begin{tabular}{|l|l}
0.07863 \\
$(0.05723)$ \\
\end{tabular} & \begin{tabular}{|c|}
-0.04557 \\
$(0.04218)$ \\
\end{tabular} & & & \begin{tabular}{|l|}
0.04359 \\
$(0.04686)$ \\
\end{tabular} & & & \begin{tabular}{|l|l|}
$0.15727^{*}$ \\
$(0.04839)$ \\
\end{tabular} & \begin{tabular}{|l|l}
$-0.1532^{\dagger}$ \\
$(0.06641)$ \\
\end{tabular} & \begin{tabular}{|l|}
0.0254 \\
0.055 \\
\end{tabular} \\
\hline
\end{tabular}

Table 96 Coefficients with individual variables for AMT - Vehicles Industry 


\begin{tabular}{|c|c|c|c|c|c|c|c|c|c|c|c|c|}
\hline $\begin{array}{l}\text { Individual variable } \\
\text { included in the } \\
\text { model in the column }\end{array}$ & 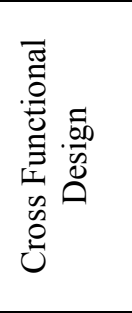 & 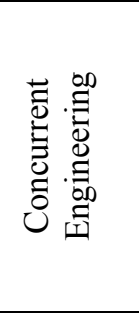 & 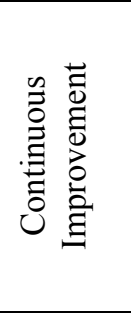 & 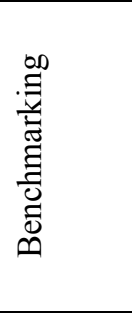 & 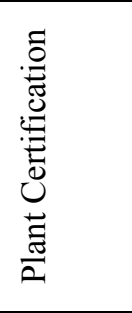 & 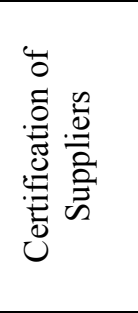 & 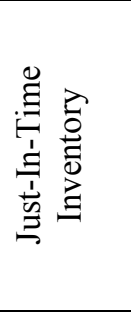 & 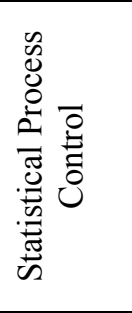 & 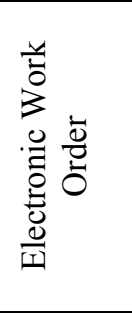 & 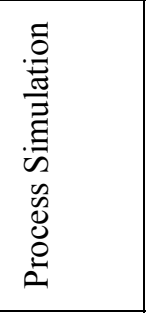 & 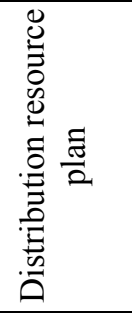 & 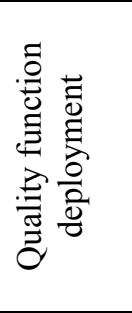 \\
\hline No fa & & $\begin{array}{l}-0.02546 \\
(0.03231)\end{array}$ & $\begin{array}{l}-0.01973 \\
(0.03187)\end{array}$ & $\begin{array}{l}-0.02252 \\
(0.03224)\end{array}$ & $\begin{array}{l}-0.0171 \\
(0.0325)\end{array}$ & $\begin{array}{l}-0.02583 \\
(0.0324)\end{array}$ & $\begin{array}{l}-0.02338 \\
(0.0319)\end{array}$ & & $\begin{array}{l}-0.02274 \\
(0.03201)\end{array}$ & $\begin{array}{l}-0.02546 \\
(0.03228)\end{array}$ & $\begin{array}{l}-0.02546 \\
(0.03226)\end{array}$ & $\begin{array}{l}-0.02574 \\
(0.0323)\end{array}$ \\
\hline Fact & $\begin{array}{l}0.13751 \\
(0.08569) \\
\end{array}$ & $\begin{array}{l}0.11645 \\
(0.09157) \\
\end{array}$ & $\begin{array}{l}0.27382^{*} \\
(0.08966) \\
\end{array}$ & $\begin{array}{l}0.16905^{\dagger} \\
(0.07974) \\
\end{array}$ & $\begin{array}{l}0.15236^{\ddagger} \\
(0.07855) \\
\end{array}$ & $\begin{array}{l}0.14505^{\ddagger} \\
(0.08076) \\
\end{array}$ & $\begin{array}{l}0.15463^{\dagger} \\
(0.07794) \\
\end{array}$ & & $\begin{array}{l}0.18074^{\dagger} \\
(0.07941) \\
\end{array}$ & \begin{tabular}{l|}
$0.14847^{\ddagger}$ \\
$(0.07886)$ \\
\end{tabular} & $\begin{array}{l}0.1498^{\ddagger} \\
(0.07883) \\
\end{array}$ & $\begin{array}{l}0.14112^{\ddagger} \\
(0.07945) \\
\end{array}$ \\
\hline Factor 2 & $\begin{array}{l}0.12012^{\ddagger} \\
(0.065) \\
\end{array}$ & $\begin{array}{l}0.11515^{\ddagger} \\
(0.06544) \\
\end{array}$ & $\begin{array}{l}0.14543^{\dagger} \\
(0.06415) \\
\end{array}$ & $\begin{array}{l}0.13084^{\dagger} \\
(0.0646)\end{array}$ & $\begin{array}{l}0.16632^{\dagger} \\
(0.06915) \\
\end{array}$ & $\begin{array}{l}0.11809^{\ddagger} \\
(0.06776) \\
\end{array}$ & $\begin{array}{l}0.18556^{*} \\
(0.06832) \\
\end{array}$ & $\begin{array}{l}0.12219^{\ddagger} \\
(0.06471)\end{array}$ & $\begin{array}{l}0.19324^{*} \\
(0.07111) \\
\end{array}$ & $\begin{array}{l}0.11476^{\ddagger} \\
(0.065) \\
\end{array}$ & $\begin{array}{l}0.14197^{\dagger} \\
(0.06695) \\
\end{array}$ & $\begin{array}{l}0.10948 \\
(0.06654) \\
\end{array}$ \\
\hline Fact & $\begin{array}{l}0.48764^{*} \\
(0.07806)\end{array}$ & $\begin{array}{l}0.45287^{*} \\
(0.09638)\end{array}$ & $\begin{array}{l}0.58286^{*} \\
(0.08046)\end{array}$ & $\begin{array}{l}0.49449^{*} \\
(0.07489)\end{array}$ & $\begin{array}{l}0.51874^{*} \\
(0.07609)\end{array}$ & $\begin{array}{l}0.49449^{*} \\
(0.07527)\end{array}$ & $\begin{array}{l}0.56857^{*} \\
(0.07954)\end{array}$ & $\begin{array}{l}0.49449^{*} \\
(0.07528)\end{array}$ & $\begin{array}{l}0.53284^{*} \\
(0.0763)\end{array}$ & $\begin{array}{l}0.49449^{*} \\
(0.07512)\end{array}$ & $\begin{array}{l}.49449^{*} \\
(0.07508)\end{array}$ & $\begin{array}{l}0.48319^{*} \\
(0.07648)\end{array}$ \\
\hline Facto & $\begin{array}{l}0.44282^{*} \\
(0.06746) \\
\end{array}$ & $\begin{array}{l}0.44115^{*} \\
(0.0673) \\
\end{array}$ & $\begin{array}{l}0.50908^{*} \\
(0.06985)\end{array}$ & $\begin{array}{l}0.47002^{*} \\
(0.06869)\end{array}$ & $\begin{array}{l}0.51963^{*} \\
(0.07942) \\
\end{array}$ & $\begin{array}{l}0.4336^{*} \\
(0.08724) \\
\end{array}$ & $\begin{array}{l}0.49638^{*} \\
(0.06908)\end{array}$ & $\begin{array}{l}0.44399^{*} \\
(0.0711) \\
\end{array}$ & $\begin{array}{l}0.45945^{*} \\
(0.06667) \\
\end{array}$ & $\begin{array}{l}0.44105^{*} \\
(0.0671)\end{array}$ & $\begin{array}{l}0.44506^{*} \\
(0.06695)\end{array}$ & $\begin{array}{l}0.44376^{*} \\
(0.06705)\end{array}$ \\
\hline Factor $1 \& 3$ & $\begin{array}{l}0.22731^{*} \\
(0.06055)\end{array}$ & $\begin{array}{l}0.20183^{*} \\
(0.07057)\end{array}$ & $\begin{array}{l}0.37386^{*} \\
(0.05989)\end{array}$ & $\begin{array}{l}0.3055^{*} \\
(0.05541)\end{array}$ & $\begin{array}{l}0.31393^{*} \\
(0.05586)\end{array}$ & $\begin{array}{l}0.23155^{*} \\
(0.06688)\end{array}$ & $\begin{array}{l}0.26746^{*} \\
(0.03873)\end{array}$ & $\begin{array}{l}0.24053^{*} \\
(0.06365)\end{array}$ & $\begin{array}{l}0.25538^{*} \\
(0.03806)\end{array}$ & $\begin{array}{l}0.2408^{*} \\
(0.03807)\end{array}$ & $\begin{array}{l}0.24287^{*} \\
(0.03799)\end{array}$ & $\begin{array}{l}0.22001^{*} \\
(0.0475)\end{array}$ \\
\hline Factor $1 \& 2$ & $\begin{array}{l}0.25176^{*} \\
(0.06877)\end{array}$ & $\begin{array}{l}0.24909^{*} \\
(0.06882)\end{array}$ & $\begin{array}{l}0.3427^{*} \\
(0.0746)\end{array}$ & $\begin{array}{l}0.29392^{*} \\
(0.07308)\end{array}$ & $\begin{array}{l}0.32388^{*} \\
(0.0794)\end{array}$ & $\begin{array}{l}0.24184^{*} \\
(0.08878)\end{array}$ & $\begin{array}{l}0.3604^{*} \\
(0.07927)\end{array}$ & $\begin{array}{l}0.25195^{*} \\
(0.07586)\end{array}$ & $\begin{array}{l}0.31551^{*} \\
(0.07306)\end{array}$ & $\begin{array}{l}0.23921^{*} \\
(0.06993)\end{array}$ & $\begin{array}{l}0.26138^{*} \\
(0.0688)\end{array}$ & $\begin{array}{l}0.23181^{*} \\
(0.07361)\end{array}$ \\
\hline $\mathrm{A}$ & $\begin{array}{l}0.27179^{*} \\
(0.05203)\end{array}$ & $\begin{array}{l}0.25022^{*} \\
(0.05876)\end{array}$ & $\begin{array}{l}0.39499^{*} \\
(0.04604)\end{array}$ & $\begin{array}{l}0.33764^{*} \\
(0.04149)\end{array}$ & $\begin{array}{l}0.34258^{*} \\
(0.04102)\end{array}$ & $\begin{array}{l}0.27554^{*} \\
(0.06028)\end{array}$ & $\begin{array}{l}0.37591^{*} \\
(0.04266)\end{array}$ & $\begin{array}{l}0.28462^{*} \\
(0.05364)\end{array}$ & $\begin{array}{l}0.36245^{*} \\
(0.0416)\end{array}$ & $\begin{array}{l}0.24742^{*} \\
(0.04701)\end{array}$ & $\begin{array}{l}0.30957^{*} \\
(0.03274)\end{array}$ & $\begin{array}{l}0.25711^{*} \\
(0.04487)\end{array}$ \\
\hline Individual Variable & $\begin{array}{l}0.01708 \\
(0.05167)\end{array}$ & $\begin{array}{l}0.04162 \\
(0.06028)\end{array}$ & $\begin{array}{l}-0.13099^{*} \\
(0.04674)\end{array}$ & $\begin{array}{l}-0.06707 \\
(0.0433)\end{array}$ & $\begin{array}{c}-0.07456^{\ddagger} \\
(0.04311)\end{array}$ & $\begin{array}{l}0.01215 \\
(0.05906)\end{array}$ & $\begin{array}{l}-0.10981^{\dagger} \\
(0.04247)\end{array}$ & $\begin{array}{l}0.0025 \\
(0.05454)\end{array}$ & $\begin{array}{l}-0.09562^{\dagger} \\
(0.04181)\end{array}$ & $\begin{array}{l}0.05074 \\
(0.05095)\end{array}$ & $\begin{array}{l}-0.05088 \\
(0.04605)\end{array}$ & $\begin{array}{l}0.03473 \\
(0.04325)\end{array}$ \\
\hline
\end{tabular}

* Significant at the 0.01 level $†$ Significant at the 0.05 level $\$$ Significant at the 0.10 level

Table 97 Coefficients with individual variables for business practices - Vehicles Industry 


\begin{tabular}{|c|c|c|c|c|c|c|c|c|c|c|c|c|c|c|c|c|c|c|}
\hline $\begin{array}{l}\text { Individual variable } \\
\text { included in the model } \\
\text { in the column }\end{array}$ & 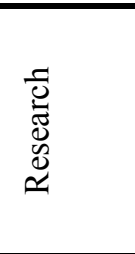 & 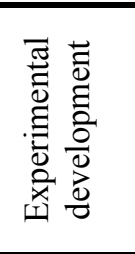 & 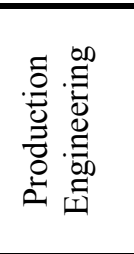 & 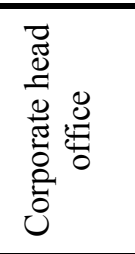 & 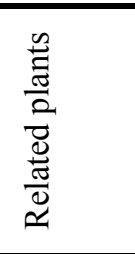 & 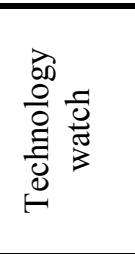 & 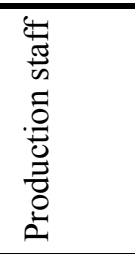 & 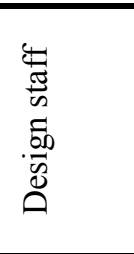 & 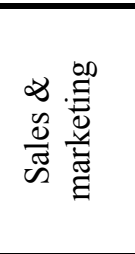 & 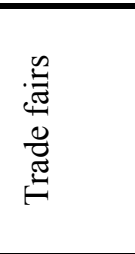 & 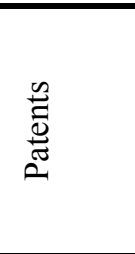 & 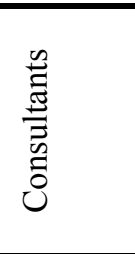 & 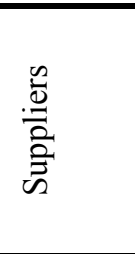 & 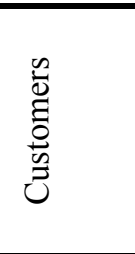 & 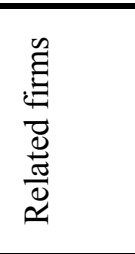 & 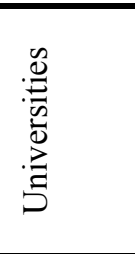 & 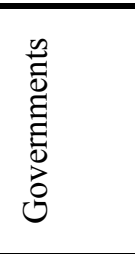 & $\begin{array}{l}0 \\
0 \\
0 \\
\bar{\Xi} \\
0 \\
0 \\
\overline{0} \\
\overline{0}\end{array}$ \\
\hline No factors & $\begin{array}{l}0.16406^{*} \\
(0.03582)\end{array}$ & $\begin{array}{l}0.16171^{*} \\
(0.03583)\end{array}$ & $\begin{array}{l}0.1661^{*} \\
(0.03664)\end{array}$ & $\begin{array}{l}0.16399^{*} \\
(0.03575)\end{array}$ & $\begin{array}{l}0.16239^{*} \\
(0.03568)\end{array}$ & $\begin{array}{l}0.16173^{*} \\
(0.03571)\end{array}$ & $\begin{array}{l}0.16931^{*} \\
(0.03652)\end{array}$ & $\begin{array}{l}0.15988^{*} \\
(0.03591)\end{array}$ & $\begin{array}{l}0.16065^{*} \\
(0.03564)\end{array}$ & $\begin{array}{l}0.16188^{*} \\
(0.03568)\end{array}$ & $\begin{array}{l}0.15907^{*} \\
(0.03663)\end{array}$ & $\begin{array}{l}0.15958^{*} \\
(0.0352)\end{array}$ & $\begin{array}{l}0.16147^{*} \\
(0.03581)\end{array}$ & $\begin{array}{l}0.16124^{*} \\
(0.03603)\end{array}$ & $\begin{array}{l}0.1598^{*} \\
(0.03576)\end{array}$ & $\begin{array}{l}0.16341^{*} \\
(0.03562)\end{array}$ & $\begin{array}{l}0.16093^{*} \\
(0.03571)\end{array}$ & $\begin{array}{l}0.15897^{*} \\
(0.03505)\end{array}$ \\
\hline Factor 4 & $\begin{array}{l}0.22629^{\dagger} \\
(0.10374)\end{array}$ & $\begin{array}{l}0.21215^{\dagger} \\
(0.10517)\end{array}$ & $\begin{array}{l}0.23013^{\dagger} \\
(0.10716)\end{array}$ & $\begin{array}{l}0.27703^{\dagger} \\
(0.12281)\end{array}$ & $\begin{array}{l}0.25915^{\dagger} \\
(0.11195)\end{array}$ & $\begin{array}{l}0.21382^{\dagger} \\
(0.10242)\end{array}$ & $\begin{array}{l}0.24822^{\dagger} \\
(0.10854)\end{array}$ & $\begin{array}{l}0.19614^{1} \\
(0.1073)\end{array}$ & $\begin{array}{l}0.20339^{\dagger} \\
(0.10252)\end{array}$ & $\begin{array}{l}0.21^{\dagger} \\
(0.1024)\end{array}$ & & $\begin{array}{l}0.21382^{\dagger} \\
(0.10093)\end{array}$ & $\begin{array}{l}0.21149^{t} \\
(0.10319)\end{array}$ & $\begin{array}{l}0.21153^{\prime} \\
(0.10377)\end{array}$ & & & & $\begin{array}{l}0.20852^{\dagger} \\
(0.10051)\end{array}$ \\
\hline Facto & $\begin{array}{l}0.065 \\
(0.12639) \\
\end{array}$ & $\begin{array}{l}0.06242 \\
(0.12671) \\
\end{array}$ & $\begin{array}{l}0.06803 \\
(0.12682) \\
\end{array}$ & $\begin{array}{l}0.06292 \\
(0.12627) \\
\end{array}$ & $\begin{array}{l}0.06292 \\
(0.12624) \\
\end{array}$ & $\begin{array}{l}0.06103 \\
(0.12639) \\
\end{array}$ & $\begin{array}{l}0.12179 \\
(0.14063) \\
\end{array}$ & $\begin{array}{l}0.0598 \\
(0.12656) \\
\end{array}$ & $\begin{array}{l}0.05159 \\
(0.1264) \\
\end{array}$ & $\begin{array}{l}0.06292 \\
(0.12625) \\
\end{array}$ & $\begin{array}{l}0.0588 \\
(0.12703) \\
\end{array}$ & $\begin{array}{l}0.05604 \\
(0.12455) \\
\end{array}$ & $\begin{array}{l}0.05607 \\
(0.13103) \\
\end{array}$ & & & & $\begin{array}{l}0.06292 \\
(0.12631) \\
\end{array}$ & $\begin{array}{l}0.06292 \\
(0.124) \\
\end{array}$ \\
\hline Factor 3\&4 & $\begin{array}{l}0.25732^{\dagger} \\
(0.10342)\end{array}$ & $\begin{array}{l}0.2466^{\dagger} \\
(0.11443)\end{array}$ & $\begin{array}{l}0.28158^{\dagger} \\
(0.11946)\end{array}$ & $\begin{array}{l}0.3088^{\dagger} \\
(0.12067)\end{array}$ & $\begin{array}{l}0.29688^{*} \\
(0.11305)\end{array}$ & $\begin{array}{l}0.25014^{t} \\
(0.10299)\end{array}$ & $\begin{array}{l}0.28451^{*} \\
(0.10907)\end{array}$ & $\begin{array}{l}0.22809^{\dagger} \\
(0.11043)\end{array}$ & $\begin{array}{l}0.20228^{\ddagger} \\
(0.10966)\end{array}$ & $\begin{array}{l}0.24627^{\dagger} \\
(0.10297)\end{array}$ & $\begin{array}{l}0.24313^{\dagger} \\
(0.105)\end{array}$ & $\begin{array}{l}0.241^{\dagger} \\
(0.10155)\end{array}$ & $\begin{array}{l}0.24689^{\dagger} \\
(0.10436)\end{array}$ & $\begin{array}{l}0.24197^{\dagger} \\
(0.11778)\end{array}$ & $\begin{array}{l}0.18105 \\
(0.12888)\end{array}$ & $\begin{array}{l}0.32294^{*} \\
(0.11591)\end{array}$ & $\begin{array}{l}0.24826^{\prime} \\
(0.10296)\end{array}$ & $\begin{array}{l}0.17001 \\
(0.10448)\end{array}$ \\
\hline Factor 2 & $\begin{array}{l}0.512 \\
0.10\end{array}$ & $\begin{array}{l}0.47071^{* \prime} \\
(0.11349)\end{array}$ & $\begin{array}{l}0.48739^{*} \\
(0.09093)\end{array}$ & $\begin{array}{l}0.4889^{\circ} \\
(0.08916)\end{array}$ & $\begin{array}{l}0.47997^{*} \\
(0.08811)\end{array}$ & $\begin{array}{l}0.4758^{*} \\
(0.08811)\end{array}$ & $\begin{array}{l}0.48556 " \\
(0.08863)\end{array}$ & $\begin{array}{l}0.4665^{*} \\
(0.08972)\end{array}$ & $\begin{array}{l}0.47144^{\prime \prime} \\
(0.08797)\end{array}$ & $\begin{array}{l}0.46971^{\prime \prime} \\
(0.08825)\end{array}$ & $\begin{array}{l}0.47079^{*} \\
(0.08933)\end{array}$ & & $\begin{array}{l}0.47403^{\prime \prime} \\
(0.08864)\end{array}$ & & $\begin{array}{l}0.46212^{\prime \prime} \\
(0.08938)\end{array}$ & $\begin{array}{l}0.4758^{*} \\
(0.08785)\end{array}$ & & $\begin{array}{l}0.46932^{\prime \prime} \\
(0.08648)\end{array}$ \\
\hline Factor $2 \& 4$ & $\begin{array}{l}0.47105^{*} \\
(0.10269)\end{array}$ & $\begin{array}{l}0.42746^{*} \\
(0.1151)\end{array}$ & $\begin{array}{l}0.46738^{*} \\
(0.11145)\end{array}$ & $\begin{array}{l}0.44393^{*} \\
(0.08994)\end{array}$ & $\begin{array}{l}0.483^{*} \\
(0.1024)\end{array}$ & $\begin{array}{l}0.42733^{*} \\
(0.08948)\end{array}$ & $\begin{array}{l}0.49981^{*} \\
(0.11373)\end{array}$ & $\begin{array}{l}0.4^{*} \\
(0.10684)\end{array}$ & $\begin{array}{l}0.4268^{\circ} \\
(0.0891)\end{array}$ & $\begin{array}{l}0.43263^{*} \\
(0.0891)\end{array}$ & $\begin{array}{l}0.41529^{\prime \prime} \\
(0.10204)\end{array}$ & $\begin{array}{l}0.42921^{*} \\
(0.08789)\end{array}$ & $\begin{array}{l}0.4324^{*} \\
(0.08929)\end{array}$ & $\begin{array}{l}0.43216^{*} \\
(0.08935)\end{array}$ & $\begin{array}{l}0.38299^{\prime \prime} \\
(0.10512)\end{array}$ & $\begin{array}{l}0.49439^{*} \\
(0.09993)\end{array}$ & $\begin{array}{l}0.42444^{\prime \prime} \\
(0.08964)\end{array}$ & $\begin{array}{l}0.4093^{*} \\
(0.08785)\end{array}$ \\
\hline $\mathrm{F}$ & & & & & & & & & Insuff & ent Data & & & & & & & & \\
\hline Factor $2,3 \&$ & & & & & & & & & $\begin{array}{l}0.3406^{\dagger} \\
(0.13177) \\
\end{array}$ & & & & & & & & $\begin{array}{l}30049^{\dagger} \\
.14354) \\
\end{array}$ & $\begin{array}{l}0.33211^{\dagger} \\
(0.12953) \\
\end{array}$ \\
\hline Factor 1 & $\begin{array}{l}0.12697 \\
(0.08964)\end{array}$ & $\begin{array}{l}0.11641 \\
(0.08897)\end{array}$ & $\begin{array}{l}0.14604 \\
(0.10493)\end{array}$ & $\begin{array}{l}0.13455 \\
(0.09059)\end{array}$ & $\begin{array}{l}0.1182 \\
(0.08856)\end{array}$ & $\begin{array}{l}0.10399 \\
(0.09039)\end{array}$ & $\begin{array}{l}0.18 \\
(0.11071)\end{array}$ & & $\begin{array}{l}0.09601 \\
(0.09)\end{array}$ & & & & & & & & & $\begin{array}{l}0.09243 \\
(0.08735)\end{array}$ \\
\hline Factor & $\begin{array}{l}0.11734 \\
(0.33326)\end{array}$ & $\begin{array}{l}0.07557 \\
(0.33312)\end{array}$ & $\begin{array}{l}0.09171 \\
(0.33048)\end{array}$ & $\begin{array}{l}0.11889 \\
(0.33191)\end{array}$ & $\begin{array}{l}0.1293 \\
(0.33288)\end{array}$ & $\begin{array}{l}0.05683 \\
(0.33077)\end{array}$ & $\begin{array}{l}0.14796 \\
(0.33701)\end{array}$ & $\begin{array}{l}0.0454 \\
(0.33503)\end{array}$ & $\begin{array}{l}0.07893 \\
(0.32861)\end{array}$ & $\begin{array}{l}0.07893 \\
(0.32907)\end{array}$ & $\begin{array}{l}0.05848 \\
(0.33479)\end{array}$ & $\begin{array}{l}-0.04806 \\
(0.32803)\end{array}$ & $\begin{array}{l}0.07036 \\
(0.33249)\end{array}$ & $\begin{array}{l}0.07013 \\
(0.33541)\end{array}$ & $\begin{array}{l}0.07893 \\
(0.32918)\end{array}$ & $\begin{array}{l}0.15174 \\
(0.33278)\end{array}$ & $\begin{array}{l}0.07893 \\
(0.32921)\end{array}$ & $\begin{array}{l}0.07893 \\
(0.32319)\end{array}$ \\
\hline $\mathrm{F}$ & $\begin{array}{l}0.19504 \\
(0.14159)\end{array}$ & $\begin{array}{l}0.17695 \\
(0.14094)\end{array}$ & $\begin{array}{l}0.21057 \\
(0.15316)\end{array}$ & $\begin{array}{l}0.2316 \\
(0.15106)\end{array}$ & $\begin{array}{l}0.17812 \\
(0.13967)\end{array}$ & $\begin{array}{l}0.16162 \\
(0.14164)\end{array}$ & $\begin{array}{l}0.24716 \\
(0.15745)\end{array}$ & $\begin{array}{l}0.14459 \\
(0.15237)\end{array}$ & $\begin{array}{l}0.10113 \\
(0.1525)\end{array}$ & $\begin{array}{l}0.17812 \\
(0.13969)\end{array}$ & $\begin{array}{l}0.15947 \\
(0.14971)\end{array}$ & $\begin{array}{l}0.16971 \\
(0.13782)\end{array}$ & $\begin{array}{l}0.17144 \\
(0.14388)\end{array}$ & $\begin{array}{l}0.16933 \\
(0.15283)\end{array}$ & $\begin{array}{l}0.11509 \\
(0.15663)\end{array}$ & $\begin{array}{l}0.1845 \\
(0.13949)\end{array}$ & $\begin{array}{l}0.17812 \\
(0.13975)\end{array}$ & $\begin{array}{l}0.17812 \\
(0.13719)\end{array}$ \\
\hline Fo & $\begin{array}{l}0.36545^{*} \\
(0.04812 \\
\end{array}$ & $\begin{array}{l}0.36158^{*} \\
(0.04831)\end{array}$ & $\begin{array}{l}0.39647^{*} \\
(0.08177)\end{array}$ & $\begin{array}{l}0.41771^{*} \\
(0.07663)\end{array}$ & $\begin{array}{l}0.38044^{*} \\
(0.0513)\end{array}$ & $\begin{array}{l}0.33899^{*} \\
(0.0574)\end{array}$ & $\begin{array}{l}0.43103^{*} \\
(0.08698)\end{array}$ & $\begin{array}{l}0.33294^{*} \\
(0.07096)\end{array}$ & $\begin{array}{l}0.30889^{*} \\
(0.06396)\end{array}$ & $\begin{array}{l}0.36199^{*} \\
(0.04786)\end{array}$ & $\begin{array}{l}0.34365^{*} \\
(0.07093)\end{array}$ & $\begin{array}{l}0.34919^{*} \\
(0.04745)\end{array}$ & $\begin{array}{l}0.35882^{*} \\
(0.0505)\end{array}$ & $\begin{array}{l}0.35385^{*} \\
(0.07431)\end{array}$ & $\begin{array}{l}0.29468^{*} \\
(0.08946)\end{array}$ & $\begin{array}{l}0.39006^{*} \\
(0.05398)\end{array}$ & $\begin{array}{l}0.36077^{*} \\
(0.0479)\end{array}$ & $\begin{array}{l}0.3593^{*} \\
(0.04701)\end{array}$ \\
\hline $\mathrm{F}$ & $\begin{array}{l}0.26556^{7} \\
(0.13723)\end{array}$ & $\begin{array}{l}0.23157 \\
(0.14733)\end{array}$ & $\begin{array}{l}0.27101^{7} \\
(0.14766)\end{array}$ & $\begin{array}{l}0.24369^{\ddagger} \\
(0.13177)\end{array}$ & $\begin{array}{l}0.24314^{7} \\
(0.13167)\end{array}$ & $\begin{array}{l}0.23068^{\ddagger} \\
(0.13185)\end{array}$ & $\begin{array}{l}0.30125^{t} \\
(0.14822)\end{array}$ & $\begin{array}{l}0.20274 \\
(0.14488)\end{array}$ & $\begin{array}{l}0.1866 \\
(0.13721)\end{array}$ & $\begin{array}{l}0.22995^{\ddagger} \\
(0.13167)\end{array}$ & $\begin{array}{l}0.22437 \\
(0.13604)\end{array}$ & $\begin{array}{l}0.22135^{5} \\
(0.12983)\end{array}$ & $\begin{array}{l}0.23067^{\ddagger} \\
(0.13469)\end{array}$ & $\begin{array}{l}0.23352^{\ddagger} \\
(0.13316)\end{array}$ & $\begin{array}{l}0.20825 \\
(0.13526)\end{array}$ & $\begin{array}{l}0.24054^{7} \\
(0.13128)\end{array}$ & $\begin{array}{l}0.21465 \\
(0.13391)\end{array}$ & $\begin{array}{l}0.13899 \\
(0.13311)\end{array}$ \\
\hline Factor $1,2 \& 4$ & $\begin{array}{l}0.32498^{\dagger} \\
(0.13179)\end{array}$ & $\begin{array}{l}0.2847^{\dagger} \\
(0.12449)\end{array}$ & $\begin{array}{l}0.32131^{\dagger} \\
(0.13875)\end{array}$ & $\begin{array}{l}0.34977^{\dagger} \\
(0.13916)\end{array}$ & 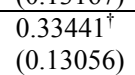 & $\begin{array}{l}0.27606^{\dagger} \\
(0.12242)\end{array}$ & $\begin{array}{l}0.3556^{\dagger} \\
(0.14152)\end{array}$ & & & & & $\begin{array}{l}0.25476 \\
(0.12038)\end{array}$ & & & & & & $\begin{array}{l}0.17494 \\
(0.12488)\end{array}$ \\
\hline Factor $1,2 \& 3$ & $\begin{array}{l}0.23662 " \\
(0.08713)\end{array}$ & $\begin{array}{l}0.19431^{\ddagger} \\
(0.10052)\end{array}$ & $\begin{array}{l}0.23152^{\prime} \\
(0.09477)\end{array}$ & $\begin{array}{l}0.20687^{\prime \prime} \\
(0.07214)\end{array}$ & $\begin{array}{l}0.2002^{*} \\
(0.07167)\end{array}$ & $\begin{array}{l}0.19473^{\prime \prime} \\
(0.07202)\end{array}$ & $\begin{array}{l}0.26177^{\prime \prime} \\
(0.09723)\end{array}$ & $\begin{array}{l}0.16579^{\ddagger} \\
(0.09381)\end{array}$ & $\begin{array}{l}0.12367 \\
(0.09376)\end{array}$ & $\begin{array}{l}0.19683^{\prime \prime} \\
(0.07172)\end{array}$ & $\begin{array}{l}0.17982^{\dagger} \\
(0.09081)\end{array}$ & $\begin{array}{l}0.14361^{\ddagger} \\
(0.0737)\end{array}$ & $\begin{array}{l}0.19531^{\prime \prime} \\
(0.07454)\end{array}$ & $\begin{array}{l}0.1949^{\dagger} \\
(0.07815)\end{array}$ & $\begin{array}{l}0.13023 \\
(0.10563)\end{array}$ & $\begin{array}{l}0.20523^{\prime \prime} \\
(0.07166)\end{array}$ & $\begin{array}{l}0.19235^{*} \\
(0.07216)\end{array}$ & $\begin{array}{l}0.17288^{\prime} \\
(0.07094)\end{array}$ \\
\hline All Factors & $\begin{array}{l}0.16125^{*} \\
(0.04864)\end{array}$ & $\begin{array}{l}0.12711 \\
(0.0794)\end{array}$ & $\begin{array}{l}0.16612^{\dagger} \\
(0.07148)\end{array}$ & $\begin{array}{l}0.19345^{*} \\
(0.07213)\end{array}$ & $\begin{array}{l}0.16672^{*} \\
(0.04564)\end{array}$ & $\begin{array}{l}0.10711^{\dagger} \\
(0.04586)\end{array}$ & $\begin{array}{l}0.20096^{\dagger} \\
(0.07814)\end{array}$ & $\begin{array}{l}0.10187 \\
(0.06255)\end{array}$ & $\begin{array}{l}0.05847 \\
(0.06627)\end{array}$ & $\begin{array}{l}0.12653^{*} \\
(0.03053)\end{array}$ & $\begin{array}{l}0.11211^{\ddagger} \\
(0.06503)\end{array}$ & $\begin{array}{l}0.08215^{\dagger} \\
(0.03499)\end{array}$ & & & & $\begin{array}{l}0.19342^{*} \\
(0.05409)\end{array}$ & & $\begin{array}{l}0.03285 \\
(0.04416)\end{array}$ \\
\hline $\begin{array}{l}\text { Individual } \\
\text { Variable }\end{array}$ & $\begin{array}{l}0.03842 \\
0.05093)\end{array}$ & $\begin{array}{l}0.00532 \\
(0.07465)\end{array}$ & $\begin{array}{l}-0.03475 \\
(0.06677)\end{array}$ & $\begin{array}{l}-0.06321 \\
(0.06789)\end{array}$ & $\begin{array}{l}-0.05037 \\
(0.05048)\end{array}$ & $\begin{array}{l}0.03495 \\
(0.04806)\end{array}$ & $\begin{array}{l}-0.06904 \\
(0.07262)\end{array}$ & $\begin{array}{l}0.03353 \\
(0.0604)\end{array}$ & $\begin{array}{l}0.07699 \\
(0.06163)\end{array}$ & $\begin{array}{l}0.10755 \\
(0.11092)\end{array}$ & $\begin{array}{l}0.02045 \\
(0.05828)\end{array}$ & $\begin{array}{l}0.12699^{*} \\
(0.04748)\end{array}$ & $\begin{array}{l}0.00857 \\
(0.04272)\end{array}$ & $\begin{array}{l}0.0088 \\
(0.06134)\end{array}$ & $\begin{array}{l}0.06909 \\
(0.07756)\end{array}$ & $\begin{array}{l}-0.07281 \\
(0.05375)\end{array}$ & $\begin{array}{l}0.05226 \\
(0.06038)\end{array}$ & $\begin{array}{l}0.14892^{*} \\
(0.04931)\end{array}$ \\
\hline
\end{tabular}

\begin{tabular}{lllllllll} 
* Significant at the 0.01 level & $(0.05093)$ & $(0.07465)$ & $(0.06677)$ & $(0.06789)$ & $(0.05048)$ & $(0.04806)$ & $(0.07262)$ & $(0.06$ \\
\hline Significant at the 0.05 level & \$ Significant at the 0.10 level
\end{tabular}

Table 98 Coefficients with individual variables for source of implementation support - Vehicles Industry 


\begin{tabular}{|c|c|c|c|c|c|c|c|c|c|c|c|c|c|}
\hline $\begin{array}{l}\text { Individual variable } \\
\text { included in the model in } \\
\text { the column }\end{array}$ & 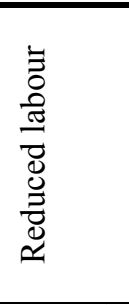 & 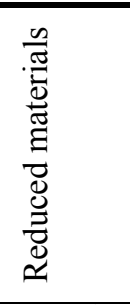 & 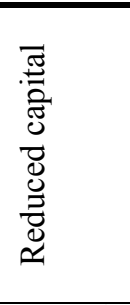 & 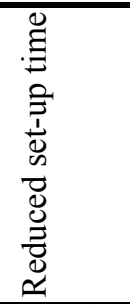 & 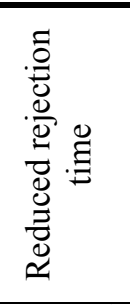 & 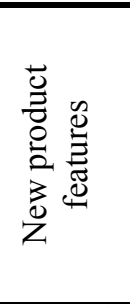 & 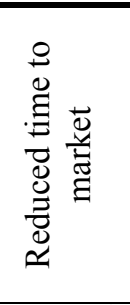 & 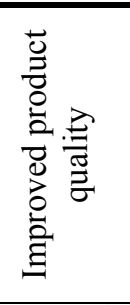 & 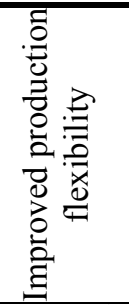 & 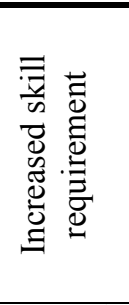 & 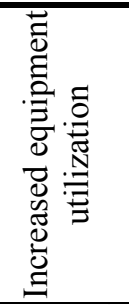 & 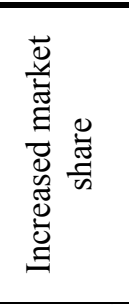 & 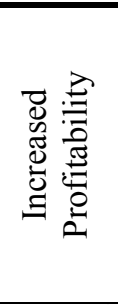 \\
\hline No factors & $\begin{array}{l}0.16508^{*} \\
(0.0395)\end{array}$ & $\begin{array}{l}0.1697^{*} \\
(0.03969)\end{array}$ & $\begin{array}{l}0.16913^{*} \\
(0.03981)\end{array}$ & $\begin{array}{l}0.1685^{*} \\
(0.03987)\end{array}$ & $\begin{array}{l}0.16794^{*} \\
(0.039)\end{array}$ & $\begin{array}{l}0.16849^{*} \\
(0.03995)\end{array}$ & $\begin{array}{l}0.16967^{*} \\
(0.03884)\end{array}$ & $\begin{array}{l}0.16774^{*} \\
(0.03965)\end{array}$ & $\begin{array}{l}0.16905^{*} \\
(0.03985)\end{array}$ & $\begin{array}{l}0.16801^{*} \\
(0.0399)\end{array}$ & $\begin{array}{l}0.16871^{*} \\
(0.03996)\end{array}$ & $\begin{array}{l}0.17079^{*} \\
(0.03916)\end{array}$ & $\begin{array}{l}0.16834^{*} \\
(0.03981)\end{array}$ \\
\hline Factor 4 & $\begin{array}{l}0.09344 \\
(0.13909)\end{array}$ & $\begin{array}{l}0.18714 \\
(0.13447)\end{array}$ & $\begin{array}{l}0.18181 \\
(0.13484)\end{array}$ & $\begin{array}{l}0.1495 \\
(0.13986)\end{array}$ & $\begin{array}{l}0.17231 \\
(0.13215)\end{array}$ & $\begin{array}{l}0.17997 \\
(0.13547)\end{array}$ & $\begin{array}{l}0.23859^{4} \\
(0.13255)\end{array}$ & $\begin{array}{l}0.16998 \\
(0.13445)\end{array}$ & $\begin{array}{l}0.21589 \\
(0.13915)\end{array}$ & $\begin{array}{l}0.16353 \\
(0.13729)\end{array}$ & $\begin{array}{l}0.17983 \\
(0.14079)\end{array}$ & $\begin{array}{l}0.2811^{\dagger} \\
(0.13674)\end{array}$ & $\begin{array}{l}0.14582 \\
(0.13822\end{array}$ \\
\hline Factor 3 & $\begin{array}{l}-0.15398^{\dagger} \\
(0.06071)\end{array}$ & $\begin{array}{l}-0.11733^{\dagger} \\
(0.05904)\end{array}$ & $\begin{array}{l}-0.11676^{\dagger} \\
(0.05923)\end{array}$ & $\begin{array}{l}-0.12027^{\dagger} \\
(0.05934)\end{array}$ & $\begin{array}{l}-0.16732^{*} \\
(0.05991)\end{array}$ & $\begin{array}{l}-0.11999^{\dagger} \\
(0.05976)\end{array}$ & $\begin{array}{l}-0.02367 \\
(0.06363)\end{array}$ & $\begin{array}{l}-0.20633^{*} \\
(0.07632)\end{array}$ & $\begin{array}{l}-0.08409 \\
(0.06833)\end{array}$ & $\begin{array}{l}-0.147^{\dagger} \\
(0.07052)\end{array}$ & $\begin{array}{c}-0.11981^{\ddagger} \\
(0.06593)\end{array}$ & $\begin{array}{l}-0.06579 \\
(0.06085)\end{array}$ & $\begin{array}{l}-0.13459 \\
(0.06078\end{array}$ \\
\hline Factor $3 \& 4$ & $\begin{array}{l}0.12497 \\
(0.07833)\end{array}$ & $\begin{array}{l}0.25735^{*} \\
(0.05403)\end{array}$ & $\begin{array}{l}0.32^{*} \\
(0.07558)\end{array}$ & $\begin{array}{l}0.25521^{*} \\
(0.05428)\end{array}$ & $\begin{array}{l}0.25468^{*} \\
(0.05309)\end{array}$ & $\begin{array}{l}0.25577^{*} \\
(0.05438)\end{array}$ & $\begin{array}{l}0.41638^{*} \\
(0.06947)\end{array}$ & $\begin{array}{l}0.25252^{*} \\
(0.054)\end{array}$ & $\begin{array}{l}0.30834^{*} \\
(0.07494)\end{array}$ & $\begin{array}{l}0.21422^{*} \\
(0.0779)\end{array}$ & $\begin{array}{l}0.25353^{*} \\
(0.07581)\end{array}$ & $\begin{array}{l}0.41251^{*} \\
(0.07468)\end{array}$ & $\begin{array}{l}0.25315^{*} \\
(0.05425\end{array}$ \\
\hline Factor 2 & $\begin{array}{l}0.14263 \\
(0.20105)\end{array}$ & $\begin{array}{l}0.32982^{\ddagger} \\
(0.19583)\end{array}$ & $\begin{array}{l}0.30959 \\
(0.19571)\end{array}$ & $\begin{array}{l}0.27138 \\
(0.19437)\end{array}$ & $\begin{array}{l}0.23848 \\
(0.19039)\end{array}$ & $\begin{array}{l}0.27779 \\
(0.19455)\end{array}$ & $\begin{array}{l}0.29903 \\
(0.18934)\end{array}$ & $\begin{array}{l}0.23229 \\
(0.19476)\end{array}$ & $\begin{array}{l}0.28454 \\
(0.19425)\end{array}$ & $\begin{array}{l}0.27779 \\
(0.19433)\end{array}$ & $\begin{array}{l}0.276 \\
(0.19774)\end{array}$ & $\begin{array}{l}0.31327 \\
(0.19114)\end{array}$ & $\begin{array}{l}0.23721 \\
(0.19695\end{array}$ \\
\hline Factor $2 \& 4$ & $\begin{array}{l}0.10994 \\
(0.31398)\end{array}$ & $\begin{array}{l}0.33021 \\
(0.31429)\end{array}$ & $\begin{array}{l}0.2671 \\
(0.31163)\end{array}$ & $\begin{array}{l}0.22862 \\
(0.31212)\end{array}$ & $\begin{array}{l}0.14394 \\
(0.30636)\end{array}$ & $\begin{array}{l}0.24509 \\
(0.31208)\end{array}$ & $\begin{array}{l}0.24509 \\
(0.30357)\end{array}$ & $\begin{array}{l}0.21362 \\
(0.31031)\end{array}$ & $\begin{array}{l}0.29722 \\
(0.31568)\end{array}$ & $\begin{array}{l}0.2311 \\
(0.31228)\end{array}$ & $\begin{array}{l}0.24233 \\
(0.31683)\end{array}$ & $\begin{array}{l}0.4044 \\
(0.31062)\end{array}$ & $\begin{array}{l}0.19289 \\
(0.31423\end{array}$ \\
\hline Factor $2 \& 3$ & $\begin{array}{l}0.03949 \\
(0.2735)\end{array}$ & $\begin{array}{l}0.25976 \\
(0.27337)\end{array}$ & $\begin{array}{l}0.20765 \\
(0.27079)\end{array}$ & $\begin{array}{l}0.17464 \\
(0.26983)\end{array}$ & $\begin{array}{l}0.09878 \\
(0.26498) \\
\end{array}$ & $\begin{array}{l}0.17204 \\
(0.27048)\end{array}$ & $\begin{array}{l}0.17464 \\
(0.2629) \\
\end{array}$ & $\begin{array}{l}0.15104 \\
(0.26863)\end{array}$ & $\begin{array}{l}0.22677 \\
(0.2746)\end{array}$ & $\begin{array}{l}0.15365 \\
(0.27141)\end{array}$ & $\begin{array}{l}0.17257 \\
(0.27337)\end{array}$ & $\begin{array}{l}0.17464 \\
(0.26501) \\
\end{array}$ & $\begin{array}{l}0.11592 \\
(0.27396\end{array}$ \\
\hline Factor $2,3 \& 4$ & $\begin{array}{l}0.04618 \\
(0.17658)\end{array}$ & $\begin{array}{l}0.25551 \\
(0.17321)\end{array}$ & $\begin{array}{l}0.2132 \\
(0.17001)\end{array}$ & $\begin{array}{l}0.17653 \\
(0.16831)\end{array}$ & $\begin{array}{l}0.13235 \\
(0.16525)\end{array}$ & $\begin{array}{l}0.17764 \\
(0.16915)\end{array}$ & $\begin{array}{l}0.27646^{\ddagger} \\
(0.16607)\end{array}$ & $\begin{array}{l}0.10527 \\
(0.17244)\end{array}$ & $\begin{array}{l}0.22006 \\
(0.17247)\end{array}$ & $\begin{array}{l}0.15291 \\
(0.1725)\end{array}$ & $\begin{array}{l}0.17893 \\
(0.17506)\end{array}$ & $\begin{array}{l}0.2892^{\ddagger} \\
(0.16912)\end{array}$ & $\begin{array}{l}0.13077 \\
(0.1733)\end{array}$ \\
\hline Factor 1 & $\begin{array}{l}0.23388 \\
(0.31865)\end{array}$ & $\begin{array}{l}0.31899 \\
(0.32442)\end{array}$ & $\begin{array}{l}0.23388 \\
(0.32138)\end{array}$ & $\begin{array}{l}0.18446 \\
(0.32664)\end{array}$ & $\begin{array}{l}0.23388 \\
(0.31488)\end{array}$ & $\begin{array}{l}0.22345 \\
(0.32512)\end{array}$ & $\begin{array}{l}0.3979 \\
(0.31699)\end{array}$ & $\begin{array}{l}0.23388 \\
(0.32007)\end{array}$ & $\begin{array}{l}0.286 \\
(0.32584)\end{array}$ & $\begin{array}{l}0.23388 \\
(0.32203)\end{array}$ & $\begin{array}{l}0.23388 \\
(0.32244)\end{array}$ & $\begin{array}{l}0.23388 \\
(0.31613)\end{array}$ & $\begin{array}{l}0.23388 \\
(0.32142\end{array}$ \\
\hline Factor $1 \& 4$ & $\begin{array}{l}0.24127 \\
(0.37777)\end{array}$ & $\begin{array}{l}0.28383 \\
(0.38072)\end{array}$ & $\begin{array}{l}0.27428 \\
(0.38199)\end{array}$ & $\begin{array}{l}0.21656 \\
(0.38261)\end{array}$ & $\begin{array}{l}0.16541 \\
(0.37403)\end{array}$ & $\begin{array}{l}0.23085 \\
(0.38451)\end{array}$ & $\begin{array}{l}0.40529 \\
(0.37465)\end{array}$ & $\begin{array}{l}0.19406 \\
(0.38034)\end{array}$ & $\begin{array}{l}0.26733 \\
(0.38227)\end{array}$ & $\begin{array}{l}0.22028 \\
(0.38281)\end{array}$ & $\begin{array}{l}0.23989 \\
(0.38324)\end{array}$ & $\begin{array}{l}0.40057 \\
(0.37856)\end{array}$ & $\begin{array}{l}0.16297 \\
(0.38673\end{array}$ \\
\hline Factor $1 \& 3$ & $\begin{array}{l}0.14517 \\
(0.16991)\end{array}$ & $\begin{array}{l}0.22244 \\
(0.16825)\end{array}$ & $\begin{array}{l}0.22056 \\
(0.16877)\end{array}$ & $\begin{array}{l}0.18988 \\
(0.17113)\end{array}$ & $\begin{array}{l}0.16949 \\
(0.16583)\end{array}$ & $\begin{array}{l}0.20673 \\
(0.17179)\end{array}$ & $\begin{array}{l}0.32662^{+} \\
(0.16762)\end{array}$ & $\begin{array}{l}0.15669 \\
(0.17094)\end{array}$ & $\begin{array}{l}0.26622 \\
(0.1766)\end{array}$ & $\begin{array}{l}0.17211 \\
(0.17802)\end{array}$ & $\begin{array}{l}0.21241 \\
(0.17244)\end{array}$ & $\begin{array}{l}0.22971 \\
(0.16601)\end{array}$ & $\begin{array}{l}0.19875 \\
(0.1692)\end{array}$ \\
\hline Factor $1,3 \& 4$ & $\begin{array}{l}0.12434 \\
(0.08316)\end{array}$ & $\begin{array}{l}0.16625^{\dagger} \\
(0.08236)\end{array}$ & $\begin{array}{l}0.17153^{\dagger} \\
(0.08314)\end{array}$ & $\begin{array}{l}0.13266 \\
(0.08776)\end{array}$ & $\begin{array}{l}0.06708 \\
(0.08555)\end{array}$ & $\begin{array}{l}0.15137^{\ddagger} \\
(0.08821)\end{array}$ & $\begin{array}{l}0.3153^{*} \\
(0.09173)\end{array}$ & $\begin{array}{l}0.07556 \\
(0.09412)\end{array}$ & $\begin{array}{l}0.19525^{\dagger} \\
(0.09008)\end{array}$ & $\begin{array}{l}0.1227 \\
(0.09571)\end{array}$ & $\begin{array}{l}0.15645 \\
(0.09646)\end{array}$ & $\begin{array}{l}0.30959^{*} \\
(0.09553)\end{array}$ & $\begin{array}{l}0.12093 \\
(0.08851)\end{array}$ \\
\hline Factor $1 \& 2$ & $\begin{array}{l}0.17312 \\
(0.14402)\end{array}$ & $\begin{array}{l}0.24597^{\ddagger} \\
(0.14414)\end{array}$ & $\begin{array}{l}0.2865^{\ddagger} \\
(0.15376)\end{array}$ & $\begin{array}{l}0.19965 \\
(0.14588)\end{array}$ & $\begin{array}{l}0.18588 \\
(0.14125)\end{array}$ & $\begin{array}{l}0.21155 \\
(0.14864)\end{array}$ & $\begin{array}{l}0.37284^{\dagger} \\
(0.14669)\end{array}$ & $\begin{array}{l}0.21377 \\
(0.14323)\end{array}$ & $\begin{array}{l}0.23058 \\
(0.14426)\end{array}$ & $\begin{array}{l}0.19621 \\
(0.14764)\end{array}$ & $\begin{array}{l}0.21996 \\
(0.14462)\end{array}$ & $\begin{array}{l}0.22049 \\
(0.14141)\end{array}$ & $\begin{array}{l}0.22049 \\
(0.14378\end{array}$ \\
\hline Factor $1,2 \& 4$ & $\begin{array}{l}0.06308 \\
(0.09412)\end{array}$ & $\begin{array}{l}0.18188^{\ddagger} \\
(0.09285)\end{array}$ & $\begin{array}{l}0.1996^{\ddagger} \\
(0.10302)\end{array}$ & $\begin{array}{l}0.09462 \\
(0.10139)\end{array}$ & $\begin{array}{l}0.03083 \\
(0.09323)\end{array}$ & $\begin{array}{l}0.13174 \\
(0.09216)\end{array}$ & $\begin{array}{l}0.22141^{\dagger} \\
(0.09022)\end{array}$ & $\begin{array}{l}0.10299 \\
(0.09082)\end{array}$ & $\begin{array}{l}0.16807^{\ddagger} \\
(0.0944)\end{array}$ & $\begin{array}{l}0.12761 \\
(0.0903)\end{array}$ & $\begin{array}{l}0.13529 \\
(0.09712)\end{array}$ & $\begin{array}{l}0.28088^{*} \\
(0.10006)\end{array}$ & $\begin{array}{l}0.06749 \\
(0.10681\end{array}$ \\
\hline Factor $1,2 \& 3$ & $\begin{array}{l}0.07028 \\
(0.11481)\end{array}$ & $\begin{array}{l}0.28239^{\dagger} \\
(0.10933)\end{array}$ & $\begin{array}{l}0.25758^{\dagger} \\
(0.10844)\end{array}$ & $\begin{array}{l}0.16803 \\
(0.1082)\end{array}$ & $\begin{array}{l}0.1026 \\
(0.10243)\end{array}$ & $\begin{array}{l}0.19791^{\ddagger} \\
(0.10433)\end{array}$ & $\begin{array}{l}0.34972^{*} \\
(0.10526)\end{array}$ & $\begin{array}{l}0.11102 \\
(0.11192)\end{array}$ & $\begin{array}{l}0.2449^{\dagger} \\
(0.10703)\end{array}$ & $\begin{array}{l}0.16632 \\
(0.11249)\end{array}$ & $\begin{array}{l}0.20403^{\ddagger} \\
(0.1036)\end{array}$ & $\begin{array}{l}0.21806^{\dagger} \\
(0.09798)\end{array}$ & $\begin{array}{l}0.19154 \\
(0.10022\end{array}$ \\
\hline All Factors & $\begin{array}{l}0.15944^{*} \\
(0.05964)\end{array}$ & $\begin{array}{l}0.34887^{*} \\
(0.04596)\end{array}$ & $\begin{array}{l}0.3378^{*} \\
(0.05085)\end{array}$ & $\begin{array}{l}0.24171^{*} \\
(0.05407)\end{array}$ & $\begin{array}{l}0.16317^{*} \\
(0.04437)\end{array}$ & $\begin{array}{l}0.27844^{*} \\
(0.03435)\end{array}$ & $\begin{array}{l}0.41027^{*} \\
(0.04309)\end{array}$ & $\begin{array}{l}0.20818^{*} \\
(0.04878)\end{array}$ & $\begin{array}{l}0.32888^{*} \\
(0.0509)\end{array}$ & $\begin{array}{l}0.24711^{*} \\
(0.05544)\end{array}$ & $\begin{array}{l}0.28176^{*} \\
(0.05519)\end{array}$ & $\begin{array}{l}0.41877^{*} \\
(0.05135)\end{array}$ & $\begin{array}{l}0.20926^{*} \\
(0.06797)\end{array}$ \\
\hline $\begin{array}{l}\text { Individual } \\
\text { Variable }\end{array}$ & $\begin{array}{l}0.13515^{\dagger} \\
(0.0587)\end{array}$ & $\begin{array}{l}-0.08512^{\ddagger} \\
(0.0508)\end{array}$ & $\begin{array}{l}-0.06601 \\
(0.05452)\end{array}$ & $\begin{array}{l}0.04942 \\
(0.05561)\end{array}$ & $\begin{array}{l}0.15172^{*} \\
(0.04629)\end{array}$ & $\begin{array}{l}0.01042 \\
(0.04193)\end{array}$ & $\begin{array}{c}-0.16402^{*} \\
(0.04615)\end{array}$ & $\begin{array}{l}0.09442 \\
(0.05199)\end{array}$ & $\begin{array}{l}-0.05213 \\
(0.05171)\end{array}$ & $\begin{array}{l}0.04199 \\
(0.05586)\end{array}$ & $\begin{array}{l}0.00276 \\
(0.05441)\end{array}$ & $\begin{array}{l}-0.1593^{*} \\
(0.05332)\end{array}$ & $\begin{array}{l}0.0783 \\
(0.06596\end{array}$ \\
\hline
\end{tabular}

* Significant at the 0.01 level

$\dagger$ Significant at the 0.05 level

+ Significant at the 0.10 level

Table 99 Coefficients with individual variables for perceived benefits of AMT - Vehicles Industry 


\begin{tabular}{|c|c|c|c|c|c|c|c|c|c|c|c|c|c|}
\hline $\begin{array}{l}\text { Individual } \\
\text { variable } \\
\text { included in the } \\
\text { model in the } \\
\text { column }\end{array}$ & 塄 & 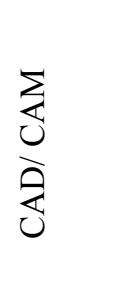 & 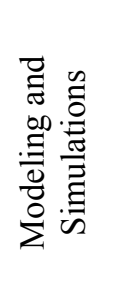 & $\begin{array}{l}\sum \\
\sum \\
4 \\
0 \\
\overrightarrow{0}\end{array}$ & $\sum_{i=1}^{\infty}$ & 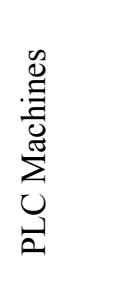 & 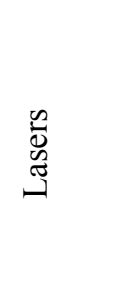 & 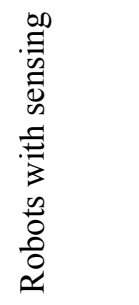 & 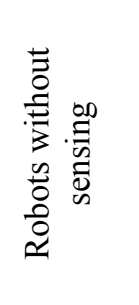 & 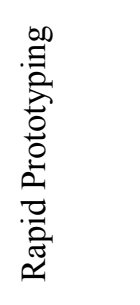 & 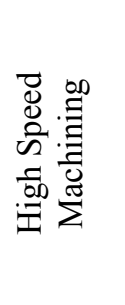 & 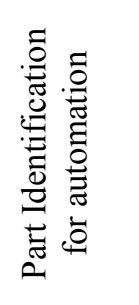 & 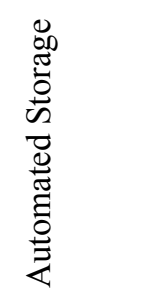 \\
\hline No factors & $\begin{array}{l}0.15712^{*} \\
(0.03667) \\
\end{array}$ & $\begin{array}{l}0.16243^{*} \\
(0.03692) \\
\end{array}$ & $\begin{array}{l}0.16754^{*} \\
(0.03598) \\
\end{array}$ & $\begin{array}{l}0.16478^{*} \\
(0.03694) \\
\end{array}$ & $\begin{array}{l}0.16804^{*} \\
(0.03711) \\
\end{array}$ & $\begin{array}{l}0.16694^{*} \\
(0.03721) \\
\end{array}$ & $\begin{array}{l}0.16754^{*} \\
(0.03720)\end{array}$ & $\begin{array}{l}0.16729^{*} \\
(0.03712)\end{array}$ & $\begin{array}{l}0.16735^{*} \\
(0.03719)\end{array}$ & $\begin{array}{l}0.16754^{*} \\
(0.03720)\end{array}$ & $\begin{array}{l}0.16754^{*} \\
(0.03697)\end{array}$ & $\begin{array}{l}0.16764^{*} \\
(0.03720)\end{array}$ & $\begin{array}{l}0.16754^{*} \\
(0.03720)\end{array}$ \\
\hline Factor 5 & $\begin{array}{l}-0.18964 \\
(0.23320)\end{array}$ & $\begin{array}{l}-0.07425 \\
(0.22954)\end{array}$ & $\begin{array}{l}-0.06537 \\
(0.22347)\end{array}$ & $\begin{array}{l}-0.09991 \\
(0.23212)\end{array}$ & $\begin{array}{l}-0.02148 \\
(0.23047)\end{array}$ & $\begin{array}{l}-0.04281 \\
(0.23242)\end{array}$ & $\begin{array}{l}-0.03115 \\
(0.23083)\end{array}$ & $\begin{array}{l}-0.04085 \\
(0.23060)\end{array}$ & $\begin{array}{l}-0.03476 \\
(0.23103)\end{array}$ & $\begin{array}{l}-0.03115 \\
(0.23081)\end{array}$ & $\begin{array}{l}-0.04839 \\
(0.22965)\end{array}$ & $\begin{array}{l}-0.03115 \\
(0.23082)\end{array}$ & $\begin{array}{l}-0.03115 \\
(0.23084)\end{array}$ \\
\hline Factor 4 & $\begin{array}{l}-0.29619 \\
(0.27371) \\
\end{array}$ & $\begin{array}{l}-0.19806 \\
(0.27211)\end{array}$ & $\begin{array}{l}-0.29696 \\
(0.26761)\end{array}$ & $\begin{array}{l}-0.22528 \\
(0.27527)\end{array}$ & $\begin{array}{l}-0.11978 \\
(0.27309)\end{array}$ & $\begin{array}{l}-0.16482 \\
(0.28069)\end{array}$ & $\begin{array}{l}-0.13499 \\
(0.27379)\end{array}$ & $\begin{array}{l}-0.15567 \\
(0.27331)\end{array}$ & $\begin{array}{l}-0.14439 \\
(0.27384)\end{array}$ & $\begin{array}{l}-0.13770 \\
(0.27315)\end{array}$ & $\begin{array}{l}-0.13770 \\
(0.27147)\end{array}$ & $\begin{array}{l}-0.13404 \\
(0.27392)\end{array}$ & $\begin{array}{l}-0.13770 \\
(0.27318)\end{array}$ \\
\hline Factor $4 \& 5$ & $\begin{array}{l}-0.17775 \\
(0.22596)\end{array}$ & $\begin{array}{l}-0.09940 \\
(0.22452)\end{array}$ & $\begin{array}{l}-0.12560 \\
(0.21777)\end{array}$ & $\begin{array}{l}-0.07774 \\
(0.22379)\end{array}$ & $\begin{array}{l}-0.01926 \\
(0.22268)\end{array}$ & $\begin{array}{l}-0.02471 \\
(0.22351)\end{array}$ & $\begin{array}{l}-0.01926 \\
(0.22321)\end{array}$ & $\begin{array}{l}-0.03740 \\
(0.22363)\end{array}$ & $\begin{array}{l}-0.02263 \\
(0.22340)\end{array}$ & $\begin{array}{l}-0.01574 \\
(0.22378)\end{array}$ & $\begin{array}{l}-0.03538 \\
(0.22206)\end{array}$ & $\begin{array}{l}-0.01682 \\
(0.22362)\end{array}$ & $\begin{array}{l}-0.01926 \\
(0.22322)\end{array}$ \\
\hline Factor 3 & $\begin{array}{l}-0.41296^{*} \\
(0.07541)\end{array}$ & $\begin{array}{l}-0.41058^{*} \\
(0.07611)\end{array}$ & $\begin{array}{l}-0.40647^{*} \\
(0.07433)\end{array}$ & $\begin{array}{l}-0.40647^{*} \\
(0.07623)\end{array}$ & $\begin{array}{l}-0.40647^{*} \\
(0.07665)\end{array}$ & $\begin{array}{l}-0.40647^{*} \\
(0.07681)\end{array}$ & $\begin{array}{l}-0.40647^{*} \\
(0.07684)\end{array}$ & $\begin{array}{l}-0.40647^{*} \\
(0.07668)\end{array}$ & $\begin{array}{l}-0.40647^{*} \\
(0.07682)\end{array}$ & $\begin{array}{l}-0.40647^{*} \\
(0.07683)\end{array}$ & $\begin{array}{l}-0.40647^{*} \\
(0.07636)\end{array}$ & $\begin{array}{l}-0.40094^{*} \\
(0.08283)\end{array}$ & $\begin{array}{l}-0.40647^{*} \\
(0.07684)\end{array}$ \\
\hline Factor & $\begin{array}{l}0.00891 \\
(0.16661)\end{array}$ & $\begin{array}{l}0.05033 \\
(0.16789)\end{array}$ & $\begin{array}{l}0.13375 \\
(0.15683)\end{array}$ & $\begin{array}{l}0.07231 \\
(0.16646)\end{array}$ & $\begin{array}{l}0.15062 \\
(0.16167)\end{array}$ & $\begin{array}{l}0.15062 \\
(0.16200)\end{array}$ & $\begin{array}{l}0.15062 \\
(0.16206)\end{array}$ & $\begin{array}{l}0.15062 \\
(0.16173)\end{array}$ & $\begin{array}{l}0.14884 \\
(0.16211)\end{array}$ & $\begin{array}{l}0.15062 \\
(0.16205)\end{array}$ & $\begin{array}{l}0.15062 \\
(0.16105)\end{array}$ & $\begin{array}{l}0.15062 \\
(0.16205)\end{array}$ & $\begin{array}{l}0.15087 \\
(0.16355)\end{array}$ \\
\hline Factor $3 \& 4$ & \multicolumn{13}{|c|}{ Insufficient Data } \\
\hline Factor $3,4 \& 5$ & $\begin{array}{l}-0.16174 \\
(0.29404)\end{array}$ & $\begin{array}{l}-0.10354 \\
(0.29556)\end{array}$ & $\begin{array}{l}-0.05889 \\
(0.28507)\end{array}$ & $\begin{array}{l}-0.09083 \\
(0.29594)\end{array}$ & $\begin{array}{l}0.01247 \\
(0.29401)\end{array}$ & $\begin{array}{l}-0.01273 \\
(0.29506)\end{array}$ & $\begin{array}{l}-0.00325 \\
(0.29430)\end{array}$ & $\begin{array}{l}-0.00325 \\
(0.29371)\end{array}$ & $\begin{array}{l}-0.00912 \\
(0.29476)\end{array}$ & $\begin{array}{l}-0.00325 \\
(0.29428)\end{array}$ & $\begin{array}{l}-0.03128 \\
(0.29299)\end{array}$ & $\begin{array}{l}-0.00005 \\
(0.29484)\end{array}$ & $\begin{array}{l}-0.00325 \\
(0.29431)\end{array}$ \\
\hline Factor 2 & $\begin{array}{l}0.17434 \\
(0.23969)\end{array}$ & $\begin{array}{l}0.20202 \\
(0.24159)\end{array}$ & $\begin{array}{l}0.24973 \\
(0.23490)\end{array}$ & $\begin{array}{l}0.24973 \\
(0.24092)\end{array}$ & $\begin{array}{l}0.24973 \\
(0.24225)\end{array}$ & $\begin{array}{l}0.23683 \\
(0.24470)\end{array}$ & $\begin{array}{l}0.24973 \\
(0.24283)\end{array}$ & $\begin{array}{l}0.23899 \\
(0.24262)\end{array}$ & $\begin{array}{l}0.24573 \\
(0.24308)\end{array}$ & $\begin{array}{l}0.24973 \\
(0.24282)\end{array}$ & $\begin{array}{l}0.24973 \\
(0.24133)\end{array}$ & $\begin{array}{l}0.25409 \\
(0.24405)\end{array}$ & $\begin{array}{l}0.24993 \\
(0.24348)\end{array}$ \\
\hline Factor $2 \& 5$ & $\begin{array}{l}0.01631 \\
(0.34988)\end{array}$ & $\begin{array}{l}0.17480 \\
(0.34864)\end{array}$ & $\begin{array}{l}0.17480 \\
(0.34060)\end{array}$ & $\begin{array}{l}0.08722 \\
(0.35264)\end{array}$ & $\begin{array}{l}0.21979 \\
(0.35409)\end{array}$ & $\begin{array}{l}0.14768 \\
(0.35791)\end{array}$ & $\begin{array}{l}0.17480 \\
(0.35210)\end{array}$ & $\begin{array}{l}0.15223 \\
(0.35224)\end{array}$ & $\begin{array}{l}0.16640 \\
(0.35293)\end{array}$ & $\begin{array}{l}0.17480 \\
(0.35207)\end{array}$ & $\begin{array}{l}0.17480 \\
(0.34992)\end{array}$ & $\begin{array}{l}0.18397 \\
(0.35581)\end{array}$ & $\begin{array}{l}0.17480 \\
(0.35212)\end{array}$ \\
\hline Factor $2 \& 4$ & $\begin{array}{l}0.09112 \\
(0.14731)\end{array}$ & $\begin{array}{l}0.19332 \\
(0.14039)\end{array}$ & $\begin{array}{l}0.19998 \\
(0.13510)\end{array}$ & $\begin{array}{l}0.16204 \\
(0.14609)\end{array}$ & $\begin{array}{l}0.27137^{\ddagger} \\
(0.14033)\end{array}$ & $\begin{array}{l}0.22250 \\
(0.15338)\end{array}$ & $\begin{array}{l}0.24962^{\ddagger} \\
(0.13899)\end{array}$ & $\begin{array}{l}0.24258^{\ddagger} \\
(0.13892)\end{array}$ & $\begin{array}{l}0.24569^{\ddagger} \\
(0.13946)\end{array}$ & $\begin{array}{l}0.25098^{*} \\
(0.13912)\end{array}$ & $\begin{array}{l}0.18814 \\
(0.14330)\end{array}$ & $\begin{array}{l}0.25104^{\ddagger} \\
(0.13922)\end{array}$ & $\begin{array}{l}0.24962^{\ddagger} \\
(0.13900)\end{array}$ \\
\hline Factor $2,4 \& 5$ & $\begin{array}{l}0.03472 \\
(0.12128)\end{array}$ & $\begin{array}{l}0.09292 \\
(0.11941)\end{array}$ & $\begin{array}{l}0.14047 \\
(0.10713)\end{array}$ & $\begin{array}{l}0.16421 \\
(0.11010)\end{array}$ & $\begin{array}{l}0.20374^{\ddagger} \\
(0.11003)\end{array}$ & $\begin{array}{l}0.16609 \\
(0.12755)\end{array}$ & $\begin{array}{l}0.19532^{\ddagger} \\
(0.11074)\end{array}$ & $\begin{array}{l}0.18046 \\
(0.11045)\end{array}$ & $\begin{array}{l}0.19010^{\ddagger} \\
(0.11017)\end{array}$ & $\begin{array}{l}0.19492^{\ddagger} \\
(0.11007)\end{array}$ & $\begin{array}{l}0.12469 \\
(0.11711)\end{array}$ & $\begin{array}{l}0.19455^{\ddagger} \\
(0.11005)\end{array}$ & $\begin{array}{l}0.19325^{\ddagger} \\
(0.10986)\end{array}$ \\
\hline Factor $2 \& 3$ & $\begin{array}{l}0.27625^{\dagger} \\
(0.12650) \\
\end{array}$ & $\begin{array}{l}0.29743^{\dagger} \\
(0.12746) \\
\end{array}$ & $\begin{array}{l}0.29743^{\dagger} \\
(0.12452) \\
\end{array}$ & $\begin{array}{l}0.28573^{\dagger} \\
(0.12787) \\
\end{array}$ & $\begin{array}{l}0.34243^{\dagger} \\
(0.13597) \\
\end{array}$ & $\begin{array}{l}0.27031^{\ddagger} \\
(0.14415) \\
\end{array}$ & $\begin{array}{l}0.29743^{\dagger} \\
(0.12872) \\
\end{array}$ & $\begin{array}{l}0.29140^{\dagger} \\
(0.12863) \\
\end{array}$ & $\begin{array}{l}0.29519^{\dagger} \\
(0.12887) \\
\end{array}$ & $\begin{array}{l}0.29743^{\dagger} \\
(0.12871) \\
\end{array}$ & $\begin{array}{l}0.29743^{\dagger} \\
(0.12793) \\
\end{array}$ & $\begin{array}{l}0.29743^{\dagger} \\
(0.12872) \\
\end{array}$ & $\begin{array}{l}0.29754^{\dagger} \\
(0.12911) \\
\end{array}$ \\
\hline Facto & $\begin{array}{l}0.21120 \\
(0.48848)\end{array}$ & $\begin{array}{l}0.11091 \\
(0.49552)\end{array}$ & $\begin{array}{l}0.21120 \\
(0.48168)\end{array}$ & $\begin{array}{l}0.12362 \\
(0.49637)\end{array}$ & $\begin{array}{l}0.21120 \\
(0.49675)\end{array}$ & $\begin{array}{l}0.18408 \\
(0.50198)\end{array}$ & $\begin{array}{l}0.21120 \\
(0.49794)\end{array}$ & $\begin{array}{l}0.21120 \\
(0.49694)\end{array}$ & $\begin{array}{l}0.21120 \\
(0.49783)\end{array}$ & $\begin{array}{l}0.21120 \\
(0.49791)\end{array}$ & $\begin{array}{l}0.21120 \\
(0.49486)\end{array}$ & $\begin{array}{l}0.21120 \\
(0.49793)\end{array}$ & $\begin{array}{l}0.21120 \\
(0.49797)\end{array}$ \\
\hline Facto & $\begin{array}{l}0.10739 \\
(0.07226) \\
\end{array}$ & $\begin{array}{l}0.16558^{\dagger} \\
(0.06776)\end{array}$ & $\begin{array}{l}0.10661^{\ddagger} \\
(0.06202)\end{array}$ & $\begin{array}{l}0.17830^{*} \\
(0.06699)\end{array}$ & $\begin{array}{l}0.27312^{*} \\
(0.04731)\end{array}$ & $\begin{array}{l}0.23900^{*} \\
(0.07964)\end{array}$ & $\begin{array}{l}0.26725^{*} \\
(0.04779)\end{array}$ & $\begin{array}{l}0.25901^{*} \\
(0.04736)\end{array}$ & $\begin{array}{l}0.25115^{*} \\
(0.06445)\end{array}$ & $\begin{array}{l}0.26588^{*} \\
(0.04686)\end{array}$ & $\begin{array}{l}0.19006^{*} \\
(0.06621)\end{array}$ & $\begin{array}{l}0.27489^{*} \\
(0.06883)\end{array}$ & $\begin{array}{l}0.26597^{*} \\
(0.04763)\end{array}$ \\
\hline Factor 2,3 & $\begin{array}{l}0.16628 \\
(0.14615) \\
\end{array}$ & $\begin{array}{l}0.21583 \\
(0.14592) \\
\end{array}$ & $\begin{array}{l}0.17818 \\
(0.13979) \\
\end{array}$ & $\begin{array}{l}0.22754 \\
(0.14585) \\
\end{array}$ & $\begin{array}{l}0.34136^{\dagger} \\
(0.14353)\end{array}$ & $\begin{array}{l}0.28820^{\ddagger} \\
(0.14794) \\
\end{array}$ & $\begin{array}{l}0.31198^{\dagger} \\
(0.14239)\end{array}$ & $\begin{array}{l}0.29748^{\dagger} \\
(0.14023)\end{array}$ & $\begin{array}{l}0.30421^{\dagger} \\
(0.14051)\end{array}$ & $\begin{array}{l}0.30958^{\dagger} \\
(0.14016) \\
\end{array}$ & $\begin{array}{l}0.27443^{\ddagger} \\
(0.14073)\end{array}$ & $\begin{array}{l}0.31641^{\dagger} \\
(0.14737)\end{array}$ & $\begin{array}{l}0.30839^{\dagger} \\
(0.14112)\end{array}$ \\
\hline Factor 1 & $\begin{array}{l}0.53046^{*} \\
(0.08911)\end{array}$ & $\begin{array}{l}0.53620^{*} \\
(0.08991)\end{array}$ & $\begin{array}{l}0.41239^{*} \\
(0.09463)\end{array}$ & $\begin{array}{l}0.54130^{*} \\
(0.08999)\end{array}$ & $\begin{array}{l}0.54610^{*} \\
(0.09045)\end{array}$ & $\begin{array}{l}0.54443^{*} \\
(0.09072)\end{array}$ & $\begin{array}{l}0.54610^{*} \\
(0.09066)\end{array}$ & $\begin{array}{l}0.54610^{*} \\
(0.09048)\end{array}$ & $\begin{array}{l}0.54610^{*} \\
(0.09064)\end{array}$ & $\begin{array}{l}0.54610^{*} \\
(0.09066)\end{array}$ & $\begin{array}{l}0.54610^{*} \\
(0.09010)\end{array}$ & $\begin{array}{l}0.54610^{*} \\
(0.09066)\end{array}$ & $\begin{array}{l}0.54610^{*} \\
(0.09067)\end{array}$ \\
\hline Factor $1 \& 5$ & $\begin{array}{l}0.09668 \\
(0.17318) \\
\end{array}$ & $\begin{array}{l}0.14593 \\
(0.17297) \\
\end{array}$ & $\begin{array}{l}0.19793 \\
(0.16712) \\
\end{array}$ & $\begin{array}{l}0.13833 \\
(0.17452) \\
\end{array}$ & $\begin{array}{l}0.20877 \\
(0.17269) \\
\end{array}$ & $\begin{array}{l}0.18274 \\
(0.17649) \\
\end{array}$ & $\begin{array}{l}0.19793 \\
(0.17276) \\
\end{array}$ & $\begin{array}{l}0.19793 \\
(0.17242) \\
\end{array}$ & $\begin{array}{l}0.19591 \\
(0.17283) \\
\end{array}$ & $\begin{array}{l}0.19793 \\
(0.17275) \\
\end{array}$ & $\begin{array}{l}0.19793 \\
(0.17169)\end{array}$ & $\begin{array}{l}0.19904 \\
(0.17287) \\
\end{array}$ & $\begin{array}{l}0.19793 \\
(0.17277) \\
\end{array}$ \\
\hline Factor $1 \& 4$ & $\begin{array}{l}0.09773 \\
(0.14689)\end{array}$ & $\begin{array}{l}0.14335 \\
(0.14744)\end{array}$ & $\begin{array}{l}0.11374 \\
(0.14004)\end{array}$ & $\begin{array}{l}0.20559 \\
(0.14077)\end{array}$ & $\begin{array}{l}0.24364^{\ddagger} \\
(0.13997)\end{array}$ & $\begin{array}{l}0.23933^{\ddagger} \\
(0.14063)\end{array}$ & $\begin{array}{l}0.24507^{\ddagger} \\
(0.14064)\end{array}$ & $\begin{array}{l}0.24005^{\ddagger} \\
(0.14008)\end{array}$ & $\begin{array}{l}0.24230^{\ddagger} \\
(0.14033)\end{array}$ & $\begin{array}{l}0.24364^{\ddagger} \\
(0.14029)\end{array}$ & $\begin{array}{l}0.23727^{\ddagger} \\
(0.13949)\end{array}$ & $\begin{array}{l}0.24509^{\ddagger} \\
(0.14054)\end{array}$ & $\begin{array}{l}0.24364^{\ddagger} \\
(0.14031)\end{array}$ \\
\hline Factor $1,4 \& 5$ & $\begin{array}{l}0.10427 \\
(0.08758)\end{array}$ & $\begin{array}{l}0.16047^{\ddagger} \\
(0.08447)\end{array}$ & $\begin{array}{l}0.16156^{\dagger} \\
(0.07272)\end{array}$ & $\begin{array}{l}0.19684^{\dagger} \\
(0.07740)\end{array}$ & $\begin{array}{l}0.25731^{*} \\
(0.07025)\end{array}$ & $\begin{array}{l}0.24604^{*} \\
(0.07540)\end{array}$ & $\begin{array}{l}0.25762^{*} \\
(0.07045)\end{array}$ & $\begin{array}{l}0.24405^{*} \\
(0.07173)\end{array}$ & $\begin{array}{l}0.25731^{*} \\
(0.07041)\end{array}$ & $\begin{array}{l}0.25731^{*} \\
(0.07042)\end{array}$ & $\begin{array}{l}0.24418^{*} \\
(0.07046)\end{array}$ & $\begin{array}{l}0.25731^{*} \\
(0.07042)\end{array}$ & $\begin{array}{l}0.25731^{*} \\
(0.07043)\end{array}$ \\
\hline $\mathrm{Fac}$ & $\begin{array}{l}-0.04469 \\
(0.11937)\end{array}$ & $\begin{array}{l}0.04306 \\
(0.11640)\end{array}$ & $\begin{array}{l}0.04306 \\
(0.11371)\end{array}$ & $\begin{array}{l}0.04306 \\
(0.11663)\end{array}$ & $\begin{array}{l}0.04306 \\
(0.11727)\end{array}$ & $\begin{array}{l}0.04306 \\
(0.11751)\end{array}$ & $\begin{array}{l}0.04306 \\
(0.11755)\end{array}$ & $\begin{array}{l}0.04306 \\
(0.11731)\end{array}$ & $\begin{array}{l}0.04043 \\
(0.11779)\end{array}$ & $\begin{array}{l}0.04306 \\
(0.11754)\end{array}$ & $\begin{array}{l}0.04306 \\
(0.11682)\end{array}$ & $\begin{array}{l}0.04306 \\
(0.11755)\end{array}$ & $\begin{array}{l}0.04306 \\
(0.11756)\end{array}$ \\
\hline Facto & $\begin{array}{l}-0.05511 \\
(0.10834) \\
\end{array}$ & $\begin{array}{l}0.01152 \\
(0.10411)\end{array}$ & $\begin{array}{l}0.09576 \\
(0.09163)\end{array}$ & $\begin{array}{l}0.09919 \\
(0.09398)\end{array}$ & $\begin{array}{l}0.10338 \\
(0.09447)\end{array}$ & $\begin{array}{l}0.10338 \\
(0.09466)\end{array}$ & $\begin{array}{l}0.10338 \\
(0.09470)\end{array}$ & $\begin{array}{l}0.10122 \\
(0.09454)\end{array}$ & $\begin{array}{l}0.10338 \\
(0.09468)\end{array}$ & $\begin{array}{l}0.10422 \\
(0.09477)\end{array}$ & $\begin{array}{l}0.10338 \\
(0.09411)\end{array}$ & $\begin{array}{l}0.10371 \\
(0.09472)\end{array}$ & $\begin{array}{l}0.10338 \\
(0.09470)\end{array}$ \\
\hline Factor $1,3 \& 4$ & $\begin{array}{l}0.03578 \\
(0.13026)\end{array}$ & $\begin{array}{l}0.11036 \\
(0.12581)\end{array}$ & $\begin{array}{l}0.11799 \\
(0.11785) \\
\end{array}$ & $\begin{array}{l}0.13294 \\
(0.12376)\end{array}$ & $\begin{array}{l}0.20946^{\ddagger} \\
(0.12067)\end{array}$ & $\begin{array}{l}0.17491 \\
(0.12862)\end{array}$ & $\begin{array}{l}0.19480 \\
(0.12006)\end{array}$ & $\begin{array}{l}0.19427 \\
(0.11977)\end{array}$ & $\begin{array}{l}0.19330 \\
(0.12002)\end{array}$ & $\begin{array}{l}0.19427 \\
(0.12000)\end{array}$ & $\begin{array}{l}0.18961 \\
(0.11930)\end{array}$ & $\begin{array}{l}0.19481 \\
(0.12004)\end{array}$ & $\begin{array}{l}0.19427 \\
(0.12002)\end{array}$ \\
\hline Factor $1,3,4 \& 5$ & $\begin{array}{l}0.00888 \\
(0.09553)\end{array}$ & $\begin{array}{l}0.08426 \\
(0.08835)\end{array}$ & $\begin{array}{l}0.13583^{\ddagger} \\
(0.07696)\end{array}$ & $\begin{array}{l}0.07979 \\
(0.09209)\end{array}$ & $\begin{array}{l}0.17377^{\dagger} \\
(0.07915)\end{array}$ & $\begin{array}{l}0.14481 \\
(0.09575)\end{array}$ & $\begin{array}{l}0.17460^{\ddagger} \\
(0.09323)\end{array}$ & $\begin{array}{l}0.16586^{\dagger} \\
(0.07894)\end{array}$ & $\begin{array}{l}0.16553^{\dagger} \\
(0.07926)\end{array}$ & $\begin{array}{l}0.16781^{\dagger} \\
(0.07910)\end{array}$ & $\begin{array}{l}0.14981^{\ddagger} \\
(0.07934)\end{array}$ & $\begin{array}{l}0.16814^{\dagger} \\
(0.07920)\end{array}$ & $\begin{array}{l}0.16737^{\dagger} \\
(0.07909)\end{array}$ \\
\hline Factor $1 \& 2$ & $\begin{array}{l}0.01213 \\
(0.17788)\end{array}$ & $\begin{array}{l}0.12661 \\
(0.17305)\end{array}$ & $\begin{array}{l}0.13167 \\
(0.16872)\end{array}$ & $\begin{array}{l}0.12973 \\
(0.17337)\end{array}$ & $\begin{array}{l}0.15671 \\
(0.17400)\end{array}$ & $\begin{array}{l}0.14122 \\
(0.17590)\end{array}$ & $\begin{array}{l}0.15120 \\
(0.17433)\end{array}$ & $\begin{array}{l}0.13460 \\
(0.17491)\end{array}$ & $\begin{array}{l}0.14502 \\
(0.17528)\end{array}$ & $\begin{array}{l}0.15334 \\
(0.17460)\end{array}$ & $\begin{array}{l}0.14136 \\
(0.17336)\end{array}$ & $\begin{array}{l}0.15457 \\
(0.17535)\end{array}$ & $\begin{array}{l}0.15140 \\
(0.17528)\end{array}$ \\
\hline Factor $1,2 \& 5$ & $\begin{array}{l}0.18024 \\
(0.16638)\end{array}$ & $\begin{array}{l}0.15595 \\
(0.17203)\end{array}$ & $\begin{array}{l}0.18673 \\
(0.16326)\end{array}$ & $\begin{array}{l}0.22488 \\
(0.16706)\end{array}$ & $\begin{array}{l}0.27148 \\
(0.16970)\end{array}$ & $\begin{array}{l}0.22876 \\
(0.17231)\end{array}$ & $\begin{array}{l}0.24483 \\
(0.16802)\end{array}$ & $\begin{array}{l}0.21808 \\
(0.17017)\end{array}$ & $\begin{array}{l}0.23487 \\
(0.17063)\end{array}$ & $\begin{array}{l}0.24483 \\
(0.16801)\end{array}$ & $\begin{array}{l}0.24483 \\
(0.16698)\end{array}$ & $\begin{array}{l}0.25131 \\
(0.17188)\end{array}$ & $\begin{array}{l}0.24492 \\
(0.16824)\end{array}$ \\
\hline Factor $1,2 \& 4$ & $\begin{array}{l}0.05287 \\
(0.19034)\end{array}$ & $\begin{array}{l}0.11107 \\
(0.19022)\end{array}$ & $\begin{array}{l}0.11843 \\
(0.18116)\end{array}$ & $\begin{array}{l}0.13594 \\
(0.18869)\end{array}$ & $\begin{array}{l}0.25636 \\
(0.19040)\end{array}$ & $\begin{array}{l}0.18425 \\
(0.19651)\end{array}$ & $\begin{array}{l}0.21537 \\
(0.18753)\end{array}$ & $\begin{array}{l}0.17249 \\
(0.18988)\end{array}$ & $\begin{array}{l}0.19923 \\
(0.18904)\end{array}$ & $\begin{array}{l}0.22160 \\
(0.19129)\end{array}$ & $\begin{array}{l}0.16455 \\
(0.18665)\end{array}$ & $\begin{array}{l}0.22054 \\
(0.19248)\end{array}$ & $\begin{array}{l}0.21197 \\
(0.19303)\end{array}$ \\
\hline Factor 1 , & $\begin{array}{l}-0.10430 \\
(0.10162)\end{array}$ & $\begin{array}{l}-0.04610 \\
(0.09896)\end{array}$ & $\begin{array}{l}0.00616 \\
(0.08475)\end{array}$ & $\begin{array}{l}-0.02532 \\
(0.09644)\end{array}$ & $\begin{array}{l}0.06869 \\
(0.08760)\end{array}$ & $\begin{array}{l}0.04546 \\
(0.08908)\end{array}$ & $\begin{array}{l}0.05546 \\
(0.08704)\end{array}$ & $\begin{array}{l}0.04102 \\
(0.08761)\end{array}$ & $\begin{array}{l}0.04929 \\
(0.08784)\end{array}$ & $\begin{array}{l}0.05596 \\
(0.08698)\end{array}$ & $\begin{array}{l}0.03000 \\
(0.08738)\end{array}$ & $\begin{array}{l}0.05707 \\
(0.08809)\end{array}$ & $\begin{array}{l}0.05428 \\
(0.08694)\end{array}$ \\
\hline Factor $1,2 \& 3$ & $\begin{array}{l}0.31473^{\ddagger} \\
(0.18557) \\
\end{array}$ & $\begin{array}{l}0.31473^{\ddagger} \\
(0.18730) \\
\end{array}$ & $\begin{array}{l}0.31473^{\ddagger} \\
(0.18298)\end{array}$ & $\begin{array}{l}0.29802 \\
(0.18790)\end{array}$ & $\begin{array}{l}0.31473^{\ddagger} \\
(0.18871)\end{array}$ & $\begin{array}{l}0.30956 \\
(0.18950)\end{array}$ & $\begin{array}{l}0.31473^{\ddagger} \\
(0.18916)\end{array}$ & $\begin{array}{l}0.30612 \\
(0.18901)\end{array}$ & $\begin{array}{l}0.31473^{\ddagger} \\
(0.18912)\end{array}$ & $\begin{array}{l}0.31473^{\ddagger} \\
(0.18915)\end{array}$ & $\begin{array}{l}0.31473^{\ddagger} \\
(0.18799)\end{array}$ & $\begin{array}{l}0.31649^{\ddagger} \\
(0.18941)\end{array}$ & $\begin{array}{l}0.31473^{\ddagger} \\
(0.18917)\end{array}$ \\
\hline Factor $1,2,3 \& 5$ & $\begin{array}{l}0.07286 \\
(0.19530)\end{array}$ & $\begin{array}{l}0.21663 \\
(0.18906)\end{array}$ & $\begin{array}{l}0.20797 \\
(0.18467)\end{array}$ & $\begin{array}{l}0.15663 \\
(0.19372)\end{array}$ & $\begin{array}{l}0.25117 \\
(0.19135)\end{array}$ & $\begin{array}{l}0.21542 \\
(0.19451)\end{array}$ & $\begin{array}{l}0.23135 \\
(0.19080)\end{array}$ & $\begin{array}{l}0.21810 \\
(0.19095)\end{array}$ & $\begin{array}{l}0.22642 \\
(0.19133)\end{array}$ & $\begin{array}{l}0.23135 \\
(0.19078)\end{array}$ & $\begin{array}{l}0.23135 \\
(0.18962)\end{array}$ & $\begin{array}{l}0.23539 \\
(0.19213)\end{array}$ & $\begin{array}{l}0.23135 \\
(0.19081)\end{array}$ \\
\hline Factor $1,2,3 \& 4$ & $\begin{array}{l}0.19643^{*} \\
(0.05114)\end{array}$ & $\begin{array}{l}0.24123^{*} \\
(0.04634)\end{array}$ & $\begin{array}{l}0.21176^{*} \\
(0.04262)\end{array}$ & $\begin{array}{l}0.24985^{*} \\
(0.04556)\end{array}$ & $\begin{array}{l}0.32323^{*} \\
(0.04412)\end{array}$ & $\begin{array}{l}0.27196^{*} \\
(0.07435)\end{array}$ & $\begin{array}{l}0.29974^{*} \\
(0.03745)\end{array}$ & $\begin{array}{l}0.29118^{*} \\
(0.03785)\end{array}$ & $\begin{array}{l}0.29583^{*} \\
(0.03810)\end{array}$ & $\begin{array}{l}0.29927^{*} \\
(0.03703)\end{array}$ & $\begin{array}{l}0.28367^{*} \\
(0.03796)\end{array}$ & $\begin{array}{l}0.30056^{*} \\
(0.03817)\end{array}$ & $\begin{array}{l}0.29890^{*} \\
(0.03703)\end{array}$ \\
\hline All Factors & $\begin{array}{l}0.15091^{\dagger} \\
(0.07004)\end{array}$ & $\begin{array}{l}0.22225^{*} \\
(0.06053)\end{array}$ & $\begin{array}{l}0.21422^{*} \\
(0.04993)\end{array}$ & $\begin{array}{l}0.22936^{*} \\
(0.06122)\end{array}$ & $\begin{array}{l}0.33437^{*} \\
(0.05367)\end{array}$ & $\begin{array}{l}0.27929^{*} \\
(0.07650)\end{array}$ & $\begin{array}{l}0.30603^{*} \\
(0.04567)\end{array}$ & $\begin{array}{l}0.26933^{*} \\
(0.05944)\end{array}$ & $\begin{array}{l}0.29147^{*} \\
(0.06090)\end{array}$ & $\begin{array}{l}0.30939^{*} \\
(0.04931)\end{array}$ & $\begin{array}{l}0.27030^{*} \\
(0.04982)\end{array}$ & $\begin{array}{l}0.31190^{*} \\
(0.05921)\end{array}$ & $\begin{array}{l}0.30519^{*} \\
(0.04656)\end{array}$ \\
\hline $\begin{array}{l}\text { Individual } \\
\text { Variable }\end{array}$ & $\begin{array}{l}0.15849^{*} \\
(0.05575)\end{array}$ & $\begin{array}{l}0.10029^{\dagger} \\
(0.04938)\end{array}$ & $\begin{array}{l}0.15927^{*} \\
(0.04232)\end{array}$ & $\begin{array}{l}0.08758^{\ddagger} \\
(0.04822)\end{array}$ & $\begin{array}{l}-0.04499 \\
(0.04471)\end{array}$ & $\begin{array}{l}0.02712 \\
(0.06497)\end{array}$ & $\begin{array}{l}-0.00900 \\
(0.06145)\end{array}$ & $\begin{array}{l}0.04514 \\
(0.04892)\end{array}$ & $\begin{array}{l}0.01680 \\
(0.05049)\end{array}$ & $\begin{array}{l}-0.01753 \\
(0.07995)\end{array}$ & $\begin{array}{l}0.08022 \\
(0.04979)\end{array}$ & $\begin{array}{l}-0.00918 \\
(0.05131)\end{array}$ & $\begin{array}{l}-0.00083731 \\
(0.07377)\end{array}$ \\
\hline
\end{tabular}

* Significant at the 0.01 level $\quad \dagger$ Significant at the 0.05 level $₫$ Significant at the 0.10 level

Table 100 Coefficients with individual variables for high-level factors - Vehicles Industry 


\begin{tabular}{|c|c|c|c|c|c|c|c|c|c|c|c|c|c|}
\hline $\begin{array}{l}\text { Individual variable } \\
\text { included in the } \\
\text { model in the } \\
\text { column }\end{array}$ & 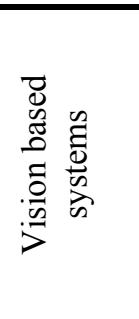 & 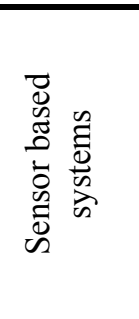 & 芯 & 孞 & $\overrightarrow{\mid \vec{I}}$ & 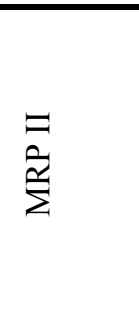 & 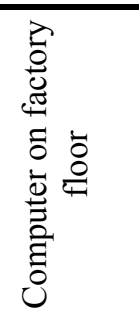 & $\sum$ & 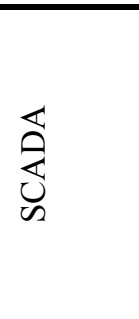 & 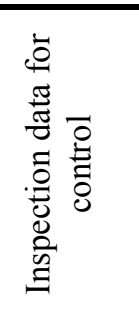 & 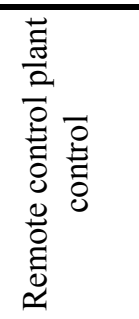 & 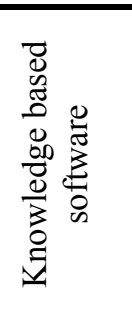 & 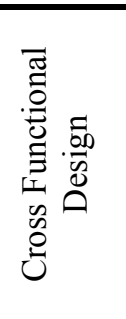 \\
\hline No factors & $\begin{array}{l}0.16743^{*} \\
(0.03719)\end{array}$ & $\begin{array}{l}0.16717^{*} \\
(0.03706)\end{array}$ & $\begin{array}{l}0.16582^{*} \\
(0.03716)\end{array}$ & $\begin{array}{l}0.17032^{*} \\
(0.03698)\end{array}$ & $(0.03717)$ & $\begin{array}{l}0.16678^{*} \\
(0.03719) \\
\end{array}$ & $\begin{array}{l}0.16701^{*} \\
(0.03718) \\
\end{array}$ & $\begin{array}{l}0.16754^{*} \\
(0.03618) \\
\end{array}$ & $\begin{array}{l}0.16754^{*} \\
(0.03703) \\
\end{array}$ & $\begin{array}{l}0.16610^{*} \\
(0.03699) \\
\end{array}$ & $\begin{array}{l}0.16754^{*} \\
(0.03630) \\
\end{array}$ & $\begin{array}{l}0.16785^{*} \\
(0.03708) \\
\end{array}$ & $\begin{array}{l}0.16407^{*} \\
(0.03702) \\
\end{array}$ \\
\hline Factor 5 & $\begin{array}{l}-0.03115 \\
(0.23075)\end{array}$ & $\begin{array}{l}-0.04538 \\
(0.23024)\end{array}$ & $\begin{array}{l}-0.07384 \\
(0.23440)\end{array}$ & $\begin{array}{l}0.01707 \\
(0.23101)\end{array}$ & $\begin{array}{l}-0.08129 \\
(0.23717)\end{array}$ & $\begin{array}{l}-0.03115 \\
(0.23066)\end{array}$ & $\begin{array}{l}-0.04485 \\
(0.23176)\end{array}$ & $\begin{array}{l}-0.06539 \\
(0.22474) \\
\end{array}$ & $\begin{array}{l}-0.03115 \\
(0.22979)\end{array}$ & $\begin{array}{l}-0.04966 \\
(0.22979)\end{array}$ & $\begin{array}{l}-0.03115 \\
(0.22523)\end{array}$ & $\begin{array}{l}-0.03115 \\
(0.23012) \\
\end{array}$ & $\begin{array}{l}-0.06863 \\
(0.23050) \\
\end{array}$ \\
\hline Factor 4 & $\begin{array}{l}-0.13770 \\
(0.27307)\end{array}$ & $\begin{array}{l}-0.13770 \\
(0.27214)\end{array}$ & $\begin{array}{l}-0.15763 \\
(0.27330)\end{array}$ & $\begin{array}{l}-0.11519 \\
(0.27162)\end{array}$ & $\begin{array}{l}-0.15278 \\
(0.27318)\end{array}$ & $\begin{array}{l}-0.14853 \\
(0.27362)\end{array}$ & $\begin{array}{l}-0.14729 \\
(0.27341)\end{array}$ & $\begin{array}{l}-0.13770 \\
(0.26570)\end{array}$ & $\begin{array}{l}-0.16796 \\
(0.27283)\end{array}$ & $\begin{array}{l}-0.17200 \\
(0.27246)\end{array}$ & $\begin{array}{l}-0.13770 \\
(0.26654)\end{array}$ & $\begin{array}{l}-0.13770 \\
(0.27232)\end{array}$ & $\begin{array}{l}-0.16394 \\
(0.27190)\end{array}$ \\
\hline Factor $4 \& 5$ & $\begin{array}{l}-0.01926 \\
(0.22314)\end{array}$ & $\begin{array}{l}-0.03257 \\
(0.22263)\end{array}$ & $\begin{array}{l}-0.06788 \\
(0.22818)\end{array}$ & $\begin{array}{l}-0.01926 \\
(0.22169) \\
\end{array}$ & $\begin{array}{l}-0.03941 \\
(0.22394)\end{array}$ & $\begin{array}{l}-0.01926 \\
(0.22305)\end{array}$ & $\begin{array}{l}-0.03207 \\
(0.22405)\end{array}$ & $\begin{array}{l}-0.01926 \\
(0.21712) \\
\end{array}$ & $\begin{array}{l}-0.03453 \\
(0.22249)\end{array}$ & $\begin{array}{l}-0.05389 \\
(0.22302)\end{array}$ & $\begin{array}{l}-0.01926 \\
(0.21780)\end{array}$ & $\begin{array}{l}-0.00806 \\
(0.22274)\end{array}$ & $\begin{array}{l}-0.05431 \\
(0.22282) \\
\end{array}$ \\
\hline Factor 3 & $\begin{array}{l}-0.41385^{*} \\
(0.07897)\end{array}$ & $\begin{array}{l}-0.43005^{*} \\
(0.07882)\end{array}$ & $\begin{array}{l}-0.40647^{*} \\
(0.07666)\end{array}$ & $\begin{array}{l}-0.40647^{*} \\
(0.07631)\end{array}$ & $\begin{array}{l}-0.40647^{*} \\
(0.07669)\end{array}$ & $\begin{array}{l}-0.42288^{*} \\
(0.08197)\end{array}$ & $\begin{array}{l}-0.41782^{*} \\
(0.07907)\end{array}$ & $\begin{array}{l}-0.40647^{*} \\
(0.07474)\end{array}$ & $\begin{array}{l}-0.40647^{*} \\
(0.07649)\end{array}$ & $\begin{array}{l}-0.40647^{*} \\
(0.07639)\end{array}$ & $\begin{array}{l}-0.40647^{*} \\
(0.07498)\end{array}$ & $\begin{array}{l}-0.40647^{*} \\
(0.07660)\end{array}$ & $\begin{array}{l}-0.41004^{*} \\
(0.07638)\end{array}$ \\
\hline Factor $3 \& 5$ & $\begin{array}{l}0.15062 \\
(0.16200)\end{array}$ & $\begin{array}{l}0.15062 \\
(0.16145)\end{array}$ & $\begin{array}{l}0.08439 \\
(0.17528)\end{array}$ & $\begin{array}{l}0.20314 \\
(0.16391)\end{array}$ & $\begin{array}{l}0.10579 \\
(0.16940)\end{array}$ & $\begin{array}{l}0.12917 \\
(0.16622)\end{array}$ & $\begin{array}{l}0.13161 \\
(0.16499)\end{array}$ & $\begin{array}{l}0.15062 \\
(0.15763)\end{array}$ & $\begin{array}{l}0.12798 \\
(0.16217)\end{array}$ & $\begin{array}{l}0.09924 \\
(0.16443)\end{array}$ & $\begin{array}{l}0.15062 \\
(0.15813)\end{array}$ & $\begin{array}{l}0.15062 \\
(0.16156)\end{array}$ & $\begin{array}{l}0.09861 \\
(0.16414) \\
\end{array}$ \\
\hline Factor $3 \& 4$ & \multicolumn{13}{|c|}{ Insufficient Data } \\
\hline Factor $3,4 \& 5$ & $\begin{array}{l}-0.00325 \\
(0.29420)\end{array}$ & $\begin{array}{l}-0.02639 \\
(0.29377)\end{array}$ & $\begin{array}{l}-0.06948 \\
(0.30133)\end{array}$ & $\begin{array}{l}0.07155 \\
(0.29560) \\
\end{array}$ & $\begin{array}{l}-0.02077 \\
(0.29441)\end{array}$ & $\begin{array}{l}-0.00325 \\
(0.29408)\end{array}$ & $\begin{array}{l}-0.01439 \\
(0.29464)\end{array}$ & $\begin{array}{l}-0.00325 \\
(0.28626) \\
\end{array}$ & $\begin{array}{l}-0.00325 \\
(0.29298)\end{array}$ & $\begin{array}{l}-0.00325 \\
(0.29258)\end{array}$ & $\begin{array}{l}-0.00325 \\
(0.28716)\end{array}$ & $\begin{array}{l}-0.00325 \\
(0.29339) \\
\end{array}$ & $\begin{array}{l}-0.03372 \\
(0.29302) \\
\end{array}$ \\
\hline Factor 2 & $\begin{array}{l}0.24973 \\
(0.24275)\end{array}$ & $\begin{array}{l}0.23398 \\
(0.24225)\end{array}$ & $\begin{array}{l}0.21823 \\
(0.24441)\end{array}$ & $\begin{array}{l}0.28531 \\
(0.24209)\end{array}$ & $\begin{array}{l}0.22588 \\
(0.24385)\end{array}$ & $\begin{array}{l}0.23679 \\
(0.24371)\end{array}$ & $\begin{array}{l}0.23456 \\
(0.24394)\end{array}$ & $\begin{array}{l}0.21183 \\
(0.23646)\end{array}$ & $\begin{array}{l}0.23165 \\
(0.24210)\end{array}$ & $\begin{array}{l}0.20874 \\
(0.24284)\end{array}$ & $\begin{array}{l}0.29288 \\
(0.23732)\end{array}$ & $\begin{array}{l}0.27623 \\
(0.24320)\end{array}$ & $\begin{array}{l}0.18326 \\
(0.24469)\end{array}$ \\
\hline Facto & $\begin{array}{l}0.16443 \\
(0.35292) \\
\end{array}$ & $\begin{array}{l}0.17480 \\
(0.35078) \\
\end{array}$ & $\begin{array}{l}0.10857 \\
(0.35776) \\
\end{array}$ & $\begin{array}{l}0.24960 \\
(0.35247) \\
\end{array}$ & $\begin{array}{l}0.12466 \\
(0.35592) \\
\end{array}$ & $\begin{array}{l}0.14759 \\
(0.35504) \\
\end{array}$ & $\begin{array}{l}0.14292 \\
(0.35579) \\
\end{array}$ & $\begin{array}{l}0.09512 \\
(0.34327) \\
\end{array}$ & $\begin{array}{l}0.17480 \\
(0.35052) \\
\end{array}$ & $\begin{array}{l}0.13172 \\
(0.35113) \\
\end{array}$ & $\begin{array}{l}0.17480 \\
(0.34356) \\
\end{array}$ & $\begin{array}{l}0.17480 \\
(0.35101) \\
\end{array}$ & $\begin{array}{l}0.08759 \\
(0.35390) \\
\end{array}$ \\
\hline Factor $2 \& 4$ & $\begin{array}{l}0.23891^{\ddagger} \\
(0.14147)\end{array}$ & $\begin{array}{l}0.23929^{\ddagger} \\
(0.13871)\end{array}$ & $\begin{array}{l}0.19992 \\
(0.14768)\end{array}$ & $\begin{array}{l}0.32441^{\dagger} \\
(0.14493)\end{array}$ & $\begin{array}{l}0.20339 \\
(0.14812)\end{array}$ & $\begin{array}{l}0.23902^{\ddagger} \\
(0.14012)\end{array}$ & $\begin{array}{l}0.21774 \\
(0.14868)\end{array}$ & $\begin{array}{l}0.18499 \\
(0.13650)\end{array}$ & $\begin{array}{l}0.18544 \\
(0.14607)\end{array}$ & $\begin{array}{l}0.20796 \\
(0.14073)\end{array}$ & $\begin{array}{l}0.24962^{\ddagger} \\
(0.13562)\end{array}$ & $\begin{array}{l}0.28797^{\dagger} \\
(0.14260)\end{array}$ & $\begin{array}{l}0.20066 \\
(0.14131)\end{array}$ \\
\hline Factor $2,4 \& 5$ & $\begin{array}{l}0.19221^{\ddagger} \\
(0.10979)\end{array}$ & $\begin{array}{l}0.14570 \\
(0.11575)\end{array}$ & $\begin{array}{l}0.12698 \\
(0.12876)\end{array}$ & $\begin{array}{l}0.26437^{\dagger} \\
(0.11686)\end{array}$ & $\begin{array}{l}0.17905 \\
(0.11074)\end{array}$ & $\begin{array}{l}0.18553^{\ddagger} \\
(0.11054)\end{array}$ & $\begin{array}{l}0.18421^{\ddagger} \\
(0.11073)\end{array}$ & $\begin{array}{l}0.04936 \\
(0.11475)\end{array}$ & $\begin{array}{l}0.17913 \\
(0.10979)\end{array}$ & $\begin{array}{l}0.16887 \\
(0.11026)\end{array}$ & $\begin{array}{l}0.32340^{*} \\
(0.11450)\end{array}$ & $\begin{array}{l}0.19592^{\ddagger} \\
(0.10949)\end{array}$ & $\begin{array}{l}0.17625 \\
(0.10959)\end{array}$ \\
\hline Factor $2 \& 3$ & $\begin{array}{l}0.29466^{\dagger} \\
(0.12886)\end{array}$ & $\begin{array}{l}0.23120^{\ddagger} \\
(0.13868)\end{array}$ & $\begin{array}{l}0.23120 \\
(0.14516)\end{array}$ & $\begin{array}{l}0.37223^{*} \\
(0.13526)\end{array}$ & $\begin{array}{l}0.24729^{\ddagger} \\
(0.14027)\end{array}$ & $\begin{array}{l}0.29379^{\dagger} \\
(0.12878)\end{array}$ & $\begin{array}{l}0.26555^{\ddagger} \\
(0.13914)\end{array}$ & $\begin{array}{l}0.29743^{\dagger} \\
(0.12521)\end{array}$ & $\begin{array}{l}0.28727^{\dagger} \\
(0.12836)\end{array}$ & $\begin{array}{l}0.21127 \\
(0.13934)\end{array}$ & $\begin{array}{l}0.32168^{\dagger} \\
(0.12583)\end{array}$ & $\begin{array}{l}0.34570^{\dagger} \\
(0.13516)\end{array}$ & $\begin{array}{l}0.28577^{\dagger} \\
(0.12810)\end{array}$ \\
\hline Factor $2,3 \& 5$ & $\begin{array}{l}0.19048 \\
(0.50043) \\
\end{array}$ & $\begin{array}{l}0.14497 \\
(0.49887) \\
\end{array}$ & $\begin{array}{l}0.14498 \\
(0.50140) \\
\end{array}$ & $\begin{array}{l}0.28600 \\
(0.49650) \\
\end{array}$ & $\begin{array}{l}0.16107 \\
(0.50019) \\
\end{array}$ & $\begin{array}{l}0.18400 \\
(0.49984) \\
\end{array}$ & $\begin{array}{l}0.21120 \\
(0.49753) \\
\end{array}$ & $\begin{array}{l}0.21120 \\
(0.48434) \\
\end{array}$ & $\begin{array}{l}0.13518 \\
(0.49880) \\
\end{array}$ & $\begin{array}{l}0.12504 \\
(0.49810) \\
\end{array}$ & $\begin{array}{l}0.21120 \\
(0.48587) \\
\end{array}$ & $\begin{array}{l}0.21120 \\
(0.49641) \\
\end{array}$ & $\begin{array}{l}0.12399 \\
(0.49764) \\
\end{array}$ \\
\hline Factor $2,3 \& 4$ & $\begin{array}{l}0.24570^{*} \\
(0.06861)\end{array}$ & $\begin{array}{l}0.20082^{*} \\
(0.06977)\end{array}$ & $\begin{array}{l}0.19965^{\dagger} \\
(0.08225)\end{array}$ & $\begin{array}{l}0.34067^{*} \\
(0.06417)\end{array}$ & $\begin{array}{l}0.21574^{*} \\
(0.07318)\end{array}$ & $\begin{array}{l}0.26126^{*} \\
(0.04752)\end{array}$ & $\begin{array}{l}0.23400^{*} \\
(0.07079)\end{array}$ & $\begin{array}{l}0.25994^{*} \\
(0.04562)\end{array}$ & $\begin{array}{l}0.26372^{*} \\
(0.04668)\end{array}$ & $\begin{array}{l}0.18048^{\dagger} \\
(0.07181)\end{array}$ & $\begin{array}{l}0.27103^{*} \\
(0.04576)\end{array}$ & $\begin{array}{l}0.26588^{*} \\
(0.04672)\end{array}$ & $\begin{array}{l}0.17866^{\dagger} \\
(0.07081)\end{array}$ \\
\hline Fact & $\begin{array}{l}0.30111^{\dagger} \\
(0.14108)\end{array}$ & $\begin{array}{l}0.26984^{\ddagger} \\
(0.14281)\end{array}$ & $\begin{array}{l}0.24196 \\
(0.15523)\end{array}$ & $\begin{array}{l}0.38299^{*} \\
(0.14591)\end{array}$ & $\begin{array}{l}0.26727^{\ddagger} \\
(0.14712)\end{array}$ & $\begin{array}{l}0.28883^{\dagger} \\
(0.14396)\end{array}$ & $\begin{array}{l}0.27631^{\ddagger} \\
(0.14964)\end{array}$ & $\begin{array}{l}0.18665 \\
(0.14074)\end{array}$ & $\begin{array}{l}0.25021^{\ddagger} \\
(0.14567)\end{array}$ & $\begin{array}{l}0.23104 \\
(0.14770)\end{array}$ & $\begin{array}{l}0.37486^{*} \\
(0.13819)\end{array}$ & $\begin{array}{l}0.33605^{\dagger} \\
(0.14173)\end{array}$ & $\begin{array}{l}0.23923 \\
(0.14539)\end{array}$ \\
\hline Factor 1 & $\begin{array}{l}0.54610^{*} \\
(0.09063)\end{array}$ & $\begin{array}{l}0.54610^{*} \\
(0.09032)\end{array}$ & $\begin{array}{l}0.53911^{*} \\
(0.09074)\end{array}$ & $\begin{array}{l}0.55348^{*} \\
(0.09015)\end{array}$ & $\begin{array}{l}0.54335^{*} \\
(0.09055)\end{array}$ & $\begin{array}{l}0.52177^{*} \\
(0.10009)\end{array}$ & $\begin{array}{l}0.54238^{*} \\
(0.09080)\end{array}$ & $\begin{array}{l}0.54610^{*} \\
(0.08819)\end{array}$ & $\begin{array}{l}0.54610^{*} \\
(0.09026)\end{array}$ & $\begin{array}{l}0.47376^{*} \\
(0.10133)\end{array}$ & $\begin{array}{l}0.54610^{*} \\
(0.08847)\end{array}$ & $\begin{array}{l}0.54610^{*} \\
(0.09038)\end{array}$ & $\begin{array}{l}0.54610^{*} \\
(0.09009)\end{array}$ \\
\hline Factor & $\begin{array}{l}0.19793 \\
(0.17270) \\
\end{array}$ & $\begin{array}{l}0.18996 \\
(0.17223) \\
\end{array}$ & $\begin{array}{l}0.16083 \\
(0.17649) \\
\end{array}$ & $\begin{array}{l}0.24884 \\
(0.17420) \\
\end{array}$ & $\begin{array}{l}0.17588 \\
(0.17421) \\
\end{array}$ & $\begin{array}{l}0.18269 \\
(0.17468) \\
\end{array}$ & $\begin{array}{l}0.18775 \\
(0.17345) \\
\end{array}$ & $\begin{array}{l}0.19793 \\
(0.16804) \\
\end{array}$ & $\begin{array}{l}0.18280 \\
(0.17234) \\
\end{array}$ & $\begin{array}{l}0.17041 \\
(0.17266) \\
\end{array}$ & $\begin{array}{l}0.19793 \\
(0.16857) \\
\end{array}$ & $\begin{array}{l}0.19793 \\
(0.17223) \\
\end{array}$ & $\begin{array}{l}0.14908 \\
(0.17424) \\
\end{array}$ \\
\hline Factor $1 \& 4$ & $\begin{array}{l}0.24364^{\ddagger} \\
(0.14026)\end{array}$ & $\begin{array}{l}0.23312^{\ddagger} \\
(0.14003)\end{array}$ & $\begin{array}{l}0.22616 \\
(0.14112)\end{array}$ & $\begin{array}{l}0.26337^{\ddagger} \\
(0.13983)\end{array}$ & $\begin{array}{l}0.23041 \\
(0.14083)\end{array}$ & $\begin{array}{l}0.23683^{\ddagger} \\
(0.14070)\end{array}$ & $\begin{array}{l}0.23314 \\
(0.14128)\end{array}$ & $\begin{array}{l}0.23099^{\ddagger} \\
(0.13652)\end{array}$ & $\begin{array}{l}0.23760^{\ddagger} \\
(0.13974)\end{array}$ & $\begin{array}{l}0.24364^{\ddagger} \\
(0.13949)\end{array}$ & $\begin{array}{l}0.24364^{\ddagger} \\
(0.13690)\end{array}$ & $\begin{array}{l}0.24364^{\ddagger} \\
(0.13987)\end{array}$ & $\begin{array}{l}0.23671^{\ddagger} \\
(0.13947)\end{array}$ \\
\hline Factor $1,4 \& 5$ & $\begin{array}{l}0.25731^{*} \\
(0.07040)\end{array}$ & $\begin{array}{l}0.25359^{*} \\
(0.07022)\end{array}$ & $\begin{array}{l}0.20631^{\dagger} \\
(0.08747)\end{array}$ & $\begin{array}{l}0.28241^{*} \\
(0.07149)\end{array}$ & $\begin{array}{l}0.24049^{*} \\
(0.07278)\end{array}$ & $\begin{array}{l}0.25090^{*} \\
(0.07126)\end{array}$ & $\begin{array}{l}0.25488^{*} \\
(0.07048)\end{array}$ & $\begin{array}{l}0.18169^{\dagger} \\
(0.07196)\end{array}$ & $\begin{array}{l}0.25731^{*} \\
(0.07011)\end{array}$ & $\begin{array}{l}0.24013^{*} \\
(0.07087)\end{array}$ & $\begin{array}{l}0.25731^{*} \\
(0.06871)\end{array}$ & $\begin{array}{l}0.25731^{*} \\
(0.07021)\end{array}$ & $\begin{array}{l}0.20607^{*} \\
(0.07667)\end{array}$ \\
\hline Facto & $\begin{array}{l}0.04306 \\
(0.11751)\end{array}$ & $\begin{array}{l}0.04306 \\
(0.11711)\end{array}$ & $\begin{array}{l}0.00312 \\
(0.12418)\end{array}$ & $\begin{array}{l}0.08817 \\
(0.11975)\end{array}$ & $\begin{array}{l}0.03521 \\
(0.11766)\end{array}$ & $\begin{array}{l}0.03226 \\
(0.11897)\end{array}$ & $\begin{array}{l}0.04306 \\
(0.11745)\end{array}$ & $\begin{array}{l}0.04306 \\
(0.11434)\end{array}$ & $\begin{array}{l}0.04306 \\
(0.11702)\end{array}$ & $\begin{array}{l}0.04306 \\
(0.11686)\end{array}$ & $\begin{array}{l}0.04306 \\
(0.11470)\end{array}$ & $\begin{array}{l}0.04306 \\
(0.11719)\end{array}$ & $\begin{array}{l}0.00844 \\
(0.11870)\end{array}$ \\
\hline Factor $1,3 \& 5$ & $\begin{array}{l}0.10338 \\
(0.09467)\end{array}$ & $\begin{array}{l}0.10338 \\
(0.09434)\end{array}$ & $\begin{array}{l}0.03715 \\
(0.11621)\end{array}$ & $\begin{array}{l}0.17547^{\ddagger} \\
(0.10324)\end{array}$ & $\begin{array}{l}0.05506 \\
(0.10898)\end{array}$ & $\begin{array}{l}0.10208 \\
(0.09466)\end{array}$ & $\begin{array}{l}0.10070 \\
(0.09473)\end{array}$ & $\begin{array}{l}0.10338 \\
(0.09211) \\
\end{array}$ & $\begin{array}{l}0.10338 \\
(0.09428)\end{array}$ & $\begin{array}{l}0.09926 \\
(0.09419)\end{array}$ & $\begin{array}{l}0.10338 \\
(0.09240)\end{array}$ & $\begin{array}{l}0.10338 \\
(0.09441) \\
\end{array}$ & $\begin{array}{l}0.09921 \\
(0.09413) \\
\end{array}$ \\
\hline Facto & $\begin{array}{l}0.19427 \\
(0.11997)\end{array}$ & $\begin{array}{l}0.19427 \\
(0.11956)\end{array}$ & $\begin{array}{l}0.14698 \\
(0.12912)\end{array}$ & $\begin{array}{l}0.22527^{\ddagger} \\
(0.12059)\end{array}$ & $\begin{array}{l}0.18460 \\
(0.12028)\end{array}$ & $\begin{array}{l}0.17790 \\
(0.12329)\end{array}$ & $\begin{array}{l}0.19057 \\
(0.12007)\end{array}$ & $\begin{array}{l}0.18203 \\
(0.11679)\end{array}$ & $\begin{array}{l}0.18986 \\
(0.11952)\end{array}$ & $\begin{array}{l}0.17926 \\
(0.11970)\end{array}$ & $\begin{array}{l}0.19427^{\ddagger} \\
(0.11710)\end{array}$ & $\begin{array}{l}0.19427 \\
(0.11964)\end{array}$ & $\begin{array}{l}0.11577 \\
(0.12855)\end{array}$ \\
\hline Factor & $\begin{array}{l}0.16684^{\dagger} \\
(0.07907)\end{array}$ & $\begin{array}{l}0.16737^{\dagger} \\
(0.07879)\end{array}$ & $\begin{array}{l}0.10281 \\
(0.10284)\end{array}$ & $\begin{array}{l}0.18457^{\dagger} \\
(0.07920)\end{array}$ & $\begin{array}{l}0.16063^{\dagger} \\
(0.07929)\end{array}$ & $\begin{array}{l}0.16452^{\dagger} \\
(0.07918)\end{array}$ & $\begin{array}{l}0.16013^{\dagger} \\
(0.07993)\end{array}$ & $\begin{array}{l}0.16737^{\dagger} \\
(0.07692)\end{array}$ & $\begin{array}{l}0.16737^{\dagger} \\
(0.07873)\end{array}$ & $\begin{array}{l}0.15727^{\dagger} \\
(0.07889)\end{array}$ & $\begin{array}{l}0.16737^{\dagger} \\
(0.07716)\end{array}$ & $\begin{array}{l}0.17204^{\dagger} \\
(0.07895)\end{array}$ & $\begin{array}{l}0.15275^{\ddagger} \\
(0.07909)\end{array}$ \\
\hline Factor $1 \& 2$ & $\begin{array}{l}0.14357 \\
(0.17530)\end{array}$ & $\begin{array}{l}0.12684 \\
(0.17476)\end{array}$ & $\begin{array}{l}0.12684 \\
(0.17571)\end{array}$ & $\begin{array}{l}0.22599 \\
(0.17868)\end{array}$ & $\begin{array}{l}0.13276 \\
(0.17523)\end{array}$ & $\begin{array}{l}0.14453 \\
(0.17459)\end{array}$ & $\begin{array}{l}0.11932 \\
(0.18210)\end{array}$ & $\begin{array}{l}0.11213 \\
(0.16995) \\
\end{array}$ & $\begin{array}{l}0.12324 \\
(0.17474)\end{array}$ & $\begin{array}{l}0.11951 \\
(0.17450)\end{array}$ & $\begin{array}{l}0.19568 \\
(0.17066)\end{array}$ & $\begin{array}{l}0.16485 \\
(0.17421) \\
\end{array}$ & $\begin{array}{l}0.11913 \\
(0.17433) \\
\end{array}$ \\
\hline Factor $1,2 \& 5$ & $\begin{array}{l}0.24247 \\
(0.16806)\end{array}$ & $\begin{array}{l}0.22974 \\
(0.16782)\end{array}$ & $\begin{array}{l}0.18614 \\
(0.17804)\end{array}$ & $\begin{array}{l}0.28914^{\ddagger} \\
(0.16891)\end{array}$ & $\begin{array}{l}0.22083 \\
(0.16985)\end{array}$ & $\begin{array}{l}0.22561 \\
(0.17122)\end{array}$ & $\begin{array}{l}0.22231 \\
(0.17202)\end{array}$ & $\begin{array}{l}0.20854 \\
(0.16377)\end{array}$ & $\begin{array}{l}0.19654 \\
(0.17093)\end{array}$ & $\begin{array}{l}0.15866 \\
(0.17590)\end{array}$ & $\begin{array}{l}0.26549 \\
(0.16407)\end{array}$ & $\begin{array}{l}0.26753 \\
(0.16869)\end{array}$ & $\begin{array}{l}0.22497 \\
(0.16739)\end{array}$ \\
\hline Factor $1,2 \& 4$ & $\begin{array}{l}0.19639 \\
(0.18915) \\
\end{array}$ & $\begin{array}{l}0.17272 \\
(0.18738) \\
\end{array}$ & $\begin{array}{l}0.14514 \\
(0.19708) \\
\end{array}$ & $\begin{array}{l}0.28616 \\
(0.18947) \\
\end{array}$ & $\begin{array}{l}0.16123 \\
(0.19354) \\
\end{array}$ & $\begin{array}{l}0.18793 \\
(0.18986) \\
\end{array}$ & $\begin{array}{l}0.18391 \\
(0.19093) \\
\end{array}$ & $\begin{array}{l}0.07414 \\
(0.18484) \\
\end{array}$ & $\begin{array}{l}0.15645 \\
(0.18898) \\
\end{array}$ & $\begin{array}{l}0.12520 \\
(0.19251) \\
\end{array}$ & $\begin{array}{l}0.28694 \\
(0.18254) \\
\end{array}$ & $\begin{array}{l}0.24388 \\
(0.18715) \\
\end{array}$ & $\begin{array}{l}0.14837 \\
(0.18833) \\
\end{array}$ \\
\hline Factor $1,2,4 \& 5$ & $\begin{array}{l}0.04920 \\
(0.08747)\end{array}$ & $\begin{array}{l}0.04152 \\
(0.08688)\end{array}$ & $\begin{array}{l}0.00530 \\
(0.09982)\end{array}$ & $\begin{array}{l}0.11241 \\
(0.09264) \\
\end{array}$ & $\begin{array}{l}0.02644 \\
(0.09189)\end{array}$ & $\begin{array}{l}0.04292 \\
(0.08877)\end{array}$ & $\begin{array}{l}0.02869 \\
(0.09641)\end{array}$ & $\begin{array}{l}-0.06848 \\
(0.09153) \\
\end{array}$ & $\begin{array}{l}0.00561 \\
(0.09322)\end{array}$ & $\begin{array}{l}0.01851 \\
(0.08909)\end{array}$ & $\begin{array}{l}0.08188 \\
(0.08495)\end{array}$ & $\begin{array}{l}0.06606 \\
(0.08698)\end{array}$ & $\begin{array}{l}0.02610 \\
(0.08776) \\
\end{array}$ \\
\hline Factor $1,2 \& 3$ & $\begin{array}{l}0.31473^{\ddagger} \\
(0.18910)\end{array}$ & $\begin{array}{l}0.31473^{\ddagger} \\
(0.18845)\end{array}$ & $\begin{array}{l}0.30209 \\
(0.18917)\end{array}$ & $\begin{array}{l}0.32901^{\ddagger} \\
(0.18806)\end{array}$ & $\begin{array}{l}0.26460 \\
(0.19702)\end{array}$ & $\begin{array}{l}0.28753 \\
(0.19492)\end{array}$ & $\begin{array}{l}0.30865 \\
(0.18928)\end{array}$ & $\begin{array}{l}0.31473^{\ddagger} \\
(0.18399) \\
\end{array}$ & $\begin{array}{l}0.23872 \\
(0.19630)\end{array}$ & $\begin{array}{l}0.22857 \\
(0.19597)\end{array}$ & $\begin{array}{l}0.31473^{\ddagger} \\
(0.18457) \\
\end{array}$ & $\begin{array}{l}0.35981^{\ddagger} \\
(0.19270) \\
\end{array}$ & $\begin{array}{l}0.29809 \\
(0.18823) \\
\end{array}$ \\
\hline Factor 1 , & $\begin{array}{l}0.21918 \\
(0.19311)\end{array}$ & $\begin{array}{l}0.19246 \\
(0.19259)\end{array}$ & $\begin{array}{l}0.16512 \\
(0.20203)\end{array}$ & $\begin{array}{l}0.27528 \\
(0.19126)\end{array}$ & $\begin{array}{l}0.18858 \\
(0.19640)\end{array}$ & $\begin{array}{l}0.21937 \\
(0.19180)\end{array}$ & $\begin{array}{l}0.22199 \\
(0.19128)\end{array}$ & $\begin{array}{l}0.18456 \\
(0.18609)\end{array}$ & $\begin{array}{l}0.22019 \\
(0.19012)\end{array}$ & $\begin{array}{l}0.19340 \\
(0.19123)\end{array}$ & $\begin{array}{l}0.23135 \\
(0.18617)\end{array}$ & $\begin{array}{l}0.25435 \\
(0.19128)\end{array}$ & $\begin{array}{l}0.18013 \\
(0.19215)\end{array}$ \\
\hline 4 & $\begin{array}{l}0.29494^{*} \\
(0.03825) \\
\end{array}$ & $\begin{array}{l}0.24037^{*} \\
(0.05943)\end{array}$ & $\begin{array}{l}0.23338^{*} \\
(0.07642) \\
\end{array}$ & $\begin{array}{l}0.34146^{*} \\
(0.04452) \\
\end{array}$ & $\begin{array}{l}0.24994^{*} \\
(0.06619) \\
\end{array}$ & $\begin{array}{l}0.27182^{*} \\
(0.06004)\end{array}$ & $\begin{array}{l}0.26987^{*} \\
(0.06083) \\
\end{array}$ & $\begin{array}{l}0.21224^{*} \\
(0.04397)\end{array}$ & $\begin{array}{l}0.23669^{*} \\
(0.05841)\end{array}$ & $\begin{array}{l}0.21951^{*} \\
(0.06270)\end{array}$ & $\begin{array}{l}0.36405^{*} \\
(0.04137) \\
\end{array}$ & $\begin{array}{l}0.32402^{*} \\
(0.04299) \\
\end{array}$ & $\begin{array}{l}0.21215^{*} \\
(0.06454) \\
\end{array}$ \\
\hline All F & $\begin{array}{l}0.29598^{*} \\
(0.05049)\end{array}$ & $\begin{array}{l}0.26272^{*} \\
(0.05626)\end{array}$ & $\begin{array}{l}0.24228^{*} \\
(0.07840)\end{array}$ & $\begin{array}{l}0.35996^{*} \\
(0.05536)\end{array}$ & $\begin{array}{l}0.26268^{*} \\
(0.06556)\end{array}$ & $\begin{array}{l}0.28315^{*} \\
(0.05922)\end{array}$ & $\begin{array}{l}0.27844^{*} \\
(0.06328)\end{array}$ & $\begin{array}{l}0.19060^{*} \\
(0.05520)\end{array}$ & $\begin{array}{l}0.24454^{*} \\
(0.06302)\end{array}$ & $\begin{array}{l}0.23143^{*} \\
(0.06510)\end{array}$ & $\begin{array}{l}0.32455^{*} \\
(0.04451)\end{array}$ & $\begin{array}{l}0.33674^{*} \\
(0.05296)\end{array}$ & $\begin{array}{l}0.23259^{*} \\
(0.06310)\end{array}$ \\
\hline $\begin{array}{l}\text { Individual } \\
\text { Variable }\end{array}$ & $\begin{array}{l}0.02073 \\
(0.05149)\end{array}$ & $\begin{array}{l}0.06623 \\
(0.05278)\end{array}$ & $\begin{array}{l}0.06623 \\
(0.06766)\end{array}$ & $\begin{array}{l}-0.07480^{\ddagger} \\
(0.04417)\end{array}$ & $\begin{array}{l}0.05014 \\
(0.05628)\end{array}$ & $\begin{array}{l}0.02721 \\
(0.04758)\end{array}$ & $\begin{array}{l}0.03188 \\
(0.05309)\end{array}$ & $\begin{array}{l}0.15935^{*} \\
(0.04648)\end{array}$ & $\begin{array}{l}0.07602 \\
(0.05543)\end{array}$ & $\begin{array}{l}0.08616 \\
(0.05513)\end{array}$ & $\begin{array}{l}-0.18146^{*} \\
(0.05630)\end{array}$ & $\begin{array}{l}-0.05571 \\
(0.04896)\end{array}$ & $\begin{array}{l}0.08721 \\
(0.05335)\end{array}$ \\
\hline
\end{tabular}

$*$ Significant at the 0.01 level $†$ Significant at the 0.05 level $\$$ Significant at the 0.10 level

\section{Table 100 Coefficients with individual variables for high-level factors - Vehicles Industry}




\begin{tabular}{|c|c|c|c|c|c|c|c|c|c|c|c|c|c|}
\hline $\begin{array}{l}\text { Individual } \\
\text { variable included } \\
\text { in the model in } \\
\text { the column }\end{array}$ & 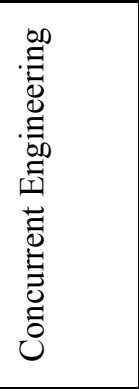 & 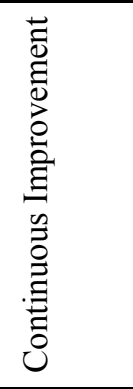 & 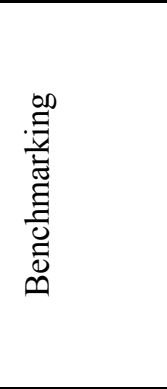 & 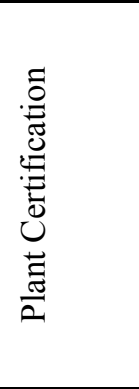 & 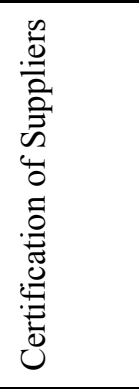 & 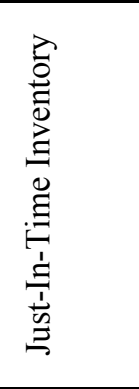 & 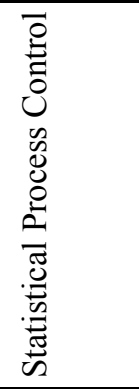 & 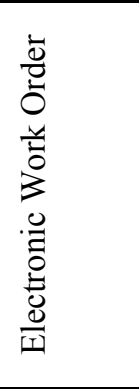 & 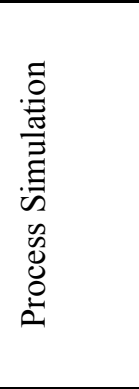 & 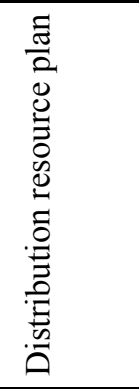 & 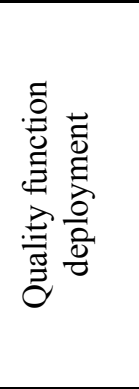 & 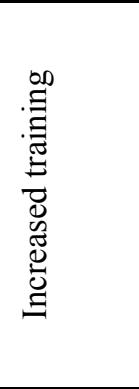 & 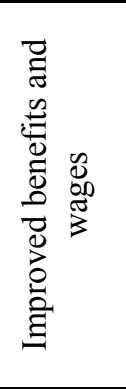 \\
\hline No factors & $\begin{array}{l}0.16483^{*} \\
(0.03703)\end{array}$ & $\begin{array}{l}0.17073^{*} \\
(0.03721)\end{array}$ & $\begin{array}{l}0.17023^{*} \\
(0.03717)\end{array}$ & $\begin{array}{l}0.15799^{*} \\
(0.03967)\end{array}$ & $\begin{array}{l}0.12879^{*} \\
(0.03819)\end{array}$ & $\begin{array}{l}0.17145^{*} \\
(0.03767)\end{array}$ & $\begin{array}{l}0.16614^{*} \\
(0.03705)\end{array}$ & $\begin{array}{l}0.16785^{*} \\
(0.03710)\end{array}$ & $\begin{array}{l}0.16530^{*} \\
(0.03716)\end{array}$ & $\begin{array}{l}0.16754^{*} \\
(0.03696)\end{array}$ & $\begin{array}{l}0.16754^{*} \\
(0.03711)\end{array}$ & $\begin{array}{l}0.16754^{*} \\
(0.03719)\end{array}$ & $\begin{array}{l}0.16754^{*} \\
(0.03707)\end{array}$ \\
\hline Factor 5 & $\begin{array}{l}-0.06842 \\
(0.23077)\end{array}$ & $\begin{array}{l}-0.00799 \\
(0.23117) \\
\end{array}$ & $\begin{array}{l}-0.02039 \\
(0.23037)\end{array}$ & $\begin{array}{l}-0.05579 \\
(0.23334)\end{array}$ & $\begin{array}{l}-0.17487 \\
(0.22943)\end{array}$ & $\begin{array}{l}-0.01937 \\
(0.23136)\end{array}$ & $\begin{array}{l}-0.06720 \\
(0.23132)\end{array}$ & $\begin{array}{l}-0.00767 \\
(0.23119)\end{array}$ & $\begin{array}{l}-0.03115 \\
(0.23021)\end{array}$ & $\begin{array}{l}-0.03115 \\
(0.22937)\end{array}$ & $\begin{array}{l}-0.03115 \\
(0.23025)\end{array}$ & $\begin{array}{l}-0.00714 \\
(0.24972)\end{array}$ & $\begin{array}{l}-0.01482 \\
(0.23040) \\
\end{array}$ \\
\hline Factor 4 & $\begin{array}{l}-0.22441 \\
(0.27733) \\
\end{array}$ & $\begin{array}{l}-0.08381 \\
(0.27688) \\
\end{array}$ & $\begin{array}{l}-0.13770 \\
(0.27238) \\
\end{array}$ & $\begin{array}{l}-0.16908 \\
(0.27666) \\
\end{array}$ & $\begin{array}{l}-0.32075 \\
(0.27231) \\
\end{array}$ & $\begin{array}{l}-0.11854 \\
(0.27457) \\
\end{array}$ & $\begin{array}{l}-0.19633 \\
(0.27534) \\
\end{array}$ & $\begin{array}{l}-0.10481 \\
(0.27407) \\
\end{array}$ & $\begin{array}{l}-0.16014 \\
(0.27326)\end{array}$ & $\begin{array}{l}-0.13770 \\
(0.27144) \\
\end{array}$ & $\begin{array}{l}-0.17604 \\
(0.27504) \\
\end{array}$ & $\begin{array}{l}-0.13770 \\
(0.27314) \\
\end{array}$ & $\begin{array}{l}-0.13770 \\
(0.27220) \\
\end{array}$ \\
\hline Factor $4 \& 5$ & $\begin{array}{l}-0.08292 \\
(0.22572) \\
\end{array}$ & $\begin{array}{l}0.02031 \\
(0.22555) \\
\end{array}$ & $\begin{array}{l}0.00086500 \\
(0.22332)\end{array}$ & $\begin{array}{l}-0.03187 \\
(0.22372)\end{array}$ & $\begin{array}{l}-0.09283 \\
(0.21888) \\
\end{array}$ & $\begin{array}{l}-0.01375 \\
(0.22317) \\
\end{array}$ & $\begin{array}{l}-0.05297 \\
(0.22360)\end{array}$ & $\begin{array}{l}-0.00828 \\
(0.22282) \\
\end{array}$ & $\begin{array}{l}-0.01926 \\
(0.22262)\end{array}$ & $\begin{array}{l}-0.01926 \\
(0.22180) \\
\end{array}$ & $\begin{array}{l}-0.03028 \\
(0.22292) \\
\end{array}$ & $\begin{array}{l}0.01799 \\
(0.26773)\end{array}$ & $\begin{array}{l}0.03148 \\
(0.22629) \\
\end{array}$ \\
\hline Factor 3 & $\begin{array}{l}-0.40647^{*} \\
(0.07640)\end{array}$ & $\begin{array}{l}-0.40427^{*} \\
(0.07665) \\
\end{array}$ & $\begin{array}{l}-0.40647^{*} \\
(0.07662)\end{array}$ & $\begin{array}{l}-0.40647^{*} \\
(0.07675) \\
\end{array}$ & $\begin{array}{l}-0.40647^{*} \\
(0.07494) \\
\end{array}$ & $\begin{array}{l}-0.40647^{*} \\
(0.07677) \\
\end{array}$ & $\begin{array}{l}-0.40647^{*} \\
(0.07650) \\
\end{array}$ & $\begin{array}{l}-0.40647^{*} \\
(0.07662) \\
\end{array}$ & $\begin{array}{l}-0.40647^{*} \\
(0.07663) \\
\end{array}$ & $\begin{array}{l}-0.40647^{*} \\
(0.07635)\end{array}$ & $\begin{array}{l}-0.40647^{*} \\
(0.07665) \\
\end{array}$ & $\begin{array}{l}-0.40647^{*} \\
(0.07683) \\
\end{array}$ & $\begin{array}{l}-0.40647^{*} \\
(0.07657) \\
\end{array}$ \\
\hline Factor $3 \& 5$ & $\begin{array}{l}0.07309 \\
(0.16874)\end{array}$ & $\begin{array}{l}0.20451 \\
(0.16905)\end{array}$ & $\begin{array}{l}0.19008 \\
(0.16553)\end{array}$ & $\begin{array}{l}0.12858 \\
(0.16502)\end{array}$ & $\begin{array}{l}0.02207 \\
(0.16293)\end{array}$ & $\begin{array}{l}0.15878 \\
(0.16242)\end{array}$ & $\begin{array}{l}0.10948 \\
(0.16414)\end{array}$ & $\begin{array}{l}0.18320 \\
(0.16436)\end{array}$ & $\begin{array}{l}0.15062 \\
(0.16163)\end{array}$ & $\begin{array}{l}0.17437 \\
(0.16169) \\
\end{array}$ & $\begin{array}{l}0.10738 \\
(0.16707) \\
\end{array}$ & $\begin{array}{l}0.18787 \\
(0.21937) \\
\end{array}$ & $\begin{array}{l}0.21856 \\
(0.17085) \\
\end{array}$ \\
\hline Factor $3 \& 4$ & \multicolumn{13}{|c|}{ Insufficient Data } \\
\hline Factor $3,4 \& 5$ & $\begin{array}{l}-0.03354 \\
(0.29327) \\
\end{array}$ & $\begin{array}{l}0.05064 \\
(0.29764) \\
\end{array}$ & $\begin{array}{l}0.01424 \\
(0.29389) \\
\end{array}$ & $\begin{array}{l}-0.03463 \\
(0.29750) \\
\end{array}$ & $\begin{array}{l}-0.00325 \\
(0.28704) \\
\end{array}$ & $\begin{array}{l}0.02416 \\
(0.29717) \\
\end{array}$ & $\begin{array}{l}-0.03255 \\
(0.29378) \\
\end{array}$ & $\begin{array}{l}0.05139 \\
(0.29775) \\
\end{array}$ & $\begin{array}{l}-0.00325 \\
(0.29352) \\
\end{array}$ & $\begin{array}{l}-0.00325 \\
(0.29244) \\
\end{array}$ & $\begin{array}{l}-0.00325 \\
(0.29357) \\
\end{array}$ & $\begin{array}{l}0.03400 \\
(0.32933) \\
\end{array}$ & $\begin{array}{l}0.02329 \\
(0.29407) \\
\end{array}$ \\
\hline Factor 2 & $\begin{array}{l}0.18364 \\
(0.24520) \\
\end{array}$ & $\begin{array}{l}0.30362 \\
(0.24719) \\
\end{array}$ & $\begin{array}{l}0.29980 \\
(0.24639) \\
\end{array}$ & $\begin{array}{l}0.21835 \\
(0.24682) \\
\end{array}$ & $\begin{array}{l}0.06668 \\
(0.24344) \\
\end{array}$ & $\begin{array}{l}0.27714 \\
(0.24641) \\
\end{array}$ & $\begin{array}{l}0.16585 \\
(0.24947) \\
\end{array}$ & $\begin{array}{l}0.29137 \\
(0.24517) \\
\end{array}$ & $\begin{array}{l}0.22016 \\
(0.24379) \\
\end{array}$ & $\begin{array}{l}0.29156 \\
(0.24267) \\
\end{array}$ & $\begin{array}{l}0.20792 \\
(0.24564) \\
\end{array}$ & $\begin{array}{l}0.24973 \\
(0.24281) \\
\end{array}$ & $\begin{array}{l}0.24973 \\
(0.24198) \\
\end{array}$ \\
\hline Factor $2 \& 5$ & $\begin{array}{l}0.08808 \\
(0.35455)\end{array}$ & $\begin{array}{l}0.22869 \\
(0.35460)\end{array}$ & $\begin{array}{l}0.22487 \\
(0.35403)\end{array}$ & $\begin{array}{l}0.14342 \\
(0.35466)\end{array}$ & $\begin{array}{l}-0.00825 \\
(0.34800)\end{array}$ & $\begin{array}{l}0.18850 \\
(0.35243)\end{array}$ & $\begin{array}{l}0.09092 \\
(0.35590)\end{array}$ & $\begin{array}{l}0.22944 \\
(0.35469)\end{array}$ & $\begin{array}{l}0.17480 \\
(0.35116)\end{array}$ & $\begin{array}{l}0.21469 \\
(0.35073)\end{array}$ & $\begin{array}{l}0.14737 \\
(0.35224)\end{array}$ & $\begin{array}{l}0.21205 \\
(0.38186)\end{array}$ & $\begin{array}{l}0.17480 \\
(0.35086)\end{array}$ \\
\hline Factor & $\begin{array}{l}0.20094 \\
(0.14173) \\
\end{array}$ & $\begin{array}{l}0.30351^{\dagger} \\
(0.14722) \\
\end{array}$ & $\begin{array}{l}0.28328^{\dagger} \\
(0.14194) \\
\end{array}$ & $\begin{array}{l}0.21824 \\
(0.14615) \\
\end{array}$ & $\begin{array}{l}0.06656 \\
(0.14679) \\
\end{array}$ & $\begin{array}{l}0.27489^{\ddagger} \\
(0.14445) \\
\end{array}$ & $\begin{array}{l}0.16574 \\
(0.15145) \\
\end{array}$ & $\begin{array}{l}0.30426^{\dagger} \\
(0.14744) \\
\end{array}$ & $\begin{array}{l}0.22488 \\
(0.14057) \\
\end{array}$ & $\begin{array}{l}0.29832^{\dagger} \\
(0.14132) \\
\end{array}$ & $\begin{array}{l}0.20758 \\
(0.14458) \\
\end{array}$ & $\begin{array}{l}0.24962^{\ddagger} \\
(0.13898) \\
\end{array}$ & $\begin{array}{l}0.24962^{\ddagger} \\
(0.13850) \\
\end{array}$ \\
\hline Fact & $\begin{array}{l}0.17293 \\
(0.10996) \\
\end{array}$ & $\begin{array}{l}0.21106^{\ddagger} \\
(0.11072) \\
\end{array}$ & $\begin{array}{l}0.20295^{\ddagger} \\
(0.10984)\end{array}$ & $\begin{array}{l}0.16183 \\
(0.11880) \\
\end{array}$ & $\begin{array}{l}0.13259 \\
(0.10870) \\
\end{array}$ & $\begin{array}{l}0.19854^{\ddagger} \\
(0.11002)\end{array}$ & $\begin{array}{l}0.17690 \\
(0.10997) \\
\end{array}$ & $\begin{array}{l}0.24520^{\dagger} \\
(0.11949) \\
\end{array}$ & $\begin{array}{l}0.18499^{\ddagger} \\
(0.10978)\end{array}$ & $\begin{array}{l}0.26136^{\dagger} \\
(0.11687)\end{array}$ & $\begin{array}{l}0.14636 \\
(0.11868) \\
\end{array}$ & $\begin{array}{l}0.23046 \\
(0.18418) \\
\end{array}$ & $\begin{array}{l}0.25143^{\dagger} \\
(0.11940) \\
\end{array}$ \\
\hline Facto & $\begin{array}{l}0.28584^{\dagger} \\
(0.12821) \\
\end{array}$ & $\begin{array}{l}0.35132^{\dagger} \\
(0.13762) \\
\end{array}$ & $\begin{array}{l}0.34750^{\dagger} \\
(0.13620) \\
\end{array}$ & $\begin{array}{l}0.26605^{\ddagger} \\
(0.13644) \\
\end{array}$ & $\begin{array}{l}0.11438 \\
(0.13759) \\
\end{array}$ & $\begin{array}{l}0.32484^{\dagger} \\
(0.13565) \\
\end{array}$ & $\begin{array}{l}0.21355 \\
(0.14217) \\
\end{array}$ & $\begin{array}{l}0.35207^{\dagger} \\
(0.13786) \\
\end{array}$ & $\begin{array}{l}0.28989^{\dagger} \\
(0.12858) \\
\end{array}$ & $\begin{array}{l}0.29743^{\dagger} \\
(0.12791) \\
\end{array}$ & $\begin{array}{l}0.24257^{\ddagger} \\
(0.13911) \\
\end{array}$ & $\begin{array}{l}0.29743^{\dagger} \\
(0.12871) \\
\end{array}$ & $\begin{array}{l}0.29743^{\dagger} \\
(0.12827) \\
\end{array}$ \\
\hline Facto & $\begin{array}{l}0.12449 \\
(0.49826) \\
\end{array}$ & $\begin{array}{l}0.26509 \\
(0.49902) \\
\end{array}$ & $\begin{array}{l}0.26127 \\
(0.49860) \\
\end{array}$ & $\begin{array}{l}0.17982 \\
(0.49949) \\
\end{array}$ & $\begin{array}{l}0.02815 \\
(0.48891) \\
\end{array}$ & $\begin{array}{l}0.23861 \\
(0.49935) \\
\end{array}$ & $\begin{array}{l}0.12732 \\
(0.49954) \\
\end{array}$ & $\begin{array}{l}0.21120 \\
(0.49655) \\
\end{array}$ & $\begin{array}{l}0.21120 \\
(0.49662) \\
\end{array}$ & $\begin{array}{l}0.21120 \\
(0.49480) \\
\end{array}$ & $\begin{array}{l}0.15635 \\
(0.49958) \\
\end{array}$ & $\begin{array}{l}0.21120 \\
(0.49789) \\
\end{array}$ & $\begin{array}{l}0.21120 \\
(0.49618) \\
\end{array}$ \\
\hline Factor $2,3 \& 4$ & $\begin{array}{l}0.17916^{\dagger} \\
(0.07289)\end{array}$ & $\begin{array}{l}0.31977^{*} \\
(0.06817)\end{array}$ & $\begin{array}{l}0.31595^{*} \\
(0.06526)\end{array}$ & $\begin{array}{l}0.23450^{*} \\
(0.06538)\end{array}$ & $\begin{array}{l}0.08282 \\
(0.07252)\end{array}$ & $\begin{array}{l}0.27029^{*} \\
(0.04734)\end{array}$ & $\begin{array}{l}0.18274^{\dagger} \\
(0.07681)\end{array}$ & $\begin{array}{l}0.26888^{*} \\
(0.04682)\end{array}$ & $\begin{array}{l}0.26328^{*} \\
(0.04681)\end{array}$ & $\begin{array}{l}0.26885^{*} \\
(0.04661)\end{array}$ & $\begin{array}{l}0.21732^{*} \\
(0.06655)\end{array}$ & $\begin{array}{l}0.26588^{*} \\
(0.04686)\end{array}$ & $\begin{array}{l}0.26588^{*} \\
(0.04670)\end{array}$ \\
\hline Factor $2,3,4 \& 5$ & $\begin{array}{l}0.23962 \\
(0.14611) \\
\end{array}$ & $\begin{array}{l}0.36208^{\dagger} \\
(0.14819) \\
\end{array}$ & $\begin{array}{l}0.34511^{\dagger} \\
(0.14361) \\
\end{array}$ & $\begin{array}{l}0.27929^{\ddagger} \\
(0.14605) \\
\end{array}$ & $\begin{array}{l}0.15876 \\
(0.14410) \\
\end{array}$ & $\begin{array}{l}0.33127^{\dagger} \\
(0.14453) \\
\end{array}$ & $\begin{array}{l}0.23309 \\
(0.14990) \\
\end{array}$ & $\begin{array}{l}0.35280^{\dagger} \\
(0.14554) \\
\end{array}$ & $\begin{array}{l}0.28000^{\ddagger} \\
(0.14216) \\
\end{array}$ & $\begin{array}{l}0.33547^{\dagger} \\
(0.14015) \\
\end{array}$ & $\begin{array}{l}0.26635^{\ddagger} \\
(0.14552) \\
\end{array}$ & $\begin{array}{l}0.34250^{\ddagger} \\
(0.19532) \\
\end{array}$ & $\begin{array}{l}0.33824^{\dagger} \\
(0.14170) \\
\end{array}$ \\
\hline Factor 1 & $\begin{array}{l}0.47330^{*} \\
(0.10169) \\
\end{array}$ & $\begin{array}{l}0.59666^{*} \\
(0.10169) \\
\end{array}$ & $\begin{array}{l}0.54919^{*} \\
(0.09045)\end{array}$ & $\begin{array}{l}0.54300^{*} \\
(0.09068)\end{array}$ & $\begin{array}{l}0.53607^{*} \\
(0.08848) \\
\end{array}$ & $\begin{array}{l}0.56911^{*} \\
(0.09755)\end{array}$ & $\begin{array}{l}0.54610^{*} \\
(0.09026)\end{array}$ & $\begin{array}{l}0.55247^{*} \\
(0.09060)\end{array}$ & $\begin{array}{l}0.54610^{*} \\
(0.09042)\end{array}$ & $\begin{array}{l}0.54610^{*} \\
(0.09009)\end{array}$ & $\begin{array}{l}0.54271^{*} \\
(0.09050)\end{array}$ & $\begin{array}{l}0.54610^{*} \\
(0.09065)\end{array}$ & $\begin{array}{l}0.54610^{*} \\
(0.09034)\end{array}$ \\
\hline $\mathrm{F}$ & $\begin{array}{l}0.17023 \\
(0.17271)\end{array}$ & $\begin{array}{l}0.23461 \\
(0.17556)\end{array}$ & $\begin{array}{l}0.23201 \\
(0.17504)\end{array}$ & $\begin{array}{l}0.17033 \\
(0.17718)\end{array}$ & $\begin{array}{l}0.09539 \\
(0.17143)\end{array}$ & $\begin{array}{l}0.20999 \\
(0.17364)\end{array}$ & $\begin{array}{l}0.16104 \\
(0.17412)\end{array}$ & $\begin{array}{l}0.23284 \\
(0.17525)\end{array}$ & $\begin{array}{l}0.19793 \\
(0.17230)\end{array}$ & $\begin{array}{l}0.19793 \\
(0.17167)\end{array}$ & $\begin{array}{l}0.18701 \\
(0.17266)\end{array}$ & $\begin{array}{l}0.23518 \\
(0.22740)\end{array}$ & $\begin{array}{l}0.27392 \\
(0.18312)\end{array}$ \\
\hline $\mathrm{Fac}$ & $\begin{array}{l}0.22884 \\
(0.13983) \\
\end{array}$ & $\begin{array}{l}0.26705^{\ddagger} \\
(0.14156)\end{array}$ & $\begin{array}{l}0.26013^{\ddagger} \\
(0.14070)\end{array}$ & $\begin{array}{l}0.24115^{\ddagger} \\
(0.14020)\end{array}$ & $\begin{array}{l}0.22910^{\ddagger} \\
(0.13692)\end{array}$ & $\begin{array}{l}0.25087^{\ddagger} \\
(0.14064)\end{array}$ & $\begin{array}{l}0.22266 \\
(0.14053)\end{array}$ & $\begin{array}{l}0.24797^{\ddagger} \\
(0.13997)\end{array}$ & $\begin{array}{l}0.24364^{\ddagger} \\
(0.13993)\end{array}$ & $\begin{array}{l}0.24364^{\ddagger} \\
(0.13942)\end{array}$ & $\begin{array}{l}0.24364^{\ddagger} \\
(0.13995)\end{array}$ & $\begin{array}{l}0.24364^{\ddagger} \\
(0.14029)\end{array}$ & $\begin{array}{l}0.24364^{\ddagger} \\
(0.13981) \\
\end{array}$ \\
\hline Factor 1 & $\begin{array}{l}0.18252^{\dagger} \\
(0.08508) \\
\end{array}$ & $\begin{array}{l}0.28415^{*} \\
(0.07445) \\
\end{array}$ & $\begin{array}{l}0.28360^{*} \\
(0.07418) \\
\end{array}$ & $\begin{array}{l}0.23821^{*} \\
(0.07563) \\
\end{array}$ & $\begin{array}{l}0.19017^{*} \\
(0.07172) \\
\end{array}$ & $\begin{array}{l}0.26497^{*} \\
(0.07138) \\
\end{array}$ & $\begin{array}{l}0.23221^{*} \\
(0.07249) \\
\end{array}$ & $\begin{array}{l}0.29556^{*} \\
(0.07855) \\
\end{array}$ & $\begin{array}{l}0.24269^{*} \\
(0.07158) \\
\end{array}$ & $\begin{array}{l}0.26180^{*} \\
(0.07003) \\
\end{array}$ & $\begin{array}{l}0.22690^{*} \\
(0.07626) \\
\end{array}$ & $\begin{array}{l}0.29247^{\ddagger} \\
(0.15632) \\
\end{array}$ & $\begin{array}{l}0.30257^{*} \\
(0.07941) \\
\end{array}$ \\
\hline Facto & $\begin{array}{l}0.04306 \\
(0.11688) \\
\end{array}$ & $\begin{array}{l}0.06445 \\
(0.11886) \\
\end{array}$ & $\begin{array}{l}0.04306 \\
(0.11721) \\
\end{array}$ & $\begin{array}{l}0.04306 \\
(0.11742) \\
\end{array}$ & $\begin{array}{l}0.04306 \\
(0.11465) \\
\end{array}$ & $\begin{array}{l}0.04306 \\
(0.11744) \\
\end{array}$ & $\begin{array}{l}0.04306 \\
(0.11703) \\
\end{array}$ & $\begin{array}{l}0.04306 \\
(0.11722) \\
\end{array}$ & $\begin{array}{l}0.04306 \\
(0.11724) \\
\end{array}$ & $\begin{array}{l}0.04306 \\
(0.11681) \\
\end{array}$ & $\begin{array}{l}0.02128 \\
(0.11917) \\
\end{array}$ & $\begin{array}{l}0.04306 \\
(0.11754) \\
\end{array}$ & $\begin{array}{l}0.04306 \\
(0.11713) \\
\end{array}$ \\
\hline Factor $1,3 \& 5$ & $\begin{array}{l}0.09609 \\
(0.09428) \\
\end{array}$ & $\begin{array}{l}0.10791 \\
(0.09453) \\
\end{array}$ & $\begin{array}{l}0.10759 \\
(0.09451) \\
\end{array}$ & $\begin{array}{l}0.10074 \\
(0.09467) \\
\end{array}$ & $\begin{array}{l}0.08800 \\
(0.09249) \\
\end{array}$ & $\begin{array}{l}0.10469 \\
(0.09463) \\
\end{array}$ & $\begin{array}{l}0.10338 \\
(0.09428) \\
\end{array}$ & $\begin{array}{l}0.10599 \\
(0.09447) \\
\end{array}$ & $\begin{array}{l}0.10338 \\
(0.09445) \\
\end{array}$ & $\begin{array}{l}0.10338 \\
(0.09410) \\
\end{array}$ & $\begin{array}{l}0.09877 \\
(0.09457) \\
\end{array}$ & $\begin{array}{l}0.10651 \\
(0.09550) \\
\end{array}$ & $\begin{array}{l}0.17937 \\
(0.11313) \\
\end{array}$ \\
\hline Factor $1,3 \& 4$ & $\begin{array}{l}0.11622 \\
(0.12955)\end{array}$ & $\begin{array}{l}0.24816^{\ddagger} \\
(0.12956) \\
\end{array}$ & $\begin{array}{l}0.20827^{\ddagger} \\
(0.12034)\end{array}$ & $\begin{array}{l}0.18419 \\
(0.12077)\end{array}$ & $\begin{array}{l}0.07764 \\
(0.12243)\end{array}$ & $\begin{array}{l}0.20097^{\ddagger} \\
(0.12036)\end{array}$ & $\begin{array}{l}0.18940 \\
(0.11953)\end{array}$ & $\begin{array}{l}0.22716^{\ddagger} \\
(0.12344)\end{array}$ & $\begin{array}{l}0.19427 \\
(0.11969)\end{array}$ & $\begin{array}{l}0.19891^{\ddagger} \\
(0.11929)\end{array}$ & $\begin{array}{l}0.19109 \\
(0.11975)\end{array}$ & $\begin{array}{l}0.19427 \\
(0.12000)\end{array}$ & $\begin{array}{l}0.19427 \\
(0.11959) \\
\end{array}$ \\
\hline Factor & $\begin{array}{l}0.14813^{\ddagger} \\
(0.07961)\end{array}$ & $\begin{array}{l}0.21690^{\dagger} \\
(0.09110) \\
\end{array}$ & $\begin{array}{l}0.21179^{\dagger} \\
(0.08861)\end{array}$ & $\begin{array}{l}0.16211^{\dagger} \\
(0.07936)\end{array}$ & $\begin{array}{l}0.13668^{\ddagger} \\
(0.07771)\end{array}$ & $\begin{array}{l}0.18913^{\dagger} \\
(0.08611)\end{array}$ & $\begin{array}{l}0.15890^{\dagger} \\
(0.07898) \\
\end{array}$ & $\begin{array}{l}0.21446^{\dagger} \\
(0.08999)\end{array}$ & $\begin{array}{l}0.16594^{\dagger} \\
(0.07888)\end{array}$ & $\begin{array}{l}0.16737^{\dagger} \\
(0.07858)\end{array}$ & $\begin{array}{l}0.16371^{\dagger} \\
(0.07897) \\
\end{array}$ & $\begin{array}{l}0.20462 \\
(0.16769) \\
\end{array}$ & $\begin{array}{l}0.23445^{\dagger} \\
(0.09615) \\
\end{array}$ \\
\hline Fac & $\begin{array}{l}0.14057 \\
(0.17347) \\
\end{array}$ & $\begin{array}{l}0.20509 \\
(0.18079) \\
\end{array}$ & $\begin{array}{l}0.16961 \\
(0.17464) \\
\end{array}$ & $\begin{array}{l}0.11982 \\
(0.18002) \\
\end{array}$ & $\begin{array}{l}-0.03186 \\
(0.17911) \\
\end{array}$ & $\begin{array}{l}0.17861 \\
(0.17943) \\
\end{array}$ & $\begin{array}{l}0.13063 \\
(0.17422) \\
\end{array}$ & $\begin{array}{l}0.15789 \\
(0.17395) \\
\end{array}$ & $\begin{array}{l}0.14428 \\
(0.17399) \\
\end{array}$ & $\begin{array}{l}0.15120 \\
(0.17323) \\
\end{array}$ & $\begin{array}{l}0.13775 \\
(0.17439) \\
\end{array}$ & $\begin{array}{l}0.15120 \\
(0.17431) \\
\end{array}$ & $\begin{array}{l}0.15120 \\
(0.17372) \\
\end{array}$ \\
\hline Fact & $\begin{array}{l}0.21521 \\
(0.16815) \\
\end{array}$ & $\begin{array}{l}0.29872^{\ddagger} \\
(0.17474) \\
\end{array}$ & $\begin{array}{l}0.27663 \\
(0.17002) \\
\end{array}$ & $\begin{array}{l}0.21345 \\
(0.17393) \\
\end{array}$ & $\begin{array}{l}0.06177 \\
(0.17328) \\
\end{array}$ & $\begin{array}{l}0.26911 \\
(0.17216) \\
\end{array}$ & $\begin{array}{l}0.16095 \\
(0.17824) \\
\end{array}$ & $\begin{array}{l}0.26349 \\
(0.16843) \\
\end{array}$ & $\begin{array}{l}0.21142 \\
(0.17051)\end{array}$ & $\begin{array}{l}0.25391 \\
(0.16705) \\
\end{array}$ & $\begin{array}{l}0.23858 \\
(0.16771)\end{array}$ & $\begin{array}{l}0.28207 \\
(0.22381)\end{array}$ & $\begin{array}{l}0.29310^{\ddagger} \\
(0.17206) \\
\end{array}$ \\
\hline Fac & $\begin{array}{l}0.13669 \\
(0.19068) \\
\end{array}$ & $\begin{array}{l}0.26526 \\
(0.19154) \\
\end{array}$ & $\begin{array}{l}0.25449 \\
(0.18911) \\
\end{array}$ & $\begin{array}{l}0.17999 \\
(0.19086) \\
\end{array}$ & $\begin{array}{l}0.07914 \\
(0.18546) \\
\end{array}$ & $\begin{array}{l}0.23497 \\
(0.18904) \\
\end{array}$ & $\begin{array}{l}0.12749 \\
(0.19469) \\
\end{array}$ & $\begin{array}{l}0.25842 \\
(0.19001) \\
\end{array}$ & $\begin{array}{l}0.17063 \\
(0.18898) \\
\end{array}$ & $\begin{array}{l}0.23352 \\
(0.18485) \\
\end{array}$ & $\begin{array}{l}0.19614 \\
(0.18566) \\
\end{array}$ & $\begin{array}{l}0.21137 \\
(0.18550) \\
\end{array}$ & $\begin{array}{l}0.21137 \\
(0.18487) \\
\end{array}$ \\
\hline Factor & $\begin{array}{l}0.00619 \\
(0.09154) \\
\end{array}$ & $\begin{array}{l}0.08565 \\
(0.09110) \\
\end{array}$ & $\begin{array}{l}0.07032 \\
(0.08760) \\
\end{array}$ & $\begin{array}{l}0.02533 \\
(0.09617) \\
\end{array}$ & $\begin{array}{l}-0.12332 \\
(0.10059) \\
\end{array}$ & $\begin{array}{l}0.07695 \\
(0.09366) \\
\end{array}$ & $\begin{array}{l}0.00522 \\
(0.09342) \\
\end{array}$ & $\begin{array}{l}0.09407 \\
(0.09385) \\
\end{array}$ & $\begin{array}{l}0.03822 \\
(0.08769) \\
\end{array}$ & $\begin{array}{l}0.06221 \\
(0.08621) \\
\end{array}$ & $\begin{array}{l}0.04084 \\
(0.08738) \\
\end{array}$ & $\begin{array}{l}0.08919 \\
(0.16371) \\
\end{array}$ & $\begin{array}{l}0.12254 \\
(0.10296) \\
\end{array}$ \\
\hline Factor $1,2 \& 3$ & $\begin{array}{l}0.31473^{\ddagger} \\
(0.18808) \\
\end{array}$ & $\begin{array}{l}0.32502^{\ddagger} \\
(0.18887) \\
\end{array}$ & $\begin{array}{l}0.32429^{\ddagger} \\
(0.18882) \\
\end{array}$ & $\begin{array}{l}0.28336 \\
(0.19439) \\
\end{array}$ & $\begin{array}{l}0.13168 \\
(0.19290) \\
\end{array}$ & $\begin{array}{l}0.34214^{\ddagger} \\
(0.19385) \\
\end{array}$ & $\begin{array}{l}0.29872 \\
(0.18869) \\
\end{array}$ & $\begin{array}{l}0.32517^{\ddagger} \\
(0.18887) \\
\end{array}$ & $\begin{array}{l}0.31473^{\ddagger} \\
(0.18866) \\
\end{array}$ & $\begin{array}{l}0.31473^{\ddagger} \\
(0.18797) \\
\end{array}$ & $\begin{array}{l}0.30426 \\
(0.18897) \\
\end{array}$ & $\begin{array}{l}0.31473^{\ddagger} \\
(0.18914) \\
\end{array}$ & $\begin{array}{l}0.31473^{\ddagger} \\
(0.18849) \\
\end{array}$ \\
\hline Factor $1,2,3 \& 5$ & $\begin{array}{l}0.20589 \\
(0.19042)\end{array}$ & $\begin{array}{l}0.28524 \\
(0.19663) \\
\end{array}$ & $\begin{array}{l}0.28142 \\
(0.19563)\end{array}$ & $\begin{array}{l}0.21292 \\
(0.19246) \\
\end{array}$ & $\begin{array}{l}0.04830 \\
(0.19442) \\
\end{array}$ & $\begin{array}{l}0.25876 \\
(0.19544)\end{array}$ & $\begin{array}{l}0.18209 \\
(0.19336) \\
\end{array}$ & $\begin{array}{l}0.28599 \\
(0.19680) \\
\end{array}$ & $\begin{array}{l}0.23135 \\
(0.19029) \\
\end{array}$ & $\begin{array}{l}0.25478 \\
(0.19014) \\
\end{array}$ & $\begin{array}{l}0.19260 \\
(0.19404) \\
\end{array}$ & $\begin{array}{l}0.26860 \\
(0.24138) \\
\end{array}$ & $\begin{array}{l}0.27387 \\
(0.19330)\end{array}$ \\
\hline Factor $1,2,3 \& 4$ & $\begin{array}{l}0.21885^{*} \\
(0.06347) \\
\end{array}$ & $\begin{array}{l}0.33371^{*} \\
(0.04888) \\
\end{array}$ & $\begin{array}{l}0.33088^{*} \\
(0.04699) \\
\end{array}$ & $\begin{array}{l}0.27883^{*} \\
(0.04708) \\
\end{array}$ & $\begin{array}{l}0.11716^{\ddagger} \\
(0.06652) \\
\end{array}$ & $\begin{array}{l}0.31640^{*} \\
(0.04611) \\
\end{array}$ & $\begin{array}{l}0.22278^{*} \\
(0.06690) \\
\end{array}$ & $\begin{array}{l}0.34871^{*} \\
(0.05885) \\
\end{array}$ & $\begin{array}{l}0.24915^{*} \\
(0.05970) \\
\end{array}$ & $\begin{array}{l}0.33625^{*} \\
(0.04334) \\
\end{array}$ & $\begin{array}{l}0.24525^{*} \\
(0.06402) \\
\end{array}$ & $\begin{array}{l}0.29909^{*} \\
(0.03700) \\
\end{array}$ & $\begin{array}{l}0.29888^{*} \\
(0.03686) \\
\end{array}$ \\
\hline All Factors & $\begin{array}{l}0.22385^{*} \\
(0.06909) \\
\end{array}$ & $\begin{array}{l}0.35768^{*} \\
(0.06617) \\
\end{array}$ & $\begin{array}{l}0.34862^{*} \\
(0.06001) \\
\end{array}$ & $\begin{array}{l}0.27428^{*} \\
(0.06357) \\
\end{array}$ & $\begin{array}{l}0.12600^{\ddagger} \\
(0.07054) \\
\end{array}$ & $\begin{array}{l}0.33074^{*} \\
(0.06060) \\
\end{array}$ & $\begin{array}{l}0.22370^{*} \\
(0.07476) \\
\end{array}$ & $\begin{array}{l}0.32812^{*} \\
(0.04981) \\
\end{array}$ & $\begin{array}{l}0.26226^{*} \\
(0.06052) \\
\end{array}$ & $\begin{array}{l}0.34396^{*} \\
(0.05087) \\
\end{array}$ & $\begin{array}{l}0.26287^{*} \\
(0.06104) \\
\end{array}$ & $\begin{array}{l}0.34178^{\dagger} \\
(0.15262) \\
\end{array}$ & $\begin{array}{l}0.34063^{*} \\
(0.05368) \\
\end{array}$ \\
\hline $\begin{array}{l}\text { Individual } \\
\text { Variable }\end{array}$ & $\begin{array}{l}0.08671 \\
(0.05605)\end{array}$ & $\begin{array}{l}-0.05389 \\
(0.04962)\end{array}$ & $\begin{array}{l}-0.05007 \\
(0.04556)\end{array}$ & $\begin{array}{l}0.03138 \\
(0.04564)\end{array}$ & $\begin{array}{l}0.18305^{*} \\
(0.05630)\end{array}$ & $\begin{array}{l}-0.02741 \\
(0.04314)\end{array}$ & $\begin{array}{l}0.08388 \\
(0.06155)\end{array}$ & $\begin{array}{l}-0.05464 \\
(0.05029)\end{array}$ & $\begin{array}{l}0.05639 \\
(0.05322)\end{array}$ & $\begin{array}{l}-0.07979 \\
(0.04904)\end{array}$ & $\begin{array}{l}0.05486 \\
(0.05352)\end{array}$ & $\begin{array}{l}-0.03725 \\
(0.14788)\end{array}$ & $\begin{array}{l}-0.07599 \\
(0.06240)\end{array}$ \\
\hline
\end{tabular}

* Significant at the 0.01 level $\quad \dagger$ Significant at the 0.05 level $\$$ Significant at the 0.10 level

Table 100 Coefficients with individual variables for high-level factors - Vehicles Industry 


\begin{tabular}{|c|c|c|c|c|c|c|c|c|c|c|c|c|c|}
\hline $\begin{array}{l}\text { Individual variable } \\
\text { included in the } \\
\text { model in the } \\
\text { column }\end{array}$ & 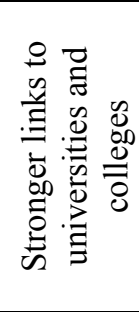 & 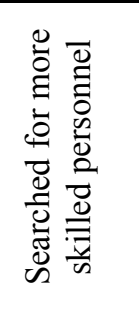 & $\begin{array}{l}20 \\
2 \\
\simeq \\
0 \\
0 \\
0 \\
\Xi\end{array}$ & 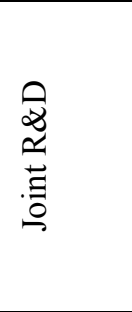 & 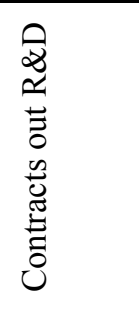 & 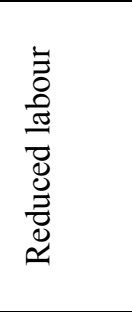 & 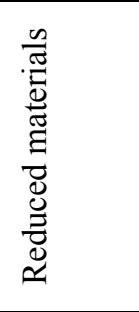 & 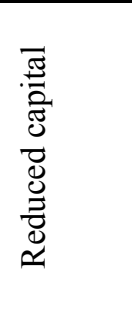 & 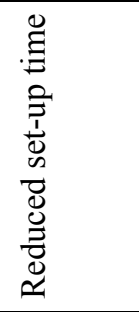 & 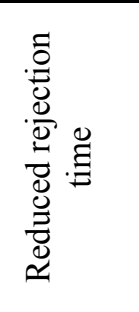 & 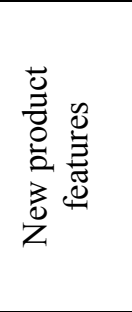 & 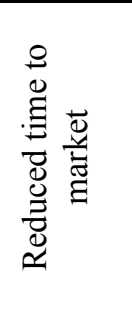 & 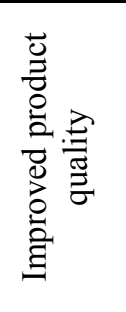 \\
\hline No factors & $\begin{array}{l}0.16754^{*} \\
(0.03719)\end{array}$ & $\begin{array}{l}0.16764^{*} \\
(0.03721)\end{array}$ & $\begin{array}{l}0.13215^{*} \\
(0.03884)\end{array}$ & $\begin{array}{l}0.16754^{*} \\
(0.03716)\end{array}$ & $\begin{array}{l}0.17728^{*} \\
(0.03677)\end{array}$ & $\begin{array}{l}0.16622^{*} \\
(0.03715) \\
\end{array}$ & $\begin{array}{l}0.16888^{*} \\
(0.03696)\end{array}$ & $\begin{array}{l}0.17029^{*} \\
(0.03715) \\
\end{array}$ & $\begin{array}{l}0.16754^{*} \\
(0.03706)\end{array}$ & $\begin{array}{l}0.16564^{*} \\
(0.03619)\end{array}$ & $\begin{array}{l}0.16941^{*} \\
(0.03717) \\
\end{array}$ & $\begin{array}{l}0.17207^{*} \\
(0.03683)\end{array}$ & $\begin{array}{l}0.16634^{*} \\
(0.03672)\end{array}$ \\
\hline Factor 5 & $\begin{array}{l}-0.01876 \\
(0.23334)\end{array}$ & $\begin{array}{l}-0.02011 \\
(0.24872) \\
\end{array}$ & $\begin{array}{l}-0.05638 \\
(0.22705)\end{array}$ & $\begin{array}{l}-0.04933 \\
(0.23221) \\
\end{array}$ & $\begin{array}{l}-0.03115 \\
(0.22704)\end{array}$ & $\begin{array}{l}-0.06397 \\
(0.23294) \\
\end{array}$ & $\begin{array}{l}0.00320 \\
(0.23025) \\
\end{array}$ & $\begin{array}{l}-0.03115 \\
(0.23006) \\
\end{array}$ & $\begin{array}{l}-0.06427 \\
(0.23145)\end{array}$ & $\begin{array}{l}-0.06778 \\
(0.22481)\end{array}$ & $\begin{array}{l}-0.00434 \\
(0.23199) \\
\end{array}$ & $\begin{array}{l}-0.00967 \\
(0.22839) \\
\end{array}$ & $\begin{array}{l}-0.05429 \\
(0.22805) \\
\end{array}$ \\
\hline Factor 4 & $\begin{array}{l}-0.13770 \\
(0.27309) \\
\end{array}$ & $\begin{array}{l}-0.13770 \\
(0.27317) \\
\end{array}$ & $\begin{array}{l}-0.21978 \\
(0.27019)\end{array}$ & $\begin{array}{l}-0.14729 \\
(0.27327)\end{array}$ & $\begin{array}{l}-0.05608 \\
(0.27046)\end{array}$ & $\begin{array}{l}-0.17328 \\
(0.27518)\end{array}$ & $\begin{array}{l}-0.10588 \\
(0.27206)\end{array}$ & $\begin{array}{l}-0.11942 \\
(0.27269)\end{array}$ & $\begin{array}{l}-0.16089 \\
(0.27275)\end{array}$ & $\begin{array}{l}-0.13770 \\
(0.26574)\end{array}$ & $\begin{array}{l}-0.11898 \\
(0.27325)\end{array}$ & $\begin{array}{l}-0.10761 \\
(0.27038)\end{array}$ & $\begin{array}{l}-0.17011 \\
(0.26998)\end{array}$ \\
\hline Factor $4 \& 5$ & $\begin{array}{l}-0.01053 \\
(0.22448)\end{array}$ & $\begin{array}{l}-0.00822 \\
(0.24167)\end{array}$ & $\begin{array}{l}-0.04285 \\
(0.21955)\end{array}$ & $\begin{array}{l}-0.02567 \\
(0.22319)\end{array}$ & $\begin{array}{l}0.01178 \\
(0.21986)\end{array}$ & $\begin{array}{l}-0.01926 \\
(0.22274) \\
\end{array}$ & $\begin{array}{l}-0.01926 \\
(0.22175)\end{array}$ & $\begin{array}{l}-0.01926 \\
(0.22247)\end{array}$ & $\begin{array}{l}-0.03474 \\
(0.22271)\end{array}$ & $\begin{array}{l}-0.05351 \\
(0.21738)\end{array}$ & $\begin{array}{l}-0.01926 \\
(0.22272) \\
\end{array}$ & $\begin{array}{l}0.02740 \\
(0.22168) \\
\end{array}$ & $\begin{array}{l}-0.06953 \\
(0.22137) \\
\end{array}$ \\
\hline Factor 3 & $\begin{array}{l}-0.40647^{*} \\
(0.07682)\end{array}$ & $\begin{array}{l}-0.40647^{*} \\
(0.07684)\end{array}$ & $\begin{array}{l}-0.48209^{*} \\
(0.08055)\end{array}$ & $\begin{array}{l}-0.40647^{*} \\
(0.07676)\end{array}$ & $\begin{array}{l}-0.40169^{*} \\
(0.07560)\end{array}$ & $\begin{array}{l}-0.40856^{*} \\
(0.07671)\end{array}$ & $\begin{array}{l}-0.40320^{*} \\
(0.07636)\end{array}$ & $\begin{array}{l}-0.40399^{*} \\
(0.07661)\end{array}$ & $\begin{array}{l}-0.40647^{*} \\
(0.07655)\end{array}$ & $\begin{array}{l}-0.40647^{*} \\
(0.07475)\end{array}$ & $\begin{array}{l}-0.40647^{*} \\
(0.07667)\end{array}$ & $\begin{array}{l}-0.31060^{*} \\
(0.08760)\end{array}$ & $\begin{array}{l}-0.51418^{*} \\
(0.08875)\end{array}$ \\
\hline Factor $3 \& 5$ & $\begin{array}{l}0.17004 \\
(0.17084)\end{array}$ & $\begin{array}{l}0.16166 \\
(0.18666)\end{array}$ & $\begin{array}{l}0.04564 \\
(0.16395)\end{array}$ & $\begin{array}{l}0.12211 \\
(0.16749) \\
\end{array}$ & $\begin{array}{l}0.18538 \\
(0.15994)\end{array}$ & $\begin{array}{l}0.12026 \\
(0.16488)\end{array}$ & $\begin{array}{l}0.18288 \\
(0.16217)\end{array}$ & $\begin{array}{l}0.15062 \\
(0.16152) \\
\end{array}$ & $\begin{array}{l}0.08171 \\
(0.17046)\end{array}$ & $\begin{array}{l}0.13256 \\
(0.15774)\end{array}$ & $\begin{array}{l}0.16462 \\
(0.16235) \\
\end{array}$ & $\begin{array}{l}0.18038 \\
(0.16077) \\
\end{array}$ & $\begin{array}{l}0.13921 \\
(0.16003)\end{array}$ \\
\hline Factor $3 \& 4$ & \multicolumn{13}{|c|}{ Insufficient Data } \\
\hline Factor $3,4 \& 5$ & $\begin{array}{l}0.00434 \\
(0.29498)\end{array}$ & $\begin{array}{l}0.00779 \\
(0.30854)\end{array}$ & $\begin{array}{l}-0.04427 \\
(0.28964)\end{array}$ & $\begin{array}{l}-0.03513 \\
(0.29789)\end{array}$ & $\begin{array}{l}-0.00325 \\
(0.28947)\end{array}$ & $\begin{array}{l}-0.02103 \\
(0.29428) \\
\end{array}$ & $\begin{array}{l}0.02467 \\
(0.29286)\end{array}$ & $\begin{array}{l}0.03626 \\
(0.29522) \\
\end{array}$ & $\begin{array}{l}-0.00325 \\
(0.29319)\end{array}$ & $\begin{array}{l}-0.00325 \\
(0.28630)\end{array}$ & $\begin{array}{l}0.04377 \\
(0.29766) \\
\end{array}$ & $\begin{array}{l}0.06179 \\
(0.29243) \\
\end{array}$ & $\begin{array}{l}-0.07333 \\
(0.29203) \\
\end{array}$ \\
\hline Factor 2 & $\begin{array}{l}0.24973 \\
(0.24277) \\
\end{array}$ & $\begin{array}{l}0.24973 \\
(0.24284) \\
\end{array}$ & $\begin{array}{l}0.24973 \\
(0.23866) \\
\end{array}$ & $\begin{array}{l}0.24973 \\
(0.24259) \\
\end{array}$ & $\begin{array}{l}0.24973 \\
(0.23885) \\
\end{array}$ & $\begin{array}{l}0.23762 \\
(0.24266) \\
\end{array}$ & $\begin{array}{l}0.26874 \\
(0.24152) \\
\end{array}$ & $\begin{array}{l}0.26417 \\
(0.24233) \\
\end{array}$ & $\begin{array}{l}0.24973 \\
(0.24192) \\
\end{array}$ & $\begin{array}{l}0.20919 \\
(0.23653) \\
\end{array}$ & $\begin{array}{l}0.24973 \\
(0.24230) \\
\end{array}$ & $\begin{array}{l}0.24973 \\
(0.24005) \\
\end{array}$ & $\begin{array}{l}0.24973 \\
(0.23969) \\
\end{array}$ \\
\hline Facto & $\begin{array}{l}0.18566 \\
(0.35331) \\
\end{array}$ & $\begin{array}{l}0.18584 \\
(0.36408) \\
\end{array}$ & $\begin{array}{l}0.11609 \\
(0.34673) \\
\end{array}$ & $\begin{array}{l}0.17480 \\
(0.35174) \\
\end{array}$ & $\begin{array}{l}0.17480 \\
(0.34632) \\
\end{array}$ & $\begin{array}{l}0.12389 \\
(0.35546) \\
\end{array}$ & $\begin{array}{l}0.25472 \\
(0.35311)\end{array}$ & $\begin{array}{l}0.20516 \\
(0.35186)\end{array}$ & $\begin{array}{l}0.13626 \\
(0.35210)\end{array}$ & $\begin{array}{l}0.08956 \\
(0.34343)\end{array}$ & $\begin{array}{l}0.17480 \\
(0.35132)\end{array}$ & $\begin{array}{l}0.17480 \\
(0.34806)\end{array}$ & $\begin{array}{l}0.17480 \\
(0.34754)\end{array}$ \\
\hline Factor & $\begin{array}{l}0.24962^{\ddagger} \\
(0.13895)\end{array}$ & $\begin{array}{l}0.25220^{\ddagger} \\
(0.14067)\end{array}$ & $\begin{array}{l}0.17981 \\
(0.13903)\end{array}$ & $\begin{array}{l}0.23917^{\ddagger} \\
(0.13974)\end{array}$ & $\begin{array}{l}0.24962^{\ddagger} \\
(0.13671)\end{array}$ & $\begin{array}{l}0.23375^{\ddagger} \\
(0.13971)\end{array}$ & $\begin{array}{l}0.24962 \\
(0.13808)\end{array}$ & $\begin{array}{l}0.24962^{\ddagger} \\
(0.13853)\end{array}$ & $\begin{array}{l}0.23761^{\ddagger} \\
(0.13879)\end{array}$ & $\begin{array}{l}0.23633^{\ddagger} \\
(0.13527)\end{array}$ & $\begin{array}{l}0.25328^{\ddagger} \\
(0.13874)\end{array}$ & $\begin{array}{l}0.27458^{\dagger} \\
(0.13787)\end{array}$ & $\begin{array}{l}0.19398 \\
(0.13924)\end{array}$ \\
\hline Factor $2,4 \& 5$ & $\begin{array}{l}0.19935^{\ddagger} \\
(0.11110)\end{array}$ & $\begin{array}{l}0.20425 \\
(0.14365)\end{array}$ & $\begin{array}{l}0.07580 \\
(0.11635)\end{array}$ & $\begin{array}{l}0.18420^{\ddagger} \\
(0.11052)\end{array}$ & $\begin{array}{l}0.20457^{\ddagger} \\
(0.10808)\end{array}$ & $\begin{array}{l}0.17883 \\
(0.11062)\end{array}$ & $\begin{array}{l}0.19710^{\ddagger} \\
(0.10911)\end{array}$ & $\begin{array}{l}0.19321^{\ddagger} \\
(0.10943)\end{array}$ & $\begin{array}{l}0.17893 \\
(0.10997)\end{array}$ & $\begin{array}{l}0.06262 \\
(0.11344)\end{array}$ & $\begin{array}{l}0.20421^{\ddagger} \\
(0.11015)\end{array}$ & $\begin{array}{l}0.20293^{\ddagger} \\
(0.10863)\end{array}$ & $\begin{array}{l}0.12118 \\
(0.11267)\end{array}$ \\
\hline Factor $2 \& 3$ & $\begin{array}{l}0.29743^{\dagger} \\
(0.12869)\end{array}$ & $\begin{array}{l}0.29743^{\dagger} \\
(0.12872)\end{array}$ & $\begin{array}{l}0.28174^{\dagger} \\
(0.12665)\end{array}$ & $\begin{array}{l}0.29743^{\dagger} \\
(0.12859)\end{array}$ & $\begin{array}{l}0.29743^{\dagger} \\
(0.12661)\end{array}$ & $\begin{array}{l}0.24653^{\ddagger} \\
(0.13929)\end{array}$ & $\begin{array}{l}0.29743^{\dagger} \\
(0.12788)\end{array}$ & $\begin{array}{l}0.29743^{\dagger} \\
(0.12829)\end{array}$ & $\begin{array}{l}0.28713^{\dagger} \\
(0.12850)\end{array}$ & $\begin{array}{l}0.12696 \\
(0.13478)\end{array}$ & $\begin{array}{l}0.34445^{\dagger} \\
(0.13737)\end{array}$ & $\begin{array}{l}0.31079^{\dagger} \\
(0.12739)\end{array}$ & $\begin{array}{l}0.20412 \\
(0.13318)\end{array}$ \\
\hline Factor $2,3 \& 5$ & $\begin{array}{l}0.23293 \\
(0.50149) \\
\end{array}$ & $\begin{array}{l}0.22224 \\
(0.50649) \\
\end{array}$ & $\begin{array}{l}0.21120 \\
(0.48939) \\
\end{array}$ & $\begin{array}{l}0.17932 \\
(0.49975) \\
\end{array}$ & $\begin{array}{l}0.21120 \\
(0.48977) \\
\end{array}$ & $\begin{array}{l}0.16030 \\
(0.49980) \\
\end{array}$ & $\begin{array}{l}0.29113 \\
(0.49704) \\
\end{array}$ & $\begin{array}{l}0.27192 \\
(0.49893) \\
\end{array}$ & $\begin{array}{l}0.13414 \\
(0.49982) \\
\end{array}$ & $\begin{array}{l}0.21120 \\
(0.48441) \\
\end{array}$ & $\begin{array}{l}0.21120 \\
(0.49684) \\
\end{array}$ & $\begin{array}{l}0.31117 \\
(0.49433) \\
\end{array}$ & $\begin{array}{l}0.21120 \\
(0.49149) \\
\end{array}$ \\
\hline Factor 2, $3 \& 4$ & $\begin{array}{l}0.26588^{*} \\
(0.04686)\end{array}$ & $\begin{array}{l}0.26597^{*} \\
(0.04688)\end{array}$ & $\begin{array}{l}0.14846^{\dagger} \\
(0.06336)\end{array}$ & $\begin{array}{l}0.26497^{*} \\
(0.04684)\end{array}$ & $\begin{array}{l}0.27126^{*} \\
(0.04614)\end{array}$ & $\begin{array}{l}0.22172^{*} \\
(0.06612)\end{array}$ & $\begin{array}{l}0.26588^{*} \\
(0.04656)\end{array}$ & $\begin{array}{l}0.31682^{*} \\
(0.06354)\end{array}$ & $\begin{array}{l}0.26369^{*} \\
(0.04672)\end{array}$ & $\begin{array}{l}0.26588^{*} \\
(0.04559)\end{array}$ & $\begin{array}{l}0.27169^{*} \\
(0.04715)\end{array}$ & $\begin{array}{l}0.36407^{*} \\
(0.06438)\end{array}$ & $\begin{array}{l}0.25045^{*} \\
(0.04673)\end{array}$ \\
\hline Fact & $\begin{array}{l}0.32648^{\dagger} \\
(0.14900)\end{array}$ & $\begin{array}{l}0.31923^{\ddagger} \\
(0.16789)\end{array}$ & $\begin{array}{l}0.21234 \\
(0.14213)\end{array}$ & $\begin{array}{l}0.29054^{\dagger} \\
(0.14238)\end{array}$ & $\begin{array}{l}0.35734^{\dagger} \\
(0.13898)\end{array}$ & $\begin{array}{l}0.28002^{\ddagger} \\
(0.14288)\end{array}$ & $\begin{array}{l}0.32715^{\dagger} \\
(0.13958)\end{array}$ & $\begin{array}{l}0.31780^{\dagger} \\
(0.13980)\end{array}$ & $\begin{array}{l}0.28991^{\dagger} \\
(0.14025)\end{array}$ & $\begin{array}{l}0.30819^{\dagger} \\
(0.13622)\end{array}$ & $\begin{array}{l}0.32055^{\dagger} \\
(0.14030)\end{array}$ & $\begin{array}{l}0.37398^{*} \\
(0.14162)\end{array}$ & $\begin{array}{l}0.27412^{\dagger} \\
(0.13898)\end{array}$ \\
\hline Factor 1 & $\begin{array}{l}0.54610^{*} \\
(0.09064)\end{array}$ & $\begin{array}{l}0.54610^{*} \\
(0.09067)\end{array}$ & $\begin{array}{l}0.43383^{*} \\
(0.09834)\end{array}$ & $\begin{array}{l}0.54610^{*} \\
(0.09057)\end{array}$ & $\begin{array}{l}0.54610^{*} \\
(0.08918)\end{array}$ & $\begin{array}{l}0.49520^{*} \\
(0.10530)\end{array}$ & $\begin{array}{l}0.62602^{*} \\
(0.10220)\end{array}$ & $\begin{array}{l}0.60682^{*} \\
(0.10393)\end{array}$ & $\begin{array}{l}0.47241^{*} \\
(0.10761)\end{array}$ & $\begin{array}{l}0.40298^{*} \\
(0.09763)\end{array}$ & $\begin{array}{l}0.59312^{*} \\
(0.10275)\end{array}$ & $\begin{array}{l}0.64606^{*} \\
(0.10052)\end{array}$ & $\begin{array}{l}0.44505^{*} \\
(0.09939)\end{array}$ \\
\hline Facto & $\begin{array}{l}0.21181 \\
(0.17701) \\
\end{array}$ & $\begin{array}{l}0.20897 \\
(0.19603) \\
\end{array}$ & $\begin{array}{l}0.14629 \\
(0.17087) \\
\end{array}$ & $\begin{array}{l}0.18391 \\
(0.17387) \\
\end{array}$ & $\begin{array}{l}0.19793 \\
(0.16993) \\
\end{array}$ & $\begin{array}{l}0.15716 \\
(0.17772) \\
\end{array}$ & $\begin{array}{l}0.26823 \\
(0.17681) \\
\end{array}$ & $\begin{array}{l}0.22464 \\
(0.17366) \\
\end{array}$ & $\begin{array}{l}0.12087 \\
(0.18266) \\
\end{array}$ & $\begin{array}{l}0.06851 \\
(0.17228) \\
\end{array}$ & $\begin{array}{l}0.20925 \\
(0.17278) \\
\end{array}$ & $\begin{array}{l}0.28586 \\
(0.17541) \\
\end{array}$ & $\begin{array}{l}0.12463 \\
(0.17339) \\
\end{array}$ \\
\hline Factor $1 \& 4$ & $\begin{array}{l}0.24364^{\ddagger} \\
(0.14027)\end{array}$ & $\begin{array}{l}0.24451^{\ddagger} \\
(0.14050)\end{array}$ & $\begin{array}{l}0.12622 \\
(0.14459)\end{array}$ & $\begin{array}{l}0.23857^{\ddagger} \\
(0.14037)\end{array}$ & $\begin{array}{l}0.26218^{\ddagger} \\
(0.13818)\end{array}$ & $\begin{array}{l}0.22152 \\
(0.14195)\end{array}$ & $\begin{array}{l}0.27836^{\dagger} \\
(0.14096)\end{array}$ & $\begin{array}{l}0.29954^{\dagger} \\
(0.14761)\end{array}$ & $\begin{array}{l}0.23752^{\ddagger} \\
(0.13986)\end{array}$ & $\begin{array}{l}0.20101 \\
(0.13706)\end{array}$ & $\begin{array}{l}0.28693^{\ddagger} \\
(0.14700)\end{array}$ & $\begin{array}{l}0.32773^{\dagger} \\
(0.14388)\end{array}$ & $\begin{array}{l}0.20539 \\
(0.13945)\end{array}$ \\
\hline Factor $1,4 \& 5$ & $\begin{array}{l}0.27116^{*} \\
(0.08032) \\
\end{array}$ & $\begin{array}{l}0.26835^{\dagger} \\
(0.11636) \\
\end{array}$ & $\begin{array}{l}0.21291^{*} \\
(0.07114)\end{array}$ & $\begin{array}{l}0.24777^{*} \\
(0.07180) \\
\end{array}$ & $\begin{array}{l}0.25731^{*} \\
(0.06927) \\
\end{array}$ & $\begin{array}{l}0.21029^{\dagger} \\
(0.08611) \\
\end{array}$ & $\begin{array}{l}0.30915^{*} \\
(0.07665) \\
\end{array}$ & $\begin{array}{l}0.27260^{*} \\
(0.07137) \\
\end{array}$ & $\begin{array}{l}0.19989^{\dagger} \\
(0.08367) \\
\end{array}$ & $\begin{array}{l}0.11113 \\
(0.08075) \\
\end{array}$ & $\begin{array}{l}0.27665^{*} \\
(0.07307) \\
\end{array}$ & $\begin{array}{l}0.32633^{*} \\
(0.07638) \\
\end{array}$ & $\begin{array}{l}0.17924^{\dagger} \\
(0.07712) \\
\end{array}$ \\
\hline Facto & $\begin{array}{l}4306 \\
11752) \\
\end{array}$ & $\begin{array}{l}0.04744 \\
(0.12317) \\
\end{array}$ & $\begin{array}{l}-0.02195 \\
(0.11801) \\
\end{array}$ & $\begin{array}{l}0.04306 \\
(0.11743) \\
\end{array}$ & $\begin{array}{l}0.06135 \\
(0.11583) \\
\end{array}$ & $\begin{array}{l}0.02034 \\
(0.11974) \\
\end{array}$ & $\begin{array}{l}0.07874 \\
(0.11875) \\
\end{array}$ & $\begin{array}{l}0.07668 \\
(0.12056) \\
\end{array}$ & $\begin{array}{l}-0.03401 \\
(0.13212) \\
\end{array}$ & $\begin{array}{l}-0.12741 \\
(0.12475) \\
\end{array}$ & $\begin{array}{l}0.06910 \\
(0.12035) \\
\end{array}$ & $\begin{array}{l}0.14302 \\
(0.12480) \\
\end{array}$ & $\begin{array}{l}-0.06465 \\
(0.12484) \\
\end{array}$ \\
\hline Factor $1,3 \& 5$ & $\begin{array}{l}0.12510 \\
(0.11242)\end{array}$ & $\begin{array}{l}0.11442 \\
(0.13247)\end{array}$ & $\begin{array}{l}-0.01404 \\
(0.10274)\end{array}$ & $\begin{array}{l}0.10070 \\
(0.09469) \\
\end{array}$ & $\begin{array}{l}0.10897 \\
(0.09317)\end{array}$ & $\begin{array}{l}0.05432 \\
(0.10783) \\
\end{array}$ & $\begin{array}{l}0.18330^{\ddagger} \\
(0.10575)\end{array}$ & $\begin{array}{l}0.16410 \\
(0.10745) \\
\end{array}$ & $\begin{array}{l}0.02631 \\
(0.11244)\end{array}$ & $\begin{array}{l}-0.06092 \\
(0.10390)\end{array}$ & $\begin{array}{l}0.15040 \\
(0.10631) \\
\end{array}$ & $\begin{array}{l}0.20334^{\ddagger} \\
(0.10409) \\
\end{array}$ & $\begin{array}{l}0.00472 \\
(0.10256) \\
\end{array}$ \\
\hline Fact & $\begin{array}{l}0.19554 \\
(0.12003)\end{array}$ & $\begin{array}{l}0.19512 \\
(0.12022)\end{array}$ & $\begin{array}{l}0.07686 \\
(0.12572)\end{array}$ & $\begin{array}{l}0.19242 \\
(0.11992)\end{array}$ & $\begin{array}{l}0.20106^{\ddagger} \\
(0.11807)\end{array}$ & $\begin{array}{l}0.14845 \\
(0.12920)\end{array}$ & $\begin{array}{l}0.23947^{\ddagger} \\
(0.12231)\end{array}$ & $\begin{array}{l}0.25147^{\ddagger} \\
(0.12902)\end{array}$ & $\begin{array}{l}0.13386 \\
(0.12882)\end{array}$ & $\begin{array}{l}0.08246 \\
(0.12124)\end{array}$ & $\begin{array}{l}0.23857^{\ddagger} \\
(0.12824)\end{array}$ & $\begin{array}{l}0.28843^{\dagger} \\
(0.12614)\end{array}$ & $\begin{array}{l}0.09282 \\
(0.12616)\end{array}$ \\
\hline Facto & $\begin{array}{l}0.16973^{\dagger} \\
(0.07934)\end{array}$ & $\begin{array}{l}0.17724 \\
(0.11450)\end{array}$ & $\begin{array}{l}0.05387 \\
(0.08837)\end{array}$ & $\begin{array}{l}0.16389^{\dagger} \\
(0.07917)\end{array}$ & $\begin{array}{l}0.26196^{*} \\
(0.08565)\end{array}$ & $\begin{array}{l}0.11945 \\
(0.09381)\end{array}$ & $\begin{array}{l}0.23827^{*} \\
(0.08948)\end{array}$ & $\begin{array}{l}0.22300^{\dagger} \\
(0.09179)\end{array}$ & $\begin{array}{l}0.14856^{\ddagger} \\
(0.08019)\end{array}$ & $\begin{array}{l}0.01613 \\
(0.08874)\end{array}$ & $\begin{array}{l}0.20947^{\dagger} \\
(0.09016)\end{array}$ & $\begin{array}{l}0.26229^{*} \\
(0.08932)\end{array}$ & $\begin{array}{l}0.06685 \\
(0.08912)\end{array}$ \\
\hline Factor $1 \& 2$ & $\begin{array}{l}0.15120 \\
(0.17429)\end{array}$ & $\begin{array}{l}0.15255 \\
(0.17470)\end{array}$ & $\begin{array}{l}0.06256 \\
(0.17446)\end{array}$ & $\begin{array}{l}0.14729 \\
(0.17425) \\
\end{array}$ & $\begin{array}{l}0.16551 \\
(0.17156)\end{array}$ & $\begin{array}{l}0.10029 \\
(0.18212) \\
\end{array}$ & $\begin{array}{l}0.23112 \\
(0.17980)\end{array}$ & $\begin{array}{l}0.20447 \\
(0.17950) \\
\end{array}$ & $\begin{array}{l}0.09302 \\
(0.17971)\end{array}$ & $\begin{array}{l}0.02252 \\
(0.17372)\end{array}$ & $\begin{array}{l}0.18093 \\
(0.17665) \\
\end{array}$ & $\begin{array}{l}0.23890 \\
(0.17690) \\
\end{array}$ & $\begin{array}{l}0.04349 \\
(0.17814) \\
\end{array}$ \\
\hline Factor $1,2 \& 5$ & $\begin{array}{l}0.25615 \\
(0.17092) \\
\end{array}$ & $\begin{array}{l}0.25586 \\
(0.19186) \\
\end{array}$ & $\begin{array}{l}0.23146 \\
(0.16521) \\
\end{array}$ & $\begin{array}{l}0.23757 \\
(0.16820) \\
\end{array}$ & $\begin{array}{l}0.25812 \\
(0.16534) \\
\end{array}$ & $\begin{array}{l}0.19972 \\
(0.17433) \\
\end{array}$ & $\begin{array}{l}0.27212 \\
(0.16773) \\
\end{array}$ & $\begin{array}{l}0.28340^{\ddagger} \\
(0.17061)\end{array}$ & $\begin{array}{l}0.19587 \\
(0.17184) \\
\end{array}$ & $\begin{array}{l}0.13654 \\
(0.16649) \\
\end{array}$ & $\begin{array}{l}0.27269 \\
(0.17012) \\
\end{array}$ & $\begin{array}{l}0.29694^{\ddagger} \\
(0.16778) \\
\end{array}$ & $\begin{array}{l}0.13712 \\
(0.17213) \\
\end{array}$ \\
\hline Factor $1,2 \& 4$ & $\begin{array}{l}0.21137 \\
(0.18548) \\
\end{array}$ & $\begin{array}{l}0.21290 \\
(0.18597) \\
\end{array}$ & $\begin{array}{l}0.09395 \\
(0.18746) \\
\end{array}$ & $\begin{array}{l}0.18391 \\
(0.18989) \\
\end{array}$ & $\begin{array}{l}0.29571 \\
(0.18526) \\
\end{array}$ & $\begin{array}{l}0.17460 \\
(0.18918) \\
\end{array}$ & $\begin{array}{l}0.29129 \\
(0.19053)\end{array}$ & $\begin{array}{l}0.25523 \\
(0.18859)\end{array}$ & $\begin{array}{l}0.13430 \\
(0.19469)\end{array}$ & $\begin{array}{l}0.04090 \\
(0.18724)\end{array}$ & $\begin{array}{l}0.25839 \\
(0.19142)\end{array}$ & $\begin{array}{l}0.29745 \\
(0.18754)\end{array}$ & $\begin{array}{l}0.10366 \\
(0.18883)\end{array}$ \\
\hline Factor $1,2,4 \& 5$ & $\begin{array}{l}0.07092 \\
(0.09837)\end{array}$ & $\begin{array}{l}0.06523 \\
(0.12682)\end{array}$ & $\begin{array}{l}-0.02654 \\
(0.09023)\end{array}$ & $\begin{array}{l}0.03420 \\
(0.09161)\end{array}$ & $\begin{array}{l}0.11681 \\
(0.08844)\end{array}$ & $\begin{array}{l}0.02602 \\
(0.09143) \\
\end{array}$ & $\begin{array}{l}0.07432 \\
(0.08690)\end{array}$ & $\begin{array}{l}0.09667 \\
(0.09350) \\
\end{array}$ & $\begin{array}{l}-0.01821 \\
(0.10368)\end{array}$ & $\begin{array}{l}-0.10945 \\
(0.09690)\end{array}$ & $\begin{array}{l}0.06034 \\
(0.08666)\end{array}$ & $\begin{array}{l}0.10250 \\
(0.08840)\end{array}$ & $\begin{array}{l}-0.00869 \\
(0.08963) \\
\end{array}$ \\
\hline Factor $1,2 \& 3$ & $\begin{array}{l}0.31473^{\ddagger} \\
(0.18911) \\
\end{array}$ & $\begin{array}{l}0.31473^{\ddagger} \\
(0.18916)\end{array}$ & $\begin{array}{l}0.31473^{\ddagger} \\
(0.18591)\end{array}$ & $\begin{array}{l}0.30865 \\
(0.18919) \\
\end{array}$ & $\begin{array}{l}0.31473^{\ddagger} \\
(0.18606)\end{array}$ & $\begin{array}{l}0.30502 \\
(0.18904) \\
\end{array}$ & $\begin{array}{l}0.39466^{\dagger} \\
(0.19403)\end{array}$ & $\begin{array}{l}0.37546^{\ddagger} \\
(0.19540) \\
\end{array}$ & $\begin{array}{l}0.30002 \\
(0.18881)\end{array}$ & $\begin{array}{l}0.28219 \\
(0.18427)\end{array}$ & $\begin{array}{l}0.32371^{\ddagger} \\
(0.18897) \\
\end{array}$ & $\begin{array}{l}0.41470^{\dagger} \\
(0.19245) \\
\end{array}$ & $\begin{array}{l}0.20703 \\
(0.19231) \\
\end{array}$ \\
\hline Factor 1 & $\begin{array}{l}0.24988 \\
(0.19764)\end{array}$ & $\begin{array}{l}0.24239 \\
(0.21210)\end{array}$ & $\begin{array}{l}0.11393 \\
(0.19250)\end{array}$ & $\begin{array}{l}0.21731 \\
(0.19177)\end{array}$ & $\begin{array}{l}0.28278 \\
(0.18867)\end{array}$ & $\begin{array}{l}0.18792 \\
(0.19586)\end{array}$ & $\begin{array}{l}0.26655 \\
(0.19074)\end{array}$ & $\begin{array}{l}0.27424 \\
(0.19359)\end{array}$ & $\begin{array}{l}0.16560 \\
(0.19711)\end{array}$ & $\begin{array}{l}0.11094 \\
(0.18892)\end{array}$ & $\begin{array}{l}0.25897 \\
(0.19251)\end{array}$ & $\begin{array}{l}0.31664 \\
(0.19257)\end{array}$ & $\begin{array}{l}0.12365 \\
(0.19388)\end{array}$ \\
\hline 4 & $\begin{array}{l}0.29888^{*} \\
(0.03698) \\
\end{array}$ & $\begin{array}{l}0.29967^{*} \\
(0.03758)\end{array}$ & $\begin{array}{l}0.19163^{*} \\
(0.05386)\end{array}$ & $\begin{array}{l}0.29114^{*} \\
(0.03875) \\
\end{array}$ & $\begin{array}{l}0.36010^{*} \\
(0.04316) \\
\end{array}$ & $\begin{array}{l}0.25212^{*} \\
(0.06174) \\
\end{array}$ & $\begin{array}{l}0.34805^{*} \\
(0.04725) \\
\end{array}$ & $\begin{array}{l}0.35893^{*} \\
(0.06275) \\
\end{array}$ & $\begin{array}{l}0.22280^{*} \\
(0.07075) \\
\end{array}$ & $\begin{array}{l}0.19306^{*} \\
(0.04746) \\
\end{array}$ & $\begin{array}{l}0.30842^{*} \\
(0.03821) \\
\end{array}$ & $\begin{array}{l}0.36056^{*} \\
(0.04610) \\
\end{array}$ & $\begin{array}{l}0.23064^{*} \\
(0.04675) \\
\end{array}$ \\
\hline All F & $\begin{array}{l}0.31479^{*} \\
(0.05271)\end{array}$ & $\begin{array}{l}0.31610^{*} \\
(0.10306)\end{array}$ & $\begin{array}{l}0.21852^{*} \\
(0.05478)\end{array}$ & $\begin{array}{l}0.28095^{*} \\
(0.05793) \\
\end{array}$ & $\begin{array}{l}0.33450^{*} \\
(0.04583)\end{array}$ & $\begin{array}{l}0.25745^{*} \\
(0.06762) \\
\end{array}$ & $\begin{array}{l}0.37715^{*} \\
(0.06255)\end{array}$ & $\begin{array}{l}0.34188^{*} \\
(0.05475) \\
\end{array}$ & $\begin{array}{l}0.24217^{*} \\
(0.06723)\end{array}$ & $\begin{array}{l}0.15226^{\dagger} \\
(0.06268)\end{array}$ & $\begin{array}{l}0.34757^{*} \\
(0.06303)\end{array}$ & $\begin{array}{l}0.38486^{*} \\
(0.05758) \\
\end{array}$ & $\begin{array}{l}0.20644^{*} \\
(0.06140)\end{array}$ \\
\hline $\begin{array}{l}\text { Individual } \\
\text { Variable }\end{array}$ & $\begin{array}{l}-0.02172 \\
(0.06063)\end{array}$ & $\begin{array}{l}-0.01104 \\
(0.09263)\end{array}$ & $\begin{array}{l}0.11742^{*} \\
(0.04350)\end{array}$ & $\begin{array}{l}0.03188 \\
(0.04801)\end{array}$ & $\begin{array}{l}-0.11676^{*} \\
(0.04427)\end{array}$ & $\begin{array}{l}0.05090 \\
(0.05388)\end{array}$ & $\begin{array}{l}-0.07992^{\ddagger} \\
(0.04828)\end{array}$ & $\begin{array}{l}-0.06072 \\
(0.05134)\end{array}$ & $\begin{array}{l}0.07707 \\
(0.06118)\end{array}$ & $\begin{array}{l}0.17047^{*} \\
(0.04985)\end{array}$ & $\begin{array}{l}-0.04702 \\
(0.04872)\end{array}$ & $\begin{array}{c}-0.09996^{\dagger} \\
(0.04551)\end{array}$ & $\begin{array}{l}0.10771^{\dagger} \\
(0.04608)\end{array}$ \\
\hline
\end{tabular}

* Significant at the 0.01 level $\dagger$ Significant at the 0.05 level $\$$ Significant at the 0.10 level

\section{Table 100 Coefficients with individual variables for high-level factors - Vehicles Industry}




\begin{tabular}{|c|c|c|c|c|c|c|c|c|c|c|c|c|c|}
\hline $\begin{array}{l}\text { Individual } \\
\text { variable included } \\
\text { in the model in } \\
\text { the column }\end{array}$ & 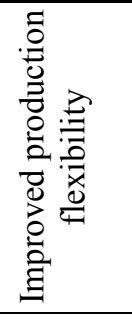 & 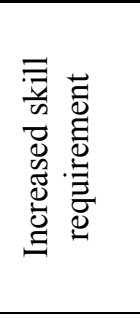 & 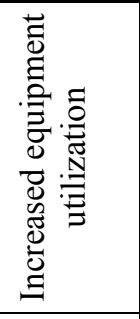 & 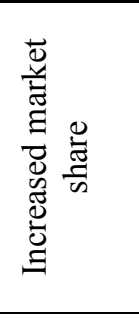 & 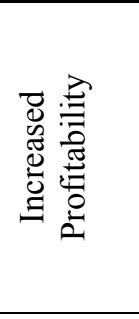 & 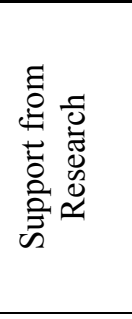 & 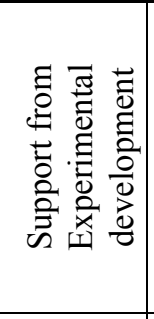 & 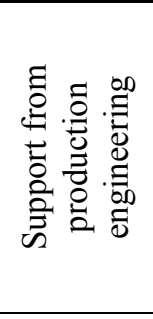 & 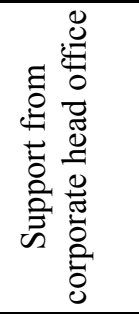 & 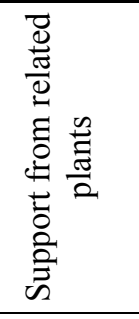 & 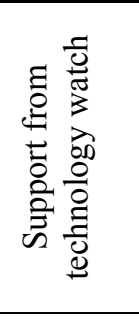 & 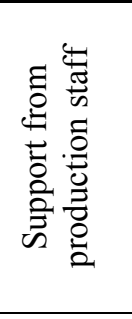 & 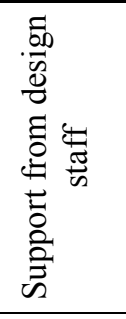 \\
\hline No factors & $\begin{array}{l}0.16735^{*} \\
(0.03716) \\
\end{array}$ & $\begin{array}{l}0.16690^{*} \\
(0.03734)\end{array}$ & $\begin{array}{l}0.16729^{*} \\
(0.03720)\end{array}$ & $\begin{array}{l}0.16754^{*} \\
(0.03708)\end{array}$ & $\begin{array}{l}0.16738^{*} \\
(0.03718)\end{array}$ & $\begin{array}{l}0.16740^{*} \\
(0.03720)\end{array}$ & $\begin{array}{l}0.16787^{*} \\
(0.03716) \\
\end{array}$ & $\begin{array}{l}0.16594^{*} \\
(0.03721)\end{array}$ & $\begin{array}{l}0.16841^{*} \\
(0.03716)\end{array}$ & $\begin{array}{l}0.16711^{*} \\
(0.03714)\end{array}$ & $\begin{array}{l}0.16754^{*} \\
(0.03710)\end{array}$ & $\begin{array}{l}0.16834^{*} \\
(0.03712) \\
\end{array}$ & $\begin{array}{l}0.16845^{*} \\
(0.03711)\end{array}$ \\
\hline Factor 5 & $\begin{array}{l}-0.05360 \\
(0.23299)\end{array}$ & $\begin{array}{l}-0.03346 \\
(0.23110)\end{array}$ & $\begin{array}{l}-0.04085 \\
(0.23343)\end{array}$ & $\begin{array}{l}-0.01901 \\
(0.23036)\end{array}$ & $\begin{array}{l}-0.03726 \\
(0.23109)\end{array}$ & $\begin{array}{l}-0.03383 \\
(0.23103)\end{array}$ & $\begin{array}{l}-0.02486 \\
(0.23079)\end{array}$ & $\begin{array}{l}-0.05292 \\
(0.23228)\end{array}$ & $\begin{array}{l}-0.00886 \\
(0.23220)\end{array}$ & $\begin{array}{l}-0.06126 \\
(0.23302)\end{array}$ & $\begin{array}{l}-0.02074 \\
(0.23043)\end{array}$ & $\begin{array}{l}0.01504 \\
(0.23479)\end{array}$ & $\begin{array}{l}-0.00468 \\
(0.23151)\end{array}$ \\
\hline Factor 4 & $\begin{array}{l}-0.17252 \\
(0.27775) \\
\end{array}$ & $\begin{array}{l}-0.14521 \\
(0.27570) \\
\end{array}$ & $\begin{array}{l}-0.14821 \\
(0.27575) \\
\end{array}$ & $\begin{array}{l}-0.09821 \\
(0.27452) \\
\end{array}$ & $\begin{array}{l}-0.13770 \\
(0.27304) \\
\end{array}$ & $\begin{array}{l}-0.14265 \\
(0.27379) \\
\end{array}$ & $\begin{array}{l}-0.11724 \\
(0.27462) \\
\end{array}$ & $\begin{array}{l}-0.18835 \\
(0.28080) \\
\end{array}$ & $\begin{array}{l}-0.11037 \\
(0.27494) \\
\end{array}$ & $\begin{array}{l}-0.16451 \\
(0.27443) \\
\end{array}$ & $\begin{array}{l}-0.11842 \\
(0.27306) \\
\end{array}$ & $\begin{array}{l}-0.08761 \\
(0.27700) \\
\end{array}$ & $\begin{array}{c}-0.07610 \\
(0.27838) \\
\end{array}$ \\
\hline Factor & $\begin{array}{l}-0.02852 \\
(0.22340)\end{array}$ & $\begin{array}{l}-0.02427 \\
(0.22460)\end{array}$ & $\begin{array}{l}-0.02228 \\
(0.22345)\end{array}$ & $\begin{array}{l}-0.00791 \\
(0.22275)\end{array}$ & $\begin{array}{l}-0.01926 \\
(0.22311)\end{array}$ & $\begin{array}{l}-0.03171 \\
(0.22822)\end{array}$ & $\begin{array}{l}-0.01926 \\
(0.22299)\end{array}$ & $\begin{array}{l}-0.02944 \\
(0.22331)\end{array}$ & $\begin{array}{l}0.01983 \\
(0.22829)\end{array}$ & $\begin{array}{l}-0.02697 \\
(0.22300)\end{array}$ & $\begin{array}{l}-0.00953 \\
(0.22282)\end{array}$ & $\begin{array}{l}0.00954 \\
(0.22450)\end{array}$ & $\begin{array}{l}0.00550 \\
(0.22379)\end{array}$ \\
\hline Factor 3 & $\begin{array}{l}-0.43987^{*} \\
(0.09142) \\
\end{array}$ & $\begin{array}{l}-0.41030^{*} \\
(0.07916)\end{array}$ & $\begin{array}{l}-0.41555^{*} \\
(0.08351)\end{array}$ & $\begin{array}{l}-0.37010^{*} \\
(0.08300)\end{array}$ & $\begin{array}{l}-0.40647^{*} \\
(0.07680)\end{array}$ & $\begin{array}{l}-0.41842^{*} \\
(0.08941) \\
\end{array}$ & $\begin{array}{l}-0.37720^{*} \\
(0.08851) \\
\end{array}$ & $\begin{array}{l}-0.45712^{*} \\
(0.10159)\end{array}$ & $\begin{array}{l}-0.36899^{*} \\
(0.09016)\end{array}$ & $\begin{array}{l}-0.44326^{*} \\
(0.08764)\end{array}$ & $\begin{array}{l}-0.37727^{*} \\
(0.08151)\end{array}$ & $\begin{array}{l}-0.33776^{*} \\
(0.10258) \\
\end{array}$ & $\begin{array}{l}-0.36680^{*} \\
(0.08505) \\
\end{array}$ \\
\hline Facto & $\begin{array}{l}0.11579 \\
(0.16997)\end{array}$ & $\begin{array}{l}0.14421 \\
(0.16516)\end{array}$ & $\begin{array}{l}0.14164 \\
(0.16524)\end{array}$ & $\begin{array}{l}0.19028 \\
(0.16527)\end{array}$ & $\begin{array}{l}0.14760 \\
(0.16211)\end{array}$ & $\begin{array}{l}0.13948 \\
(0.16755)\end{array}$ & $\begin{array}{l}0.17369 \\
(0.16558)\end{array}$ & $\begin{array}{l}0.09997 \\
(0.17500)\end{array}$ & $\begin{array}{l}0.17054 \\
(0.16376)\end{array}$ & $\begin{array}{l}0.12368 \\
(0.16472)\end{array}$ & $\begin{array}{l}0.17950 \\
(0.16395)\end{array}$ & $\begin{array}{l}0.20093 \\
(0.16920)\end{array}$ & $\begin{array}{l}0.18201 \\
(0.16423)\end{array}$ \\
\hline Factor $3 \& 4$ & \multicolumn{13}{|c|}{ Insufficient Data } \\
\hline Factor $3,4 \& 5$ & $\begin{array}{l}-0.02591 \\
(0.29592)\end{array}$ & $\begin{array}{l}-0.01024 \\
(0.29634)\end{array}$ & $\begin{array}{l}-0.00851 \\
(0.29487) \\
\end{array}$ & $\begin{array}{l}-0.00325 \\
(0.29339)\end{array}$ & $\begin{array}{l}-0.02176 \\
(0.29686)\end{array}$ & $\begin{array}{l}-0.00760 \\
(0.29474)\end{array}$ & $\begin{array}{l}0.02602 \\
(0.29728)\end{array}$ & $\begin{array}{l}-0.05390 \\
(0.30135)\end{array}$ & $\begin{array}{l}0.03584 \\
(0.29799) \\
\end{array}$ & $\begin{array}{l}-0.01665 \\
(0.29418)\end{array}$ & $\begin{array}{l}0.04518 \\
(0.29711) \\
\end{array}$ & $\begin{array}{l}0.06839 \\
(0.30207)\end{array}$ & $\begin{array}{l}0.05835 \\
(0.29904)\end{array}$ \\
\hline Factor 2 & $\begin{array}{l}0.24144 \\
(0.24289) \\
\end{array}$ & $\begin{array}{l}0.24973 \\
(0.24282) \\
\end{array}$ & $\begin{array}{l}0.24615 \\
(0.24314) \\
\end{array}$ & $\begin{array}{l}0.24973 \\
(0.24209) \\
\end{array}$ & $\begin{array}{l}0.24296 \\
(0.24316) \\
\end{array}$ & $\begin{array}{l}0.24973 \\
(0.24281) \\
\end{array}$ & \begin{tabular}{|l}
0.24973 \\
$(0.24259)$ \\
\end{tabular} & $\begin{array}{l}0.22564 \\
(0.24456) \\
\end{array}$ & $\begin{array}{l}0.26832 \\
(0.24361) \\
\end{array}$ & $\begin{array}{l}0.23148 \\
(0.24331) \\
\end{array}$ & $\begin{array}{l}0.24973 \\
(0.24220) \\
\end{array}$ & $\begin{array}{l}0.28381 \\
(0.24460) \\
\end{array}$ & $\begin{array}{l}0.26438 \\
(0.24255) \\
\end{array}$ \\
\hline Fact & $\begin{array}{l}0.15738 \\
(0.35268) \\
\end{array}$ & $\begin{array}{l}0.17480 \\
(0.35208) \\
\end{array}$ & $\begin{array}{l}0.15975 \\
(0.35621) \\
\end{array}$ & $\begin{array}{l}0.20304 \\
(0.35189) \\
\end{array}$ & $\begin{array}{l}0.16058 \\
(0.35327) \\
\end{array}$ & $\begin{array}{l}0.17480 \\
(0.35206) \\
\end{array}$ & \begin{tabular}{|l}
0.17480 \\
$(0.35174)$ \\
\end{tabular} & $\begin{array}{l}0.12415 \\
(0.35787) \\
\end{array}$ & $\begin{array}{l}0.19434 \\
(0.35245) \\
\end{array}$ & $\begin{array}{l}0.15562 \\
(0.35217) \\
\end{array}$ & $\begin{array}{l}0.17480 \\
(0.35117) \\
\end{array}$ & $\begin{array}{l}0.21062 \\
(0.35304) \\
\end{array}$ & $\begin{array}{l}0.17480 \\
(0.35113) \\
\end{array}$ \\
\hline $\mathrm{F}$ & $\begin{array}{l}0.23162 \\
(0.14140)\end{array}$ & $\begin{array}{l}0.24574^{\ddagger} \\
(0.14032)\end{array}$ & $\begin{array}{l}0.24610^{\ddagger} \\
(0.13955)\end{array}$ & $\begin{array}{l}0.25842^{\ddagger} \\
(0.13878)\end{array}$ & $\begin{array}{l}0.23808^{\ddagger} \\
(0.14113)\end{array}$ & $\begin{array}{l}0.24318^{\ddagger} \\
(0.14114)\end{array}$ & $\begin{array}{l}0.26473^{\ddagger} \\
(0.14070)\end{array}$ & $\begin{array}{l}0.20291 \\
(0.15177)\end{array}$ & $\begin{array}{l}0.27652^{\ddagger} \\
(0.14289)\end{array}$ & $\begin{array}{l}0.22023 \\
(0.14282)\end{array}$ & $\begin{array}{l}0.27086^{\ddagger} \\
(0.14009)\end{array}$ & $\begin{array}{l}0.31568^{\dagger} \\
(0.15336)\end{array}$ & $\begin{array}{l}0.29202^{\dagger} \\
(0.14411)\end{array}$ \\
\hline Facto & $\begin{array}{l}0.18337^{\ddagger} \\
(0.11066)\end{array}$ & $\begin{array}{l}0.18446 \\
(0.11813)\end{array}$ & $\begin{array}{l}0.18969^{\ddagger} \\
(0.11052)\end{array}$ & $\begin{array}{l}0.19871^{\ddagger} \\
(0.10957)\end{array}$ & $\begin{array}{l}0.18794^{\ddagger} \\
(0.11033)\end{array}$ & $\begin{array}{l}0.18909^{\ddagger} \\
(0.11092)\end{array}$ & $\begin{array}{l}0.20006^{\ddagger} \\
(0.11017)\end{array}$ & $\begin{array}{l}0.14256 \\
(0.12828)\end{array}$ & $\begin{array}{l}0.19701^{\ddagger} \\
(0.10974)\end{array}$ & $\begin{array}{l}0.18424^{\ddagger} \\
(0.11009)\end{array}$ & $\begin{array}{l}0.19321^{\ddagger} \\
(0.10951)\end{array}$ & $\begin{array}{l}0.25789^{\dagger} \\
(0.12694)\end{array}$ & $\begin{array}{l}0.21062^{\ddagger} \\
(0.11069)\end{array}$ \\
\hline Factor & $\begin{array}{l}0.29278^{\dagger} \\
(0.12877) \\
\end{array}$ & $\begin{array}{l}0.28669^{\dagger} \\
(0.13939) \\
\end{array}$ & $\begin{array}{l}0.28238^{\dagger} \\
(0.13969) \\
\end{array}$ & $\begin{array}{l}0.30498^{\dagger} \\
(0.12850) \\
\end{array}$ & $\begin{array}{l}0.27279^{\ddagger} \\
(0.13921) \\
\end{array}$ & $\begin{array}{l}0.29743^{\dagger} \\
(0.12871) \\
\end{array}$ & $\begin{array}{l}0.29743^{\dagger} \\
(0.12859) \\
\end{array}$ & $\begin{array}{l}0.24678^{\ddagger} \\
(0.14477) \\
\end{array}$ & $\begin{array}{l}0.33652^{\dagger} \\
(0.13769) \\
\end{array}$ & $\begin{array}{l}0.25908^{\ddagger} \\
(0.13589) \\
\end{array}$ & $\begin{array}{l}0.30390^{\dagger} \\
(0.12853) \\
\end{array}$ & $\begin{array}{l}0.36908^{\dagger} \\
(0.14677) \\
\end{array}$ & $\begin{array}{l}0.30566^{\dagger} \\
(0.12860) \\
\end{array}$ \\
\hline Factor 2 & $\begin{array}{l}0.17638 \\
(0.50011) \\
\end{array}$ & $\begin{array}{l}0.21120 \\
(0.49792) \\
\end{array}$ & $\begin{array}{l}0.19616 \\
(0.50083) \\
\end{array}$ & $\begin{array}{l}0.21120 \\
(0.49641) \\
\end{array}$ & $\begin{array}{l}0.21120 \\
(0.49771) \\
\end{array}$ & $\begin{array}{l}0.19875 \\
(0.50016) \\
\end{array}$ & \begin{tabular}{|l}
0.24047 \\
$(0.49938)$ \\
\end{tabular} & $\begin{array}{l}0.21120 \\
(0.49727) \\
\end{array}$ & $\begin{array}{l}0.21120 \\
(0.49721) \\
\end{array}$ & $\begin{array}{l}0.17285 \\
(0.49902) \\
\end{array}$ & $\begin{array}{l}0.21120 \\
(0.49663) \\
\end{array}$ & $\begin{array}{l}0.28285 \\
(0.50180) \\
\end{array}$ & $\begin{array}{l}0.27280 \\
(0.49987) \\
\end{array}$ \\
\hline Factor $2,3 \& 4$ & $\begin{array}{l}0.23567^{*} \\
(0.06488)\end{array}$ & $\begin{array}{l}0.25513^{*} \\
(0.07112)\end{array}$ & $\begin{array}{l}0.25110^{*} \\
(0.07100) \\
\end{array}$ & $\begin{array}{l}0.32026^{*} \\
(0.06682) \\
\end{array}$ & $\begin{array}{l}0.26507^{*} \\
(0.04688) \\
\end{array}$ & $\begin{array}{l}0.25364^{*} \\
(0.06625)\end{array}$ & $\begin{array}{l}0.29463^{*} \\
(0.06376) \\
\end{array}$ & $\begin{array}{l}0.21523^{*} \\
(0.08139) \\
\end{array}$ & $\begin{array}{l}0.30013^{*} \\
(0.06374)\end{array}$ & $\begin{array}{l}0.23193^{*} \\
(0.06099)\end{array}$ & $\begin{array}{l}0.30651^{*} \\
(0.06064)\end{array}$ & $\begin{array}{l}0.33752^{*} \\
(0.08507)\end{array}$ & $\begin{array}{l}0.32518^{*} \\
(0.07231) \\
\end{array}$ \\
\hline Factor2 & $\begin{array}{l}0.28616^{\dagger} \\
(0.14366) \\
\end{array}$ & $\begin{array}{l}0.30085^{\dagger} \\
(0.14472) \\
\end{array}$ & $\begin{array}{l}0.30067^{\dagger} \\
(0.14261) \\
\end{array}$ & $\begin{array}{l}0.32894^{\dagger} \\
(0.14078) \\
\end{array}$ & $\begin{array}{l}0.29847^{\dagger} \\
(0.14152) \\
\end{array}$ & $\begin{array}{l}0.29704^{\dagger} \\
(0.14637)\end{array}$ & $\begin{array}{l}0.32671^{\dagger} \\
(0.14263)\end{array}$ & $\begin{array}{l}0.26284^{\ddagger} \\
(0.15201)\end{array}$ & $\begin{array}{l}0.33801^{\dagger} \\
(0.14480)\end{array}$ & $\begin{array}{l}0.27992^{\ddagger} \\
(0.14353)\end{array}$ & $\begin{array}{l}0.33883^{\dagger} \\
(0.14266)\end{array}$ & $\begin{array}{l}0.37984^{\dagger} \\
(0.15673)\end{array}$ & $\begin{array}{l}0.36492^{\dagger} \\
(0.14928)\end{array}$ \\
\hline $\mathrm{F}_{2}$ & $\begin{array}{l}0.51342^{*} \\
(0.10278)\end{array}$ & $\begin{array}{l}0.54504^{*} \\
(0.09081)\end{array}$ & $\begin{array}{l}0.53198^{*} \\
(0.10398)\end{array}$ & $\begin{array}{l}0.60258^{*} \\
(0.10311)\end{array}$ & $\begin{array}{l}0.51766^{*} \\
(0.10943)\end{array}$ & $\begin{array}{l}0.53365^{*} \\
(0.10243)\end{array}$ & $\begin{array}{l}0.57067^{*} \\
(0.09784) \\
\end{array}$ & $\begin{array}{l}0.54020^{*} \\
(0.09087)\end{array}$ & $\begin{array}{l}0.54782^{*} \\
(0.09056)\end{array}$ & $\begin{array}{l}0.54442^{*} \\
(0.09052)\end{array}$ & $\begin{array}{l}0.54909^{*} \\
(0.09047)\end{array}$ & $\begin{array}{l}0.55445^{*} \\
(0.09082)\end{array}$ & $\begin{array}{l}0.55328^{*} \\
(0.09066)\end{array}$ \\
\hline $\mathrm{F}$ & $\begin{array}{l}0.16310 \\
(0.18018)\end{array}$ & $\begin{array}{l}0.18719 \\
(0.18085) \\
\end{array}$ & $\begin{array}{l}0.18651 \\
(0.17759) \\
\end{array}$ & $\begin{array}{l}0.25442 \\
(0.17924) \\
\end{array}$ & $\begin{array}{l}0.17515 \\
(0.17954) \\
\end{array}$ & $\begin{array}{l}0.19245 \\
(0.17401) \\
\end{array}$ & \begin{tabular}{|l}
0.20728 \\
$(0.17316)$ \\
\end{tabular} & $\begin{array}{l}0.15338 \\
(0.18220)\end{array}$ & $\begin{array}{l}0.20734 \\
(0.17292) \\
\end{array}$ & $\begin{array}{l}0.18106 \\
(0.17355)\end{array}$ & $\begin{array}{l}0.20376 \\
(0.17240) \\
\end{array}$ & $\begin{array}{l}0.24669 \\
(0.17901) \\
\end{array}$ & $\begin{array}{l}0.23729 \\
(0.17614) \\
\end{array}$ \\
\hline Fact & $\begin{array}{l}0.23445^{\ddagger} \\
(0.14082)\end{array}$ & $\begin{array}{l}0.23402 \\
(0.14824) \\
\end{array}$ & $\begin{array}{l}0.24125^{\ddagger} \\
(0.14055) \\
\end{array}$ & $\begin{array}{l}0.28600^{\dagger} \\
(0.14474)\end{array}$ & $\begin{array}{l}0.23128 \\
(0.14275) \\
\end{array}$ & $\begin{array}{l}0.24052^{\ddagger} \\
(0.14079)\end{array}$ & $\begin{array}{l}0.25095^{\ddagger} \\
(0.14059) \\
\end{array}$ & $\begin{array}{l}0.22695 \\
(0.14182) \\
\end{array}$ & $\begin{array}{l}0.25085^{\ddagger} \\
(0.14039)\end{array}$ & $\begin{array}{l}0.23002 \\
(0.14093) \\
\end{array}$ & $\begin{array}{l}0.24364^{\ddagger} \\
(0.13994) \\
\end{array}$ & $\begin{array}{l}0.25501^{\text {\# }} \\
(0.14042)\end{array}$ & $\begin{array}{l}0.24853^{\ddagger} \\
(0.13999) \\
\end{array}$ \\
\hline Factor 1 & $\begin{array}{l}0.22744^{*} \\
(0.08319) \\
\end{array}$ & $\begin{array}{l}0.24996^{*} \\
(0.07937) \\
\end{array}$ & $\begin{array}{l}0.24575^{*} \\
(0.08184) \\
\end{array}$ & $\begin{array}{l}0.29293^{*} \\
(0.07686) \\
\end{array}$ & $\begin{array}{l}0.23736^{*} \\
(0.08249) \\
\end{array}$ & $\begin{array}{l}0.25355^{*} \\
(0.07187) \\
\end{array}$ & $\begin{array}{l}0.27209^{*} \\
(0.07378) \\
\end{array}$ & $\begin{array}{l}0.21410^{\dagger} \\
(0.09040) \\
\end{array}$ & $\begin{array}{l}0.27502^{*} \\
(0.07379) \\
\end{array}$ & $\begin{array}{l}0.24610^{*} \\
(0.07148) \\
\end{array}$ & $\begin{array}{l}0.25828^{*} \\
(0.07024) \\
\end{array}$ & $\begin{array}{l}0.29650^{*} \\
(0.08029) \\
\end{array}$ & $\begin{array}{l}0.31891^{*} \\
(0.09065) \\
\end{array}$ \\
\hline Factor $1 \& 3$ & $\begin{array}{l}0.01369 \\
(0.12528) \\
\end{array}$ & $\begin{array}{l}0.03400 \\
(0.12590)\end{array}$ & $\begin{array}{l}0.03037 \\
(0.12614) \\
\end{array}$ & $\begin{array}{l}0.09954 \\
(0.12726) \\
\end{array}$ & $\begin{array}{l}0.01462 \\
(0.13255) \\
\end{array}$ & $\begin{array}{l}0.03555 \\
(0.12100) \\
\end{array}$ & \begin{tabular}{|l}
0.05613 \\
$(0.11907)$ \\
\end{tabular} & $\begin{array}{l}0.00034540 \\
(0.13013)\end{array}$ & $\begin{array}{l}0.07603 \\
(0.12454) \\
\end{array}$ & $\begin{array}{l}0.02594 \\
(0.11899) \\
\end{array}$ & $\begin{array}{l}0.06229 \\
(0.11866)\end{array}$ & $\begin{array}{l}0.11471 \\
(0.13712) \\
\end{array}$ & $\begin{array}{l}0.07717 \\
(0.12145) \\
\end{array}$ \\
\hline Factor 1 & $\begin{array}{l}0.06855 \\
(0.10785) \\
\end{array}$ & $\begin{array}{l}0.09303 \\
(0.10782) \\
\end{array}$ & $\begin{array}{l}0.08833 \\
(0.10915) \\
\end{array}$ & $\begin{array}{l}0.15986 \\
(0.10665) \\
\end{array}$ & $\begin{array}{l}0.07596 \\
(0.11161) \\
\end{array}$ & $\begin{array}{l}0.09093 \\
(0.10602)\end{array}$ & \begin{tabular}{|l}
0.13265 \\
$(0.10436)$ \\
\end{tabular} & $\begin{array}{l}0.05273 \\
(0.11566)\end{array}$ & $\begin{array}{l}0.10666 \\
(0.09465)\end{array}$ & $\begin{array}{l}0.10016 \\
(0.09461)\end{array}$ & $\begin{array}{l}0.10570 \\
(0.09448)\end{array}$ & $\begin{array}{l}0.17244 \\
(0.11670) \\
\end{array}$ & $\begin{array}{l}0.16275 \\
(0.10941) \\
\end{array}$ \\
\hline Factor $1,3 \& 4$ & $\begin{array}{l}0.16495 \\
(0.12757) \\
\end{array}$ & $\begin{array}{l}0.18737 \\
(0.12483) \\
\end{array}$ & $\begin{array}{l}0.18973 \\
(0.12111) \\
\end{array}$ & $\begin{array}{l}0.23055^{\ddagger} \\
(0.12381) \\
\end{array}$ & $\begin{array}{l}0.16748 \\
(0.13315) \\
\end{array}$ & $\begin{array}{l}0.18723 \\
(0.12299) \\
\end{array}$ & $\begin{array}{l}0.20708^{\ddagger} \\
(0.12143) \\
\end{array}$ & $\begin{array}{l}0.16132 \\
(0.12744) \\
\end{array}$ & $\begin{array}{l}0.23336^{\ddagger} \\
(0.12961) \\
\end{array}$ & $\begin{array}{l}0.18355 \\
(0.12043) \\
\end{array}$ & $\begin{array}{l}0.20610^{\ddagger} \\
(0.12022) \\
\end{array}$ & $\begin{array}{l}0.24843^{\ddagger} \\
(0.13122) \\
\end{array}$ & $\begin{array}{l}0.25587^{\ddagger} \\
(0.13270) \\
\end{array}$ \\
\hline Fact & $\begin{array}{l}0.15654^{\ddagger} \\
(0.08062) \\
\end{array}$ & $\begin{array}{l}0.15752^{\ddagger} \\
(0.09303)\end{array}$ & $\begin{array}{l}0.15446^{\ddagger} \\
(0.09176) \\
\end{array}$ & $\begin{array}{l}0.22008^{\dagger} \\
(0.09143)\end{array}$ & $\begin{array}{l}0.14154 \\
(0.09670) \\
\end{array}$ & $\begin{array}{l}0.15632^{\ddagger} \\
(0.08968)\end{array}$ & $\begin{array}{l}0.19492^{\dagger} \\
(0.08923) \\
\end{array}$ & $\begin{array}{l}0.12209 \\
(0.09889) \\
\end{array}$ & $\begin{array}{l}0.20231^{\dagger} \\
(0.09047)\end{array}$ & $\begin{array}{l}0.16287^{\dagger} \\
(0.07911)\end{array}$ & $\begin{array}{l}0.17552^{\dagger} \\
(0.07925)\end{array}$ & $\begin{array}{l}0.23540^{\dagger} \\
(0.10382) \\
\end{array}$ & $\begin{array}{l}0.22897^{\dagger} \\
(0.09749) \\
\end{array}$ \\
\hline $\mathrm{Fa}$ & $\begin{array}{l}0.11637 \\
(0.18169) \\
\end{array}$ & $\begin{array}{l}0.14309 \\
(0.17894) \\
\end{array}$ & $\begin{array}{l}0.13615 \\
(0.18257) \\
\end{array}$ & $\begin{array}{l}0.20076 \\
(0.17916) \\
\end{array}$ & $\begin{array}{l}0.12624 \\
(0.18237) \\
\end{array}$ & $\begin{array}{l}0.14814 \\
(0.17470)\end{array}$ & \begin{tabular}{|l}
0.15478 \\
$(0.17424)$ \\
\end{tabular} & $\begin{array}{l}0.13878 \\
(0.17486) \\
\end{array}$ & $\begin{array}{l}0.16078 \\
(0.17450) \\
\end{array}$ & $\begin{array}{l}0.13709 \\
(0.17478)\end{array}$ & $\begin{array}{l}0.15120 \\
(0.17387) \\
\end{array}$ & $\begin{array}{l}0.16876 \\
(0.17478) \\
\end{array}$ & $\begin{array}{l}0.17385 \\
(0.17513) \\
\end{array}$ \\
\hline Facto & $\begin{array}{l}0.22419 \\
(0.17063) \\
\end{array}$ & $\begin{array}{l}0.23408 \\
(0.17632) \\
\end{array}$ & $\begin{array}{l}0.23420 \\
(0.17232) \\
\end{array}$ & $\begin{array}{l}0.26412 \\
(0.16836) \\
\end{array}$ & $\begin{array}{l}0.22676 \\
(0.17240) \\
\end{array}$ & $\begin{array}{l}0.23886 \\
(0.16954) \\
\end{array}$ & \begin{tabular}{|l|}
0.25149 \\
$(0.16815)$ \\
\end{tabular} & $\begin{array}{l}0.21482 \\
(0.17237) \\
\end{array}$ & $\begin{array}{l}0.27243 \\
(0.17136) \\
\end{array}$ & $\begin{array}{l}0.23609 \\
(0.16802)\end{array}$ & $\begin{array}{l}0.24483 \\
(0.16758) \\
\end{array}$ & $\begin{array}{l}0.28728^{\ddagger} \\
(0.17282) \\
\end{array}$ & $\begin{array}{l}0.26730 \\
(0.16886) \\
\end{array}$ \\
\hline Factor & $\begin{array}{l}0.17654 \\
(0.19243) \\
\end{array}$ & $\begin{array}{l}0.20838 \\
(0.18611) \\
\end{array}$ & $\begin{array}{l}0.20050 \\
(0.18960) \\
\end{array}$ & $\begin{array}{l}0.26785 \\
(0.19149) \\
\end{array}$ & $\begin{array}{l}0.18292 \\
(0.19532) \\
\end{array}$ & $\begin{array}{l}0.20618 \\
(0.18656) \\
\end{array}$ & $\begin{array}{l}0.21949 \\
(0.18574) \\
\end{array}$ & $\begin{array}{l}0.19027 \\
(0.18734) \\
\end{array}$ & $\begin{array}{l}0.22222 \\
(0.18576) \\
\end{array}$ & $\begin{array}{l}0.20072 \\
(0.18560) \\
\end{array}$ & $\begin{array}{l}0.21137 \\
(0.18504) \\
\end{array}$ & $\begin{array}{l}0.24120 \\
(0.18743) \\
\end{array}$ & $\begin{array}{l}0.23702 \\
(0.18655) \\
\end{array}$ \\
\hline Factor $1,2,4 \& 5$ & $\begin{array}{l}0.02042 \\
(0.10004)\end{array}$ & $\begin{array}{l}0.04857 \\
(0.09102)\end{array}$ & $\begin{array}{l}0.04126 \\
(0.09837)\end{array}$ & $\begin{array}{l}0.10213 \\
(0.09607)\end{array}$ & $\begin{array}{l}0.02947 \\
(0.10168)\end{array}$ & $\begin{array}{l}0.05219 \\
(0.08694)\end{array}$ & $\begin{array}{l}0.05983 \\
(0.08694)\end{array}$ & $\begin{array}{l}0.02922 \\
(0.09252)\end{array}$ & $\begin{array}{l}0.06332 \\
(0.08725)\end{array}$ & $\begin{array}{l}0.04532 \\
(0.08706)\end{array}$ & $\begin{array}{l}0.06241 \\
(0.08674)\end{array}$ & $\begin{array}{l}0.11647 \\
(0.10622)\end{array}$ & $\begin{array}{l}0.08270 \\
(0.09036)\end{array}$ \\
\hline Factor $1,2 \& 3$ & $\begin{array}{l}0.27991 \\
(0.19593)\end{array}$ & $\begin{array}{l}0.30399 \\
(0.19657)\end{array}$ & $\begin{array}{l}0.29969 \\
(0.19677)\end{array}$ & $\begin{array}{l}0.37122^{\ddagger} \\
(0.19500)\end{array}$ & $\begin{array}{l}0.28629 \\
(0.19878)\end{array}$ & $\begin{array}{l}0.30228 \\
(0.19506)\end{array}$ & $\begin{array}{l}0.34400^{\ddagger} \\
(0.19404)\end{array}$ & $\begin{array}{l}0.30507 \\
(0.18933)\end{array}$ & $\begin{array}{l}0.35382^{\ddagger} \\
(0.19523)\end{array}$ & $\begin{array}{l}0.30741 \\
(0.18901)\end{array}$ & $\begin{array}{l}0.32398^{\ddagger} \\
(0.18887)\end{array}$ & $\begin{array}{l}0.38638^{\ddagger} \\
(0.20165)\end{array}$ & $\begin{array}{l}0.37633^{\ddagger} \\
(0.19716)\end{array}$ \\
\hline Factor & $\begin{array}{l}0.20164 \\
(0.19565) \\
\end{array}$ & $\begin{array}{l}0.22061 \\
(0.19815) \\
\end{array}$ & $\begin{array}{l}0.21851 \\
(0.19631) \\
\end{array}$ & $\begin{array}{l}0.23964 \\
(0.19035) \\
\end{array}$ & $\begin{array}{l}0.22300 \\
(0.19156) \\
\end{array}$ & $\begin{array}{l}0.21890 \\
(0.19664) \\
\end{array}$ & $\begin{array}{l}0.26062 \\
(0.19563) \\
\end{array}$ & $\begin{array}{l}0.18070 \\
(0.20184) \\
\end{array}$ & $\begin{array}{l}0.26470 \\
(0.19512) \\
\end{array}$ & $\begin{array}{l}0.22009 \\
(0.19090) \\
\end{array}$ & $\begin{array}{l}0.24557 \\
(0.19078) \\
\end{array}$ & $\begin{array}{l}0.29248 \\
(0.19976) \\
\end{array}$ & $\begin{array}{l}0.29295 \\
(0.19872) \\
\end{array}$ \\
\hline Factor $1,2,3 \& 4$ & $\begin{array}{l}0.26751^{*} \\
(0.05950)\end{array}$ & $\begin{array}{l}0.28838^{*} \\
(0.06407) \\
\end{array}$ & $\begin{array}{l}0.28406^{*} \\
(0.06502)\end{array}$ & $\begin{array}{l}0.35019^{*} \\
(0.05823) \\
\end{array}$ & $\begin{array}{l}0.27117^{*} \\
(0.07029) \\
\end{array}$ & $\begin{array}{l}0.29631^{*} \\
(0.03827)\end{array}$ & $\begin{array}{l}0.31726^{*} \\
(0.04617) \\
\end{array}$ & $\begin{array}{l}0.24888^{*} \\
(0.07540) \\
\end{array}$ & $\begin{array}{l}0.33688^{*} \\
(0.06057) \\
\end{array}$ & $\begin{array}{l}0.28900^{*} \\
(0.03864) \\
\end{array}$ & $\begin{array}{l}0.33860^{*} \\
(0.05279) \\
\end{array}$ & $\begin{array}{l}0.37053^{*} \\
(0.08008) \\
\end{array}$ & $\begin{array}{l}0.35607^{*} \\
(0.06475) \\
\end{array}$ \\
\hline All $\mathrm{F}$ & $\begin{array}{l}0.27611^{*} \\
(0.06237)\end{array}$ & $\begin{array}{l}0.29555^{*} \\
(0.06546) \\
\end{array}$ & $\begin{array}{l}0.29278^{*} \\
(0.06328) \\
\end{array}$ & $\begin{array}{l}0.33621^{*} \\
(0.05271) \\
\end{array}$ & $\begin{array}{l}0.28060^{*} \\
(0.06945) \\
\end{array}$ & $\begin{array}{l}0.29607^{*} \\
(0.05680)\end{array}$ & $\begin{array}{l}0.32403^{*} \\
(0.05341) \\
\end{array}$ & $\begin{array}{l}0.25665^{*} \\
(0.07802) \\
\end{array}$ & $\begin{array}{l}0.32834^{*} \\
(0.05386) \\
\end{array}$ & $\begin{array}{l}0.27357^{*} \\
(0.05790) \\
\end{array}$ & $\begin{array}{l}0.32108^{*} \\
(0.04757) \\
\end{array}$ & $\begin{array}{l}0.37612^{*} \\
(0.08367) \\
\end{array}$ & $\begin{array}{l}0.36319^{*} \\
(0.07040) \\
\end{array}$ \\
\hline $\begin{array}{l}\text { Individual } \\
\text { Variable }\end{array}$ & $\begin{array}{l}0.03483 \\
(0.05178)\end{array}$ & $\begin{array}{l}0.01074 \\
(0.05349)\end{array}$ & $\begin{array}{l}0.01505 \\
(0.05429)\end{array}$ & $\begin{array}{l}-0.05648 \\
(0.04962)\end{array}$ & $\begin{array}{l}0.02844 \\
(0.06135)\end{array}$ & $(0.04768)$ & $\begin{array}{l}-0.02927 \\
(0.04406)\end{array}$ & $\begin{array}{l}0.05065 \\
(0.06658)\end{array}$ & $\begin{array}{l}-0.03909 \\
(0.04938)\end{array}$ & $\begin{array}{l}0.03835 \\
(0.04421)\end{array}$ & $\begin{array}{l}-0.04843 \\
(0.04603)\end{array}$ & $\begin{array}{l}-0.07165 \\
(0.07108)\end{array}$ & $\begin{array}{l}-0.06160 \\
(0.05731)\end{array}$ \\
\hline
\end{tabular}

* Significant at the 0.01 level $\dagger$ Significant at the 0.05 level $\$$ Significant at the 0.10 level

Table 100 Coefficients with individual variables for high-level factors - Vehicles Industry 


\begin{tabular}{|c|c|c|c|c|c|c|c|c|c|c|}
\hline $\begin{array}{l}\text { Individual variable } \\
\text { included in the model in } \\
\text { the column }\end{array}$ & 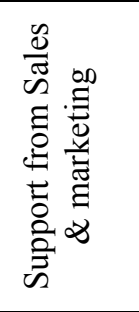 & 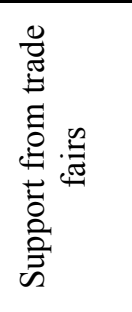 & 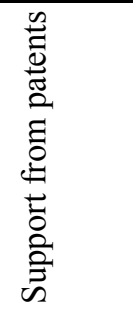 & 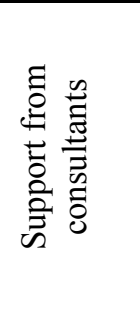 & 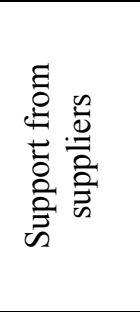 & 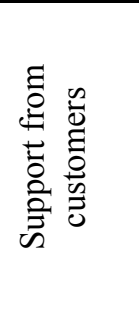 & 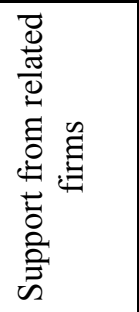 & 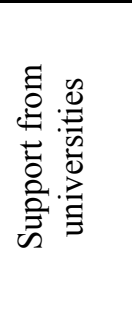 & 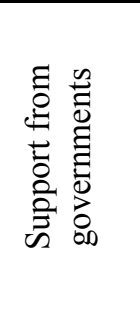 & 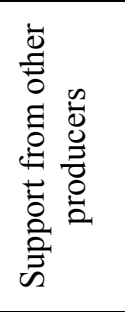 \\
\hline No factors & $\begin{array}{l}0.16753^{*} \\
(0.03720)\end{array}$ & $\begin{array}{l}0.16754^{*} \\
(0.03720)\end{array}$ & $\begin{array}{l}0.16751^{*} \\
(0.03721)\end{array}$ & $\begin{array}{l}0.16754^{*} \\
(0.03719)\end{array}$ & $\begin{array}{l}0.16945^{*} \\
(0.03631)\end{array}$ & $\begin{array}{l}0.16777^{*} \\
(0.03715)\end{array}$ & $\begin{array}{l}0.16579^{*} \\
(0.03707)\end{array}$ & $\begin{array}{l}0.16815^{*} \\
(0.03700)\end{array}$ & $\begin{array}{l}0.16754^{*} \\
(0.03720)\end{array}$ & $\begin{array}{l}0.16754^{*} \\
(0.03665) \\
\end{array}$ \\
\hline Factor 5 & $\begin{array}{l}-0.03159 \\
(0.23109)\end{array}$ & $\begin{array}{l}-0.03115 \\
(0.23084) \\
\end{array}$ & $\begin{array}{l}-0.03185 \\
(0.23227) \\
\end{array}$ & $\begin{array}{l}-0.03115 \\
(0.23080)\end{array}$ & $\begin{array}{l}0.02433 \\
(0.22593) \\
\end{array}$ & $\begin{array}{l}-0.00434 \\
(0.23337) \\
\end{array}$ & $\begin{array}{l}-0.06800 \\
(0.23161) \\
\end{array}$ & $\begin{array}{l}-0.01690 \\
(0.22977) \\
\end{array}$ & $\begin{array}{l}-0.02931 \\
(0.23112) \\
\end{array}$ & $\begin{array}{l}-0.03115 \\
(0.22742) \\
\end{array}$ \\
\hline Factor 4 & $\begin{array}{l}-0.13851 \\
(0.27391)\end{array}$ & $\begin{array}{l}-0.13770 \\
(0.27318)\end{array}$ & $\begin{array}{l}-0.13770 \\
(0.27318)\end{array}$ & $\begin{array}{l}-0.13185 \\
(0.27405)\end{array}$ & $\begin{array}{l}-0.05999 \\
(0.26768)\end{array}$ & $\begin{array}{l}-0.10863 \\
(0.27564)\end{array}$ & $\begin{array}{l}-0.17183 \\
(0.27330)\end{array}$ & $\begin{array}{l}-0.13770 \\
(0.27169)\end{array}$ & $\begin{array}{l}-0.13512 \\
(0.27366)\end{array}$ & $\begin{array}{l}-0.13770 \\
(0.26913)\end{array}$ \\
\hline Factor $4 \& 5$ & $\begin{array}{l}-0.01967 \\
(0.22345)\end{array}$ & $\begin{array}{l}-0.01926 \\
(0.22322)\end{array}$ & $\begin{array}{l}-0.02013 \\
(0.22551)\end{array}$ & $\begin{array}{l}-0.01926 \\
(0.22319)\end{array}$ & $\begin{array}{l}0.00668 \\
(0.21798)\end{array}$ & $\begin{array}{l}0.00291 \\
(0.22494)\end{array}$ & $\begin{array}{l}-0.03649 \\
(0.22270) \\
\end{array}$ & $\begin{array}{l}-0.01926 \\
(0.22201)\end{array}$ & $\begin{array}{l}-0.01754 \\
(0.22348)\end{array}$ & $\begin{array}{l}0.00253 \\
(0.22009) \\
\end{array}$ \\
\hline Factor 3 & $\begin{array}{l}-0.40852^{*} \\
(0.09190)\end{array}$ & $\begin{array}{l}-0.40647^{*} \\
(0.07684) \\
\end{array}$ & $\begin{array}{l}-0.40810^{*} \\
(0.09749) \\
\end{array}$ & $\begin{array}{l}-0.40647^{*} \\
(0.07683)\end{array}$ & $\begin{array}{l}-0.36051^{*} \\
(0.07634)\end{array}$ & $\begin{array}{l}-0.36659^{*} \\
(0.09383)\end{array}$ & $\begin{array}{l}-0.49221^{*} \\
(0.10081)\end{array}$ & $\begin{array}{l}-0.34014^{*} \\
(0.08822) \\
\end{array}$ & $\begin{array}{l}-0.40647^{*} \\
(0.07684)\end{array}$ & $\begin{array}{l}-0.40647^{*} \\
(0.07570) \\
\end{array}$ \\
\hline Factor $3 \& 5$ & $\begin{array}{l}0.14979 \\
(0.16334)\end{array}$ & $\begin{array}{l}0.15062 \\
(0.16207)\end{array}$ & $\begin{array}{l}0.14916 \\
(0.17071) \\
\end{array}$ & $\begin{array}{l}0.15062 \\
(0.16204)\end{array}$ & $\begin{array}{l}0.26604 \\
(0.16218)\end{array}$ & $\begin{array}{l}0.19220 \\
(0.17136)\end{array}$ & $\begin{array}{l}0.06488 \\
(0.17423)\end{array}$ & $\begin{array}{l}0.19720 \\
(0.16413)\end{array}$ & $\begin{array}{l}0.15572 \\
(0.16532)\end{array}$ & $\begin{array}{l}0.24756 \\
(0.16431)\end{array}$ \\
\hline Factor $3 \& 4$ & \multicolumn{10}{|c|}{ Insufficient Data } \\
\hline Factor $3,4 \& 5$ & $\begin{array}{l}-0.00325 \\
(0.29431)\end{array}$ & $\begin{array}{l}-0.00325 \\
(0.29431)\end{array}$ & $\begin{array}{l}-0.00488 \\
(0.30037)\end{array}$ & $\begin{array}{l}0.00188 \\
(0.29492)\end{array}$ & $\begin{array}{l}-0.00325 \\
(0.28721)\end{array}$ & $\begin{array}{l}0.03833 \\
(0.29927)\end{array}$ & $\begin{array}{l}-0.08899 \\
(0.30036) \\
\end{array}$ & $\begin{array}{l}0.03991 \\
(0.29411)\end{array}$ & $\begin{array}{l}-0.00026 \\
(0.29492)\end{array}$ & $\begin{array}{l}-0.00325 \\
(0.28995) \\
\end{array}$ \\
\hline Factor 2 & $\begin{array}{l}0.24973 \\
(0.24284)\end{array}$ & $\begin{array}{l}0.25056 \\
(0.24413)\end{array}$ & $\begin{array}{l}0.24973 \\
(0.24285)\end{array}$ & $\begin{array}{l}0.24973 \\
(0.24281)\end{array}$ & $\begin{array}{l}0.31113 \\
(0.23775)\end{array}$ & $\begin{array}{l}0.26951 \\
(0.24400)\end{array}$ & $\begin{array}{l}0.20895 \\
(0.24385)\end{array}$ & $\begin{array}{l}0.28128 \\
(0.24243)\end{array}$ & $\begin{array}{l}0.24973 \\
(0.24283)\end{array}$ & $\begin{array}{l}0.27551 \\
(0.23947)\end{array}$ \\
\hline Factor $2 \& 5$ & $\begin{array}{l}0.17377 \\
(0.35302)\end{array}$ & $\begin{array}{l}0.17480 \\
(0.35211)\end{array}$ & $\begin{array}{l}0.17398 \\
(0.35339)\end{array}$ & $\begin{array}{l}0.17480 \\
(0.35206)\end{array}$ & $\begin{array}{l}0.23935 \\
(0.34420)\end{array}$ & $\begin{array}{l}0.19559 \\
(0.35277)\end{array}$ & $\begin{array}{l}0.08906 \\
(0.35675)\end{array}$ & $\begin{array}{l}0.17480 \\
(0.35020)\end{array}$ & $\begin{array}{l}0.17480 \\
(0.35209)\end{array}$ & $\begin{array}{l}0.22901 \\
(0.34758)\end{array}$ \\
\hline Factor $2 \& 4$ & $\begin{array}{l}0.24946^{\ddagger} \\
(0.13905) \\
\end{array}$ & $\begin{array}{l}0.24962^{\ddagger} \\
(0.13900)\end{array}$ & $\begin{array}{l}0.24911^{\ddagger} \\
(0.14025) \\
\end{array}$ & $\begin{array}{l}0.24962^{\ddagger} \\
(0.13897) \\
\end{array}$ & $\begin{array}{l}0.25967^{\ddagger} \\
(0.13568)\end{array}$ & $\begin{array}{l}0.24962^{\ddagger} \\
(0.13881) \\
\end{array}$ & $\begin{array}{l}0.24962^{\ddagger} \\
(0.13843) \\
\end{array}$ & $\begin{array}{l}0.24962^{\ddagger} \\
(0.13824) \\
\end{array}$ & $\begin{array}{l}0.24962^{\ddagger} \\
(0.13899) \\
\end{array}$ & $\begin{array}{l}0.24962^{\ddagger} \\
(0.13694) \\
\end{array}$ \\
\hline Factor $2,4 \& 5$ & $\begin{array}{l}0.19283^{\ddagger} \\
(0.11020) \\
\end{array}$ & $\begin{array}{l}0.19321^{\ddagger} \\
(0.10980)\end{array}$ & $\begin{array}{l}0.19159 \\
(0.12513)\end{array}$ & $\begin{array}{l}0.19464^{\sharp} \\
(0.10992)\end{array}$ & $\begin{array}{l}0.19949^{\ddagger} \\
(0.10717)\end{array}$ & $\begin{array}{l}0.20130^{\ddagger} \\
(0.11020)\end{array}$ & $\begin{array}{l}0.18488^{\ddagger} \\
(0.10954)\end{array}$ & $\begin{array}{l}0.20228^{\ddagger} \\
(0.10937)\end{array}$ & $\begin{array}{l}0.19438^{\ddagger} \\
(0.11005)\end{array}$ & $\begin{array}{l}0.20803^{\ddagger} \\
(0.10834)\end{array}$ \\
\hline Factor $2 \& 3$ & $\begin{array}{l}0.29743^{\dagger} \\
(0.12873)\end{array}$ & $\begin{array}{l}0.29743^{\dagger} \\
(0.12873)\end{array}$ & $\begin{array}{l}0.29580^{\dagger} \\
(0.14202)\end{array}$ & $\begin{array}{l}0.29743^{\dagger} \\
(0.12871)\end{array}$ & $\begin{array}{l}0.42653^{*} \\
(0.13189)\end{array}$ & $\begin{array}{l}0.33901^{\dagger} \\
(0.14034)\end{array}$ & $\begin{array}{l}0.21170 \\
(0.14402)\end{array}$ & $\begin{array}{l}0.36376^{*} \\
(0.13540)\end{array}$ & $\begin{array}{l}0.29743^{\dagger} \\
(0.12872)\end{array}$ & $\begin{array}{l}0.29743^{\dagger} \\
(0.12682)\end{array}$ \\
\hline Factor $2,3 \& 5$ & $\begin{array}{l}0.21120 \\
(0.49796)\end{array}$ & $\begin{array}{l}0.21120 \\
(0.49797)\end{array}$ & $\begin{array}{l}0.20958 \\
(0.50157)\end{array}$ & $\begin{array}{l}0.22590 \\
(0.50104)\end{array}$ & $\begin{array}{l}0.34030 \\
(0.48760)\end{array}$ & $\begin{array}{l}0.25278 \\
(0.50048)\end{array}$ & $\begin{array}{l}0.12547 \\
(0.50024)\end{array}$ & $\begin{array}{l}0.27753 \\
(0.49721)\end{array}$ & $\begin{array}{l}0.21120 \\
(0.49794)\end{array}$ & $\begin{array}{l}0.31963 \\
(0.49251)\end{array}$ \\
\hline Factor $2,3 \& 4$ & $\begin{array}{l}0.26392^{*} \\
(0.06715)\end{array}$ & $\begin{array}{l}0.26588^{*} \\
(0.04687)\end{array}$ & $\begin{array}{l}0.26426^{*} \\
(0.07572)\end{array}$ & $\begin{array}{l}0.27976^{*} \\
(0.07082)\end{array}$ & $\begin{array}{l}0.39383^{*} \\
(0.06065)\end{array}$ & $\begin{array}{l}0.30709^{*} \\
(0.07282)\end{array}$ & $\begin{array}{l}0.18166^{\dagger} \\
(0.07959)\end{array}$ & $\begin{array}{l}0.32341^{*} \\
(0.06028)\end{array}$ & $\begin{array}{l}0.26603^{*} \\
(0.04688)\end{array}$ & $\begin{array}{l}0.35685^{*} \\
(0.05881)\end{array}$ \\
\hline Factor2, & $\begin{array}{l}0.30717^{\dagger} \\
(0.14228)\end{array}$ & $\begin{array}{l}0.30883^{\dagger} \\
(0.14136)\end{array}$ & $\begin{array}{l}0.30674^{\dagger} \\
(0.14998)\end{array}$ & $\begin{array}{l}0.31786^{\dagger} \\
(0.14481)\end{array}$ & $\begin{array}{l}0.40667^{*} \\
(0.14005)\end{array}$ & $\begin{array}{l}0.34649^{\dagger} \\
(0.14914)\end{array}$ & $\begin{array}{l}0.23602 \\
(0.15000)\end{array}$ & $\begin{array}{l}0.35709^{\dagger} \\
(0.14301)\end{array}$ & $\begin{array}{l}0.31450^{\dagger} \\
(0.14573)\end{array}$ & $\begin{array}{l}0.36240^{\dagger} \\
(0.13965)\end{array}$ \\
\hline Factor 1 & $\begin{array}{l}0.54597^{*} \\
(0.09072)\end{array}$ & $\begin{array}{l}0.54610^{*} \\
(0.09067)\end{array}$ & $\begin{array}{l}0.54593^{*} \\
(0.09089)\end{array}$ & $\begin{array}{l}0.54781^{*} \\
(0.09089)\end{array}$ & $\begin{array}{l}0.56681^{*} \\
(0.08871)\end{array}$ & $\begin{array}{l}0.54838^{*} \\
(0.09060)\end{array}$ & $\begin{array}{l}0.54140^{*} \\
(0.09037)\end{array}$ & $\begin{array}{l}0.54610^{*} \\
(0.09017)\end{array}$ & $\begin{array}{l}0.54610^{*} \\
(0.09066)\end{array}$ & $\begin{array}{l}0.55204^{*} \\
(0.08936)\end{array}$ \\
\hline Factor $1 \& 5$ & $\begin{array}{l}0.19687 \\
(0.17474) \\
\end{array}$ & $\begin{array}{l}0.19793 \\
(0.17277) \\
\end{array}$ & $\begin{array}{l}0.19702 \\
(0.17601) \\
\end{array}$ & $\begin{array}{l}0.20086 \\
(0.17311) \\
\end{array}$ & $\begin{array}{l}0.29595^{\ddagger} \\
(0.17134) \\
\end{array}$ & $\begin{array}{l}0.22950 \\
(0.17776) \\
\end{array}$ & $\begin{array}{l}0.13958 \\
(0.17776) \\
\end{array}$ & $\begin{array}{l}0.25628 \\
(0.17615) \\
\end{array}$ & $\begin{array}{l}0.19896 \\
(0.17289) \\
\end{array}$ & $\begin{array}{l}0.19793 \\
(0.17021) \\
\end{array}$ \\
\hline Factor $1 \& 4$ & $\begin{array}{l}0.24312^{\ddagger} \\
(0.14088)\end{array}$ & $\begin{array}{l}0.24451^{\ddagger} \\
(0.14275)\end{array}$ & $\begin{array}{l}0.24306^{\ddagger} \\
(0.14192)\end{array}$ & $\begin{array}{l}0.24614^{\sharp} \\
(0.14062)\end{array}$ & $\begin{array}{l}0.26745^{\ddagger} \\
(0.13712)\end{array}$ & $\begin{array}{l}0.24800^{\ddagger} \\
(0.14025)\end{array}$ & $\begin{array}{l}0.22220 \\
(0.14069)\end{array}$ & $\begin{array}{l}0.25417^{\ddagger} \\
(0.13972)\end{array}$ & $\begin{array}{l}0.24364^{\ddagger} \\
(0.14030)\end{array}$ & $\begin{array}{l}0.25224^{4} \\
(0.13828)\end{array}$ \\
\hline Factor $1,4 \& 5$ & $\begin{array}{l}0.25631^{*} \\
(0.07462)\end{array}$ & $\begin{array}{l}0.25731^{*} \\
(0.07043) \\
\end{array}$ & $\begin{array}{l}0.25639^{*} \\
(0.07816) \\
\end{array}$ & $\begin{array}{l}0.25731^{*} \\
(0.07041)\end{array}$ & $\begin{array}{l}0.29161^{*} \\
(0.06955) \\
\end{array}$ & $\begin{array}{l}0.28468^{*} \\
(0.07949) \\
\end{array}$ & $\begin{array}{l}0.20806^{*} \\
(0.07962) \\
\end{array}$ & $\begin{array}{l}0.28043^{*} \\
(0.07171) \\
\end{array}$ & $\begin{array}{l}0.25748^{*} \\
(0.07043) \\
\end{array}$ & $\begin{array}{l}0.30304^{*} \\
(0.07176) \\
\end{array}$ \\
\hline Factor $1 \& 3$ & $\begin{array}{l}0.04133 \\
(0.12501)\end{array}$ & $\begin{array}{l}0.04306 \\
(0.11756)\end{array}$ & $\begin{array}{l}0.04143 \\
(0.13198)\end{array}$ & $\begin{array}{l}0.04306 \\
(0.11754)\end{array}$ & $\begin{array}{l}0.11454 \\
(0.11686)\end{array}$ & $\begin{array}{l}0.08464 \\
(0.13020)\end{array}$ & $\begin{array}{l}-0.04267 \\
(0.13421)\end{array}$ & $\begin{array}{l}0.04306 \\
(0.11691)\end{array}$ & $\begin{array}{l}0.04306 \\
(0.11755)\end{array}$ & $\begin{array}{l}0.04306 \\
(0.11581)\end{array}$ \\
\hline Factor $1,3 \& 5$ & $\begin{array}{l}0.10133 \\
(0.10728)\end{array}$ & $\begin{array}{l}0.10338 \\
(0.09470)\end{array}$ & $\begin{array}{l}0.10175 \\
(0.11211)\end{array}$ & $\begin{array}{l}0.10391 \\
(0.09471)\end{array}$ & $\begin{array}{l}0.11423 \\
(0.09248)\end{array}$ & $\begin{array}{l}0.10687 \\
(0.09470)\end{array}$ & $\begin{array}{l}0.01765 \\
(0.11490)\end{array}$ & $\begin{array}{l}0.10895 \\
(0.09426)\end{array}$ & $\begin{array}{l}0.10410 \\
(0.09481)\end{array}$ & $\begin{array}{l}0.10857 \\
(0.09333)\end{array}$ \\
\hline Factor $1,3 \& 4$ & $\begin{array}{l}0.19284 \\
(0.12506)\end{array}$ & $\begin{array}{l}0.19427 \\
(0.12002)\end{array}$ & $\begin{array}{l}0.19277 \\
(0.13218)\end{array}$ & $\begin{array}{l}0.19598 \\
(0.12017)\end{array}$ & $\begin{array}{l}0.29479^{\dagger} \\
(0.12123)\end{array}$ & $\begin{array}{l}0.22907^{\ddagger} \\
(0.12878)\end{array}$ & $\begin{array}{l}0.14823 \\
(0.12461)\end{array}$ & $\begin{array}{l}0.20198^{\ddagger} \\
(0.11947)\end{array}$ & $\begin{array}{l}0.19477 \\
(0.12005)\end{array}$ & $\begin{array}{l}0.25019^{\dagger} \\
(0.12034)\end{array}$ \\
\hline Factor $1,3,4 \& 5$ & $\begin{array}{l}0.16554^{\ddagger} \\
(0.09095)\end{array}$ & $\begin{array}{l}0.16737^{\dagger} \\
(0.07909)\end{array}$ & $\begin{array}{l}0.16574^{\ddagger} \\
(0.09927)\end{array}$ & $\begin{array}{l}0.16929^{\dagger} \\
(0.07942)\end{array}$ & $\begin{array}{l}0.19331^{\dagger} \\
(0.07760)\end{array}$ & $\begin{array}{l}0.20333^{\dagger} \\
(0.09278)\end{array}$ & $\begin{array}{l}0.08379 \\
(0.10146)\end{array}$ & $\begin{array}{l}0.17794^{\dagger} \\
(0.07897)\end{array}$ & $\begin{array}{l}0.16830^{\dagger} \\
(0.07931)\end{array}$ & $\begin{array}{l}0.18554^{\dagger} \\
(0.07825)\end{array}$ \\
\hline Factor $1 \& 2$ & $\begin{array}{l}0.15120 \\
(0.17434)\end{array}$ & $\begin{array}{l}0.15120 \\
(0.17434) \\
\end{array}$ & $\begin{array}{l}0.15080 \\
(0.17496) \\
\end{array}$ & $\begin{array}{l}0.15300 \\
(0.17445)\end{array}$ & $\begin{array}{l}0.18284 \\
(0.17042)\end{array}$ & $\begin{array}{l}0.16139 \\
(0.17466)\end{array}$ & $\begin{array}{l}0.15120 \\
(0.17362)\end{array}$ & $\begin{array}{l}0.16746 \\
(0.17373)\end{array}$ & $\begin{array}{l}0.15120 \\
(0.17433)\end{array}$ & $\begin{array}{l}0.15120 \\
(0.17176)\end{array}$ \\
\hline Factor $1,2 \& 5$ & $\begin{array}{l}0.24459 \\
(0.16813) \\
\end{array}$ & $\begin{array}{l}0.24483 \\
(0.16803) \\
\end{array}$ & $\begin{array}{l}0.24338 \\
(0.17624) \\
\end{array}$ & $\begin{array}{l}0.24483 \\
(0.16800) \\
\end{array}$ & $\begin{array}{l}0.33601^{\dagger} \\
(0.16641)\end{array}$ & $\begin{array}{l}0.26473 \\
(0.16995) \\
\end{array}$ & $\begin{array}{l}0.18427 \\
(0.17364) \\
\end{array}$ & $\begin{array}{l}0.28412^{\ddagger} \\
(0.16914) \\
\end{array}$ & $\begin{array}{l}0.24483 \\
(0.16802) \\
\end{array}$ & $\begin{array}{l}0.25717 \\
(0.16561) \\
\end{array}$ \\
\hline Factor $1,2 \& 4$ & $\begin{array}{l}0.21080 \\
(0.18606)\end{array}$ & $\begin{array}{l}0.21185 \\
(0.18611)\end{array}$ & $\begin{array}{l}0.21069 \\
(0.18721)\end{array}$ & $\begin{array}{l}0.21994 \\
(0.18838)\end{array}$ & $\begin{array}{l}0.30462^{\ddagger} \\
(0.18337)\end{array}$ & $\begin{array}{l}0.24718 \\
(0.19152)\end{array}$ & $\begin{array}{l}0.16376 \\
(0.18833)\end{array}$ & $\begin{array}{l}0.25007 \\
(0.18630)\end{array}$ & $\begin{array}{l}0.21517 \\
(0.18712)\end{array}$ & $\begin{array}{l}0.25958 \\
(0.18380)\end{array}$ \\
\hline Factor $1,2,4 \& 5$ & $\begin{array}{l}0.05407 \\
(0.08667)\end{array}$ & $\begin{array}{l}0.05419 \\
(0.08662)\end{array}$ & $\begin{array}{l}0.05372 \\
(0.08837)\end{array}$ & $\begin{array}{l}0.05553 \\
(0.08675)\end{array}$ & $\begin{array}{l}0.12607 \\
(0.08744)\end{array}$ & $\begin{array}{l}0.08746 \\
(0.09753)\end{array}$ & $\begin{array}{l}-0.00319 \\
(0.09680)\end{array}$ & $\begin{array}{l}0.09850 \\
(0.09104)\end{array}$ & $\begin{array}{l}0.05464 \\
(0.08666)\end{array}$ & $\begin{array}{l}0.05853 \\
(0.08535)\end{array}$ \\
\hline Factor $1,2 \& 3$ & $\begin{array}{l}0.31308 \\
(0.19352)\end{array}$ & $\begin{array}{l}0.31473^{\ddagger} \\
(0.18917) \\
\end{array}$ & $\begin{array}{l}0.31311 \\
(0.19846) \\
\end{array}$ & $\begin{array}{l}0.31473^{\ddagger} \\
(0.18914)\end{array}$ & $\begin{array}{l}0.33938^{\ddagger} \\
(0.18476)\end{array}$ & $\begin{array}{l}0.32267^{\ddagger} \\
(0.18923)\end{array}$ & $\begin{array}{l}0.22900 \\
(0.19949)\end{array}$ & $\begin{array}{l}0.38106^{\dagger} \\
(0.19323)\end{array}$ & $\begin{array}{l}0.31637^{\ddagger} \\
(0.18945)\end{array}$ & $\begin{array}{l}0.31473^{\ddagger} \\
(0.18637) \\
\end{array}$ \\
\hline Factor $1,2,3 \& 5$ & $\begin{array}{l}0.22960 \\
(0.19559)\end{array}$ & $\begin{array}{l}0.23135 \\
(0.19081)\end{array}$ & $\begin{array}{l}0.22972 \\
(0.20002)\end{array}$ & $\begin{array}{l}0.23567 \\
(0.19149)\end{array}$ & $\begin{array}{l}0.32254^{\ddagger} \\
(0.18835)\end{array}$ & $\begin{array}{l}0.27293 \\
(0.19869)\end{array}$ & $\begin{array}{l}0.15820 \\
(0.19810)\end{array}$ & $\begin{array}{l}0.26846 \\
(0.19136)\end{array}$ & $\begin{array}{l}0.23865 \\
(0.19644)\end{array}$ & $\begin{array}{l}0.29202 \\
(0.18954)\end{array}$ \\
\hline Factor $1,2,3 \& 4$ & $\begin{array}{l}0.29701^{*} \\
(0.05906)\end{array}$ & $\begin{array}{l}0.29915^{*} \\
(0.03784) \\
\end{array}$ & $\begin{array}{l}0.29738^{*} \\
(0.06659) \\
\end{array}$ & $\begin{array}{l}0.30061^{*} \\
(0.03757)\end{array}$ & $\begin{array}{l}0.35955^{*} \\
(0.04074)\end{array}$ & $\begin{array}{l}0.34007^{*} \\
(0.06688) \\
\end{array}$ & $\begin{array}{l}0.21487^{*} \\
(0.07411) \\
\end{array}$ & $\begin{array}{l}0.33418^{*} \\
(0.04363)\end{array}$ & $\begin{array}{l}0.30056^{*} \\
(0.03853) \\
\end{array}$ & $\begin{array}{l}0.35043^{*} \\
(0.04188) \\
\end{array}$ \\
\hline All Factors & $\begin{array}{l}0.30408^{*} \\
(0.05125)\end{array}$ & $\begin{array}{l}0.30518^{*} \\
(0.04532)\end{array}$ & $\begin{array}{l}0.30362^{*} \\
(0.06986)\end{array}$ & $\begin{array}{l}0.31017^{*} \\
(0.04922)\end{array}$ & $\begin{array}{l}0.36222^{*} \\
(0.04755)\end{array}$ & $\begin{array}{l}0.33101^{*} \\
(0.05719)\end{array}$ & $\begin{array}{l}0.22359^{*} \\
(0.07690)\end{array}$ & $\begin{array}{l}0.35784^{*} \\
(0.05701)\end{array}$ & $\begin{array}{l}0.30810^{*} \\
(0.04921)\end{array}$ & $\begin{array}{l}0.34242^{*} \\
(0.04697)\end{array}$ \\
\hline $\begin{array}{l}\text { Individual } \\
\text { Variable }\end{array}$ & $\begin{array}{l}0.00205 \\
(0.05041)\end{array}$ & $\begin{array}{l}-0.00349 \\
(0.10507)\end{array}$ & $\begin{array}{l}0.00163 \\
(0.06000)\end{array}$ & $\begin{array}{l}-0.01470 \\
(0.05619)\end{array}$ & $\begin{array}{l}-0.12910^{*} \\
(0.04019)\end{array}$ & $\begin{array}{l}-0.04158 \\
(0.05629)\end{array}$ & $\begin{array}{l}0.08573 \\
(0.06562)\end{array}$ & $\begin{array}{l}-0.06633 \\
(0.04407)\end{array}$ & $\begin{array}{l}-0.00856 \\
(0.05478)\end{array}$ & $\begin{array}{l}-0.10842^{\dagger} \\
(0.04340)\end{array}$ \\
\hline
\end{tabular}

$*$ Significant at the 0.01 level $\quad \dagger$ Significant at the 0.05 level $\$$ Significant at the 0.10 level

\section{Table 100 Coefficients with individual variables for high-level factors - Vehicles Industry}




\subsubsection{Wood Industry}

The analysis of the wood industry is more similar to that of the medium plants than to any of the other industry analyses. There are still a small number of states that have insignificant coefficients but the significant coefficients on the individual variables are much smaller. On average, the significant coefficients on the individual variables are only $15 \%-30 \%$ of the coefficients for the state variables. The sample size of the wood industry is the largest for any of the industries analysed that may have contributed to these results.

There are a few states that have insignificant coefficients in the analysis of the AMT model (refer to Table 101). The state consisting of the inspection and storage and the robotics factors, the production design technologies and the robotics factors, and the production design, inspection and storage, and the robotics factors all have insignificant coefficients. These results demonstrate that the use of robotics is not common or very important in the wood industry, and that once again, robotics are only implemented after many other forms of AMT have been integrated into the manufacturing process.

There are a large number of individual AMT variables that have significant coefficients. The use of modeling and simulations, EDI of CAM designs, lasers, robots with sensing, and rapid prototyping all have positive coefficients while the use of CAD/CAE, CIM, and SCADA all have negative coefficients. These results demonstrate that the use of the smaller, more design oriented forms of technology are understated by the factor state variables but that the more complex and integrated technologies have over stated benefits.

The analysis of the business practices using the individual and state variables has no insignificant states (refer to Table 102). There are, however, a number of individual variables that have 
negative coefficients that are significant. The use of plant certifications, JIT inventory control, statistical process control, and electronic work orders all have negative coefficients that are $20 \%$ $30 \%$ of the coefficients for the state variables. These results are very similar to those found in the vehicles industry and medium plant analysis.

The model of the sources of implementation support has only one state with an insignificant coefficient, consisting of the research and development group, the external information sources and the corporate organizations (refer to Table 103). There are also a large number of individual variables that have significant coefficients. The use of the corporate head office, the design staff, sales and marketing, suppliers, related firms, and universities all have negative coefficients. These results demonstrate that the makeup of the factors has an effect on the value that the state should possess and that the determination of the state of a plant may require some form of weighted decision and not a simple use of the average value for the factor. Once again, the use of the government and the use of other producers for implementation support have positive coefficients.

The model of the perceived benefits has only one state with an insignificant coefficient, the state consisting of improved time usage (refer to Table 104). The perceived benefit of reduced materials, reduced time to market, increased quality, and increased profitability all have negative coefficients that are significant. The perceived benefit of reduced rejection time and increased skill requirement both have positive and significant coefficients. All of these coefficients are relatively small as they are only $10 \%-30 \%$ of the state variable coefficients.

The analysis of the high-level factor model using the state variables and individual variables has only four states with insignificant coefficients; the state consisting of the implementation support and research and development factor, the state consisting of the AMT 
and business practices and the human resources factor, the state consisting of the AMT and business practices, design, and human resources factor, and the state consisting of the perceived benefits, design, and human resources factor (refer to Table 105). These results demonstrate that the use of the human resources techniques is not common in the wood industry.

The individual AMT variables have similar coefficients to those found in the AMT analysis, as the business practices have with the variables in their respective analysis. This is also true for the implementation support analysis with the exception of support from the research and development group and experimental development. These two variables now have significant coefficients that are negative demonstrating that the effect from $R \& D$ is overstated in the state variables. This is further supported by the fact that both the use of joint R\&D and contracted out R\&D have small negative coefficients that are significant.

Finally, two human resources variables have significant coefficients. Creating stronger links to higher education institutions has a negative coefficient and the search for more skilled personnel has a positive coefficient. In particular, the coefficient for the search for higher skilled personnel is twice as large as that for links to higher education. This may be because most of the labour force in the wood industry would not come from universities or colleges but would be people who undergo an apprenticeship type program after high school and learn most of the required skills while on the job.

The large sample size, and rather even distribution, of the plants in the wood industry helped to reduce the number of states with insignificant coefficients in the analysis. The analysis of the wood industry supports complementarity, as the interaction effects included in the state variables are much larger than any of the significant individual variable coefficients. The only area where there may be a significant overstatement of benefits is with respect to research and 
development since many of the individual variables that are related to $R \& D$ had negative coefficients indicating a consistent overstatement of the benefits from R\&D in the wood industry. As this is considered a low-technology or low-innovation type of industry, this supports the results of the overstatement of benefits from $R \& D$. 


\begin{tabular}{|c|c|c|c|c|c|c|c|c|c|c|c|c|c|c|c|c|c|c|c|c|c|c|c|c|c|}
\hline $\begin{array}{l}\text { Individual variable } \\
\text { included in the } \\
\text { model in the } \\
\text { column }\end{array}$ & 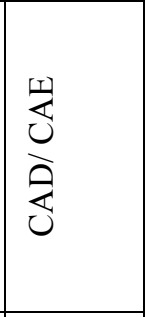 & 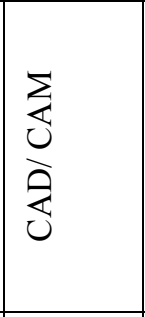 & 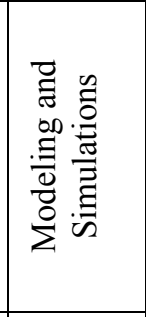 & 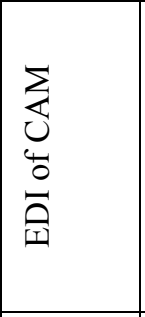 & $\sum_{i=1}^{n}$ & 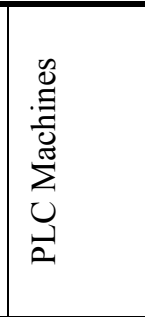 & 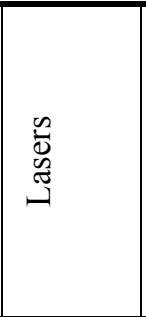 & 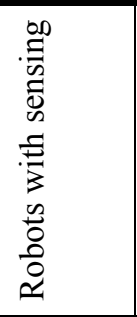 & 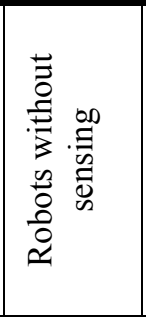 & 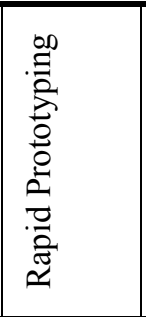 & 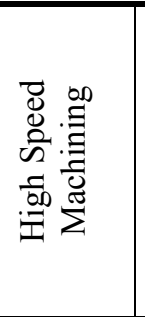 & 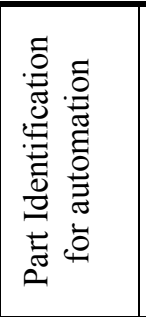 & 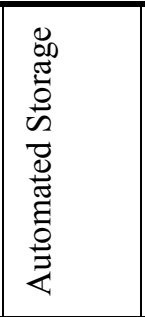 & 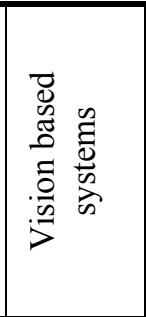 & 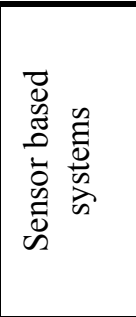 & Z & $\frac{z}{z}$ & 公 & $\stackrel{F}{a}$ & 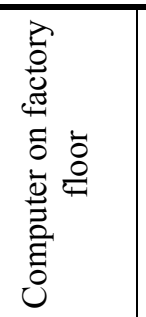 & $\sum$ & 蔗 & 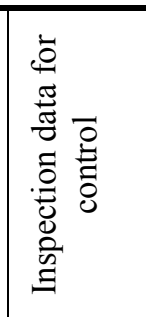 & 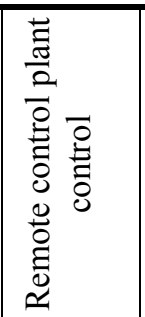 & 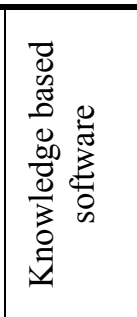 \\
\hline No factors & & & \begin{tabular}{|l|l|}
$0.26629^{*}$ \\
$(0.01363)$ \\
\end{tabular} & & & & & & \begin{tabular}{|l|l|}
$0.26723^{*}$ \\
$(0.01379)$ \\
\end{tabular} & & & & & & \begin{tabular}{|l|}
$0.26732^{*}$ \\
$(0.01380)$ \\
\end{tabular} & & & & & & & & & & \\
\hline tor 4 & & \begin{tabular}{|l|l|}
$0.14257^{*}$ \\
$(0.04593)$ \\
\end{tabular} & 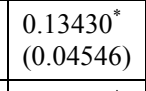 & & & 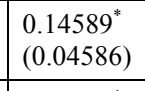 & & & \begin{tabular}{|l|l|}
$0.11611^{\ddagger}$ \\
$(0.06622)$ \\
\end{tabular} & & $\begin{array}{l}4315^{*} \\
44591) \\
\end{array}$ & \begin{tabular}{|l|}
$0.14298^{*}$ \\
$(0.04593)$ \\
\end{tabular} & \begin{tabular}{|l|}
$0.14282^{*}$ \\
$(0.04585)$ \\
\end{tabular} & $\begin{array}{l}14180^{*} \\
04592) \\
\end{array}$ & \begin{tabular}{|l|l}
$0.14282^{*}$ \\
$(0.04595)$ \\
\end{tabular} & & & & & $\begin{array}{l}14618^{\circ} \\
0.4672) \\
\end{array}$ & & & & \begin{tabular}{|l|}
$0.14282^{*}$ \\
$(0.04593)$ \\
\end{tabular} & $\begin{array}{l}14282^{*} \\
.04595) \\
\end{array}$ \\
\hline & & \begin{tabular}{|l|l}
$0.29284^{*}$ \\
$(0.02869)$
\end{tabular} & \begin{tabular}{|l}
$0.29190^{*}$ \\
$(0.02833)$
\end{tabular} & & & \begin{tabular}{|l|l}
$0.30603^{*}$ \\
$(0.02973)$
\end{tabular} & \begin{tabular}{|l|l|}
$219^{*}$ \\
$2852)$ \\
\end{tabular} & & \begin{tabular}{|l|l}
$0.29350^{\circ}$ \\
$(0.02867)$ \\
\end{tabular} & & \begin{tabular}{l|l|}
$9978^{*}$ \\
$92949)$ \\
\end{tabular} & \begin{tabular}{|l|}
$0.31173^{*}$ \\
$(0.04057)$
\end{tabular} & & \begin{tabular}{|l|}
$0.28842^{*}$ \\
$(0.02921)$
\end{tabular} & \begin{tabular}{|l|l|l}
$0.29276^{*}$ \\
$(0.02913)$ \\
\end{tabular} & & & & & $29247^{*}$ & & & & \begin{tabular}{|l|}
$0.29422^{*}$ \\
$(0.02868)$
\end{tabular} & \begin{tabular}{|l|l}
$0.29372^{*}$ \\
$(0.02868)$
\end{tabular} \\
\hline & & & & & & & & & & & & & & & $\begin{array}{l}\begin{array}{l}0.12948 \\
(0.18303)\end{array} \\
\end{array}$ & & & & & & & & & $\begin{array}{l}0.13440 \\
(0.18046)\end{array}$ & \begin{tabular}{|l|l}
$\begin{array}{l}0.13440 \\
(0.18054)\end{array}$ \\
\end{tabular} \\
\hline & & (52557) & $\begin{array}{l}6582^{\circ} \\
6203) \\
\end{array}$ & & 977" & $\begin{array}{l}0.15963^{*} \\
(0.02532) \\
\end{array}$ & $5580^{\circ}$ & \begin{tabular}{|l|}
$7202 *$ \\
$217)$ \\
\end{tabular} & \begin{tabular}{|l|l}
$0.15724^{*}$ \\
$(0.02534)$ \\
\end{tabular} & $\begin{array}{l}5724^{*} \\
72516) \\
\end{array}$ & 25134) & \begin{tabular}{l|}
$5763^{*}$ \\
$02534)$ \\
\end{tabular} & \begin{tabular}{|l|l}
$0.15785^{*}$ \\
$(0.02529)$ \\
\end{tabular} & $\begin{array}{l}5724^{*} \\
02532) \\
\end{array}$ & \begin{tabular}{|l|l}
$0.15720^{*}$ \\
$(0.02535)$ \\
\end{tabular} & & & & & \begin{tabular}{|l}
$0.16169^{*}$ \\
$(0.02774)$
\end{tabular} & & & & & \begin{tabular}{|l}
$0.15979^{*}$ \\
$(0.02822)$ \\
\end{tabular} \\
\hline & & & & & & \begin{tabular}{|l|l|}
$0.27995^{*}$ \\
$(0.06697)$
\end{tabular} & & $\begin{array}{ll}5^{*} \\
711) \\
\end{array}$ & \begin{tabular}{|l|}
$0.27995^{*}$ \\
$(0.06714)$
\end{tabular} & & & \begin{tabular}{|l|}
$0.27995^{*}$ \\
$(0.06713)$ \\
\end{tabular} & & & \begin{tabular}{|l|l}
$0.27995^{*}$ \\
$(0.06716)$ \\
\end{tabular} & & & & & & & & & & \begin{tabular}{|l|l|}
$0.27995^{*}$ \\
$(0.06716)$ \\
\end{tabular} \\
\hline & & & 550" & & & $\begin{array}{l}0.38526^{*} \\
(0.004152) \\
\end{array}$ & 20077) & & \begin{tabular}{|l|}
$0.36917^{*}$ \\
$(0.04029)$ \\
\end{tabular} & & & \begin{tabular}{|l|}
$0.38891^{*}$ \\
$(0.05081)$
\end{tabular} & & & \begin{tabular}{|l|l|}
$0.36762^{*}$ \\
$(0.04167)$ \\
\end{tabular} & & & & & & & & & & $\begin{array}{l}0.37042^{*} \\
(0.04065) \\
\end{array}$ \\
\hline & & & & & & & & & & & & \begin{tabular}{|l|}
$0.49452^{*}$ \\
$(0.04867)$ \\
\end{tabular} & & & & & & & & & & 30) & & & \\
\hline & & $807^{*}$ & $\begin{array}{l}0 \\
(1 \\
\end{array}$ & & $\begin{array}{l}896^{*} \\
96) \\
\end{array}$ & $\begin{array}{l}0.27288^{*} \\
(0.03141) \\
\end{array}$ & 32436) & $2982)$ & $30001)$ & \begin{tabular}{|l|}
$0.25836^{*}$ \\
$(0.02981)$ \\
\end{tabular} & \begin{tabular}{l|l|}
$4^{*}$ \\
$28)$
\end{tabular} & \begin{tabular}{|l|}
$0.25836^{*}$ \\
$(0.03001)$ \\
\end{tabular} & \begin{tabular}{|l|}
$0.25836^{*}$ \\
$(0.02996)$ \\
\end{tabular} & & $\begin{array}{l}0.25835 \\
(0.03002) \\
\end{array}$ & & & & & & & & & & \begin{tabular}{|l}
$0.25840^{*}$ \\
$(0.03002)$ \\
\end{tabular} \\
\hline & & & & & & & & & & & & & & & & & & & & & & & & & \\
\hline artact & & $\begin{array}{l}0 \\
1 \\
\end{array}$ & & & & $\begin{array}{l}0 \\
0 \\
0\end{array}$ & 111) & 5099) & $\begin{array}{l}214^{*} \\
132) \\
\end{array}$ & & 621" & \begin{tabular}{|l|}
$0.23244^{*}$ \\
$(0.05370)$ \\
\end{tabular} & \begin{tabular}{|l|}
$0.22575^{*}$ \\
$(0.05128)$ \\
\end{tabular} & & \begin{tabular}{|l|l}
$0.22215^{*}$ \\
$(0.05137)$ \\
\end{tabular} & & & & & & & & & & \begin{tabular}{|l}
$0.22244^{*}$ \\
$(0.05134)$ \\
\end{tabular} \\
\hline & & & & & & & & & \begin{tabular}{|l|}
0.05626 \\
$(0.19017)$ \\
\end{tabular} & & & & & & & & & & & & & & & & \\
\hline - & & & \begin{tabular}{l|l|}
$0.17325^{*}$ \\
$(0.02984)$
\end{tabular} & & & \begin{tabular}{|l|l}
$0.19748^{*}$ \\
$(0.03177)$
\end{tabular} & \begin{tabular}{|l|}
$0.16500^{*}$ \\
$(0.03085)$ \\
\end{tabular} & & \begin{tabular}{|l|}
$0.18155^{*}$ \\
$(0.03008)$ \\
\end{tabular} & \begin{tabular}{|l|}
$0.18120^{*}$ \\
$(0.02988)$ \\
\end{tabular} & & \begin{tabular}{|l|}
$0.18168^{*}$ \\
$(0.03008)$ \\
\end{tabular} & & & & & & & & & & & & & \\
\hline & & & \begin{tabular}{|l}
$0.33759^{*}$ \\
$(0.04416)$ \\
\end{tabular} & & 2608* & \begin{tabular}{|l|l}
$0.38764^{*}$ \\
$(0.04565)$
\end{tabular} & \begin{tabular}{|l|}
$0.34917^{*}$ \\
$(0.04424)$ \\
\end{tabular} & $4344^{*}$ & \begin{tabular}{|l|}
$0.36625^{*}$ \\
$(0.04375)$ \\
\end{tabular} & \begin{tabular}{|l|}
$0.29599^{*}$ \\
$(0.05227)$ \\
\end{tabular} & \begin{tabular}{|l|l|}
$3776^{\circ}$ \\
$4436)$
\end{tabular} & \begin{tabular}{|l|l}
$0.36719^{*}$ \\
$(0.04372)$ \\
\end{tabular} & $\begin{array}{l}0.36705^{*} \\
(0.04365) \\
\end{array}$ & & $\begin{array}{l}0.36705^{*} \\
(0.04374)\end{array}$ & & & & & & & & & & \\
\hline Factor $1,2 \& 3$ & & & & & & $\begin{array}{l}0.22678^{*} \\
(0.03332)\end{array}$ & & & \begin{tabular}{|l|}
$0.20825^{*}$ \\
$(0.03113)$
\end{tabular} & & & \begin{tabular}{|l|}
$0.22581^{*}$ \\
$(0.04163)$
\end{tabular} & & & $\begin{array}{l}0.20616^{*} \\
(0.03392)\end{array}$ & & & & & & & \begin{tabular}{|l|l}
$0.23656^{*}$ \\
$(0.03274)$
\end{tabular} & & & \\
\hline & & & $\begin{array}{l}0.20610^{*} \\
(0.03236) \\
\end{array}$ & & $\begin{array}{l}0.31271^{*} \\
(0.05222) \\
\end{array}$ & $\begin{array}{l}0.28105^{*} \\
(0.03289) \\
\end{array}$ & \begin{tabular}{|l|l|}
$0.24387^{*}$ \\
$(0.02910)$ \\
\end{tabular} & $\begin{array}{l}0.22632^{*} \\
(0.03121)\end{array}$ & \begin{tabular}{|l|l}
$0.25241^{*}$ \\
$(0.02954)$ \\
\end{tabular} & & $\begin{array}{l}.27367^{\circ} \\
0.03475) \\
\end{array}$ & \begin{tabular}{|l|}
$0.27246^{*}$ \\
$(0.03843)$
\end{tabular} & \begin{tabular}{|l|}
$0.29987^{*}$ \\
$(0.04310)$ \\
\end{tabular} & $\begin{array}{l}0.24232^{*} \\
(0.03258) \\
\end{array}$ & \begin{tabular}{|l|l}
$0.25378^{*}$ \\
$(0.03263)$ \\
\end{tabular} & & & & & & & & & & \\
\hline $\mathrm{I}_{1}$ & .02537) & & $\begin{array}{l}9416^{*} \\
02924) \\
\end{array}$ & $(0.02644)$ & $\begin{array}{c}-0.06062 \\
(0.04692) \\
\end{array}$ & \begin{tabular}{|l|l}
-0.02968 \\
$(0.01942)$ \\
\end{tabular} & 02446) & $\begin{array}{l}0.10102^{\dagger} \\
(0.04267) \\
\end{array}$ & \begin{tabular}{|l|}
0.03061 \\
$(0.05465)$ \\
\end{tabular} & $(0.04600)$ & .02477) & $(0.03360)$ & $(0.04066)$ & $(0.03065)$ & $(0.03008)$ & & & $(0.02106)$ & 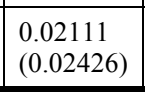 & \begin{tabular}{|l|l}
-0.00862 \\
$(0.02184)$ \\
\end{tabular} & $(0.02297)$ & \begin{tabular}{|l|l}
$-0.0669^{*}$ \\
$(0.02566)$ \\
\end{tabular} & \begin{tabular}{|l|}
0.02295 \\
0.02450 \\
\end{tabular} & $(0.03756)$ & $\mid 0.0250$ \\
\hline
\end{tabular}

Significant at the 0.01 level $\dagger$ Significant at the 0.05 level $\$$ Significant at the 0.10 level

Table 101 Coefficients with individual variables for AMT - Wood Industry 


\begin{tabular}{|c|c|c|c|c|c|c|c|c|c|c|c|c|}
\hline $\begin{array}{l}\text { Individual variable } \\
\text { included in the } \\
\text { model in the } \\
\text { column }\end{array}$ & 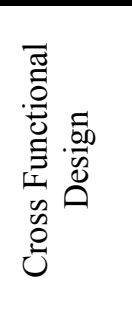 & 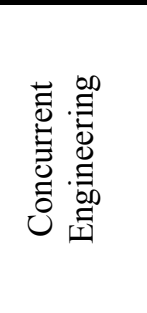 & 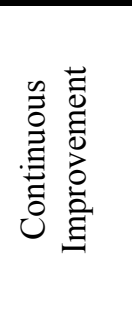 & 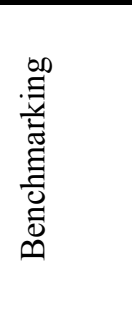 & 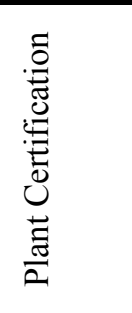 & 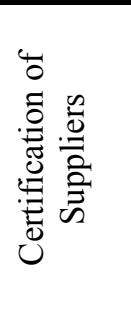 & 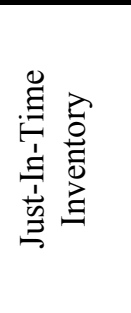 & 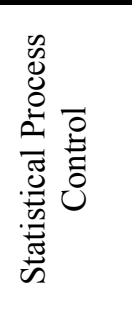 & 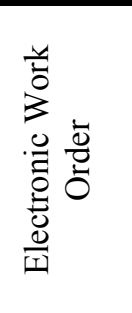 & 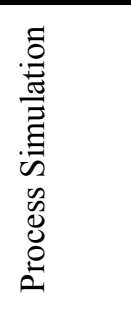 & 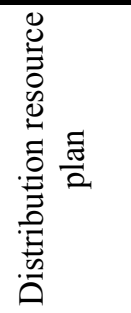 & 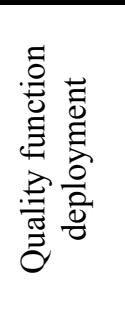 \\
\hline No fa & $\begin{array}{l}0.26095^{*} \\
(0.01352)\end{array}$ & $\begin{array}{l}0.26072^{*} \\
(0.01351)\end{array}$ & $\begin{array}{l}0.26187^{*} \\
(0.01399)\end{array}$ & $\begin{array}{l}0.26103^{*} \\
(0.01362)\end{array}$ & $\begin{array}{l}0.26768^{*} \\
(0.01348)\end{array}$ & $\begin{array}{l}0.26125^{*} \\
(0.0137)\end{array}$ & $\begin{array}{l}0.26731^{*} \\
(0.01375)\end{array}$ & $\begin{array}{l}0.26251^{*} \\
(0.01336)\end{array}$ & $\begin{array}{l}0.26329^{*} \\
(0.01343)\end{array}$ & $\begin{array}{l}0.2606^{*} \\
(0.01352)\end{array}$ & $\begin{array}{l}0.26108^{*} \\
(0.01348)\end{array}$ & $\begin{array}{l}0.26036^{*} \\
(0.01346)\end{array}$ \\
\hline $\mathrm{Fac}$ & $\begin{array}{l}0.22835^{*} \\
(0.04709)\end{array}$ & \begin{tabular}{|l|}
$0.21334^{*}$ \\
$(0.04674)$
\end{tabular} & $\begin{array}{l}.23035^{*} \\
(0.04657)\end{array}$ & $\begin{array}{l}0.22606^{*} \\
(0.04349)\end{array}$ & $\begin{array}{l}0.23709^{*} \\
(0.04286)\end{array}$ & $\begin{array}{l}.22607^{*} \\
(0.04338)\end{array}$ & $\begin{array}{l}.22766^{*} \\
(0.04309)\end{array}$ & $\begin{array}{l}.22902^{*} \\
(0.04281)\end{array}$ & $\begin{array}{l}0.24375^{*} \\
(0.04347)\end{array}$ & $\begin{array}{l}0.22412^{*} \\
(0.04337)\end{array}$ & $\begin{array}{l}.22573^{*} \\
(0.04322)\end{array}$ & $\begin{array}{l}0.22573^{*} \\
(0.04314)\end{array}$ \\
\hline Factor 2 & $\begin{array}{l}0.19419^{*} \\
(0.02841)\end{array}$ & $\begin{array}{l}0.19379^{*} \\
(0.0284)\end{array}$ & $\begin{array}{l}0.19492^{*} \\
(0.02854)\end{array}$ & $\begin{array}{l}0.19428^{*} \\
(0.02844)\end{array}$ & $\begin{array}{l}0.21689^{*} \\
(0.02879)\end{array}$ & $\begin{array}{l}0.19431^{*} \\
(0.02843)\end{array}$ & $\begin{array}{l}0.22145^{*} \\
(0.03081)\end{array}$ & $\begin{array}{l}0.19899^{*} \\
(0.0281)\end{array}$ & $\begin{array}{l}0.21846^{*} \\
(0.02956)\end{array}$ & $\begin{array}{l}0.18944^{*} \\
(0.02927)\end{array}$ & $\begin{array}{l}0.19793^{*} \\
(0.02845)\end{array}$ & $\begin{array}{l}0.1773^{*} \\
(0.02959)\end{array}$ \\
\hline Facto & $\begin{array}{l}0.27213^{*} \\
(0.03936)\end{array}$ & \begin{tabular}{|l|}
$0.26271^{*}$ \\
$(0.03545)$
\end{tabular} & $\begin{array}{l}.27327^{*} \\
(0.03712)\end{array}$ & $\begin{array}{l}0.26974^{*} \\
(0.03445)\end{array}$ & $\begin{array}{l}0.27089^{*} \\
(0.03372)\end{array}$ & $\begin{array}{l}0.26954^{*} \\
(0.03421)\end{array}$ & $\begin{array}{l}0.30682^{*} \\
(0.03796)\end{array}$ & $\begin{array}{l}.27143^{*} \\
(0.03378)\end{array}$ & $\begin{array}{l}0.29969^{*} \\
(0.0357)\end{array}$ & $\begin{array}{l}0.26912^{*} \\
(0.03417)\end{array}$ & $\begin{array}{l}0.27606^{*} \\
(0.0344)\end{array}$ & $\begin{array}{l}0.24775^{*} \\
(0.03581)\end{array}$ \\
\hline $\mathrm{F}$ & $\begin{array}{l}0.14334^{*} \\
(0.03362)\end{array}$ & \begin{tabular}{|l|}
$0.1426^{*}$ \\
$(0.03356)$
\end{tabular} & $\begin{array}{l}0.14752^{*} \\
(0.03739)\end{array}$ & $\begin{array}{l}0.14463^{*} \\
(0.0377)\end{array}$ & $\begin{array}{l}0.20911^{*} \\
(0.03828)\end{array}$ & $\begin{array}{l}0.14706^{*} \\
(0.04128)\end{array}$ & $\begin{array}{l}0.16163^{*} \\
(0.03441)\end{array}$ & $\begin{array}{l}0.17457^{*} \\
(0.03456)\end{array}$ & $\begin{array}{l}0.15184^{*} \\
(0.03344)\end{array}$ & $\begin{array}{l}0.14241^{*} \\
(0.03357)\end{array}$ & $\begin{array}{l}0.14307^{*} \\
(0.03348)\end{array}$ & $\begin{array}{l}0.1406^{*} \\
(0.03344)\end{array}$ \\
\hline $\mathrm{F}$ & $\begin{array}{l}0.31302^{*} \\
(0.03559)\end{array}$ & \begin{tabular}{|l|}
$0.29546^{*}$ \\
$(0.04013)$
\end{tabular} & $\begin{array}{l}.31792^{*} \\
(0.04146)\end{array}$ & $\begin{array}{l}.31342^{*} \\
(0.04113)\end{array}$ & $\begin{array}{l}0.3493^{*} \\
(0.03467)\end{array}$ & $\begin{array}{l}0.31418^{*} \\
(0.03774)\end{array}$ & $\begin{array}{l}.31602^{*} \\
(0.03319)\end{array}$ & $\begin{array}{l}.32504^{*} \\
(0.03318)\end{array}$ & $\begin{array}{l}0.31774^{*} \\
(0.03311)\end{array}$ & $\begin{array}{l}0.31029^{*} \\
(0.03332)\end{array}$ & $\begin{array}{l}0.31124^{*} \\
(0.03323)\end{array}$ & $\begin{array}{l}0.3068^{*} \\
(0.03324)\end{array}$ \\
\hline Fact & $\begin{array}{l}0.35791^{*} \\
(0.03693) \\
\end{array}$ & \begin{tabular}{|l|}
$0.35692^{*}$ \\
$(0.03685)$ \\
\end{tabular} & $\begin{array}{l}0.36288^{*} \\
(0.04173)\end{array}$ & $\begin{array}{l}0.35938^{*} \\
(0.04184)\end{array}$ & $\begin{array}{l}0.43399^{*} \\
(0.04262)\end{array}$ & $\begin{array}{l}0.36086^{*} \\
(0.04178)\end{array}$ & $\begin{array}{l}0.39581^{*} \\
(0.0405)\end{array}$ & $\begin{array}{l}0.40774^{*} \\
(0.03957)\end{array}$ & $\begin{array}{l}0.39869^{*} \\
(0.03957)\end{array}$ & $\begin{array}{l}0.35282^{*} \\
(0.03752)\end{array}$ & $\begin{array}{l}0.36166^{*} \\
(0.03687)\end{array}$ & $\begin{array}{l}0.3115^{*} \\
(0.04369)\end{array}$ \\
\hline $\bar{A}$ & $\begin{array}{l}0.24232^{*} \\
(0.02247)\end{array}$ & \begin{tabular}{|l|}
$0.22989^{*}$ \\
$(0.02297)$ \\
\end{tabular} & $\begin{array}{l}0.24659^{*} \\
(0.02904)\end{array}$ & $\begin{array}{l}0.24235^{*} \\
(0.0284) \\
\end{array}$ & $\begin{array}{l}0.29559^{*} \\
(0.02363)\end{array}$ & $\begin{array}{l}0.24353^{*} \\
(0.02611)\end{array}$ & $\begin{array}{l}0.27273^{*} \\
(0.02276)\end{array}$ & $\begin{array}{l}0.28058^{*} \\
(0.02134)\end{array}$ & $\begin{array}{l}0.26854^{*} \\
(0.02029)\end{array}$ & $\begin{array}{l}0.2366^{*} \\
(0.01841)\end{array}$ & $\begin{array}{l}0.25269^{*} \\
(0.01941)\end{array}$ & $\begin{array}{l}0.19649^{*} \\
(0.02853) \\
\end{array}$ \\
\hline Indiv & $\begin{array}{l}-0.00365 \\
(0.02567)\end{array}$ & \begin{tabular}{|l|}
0.01905 \\
$(0.02705)$
\end{tabular} & $\begin{array}{l}-0.00668 \\
(0.02469)\end{array}$ & $\begin{array}{l}-0.00241 \\
(0.02663)\end{array}$ & $\begin{array}{l}-0.07932^{*} \\
(0.02309)\end{array}$ & $\begin{array}{l}-0.00425 \\
(0.02561)\end{array}$ & $\begin{array}{l}-0.04693^{\dagger} \\
(0.02117)\end{array}$ & $\begin{array}{l}-0.08147^{*} \\
(0.02517)\end{array}$ & $\begin{array}{l}-0.06002^{*} \\
(0.02217)\end{array}$ & $\begin{array}{l}0.02112 \\
(0.03158)\end{array}$ & $\begin{array}{l}-0.05009 \\
(0.03391)\end{array}$ & $\begin{array}{l}0.05484^{\ddagger} \\
(0.02822)\end{array}$ \\
\hline
\end{tabular}

* Significant at the 0.01 level † Significant at the 0.05 level $\quad$ Significant at the 0.10 level

Table 102 Coefficients with individual variables for business practices - Wood Industry 


\begin{tabular}{|c|c|c|c|c|c|c|c|c|c|c|c|c|c|c|c|c|c|c|}
\hline $\begin{array}{l}\text { Individual variable included } \\
\text { in the model in the column }\end{array}$ & 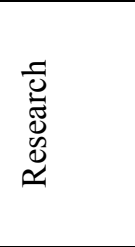 & 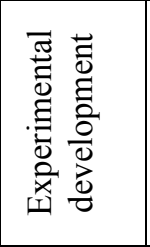 & 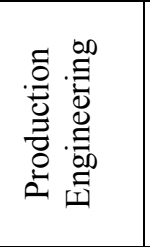 & 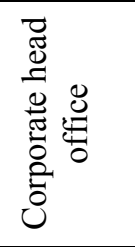 & 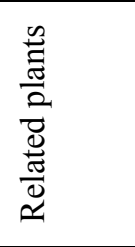 & 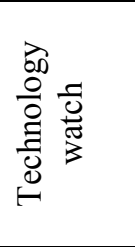 & 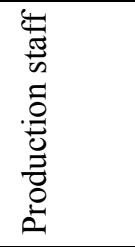 & 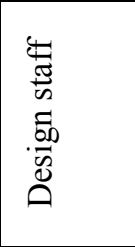 & 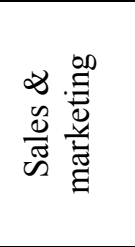 & 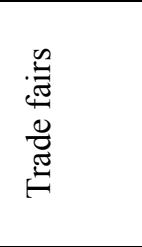 & 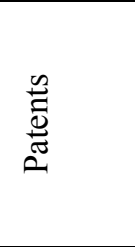 & 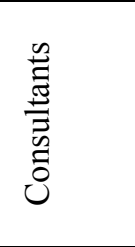 & 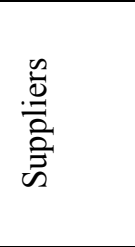 & $\begin{array}{l}0 \\
0 \\
0 \\
0 \\
0 \\
0 \\
0 \\
0\end{array}$ & 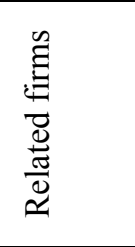 & 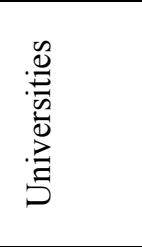 & 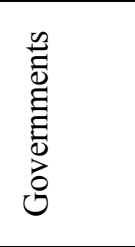 & 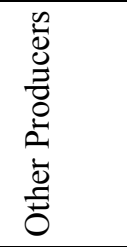 \\
\hline No fac & $\begin{array}{l}0.24884^{*} \\
(0.01598)\end{array}$ & \begin{tabular}{|l|}
$0.24688^{*}$ \\
$(0.01585)$ \\
\end{tabular} & $\begin{array}{l}0.24569^{*} \\
(0.01574) \\
\end{array}$ & $\begin{array}{l}0.24581^{*} \\
(0.01574)\end{array}$ & $\begin{array}{l}0.24729^{*} \\
(0.01573)\end{array}$ & $\begin{array}{l}0.24507^{*} \\
(0.01577)\end{array}$ & $\begin{array}{l}0.24803^{*} \\
(0.01615)\end{array}$ & $\begin{array}{l}0.24808^{*} \\
(0.0158) \\
\end{array}$ & $\begin{array}{l}0.25041^{*} \\
(0.01596)\end{array}$ & $\begin{array}{l}0.24491^{*} \\
(0.01578)\end{array}$ & $\begin{array}{l}0.24665^{*} \\
(0.01637)\end{array}$ & $\begin{array}{l}0.24487^{*} \\
(0.01577)\end{array}$ & $\begin{array}{l}0.24708^{*} \\
(0.01552)\end{array}$ & \begin{tabular}{|l|}
$0.24936^{*}$ \\
$(0.01614)$ \\
\end{tabular} & $\begin{array}{l}0.25795^{*} \\
(0.01576)\end{array}$ & $\begin{array}{l}0.24614^{*} \\
(0.01568)\end{array}$ & $\begin{array}{l}0.24442^{*} \\
(0.01562)\end{array}$ & $\begin{array}{l}0.24422^{*} \\
(0.01561)\end{array}$ \\
\hline Factor 4 & $\begin{array}{l}0.14405^{*} \\
(0.0402)\end{array}$ & \begin{tabular}{|l|}
$0.13289^{*}$ \\
$(0.03918)$ \\
\end{tabular} & $\begin{array}{l}0.13472^{*} \\
(0.03917) \\
\end{array}$ & $\begin{array}{l}0.16774^{*} \\
(0.04552)\end{array}$ & $\begin{array}{l}0.16298^{*} \\
(0.04179)\end{array}$ & $\begin{array}{l}0.13162^{*} \\
(0.03917)\end{array}$ & $\begin{array}{l}0.14356^{*} \\
(0.04171)\end{array}$ & $\begin{array}{l}0.13276^{*} \\
(0.03903)\end{array}$ & $\begin{array}{l}0.1462^{*} \\
(0.03981)\end{array}$ & $\begin{array}{l}0.13073^{*} \\
(0.03918)\end{array}$ & $\begin{array}{l}0.13532^{*} \\
(0.04093)\end{array}$ & $\begin{array}{l}0.13051^{*} \\
(0.03917)\end{array}$ & $\begin{array}{l}0.14374 \\
(0.03867)\end{array}$ & $\begin{array}{l}0.14917^{*} \\
(0.04178)\end{array}$ & $\begin{array}{l}0.13699^{*} \\
(0.0384)\end{array}$ & $\begin{array}{l}0.1419^{*} \\
(0.03921)\end{array}$ & $\begin{array}{l}0.12911^{*} \\
(0.0388)\end{array}$ & $\begin{array}{l}0.12522^{*} \\
(0.0388)\end{array}$ \\
\hline Factor 3 & $\begin{array}{l}0.23323^{*} \\
(0.04261)\end{array}$ & \begin{tabular}{|l|}
$0.23297^{*}$ \\
$(0.04265)$ \\
\end{tabular} & $\begin{array}{l}0.23512^{*} \\
(0.04264)\end{array}$ & $\begin{array}{l}0.23515^{*} \\
(0.04262)\end{array}$ & $\begin{array}{l}0.23236^{*} \\
(0.04248)\end{array}$ & $\begin{array}{l}0.23249^{*} \\
(0.04268)\end{array}$ & $\begin{array}{l}0.2344^{*} \\
(0.04273)\end{array}$ & \begin{tabular}{|l|l|}
$0.2326^{*}$ \\
$(0.04253)$ \\
\end{tabular} & $\begin{array}{l}0.25912^{*} \\
(0.04465)\end{array}$ & $\begin{array}{l}0.23236^{*} \\
(0.04271) \\
\end{array}$ & $\begin{array}{l}0.23487^{*} \\
(0.04315) \\
\end{array}$ & $\begin{array}{l}0.23236^{*} \\
(0.0427) \\
\end{array}$ & $\begin{array}{l}0.2436^{*} \\
(0.04209)\end{array}$ & $\begin{array}{l}0.26611^{*} \\
(0.05026) \\
\end{array}$ & $\begin{array}{l}0.30386^{*} \\
(0.04517)\end{array}$ & $\begin{array}{l}0.24614^{*} \\
(0.04283) \\
\end{array}$ & $\begin{array}{l}0.23236^{*} \\
(0.0423)\end{array}$ & $\begin{array}{l}0.23143^{*} \\
(0.04226) \\
\end{array}$ \\
\hline Facto & $\begin{array}{l}0.35563^{*} \\
(0.0427)\end{array}$ & \begin{tabular}{|l|}
$0.34731^{*}$ \\
$(0.04205)$ \\
\end{tabular} & $\begin{array}{l}0.35426^{*} \\
(0.04246)\end{array}$ & $\begin{array}{l}0.3712^{*} \\
(0.04529)\end{array}$ & $\begin{array}{l}0.3874^{*} \\
(0.04645)\end{array}$ & $\begin{array}{l}0.3458^{*} \\
(0.04203)\end{array}$ & $\begin{array}{l}0.35102^{*} \\
(0.04273)\end{array}$ & $\begin{array}{l}0.34739^{*} \\
(0.04186)\end{array}$ & $\begin{array}{l}0.36873^{*} \\
(0.0437)\end{array}$ & $\begin{array}{l}0.34397^{*} \\
(0.042)\end{array}$ & $\begin{array}{l}0.34905^{*} \\
(0.044)\end{array}$ & $\begin{array}{l}0.34371^{*} \\
(0.04198)\end{array}$ & $\begin{array}{l}0.37955^{*} \\
(0.04234)\end{array}$ & $\begin{array}{l}0.36878^{*} \\
(0.04635) \\
\end{array}$ & $\begin{array}{l}0.44863^{*} \\
(0.04814)\end{array}$ & $\begin{array}{l}0.39115^{*} \\
(0.04627)\end{array}$ & $\begin{array}{l}0.34253^{*} \\
(0.04159)\end{array}$ & $\begin{array}{l}0.31542^{*} \\
(0.04262) \\
\end{array}$ \\
\hline Factor 2 & $\begin{array}{l}0.19892^{\dagger} \\
(0.09509)\end{array}$ & \begin{tabular}{|l|}
$0.19542^{\dagger}$ \\
$(0.09589)$ \\
\end{tabular} & \begin{tabular}{l|}
$0.2074^{\dagger}$ \\
$(0.09622)$ \\
\end{tabular} & $\begin{array}{l}0.16722^{\ddagger} \\
(0.09247)\end{array}$ & $\begin{array}{l}0.18273^{\dagger} \\
(0.09252)\end{array}$ & $\begin{array}{l}0.16722^{\ddagger} \\
(0.09267)\end{array}$ & $\begin{array}{l}0.18112^{\ddagger} \\
(0.09392)\end{array}$ & \begin{tabular}{|l|l|}
$0.1809^{\ddagger}$ \\
$(0.09264)$ \\
\end{tabular} & $\begin{array}{l}0.18203^{\ddagger} \\
(0.09263)\end{array}$ & $\begin{array}{l}0.16722^{\ddagger} \\
(0.09274) \\
\end{array}$ & $\begin{array}{l}0.1699^{\ddagger} \\
(0.09297)\end{array}$ & $\begin{array}{l}0.16443^{\ddagger} \\
(0.09283)\end{array}$ & $\begin{array}{l}0.18018^{\dagger} \\
(0.09122)\end{array}$ & $\begin{array}{l}0.17181^{\ddagger} \\
(0.09264) \\
\end{array}$ & $\begin{array}{l}0.20435^{\dagger} \\
(0.09126)\end{array}$ & $\begin{array}{l}0.16722^{\ddagger} \\
(0.09212) \\
\end{array}$ & $\begin{array}{l}0.15181^{\ddagger} \\
(0.092)\end{array}$ & $\begin{array}{l}0.14287 \\
(0.09212) \\
\end{array}$ \\
\hline $\mathrm{Fa}$ & $\begin{array}{l}0.30734^{\dagger} \\
(0.12421)\end{array}$ & \begin{tabular}{|l|}
$0.28584^{\dagger}$ \\
$(0.12299)$ \\
\end{tabular} & $\begin{array}{l}0.32029^{\dagger} \\
(0.12556) \\
\end{array}$ & $\begin{array}{l}0.32071^{\dagger} \\
(0.12531) \\
\end{array}$ & $\begin{array}{l}0.33193^{*} \\
(0.12474)\end{array}$ & $\begin{array}{l}0.28112^{\dagger} \\
(0.12297) \\
\end{array}$ & $\begin{array}{l}0.29464^{\dagger} \\
(0.12399) \\
\end{array}$ & $\begin{array}{l}0.28659^{\dagger} \\
(0.12259) \\
\end{array}$ & $\begin{array}{l}0.3146^{\dagger} \\
(0.12375) \\
\end{array}$ & $\begin{array}{l}0.2867^{\dagger} \\
(0.12448) \\
\end{array}$ & $\begin{array}{l}0.28193^{\dagger} \\
(0.12313) \\
\end{array}$ & $\begin{array}{l}0.26443^{\dagger} \\
(0.12581) \\
\end{array}$ & $\begin{array}{l}0.33973^{*} \\
(0.12197)\end{array}$ & $\begin{array}{l}0.28332^{\dagger} \\
(0.12286) \\
\end{array}$ & $\begin{array}{l}0.35435^{*} \\
(0.12182) \\
\end{array}$ & $\begin{array}{l}0.29551^{\dagger} \\
(0.12241) \\
\end{array}$ & $\begin{array}{l}0.19018 \\
(0.12584) \\
\end{array}$ & $\begin{array}{l}0.21898^{+} \\
(0.12346) \\
\end{array}$ \\
\hline Factor $2 \& 3$ & & & & & & & & & Insuffic & ent Data & & & & & & & & \\
\hline Factor $2,3 \& 4$ & $\begin{array}{l}-0.02332 \\
(0.08009)\end{array}$ & \begin{tabular}{|l|}
-0.02735 \\
$(0.08086)$ \\
\end{tabular} & \begin{tabular}{l|}
-0.01862 \\
$(0.08065)$ \\
\end{tabular} & $\begin{array}{l}-0.01518 \\
(0.08093)\end{array}$ & $\begin{array}{l}-0.00321 \\
(0.08047)\end{array}$ & $\begin{array}{l}-0.05502 \\
(0.07713)\end{array}$ & $\begin{array}{l}-0.05461 \\
(0.07713)\end{array}$ & \begin{tabular}{|l|}
-0.05502 \\
$(0.07687)$ \\
\end{tabular} & $\begin{array}{l}-0.01157 \\
(0.07996)\end{array}$ & $\begin{array}{l}-0.05502 \\
(0.07719) \\
\end{array}$ & $\begin{array}{l}-0.05384 \\
(0.07724)\end{array}$ & $\begin{array}{l}-0.05502 \\
(0.07717)\end{array}$ & $\begin{array}{l}-0.05354 \\
(0.07588)\end{array}$ & \begin{tabular}{|l|}
-0.05058 \\
$(0.07713)$ \\
\end{tabular} & $\begin{array}{l}0.05814 \\
(0.08027)\end{array}$ & \begin{tabular}{|l|}
0.0102 \\
$(0.08147)$ \\
\end{tabular} & $\begin{array}{l}-0.06568 \\
(0.07654)\end{array}$ & $\begin{array}{c}-0.05654 \\
(0.07638) \\
\end{array}$ \\
\hline Factor 1 & $\begin{array}{l}0.24735^{*} \\
(0.0394)\end{array}$ & \begin{tabular}{|l|}
$0.22967^{*}$ \\
$(0.03651)$ \\
\end{tabular} & $\begin{array}{l}0.23895^{*} \\
(0.03737) \\
\end{array}$ & $\begin{array}{l}0.2388^{*} \\
(0.03723) \\
\end{array}$ & $\begin{array}{l}0.22924^{*} \\
(0.03619)\end{array}$ & $\begin{array}{l}0.22965^{*} \\
(0.03668)\end{array}$ & $\begin{array}{l}0.24614^{*} \\
(0.0431)\end{array}$ & $\begin{array}{l}0.24997^{*} \\
(0.03851) \\
\end{array}$ & $\begin{array}{l}0.23091^{*} \\
(0.03629) \\
\end{array}$ & $\begin{array}{l}0.22511^{*} \\
(0.03634)\end{array}$ & $\begin{array}{l}0.23407^{*} \\
(0.04252) \\
\end{array}$ & $\begin{array}{l}0.22511^{*} \\
(0.03633)\end{array}$ & $\begin{array}{l}0.28526^{*} \\
(0.03906)\end{array}$ & $\begin{array}{l}0.25622^{*} \\
(0.04379) \\
\end{array}$ & $\begin{array}{l}0.22605^{*} \\
(0.03559) \\
\end{array}$ & $\begin{array}{l}0.22538^{*} \\
(0.0361)\end{array}$ & $\begin{array}{l}0.22433^{*} \\
(0.03599)\end{array}$ & $\begin{array}{l}0.21758^{*} \\
(0.03604) \\
\end{array}$ \\
\hline $\mathrm{Fa}$ & $\begin{array}{l}0.1941^{*} \\
(0.05109)\end{array}$ & \begin{tabular}{|l|}
$0.19686^{*}$ \\
$(0.05128)$ \\
\end{tabular} & $\begin{array}{l}0.20747^{*} \\
(0.05204) \\
\end{array}$ & $\begin{array}{l}0.22879^{*} \\
(0.05596)\end{array}$ & $\begin{array}{l}0.21239^{*} \\
(0.05177)\end{array}$ & $\begin{array}{l}0.20612^{*} \\
(0.05362)\end{array}$ & $\begin{array}{l}0.21347^{*} \\
(0.05628)\end{array}$ & $\begin{array}{l}0.22832^{*} \\
(0.05445) \\
\end{array}$ & $\begin{array}{l}0.21893^{*} \\
(0.05274)\end{array}$ & $\begin{array}{l}0.19222^{*} \\
(0.05119)\end{array}$ & $\begin{array}{l}0.20078^{*} \\
(0.05537)\end{array}$ & $\begin{array}{l}0.19137^{*} \\
(0.0512)\end{array}$ & $\begin{array}{l}0.26837^{*} \\
(0.05415)\end{array}$ & \begin{tabular}{|l|}
$0.2063^{*}$ \\
$(0.05229)$ \\
\end{tabular} & $\begin{array}{l}0.19322^{2} \\
(0.05014)\end{array}$ & $\begin{array}{l}0.20846 " \\
(0.05131)\end{array}$ & $\begin{array}{l}0.18201^{*} \\
(0.05082)\end{array}$ & $\begin{array}{l}0.19155^{*} \\
(0.05065) \\
\end{array}$ \\
\hline Factor $1 \& 3$ & $\begin{array}{l}0.27028^{*} \\
(0.02201) \\
\end{array}$ & \begin{tabular}{|l|}
$0.27029^{*}$ \\
$(0.02212)$ \\
\end{tabular} & $\begin{array}{l}0.26967^{*} \\
(0.02194) \\
\end{array}$ & $\begin{array}{l}0.27253^{*} \\
(0.02216) \\
\end{array}$ & $\begin{array}{l}0.26699^{*} \\
(0.02178)\end{array}$ & $\begin{array}{l}0.2712^{*} \\
(0.02255)\end{array}$ & $\begin{array}{l}0.286^{*} \\
(0.0307) \\
\end{array}$ & \begin{tabular}{|l|}
$0.2897^{*}$ \\
$(0.02503)$ \\
\end{tabular} & $\begin{array}{l}0.30815^{*} \\
(0.03041)\end{array}$ & $\begin{array}{l}0.26651^{*} \\
(0.0219) \\
\end{array}$ & $\begin{array}{l}0.273^{*} \\
(0.02713) \\
\end{array}$ & $\begin{array}{l}0.26489^{*} \\
(0.02206) \\
\end{array}$ & $\begin{array}{l}0.31299^{*} \\
(0.02475) \\
\end{array}$ & $\begin{array}{l}0.30025^{*} \\
(0.03444) \\
\end{array}$ & $\begin{array}{l}0.3673^{*} \\
(0.03221) \\
\end{array}$ & $\begin{array}{l}0.2696^{*} \\
(0.0218) \\
\end{array}$ & $\begin{array}{l}0.25619^{*} \\
(0.02199)\end{array}$ & $\begin{array}{l}0.25618^{*} \\
(0.02194) \\
\end{array}$ \\
\hline Factor & $\begin{array}{l}0.30205^{*} \\
(0.02429)\end{array}$ & \begin{tabular}{|l|}
$0.29774^{*}$ \\
$(0.02401)$ \\
\end{tabular} & $\begin{array}{l}0.30969^{*} \\
(0.02554) \\
\end{array}$ & $\begin{array}{l}0.32609^{*} \\
(0.03039)\end{array}$ & $\begin{array}{l}0.32981^{*} \\
(0.02853) \\
\end{array}$ & $\begin{array}{l}0.30092^{*} \\
(0.02459)\end{array}$ & $\begin{array}{l}0.31841^{*} \\
(0.03426)\end{array}$ & $\begin{array}{l}0.33217^{*} \\
(0.03056) \\
\end{array}$ & $\begin{array}{l}0.33551^{*} \\
(0.03115) \\
\end{array}$ & $\begin{array}{l}0.29635^{*} \\
(0.02401)\end{array}$ & $\begin{array}{l}0.30435^{*} \\
(0.03121)\end{array}$ & $\begin{array}{l}0.29556^{*} \\
(0.02403) \\
\end{array}$ & $\begin{array}{l}0.33549^{*} \\
(0.02575)\end{array}$ & \begin{tabular}{|l}
$0.32994^{*}$ \\
$(0.03578)$ \\
\end{tabular} & $\begin{array}{l}0.38322^{*} \\
(0.03135) \\
\end{array}$ & $\begin{array}{l}0.35397^{*} \\
(0.03408)\end{array}$ & $\begin{array}{l}0.29284^{*} \\
(0.02381)\end{array}$ & $\begin{array}{l}0.28479^{*} \\
(0.02407) \\
\end{array}$ \\
\hline Factor $1 \& 2$ & $\begin{array}{l}0.22751^{\dagger} \\
(0.11373)\end{array}$ & \begin{tabular}{|l|}
0.22661 \\
$(0.11429)$ \\
\end{tabular} & $\begin{array}{l}0.24046 \\
(0.11497) \\
\end{array}$ & $\begin{array}{l}0.208^{\ddagger} \\
(0.1272)\end{array}$ & $\begin{array}{l}0.21317^{\ddagger} \\
(0.11248)\end{array}$ & $\begin{array}{l}0.20831^{\ddagger} \\
(0.11298)\end{array}$ & $\begin{array}{l}0.22405^{7} \\
(0.11463)\end{array}$ & $\begin{array}{l}0.24259^{\dagger} \\
(0.1142) \\
\end{array}$ & $\begin{array}{l}0.20911^{\ddagger} \\
(0.11255)\end{array}$ & $\begin{array}{l}0.20637^{+} \\
(0.11305)\end{array}$ & & & $\begin{array}{l}0.26647^{\dagger} \\
(0.11224)\end{array}$ & & $\begin{array}{l}0.21927^{7} \\
(0.11077)\end{array}$ & $\begin{array}{l}0.20637^{+} \\
(0.1123)\end{array}$ & & $\begin{array}{l}0.16202 \\
(0.11284)\end{array}$ \\
\hline Factor $1,2 \& 4$ & $\begin{array}{l}0.27929^{*} \\
(0.0353)\end{array}$ & \begin{tabular}{|l}
$0.27633^{*}$ \\
$(0.0358)$
\end{tabular} & $\begin{array}{l}0.29956^{*} \\
(0.04193)\end{array}$ & $\begin{array}{l}0.29115^{*} \\
(0.0381)\end{array}$ & $\begin{array}{l}0.29123^{*} \\
(0.03562)\end{array}$ & $\begin{array}{l}0.27014^{*} \\
(0.03484)\end{array}$ & $\begin{array}{l}0.27663^{*} \\
(0.03771)\end{array}$ & $\begin{array}{l}0.29001^{*} \\
(0.03626)\end{array}$ & $\begin{array}{l}0.28452^{*} \\
(0.03485)\end{array}$ & $\begin{array}{l}0.25938^{*} \\
(0.03256)\end{array}$ & $\begin{array}{l}0.26603^{*} \\
(0.03646)\end{array}$ & $\begin{array}{l}0.25068^{*} \\
(0.03568)\end{array}$ & $\begin{array}{l}0.30306^{*} \\
(0.034)\end{array}$ & $\begin{array}{l}0.27489^{*} \\
(0.03473)\end{array}$ & $\begin{array}{l}0.26664^{*} \\
(0.03194)\end{array}$ & $\begin{array}{l}0.26405^{*} \\
(0.03241)\end{array}$ & $\begin{array}{l}0.25645^{*} \\
(0.03226)\end{array}$ & $\begin{array}{l}0.25745^{*} \\
(0.03223)\end{array}$ \\
\hline Factor $1,2 \& 3$ & $\begin{array}{l}0.19553^{*} \\
(0.05552)\end{array}$ & \begin{tabular}{|l|}
$0.1961^{*}$ \\
$(0.05716)$ \\
\end{tabular} & $\begin{array}{l}0.20131^{*} \\
(0.05646) \\
\end{array}$ & $\begin{array}{l}0.17533^{*} \\
(0.05315)\end{array}$ & $\begin{array}{l}0.18343^{*} \\
(0.05323)\end{array}$ & $\begin{array}{l}0.17577^{*} \\
(0.05338)\end{array}$ & $\begin{array}{l}0.19454^{*} \\
(0.05858)\end{array}$ & $\begin{array}{l}0.20058^{*} \\
(0.05511) \\
\end{array}$ & $\begin{array}{l}0.21594^{*} \\
(0.05748)\end{array}$ & $\begin{array}{l}0.17239^{*} \\
(0.05327)\end{array}$ & $\begin{array}{l}0.18078^{*} \\
(0.05715)\end{array}$ & $\begin{array}{l}0.16947^{*} \\
(0.05348)\end{array}$ & $\begin{array}{l}0.23836^{*} \\
(0.05516)\end{array}$ & $\begin{array}{l}0.20613^{*} \\
(0.05946)\end{array}$ & $\begin{array}{l}0.27222^{2} \\
(0.05735)\end{array}$ & $\begin{array}{l}0.19443^{\circ} \\
(0.05373)\end{array}$ & $\begin{array}{l}0.12638^{\dagger} \\
(0.05515)\end{array}$ & $\begin{array}{l}0.12675^{+} \\
(0.05488) \\
\end{array}$ \\
\hline All Factors & $\begin{array}{l}0.3148^{*} \\
(0.02902)\end{array}$ & \begin{tabular}{|l|}
$0.30484^{*}$ \\
$(0.02684)$ \\
\end{tabular} & $\begin{array}{l}0.3252^{*} \\
(0.03283)\end{array}$ & $\begin{array}{l}0.32514^{*} \\
(0.03206)\end{array}$ & $\begin{array}{l}0.32174^{*} \\
(0.0278)\end{array}$ & $\begin{array}{l}0.29768^{*} \\
(0.02515)\end{array}$ & $\begin{array}{l}0.30701^{*} \\
(0.02969)\end{array}$ & $\begin{array}{l}0.30748^{*} \\
(0.02543) \\
\end{array}$ & $\begin{array}{l}0.33012^{*} \\
(0.03105)\end{array}$ & $\begin{array}{l}0.29196^{*} \\
(0.02412)\end{array}$ & $\begin{array}{l}0.29932^{*} \\
(0.0313)\end{array}$ & $\begin{array}{l}0.28649^{*} \\
(0.02526)\end{array}$ & $\begin{array}{l}0.34361^{*} \\
(0.02733)\end{array}$ & $\begin{array}{l}0.31966^{\prime \prime} \\
(0.03285) \\
\end{array}$ & $\begin{array}{l}0.40211^{*} \\
(0.03539)\end{array}$ & $\begin{array}{l}0.32888^{*} \\
(0.02867)\end{array}$ & $\begin{array}{l}0.26061^{*} \\
(0.02606)\end{array}$ & $\begin{array}{l}0.24844^{*} \\
(0.02773)\end{array}$ \\
\hline Individual Variable & $\begin{array}{l}-0.0317 \\
(0.02199)\end{array}$ & \begin{tabular}{|c|}
-0.02819 \\
$(0.02488)$ \\
\end{tabular} & $\begin{array}{l}-0.04018 \\
(0.02652)\end{array}$ & $\begin{array}{l}-0.04059 \\
(0.02548)\end{array}$ & $\begin{array}{l}-0.05181^{\dagger} \\
(0.0241)\end{array}$ & $\begin{array}{l}-0.02124 \\
(0.02458) \\
\end{array}$ & $\begin{array}{l}-0.02215 \\
(0.02446)\end{array}$ & $\begin{array}{l}-0.04168^{\ddagger} \\
(0.02209) \\
\end{array}$ & $\begin{array}{l}-0.04514^{*} \\
(0.02298)\end{array}$ & $\begin{array}{l}-0.04674 \\
(0.13329)\end{array}$ & $\begin{array}{l}-0.00896 \\
(0.02209)\end{array}$ & $\begin{array}{l}0.02053 \\
(0.03448) \\
\end{array}$ & $\begin{array}{l}-0.07936^{*} \\
(0.02084)\end{array}$ & \begin{tabular}{|l|}
-0.03374 \\
$(0.02661)$ \\
\end{tabular} & $\begin{array}{l}-0.11316^{*} \\
(0.02698)\end{array}$ & $\begin{array}{l}-0.06522^{\dagger} \\
(0.02751)\end{array}$ & $\begin{array}{l}0.09442^{*} \\
(0.03296)\end{array}$ & $\begin{array}{l}0.08139^{*} \\
(0.02722)\end{array}$ \\
\hline
\end{tabular}

Table 103 Coefficients with individual variables for source of implementation support - Wood Industry 


\begin{tabular}{|c|c|c|c|c|c|c|c|c|c|c|c|c|c|}
\hline $\begin{array}{l}\text { Individual variable included } \\
\text { in the model in the column }\end{array}$ & 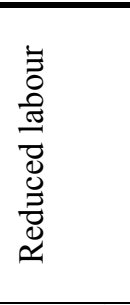 & 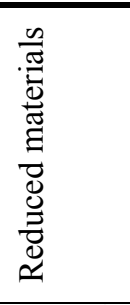 & 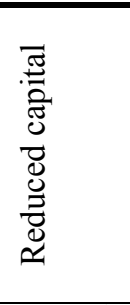 & 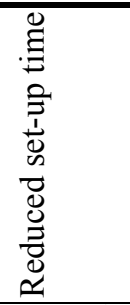 & 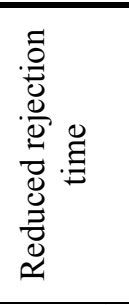 & 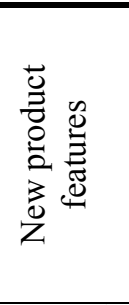 & 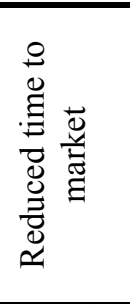 & 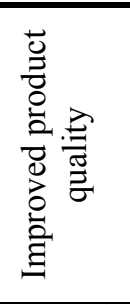 & 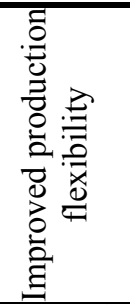 & 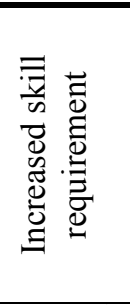 & 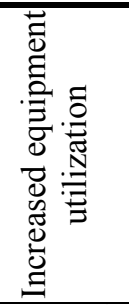 & 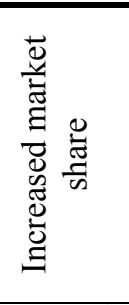 & 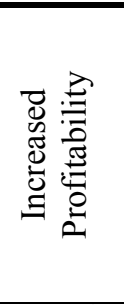 \\
\hline No factors & $\begin{array}{l}0.21221^{*} \\
(0.0157)\end{array}$ & $\begin{array}{l}0.21499^{*} \\
(0.0154)\end{array}$ & $\begin{array}{l}0.21234^{*} \\
(0.0157)\end{array}$ & $\begin{array}{l}0.21256^{*} \\
(0.01569)\end{array}$ & $\begin{array}{l}0.21187^{*} \\
(0.01559)\end{array}$ & $\begin{array}{l}0.21437^{*} \\
(0.0158)\end{array}$ & $\begin{array}{l}0.2124^{*} \\
(0.01562)\end{array}$ & $\begin{array}{l}0.21609^{*} \\
(0.01567)\end{array}$ & $\begin{array}{l}0.21369^{*} \\
(0.01575)\end{array}$ & $\begin{array}{l}0.20887^{*} \\
(0.01577)\end{array}$ & $\begin{array}{l}0.21205^{*} \\
(0.0157)\end{array}$ & $\begin{array}{l}0.21225^{*} \\
(0.0157)\end{array}$ & $\begin{array}{l}0.2124^{*} \\
(0.01559)\end{array}$ \\
\hline Factor 4 & $\begin{array}{l}0.222^{*} \\
(0.04718)\end{array}$ & $\begin{array}{l}0.28592^{*} \\
(0.0476)\end{array}$ & $\begin{array}{l}0.22351^{*} \\
(0.04602)\end{array}$ & $\begin{array}{l}0.23138^{*} \\
(0.04735)\end{array}$ & $\begin{array}{l}0.22278^{*} \\
(0.0457)\end{array}$ & $\begin{array}{l}0.22523^{*} \\
(0.04599)\end{array}$ & $\begin{array}{l}0.25666^{*} \\
(0.04876)\end{array}$ & $\begin{array}{l}0.25542^{*} \\
(0.04762)\end{array}$ & $\begin{array}{l}0.22388^{*} \\
(0.04597)\end{array}$ & $\begin{array}{l}0.2184^{*} \\
(0.04594)\end{array}$ & $\begin{array}{l}0.21371^{*} \\
(0.0515)\end{array}$ & $\begin{array}{l}0.22159^{*} \\
(0.05397)\end{array}$ & $\begin{array}{l}0.30673^{*} \\
(0.05761)\end{array}$ \\
\hline Factor 3 & $\begin{array}{l}0.22741^{*} \\
(0.05299)\end{array}$ & $\begin{array}{l}0.30084^{*} \\
(0.05462)\end{array}$ & $\begin{array}{l}0.22805^{*} \\
(0.05272)\end{array}$ & $\begin{array}{l}0.24153^{*} \\
(0.05591)\end{array}$ & $\begin{array}{l}0.1842^{*} \\
(0.05545)\end{array}$ & $\begin{array}{l}0.23047^{*} \\
(0.0527)\end{array}$ & $\begin{array}{l}0.23587^{*} \\
(0.05262)\end{array}$ & $\begin{array}{l}0.28405^{*} \\
(0.05732)\end{array}$ & $\begin{array}{l}0.25208^{*} \\
(0.05836)\end{array}$ & $\begin{array}{l}0.19018^{*} \\
(0.05705)\end{array}$ & $\begin{array}{l}0.22745^{*} \\
(0.05273)\end{array}$ & $\begin{array}{l}0.22797^{*} \\
(0.05274)\end{array}$ & $\begin{array}{l}0.23293^{*} \\
(0.05241)\end{array}$ \\
\hline Factor $3 \& 4$ & $\begin{array}{l}0.3751^{*} \\
(0.0531)\end{array}$ & $\begin{array}{l}0.38395^{*} \\
(0.05198)\end{array}$ & $\begin{array}{l}0.37636^{*} \\
(0.05309)\end{array}$ & $\begin{array}{l}0.3768^{*} \\
(0.05299)\end{array}$ & $\begin{array}{l}0.36207^{*} \\
(0.05292)\end{array}$ & $\begin{array}{l}0.3939^{*} \\
(0.0557)\end{array}$ & $\begin{array}{l}0.38771^{*} \\
(0.05309)\end{array}$ & $\begin{array}{l}0.42802^{*} \\
(0.05699)\end{array}$ & $\begin{array}{l}0.39954^{*} \\
(0.05861)\end{array}$ & $\begin{array}{l}0.3313^{*} \\
(0.05884)\end{array}$ & $\begin{array}{l}0.36829^{*} \\
(0.05579)\end{array}$ & $\begin{array}{l}0.37432^{*} \\
(0.05671)\end{array}$ & $\begin{array}{l}0.42076^{*} \\
(0.05596)\end{array}$ \\
\hline Factor 2 & $\begin{array}{l}0.25221^{\dagger} \\
(0.11263)\end{array}$ & $\begin{array}{l}0.36461^{*} \\
(0.11084)\end{array}$ & $\begin{array}{l}0.25706^{\dagger} \\
(0.11002)\end{array}$ & $\begin{array}{l}0.26091^{\dagger} \\
(0.11001)\end{array}$ & $\begin{array}{l}0.23614 \\
(0.10933)\end{array}$ & $\begin{array}{l}0.25737^{\dagger} \\
(0.10967)\end{array}$ & $\begin{array}{l}0.26848^{\dagger} \\
(0.10947)\end{array}$ & $\begin{array}{l}0.27665^{\dagger} \\
(0.1094)\end{array}$ & $\begin{array}{l}0.2707^{\dagger} \\
(0.11087)\end{array}$ & $\begin{array}{l}0.25002^{\dagger} \\
(0.10946)\end{array}$ & $\begin{array}{l}0.24718^{\dagger} \\
(0.11149)\end{array}$ & $\begin{array}{l}0.25522^{\dagger} \\
(0.1098)\end{array}$ & $\begin{array}{l}0.28335^{\dagger} \\
(0.10969)\end{array}$ \\
\hline Fact & $\begin{array}{l}0.18958^{*} \\
(0.06675)\end{array}$ & $\begin{array}{l}0.29903^{*} \\
(0.06639)\end{array}$ & $\begin{array}{l}0.19815^{*} \\
(0.06611)\end{array}$ & $\begin{array}{l}0.20657^{*} \\
(0.06534)\end{array}$ & $\begin{array}{l}0.17638^{*} \\
(0.0623)\end{array}$ & $\begin{array}{l}0.19544^{*} \\
(0.06235)\end{array}$ & $\begin{array}{l}0.19828^{*} \\
(0.06214)\end{array}$ & $\begin{array}{l}0.1933^{*} \\
(0.06194)\end{array}$ & $\begin{array}{l}0.19342^{*} \\
(0.06231)\end{array}$ & $\begin{array}{l}0.18629^{*} \\
(0.06225)\end{array}$ & $\begin{array}{l}0.18227^{*} \\
(0.06705)\end{array}$ & $\begin{array}{l}0.19076^{*} \\
(0.0686)\end{array}$ & $\begin{array}{l}0.27414^{*} \\
(0.07082)\end{array}$ \\
\hline Fact & $\begin{array}{l}0.11252 \\
(0.10418)\end{array}$ & $\begin{array}{l}0.22561^{\dagger} \\
(0.1026)\end{array}$ & $\begin{array}{l}0.12026 \\
(0.10267)\end{array}$ & $\begin{array}{l}0.11881 \\
(0.10111)\end{array}$ & $\begin{array}{l}0.08998 \\
(0.10093)\end{array}$ & $\begin{array}{l}0.12271 \\
(0.10116)\end{array}$ & $\begin{array}{l}0.12152 \\
(0.10063)\end{array}$ & $\begin{array}{l}0.17175^{t} \\
(0.10306)\end{array}$ & $\begin{array}{l}0.13191 \\
(0.1024)\end{array}$ & $\begin{array}{l}0.07447 \\
(0.10356)\end{array}$ & $\begin{array}{l}0.10902 \\
(0.10228)\end{array}$ & $\begin{array}{l}0.11523 \\
(0.10122)\end{array}$ & $\begin{array}{l}0.15125 \\
(0.10149)\end{array}$ \\
\hline Factor $2,3 \& 4$ & $\begin{array}{l}0.18551^{*} \\
(0.0646)\end{array}$ & $\begin{array}{l}0.27712^{*} \\
(0.06201)\end{array}$ & $\begin{array}{l}0.19518^{*} \\
(0.06455)\end{array}$ & $\begin{array}{l}0.20041^{*} \\
(0.06154)\end{array}$ & $\begin{array}{l}0.17806^{*} \\
(0.05909)\end{array}$ & $\begin{array}{l}0.19192^{*} \\
(0.05935)\end{array}$ & $\begin{array}{l}0.19537^{*} \\
(0.05915)\end{array}$ & $\begin{array}{l}0.20683^{*} \\
(0.05942)\end{array}$ & $\begin{array}{l}0.19679^{*} \\
(0.0599)\end{array}$ & $\begin{array}{l}0.14551^{\dagger} \\
(0.06431)\end{array}$ & $\begin{array}{l}0.17938^{*} \\
(0.06338)\end{array}$ & $\begin{array}{l}0.188^{*} \\
(0.06011)\end{array}$ & $\begin{array}{l}0.2727^{*} \\
(0.06872)\end{array}$ \\
\hline Fact & $\begin{array}{l}0.28511 \\
(0.18105)\end{array}$ & $\begin{array}{l}0.29813^{t} \\
(0.17746)\end{array}$ & $\begin{array}{l}0.29194 \\
(0.18294)\end{array}$ & $\begin{array}{l}0.30254^{\ddagger} \\
(0.18255)\end{array}$ & $\begin{array}{l}0.2406 \\
(0.18075)\end{array}$ & $\begin{array}{l}0.30507^{\ddagger} \\
(0.18179)\end{array}$ & $\begin{array}{l}0.32864^{\ddagger} \\
(0.1815)\end{array}$ & $\begin{array}{l}0.29288 \\
(0.17983)\end{array}$ & $\begin{array}{l}0.30383^{\ddagger} \\
(0.18191)\end{array}$ & $\begin{array}{l}0.28511 \\
(0.18042)\end{array}$ & $\begin{array}{l}0.28129 \\
(0.18125)\end{array}$ & $\begin{array}{l}0.28511 \\
(0.18105)\end{array}$ & $\begin{array}{l}0.28511 \\
(0.17982)\end{array}$ \\
\hline Factor $1 \& 4$ & $\begin{array}{l}0.28532^{\dagger} \\
(0.14015)\end{array}$ & $\begin{array}{l}0.33785^{\dagger} \\
(0.13699)\end{array}$ & $\begin{array}{l}0.28726^{\dagger} \\
(0.13921)\end{array}$ & $\begin{array}{l}0.29916^{\dagger} \\
(0.1401)\end{array}$ & $\begin{array}{l}0.25654^{t} \\
(0.13884)\end{array}$ & $\begin{array}{l}0.3136^{\dagger} \\
(0.14124)\end{array}$ & $\begin{array}{l}0.34315^{\dagger} \\
(0.14135)\end{array}$ & $\begin{array}{l}0.31747^{\dagger} \\
(0.13882)\end{array}$ & $\begin{array}{l}0.28726^{\dagger} \\
(0.13906)\end{array}$ & $\begin{array}{l}0.2627^{\ddagger} \\
(0.13948)\end{array}$ & $\begin{array}{l}0.27656^{\ddagger} \\
(0.14159)\end{array}$ & $\begin{array}{l}0.28653^{\dagger} \\
(0.13978)\end{array}$ & $\begin{array}{l}0.37572^{*} \\
(0.14317)\end{array}$ \\
\hline $1 \& 3$ & $\begin{array}{l}0.23024^{\dagger} \\
(0.09032)\end{array}$ & $\begin{array}{l}0.32416^{*} \\
(0.09129)\end{array}$ & $\begin{array}{l}0.23151^{\dagger} \\
(0.09026)\end{array}$ & $\begin{array}{l}0.23902^{*} \\
(0.09089)\end{array}$ & $\begin{array}{l}0.22383^{\dagger} \\
(0.08964)\end{array}$ & $\begin{array}{l}0.25357^{*} \\
(0.09264)\end{array}$ & $\begin{array}{l}0.28515^{*} \\
(0.09384)\end{array}$ & $\begin{array}{l}0.27685^{*} \\
(0.09163)\end{array}$ & $\begin{array}{l}0.25237^{*} \\
(0.09292)\end{array}$ & $\begin{array}{l}0.20503^{\dagger} \\
(0.09116)\end{array}$ & $\begin{array}{l}0.22645^{\dagger} \\
(0.0908)\end{array}$ & $\begin{array}{l}0.23074^{\dagger} \\
(0.09022)\end{array}$ & $\begin{array}{l}0.23074^{\dagger} \\
(0.0896)\end{array}$ \\
\hline Factor $1,3 \& 4$ & $\begin{array}{l}0.36158^{*} \\
(0.02542)\end{array}$ & $\begin{array}{l}0.38729^{*} \\
(0.02404)\end{array}$ & $\begin{array}{l}0.36346^{*} \\
(0.02395)\end{array}$ & $\begin{array}{l}0.37655^{*} \\
(0.03058)\end{array}$ & $\begin{array}{l}0.29441^{*} \\
(0.03694)\end{array}$ & $\begin{array}{l}0.38822^{*} \\
(0.03385)\end{array}$ & $\begin{array}{l}0.40422^{*} \\
(0.03151)\end{array}$ & $\begin{array}{l}0.4241^{*} \\
(0.03481)\end{array}$ & $\begin{array}{l}0.38233^{*} \\
(0.03141)\end{array}$ & $\begin{array}{l}0.32376^{*} \\
(0.0329)\end{array}$ & $\begin{array}{l}0.35483^{*} \\
(0.0304)\end{array}$ & $\begin{array}{l}0.36091^{*} \\
(0.03805)\end{array}$ & $\begin{array}{l}0.4498^{*} \\
(0.04355)\end{array}$ \\
\hline Factor $1 \& 2$ & $\begin{array}{l}0.26261^{\dagger} \\
(0.125)\end{array}$ & $\begin{array}{l}0.29514^{\dagger} \\
(0.12254)\end{array}$ & $\begin{array}{l}0.27122^{\dagger} \\
(0.12826)\end{array}$ & $\begin{array}{l}0.26846^{\dagger} \\
(0.1249)\end{array}$ & $\begin{array}{l}0.25179^{\dagger} \\
(0.12402)\end{array}$ & $\begin{array}{l}0.28378^{\dagger} \\
(0.12608)\end{array}$ & $\begin{array}{l}0.31296^{\dagger} \\
(0.12664)\end{array}$ & $\begin{array}{l}0.27132^{\dagger} \\
(0.12396)\end{array}$ & $\begin{array}{l}0.27033^{\dagger} \\
(0.12485)\end{array}$ & $\begin{array}{l}0.23426^{\ddagger} \\
(0.12553)\end{array}$ & $\begin{array}{l}0.2635^{\dagger} \\
(0.12476)\end{array}$ & $\begin{array}{l}0.26328^{\dagger} \\
(0.12484)\end{array}$ & $\begin{array}{l}0.27311^{\dagger} \\
(0.124)\end{array}$ \\
\hline Factor $1,2 \& 4$ & $\begin{array}{l}0.51193^{*} \\
(0.05534)\end{array}$ & $\begin{array}{l}0.55097^{*} \\
(0.05472)\end{array}$ & $\begin{array}{l}0.51899^{*} \\
(0.06019)\end{array}$ & $\begin{array}{l}0.52775^{*} \\
(0.05885)\end{array}$ & $\begin{array}{l}0.45456^{*} \\
(0.05976)\end{array}$ & $\begin{array}{l}0.53224^{*} \\
(0.05798)\end{array}$ & $\begin{array}{l}0.53035^{*} \\
(0.05547)\end{array}$ & $\begin{array}{l}0.52273^{*} \\
(0.0548)\end{array}$ & $\begin{array}{l}0.51391^{*} \\
(0.05498)\end{array}$ & $\begin{array}{l}0.48129^{*} \\
(0.05784)\end{array}$ & $\begin{array}{l}0.50246^{*} \\
(0.06029)\end{array}$ & $\begin{array}{l}0.51075^{*} \\
(0.06372)\end{array}$ & $\begin{array}{l}0.5294^{*} \\
(0.0551)\end{array}$ \\
\hline Factor $1,2 \& 3$ & $\begin{array}{l}0.23368^{*} \\
(0.06364)\end{array}$ & $\begin{array}{l}0.31762^{*} \\
(0.06411)\end{array}$ & $\begin{array}{l}0.24249^{*} \\
(0.06798)\end{array}$ & $\begin{array}{l}0.24599^{*} \\
(0.06385)\end{array}$ & $\begin{array}{l}0.20109^{*} \\
(0.06335)\end{array}$ & $\begin{array}{l}0.24728^{*} \\
(0.06309)\end{array}$ & $\begin{array}{l}0.28489^{*} \\
(0.06665)\end{array}$ & $\begin{array}{l}0.28246^{*} \\
(0.06477)\end{array}$ & $\begin{array}{l}0.25275^{*} \\
(0.06471)\end{array}$ & $\begin{array}{l}0.19112^{*} \\
(0.06715)\end{array}$ & $\begin{array}{l}0.23299^{*} \\
(0.06239)\end{array}$ & $\begin{array}{l}0.23515^{*} \\
(0.06222)\end{array}$ & $\begin{array}{l}0.26253^{*} \\
(0.06278)\end{array}$ \\
\hline All Factors & $\begin{array}{l}0.22961^{*} \\
(0.02657)\end{array}$ & $\begin{array}{l}0.33845^{*} \\
(0.02866)\end{array}$ & $\begin{array}{l}0.23915^{*} \\
(0.02892)\end{array}$ & $\begin{array}{l}0.2469^{*} \\
(0.02382)\end{array}$ & $\begin{array}{l}0.17281^{*} \\
(0.02788)\end{array}$ & $\begin{array}{l}0.25415^{*} \\
(0.02418)\end{array}$ & $\begin{array}{l}0.28322^{*} \\
(0.02846)\end{array}$ & $\begin{array}{l}0.29574^{*} \\
(0.02927)\end{array}$ & $\begin{array}{l}0.25236^{*} \\
(0.02448)\end{array}$ & $\begin{array}{l}0.19434^{*} \\
(0.02572)\end{array}$ & $\begin{array}{l}0.22319^{*} \\
(0.02573)\end{array}$ & $\begin{array}{l}0.23063^{*} \\
(0.03271)\end{array}$ & $\begin{array}{l}0.31526^{*} \\
(0.03704)\end{array}$ \\
\hline Individual Variable & $\begin{array}{l}0.00314 \\
(0.02613)\end{array}$ & $\begin{array}{l}-0.11385^{*} \\
(0.02768)\end{array}$ & $\begin{array}{l}-0.00772 \\
(0.02968)\end{array}$ & $\begin{array}{l}-0.01743 \\
(0.02419)\end{array}$ & $\begin{array}{l}0.06915^{\dagger} \\
(0.0288)\end{array}$ & $\begin{array}{l}-0.02634 \\
(0.02488)\end{array}$ & $\begin{array}{l}-0.05588^{\dagger} \\
(0.02801)\end{array}$ & $\begin{array}{l}-0.06798^{\dagger} \\
(0.02832)\end{array}$ & $\begin{array}{l}-0.02403 \\
(0.02515)\end{array}$ & $\begin{array}{l}0.0442^{\ddagger} \\
(0.02595)\end{array}$ & $\begin{array}{l}0.01071 \\
(0.02595)\end{array}$ & $\begin{array}{l}0.00193 \\
(0.03282)\end{array}$ & $\begin{array}{l}-0.08846 \\
(0.03716)\end{array}$ \\
\hline
\end{tabular}

* Significant at the 0.01 level $\quad$ Significant at the 0.05 level $\$$ Significant at the 0.10 level

Table 104 Coefficients with individual variables for perceived benefits of AMT - Wood Industry 


\begin{tabular}{|c|c|c|c|c|c|c|c|c|c|c|c|c|c|}
\hline $\begin{array}{l}\text { Individual variable } \\
\text { included in the } \\
\text { model in the } \\
\text { column }\end{array}$ & 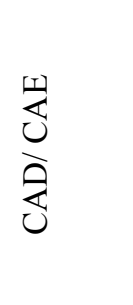 & 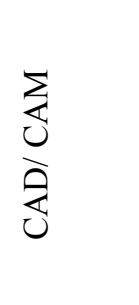 & 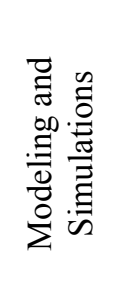 & 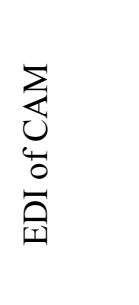 & $\sum_{i=1}^{\infty}$ & 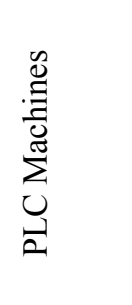 & 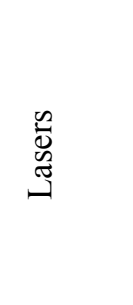 & $\begin{array}{l}0 \\
.0 \\
0 \\
0 \\
0 \\
0 \\
0 \\
0 \\
3 \\
0 \\
0 \\
0 \\
0 \\
0\end{array}$ & 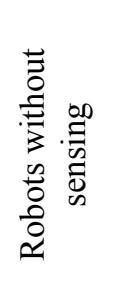 & 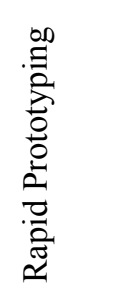 & 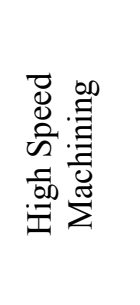 & 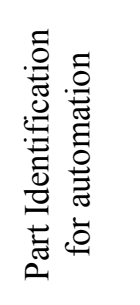 & 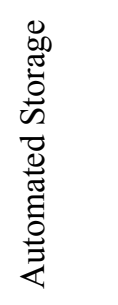 \\
\hline No factors & $\begin{array}{l}0.26087^{*} \\
(0.01824)\end{array}$ & $\begin{array}{l}0.26059^{*} \\
(0.01821)\end{array}$ & $\begin{array}{l}0.25949^{*} \\
(0.01801)\end{array}$ & $\begin{array}{l}0.25923^{*} \\
(0.0181)\end{array}$ & $\begin{array}{l}0.26008^{*} \\
(0.01824)\end{array}$ & $\begin{array}{l}0.26179^{*} \\
(0.01835)\end{array}$ & $\begin{array}{l}0.25992^{*} \\
(0.01819)\end{array}$ & $\begin{array}{l}0.26009^{*} \\
(0.01823)\end{array}$ & $\begin{array}{l}0.26025^{*} \\
(0.0182)\end{array}$ & $\begin{array}{l}0.26012^{*} \\
(0.0176)\end{array}$ & \begin{tabular}{|l|}
$0.26007^{*}$ \\
$(0.0182)$
\end{tabular} & $\begin{array}{l}0.25758^{*} \\
(0.01807)\end{array}$ & $\begin{array}{l}0.25999^{*} \\
(0.0182)\end{array}$ \\
\hline Factor 5 & $\begin{array}{l}0.26019^{*} \\
(0.0941)\end{array}$ & $\begin{array}{l}0.25772^{*} \\
(0.09379)\end{array}$ & $\begin{array}{l}0.24965^{*} \\
(0.0927)\end{array}$ & $\begin{array}{l}0.24285^{*} \\
(0.09323)\end{array}$ & $\begin{array}{l}0.2521^{*} \\
(0.0939)\end{array}$ & $\begin{array}{l}0.25948^{*} \\
(0.09422)\end{array}$ & $\begin{array}{l}0.24993^{*} \\
(0.0936)\end{array}$ & $\begin{array}{l}0.25177^{*} \\
(0.09382)\end{array}$ & $\begin{array}{l}0.2629^{*} \\
(0.09394)\end{array}$ & $\begin{array}{l}0.2526^{*} \\
(0.09059)\end{array}$ & \begin{tabular}{|l|}
$0.24358^{*}$ \\
$(0.09385)$
\end{tabular} & $\begin{array}{l}0.24403^{*} \\
(0.09295)\end{array}$ & $\begin{array}{l}0.25097^{*} \\
(0.09365)\end{array}$ \\
\hline Factor 4 & $\begin{array}{l}0.1649^{*} \\
(0.04988)\end{array}$ & $\begin{array}{l}0.1691^{*} \\
(0.04863)\end{array}$ & $\begin{array}{l}0.13542^{*} \\
(0.04226)\end{array}$ & $\begin{array}{l}0.1275^{*} \\
(0.04273)\end{array}$ & $\begin{array}{l}0.13456^{*} \\
(0.04689)\end{array}$ & $\begin{array}{l}0.14922^{*} \\
(0.04442)\end{array}$ & $\begin{array}{l}0.13892^{*} \\
(0.04265)\end{array}$ & $\begin{array}{l}0.13974^{*} \\
(0.04275)\end{array}$ & $\begin{array}{l}0.13974^{*} \\
(0.04268)\end{array}$ & $\begin{array}{l}0.13974^{*} \\
(0.04128)\end{array}$ & \begin{tabular}{|l|}
$0.11979^{*}$ \\
$(0.04482)$
\end{tabular} & $\begin{array}{l}0.13974^{*} \\
(0.04233)\end{array}$ & $\begin{array}{l}0.13974^{*} \\
(0.04267)\end{array}$ \\
\hline Factor $4 \& 5$ & $\begin{array}{l}0.35445^{\ddagger} \\
(0.19731)\end{array}$ & $\begin{array}{l}0.34001^{\ddagger} \\
(0.19591)\end{array}$ & $\begin{array}{l}0.30521 \\
(0.19389)\end{array}$ & $\begin{array}{l}0.27746 \\
(0.19587)\end{array}$ & $\begin{array}{l}0.33099^{\ddagger} \\
(0.19622)\end{array}$ & $\begin{array}{l}0.33833^{\ddagger} \\
(0.19622)\end{array}$ & $\begin{array}{l}0.32069 \\
(0.1957)\end{array}$ & $\begin{array}{l}0.32859^{\ddagger} \\
(0.1961)\end{array}$ & $\begin{array}{l}0.33933^{\ddagger} \\
(0.19579)\end{array}$ & $\begin{array}{l}0.33196^{\ddagger} \\
(0.18931)\end{array}$ & \begin{tabular}{|l|}
0.30519 \\
$(0.19655)$
\end{tabular} & $\begin{array}{l}0.31494 \\
(0.19423)\end{array}$ & $\begin{array}{l}0.33196^{\ddagger} \\
(0.1957)\end{array}$ \\
\hline Factor 3 & $\begin{array}{l}0.12528 \\
(0.10563)\end{array}$ & $\begin{array}{l}0.12581 \\
(0.10554)\end{array}$ & $\begin{array}{l}0.11445 \\
(0.1044)\end{array}$ & $\begin{array}{l}0.11223 \\
(0.10496)\end{array}$ & $\begin{array}{l}0.1205 \\
(0.1059)\end{array}$ & $\begin{array}{l}0.12957 \\
(0.10604)\end{array}$ & $\begin{array}{l}0.11267 \\
(0.10554)\end{array}$ & $\begin{array}{l}0.12117 \\
(0.10564)\end{array}$ & $\begin{array}{l}0.12451 \\
(0.10546)\end{array}$ & $\begin{array}{l}0.12222 \\
(0.102)\end{array}$ & \begin{tabular}{|l|}
0.12072 \\
$(0.10544)$
\end{tabular} & $\begin{array}{l}0.12222 \\
(0.1046)\end{array}$ & $\begin{array}{l}0.12222 \\
(0.10544)\end{array}$ \\
\hline Factor $3 \& 5$ & $\begin{array}{l}0.16178^{*} \\
(0.05102)\end{array}$ & $\begin{array}{l}0.16132^{*} \\
(0.05098)\end{array}$ & $\begin{array}{l}0.16045^{*} \\
(0.05043)\end{array}$ & $\begin{array}{l}0.16076^{*} \\
(0.05068)\end{array}$ & $\begin{array}{l}0.16132^{*} \\
(0.05107)\end{array}$ & $\begin{array}{l}0.16411^{*} \\
(0.05116)\end{array}$ & $\begin{array}{l}0.16132^{*} \\
(0.05091)\end{array}$ & $\begin{array}{l}0.16132^{*} \\
(0.05104)\end{array}$ & $\begin{array}{l}0.16132^{*} \\
(0.05095)\end{array}$ & $\begin{array}{l}0.16132^{*} \\
(0.04928)\end{array}$ & $\begin{array}{l}0.1555^{*} \\
(0.0511)\end{array}$ & $\begin{array}{l}0.16132^{*} \\
(0.05054)\end{array}$ & $\begin{array}{l}0.16132^{*} \\
(0.05095)\end{array}$ \\
\hline Facto & $\begin{array}{l}0.17907^{\ddagger} \\
(0.1047) \\
\end{array}$ & $\begin{array}{l}0.18364^{\ddagger} \\
(0.10404)\end{array}$ & $\begin{array}{l}0.15291 \\
(0.10007) \\
\end{array}$ & $\begin{array}{l}0.1022 \\
(0.10257)\end{array}$ & $\begin{array}{l}0.14827 \\
(0.10279) \\
\end{array}$ & $\begin{array}{l}0.16278 \\
(0.10205) \\
\end{array}$ & $\begin{array}{l}0.15291 \\
(0.10103)\end{array}$ & $\begin{array}{l}0.15291 \\
(0.10128)\end{array}$ & $\begin{array}{l}0.15291 \\
(0.1011) \\
\end{array}$ & $\begin{array}{l}0.15291 \\
(0.09779)\end{array}$ & \begin{tabular}{|l|}
0.12998 \\
$(0.10231)$ \\
\end{tabular} & $\begin{array}{l}0.15291 \\
(0.10029)\end{array}$ & $\begin{array}{l}0.15291 \\
(0.10109) \\
\end{array}$ \\
\hline Factor $3,4 \& 5$ & \multicolumn{13}{|c|}{ Insufficient Data } \\
\hline Factor 2 & $\begin{array}{l}0.16108^{*} \\
(0.03545)\end{array}$ & $\begin{array}{l}0.16084^{*} \\
(0.03541)\end{array}$ & $\begin{array}{l}0.16018^{*} \\
(0.03503)\end{array}$ & $\begin{array}{l}0.16018^{*} \\
(0.0352)\end{array}$ & $\begin{array}{l}0.16018^{*} \\
(0.03548)\end{array}$ & $\begin{array}{l}0.16046^{*} \\
(0.03546)\end{array}$ & $\begin{array}{l}0.15929^{*} \\
(0.03537)\end{array}$ & $\begin{array}{l}0.16018^{*} \\
(0.03546)\end{array}$ & $\begin{array}{l}0.16018^{*} \\
(0.03539)\end{array}$ & $\begin{array}{l}0.16018^{*} \\
(0.03424)\end{array}$ & $\begin{array}{l}0.15922^{*} \\
(0.03539)\end{array}$ & $\begin{array}{l}0.15883^{*} \\
(0.03511)\end{array}$ & $\begin{array}{l}0.15887^{*} \\
(0.0354)\end{array}$ \\
\hline Factor $2 \& 5$ & $\begin{array}{l}0.23954 \\
(0.17797)\end{array}$ & $\begin{array}{l}0.24747 \\
(0.17811)\end{array}$ & \begin{tabular}{|l|}
0.21694 \\
$(0.17553)$
\end{tabular} & $\begin{array}{l}0.16414 \\
(0.17817)\end{array}$ & $\begin{array}{l}0.22573 \\
(0.17787)\end{array}$ & $\begin{array}{l}0.24336 \\
(0.17876)\end{array}$ & $\begin{array}{l}0.22754 \\
(0.17719)\end{array}$ & $\begin{array}{l}0.22477 \\
(0.17766)\end{array}$ & $\begin{array}{l}0.22754 \\
(0.17732)\end{array}$ & $\begin{array}{l}0.22754 \\
(0.17151)\end{array}$ & \begin{tabular}{|l|}
0.22754 \\
$(0.17729)$
\end{tabular} & $\begin{array}{l}0.16952 \\
(0.17702)\end{array}$ & $\begin{array}{l}0.22754 \\
(0.1773)\end{array}$ \\
\hline Factor $2 \& 4$ & $\begin{array}{l}0.15865^{\ddagger} \\
(0.08095)\end{array}$ & $\begin{array}{l}0.17057^{\dagger} \\
(0.08224)\end{array}$ & $\begin{array}{l}0.10286 \\
(0.0796)\end{array}$ & $\begin{array}{l}0.08027 \\
(0.08291)\end{array}$ & $\begin{array}{l}0.14297^{\ddagger} \\
(0.07962)\end{array}$ & $\begin{array}{l}0.1535^{\ddagger} \\
(0.0805)\end{array}$ & $\begin{array}{l}0.13724^{\ddagger} \\
(0.07943)\end{array}$ & $\begin{array}{l}0.14122^{\ddagger} \\
(0.07959)\end{array}$ & $\begin{array}{l}0.14781^{\ddagger} \\
(0.07945)\end{array}$ & $\begin{array}{l}0.14368^{\ddagger} \\
(0.07679)\end{array}$ & \begin{tabular}{|l|}
$0.13734^{\ddagger}$ \\
$(0.0795)$
\end{tabular} & $\begin{array}{l}0.11148 \\
(0.07952)\end{array}$ & $\begin{array}{l}0.13994^{\ddagger} \\
(0.07943)\end{array}$ \\
\hline Factor $2,4 \& 5$ & $\begin{array}{l}0.07649 \\
(0.09715) \\
\end{array}$ & $\begin{array}{l}0.07384 \\
(0.09625) \\
\end{array}$ & \begin{tabular}{|l|l}
0.05718 \\
$(0.09462)$ \\
\end{tabular} & $\begin{array}{l}0.03223 \\
(0.09574) \\
\end{array}$ & $\begin{array}{l}0.06026 \\
(0.09583) \\
\end{array}$ & $\begin{array}{l}0.06725 \\
(0.09617) \\
\end{array}$ & $\begin{array}{l}0.05896 \\
(0.09553) \\
\end{array}$ & $\begin{array}{l}0.05865 \\
(0.09579) \\
\end{array}$ & $\begin{array}{l}0.06378 \\
(0.09563) \\
\end{array}$ & $\begin{array}{l}0.06026 \\
(0.09247) \\
\end{array}$ & \begin{tabular}{|l|}
0.03861 \\
$(0.09674)$ \\
\end{tabular} & $\begin{array}{l}-0.00474 \\
(0.09743)\end{array}$ & $\begin{array}{l}0.03194 \\
(0.09762) \\
\end{array}$ \\
\hline Factor $2 \& 3$ & $\begin{array}{l}0.26271^{\ddagger} \\
(0.13525)\end{array}$ & $\begin{array}{l}0.26165^{\ddagger} \\
(0.13508)\end{array}$ & $\begin{array}{l}0.2451^{\ddagger} \\
(0.13366)\end{array}$ & $\begin{array}{l}0.24993^{\ddagger} \\
(0.13429)\end{array}$ & $\begin{array}{l}0.25733^{\ddagger} \\
(0.13532)\end{array}$ & $\begin{array}{l}0.26184^{\ddagger} \\
(0.13531)\end{array}$ & $\begin{array}{l}0.25783^{\ddagger} \\
(0.13488)\end{array}$ & $\begin{array}{l}0.25783^{\ddagger} \\
(0.13522)\end{array}$ & $\begin{array}{l}0.25783^{\ddagger} \\
(0.13498)\end{array}$ & $\begin{array}{l}0.25783^{\dagger} \\
(0.13056)\end{array}$ & \begin{tabular}{|l|}
$0.25537^{\ddagger}$ \\
$(0.13497)$
\end{tabular} & $\begin{array}{l}0.24944^{\ddagger} \\
(0.13393)\end{array}$ & $\begin{array}{l}0.25783^{\ddagger} \\
(0.13497)\end{array}$ \\
\hline Factor $2,3 \& 5$ & $\begin{array}{l}0.27514^{\ddagger} \\
(0.16131)\end{array}$ & $\begin{array}{l}0.29057^{\ddagger} \\
(0.16211)\end{array}$ & $\begin{array}{l}0.24785 \\
(0.15924) \\
\end{array}$ & $\begin{array}{l}0.20188 \\
(0.16191) \\
\end{array}$ & $\begin{array}{l}0.26528 \\
(0.16117) \\
\end{array}$ & $\begin{array}{l}0.27264^{\ddagger} \\
(0.16134)\end{array}$ & $\begin{array}{l}0.2616 \\
(0.16069) \\
\end{array}$ & $\begin{array}{l}0.263 \\
(0.16109)\end{array}$ & $\begin{array}{l}0.26528^{\ddagger} \\
(0.16079) \\
\end{array}$ & $\begin{array}{l}0.11636 \\
(0.15793)\end{array}$ & \begin{tabular}{|l|}
0.24564 \\
$(0.16133)$ \\
\end{tabular} & $\begin{array}{l}0.20602 \\
(0.16079)\end{array}$ & $\begin{array}{l}0.22917 \\
(0.16274) \\
\end{array}$ \\
\hline Factor & $\begin{array}{l}0.33504^{*} \\
(0.08604)\end{array}$ & $\begin{array}{l}0.34268^{*} \\
(0.08619)\end{array}$ & $\begin{array}{l}0.22983^{*} \\
(0.08572)\end{array}$ & $\begin{array}{l}0.284^{*} \\
(0.08294)\end{array}$ & $\begin{array}{l}0.30612^{*} \\
(0.08561)\end{array}$ & $\begin{array}{l}0.32777^{*} \\
(0.08518)\end{array}$ & $\begin{array}{l}0.30886^{*} \\
(0.0826)\end{array}$ & $\begin{array}{l}0.2988^{*} \\
(0.0845)\end{array}$ & $\begin{array}{l}0.3343^{*} \\
(0.08416)\end{array}$ & $\begin{array}{l}0.31195^{*} \\
(0.07993)\end{array}$ & $\begin{array}{l}0.29157^{*} \\
(0.0838)\end{array}$ & $\begin{array}{l}0.26513^{*} \\
(0.08354)\end{array}$ & $\begin{array}{l}0.29202^{*} \\
(0.08379)\end{array}$ \\
\hline Facto & $\begin{array}{l}0.29873^{\dagger} \\
(0.12316) \\
\end{array}$ & $\begin{array}{l}0.28342^{\dagger} \\
(0.12044)\end{array}$ & $\begin{array}{l}0.26258^{\dagger} \\
(0.11888)\end{array}$ & $\begin{array}{l}0.2249^{\ddagger} \\
(0.12093)\end{array}$ & $\begin{array}{l}0.2714^{\dagger} \\
(0.12044)\end{array}$ & $\begin{array}{l}0.28446^{\dagger} \\
(0.12122) \\
\end{array}$ & $\begin{array}{l}0.256^{\dagger} \\
(0.12043)\end{array}$ & $\begin{array}{l}0.27123^{\dagger} \\
(0.12029)\end{array}$ & $\begin{array}{l}0.2755^{\dagger} \\
(0.12009)\end{array}$ & $\begin{array}{l}0.25884^{\dagger} \\
(0.11617)\end{array}$ & \begin{tabular}{|l|}
$0.26151^{\dagger}$ \\
$(0.12029)$
\end{tabular} & $\begin{array}{l}0.2466^{\dagger} \\
(0.11944)\end{array}$ & $\begin{array}{l}0.25733^{\dagger} \\
(0.12053)\end{array}$ \\
\hline Factor 1 & $\begin{array}{l}0.22118^{*} \\
(0.03879)\end{array}$ & $\begin{array}{l}0.22281^{*} \\
(0.03881)\end{array}$ & $\begin{array}{l}0.21758^{*} \\
(0.03821)\end{array}$ & $\begin{array}{l}0.21322^{*} \\
(0.03845)\end{array}$ & $\begin{array}{l}0.21809^{*} \\
(0.0387)\end{array}$ & $\begin{array}{l}0.22673^{*} \\
(0.04021)\end{array}$ & $\begin{array}{l}0.21441^{*} \\
(0.03865)\end{array}$ & $\begin{array}{l}0.21717^{*} \\
(0.03869)\end{array}$ & $\begin{array}{l}0.21932^{*} \\
(0.03862)\end{array}$ & $\begin{array}{l}0.21094^{*} \\
(0.03737)\end{array}$ & $\begin{array}{l}0.21212^{*} \\
(0.03882)\end{array}$ & $\begin{array}{l}0.18483^{*} \\
(0.03997)\end{array}$ & $\begin{array}{l}0.21809^{*} \\
(0.03861)\end{array}$ \\
\hline Factor $1 \& 5$ & $\begin{array}{l}0.31703^{*} \\
(0.03715)\end{array}$ & $\begin{array}{l}0.32675^{*} \\
(0.038)\end{array}$ & $\begin{array}{l}0.31647^{*} \\
(0.03672)\end{array}$ & $\begin{array}{l}0.3158^{*} \\
(0.0369)\end{array}$ & $\begin{array}{l}0.31647^{*} \\
(0.03719)\end{array}$ & $\begin{array}{l}0.32242^{*} \\
(0.03793)\end{array}$ & $\begin{array}{l}0.30105^{*} \\
(0.0383)\end{array}$ & $\begin{array}{l}0.31559^{*} \\
(0.03718)\end{array}$ & $\begin{array}{l}0.31781^{*} \\
(0.03711)\end{array}$ & $\begin{array}{l}0.31647^{*} \\
(0.03589)\end{array}$ & $\begin{array}{l}0.31353^{*} \\
(0.03715)\end{array}$ & $\begin{array}{l}0.31211^{*} \\
(0.03683)\end{array}$ & $\begin{array}{l}0.31361^{*} \\
(0.03715)\end{array}$ \\
\hline Factor $1 \& 4$ & $\begin{array}{l}0.16706^{\dagger} \\
(0.08078)\end{array}$ & $\begin{array}{l}0.16806^{\dagger} \\
(0.08004)\end{array}$ & $\begin{array}{l}0.13231^{\ddagger} \\
(0.07796)\end{array}$ & $\begin{array}{l}0.1298^{\ddagger} \\
(0.07855)\end{array}$ & $\begin{array}{l}0.14773^{\ddagger} \\
(0.07897)\end{array}$ & $\begin{array}{l}0.16467^{\dagger} \\
(0.08115)\end{array}$ & $\begin{array}{l}0.13145^{\ddagger} \\
(0.07931)\end{array}$ & $\begin{array}{l}0.14865^{\ddagger} \\
(0.07873)\end{array}$ & $\begin{array}{l}0.15038^{\ddagger} \\
(0.0786)\end{array}$ & $\begin{array}{l}0.14331^{\ddagger} \\
(0.07603)\end{array}$ & \begin{tabular}{|l|}
$0.14221^{\ddagger}$ \\
$(0.07872)$
\end{tabular} & $\begin{array}{l}0.14375^{\ddagger} \\
(0.07798)\end{array}$ & $\begin{array}{l}0.14379^{\ddagger} \\
(0.07867)\end{array}$ \\
\hline Factor $1,4 \& 5$ & $\begin{array}{l}0.11468 \\
(0.12437) \\
\end{array}$ & $\begin{array}{l}0.11951 \\
(0.12423) \\
\end{array}$ & \begin{tabular}{|l|}
0.04007 \\
$(0.12288)$ \\
\end{tabular} & $\begin{array}{l}0.0645 \\
(0.12287) \\
\end{array}$ & $\begin{array}{l}0.09571 \\
(0.12321) \\
\end{array}$ & $\begin{array}{l}0.10755 \\
(0.12382) \\
\end{array}$ & $\begin{array}{l}0.07073 \\
(0.12383) \\
\end{array}$ & $\begin{array}{l}0.09534 \\
(0.12308) \\
\end{array}$ & $\begin{array}{l}0.09988 \\
(0.12287) \\
\end{array}$ & $\begin{array}{l}0.09677 \\
(0.11883) \\
\end{array}$ & \begin{tabular}{|l|}
0.06394 \\
$(0.12489)$ \\
\end{tabular} & $\begin{array}{l}0.09317 \\
(0.12187) \\
\end{array}$ & $\begin{array}{l}0.09677 \\
(0.12284) \\
\end{array}$ \\
\hline Factor $1 \& 3$ & $\begin{array}{l}0.33099^{*} \\
(0.03106)\end{array}$ & $\begin{array}{l}0.33537^{*} \\
(0.03125)\end{array}$ & $\begin{array}{l}0.33068^{*} \\
(0.0307)\end{array}$ & $\begin{array}{l}0.32045^{*} \\
(0.03112)\end{array}$ & $\begin{array}{l}0.33062^{*} \\
(0.03109)\end{array}$ & $\begin{array}{l}0.33131^{*} \\
(0.03108)\end{array}$ & $\begin{array}{l}0.33068^{*} \\
(0.031)\end{array}$ & $\begin{array}{l}0.33059^{*} \\
(0.03107)\end{array}$ & $\begin{array}{l}0.33157^{*} \\
(0.03103)\end{array}$ & $\begin{array}{l}0.33068^{*} \\
(0.03)\end{array}$ & \begin{tabular}{|l|}
$0.3286^{*}$ \\
$(0.03105)$
\end{tabular} & $\begin{array}{l}0.31516^{*} \\
(0.03123)\end{array}$ & $\begin{array}{l}0.33068^{*} \\
(0.03102)\end{array}$ \\
\hline Factor $1,3 \& 5$ & $\begin{array}{l}0.29456^{*} \\
(0.04036)\end{array}$ & $\begin{array}{l}0.29442^{*} \\
(0.04031)\end{array}$ & $\begin{array}{l}0.29056^{*} \\
(0.03988)\end{array}$ & $\begin{array}{l}0.29122^{*} \\
(0.04008)\end{array}$ & $\begin{array}{l}0.29332^{*} \\
(0.04038)\end{array}$ & $\begin{array}{l}0.30305^{*} \\
(0.04221)\end{array}$ & $\begin{array}{l}0.29216^{*} \\
(0.04026)\end{array}$ & $\begin{array}{l}0.28668^{*} \\
(0.04125)\end{array}$ & $\begin{array}{l}0.3078^{*} \\
(0.04159)\end{array}$ & $\begin{array}{l}0.29332^{*} \\
(0.03897)\end{array}$ & \begin{tabular}{|l|}
$0.29209^{*}$ \\
$(0.04029)$
\end{tabular} & $\begin{array}{l}0.2926^{*} \\
(0.03996)\end{array}$ & $\begin{array}{l}0.29332^{*} \\
(0.04028)\end{array}$ \\
\hline Factor & $\begin{array}{l}0.30063^{*} \\
(0.03448)\end{array}$ & $\begin{array}{l}0.29602^{*} \\
(0.03078)\end{array}$ & $\begin{array}{l}0.23567^{*} \\
(0.03185)\end{array}$ & $\begin{array}{l}0.24387^{*} \\
(0.03231)\end{array}$ & $\begin{array}{l}0.28083^{*} \\
(0.02904)\end{array}$ & $\begin{array}{l}0.29298^{*} \\
(0.03197)\end{array}$ & $\begin{array}{l}0.26728^{*} \\
(0.03008)\end{array}$ & $\begin{array}{l}0.28108^{*} \\
(0.02875)\end{array}$ & $\begin{array}{l}0.28401^{*} \\
(0.02871)\end{array}$ & $\begin{array}{l}0.2456^{*} \\
(0.02854)\end{array}$ & \begin{tabular}{|l|}
$0.28173^{*}$ \\
$(0.02867)$
\end{tabular} & $\begin{array}{l}0.27721^{*} \\
(0.02849)\end{array}$ & $\begin{array}{l}0.28108^{*} \\
(0.02868)\end{array}$ \\
\hline Factor $1,3,4 \& 5$ & $\begin{array}{l}0.27842^{*} \\
(0.05549)\end{array}$ & $\begin{array}{l}0.27379^{*} \\
(0.05155)\end{array}$ & $\begin{array}{l}0.2459^{*} \\
(0.04823)\end{array}$ & $\begin{array}{l}0.21059^{*} \\
(0.05123)\end{array}$ & $\begin{array}{l}0.25129^{*} \\
(0.04901)\end{array}$ & $\begin{array}{l}0.2614^{*} \\
(0.05007)\end{array}$ & $\begin{array}{l}0.23527^{*} \\
(0.04983)\end{array}$ & $\begin{array}{l}0.25227^{*} \\
(0.04877)\end{array}$ & $\begin{array}{l}0.25298^{*} \\
(0.04869)\end{array}$ & $\begin{array}{l}0.2525^{*} \\
(0.04709)\end{array}$ & $\begin{array}{l}0.23109^{*} \\
(0.05086)\end{array}$ & $\begin{array}{l}0.22989^{*} \\
(0.04892)\end{array}$ & $\begin{array}{l}0.25062^{*} \\
(0.0487)\end{array}$ \\
\hline Factor $1 \& 2$ & $\begin{array}{l}0.1951^{*} \\
(0.03288)\end{array}$ & $\begin{array}{l}0.19023^{*} \\
(0.03221)\end{array}$ & $\begin{array}{l}0.18799^{*} \\
(0.03185)\end{array}$ & $\begin{array}{l}0.18734^{*} \\
(0.03201)\end{array}$ & $\begin{array}{l}0.187^{*} \\
(0.03286)\end{array}$ & $\begin{array}{l}0.19349^{*} \\
(0.0328)\end{array}$ & $\begin{array}{l}0.18869^{*} \\
(0.03215)\end{array}$ & $\begin{array}{l}0.18255^{*} \\
(0.03319)\end{array}$ & $\begin{array}{l}0.20208^{*} \\
(0.03356)\end{array}$ & $\begin{array}{l}0.18869^{*} \\
(0.03112)\end{array}$ & \begin{tabular}{|l|}
$0.17197^{*}$ \\
$(0.03417)$
\end{tabular} & $\begin{array}{l}0.15447^{*} \\
(0.03401)\end{array}$ & $\begin{array}{l}0.18778^{*} \\
(0.03218)\end{array}$ \\
\hline Factor $1,2 \& 5$ & $\begin{array}{l}0.29691^{*} \\
(0.08714)\end{array}$ & $\begin{array}{l}0.30623^{*} \\
(0.08747)\end{array}$ & $\begin{array}{l}0.29282^{*} \\
(0.08613)\end{array}$ & $\begin{array}{l}0.29391^{*} \\
(0.08655)\end{array}$ & $\begin{array}{l}0.29325^{*} \\
(0.08764)\end{array}$ & $\begin{array}{l}0.30097^{*} \\
(0.08743)\end{array}$ & $\begin{array}{l}0.2879^{*} \\
(0.08708)\end{array}$ & $\begin{array}{l}0.29404^{*} \\
(0.08719)\end{array}$ & $\begin{array}{l}0.29968^{*} \\
(0.08707)\end{array}$ & $\begin{array}{l}0.29555^{*} \\
(0.08416)\end{array}$ & $\begin{array}{l}0.27738^{*} \\
(0.08789)\end{array}$ & $\begin{array}{l}0.26331^{*} \\
(0.08702)\end{array}$ & $\begin{array}{l}0.2862^{*} \\
(0.08725)\end{array}$ \\
\hline Factor $1,2 \& 4$ & $\begin{array}{l}0.47905^{*} \\
(0.03839)\end{array}$ & $\begin{array}{l}0.4891^{*} \\
(0.0396)\end{array}$ & $\begin{array}{l}0.47267^{*} \\
(0.03784)\end{array}$ & $\begin{array}{l}0.4694^{*} \\
(0.03811)\end{array}$ & $\begin{array}{l}0.47136^{*} \\
(0.04221)\end{array}$ & $\begin{array}{l}0.48591^{*} \\
(0.04026)\end{array}$ & $\begin{array}{l}0.46462^{*} \\
(0.03886)\end{array}$ & $\begin{array}{l}0.46734^{*} \\
(0.03993)\end{array}$ & $\begin{array}{l}0.47727^{*} \\
(0.03823)\end{array}$ & $\begin{array}{l}0.47201^{*} \\
(0.03698)\end{array}$ & \begin{tabular}{|l|}
$0.46414^{*}$ \\
$(0.0391)$
\end{tabular} & $\begin{array}{l}0.45878^{*} \\
(0.03838)\end{array}$ & $\begin{array}{l}0.47586^{*} \\
(0.03822)\end{array}$ \\
\hline Factor $1,2,4 \& 5$ & $\begin{array}{l}0.32084^{*} \\
(0.05659)\end{array}$ & $\begin{array}{l}0.30844^{*} \\
(0.05496)\end{array}$ & $\begin{array}{l}0.30356^{*} \\
(0.05438)\end{array}$ & $\begin{array}{l}0.27678^{*} \\
(0.05601)\end{array}$ & $\begin{array}{l}0.30058^{*} \\
(0.06118)\end{array}$ & $\begin{array}{l}0.3236^{*} \\
(0.05858)\end{array}$ & $\begin{array}{l}0.28654^{*} \\
(0.05646)\end{array}$ & $\begin{array}{l}0.30778^{*} \\
(0.05502)\end{array}$ & $\begin{array}{l}0.30836^{*} \\
(0.05493)\end{array}$ & $\begin{array}{l}0.04102 \\
(0.07242)\end{array}$ & \begin{tabular}{|l|}
$0.27219^{*}$ \\
$(0.06013)$
\end{tabular} & $\begin{array}{l}0.27528^{*} \\
(0.05562)\end{array}$ & $\begin{array}{l}0.30722^{*} \\
(0.05492)\end{array}$ \\
\hline Factor $1,2 \& 3$ & $\begin{array}{l}0.10169^{\dagger} \\
(0.04985)\end{array}$ & $\begin{array}{l}0.10235^{\dagger} \\
(0.0498)\end{array}$ & $\begin{array}{l}0.09142^{\ddagger} \\
(0.04927)\end{array}$ & $\begin{array}{l}0.09313^{\ddagger} \\
(0.04951)\end{array}$ & $\begin{array}{l}0.09923^{\dagger} \\
(0.04984)\end{array}$ & $\begin{array}{l}0.10314^{\dagger} \\
(0.05005)\end{array}$ & $\begin{array}{l}0.09923^{\dagger} \\
(0.04968)\end{array}$ & $\begin{array}{l}0.09874^{\dagger} \\
(0.04981)\end{array}$ & $\begin{array}{l}0.10031^{\dagger} \\
(0.04973)\end{array}$ & $\begin{array}{l}0.09923^{\dagger} \\
(0.04809)\end{array}$ & $\begin{array}{l}0.09853^{\dagger} \\
(0.04971)\end{array}$ & $\begin{array}{l}0.08372^{\ddagger} \\
(0.04961)\end{array}$ & $\begin{array}{l}0.09114^{\ddagger} \\
(0.05004)\end{array}$ \\
\hline Factor $1,2,3 \& 5$ & $\begin{array}{l}0.32181^{*} \\
(0.04513)\end{array}$ & $\begin{array}{l}0.32177^{*} \\
(0.04507)\end{array}$ & $\begin{array}{l}0.31847^{*} \\
(0.04456)\end{array}$ & $\begin{array}{l}0.31285^{*} \\
(0.04487)\end{array}$ & $\begin{array}{l}0.31964^{*} \\
(0.04514)\end{array}$ & $\begin{array}{l}0.32356^{*} \\
(0.04535)\end{array}$ & $\begin{array}{l}0.31953^{*} \\
(0.04499)\end{array}$ & $\begin{array}{l}0.31729^{*} \\
(0.04522)\end{array}$ & $\begin{array}{l}0.32457^{*} \\
(0.04515)\end{array}$ & $\begin{array}{l}0.31983^{*} \\
(0.04355)\end{array}$ & \begin{tabular}{|l|}
$0.31956^{*}$ \\
$(0.04502)$
\end{tabular} & $\begin{array}{l}0.28078^{*} \\
(0.04663)\end{array}$ & $\begin{array}{l}0.3157^{*} \\
(0.04511)\end{array}$ \\
\hline Factor $1,2,3 \& 4$ & $\begin{array}{l}0.30418^{*} \\
(0.04421)\end{array}$ & $\begin{array}{l}0.30522^{*} \\
(0.04222)\end{array}$ & $\begin{array}{l}0.21765^{*} \\
(0.04277)\end{array}$ & $\begin{array}{l}0.24181^{*} \\
(0.04119)\end{array}$ & $\begin{array}{l}0.27804^{*} \\
(0.04135)\end{array}$ & $\begin{array}{l}0.29527^{*} \\
(0.04156)\end{array}$ & $\begin{array}{l}0.2685^{*} \\
(0.03903)\end{array}$ & $\begin{array}{l}0.27986^{*} \\
(0.03829)\end{array}$ & $\begin{array}{l}0.28539^{*} \\
(0.03816)\end{array}$ & $\begin{array}{l}0.19057^{*} \\
(0.04055)\end{array}$ & \begin{tabular}{|l|}
$0.26503^{*}$ \\
$(0.0399)$
\end{tabular} & $\begin{array}{l}0.24398^{*} \\
(0.04002)\end{array}$ & $\begin{array}{l}0.26071^{*} \\
(0.04098)\end{array}$ \\
\hline All F & $\begin{array}{l}0.1983^{*} \\
(0.03608)\end{array}$ & $\begin{array}{l}0.20184^{*} \\
(0.03499)\end{array}$ & $\begin{array}{l}0.14407^{*} \\
(0.03089)\end{array}$ & $\begin{array}{l}0.13097^{*} \\
(0.03465)\end{array}$ & $\begin{array}{l}0.17352^{*} \\
(0.03345)\end{array}$ & $\begin{array}{l}0.18848^{*} \\
(0.03236)\end{array}$ & $\begin{array}{l}0.15891^{*} \\
(0.03161)\end{array}$ & $\begin{array}{l}0.17352^{*} \\
(0.02991)\end{array}$ & $\begin{array}{l}0.17948^{*} \\
(0.02937)\end{array}$ & $\begin{array}{l}0.17607^{*} \\
(0.02838)\end{array}$ & $\begin{array}{l}0.14962^{*} \\
(0.03518)\end{array}$ & $\begin{array}{l}0.13738^{*} \\
(0.03225)\end{array}$ & $\begin{array}{l}0.14642^{*} \\
(0.03664)\end{array}$ \\
\hline $\begin{array}{l}\text { Individual } \\
\text { Variable }\end{array}$ & $\begin{array}{l}-0.02616 \\
(0.02674)\end{array}$ & $\begin{array}{l}-0.03072 \\
(0.02435)\end{array}$ & $\begin{array}{l}0.09862^{*} \\
(0.0308)\end{array}$ & $\begin{array}{l}0.0634^{\dagger} \\
(0.02527)\end{array}$ & $\begin{array}{l}0.00744 \\
(0.02758)\end{array}$ & $\begin{array}{l}-0.01582 \\
(0.02011)\end{array}$ & $\begin{array}{l}0.04156 \\
(0.02594)\end{array}$ & $\begin{array}{l}0.02579 \\
(0.03327)\end{array}$ & $\begin{array}{l}-0.05624 \\
(0.04015)\end{array}$ & $\begin{array}{l}0.27867^{*} \\
(0.05142)\end{array}$ & $\begin{array}{l}0.03675 \\
(0.0253)\end{array}$ & $\begin{array}{l}0.065^{*} \\
(0.02235)\end{array}$ & $\begin{array}{l}0.05077 \\
(0.03549)\end{array}$ \\
\hline
\end{tabular}

* Significant at the 0.01 level $\dagger$ Significant at the 0.05 level $\$$ Significant at the 0.10 level

Table 105 Coefficients with individual variables for high-level factors - Wood Industry 


\begin{tabular}{|c|c|c|c|c|c|c|c|c|c|c|c|c|c|}
\hline $\begin{array}{l}\text { Individual variable } \\
\text { included in the } \\
\text { model in the } \\
\text { column }\end{array}$ & 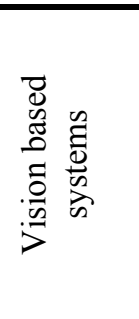 & 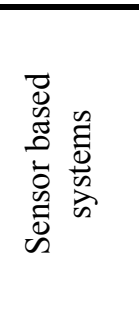 & 芯 & $\underset{3}{3}$ & $\overrightarrow{\mid \vec{I}}$ & 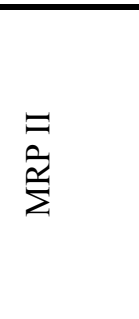 & 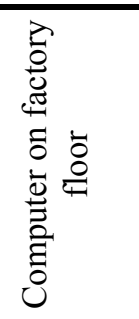 & $\sum_{U}$ & 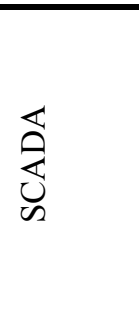 & 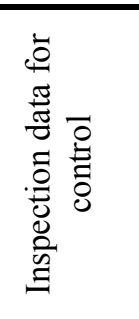 & 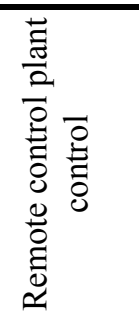 & 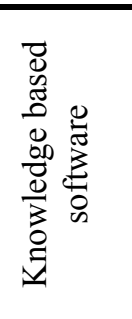 & 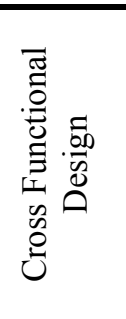 \\
\hline No factors & $\begin{array}{l}0.25944^{*} \\
(0.01808)\end{array}$ & $\begin{array}{l}0.2594^{*} \\
(0.01808)\end{array}$ & $\begin{array}{l}0.26109^{*} \\
(0.01825)\end{array}$ & $\begin{array}{l}0.26082^{*} \\
(0.01829)\end{array}$ & $(0.01827)$ & $\begin{array}{l}0.26009^{*} \\
(0.01825)\end{array}$ & $\begin{array}{l}0.26079^{*} \\
(0.01828)\end{array}$ & $\begin{array}{l}0.26078^{*} \\
(0.01814)\end{array}$ & $\begin{array}{l}0.26096^{*} \\
(0.01812)\end{array}$ & $\begin{array}{l}0.25904^{*} \\
(0.01818)\end{array}$ & $\begin{array}{l}0.2601^{*} \\
(0.01825)\end{array}$ & $\begin{array}{l}0.26005^{*} \\
(0.01823)\end{array}$ & $\begin{array}{l}0.259^{*} \\
(0.01826)\end{array}$ \\
\hline Factor 5 & $\begin{array}{l}0.23196^{\dagger} \\
(0.09333)\end{array}$ & $\begin{array}{l}0.24296^{*} \\
(0.09312)\end{array}$ & $\begin{array}{l}0.2601^{*} \\
(0.09407)\end{array}$ & $\begin{array}{l}0.25715^{*} \\
(0.09428)\end{array}$ & $\begin{array}{l}0.2539^{*} \\
(0.09442)\end{array}$ & $\begin{array}{l}0.25205^{*} \\
(0.09432)\end{array}$ & $\begin{array}{l}0.25981^{*} \\
(0.09476)\end{array}$ & $\begin{array}{l}0.2541^{*} \\
(0.09335)\end{array}$ & $\begin{array}{l}0.26467^{*} \\
(0.09335)\end{array}$ & $\begin{array}{l}0.24729^{*} \\
(0.09356)\end{array}$ & $\begin{array}{l}0.2526^{*} \\
(0.09389)\end{array}$ & $\begin{array}{l}0.24861^{*} \\
(0.09396)\end{array}$ & $\begin{array}{l}0.24354^{\dagger} \\
(0.0943)\end{array}$ \\
\hline Factor 4 & $\begin{array}{l}0.13742^{*} \\
(0.0424) \\
\end{array}$ & $\begin{array}{l}0.13871^{*} \\
(0.0424) \\
\end{array}$ & $\begin{array}{l}0.14524^{*} \\
(0.04308) \\
\end{array}$ & $\begin{array}{l}0.14253^{*} \\
(0.04312) \\
\end{array}$ & $\begin{array}{l}0.1401^{*} \\
(0.04287) \\
\end{array}$ & $\begin{array}{l}0.13973^{*} \\
(0.04278) \\
\end{array}$ & $\begin{array}{l}0.14361^{*} \\
(0.04334)\end{array}$ & $\begin{array}{l}0.15898^{*} \\
(0.04347)\end{array}$ & $\begin{array}{l}0.14212^{*} \\
(0.04249)\end{array}$ & $\begin{array}{l}0.13947^{*} \\
(0.04261)\end{array}$ & $\begin{array}{l}0.13973^{*} \\
(0.04278)\end{array}$ & $\begin{array}{l}0.13961^{*} \\
(0.04275)\end{array}$ & $\begin{array}{l}0.13947^{*} \\
(0.04274)\end{array}$ \\
\hline Factor $4 \& 5$ & $\begin{array}{l}0.32158^{\ddagger} \\
(0.19443)\end{array}$ & $\begin{array}{l}0.31046 \\
(0.19462)\end{array}$ & $\begin{array}{l}0.33744^{\ddagger} \\
(0.19603)\end{array}$ & $\begin{array}{l}0.33196^{\ddagger} \\
(0.19614)\end{array}$ & $\begin{array}{l}0.33196^{\ddagger} \\
(0.1962)\end{array}$ & $\begin{array}{l}0.33177^{\ddagger} \\
(0.19623)\end{array}$ & $\begin{array}{l}0.33513^{\ddagger} \\
(0.19621)\end{array}$ & $\begin{array}{l}0.3385^{\ddagger} \\
(0.1951)\end{array}$ & $\begin{array}{l}0.36991^{\ddagger} \\
(0.19543)\end{array}$ & $\begin{array}{l}0.29483 \\
(0.19652)\end{array}$ & $\begin{array}{l}0.33196^{\ddagger} \\
(0.1962)\end{array}$ & $\begin{array}{l}0.33196^{\ddagger} \\
(0.19606)\end{array}$ & $\begin{array}{l}0.32928^{\ddagger} \\
(0.19601)\end{array}$ \\
\hline Factor 3 & $\begin{array}{l}0.12222 \\
(0.10474) \\
\end{array}$ & $\begin{array}{l}0.12222 \\
(0.10477) \\
\end{array}$ & $\begin{array}{l}0.13855 \\
(0.10683) \\
\end{array}$ & $\begin{array}{l}0.12855 \\
(0.1064) \\
\end{array}$ & $\begin{array}{l}0.12306 \\
(0.10591) \\
\end{array}$ & $\begin{array}{l}0.12217 \\
(0.10572) \\
\end{array}$ & $\begin{array}{l}0.12894 \\
(0.10637) \\
\end{array}$ & $\begin{array}{l}0.12399 \\
(0.10511) \\
\end{array}$ & $\begin{array}{l}0.13923 \\
(0.10519) \\
\end{array}$ & $\begin{array}{l}0.11242 \\
(0.10543) \\
\end{array}$ & $\begin{array}{l}0.12216 \\
(0.10572) \\
\end{array}$ & $\begin{array}{l}0.11291 \\
(0.10632) \\
\end{array}$ & $\begin{array}{l}0.12055 \\
(0.10561) \\
\end{array}$ \\
\hline Factor $3 \& 5$ & $\begin{array}{l}0.16132^{*} \\
(0.05061)\end{array}$ & $\begin{array}{l}0.16132^{*} \\
(0.05062)\end{array}$ & $\begin{array}{l}0.18151^{*} \\
(0.05485)\end{array}$ & $\begin{array}{l}0.16313^{*} \\
(0.05119)\end{array}$ & $\begin{array}{l}0.16176^{*} \\
(0.05119)\end{array}$ & $\begin{array}{l}0.16132^{*} \\
(0.05108)\end{array}$ & $\begin{array}{l}0.16142^{*} \\
(0.05106)\end{array}$ & $\begin{array}{l}0.16132^{*} \\
(0.05079)\end{array}$ & $\begin{array}{l}0.18747^{*} \\
(0.05187)\end{array}$ & $\begin{array}{l}0.16094^{*} \\
(0.05088)\end{array}$ & $\begin{array}{l}0.16132^{*} \\
(0.05108)\end{array}$ & $\begin{array}{l}0.15063^{*} \\
(0.05289)\end{array}$ & $\begin{array}{l}0.15269^{*} \\
(0.05187)\end{array}$ \\
\hline Factor & $\begin{array}{l}0.15291 \\
(0.10042) \\
\end{array}$ & $\begin{array}{l}0.15291 \\
(0.10045) \\
\end{array}$ & $\begin{array}{l}0.16906^{\ddagger} \\
(0.1025) \\
\end{array}$ & $\begin{array}{l}0.15677 \\
(0.1016) \\
\end{array}$ & $\begin{array}{l}0.15394 \\
(0.10167) \\
\end{array}$ & $\begin{array}{l}0.15266 \\
(0.10144) \\
\end{array}$ & $\begin{array}{l}0.16019 \\
(0.10217) \\
\end{array}$ & $\begin{array}{l}0.15291 \\
(0.10077) \\
\end{array}$ & $\begin{array}{l}0.15291 \\
(0.10062) \\
\end{array}$ & $\begin{array}{l}0.15291 \\
(0.10095) \\
\end{array}$ & $\begin{array}{l}0.15291 \\
(0.10135) \\
\end{array}$ & $\begin{array}{l}0.15291 \\
(0.10128) \\
\end{array}$ & $\begin{array}{l}0.15291 \\
(0.10124) \\
\end{array}$ \\
\hline Factor $3,4 \& 5$ & \multicolumn{13}{|c|}{ Insufficient Data } \\
\hline Factor 2 & $\begin{array}{l}0.15955^{*} \\
(0.03515)\end{array}$ & $\begin{array}{l}0.15882^{*} \\
(0.03517)\end{array}$ & $\begin{array}{l}0.16454^{*} \\
(0.0357)\end{array}$ & $\begin{array}{l}0.16355^{*} \\
(0.03608)\end{array}$ & $\begin{array}{l}0.16107^{*} \\
(0.03615)\end{array}$ & $\begin{array}{l}0.16016^{*} \\
(0.03548)\end{array}$ & $\begin{array}{l}0.1637^{*} \\
(0.03604)\end{array}$ & $\begin{array}{l}0.17064^{*} \\
(0.03561)\end{array}$ & $\begin{array}{l}0.19273^{*} \\
(0.03774)\end{array}$ & $\begin{array}{l}0.13827^{*} \\
(0.03741)\end{array}$ & $\begin{array}{l}0.16016^{*} \\
(0.03549)\end{array}$ & $\begin{array}{l}0.15437^{*} \\
(0.03625)\end{array}$ & $\begin{array}{l}0.15436^{*} \\
(0.036)\end{array}$ \\
\hline Fact & $\begin{array}{l}0.2095 \\
(0.17624) \\
\end{array}$ & $\begin{array}{l}0.19969 \\
(0.17648) \\
\end{array}$ & $\begin{array}{l}0.2368 \\
(0.17778) \\
\end{array}$ & $\begin{array}{l}0.23225 \\
(0.17794) \\
\end{array}$ & $\begin{array}{l}0.2288 \\
(0.17803) \\
\end{array}$ & $\begin{array}{l}0.22676 \\
(0.17822) \\
\end{array}$ & $\begin{array}{l}0.2392 \\
(0.17895) \\
\end{array}$ & $\begin{array}{l}0.26911 \\
(0.17779) \\
\end{array}$ & $\begin{array}{l}0.27554 \\
(0.17761) \\
\end{array}$ & $\begin{array}{l}0.19524 \\
(0.17797) \\
\end{array}$ & $\begin{array}{l}0.22647 \\
(0.17962)\end{array}$ & $\begin{array}{l}0.21539 \\
(0.17832)\end{array}$ & $\begin{array}{l}0.22313 \\
(0.17763)\end{array}$ \\
\hline Factor $2 \& 4$ & $\begin{array}{l}0.13252^{\ddagger} \\
(0.07896)\end{array}$ & $\begin{array}{l}0.13443^{\ddagger} \\
(0.07895)\end{array}$ & $\begin{array}{l}0.15622^{\ddagger} \\
(0.08047)\end{array}$ & $\begin{array}{l}0.15088^{\ddagger} \\
(0.0808)\end{array}$ & $\begin{array}{l}0.14538^{\ddagger} \\
(0.08068)\end{array}$ & $\begin{array}{l}0.14351^{\ddagger} \\
(0.07963)\end{array}$ & $\begin{array}{l}0.15092 \\
(0.08064)\end{array}$ & $\begin{array}{l}0.16555^{\dagger} \\
(0.07978)\end{array}$ & $\begin{array}{l}0.17486^{\dagger} \\
(0.08007)\end{array}$ & $\begin{array}{l}0.12269 \\
(0.08014)\end{array}$ & $\begin{array}{l}0.14339^{\ddagger} \\
(0.07988)\end{array}$ & $\begin{array}{l}0.13447^{\ddagger} \\
(0.08042)\end{array}$ & $\begin{array}{l}0.14165^{\ddagger} \\
(0.07953)\end{array}$ \\
\hline Factor 2 & $\begin{array}{l}0.01668 \\
(0.09629) \\
\end{array}$ & $\begin{array}{l}-0.019 \\
(0.0995) \\
\end{array}$ & $\begin{array}{l}0.06919 \\
(0.09613) \\
\end{array}$ & $\begin{array}{l}0.07052 \\
(0.09789) \\
\end{array}$ & $\begin{array}{l}0.06301 \\
(0.09818) \\
\end{array}$ & $\begin{array}{l}0.05937 \\
(0.09694) \\
\end{array}$ & $\begin{array}{l}0.07192 \\
(0.09811) \\
\end{array}$ & $\begin{array}{l}0.10684 \\
(0.09773) \\
\end{array}$ & $\begin{array}{l}0.08833 \\
(0.09586) \\
\end{array}$ & $\begin{array}{l}0.03376 \\
(0.0966) \\
\end{array}$ & $\begin{array}{l}0.06026 \\
(0.09584) \\
\end{array}$ & $\begin{array}{l}0.04981 \\
(0.09672) \\
\end{array}$ & $\begin{array}{l}0.05898 \\
(0.09574) \\
\end{array}$ \\
\hline Factor $2 \& 3$ & $\begin{array}{l}0.24401^{\ddagger} \\
(0.13416)\end{array}$ & $\begin{array}{l}0.23773^{\ddagger} \\
(0.13432)\end{array}$ & $\begin{array}{l}0.27801^{\dagger} \\
(0.13664)\end{array}$ & $\begin{array}{l}0.26808^{\ddagger} \\
(0.13676)\end{array}$ & $\begin{array}{l}0.26057^{\ddagger} \\
(0.13699)\end{array}$ & $\begin{array}{l}0.25764^{\ddagger} \\
(0.13535)\end{array}$ & $\begin{array}{l}0.26948^{\dagger} \\
(0.13691)\end{array}$ & $\begin{array}{l}0.29839^{\dagger} \\
(0.13586)\end{array}$ & $\begin{array}{l}0.31736^{\dagger} \\
(0.13661)\end{array}$ & $\begin{array}{l}0.21511 \\
(0.13689)\end{array}$ & $\begin{array}{l}0.25752^{\ddagger} \\
(0.13552)\end{array}$ & $\begin{array}{l}0.25658^{\ddagger} \\
(0.13523)\end{array}$ & $\begin{array}{l}0.25262^{\ddagger} \\
(0.13529)\end{array}$ \\
\hline Factor $2,3 \& 5$ & $\begin{array}{l}0.20613 \\
(0.16117) \\
\end{array}$ & $\begin{array}{l}0.24426 \\
(0.15995) \\
\end{array}$ & $\begin{array}{l}0.28546^{\ddagger} \\
(0.16224)\end{array}$ & $\begin{array}{l}0.27348^{\ddagger} \\
(0.16194) \\
\end{array}$ & $\begin{array}{l}0.26802 \\
(0.16259) \\
\end{array}$ & $\begin{array}{l}0.26409 \\
(0.16235) \\
\end{array}$ & $\begin{array}{l}0.2759^{\ddagger} \\
(0.16228) \\
\end{array}$ & $\begin{array}{l}0.30362^{\ddagger} \\
(0.16125) \\
\end{array}$ & $\begin{array}{l}0.30481^{\ddagger} \\
(0.16087) \\
\end{array}$ & $\begin{array}{l}0.22255 \\
(0.16232) \\
\end{array}$ & $\begin{array}{l}0.26484 \\
(0.16153) \\
\end{array}$ & $\begin{array}{l}0.2482 \\
(0.16258) \\
\end{array}$ & $\begin{array}{l}0.25935 \\
(0.16114) \\
\end{array}$ \\
\hline Factor 2, $3 \& 4$ & $\begin{array}{l}0.29306^{*} \\
(0.08237)\end{array}$ & $\begin{array}{l}0.28791^{*} \\
(0.08259)\end{array}$ & $\begin{array}{l}0.32372^{*} \\
(0.08357)\end{array}$ & $\begin{array}{l}0.32195^{*} \\
(0.0851)\end{array}$ & $\begin{array}{l}0.31349^{*} \\
(0.08369)\end{array}$ & $\begin{array}{l}0.31119^{*} \\
(0.08379)\end{array}$ & $\begin{array}{l}0.31549^{*} \\
(0.08306)\end{array}$ & $\begin{array}{l}0.33797^{*} \\
(0.08325)\end{array}$ & $\begin{array}{l}0.37544^{*} \\
(0.08638)\end{array}$ & $\begin{array}{l}0.27023^{*} \\
(0.08576)\end{array}$ & $\begin{array}{l}0.31169^{*} \\
(0.08309)\end{array}$ & $\begin{array}{l}0.30671^{*} \\
(0.08306)\end{array}$ & $\begin{array}{l}0.29194^{*} \\
(0.08553)\end{array}$ \\
\hline Fact & $\begin{array}{l}0.2426^{\dagger} \\
(0.11976)\end{array}$ & $\begin{array}{l}0.21688^{\ddagger} \\
(0.1211)\end{array}$ & $\begin{array}{l}0.29276^{\dagger} \\
(0.1219)\end{array}$ & $\begin{array}{l}0.28283^{\dagger} \\
(0.122)\end{array}$ & $\begin{array}{l}0.27449^{\dagger} \\
(0.12129)\end{array}$ & $\begin{array}{l}0.27156^{\dagger} \\
(0.1215)\end{array}$ & $\begin{array}{l}0.28423^{\dagger} \\
(0.12217)\end{array}$ & $\begin{array}{l}0.275^{\dagger} \\
(0.11968)\end{array}$ & $\begin{array}{l}0.30119^{\dagger} \\
(0.1201)\end{array}$ & $\begin{array}{l}0.24045^{\dagger} \\
(0.12123)\end{array}$ & $\begin{array}{l}0.27174^{\dagger} \\
(0.12203)\end{array}$ & $\begin{array}{l}0.25666^{\dagger} \\
(0.12204)\end{array}$ & $\begin{array}{l}0.25714^{\dagger} \\
(0.12139)\end{array}$ \\
\hline Fact & $\begin{array}{l}0.21419^{*} \\
(0.03837)\end{array}$ & $\begin{array}{l}0.20867^{*} \\
(0.03852)\end{array}$ & $\begin{array}{l}0.22313^{*} \\
(0.03898)\end{array}$ & $\begin{array}{l}0.22117^{*} \\
(0.03916)\end{array}$ & $\begin{array}{l}0.21873^{*} \\
(0.03903)\end{array}$ & $\begin{array}{l}0.21808^{*} \\
(0.03871)\end{array}$ & $\begin{array}{l}0.21931^{*} \\
(0.03875)\end{array}$ & $\begin{array}{l}0.22025^{*} \\
(0.0385)\end{array}$ & $\begin{array}{l}0.22079^{*} \\
(0.03844)\end{array}$ & $\begin{array}{l}0.21321^{*} \\
(0.03865)\end{array}$ & $\begin{array}{l}0.21804^{*} \\
(0.03872)\end{array}$ & $\begin{array}{l}0.2176^{*} \\
(0.03868)\end{array}$ & $\begin{array}{l}0.21652^{*} \\
(0.0387)\end{array}$ \\
\hline Factor $1 \& 5$ & $\begin{array}{l}0.31314^{*} \\
(0.03687)\end{array}$ & $\begin{array}{l}0.31563^{*} \\
(0.03686)\end{array}$ & $\begin{array}{l}0.32314^{*} \\
(0.03774)\end{array}$ & $\begin{array}{l}0.31928^{*} \\
(0.03759)\end{array}$ & $\begin{array}{l}0.31732^{*} \\
(0.03777)\end{array}$ & $\begin{array}{l}0.31644^{*} \\
(0.0372)\end{array}$ & $\begin{array}{l}0.32175^{*} \\
(0.03839)\end{array}$ & $\begin{array}{l}0.33166^{*} \\
(0.03765)\end{array}$ & $\begin{array}{l}0.33101^{*} \\
(0.03742)\end{array}$ & $\begin{array}{l}0.31214^{*} \\
(0.03712)\end{array}$ & $\begin{array}{l}0.31647^{*} \\
(0.03719)\end{array}$ & $\begin{array}{l}0.31482^{*} \\
(0.03723)\end{array}$ & $\begin{array}{l}0.31647^{*} \\
(0.03715)\end{array}$ \\
\hline Factor $1 \& 4$ & $\begin{array}{l}0.14752^{\ddagger} \\
(0.07806)\end{array}$ & $\begin{array}{l}0.14076^{\ddagger} \\
(0.07815)\end{array}$ & $\begin{array}{l}0.15563^{\dagger} \\
(0.07895)\end{array}$ & $\begin{array}{l}0.15338^{\ddagger} \\
(0.07919)\end{array}$ & $\begin{array}{l}0.15018^{\ddagger} \\
(0.07916)\end{array}$ & $\begin{array}{l}0.14916^{\ddagger} \\
(0.07879)\end{array}$ & $\begin{array}{l}0.15437^{\ddagger} \\
(0.07932)\end{array}$ & $\begin{array}{l}0.16405^{\dagger} \\
(0.07864)\end{array}$ & $\begin{array}{l}0.15341^{\ddagger} \\
(0.07824)\end{array}$ & $\begin{array}{l}0.14732^{\ddagger} \\
(0.07848)\end{array}$ & $\begin{array}{l}0.14916^{\ddagger} \\
(0.07879)\end{array}$ & $\begin{array}{l}0.14717^{\ddagger} \\
(0.07877)\end{array}$ & $\begin{array}{l}0.14826^{\ddagger} \\
(0.07871)\end{array}$ \\
\hline Factor $1,4 \& 5$ & $\begin{array}{l}0.09677 \\
(0.12202)\end{array}$ & $\begin{array}{l}0.07209 \\
(0.12241)\end{array}$ & $\begin{array}{l}0.10668 \\
(0.1234)\end{array}$ & $\begin{array}{l}0.10233 \\
(0.1236)\end{array}$ & $\begin{array}{l}0.09825 \\
(0.1237)\end{array}$ & $\begin{array}{l}0.09669 \\
(0.12316)\end{array}$ & $\begin{array}{l}0.10407 \\
(0.12382)\end{array}$ & $\begin{array}{l}0.12354 \\
(0.12308)\end{array}$ & $\begin{array}{l}0.13302 \\
(0.1232)\end{array}$ & $\begin{array}{l}0.06764 \\
(0.12375)\end{array}$ & $\begin{array}{l}0.09625 \\
(0.12378)\end{array}$ & $\begin{array}{l}0.08277 \\
(0.1244)\end{array}$ & $\begin{array}{l}0.09143 \\
(0.12316)\end{array}$ \\
\hline Factor $1 \& 3$ & $\begin{array}{l}0.33012^{*} \\
(0.03081)\end{array}$ & $\begin{array}{l}0.32941^{*} \\
(0.03082)\end{array}$ & $\begin{array}{l}0.3378^{*} \\
(0.03186)\end{array}$ & $\begin{array}{l}0.33432^{*} \\
(0.0319)\end{array}$ & $\begin{array}{l}0.33168^{*} \\
(0.03206)\end{array}$ & $\begin{array}{l}0.33037^{*} \\
(0.0315)\end{array}$ & $\begin{array}{l}0.3316^{*} \\
(0.03113)\end{array}$ & $\begin{array}{l}0.33237^{*} \\
(0.03093)\end{array}$ & $\begin{array}{l}0.33136^{*} \\
(0.03087)\end{array}$ & $\begin{array}{l}0.32795^{*} \\
(0.03101)\end{array}$ & $\begin{array}{l}0.33065^{*} \\
(0.0311)\end{array}$ & $\begin{array}{l}0.32658^{*} \\
(0.03152)\end{array}$ & $\begin{array}{l}0.3218^{*} \\
(0.03251)\end{array}$ \\
\hline Factor $1,3 \& 5$ & $\begin{array}{l}0.2925^{*} \\
(0.04001)\end{array}$ & $\begin{array}{l}0.29023^{*} \\
(0.04004)\end{array}$ & $\begin{array}{l}0.29616^{*} \\
(0.04043)\end{array}$ & $\begin{array}{l}0.29488^{*} \\
(0.04049)\end{array}$ & $\begin{array}{l}0.29341^{*} \\
(0.04039)\end{array}$ & $\begin{array}{l}0.29321^{*} \\
(0.04042)\end{array}$ & $\begin{array}{l}0.29758^{*} \\
(0.0411) \\
\end{array}$ & $\begin{array}{l}0.31555^{*} \\
(0.04147) \\
\end{array}$ & $\begin{array}{l}0.29507^{*} \\
(0.0401) \\
\end{array}$ & $\begin{array}{l}0.2892^{*} \\
(0.04029)\end{array}$ & $\begin{array}{l}0.29326^{*} \\
(0.04041)\end{array}$ & $\begin{array}{l}0.29332^{*} \\
(0.04035) \\
\end{array}$ & $\begin{array}{l}0.28486^{*} \\
(0.04136) \\
\end{array}$ \\
\hline Factor & $\begin{array}{l}0.27207^{*} \\
(0.02871)\end{array}$ & $\begin{array}{l}0.28059^{*} \\
(0.02849)\end{array}$ & $\begin{array}{l}0.29273^{*} \\
(0.03066)\end{array}$ & $\begin{array}{l}0.28626^{*} \\
(0.02995)\end{array}$ & $\begin{array}{l}0.28208^{*} \\
(0.02876)\end{array}$ & $\begin{array}{l}0.28166^{*} \\
(0.02912)\end{array}$ & $\begin{array}{l}0.28432^{*} \\
(0.02906)\end{array}$ & $\begin{array}{l}0.29539^{*} \\
(0.02926)\end{array}$ & $\begin{array}{l}0.28285^{*} \\
(0.02854)\end{array}$ & $\begin{array}{l}0.28049^{*} \\
(0.02864)\end{array}$ & $\begin{array}{l}0.28195^{*} \\
(0.02875)\end{array}$ & $\begin{array}{l}0.2795^{*} \\
(0.0289)\end{array}$ & $\begin{array}{l}0.27811^{*} \\
(0.02901)\end{array}$ \\
\hline Factor & $\begin{array}{l}0.25185^{*} \\
(0.04836)\end{array}$ & $\begin{array}{l}0.25112^{*} \\
(0.04838)\end{array}$ & $\begin{array}{l}0.26125^{*} \\
(0.04952)\end{array}$ & $\begin{array}{l}0.25476^{*} \\
(0.04899)\end{array}$ & $\begin{array}{l}0.25266^{*} \\
(0.04882)\end{array}$ & $\begin{array}{l}0.25238^{*} \\
(0.04885)\end{array}$ & $\begin{array}{l}0.25617^{*} \\
(0.04925)\end{array}$ & $\begin{array}{l}0.25964^{*} \\
(0.04864)\end{array}$ & $\begin{array}{l}0.26168^{*} \\
(0.04861)\end{array}$ & $\begin{array}{l}0.23864^{*} \\
(0.04923)\end{array}$ & $\begin{array}{l}0.25223^{*} \\
(0.04923)\end{array}$ & $\begin{array}{l}0.24962^{*} \\
(0.04891)\end{array}$ & $\begin{array}{l}0.24406^{*} \\
(0.0496)\end{array}$ \\
\hline Facto & $\begin{array}{l}0.18394^{*} \\
(0.032) \\
\end{array}$ & $\begin{array}{l}0.1824^{*} \\
(0.03205)\end{array}$ & $\begin{array}{l}0.19051^{*} \\
(0.03227)\end{array}$ & $\begin{array}{l}0.19184^{*} \\
(0.03283)\end{array}$ & $\begin{array}{l}0.19017^{*} \\
(0.03423)\end{array}$ & $\begin{array}{l}0.18827^{*} \\
(0.03298)\end{array}$ & $\begin{array}{l}0.19231^{*} \\
(0.03291)\end{array}$ & $\begin{array}{l}0.20295^{*} \\
(0.03275) \\
\end{array}$ & $\begin{array}{l}0.19277^{*} \\
(0.03207)\end{array}$ & $\begin{array}{l}0.18484^{*} \\
(0.0322)\end{array}$ & $\begin{array}{l}0.18859^{*} \\
(0.03234)\end{array}$ & $\begin{array}{l}0.18335^{*} \\
(0.03296) \\
\end{array}$ & $\begin{array}{l}0.18284^{*} \\
(0.03283)\end{array}$ \\
\hline Factor $1,2 \& 5$ & $\begin{array}{l}0.26077^{*} \\
(0.08736)\end{array}$ & $\begin{array}{l}0.25069^{*} \\
(0.08807)\end{array}$ & $\begin{array}{l}0.30408^{*} \\
(0.08754)\end{array}$ & $\begin{array}{l}0.29831^{*} \\
(0.08737)\end{array}$ & $\begin{array}{l}0.29621^{*} \\
(0.08738)\end{array}$ & $\begin{array}{l}0.29503^{*} \\
(0.08765)\end{array}$ & $\begin{array}{l}0.30567^{*} \\
(0.08911)\end{array}$ & $\begin{array}{l}0.32321^{*} \\
(0.08768)\end{array}$ & $\begin{array}{l}0.32029^{*} \\
(0.08721)\end{array}$ & $\begin{array}{l}0.27387^{*} \\
(0.08773)\end{array}$ & $\begin{array}{l}0.29499^{*} \\
(0.08826)\end{array}$ & $\begin{array}{l}0.28764^{*} \\
(0.08777)\end{array}$ & $\begin{array}{l}0.29555^{*} \\
(0.08714)\end{array}$ \\
\hline Factor $1,2 \& 4$ & $\begin{array}{l}0.44488^{*} \\
(0.03966) \\
\end{array}$ & $\begin{array}{l}0.46769^{*} \\
(0.03811) \\
\end{array}$ & $\begin{array}{l}0.47868^{*} \\
(0.03835)\end{array}$ & $\begin{array}{l}0.4831^{*} \\
(0.04066)\end{array}$ & $\begin{array}{l}0.47699^{*} \\
(0.03889)\end{array}$ & $\begin{array}{l}0.47527^{*} \\
(0.04082)\end{array}$ & $\begin{array}{l}0.48756^{*} \\
(0.04356)\end{array}$ & $\begin{array}{l}0.47871^{*} \\
(0.03811)\end{array}$ & $\begin{array}{l}0.48287^{*} \\
(0.03814)\end{array}$ & $\begin{array}{l}0.4581^{*} \\
(0.03948)\end{array}$ & $\begin{array}{l}0.47608^{*} \\
(0.03833)\end{array}$ & $\begin{array}{l}0.4751^{*} \\
(0.03831)\end{array}$ & $\begin{array}{l}0.47531^{*} \\
(0.03828)\end{array}$ \\
\hline Factor $1,2,4 \& 5$ & $\begin{array}{l}0.27003^{*} \\
(0.05629)\end{array}$ & $\begin{array}{l}0.26733^{*} \\
(0.05663)\end{array}$ & $\begin{array}{l}0.31808^{*} \\
(0.05595)\end{array}$ & $\begin{array}{l}0.31804^{*} \\
(0.0586)\end{array}$ & $\begin{array}{l}0.31049^{*} \\
(0.05896)\end{array}$ & $\begin{array}{l}0.30706^{*} \\
(0.05631)\end{array}$ & $\begin{array}{l}0.31943^{*} \\
(0.05897)\end{array}$ & $\begin{array}{l}0.33158^{*} \\
(0.05586)\end{array}$ & $\begin{array}{l}0.3692^{*} \\
(0.06035)\end{array}$ & $\begin{array}{l}0.28595^{*} \\
(0.05619)\end{array}$ & $\begin{array}{l}0.30693^{*} \\
(0.0587)\end{array}$ & $\begin{array}{l}0.28944^{*} \\
(0.05994)\end{array}$ & $\begin{array}{l}0.30711^{*} \\
(0.05501)\end{array}$ \\
\hline Factor 1, & $\begin{array}{l}0.08473^{\ddagger} \\
(0.04967)\end{array}$ & $\begin{array}{l}0.08369^{\ddagger} \\
(0.04974)\end{array}$ & $\begin{array}{l}0.10763^{\dagger} \\
(0.05048)\end{array}$ & $\begin{array}{l}0.10204^{\dagger} \\
(0.05013)\end{array}$ & $\begin{array}{l}0.10014^{\dagger} \\
(0.05034)\end{array}$ & $\begin{array}{l}0.0988^{\ddagger} \\
(0.05033)\end{array}$ & $\begin{array}{l}0.10991^{\dagger} \\
(0.05347)\end{array}$ & $\begin{array}{l}0.13489^{\dagger} \\
(0.05227) \\
\end{array}$ & $\begin{array}{l}0.14784^{*} \\
(0.05346)\end{array}$ & $\begin{array}{l}0.08546^{\ddagger} \\
(0.05024)\end{array}$ & $\begin{array}{l}0.0992^{\dagger} \\
(0.04985)\end{array}$ & $\begin{array}{l}0.09776^{\ddagger} \\
(0.04984) \\
\end{array}$ & $\begin{array}{l}0.09674^{\ddagger} \\
(0.04986) \\
\end{array}$ \\
\hline Factor $1,2,3 \& 5$ & $\begin{array}{l}0.26834^{*} \\
(0.04855)\end{array}$ & $\begin{array}{l}0.30069^{*} \\
(0.0453)\end{array}$ & $\begin{array}{l}0.33641^{*} \\
(0.04802)\end{array}$ & $\begin{array}{l}0.32831^{*} \\
(0.04808)\end{array}$ & $\begin{array}{l}0.32028^{*} \\
(0.04527)\end{array}$ & $\begin{array}{l}0.31904^{*} \\
(0.04697)\end{array}$ & $\begin{array}{l}0.32763^{*} \\
(0.04729)\end{array}$ & $\begin{array}{l}0.34457^{*} \\
(0.04633)\end{array}$ & $\begin{array}{l}0.32312^{*} \\
(0.04483)\end{array}$ & $\begin{array}{l}0.29542^{*} \\
(0.04699)\end{array}$ & $\begin{array}{l}0.31975^{*} \\
(0.04517)\end{array}$ & $\begin{array}{l}0.31763^{*} \\
(0.04519)\end{array}$ & $\begin{array}{l}0.31252^{*} \\
(0.04577)\end{array}$ \\
\hline Facto & $\begin{array}{l}0.232^{*} \\
(0.0421) \\
\end{array}$ & $\begin{array}{l}0.23005^{*} \\
(0.0426) \\
\end{array}$ & $\begin{array}{l}0.29756^{*} \\
(0.04106) \\
\end{array}$ & $\begin{array}{l}0.29132^{*} \\
(0.04205) \\
\end{array}$ & $\begin{array}{l}0.28374^{*} \\
(0.03975) \\
\end{array}$ & $\begin{array}{l}0.28135^{*} \\
(0.04137) \\
\end{array}$ & $\begin{array}{l}0.28703^{*} \\
(0.03912) \\
\end{array}$ & $\begin{array}{l}0.3065^{*} \\
(0.03961) \\
\end{array}$ & $\begin{array}{l}0.31519^{*} \\
(0.04031)\end{array}$ & $\begin{array}{l}0.24599^{*} \\
(0.04314)\end{array}$ & $\begin{array}{l}0.28213^{*} \\
(0.03845) \\
\end{array}$ & $\begin{array}{l}0.27624^{*} \\
(0.03897) \\
\end{array}$ & $\begin{array}{l}0.27001^{*} \\
(0.0404) \\
\end{array}$ \\
\hline All F & $\begin{array}{l}0.15207^{*} \\
(0.03064)\end{array}$ & $\begin{array}{l}0.15506^{*} \\
(0.03037)\end{array}$ & $\begin{array}{l}0.18836^{*} \\
(0.03121)\end{array}$ & $\begin{array}{l}0.18585^{*} \\
(0.03335)\end{array}$ & $\begin{array}{l}0.17995^{*} \\
(0.03375)\end{array}$ & $\begin{array}{l}0.17729^{*} \\
(0.03067)\end{array}$ & $\begin{array}{l}0.18414^{*} \\
(0.03157)\end{array}$ & $\begin{array}{l}0.21161^{*} \\
(0.03321)\end{array}$ & $\begin{array}{l}0.19296^{*} \\
(0.02987)\end{array}$ & $\begin{array}{l}0.16087^{*} \\
(0.0308)\end{array}$ & $\begin{array}{l}0.17767^{*} \\
(0.02963)\end{array}$ & $\begin{array}{l}0.16992^{*} \\
(0.03113)\end{array}$ & $\begin{array}{l}0.16223^{*} \\
(0.03386)\end{array}$ \\
\hline $\begin{array}{l}\text { Individual } \\
\text { Variable }\end{array}$ & $\begin{array}{l}0.07398^{*} \\
(0.02715)\end{array}$ & $\begin{array}{l}0.07926^{*} \\
(0.02964)\end{array}$ & $\begin{array}{l}-0.02019 \\
(0.02016)\end{array}$ & $\begin{array}{l}-0.01026 \\
(0.0201)\end{array}$ & $\begin{array}{l}-0.00274 \\
(0.02133)\end{array}$ & $\begin{array}{l}0.00144 \\
(0.02355)\end{array}$ & $\begin{array}{l}-0.01165 \\
(0.02117)\end{array}$ & $\begin{array}{l}-0.04658^{\dagger} \\
(0.02171)\end{array}$ & $\begin{array}{l}-0.06348^{\dagger} \\
(0.02642)\end{array}$ & $\begin{array}{l}0.04272^{\ddagger} \\
(0.02394)\end{array}$ & $\begin{array}{l}0.00165 \\
(0.03978)\end{array}$ & $\begin{array}{l}0.01874 \\
(0.02429)\end{array}$ & $\begin{array}{l}0.02052 \\
(0.02216)\end{array}$ \\
\hline
\end{tabular}

* Significant at the 0.01 level $†$ Significant at the 0.05 level $\$$ Significant at the 0.10 level

Table 105 Coefficients with individual variables for high-level factors - Wood Industry 


\begin{tabular}{|c|c|c|c|c|c|c|c|c|c|c|c|c|c|}
\hline $\begin{array}{l}\text { Individual variable } \\
\text { included in the } \\
\text { model in the } \\
\text { column }\end{array}$ & 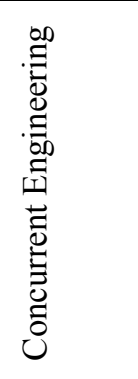 & 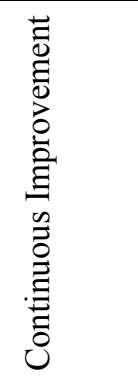 & 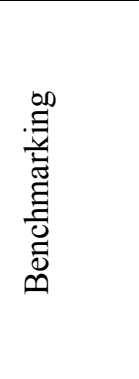 & 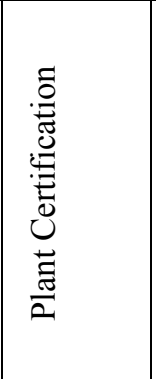 & 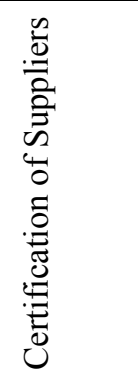 & 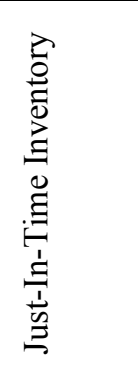 & 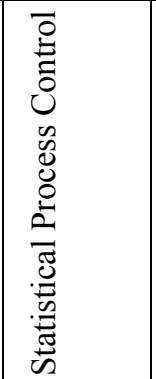 & 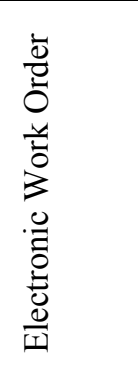 & 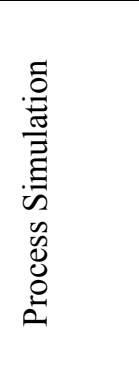 & 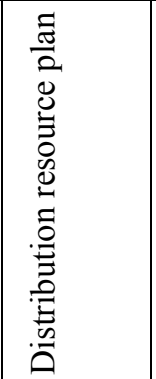 & 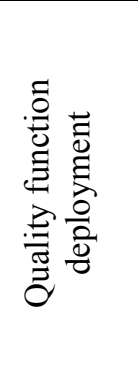 & 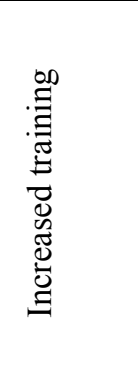 & 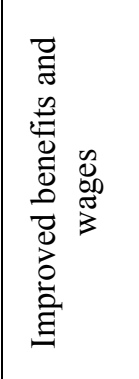 \\
\hline No factors & $\begin{array}{l}0.26022^{*} \\
(0.01824)\end{array}$ & $\begin{array}{l}0.26059^{*} \\
(0.01826)\end{array}$ & $\begin{array}{l}0.2595^{*} \\
(0.01824)\end{array}$ & \begin{tabular}{|l|}
$0.27232^{*}$ \\
$(0.01851)$ \\
\end{tabular} & $\begin{array}{l}0.26184^{*} \\
(0.01843)\end{array}$ & $\begin{array}{l}0.26626^{*} \\
(0.01886)\end{array}$ & \begin{tabular}{|l|}
$0.26136^{*}$ \\
$(0.01827)$ \\
\end{tabular} & $\begin{array}{l}0.26058^{*} \\
(0.01822)\end{array}$ & $\begin{array}{l}0.25878^{*} \\
(0.01813)\end{array}$ & \begin{tabular}{|l|}
$0.26024^{*}$ \\
$(0.01824)$ \\
\end{tabular} & $\begin{array}{l}0.25527^{*} \\
(0.01817)\end{array}$ & $\begin{array}{l}0.26012^{*} \\
(0.01821)\end{array}$ & \begin{tabular}{|l|l}
$0.26012^{*}$ \\
$(0.01823)$
\end{tabular} \\
\hline Factor 5 & $\begin{array}{ll}0.2561^{*} \\
(0.09435)\end{array}$ & $\begin{array}{l}0.259^{*} \\
(0.09473)\end{array}$ & $\begin{array}{l}0.24646^{*} \\
(0.09411)\end{array}$ & \begin{tabular}{|l|}
$0.29285^{*}$ \\
$(0.09387)$ \\
\end{tabular} & $\begin{array}{l}0.25856^{*} \\
(0.0943)\end{array}$ & $\begin{array}{l}0.26318^{*} \\
(0.09409)\end{array}$ & \begin{tabular}{|l|l}
$0.26095^{*}$ \\
$(0.0942)$
\end{tabular} & $\begin{array}{l}0.26628^{*} \\
(0.09462)\end{array}$ & $\begin{array}{ll}0.2526^{*} \\
(0.09327)\end{array}$ & \begin{tabular}{|l|}
$0.2526^{*}$ \\
$(0.09386)$ \\
\end{tabular} & $\begin{array}{l}0.24719^{*} \\
(0.09307)\end{array}$ & $\begin{array}{l}0.11246 \\
(0.15049)\end{array}$ & \begin{tabular}{|l|l|}
$0.26814^{*}$ \\
$(0.09577)$
\end{tabular} \\
\hline ctor 4 & $\begin{array}{l}0.13979^{*} \\
(0.04278)\end{array}$ & $\begin{array}{l}0.14178^{*} \\
(0.04297)\end{array}$ & $\begin{array}{l}0.13695^{*} \\
(0.04288)\end{array}$ & \begin{tabular}{|l|}
$0.14238^{*}$ \\
$(0.04233)$
\end{tabular} & $\begin{array}{l}0.14026^{*} \\
(0.04277)\end{array}$ & $\begin{array}{l}0.15296^{*} \\
(0.044)\end{array}$ & \begin{tabular}{|l|}
$0.1399^{*}$ \\
$(0.04274)$ \\
\end{tabular} & $\begin{array}{l}0.14082^{*} \\
(0.04273)\end{array}$ & $\begin{array}{l}0.13882^{*} \\
(0.0425)\end{array}$ & \begin{tabular}{|l|}
$0.13983^{*}$ \\
$(0.04277)$ \\
\end{tabular} & $\begin{array}{l}0.13974^{*} \\
(0.0424)\end{array}$ & $\begin{array}{l}0.13974^{*} \\
(0.04271)\end{array}$ & \begin{tabular}{|l}
$0.13974^{*}$ \\
$(0.04275)$
\end{tabular} \\
\hline Factor $4 \& 5$ & $\begin{array}{l}0.3342^{\ddagger} \\
(0.19626)\end{array}$ & $\begin{array}{l}0.33483^{\ddagger} \\
(0.19623)\end{array}$ & $\begin{array}{l}0.32725^{\ddagger} \\
(0.19612)\end{array}$ & \begin{tabular}{|l|}
$0.33986^{\ddagger}$ \\
$(0.1941)$
\end{tabular} & $\begin{array}{l}0.33564^{\ddagger} \\
(0.19619)\end{array}$ & $\begin{array}{l}0.33196^{\ddagger} \\
(0.19582)\end{array}$ & \begin{tabular}{|l|}
$0.33196^{\ddagger}$ \\
$(0.19598)$
\end{tabular} & $\begin{array}{l}0.3352^{\ddagger} \\
(0.19594)\end{array}$ & $\begin{array}{l}0.32164^{\ddagger} \\
(0.19497)\end{array}$ & \begin{tabular}{|l|}
$0.33196^{\ddagger}$ \\
$(0.19615)$ \\
\end{tabular} & $\begin{array}{l}0.32427^{\ddagger} \\
(0.19447)\end{array}$ & $\begin{array}{l}0.27559 \\
(0.2015)\end{array}$ & \begin{tabular}{|l|l}
$0.33589^{\ddagger}$ \\
$(0.1961)$
\end{tabular} \\
\hline Factor 3 & $\begin{array}{l}2413 \\
10582)\end{array}$ & $\begin{array}{ll} & 0.12518 \\
(0.10585)\end{array}$ & $\begin{array}{ll}0.11805 \\
(0.10575)\end{array}$ & \begin{tabular}{|l|}
0.12681 \\
$(0.10458)$ \\
\end{tabular} & $\begin{array}{ll} & 0.12329 \\
(0.10567)\end{array}$ & $\begin{array}{l}0.12866 \\
(0.10563)\end{array}$ & \begin{tabular}{|l|}
0.13045 \\
$(0.10595)$ \\
\end{tabular} & $\begin{array}{l}0.12417 \\
(0.10558)\end{array}$ & $\begin{array}{l}0.12222 \\
(0.10502)\end{array}$ & \begin{tabular}{|l|}
0.12222 \\
$(0.10568)$ \\
\end{tabular} & $\begin{array}{l}0.10875 \\
(0.10489)\end{array}$ & $\begin{array}{l}0.12222 \\
(0.10552)\end{array}$ & \begin{tabular}{|l|l|}
0.12222 \\
$(0.10563)$
\end{tabular} \\
\hline Factor 3 & $\begin{array}{l}0.16139^{*} \\
(0.05107)\end{array}$ & $\begin{array}{l}0.16592^{*} \\
(0.05189)\end{array}$ & $\begin{array}{l}0.15831^{*} \\
(0.05117)\end{array}$ & \begin{tabular}{|l|}
$0.17195^{*}$ \\
$(0.05065)$ \\
\end{tabular} & $\begin{array}{l}0.16132^{*} \\
(0.05105)\end{array}$ & $\begin{array}{l}0.17526^{*} \\
(0.05219)\end{array}$ & \begin{tabular}{|l|}
$0.16132^{*}$ \\
$(0.05102)$ \\
\end{tabular} & $\begin{array}{l}0.17171^{*} \\
(0.05192)\end{array}$ & $\begin{array}{l}0.13105^{\dagger} \\
(0.05243)\end{array}$ & \begin{tabular}{|l|}
$0.16146^{*}$ \\
$(0.05107)$ \\
\end{tabular} & $\begin{array}{l}0.1366^{*} \\
(0.05146)\end{array}$ & $\begin{array}{l}0.02118 \\
(0.12832)\end{array}$ & \begin{tabular}{|l|l}
$0.17779^{*}$ \\
$(0.05498)$
\end{tabular} \\
\hline Factor $3 \& 4$ & $\begin{array}{l}8456 \\
0144)\end{array}$ & $\begin{array}{ll}0.16386 \\
(0.10367)\end{array}$ & $\begin{array}{l}4931 \\
10136)\end{array}$ & \begin{tabular}{|l|}
$0.17559^{\ddagger}$ \\
$0.10055)$
\end{tabular} & $\begin{array}{l}.15572 \\
(0.1014)\end{array}$ & $\begin{array}{l}15773 \\
.10123) \\
\end{array}$ & \begin{tabular}{|l|}
$0.16957^{\ddagger}$ \\
$(0.10276)$
\end{tabular} & $\begin{array}{ll}0.15726 \\
(0.10129)\end{array}$ & $\begin{array}{ll}0.10705 \\
(0.10265)\end{array}$ & \begin{tabular}{|l|}
0.16237 \\
$0.10336)$ \\
\end{tabular} & $\begin{array}{ll}0.11626 \\
(0.10138)\end{array}$ & $\begin{array}{l}15291 \\
.10117) \\
\end{array}$ & \begin{tabular}{|l|l|}
0.15291 \\
$(0.10127)$
\end{tabular} \\
\hline Factor 3, & \multicolumn{13}{|c|}{ Insufficient Dat } \\
\hline Factor 2 & $\begin{array}{l}0.16189^{*} \\
(0.03578)\end{array}$ & $\begin{array}{l}0.17097^{*} \\
(0.04155)\end{array}$ & $\begin{array}{l}0.14343^{*} \\
(0.04093)\end{array}$ & \begin{tabular}{|l|}
$0.20382^{*}$ \\
$(0.03808)$
\end{tabular} & $\begin{array}{l}0.17035^{*} \\
(0.03888)\end{array}$ & $\begin{array}{l}18414^{*} \\
.0403)\end{array}$ & \begin{tabular}{|l|}
$0.17372^{*}$ \\
$(0.03823)$
\end{tabular} & $\begin{array}{l}0.17452^{*} \\
(0.03789)\end{array}$ & $\begin{array}{l}0.14526^{*} \\
(0.03584)\end{array}$ & \begin{tabular}{|l|l}
$0.16354^{*}$ \\
$(0.0362)$
\end{tabular} & $\begin{array}{l}0.11864^{*} \\
(0.03844)\end{array}$ & $\begin{array}{l}0.16018^{*} \\
(0.03542)\end{array}$ & \begin{tabular}{|l|l}
$0.16018^{*}$ \\
$(0.03545)$
\end{tabular} \\
\hline Factor $2 \& 5$ & $\begin{array}{l}0.22931 \\
(0.1778) \\
\end{array}$ & $\begin{array}{ll}0.23256 \\
(0.17799)\end{array}$ & 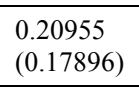 & \begin{tabular}{|l|}
0.28785 \\
$(0.17702)$ \\
\end{tabular} & $\begin{array}{ll}0.24158 \\
(0.17903)\end{array}$ & $\begin{array}{l}0.24902 \\
(0.17825)\end{array}$ & \begin{tabular}{|l|}
0.25423 \\
$(0.17979)$ \\
\end{tabular} & $\begin{array}{l}0.23887 \\
(0.17782) \\
\end{array}$ & $\begin{array}{l}0.20172 \\
(0.17695)\end{array}$ & \begin{tabular}{|l|}
0.22754 \\
$(0.17771)$ \\
\end{tabular} & $\begin{array}{ll}0.18944 \\
(0.17675)\end{array}$ & \begin{tabular}{|l|l}
0.11752 \\
$(0.20008)$
\end{tabular} & \begin{tabular}{|l}
0.24039 \\
$(0.17833)$
\end{tabular} \\
\hline Factor $2 \& 4$ & $\begin{array}{l}4711^{\ddagger} \\
08012) \\
\end{array}$ & $\begin{array}{l}0.15358^{\ddagger} \\
(0.08201) \\
\end{array}$ & 12739 & \begin{tabular}{|l|l}
$0.18032^{\dagger}$ \\
$(0.0797)$ \\
\end{tabular} & $\begin{array}{l}0.15608^{\ddagger} \\
(0.08189) \\
\end{array}$ & $\begin{array}{l}0.1635^{\dagger} \\
(0.08101) \\
\end{array}$ & \begin{tabular}{|l|}
$0.16725^{\dagger}$ \\
$0.08332)$ \\
\end{tabular} & $\begin{array}{l}0.16517^{\dagger} \\
(0.08198) \\
\end{array}$ & $\begin{array}{l}0.14051^{\ddagger} \\
(0.07908) \\
\end{array}$ & \begin{tabular}{|l|}
$0.14452^{\ddagger}$ \\
$(0.07959)$ \\
\end{tabular} & $\begin{array}{l}0.13436^{\ddagger} \\
(0.07895) \\
\end{array}$ & $\begin{array}{l}0.14368^{\ddagger} \\
(0.07945) \\
\end{array}$ & \begin{tabular}{|l}
$0.14368^{\ddagger}$ \\
$(0.07952)$ \\
\end{tabular} \\
\hline Factor 2 & $\begin{array}{ll}0.06511 \\
(0.09673)\end{array}$ & $\begin{array}{ll}0.0712 \\
(0.09829)\end{array}$ & $\begin{array}{l}.04227 \\
0.09824)\end{array}$ & \begin{tabular}{|l|}
0.12057 \\
$(0.09697)$ \\
\end{tabular} & $\begin{array}{ll}0.0743 \\
(0.09829)\end{array}$ & $\begin{array}{ll}0.08358 \\
(0.09746)\end{array}$ & \begin{tabular}{|l|}
0.08695 \\
$(0.0998)$
\end{tabular} & $\begin{array}{ll}0.07558 \\
(0.09677)\end{array}$ & $\begin{array}{ll}0.03007 \\
(0.09611)\end{array}$ & \begin{tabular}{|l|}
0.06872 \\
$(0.09753)$ \\
\end{tabular} & $\begin{array}{ll}0.00519 \\
(0.09719)\end{array}$ & $\begin{array}{l}-0.07988 \\
(0.15171)\end{array}$ & \begin{tabular}{|l|l|}
0.07676 \\
$(0.09792)$
\end{tabular} \\
\hline Factor $2 \& 3$ & $\begin{array}{l}0.25937^{\ddagger} \\
(0.13536) \\
\end{array}$ & $\begin{array}{l}0.26877^{\ddagger} \\
(0.13705) \\
\end{array}$ & $\begin{array}{l}0.25438^{\ddagger} \\
(0.13527) \\
\end{array}$ & \begin{tabular}{|l|}
$0.31411^{\dagger}$ \\
$(0.1352)$ \\
\end{tabular} & $\begin{array}{l}0.27093^{\dagger} \\
(0.1368) \\
\end{array}$ & $\begin{array}{l}0.28039^{\dagger} \\
(0.13626) \\
\end{array}$ & \begin{tabular}{|l|}
$0.26281^{\ddagger}$ \\
$(0.13527)$
\end{tabular} & $\begin{array}{l}0.26409^{\ddagger} \\
(0.13525)\end{array}$ & $\begin{array}{l}0.23919^{\ddagger} \\
(0.13467)\end{array}$ & \begin{tabular}{|l|}
$0.25884^{\ddagger}$ \\
$(0.1353)$
\end{tabular} & $\begin{array}{l}0.24659^{\ddagger} \\
(0.13418)\end{array}$ & $\begin{array}{l}0.25783^{\ddagger} \\
(0.13508)\end{array}$ & \begin{tabular}{|l|l}
$0.25783^{\ddagger}$ \\
$(0.1321)$
\end{tabular} \\
\hline Factor $2,3 \& 5$ & $\begin{array}{ll}0.26693^{\ddagger} \\
(0.16122) \\
\end{array}$ & $\begin{array}{l}0.27622^{\ddagger} \\
(0.16263) \\
\end{array}$ & $\begin{array}{l}0.24887 \\
(0.16229) \\
\end{array}$ & \begin{tabular}{|l|}
$0.31349^{\ddagger}$ \\
$(0.16028)$ \\
\end{tabular} & $\begin{array}{l}0.27932^{\ddagger} \\
(0.1626) \\
\end{array}$ & $\begin{array}{l}0.28722^{\ddagger} \\
(0.16183) \\
\end{array}$ & \begin{tabular}{|l|}
$0.27535^{\ddagger}$ \\
$0.16136)$ \\
\end{tabular} & $\begin{array}{l}0.28997^{\ddagger} \\
(0.16261) \\
\end{array}$ & $\begin{array}{l}0.23106 \\
(0.16082) \\
\end{array}$ & \begin{tabular}{|l|}
$0.26966^{\ddagger}$ \\
$(0.16142)$ \\
\end{tabular} & $\begin{array}{l}\begin{array}{l}0.21172 \\
(0.161)\end{array} \\
\end{array}$ & $\begin{array}{ll}0.12514 \\
(0.19939) \\
\end{array}$ & \begin{tabular}{|l|l|}
$0.28024^{\ddagger}$ \\
$(0.16212)$ \\
\end{tabular} \\
\hline Factor & $\begin{array}{l}0.31978^{*} \\
(0.08553)\end{array}$ & $\begin{array}{l}0.3229^{*} \\
(0.08568)\end{array}$ & $\begin{array}{l}0.30146^{\circ} \\
(0.08376)\end{array}$ & \begin{tabular}{|l|}
$0.36926^{*}$ \\
$(0.08421)$
\end{tabular} & $\begin{array}{l}0.32399^{*} \\
(0.08492)\end{array}$ & $\begin{array}{l}0.3327^{*} \\
(0.08434)\end{array}$ & \begin{tabular}{|l|}
$0.32685^{*}$ \\
$(0.08423)$
\end{tabular} & $\begin{array}{l}0.32974^{*} \\
(0.08438)\end{array}$ & $\begin{array}{l}0.26734^{*} \\
(0.08457)\end{array}$ & \begin{tabular}{|l|}
$0.31411^{*}$ \\
$(0.08295)$
\end{tabular} & $\begin{array}{l}0.25613^{*} \\
(0.08471)\end{array}$ & $\begin{array}{l}0.31195^{*} \\
(0.08269)\end{array}$ & \begin{tabular}{|l|l}
$0.31195^{*}$ \\
$(0.08277)$
\end{tabular} \\
\hline Facto & $\begin{array}{ll}0.27876^{\dagger} \\
(0.12153)\end{array}$ & $\begin{array}{l}0.2808^{\dagger} \\
(0.12146)\end{array}$ & $\begin{array}{l}0.25904^{\dagger} \\
(0.1214)\end{array}$ & \begin{tabular}{|l|}
$0.30295^{\dagger}$ \\
$(0.11951)$ \\
\end{tabular} & $\begin{array}{l}0.27821^{\dagger} \\
(0.12063)\end{array}$ & $\begin{array}{l}0.28232^{\dagger} \\
(0.12039)\end{array}$ & \begin{tabular}{|l|}
$0.29264^{\dagger}$ \\
$(0.12209)$
\end{tabular} & $\begin{array}{l}0.28983^{\dagger} \\
(0.12128)\end{array}$ & $\begin{array}{l}0.25767^{\dagger} \\
(0.11976)\end{array}$ & \begin{tabular}{|l|l|l}
$0.2757^{\dagger}$ \\
$(0.12053)$
\end{tabular} & $\begin{array}{l}0.23419^{\ddagger} \\
(0.12016)\end{array}$ & $\begin{array}{l}0.20928 \\
(0.1314)\end{array}$ & $\begin{array}{l}0.28785^{\dagger} \\
(0.12176)\end{array}$ \\
\hline Factor 1 & $\begin{array}{l}0.21817^{*} \\
(0.0387) \\
\end{array}$ & $\begin{array}{l}0.21943^{*} \\
(0.03879) \\
\end{array}$ & $\begin{array}{l}0.21622^{*} \\
(0.03874) \\
\end{array}$ & \begin{tabular}{|l|l|}
$0.22551^{*}$ \\
$(0.03837)$ \\
\end{tabular} & $\begin{array}{l}0.21979^{*} \\
(0.03878) \\
\end{array}$ & $\begin{array}{l}0.22151^{*} \\
(0.03873) \\
\end{array}$ & \begin{tabular}{|l|}
$0.22013^{*}$ \\
$(0.03872)$ \\
\end{tabular} & $\begin{array}{l}0.22176^{*} \\
(0.0388)\end{array}$ & $\begin{array}{l}0.21694^{*} \\
(0.03845) \\
\end{array}$ & \begin{tabular}{|l|}
$0.211809^{*}$ \\
$(0.03869)$ \\
\end{tabular} & $\begin{array}{l}0.21329^{*} \\
(0.0384)\end{array}$ & $\begin{array}{l}0.21809^{*} \\
(0.03864)\end{array}$ & \begin{tabular}{|l|l}
$0.21809^{*}$ \\
$(0.03867)$ \\
\end{tabular} \\
\hline Factor $1 \& 5$ & $\begin{array}{l}0.31647^{*} \\
(0.03719) \\
\end{array}$ & $\begin{array}{l}0.31941^{*} \\
(0.03765) \\
\end{array}$ & $\begin{array}{l}0.31583^{*} \\
(0.03717) \\
\end{array}$ & \begin{tabular}{|l|l|}
$0.33861^{*}$ \\
$(0.03755)$ \\
\end{tabular} & $\begin{array}{l}0.31705^{*} \\
(0.03718) \\
\end{array}$ & $\begin{array}{l}0.32427^{*} \\
(0.03764) \\
\end{array}$ & \begin{tabular}{|l|l|}
$0.31647^{*}$ \\
$(0.03715)$ \\
\end{tabular} & $\begin{array}{l}0.31759^{*} \\
(0.03715) \\
\end{array}$ & $\begin{array}{l}0.31647^{*} \\
(0.03695) \\
\end{array}$ & \begin{tabular}{|l|}
$0.31647^{*}$ \\
$(0.03718)$ \\
\end{tabular} & $\begin{array}{l}0.31585^{*} \\
(0.03686) \\
\end{array}$ & \begin{tabular}{|l|l|l|l}
$0.1227)$ \\
$(0.127)$ \\
\end{tabular} & \begin{tabular}{|l}
$0.34193^{*}$ \\
$(0.04878)$ \\
\end{tabular} \\
\hline Factor & $\begin{array}{l}0.14996^{\ddagger} \\
(0.0788)\end{array}$ & $\begin{array}{l}0.15127^{\ddagger} \\
(0.07887)\end{array}$ & $\begin{array}{l}14533^{\ddagger} \\
.07886)\end{array}$ & \begin{tabular}{|l|l|}
$0.1649^{\dagger}$ \\
$(0.07811)$ \\
\end{tabular} & $\begin{array}{l}0.15197^{\ddagger} \\
(0.07887)\end{array}$ & $\begin{array}{l}0.15226^{\ddagger} \\
(0.07867)\end{array}$ & \begin{tabular}{|l|l}
$0.14919^{*}$ \\
$(0.0787)$
\end{tabular} & $\begin{array}{l}0.15027^{\ddagger} \\
(0.07868)\end{array}$ & $\begin{array}{l}0.13971^{\ddagger} \\
(0.07838)\end{array}$ & \begin{tabular}{|l|}
$0.14919^{\ddagger}$ \\
$(0.07877)$ \\
\end{tabular} & $\begin{array}{l}0.14171^{\ddagger} \\
(0.07813)\end{array}$ & $\begin{array}{l}0.14602^{*} \\
(0.07869)\end{array}$ & \begin{tabular}{|l|l}
$0.14919^{\ddagger}$ \\
$(0.07872)$
\end{tabular} \\
\hline $\mathrm{Fa}$ & $\begin{array}{ll}0.09978 \\
(0.12341)\end{array}$ & $\begin{array}{l}0.1027 \\
(0.1237)\end{array}$ & $\begin{array}{l}0.08917 \\
(0.1234)\end{array}$ & \begin{tabular}{|l|}
0.11577 \\
$(0.12199)$ \\
\end{tabular} & $\begin{array}{ll}0.10041 \\
(0.12323)\end{array}$ & $\begin{array}{ll}0.10768 \\
(0.12323)\end{array}$ & \begin{tabular}{|l|}
0.11063 \\
$(0.12389)$ \\
\end{tabular} & $\begin{array}{l}0.10454 \\
(0.1232)\end{array}$ & $\begin{array}{ll}0.07361 \\
(0.12277)\end{array}$ & \begin{tabular}{|l|}
0.1007 \\
$(0.12342)$ \\
\end{tabular} & $\begin{array}{ll}0.07825 \\
(0.12226)\end{array}$ & $\begin{array}{l}-0.04338 \\
(0.17023)\end{array}$ & \begin{tabular}{|l|l|}
0.11684 \\
$(0.12556)$ \\
\end{tabular} \\
\hline Factor $1 \& 3$ & $\begin{array}{l}0.33277^{*} \\
(0.03161)\end{array}$ & $\begin{array}{l}0.33716^{*} \\
(0.0337)\end{array}$ & $\begin{array}{l}0.32364^{*} \\
(0.03223)\end{array}$ & \begin{tabular}{|l|}
$0.33185^{*}$ \\
$(0.03076)$
\end{tabular} & $\begin{array}{l}0.33329^{*} \\
(0.03135)\end{array}$ & $\begin{array}{l}0.335^{*} \\
(0.03123)\end{array}$ & \begin{tabular}{|l|}
$0.3309^{*}$ \\
$(0.03106)$ \\
\end{tabular} & $\begin{array}{l}0.33604^{*} \\
(0.03145)\end{array}$ & $\begin{array}{l}0.33042^{*} \\
(0.03089)\end{array}$ & \begin{tabular}{|l|}
$0.33299^{*}$ \\
$(0.03149)$ \\
\end{tabular} & $\begin{array}{l}0.32152^{*} \\
(0.03101)\end{array}$ & $\begin{array}{l}0.33068^{*} \\
(0.03104) \\
\end{array}$ & \begin{tabular}{|l|l}
$0.33068^{*}$ \\
$(0.03107)$
\end{tabular} \\
\hline Factor $1,3 \& 5$ & $\begin{array}{l}0.29648^{*} \\
(0.04129)\end{array}$ & $\begin{array}{l}0.29523^{*} \\
(0.04055)\end{array}$ & $\begin{array}{l}0.29083^{*} \\
(0.04046)\end{array}$ & \begin{tabular}{|l|}
$0.29399^{*}$ \\
$(0.03995)$
\end{tabular} & $\begin{array}{l}0.2934^{*} \\
(0.04036)\end{array}$ & $\begin{array}{l}0.30256^{*} \\
(0.04098)\end{array}$ & \begin{tabular}{|l|}
$0.29421^{*}$ \\
$(0.04035)$
\end{tabular} & $\begin{array}{l}0.29359^{*} \\
(0.04033)\end{array}$ & $\begin{array}{l}0.29127^{*} \\
(0.04013)\end{array}$ & \begin{tabular}{|l|}
$0.2934^{*}$ \\
$(0.04037)$ \\
\end{tabular} & $\begin{array}{l}0.2875^{*} \\
(0.04008)\end{array}$ & $\begin{array}{l}0.15513 \\
(0.12291)\end{array}$ & \begin{tabular}{|l|l}
$0.29843^{*}$ \\
$(0.04085)$
\end{tabular} \\
\hline Fact & $\begin{array}{l}0.28556^{*} \\
(0.03038)\end{array}$ & $\begin{array}{l}0.28913^{*} \\
(0.03215)\end{array}$ & $\begin{array}{l}0.27589^{*} \\
(0.02966)\end{array}$ & \begin{tabular}{|l|}
$0.29102^{*}$ \\
$(0.0286)$
\end{tabular} & $\begin{array}{l}0.28473^{*} \\
(0.02906)\end{array}$ & $\begin{array}{l}0.28523^{*} \\
(0.02881)\end{array}$ & \begin{tabular}{|l|}
$0.28278^{*}$ \\
$(0.02873)$
\end{tabular} & $\begin{array}{l}0.29218^{*} \\
(0.03026)\end{array}$ & $\begin{array}{l}0.28195^{*} \\
(0.02856)\end{array}$ & \begin{tabular}{|l|}
$0.28195^{*}$ \\
$(0.02874)$
\end{tabular} & $\begin{array}{l}0.27328^{*} \\
(0.02867)\end{array}$ & $\begin{array}{l}0.28195^{\circ} \\
(0.02869)\end{array}$ & \begin{tabular}{|l|l}
$0.28195^{*}$ \\
$(0.02872)$
\end{tabular} \\
\hline Factor $1,3,4 \& 5$ & $\begin{array}{l}0.25543^{*} \\
(0.04945)\end{array}$ & $\begin{array}{l}0.25896^{*} \\
(0.05049)\end{array}$ & $\begin{array}{l}0.24847^{*} \\
(0.04902)\end{array}$ & \begin{tabular}{|l|}
$0.27293^{*}$ \\
$(0.04877)$ \\
\end{tabular} & $\begin{array}{l}0.2551^{*} \\
(0.04895)\end{array}$ & $\begin{array}{l}0.2538^{*} \\
(0.04873)\end{array}$ & \begin{tabular}{|l|}
$0.25705^{*}$ \\
$(0.04899)$ \\
\end{tabular} & $\begin{array}{l}0.25729^{*} \\
(0.04894)\end{array}$ & $\begin{array}{l}0.24822^{*} \\
(0.04852)\end{array}$ & \begin{tabular}{|l|}
$0.2525^{*}$ \\
$(0.0488)$ \\
\end{tabular} & $\begin{array}{l}0.24131^{*} \\
(0.04855) \\
\end{array}$ & 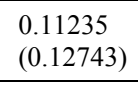 & \begin{tabular}{|l}
$0.25942^{*}$ \\
$(0.04952)$ \\
\end{tabular} \\
\hline Factor $1 \& 2$ & $\begin{array}{l}0.19069^{*} \\
(0.03271)\end{array}$ & $\begin{array}{l}0.19462^{*} \\
(0.03437)\end{array}$ & $\begin{array}{l}0.1831^{*} \\
(0.03294)\end{array}$ & \begin{tabular}{|l|}
$0.23203^{*}$ \\
$(0.03512)$
\end{tabular} & $\begin{array}{l}0.19934^{*} \\
(0.0363)\end{array}$ & $\begin{array}{l}0.19513^{*} \\
(0.0326)\end{array}$ & \begin{tabular}{|l|}
$0.18966^{*}$ \\
$(0.03223)$
\end{tabular} & $\begin{array}{l}0.18948^{*} \\
(0.03222)\end{array}$ & $\begin{array}{l}0.18765^{*} \\
(0.03205)\end{array}$ & \begin{tabular}{|l|}
$0.19219^{*}$ \\
$(0.03312)$
\end{tabular} & $\begin{array}{l}0.14666^{*} \\
(0.03562)\end{array}$ & & \begin{tabular}{|l|l}
$0.18869^{*}$ \\
$(0.03223)$
\end{tabular} \\
\hline Factor $1,2 \& 5$ & $\begin{array}{l}0.29555^{*} \\
(0.08722) \\
\end{array}$ & $\begin{array}{l}0.30298^{*} \\
(0.08847) \\
\end{array}$ & $\begin{array}{l}0.28893^{*} \\
(0.08753) \\
\end{array}$ & \begin{tabular}{|l|}
$0.31776^{*}$ \\
$(0.08661)$ \\
\end{tabular} & $\begin{array}{l}0.30384^{*} \\
(0.08815) \\
\end{array}$ & $\begin{array}{l}0.31785^{*} \\
(0.08888) \\
\end{array}$ & \begin{tabular}{|l|}
$0.30116^{*}$ \\
$(0.08733)$ \\
\end{tabular} & $\begin{array}{l}0.3123^{*} \\
(0.0885)\end{array}$ & $\begin{array}{l}0.26882^{*} \\
(0.08744) \\
\end{array}$ & \begin{tabular}{|l|}
$0.29874^{*}$ \\
$(0.08748)$ \\
\end{tabular} & $\begin{array}{l}0.24264^{*} \\
(0.08869) \\
\end{array}$ & 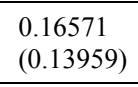 & \begin{tabular}{|l}
$0.31026^{*}$ \\
$(0.08905)$ \\
\end{tabular} \\
\hline Factor & $\begin{array}{l}.4815^{*} \\
(0.04101) \\
\end{array}$ & $\begin{array}{l}.48708^{*} \\
(0.04415) \\
\end{array}$ & $\begin{array}{l}0.4634^{*} \\
(0.04132) \\
\end{array}$ & \begin{tabular}{|l|}
$0.51644^{*}$ \\
$(0.04028)$ \\
\end{tabular} & $\begin{array}{l}0.48982^{*} \\
(0.0439) \\
\end{array}$ & $\begin{array}{l}\begin{array}{l}0.49102^{*} \\
(0.04006)\end{array} \\
\end{array}$ & \begin{tabular}{|l|l|}
$0.47654^{*}$ \\
$(0.03828)$ \\
\end{tabular} & $\begin{array}{l}0.47677^{*} \\
(0.03827) \\
\end{array}$ & $\begin{array}{l}0.47246^{*} \\
(0.0381) \\
\end{array}$ & \begin{tabular}{|l|l|}
$0.47621^{*}$ \\
$(0.03831)$ \\
\end{tabular} & $\begin{array}{l}0.43981^{*} \\
(0.04033) \\
\end{array}$ & $\begin{array}{l}0.47613^{*} \\
(0.03825) \\
\end{array}$ & \begin{tabular}{|l}
$0.47613^{*}$ \\
$(0.03828)$ \\
\end{tabular} \\
\hline Factor $1,2,4 \& 5$ & $\begin{array}{l}0.30786^{*} \\
(0.05505)\end{array}$ & $\begin{array}{l}0.31861^{*} \\
(0.05918)\end{array}$ & $\begin{array}{l}0.2984^{*} \\
(0.0562)\end{array}$ & \begin{tabular}{|l|}
$0.30907^{*}$ \\
$(0.05447)$ \\
\end{tabular} & $\begin{array}{l}0.31464^{*} \\
(0.05608)\end{array}$ & $\begin{array}{l}0.30829^{*} \\
(0.05496)\end{array}$ & \begin{tabular}{|l|}
$0.30835^{*}$ \\
$(0.055)$
\end{tabular} & $\begin{array}{l}0.30856^{*} \\
(0.05499)\end{array}$ & $\begin{array}{l}0.30621^{*} \\
(0.05471)\end{array}$ & \begin{tabular}{|l|}
$0.3081^{*}$ \\
$(0.05505)$ \\
\end{tabular} & $\begin{array}{l}0.30652^{*} \\
(0.05457)\end{array}$ & & \begin{tabular}{|l|l}
$0.33516^{*}$ \\
$(0.06467)$
\end{tabular} \\
\hline Factor $1,2 \& 3$ & $\begin{array}{l}0.10165^{\dagger} \\
(0.05027)\end{array}$ & $\begin{array}{l}0.10414^{\dagger} \\
(0.05079)\end{array}$ & $\begin{array}{l}0.09116^{\ddagger} \\
(0.05077)\end{array}$ & \begin{tabular}{|l|}
$0.14125^{*}$ \\
$(0.05132)$ \\
\end{tabular} & $\begin{array}{ll}0.1089^{\dagger} \\
(0.05207)\end{array}$ & $\begin{array}{ll}0.10735^{\dagger} \\
(0.05017)\end{array}$ & \begin{tabular}{|l|}
$0.10173^{\dagger}$ \\
$(0.04986)$ \\
\end{tabular} & $\begin{array}{ll}0.1078^{\dagger} \\
(0.05041)\end{array}$ & $\begin{array}{ll}0.0968^{\ddagger} \\
(0.04953)\end{array}$ & \begin{tabular}{|l|}
$0.10214^{\dagger}$ \\
$(0.05022)$ \\
\end{tabular} & $\begin{array}{ll}0.07898 \\
(0.04997)\end{array}$ & $\begin{array}{l}0.09923^{\dagger} \\
(0.04975)\end{array}$ & \begin{tabular}{|l|}
$0.09923^{\dagger}$ \\
$(0.0498)$
\end{tabular} \\
\hline & $\begin{array}{l}0.32078^{*} \\
(0.0452)\end{array}$ & $\begin{array}{l}0.32616^{*} \\
(0.04687)\end{array}$ & $\begin{array}{l}0.30417^{*} \\
(0.04898)\end{array}$ & \begin{tabular}{|l|}
$0.36876^{*}$ \\
$(0.04762)$
\end{tabular} & $\begin{array}{l}0.32824^{*} \\
(0.047)\end{array}$ & $\begin{array}{l}0.33391^{*} \\
(0.04644)\end{array}$ & \begin{tabular}{|l|}
$0.33501^{*}$ \\
$(0.04786)$
\end{tabular} & $\begin{array}{l}0.34164^{*} \\
(0.04947)\end{array}$ & $\begin{array}{l}0.31763^{*} \\
(0.04485)\end{array}$ & \begin{tabular}{|l|}
$0.32282^{*}$ \\
$(0.04558)$
\end{tabular} & $\begin{array}{l}0.28186^{*} \\
(0.04693)\end{array}$ & $\begin{array}{ll}0.17969 \\
(0.12608)\end{array}$ & $\begin{array}{l}0.34298^{*} \\
(0.05348)\end{array}$ \\
\hline Factor $1,2,3 \& 4$ & $\begin{array}{l}0.28834^{*} \\
(0.04156)\end{array}$ & $\begin{array}{l}0.29248^{*} \\
(0.04328)\end{array}$ & $\begin{array}{l}0.26666^{*} \\
(0.04268)\end{array}$ & \begin{tabular}{|l|}
$0.3313^{*}$ \\
$(0.04126)$
\end{tabular} & $\begin{array}{l}0.294^{*} \\
(0.04234)\end{array}$ & $\begin{array}{l}0.29856^{*} \\
(0.04028)\end{array}$ & \begin{tabular}{|l|}
$0.30376^{*}$ \\
$(0.04438)$
\end{tabular} & $\begin{array}{l}0.29404^{*} \\
(0.03968)\end{array}$ & $\begin{array}{l}0.26532^{*} \\
(0.03866)\end{array}$ & \begin{tabular}{|l|}
$0.28557^{*}$ \\
$(0.03882)$
\end{tabular} & $\begin{array}{l}0.23813^{*} \\
(0.0413)\end{array}$ & $\begin{array}{l}0.28232^{*} \\
(0.03812)\end{array}$ & \begin{tabular}{|l|l}
$0.28232^{*}$ \\
$(0.03816)$
\end{tabular} \\
\hline A & $\begin{array}{l}1808^{*} \\
.03051)\end{array}$ & $\begin{array}{l}0.18674^{*} \\
(0.03443) \\
\end{array}$ & $\begin{array}{l}0.16315^{*} \\
(0.03441)\end{array}$ & \begin{tabular}{|l|}
$0.20532^{*}$ \\
$(0.03055)$ \\
\end{tabular} & $\begin{array}{l}0.18523^{*} \\
(0.03161) \\
\end{array}$ & $\begin{array}{l}0.19235^{*} \\
(0.03159) \\
\end{array}$ & \begin{tabular}{|l|}
$0.19638^{*}$ \\
$(0.03534)$
\end{tabular} & $\begin{array}{l}0.19327^{*} \\
(0.03274) \\
\end{array}$ & $\begin{array}{l}0.15784^{*} \\
(0.03049) \\
\end{array}$ & \begin{tabular}{|l|}
$0.17865^{*}$ \\
$(0.02946)$ \\
\end{tabular} & $\begin{array}{l}0.16193^{*} \\
(0.02975) \\
\end{array}$ & $\begin{array}{ll}0.03906 \\
(0.12023)\end{array}$ & \begin{tabular}{|l}
$0.19201^{*}$ \\
$(0.03427)$
\end{tabular} \\
\hline Variable & $\begin{array}{l}-0.00823 \\
(0.02245)\end{array}$ & $(0.02196)$ & $(0.02196)$ & $\mid$\begin{tabular}{|c|}
$-0.060031^{*}$ \\
$(0.02042)$
\end{tabular} & $(0.02202)$ & $(0.01931)$ & $(0.02823)$ & $(0.0231)$ & $(0.03206)$ & $\left|\begin{array}{l}-0.01516 \\
(0.03271)\end{array}\right|$ & $(0.02196)$ & $\begin{array}{l}0.14014 \\
(0.11775)\end{array}$ & \begin{tabular}{|l|l}
-0.028 \\
$(0.03475)$
\end{tabular} \\
\hline
\end{tabular}

* Significant at the 0.01 level $†$ Significant at the 0.05 level $\$$ Significant at the 0.10 level

Table 105 Coefficients with individual variables for high-level factors - Wood Industry 


\begin{tabular}{|c|c|c|c|c|c|c|c|c|c|c|c|c|c|}
\hline $\begin{array}{l}\text { Individual variable } \\
\text { included in the } \\
\text { model in the } \\
\text { column }\end{array}$ & 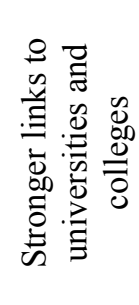 & 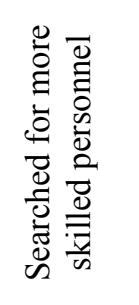 & 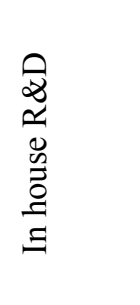 & 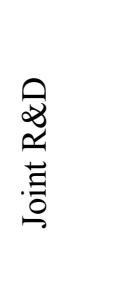 & 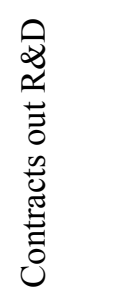 & 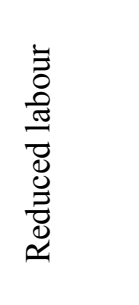 & 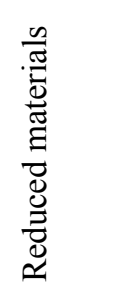 & 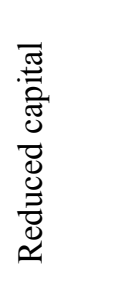 & 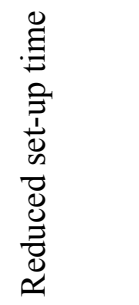 & 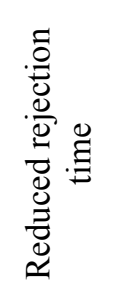 & 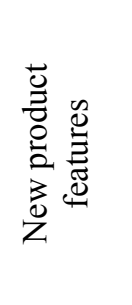 & 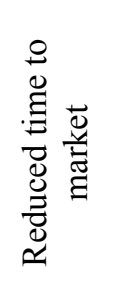 & 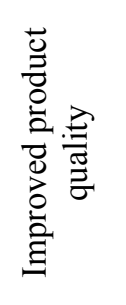 \\
\hline No factors & $\begin{array}{l}0.26012^{*} \\
(0.01817)\end{array}$ & $\begin{array}{l}0.2594^{*} \\
(0.01813)\end{array}$ & $\begin{array}{l}0.2567^{*} \\
(0.0186) \\
\end{array}$ & $\begin{array}{l}0.26127^{*} \\
(0.01817) \\
\end{array}$ & $\begin{array}{l}0.26296^{*} \\
(0.01819) \\
\end{array}$ & $\begin{array}{l}0.26197^{*} \\
(0.01818)\end{array}$ & $\begin{array}{l}0.27373^{*} \\
(0.01766) \\
\end{array}$ & $\begin{array}{l}0.26118^{*} \\
(0.01784)\end{array}$ & $\begin{array}{l}0.26427^{*} \\
(0.01836)\end{array}$ & $\begin{array}{l}0.25421^{*} \\
(0.01826)\end{array}$ & $\begin{array}{l}0.25993^{*} \\
(0.01824) \\
\end{array}$ & $\begin{array}{l}0.2615^{*} \\
(0.01818) \\
\end{array}$ & $\begin{array}{l}0.26369^{*} \\
(0.01836)\end{array}$ \\
\hline Factor 5 & $\begin{array}{l}0.30256^{*} \\
(0.09786)\end{array}$ & $\begin{array}{l}0.13106 \\
(0.10828)\end{array}$ & $\begin{array}{l}0.24323^{\dagger} \\
(0.09434)\end{array}$ & $\begin{array}{l}0.26144^{*} \\
(0.09356)\end{array}$ & $\begin{array}{l}0.2526^{*} \\
(0.09337)\end{array}$ & $\begin{array}{l}0.27506^{*} \\
(0.09418)\end{array}$ & $\begin{array}{l}0.34674^{*} \\
(0.0915)\end{array}$ & $\begin{array}{l}0.26463^{*} \\
(0.09186)\end{array}$ & $\begin{array}{l}0.27091^{*} \\
(0.09425)\end{array}$ & $\begin{array}{l}0.24685^{*} \\
(0.0932)\end{array}$ & $\begin{array}{l}0.2511^{*} \\
(0.0939)\end{array}$ & $\begin{array}{l}0.26344^{*} \\
(0.09367)\end{array}$ & $\begin{array}{l}0.26069^{*} \\
(0.09381)\end{array}$ \\
\hline Factor 4 & $\begin{array}{l}0.13974^{*} \\
(0.04262)\end{array}$ & $\begin{array}{l}0.13974^{*} \\
(0.04252)\end{array}$ & $\begin{array}{l}0.12811^{*} \\
(0.04455)\end{array}$ & $\begin{array}{l}0.14032^{*} \\
(0.04258)\end{array}$ & $\begin{array}{l}0.13974^{*} \\
(0.04255)\end{array}$ & $\begin{array}{l}0.15397^{*} \\
(0.04322)\end{array}$ & $\begin{array}{l}0.14517^{*} \\
(0.04106)\end{array}$ & $\begin{array}{l}0.14456^{*} \\
(0.04185)\end{array}$ & $\begin{array}{l}0.13974^{*} \\
(0.04264)\end{array}$ & $\begin{array}{l}0.13777^{*} \\
(0.04246)\end{array}$ & $\begin{array}{l}0.13966^{*} \\
(0.04277)\end{array}$ & $\begin{array}{l}0.13974^{*} \\
(0.0426)\end{array}$ & $\begin{array}{l}0.14087^{*} \\
(0.04268)\end{array}$ \\
\hline Factor $4 \& 5$ & $\begin{array}{l}0.33948^{\ddagger} \\
(0.19551) \\
\end{array}$ & $\begin{array}{l}0.20667 \\
(0.20306) \\
\end{array}$ & $\begin{array}{l}0.32495^{\ddagger} \\
(0.19614) \\
\end{array}$ & $\begin{array}{l}0.3442^{\ddagger} \\
(0.19538) \\
\end{array}$ & $\begin{array}{l}0.33196^{\ddagger} \\
(0.19512) \\
\end{array}$ & $\begin{array}{l}0.37555^{\ddagger} \\
(0.1966) \\
\end{array}$ & $\begin{array}{l}0.43979^{\dagger} \\
(0.18913) \\
\end{array}$ & $\begin{array}{l}0.33196^{\ddagger} \\
(0.19187) \\
\end{array}$ & $\begin{array}{l}0.36746^{\ddagger} \\
(0.19677) \\
\end{array}$ & $\begin{array}{l}0.32375^{\ddagger} \\
(0.19472)\end{array}$ & $\begin{array}{l}0.32869^{\ddagger} \\
(0.19624) \\
\end{array}$ & $\begin{array}{l}0.33196^{\ddagger} \\
(0.19536) \\
\end{array}$ & $\begin{array}{l}0.35355^{\ddagger} \\
(0.19625) \\
\end{array}$ \\
\hline Factor 3 & $\begin{array}{l}0.12222 \\
(0.10531)\end{array}$ & $\begin{array}{l}0.12222 \\
(0.10507)\end{array}$ & $\begin{array}{l}0.11213 \\
(0.10616)\end{array}$ & $\begin{array}{l}0.13441 \\
(0.1054)\end{array}$ & $\begin{array}{l}0.13828 \\
(0.1054)\end{array}$ & $\begin{array}{l}0.14567 \\
(0.10592)\end{array}$ & $\begin{array}{l}0.17477^{\ddagger} \\
(0.10181)\end{array}$ & $\begin{array}{l}0.1405 \\
(0.10347)\end{array}$ & $\begin{array}{l}0.12222 \\
(0.10536)\end{array}$ & $\begin{array}{l}0.11021 \\
(0.10501)\end{array}$ & $\begin{array}{l}0.11436 \\
(0.10678)\end{array}$ & $\begin{array}{l}0.14362 \\
(0.1059)\end{array}$ & $\begin{array}{l}0.12913 \\
(0.10554)\end{array}$ \\
\hline Factor $3 \& 5$ & $\begin{array}{l}0.21822^{*} \\
(0.06053) \\
\end{array}$ & $\begin{array}{l}0.03602 \\
(0.07606) \\
\end{array}$ & $\begin{array}{l}0.1584^{*} \\
(0.05112) \\
\end{array}$ & $\begin{array}{l}0.16172^{*} \\
(0.05084) \\
\end{array}$ & $\begin{array}{l}0.16132^{*} \\
(0.0508) \\
\end{array}$ & $\begin{array}{l}0.17927^{*} \\
(0.05169) \\
\end{array}$ & $\begin{array}{l}0.21352^{*} \\
(0.04981)\end{array}$ & $\begin{array}{l}0.20149^{*} \\
(0.05084) \\
\end{array}$ & $\begin{array}{l}0.18488^{*} \\
(0.05294) \\
\end{array}$ & $\begin{array}{l}0.15138^{*} \\
(0.05084) \\
\end{array}$ & $\begin{array}{l}0.15941^{*} \\
(0.0512) \\
\end{array}$ & $\begin{array}{l}0.16132^{*} \\
(0.05086) \\
\end{array}$ & $\begin{array}{l}0.16704^{*} \\
(0.0511) \\
\end{array}$ \\
\hline Factor $3 \& 4$ & $\begin{array}{l}0.15291 \\
(0.10097) \\
\end{array}$ & $\begin{array}{l}0.15291 \\
(0.10073) \\
\end{array}$ & $\begin{array}{l}0.14636 \\
(0.10149) \\
\end{array}$ & $\begin{array}{l}0.16085 \\
(0.10096)\end{array}$ & $\begin{array}{l}0.16337 \\
(0.10091) \\
\end{array}$ & $\begin{array}{l}0.15291 \\
(0.10088)\end{array}$ & $\begin{array}{l}0.15291 \\
(0.09723) \\
\end{array}$ & $\begin{array}{l}0.16971^{\ddagger} \\
(0.0992)\end{array}$ & $\begin{array}{l}0.15291 \\
(0.10102) \\
\end{array}$ & $\begin{array}{l}0.15291 \\
(0.10057) \\
\end{array}$ & $\begin{array}{l}0.14325 \\
(0.10305) \\
\end{array}$ & $\begin{array}{l}0.18199^{\ddagger} \\
(0.10214)\end{array}$ & $\begin{array}{l}0.17545^{\ddagger} \\
(0.10227) \\
\end{array}$ \\
\hline Factor $3,4 \& 5$ & \multicolumn{13}{|c|}{ Insufficient Data } \\
\hline Factor 2 & $\begin{array}{l}0.16018^{*} \\
(0.03535)\end{array}$ & $\begin{array}{l}0.15964^{*} \\
(0.03527)\end{array}$ & $\begin{array}{l}0.14861^{*} \\
(0.0376)\end{array}$ & $\begin{array}{l}0.16973^{*} \\
(0.03566)\end{array}$ & $\begin{array}{l}0.16044^{*} \\
(0.03529)\end{array}$ & $\begin{array}{l}0.16165^{*} \\
(0.03533)\end{array}$ & $\begin{array}{l}0.17249^{*} \\
(0.03411)\end{array}$ & $\begin{array}{l}0.16059^{*} \\
(0.0347)\end{array}$ & $\begin{array}{l}0.1614^{*} \\
(0.03537)\end{array}$ & $\begin{array}{l}0.15915^{*} \\
(0.03521)\end{array}$ & $\begin{array}{l}0.15584^{*} \\
(0.03646)\end{array}$ & $\begin{array}{l}0.16435^{*} \\
(0.0354)\end{array}$ & $\begin{array}{l}0.17043^{*} \\
(0.03609)\end{array}$ \\
\hline Facto & $\begin{array}{l}0.25861 \\
(0.17799) \\
\end{array}$ & $\begin{array}{l}0.10224 \\
(0.18553) \\
\end{array}$ & $\begin{array}{l}0.22329 \\
(0.17763) \\
\end{array}$ & $\begin{array}{l}0.23239 \\
(0.17694) \\
\end{array}$ & $\begin{array}{l}0.23393 \\
(0.1768) \\
\end{array}$ & $\begin{array}{l}0.23817 \\
(0.17702) \\
\end{array}$ & $\begin{array}{l}0.33828^{\dagger} \\
(0.17158) \\
\end{array}$ & $\begin{array}{l}0.22754 \\
(0.17384) \\
\end{array}$ & $\begin{array}{l}0.24205 \\
(0.1774) \\
\end{array}$ & $\begin{array}{l}0.21224 \\
(0.1765) \\
\end{array}$ & $\begin{array}{l}0.22754 \\
(0.1777) \\
\end{array}$ & $\begin{array}{l}0.22754 \\
(0.177) \\
\end{array}$ & $\begin{array}{l}0.24023 \\
(0.17751) \\
\end{array}$ \\
\hline Factor $2 \& 4$ & $\begin{array}{l}0.14368^{\ddagger} \\
(0.07929)\end{array}$ & $\begin{array}{l}0.14368^{\ddagger} \\
(0.0791)\end{array}$ & $\begin{array}{l}0.14292^{\ddagger} \\
(0.07951)\end{array}$ & $\begin{array}{l}0.14368^{\ddagger} \\
(0.07921) \\
\end{array}$ & $\begin{array}{l}0.14368^{\ddagger} \\
(0.07915) \\
\end{array}$ & $\begin{array}{l}0.14704^{\ddagger} \\
(0.07924)\end{array}$ & $\begin{array}{l}0.14902^{\ddagger} \\
(0.07636) \\
\end{array}$ & $\begin{array}{l}0.15105^{\ddagger} \\
(0.07785)\end{array}$ & $\begin{array}{l}0.14368^{\ddagger} \\
(0.07932)\end{array}$ & $\begin{array}{l}0.14368^{\ddagger} \\
(0.07898)\end{array}$ & $\begin{array}{l}0.14342^{\ddagger} \\
(0.07956) \\
\end{array}$ & $\begin{array}{l}0.14468^{\ddagger} \\
(0.07925) \\
\end{array}$ & $\begin{array}{l}0.15519^{\ddagger} \\
(0.07977) \\
\end{array}$ \\
\hline Factor & $\begin{array}{l}0.09588 \\
(0.09765) \\
\end{array}$ & $\begin{array}{l}-0.06503 \\
(0.11081) \\
\end{array}$ & $\begin{array}{l}0.0531 \\
(0.09605)\end{array}$ & $\begin{array}{l}0.10255 \\
(0.09784) \\
\end{array}$ & $\begin{array}{l}0.09343 \\
(0.0966) \\
\end{array}$ & $\begin{array}{l}0.07953 \\
(0.09591) \\
\end{array}$ & $\begin{array}{l}0.06801 \\
(0.09195) \\
\end{array}$ & $\begin{array}{l}0.06324 \\
(0.09372) \\
\end{array}$ & $\begin{array}{l}0.07594 \\
(0.09601) \\
\end{array}$ & $\begin{array}{l}0.0583 \\
(0.0951) \\
\end{array}$ & $\begin{array}{l}0.05352 \\
(0.0967) \\
\end{array}$ & $\begin{array}{l}0.07795 \\
(0.09591) \\
\end{array}$ & $\begin{array}{l}0.08041 \\
(0.09659) \\
\end{array}$ \\
\hline Factor $2 \& 3$ & $\begin{array}{l}0.25783^{\ddagger} \\
(0.13481) \\
\end{array}$ & $\begin{array}{l}0.25783^{\ddagger} \\
(0.13449) \\
\end{array}$ & $\begin{array}{l}0.25566^{\ddagger} \\
(0.13519) \\
\end{array}$ & $\begin{array}{l}0.26365^{\ddagger} \\
(0.13471) \\
\end{array}$ & $\begin{array}{l}0.2692^{\dagger} \\
(0.13468) \\
\end{array}$ & $\begin{array}{l}0.26345^{\ddagger} \\
(0.13472)\end{array}$ & $\begin{array}{l}0.27384^{\dagger} \\
(0.12985) \\
\end{array}$ & $\begin{array}{l}0.32909^{\dagger} \\
(0.13339)\end{array}$ & $\begin{array}{l}0.25783^{\ddagger} \\
(0.13487) \\
\end{array}$ & $\begin{array}{l}0.25392^{\ddagger} \\
(0.13429) \\
\end{array}$ & $\begin{array}{l}0.24731^{\ddagger} \\
(0.13682) \\
\end{array}$ & $\begin{array}{l}0.29572^{\dagger} \\
(0.13629) \\
\end{array}$ & $\begin{array}{l}0.26458^{\ddagger} \\
(0.13504) \\
\end{array}$ \\
\hline Factor $2,3 \& 5$ & $\begin{array}{l}0.31761^{\ddagger} \\
(0.16338)\end{array}$ & $\begin{array}{l}0.13998 \\
(0.16992) \\
\end{array}$ & $\begin{array}{l}0.24784 \\
(0.16212) \\
\end{array}$ & $\begin{array}{l}0.27724^{\ddagger} \\
(0.16055) \\
\end{array}$ & $\begin{array}{l}0.28104^{\ddagger} \\
(0.16047) \\
\end{array}$ & $\begin{array}{l}0.29242^{\ddagger} \\
(0.16106)\end{array}$ & $\begin{array}{l}0.34254^{\dagger} \\
(0.1552) \\
\end{array}$ & $\begin{array}{l}0.28442^{\ddagger} \\
(0.1577)\end{array}$ & $\begin{array}{l}0.29563^{\ddagger} \\
(0.16174)\end{array}$ & $\begin{array}{l}0.25973 \\
(0.15996) \\
\end{array}$ & $\begin{array}{l}0.26421 \\
(0.16115) \\
\end{array}$ & $\begin{array}{l}0.27462^{\ddagger} \\
(0.16058) \\
\end{array}$ & $\begin{array}{l}0.26847^{\ddagger} \\
(0.16078) \\
\end{array}$ \\
\hline Factor & $\begin{array}{l}0.31195^{*} \\
(0.08253)\end{array}$ & $\begin{array}{l}0.31195^{*} \\
(0.08233)\end{array}$ & $\begin{array}{l}0.29646^{*} \\
(0.08444)\end{array}$ & $\begin{array}{l}0.35377^{*} \\
(0.08522) \\
\end{array}$ & $\begin{array}{l}0.36993^{*} \\
(0.08687) \\
\end{array}$ & $\begin{array}{l}0.31791^{*} \\
(0.08251)\end{array}$ & $\begin{array}{l}0.32102^{*} \\
(0.07949) \\
\end{array}$ & $\begin{array}{l}0.31195^{*} \\
(0.08101)\end{array}$ & $\begin{array}{l}0.31661^{*} \\
(0.08262)\end{array}$ & $\begin{array}{l}0.31195^{*} \\
(0.0822)\end{array}$ & $\begin{array}{l}0.30947^{*} \\
(0.08295) \\
\end{array}$ & $\begin{array}{l}0.32384^{*} \\
(0.08274) \\
\end{array}$ & $\begin{array}{l}0.31284^{*} \\
(0.08262)\end{array}$ \\
\hline $\mathrm{Fa}$ & $\begin{array}{l}0.29567^{\dagger} \\
(0.12065)\end{array}$ & $\begin{array}{l}0.14728 \\
(0.13236) \\
\end{array}$ & $\begin{array}{l}0.26471^{\dagger} \\
(0.12054)\end{array}$ & $\begin{array}{l}0.30414^{\dagger} \\
(0.1209) \\
\end{array}$ & $\begin{array}{l}0.29942^{\dagger} \\
(0.12038) \\
\end{array}$ & $\begin{array}{l}0.28781^{\dagger} \\
(0.12007)\end{array}$ & $\begin{array}{l}0.28524^{\dagger} \\
(0.1155)\end{array}$ & $\begin{array}{l}0.29626^{\dagger} \\
(0.11785)\end{array}$ & $\begin{array}{l}0.29525^{\dagger} \\
(0.12078)\end{array}$ & $\begin{array}{l}0.25064^{\dagger} \\
(0.11977)\end{array}$ & $\begin{array}{l}0.26594^{\dagger} \\
(0.12102) \\
\end{array}$ & $\begin{array}{l}0.28414^{\dagger} \\
(0.12002) \\
\end{array}$ & $\begin{array}{l}0.28532^{\dagger} \\
(0.12037) \\
\end{array}$ \\
\hline Factor 1 & $\begin{array}{l}0.21809^{*} \\
(0.03856)\end{array}$ & $\begin{array}{l}0.21471^{*} \\
(0.0385) \\
\end{array}$ & $\begin{array}{l}0.21706^{*} \\
(0.03868)\end{array}$ & $\begin{array}{l}0.22063^{*} \\
(0.03855) \\
\end{array}$ & $\begin{array}{l}0.21959^{*} \\
(0.0385) \\
\end{array}$ & $\begin{array}{l}0.24096^{*} \\
(0.04032)\end{array}$ & $\begin{array}{l}0.28706^{*} \\
(0.03896) \\
\end{array}$ & $\begin{array}{l}0.2687^{*} \\
(0.03968)\end{array}$ & $\begin{array}{l}0.25792^{*} \\
(0.04571) \\
\end{array}$ & $\begin{array}{l}0.17095^{*} \\
(0.04284)\end{array}$ & $\begin{array}{l}0.20917^{*} \\
(0.04241) \\
\end{array}$ & $\begin{array}{l}0.25488^{*} \\
(0.04338) \\
\end{array}$ & $\begin{array}{l}0.24975^{*} \\
(0.04435) \\
\end{array}$ \\
\hline Factor $1 \& 5$ & $\begin{array}{l}0.33456^{*} \\
(0.03849)\end{array}$ & $\begin{array}{l}0.22907^{*} \\
(0.0541)\end{array}$ & $\begin{array}{l}0.31035^{*} \\
(0.03774)\end{array}$ & $\begin{array}{l}0.33997^{*} \\
(0.03895)\end{array}$ & $\begin{array}{l}0.33586^{*} \\
(0.03812) \\
\end{array}$ & $\begin{array}{l}0.34688^{*} \\
(0.04025)\end{array}$ & $\begin{array}{l}0.39856^{*} \\
(0.03834) \\
\end{array}$ & $\begin{array}{l}0.37841^{*} \\
(0.03918)\end{array}$ & $\begin{array}{l}0.34312^{*} \\
(0.04054)\end{array}$ & $\begin{array}{l}0.25553^{*} \\
(0.04432)\end{array}$ & $\begin{array}{l}0.30572^{*} \\
(0.04267) \\
\end{array}$ & $\begin{array}{l}0.34715^{*} \\
(0.04059) \\
\end{array}$ & $\begin{array}{l}0.35117^{*} \\
(0.04414)\end{array}$ \\
\hline Factor $1 \& 4$ & $\begin{array}{l}0.14919^{\ddagger} \\
(0.07849) \\
\end{array}$ & $\begin{array}{l}0.14919^{\ddagger} \\
(0.0783) \\
\end{array}$ & $\begin{array}{l}0.1392^{\ddagger} \\
(0.07944) \\
\end{array}$ & $\begin{array}{l}0.15201^{\ddagger} \\
(0.07843) \\
\end{array}$ & $\begin{array}{l}0.14919^{\ddagger} \\
(0.07835) \\
\end{array}$ & $\begin{array}{l}0.1845^{\dagger} \\
(0.08054) \\
\end{array}$ & $\begin{array}{l}0.22129^{*} \\
(0.07658) \\
\end{array}$ & $\begin{array}{l}0.23195^{*} \\
(0.07947) \\
\end{array}$ & $\begin{array}{l}0.17812^{\dagger} \\
(0.08052) \\
\end{array}$ & $\begin{array}{l}0.09454 \\
(0.08122) \\
\end{array}$ & $\begin{array}{l}0.13997^{\ddagger} \\
(0.08078) \\
\end{array}$ & $\begin{array}{l}0.18005^{\dagger} \\
(0.08021) \\
\end{array}$ & $\begin{array}{l}0.17997^{\dagger} \\
(0.08139) \\
\end{array}$ \\
\hline Factor $1,4 \& 5$ & $\begin{array}{l}0.13772 \\
(0.12494) \\
\end{array}$ & $\begin{array}{l}-0.02853 \\
(0.13487) \\
\end{array}$ & $\begin{array}{l}0.09031 \\
(0.12322) \\
\end{array}$ & $\begin{array}{l}0.11098 \\
(0.1228) \\
\end{array}$ & $\begin{array}{l}0.11856 \\
(0.12291) \\
\end{array}$ & $\begin{array}{l}0.13794 \\
(0.12444) \\
\end{array}$ & $\begin{array}{l}0.199^{\ddagger} \\
(0.11944) \\
\end{array}$ & $\begin{array}{l}0.19224 \\
(0.12252) \\
\end{array}$ & $\begin{array}{l}0.12851 \\
(0.1243) \\
\end{array}$ & $\begin{array}{l}0.06484 \\
(0.12289) \\
\end{array}$ & $\begin{array}{l}0.09296 \\
(0.12334) \\
\end{array}$ & $\begin{array}{l}0.10995 \\
(0.12284) \\
\end{array}$ & $\begin{array}{l}0.11939 \\
(0.12382) \\
\end{array}$ \\
\hline Factor $1 \& 3$ & $\begin{array}{l}0.33068^{*} \\
(0.03098)\end{array}$ & $\begin{array}{l}0.33026^{*} \\
(0.03091) \\
\end{array}$ & $\begin{array}{l}0.32603^{*} \\
(0.03147)\end{array}$ & $\begin{array}{l}0.33344^{*} \\
(0.03098)\end{array}$ & $\begin{array}{l}0.34331^{*} \\
(0.0315)\end{array}$ & $\begin{array}{l}0.3734^{*} \\
(0.03808)\end{array}$ & $\begin{array}{l}0.42637^{*} \\
(0.03402)\end{array}$ & $\begin{array}{l}0.40494^{*} \\
(0.03507)\end{array}$ & $\begin{array}{l}0.36489^{*} \\
(0.03747)\end{array}$ & $\begin{array}{l}0.293^{*} \\
(0.03439)\end{array}$ & $\begin{array}{l}0.32076^{*} \\
(0.03659)\end{array}$ & $\begin{array}{l}0.36562^{*} \\
(0.03628)\end{array}$ & $\begin{array}{l}0.36666^{*} \\
(0.03972)\end{array}$ \\
\hline Factor & $\begin{array}{l}0.30428^{*} \\
(0.04072)\end{array}$ & $\begin{array}{l}0.16802^{\dagger} \\
(0.06941)\end{array}$ & $\begin{array}{l}0.28762^{*} \\
(0.04081)\end{array}$ & $\begin{array}{l}0.30768^{*} \\
(0.04087) \\
\end{array}$ & $\begin{array}{l}0.29564^{*} \\
(0.04018)\end{array}$ & $\begin{array}{l}0.33569^{*} \\
(0.04583)\end{array}$ & $\begin{array}{l}0.41324^{*} \\
(0.04383)\end{array}$ & $\begin{array}{l}0.3788^{*} \\
(0.04432)\end{array}$ & $\begin{array}{l}0.33231^{*} \\
(0.04687)\end{array}$ & $\begin{array}{l}0.23268^{*} \\
(0.04693)\end{array}$ & $\begin{array}{l}0.2825^{*} \\
(0.04553) \\
\end{array}$ & $\begin{array}{l}0.33966^{*} \\
(0.0474) \\
\end{array}$ & $\begin{array}{l}0.31099^{*} \\
(0.04208)\end{array}$ \\
\hline $\mathrm{Fc}$ & $\begin{array}{l}0.28195^{*} \\
(0.02864)\end{array}$ & $\begin{array}{l}0.27319^{*} \\
(0.02884)\end{array}$ & $\begin{array}{l}0.27302^{*} \\
(0.0303)\end{array}$ & $\begin{array}{l}0.29099^{*} \\
(0.02899) \\
\end{array}$ & $\begin{array}{l}0.29404^{*} \\
(0.02916)\end{array}$ & $\begin{array}{l}0.29709^{*} \\
(0.02967)\end{array}$ & $\begin{array}{l}0.35261^{*} \\
(0.0301)\end{array}$ & $\begin{array}{l}0.30901^{*} \\
(0.02882)\end{array}$ & $\begin{array}{l}0.31773^{*} \\
(0.03614) \\
\end{array}$ & $\begin{array}{l}0.24799^{*} \\
(0.03164)\end{array}$ & $\begin{array}{l}0.27541^{*} \\
(0.03143)\end{array}$ & $\begin{array}{l}0.30965^{*} \\
(0.03232)\end{array}$ & $\begin{array}{l}0.31263^{*} \\
(0.03563)\end{array}$ \\
\hline Factor 1 , & $\begin{array}{l}0.2755^{*} \\
(0.0504) \\
\end{array}$ & $\begin{array}{l}0.1272^{\ddagger} \\
(0.07457) \\
\end{array}$ & $\begin{array}{l}0.23838^{*} \\
(0.0511) \\
\end{array}$ & $\begin{array}{l}0.25981^{*} \\
(0.04872)\end{array}$ & $\begin{array}{l}0.2656^{*} \\
(0.04894) \\
\end{array}$ & $\begin{array}{l}0.29288^{*} \\
(0.05292)\end{array}$ & $\begin{array}{l}0.35455^{*} \\
(0.04997) \\
\end{array}$ & $\begin{array}{l}0.29742^{*} \\
(0.04889) \\
\end{array}$ & $\begin{array}{l}0.26304^{*} \\
(0.04908)\end{array}$ & $\begin{array}{l}0.19345^{*} \\
(0.05396)\end{array}$ & $\begin{array}{l}0.24227^{*} \\
(0.0527) \\
\end{array}$ & $\begin{array}{l}0.29869^{*} \\
(0.05466) \\
\end{array}$ & $\begin{array}{l}0.28743^{*} \\
(0.05431) \\
\end{array}$ \\
\hline Facto & $\begin{array}{l}0.18869^{*} \\
(0.03213)\end{array}$ & $\begin{array}{l}0.18869^{*} \\
(0.03206)\end{array}$ & $\begin{array}{l}0.18782^{*} \\
(0.03223)\end{array}$ & $\begin{array}{l}0.19959^{*} \\
(0.03259)\end{array}$ & $\begin{array}{l}0.18953^{*} \\
(0.03208)\end{array}$ & $\begin{array}{l}0.21164^{*} \\
(0.03425)\end{array}$ & $\begin{array}{l}0.31141^{*} \\
(0.03738)\end{array}$ & $\begin{array}{l}0.26211^{*} \\
(0.03596)\end{array}$ & $\begin{array}{l}0.22862^{*} \\
(0.04047)\end{array}$ & $\begin{array}{l}0.12684^{*} \\
(0.04056)\end{array}$ & $\begin{array}{l}0.17949^{*} \\
(0.03689)\end{array}$ & $\begin{array}{l}0.23462^{*} \\
(0.04062)\end{array}$ & $\begin{array}{l}0.22348^{*} \\
(0.04013)\end{array}$ \\
\hline Factor $1,2 \& 5$ & $\begin{array}{l}0.30622^{*} \\
(0.08712)\end{array}$ & $\begin{array}{l}0.21056^{\dagger} \\
(0.09483)\end{array}$ & $\begin{array}{l}0.28565^{*} \\
(0.08779)\end{array}$ & $\begin{array}{l}0.30835^{*} \\
(0.08707)\end{array}$ & $\begin{array}{l}0.29874^{*} \\
(0.08676)\end{array}$ & $\begin{array}{l}0.33801^{*} \\
(0.08958)\end{array}$ & $\begin{array}{l}0.36881^{*} \\
(0.08462)\end{array}$ & $\begin{array}{l}0.36032^{*} \\
(0.08666)\end{array}$ & $\begin{array}{l}0.33578^{*} \\
(0.0904)\end{array}$ & $\begin{array}{l}0.23904^{*} \\
(0.0895)\end{array}$ & $\begin{array}{l}0.29118^{*} \\
(0.08762)\end{array}$ & $\begin{array}{l}0.32245^{*} \\
(0.08807)\end{array}$ & $\begin{array}{l}0.32005^{*} \\
(0.08862)\end{array}$ \\
\hline Factor $1,2 \& 4$ & $\begin{array}{l}0.47613^{*} \\
(0.03817) \\
\end{array}$ & $\begin{array}{l}0.47551^{*} \\
(0.03808)\end{array}$ & $\begin{array}{l}0.47147^{*} \\
(0.03861)\end{array}$ & $\begin{array}{l}0.47658^{*} \\
(0.03814) \\
\end{array}$ & $\begin{array}{l}0.47613^{*} \\
(0.0381) \\
\end{array}$ & $\begin{array}{l}0.49477^{*} \\
(0.03935)\end{array}$ & $\begin{array}{l}0.52348^{*} \\
(0.03764) \\
\end{array}$ & $\begin{array}{l}0.57062^{*} \\
(0.04357)\end{array}$ & $\begin{array}{l}0.517^{*} \\
(0.04574) \\
\end{array}$ & $\begin{array}{l}0.4159^{*} \\
(0.0451) \\
\end{array}$ & $\begin{array}{l}0.46429^{*} \\
(0.04471) \\
\end{array}$ & $\begin{array}{l}0.5066^{*} \\
(0.04157) \\
\end{array}$ & $\begin{array}{l}0.50046^{*} \\
(0.04173) \\
\end{array}$ \\
\hline Factor $1,2,4 \& 5$ & $\begin{array}{l}0.33585^{*} \\
(0.05719) \\
\end{array}$ & $\begin{array}{l}0.18248^{\dagger} \\
(0.07875)\end{array}$ & $\begin{array}{l}0.29925^{*} \\
(0.05577) \\
\end{array}$ & $\begin{array}{l}0.30874^{*} \\
(0.05481)\end{array}$ & $\begin{array}{l}0.30844^{*} \\
(0.05476) \\
\end{array}$ & $\begin{array}{l}0.32957^{*} \\
(0.05596)\end{array}$ & $\begin{array}{l}0.37119^{*} \\
(0.05392) \\
\end{array}$ & $\begin{array}{l}0.35657^{*} \\
(0.05506)\end{array}$ & $\begin{array}{l}0.30909^{*} \\
(0.05489) \\
\end{array}$ & $\begin{array}{l}0.24568^{*} \\
(0.06009) \\
\end{array}$ & $\begin{array}{l}0.29596^{*} \\
(0.05966) \\
\end{array}$ & $\begin{array}{l}0.3539^{*} \\
(0.06025) \\
\end{array}$ & $\begin{array}{l}0.32661^{*} \\
(0.05643) \\
\end{array}$ \\
\hline Factor 1 & $\begin{array}{l}0.09923^{\dagger} \\
(0.04965) \\
\end{array}$ & $\begin{array}{l}0.09923^{\dagger} \\
(0.04954) \\
\end{array}$ & $\begin{array}{l}0.09424^{\ddagger} \\
(0.05008) \\
\end{array}$ & $\begin{array}{l}0.12452^{\dagger} \\
(0.05129) \\
\end{array}$ & $\begin{array}{l}0.13306^{\dagger} \\
(0.05211)\end{array}$ & $\begin{array}{l}0.14282^{*} \\
(0.05453)\end{array}$ & $\begin{array}{l}0.20248^{*} \\
(0.05097) \\
\end{array}$ & $\begin{array}{l}0.1872^{*} \\
(0.05295) \\
\end{array}$ & $\begin{array}{l}0.11386^{\dagger} \\
(0.05049) \\
\end{array}$ & $\begin{array}{l}0.07554 \\
(0.05037) \\
\end{array}$ & $\begin{array}{l}0.08725 \\
(0.05501) \\
\end{array}$ & $\begin{array}{l}0.14541^{*} \\
(0.05557) \\
\end{array}$ & $\begin{array}{l}0.13281^{\dagger} \\
(0.05484) \\
\end{array}$ \\
\hline Factor $1,2,3 \& 5$ & $\begin{array}{l}0.34352^{*} \\
(0.04699)\end{array}$ & $\begin{array}{l}0.19454^{*} \\
(0.07225)\end{array}$ & $\begin{array}{l}0.30722^{*} \\
(0.04711)\end{array}$ & $\begin{array}{l}0.34347^{*} \\
(0.04654)\end{array}$ & $\begin{array}{l}0.32619^{*} \\
(0.04499)\end{array}$ & $\begin{array}{l}0.35762^{*} \\
(0.04903)\end{array}$ & $\begin{array}{l}0.41285^{*} \\
(0.04612)\end{array}$ & $\begin{array}{l}0.40457^{*} \\
(0.04843)\end{array}$ & $\begin{array}{l}0.35221^{*} \\
(0.0492) \\
\end{array}$ & $\begin{array}{l}0.26015^{*} \\
(0.05083)\end{array}$ & $\begin{array}{l}0.30885^{*} \\
(0.04993)\end{array}$ & $\begin{array}{l}0.36081^{*} \\
(0.05012)\end{array}$ & $\begin{array}{l}0.35227^{*} \\
(0.05027)\end{array}$ \\
\hline Factor & $\begin{array}{l}0.28232^{*} \\
(0.03804) \\
\end{array}$ & $\begin{array}{l}0.28232^{*} \\
(0.03796) \\
\end{array}$ & $\begin{array}{l}0.26907^{*} \\
(0.04076) \\
\end{array}$ & $\begin{array}{l}0.29859^{*} \\
(0.03892) \\
\end{array}$ & $\begin{array}{l}0.29468^{*} \\
(0.03843) \\
\end{array}$ & $\begin{array}{l}0.31506^{*} \\
(0.04164) \\
\end{array}$ & $\begin{array}{l}0.39103^{*} \\
(0.04108) \\
\end{array}$ & $\begin{array}{l}0.36414^{*} \\
(0.04201) \\
\end{array}$ & $\begin{array}{l}0.3146^{*} \\
(0.04294) \\
\end{array}$ & $\begin{array}{l}0.23391^{*} \\
(0.04262) \\
\end{array}$ & $\begin{array}{l}0.27301^{*} \\
(0.04226) \\
\end{array}$ & $\begin{array}{l}0.32403^{*} \\
(0.04423) \\
\end{array}$ & $\begin{array}{l}0.31233^{*} \\
(0.04335) \\
\end{array}$ \\
\hline A & $\begin{array}{l}0.21717^{*} \\
(0.03704)\end{array}$ & $\begin{array}{l}0.05252 \\
(0.06373)\end{array}$ & $\begin{array}{l}0.17203^{*} \\
(0.03004)\end{array}$ & $\begin{array}{l}0.19051^{*} \\
(0.03)\end{array}$ & $\begin{array}{l}0.18121^{*} \\
(0.0293)\end{array}$ & $\begin{array}{l}0.2188^{*} \\
(0.0362)\end{array}$ & $\begin{array}{l}0.29847^{*} \\
(0.03494)\end{array}$ & $\begin{array}{l}0.23833^{*} \\
(0.03209)\end{array}$ & $\begin{array}{l}0.21652^{*} \\
(0.03778)\end{array}$ & $\begin{array}{l}0.11997^{*} \\
(0.03735)\end{array}$ & $\begin{array}{l}0.16827^{*} \\
(0.03479)\end{array}$ & $\begin{array}{l}0.21905^{*} \\
(0.03683)\end{array}$ & $\begin{array}{l}0.21319^{*} \\
(0.03815)\end{array}$ \\
\hline $\begin{array}{l}\text { Individual } \\
\text { Variable }\end{array}$ & $\begin{array}{l}-0.05741^{\ddagger} \\
(0.03306)\end{array}$ & $\begin{array}{l}0.1253^{\dagger} \\
(0.05663)\end{array}$ & $\begin{array}{l}0.01744 \\
(0.01889)\end{array}$ & $\begin{array}{l}-0.04511^{\ddagger} \\
(0.02325)\end{array}$ & $\begin{array}{l}-0.05945^{\dagger} \\
(0.02827)\end{array}$ & $\begin{array}{l}-0.04359^{\ddagger} \\
(0.02264)\end{array}$ & $\begin{array}{l}-0.12407^{*} \\
(0.0212)\end{array}$ & $\begin{array}{l}-0.09547^{*} \\
(0.02246)\end{array}$ & $\begin{array}{l}-0.0413 \\
(0.02543)\end{array}$ & $\begin{array}{l}0.06274^{\dagger} \\
(0.02527)\end{array}$ & $\begin{array}{l}0.01208 \\
(0.02352)\end{array}$ & $\begin{array}{l}-0.0466^{\ddagger} \\
(0.02523)\end{array}$ & $\begin{array}{l}-0.03612 \\
(0.0249)\end{array}$ \\
\hline
\end{tabular}

* Significant at the 0.01 level $\quad \dagger$ Significant at the 0.05 level $\$$ Significant at the 0.10 level

Table 105 Coefficients with individual variables for high-level factors - Wood Industry 


\begin{tabular}{|c|c|c|c|c|c|c|c|c|c|c|c|c|c|}
\hline $\begin{array}{l}\text { Individual variable } \\
\text { included in the } \\
\text { model in the } \\
\text { column }\end{array}$ & 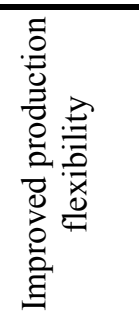 & 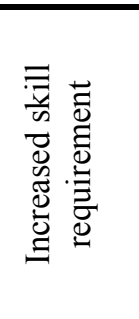 & 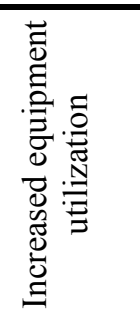 & 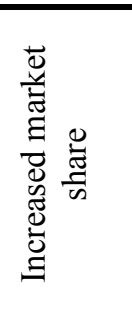 & 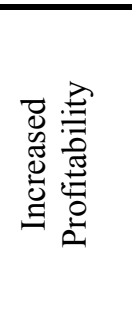 & 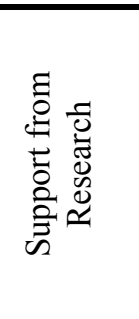 & 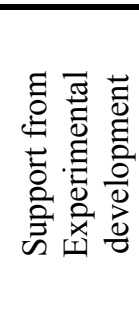 & 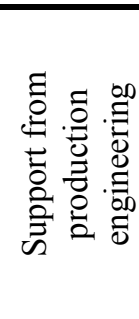 & 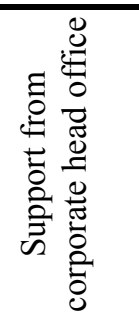 & 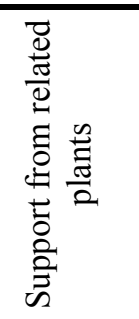 & 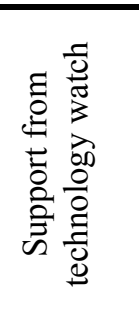 & 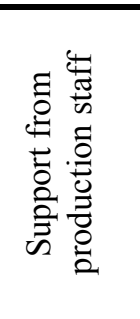 & 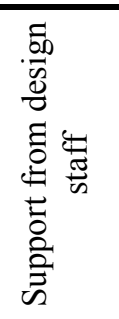 \\
\hline No factors & $\begin{array}{l}0.26131^{*} \\
(0.01839)\end{array}$ & $\begin{array}{l}0.25778^{*} \\
(0.01843)\end{array}$ & $\begin{array}{l}0.25929^{\ddagger} \\
(0.01824)\end{array}$ & $\begin{array}{l}0.25933^{\ddagger} \\
(0.01822)\end{array}$ & $(0.01823)$ & $\begin{array}{l}0.26138^{\ddagger} \\
(0.01817)\end{array}$ & $\begin{array}{l}0.26087^{\ddagger} \\
(0.01816)\end{array}$ & $\begin{array}{l}0.2616^{\ddagger} \\
(0.01821)\end{array}$ & $\begin{array}{l}0.26144^{\ddagger} \\
(0.01824)\end{array}$ & $\begin{array}{l}0.26097^{\ddagger} \\
(0.01824)\end{array}$ & $\begin{array}{l}0.26042^{\ddagger} \\
(0.01823)\end{array}$ & $\begin{array}{l}0.26237^{\ddagger} \\
(0.01845)\end{array}$ & $\begin{array}{l}0.25977^{\ddagger} \\
(0.01835)\end{array}$ \\
\hline Factor 5 & $\begin{array}{l}0.25548^{*} \\
(0.09403)\end{array}$ & $\begin{array}{l}0.24193^{\dagger} \\
(0.09461)\end{array}$ & $\begin{array}{l}0.24092^{\dagger} \\
(0.09458)\end{array}$ & $\begin{array}{l}0.24409^{\ddagger} \\
(0.09397)\end{array}$ & $\begin{array}{l}0.25932^{\ddagger} \\
(0.09372)\end{array}$ & $\begin{array}{l}0.27516^{\ddagger} \\
(0.09413)\end{array}$ & $\begin{array}{l}0.25718^{\ddagger} \\
(0.09347)\end{array}$ & $\begin{array}{l}0.26445^{\ddagger} \\
(0.09392)\end{array}$ & $\begin{array}{l}0.26202^{\ddagger} \\
(0.09404)\end{array}$ & $\begin{array}{l}0.25958^{\ddagger} \\
(0.09402)\end{array}$ & $\begin{array}{l}0.2568^{\ddagger} \\
(0.09388)\end{array}$ & $\begin{array}{l}0.26984^{\ddagger} \\
(0.09633)\end{array}$ & $\begin{array}{l}0.25084^{\ddagger} \\
(0.09441)\end{array}$ \\
\hline Factor 4 & $\begin{array}{l}0.14015^{*} \\
(0.04278)\end{array}$ & $\begin{array}{l}0.13689^{*} \\
(0.04287)\end{array}$ & $\begin{array}{l}0.13128^{\ddagger} \\
(0.04365)\end{array}$ & $\begin{array}{l}0.12963^{\ddagger} \\
(0.0435)\end{array}$ & $\begin{array}{l}0.15352^{\ddagger} \\
(0.04362)\end{array}$ & $\begin{array}{l}0.15615^{\ddagger} \\
(0.04338)\end{array}$ & $\begin{array}{l}0.1631^{\ddagger} \\
(0.04422)\end{array}$ & $\begin{array}{l}0.14123^{\ddagger} \\
(0.04267)\end{array}$ & $\begin{array}{l}0.14005^{\ddagger} \\
(0.0427)\end{array}$ & $\begin{array}{l}0.14041^{\ddagger} \\
(0.04273)\end{array}$ & $\begin{array}{l}0.13974^{\ddagger} \\
(0.04273)\end{array}$ & $\begin{array}{l}0.14804 \\
(0.04403)\end{array}$ & $\begin{array}{l}0.13788^{\ddagger} \\
(0.04403)\end{array}$ \\
\hline Factor $4 \& 5$ & $\begin{array}{l}0.33638^{\ddagger} \\
(0.19634)\end{array}$ & $\begin{array}{l}0.31222 \\
(0.19735)\end{array}$ & $\begin{array}{l}0.32195 \\
(0.19626)\end{array}$ & $\begin{array}{l}0.32749^{\ddagger} \\
(0.19587)\end{array}$ & $\begin{array}{l}0.33196^{\ddagger} \\
(0.19564)\end{array}$ & $\begin{array}{l}0.33725^{\ddagger} \\
(0.19527)\end{array}$ & $\begin{array}{l}0.33196^{\ddagger} \\
(0.19526)\end{array}$ & $\begin{array}{l}0.3411^{\ddagger} \\
(0.1957) \\
\end{array}$ & $\begin{array}{l}0.35048^{\ddagger} \\
(0.19643)\end{array}$ & $\begin{array}{l}0.33196^{\ddagger} \\
(0.19595)\end{array}$ & $\begin{array}{l}0.335^{\ddagger} \\
(0.196)\end{array}$ & $\begin{array}{l}0.3389^{\ddagger} \\
(0.19625)\end{array}$ & $\begin{array}{l}0.33102^{\ddagger} \\
(0.19627)\end{array}$ \\
\hline Factor 3 & $\begin{array}{l}0.12685 \\
(0.10608) \\
\end{array}$ & $\begin{array}{l}0.10288 \\
(0.10798) \\
\end{array}$ & $\begin{array}{l}0.09935 \\
(0.10829) \\
\end{array}$ & $\begin{array}{l}0.12092 \\
(0.10552) \\
\end{array}$ & $\begin{array}{l}0.13807 \\
(0.10593) \\
\end{array}$ & $\begin{array}{l}0.13467 \\
(0.10539) \\
\end{array}$ & $\begin{array}{l}0.13457 \\
(0.10539) \\
\end{array}$ & $\begin{array}{l}0.14171 \\
(0.10615) \\
\end{array}$ & $\begin{array}{l}0.14154 \\
(0.10671) \\
\end{array}$ & $\begin{array}{l}0.13211 \\
(0.10603) \\
\end{array}$ & $\begin{array}{l}0.12842 \\
(0.10579) \\
\end{array}$ & $\begin{array}{l}0.13946 \\
(0.10787) \\
\end{array}$ & $\begin{array}{l}0.12087 \\
(0.10598) \\
\end{array}$ \\
\hline Factor $3 \& 5$ & $\begin{array}{l}0.16306^{*} \\
(0.05118)\end{array}$ & $\begin{array}{l}0.14802^{*} \\
(0.05332)\end{array}$ & $\begin{array}{l}0.15084^{\ddagger} \\
(0.05219)\end{array}$ & $\begin{array}{l}0.14152^{\ddagger} \\
(0.05352)\end{array}$ & $\begin{array}{l}0.18531^{\ddagger} \\
(0.05334)\end{array}$ & $\begin{array}{l}0.17831^{\ddagger} \\
(0.05156)\end{array}$ & $\begin{array}{l}0.16856^{\ddagger} \\
(0.05097)\end{array}$ & $\begin{array}{l}0.16725^{\ddagger} \\
(0.05107)\end{array}$ & $\begin{array}{l}0.1721^{\ddagger} \\
(0.05175)\end{array}$ & $\begin{array}{l}0.16493^{\ddagger} \\
(0.05114)\end{array}$ & $\begin{array}{l}0.16153^{\ddagger} \\
(0.05102)\end{array}$ & $\begin{array}{l}0.17856^{\ddagger} \\
(0.05553)\end{array}$ & $\begin{array}{l}0.1593^{\ddagger} \\
(0.05233)\end{array}$ \\
\hline Facto & $\begin{array}{l}0.15976 \\
(0.10223) \\
\end{array}$ & $\begin{array}{l}0.13858 \\
(0.10262) \\
\end{array}$ & $\begin{array}{l}0.15291 \\
(0.10124) \\
\end{array}$ & $\begin{array}{l}0.13158 \\
(0.10267) \\
\end{array}$ & $\begin{array}{l}0.17875^{\ddagger} \\
(0.10249) \\
\end{array}$ & $\begin{array}{l}0.15291 \\
(0.10086) \\
\end{array}$ & $\begin{array}{l}0.18142^{\ddagger} \\
(0.10191) \\
\end{array}$ & $\begin{array}{l}0.15884 \\
(0.10112) \\
\end{array}$ & $\begin{array}{l}0.17358^{\ddagger} \\
(0.10259) \\
\end{array}$ & $\begin{array}{l}0.15722 \\
(0.10131) \\
\end{array}$ & $\begin{array}{l}0.17199^{*} \\
(0.10319) \\
\end{array}$ & $\begin{array}{l}0.16712 \\
(0.10286)\end{array}$ & $\begin{array}{l}0.14942 \\
(0.10323) \\
\end{array}$ \\
\hline Factor & \multicolumn{13}{|c|}{ Insufficient Data } \\
\hline Factor 2 & $\begin{array}{l}0.16428^{*} \\
(0.03639)\end{array}$ & $\begin{array}{l}0.15949^{*} \\
(0.03546)\end{array}$ & $\begin{array}{l}0.15997^{\ddagger} \\
(0.03544)\end{array}$ & $\begin{array}{l}0.16018^{\ddagger} \\
(0.03542)\end{array}$ & $\begin{array}{l}0.16018^{\ddagger} \\
(0.03538)\end{array}$ & $\begin{array}{l}0.18275^{\ddagger} \\
(0.03712)\end{array}$ & $\begin{array}{l}0.17295^{\ddagger} \\
(0.03591)\end{array}$ & $\begin{array}{l}0.16973^{\ddagger} \\
(0.03591)\end{array}$ & $\begin{array}{l}0.16569^{\ddagger} \\
(0.03571)\end{array}$ & $\begin{array}{l}0.16483^{\ddagger} \\
(0.03573)\end{array}$ & $\begin{array}{l}0.16038^{\ddagger} \\
(0.03544)\end{array}$ & $\begin{array}{l}0.16605^{\ddagger} \\
(0.03623)\end{array}$ & $\begin{array}{l}0.16015^{\ddagger} \\
(0.03548)\end{array}$ \\
\hline $\mathrm{F}$ & $\begin{array}{l}0.23616 \\
(0.17852)\end{array}$ & $\begin{array}{l}0.22754 \\
(0.17759)\end{array}$ & $\begin{array}{l}0.21611 \\
(0.17796)\end{array}$ & $\begin{array}{l}0.2192 \\
(0.17756)\end{array}$ & $\begin{array}{l}0.22754 \\
(0.17725)\end{array}$ & $\begin{array}{l}0.23739 \\
(0.17697)\end{array}$ & $\begin{array}{l}0.23868 \\
(0.177)\end{array}$ & $\begin{array}{l}0.25759 \\
(0.17829)\end{array}$ & $\begin{array}{l}0.24993 \\
(0.17839)\end{array}$ & $\begin{array}{l}0.24908 \\
(0.1788)\end{array}$ & $\begin{array}{l}0.23003 \\
(0.17758)\end{array}$ & $\begin{array}{l}0.24478 \\
(0.17896)\end{array}$ & $\begin{array}{l}0.22528 \\
(0.17821)\end{array}$ \\
\hline Factc & $\begin{array}{l}0.14718^{\ddagger} \\
(0.07986) \\
\end{array}$ & $\begin{array}{l}0.14318^{\ddagger} \\
(0.07951)\end{array}$ & $\begin{array}{l}0.14175^{\ddagger} \\
(0.07952) \\
\end{array}$ & $\begin{array}{l}0.14368^{\ddagger} \\
(0.07944) \\
\end{array}$ & $\begin{array}{l}0.14368^{\ddagger} \\
(0.07936) \\
\end{array}$ & $\begin{array}{l}0.14368^{\ddagger} \\
(0.0792) \\
\end{array}$ & $\begin{array}{l}0.14721^{\ddagger} \\
(0.07922)\end{array}$ & $\begin{array}{l}0.14773^{\ddagger} \\
(0.07939) \\
\end{array}$ & $\begin{array}{l}0.15565^{\ddagger} \\
(0.08005) \\
\end{array}$ & $\begin{array}{l}0.1535^{\ddagger} \\
(0.08008) \\
\end{array}$ & $\begin{array}{l}0.14596^{\ddagger} \\
(0.07953) \\
\end{array}$ & $\begin{array}{l}0.15228^{\ddagger} \\
(0.08027)\end{array}$ & $\begin{array}{l}0.14324^{\ddagger} \\
(0.07962)\end{array}$ \\
\hline Factor & $\begin{array}{l}0.06477 \\
(0.09622) \\
\end{array}$ & $\begin{array}{l}0.05083 \\
(0.09637) \\
\end{array}$ & $\begin{array}{l}0.05871 \\
(0.09574) \\
\end{array}$ & $\begin{array}{l}0.06026 \\
(0.09566) \\
\end{array}$ & $\begin{array}{l}0.06026 \\
(0.09556) \\
\end{array}$ & $\begin{array}{l}0.06026 \\
(0.09537) \\
\end{array}$ & $\begin{array}{l}0.06026 \\
(0.09538) \\
\end{array}$ & $\begin{array}{l}0.07515 \\
(0.09603) \\
\end{array}$ & $\begin{array}{l}0.07504 \\
(0.09643) \\
\end{array}$ & $\begin{array}{l}0.06979 \\
(0.09617) \\
\end{array}$ & $\begin{array}{l}0.06171 \\
(0.09574) \\
\end{array}$ & $\begin{array}{l}0.07042 \\
(0.09662) \\
\end{array}$ & $\begin{array}{l}0.05894 \\
(0.09612) \\
\end{array}$ \\
\hline Factor $2 \& 3$ & $\begin{array}{l}0.25851^{\ddagger} \\
(0.13528)\end{array}$ & $\begin{array}{l}0.23629^{\ddagger} \\
(0.13748)\end{array}$ & $\begin{array}{l}0.25461^{\ddagger} \\
(0.13521)\end{array}$ & $\begin{array}{l}0.25554^{\ddagger} \\
(0.13508)\end{array}$ & $\begin{array}{l}0.26317^{\ddagger} \\
(0.13497)\end{array}$ & $\begin{array}{l}0.26555^{\dagger} \\
(0.13472)\end{array}$ & $\begin{array}{l}0.30067^{\dagger} \\
(0.13643)\end{array}$ & $\begin{array}{l}0.26427^{\ddagger} \\
(0.13498)\end{array}$ & $\begin{array}{l}0.28291^{\dagger} \\
(0.13664)\end{array}$ & $\begin{array}{l}0.27669^{\dagger} \\
(0.13642)\end{array}$ & $\begin{array}{l}0.26226^{\ddagger} \\
(0.13524)\end{array}$ & $\begin{array}{l}0.2622^{\$} \\
(0.13533)\end{array}$ & $\begin{array}{l}0.25718^{\ddagger} \\
(0.13536)\end{array}$ \\
\hline Factor $2,3 \& 5$ & $\begin{array}{l}0.26845^{\ddagger} \\
(0.16126)\end{array}$ & $\begin{array}{l}0.25919 \\
(0.16119) \\
\end{array}$ & $\begin{array}{l}0.24037 \\
(0.16311) \\
\end{array}$ & $\begin{array}{l}0.26226 \\
(0.16091) \\
\end{array}$ & $\begin{array}{l}0.26894^{\ddagger} \\
(0.16074) \\
\end{array}$ & $\begin{array}{l}0.30565^{\ddagger} \\
(0.16171)\end{array}$ & $\begin{array}{l}0.30693^{\ddagger} \\
(0.16182) \\
\end{array}$ & $\begin{array}{l}0.27798^{\ddagger} \\
(0.16092) \\
\end{array}$ & $\begin{array}{l}0.29036^{\ddagger} \\
(0.16221) \\
\end{array}$ & $\begin{array}{l}0.2825^{\ddagger} \\
(0.16188) \\
\end{array}$ & $\begin{array}{l}0.2797^{\ddagger} \\
(0.16171) \\
\end{array}$ & $\begin{array}{l}0.28099^{\ddagger} \\
(0.16229)\end{array}$ & $\begin{array}{l}0.26458 \\
(0.16123) \\
\end{array}$ \\
\hline Factor 2 & $\begin{array}{l}0.31735^{*} \\
(0.0835) \\
\end{array}$ & $\begin{array}{l}0.29066^{*} \\
(0.08638)\end{array}$ & $\begin{array}{l}0.30622^{\ddagger} \\
(0.08297)\end{array}$ & $\begin{array}{l}0.31111^{\ddagger} \\
(0.08269)\end{array}$ & $\begin{array}{l}0.33877^{\ddagger} \\
(0.08448)\end{array}$ & $\begin{array}{l}0.33194^{\ddagger} \\
(0.08306)\end{array}$ & $\begin{array}{l}0.35545^{\ddagger} \\
(0.08538)\end{array}$ & $\begin{array}{l}0.34562^{\ddagger} \\
(0.08542)\end{array}$ & $\begin{array}{l}0.326^{\ddagger} \\
(0.08349)\end{array}$ & $\begin{array}{l}0.32452^{\ddagger} \\
(0.08366)\end{array}$ & $\begin{array}{l}0.32434^{\ddagger} \\
(0.08376)\end{array}$ & $\begin{array}{l}0.32876^{\ddagger} \\
(0.08548)\end{array}$ & $\begin{array}{l}0.30863^{\ddagger} \\
(0.08492)\end{array}$ \\
\hline $\mathrm{F}$ & $\begin{array}{l}0.27859^{\dagger} \\
(0.12092)\end{array}$ & $\begin{array}{l}0.26568^{\dagger} \\
(0.12052)\end{array}$ & $\begin{array}{l}0.27003^{\dagger} \\
(0.12026)\end{array}$ & $\begin{array}{l}0.27257^{\dagger} \\
(0.12015)\end{array}$ & $\begin{array}{l}0.28489^{\dagger} \\
(0.1203)\end{array}$ & $\begin{array}{l}0.30886^{\dagger} \\
(0.12119)\end{array}$ & $\begin{array}{l}0.31363^{\dagger} \\
(0.12161)\end{array}$ & $\begin{array}{l}0.30624^{\dagger} \\
(0.12198)\end{array}$ & $\begin{array}{l}0.29143^{\dagger} \\
(0.12115)\end{array}$ & $\begin{array}{l}0.28877^{\dagger} \\
(0.12128)\end{array}$ & $\begin{array}{l}0.28763^{\dagger} \\
(0.12126)\end{array}$ & $\begin{array}{l}0.28981^{\dagger} \\
(0.12225)\end{array}$ & $\begin{array}{l}0.26925^{\dagger} \\
(0.12181)\end{array}$ \\
\hline $\mathrm{F}$ & $\begin{array}{l}0.22562^{*} \\
(0.04148)\end{array}$ & $\begin{array}{l}0.20454^{*} \\
(0.04174)\end{array}$ & $\begin{array}{l}0.20465^{\ddagger} \\
(0.04115)\end{array}$ & $\begin{array}{l}0.19339^{\ddagger} \\
(0.04364)\end{array}$ & $\begin{array}{l}0.24999^{\ddagger} \\
(0.04397)\end{array}$ & $\begin{array}{l}0.22196^{\ddagger} \\
(0.03857)\end{array}$ & $\begin{array}{l}0.22014 \\
(0.03853)\end{array}$ & $\begin{array}{l}0.21901^{\ddagger} \\
(0.03859)\end{array}$ & $\begin{array}{l}0.23089^{\ddagger} \\
(0.04005)\end{array}$ & $\begin{array}{l}0.22763^{\ddagger} \\
(0.03979)\end{array}$ & $\begin{array}{l}0.21986^{\ddagger} \\
(0.03871)\end{array}$ & $\begin{array}{l}0.22242^{\ddagger} \\
(0.03906)\end{array}$ & $\begin{array}{l}0.21769^{\ddagger} \\
(0.03877)\end{array}$ \\
\hline Factol & $\begin{array}{l}0.32693^{*} \\
(0.04258)\end{array}$ & $\begin{array}{l}0.29375^{*} \\
(0.04557)\end{array}$ & $\begin{array}{l}0.3069^{\ddagger} \\
(0.03849)\end{array}$ & $\begin{array}{l}0.28346^{\ddagger} \\
(0.04598) \\
\end{array}$ & $\begin{array}{l}0.35548^{\ddagger} \\
(0.04515) \\
\end{array}$ & $\begin{array}{l}0.32115^{\ddagger} \\
(0.03709)\end{array}$ & $\begin{array}{l}0.32868^{\ddagger} \\
(0.03753)\end{array}$ & $\begin{array}{l}0.31951^{\ddagger} \\
(0.03713)\end{array}$ & $\begin{array}{l}0.3182^{\ddagger} \\
(0.03715)\end{array}$ & $\begin{array}{l}0.31783^{\ddagger} \\
(0.03717)\end{array}$ & $\begin{array}{l}0.32178^{\ddagger} \\
(0.03756)\end{array}$ & $\begin{array}{l}0.32742^{\ddagger} \\
(0.03968)\end{array}$ & $\begin{array}{l}0.31534^{\ddagger} \\
(0.03774) \\
\end{array}$ \\
\hline Factor & $\begin{array}{l}0.1583^{\ddagger} \\
(0.08081)\end{array}$ & $\begin{array}{l}0.13011 \\
(0.08177)\end{array}$ & $\begin{array}{l}0.12641 \\
(0.08225)\end{array}$ & $\begin{array}{l}0.12519 \\
(0.08108)\end{array}$ & $\begin{array}{l}0.1906^{\dagger} \\
(0.08318)\end{array}$ & $\begin{array}{l}0.15102^{\ddagger} \\
(0.07841)\end{array}$ & $\begin{array}{l}0.15126^{\ddagger} \\
(0.07841)\end{array}$ & $\begin{array}{l}0.17116^{\dagger} \\
(0.07983)\end{array}$ & $\begin{array}{l}0.16624^{\dagger} \\
(0.07989)\end{array}$ & $\begin{array}{l}0.15377^{\ddagger} \\
(0.07882)\end{array}$ & $\begin{array}{l}0.14919^{\ddagger} \\
(0.0787)\end{array}$ & $\begin{array}{l}0.16207^{\dagger} \\
(0.0804)\end{array}$ & $\begin{array}{l}0.14888^{\ddagger} \\
(0.0788)\end{array}$ \\
\hline Factor $1,4 \& 5$ & $\begin{array}{l}0.10775 \\
(0.12503)\end{array}$ & $\begin{array}{l}0.0738 \\
(0.1259)\end{array}$ & $\begin{array}{l}0.07762 \\
(0.12465)\end{array}$ & $\begin{array}{l}0.095 \\
(0.12294)\end{array}$ & $\begin{array}{l}0.13818 \\
(0.12581)\end{array}$ & $\begin{array}{l}0.11157 \\
(0.12279)\end{array}$ & $\begin{array}{l}0.09912 \\
(0.12257)\end{array}$ & $\begin{array}{l}0.11982 \\
(0.12369)\end{array}$ & $\begin{array}{l}0.09945 \\
(0.12295)\end{array}$ & $\begin{array}{l}0.10846 \\
(0.12354)\end{array}$ & $\begin{array}{l}0.10278 \\
(0.12318)\end{array}$ & $\begin{array}{l}0.10912 \\
(0.12406)\end{array}$ & $\begin{array}{l}0.09408 \\
(0.12408)\end{array}$ \\
\hline Factor $1 \& 3$ & $\begin{array}{l}0.33987^{*} \\
(0.03605)\end{array}$ & $\begin{array}{l}0.31122^{*} \\
(0.03841)\end{array}$ & $\begin{array}{l}0.30957^{\ddagger} \\
(0.03815)\end{array}$ & $\begin{array}{l}0.2974^{\ddagger} \\
(0.04137)\end{array}$ & $\begin{array}{l}0.37167^{\ddagger} \\
(0.04115)\end{array}$ & $\begin{array}{l}0.35318^{\ddagger} \\
(0.03298)\end{array}$ & $\begin{array}{l}0.3428^{\ddagger} \\
(0.03156)\end{array}$ & $\begin{array}{l}0.35162^{\ddagger} \\
(0.03384)\end{array}$ & $\begin{array}{l}0.35116^{\ddagger} \\
(0.03533)\end{array}$ & $\begin{array}{l}0.34085^{\ddagger} \\
(0.03264)\end{array}$ & $\begin{array}{l}0.33963^{\ddagger} \\
(0.03244)\end{array}$ & $\begin{array}{l}0.34427^{\ddagger} \\
(0.03554)\end{array}$ & $\begin{array}{l}0.32916^{\ddagger} \\
(0.03224)\end{array}$ \\
\hline Factor $1,3 \& 5$ & $\begin{array}{l}0.30393^{*} \\
(0.04553)\end{array}$ & $\begin{array}{l}0.27048^{*} \\
(0.04828)\end{array}$ & $\begin{array}{l}0.26842^{\ddagger} \\
(0.04807)\end{array}$ & $\begin{array}{l}0.26104^{\ddagger} \\
(0.04825) \\
\end{array}$ & $\begin{array}{l}0.33334^{\ddagger} \\
(0.04816) \\
\end{array}$ & $\begin{array}{l}0.31483^{\ddagger} \\
(0.04164)\end{array}$ & $\begin{array}{l}0.29458^{\ddagger} \\
(0.0402) \\
\end{array}$ & $\begin{array}{l}0.32448^{\ddagger} \\
(0.04504)\end{array}$ & $\begin{array}{l}0.31597^{\ddagger} \\
(0.04442)\end{array}$ & $\begin{array}{l}0.29552^{\ddagger} \\
(0.04039)\end{array}$ & $\begin{array}{l}0.29582^{\ddagger} \\
(0.04042)\end{array}$ & $\begin{array}{l}0.30219^{\ddagger} \\
(0.04189)\end{array}$ & $\begin{array}{l}0.29175^{\ddagger} \\
(0.04133) \\
\end{array}$ \\
\hline $\mathrm{F}$ & $\begin{array}{l}0.28808^{*} \\
(0.03121)\end{array}$ & $\begin{array}{l}0.26313^{*} \\
(0.03608)\end{array}$ & $\begin{array}{l}0.25747^{\ddagger} \\
(0.03854)\end{array}$ & $\begin{array}{l}0.25327^{\ddagger} \\
(0.03713)\end{array}$ & $\begin{array}{l}0.31173^{\ddagger} \\
(0.03476)\end{array}$ & $\begin{array}{l}0.28758^{\ddagger} \\
(0.02875)\end{array}$ & $\begin{array}{l}0.29439^{\ddagger} \\
(0.0293)\end{array}$ & $\begin{array}{l}0.29272^{\ddagger} \\
(0.0295)\end{array}$ & $\begin{array}{l}0.29979^{\ddagger} \\
(0.03224)\end{array}$ & $\begin{array}{l}0.29059^{\ddagger} \\
(0.02995)\end{array}$ & $\begin{array}{l}0.28861^{\ddagger} \\
(0.02955)\end{array}$ & $\begin{array}{l}0.29918^{\ddagger} \\
(0.0361)\end{array}$ & $\begin{array}{l}0.28009^{\ddagger} \\
(0.03058)\end{array}$ \\
\hline $\mathrm{Fr}$ & $\begin{array}{l}0.26303^{*} \\
(0.05308)\end{array}$ & $\begin{array}{l}0.22953^{*} \\
(0.05557)\end{array}$ & $\begin{array}{l}0.22759^{\ddagger} \\
(0.05532)\end{array}$ & $\begin{array}{l}0.23288^{\ddagger} \\
(0.05132)\end{array}$ & $\begin{array}{l}0.27698^{\ddagger} \\
(0.05128)\end{array}$ & $\begin{array}{l}0.26564^{\ddagger} \\
(0.04903)\end{array}$ & $\begin{array}{l}0.26569^{\ddagger} \\
(0.04904)\end{array}$ & $\begin{array}{l}0.27103^{\ddagger} \\
(0.05012)\end{array}$ & $\begin{array}{l}0.26426^{\ddagger} \\
(0.04967)\end{array}$ & $\begin{array}{l}0.26995^{\ddagger} \\
(0.05171)\end{array}$ & $\begin{array}{l}0.26066^{\ddagger} \\
(0.0495)\end{array}$ & $\begin{array}{l}0.26932^{\ddagger} \\
(0.05324)\end{array}$ & $\begin{array}{l}0.249^{\ddagger} \\
(0.05261)\end{array}$ \\
\hline Facto & $\begin{array}{l}0.19447^{*} \\
(0.03422)\end{array}$ & $\begin{array}{l}0.18215^{*} \\
(0.03311)\end{array}$ & $\begin{array}{l}0.16945^{\ddagger} \\
(0.03803)\end{array}$ & $\begin{array}{l}0.15451^{\ddagger} \\
(0.04273) \\
\end{array}$ & $\begin{array}{l}0.2301^{\ddagger} \\
(0.04221)\end{array}$ & $\begin{array}{l}0.20018^{\ddagger} \\
(0.03262)\end{array}$ & $\begin{array}{l}0.19098^{\ddagger} \\
(0.03212)\end{array}$ & $\begin{array}{l}0.19085^{\ddagger} \\
(0.03219)\end{array}$ & $\begin{array}{l}0.19495^{\ddagger} \\
(0.03261)\end{array}$ & $\begin{array}{l}0.18924^{\ddagger} \\
(0.03222)\end{array}$ & $\begin{array}{l}0.18918^{\ddagger} \\
(0.03222)\end{array}$ & $\begin{array}{l}0.19404^{\ddagger} \\
(0.03294)\end{array}$ & $\begin{array}{l}0.18773^{\ddagger} \\
(0.0327) \\
\end{array}$ \\
\hline Factor $1,2 \& 5$ & $\begin{array}{l}0.30219^{*} \\
(0.08819)\end{array}$ & $\begin{array}{l}0.27997^{*} \\
(0.08901)\end{array}$ & $\begin{array}{l}0.27248^{\ddagger} \\
(0.09044)\end{array}$ & $\begin{array}{l}0.26137^{\ddagger} \\
(0.09149)\end{array}$ & $\begin{array}{l}0.33048^{\ddagger} \\
(0.08998)\end{array}$ & $\begin{array}{l}0.30641^{\ddagger} \\
(0.08698)\end{array}$ & $\begin{array}{l}0.31368^{\ddagger} \\
(0.0873) \\
\end{array}$ & $\begin{array}{l}0.30971^{\ddagger} \\
(0.08745)\end{array}$ & $\begin{array}{l}0.31192^{\ddagger} \\
(0.08811)\end{array}$ & $\begin{array}{l}0.29956^{\ddagger} \\
(0.08721)\end{array}$ & $\begin{array}{l}0.2962^{\ddagger} \\
(0.08713)\end{array}$ & $\begin{array}{l}0.30724^{\ddagger} \\
(0.08842)\end{array}$ & $\begin{array}{l}0.29438^{\ddagger} \\
(0.08748)\end{array}$ \\
\hline Factor $1,2 \& 4$ & $\begin{array}{l}0.48337^{*} \\
(0.04091)\end{array}$ & $\begin{array}{l}0.45352^{*} \\
(0.04641)\end{array}$ & $\begin{array}{l}0.45324^{\ddagger} \\
(0.04519)\end{array}$ & $\begin{array}{l}0.44299^{\ddagger} \\
(0.04695)\end{array}$ & $\begin{array}{l}0.50425^{\ddagger} \\
(0.04248)\end{array}$ & 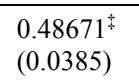 & $\begin{array}{l}0.47636^{\ddagger} \\
(0.03813)\end{array}$ & $\begin{array}{l}0.48513^{\ddagger} \\
(0.03864)\end{array}$ & $\begin{array}{l}0.47714^{\ddagger} \\
(0.03825)\end{array}$ & $\begin{array}{l}0.48232^{\ddagger} \\
(0.03875)\end{array}$ & $\begin{array}{l}0.4766^{\ddagger} \\
(0.03827)\end{array}$ & $\begin{array}{l}0.48824^{\ddagger} \\
(0.04125)\end{array}$ & $\begin{array}{l}0.475^{\ddagger} \\
(0.03885)\end{array}$ \\
\hline Factor $1,2,4 \& 5$ & $\begin{array}{l}0.30813^{*} \\
(0.05505)\end{array}$ & $\begin{array}{l}0.28505^{*} \\
(0.06101)\end{array}$ & $\begin{array}{l}0.2948^{\ddagger} \\
(0.05666)\end{array}$ & $\begin{array}{l}0.29069^{\ddagger} \\
(0.05673)\end{array}$ & $\begin{array}{l}0.34919^{\ddagger} \\
(0.06133)\end{array}$ & $\begin{array}{l}0.34688^{\ddagger} \\
(0.05828)\end{array}$ & $\begin{array}{l}0.30876^{\ddagger} \\
(0.0548) \\
\end{array}$ & $\begin{array}{l}0.30847^{\ddagger} \\
(0.0549) \\
\end{array}$ & $\begin{array}{l}0.3206^{\ddagger} \\
(0.05597)\end{array}$ & $\begin{array}{l}0.31833^{\ddagger} \\
(0.05597)\end{array}$ & $\begin{array}{l}0.31886^{\ddagger} \\
(0.05621)\end{array}$ & $\begin{array}{l}0.32465^{\ddagger} \\
(0.05904)\end{array}$ & $\begin{array}{l}0.30607^{\ddagger} \\
(0.05589)\end{array}$ \\
\hline Factor & $\begin{array}{l}0.11021^{\dagger} \\
(0.05438)\end{array}$ & $\begin{array}{l}0.07671 \\
(0.05624)\end{array}$ & $\begin{array}{l}0.07539 \\
(0.05572)\end{array}$ & $\begin{array}{l}0.06536 \\
(0.05701) \\
\end{array}$ & $\begin{array}{l}0.13856^{\dagger} \\
(0.05607) \\
\end{array}$ & $\begin{array}{l}0.13317^{\dagger} \\
(0.0525)\end{array}$ & $\begin{array}{l}0.11736^{\dagger} \\
(0.05046)\end{array}$ & $\begin{array}{l}0.12569^{\dagger} \\
(0.05257)\end{array}$ & $\begin{array}{l}0.10941^{\dagger} \\
(0.05045)\end{array}$ & $\begin{array}{l}0.11789^{\dagger} \\
(0.05309)\end{array}$ & $\begin{array}{l}0.10223^{\dagger} \\
(0.04988)\end{array}$ & $\begin{array}{l}0.10696^{\dagger} \\
(0.05076)\end{array}$ & $\begin{array}{l}0.09616^{\ddagger} \\
(0.05274) \\
\end{array}$ \\
\hline Factor $1,2,3 \& 5$ & $\begin{array}{l}0.33008^{*} \\
(0.04949)\end{array}$ & $\begin{array}{l}0.29687^{*} \\
(0.05238)\end{array}$ & $\begin{array}{l}0.29615^{\ddagger} \\
(0.05149)\end{array}$ & $\begin{array}{l}0.2902^{\ddagger} \\
(0.05121)\end{array}$ & $\begin{array}{l}0.3592^{\ddagger} \\
(0.05197)\end{array}$ & $\begin{array}{l}0.34359^{\ddagger} \\
(0.0465)\end{array}$ & $\begin{array}{l}0.3464^{\ddagger} \\
(0.04692)\end{array}$ & $\begin{array}{l}0.33305^{\ddagger} \\
(0.04581)\end{array}$ & $\begin{array}{l}0.33888^{\ddagger} \\
(0.04771)\end{array}$ & $\begin{array}{l}0.33705^{\ddagger} \\
(0.04818)\end{array}$ & $\begin{array}{l}0.32313^{\ddagger} \\
(0.04521)\end{array}$ & $\begin{array}{l}0.33153^{\ddagger} \\
(0.04748)\end{array}$ & $\begin{array}{l}0.31833^{\ddagger} \\
(0.04592)\end{array}$ \\
\hline$\& 4$ & $\begin{array}{l}0.29252^{*} \\
(0.04321) \\
\end{array}$ & $\begin{array}{l}0.26003^{*} \\
(0.0461) \\
\end{array}$ & $\begin{array}{l}0.26302^{\ddagger} \\
(0.04319) \\
\end{array}$ & $\begin{array}{l}0.25974^{\ddagger} \\
(0.04239) \\
\end{array}$ & $\begin{array}{l}0.31735^{\ddagger} \\
(0.04455) \\
\end{array}$ & $\begin{array}{l}0.31346^{\ddagger} \\
(0.04116) \\
\end{array}$ & $\begin{array}{l}0.3167^{\ddagger} \\
(0.04186) \\
\end{array}$ & $\begin{array}{l}0.30929^{\ddagger} \\
(0.04189) \\
\end{array}$ & $\begin{array}{l}0.29627^{\ddagger} \\
(0.03981) \\
\end{array}$ & $\begin{array}{l}0.28797^{\ddagger} \\
(0.03855) \\
\end{array}$ & $\begin{array}{l}0.29403^{\ddagger} \\
(0.04007) \\
\end{array}$ & $\begin{array}{l}0.29861^{\ddagger} \\
(0.0434) \\
\end{array}$ & $\begin{array}{l}0.28005^{\ddagger} \\
(0.04027)\end{array}$ \\
\hline All I & $\begin{array}{l}0.18684^{*} \\
(0.03443)\end{array}$ & $\begin{array}{l}0.1559^{*} \\
(0.03887)\end{array}$ & $\begin{array}{l}0.15405^{\ddagger} \\
(0.03855)\end{array}$ & $\begin{array}{l}0.14637^{\ddagger} \\
(0.03911) \\
\end{array}$ & $\begin{array}{l}0.21733^{\ddagger} \\
(0.03925) \\
\end{array}$ & $\begin{array}{l}0.19547^{\ddagger} \\
(0.03061)\end{array}$ & $\begin{array}{l}0.18677^{\ddagger} \\
(0.02963)\end{array}$ & $\begin{array}{l}0.19568^{\ddagger} \\
(0.03152)\end{array}$ & $\begin{array}{l}0.1932^{\ddagger} \\
(0.03198)\end{array}$ & $\begin{array}{l}0.19264^{\ddagger} \\
(0.03283)\end{array}$ & $\begin{array}{l}0.18665^{\ddagger} \\
(0.0308) \\
\end{array}$ & $\begin{array}{l}0.19459^{\ddagger} \\
(0.03629)\end{array}$ & $\begin{array}{l}0.17528^{\ddagger} \\
(0.0327) \\
\end{array}$ \\
\hline $\begin{array}{l}\text { Individual } \\
\text { Variable }\end{array}$ & $\begin{array}{l}-0.01098 \\
(0.02179)\end{array}$ & $\begin{array}{l}0.02297 \\
(0.02666)\end{array}$ & $\begin{array}{l}0.0249 \\
(0.02614)\end{array}$ & $\begin{array}{l}0.03418 \\
(0.02809)\end{array}$ & $\begin{array}{l}-0.04141 \\
(0.02734)\end{array}$ & $\begin{array}{c}-0.04038^{\dagger} \\
(0.02049)\end{array}$ & $\begin{array}{l}-0.04569^{\ddagger} \\
(0.02333)\end{array}$ & $\begin{array}{l}-0.03367 \\
(0.02181)\end{array}$ & $\begin{array}{l}-0.02509 \\
(0.02068)\end{array}$ & $\begin{array}{l}-0.02154 \\
(0.02129)\end{array}$ & $\begin{array}{l}-0.02315 \\
(0.02424)\end{array}$ & $\begin{array}{l}-0.01724 \\
(0.02187)\end{array}$ & $\begin{array}{l}0.00349 \\
(0.01964)\end{array}$ \\
\hline
\end{tabular}

* Significant at the 0.01 level $\dagger$ Significant at the 0.05 level $\$$ Significant at the 0.10 level

\section{Table 105 Coefficients with individual variables for high-level factors - Wood Industry}




\begin{tabular}{|c|c|c|c|c|c|c|c|c|c|c|}
\hline $\begin{array}{l}\text { Individual variable } \\
\text { included in the model in } \\
\text { the column }\end{array}$ & 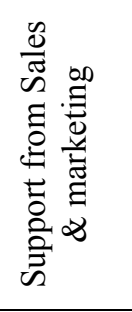 & 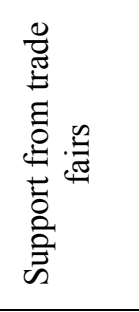 & 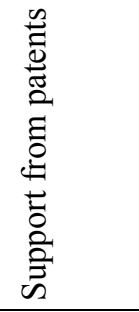 & 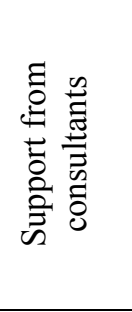 & 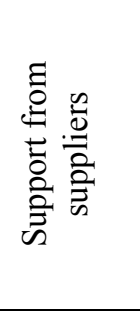 & 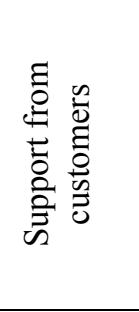 & 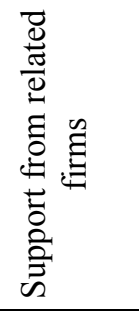 & 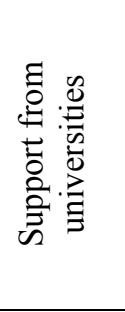 & 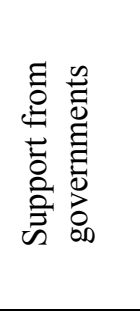 & 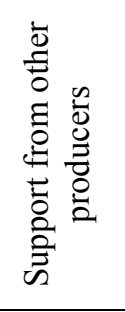 \\
\hline No factors & $\begin{array}{l}0.2655^{\ddagger} \\
(0.01831)\end{array}$ & $\begin{array}{l}0.26012^{\ddagger} \\
(0.01824)\end{array}$ & $\begin{array}{l}0.2626^{*} \\
(0.01851)\end{array}$ & $\begin{array}{l}0.26003^{*} \\
(0.01822) \\
\end{array}$ & $\begin{array}{l}0.26201^{*} \\
(0.01813)\end{array}$ & $\begin{array}{l}0.25927^{*} \\
(0.01851)\end{array}$ & $\begin{array}{l}0.26233^{*} \\
(0.01822)\end{array}$ & $\begin{array}{l}0.26031^{*} \\
(0.01826)\end{array}$ & $\begin{array}{l}0.25989^{*} \\
(0.01821)\end{array}$ & $\begin{array}{l}0.25951^{*} \\
(0.01812)\end{array}$ \\
\hline Factor 5 & $\begin{array}{l}0.27928^{\ddagger} \\
(0.09417) \\
\end{array}$ & $\begin{array}{l}0.25426^{\ddagger} \\
(0.09396)\end{array}$ & $\begin{array}{l}0.2695^{*} \\
(0.09632)\end{array}$ & $\begin{array}{l}0.2526^{*} \\
(0.09375) \\
\end{array}$ & $\begin{array}{l}0.26726^{*} \\
(0.0934)\end{array}$ & $\begin{array}{l}0.24805^{*} \\
(0.09539)\end{array}$ & $\begin{array}{l}0.27219^{*} \\
(0.09418)\end{array}$ & $\begin{array}{l}0.25455^{*} \\
(0.09425)\end{array}$ & $\begin{array}{l}0.25146^{*} \\
(0.09371)\end{array}$ & $\begin{array}{l}0.2508^{*} \\
(0.09324) \\
\end{array}$ \\
\hline Factor 4 & $\begin{array}{l}0.1641^{\ddagger} \\
(0.04402)\end{array}$ & $\begin{array}{l}0.13974^{\ddagger} \\
(0.04277)\end{array}$ & $\begin{array}{l}0.1445^{*} \\
(0.04319)\end{array}$ & $\begin{array}{l}0.13974^{*} \\
(0.04272)\end{array}$ & $\begin{array}{l}0.14797^{*} \\
(0.04261)\end{array}$ & $\begin{array}{l}0.13709^{*} \\
(0.0439)\end{array}$ & $\begin{array}{l}0.1699^{*} \\
(0.04593)\end{array}$ & $\begin{array}{l}0.14103^{*} \\
(0.04314)\end{array}$ & $\begin{array}{l}0.1383^{*} \\
(0.04272)\end{array}$ & $\begin{array}{l}0.13936^{*} \\
(0.04248)\end{array}$ \\
\hline Factor $4 \& 5$ & $\begin{array}{l}0.33784^{\ddagger} \\
(0.19509)\end{array}$ & $\begin{array}{l}0.33196^{\ddagger} \\
(0.19616)\end{array}$ & $\begin{array}{l}0.34714^{\ddagger} \\
(0.19703)\end{array}$ & $\begin{array}{l}0.33196^{\ddagger} \\
(0.19591)\end{array}$ & $\begin{array}{l}0.36242^{\ddagger} \\
(0.19519)\end{array}$ & $\begin{array}{l}0.32963^{\ddagger} \\
(0.19638)\end{array}$ & $\begin{array}{l}0.33686^{\ddagger} \\
(0.19546)\end{array}$ & $\begin{array}{l}0.33196^{\ddagger} \\
(0.19619) \\
\end{array}$ & $\begin{array}{l}0.32661^{\ddagger} \\
(0.19587)\end{array}$ & $\begin{array}{l}0.28769 \\
(0.19573) \\
\end{array}$ \\
\hline Factor 3 & $\begin{array}{l}0.15681 \\
(0.10633) \\
\end{array}$ & $\begin{array}{l}0.12222 \\
(0.10569) \\
\end{array}$ & $\begin{array}{l}0.13968 \\
(0.10801) \\
\end{array}$ & $\begin{array}{l}0.12086 \\
(0.10556) \\
\end{array}$ & $\begin{array}{l}0.16342 \\
(0.10633) \\
\end{array}$ & $\begin{array}{l}0.11643 \\
(0.10787) \\
\end{array}$ & $\begin{array}{l}0.15244 \\
(0.10669) \\
\end{array}$ & $\begin{array}{l}0.12604 \\
(0.10697) \\
\end{array}$ & $\begin{array}{l}0.11932 \\
(0.10554) \\
\end{array}$ & $\begin{array}{l}0.10618 \\
(0.10519) \\
\end{array}$ \\
\hline Factor $3 \& 5$ & $\begin{array}{l}0.18732^{\ddagger} \\
(0.05221)\end{array}$ & $\begin{array}{l}0.16132^{\ddagger} \\
(0.05107)\end{array}$ & $\begin{array}{l}0.17878^{*} \\
(0.0558)\end{array}$ & $\begin{array}{l}0.161^{*} \\
(0.05101)\end{array}$ & $\begin{array}{l}0.20419^{*} \\
(0.05374)\end{array}$ & $\begin{array}{l}0.15553^{*} \\
(0.05543)\end{array}$ & $\begin{array}{l}0.1987^{*} \\
(0.05514)\end{array}$ & $\begin{array}{l}0.16141^{*} \\
(0.05108)\end{array}$ & $\begin{array}{l}0.16064^{*} \\
(0.05098)\end{array}$ & $\begin{array}{l}0.15075^{*} \\
(0.05092)\end{array}$ \\
\hline Factor $3 \& 4$ & $\begin{array}{l}0.1899^{\ddagger} \\
(0.10223) \\
\end{array}$ & $\begin{array}{l}0.15291 \\
(0.10133) \\
\end{array}$ & $\begin{array}{l}0.17037 \\
(0.10376) \\
\end{array}$ & $\begin{array}{l}0.15291 \\
(0.1012) \\
\end{array}$ & $\begin{array}{l}0.17207^{\ddagger} \\
(0.10093) \\
\end{array}$ & $\begin{array}{l}0.14712 \\
(0.1036) \\
\end{array}$ & $\begin{array}{l}0.18371^{\ddagger} \\
(0.10246)\end{array}$ & $\begin{array}{l}0.15391 \\
(0.10144) \\
\end{array}$ & $\begin{array}{l}0.1462 \\
(0.1013) \\
\end{array}$ & $\begin{array}{l}0.13036 \\
(0.10109) \\
\end{array}$ \\
\hline Factor $3,4 \& 5$ & \multicolumn{10}{|c|}{ Insufficient Data } \\
\hline Factor 2 & $\begin{array}{l}0.17126^{\ddagger} \\
(0.03565) \\
\end{array}$ & $\begin{array}{l}0.16041^{\ddagger} \\
(0.03548) \\
\end{array}$ & $\begin{array}{l}0.16574^{*} \\
(0.03618) \\
\end{array}$ & $\begin{array}{l}0.16003^{*} \\
(0.03543) \\
\end{array}$ & $\begin{array}{l}0.17596^{*} \\
(0.03583) \\
\end{array}$ & $\begin{array}{l}0.15834^{*} \\
(0.03614) \\
\end{array}$ & $\begin{array}{l}0.16761^{*} \\
(0.0356) \\
\end{array}$ & $\begin{array}{l}0.16121^{*} \\
(0.03575) \\
\end{array}$ & $\begin{array}{l}0.16002^{*} \\
(0.03541) \\
\end{array}$ & $\begin{array}{l}0.15992^{*} \\
(0.03523) \\
\end{array}$ \\
\hline Factor $2 \& 5$ & $\begin{array}{l}0.26278 \\
(0.17749) \\
\end{array}$ & $\begin{array}{l}0.22754 \\
(0.17772) \\
\end{array}$ & $\begin{array}{l}0.245 \\
(0.17905) \\
\end{array}$ & $\begin{array}{l}0.22754 \\
(0.1775) \\
\end{array}$ & $\begin{array}{l}0.27301 \\
(0.17747) \\
\end{array}$ & $\begin{array}{l}0.22551 \\
(0.1779) \\
\end{array}$ & $\begin{array}{l}0.23666 \\
(0.17715) \\
\end{array}$ & $\begin{array}{l}0.23023 \\
(0.17812) \\
\end{array}$ & $\begin{array}{l}0.2028 \\
(0.17854) \\
\end{array}$ & $\begin{array}{l}0.22754 \\
(0.17652) \\
\end{array}$ \\
\hline Facto & $\begin{array}{l}0.15176^{\ddagger} \\
(0.07922) \\
\end{array}$ & $\begin{array}{l}0.14368^{\ddagger} \\
(0.07957)\end{array}$ & $\begin{array}{l}0.14405^{\ddagger} \\
(0.07953) \\
\end{array}$ & $\begin{array}{l}0.14368^{\ddagger} \\
(0.07947) \\
\end{array}$ & $\begin{array}{l}0.14871^{\ddagger} \\
(0.07904)\end{array}$ & $\begin{array}{l}0.14078^{\ddagger} \\
(0.0803) \\
\end{array}$ & $\begin{array}{l}0.14961^{\ddagger} \\
(0.07935) \\
\end{array}$ & $\begin{array}{l}0.14419^{\ddagger} \\
(0.07961)\end{array}$ & $\begin{array}{l}0.14203^{\ddagger} \\
(0.07944) \\
\end{array}$ & $\begin{array}{l}0.13416^{\ddagger} \\
(0.07913) \\
\end{array}$ \\
\hline Factor $2,4 \& 5$ & $\begin{array}{l}0.06026 \\
(0.09528)\end{array}$ & $\begin{array}{l}0.06026 \\
(0.09582)\end{array}$ & $\begin{array}{l}0.06798 \\
(0.09628)\end{array}$ & $\begin{array}{l}0.06026 \\
(0.0957)\end{array}$ & $\begin{array}{l}0.11121 \\
(0.09746)\end{array}$ & $\begin{array}{l}0.05447 \\
(0.09822)\end{array}$ & $\begin{array}{l}0.08111 \\
(0.0962)\end{array}$ & $\begin{array}{l}0.06318 \\
(0.09665)\end{array}$ & $\begin{array}{l}0.05907 \\
(0.09566)\end{array}$ & $\begin{array}{l}0.03749 \\
(0.09565)\end{array}$ \\
\hline Factor $2 \& 3$ & $\begin{array}{l}0.29972^{\dagger} \\
(0.13594) \\
\end{array}$ & $\begin{array}{l}0.25783^{\ddagger} \\
(0.13529) \\
\end{array}$ & $\begin{array}{l}0.27529^{\dagger} \\
(0.13708)\end{array}$ & $\begin{array}{l}0.2556^{\ddagger} \\
(0.13514) \\
\end{array}$ & $\begin{array}{l}0.30878^{\dagger} \\
(0.13599) \\
\end{array}$ & $\begin{array}{l}0.25203^{\ddagger} \\
(0.13701) \\
\end{array}$ & $\begin{array}{l}0.2952^{\dagger} \\
(0.13646) \\
\end{array}$ & $\begin{array}{l}0.26153^{\ddagger} \\
(0.13624) \\
\end{array}$ & $\begin{array}{l}0.2507^{\ddagger} \\
(0.13518) \\
\end{array}$ & $\begin{array}{l}0.24662^{\ddagger} \\
(0.13446) \\
\end{array}$ \\
\hline Factor $2,3 \& 5$ & $\begin{array}{l}0.30223^{\ddagger} \\
(0.16118) \\
\end{array}$ & $\begin{array}{l}0.26528 \\
(0.16116) \\
\end{array}$ & $\begin{array}{l}0.28274^{\ddagger} \\
(0.16264) \\
\end{array}$ & $\begin{array}{l}0.26528 \\
(0.16095) \\
\end{array}$ & $\begin{array}{l}0.289^{\ddagger} \\
(0.16032)\end{array}$ & $\begin{array}{l}0.25948 \\
(0.16261) \\
\end{array}$ & $\begin{array}{l}0.29605^{\ddagger} \\
(0.16151)\end{array}$ & $\begin{array}{l}0.26924^{\ddagger} \\
(0.16207) \\
\end{array}$ & $\begin{array}{l}0.25854 \\
(0.16097) \\
\end{array}$ & $\begin{array}{l}0.22263 \\
(0.16107) \\
\end{array}$ \\
\hline Factor $2,3 \& 4$ & $\begin{array}{l}0.34213^{\ddagger} \\
(0.08355)\end{array}$ & $\begin{array}{l}0.31327^{\ddagger} \\
(0.08289)\end{array}$ & $\begin{array}{l}0.32941^{*} \\
(0.0858)\end{array}$ & $\begin{array}{l}0.29595^{*} \\
(0.08403)\end{array}$ & $\begin{array}{l}0.3629^{*} \\
(0.08491)\end{array}$ & $\begin{array}{l}0.30858^{*} \\
(0.08378) \\
\end{array}$ & $\begin{array}{l}0.33375^{*} \\
(0.08344) \\
\end{array}$ & $\begin{array}{l}0.31346^{*} \\
(0.08309)\end{array}$ & $\begin{array}{l}0.30563^{*} \\
(0.08284)\end{array}$ & $\begin{array}{l}0.30203^{*} \\
(0.08237) \\
\end{array}$ \\
\hline Factor $2,3,4 \& 5$ & $\begin{array}{l}0.30632^{\dagger} \\
(0.1207)\end{array}$ & $\begin{array}{l}0.2753^{\dagger} \\
(0.12053)\end{array}$ & $\begin{array}{l}0.29003^{\dagger} \\
(0.12238)\end{array}$ & $\begin{array}{l}0.26192^{\dagger} \\
(0.1206)\end{array}$ & $\begin{array}{l}0.31088^{\dagger} \\
(0.12055)\end{array}$ & $\begin{array}{l}0.26822^{\dagger} \\
(0.12144)\end{array}$ & $\begin{array}{l}0.28751^{\dagger} \\
(0.1202)\end{array}$ & $\begin{array}{l}0.27384^{\dagger} \\
(0.12049)\end{array}$ & $\begin{array}{l}0.25524^{\dagger} \\
(0.12095)\end{array}$ & $\begin{array}{l}0.23043^{\ddagger} \\
(0.12085)\end{array}$ \\
\hline Factor 1 & $\begin{array}{l}0.22707^{\ddagger} \\
(0.03871)\end{array}$ & $\begin{array}{l}0.21862^{\ddagger} \\
(0.03872)\end{array}$ & $\begin{array}{l}0.22847^{*} \\
(0.04094)\end{array}$ & $\begin{array}{l}0.21789^{*} \\
(0.03865)\end{array}$ & $\begin{array}{l}0.22461^{*} \\
(0.03852)\end{array}$ & $\begin{array}{l}0.21432^{*} \\
(0.04116)\end{array}$ & $\begin{array}{l}0.22591^{*} \\
(0.03881)\end{array}$ & $\begin{array}{l}0.21872^{*} \\
(0.0388)\end{array}$ & $\begin{array}{l}0.21788^{*} \\
(0.03863)\end{array}$ & $\begin{array}{l}0.21281^{*} \\
(0.0385)\end{array}$ \\
\hline Factor $1 \& 5$ & $\begin{array}{l}0.33189^{\ddagger} \\
(0.03767) \\
\end{array}$ & $\begin{array}{l}0.31703^{\ddagger} \\
(0.03721)\end{array}$ & $\begin{array}{l}0.33347^{*} \\
(0.04317)\end{array}$ & $\begin{array}{l}0.30865^{*} \\
(0.03784) \\
\end{array}$ & $\begin{array}{l}0.31951^{*} \\
(0.03694)\end{array}$ & $\begin{array}{l}0.31094^{*} \\
(0.04249)\end{array}$ & $\begin{array}{l}0.32793^{*} \\
(0.03762)\end{array}$ & $\begin{array}{l}0.31825^{*} \\
(0.03796)\end{array}$ & $\begin{array}{l}0.31607^{*} \\
(0.03712)\end{array}$ & $\begin{array}{l}0.31583^{*} \\
(0.03693) \\
\end{array}$ \\
\hline Factor $1 \& 4$ & $\begin{array}{l}0.15319^{\ddagger} \\
(0.07835)\end{array}$ & $\begin{array}{l}0.15247^{\ddagger} \\
(0.07918)\end{array}$ & $\begin{array}{l}0.16182^{\dagger} \\
(0.0804)\end{array}$ & $\begin{array}{l}0.14919^{\ddagger} \\
(0.07867) \\
\end{array}$ & $\begin{array}{l}0.19376^{\dagger} \\
(0.08037) \\
\end{array}$ & $\begin{array}{l}0.14502^{\ddagger} \\
(0.08029)\end{array}$ & $\begin{array}{l}0.14998^{\ddagger} \\
(0.07848)\end{array}$ & $\begin{array}{l}0.15036^{\ddagger} \\
(0.07894)\end{array}$ & $\begin{array}{l}0.1468^{\ddagger} \\
(0.07866)\end{array}$ & $\begin{array}{l}0.14281^{\ddagger} \\
(0.07828)\end{array}$ \\
\hline Factor $1,4 \& 5$ & $\begin{array}{l}0.11091 \\
(0.12262) \\
\end{array}$ & $\begin{array}{l}0.09677 \\
(0.12313) \\
\end{array}$ & $\begin{array}{l}0.11174 \\
(0.12458) \\
\end{array}$ & $\begin{array}{l}0.09677 \\
(0.12298) \\
\end{array}$ & $\begin{array}{l}0.1315 \\
(0.12311) \\
\end{array}$ & $\begin{array}{l}0.09129 \\
(0.12481) \\
\end{array}$ & $\begin{array}{l}0.09677 \\
(0.12268) \\
\end{array}$ & $\begin{array}{l}0.10016 \\
(0.12401) \\
\end{array}$ & $\begin{array}{l}0.0948 \\
(0.12293) \\
\end{array}$ & $\begin{array}{l}0.07809 \\
(0.12255) \\
\end{array}$ \\
\hline Factor $1 \& 3$ & $\begin{array}{l}0.36157^{\ddagger} \\
(0.03409) \\
\end{array}$ & $\begin{array}{l}0.33068^{\ddagger} \\
(0.03109)\end{array}$ & $\begin{array}{l}0.34779^{*} \\
(0.03813)\end{array}$ & $\begin{array}{l}0.32132^{*} \\
(0.03223) \\
\end{array}$ & $\begin{array}{l}0.37265^{*} \\
(0.03544)\end{array}$ & $\begin{array}{l}0.32613^{*} \\
(0.03539)\end{array}$ & $\begin{array}{l}0.3542^{*} \\
(0.03374)\end{array}$ & $\begin{array}{l}0.33182^{*} \\
(0.03148)\end{array}$ & $\begin{array}{l}0.32227^{*} \\
(0.03177)\end{array}$ & $\begin{array}{l}0.30493^{*} \\
(0.03274) \\
\end{array}$ \\
\hline Factor $1,3 \& 5$ & $\begin{array}{l}0.3264^{\ddagger} \\
(0.043) \\
\end{array}$ & $\begin{array}{l}0.29332^{\ddagger} \\
(0.04038)\end{array}$ & $\begin{array}{l}0.31078^{*} \\
(0.04623)\end{array}$ & $\begin{array}{l}0.2841^{*} \\
(0.04122) \\
\end{array}$ & $\begin{array}{l}0.30499^{*} \\
(0.04038)\end{array}$ & $\begin{array}{l}0.2905^{*} \\
(0.04172)\end{array}$ & $\begin{array}{l}0.31714^{*} \\
(0.04244)\end{array}$ & $\begin{array}{l}0.29425^{*} \\
(0.04058)\end{array}$ & $\begin{array}{l}0.29183^{*} \\
(0.04032)\end{array}$ & $\begin{array}{l}0.29131^{*} \\
(0.04011)\end{array}$ \\
\hline Factor $1,3 \& 4$ & $\begin{array}{l}0.3262^{\ddagger} \\
(0.03523) \\
\end{array}$ & $\begin{array}{l}0.28195^{\ddagger} \\
(0.02874)\end{array}$ & $\begin{array}{l}0.29713^{*} \\
(0.03478) \\
\end{array}$ & $\begin{array}{l}0.28144^{*} \\
(0.02871)\end{array}$ & $\begin{array}{l}0.30957^{*} \\
(0.03075) \\
\end{array}$ & $\begin{array}{l}0.2772^{*} \\
(0.03372) \\
\end{array}$ & $\begin{array}{l}0.30594^{*} \\
(0.03172) \\
\end{array}$ & $\begin{array}{l}0.28403^{*} \\
(0.0301)\end{array}$ & $\begin{array}{l}0.28129^{*} \\
(0.0287) \\
\end{array}$ & $\begin{array}{l}0.26958^{*} \\
(0.02902) \\
\end{array}$ \\
\hline Factor $1,3,4 \& 5$ & $\begin{array}{l}0.2966^{\ddagger} \\
(0.05269)\end{array}$ & $\begin{array}{l}0.2525^{\ddagger} \\
(0.0488) \\
\end{array}$ & $\begin{array}{l}0.26995^{*} \\
(0.05374)\end{array}$ & $\begin{array}{l}0.24408^{*} \\
(0.04936)\end{array}$ & $\begin{array}{l}0.28177^{*} \\
(0.04995)\end{array}$ & $\begin{array}{l}0.2467^{*} \\
(0.05334)\end{array}$ & $\begin{array}{l}0.28941^{*} \\
(0.05295)\end{array}$ & $\begin{array}{l}0.25656^{*} \\
(0.05183)\end{array}$ & $\begin{array}{l}0.24994^{*} \\
(0.04876)\end{array}$ & $\begin{array}{l}0.24775^{*} \\
(0.04851) \\
\end{array}$ \\
\hline Factor $1 \& 2$ & $\begin{array}{l}0.22206^{\ddagger} \\
(0.03563)\end{array}$ & $\begin{array}{l}0.18869^{\ddagger} \\
(0.03225)\end{array}$ & $\begin{array}{l}0.19317^{*} \\
(0.03275)\end{array}$ & $\begin{array}{l}0.18856^{*} \\
(0.03221)\end{array}$ & $\begin{array}{l}0.20154^{*} \\
(0.03246)\end{array}$ & $\begin{array}{l}0.1858^{*} \\
(0.03399)\end{array}$ & $\begin{array}{l}0.2084^{*} \\
(0.03403)\end{array}$ & $\begin{array}{l}0.1888^{*} \\
(0.03226)\end{array}$ & $\begin{array}{l}0.18855^{*} \\
(0.03219)\end{array}$ & $\begin{array}{l}0.18763^{*} \\
(0.03203)\end{array}$ \\
\hline Factor $1,2 \& 5$ & $\begin{array}{l}0.32411^{\ddagger} \\
(0.08774) \\
\end{array}$ & $\begin{array}{l}0.29555^{\ddagger} \\
(0.08721)\end{array}$ & $\begin{array}{l}0.31173^{*} \\
(0.08964)\end{array}$ & $\begin{array}{l}0.29555^{*} \\
(0.0871) \\
\end{array}$ & $\begin{array}{l}0.30128^{*} \\
(0.08663) \\
\end{array}$ & $\begin{array}{l}0.28991^{*} \\
(0.08971)\end{array}$ & $\begin{array}{l}0.3203^{*} \\
(0.08802)\end{array}$ & $\begin{array}{l}0.29701^{*} \\
(0.08745)\end{array}$ & $\begin{array}{l}0.29555^{*} \\
(0.08706)\end{array}$ & $\begin{array}{l}0.294^{*} \\
(0.08662)\end{array}$ \\
\hline Factor $1,2 \& 4$ & $\begin{array}{l}0.50767^{\ddagger} \\
(0.04082) \\
\end{array}$ & $\begin{array}{l}0.47613^{\ddagger} \\
(0.03831) \\
\end{array}$ & $\begin{array}{l}0.48623^{*} \\
(0.04045) \\
\end{array}$ & $\begin{array}{l}0.47613^{*} \\
(0.03826) \\
\end{array}$ & $\begin{array}{l}0.50636^{*} \\
(0.04005) \\
\end{array}$ & $\begin{array}{l}0.47057^{*} \\
(0.04353) \\
\end{array}$ & $\begin{array}{l}0.51124^{*} \\
(0.04307) \\
\end{array}$ & $\begin{array}{l}0.47761^{*} \\
(0.03883)\end{array}$ & $\begin{array}{l}0.4637^{*} \\
(0.03954) \\
\end{array}$ & $\begin{array}{l}0.45594^{*} \\
(0.03899) \\
\end{array}$ \\
\hline Factor $1,2,4 \& 5$ & $\begin{array}{l}0.32973^{\ddagger} \\
(0.05569)\end{array}$ & $\begin{array}{l}0.30778^{\ddagger} \\
(0.05505)\end{array}$ & $\begin{array}{l}0.32485^{*} \\
(0.05928)\end{array}$ & $\begin{array}{l}0.30778^{*} \\
(0.05498)\end{array}$ & $\begin{array}{l}0.30831^{*} \\
(0.05466)\end{array}$ & $\begin{array}{l}0.30199^{*} \\
(0.05912)\end{array}$ & $\begin{array}{l}0.30858^{*} \\
(0.05485)\end{array}$ & $\begin{array}{l}0.30788^{*} \\
(0.05506)\end{array}$ & $\begin{array}{l}0.30739^{*} \\
(0.05496)\end{array}$ & $\begin{array}{l}0.30716^{*} \\
(0.05468) \\
\end{array}$ \\
\hline Factor $1,2 \& 3$ & $\begin{array}{l}0.11259^{\dagger} \\
(0.04994) \\
\end{array}$ & $\begin{array}{l}0.09923^{\dagger} \\
(0.04983) \\
\end{array}$ & $\begin{array}{l}0.11627^{\dagger} \\
(0.05445) \\
\end{array}$ & $\begin{array}{l}0.07977 \\
(0.05293) \\
\end{array}$ & $\begin{array}{l}0.14635^{*} \\
(0.0532) \\
\end{array}$ & $\begin{array}{l}0.09344^{\ddagger} \\
(0.05429) \\
\end{array}$ & $\begin{array}{l}0.11067^{\dagger} \\
(0.05007) \\
\end{array}$ & $\begin{array}{l}0.10009^{\dagger} \\
(0.04997) \\
\end{array}$ & $\begin{array}{l}0.09856^{\dagger} \\
(0.04975) \\
\end{array}$ & $\begin{array}{l}0.09567^{\ddagger} \\
(0.04952) \\
\end{array}$ \\
\hline Factor $1,2,3 \& 5$ & $\begin{array}{l}0.36183^{\ddagger} \\
(0.04894)\end{array}$ & $\begin{array}{l}0.31983^{\ddagger} \\
(0.04513)\end{array}$ & $\begin{array}{l}0.33155^{*} \\
(0.04757)\end{array}$ & $\begin{array}{l}0.31957^{*} \\
(0.04507)\end{array}$ & $\begin{array}{l}0.34931^{*} \\
(0.04644)\end{array}$ & $\begin{array}{l}0.31663^{*} \\
(0.04667)\end{array}$ & $\begin{array}{l}0.35111^{*} \\
(0.04835)\end{array}$ & $\begin{array}{l}0.32222^{*} \\
(0.04628)\end{array}$ & $\begin{array}{l}0.31049^{*} \\
(0.04568)\end{array}$ & $\begin{array}{l}0.28785^{*} \\
(0.04681)\end{array}$ \\
\hline Factor $1,2,3 \& 4$ & $\begin{array}{l}0.32096^{\ddagger} \\
(0.04201) \\
\end{array}$ & $\begin{array}{l}0.28416^{\ddagger} \\
(0.03845) \\
\end{array}$ & $\begin{array}{l}0.29378^{*} \\
(0.04093) \\
\end{array}$ & $\begin{array}{l}0.27566^{*} \\
(0.03863) \\
\end{array}$ & $\begin{array}{l}0.31158^{*} \\
(0.03981) \\
\end{array}$ & $\begin{array}{l}0.2785^{*} \\
(0.04073) \\
\end{array}$ & $\begin{array}{l}0.30298^{*} \\
(0.03981) \\
\end{array}$ & $\begin{array}{l}0.28443^{*} \\
(0.03925) \\
\end{array}$ & $\begin{array}{l}0.26999^{*} \\
(0.0394) \\
\end{array}$ & $\begin{array}{l}0.26088^{*} \\
(0.03899) \\
\end{array}$ \\
\hline All Factors & $\begin{array}{l}0.21677^{\ddagger} \\
(0.0344) \\
\end{array}$ & $\begin{array}{l}0.17782^{\ddagger} \\
(0.02941) \\
\end{array}$ & $\begin{array}{l}0.18913^{*} \\
(0.03283) \\
\end{array}$ & $\begin{array}{l}0.17481^{*} \\
(0.0295) \\
\end{array}$ & $\begin{array}{l}0.2163^{*} \\
(0.03328) \\
\end{array}$ & $\begin{array}{l}0.17248^{*} \\
(0.03547) \\
\end{array}$ & $\begin{array}{l}0.2124^{*} \\
(0.03528) \\
\end{array}$ & $\begin{array}{l}0.1815^{*} \\
(0.03338) \\
\end{array}$ & $\begin{array}{l}0.16738^{*} \\
(0.03055) \\
\end{array}$ & $\begin{array}{l}0.16195^{*} \\
(0.02997) \\
\end{array}$ \\
\hline $\begin{array}{l}\text { Individual } \\
\text { Variable }\end{array}$ & $\begin{array}{l}-0.04489^{\dagger} \\
(0.02089)\end{array}$ & $\begin{array}{l}-0.05241 \\
(0.12809)\end{array}$ & $\begin{array}{l}-0.01746 \\
(0.02256)\end{array}$ & $\begin{array}{l}0.03583 \\
(0.03316)\end{array}$ & $\begin{array}{c}-0.05095^{\dagger} \\
(0.02113)\end{array}$ & $\begin{array}{l}0.00579 \\
(0.02153)\end{array}$ & $\begin{array}{l}-0.03738^{\ddagger} \\
(0.02124)\end{array}$ & $\begin{array}{l}-0.00496 \\
(0.02129)\end{array}$ & $\begin{array}{l}0.03814 \\
(0.03083)\end{array}$ & $\begin{array}{l}0.05998^{\dagger} \\
(0.02536)\end{array}$ \\
\hline
\end{tabular}

* Significant at the 0.01 level + Significant at the 0.05 level Significant at the 0.10 level

\section{Table 105 Coefficients with individual variables for high-level factors - Wood Industry}




\subsection{Solution Lattice}

A sample set of solution lattices has been created for the medium plant size and the vehicles industry. These two were chosen since they have large sample sizes and few empty states. The analysis of the lattice is dependent on having values for each node of the structure (the nodes represent the various factor states). The lattices are represented as scatter-plot diagrams as it is impossible to present the four and five dimensions in any other format. From the diagrams, the optimal plant states can be observed, and analysis about the importance of the complementarities can be performed.

The analysis focuses on the top tier and bottom tier states and not specific states since many of the top tier and bottom tier states contain small percentages of the population. Since these states are not highly represented, it is impossible to know if the plant surveyed had an exceptionally good (or poor) year in terms of profitability. This is an additional limitation due to the sample sizes of the industries and size classes and the static nature of the survey (profit values are for 1998 only). The profit margin displayed as the dependent variable (y-axis) in the graphs is manufacturing value added per shipment dollar. Therefore, it represents the profit margin as a percentage in decimal form. The independent variable (x-axis) is the state of the plant. The positioning of the plant state on the x-axis is determined by an algorithm that adds or subtracts from the initial base position based on the factors included in the state. For example in the AMT analysis where there are four factors, if the state includes factor 4 then 1 is added to the base state (state 0 ) $\mathrm{x}$ value. If the state includes factor 3 then 1 is subtracted from the $\mathrm{x}$ value. If the state includes factor 2 then 2 is added to the $\mathrm{x}$ value and if the state includes factor 1 then 2 is subtracted from the $\mathrm{x}$ value. A similar algorithm is used for analysis with three or five factors. 
Each figure includes an example path that a manager might traverse in order to move from the worst state to the optimal state. This path demonstrates the optimal one-step movements only. It is possible for a manager to move states by changing more than one of their factor attributes simultaneously if they had no other business constraints affecting their decision. In that case, a different path movement through the lattice would be optimal. A plant manager should always attempt to move as quickly as possible from their current state in the lattice to the optimal state. The speed at which a plant can traverse the lattice will depend on the financial constraints as well as the human resources and other business issues constraining the plant at the time of the change process.

\subsubsection{Medium Plants}

There are no empty states in the medium plant analysis therefore all of the complementarities and substitutes identified in the previous analysis should be apparent in the lattice plots. There are instances, however, that the complementarity or substitute pairing does not appear to be a complement or substitute from the zero state but is from many of the other possible starting states. This is due to the statistical significance level used in the regression analysis and the size of the difference between the profit measures in the various states. It is possible for the constraints to be met because one of the state coefficients is very small or very large so that the factors may not appear to be complementary in the lattice plots.

The plot of the lattice of the AMT implemented by medium plants supports both the complementary pairing of communications and robotics technologies (factors 2 and 4) and the substitute pairing of production design and robotics technologies (factors 1 and 4) (refer to Figure 4). Considering the upper tier of states as those with an average profit margin greater than $30 \%$, then these states represent $17.5 \%$ of medium plants. The majority of medium plants are 
found in states with average profit margins between $22 \%$ and $28 \%$. The state consisting of only the substitute pairing of production design and robotics technologies (state 1001) performs on average much worse, with an average profit margin of only $12 \%$. The plot of the lattice for the AMT in medium plants demonstrates that the optimal set of AMT would be communications, inspection and storage, and robotics technologies but not many production design technologies (state 0111 ) so as not to exhibit the first factor.

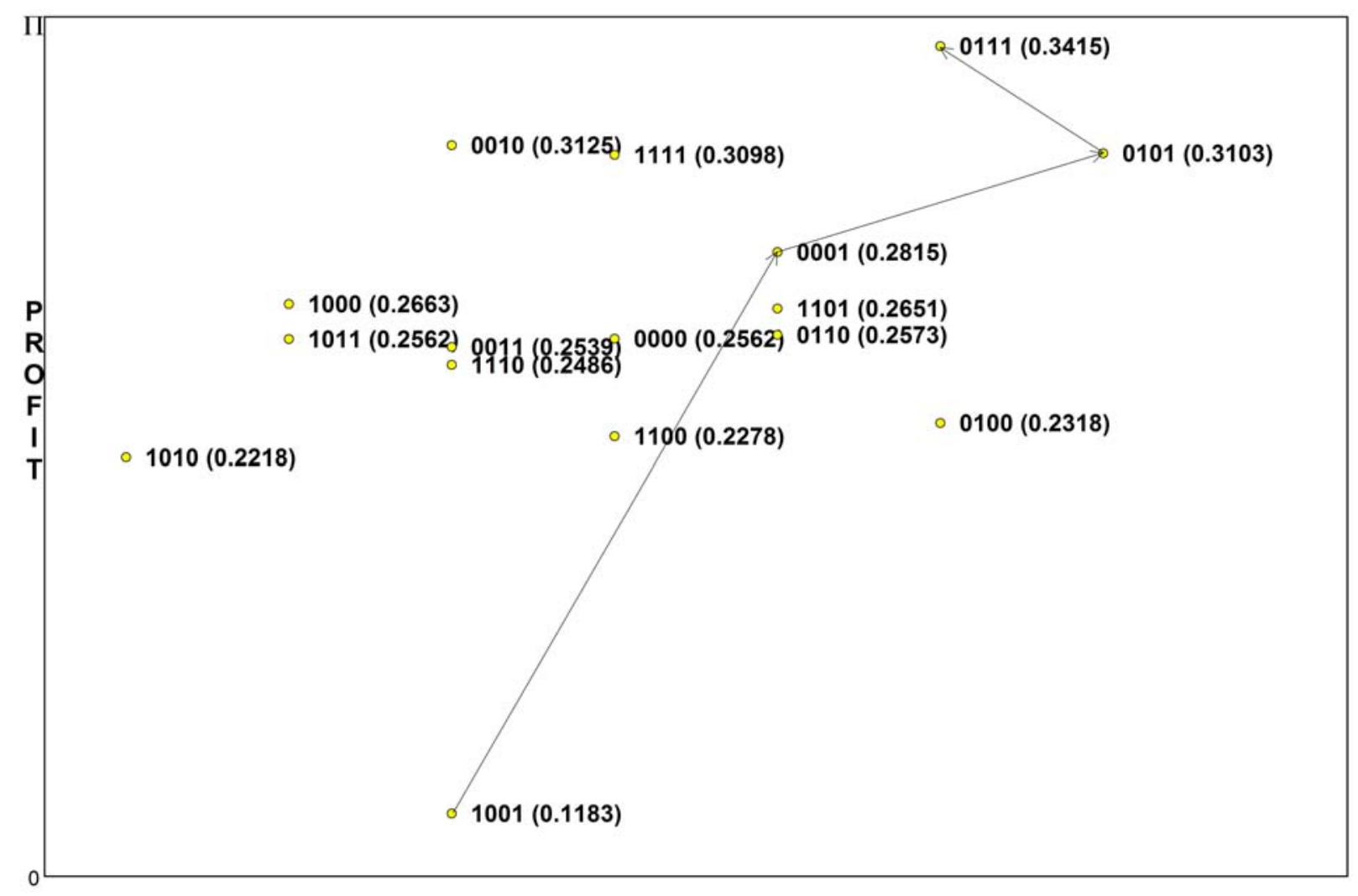

Figure 4 Plot of lattice for AMT - Medium Plants ${ }^{10}$

${ }^{10}$ The example path in this case begins at the worst state (state $\left.9-1001\right)$. It then stops having the substitute pairing by eliminating the excess production design technologies, moving to state $1(0001)$. The next node in the path is state 5 (0101) and then finally the optimal state (state $7-0111)$. If there were no financial or other business constraints on a plant then the plant could move directly from state 9 to state 7 . 
The plot of the lattice of business practices used in medium plants does not appear to support the conclusions from the constrained regression analysis (refer to Figure 5). The constrained regression analysis did have many inconclusive results but the substitute pairing of quality control and design (factor 1 and 3 ) is in the upper tier of plant states in the lattice plot. This contradiction may be because of the tight range in which all of the average profit margins fall and the level of significance chosen in the regression analysis. The top three states in the plot represent $29.53 \%$ of medium plants while the lower two states represent $41.46 \%$. The entire range of average profit margins is only $10.5 \%$, which is very small compared to the rest of the lattice plots. From this analysis the optimal set of business practices for medium plants is either to use only a large number of quality control practices and very few of the others or to use a combination of quality control and planning practices.

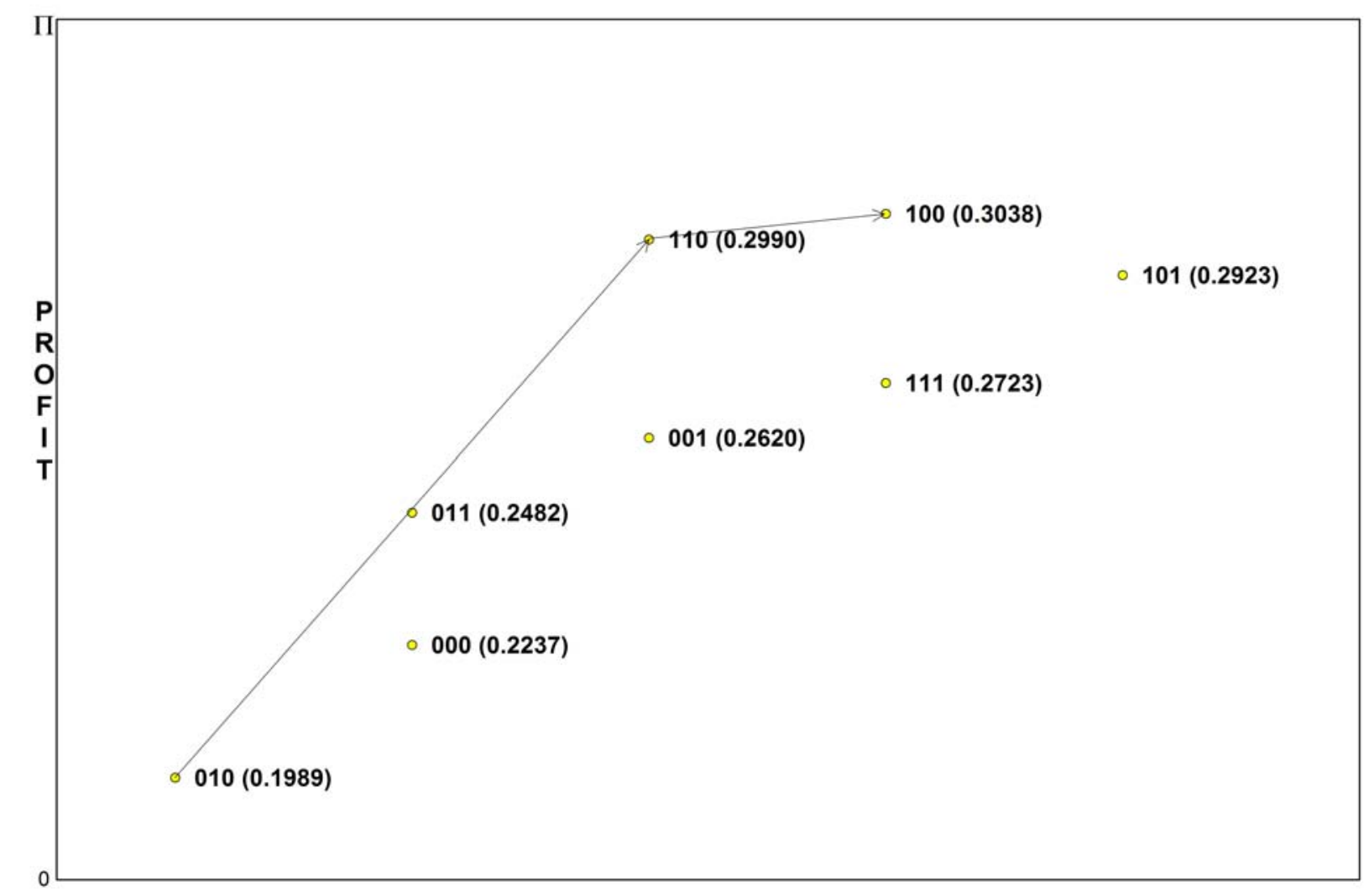

Figure 5 Plot of lattice of business practices - Medium Plants 
The plot of the lattice of the source of implementation support for AMT supports many of the results from the constrained regression analysis (refer to Figure 6). The complementary pairs of the primary stakeholders and the external information sources (factors 1 and 3), external information sources and corporate organizations (factors 3 and 4), and research and development and corporate organizations (factors 2 and 4) all have better average profit margins when compared to the initial zero state. The substitute pairing of the research and development group and external information sources (factors 2 and 3 ) is marginally supported as even though the state consisting of only these two factors (state 0110) performs slightly better that state zero; none of the top tier states (those with an average profit margin greater than $30 \%$ ) contains the two factors. The lower tier states (those with profit margins less than $20 \%$ ) represent $8.02 \%$ of medium plants, almost double the $4.25 \%$ represented by top tier states. In this case, plants in the optimal state of research and development and corporate organizations (state 0101) perform much better (almost three times better) than the worst state (state 0111). 


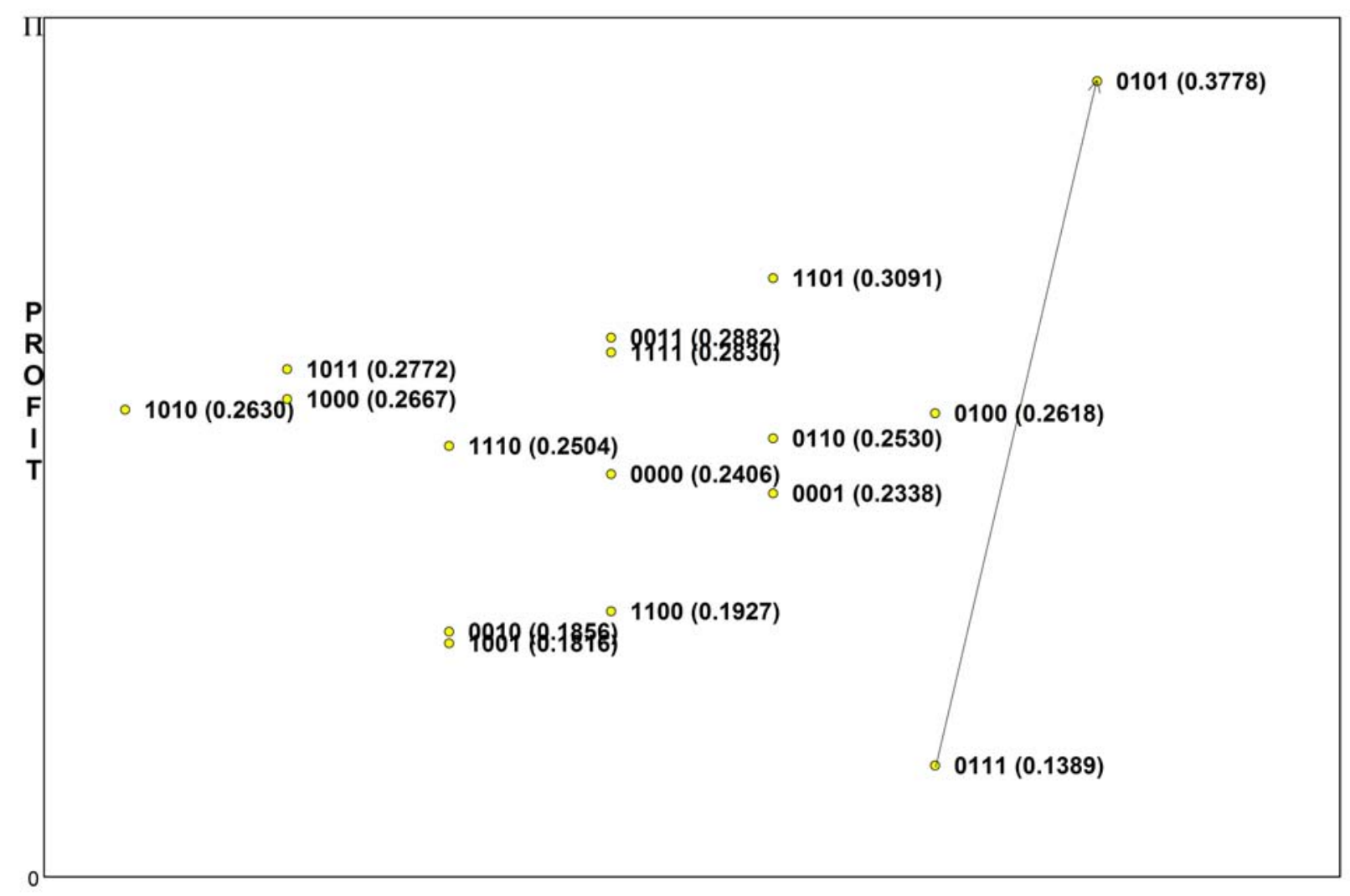

Figure 6 Plot of lattice of source of implementation support - Medium Plants

The plot of the lattice of the perceived benefits of AMT support the substitute pairing of the perceived benefits of increased productivity and increased flexibility (factors 2 and 3) found in the constrained regression analysis (refer to Figure 7). The states in this plot form four distinct clusters. The first cluster is the upper group consisting of states with average profit margins greater than $30 \%$ (states 1011 and 0100 ). These states represent just over 10\% of the medium plants. It is interesting to note that the states in the upper cluster are complete opposites of one another indicating the best alternative for medium plants is to either perceive the benefits of AMT as relating to increased productivity or as everything except increased productivity. The second cluster is of states with average profit margins between $26 \%$ and $30 \%$ (states 1010,1110 , $1111,1101,0111$, and 0101). The next cluster is states with average profit margins between $19 \%$ and $23 \%$ (states $0010,1001,0000,1100$, and 0001 ). The final cluster is states with average profit 
margins between $17 \%$ and $14 \%$ (states 1000,0011 , and 0110 ). This last cluster represents only $2.81 \%$ of medium plants demonstrating that the large majority is in the middle groupings of states.

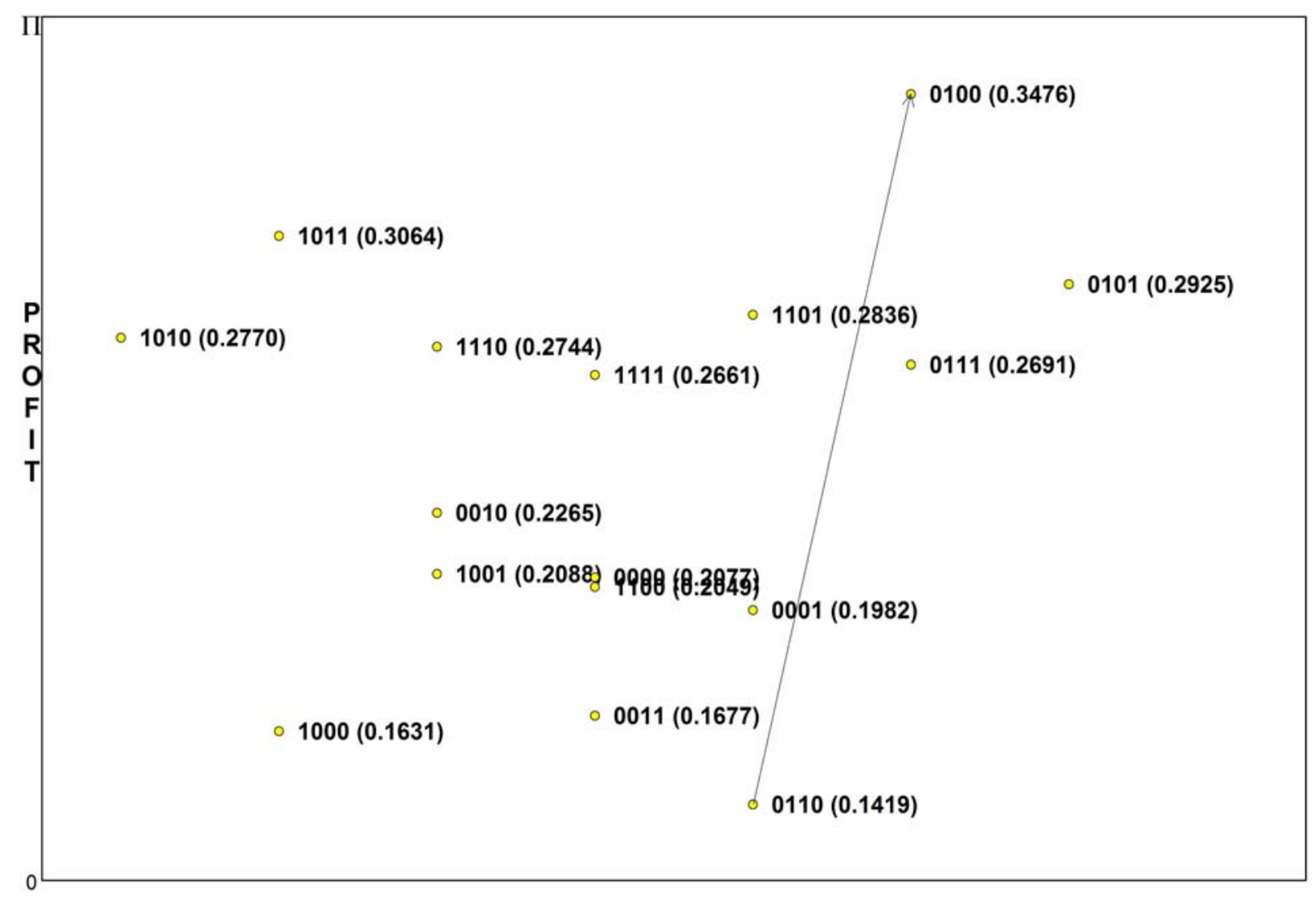

Figure 7 Plot of lattice of perceived benefits of AMT - Medium Plants

The plot of the lattice of the high-level factors for medium plants strongly supports all of the complementarities and substitutes identified in the constrained regression analysis except for the complementary pairing of the implementation support and $R \& D$ factor and the human resources factor (factors 3 and 5) (refer to Figure 8). This pairing is supported from many of the initial starting states but not from the zero state. Defining the top tier once again as those states with an average profit margin greater than $30 \%$ (states 11010, 01001, 01101, 11111, and 11101), this grouping represents $15 \%$ of medium plants. The lower tier, those states with an average 
profit margin less than 20\% (states 01000, 10110, 00111, 00010, 00101, 01011, and 00001), represents $19.41 \%$ of medium plants.

There is one state in the high-level factors that consists of two complementary pairs (factors 1 and 4 and factors 2 and 4 ) that performs significantly better than any other state. The optimal state for medium plants to occupy appears to be the state consisting of the perceived benefits factors, the AMT and business practices factor, and the design factor (state 11010). This state out-performs the worst state (state 00001 ) by over $400 \%$.

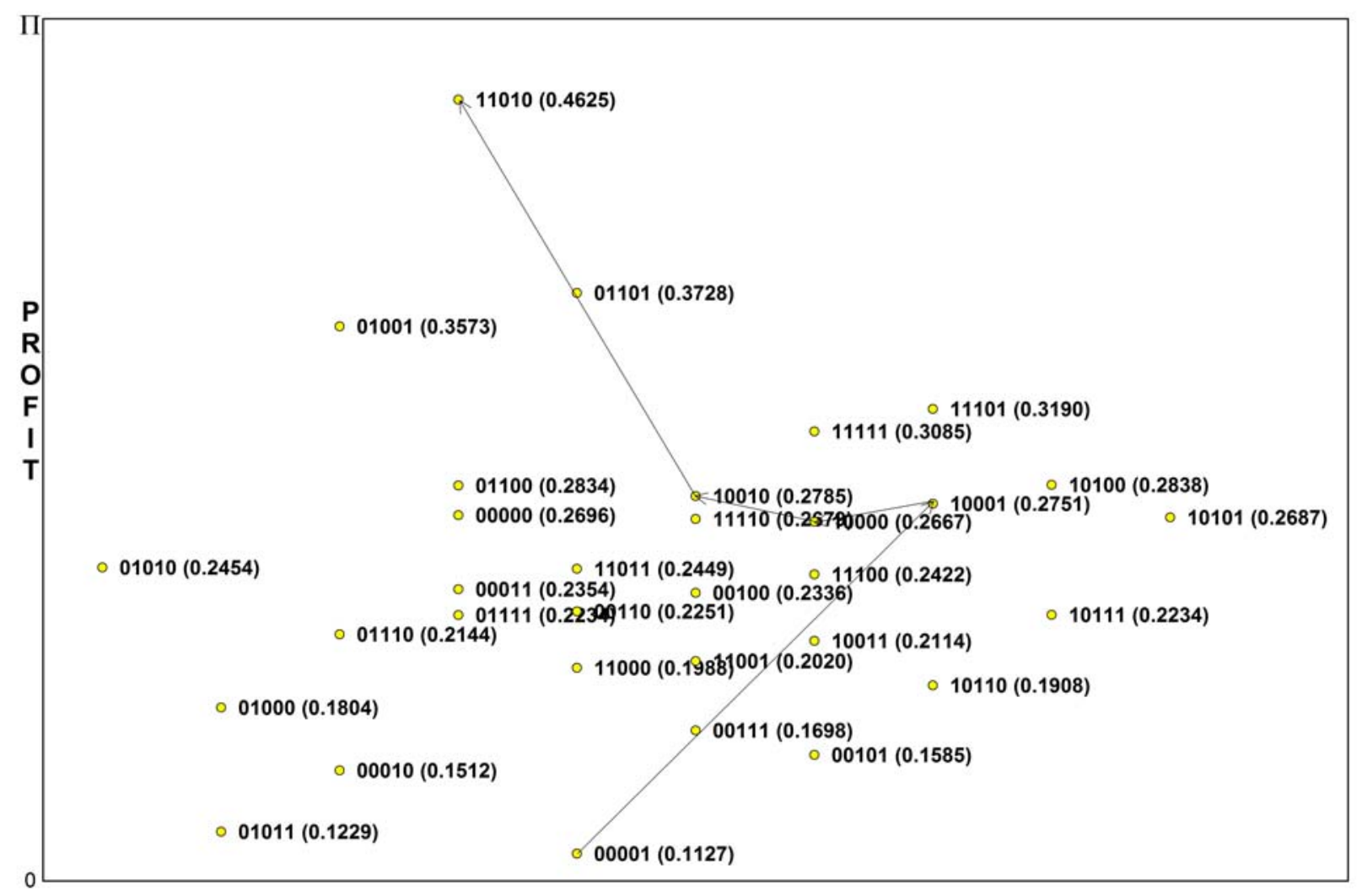

Figure 8 Plot of lattice of high-level factors - Medium Plants

\subsubsection{Vehicles Industry}

There is only one empty state in the vehicles industry analysis. Therefore, most of the complementarities and substitutes identified in the constrained regression analysis are available 
for analysis. The vehicles industry has very different results from those in the medium plant analysis and some of the plots of the lattices contain negative average profit margins. These plots demonstrate that there are many states where choosing the appropriate state can lead to a large competitive advantage.

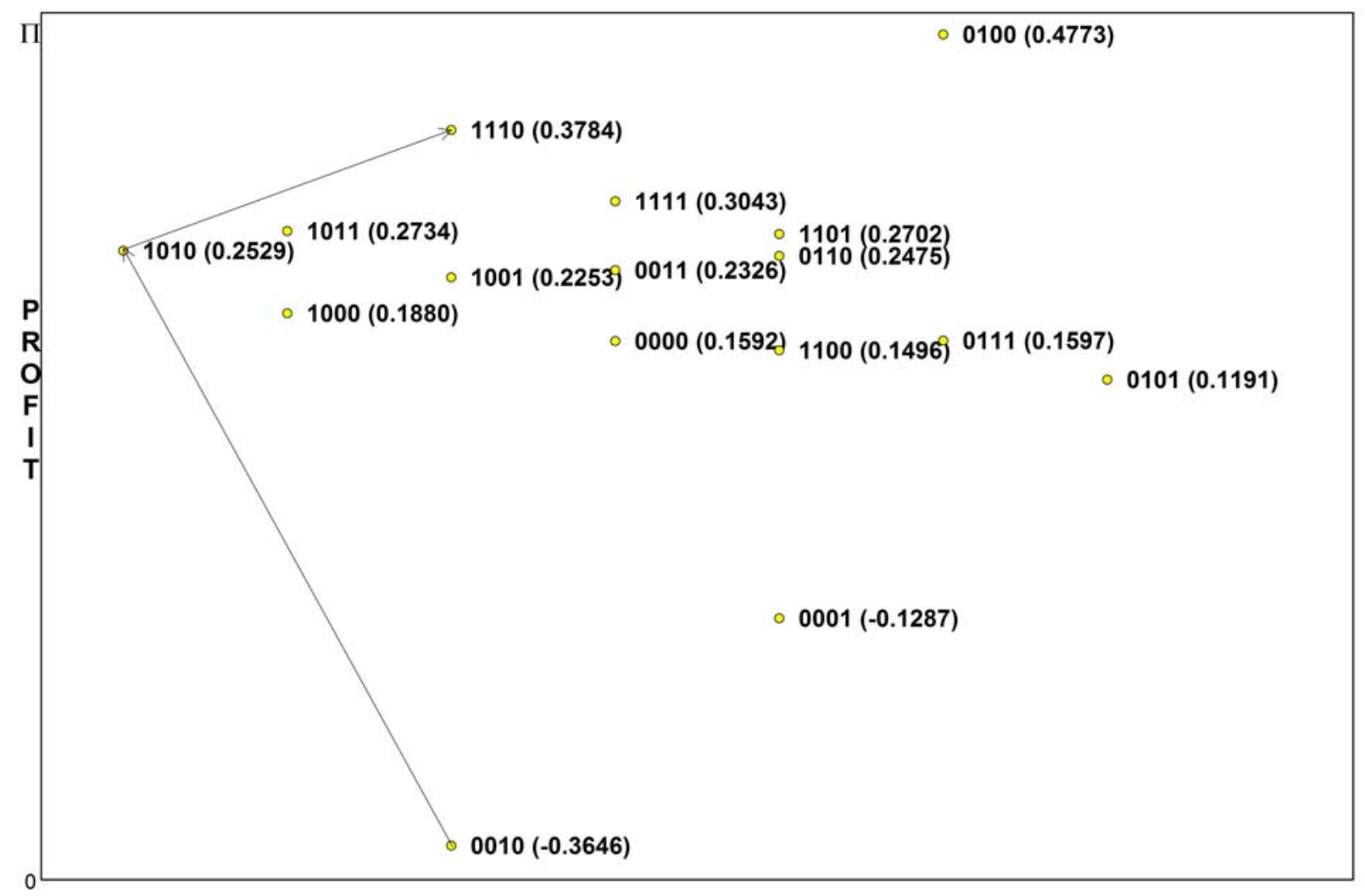

Figure 9 Plot of lattice of AMT - Vehicles Industry

The plot of the lattice of AMT in the vehicles industry supports the complementary pairing of production design and inspection and storage technologies (factors 1 and 3). It also clarifies the substitute pairing of production design and communications technologies (factors 1 and 2) identified in the constrained regression analysis (refer to Figure 9). Due to the extremely high average profit margin of the plants with only the communications technology $(47.73 \%)$ it is very difficult to find variables that will complement the use of communications technologies, even though the remaining states in the top tier include both communications and production 
design technologies. The lattice demonstrates that it is likely that communications and production design technologies are complementary from many of the initial states.

The top grouping, consisting of states with average profit margins greater than $30 \%$ (states 0100,1110 , and 1111 ), represents $29.35 \%$ of vehicles plants. On the other hand, the lower cluster (states 0001 and 0010 ), which has negative average profit margins, represents 5.53\% of the vehicles industry. The remaining plants are split almost evenly between the grouping of states with average profit margins between $22 \%$ and $28 \%$ and the grouping of states with average profit margins between $19 \%$ and $12 \%$. There is one state that does perform significantly better than the others. The optimal state appears to be the one consisting of only the communications technologies (state 0100) although the remaining top tier states do perform very well. This could be because the communications technology is more widely accepted throughout the industry and has many different sources of support.

The analysis of the lattice of business practices of the vehicles industry strongly support the complementarity between the planning and design business practices but has only weak support for the substitute pairing of quality control and design (factors 1 and 3) as well as quality control and planning business practices (factors 1 and 2) (refer to Figure 10). The top two performing states (states 011 and 100) do not contain either of the two substitute pairings supporting the conclusions from the constrained regression analysis. These top two states represent $9.5 \%$ of vehicle plants, and performed significantly better than not doing any of the business practices which has a small negative average profit margin. The zero state still represents a significant number of vehicle plants at $22.87 \%$ of the industry. This result demonstrates just how important having an appropriate set of business practices can be for 
gaining and maintaining a competitive advantage since the average profit margin in the upper two states is over $44 \%$.

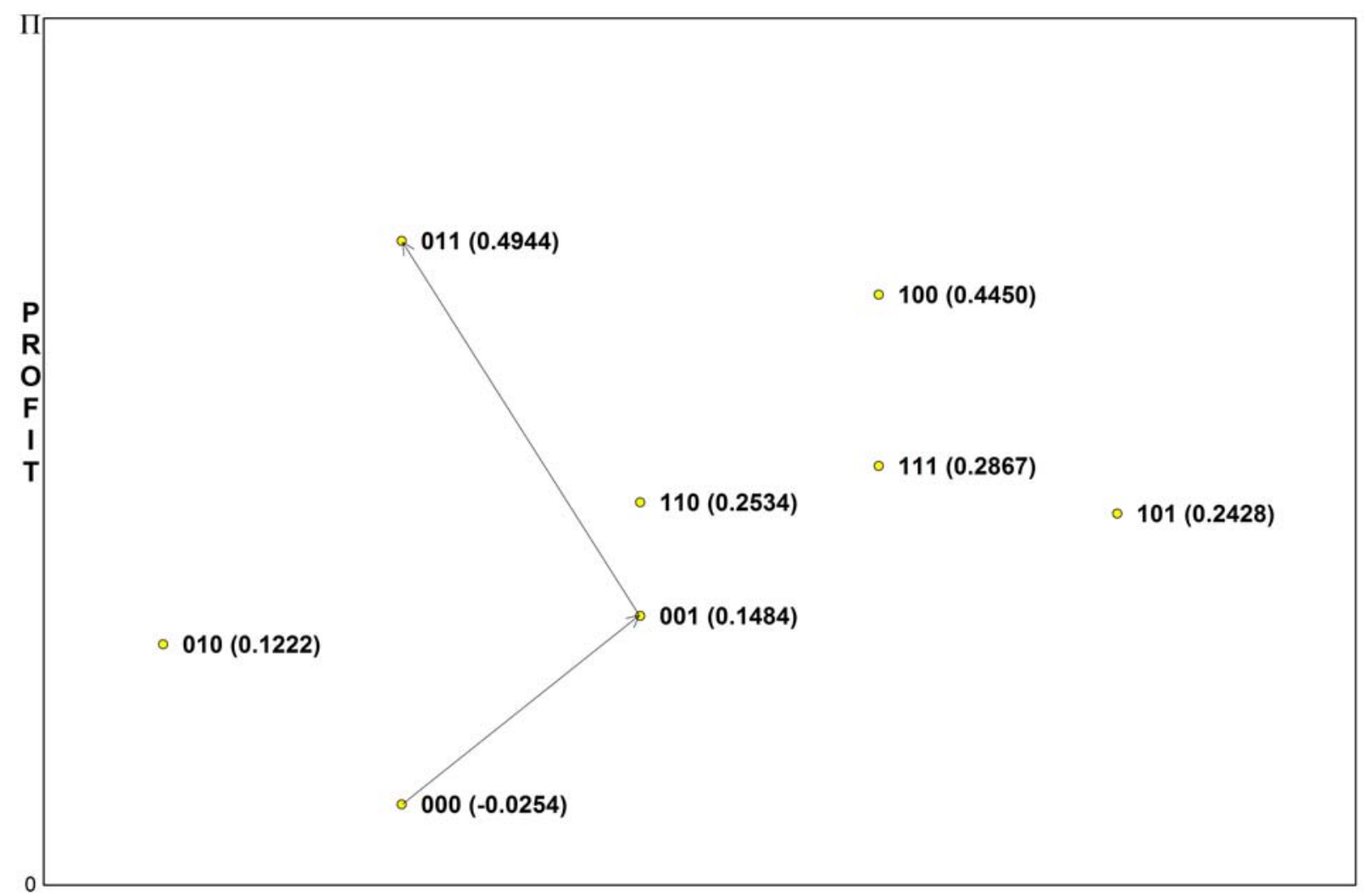

Figure 10 Plot of lattice of business practices - Vehicles Industry

The analysis of the lattice of sources of implementation support for the vehicles industry confirms the substitute pairing of support from primary stakeholders and corporate organizations (factors 1 and 4) (refer to Figure 11). The upper tier of states, those with average profit margins above 0.3 (states 1011, 0100, 0101, and 0111), represents $21.93 \%$ of vehicle plants. In particular, the two states with average profit margins grater than 0.4 (states 0100 and 0101 ) represent $7.47 \%$ of the vehicle industry. These two states both contain support from the research and development group indicating the importance of new designs and innovations to the vehicles industry. The lower tier of states, those with average profit margins less than 0.12 (states 1000, 1001, and 
0010), represents over $38.62 \%$ of vehicle plants. These results demonstrate that many plants are not in states that have even moderate success and that many improvements can be made.

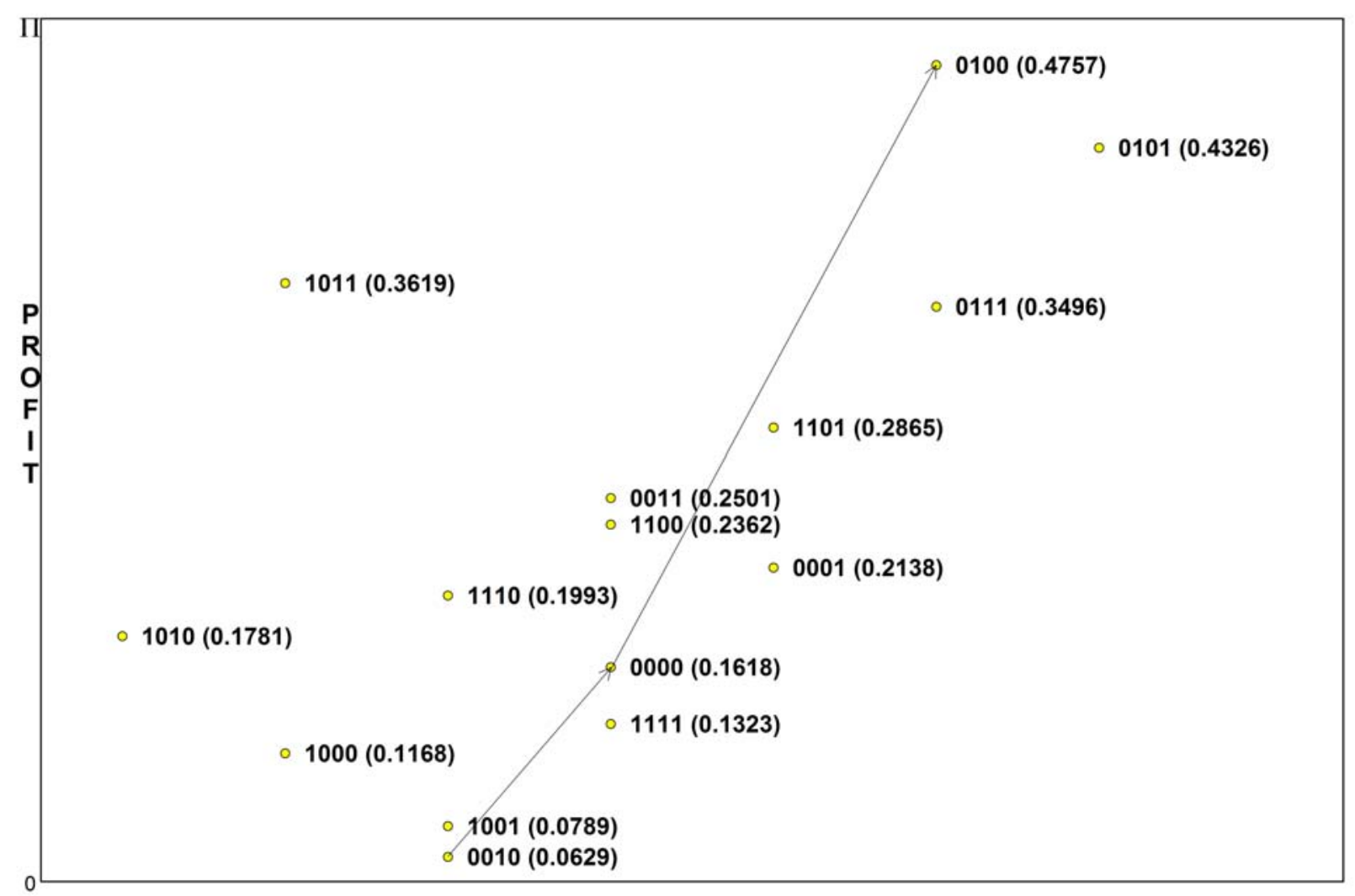

Figure 11 Plot of lattice of sources of implementation support - Vehicles Industry

The plot of the lattice of the perceived benefits of AMT supports the complementary pairing of the perceived benefit of increased productivity and the perceived benefit of increased flexibility (factors 2 and 3) (refer to Figure 12). The state consisting of this grouping (state 0110) performs moderately better than the zero state and only having the perceived benefit of increased flexibility results in a negative average profit (state 0010). There is not a large discrepancy between the average profit margins of most of the states and there is no clear-cut optimal state. The state consisting of only the perceived benefit of increased flexibility is definitely to be avoided yet still represents $8.21 \%$ of the vehicle industry. 


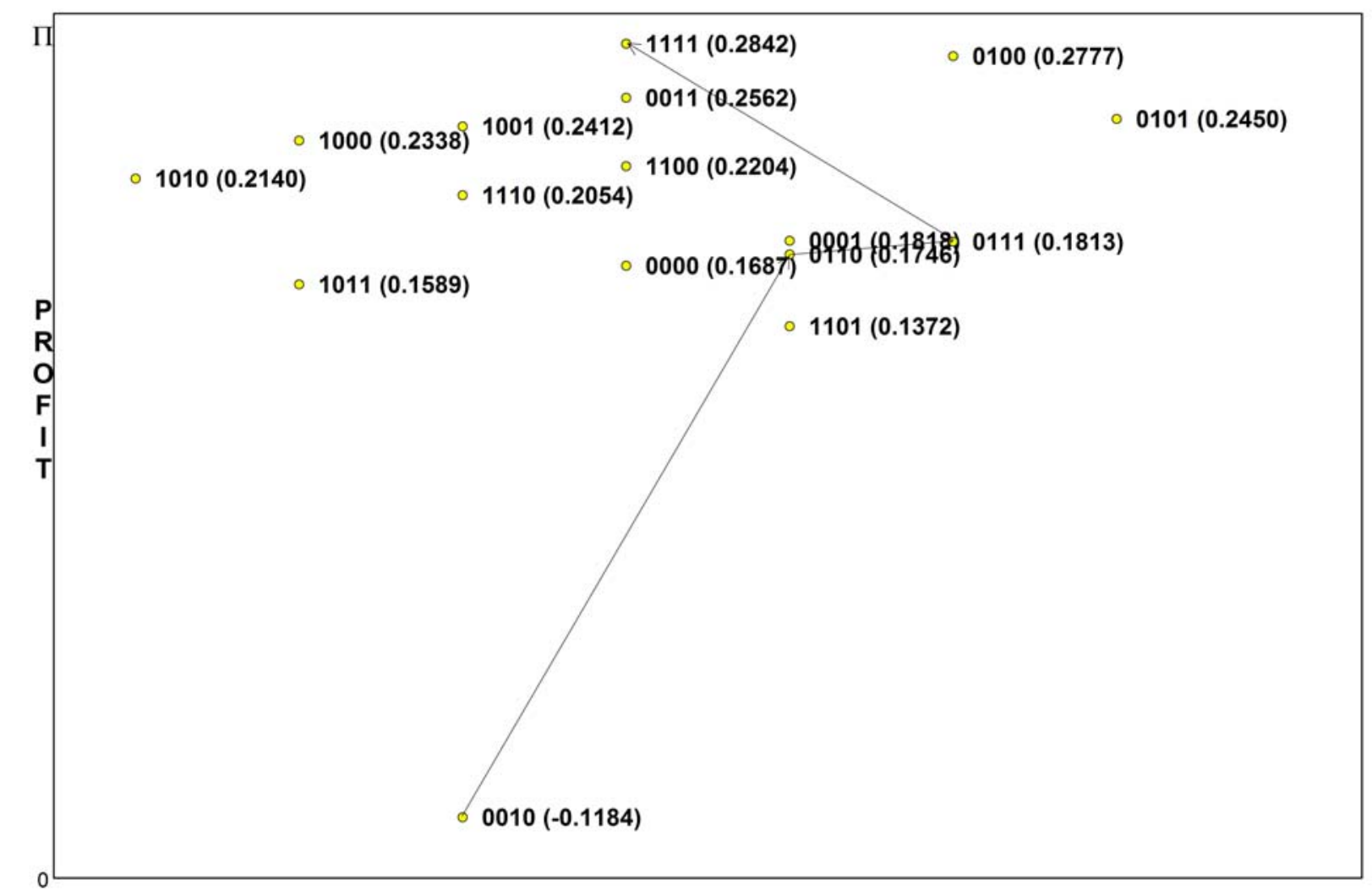

Figure 12 Plot of lattice of perceived benefits of AMT - Vehicles Industry

Finally, the plot of the lattice of high-level factors for the vehicles industry supports all the complementarities and substitutes identified in the constrained regression analysis, except the substitute pairing of the AMT and business practices factor and the design factor (factors 2 and 4) (refer to Figure 13). The combination of these two factors is found in many of the states in the upper tier and in particular the state with only these two factors (state 01010) performs significantly better than the zero state. The reason that these two factors may have been found to be substitutes is due to the extremely large negative average profit margin for the state with only the design factor (state 00010) and large average profit margin for the state with only the AMT and business practices factor (state 01000). The plot shows that there are a large number of states with negative average profit margins (states 00111, 00011, 00001, 00010, and 00100). A total of $5.61 \%$ of vehicle plants are located in states with negative average performance. There are also 
another $7.56 \%$ of plants in the vehicles industry that have average profit margins less than $11 \%$ (found in states 11011, 10100, and 10101).

The upper tier, plants with average profit margins greater than 29\% (states 10000, 01100, 01111, 11110, 11100, and 11111), represents 36.97\% of the vehicle industry. The state consisting of only the perceived benefits factor (state 10000) performs extremely well compared to the average profit margins of the other states. The remaining upper tier states (states 01100, 01111, 11110, 11100, and 11111) all have the complementary pairing of the AMT and business practices factor and the sources of implementation support and R\&D factor indicating the importance of theses factor to the competitive advantage of plants in the vehicles industry.

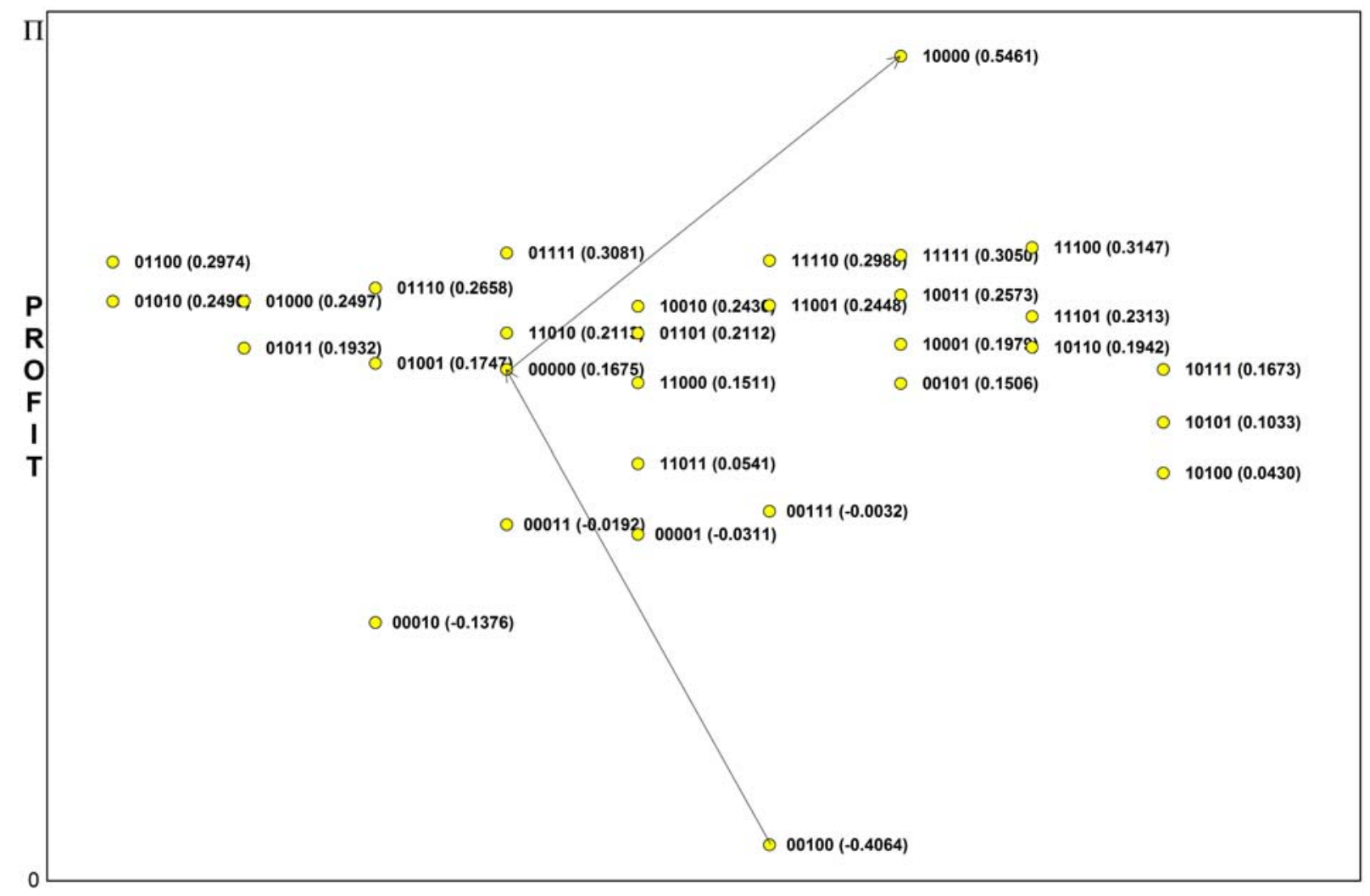

Figure 13 Plot of lattice of high-level factors - Vehicles Industry 


\subsubsection{Conclusion}

The plots of the lattices for the various factor types support the conclusions about substitutes and complements presented in the previous sections. The majority of the substitutes and complements identified in the constrained regression analysis appear in the plot of the average profit margins by factor state. These plots also aid in the identification of the intensity or importance of the various complements and substitutes identified. Some of the pairings seem to make little difference to the profit margins while others have dramatic effects.

The cost and time involved in moving from one of the lower tier states to the optimal state may be quite large, so the plots identify the path that should be taken to have the greatest impact with the smallest amount of effort and cost. Managers will be able to use the plots to identify better performing states and then determine the optimal state they can achieve given their budgetary and time constraints. The optimal state may also not be possible for many firms due to other organizational constraints; in which case they can identify the best possible state that they can attain. These figures will assist organizations in determining the specific set of policies they should create in their organization given a restricted budget by prioritizing the changes that need to be made in order to exhibit the optimal state or at least one of the top tier states.

These plots also help to explain why some plants are not performing well in the context of their implementation of AMT. Plants that are in the lower tier or lower groupings of states perform much worse than those in the top tier states. Managers can use the identification of poor performing states to support the need for change and aid them in choosing the optimal direction for change as well. 


\section{CONCLUSION AND FUTURE RESEARCH}

The main objective of this dissertation was to determine the complementary and substitute pairings of variables for the implementation of AMT. Current literature focusing on the use of AMT as well as complementary business practices was explored. The study focused on the analysis of business practices, sources of information support, perceived benefits of the implementation and the forms of AMT implemented by Canadian manufacturing plants. The analysis was performed by size class and industry in order to isolate differences due to the competitive markets in which the plants reside.

A number of methodological enhancements were introduced over the course of the study. These include using a large number of variables and factors for variable reduction in the complementarity analysis, as well as determining size and industry specific complementarities. Another innovation included in the thesis was the creation of the lattice for the medium sized plants and the vehicles industry.

\subsection{Conclusions}

The dissertation begins with factor analysis conducted in order to reduce the number of variables for the complementarity research that follows. The results of the factor analysis and the distribution of plants within the various factor states identify unique characteristics about the size classes and industries. Most importantly, the distribution of the plants demonstrates that there are some industries where the number of different sets of policy or business practice decisions is not very broad, while in other industries every possible state represents a significant proportion of the population. This analysis was the first indication of differences that were to emerge based both on size class and industry classification. 
Based on the complementarity analysis, the results support the hypotheses from Meredith (1987) that small plants will use different forms of AMT and will obtain different benefits from the use of AMT than large plants. The complementarities exhibited in the small and large plants are quite different. In particular, the complementarities in the use of AMT and business practices are almost completely opposites. Rarely is there a pairing in the analysis that is a substitute or complement in all three size classes.

The effect of the industry classification is even more pronounced than those exhibited in the size class analysis. Each industry exhibits a unique set of complementary and substitute pairings. The similarities found between industries cannot be explained by standard classifications such as high-technology and low-technology industries. In some instances the electronics industry exhibits results similar to those found in the wood or machinery industry and at other times results unique to it. This may be due to competition pressures or government regulations and tariffs that were not examined in the study. The industry effect on the complementarities exhibited by the plants is significant and should be taken into account in all future research.

A number of hypotheses from the literature review (presented in chapter three) were supported by the complementarity analysis. These are discussed in detail in chapter five during the analysis. The hypothesis of Chenhall and Langfield-Smith (1998) that the use of AMT and R\&D are complementary is supported in all size classes and all industries that have a significant result except for the wood industry. The use of AMT and human resource incentives as complementary, hypothesised by Alcorta (1994), is supported by all significant results except those found in the chemical and machinery industries. As discussed in chapter five, the hypothesis that all forms of AMT are complementary (Alcorta, 1994) is rejected in all cases. The 
hypothesis of Schlie and Goldhar (1995) that information exchange and design technologies are complementary is also not entirely supported by the analysis (refer to Table 106 for a summary of the hypotheses from the literature review and the conclusions from the study).

\begin{tabular}{|c|c|c|}
\hline Author & Hypothesis/Test Results & Results from this Study \\
\hline $\begin{array}{l}\text { Teece et al. }(1997)^{*}, \\
\text { Tyler }(2001)^{*}, \\
\text { Rivkin }(2000)^{*}\end{array}$ & $\begin{array}{l}\text { More complex strategies give } \\
\text { firms a competitive advantage }\end{array}$ & $\begin{array}{l}\text { A complex strategy is beneficial only if the } \\
\text { strategies are complementary }\end{array}$ \\
\hline Meredith $(1987)^{\ddagger}$ & $\begin{array}{l}\text { Small plants use different AMT } \\
\text { than large plants }\end{array}$ & $\begin{array}{l}\text { This is supported as the distribution of the use } \\
\text { of AMT is different for small and large plants } \\
\text { as are the complementary pairs in the AMT } \\
\text { analysis }\end{array}$ \\
\hline $\begin{array}{l}\text { Small and Chen } \\
(1995)^{\dagger} \text {, Mohnen } \\
\text { and Roller }(2003)^{\dagger}\end{array}$ & $\begin{array}{l}\text { Industry effect is an important } \\
\text { determinant of complementarities }\end{array}$ & $\begin{array}{l}\text { This is supported as the distribution of the } \\
\text { states for all of the analyses are different for } \\
\text { each industry as are the complementary pairs }\end{array}$ \\
\hline $\begin{array}{l}\text { Chenhall and } \\
\text { Langsfield-Smith } \\
(1998)^{\dagger}\end{array}$ & $\begin{array}{l}\text { Cross-functional teams, } \\
\text { benchmarking, and training are all } \\
\text { complementary }\end{array}$ & $\begin{array}{l}66 \% \text { of the significant results support business } \\
\text { practices and human resources practices as } \\
\text { complementary }\end{array}$ \\
\hline $\begin{array}{l}\text { Ichniowski, Shaw, } \\
\text { and Prennushi } \\
(1997)^{\dagger}\end{array}$ & $\begin{array}{l}\text { HR incentives and training are } \\
\text { complementary }\end{array}$ & $\begin{array}{l}\text { All human resources practices are so } \\
\text { correlated that they are all one factor }\end{array}$ \\
\hline Teece et al. (1997) ${ }^{*}$ & $\begin{array}{l}\text { Cross-functional teams and } \\
\text { product improvements are } \\
\text { complementary }\end{array}$ & $\begin{array}{l}50 \% \text { of the significant results support business } \\
\text { practices and perceived benefits as } \\
\text { complementary }\end{array}$ \\
\hline Udo and Ehie (1996) & $\begin{array}{l}\text { A project champion and internal } \\
\text { stakeholder support is critical for } \\
\text { successful AMT implementations }\end{array}$ & $\begin{array}{l}\text { This is supported as primary stakeholders are } \\
\text { complementary to most of the other forms of } \\
\text { implementation support for all size classes } \\
\text { and industries }\end{array}$ \\
\hline Helfat (1997) ${ }^{\dagger}$ & $\begin{array}{l}\text { Diverse forms of R\&D are } \\
\text { complementary (internal } R \& D \text {, } \\
\text { joint } R \& D \text {, etc.) }\end{array}$ & $\begin{array}{l}\text { All forms of R\&D are so correlated that they } \\
\text { are all one factor }\end{array}$ \\
\hline $\begin{array}{l}\text { Moorman and } \\
\text { Slotegraaf }(1999)\end{array}$ & $\begin{array}{l}\text { Increased levels of technology } \\
\text { and quality improvements are } \\
\text { complementary }\end{array}$ & $\begin{array}{l}50 \% \text { of the significant results support AMT } \\
\text { and perceived benefits as complementary }\end{array}$ \\
\hline $\begin{array}{l}\text { Chenhall and } \\
\text { Langsfield-Smith } \\
(1998)^{\dagger}\end{array}$ & $\begin{array}{l}\text { Internal R\&D and AMT are } \\
\text { complementary }\end{array}$ & $\begin{array}{l}100 \% \text { of the results by size class and } 60 \% \text { of } \\
\text { the results by industry support this hypothesis }\end{array}$ \\
\hline Alcorta $(1994)^{*}$ & $\begin{array}{l}\text { All forms of AMT are } \\
\text { complementary } \\
\text { CAD/CAM, automated storage } \\
\text { and retrieval systems, CNC } \\
\text { machines, FMS, and CIM) }\end{array}$ & $\begin{array}{l}\text { This hypothesis is not supported. Only } \\
45.28 \% \text { of the significant results for AMT } \\
\text { support complementarity. }\end{array}$ \\
\hline
\end{tabular}

*Theoretical argument

$\dagger$ Empirical tests - survey

$\$$ Empirical tests - case study

Table 106 Hypotheses Tested from the Literature Review 


\begin{tabular}{|c|c|c|}
\hline Author & Hypothesis/Test Results & Results from this Study \\
\hline $\begin{array}{l}\text { Lei, Hitt, Goldhar } \\
(1996) *\end{array}$ & $\begin{array}{l}\text { Design technologies, external } \\
\text { linkages, and plant organizational } \\
\text { changes are complementary }\end{array}$ & $\begin{array}{l}100 \% \text { of the results by size class and } 60 \% \text { of } \\
\text { the results by industry support sources of } \\
\text { implementation support and AMT as } \\
\text { complementary }\end{array}$ \\
\hline $\begin{array}{l}\text { Schlie and Goldhar } \\
(1995)^{*}\end{array}$ & $\begin{array}{l}\text { Design technologies, product } \\
\text { improvements, and information } \\
\text { exchange are complementary }\end{array}$ & $\begin{array}{l}50 \% \text { of the significant results support AMT } \\
\text { and perceived benefits as complementary }\end{array}$ \\
\hline $\begin{array}{l}\text { Milgrom } \\
\text { Roberts }(1995) *\end{array}$ & $\begin{array}{l}\text { Internal R\&D, AMT, cross- } \\
\text { functional teams, training, } \\
\text { incentives, and product } \\
\text { improvements are complementary }\end{array}$ & $\begin{array}{l}62.5 \% \text { of the significant results support AMT } \\
\text { and HR practices as complementary; } 100 \% \text { of } \\
\text { the results by size class and } 60 \% \text { of the results } \\
\text { by industry support R\&D and AMT as } \\
\text { complementary; } 50 \% \text { of the significant results } \\
\text { support AMT and perceived benefits as } \\
\text { complementary }\end{array}$ \\
\hline $\begin{array}{l}\text { Milgrom } \\
\text { Roberts }(1990)\end{array}$ & $\begin{array}{l}\text { Training, process technologies, } \\
\text { and external linkages are } \\
\text { complementary }\end{array}$ & $\begin{array}{l}62.5 \% \text { of the significant results support AMT } \\
\text { and HR practices as complementary; } 100 \% \text { of } \\
\text { the results by size class and } 60 \% \text { of the results } \\
\text { by industry support sources of implementation } \\
\text { support and AMT as complementary }\end{array}$ \\
\hline
\end{tabular}

Table 106 Hypotheses Tested from the Literature Review Continued

The analysis of the OLS model including the factor state variables as well as the individual variables (presented in section 5.2) supported the concept of complementarity. The interaction effects included as part of the state variables were much larger than most of the significant coefficients in the individual variables. In particular, the majority of the variables had insignificant coefficients when added to the model containing the factor state variables. This method demonstrated, however, that the use of factors in the complementarity analysis might require the addition of individual variables as well. In cases where the sample size is small, such as the electronics industry, some of the individual variables had coefficients as large as those of the factor state variables. Some of these significances may be due to the composition of the factors. A plant can exhibit a factor with a number of different combinations since exhibiting the factor is based on the mean value of practices, technologies, or sources of support exhibited by 
the plant. If a specific combination does not occur in many plants, then the effect of the individual variable that is rarely included will be understated and thus the variable will have a significant coefficient. For this reason, the use of factors in variable reduction for this type of analysis should be used in conjunction with analysis of the individual variables in future research in order to ensure that there are not individual variables that have a large impact on the profit or productivity function not included in the interaction effects.

The lattice plots of the medium size class and the vehicles industry demonstrate that it is not the most complex set of policies that results in the optimal state. Due to the number of substitute pairs, the use of all of the factors never has the highest average profit margin in the lattice plots. These results imply that a complex set of strategies is beneficial for a competitive advantage but only if these strategies are complementary. The hypothesis that a complex set of business practices and technology usages by Amit and Zott (2001), Teece (1986), and Rivkin (2000) is supported as most of the upper tier states include multiple factors. The lattice plots support that there is a difference between size class and industry, and that global conclusions cannot effectively represent the different sized plants or industries.

Managers will be able to use the results from this study to improve their implementations of AMT. By considering their current position on the lattice, they will be able to determine the optimal path for improvement given their budgetary restrictions. The lattices will also demonstrate to managers the benefits of changing their current business practices and technology usage by displaying the current position of the plant relative to those within their size class and industry.

There are a number of limitations to this study. One limitation relates to the available sample size. The small sample size of the industry analysis forced the number of available 
factors to be constrained to five (due to the exponential increase in the number of factor states). As a result, the high-level analysis has some extremely large factors. The second limitation due to sample size was that the analysis by industry could not be done at the 3-digit SIC level but had to be condensed to the 2-digit SIC level. This forced diverse sub-industries such as those in the chemical industry (pharmaceuticals, pesticides, etc.) to be analysed together. Finally, the small sample sizes, in combination with the non-uniform plant distributions, caused a number of empty factor states. Thus, the complementarity restrictions for the states had to be removed.

Another limitation of the study is the use of secondary data linked by plant. The economic data from the Annual Survey of Manufactures (ASM) is only available for roughly $60 \%$ of the plants. This reduced the sample size by $38.6 \%$ to 2,273 plants and increased the variability in the estimates. The ASM data only includes aggregate economic plant variables so only basic profit and labour productivity measures were constructed.

The use of factors and not the exact survey responses themselves also influences the results of the study. By using factors, plants may exhibit a factor by using different individual technologies or business practices. In this case, if there is a particular grouping of technologies that is widely used, then this grouping will have a significant impact in determining complementarity. If there is a plant that uses a very unusual set of technologies in order to exhibit the factor, then it may not be well represented by the results. This is especially true in the diverse industries where two different sub industries may use opposite sets of technologies to represent the same factor. The use of factors is important for the inclusion of multiple variables but the composition of the factor must be taken into consideration as demonstrated in the OLS modelling of Chapter 5.2. 


\subsection{Future Research}

The analysis has suggested several possibilities for future research. There are two main areas where future research could be conducted. The first is with respect to the method chosen for the complementarity analysis and the construction of solution lattices. The second is with respect to the nature of the data chosen to analyse. Both of these areas offer many different possibilities for future research and the growth of this topic area in the literature.

From the point of view of the methodology, the ability to include a greater number of variables in the modelling may have first priority for future research. The large amount of data required for this analysis is a large limiting factor primarily due to the exponential nature of the regression constraints. If a new method could be developed that would allow for a greater number of variables to be studied either by creating probability functions for estimating missing state coefficients or by creating an iterative model allowing individual and not factor variables to be used, this would greatly enhance the ability to determine the optimal set of policies and technologies for managers.

From a practical point of view, the use of different data sources and dependent variables could introduce more insight into the effect that the complementarities have on the profit and labour productivity measures of the plants. The use of growth measures for profit and labour productivity may be more appropriate than the static measures used in this thesis. This is only true if the time of implementation of the technology or business practice is known so the impact of the implementation can be more readily isolated. Another way in which to look at growth measures would be to use a longitudinal dataset. This would complicate the analysis and the model with the changing of a plant from one state to another but the effect of the change would 
be more easily isolated. This would make it much easier to create a plot of the lattice structure of the industry or size class.

As discussed in the previous section, the inclusion of the significant individual variables may be necessary in order to obtain better fitting models. The choice of which individual variable to include would depend on the size class or industry chosen. There does not appear to be individual variables that should be globally included. The addition of the individual variables may confound the complementarity analysis, as the variable would be included individually and as part of the state variables for some plants.

The use of a more detailed, perhaps industry specific, survey would also allow for results that are more detailed. The current data has broadly defined business practices and technologies, many of which are used in only a subset of the industries. If a more specific survey was developed then there would be a greater ability to use industry sub-classes and to deal with the more pervasive technologies and practices that occur in the industry.

Due to the hypothesized importance of R\&D to the use of AMT a further analysis of this complementarity could also be undertaken in the future. A linkage between the existing dataset and a survey on innovation or research and development that are currently maintained by Statistics Canada could provide greater insight into how the various forms of technology support the innovation process. This research could offer valuable insight to the government on possible policy strategies that could be used to encourage innovation in Canadian manufacturing.

Finally, a study of the complementarities that exist between business practices and technology usage of plants within a supply chain is an area where future research could be extremely productive. The ability to maintain a highly efficient supply chain is a source of 
competitive advantage for many firms. Many contracts stipulate the types of business practices, form of information exchange, and even nature of technologies that must be implemented in order to be a member of the supply chain implying that there must be some complementarities expected by such restrictions. A study of different supply chains and the complementarities that exist between the business practices used by the various members could offer insight into the fit required to create a superior supply chain environment. 


\section{REFERENCES}

Abdel-Kader, M.G., Dugdale, D. (1998) Investment in Advanced Manufacturing Technology: a Study of Practice in Large U.K. Companies. Management Accounting Research 9:261-284.

Acton, G.S. (2002) Interpersonal Complementarity [on-line]. University of California, San Francisco [cited 2002-10-30], Available at http://www.personalityresearch.org/interpersonal/complementarity.html.

Alcorta, L. (1994) The Impact of new Technologies on Scale in Manufacturing-Industries Issues and Evidence. World Development 22 (5): 755-769.

Allen, R.G.D. (1934) A Comparison Between Different Definitions of Complementary and Competitive Goods. Econometrica 2(2): 168 - 175.

Amit, R., Zott, C. (2001) Value Creation in E-business. Strategic Management Journal 22(6-7): 493-520.

Antonelli, C. (1993) Externalities and Complementarities in Telecommunications Dynamics. International Journal of Industrial Organization 11 (3): 437-447.

Antonelli, C. (1997) The Economics of Path-Dependence in Industrial Organization. International Journal of Industrial Organization 15 (6): 643-675.

Argyres, N.S. (1995) Technology Strategy, Governance Structure and Interdivisional Coordination. Journal of Economic Behavior \& Organization 28 (3): 337-358.

Arora, A. (1996) Testing for Complementarities in Reduced-form Regressions: A note. Economics Letters 50 (1): 51-55.

Arora, A., Gambardella, A. (1990) Complementarity and External Linkages: The Strategies of the Large Firms in Biotechnology. The Journal of Industrial Economics 38 (4): 361-379.

Arundel, A., Sonntag, V. (1999) Patterns of Advanced Manufacturing Technology use in Canadian Manufacturing. Statistics Canada 88F0017MIE - 12.

Athey, S., Stern, S. (1998) An Empirical Framework for Testing Theories about Complementarity in Organizational Design. Stanford University, Working Paper.

Athey, S. (2002) Monotone Comparative Statics under Uncertainty. Quarterly Journal of Economics 117 (1): 187-223.

Athey, S., Roberts, J. (2001) Organizational Design: Decision Rights and Incentive Contracts. American Economic Review 91 (2): 200-205.

Athey, S., Schmutzler, A. (1995) Product and Process Flexibility in an Innovative Environment. RAND Journal of Economics 26 (4): 557-574. 
Barua, A., Lee, C.H.S., Whinston, A.B. (1996) The Calculus of Reengineering. Information Systems Research 7 (4): 409-428.

Barua, A., Lee, B. (1997) The Information Technology Productivity Paradox Revisited: A Theoretical and Empirical Investigation in the Manufacturing Sector. International Journal of Flexible Manufacturing Systems 9 (2): 145-166.

Berndt, Ernst, Savin, Eugene (1977) Conflict Among Criteria for Testing Hypotheses in the Multivariate Linear Regression Model. Econometrica 45(5): 1263 -1277.

Birkoff, G. (1948) Lattice Theory. American Mathematical Society, Rhode Island.

Boyer, K., Leong, G., Ward, P., and Krajewski, L. (1997) Unlocking the Potential of Advanced Manufacturing Technologies. Journal of Operations Management 15: 331-347.

Brynjolfsson, E., Hitt, L. (1996) Paradox lost? Firm-level Evidence on the Returns to Information Systems Spending. Management Science 42 (4): 541-558.

Bushnell, P.T., Shepard, A.D. (1995) The Economics of Modern Manufacturing - Comment. American Economic Review 85 (4): 987-900.

Cahill, S.A. (1999) On the Various Measures of the Elasticity of Substitution. Policy Analysis Division, Agriculture and Agri-Food Canada, Working Paper.

Chambers, R.G. (1988) Applied Production Analysis: A Dual Approach. Cambridge University Press, Cambridge U.K.

Chang, M.H. (1993) Flexible Manufacturing, Uncertain Consumer Tastes, and Strategic Entry Deterrence. Journal of Industrial Economics 41 (1): 77-90.

Chenhall, R.H., Langfield-Smith, K. (1998) The Relationship Between Strategic Priorities, Management Techniques and Management Accounting: An Empirical Investigation using a Systems Approach. Accounting, Organizations and Society 23 (3): 243-264.

Chung, C. (1996) Human Issues Influencing the Successful Implementation of Advanced Manufacturing Technology. Journal of Engineering and Technology Management Jet-M 13: 283-299.

Davern, M.J., Kauffman, R.J. (2000) Discovering Potential and Realizing Value from Information Technology Investments. Journal of Management Information Systems 16 (4): 121143.

Dietl, H. (1999) Coordinating Complementary R\&D Activities Across Industries. Neue Folge Nr. 60, University of Paderborn.

Doms, M., Dunne, T., and Roberts, M.J. (1995) The role of Technology use in the Survival and Growth of Manufacturing Plants. International Journal of Industrial Organization 13:523-542. 
Donnellan, T. (1968) Lattice Theory. Pergamon Press, Toronto.

Drake, A.R., Haka, S.F., Ravenscroft, S.P. (1999) Cost System and Incentive Structure Effects on Innovation, Efficiency and Profitability in Teams. The Accounting Review 74 (3): 323-345.

Ettlie, J.E., Pennerhahn, J.D. (1994) Flexibility Ratios and Manufacturing Strategy. Management Science 40 (11): 1444-1454.

Ettlie, J.E., Reza, E.M. (1992) Organizational Integration and Process Innovation. Academy of Management Journal 35 (4): 795-827.

Frohlich, M. (1998) How do you Successfully Adopt an Advanced Manufacturing Technology? European Management Journal 16(2): 151-159.

Ghosh, D. (2000) Complementary Arrangements of Organizational Factors and Outcomes of Negotiated Transfer Price. Accounting Organizations and Society 25 (7): 661-682.

Goldhar, J.D., Jelinek, M. (1985) Computer Integrated Flexible Manufacturing: Organizational, Economic, and Strategic Implications. Interfaces 15(3): 94-105.

Gonzalez-Benito, Javier, Suarez-Gonzalez, Isabel, Spring, Martin (2000) Complementarities Between JIT Purchasing Practices. International Journal of Production Economics 67: 279-293.

Gourieroux, Christian, Holly, Alberto, Monfort, Alain (1982) Likelihood Ratio Test, Wald Test, and Kuhn-Tucker Test in Linear Models with Inequality Constraints on the Regression Parameters. Econometrica 50(1): 63-80.

Gupta, A., Chen, I.J., and Chiang, D. (1997) Determining Organizational Structure Choices in Advanced Manufacturing Technology Management. Omega, International Journal of Management Science 25(5): 511 - 521.

Helfat, C.E. (1997) Know-how and asset Complementarity and Dynamic Capability Accumulation: The case of R\&D. Strategic Management Journal 18 (5): 339-360.

Hicks, J.R. (1970) Elasticity of Substitution Again: Substitutes and Complements. Oxford Economic Papers 22: 289 - 296.

Hicks, J.R., Allen, R.G.D. (1934) A Reconsideration of the Theory of Value. Parts I and II, Economica, N.S., 1(1): $52-76 ; 1(2): 196-219$.

Hitchcock, J. (2002) Complementarity [on-line]. International Society for the Systems Sciences, U.S. [cited 2002-10-30]. Available at http://www.newciv.org/ISSS_Primer/asem20jh.html.

Ichiniowski, C., Shaw, K., Prennush, G. (1997) The Effects of Human Resource Management Practices on Productivity: A Study of Steel Finishing Lines. The American Economic Review 87(3): 291-313. 
Kakati, M. (1997) Strategic Evaluation of Advanced Manufacturing Technology. International Journal of Production Economics 53: 141-156.

Kim, G., Park, C.S., Yoon, K.P. (1997) Identifying Investment Opportunities for Advanced Manufacturing Systems with Comparative-Integrated Performance Measurement. International Journal of Production Economics 50:23-33.

Kodde D. A., Palm, P.C. (1986) Wald Criteria for Jointly Testing Equality and Inequality Restrictions. Econometrica, 54 (5): 1243-1248.

Koste, L.L., Malhotra, M.K. (1999) A Theoretical Framework for Analyzing the Dimensions of Manufacturing Flexibility. Journal of Operations Management 18 (1): 75-93.

Kotha, S., Swamidass, P.M. (2000) Strategy, Advanced Manufacturing Technology and Performance: Empirical Evidence from U.S. Manufacturing Firms. Journal of Operations Management 18: 257-277.

Knupling, F. (1997) Testing Between Different Types of Switching Regression Models. Institut fur Allgemeine Wirschaftsforschung, Germany.

Lee, C.S. (2001) Modeling the Business Value of Information Technology. Information \& Management 39 (3): 191-210.

Lei, D., Hitt, M.A., Goldhar, J.D. (1996) Advanced Manufacturing Technology: Organizational Design and Strategic Flexibility. Organization Studies 17 (3): 501-523.

Meredith, J. (1987) The Strategic Advantages of new Manufacturing Technologies for Small Firms. Strategic Management Journal 8: 249-258.

Meredith, J., Suresh, N. (1986) Justification Techniques for Advanced Manufacturing Technologies. International Journal of Production Research 24(5): 1043-1057.

Milgrom, P., Roberts, J. (1990) Rationalizability, Learning, and Equilibrium in Games with Strategic Complmentarities. Econometrica 58 (6): 1255-1277.

Milgrom, P., Roberts, J. (1994) Comparing Equilibria. American Economic Review 84 (3): 441459.

Milgrom, P., Roberts, J. (1995) Complementarities and Fit - Strategy, Structure, and Organizational-Change in Manufacturing. Journal of Accounting Economics 19 (2-3): 179-208.

Milgrom, P., Roberts, J. (1995b) The Economics of Modern Manufacturing - Reply. American Economic Review 85 (4): 997- 999.

Milgrom, P., Roberts, J. (1996) The LeChatelier Principle. American Economic Review 86 (1): 173-179. 
Milgrom, P., Qian, Y.Y., Roberts, J. (1991) Complementarities, Momentum, and the Evolution of Modern Manufacturing. American Economic Review 81 (2):84-88.

Milgrom, P., Shannon, C. (1994) Monotone Comparative Statics. Econometrica 62 (1): 157-180.

Miller, P., Oleary, T. (1997) Capital Budgeting Practices and Complementarity Relations in the Transition to Modern Manufacture: A Field-Based Analysis. Journal of Accounting Research 35 (2): $257-271$.

Mohnen, P., Roller, L.H. (2003) Complementarities in Innovation Policy. CIRANO, Under Review.

Moorman, C., Slotegraaf, R.J. (1999) The Contingency Value of Complementary Capabilities in Product Development. Journal of Marketing Research 36 (2): 239-257.

Park, C.S., Kim, G. (1995) An Economic Evaluation Model for Advanced Manufacturing Systems Using Activity-Based Costing. Journal of Manufacturing Systems 14(6): 439-451.

Porter, M.E. (1996) What is Strategy? Harvard Business Review 74 (6): 61-78.

Rivkin, J.W. (2000) Imitation of Complex Strategies. Management Science 46 (6): 824-844.

Roberts, Georgia, Kovacevic, Milorad, Phillips, Owen, Gentleman, Jane. (2003) Bridging the Gap Between Theory and Practice of Analysis of Data from Complex Surveys. Statistics Canada DARC Publication.

Roller, L.H, Tombak, M.M. (1993) Competition and Investment in Flexible Technologies. Management Science 39 (1):107-114.

Sabourin, D., Beckstead, D. (1999) Technology Adoption in Canadian Manufacturing, Statistics Canada $88 F 0006 X P B-05$.

Samuelson, P. (1974) Complementarity: An Essay on the $40^{\text {th }}$ Anniversary of the Hicks-Allen Revolution in Demand Theory. Journal of Economic Literature 12: 1255-1289.

Sanchez, A.M. (1996) Adopting Advanced Manufacturing Technologies: Experience from Spain. Journal of Manufacturing Systems 15(2): 133-140.

Schlie, T.W, Goldhar, J.D. (1995) Advanced Manufacturing and new Directions for Competitive Strategy. Journal of Business Research 33 (2): 103-114.

Shephard, D., McDermott, C., Stock, G. (2000) Advanced Manufacturing Technology: Does Radicalness mean more Perceived Benefits? The Journal of High Technology Management Research 11(1):19-33.

Siggelkow, N. (2001) Misperceiving Interactions: Organizational Consequences. Wharton School, University of Pennsylvania, Unpublished. 
Small, M., Chen, I. (1995) Investment Justification of Advanced Manufacturing Technology: An Empirical Analysis. Journal of Engineering Technology Management 12: 27-55.

Small, M., Yasin, M. (1997) Advanced Manufacturing Technology: Implementation Policy and Performanace. Journal of Operations Management 15: 349-370.

Stevenson, William, Hojati, Mehran (2001) Production Operations Management McGraw-Hill, Toronto.

Sun, H., Gertsen, F. (1995) Organizational Changes Related to Advanced Manufacturing Technology in the Production area. International Journal of Production Economics 41: 369-375.

Takahashi, T. (2001) The role of Knowledge and Organization in the Competitiveness of Japanese High-tech Industry. International Journal of Technology Management 22 (5-6): 480502.

Teece, D.J., Pisano, G., Shuen, A. (1997) Dynamic Capabilities and Strategic Management. Strategic Management Journal 18 (7): 509-533.

Topkis, D.M. (1978) Minimizing a Submodular Function on a Lattice. Operations Research 26 (2): 305- 321.

Topkis, D.M. (1995) Comparative Statics of the Firm. Journal of Economic Theory 67(2): 370401.

Topkis, D.M. (1995b) The Economics of Modern Manufacturing - Comment. American Economic Review 85 (4): 991- 996.

Troxler, J. (1990) Estimating the Cost Impact of Flexible Manufacturing. Journal of Cost Management 4(2): 26-32.

Tyler, B.B. (2001) The Complementarity of Cooperative and Technological Competencies: a Resource-based Perspective. Journal of Engineering and Technology Management 18 (1): 1-27.

Udo, G., Ehie, I. (1996) Critical Success Factors for Advanced Manufacturing Systems. Computers and Industrial Engineering 31(2): 91-94.

Uzawa, H. (1962) Production Functions with Constant Elasticities of Substitution. The Review of Economic Studies 29: 291 - 299.

Verbeek, M. (2000) A Guide to Modern Econometrics John Wiley \& Sons, Ltd., West Sussex, England.

Whittington, R., Pettigrew, A., Peck, S., et al. (1999) Change and Complementarities in the new Competitive Landscape: A European Panel Study, 1992-1996. Organization Science 10 (5): 583600 . 


\title{
APPENDIX A - DEFINITION OF ADVANCED TECHNOLOGIES
}

\author{
Advanced Technologies by Functional Group
}

\begin{tabular}{|c|c|}
\hline TECHNOLOGIES & DESCRIPTION \\
\hline \multicolumn{2}{|l|}{ Design and Engineering } \\
\hline $\begin{array}{l}\text { a) Computer-aided design and engineering } \\
\text { (CAD/CAE) }\end{array}$ & $\begin{array}{l}\text { Use of computer-based software for designing and } \\
\text { testing new products }\end{array}$ \\
\hline $\begin{array}{l}\text { b) CAD output to control manufacturing machines } \\
\text { (CAD/CAM) }\end{array}$ & $\begin{array}{l}\text { Computer-aided manufacturing uses the output } \\
\text { produced by CAD systems to control the machines } \\
\text { that manufacture the part or the product }\end{array}$ \\
\hline c) Modeling or simulation technologies & $\begin{array}{l}\text { Used to provide a computer-based visualization of } \\
\text { the performance of a computer-aided design, e.g., } \\
\text { the simulation of the flow of molten plastic into an } \\
\text { injection mould }\end{array}$ \\
\hline d) Electronic exchange of CAD files & Electronic transfer of computer-aided design files \\
\hline \multicolumn{2}{|l|}{ Processing, Fabrication, Assembly } \\
\hline a) Flexible manufacturing systems & $\begin{array}{l}\text { Collections of computer-controlled machine tools, } \\
\text { serviced by robots and/or automated material } \\
\text { handling systems and overseen by computers }\end{array}$ \\
\hline b) Programmable logic controllers & $\begin{array}{l}\text { Programmable solid state units that are used as } \\
\text { switching devices }\end{array}$ \\
\hline c) Lasers for materials processing & $\begin{array}{l}\text { These are used for such processes as welding, } \\
\text { cutting, treating, scribing and marking }\end{array}$ \\
\hline d) Robots with sensing & $\begin{array}{l}\text { Robots programmed to alter their function based } \\
\text { on input from sensors-more sophisticated robots }\end{array}$ \\
\hline e) Robots without sensing & $\begin{array}{l}\text { Robots programmed to undertake simple tasks } \\
\text { such as pick and place-less sophisticated robots }\end{array}$ \\
\hline f) Rapid prototyping systems & $\begin{array}{l}\text { Systems capable of producing a prototype part } \\
\text { from the output of a computer-aided design }\end{array}$ \\
\hline g) High speed machining & $\begin{array}{l}\text { Metal cutting machines operating at speeds of } \\
10,000 \mathrm{rpm} \text { or higher }\end{array}$ \\
\hline h) Near net shape technologies & $\begin{array}{l}\text { Technologies that produce finished plastic, metal } \\
\text { or composite parts in a single production stage with } \\
\text { a minimum of final machining }\end{array}$ \\
\hline \multicolumn{2}{|l|}{ Automated Material Handling } \\
\hline a) Part identification for manufacturing automation & $\begin{array}{l}\text { Use of machine readable labels for monitoring } \\
\text { parts during the manufacturing process and } \\
\text { afterwards when they are being stored }\end{array}$ \\
\hline
\end{tabular}




\begin{tabular}{|c|c|}
\hline b) Automated storage/retrieval system & $\begin{array}{l}\text { Use computer-controlled equipment to handle and } \\
\text { store goods and materials }\end{array}$ \\
\hline \multicolumn{2}{|l|}{ Inspection } \\
\hline $\begin{array}{l}\text { a) Automated vision-based systems used for } \\
\text { inspection/testing }\end{array}$ & $\begin{array}{l}\text { Systems typically using a computer-controlled } \\
\text { video camera for inspecting products for defects, } \\
\text { blemishes, colour, orientation, etc. }\end{array}$ \\
\hline $\begin{array}{l}\text { b) Other automated sensor-based systems used for } \\
\text { inspection/testing }\end{array}$ & $\begin{array}{l}\text { Automated sensor-based equipment used for } \\
\text { inspecting/testing incoming materials or final } \\
\text { products }\end{array}$ \\
\hline \multicolumn{2}{|l|}{ Network Communications } \\
\hline $\begin{array}{l}\text { a) Local area network (LAN) for engineering or } \\
\text { production }\end{array}$ & $\begin{array}{l}\text { Communications networks within a plant used for } \\
\text { exchanging information on the 'shop floor', and } \\
\text { within design and engineering departments }\end{array}$ \\
\hline b) Company-wide computer networks & $\begin{array}{l}\text { Communications networks within an enterprise } \\
\text { extending beyond a single site includes Intranets } \\
\text { and Wide Area Networks (WAN) }\end{array}$ \\
\hline c) Inter-company computer networks & $\begin{array}{l}\text { Wide area communications networks that connect } \\
\text { establishments with their subcontractors, suppliers, } \\
\text { and customers }\end{array}$ \\
\hline \multicolumn{2}{|l|}{ Integration and Control } \\
\hline a) Manufacturing Resource Planning & $\begin{array}{l}\text { Information system used to keep track of machine } \\
\text { loading, production scheduling, inventory control, } \\
\text { and material handling }\end{array}$ \\
\hline b) Computers used for control on the factory floor & $\begin{array}{l}\text { These are 'stand-alone' machines dedicated to } \\
\text { controlling the manufacturing process but are also } \\
\text { capable of other functions }\end{array}$ \\
\hline c) Computer integrated manufacturing & $\begin{array}{l}\text { Totally automated factory, where all activities, } \\
\text { from start to finish, are co-ordinated by computers }\end{array}$ \\
\hline $\begin{array}{l}\text { d) Supervisory control and data acquisition } \\
\text { (SCADA) }\end{array}$ & $\begin{array}{l}\text { Technology which involves 'real-time' monitoring } \\
\text { and controlling of production processes }\end{array}$ \\
\hline e) Use of inspection data for manufacturing control & $\begin{array}{l}\text { Inspection data is used to discriminate between } \\
\text { good and defective parts, and monitoring } \\
\text { production throughput }\end{array}$ \\
\hline f) Digital, remote controlled process plant control & $\begin{array}{l}\text { Local area network used to connect measurement } \\
\text { and control equipment (such as sensors and } \\
\text { controllers) }\end{array}$ \\
\hline g) Knowledge-based software & $\begin{array}{l}\text { Software systems that use artificial intelligence or } \\
\text { rules based on process knowledge to control } \\
\text { manufacturing processes }\end{array}$ \\
\hline
\end{tabular}

Source: Sabourin and Beckstead, 1999 


\section{APPENDIX B - SURVEYS}

\section{B1 Survey of Advanced Technology in Canadian Manufacturing - 1998}

This Appendix provides a copy of the questionnaire used in the survey.

\section{Section A - General Questions}

A1. Please indicate the geographic region of the head office of your controlling firm.

\begin{tabular}{|l|l|}
\hline Canada & \\
\hline U.S.A. & \\
\hline Europe & \\
\hline Pacific Rim & \\
\hline Other foreign & \\
\hline
\end{tabular}

A2. Please indicate the average number of employees working in your plant.

\begin{tabular}{|l|l|}
\hline Less than 50 & \\
\hline 50 to 99 & \\
\hline 100 to 249 & \\
\hline 250 or more & \\
\hline
\end{tabular}

A3. Please indicate in which of the following markets your plant's primary product is sold.

\begin{tabular}{|l|l|}
\hline Canadian markets & \\
\hline U.S. markets & \\
\hline European markets & \\
\hline Pacific Rim markets & \\
\hline Other foreign markets & \\
\hline
\end{tabular}

A4. Please indicate how many firms (both domestic and foreign owned) offer products directly competing with your plant's primary product.

\begin{tabular}{|l|l|}
\hline None & \\
\hline 1 to 5 & \\
\hline 6 to 20 & \\
\hline Over 20 & \\
\hline
\end{tabular}


A5. Please rate the importance of the following factors in your firm's business strategy.

\begin{tabular}{|l|l|l|l|l|l|}
\hline & \multicolumn{3}{|l}{ IMPORTANCE } \\
\hline & low & & & & high \\
\hline & 1 & 2 & 3 & 4 & 5 \\
\hline Products and Marketing & & & & & \\
\hline a) Developing new products & & & & & \\
\hline b) Entering new markets & & & & & \\
\hline Technology & & & & & \\
\hline c) Reducing manufacturing costs & & & & & \\
\hline $\begin{array}{l}\text { d) Developing new } \\
\text { manufacturing technology }\end{array}$ & & & & & \\
\hline e) Using new materials & & & & & \\
\hline Human Resources & & & & & \\
\hline $\begin{array}{l}\text { f) Using teams (e.g.,, cross } \\
\text { functional, quality improvement) }\end{array}$ & & & & & \\
\hline g) Ongoing technical training & & & & & \\
\hline
\end{tabular}

\section{$\underline{\text { Section B - Advanced Technologies }}$}

B1. Please indicate whether you are currently using, plan to use (within two years), or have no plans to use the following advanced technologies in your plant.

\begin{tabular}{|l|l|l|l|}
\hline TECHNOLOGIES & In Use & Plan to Use & No Plans \\
\hline Design and Engineering & & & \\
\hline a) Computer-Aided Design/Engineering (CAD/CAE) & & & \\
\hline $\begin{array}{l}\text { b) Computer-Aided Design/Manufacturing } \\
\text { (CAD/CAM) }\end{array}$ & & & \\
\hline c) Modelling or simulation technologies & & & \\
\hline d) Electronic exchange of CAD files & & & \\
\hline Processing, Fabrication and Assembly & & & \\
\hline $\begin{array}{l}\text { a) Flexible Manufacturing Cells or Systems } \\
\text { (FMC/FMS) }\end{array}$ & & & \\
\hline $\begin{array}{l}\text { b) Programmable Logic Control (PLC) machines or } \\
\text { processes }\end{array}$ & & & \\
\hline $\begin{array}{l}\text { c) Lasers used in materials processing (including } \\
\text { surface modification) }\end{array}$ & & & \\
\hline
\end{tabular}




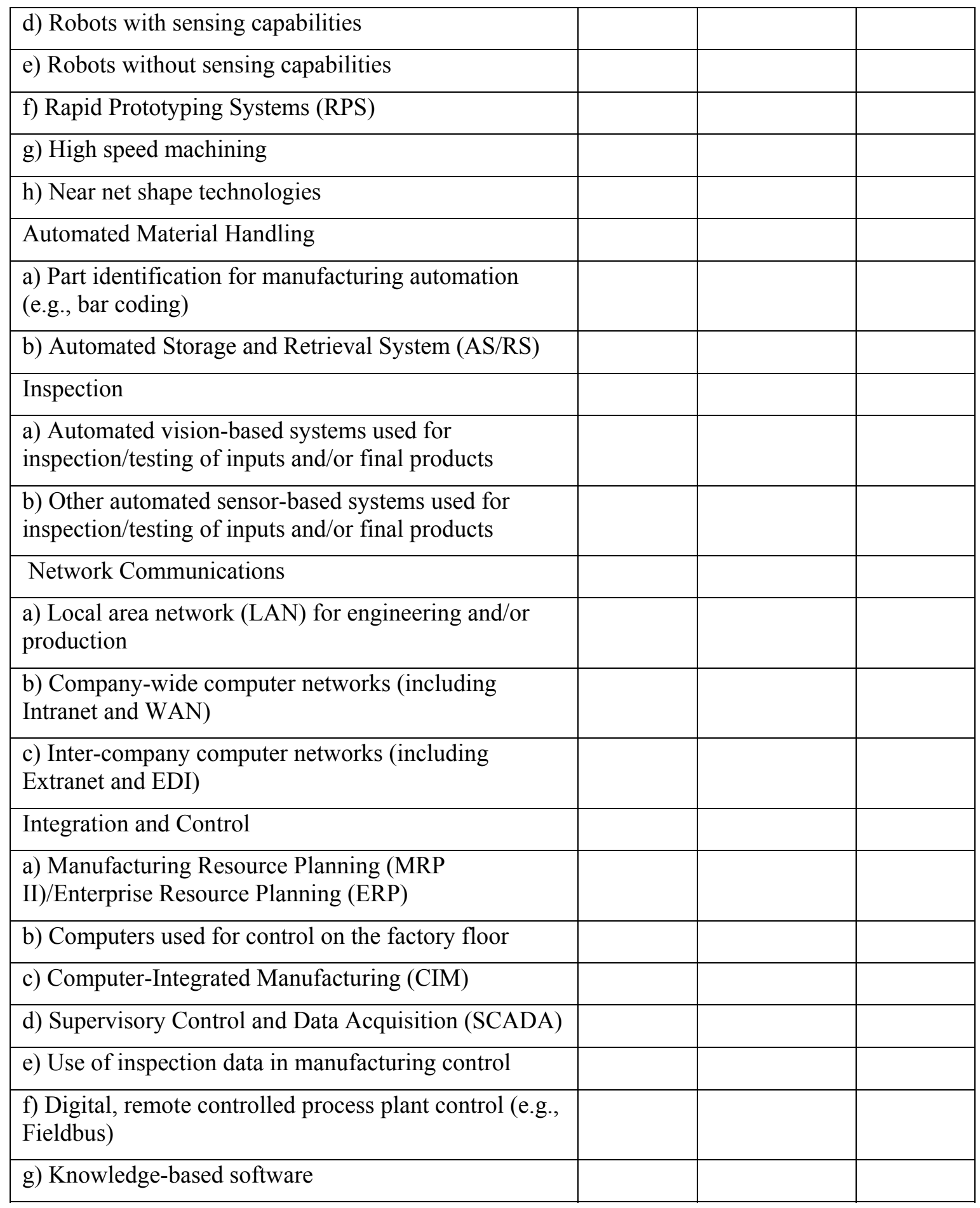


B2. Over the last three years, what percentage of your plant's investment in machinery and equipment was spent on advanced equipment (as listed in question B1 above)?

\begin{tabular}{|l|l|}
\hline Zero percent & \\
\hline $1 \%$ to $25 \%$ & \\
\hline $26 \%$ to $50 \%$ & \\
\hline $51 \%$ to $75 \%$ & \\
\hline $76 \%$ to $100 \%$ & \\
\hline
\end{tabular}

B3. How would you compare your plant's production technology with that of your most significant competitors?

\begin{tabular}{|l|l|l|l|l|l|l|}
\hline COMPETITORS & \multicolumn{2}{l|}{ less advanced } & & \multicolumn{2}{l|}{ more advanced } & \\
\hline & 1 & 2 & 3 & 4 & 5 & N/A \\
\hline a) Other producers in Canada & & & & & & \\
\hline b) Producers in the U.S. & & & & & & \\
\hline
\end{tabular}

B4. For what purposes does your plant use communications networks (Internet, Intranet, Extranet, VAN)? Check all that apply.

\begin{tabular}{|l|l|l|l|}
\hline & YES & NO & NA \\
\hline a) ordering products & & & \\
\hline b) tracking production flow & & & \\
\hline c) on-line maintenance & & & \\
\hline d) tracking sales and inventory & & & \\
\hline e) tracking distribution & & & \\
\hline f) sharing technology information & & & \\
\hline g) accounting and financing & & & \\
\hline h) human resources purposes & & & \\
\hline i) management planning system & & & \\
\hline j) marketing/customer information & & & \\
\hline k) financial transactions (e.g.,, banking) & & & \\
\hline l) consumer information & & & \\
\hline m) production status information & & & \\
\hline $\begin{array}{l}\text { n) general reference (e.g., phone } \\
\text { numbers) }\end{array}$ & & & \\
\hline o) other & & & \\
\hline
\end{tabular}




\section{$\underline{\text { Section C - Business Practices }}$}

$\mathrm{C} 1$. Are the following practices or techniques regularly used in your plant?

\begin{tabular}{|l|l|l|l|}
\hline & YES & NO & NA \\
\hline a) cross-functional design teams & & & \\
\hline b) concurrent engineering & & & \\
\hline $\begin{array}{l}\text { c) continuous improvement (including } \\
\text { TQM) }\end{array}$ & & & \\
\hline d) benchmarking & & & \\
\hline e) plant certification (e.g., ISO9000) & & & \\
\hline f) certification of suppliers & & & \\
\hline g) just-in-time inventory control & & & \\
\hline h) statistical process control & & & \\
\hline i) electronic work order management & & & \\
\hline j) process simulation & & & \\
\hline k) distribution resource planning & & & \\
\hline 1) quality function deployment & & & \\
\hline
\end{tabular}

\section{$\underline{\text { Section D - Development and Implementation of Advanced Technologies }}$}

D1. Have any advanced technologies (as listed in question B1) been introduced into your plant? Yes No

If $\mathrm{NO}$, then please go to question G1.

D2. If YES, by which method does your plant introduce advanced technologies?

\begin{tabular}{|l|l|l|}
\hline & YES & NO \\
\hline a) by purchasing off-the-shelf equipment & & \\
\hline b) by licensing new technology & & \\
\hline $\begin{array}{l}\text { c) by customizing or significantly modifying } \\
\text { existing technology }\end{array}$ & & \\
\hline $\begin{array}{l}\text { d) by developing brand new advanced technologies } \\
\text { (either alone or in conjunction with others) }\end{array}$ & & \\
\hline
\end{tabular}


D3. Please indicate which of the following sources play an important role in providing ideas for the adoption of advanced technology in your plant. Please check all that apply.

\begin{tabular}{|l|l|l|l|}
\hline & YES & NO & NA \\
\hline INTERNAL to your firm & & & \\
\hline a) research & & & \\
\hline b) experimental development & & & \\
\hline c) production engineering & & & \\
\hline d) corporate head office & & & \\
\hline e) related plants & & & \\
\hline f) technology watch program & & & \\
\hline g) production staff & & & \\
\hline h) design staff & & & \\
\hline i) sales and marketing & & & \\
\hline j) other & & & \\
\hline EXTERNAL to your firm & & & \\
\hline k) trade fairs, conferences, publications & & & \\
\hline 1) patents & & & \\
\hline m) consultants/service firms & & & \\
\hline n) suppliers & & & \\
\hline o) customers & & & \\
\hline p) related firms & & \\
\hline q) universities & & \\
\hline r) governments, institutes, associations & & \\
\hline s) other producers in your industry & & \\
\hline t) other & & \\
\hline
\end{tabular}

Section E - Skill Requirements

E1. Have your plant employees received any training pertaining to the adoption of advanced technology in the last three years?
Yes
No

If $\mathrm{NO}$, then please go to question $\mathrm{E} 3$. 
E2. If YES, please indicate in which of the following areas training was provided. Please include both on-site and off-site training. Check all that apply.

\begin{tabular}{|l|l|l|l|}
\hline & YES & NO & NA \\
\hline a) basic literacy/numeracy & & & \\
\hline b) computer literacy & & & \\
\hline c) technical skills & & & \\
\hline d) quality control skills & & & \\
\hline e) safety skills & & & \\
\hline f) other & & & \\
\hline
\end{tabular}

E3. In the operation of advanced technology, for which types of skilled personnel have you experienced shortages at your plant during the past year? Please check all that apply.

\begin{tabular}{|l|l|l|l|}
\hline & YES & NO & NA \\
\hline Professionals with university degree: & & & \\
\hline a) mechanical/aerospace & & & \\
\hline b) electronic/computer & & & \\
\hline c) chemical/chemical process & & & \\
\hline d) industrial/manufacturing process & & & \\
\hline e) science professionals & & & \\
\hline f) computer scientists & & & \\
\hline Management: & & & \\
\hline g) production management & & & \\
\hline h) design management & & & \\
\hline i) human resources management & & & \\
\hline $\begin{array}{l}\text { Technicians/Technologists (Community } \\
\text { College/CEGEP): }\end{array}$ & & & \\
\hline j) electronics/computer hardware & & & \\
\hline k) science technicians & & & \\
\hline 1) engineering science technicians & & & \\
\hline m) computer programmers & & & \\
\hline administration communications network & & & \\
\hline o) computer aided design & & \\
\hline p) instrumentation & & \\
\hline Skilled Trades: & & \\
\hline
\end{tabular}




\begin{tabular}{|l|l|l|l|}
\hline q) machinist ( including tool, die mould) & & & \\
\hline r) machine operator & & & \\
\hline s) Electronic equipment operator & & & \\
\hline t) process plant operator & & & \\
\hline Other: & & & \\
\hline u) other & & & \\
\hline
\end{tabular}

If you are NOT experiencing any skill shortages at your plant, then please go to question F1.

E4. Have you taken any steps at your plant to deal with these shortages?

Yes No

If $\mathrm{NO}$, then please go to question $\mathrm{F} 1$.

E5. If YES, what steps have you taken? Check all that apply.

\begin{tabular}{|l|l|l|l|}
\hline & YES & NO & NA \\
\hline a) provided training & & & \\
\hline b) improved wages and benefits & & & \\
\hline $\begin{array}{l}\text { c) established stronger links with } \\
\text { educational institutions (e.g.,, research } \\
\text { scholarships, hired summer students) }\end{array}$ & & & \\
\hline d) searched for skilled personnel & & & \\
\hline e) other & & & \\
\hline
\end{tabular}

E6. In order to deal with these skill shortages, did you search for personnel

\begin{tabular}{|l|l|l|}
\hline & YES & NO \\
\hline a) within your region & & \\
\hline b) outside your region (in Canada) & & \\
\hline c) outside Canada & & \\
\hline
\end{tabular}




\section{Section F - Results of Adoption}

F1. Rate the importance of the following effects related to the adoption of advanced technology by your plant.

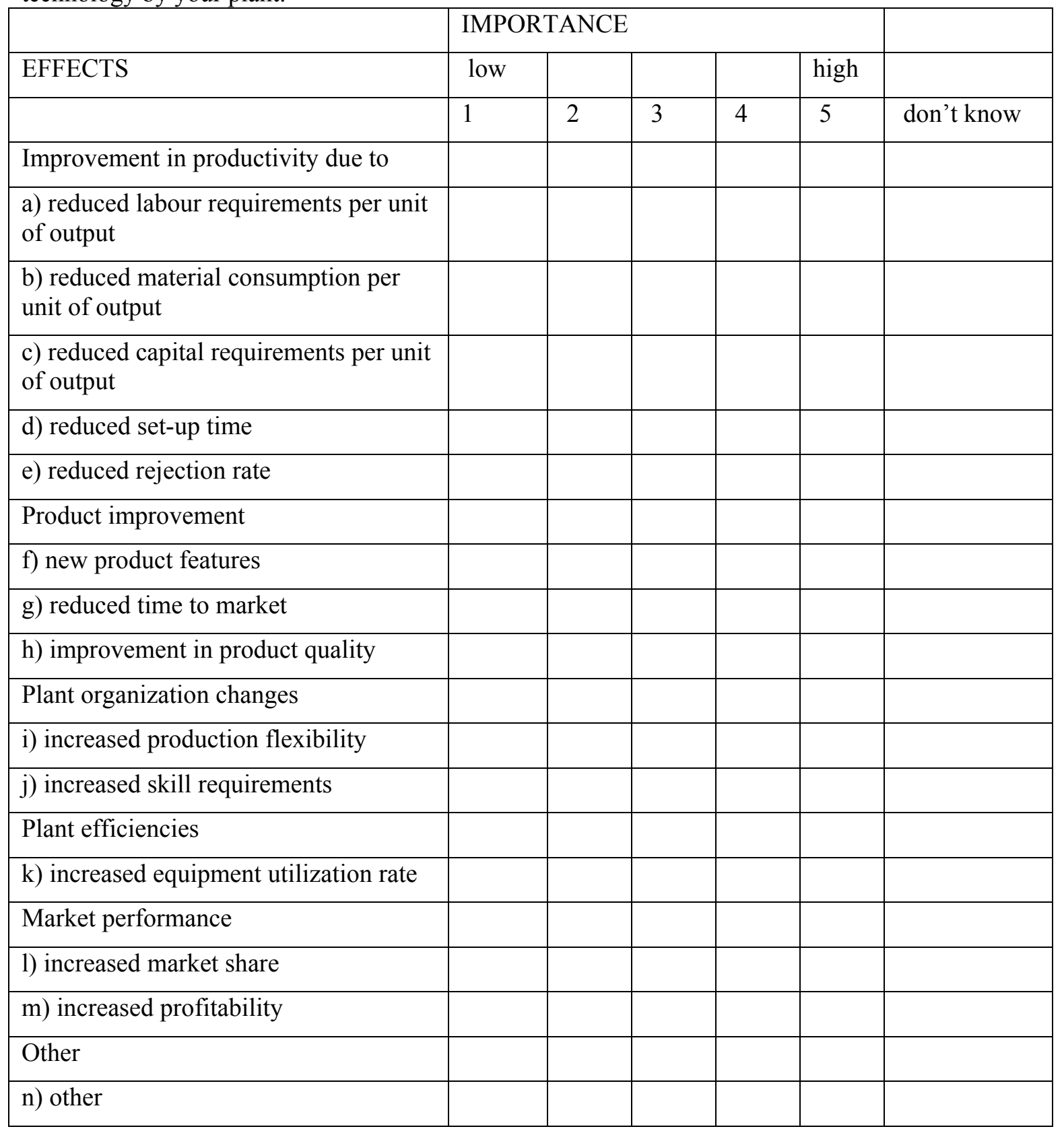




\section{$\underline{\text { Section G - Obstacles to Adoption }}$}

G1. Rate the importance of the following factors as obstacles to advanced technology adoption by your plant.

\begin{tabular}{|l|l|l|l|l|l|}
\hline & \multicolumn{3}{|l|}{ IMPORTANCE } \\
\hline OBSTACLES & low & & & & high \\
\hline & 1 & 2 & 3 & 4 & 5 \\
\hline Lack of financial justification due to & & & & & \\
\hline a) small market size & & & & & \\
\hline b) high cost of equipment & & & & & \\
\hline c) cost of capital & & & & & \\
\hline d) costs to develop software & & & & & \\
\hline $\begin{array}{l}\text { e) cost of integration of new } \\
\text { technology }\end{array}$ & & & & & \\
\hline Human resources & & & & & \\
\hline f) shortage of skills & & & & & \\
\hline g) worker resistance & & & & & \\
\hline Management & & & & & \\
\hline $\begin{array}{l}\text { h) resistance to introduction of new } \\
\text { technology }\end{array}$ & & & & & \\
\hline i) inability to evaluate new technology & & & & & \\
\hline External support services & & & & & \\
\hline $\begin{array}{l}\text { j) lack of technical support or service } \\
\text { (from consultants or vendors) }\end{array}$ & & & & & \\
\hline Other & & & & & \\
\hline k) other & & & & & \\
\hline
\end{tabular}

\section{$\underline{\text { Section H - Research and Development Activity }}$}

H1. Please indicate whether or not your firm has been involved in any of the following R\&D activities over the last three years. Please exclude quality control, routine testing, style changes, minor adaptations and market research.

\begin{tabular}{|l|l|l|}
\hline & YES & NO \\
\hline a) does your firm do R\&D in-house? & & \\
\hline b) does your firm do R\&D jointly with another firm? & & \\
\hline c) does your firm contract out R\&D? & & \\
\hline
\end{tabular}

If you answered $\mathrm{NO}$ to all three parts of question $\mathrm{H} 1$, then please go to question I1. 
$\mathrm{H} 2$. Please indicate the frequency of R\&D in your firm.

\begin{tabular}{|l|l|l|}
\hline & YES & NO \\
\hline a) R\&D are performed on an ongoing basis & 1.9 & 1.9 \\
\hline b) R\&D are performed on an occasional basis & 1.9 & 1.9 \\
\hline
\end{tabular}

H3. What is your firm's R\&D program responsible for?

\begin{tabular}{|l|l|l|}
\hline & YES & NO \\
\hline a) creating original products & 1.7 & 1.7 \\
\hline $\begin{array}{l}\text { b) creating original production equipment or new process } \\
\text { technology }\end{array}$ & 1.8 & 1.8 \\
\hline c) substantially adapting technology acquired from others & 1.8 & 1.8 \\
\hline $\begin{array}{l}\text { d) introducing off-the-shelf equipment or process } \\
\text { technology }\end{array}$ & 1.8 & 1.8 \\
\hline
\end{tabular}

\section{$\underline{\text { Section I - Electronic Communication }}$}

I1. Does your firm use e-mail?

Yes No

I2. Does your firm use Internet?

Yes No

If NO, then please go to question I5.

I3. If YES, please indicate for what purposes your firm uses the Internet.

\begin{tabular}{|l|l|l|}
\hline & YES & NO \\
\hline a) searching on the World Wide Web & & \\
\hline b) selling your goods and services & & \\
\hline c) advertising/marketing your goods and services & & \\
\hline d) purchasing goods and services & & \\
\hline e) secure electronic transactions & & \\
\hline f) sharing research and development (R\&D) & & \\
\hline g) other & & \\
\hline
\end{tabular}


I4. Does your firm have a home page on the World Wide Web?

Yes No

I5. Does your firm use electronic data interchange (EDI)?

Yes No

If $\mathrm{NO}$, then please go to the end of the questionnaire.

I6. If YES, what type of communication network setup does your firm use for EDI? Check all that apply.

\begin{tabular}{|l|l|l|}
\hline & YES & NO \\
\hline a) Value Added Network (VAN) & & \\
\hline b) Internet & & \\
\hline c) Extranet & & \\
\hline
\end{tabular}




\section{B2 Annual Survey of Manufactures}

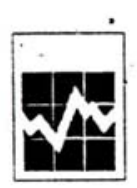

Manufacturing. Construction and Energy Division

Annual Survey of Manufactures, 1999

Head Office and Other Business

Support Units

REFEREMCE NUMBER
口 Si vous préférez recevoir ce questionnaire en français, veuillez cocher la case et retourner à la Division des operations et de l'intégration, Statistique Canada Ottawa, K1A OT

ou

éléphoner au 1-800-386-1276 $1-800-386-1277$

\section{$\uparrow$}

Mailing Address (Please correct if necessary)

Physical Location of Establishment (Please correct if necessary)

PURPOSE OF THE SURVEY:

This survey of head offices and other business support units is conducted to improve the accuracy and completeness of statistics on businesses in Canada. Head Thtrice units, by purchasing sevices and performing general administrative functions for the enterprise as a whole, account for significant expenses and employment. These data must be included in the statistics for the whole economy.

Other business support units (ie, those which provide specialized functions in support $d$ other units of the firm) have a similar importance to general economic statistics. Such support units include warehouses, sales centres, trucking faciltiles, data processing faciltiles, research and development centres, efc.

Some head offices and support units undertake additional actions, such as allocating their expenses to other operating units, or generating revenue, either through transactions with other operating units, or with external cllents. These issues are vital to providing a complete picture of how the firm performs and they are covered by the content of this survey form.

These data will be aggregated with information from other sources to produce official estimates of national and provincial ecomomic production in Canada, as well as offlicial estimates of activity by industry. Those estimates are used by government for national and regional programs and policy planning and by the private sector for Industry performance measurement and market development.

COVERAGE:

Please complete this questionnaire for the operation(s) and location(s) described on the address label above. You should only report for those operations located in Canada.

PLEASE COMPLETE AND RETURN BY: MAY 15, 2000

If necessary, please provide your best estimates.

Please keep a completed copy for future reference.

For assistance, call 1-800-386-1276 or 1-800-386-1277.

1.9 REPORTING YEAR - This report covers your most recent financial year ending between January 1, 1999 and December 31, 1909.

from

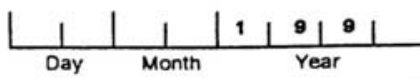

to

\begin{tabular}{|l|l|l|l|l|l|}
$\mid$ & $\mid$ & 1 & 9 & 9 & 9 \\
\hline Day & Menth
\end{tabular}

CONTACT INFORMATION

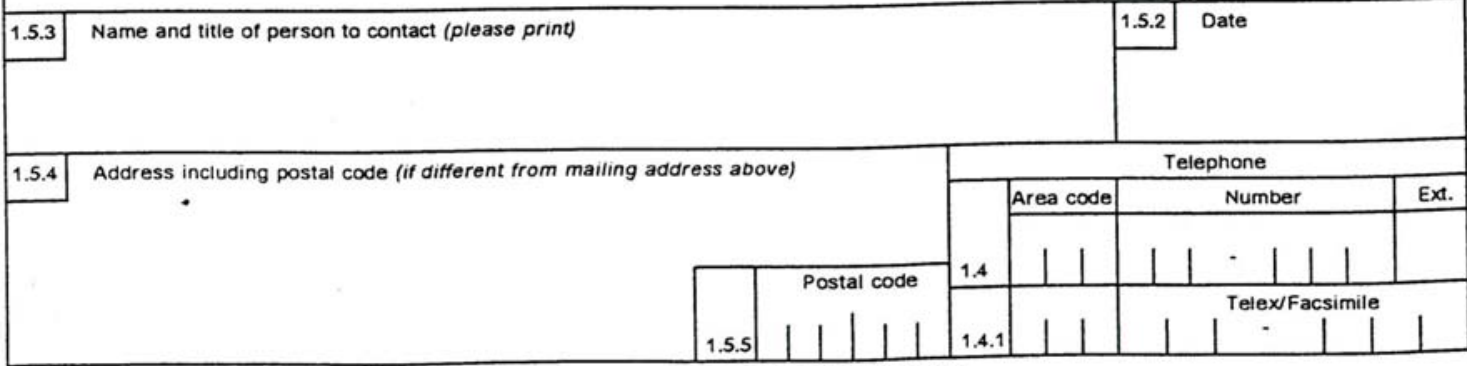

STC / MCED-310-60195 


\section{AUTHORITY:}

This annual survey is conducted under the authority of the Statistics Act, Revised Statutes of Canada 1985, Chapter S19. Completion of this questionnaire is a legal requirement under the Statistics Act.

\section{CONFIDENTIALITY:}

Statistics Canada is prohibited by law from publishing any statistics which would divulge information obtained from this survey that relates to any identifiable business without the previous written consent f that business. The treated in strict confidence, used for for statistical purposes and published in aggregate form only.

\section{DATA SHARING AGREEMENTS:}

To avoid duplication of information collection and to ensure more uniform statistics. Statistics Canada has entered into agreements with various government departments and agencies for the sharing of data from this survey for statistical purposes only. Please note that Statistics Canada does not share any individual responses with Revenue Canada. See the Reporting Guide for details.

\section{TO RETURN THIS BY FAX, CALL: 1-800-386-1278.}

Statistics Canada advises you that there is a risk of disclosure during the facsimile communication process.

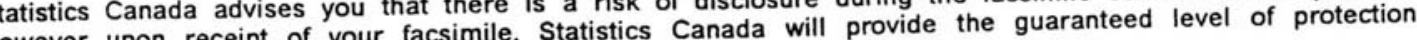
afforded all information collected under the authority of the Statistics Act.

\section{REPORTING INSTRUCTIONS:}

1. All dollar amounts reported should be rounded to THOUSANDS OF CANADIAN DOLLARS (\$'000 CDN) (e.g. $\$ 8,555,400.00$ should be rounded to $\$ 8,555$ ).

2. Your best estimates are acceptable when precise figures are not available.

3. DO NOT COMPLETE SHADED AREAS.

$\begin{array}{lrl}\text { 1.6.1 Type of organization } & 1 \\ \text { (Check one) } & { }^{1} & \begin{array}{l}\text { Incorporated } \\ \text { company } \\ \text { Unincorporated } \\ \text { company }\end{array} \\ & { }^{3} \square\end{array}$

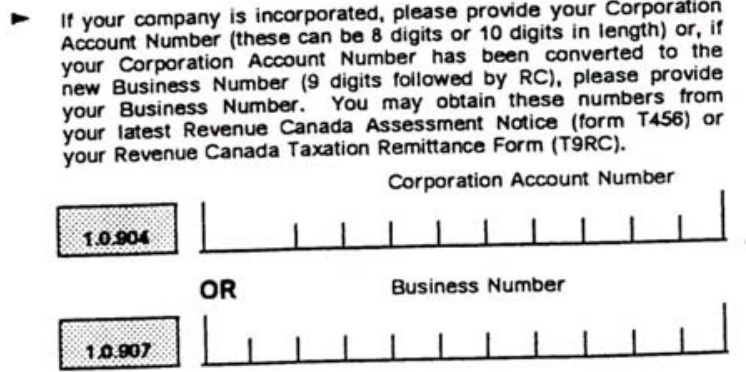

\section{MAIN BUSINESS ACTIVITY}

1.8.1 Check only ONE item which BEST describes the principal activity at this location;

1. $\square$ Centralized administration(corporate, general home or central office)

2. $\square$ Other administration(divisional, branch or district office)

3. $\square$ Research, development and testing laboratories

4. $\square$ Ancillary operations(sales, distribution, warehousing, garage, etc.) Please specify 
$\cdot 3$.

\section{B Revenue of this Head Office (or other business support unit)}

Did the accounts of this head office (or other business support unit) record revenue during the reporting period?
9.0 .10
$1 \square$ Yes
$2 \square$ No

If No, please go to Section C (Expenses).

\section{PLEASE INCLUDE:}

- Revenue recorded in your accounts for sales or transfers to other businesses and to other units of your business.

- All foreign revenue recorded by this head office (or other business support unit).

EXCLUDE:

- Federal or provincial sales taxes collected for remittance to a government agency.

Operating Revenue from transactions with external clients (e.g. third party or non-affiliated businesses) (Exclude receipts from billings to other units of the firm)

\begin{tabular}{|c|c|c|c|}
\hline 1. & Sales of Goods manufactured and/or as & 9.11 & \\
\hline & external cllents and transferred to this head office (or other bi & 0.1 .1 & \\
\hline 2. & $\begin{array}{l}\text { Sales of Goods purchased from outside the firm for resale to external clients } \\
\text { in the same condition as purchased. }\end{array}$ & 9.1 .2 & \\
\hline 3. & $\begin{array}{l}\text { Rental revenue from assets on your books produced by manufacturing and/or logging } \\
\text { establishments of your firm (unless already included in other Survey of Manufactures and/or } \\
\text { Survey of Forestry reports of your firm). }\end{array}$ & 9.5 & \\
\hline 4. & Sales of Services produced by the firm for external clients & 9.6 & \\
\hline 5. & Revenue from lease or rental of property (lands, buildings, offices, etc.) & 11 & \\
\hline 6. & $\begin{array}{l}\text { Revenue from lease or rental of machinery and equipment other than that included in section B, } \\
\text { line } 3 \text { (i.e. from machinery of all kinds, engines, trucks of all types, trailers, other equipment, etc.) }\end{array}$ & 12 & - \\
\hline 7. & $\begin{array}{l}\text { Other operating revenue from transactions with external cllents } \\
\text { (e.g. commissions, royalties and franchise fees, etc.) }\end{array}$ & 9.6 .1 & $=$ \\
\hline 8. & $\begin{array}{l}\text { Total Operating Revenue from transactions with other businesses (i.e. external clients) } \\
\text { (Sum of lines } 1 \text { to } 7 \text { ) }\end{array}$ & 9.8 & \\
\hline
\end{tabular}

Operating Revenue from transactions with internal clients (e.g. other units of this firm)

\begin{tabular}{|c|c|c|}
\hline $\begin{array}{l}\text { 9. Management fees (only) paid to this unit by other units of the firm } \\
\text { Report all other fees for services in section } B \text {, line } 10 \text {. }\end{array}$ & 9.9 .1 & \\
\hline $\begin{array}{l}\text { 10. Other operating revenue from transactions with other units of the firm } \\
\text { (e.g. intra-company sales and other fees) }\end{array}$ & 9.9 .2 & \\
\hline $\begin{array}{l}\text { 11. Total Operating Revenue from transactions with other units of the firm (i.e. internal } \\
\text { clients) (Sum of lines } 9 \text { and 10) }\end{array}$ & 9.10 & \\
\hline 12. Total Operating Revenue (Sum of lines 8 and 11) & 9.11 & \\
\hline 13. Non-operating revenue (e.g.interest income and dividends) & 9.12 & \\
\hline $\begin{array}{l}\text { 14. Total Revenue of this Head Office (or other business support unit) } \\
\text { (Sum of lines } 12 \text { and 13) }\end{array}$ & 9.13 & \\
\hline
\end{tabular}
7-6100-6.1 1999 
$-4$.

\section{Expenses of this Head Office (or other business support unit)}

Please include: all expenses within or outside Canada recorded by this head office (or other business support unit). exclude: income tax and the portion of federal or provincial sales taxes refunded by government.

\section{Expenses for purchased services}

\begin{tabular}{|c|c|c|c|}
\hline \multicolumn{2}{|c|}{ 1. Goods transportation, warehousing and storage expenses. } & 7.8 .1 & \\
\hline \multicolumn{2}{|c|}{ 2. Postage and courier expenses } & 7.8 .2 & \\
\hline \multicolumn{2}{|c|}{ 3. Telephone and other telecommunications expenses } & 7.8 .3 & \\
\hline \multicolumn{2}{|c|}{ 4. Rental and leasing expenses } & 7.8 .4 & \\
\hline \multicolumn{2}{|c|}{ 5. Purchased maintenance and repair services expenses } & 7.8 .5 & \\
\hline \multicolumn{2}{|c|}{ 6. Professional and business service fees } & 7.8 .6 & \\
\hline \multicolumn{2}{|c|}{$\begin{array}{l}\text { 7. Payments to employment agency or personnel supplier. } \\
\text { (e.g. pay for temporary workers paid through an agency and / or charges for } \\
\text { personnel search services) }\end{array}$} & 7.8 .7 & \\
\hline \multicolumn{2}{|c|}{$\begin{array}{l}\text { 8. Financial service fees (e.g. bank charges, etc.) } \\
\text { Exclude interest expenses (report on section C, line 30) }\end{array}$} & 7.8 .8 & \\
\hline \multicolumn{2}{|c|}{ 9. Insurance premiums (liability, auto, building, equipment, etc.) } & 7.8.9 & \\
\hline \multicolumn{2}{|l|}{ 10. Advertising expenses } & 7.8 .11 & \\
\hline \multicolumn{2}{|l|}{ 11. Travel expenses } & 7.8 .12 & \\
\hline \multicolumn{2}{|c|}{ 12. Meals and entertainment expenses } & 7.8.13 & - \\
\hline \multicolumn{2}{|c|}{ 13. Property and business taxes, licenses and permits } & 7.8.14 & \\
\hline \multicolumn{2}{|l|}{ 14. Royalties and franchise fees } & 7.8 .15 & \\
\hline $\begin{array}{l}\begin{array}{l}\text { 15. Other purchased service } \\
\text { expenses }\end{array} \\
\end{array}$ & (p/ease specify) 7.8 .17 & 7.8.16 & \\
\hline \multicolumn{2}{|c|}{ 16. Total expenses for purchased services (Sum of lines 1 to 15) } & 7.9 & \\
\hline
\end{tabular}


Other Expenses

-5 .

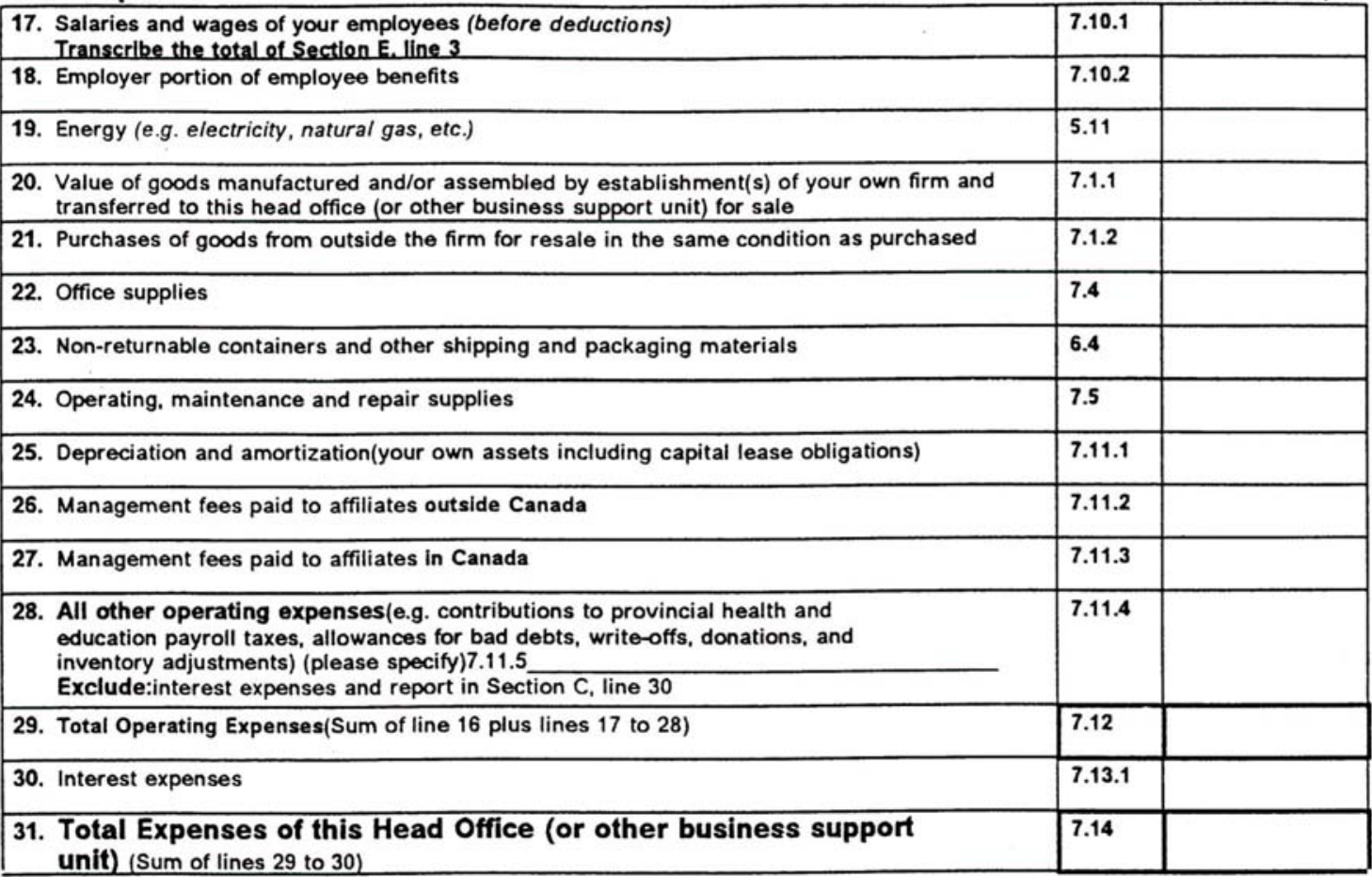

32. Excluding purchased services (which were itemized above), did you allocate any other expenses (e.g. overhead, general administration, etc.) to other units of the firm?

7.15 $1 \square$ Yes - Please enter the amount allocated

$2 \square$ No - Please go to Section D.

\section{Inventory of this Head Office (or other business support unit)}

Inventories are to be reported at book values (the value maintained in the accounting records).

The valuation should:

Include: inventory at this head office (or other business support unit) and at any warehouse or selling outlet which is treated as part of this head office (or other business support unit) and inventory in transit in Canada or on consignment in Canada.

Exclude: inventory held abroad or held on consignment for others. transferred to head office (or other business support unit) for sale 2. Goods purchased for resale in the same condition as purchased,
including raw materials held for manufacturing operations of your own company

\begin{tabular}{|c|c|c|}
\hline & \multicolumn{2}{|c|}{$\left(\$^{\prime} 000 \mathrm{CDN}\right)$} \\
\hline & $\begin{array}{l}\text { Value of opening } \\
\text { inventory }\end{array}$ & $\begin{array}{l}\text { Value of closing } \\
\text { inventory }\end{array}$ \\
\hline 2.1 & & \\
\hline 2.2 & & \\
\hline
\end{tabular}


- 6 .

\section{Inventory of this Head Office (or other business support unit)}

Include inventory in transit in Canada or on consignment in Canada.

Exclude inventory held abroad or held on consignment for others.

Inventories are to be reported at book values (the values maintained in the accounting records).

\begin{tabular}{|c|c|c|c|}
\hline \multirow[b]{2}{*}{ Head Office Inventories } & & \multicolumn{2}{|c|}{$\left(\$^{\prime} 000 \mathrm{CDN}\right)$} \\
\hline & & $\begin{array}{l}\text { Value of opening } \\
\text { inventory }\end{array}$ & $\begin{array}{l}\text { Value of closing } \\
\text { inventory }\end{array}$ \\
\hline $\begin{array}{l}\text { 1. Goods manufactured and/or produced by your firm and } \\
\text { transferred to Head Office for sale }\end{array}$ & 2.1 & & \\
\hline 2. Goods purchased for resale in the same condition as purchased & 2.2 & & \\
\hline
\end{tabular}

Inventories at plants or warehouses that are on the accounts of this Head Office

\begin{tabular}{|l|l|l|l|}
\hline 3. Raw Materials & 2.1 .3 & & \\
\hline 4. Goods in Process & 2.1 .5 & & \\
\hline 5. Other Inventory (specify) & 2.3 & & \\
\hline
\end{tabular}

$7-6100-6.11999$ 
$\cdot 7 \cdot$

\section{E Employment at this Head office (or other business support unit)}

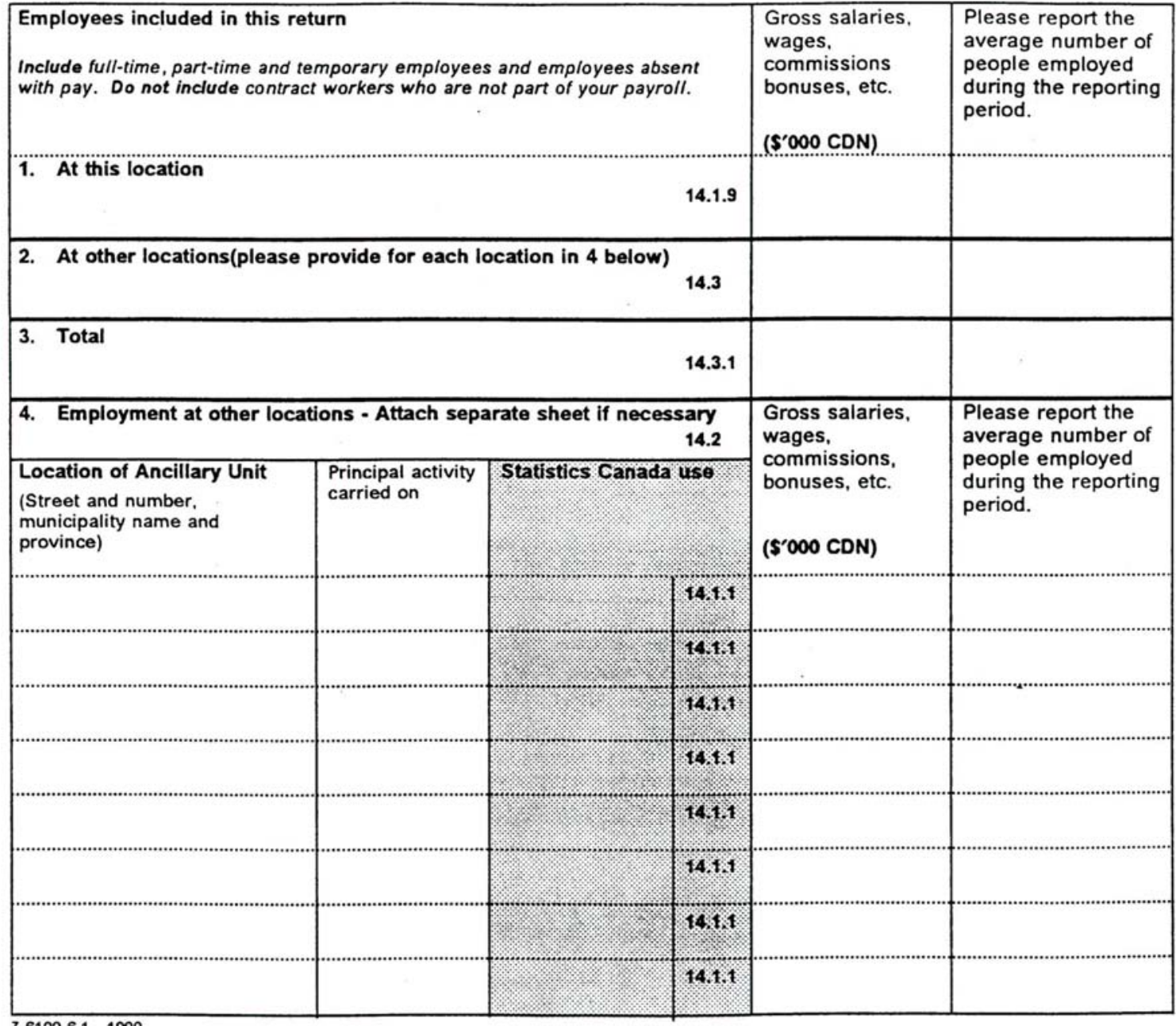


- 8 -

\section{F Significant changes from the previous year}

Compared to last fiscal year, was there any event(s) which have significantly affected your business during the reported period?

If yes, please check as many boxes as apply to explain significant changes in the numbers from last year.

\begin{tabular}{|l|l|l|l|}
\hline & & $\begin{array}{l}\text { Employment } \\
\text { Wages \& } \\
\text { Salaries }\end{array}$ & Output \\
\hline 15.1 & 1. Change in legal structure & & \\
\hline 15.2 & 2. New plant/closed plant & & \\
\hline 15.3 & 3. Plant expansion/contraction & & \\
\hline 15.4 & $\begin{array}{l}\text { 4. Organizational change (downsizing, } \\
\text { reengineering, integration, delayering) }\end{array}$ & & \\
\hline 15.5 & 5. Adverse weather, natural disaster or fire & & \\
\hline 15.6 & 6. Change in competitive environment & & \\
\hline 15.7 & 7. New contract/Loss of contract & & \\
\hline 15.8 & 8. Foreign exchange & & \\
\hline 15.9 & 9. Change in the overtime & & \\
\hline 15.10 & 10. Shutdown & & \\
\hline 15.11 & 11. Contracting out & & \\
\hline 15.12 & 12. Automation & & \\
\hline 15.13 & 13. Labour dispute & & \\
\hline 15.14 & $\begin{array}{l}\text { 14. Changes in bonus payments, drawings, or } \\
\text { severance payments }\end{array}$ & & \\
\hline 15.15 & 15. Change in product line & & \\
\hline 15.16 & 16. Price changes, goods and/or services sold & & \\
\hline 15.17 & 17. Price changes, labour and/or raw materials & & \\
\hline 15.18 & \begin{tabular}{l} 
18. Other, please specify \\
\hline
\end{tabular} & & \\
\hline
\end{tabular}

\section{G Respondent's comments section}

1. How long did you spend collecting the data and completing this form? 15.0 .1 hours

Comments?
We invite your help in improving our business survey program. Your comments on the following range of suggested We invite your help in improving our business survey program. Your

- questionnaire content

- new questions of interest to your industry

- questionnaire language

- use of business terminology

- comprehension of questions (through definitions. examples of inclusions and exclusions, code sheets, instruction sheets, reporting guides, etc.)

- order and flow of questions
- timing of receipt of questionnaire and the period given for response

- other sources of data to further reduce response burden

- potential for electronic data reporting

- general (non-proprietary) business software packages in use.

Lost the postpaid envelope? Our mailing address is:

Operations and Integration Division, Statistics Canada, Ottawa, K1A OT6.

Or call 1-800-386-1276 and we'll send you another envelope.

Or see page -2 - to FAX us the completed questionnaire

Thank you for completing the questionnaire. 


\section{APPENDIX C - INDUSTRY STRATIFICATION}

Industry strata used for sample selection for the survey

\begin{tabular}{|c|c|}
\hline 1980 SIC-E & Description \\
\hline 11 & Beverage Industries \\
\hline 12 & Tobacco Product Industries \\
\hline 15 & Rubber Products Industries \\
\hline 16 & Plastic Products Industries \\
\hline 17 & Leather and Allied Products Industries \\
\hline 18 & Primary Textile Industries \\
\hline 19 & Textile Products Industries \\
\hline 24 & Clothing Industries \\
\hline 25 & Wood Industries \\
\hline 26 & Furniture and Fixture Industries \\
\hline 27 & Paper and Allied Products Industries \\
\hline 28 & Printing, Publishing and Allied Industries \\
\hline 29 & Primary Metal Industries \\
\hline 30 & Fabricated Metal Products Industries (except Machinery and Transportation Equipment) \\
\hline 31 & Machinery Industries (except Electronic Machinery) \\
\hline 321 & Aircraft and Aircraft Parts Industries \\
\hline 323 & Motor Vehicle Industries \\
\hline 324 & Truck and Bus Body and Trailer Industries \\
\hline 325 & Motor Vehicle Parts and Accessories Industries \\
\hline 326 & Railroad Rolling Stock Industries \\
\hline 327 & Shipbuilding and Repair Industry \\
\hline 328 & Boatbuilding and Repair industry \\
\hline 329 & Other Transportation Equipment Industries \\
\hline 331 & Small Electronic Appliance Industry \\
\hline 332 & Major Appliance Industry (Electric and Non-Electric) \\
\hline 333 & Electric Lighting Industries \\
\hline 334 & Record Player, Radio and Television Receiver Industry \\
\hline 335 & Communication and Other Electronic Equipment Industries \\
\hline 336 & Office, Store and Business Machine Industries \\
\hline 337 & Electronic Industrial Equipment Industries \\
\hline 338 & Communications and Energy Wire and Cable Industry \\
\hline 339 & Other Electronic Products Industries \\
\hline 35 & Non-Metallic Mineral Products Industries \\
\hline 36 & Refined Petroleum and Coal Products Industries \\
\hline 371 & Industrial Chemicals Industries (not elsewhere classified) \\
\hline 372 & Agricultural Chemical Industries \\
\hline 373 & Plastic and Synthetic Resin Industry \\
\hline 374 & Pharmaceutical and Medicine Industry \\
\hline 375 & Paint and Varnish Industry \\
\hline 376 & Soap and Cleaning Compounds Industry \\
\hline 377 & Total Preparations Industry \\
\hline 379 & Other Chemical Products Industries \\
\hline 39 & Other Manufacturing Products Industries \\
\hline
\end{tabular}

Source: Sabourin and Beckstead, 1999 


\section{APPENDIX D - ADVANCED TECHNOLOGY USE}

Advanced Technology Use by Employment Size (Establishment Weighted)

\begin{tabular}{|c|c|c|c|c|}
\hline TECHNOLOGIES (percentage of establishments) & Small & Medium & Large & All \\
\hline \multicolumn{5}{|l|}{ Design and Engineering } \\
\hline a) Computer-Aided Design/Engineering (CAD/CAE) & 37 & 57 & 81 & 44 \\
\hline b) Computer-Aided Design/Manufacturing (CAD/CAM) & 31 & 43 & 67 & 36 \\
\hline c) Modelling or simulation technologies & 14 & 20 & 49 & 17 \\
\hline d) Electronic exchange of CAD files & 28 & 42 & 74 & 34 \\
\hline \multicolumn{5}{|l|}{ Processing, Fabrication and Assembly } \\
\hline a) Flexible Manufacturing Cells or Systems (FMC/FMS) & 12 & 20 & 40 & 15 \\
\hline b) Programmable Logic Control (PLC) machines or processes & 27 & 54 & 82 & 37 \\
\hline $\begin{array}{l}\text { c) Lasers used in materials processing (including surface } \\
\text { modification) }\end{array}$ & 5 & 13 & 26 & 9 \\
\hline d) Robots with sensing capabilities & 4 & 11 & 34 & 8 \\
\hline e) Robots without sensing capabilities & 4 & 10 & 32 & 7 \\
\hline f) Rapid Prototyping Systems (RPS) & 4 & 6 & 14 & 5 \\
\hline g) High speed machining & 14 & 21 & 35 & 17 \\
\hline h) Near net shape technologies & 6 & 8 & 13 & 7 \\
\hline \multicolumn{5}{|l|}{ Automated Material Handling } \\
\hline a) Part identification for manufacturing automation (e.g., bar coding) & 11 & 27 & 57 & 18 \\
\hline b) Automated Storage and Retrieval System (AS/RS) & 4 & 6 & 17 & 5 \\
\hline \multicolumn{5}{|l|}{ Inspection } \\
\hline $\begin{array}{l}\text { a) Automated vision-based systems used for inspection/testing of } \\
\text { inputs and/or final products }\end{array}$ & 7 & 15 & 35 & 11 \\
\hline $\begin{array}{l}\text { b) Other automated sensor-based systems used for inspection/testing } \\
\text { of inputs and/or final products }\end{array}$ & 8 & 18 & 45 & 13 \\
\hline \multicolumn{5}{|l|}{ Network Communications } \\
\hline a) Local area network (LAN) for engineering and/or production & 24 & 57 & 86 & 36 \\
\hline b) Company-wide computer networks (including Intranet and WAN) & 24 & 54 & 83 & 35 \\
\hline c) Inter-company computer networks (including Extranet and EDI) & 20 & 42 & 74 & 29 \\
\hline \multicolumn{5}{|l|}{ Integration and Control } \\
\hline $\begin{array}{l}\text { a) Manufacturing Resource Planning (MRP II)/Enterprise Resource } \\
\text { Planning (ERP) }\end{array}$ & 13 & 34 & 52 & 21 \\
\hline b) Computers used for control on the factory floor & 21 & 47 & 79 & 31 \\
\hline c) Computer-Integrated Manufacturing (CIM) & 15 & 23 & 42 & 18 \\
\hline d) Supervisory Control and Data Acquisition (SCADA) & 11 & 23 & 43 & 16 \\
\hline e) Use of inspection data in manufacturing control & 18 & 39 & 70 & 26 \\
\hline f) Digital, remote controlled process plant control (e.g., Fieldbus) & 3 & 8 & 20 & 5 \\
\hline g) Knowledge-based software & 16 & 20 & 32 & 18 \\
\hline
\end{tabular}

Source: Sabourin and Beckstead, 1999 
Functional Technology Use by Industry (Establishment Weighted)

\begin{tabular}{|c|c|c|c|c|c|c|}
\hline INDUSTRY & $\begin{array}{l}\text { Design and } \\
\text { Engineering }\end{array}$ & $\begin{array}{l}\text { Processing, } \\
\text { Fabrication, } \\
\text { Assembly }\end{array}$ & $\begin{array}{l}\text { Automated } \\
\text { Material } \\
\text { Handling }\end{array}$ & Inspection & $\begin{array}{l}\text { Network } \\
\text { Communications }\end{array}$ & $\begin{array}{r}\text { Integration } \\
\text { and Control }\end{array}$ \\
\hline \multicolumn{7}{|c|}{ (percentage of establishments) } \\
\hline Beverage & 34 & 65 & 40 & 34 & 77 & 77 \\
\hline Rubber Products & 50 & 64 & 23 & 18 & 49 & 51 \\
\hline Plastic Products & 51 & 68 & 23 & 21 & 54 & 59 \\
\hline $\begin{array}{l}\text { Leather and } \\
\text { Allied Products }\end{array}$ & 38 & 33 & 19 & 3 & 37 & 42 \\
\hline Primary Textile & 49 & 54 & 45 & 38 & 69 & 70 \\
\hline Textile Products & 29 & 39 & 18 & 13 & 48 & 51 \\
\hline Clothing & 33 & 22 & 17 & 6 & 34 & 36 \\
\hline Wood & 37 & 53 & 18 & 13 & 34 & 43 \\
\hline $\begin{array}{l}\text { Furniture and } \\
\text { Fixture }\end{array}$ & 43 & 37 & 16 & 6 & 36 & 37 \\
\hline $\begin{array}{l}\text { Paper and Allied } \\
\text { Products }\end{array}$ & 55 & 63 & 41 & 33 & 66 & 66 \\
\hline $\begin{array}{l}\text { Printing, } \\
\text { Publishing and } \\
\text { Allied }\end{array}$ & 36 & 30 & 16 & 12 & 58 & 45 \\
\hline Primary Metal & 80 & 67 & 30 & 29 & 62 & 61 \\
\hline $\begin{array}{l}\text { Fabricated Metal } \\
\text { Products }\end{array}$ & 71 & 56 & 10 & 14 & 52 & 54 \\
\hline Machinery & 70 & 60 & 19 & 20 & 61 & 60 \\
\hline $\begin{array}{l}\text { Transportation } \\
\text { Equipment }\end{array}$ & 66 & 55 & 40 & 33 & 57 & 58 \\
\hline $\begin{array}{l}\text { Electronic and } \\
\text { Electronic } \\
\text { Products }\end{array}$ & 79 & 59 & 27 & 23 & 71 & 72 \\
\hline $\begin{array}{l}\text { Non-Metallic } \\
\text { Mineral } \\
\text { Products }\end{array}$ & 38 & 49 & 15 & 11 & 39 & 53 \\
\hline $\begin{array}{l}\text { Refined } \\
\text { Petroleum and } \\
\text { Coal Products }\end{array}$ & 42 & 33 & 16 & 21 & 33 & 51 \\
\hline $\begin{array}{l}\text { Chemical and } \\
\text { Chemical } \\
\text { Products }\end{array}$ & 36 & 48 & 22 & 24 & 65 & 58 \\
\hline $\begin{array}{l}\text { Other } \\
\text { Manufacturing }\end{array}$ & 52 & 42 & 15 & 10 & 49 & 43 \\
\hline
\end{tabular}

Source: Sabourin and Beckstead, 1999 
APPENDIX E - SIGNIFICANCE VALUES

\begin{tabular}{|l|l|l|l|}
\hline & $\boldsymbol{\alpha}=\mathbf{0 . 0 1}$ & $\boldsymbol{\alpha}=\mathbf{0 . 0 5}$ & $\boldsymbol{\alpha}=\mathbf{0 . 1}$ \\
\hline 1 & 3.32 & 2.37 & 1.94 \\
\hline 2 & 3.78 & 2.61 & 2.08 \\
\hline 3 & 3.96 & 2.68 & 2.13 \\
\hline 4 & 7.00 & 3.97 & 2.78 \\
\hline 5 & 4.91 & 3.12 & 2.38 \\
\hline 6 & 4.07 & 2.73 & 2.16 \\
\hline 7 & 3.53 & 2.48 & 2.01 \\
\hline
\end{tabular}

Table 107 Significance values for likelihood ratios of AMT

1. These significance values are for all tests by size class and all tests for the electronics, furniture and other, metals, vehicle and wood industries.

2. These values are for all tests in the chemicals and textiles industries.

3. These values are for all tests in the plastics industry.

4. These values are for the test of the first and fourth factors in the machinery industry.

5. These values are for the tests of the second and fourth, third and fourth, the first and third, and the first and second factors in the machinery industry.

6. These values are for the test of the second and third factors in the machinery industry.

7. These factors are for all tests in the non-metals industry.

\begin{tabular}{|l|l|l|l|}
\hline & $\boldsymbol{\alpha}=\mathbf{0 . 0 1}$ & $\boldsymbol{\alpha}=\mathbf{0 . 0 5}$ & $\mathbf{A}=\mathbf{0 . 1}$ \\
\hline 1 & 4.61 & 3.00 & 2.30 \\
\hline 2 & 4.90 & 3.12 & 2.37 \\
\hline 3 & 4.83 & 3.09 & 2.36 \\
\hline
\end{tabular}

Table 108 Significance values for likelihood ratios of business practices

1. These significance values are for all tests by size class and all tests for all industries except the machinery and non-metals industries.

2. These values are for all tests in the machinery industry.

3. These values are for all tests in the non-metals industry. 


\begin{tabular}{|l|l|l|l|}
\hline & $\boldsymbol{\alpha}=\mathbf{0 . 0 1}$ & $\boldsymbol{\alpha}=\mathbf{0 . 0 5}$ & $\boldsymbol{\alpha}=\mathbf{0 . 1}$ \\
\hline 1 & 3.78 & 2.61 & 2.08 \\
\hline 2 & 4.92 & 3.12 & 2.38 \\
\hline 3 & 4.07 & 2.73 & 2.16 \\
\hline 4 & 3.99 & 2.70 & 2.14 \\
\hline 5 & 4.61 & 3.00 & 2.30 \\
\hline
\end{tabular}

Table 109 Significance values for likelihood ratios of sources of implementation support

1. These significance values are for all tests by size class and all industries except the machinery and non-metals industries, and tests of the second and third, first and second, and first and third factors of the textiles industry.

2. These values are for all tests involving the fourth factor in the machinery industry.

3. These values are for tests of the second and third, first and second, and first and third factors of the machinery industry.

4. These values are for all tests in the non-metals industry.

5. These values are for the tests of the second and third, first and second, and first and third factors of the textiles industry.

\begin{tabular}{|l|l|l|l|}
\hline & $\boldsymbol{\alpha}=\mathbf{0 . 0 1}$ & $\boldsymbol{\alpha}=\mathbf{0 . 0 5}$ & $\boldsymbol{\alpha}=\mathbf{0 . 1}$ \\
\hline 1 & 3.78 & 2.61 & 2.08 \\
\hline 2 & 7.00 & 3.97 & 2.78 \\
\hline 3 & 4.91 & 3.12 & 2.38 \\
\hline 4 & 3.32 & 2.37 & 1.94 \\
\hline 5 & 6.92 & 3.94 & 2.76 \\
\hline 6 & 4.85 & 3.09 & 2.36 \\
\hline 7 & 4.79 & 3.07 & 2.35 \\
\hline 8 & 6.85 & 3.92 & 2.75 \\
\hline
\end{tabular}

Table 110 Significance values for likelihood ratios of perceived benefits

1. These significance values are for all tests by size class and all tests for the chemical, electronics, and furniture and other industries.

2 These values are for all tests not including the third factor in the machinery industry.

3 These values are for all the tests involving the third factor in the machinery industry.

4 These values are for all the tests in the metals, textiles, vehicles, and wood industries.

5 These values are for the test of the first and third factors in the non-metals industry.

6 These values are for tests except the first and third factors in the non-metals industry.

7 These factors are for all tests involving the first factor in the plastics industry.

8 These factors are for all tests not involving the first factor in the plastics industry. 


\begin{tabular}{|l|l|l|l|}
\hline & $\boldsymbol{\alpha}=\mathbf{0 . 0 1}$ & $\boldsymbol{\alpha}=\mathbf{0 . 0 5}$ & $\boldsymbol{\alpha}=\mathbf{0 . 1}$ \\
\hline 1 & 2.51 & 1.94 & 1.67 \\
\hline 2 & 4.12 & 2.75 & 2.17 \\
\hline 3 & 3.62 & 2.52 & 2.03 \\
\hline 4 & 3.57 & 2.49 & 2.02 \\
\hline 5 & 3.26 & 2.33 & 1.93 \\
\hline 6 & 3.52 & 2.47 & 2.00 \\
\hline 7 & 3.21 & 2.31 & 1.91 \\
\hline 8 & 3.00 & 2.20 & 1.83 \\
\hline 9 & 3.32 & 2.31 & 1.94 \\
\hline 10 & 2.80 & 2.10 & 1.77 \\
\hline 11 & 3.02 & 2.21 & 1.85 \\
\hline 12 & 2.64 & 2.01 & 1.72 \\
\hline
\end{tabular}

Table 111 Significance values for likelihood ratios of high-level factors

1. These significance values are for all tests by size class and all tests for the metals, vehicle and wood industries.

2. These values are for the tests of the first and third, first and fifth, and third and fifth factors in the machinery industry.

3. These values are for all tests except those mentioned in 2 in the machinery industry.

4. These values are for the tests of the first and third, first and fourth, and first and fifth factors in the non-metals industry.

5. These values are for all the tests except those mentioned in 4 in the non-metals industry.

6. These values are for the tests of the first and second, first and third, and second and third factors in the plastics industry.

7. These factors are for the tests of the first and fourth, first and fifth, third and fourth, and third and fifth factors in the plastics industry.

8. These factors are for the tests of the second and fourth, second and fifth, and fourth and fifth factors in the plastics industry.

9. These factors are for the tests of the first and second, first and fourth, second and third, second and fourth, and third and fourth factors in the chemical industry. These are also used for the tests of the first, and fourth and second and fourth factors in the electronics industry.

10. These factors are used for all tests in the chemical industry not mentioned in 9, as well as for the test of the third and fifth factors in the electronics industry. These factors are also used for all tests in the furniture industry except the tests of the fourth and fifth factors.

11. These factors are used for all tests in the electronics industry not mentioned in 9 or 10.

12. These factors are used for the test of the fourth and fifth factors in the furniture industry and all tests in the wood industry. 
APPENDIX F - EXAMPLE SAS CODE

\section{F1 Main Database Program}

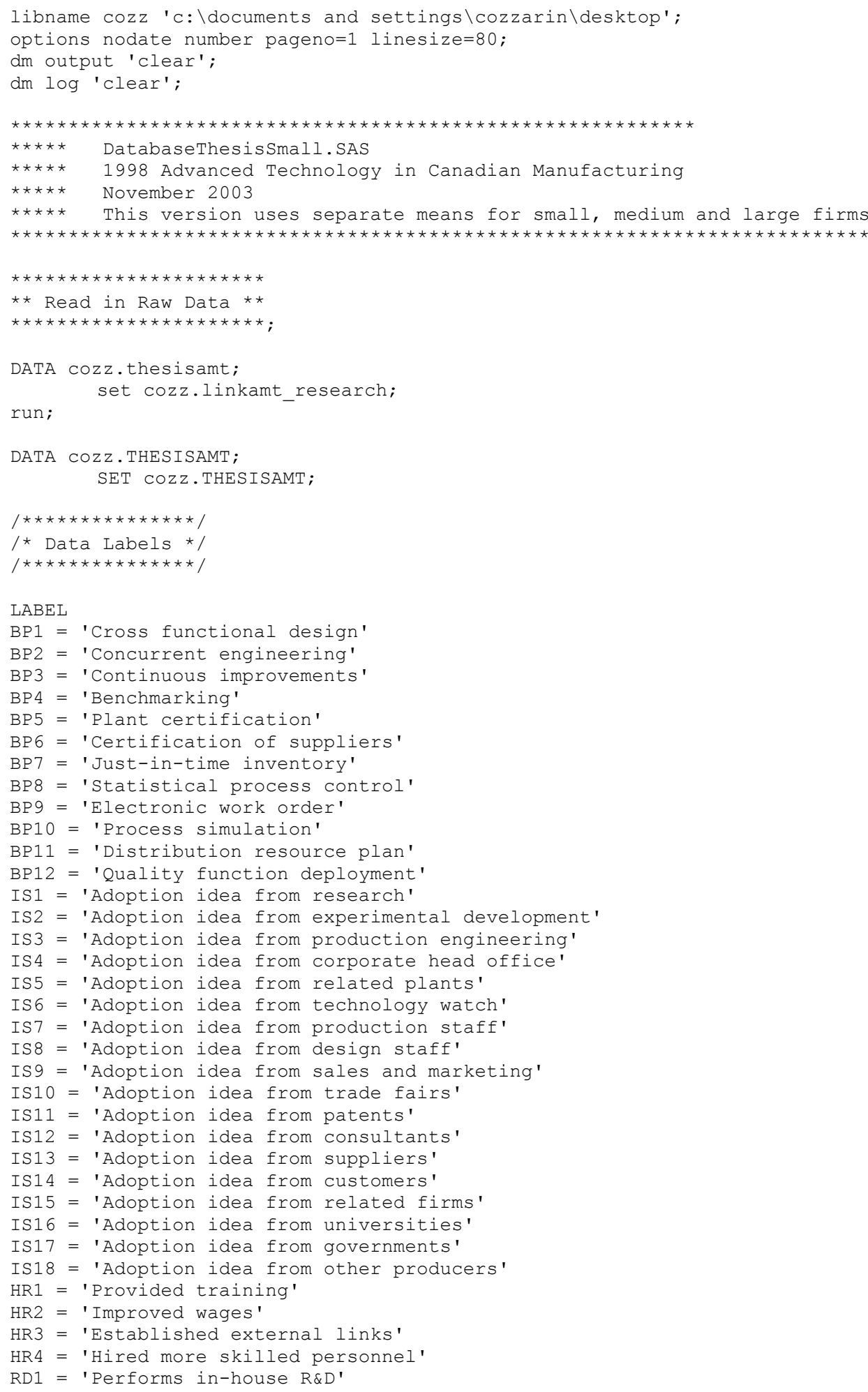




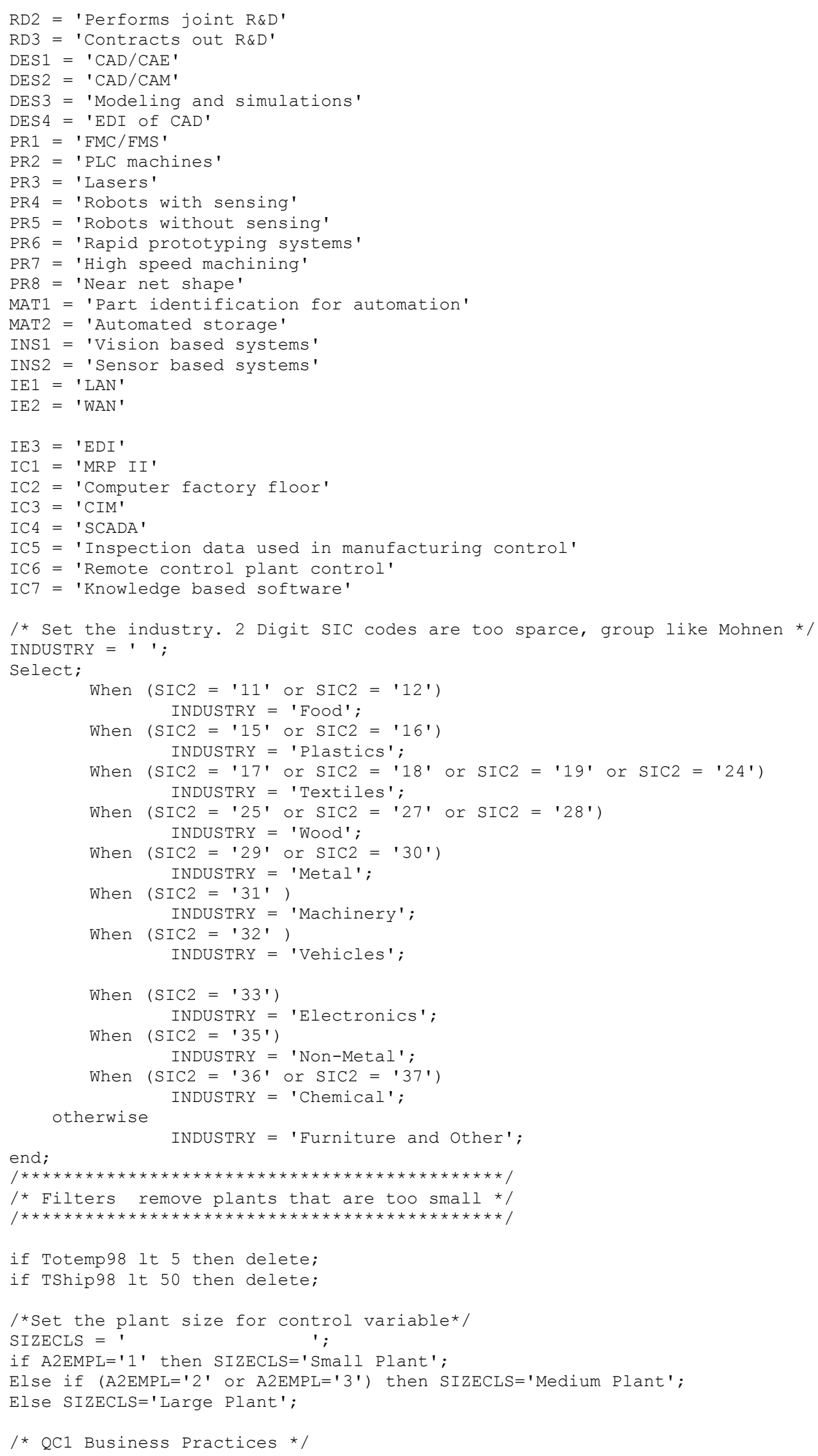




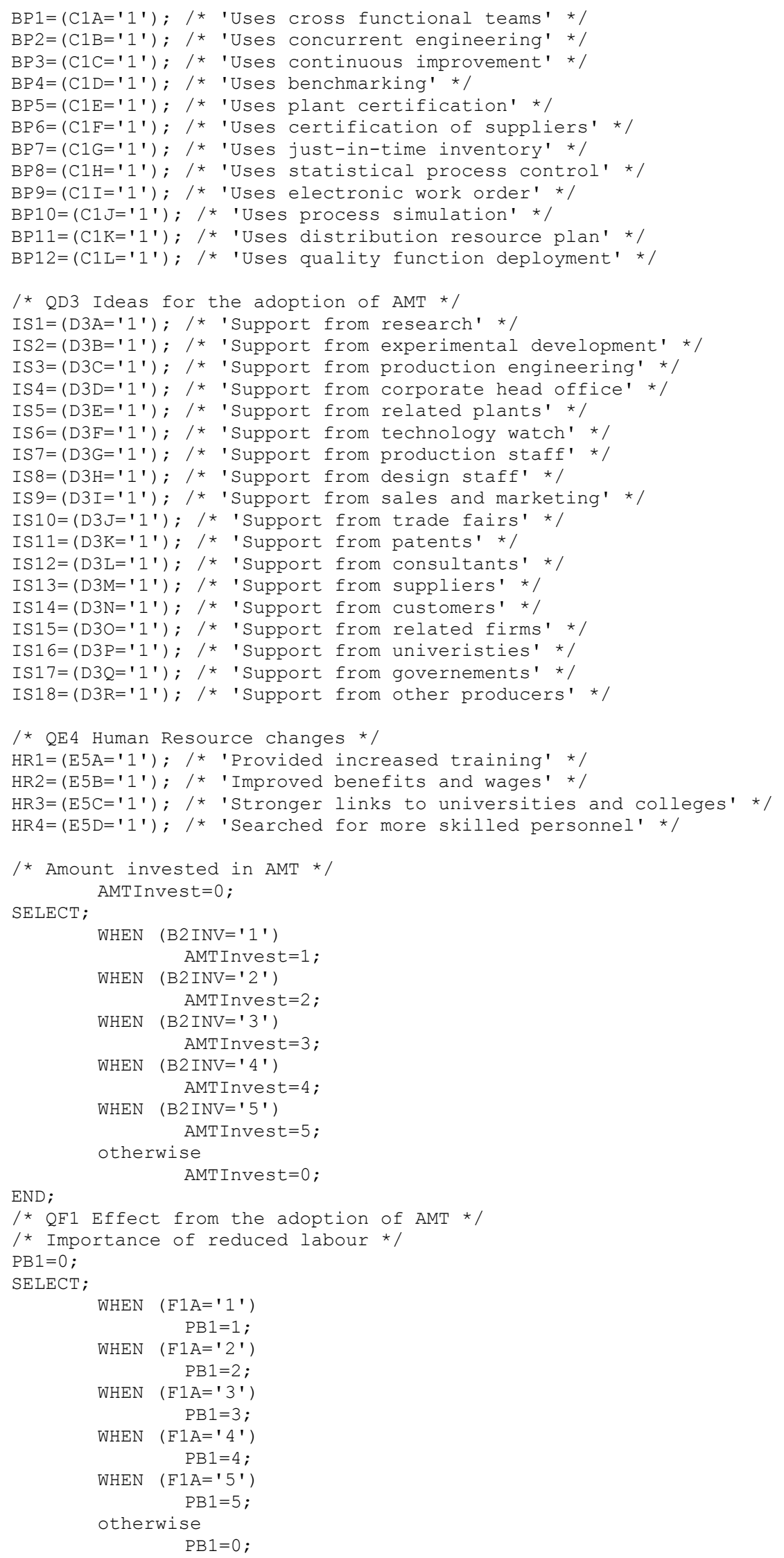




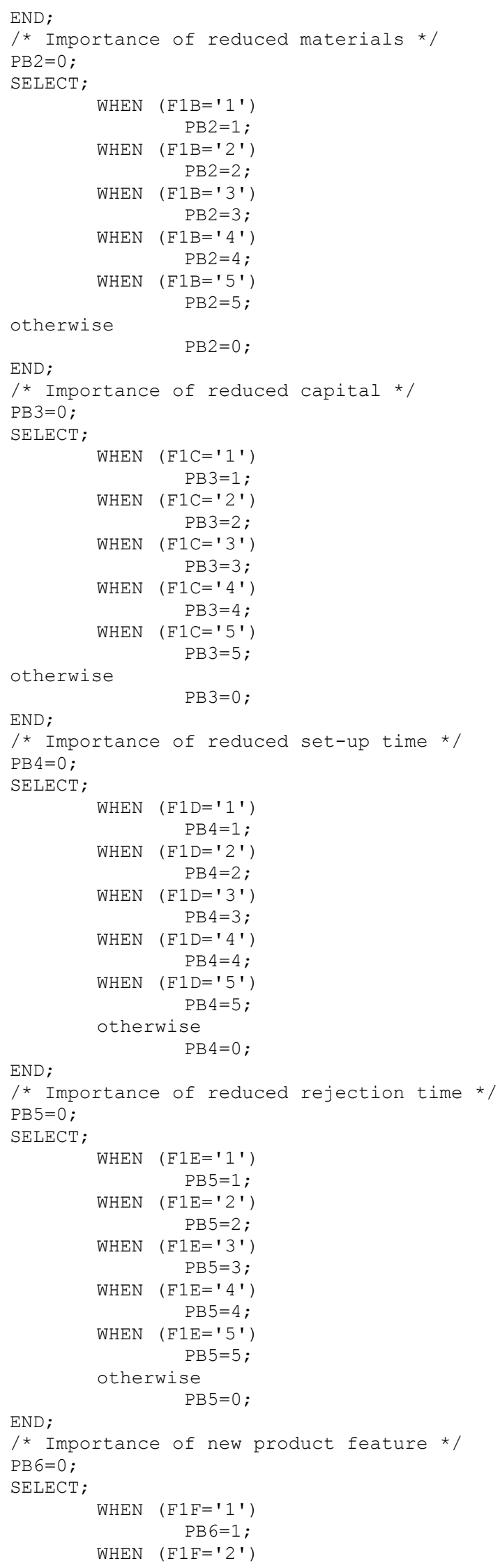




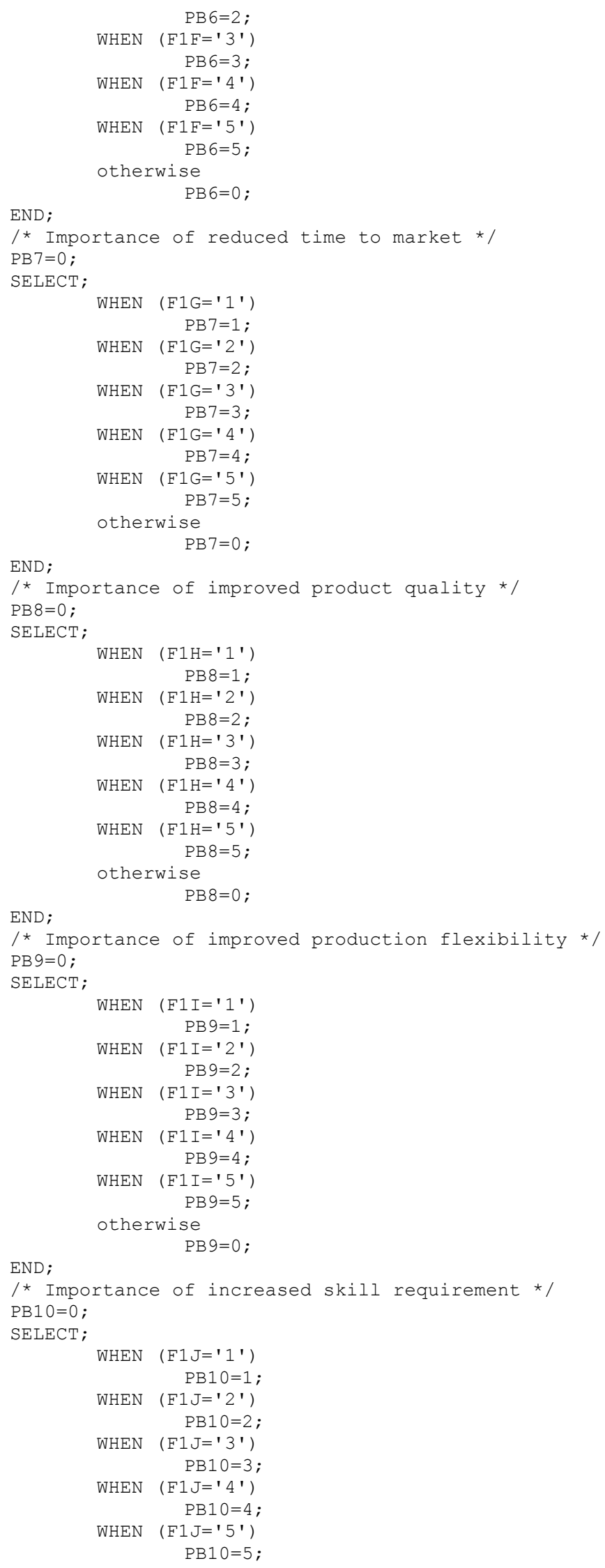




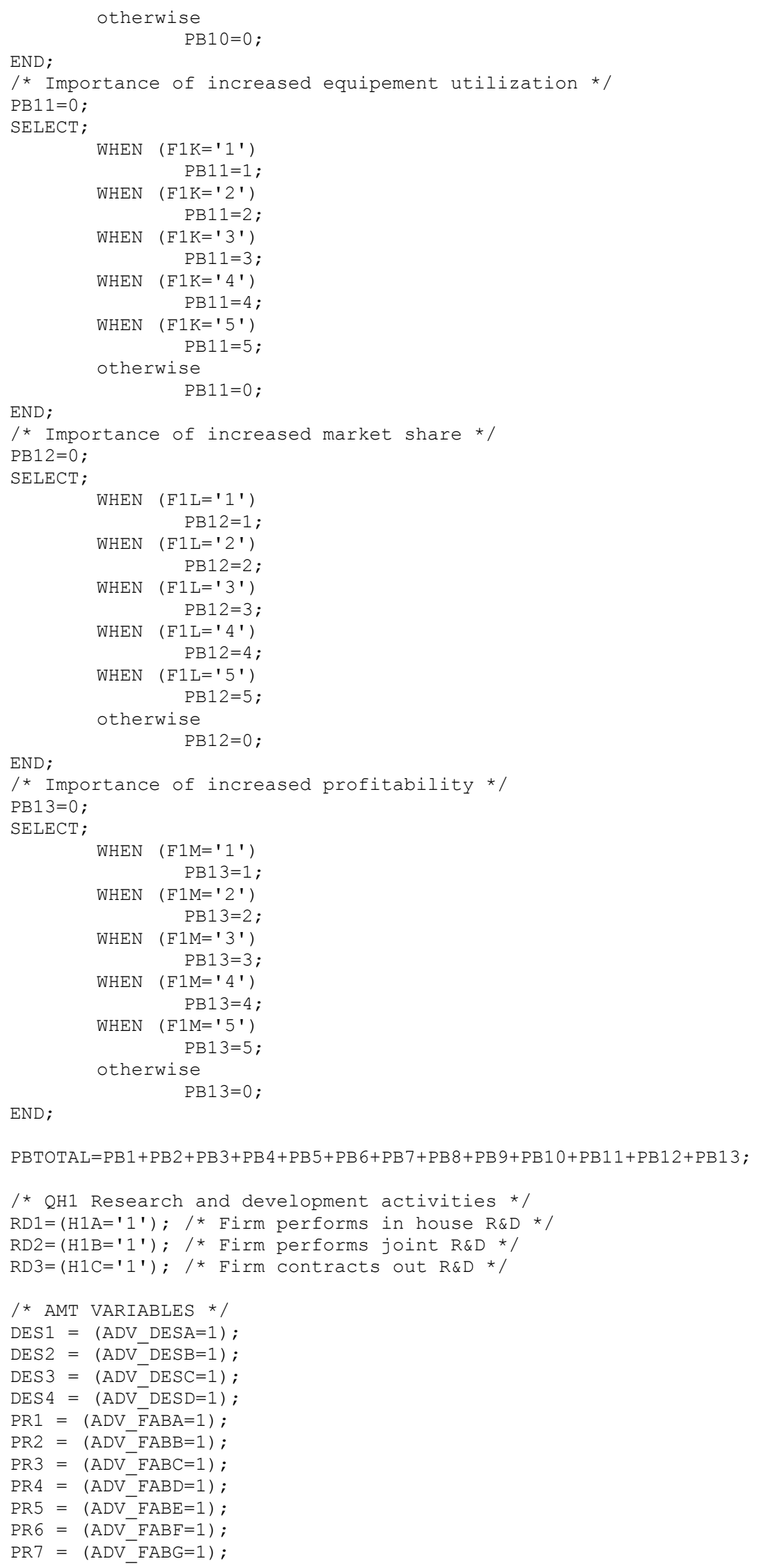




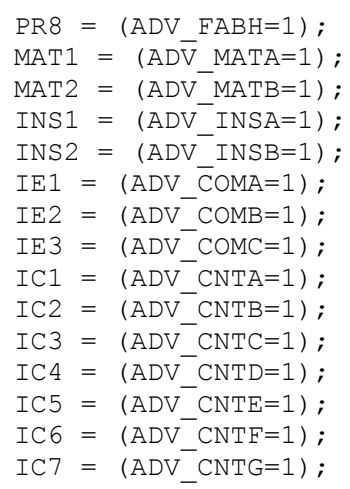


where sizecls= 'Large Plant';

OUTPUT OUT=LPBMEANS MEAN= LMPB1 LMPB2 LMPB3 LMPB4 LMPB5 LMPB6 LMPB7 LMPB8

LMPB10 LMPB11 LMPB12 LMPB13 LMPBTOTAL;

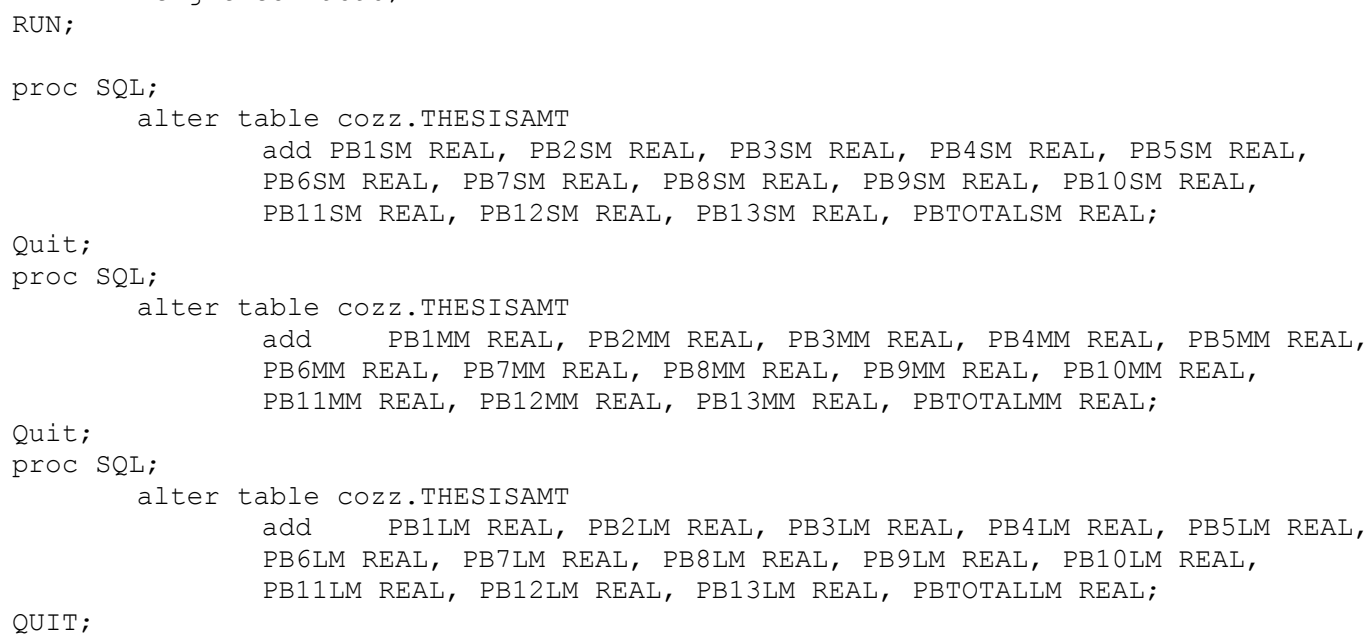

PROC sql

UPDATE COZZ.THESISAMT

set $\mathrm{PB} 1 \mathrm{SM}=$ (select SMPB1 from SPBMEANS), $\mathrm{PB} 2 \mathrm{SM}=$ (select SMPB2 from SPBMEANS), $\mathrm{PB} 3 \mathrm{SM}=$ (select SMPB3 from SPBMEANS), $\mathrm{PB} 4 \mathrm{SM}=$ (select SMPB4 from SPBMEANS), PB5SM = (select SMPB5 from SPBMEANS), PB6SM $=$ (select SMPB6 from SPBMEANS), $\mathrm{PB} 7 \mathrm{SM}=$ (select SMPB7 from SPBMEANS), PB8SM $=$ (select SMPB8 from SPBMEANS), PB9SM = (select SMPB9 from SPBMEANS), PB10SM = (select SMPB10 from SPBMEANS), $\mathrm{PB1} 1 \mathrm{SM}=$ (select SMPB11 from SPBMEANS), $\mathrm{PB} 12 \mathrm{SM}=$ (select SMPB12 from SPBMEANS), PB13SM = (select SMPB13 from SPBMEANS), PBTOTALSM = (select SMPBTOTAL from SPBMEANS), PB1MM = (select MMPB1 from MPBMEANS), PB2MM = (select MMPB2 from MPBMEANS), PB3MM = (select MMPB3 from MPBMEANS), $\mathrm{PB} 4 \mathrm{MM}=$ (select MMPB4 from MPBMEANS), PB5MM $=$ (select MMPB5 from MPBMEANS), $\mathrm{PB} 6 \mathrm{MM}=$ (select MMPB6 from MPBMEANS), $\mathrm{PB} 7 \mathrm{MM}=$ (select MMPB7 from MPBMEANS) PB8MM $=$ (select MMPB8 from MPBMEANS), $\mathrm{PB} 9 \mathrm{MM}=$ (select MMPB9 from MPBMEANS) PB10MM = (select MMPB10 from MPBMEANS), PB11MM = (select MMPB11 from MPBMEANS), $\mathrm{PB} 12 \mathrm{MM}=$ (select MMPB12 from MPBMEANS), PB13MM $=$ (select MMPB13 from MPBMEANS), PBTOTALMM = (select MMPBTOTAL from MPBMEANS);

Quit;

PROC sql;

UPDATE COZZ.THESISAMT

set $\quad$ PBILM $=$ (select LMPB1 from LPBMEANS) PB2LM = (select LMPB2 from LPBMEANS), PB3LM $=$ (select LMPB3 from LPBMEANS), PB4LM $=$ (select LMPB4 from LPBMEANS), PB5LM $=$ (select LMPB5 from LPBMEANS), PB6LM $=$ (select LMPB6 from LPBMEANS), PB7LM $=$ (select LMPB7 from LPBMEANS), PB8LM $=$ (select LMPB8 from LPBMEANS), PB9LM = (select LMPB9 from LPBMEANS), PB10LM = (select LMPB10 from LPBMEANS), PB11LM = (select LMPB11 from LPBMEANS), PB12LM $=$ (select LMPB12 from LPBMEANS) PB13LM = (select LMPB13 from LPBMEANS), 
QUIT;

PBTOTALLM $=$ ( select LMPBTOTAL from LPBMEANS);

/* Create binary variables for the perceived benefits * /

/* For now enter the means for each sizeclass by hand from the above output */ data cozz.THESISAMT; set COZZ.THESISAMT;

label

$\mathrm{PB} 1 \mathrm{~B}=$ 'Improvement reduced labour'

$\mathrm{PB} 2 \mathrm{~B}=$ 'Improvement reduced material'

$\mathrm{PB} 3 \mathrm{~B}=$ 'Improvement reduced capital'

PB4B = 'Improvement reduced set-up time'

$\mathrm{PB} 5 \mathrm{~B}=$ 'Improvement reduced rejection time'

$\mathrm{PB} 6 \mathrm{~B}=$ 'Improvement new product features'

$\mathrm{PB} 7 \mathrm{~B}=$ 'Improvement reduced time to market'

PB8B = 'Improvement product quality'

$\mathrm{PB} 9 \mathrm{~B}=$ 'Improvement flexibility'

$\mathrm{PB} 10 \mathrm{~B}=$ 'Improvement skill requirements'

$\mathrm{PB} 11 \mathrm{~B}=$ 'Improvement increased equipment utilization'

$\mathrm{PB} 12 \mathrm{~B}=$ 'Improvement increased market share'

$\mathrm{PB} 13 \mathrm{~B}=$ 'Improvement increased profitability';

if SizeCls='Small Plant' then do;

$\mathrm{PB} 1 \mathrm{~B}=(\mathrm{PB} 1>=\mathrm{PB} 1 \mathrm{SM})$;

$\mathrm{PB} 2 \mathrm{~B}=(\mathrm{PB} 2>=\mathrm{PB} 2 \mathrm{SM})$;

$\mathrm{PB} 3 \mathrm{~B}=(\mathrm{PB} 3>=\mathrm{PB} 3 \mathrm{SM})$

$\mathrm{PB} 4 \mathrm{~B}=(\mathrm{PB} 4>=\mathrm{PB} 4 \mathrm{SM})$;

$\mathrm{PB} 5 \mathrm{~B}=(\mathrm{PB} 5>=\mathrm{PB} 5 \mathrm{SM})$

$\mathrm{PB} 6 \mathrm{~B}=(\mathrm{PB} 6>=\mathrm{PB} 6 \mathrm{SM})$

$\mathrm{PB} 7 \mathrm{~B}=(\mathrm{PB} 7>=\mathrm{PB} 7 \mathrm{SM})$

$\mathrm{PB} 8 \mathrm{~B}=(\mathrm{PB} 8>=\mathrm{PB} 8 \mathrm{SM})$;

$\mathrm{PB} 9 \mathrm{~B}=(\mathrm{PB} 9>=\mathrm{PB} 9 \mathrm{SM})$;

$\mathrm{PB} 10 \mathrm{~B}=(\mathrm{PB} 10>=\mathrm{PB} 10 \mathrm{SM})$;

$\mathrm{PB} 11 \mathrm{~B}=(\mathrm{PB} 11>=\mathrm{PB} 11 \mathrm{SM})$;

$\mathrm{PB} 12 \mathrm{~B}=(\mathrm{PB} 12>=\mathrm{PB} 12 \mathrm{SM})$;

$\mathrm{PB} 13 \mathrm{~B}=(\mathrm{PB} 13>=\mathrm{PB} 13 \mathrm{SM})$;

end;

PBTOTALB $=($ PBTOTAL $>=$ PBTOTALSM $)$

else if SizeCls = 'Medium Plant' then do;

$\mathrm{PB} 1 \mathrm{~B}=(\mathrm{PB} 1>=\mathrm{PB} 1 \mathrm{MM})$;

$\mathrm{PB} 2 \mathrm{~B}=(\mathrm{PB} 2>=\mathrm{PB} 2 \mathrm{MM})$

$\mathrm{PB} 3 \mathrm{~B}=(\mathrm{PB} 3>=\mathrm{PB} 3 \mathrm{MM})$;

$\mathrm{PB} 4 \mathrm{~B}=(\mathrm{PB} 4>=\mathrm{PB} 4 \mathrm{MM})$;

$\mathrm{PB} 5 \mathrm{~B}=(\mathrm{PB} 5>=\mathrm{PB} 5 \mathrm{MM})$;

$\mathrm{PB} 6 \mathrm{~B}=(\mathrm{PB} 6>=\mathrm{PB} 6 \mathrm{MM})$;

$\mathrm{PB} 7 \mathrm{~B}=(\mathrm{PB} 7>=\mathrm{PB} 7 \mathrm{MM})$

$\mathrm{PB} 8 \mathrm{~B}=(\mathrm{PB} 8>=\mathrm{PB} 8 \mathrm{MM})$;

$\mathrm{PB} 9 \mathrm{~B}=(\mathrm{PB} 9>=\mathrm{PB} 9 \mathrm{MM})$;

$\mathrm{PB} 10 \mathrm{~B}=(\mathrm{PB} 10>=\mathrm{PB} 10 \mathrm{MM})$;

$\mathrm{PB} 11 \mathrm{~B}=(\mathrm{PB} 11>=\mathrm{PB} 11 \mathrm{MM})$

$\mathrm{PB} 12 \mathrm{~B}=(\mathrm{PB} 12>=\mathrm{PB} 12 \mathrm{MM})$;

$\mathrm{PB} 13 \mathrm{~B}=(\mathrm{PB} 13>=\mathrm{PB} 13 \mathrm{MM})$;

PBTOTALB $=($ PBTOTAL $>=$ PBTOTALMM $)$;

end;

else do;

/* The Plant is a Large Plant */

$\mathrm{PB} 1 \mathrm{~B}=(\mathrm{PB} 1>=\mathrm{PB} 1 \mathrm{LM})$;

$\mathrm{PB} 2 \mathrm{~B}=(\mathrm{PB} 2>=\mathrm{PB} 2 \mathrm{LM})$;

$\mathrm{PB} 3 \mathrm{~B}=(\mathrm{PB} 3>=\mathrm{PB} 3 \mathrm{LM})$;

$\mathrm{PB} 4 \mathrm{~B}=(\mathrm{PB} 4>=\mathrm{PB} 4 \mathrm{LM})$;

$\mathrm{PB} 5 \mathrm{~B}=(\mathrm{PB} 5>=\mathrm{PB} 5 \mathrm{LM})$

$\mathrm{PB} 6 \mathrm{~B}=(\mathrm{PB} 6>=\mathrm{PB} 6 \mathrm{LM})$

$\mathrm{PB} 7 \mathrm{~B}=(\mathrm{PB} 7>=\mathrm{PB} 7 \mathrm{LM})$;

$\mathrm{PB} 8 \mathrm{~B}=(\mathrm{PB} 8>=\mathrm{PB} 8 \mathrm{LM})$;

$\mathrm{PB} 9 \mathrm{~B}=(\mathrm{PB} 9>=\mathrm{PB} 9 \mathrm{LM})$;

$\mathrm{PB} 10 \mathrm{~B}=(\mathrm{PB} 10>=\mathrm{PB} 10 \mathrm{LM})$

$\mathrm{PB} 11 \mathrm{~B}=(\mathrm{PB} 11>=\mathrm{PB} 11 \mathrm{LM})$;

$\mathrm{PB} 12 \mathrm{~B}=(\mathrm{PB} 12>=\mathrm{PB} 12 \mathrm{LM})$

$\mathrm{PB} 13 \mathrm{~B}=(\mathrm{PB} 13>=\mathrm{PB} 13 \mathrm{LM})$; 


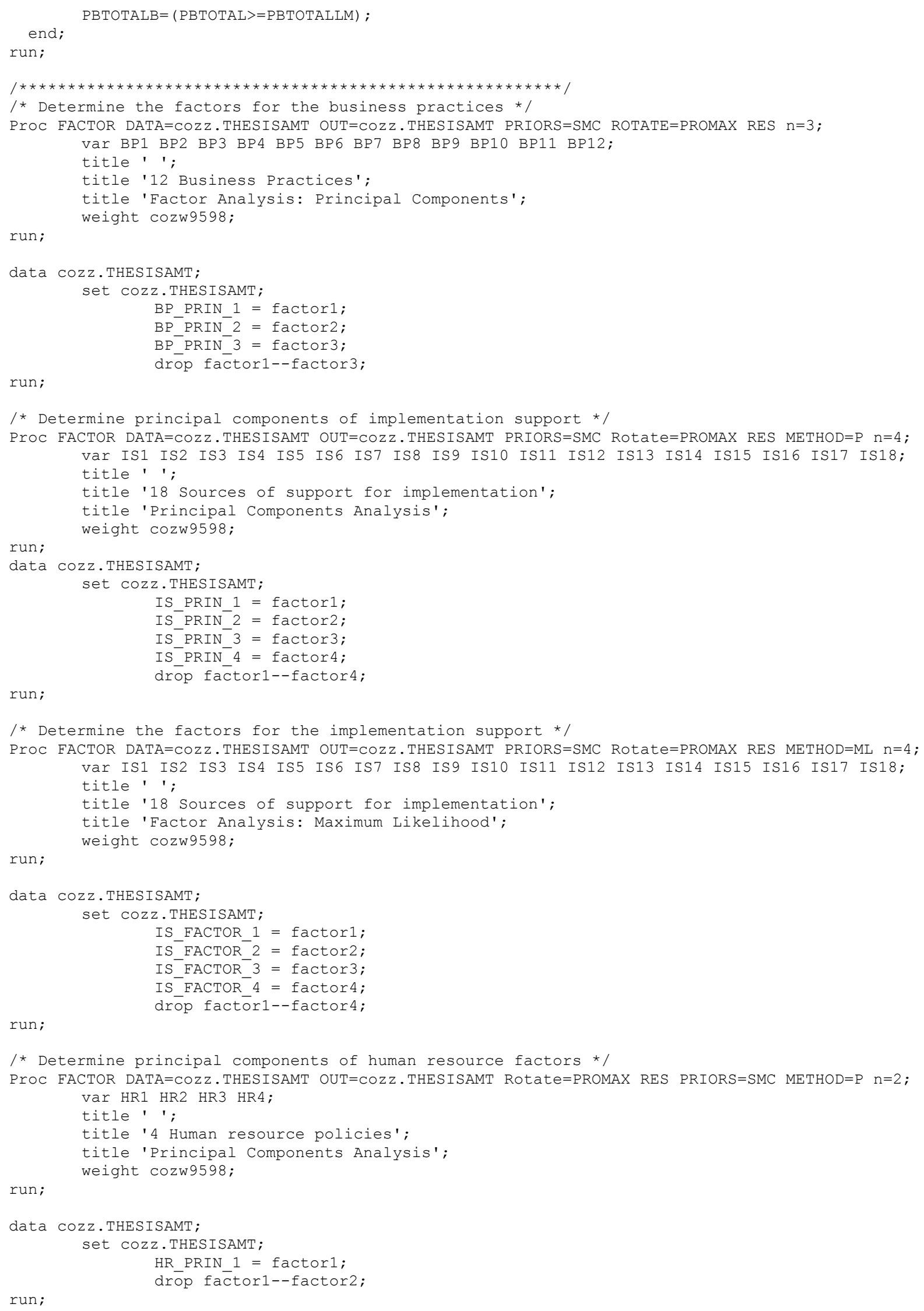

run; 


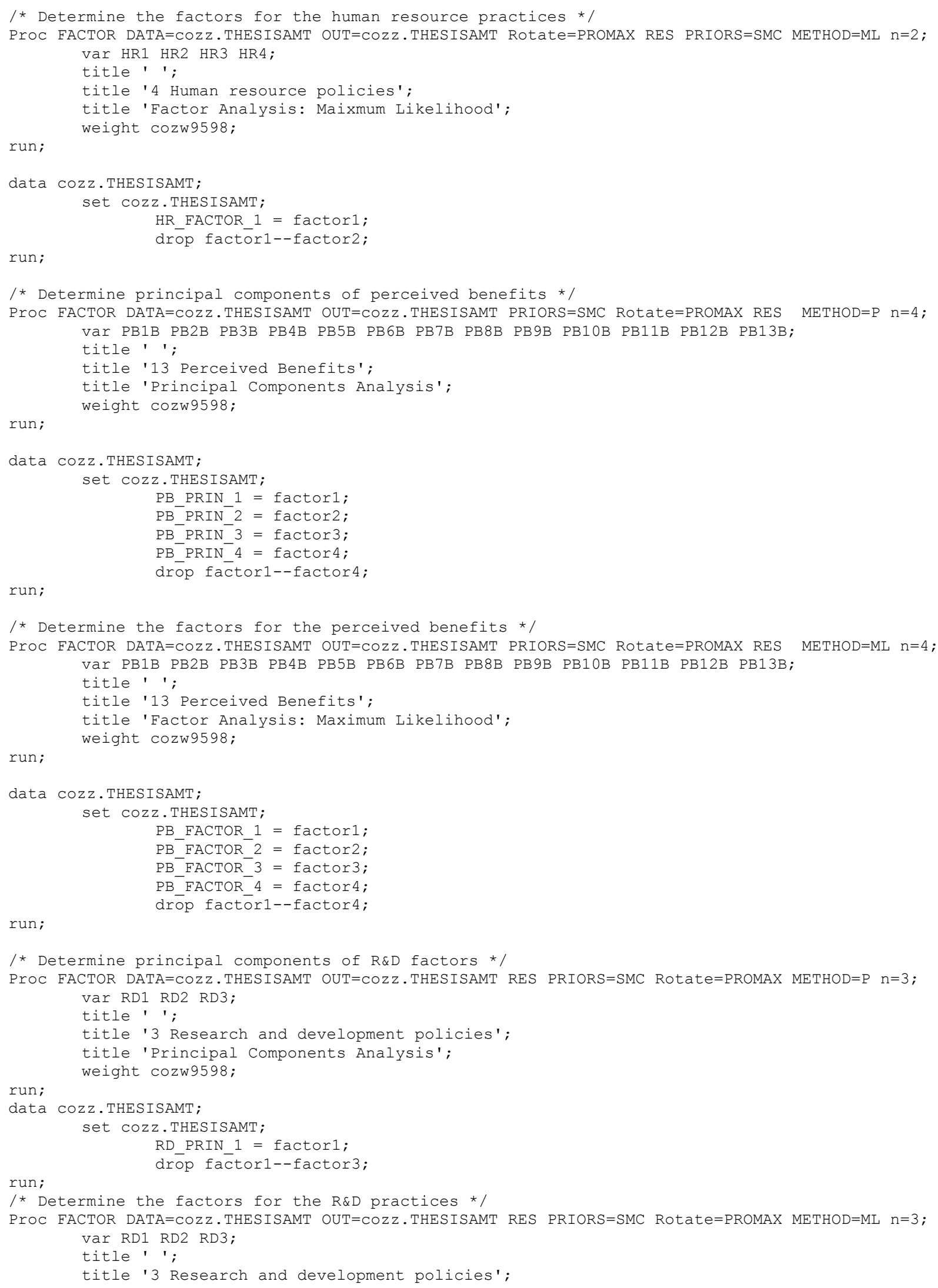




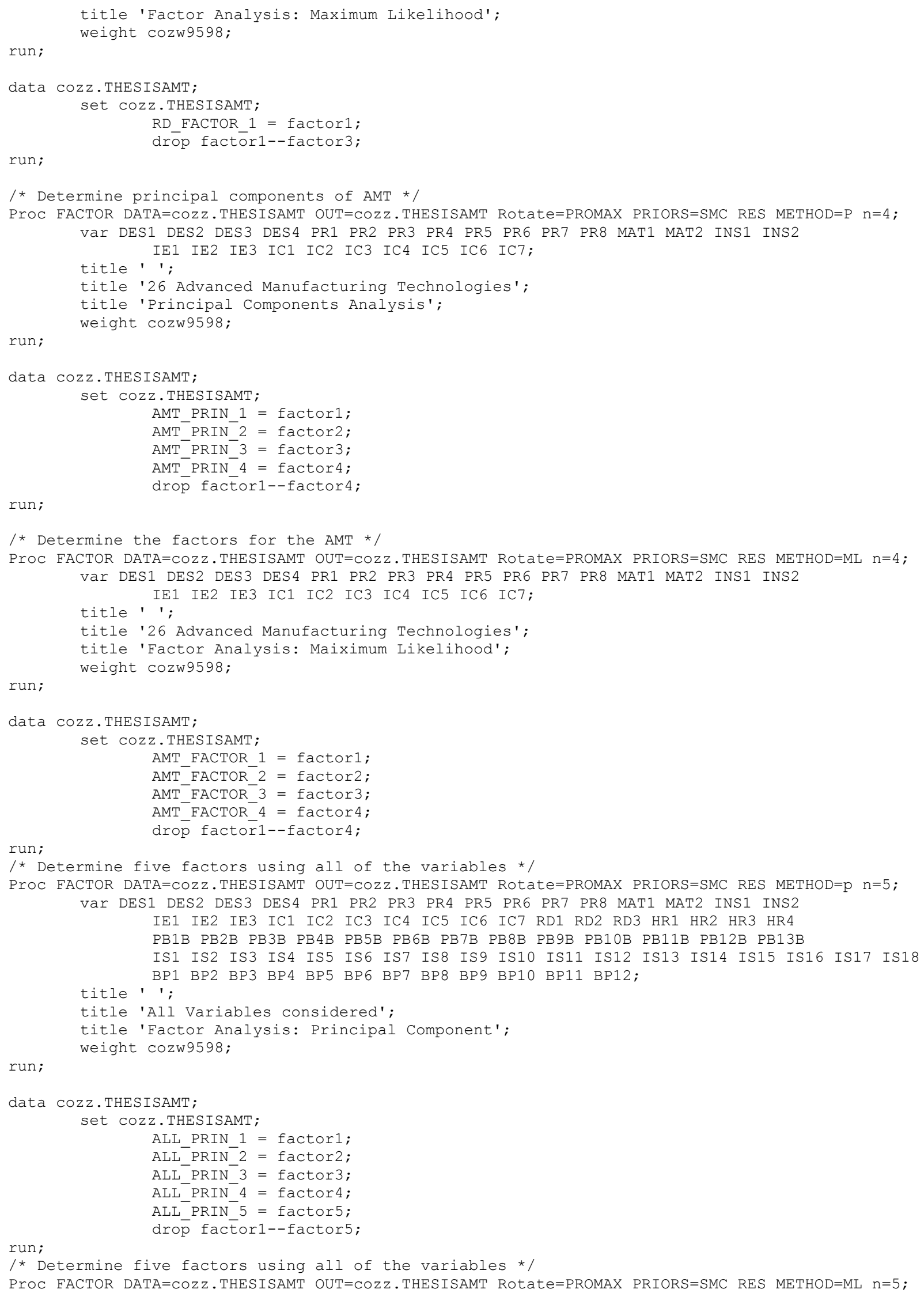


var DES1 DES2 DES3 DES4 PR1 PR2 PR3 PR4 PR5 PR6 PR7 PR8 MAT1 MAT2 INS1 INS2 IE1 IE2 IE3 IC1 IC2 IC3 IC4 IC5 IC6 IC7 RD1 RD2 RD3 HR1 HR2 HR3 HR4 PB1B PB2B PB3B PB4B PB5B PB6B PB7B PB8B PB9B PB10B PB11B PB12B PB13B IS1 IS2 IS3 IS4 IS5 IS6 IS7 IS8 IS9 IS10 IS11 IS12 IS13 IS14 IS15 IS16 IS17 IS18

title' '; BP1 BP2 BP3 BP4 BP5 BP6 BP7 BP8 BP9 BP10 BP11 BP12;

title 'All Variables considered';

title 'Factor Analysis: Maximum Likelihood';

weight cozw9598;

run;

data cozz.THESISAMT;

set COZz.THESISAMT;

ALL_FACTOR_1 = factor 1 ;

ALL FACTOR 2 = factor2;

ALL_FACTOR_3 = factor 3 ;

ALL FACTOR $4=$ factor 4

ALL_EACTOR_5 = factor5;

run;

drop factori--factor5;

/**** Using Factor Results determine the mean value of each factor $\star * /$

/** Factors were determine by hand from the factor procedures **/

/** A reduced number of factors was needed due to the sparceness of the data $* /$

data cozz.THESISAMT;

set COZZ.THESISAMT;

$\mathrm{BPF} 1 \mathrm{Total}=\mathrm{BP} 3+\mathrm{BP} 4+\mathrm{BP} 5+\mathrm{BP} 6+\mathrm{BP} 8$;

$\mathrm{BPF} 3$ Total $=\mathrm{BP} 1+\mathrm{BP} 2+\mathrm{BP} 3$;

$\mathrm{BPF} 2$ Total $=\mathrm{BP} 7+\mathrm{BP} 9+\mathrm{BP} 10+\mathrm{BP} 11+\mathrm{BP} 12$;

ISF1Total = IS11+IS3+IS6+IS7+IS8+IS9+IS13+IS14

ISF4Total = IS4+IS5+IS16;

ISF2Total = IS1+IS2+IS3+IS10+IS12+IS17+IS18;

ISF3Total = IS9+IS15+IS16+IS14;

HRFTotal = HR1+HR2+HR3+HR4;

RDFTotal = RD1+RD2+RD3;

$\mathrm{PBF} 2 \mathrm{Total}=\mathrm{PB} 1+\mathrm{PB} 2+\mathrm{PB} 3$;

PBF3Total $=\mathrm{PB} 8+\mathrm{PB} 9+\mathrm{PB} 10 ;$

$\mathrm{PBF} 4$ Total $=\mathrm{PB} 12+\mathrm{PB} 13+\mathrm{PB} 11$

$\mathrm{PBF} 1$ Total $=\mathrm{PB} 3+\mathrm{PB} 4+\mathrm{PB} 5+\mathrm{PB} 6+\mathrm{PB} 7+\mathrm{PB} 8$;

AMTF1Total = DES1+DES2+DES3+DES4+PR2+IE1+PR3+PR7+PR8;

AMTF2Total = IE2+IE3+IC1+IC2+IC3+IC4+IC5+IC6+IC7+PR6;

AMTF3Total $=$ INS1+INS2+MAT1+MAT2;

AMTF4 Total = PR4+PR5+PR1

$\mathrm{ALLF} 2 \mathrm{~T}=\mathrm{BP} 3+\mathrm{BP} 4+\mathrm{BP} 5+\mathrm{BP} 6+\mathrm{BP} 7+\mathrm{BP} 8+\mathrm{BP} 9+\mathrm{BP} 10+\mathrm{BP} 11+\mathrm{BP} 12+\mathrm{AMTF} 2 \mathrm{Total}+$ AMTF3Total+PR4+PR5-PR6;

ALLF1T = PBF1Total+PBF2Total+PBF3Total+PBF4Total;

ALLF3T = IE1+BP1+RDFTOTAL-RD2+IS1+IS2+IS3+IS4+IS5+IS6+IS7+IS 8+IS9+IS11+

IS12+IS13+IS14+IS11+IS15+IS16+IS17+IS18;

ALLF4T = AMTF1Total-IE1+PR1+PR3+PR6+BP2+IS10;

ALLF5T = HRFTotal;

run;

Proc means data $=$ cozz.THESISAMT MEAN;

VAR BPF1Total BPF2Total BPF3Total ISF1Total ISF2Total

ISF3Total ISF4Total HRFTotal RDFTotal PBF1Total PBF2Total

PBF3Total PBF4Total AMTF1Total AMTF2Total AMTF3Total

AMTF4Total ALLF1T ALLF2T ALLF3T ALLF4T ALLF5T;

WHERE SizeCls = 'Small Plant'.

weight cozw9598;

OUTPUT OUT=SFactorMEANS Mean= SMBPF1Total SMBPF2Total SMBPF3Total

SMISF1Total SMISF2Total SMISF3Total SMISF4Total SMHRFTotal

SMRDFTotal SMPBF1Total SMPBF2Total

SMPBF3Total SMPBF4Total SMAMTF1Total SMAMTF2Total SMAMTF3Total

RUN ;

SMAMTF4Total SMALLF1T SMALLF2T SMALLF3T SMALLF4T SMALLF5T;

Proc means data=cozz.THESISAMT MEAN;

VAR BPF1Total BPF2Total BPF3Total ISF1Total ISF2Total

ISF3Total ISF4Total HRFTotal RDFTotal PBF1Total PBF2Total

PBF3Total PBF4Total AMTF1Total AMTF2Total AMTF3Total 
AMTF4Total ALLF1T ALLF2T ALLF3T ALLF4T ALLF5T;

WHERE SizeCls = 'Medium Plant';

weight cozw9598;

OUTPUT OUT=MFactorMEANS Mean= MMBPF1Total MMBPF2Total MMBPF3Total

MMISF1Total MMISF2Total MMISF3Total MMISF4Total MMHRFTotal

MMRDFTotal MMPBF1Total MMPBF2Total

MMPBF3Total MMPBF4Total MMAMTF1Total MMAMTF2Total MMAMTF3Total

MMAMTF4Total MMALLF1T MMALLF2T MMALLF3T MMALLF4T MMALLF5T;

RUN；

Proc means data=cozz.THESISAMT MEAN;

VAR BPF1Total BPF2Total BPF3Total ISF1Total ISF2Total

ISF3Total ISF4Total HRFTotal RDFTotal PBF1Total PBF2Total

PBF3Total PBF4Total AMTF1Total AMTF2Total AMTF3Total

AMTF4Total ALLF1T ALLF2T ALLF3T ALLF4T ALLF5T;

WHERE SizeCls = 'Large Plant';

weight cozw9598;

OUTPUT OUT=LFactorMEANS Mean= LMBPF1Total LMBPF2Total LMBPF3Total

LMISF1Total LMISF2Total LMISF3Total LMISF4Total LMHRFTotal

LMRDFTotal LMPBF1Total LMPBF2Total

LMPBF3Total LMPBF4Total LMAMTF1Total LMAMTF2Total LMAMTF3Total

RUN;

LMAMTF4Total LMALLF1T LMALLF2T LMALLF3T LMALLF4T LMALLF5T;

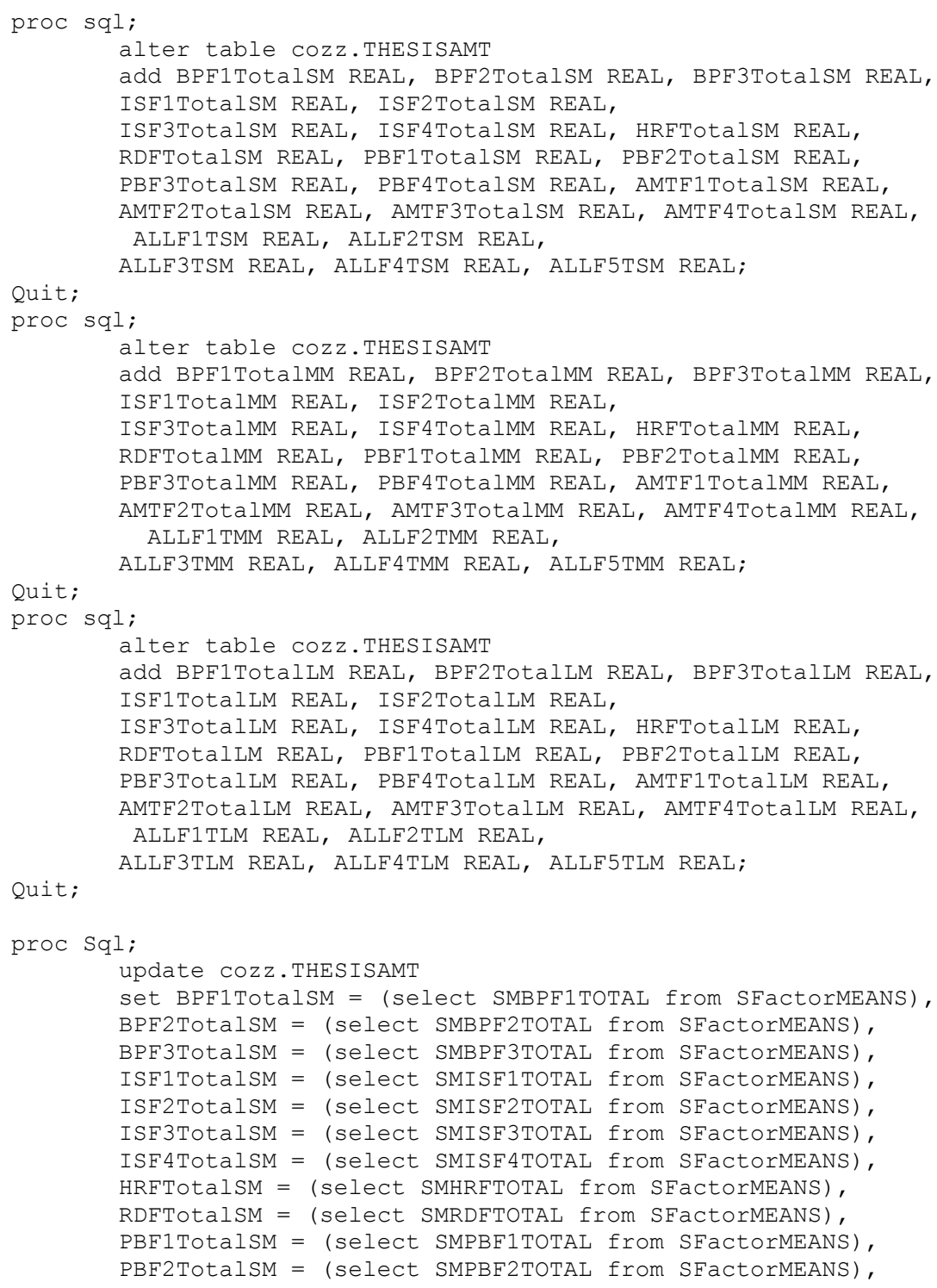


PBF3TotalSM $=$ (select SMPBF3TOTAL from SFactorMEANS), PBF4TotalSM = (select SMPBF4TOTAL from SFactorMEANS), AMTF1TotalSM = (select SMAMTF1TOTAL from SFactorMEANS), AMTF2TotalSM = (select SMAMTF2TOTAL from SFactorMEANS), AMTF3TotalSM = (select SMAMTF3TOTAL from SFactorMEANS), AMTF4TotalSM = (select SMAMTF4TOTAL from SFactorMEANS), ALLF1TSM = (select SMALLF1T from SFactorMEANS), ALLF2TSM $=$ (select SMALLF2T from SFactorMEANS) ALLF3TSM $=$ (select SMALLF3T from SFactorMEANS), ALLF4TSM = (select SMALLF4T from SFactorMEANS), ALLF5TSM = (select SMALLF5T from SFactorMEANS);

Quit; proc Sql; update cozz.THESISAMT set ALLFITSM = (select SMALLF1T from SFactorMEANS), ALLF2TSM $=$ (select SMALLF2T from SFactorMEANS), ALLF3TSM $=$ (select SMALLF3T from SFactorMEANS), ALLF4TSM = (select SMALLF4T from SFactorMEANS), ALLF5TSM = (select SMALLF5T from SFactorMEANS), BPF1TotalMM = (select MMBPF1TOTAL from MFactorMEANS), BPF2TotalMM = (select MMBPF2TOTAL from MFactorMEANS), BPF3TotalMM = (select MMBPF3TOTAL from MFactorMEANS), ISF1TotalMM = (select MMISF1TOTAL from MFactorMEANS), ISF2TotalMM = (select MMISF2TOTAL from MFactorMEANS), ISF3TotalMM = (select MMISF3TOTAL from MFactorMEANS), ISF4TotalMM = (select MMISF4TOTAL from MFactorMEANS), HRFTotalMM = (select MMHRFTOTAL from MFactorMEANS) RDFTotalMM = (select MMRDFTOTAL from MFactorMEANS), PBF1TotalMM = (select MMPBF1TOTAL from MFactorMEANS), PBF2TotalMM = (select MMPBF2TOTAL from MFactorMEANS), PBF3TotalMM = (select MMPBF3TOTAL from MFactorMEANS), PBF4TotalMM = (select MMPBF4TOTAL from MFactorMEANS), AMTF1TotalMM = (select MMAMTF1TOTAL from MFactorMEANS), AMTF2TotalMM = (select MMAMTF2TOTAL from MFactorMEANS), AMTF3TotalMM = (select MMAMTF3TOTAL from MFactorMEANS), AMTF4TotalMM = (select MMAMTF4TOTAL from MFactorMEANS), ALLF1TMM $=$ (select MMALLF1T from MFactorMEANS), ALLF2TMM = (select MMALLF2T from MFactorMEANS) ALLF3TMM $=$ (select MMALLF3T from MFactorMEANS), ALLF4TMM $=$ (select MMALLF4T from MFactorMEANS), Quit; ALLF5TMM $=$ (select MMALLF5T from MFactorMEANS);

proc Sql;

update cozz.THESISAMT

set BPF1TotalLM = (select LMBPF1TOTAL from LFactorMEANS), BPF2TotalLM = (select LMBPF2TOTAL from LFactorMEANS), BPF3TotalLM $=$ (select LMBPF3TOTAL from LFactorMEANS), ISF1TotalLM = (select LMISF1TOTAL from LFactorMEANS), ISF2TotalLM $=$ (select LMISF2TOTAL from LFactorMEANS), ISF3TotalLM $=$ (select LMISF3TOTAL from LFactorMEANS), ISF4TotalLM = (select LMISF4TOTAL from LFactorMEANS), HRFTotalLM = (select LMHRFTOTAL from LFactorMEANS), RDFTotalLM = (select LMRDFTOTAL from LFactorMEANS), PBF1TotalLM $=$ (select LMPBF1TOTAL from LFactorMEANS), PBF2TotalLM = (select LMPBF2TOTAL from LFactorMEANS), PBF3TotalLM $=$ (select LMPBF3TOTAL from LFactorMEANS), PBF4TotalLM = (select LMPBF4TOTAL from LFactorMEANS), AMTF1TotalLM = (select LMAMTF1TOTAL from LFactorMEANS), AMTF2TotalLM = (select LMAMTF2TOTAL from LFactorMEANS), AMTF3TotalLM = (select LMAMTF3TOTAL from LFactorMEANS), AMTF4TotalLM = (select LMAMTF4TOTAL from LFactorMEANS), ALLF1TLM = (select LMALLF1T from LFactorMEANS), ALLF2TLM = (select LMALLF2T from LFactorMEANS), ALLF3TLM $=$ (select LMALLF3T from LFactorMEANS), ALLF4TLM = (select LMALLF4T from LFactorMEANS), ALLF5TLM $=($ select LMALLF5T from LFactorMEANS $) ;$

QUIT;

/*** Use the means from the factors in order to create binary variables */

/*** This is done by SizeCls since small plants have a smaller value for the variables */ 
data cozz.THESISAMT;

set COZz.THESISAMT;

if SizeCls = 'Small Plant' then do;

BPF $1=($ BPF1Total $>=$ BPF1TotalSM $)$; $\mathrm{BPF} 2=(\mathrm{BPF} 2 \mathrm{Total}>=\mathrm{BPF} 2 \mathrm{TotalSM})$;

BPF3=(BPF3Total >=BPF3TotalSM) ;

ISF1 $=($ ISF1Total $>=$ ISF1TotalSM)

ISF2=(ISF2Total >=ISF2TotalSM $)$;

ISF3 $=($ ISF3Total >=ISF3TotalSM $)$;

ISF $4=($ ISF4Total >=ISF4TotalSM $)$;

$\mathrm{HRF}=($ HRFTotal $>=$ HRFTotalSM $)$;

$\mathrm{RDF}=($ RDFTotal $>=$ RDFTotalSM $)$;

$\mathrm{PBF} 1=(\mathrm{PBF} 1 \mathrm{Total}>=\mathrm{PBF}$ TotalsM $)$;

PBF2 $=($ PBF2Total > $>$ PBF2TotalSM $)$;

PBF3=(PBF3Total >=PBF3TotalsM) ;

$\mathrm{PBF} 4=(\mathrm{PBF} 4 \mathrm{Total}>=\mathrm{PBF} 4$ TotalsM $)$;

AMTF1 $=($ AMTF1Total > =AMTF1TotalSM $) ;$

AMTF2 $=($ AMTF2Total $>=$ AMTF2TotalSM $) ;$

AMTF3=(AMTF3Total >=AMTF3TotalSM $)$;

AMTF $4=($ AMTF 4 Total $>=$ AMTF4TotalSM $)$;

ALLF $1=($ ALLF1T $>=$ ALLF1TSM $)$;

ALLF2 $=(A L L F 2 T>=A L L F 2 T S M) ;$

ALLF3 $=($ ALLF3T $>=$ ALLF3TSM $)$;

ALLF $4=($ ALLF $4 \mathrm{~T}>=$ ALLF $4 \mathrm{TSM})$;

ALLF5 $=($ ALLF5T $>=$ ALLF5TSM $)$

end;

else if SizeCls = 'Medium Plant' then do; BPF1=(BPF1Total >=BPF1TotalMM); $\mathrm{BPF} 2=(\mathrm{BPF} 2 \mathrm{Total}>=\mathrm{BPF} 2 \mathrm{Total} M \mathrm{M}) ;$ $\mathrm{BPF} 3=(\mathrm{BPF} 3 \mathrm{Total}>=\mathrm{BPF} 3 \mathrm{TotalMM})$; ISF $1=($ ISF1Total >=ISF1TotalMM) ; ISF2 $=($ ISF2Total >=ISF2TotalMM $)$; ISF3 $=($ ISF3Total >=ISF3TotalMM) ; ISF4 $=($ ISF4Total >=ISF4TotalMM $)$; HRF $=($ HRFTotal $>=$ HRFTotalMM $)$; $\mathrm{RDF}=($ RDFTotal $>=$ RDFTotalMM $)$; PBF1 $=($ PBF1Total > =PBF1TotalMM $)$; PBF2 $=($ PBF2Total $>=$ PBF2TotalMM $) ;$ PBF3=(PBF3Total >=PBF3TotalMM) ; PBF4 $=($ PBF4Total $>=$ PBF4TotalMM $) ;$ AMTF1=(AMTF1Total >=AMTF1TotalMM); AMTF2 $=($ AMTF2Total >=AMTF2TotalMM $) ;$ AMTF3=(AMTF3Total >=AMTF3TotalMM) ; AMTF4 $=($ AMTF4Total >=AMTF4TotalMM $)$; $A L L F 1=(A L L F 1 T>=A L L F 1 T M M)$; ALLF2 $=($ ALLF2T $>=$ ALLF2TMM $)$; $A L L F 3=(A L L F 3 T>=A L L F 3 T M M)$; ALLF4 $=($ ALLF 4T $>=A L L F 4 T M M) ;$ ALLF5 $=($ ALLF5T $>=$ ALLF5TMM $)$

end;

else do;

/* The Plant is a Large Plant */ BPF1 $=($ BPF1Total > $>$ BPF1TotalLM $)$; BPF2 $=($ BPF2Total > $=$ BPF2TotalLM $)$; BPF3=(BPF3Total >=BPF3TotalLM); ISF1 $=($ ISF1Total >=ISF1TotalLM); ISF2 $=($ ISF2Total >=ISF2TotalLM $)$; ISF3 $=($ ISF3Total >=ISF3TotalLM $)$; ISF4 $=($ ISF4Total >=ISF4TotalLM); HRF $=($ HRFTotal $>=$ HRFTotalLM) RDF $=($ RDFTotal > =RDFTotalLM); PBF1 $=($ PBF1Total > $>$ PBF1TotalLM $)$; PBF2 $=($ PBF2Total $>=$ PBF2TotalLM $) ;$ PBF3 $=($ PBF3Total $>=$ PBF3TotalLM $)$; $\mathrm{PBF} 4=(\mathrm{PBF} 4 \mathrm{Total}>=\mathrm{PBF} 4 \mathrm{TotalLM})$; AMTF1=(AMTF1Total >=AMTF1TotalLM) ; AMTF2 $=($ AMTF2Total >=AMTF2TotalLM $) ;$ AMTF3=(AMTF3Total >=AMTF3TotalLM $)$; AMTF4 $=($ AMTF 4 Total >=AMTF4TotalLM $)$; $A L L F 1=(A L L F 1 T>=A L L F 1 T L M)$; 


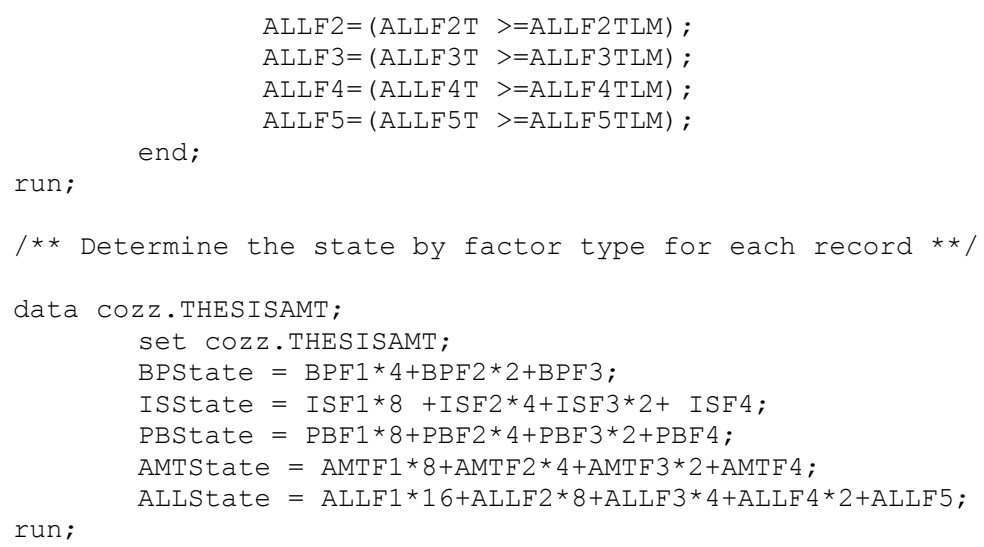

\section{F2 State Information Program}

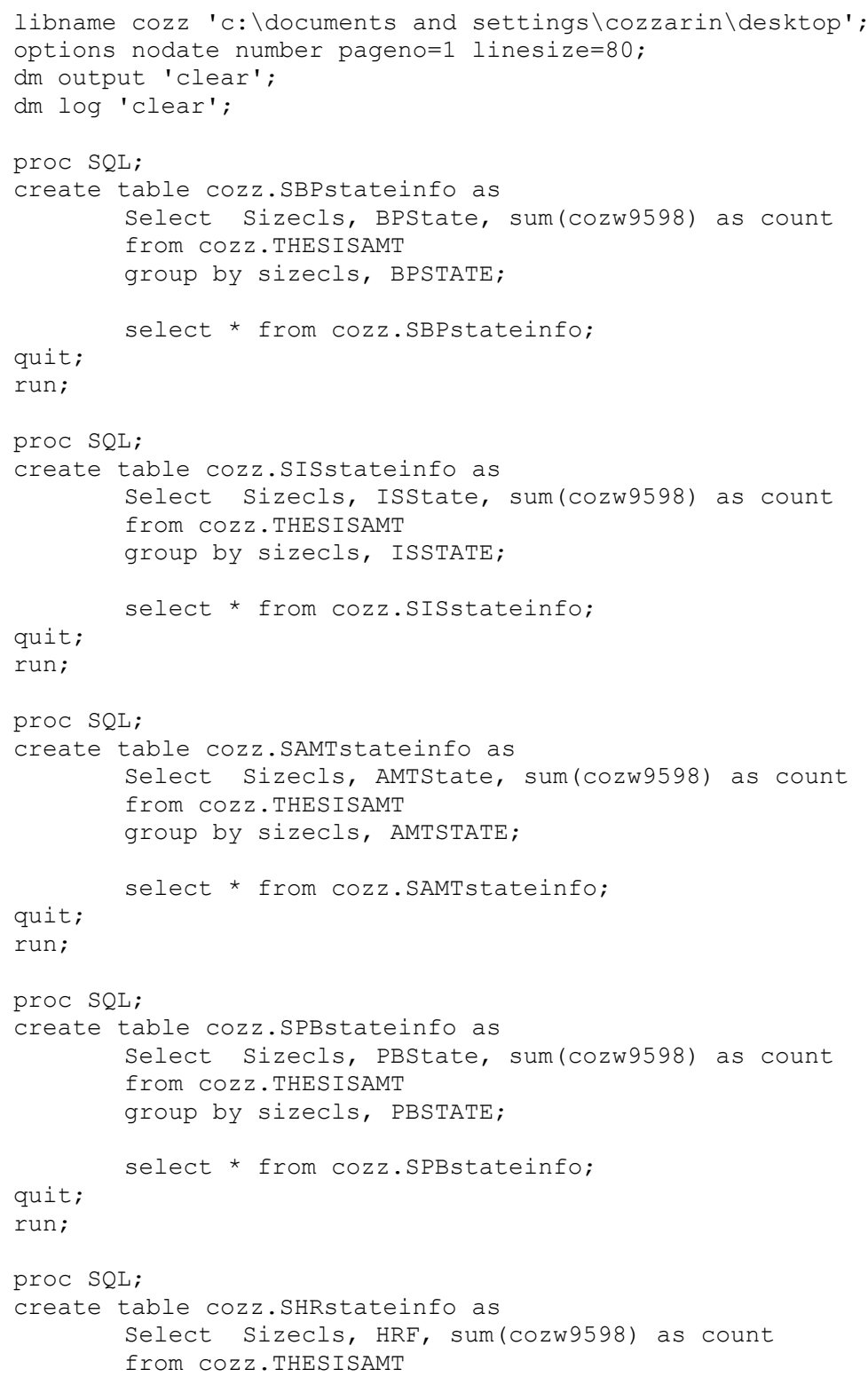




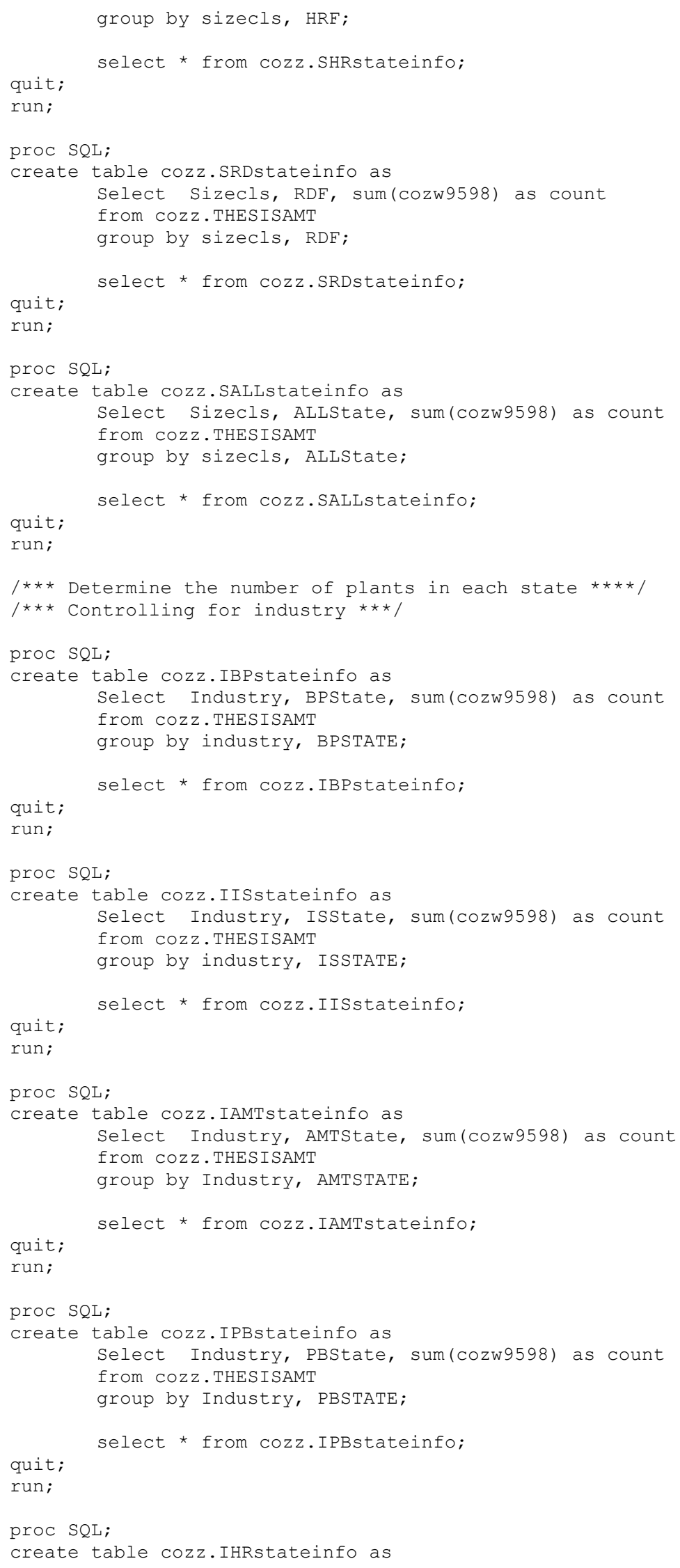




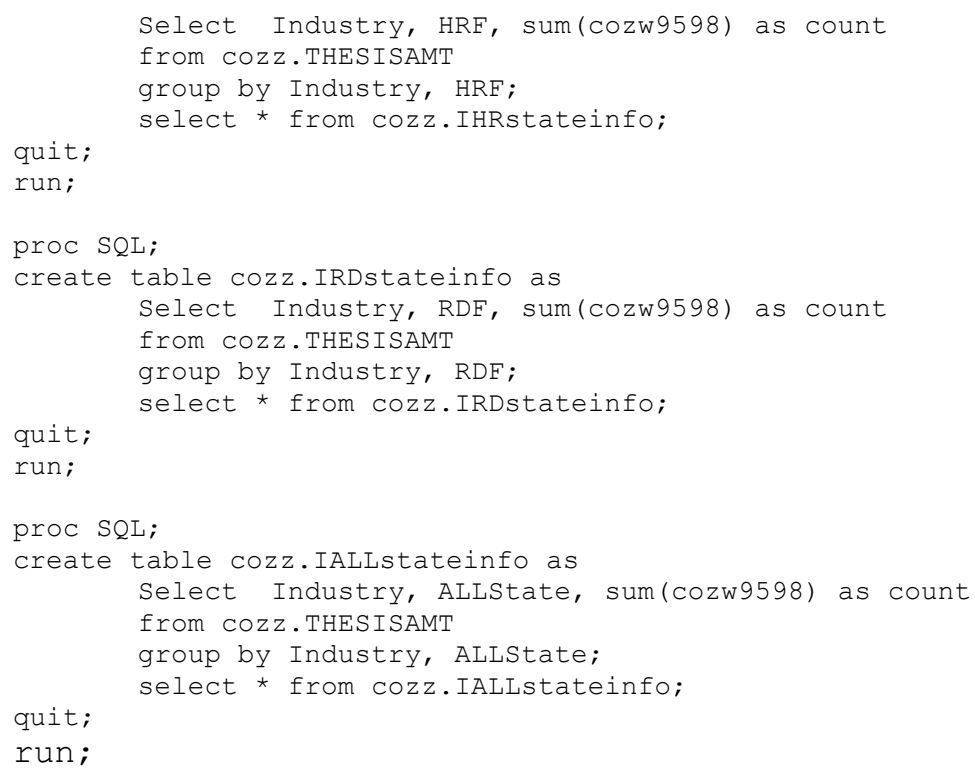

run;

\section{F3 Business Practices Constrained Regression - Supermodularity Constraints}

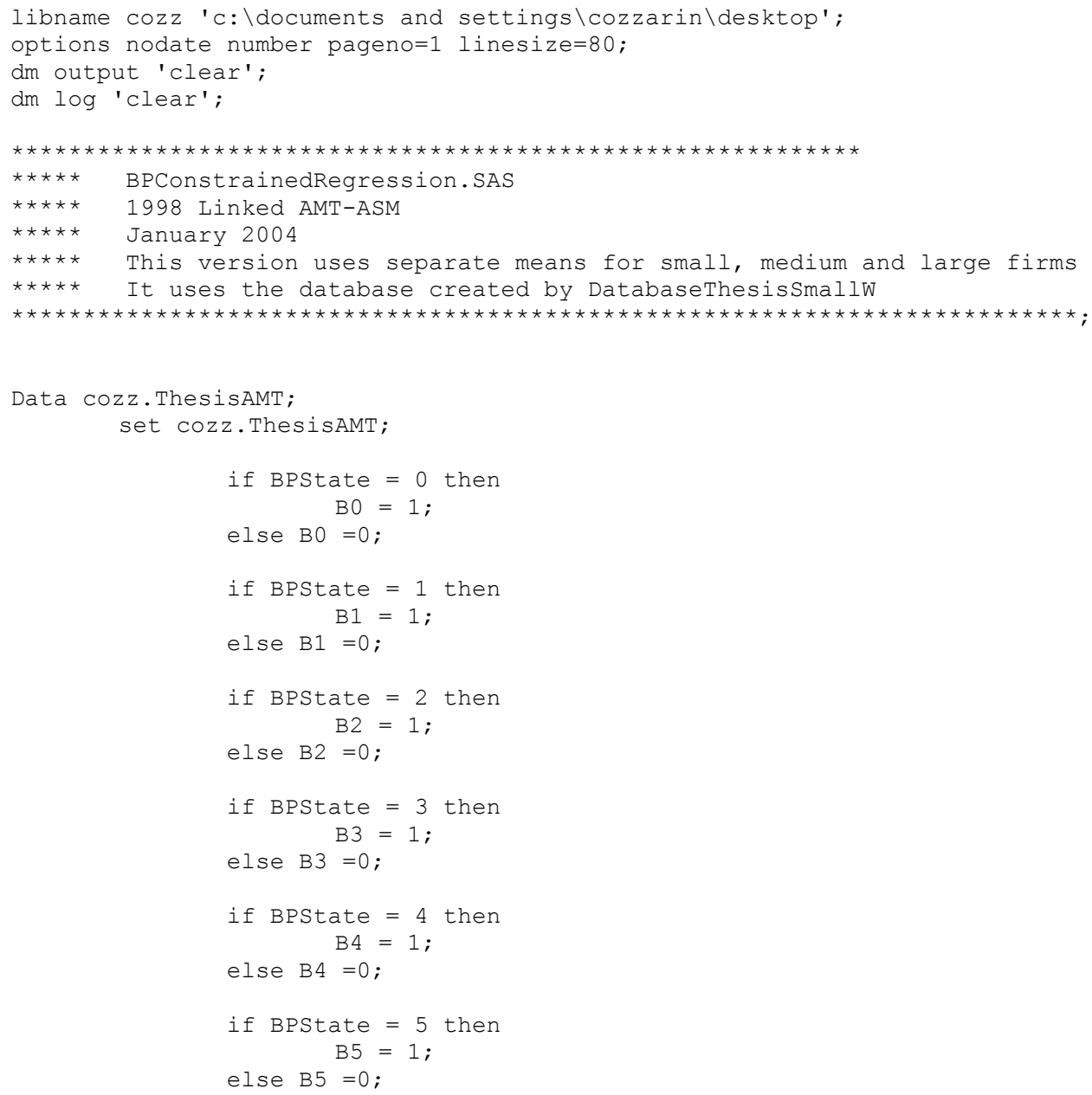




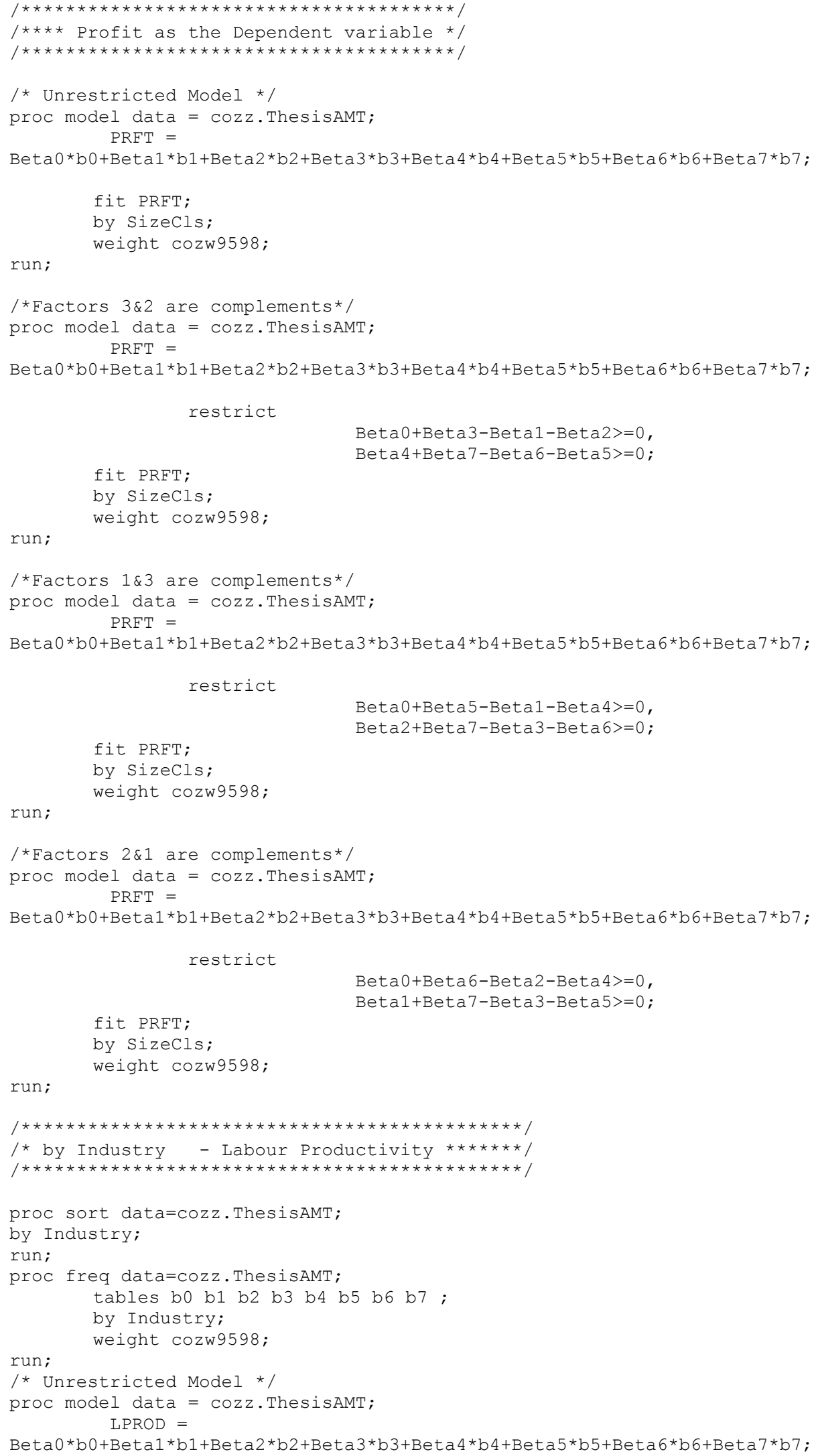




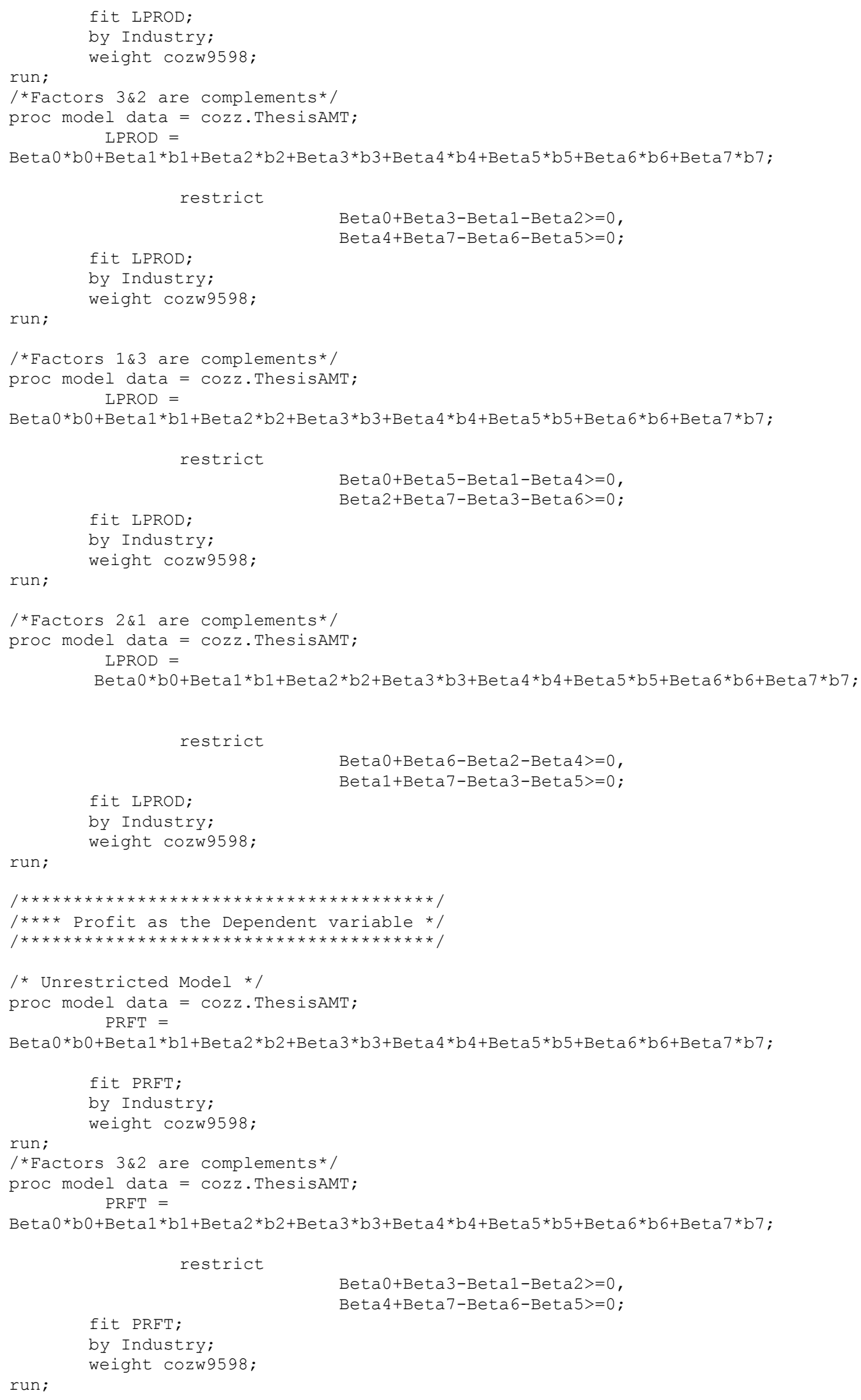




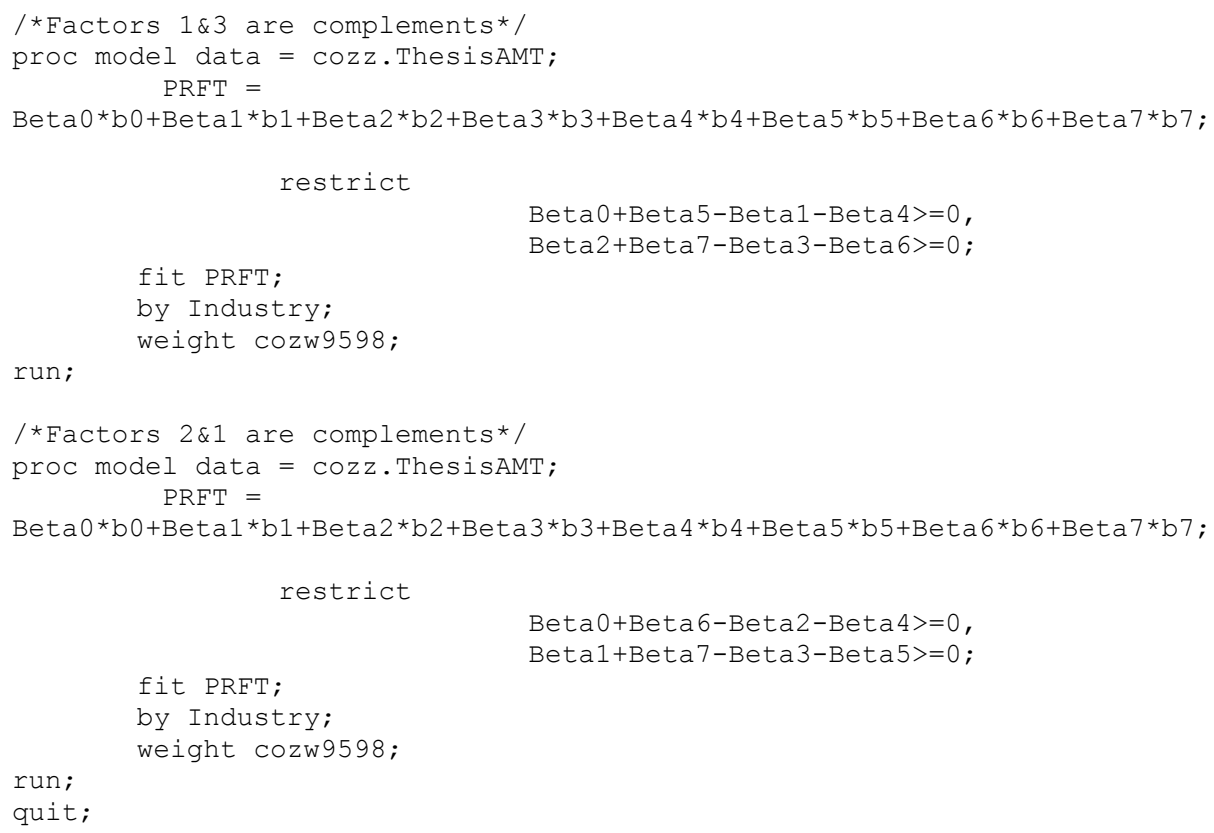

\section{F4 AMT Constrained Regression - Submodularity Constraints}

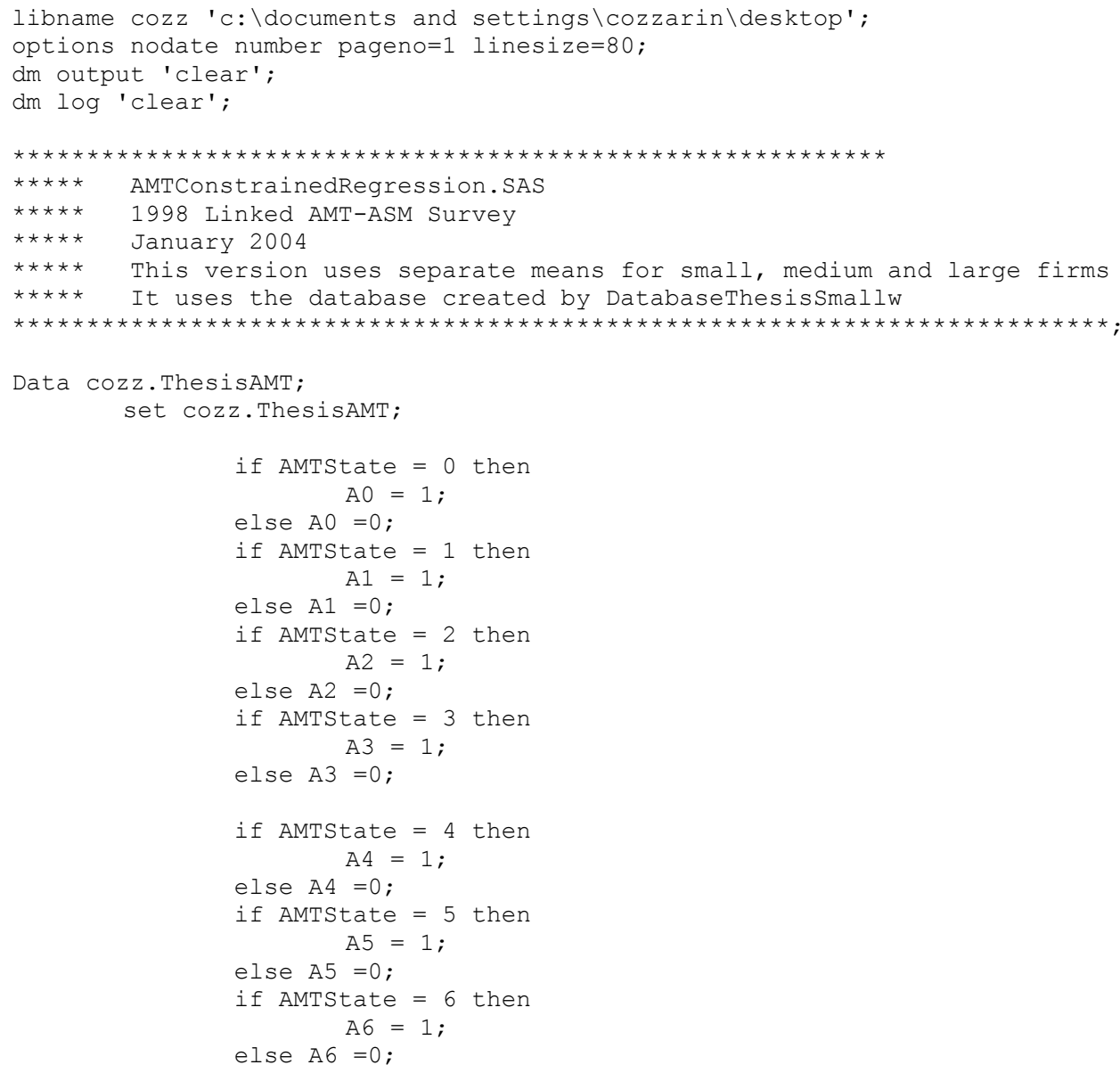




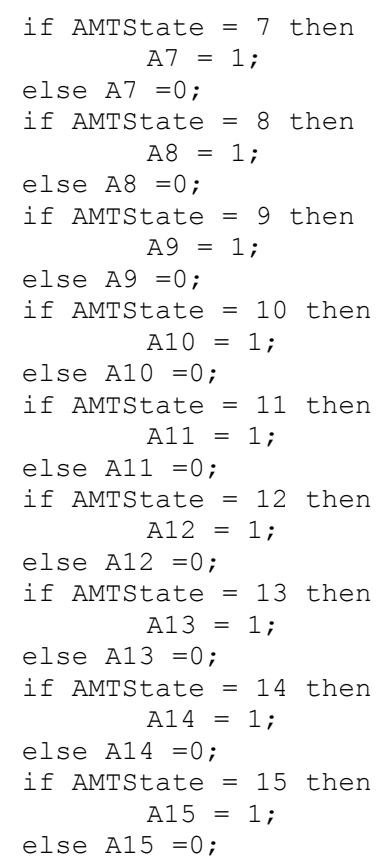

run;

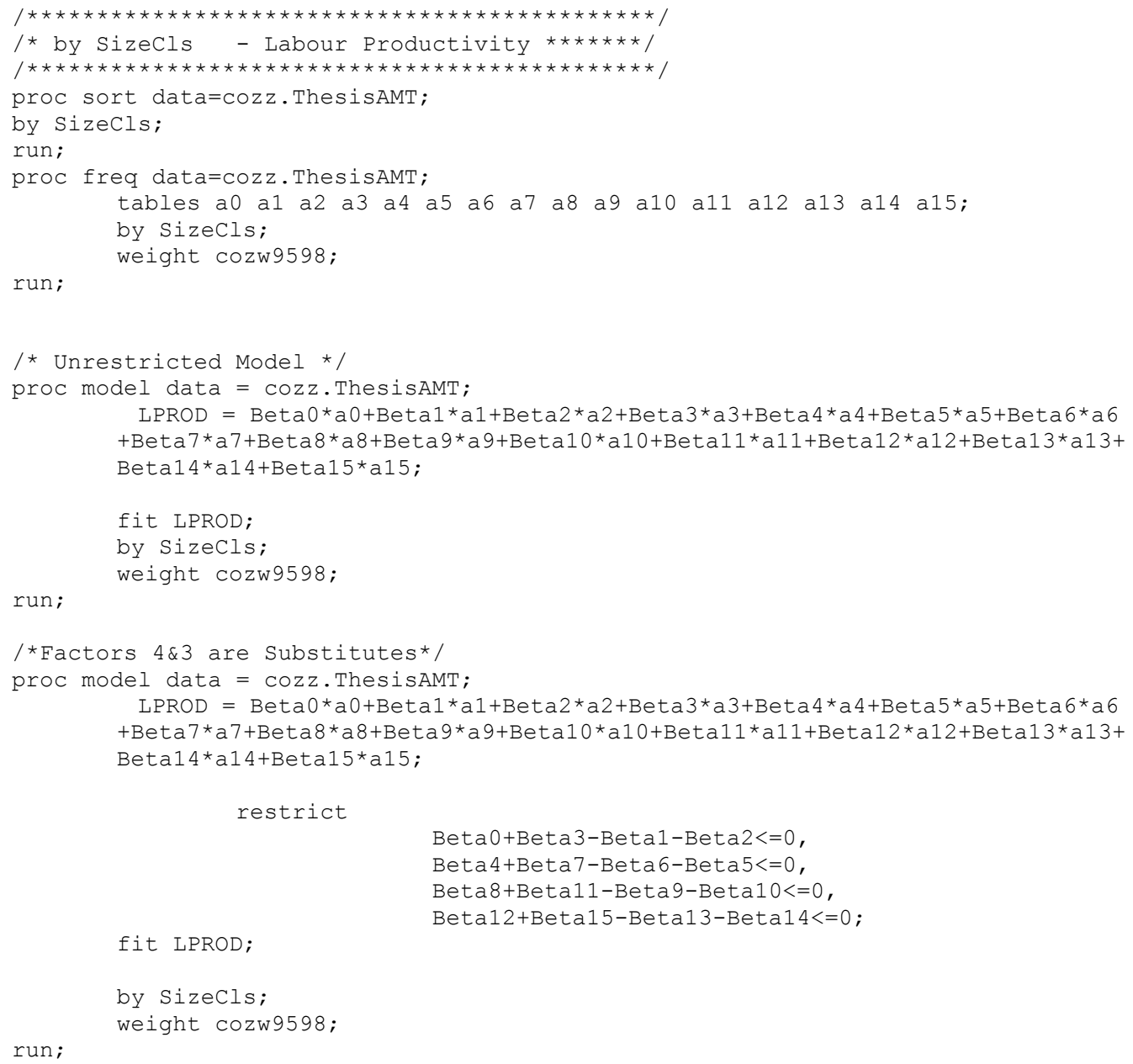




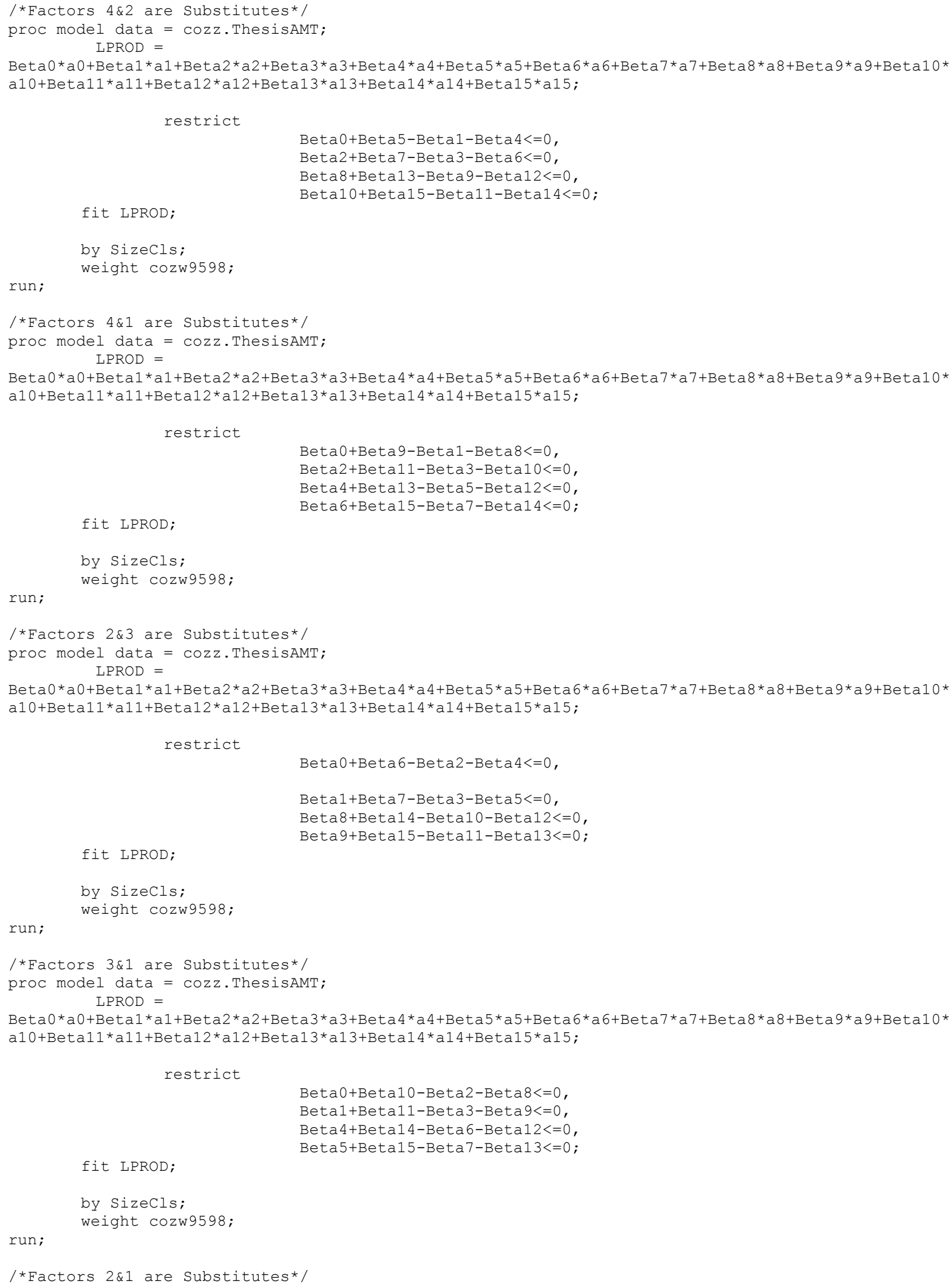


proc model data $=$ cozz.ThesisAMT; $\mathrm{LPROD}=$

Beta $0 * a 0+B e t a 1 * a 1+B e t a 2 * a 2+$ Beta3*a3+Beta $4 * a 4+B e t a 5 * a 5+B e t a 6 * a 6+B e t a 7 * a 7+B e t a 8 * a 8+B e t a 9 * a 9+B e t a 10 *$ a10+Beta11*a11+Beta12*a12+Beta13*a13+Beta14*a14+Beta15*a15;

restrict

fit LPROD;

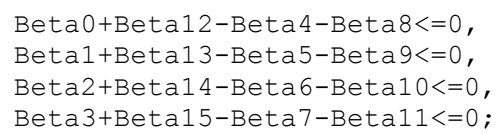




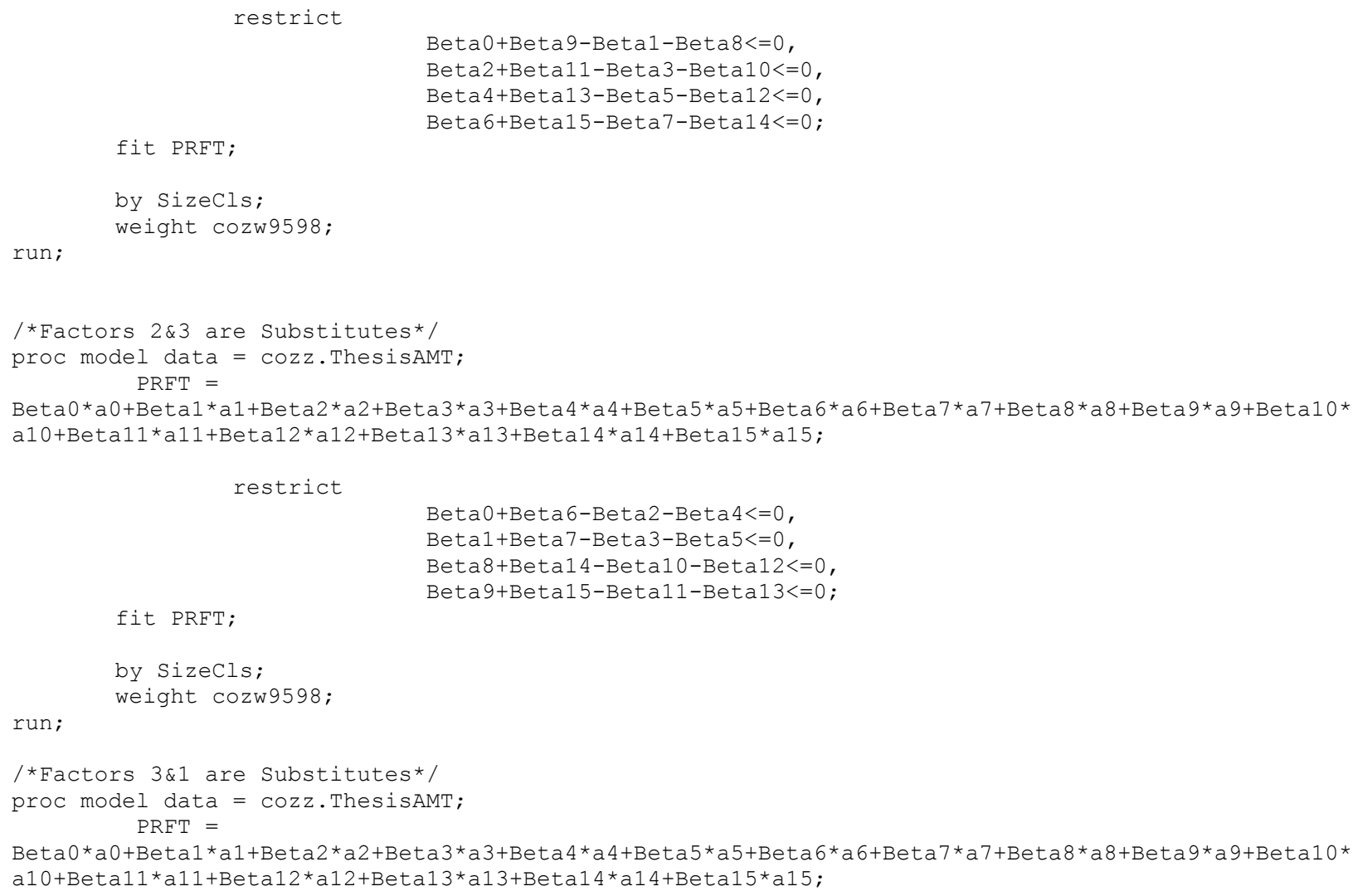




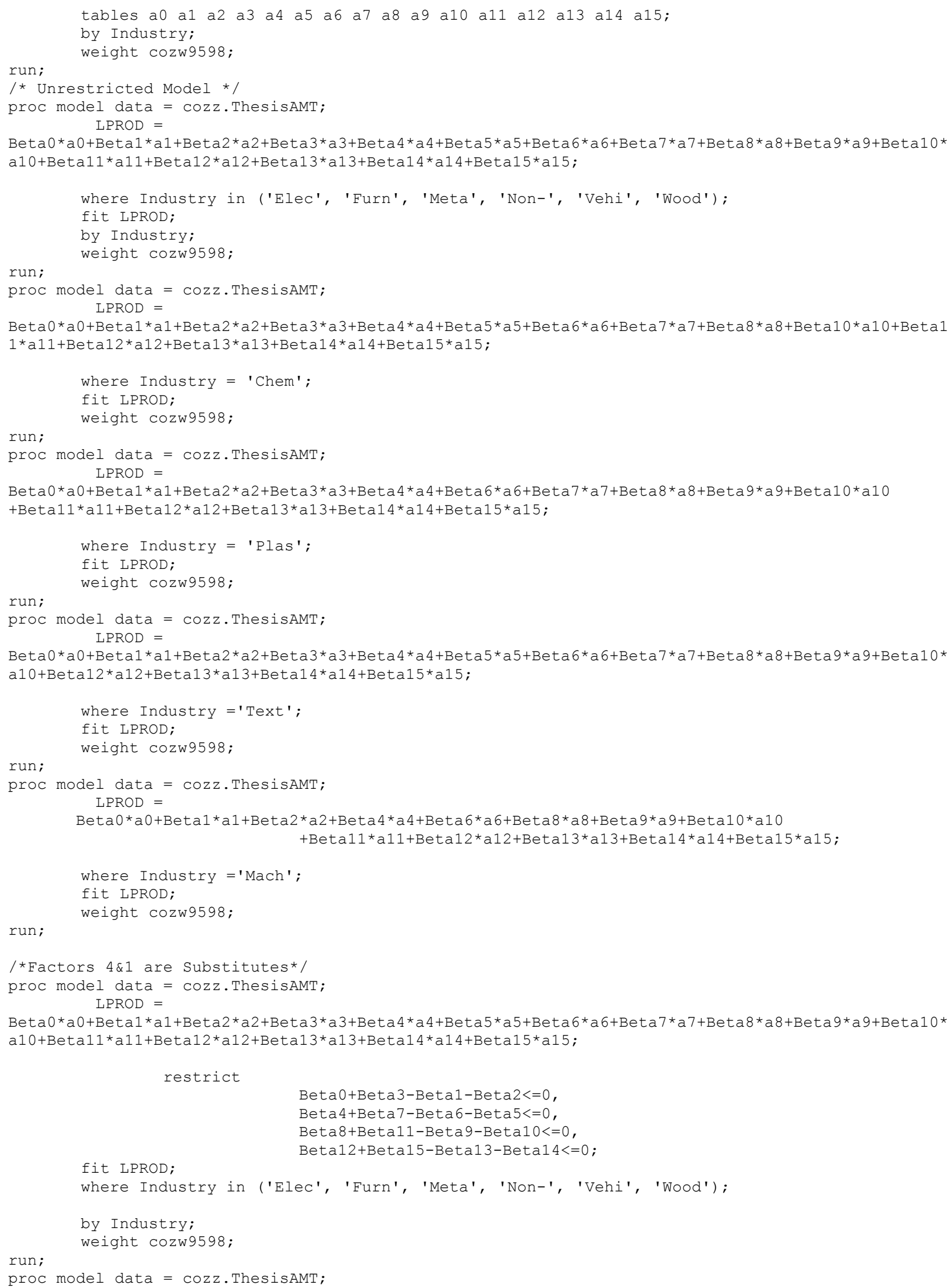


$\mathrm{LPROD}=$

Beta0*a0+Beta1*a1+Beta2*a2+Beta3*a3+Beta4*a4+Beta5*a5+Beta6*a6+Beta7*a7+Beta8*a8+Beta10*a10+Beta1 $1 * a 11+$ Beta $12 * a 12+$ Beta13*a13+Beta14*a14+Beta15*a15;

restrict

Beta0+Beta3-Beta1-Beta $2<=0$, Beta $4+$ Beta $7-$ Beta $6-$ Beta $5<=0$, fit LPROD; Beta12+Beta15-Beta13-Beta14<=0;

where Industry = 'Chem';

run;

weight cozw9598;

proc model data $=$ cozz.ThesisAMT;

LPROD $=$

Beta $0 * a 0+B e t a 1 * a 1+B e t a 2 * a 2+B e t a 3 * a 3+B e t a 4 * a 4+B e t a 6 * a 6+B e t a 7 * a 7+B e t a 8 * a 8+B e t a 9 * a 9+B e t a 10 * a 10+B e t a 1$ $1 * a 11+$ Beta $12 * a 12+$ Beta13*a13+Beta14*a14+Beta15*a15;

restrict

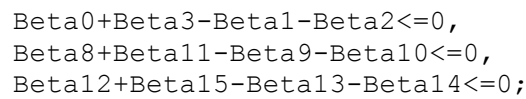


$\mathrm{LPROD}=$

Beta $0 * a 0+B e t a 1 * a 1+B e t a 2 * a 2+B e t a 3 * a 3+B e t a 4 * a 4+B e t a 5 * a 5+B e t a 6 * a 6+B e t a 7 * a 7+B e t a 8 * a 8+B e t a 10 * a 10+B e t a 1$ $1 * a 11+$ Beta $12 * a 12+$ Beta13*a13+Beta14*a14+Beta15*a15;

restrict

Beta0+Beta5-Beta1-Beta $4<=0$, Beta $2+$ Beta $7-$ Beta $3-$ Beta $6<=0$, fit LPROD; Beta10+Beta15-Beta11-Beta14<=0

where Industry = 'Chem';

run;

weight cozw9598;

proc model data $=$ cozz.ThesisAMT;

LPROD $=$

Beta $0 * a 0+B e t a 1 * a 1+B e t a 2 * a 2+B e t a 3 * a 3+$ Beta $4 * a 4+B e t a 6 * a 6+$ Beta $7 * a 7+B e t a 8 * a 8+B e t a 9 * a 9+B e t a 10 * a 10+B e t a 1$ $1 * a 11+$ Beta $12 * a 12+$ Beta13*a13+Beta14*a14+Beta15*a15;

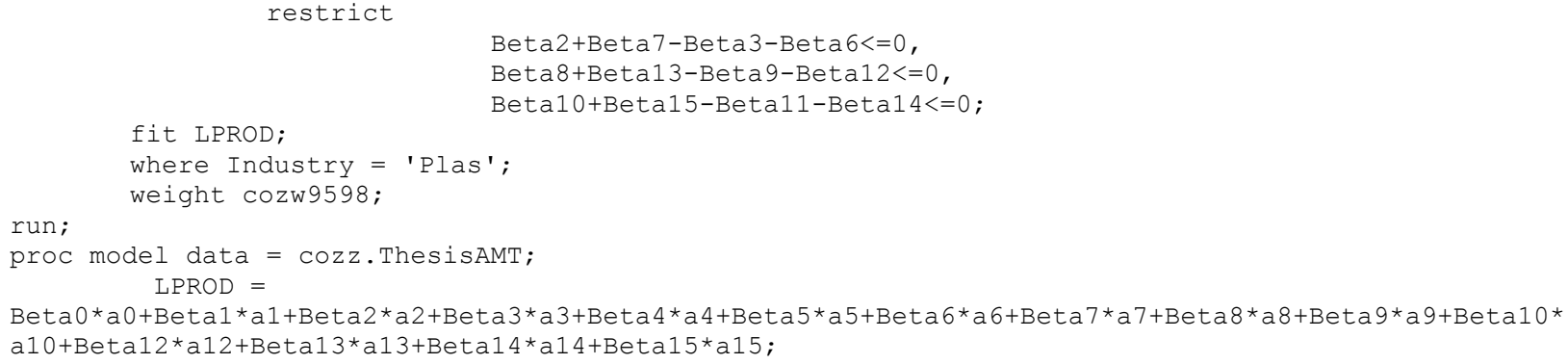


LPROD $=$

Beta0*a0+Beta1*a1+Beta2*a2+Beta3*a3+Beta4*a4+Beta5*a5+Beta6*a6+Beta7*a7+Beta8*a8+Beta10*a10+Beta1 $1 * a 11+$ Beta12*a12+Beta13*a13+Beta14*a14+Beta15*a15;

restrict

Beta2+Beta11-Beta3-Beta10<=0, Beta4+Beta13-Beta5-Beta12<=0,

fit LPROD; Beta6+Beta15-Beta7-Beta14<=0;

where Industry = 'Chem';

run;

weight cozw9598;

proc model data $=$ cozz.ThesisAMT;

LPROD $=$

Beta0*a0+Beta1*a1+Beta2*a2+Beta3*a3+Beta4*a4+Beta6*a6+Beta7*a7+Beta8*a8+Beta9*a9+Beta10*a10+Beta1

$1 * a 11+$ Beta12*a12+Beta13*a13+Beta14*a14+Beta15*a15;

restrict

Beta0+Beta9-Beta1-Beta $8<=0$,
Beta2+Beta11-Beta3-Beta10<=0,
Beta6+Beta15-Beta7-Beta14<=0;

fit LPROD;

where Industry = 'Plas';

run;

weight cozw9598;

proc model data $=$ cozz. ThesisAMT;

LPROD $=$

Beta $0 * a 0+B e t a 1 * a 1+B e t a 2 * a 2+B e t a 3 * a 3+B e t a 4 * a 4+B e t a 5 * a 5+B e t a 6 * a 6+B e t a 7 * a 7+B e t a 8 * a 8+B e t a 9 * a 9+B e t a 10 *$ a10+Beta12*a12+Beta13*a13+Beta14*a14+Beta15*a15;

restrict

Beta0+Beta9-Beta1-Beta $8<=0$,

Beta4+Beta13-Beta5-Beta12<=0,

fit LPROD;

Beta6+Beta15-Beta7-Beta14<=0;

where Industry ='Text';

weight cozw9598;

run;

proc model data $=$ cozz. ThesisAMT;

LPROD $=$

Beta $0 * a 0+$ Beta $1 * a 1+$ Beta $2 * a 2+$ Beta $4 * a 4+$ Beta $6 * a 6+$ Beta $8 * a 8+$ Beta $9 * a 9+$ Beta $10 * a 10$

+Beta11*a11+Beta12*a12+Beta13*a13+Beta14*a14+Beta15*a15;

restrict

fit LPROD;

Beta0+Beta9-Beta1-Beta $8<=0 ;$

where Industry ='Mach';

weight cozw9598;

run;

/*Factors $2 \& 3$ are Substitutes*/

proc model data $=$ cozz.ThesisAMT;

LPROD $=$

Beta0*a0+Beta1*a1+Beta2*a2+Beta3*a3+Beta4*a4+Beta5*a5+Beta6*a6+Beta7*a 7+Beta 8*a8+Beta9*a9+Beta10* a10+Beta11*a11+Beta12*a12+Beta13*a13+Beta14*a14+Beta15*a15;

restrict

Beta0+Beta6-Beta2-Beta $4<=0$,

Beta1+Beta7-Beta3-Beta $5<=0$,

Beta8+Beta14-Beta10-Beta12<=0,

fit LPROD;

Beta9+Beta15-Beta11-Beta13<=0;

where Industry in ('Elec', 'Furn', 'Meta', 'Non-', 'Vehi', 'Wood');

by Industry;

weight cozw9598;

run;

proc model data $=$ cozz.ThesisAMT;

$\mathrm{LPROD}=$

Beta $0 * a 0+B e t a 1 * a 1+B e t a 2 * a 2+B e t a 3 * a 3+B e t a 4 * a 4+B e t a 5 * a 5+B e t a 6 * a 6+B e t a 7 * a 7+B e t a 8 * a 8+B e t a 10 * a 10+B e t a 1$ $1 * a 11+$ Beta12*a12+Beta13*a13+Beta14*a14+Beta15*a15; 


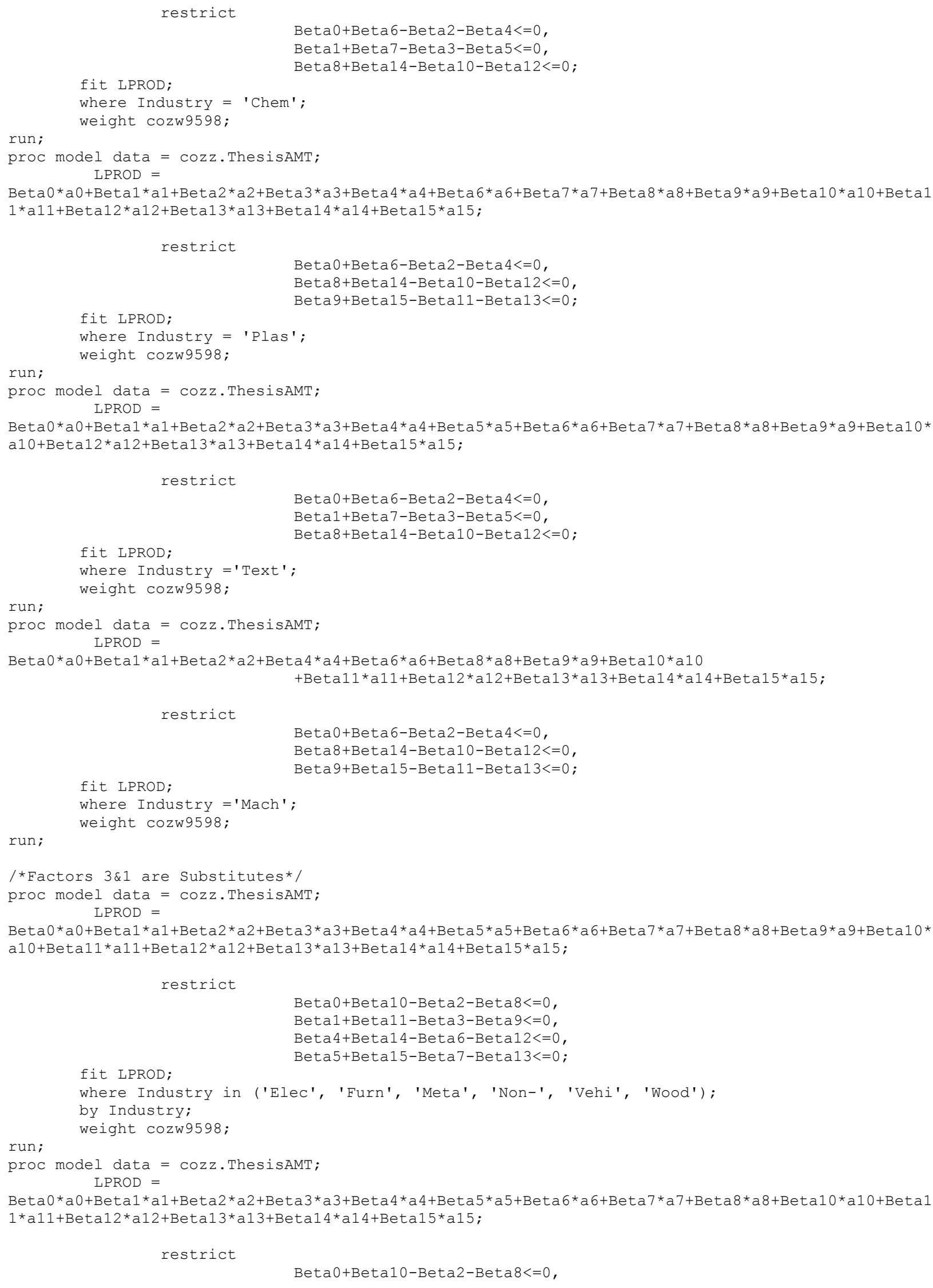




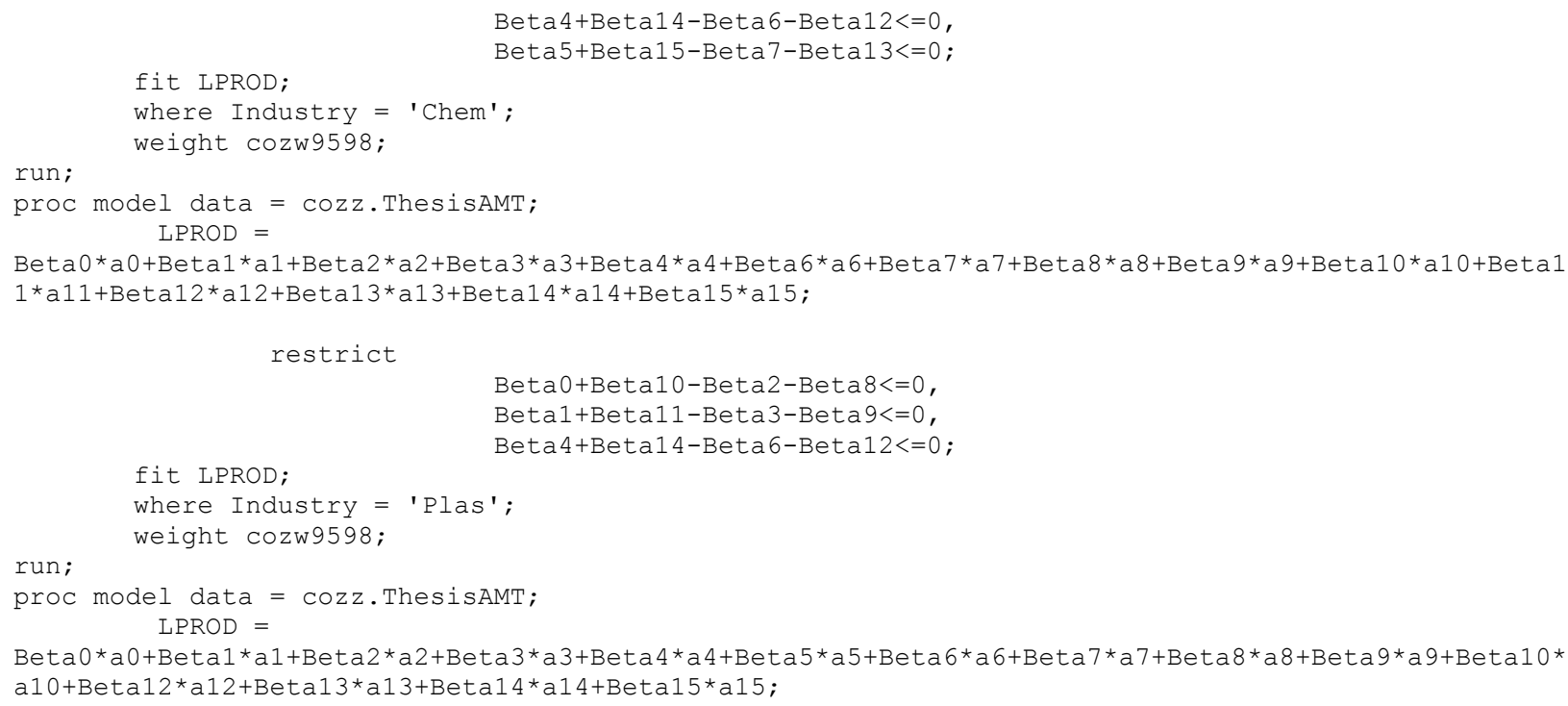

fit LPROD; 


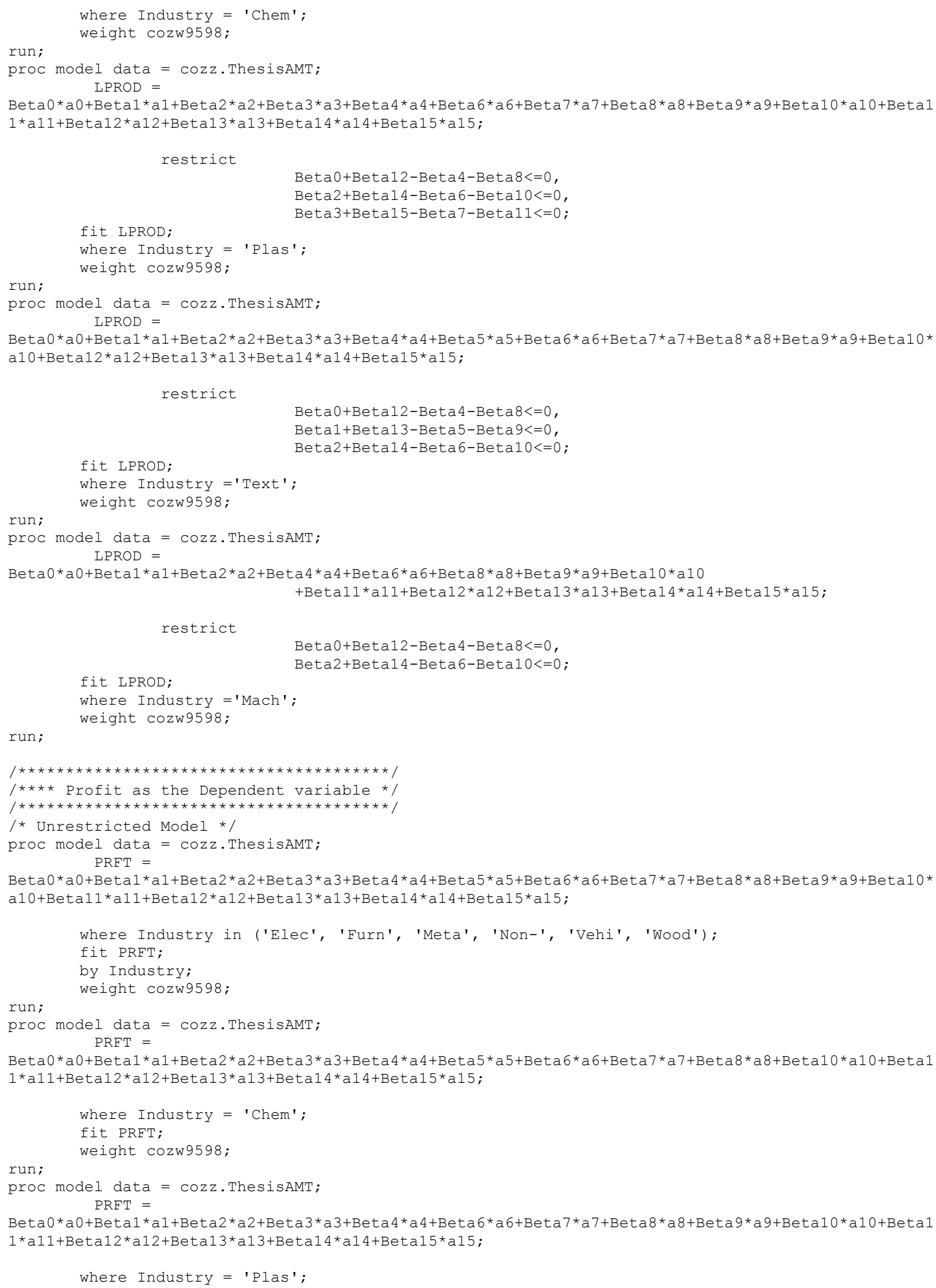




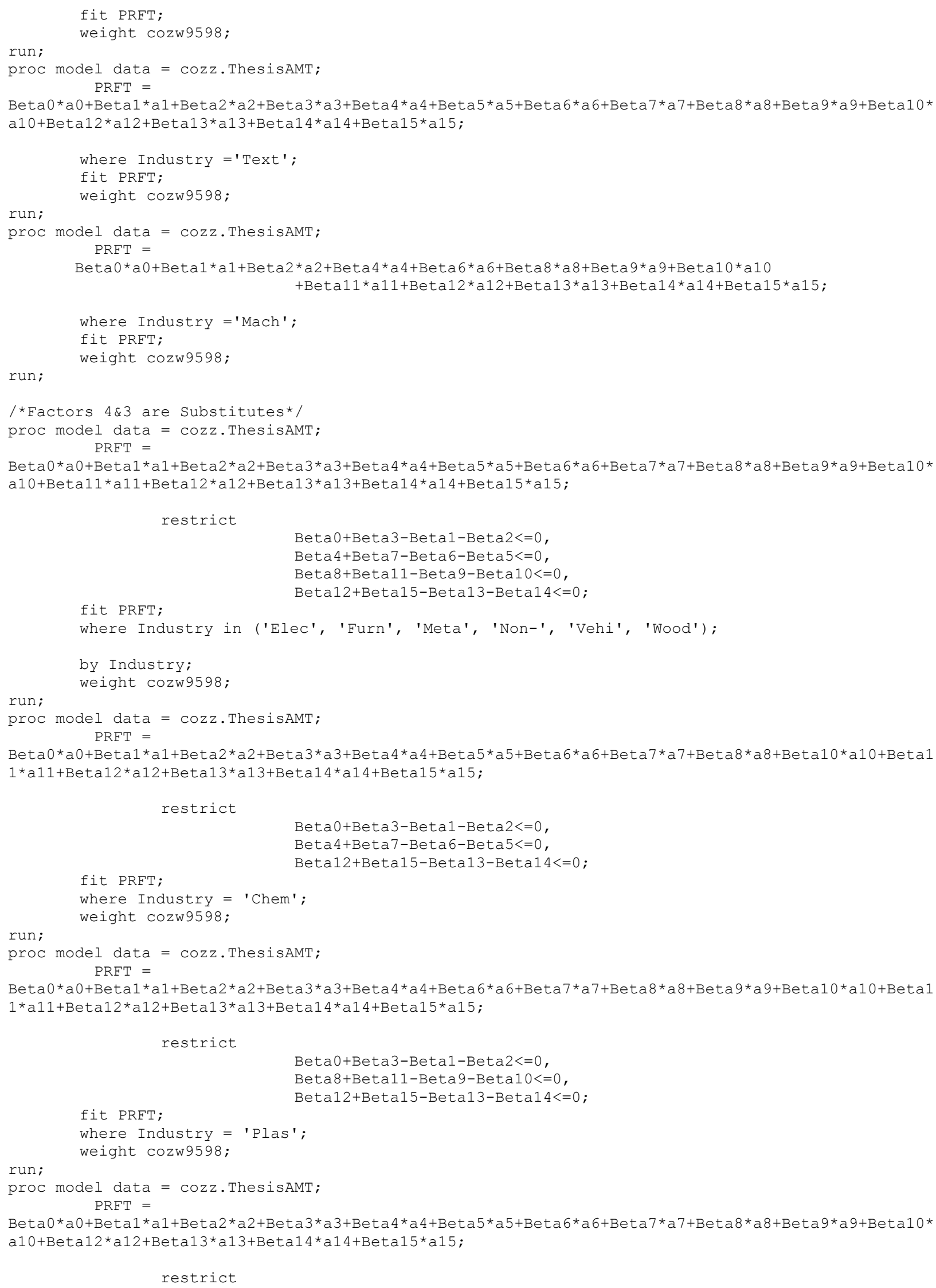


Beta $0+$ Beta $3-$ Beta $1-$ Beta $2<=0$,

Beta $4+$ Beta $7-$ Beta $6-$ Beta $5<=0$,

fit PRFT;

Beta12+Beta15-Beta13-Beta14<=0;

where Industry =' Text' ;

weight cozw9598;

run;

proc model data $=$ cozz. ThesisAMT;

$\mathrm{PRFT}=$

Beta $0 * a 0+$ Beta $1 * a 1+$ Beta2*a2+Beta $4 * a 4+$ Beta $6 * a 6+$ Beta $8 * a 8+$ Beta $9 * a 9+$ Beta $10 * a 10$

+Beta11*a11+Beta12*a12+Beta13*a13+Beta14*a14+Beta15*a15;

restrict

Beta8+Beta11-Beta9-Beta10<=0,

fit PRFT;

Beta12+Beta15-Beta13-Beta14<=0

where Industry ='Mach';

weight cozw9598;

run;

/*Factors $4 \& 2$ are Substitutes*/

proc model data $=$ cozz.ThesisAMT;

PRFT $=$

Beta $0 * a 0+B e t a 1 * a 1+B e t a 2 * a 2+B e t a 3 * a 3+B e t a 4 * a 4+B e t a 5 * a 5+B e t a 6 * a 6+B e t a 7 * a 7+B e t a 8 * a 8+B e t a 9 * a 9+B e t a 10 *$ a10+Beta11*a11+Beta12*a12+Beta13*a13+Beta14*a14+Beta15*a15;

restrict

fit PRFT;

where Industry in ('Elec', 'Furn', 'Meta', 'Non-', 'Vehi', 'Wood');

by Industry;

weight cozw9598;

run;

proc model data = cozz.ThesisAMT;

PRFT $=$

Beta $0 * a 0+B e t a 1 * a 1+B e t a 2 * a 2+B e t a 3 * a 3+B e t a 4 * a 4+B e t a 5 * a 5+B e t a 6 * a 6+B e t a 7 * a 7+B e t a 8 * a 8+B e t a 10 * a 10+B e t a 1$

$1 * a 11+$ Beta12*a12+Beta13*a13+Beta14*a14+Beta15*a15;

Beta0+Beta5-Beta1-Beta $4<=0$,

Beta2+Beta $7-$ Beta3-Beta $6<=0$,

Beta8+Beta13-Beta9-Beta12<=0,

Beta10+Beta15-Beta11-Beta14<=0

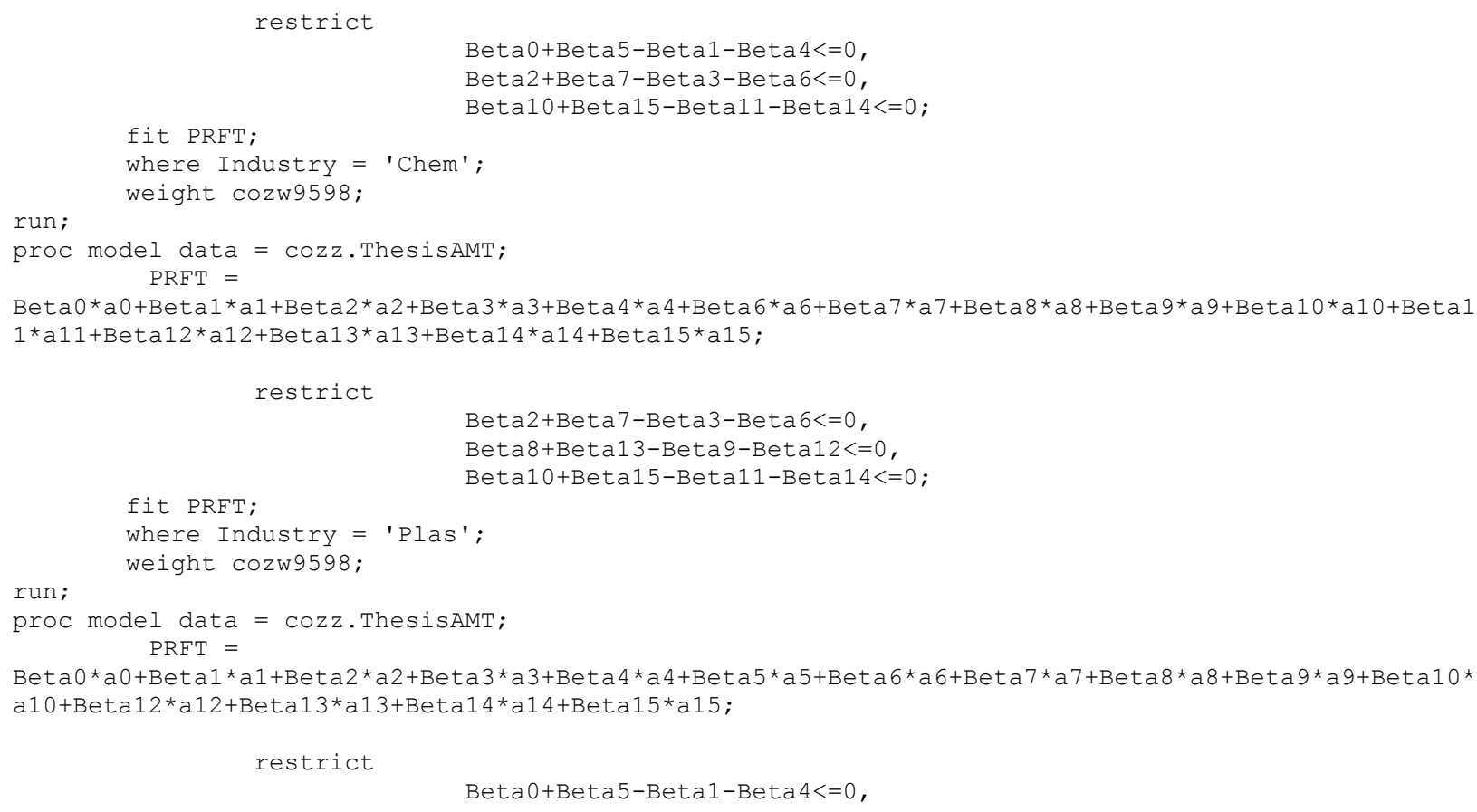

restrict 
Beta2+Beta $7-$ Beta $3-$ Beta $6<=0$,

Beta8+Beta13-Beta9-Beta12<=0;

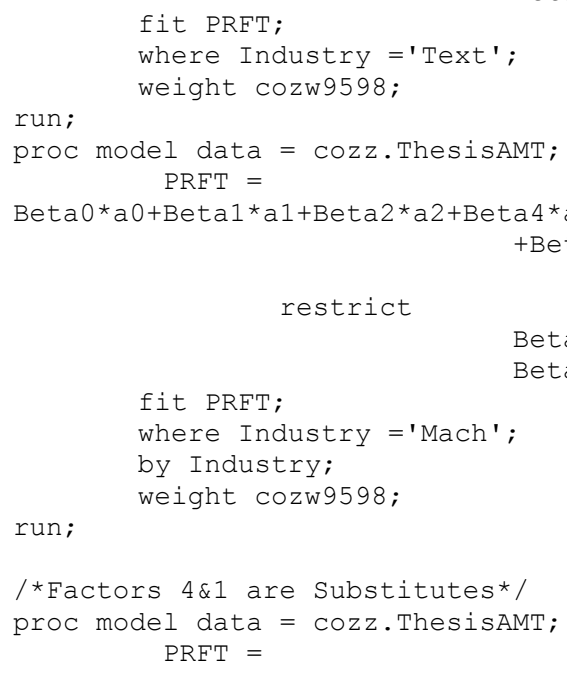

fit PRFT; 


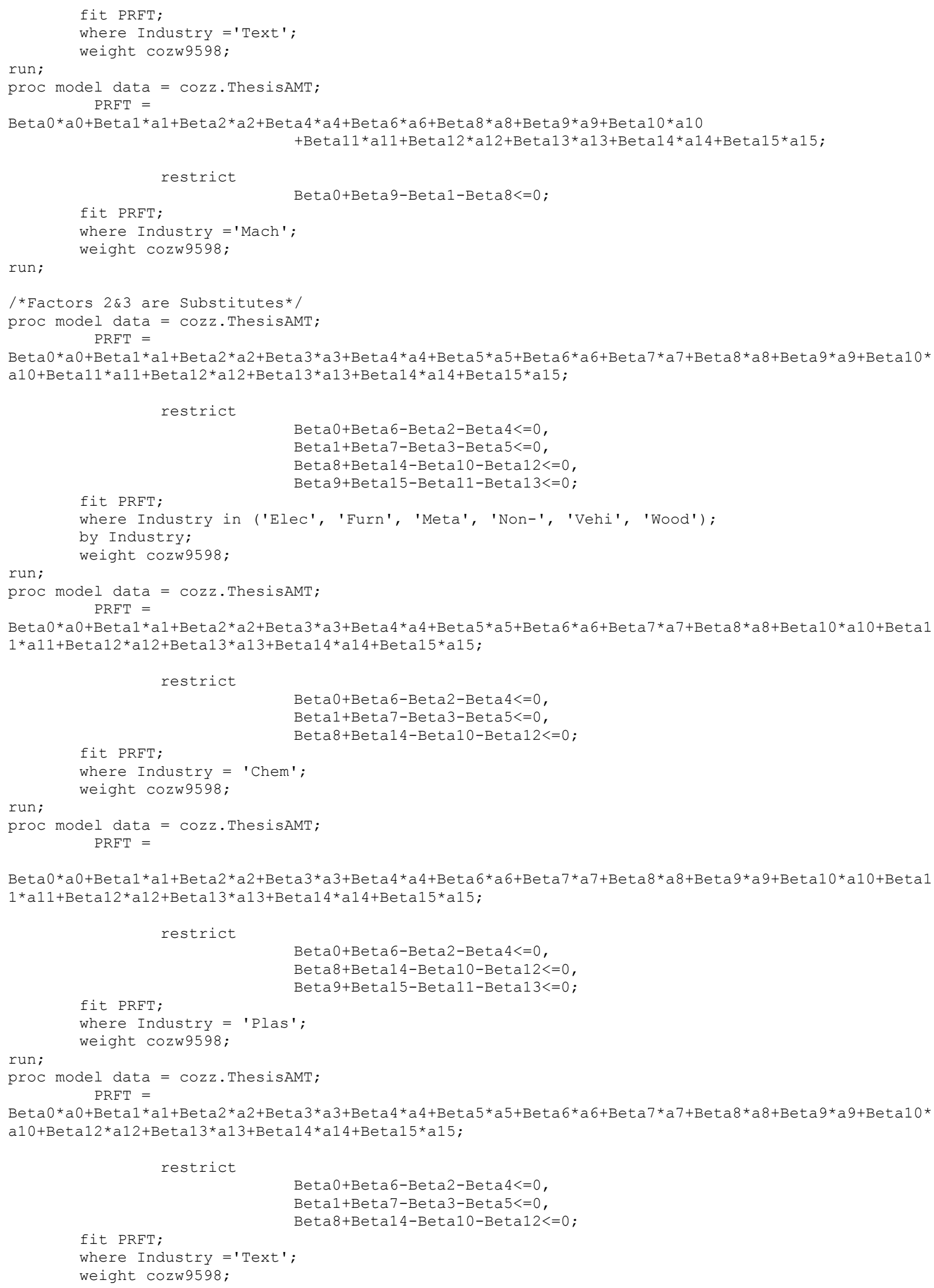


run;

proc model data = cozz. ThesisAMT;

PRFT $=$

Beta $0 * a 0+B e t a 1 * a 1+B e t a 2 * a 2+B e t a 4 * a 4+B e t a 6 * a 6+B e t a 8 * a 8+B e t a 9 * a 9+\operatorname{Beta} 10 * a 10$

+Beta11*a11+Beta12*a12+Beta13*a13+Beta14*a14+Beta15*a15;

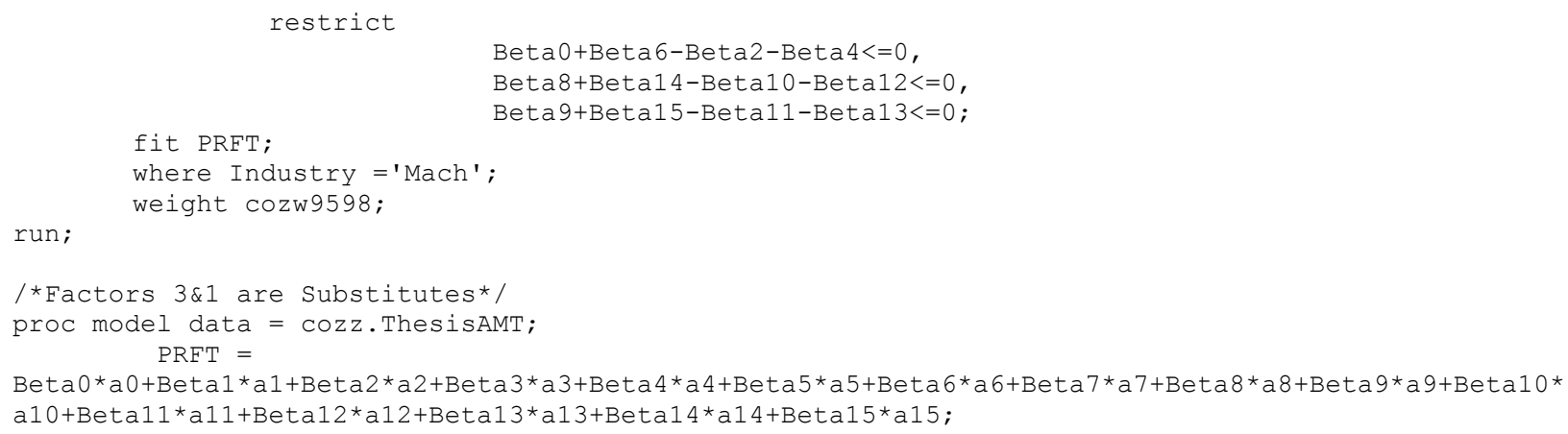




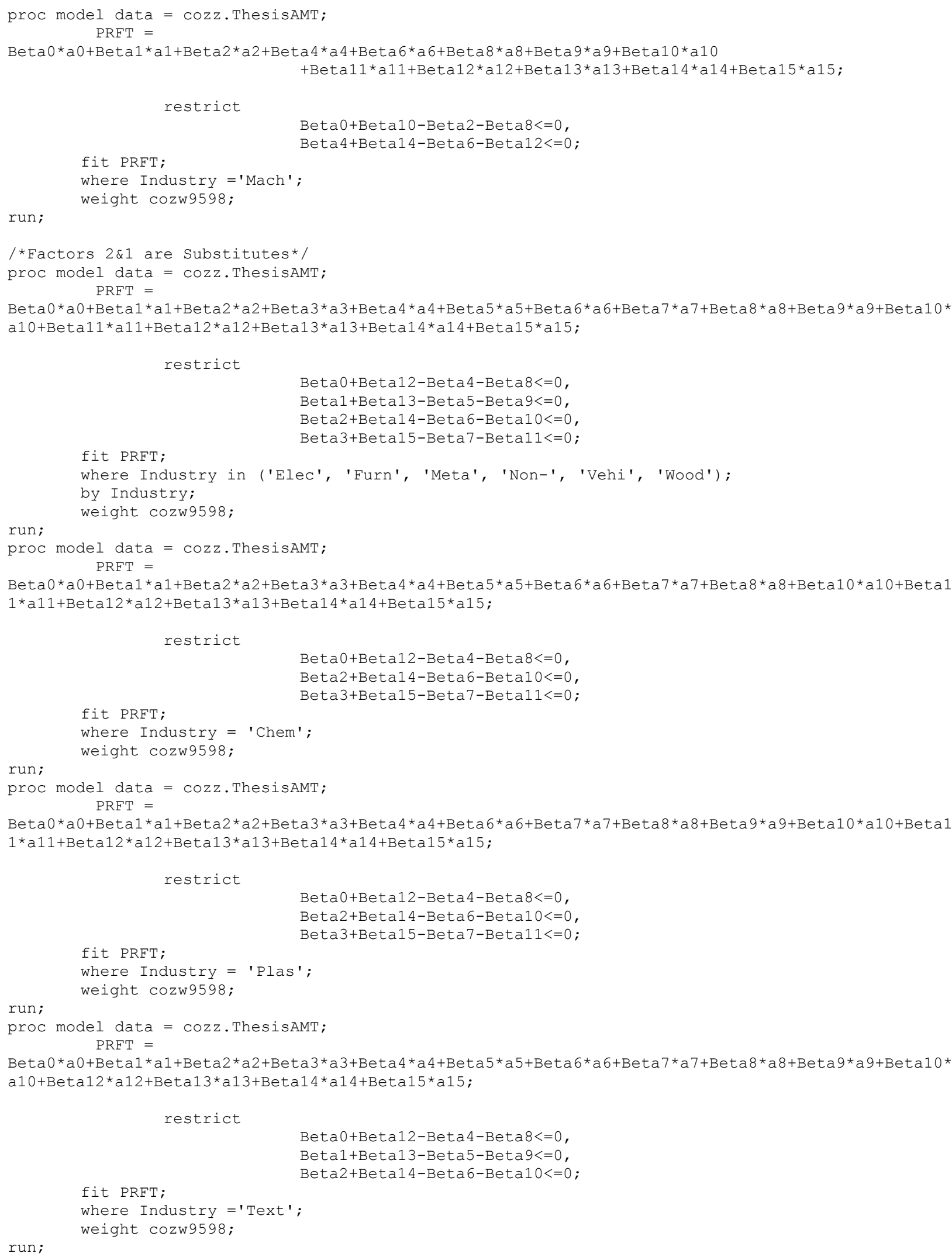




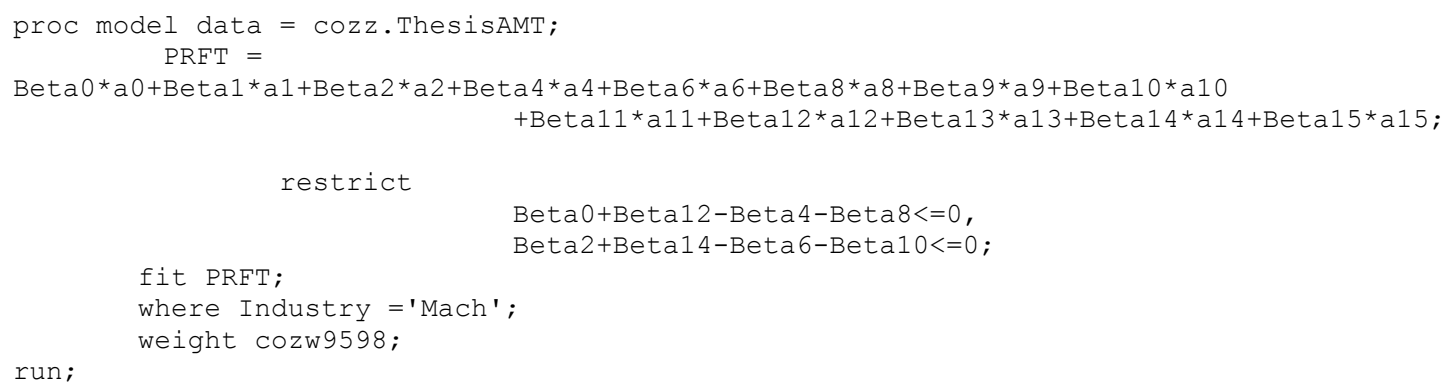

\section{F5 Sources of Implementation Support OLS Regression - Medium Plants}

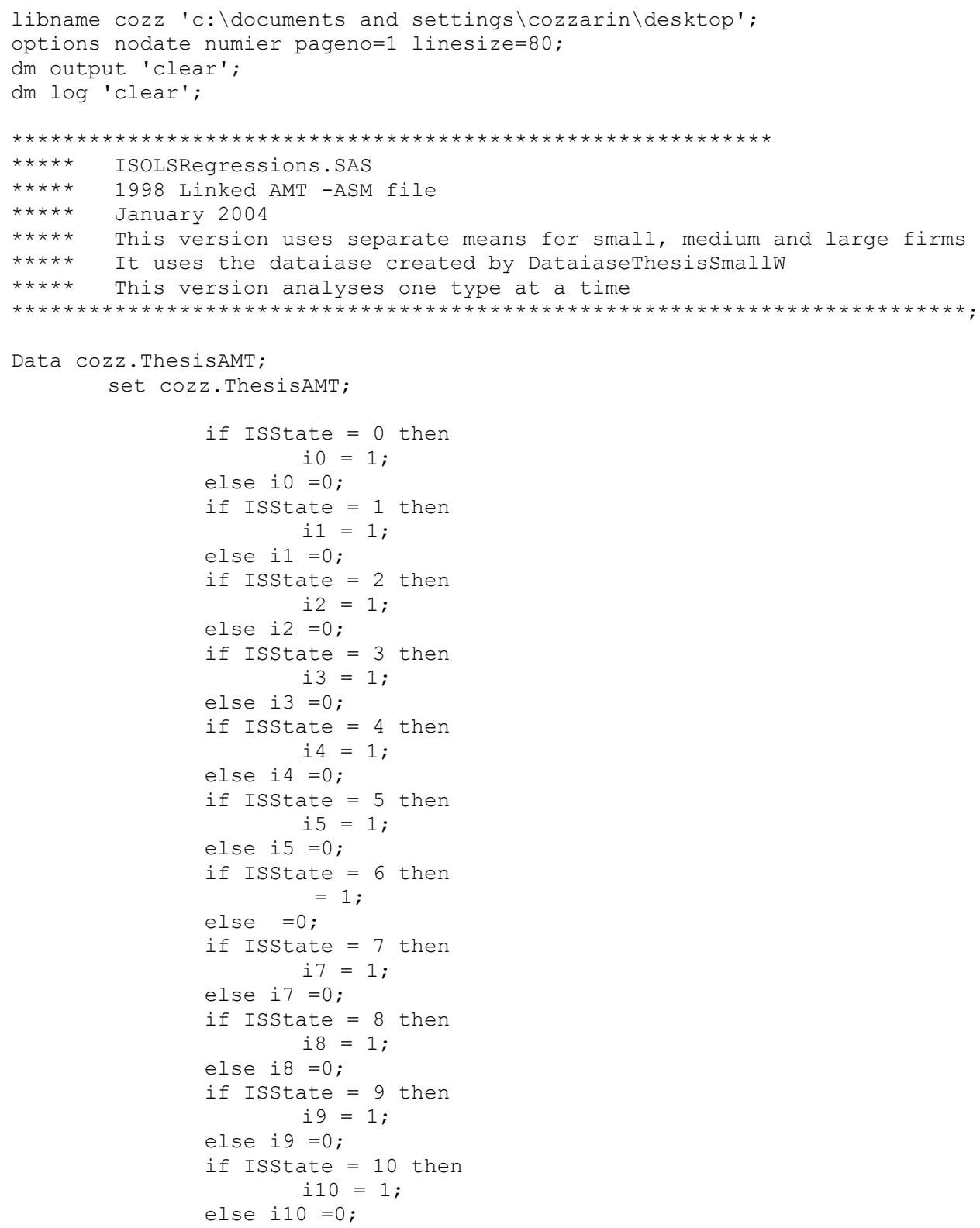


run;

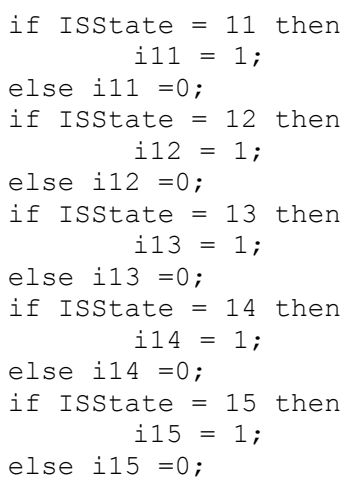




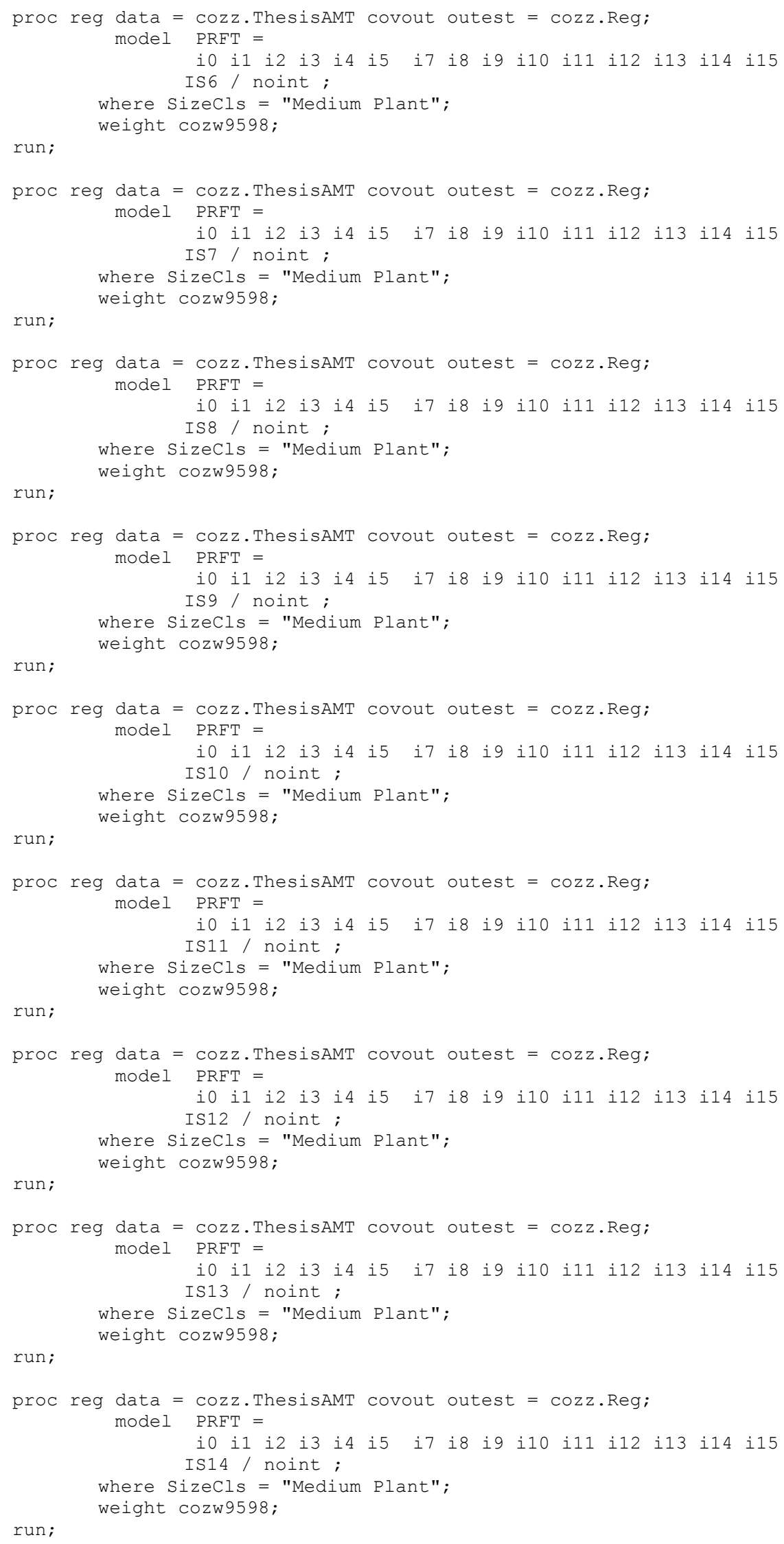




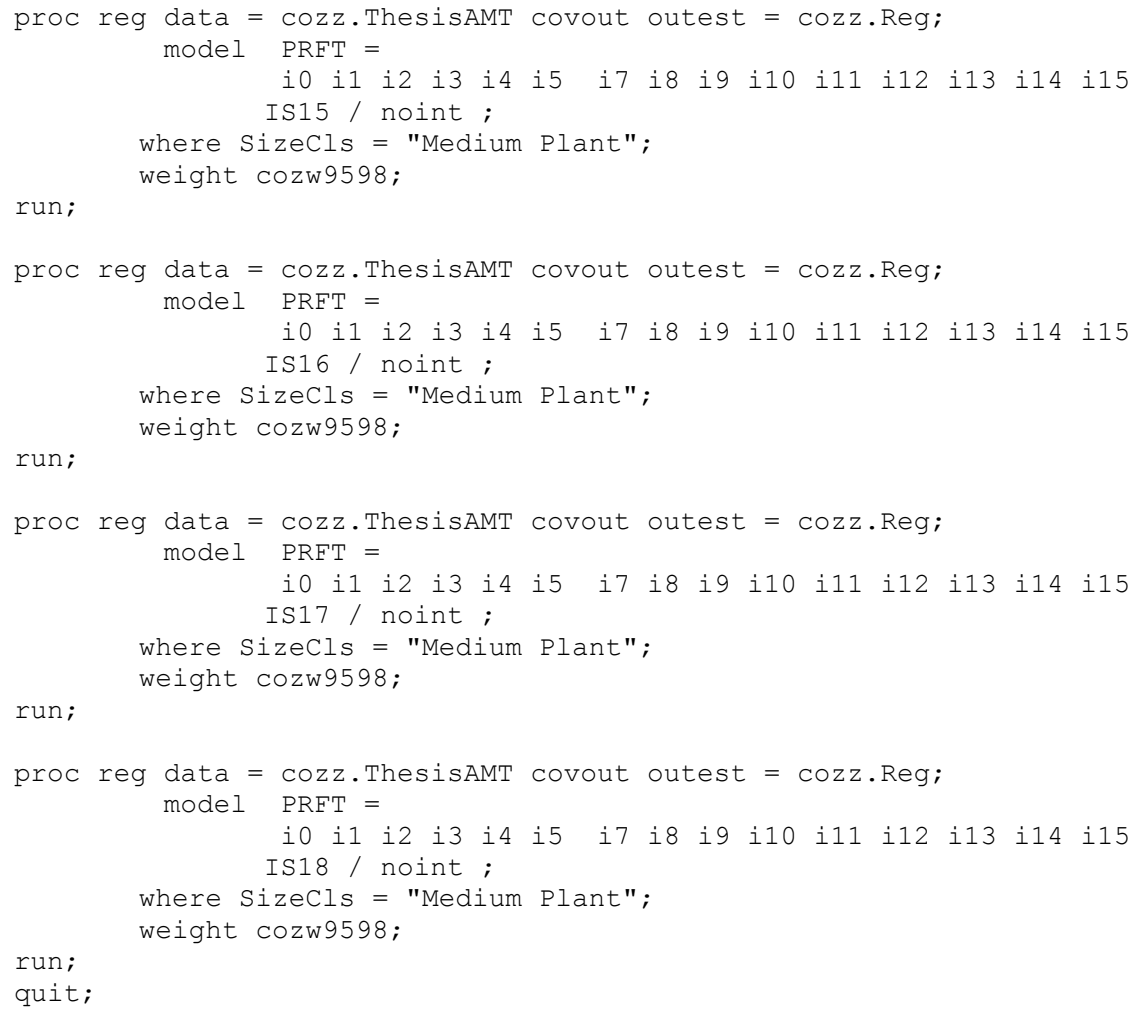

\section{F6 Perceived Benefits of AMT OLS Regression - Wood Industry}

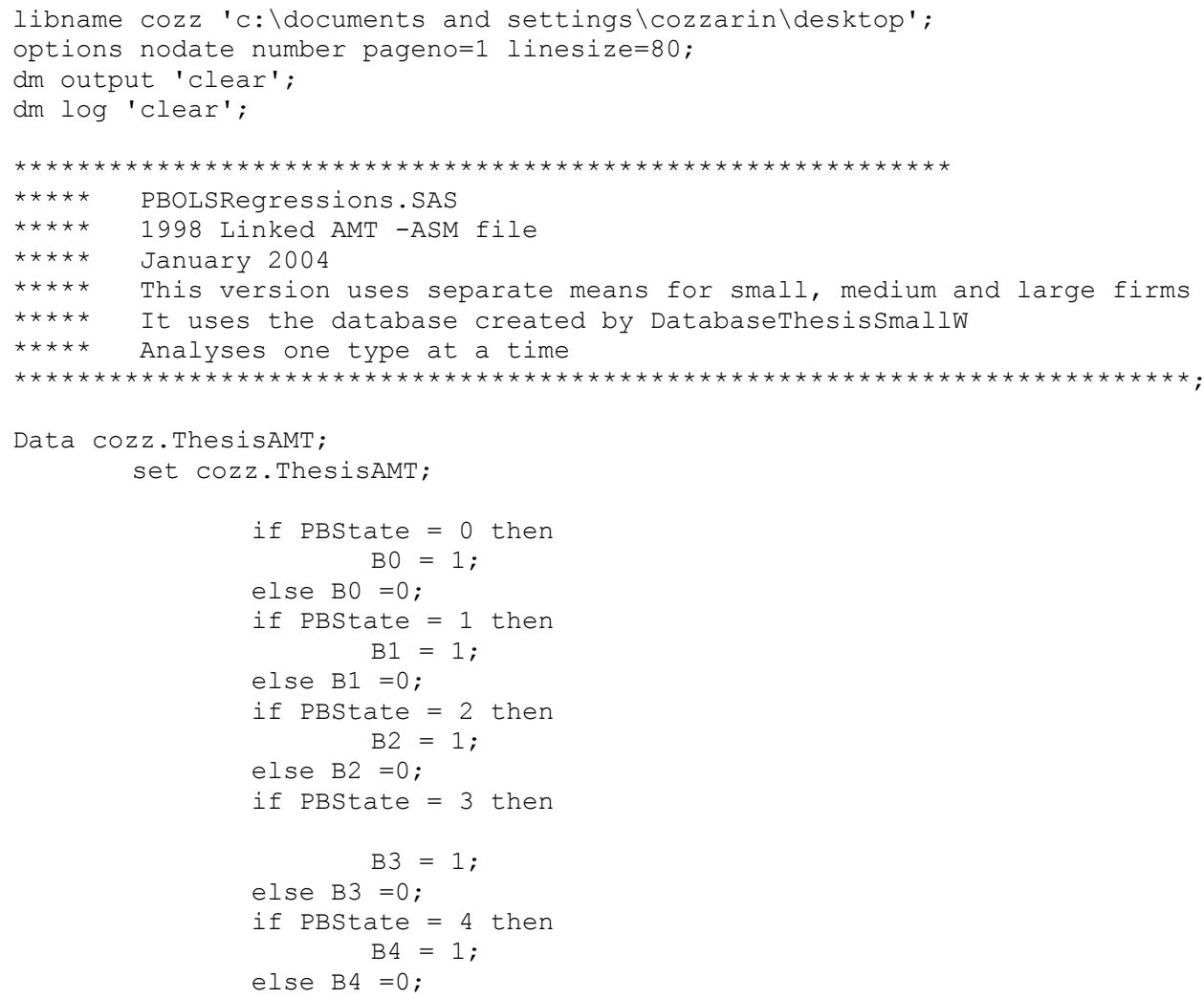




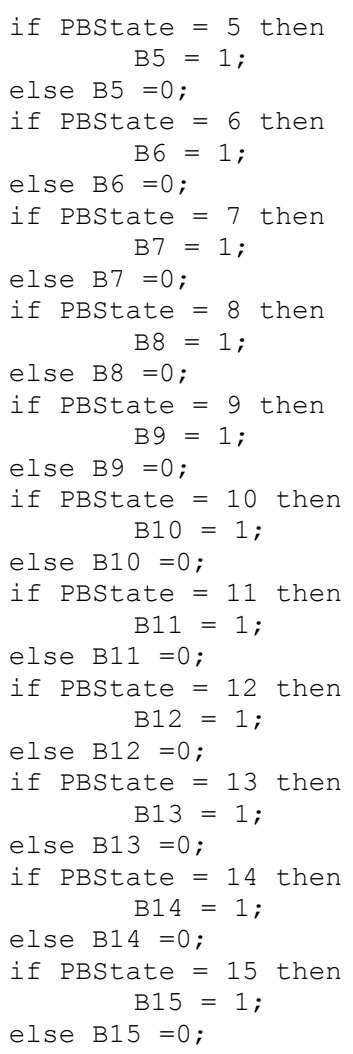

run;

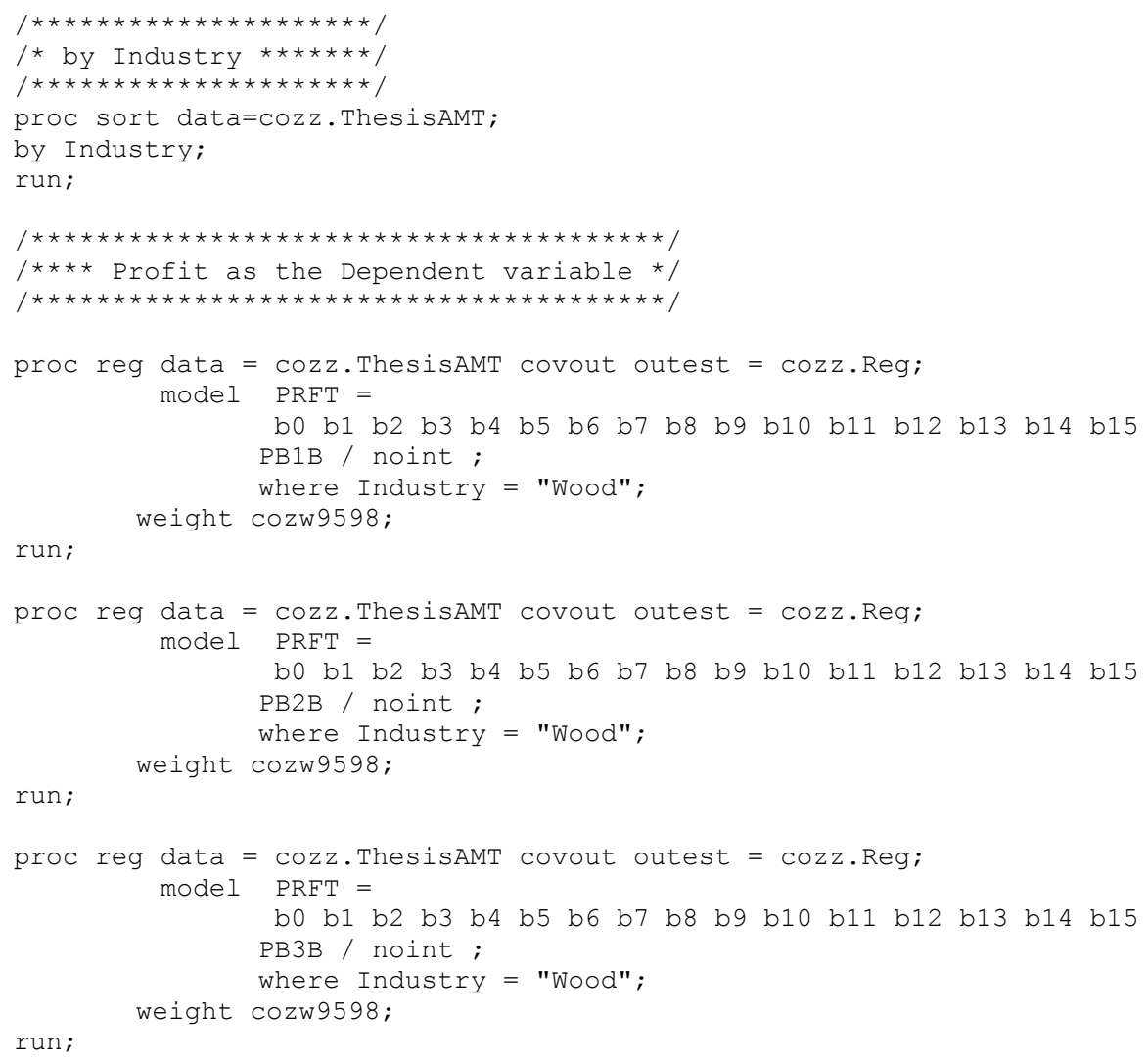

run; 


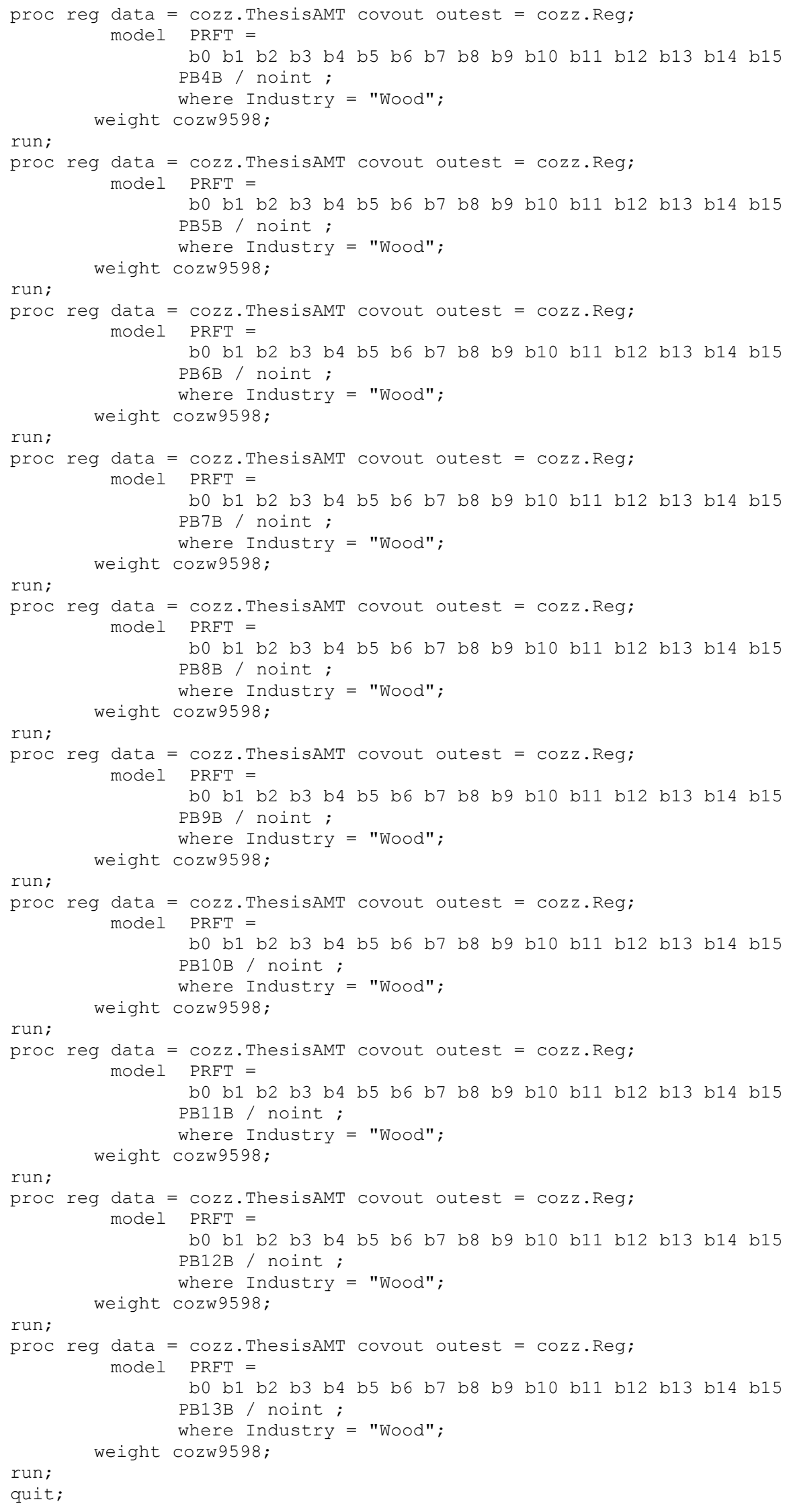

